\title{
Acetamide Herbicides and Their Degradation Products in Ground Water and Surface Water of the United States, 1993-2003
}

By Elisabeth A. Scribner, Julie E. Dietze, and E. Michael Thurman

Data Series 88 


\title{
U.S. Department of the Interior Gale A. Norton, Secretary
}

\author{
U.S. Geological Survey \\ Charles G. Groat, Director
}

\section{U.S. Geological Survey, Reston, Virginia: 2004}

\author{
For sale by U.S. Geological Survey, Information Services \\ Box 25286, Denver Federal Center \\ Denver, CO 80225 \\ For more information about the USGS and its products: \\ Telephone: 1-888-ASK-USGS \\ World Wide Web: http://www.usgs.gov/
}

Any use of trade, product, or firm names in this publication is for descriptive purposes only and does not imply endorsement by the U.S. Government.

Although this report is in the public domain, permission must be secured from the individual copyright owners to reproduce any copyrighted materials contained within this report.

Suggested citation:

Scribner, E.A., Dietze, J.E., and Thurman, E.M., 2004, Acetamide herbicides and their degradation products in ground water and surface water of the United States, 1993-2003: U.S. Geological Survey Data Series 88, 252 p. 


\section{Contents}

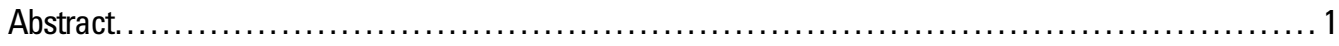

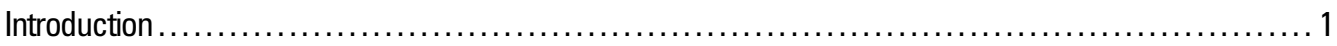

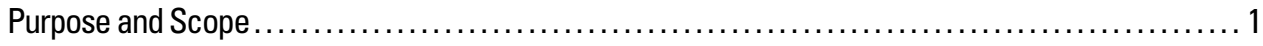

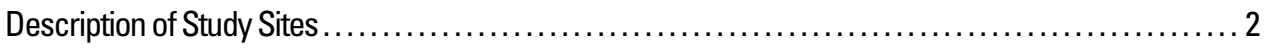

Description of Acetamide Herbicides and Their Degradation Products ......................... 3

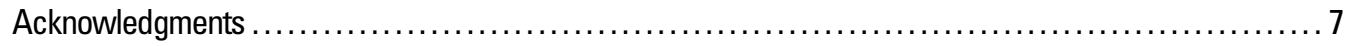

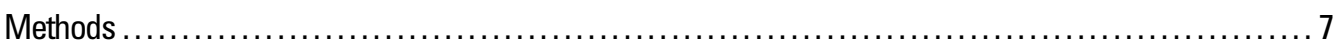

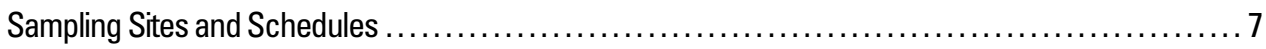

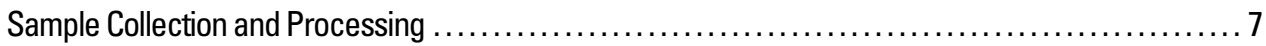

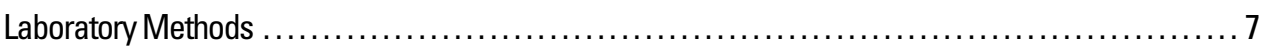

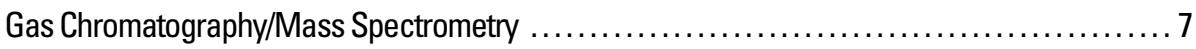

High-Performance Liquid Chromatography/Diode Array Detection ...................... 9

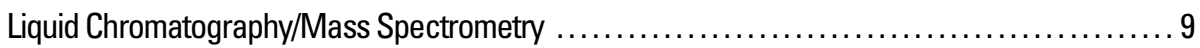

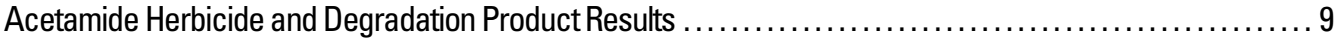

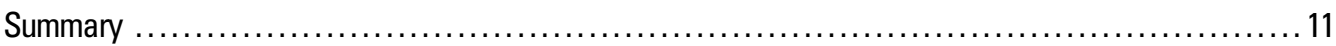

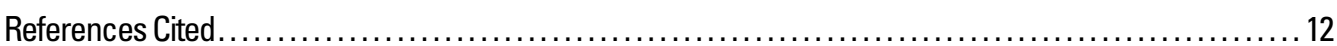

\section{Figures}

1. Location of area studied as part of the U.S. Geological Survey's Midcontinent Agricultural

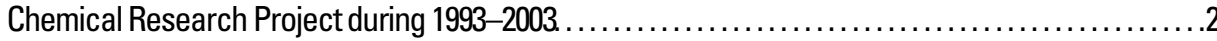

2. Location of streamflow-gaging stations where samples were collected for study of acetamide herbicides and their degradation products as part of the U.S. Geological Survey's National

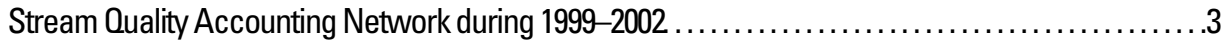

3. Location of National Water-Quality Assessment study units where samples were collected for study of acetamide herbicides and their degradation products during $1996-2003 \ldots \ldots \ldots \ldots \ldots \ldots$

4. Chemical structures and pathways for degradation of acetochlor, alachlor, dimethenamid,

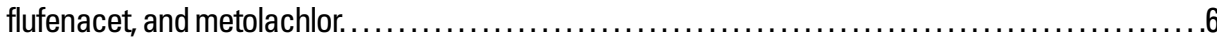

5. Percentage of detections for acetamide herbicides and their degradation products determined by laboratory methods in ground-water samples collected for selected U.S. Geological Survey studies, 1994-2002.

6. Percentage of detections for acetamide herbicides and their degradation products determined by laboratory methods in surface-water samples collected for selected U.S. Geological Survey studies, 1993-2003.

\section{Tables}

1. Acetamide herbicide use for crop production in the United States during $1997 \ldots \ldots \ldots \ldots \ldots \ldots . \ldots$

2. Methods of analysis for acetamide herbicides and their degradation products . ...............8 
3. Statistical summary of concentrations of acetamide herbicides and their degradation products detected in ground-water samples collected as part of selected U.S. Geological Survey studies, 1994-2002.

4. Statistical summary of concentrations of acetamide herbicides and degradation products detected in surface-water samples collected as part of selected U.S. Geological Survey studies, 1993-2003. .

5. Analytical results for selected acetamide herbicides and their degradation products, ethanesulfonic acid, oxanilic acid, and sulfinyl acetic acid, in ground-water samples, 1994-2002 .. 16

6. Analytical results for selected acetamide herbicides and their degradation products, ethanesulfonic acid, oxanilic acid, and sulfinyl acetic acid, in surface-water samples, 1993-2003. 117

\section{Conversion Factors, Abbreviated Water-Quality Units, and Datum}

\begin{tabular}{lll}
\hline Multiply & \multicolumn{1}{c}{ By } & To obtain \\
\hline acre & 0.4047 & hectare (ha) \\
foot $(\mathrm{ft})$ & 0.3048 & meter $(\mathrm{m})$ \\
hectare $(\mathrm{ha})$ & 2.471 & acre \\
kilogram $(\mathrm{kg})$ & 2.205 & pound $(\mathrm{lb})$ \\
kilogram per hectare per year & 0.8922 & pound per acre per year \\
$\quad[(\mathrm{kg} / \mathrm{ha}) / \mathrm{yr}]$ & & [(lb/acre)/yr] \\
liter $(\mathrm{L})$ & $2.642 \times 10^{-1}$ & gallon $(\mathrm{gal})$ \\
meter $(\mathrm{m})$ & 3.281 & foot $(\mathrm{ft})$ \\
microliter $(\mu \mathrm{L})$ & $2.642 \times 10^{-7}$ & gallon $(\mathrm{gal})$ \\
micrometer $(\mu \mathrm{m})$ & $3.937 \times 10^{-2}$ & inch (in.) \\
millimeter $(\mathrm{mm})$ & 0.03937 & inch (in.) \\
pound $(\mathrm{lb})$ & 453.6 & gram $(\mathrm{g})$ \\
pound per acre $(\mathrm{lb} / \mathrm{acre})$ & 1.121 & kilogram per hectare $(\mathrm{kg} / \mathrm{ha})$ \\
\hline
\end{tabular}

Temperature in degrees Celsius $\left({ }^{\circ} \mathrm{C}\right)$ may be converted to degrees Fahrenheit $\left({ }^{\circ} \mathrm{F}\right)$ as follows:

$$
\begin{aligned}
& { }^{\circ} \mathrm{F}=\left(1.8 \mathrm{x}^{\circ} \mathrm{C}\right)+32 \\
& { }^{\circ} \mathrm{C}=\left({ }^{\circ} \mathrm{F}-32\right) / 1.8
\end{aligned}
$$

\section{Abbreviated Water-Quality Units}

microgram $(\mu \mathrm{g})$

microgram per liter $(\mu \mathrm{g} / \mathrm{L})$

milligram per liter (mg/L)

milliliter (mL)

\section{Datum}

Horizontal coordinate information is referenced to the North American Datum of 1983 (NAD 83). 


\title{
Acetamide Herbicides and Their Degradation Products in Ground Water and Surface Water of the United States, 1993-2003
}

\author{
By Elisabeth A. Scribner, Julie E. Dietze, and E. Michael Thurman
}

\section{Abstract}

During 1993 through 2003, the U.S. Geological Survey conducted a number of studies to investigate and document the occurrence, fate, and transport of acetamide herbicides and their degradation products in ground and surface water. As part of these studies, approximately 5,100 water samples were collected and analyzed for the acetamide parent herbicides acetochlor, alachlor, dimethenamid, flufenacet, and metolachlor and their degradation products ethanesulfonic acid, oxanilic acid, and sulfinyl acetic acid. During this period, various analytical methods were developed to detect and measure concentrations of acetamide herbicides and their degradation products in ground water and surface water. Results showed that the degradation products of acetamide herbicides in ground water were detected more frequently and occurred at higher concentrations than their parent compounds. Further study showed that the acetamide herbicides and their degradation products were detected more frequently in surface water than in ground water. In general, the parent compounds were detected at similar or greater frequencies than the degradation products in surface water. The developed methods and data were valuable for acquiring information about the occurrence, fate, and transport of the herbicides and their degradation products and the importance of analyzing for both parent compounds and their degradate products in water-quality studies.

\section{Introduction}

During the 1990s and early 2000s, the occurrence, fate, and transport of herbicides in ground and surface water became a major water-quality issue throughout the United States.

Numerous studies were completed by various government agencies, including the U.S. Geological Survey (USGS), academic institutions, and chemical manufacturers. The Organic Geochemistry Research Laboratory (OGRL) was established by the USGS in Lawrence, Kansas, to provide new knowledge of herbicides and their degradation products in both agriculturally affected and natural water. Essential analytical methods have been and continue to be developed to detect and measure herbicides and degradation-product concentrations from several major classes of organic compounds, including acetamide herbicides and their degradation products identified in this report in environmental waters.

In understanding the fate and transport of parent compounds, methods for the analysis of herbicides and their degradation products are very important (Meyer and others, 1993; Aga and others, 1994; Ferrer and others, 1997; Zimmerman and Thurman, 1999; Hostetler and Thurman, 2000; Kish and others, 2000; Scribner and others, 2000b; Lee and others, 2001; Thurman and others, 2002; Lee and Strahan, 2003; Vargo and others, 2003). More than 15 journal articles and USGS reports have been published describing the occurrence and fate of acetamide herbicides and their degradation products in the environment (Aga and others, 1996; Field and Thurman, 1996; Kolpin and others, 1996, 1998, 2000, in press; Thurman and others, 1996; Kalkhoff and others, 1998, 2003; Scribner and others, 1998, 2000a, 2003; Graham and others, 1999; Phillips and others, 1999; Tauler and others, 2000; Thurman and Cromwell, 2000; Aga and Thurman, 2001; Zimmerman and others, 2002; Battaglin and others, 2003; Schnoebelen and others, 2003; Mills and others, 2004).

\section{Purpose and Scope}

The purpose of this report is to present results of analyses for acetamide herbicides and their degradation products in more than 5,100 ground- and surface-water samples collected during the 1990s and early 2000s. The samples were collected as part of various ongoing USGS studies being conducted throughout the United States that include the Midcontinent Agricultural Chemical Research Project, the National Stream Quality Accounting Network (NASQAN), several National WaterQuality Assessments (NAWQA), and USGS studies with Federal-State cooperative agencies. The data included in this report were collected from studies funded, in part or whole, by these programs.

The samples were analyzed at the OGRL, and the data are being used to document the occurrence, distribution, and concentrations of five acetamide herbicides, alachlor, acetochlor, dimethenamid, flufenacet, and metolachlor, and two of their degradation products, ethanesulfonic acid (ESA) and oxanilic 
acid (OXA). In 2001, the OGRL began analyzing concentrations of the acetochlor and alachlor degradation product, sulfinyl acetic acid (SAA). This report also includes a description of acetamide herbicides and documents the sample-collection and processing methods in addition to laboratory and analytical methods.

\section{Description of Study Sites}

Water samples were collected and analyzed from four distinct types of studies described as follows:

1. The Midcontinent Agricultural Chemical Research Project (fig. 1) is a study of the occurrence, movement, flux, fate, and effects of agricultural chemicals in 25 States. The study area includes the Midwest Corn Belt, which accounts for about 65 percent of the total harvested cropland and 60 percent of herbicide use in the United States (Buxton, 2000). The Midcontinent Agricultural Chemical Research Project is part of the USGS Toxic Substances Hydrology Program. Studies included in the project have provided the general scientific basis needed to develop agricultural-management practices that protect the quality of the region's water resources (Anderson and others, 1995).
2. National Stream Quality Accounting Network

(NASQAN) is the Nation's only large-scale program that measures the amount of chemicals and sediment that flow past streamflow-gaging stations on large rivers throughout the United States. Data are collected from four major river basins - the Colorado, Columbia, Mississippi, and Rio Grande (fig. 2). These data are important in characterizing variations in chemical and sediment concentrations that occur throughout the year, such as during periods of high and low streamflow, during different seasons, and under different conditions of land use (Hooper and others, 1997).

3. The National Water-Quality Assessment (NAWQA) Program includes investigations of 59 study units (fig. 3) that provide the framework for national and regional status and trends in the quality of the Nation's groundand surface-water resources (U.S. Geological Survey, 2003). Data for acetamide herbicides and their degradation products from 11 of the study units are included in this report.

4. The USGS cooperates with various Federal-State agencies to investigate and study aquatic ecosystems. In some cases, these cooperative studies evaluate agricultural contamination of ground and surface water from nonpoint and distributed point sources. For

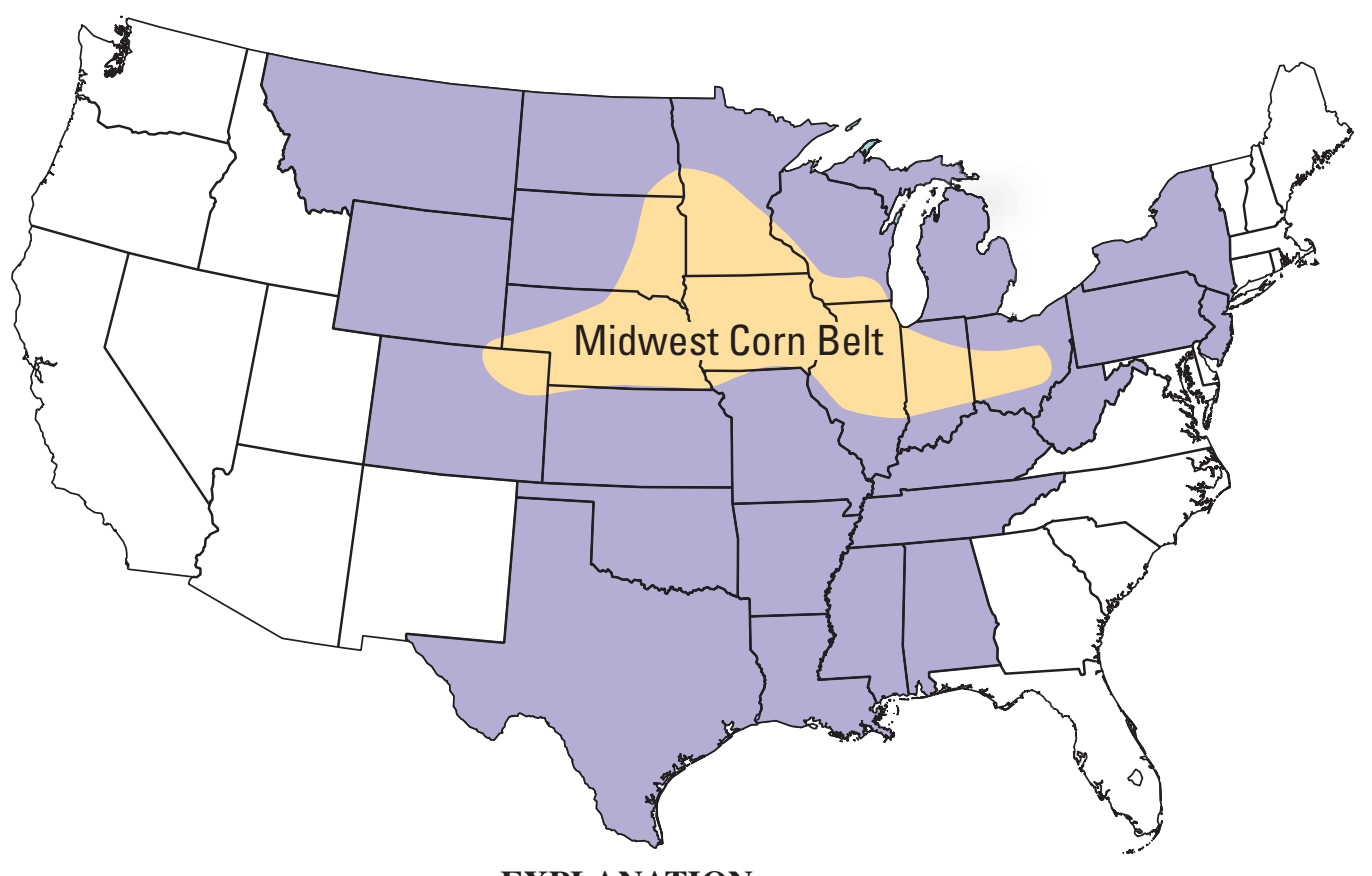

EXPLANATION

Study area of Midcontinent Agricultural

Chemical Research Project

Figure 1. Location of area studied as part of the U.S. Geological Survey's Midcontinent Agricultural Chemical Research Project during 1993-2003. 


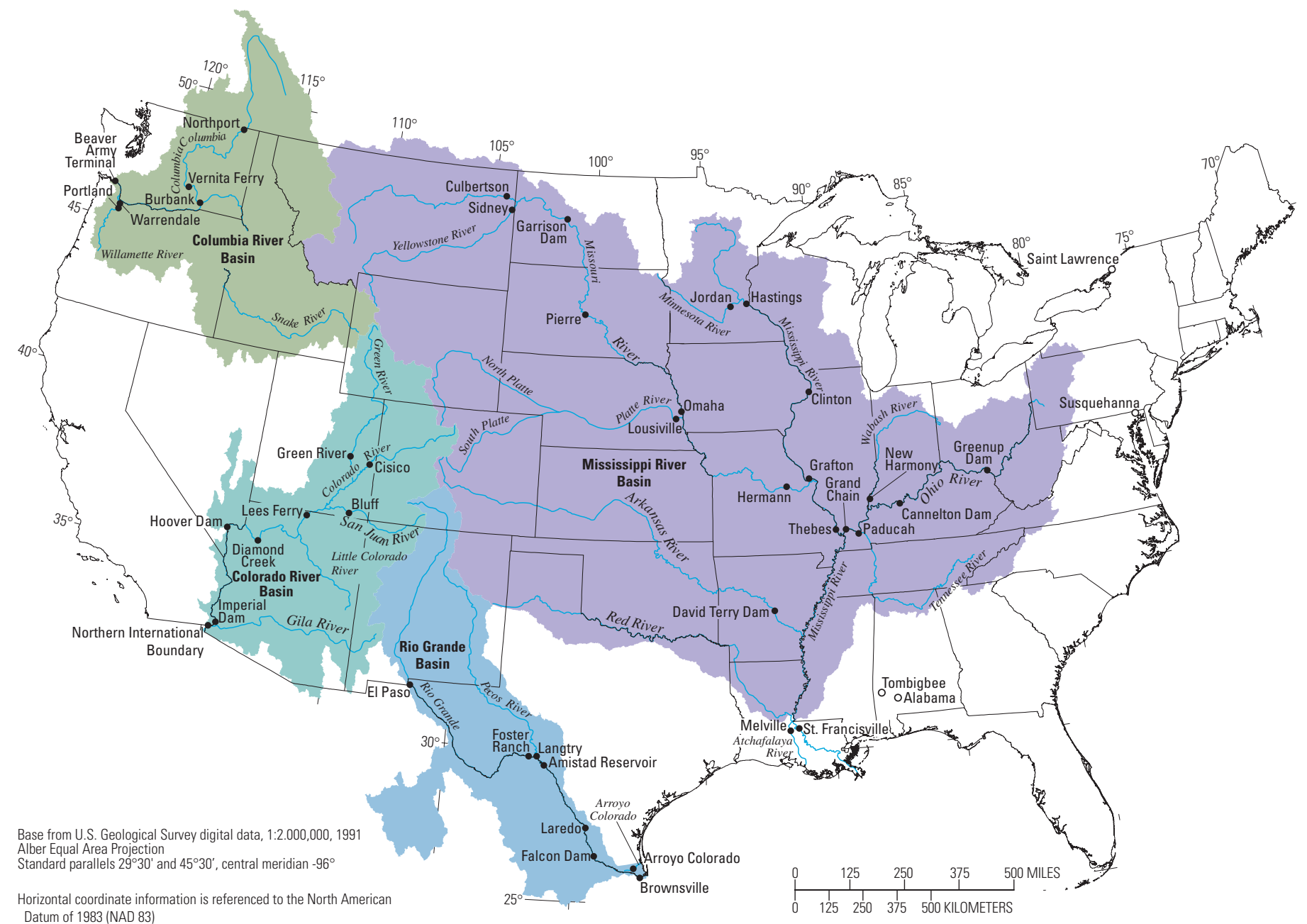

EXPLANATION

Tombigbee ${ }_{\circ}$ Inactive station

Brownsville. Active station

Figure 2. Location of streamflow-gaging stations where samples were collected for study of acetamide herbicides and their degradation products as part of the U.S. Geological Survey's National Stream Quality Accounting Network during 1999-2002.

example, herbicide-related ground- and surface-waterquality data were collected in 1999 in cooperation with the New York State Department of Environmental Conservation (Phillips and others, 2000; Eckhardt and others, 2001).

\section{Description of Acetamide Herbicides and Their Degradation Products}

The acetamide herbicides, acetochlor, alachlor, dimethenamid, flufenacet, and metolachlor, constitute a major class of herbicides used throughout the United States. As shown in table 1, during 1997 the acetamide herbicides accounted for more than 121 million pounds applied in the United States for weed control on crops (Gianessi and Marcelli, 2000). Flufenacet has been used only since 1998; therefore, usage data for flufenacet are not included in table 1.

Acetamide herbicides and their degradation products are just now being realized as important to agricultural chemistry because of their solubility and ease of transport. At this time (2003), they are thought to be of low toxicity (Yokley and others, 2002). Figure 4 shows the structures and degradation 


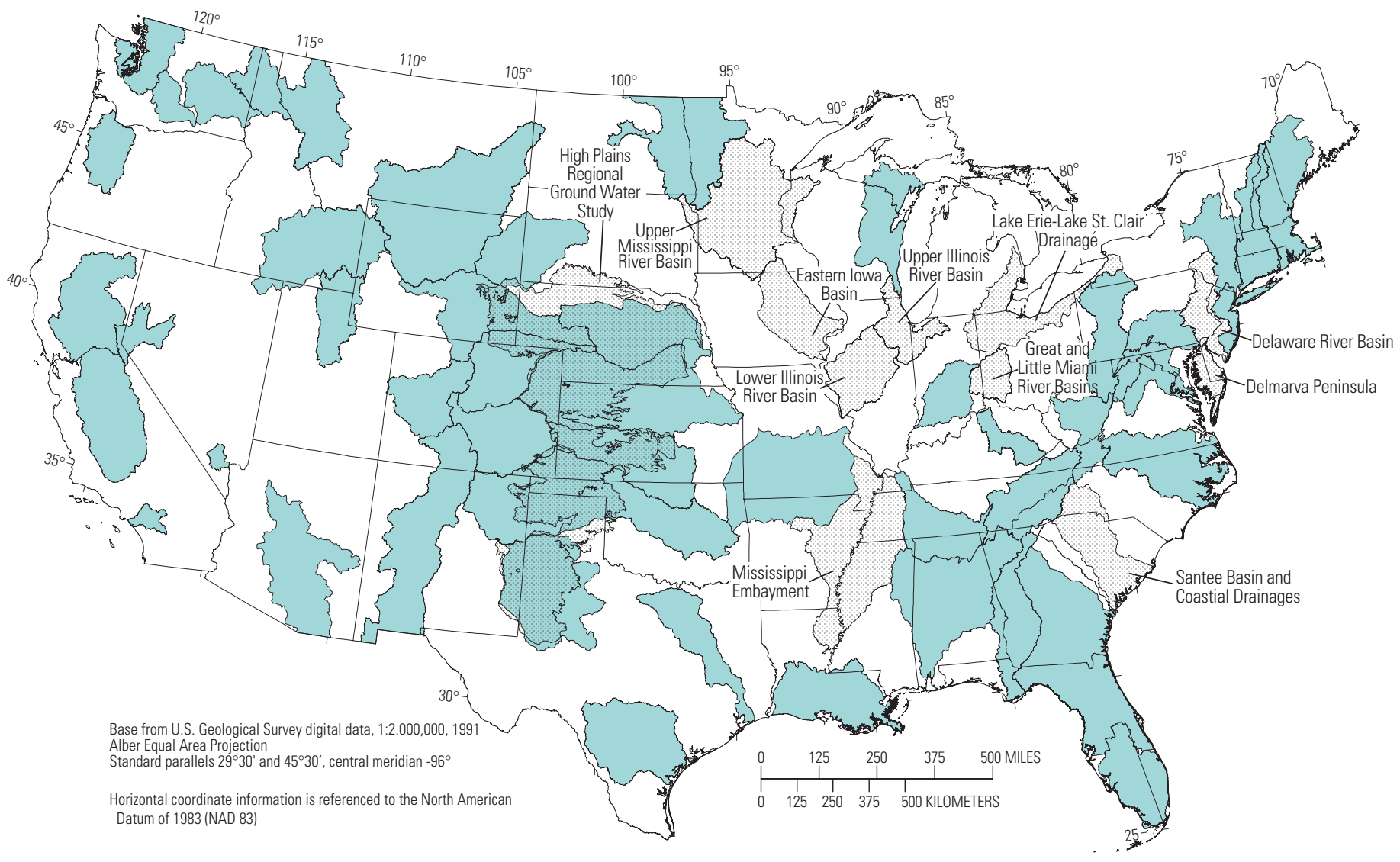

EXPLANATION

Study unit

Study unit for which data are included in this report

Figure 3. Location of National Water-Quality Assessment study units where samples were collected for study of acetamide herbicides and their degradation products during 1996-2003.

pathways of the acetamide parent compounds to ESA, OXA, and SAA. As shown, the parent compounds acetochlor and alachlor degrade to SAA then to ESA. Acetochlor and alachlor also degrade directly to OXA. Dimethenamid, flufenacet, and metolachlor degrade to ESA and OXA.

Acetochlor has a conditional registration that was granted in 1994 to a partnership composed of Zeneca Agricultural Products (Wilmington, Delaware) and Monsanto Company (St. Louis, Missouri; Meister, 2000). In November 2000, Dow AgroSciences LLC (Indianapolis, Indiana) purchased the acetochlor product line from Zeneca Limited (SeedQuest, 2000). As a preemergent herbicide, acetochlor is rapidly replacing alachlor. It is used for the control of most annual grasses and certain broadleaf weeds in corn. It has a solubility in water of
$223 \mathrm{mg} / \mathrm{L}$ at $25^{\circ} \mathrm{C}$ and generally provides 8 to 12 weeks of weed control, depending on soil type and weather conditions (Meister, 2000). Common herbicides that contain acetochlor are Guardian ${ }^{\circledR}$, Harness ${ }^{\circledR}$ (Monsanto Company), and Surpass ${ }^{\circledR}$ (Zeneca Agricultural Products/Dow AgroSciences LLC).

Alachlor is a restricted-use herbicide, which means it may be purchased and used only by certified applicators. Alachlor was developed in 1952 by Monsanto Company, first reported in 1966, and then commercialized as Lasso ${ }^{\circledR}$ in 1969 (Vencill, 2002). Alachlor is used mostly to control annual grasses and broadleaf weeds in corn, sorghum, and soybeans as well as dry beans, dry peas, peanuts, and sweet corn. Alachlor breaks down rapidly in natural water primarily due to the action of 
Table 1. Acetamide herbicide use for crop production in the United States during 1997.

[All values given in pounds. Source of data: Gianessi and Marcelli (2000). --, not available]

\begin{tabular}{|c|c|c|c|c|c|}
\hline Crop & Acetochlor & Alachlor & Dimethenamid & Metolachlor & Grand total \\
\hline Cabbage & -- & -- & -- & 19,995 & \\
\hline Celery & -- & -- & -- & 1,873 & \\
\hline Cotton & -- & -- & -- & 498,189 & \\
\hline Dry beans & -- & 281,477 & 67,350 & 395,098 & \\
\hline Green beans & -- & -- & -- & 212,298 & \\
\hline Green peas & -- & -- & -- & 23,081 & \\
\hline Hot peppers & -- & -- & -- & 18,475 & \\
\hline Onions & -- & -- & -- & 35,037 & \\
\hline Sod & -- & -- & -- & 2,072 & \\
\hline Sorghum & -- & $1,449,463$ & 416,937 & $4,599,824$ & \\
\hline Soybeans & -- & $7,233,638$ & 938,327 & $9,437,847$ & \\
\hline Spinach & -- & -- & -- & 9,529 & \\
\hline Sweet corn & -- & 319,254 & 94,964 & 542,577 & \\
\hline Sweet peppers & -- & -- & -- & 12,140 & \\
\hline Tomatoes & -- & -- & -- & 16,459 & \\
\hline Watermelons & -- & -- & -- & 4,193 & \\
\hline
\end{tabular}

microorganisms. It has a solubility in water of $242 \mathrm{mg} / \mathrm{L}$ at $25^{\circ} \mathrm{C}$ and is absorbed by germinating shoots and works by interfering with a plant's ability to produce protein and by interfering with root elongation. Common herbicides that contain alachlor include Lasso $®$, Freedom $\AA$, and Lariat $\AA$ (Meister, 2000).

Dimethenamid was registered for corn in March 1993 and for soybeans in March 1994 by Sandoz Agro, Inc. (Des Plaines, Illinois). It is presently marketed by BASF (Mt. Olive, New Jersey) as the active ingredient in the herbicides Frontier $\circledast$ and Guardsman ${ }^{\circledR}$ (Meister, 2000). In addition to control of broadleaf and annual weeds in corn and soybeans, dimethenamid also is registered for use on dry beans, peanuts, sorghum, and sweet corn. Dimethenamid has a solubility in water of $1,174 \mathrm{mg} / \mathrm{L}$ at $25^{\circ} \mathrm{C}$ and has a half-life of 1 to 2 weeks in the southern United States and 5 to 6 weeks in the northern United States (Vencill, 2002).

Flufenacet, initially synthesized by Bayer Corporation in 1988, received conditional registration by USEPA in April 1998 (U.S. Environmental Protection Agency, 1998). Flufenacet is in the thiadiazole chemical family but is also an acetamide. It is one of the active ingredients in the herbicides
Axiom $\AA$, Define $\AA$, and Epic $\AA$ from Bayer Corporation (Pittsburgh, Pennsylvania). Flufenacet controls most annual grasses and certain small-seeded broadleaf weeds. It is used for the corn and soybean markets under the trade name Axiom ${ }^{\circledR}$. Application rate of flufenacet ranges from 0.6 to $1.1(\mathrm{~kg} / \mathrm{ha}) / \mathrm{yr}$. It has a solubility of $56 \mathrm{mg} / \mathrm{L}$ at $25^{\circ} \mathrm{C}$ in water (Vencill, 2002). Usage data presently are not available.

Metolachlor was synthesized in 1972 and made available commercially for use on corn in 1977 by Ciba Geigy Ltd (now Syngenta, Basel, Switzerland). Metolachlor is a preemergent selective herbicide used to control certain broadleaf and annual grassy weeds primarily in crops. Its solubility is $488 \mathrm{mg} / \mathrm{L}$ at $20{ }^{\circ} \mathrm{C}$ (Vencill, 2002). Soil studies of metolachlor oxidation have reported that the major degradation products include metolachlor ESA and OXA (Phillips and others, 1999). Commonly used commercial products that contain metolachlor are Dual Magnum ${ }^{\circledR}$ and Bicep II Magnum $\AA$. $S$-metolachlor was registered for use in the United States on March 14, 1977. $S$ metolachlor is physically and chemically equivalent to metolachlor but is more active at the site of application in susceptible plants and allows for lower use rates (Vencill, 2002). 


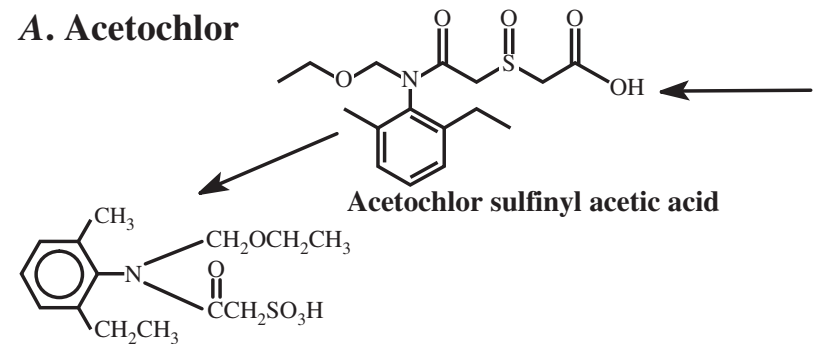

Acetochlor ethanesulfonic acid

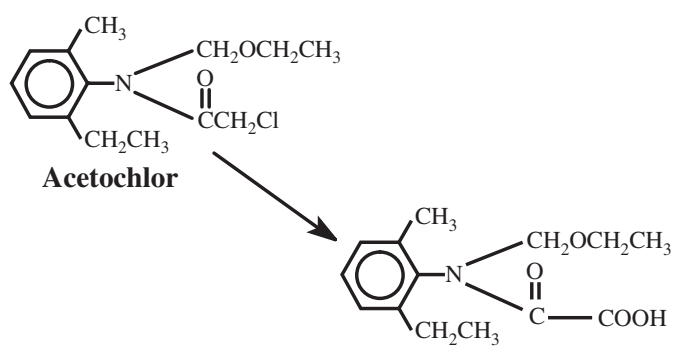

Acetochlor oxanilic acid

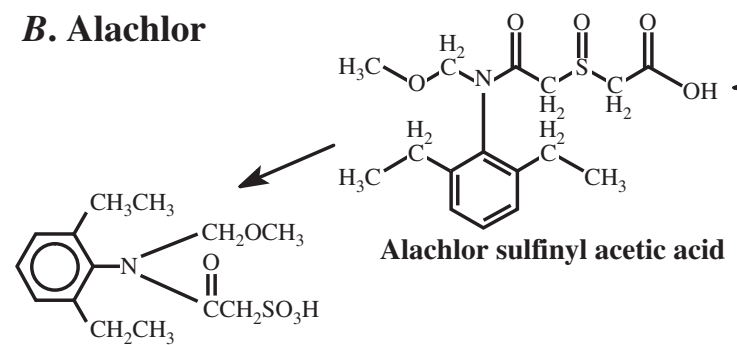

Alachlor ethanesulfonic acid

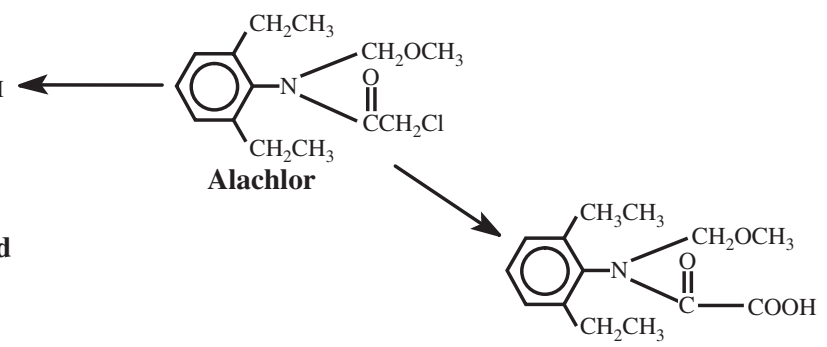

Alachlor oxanilic acid

\section{Dimethenamid}
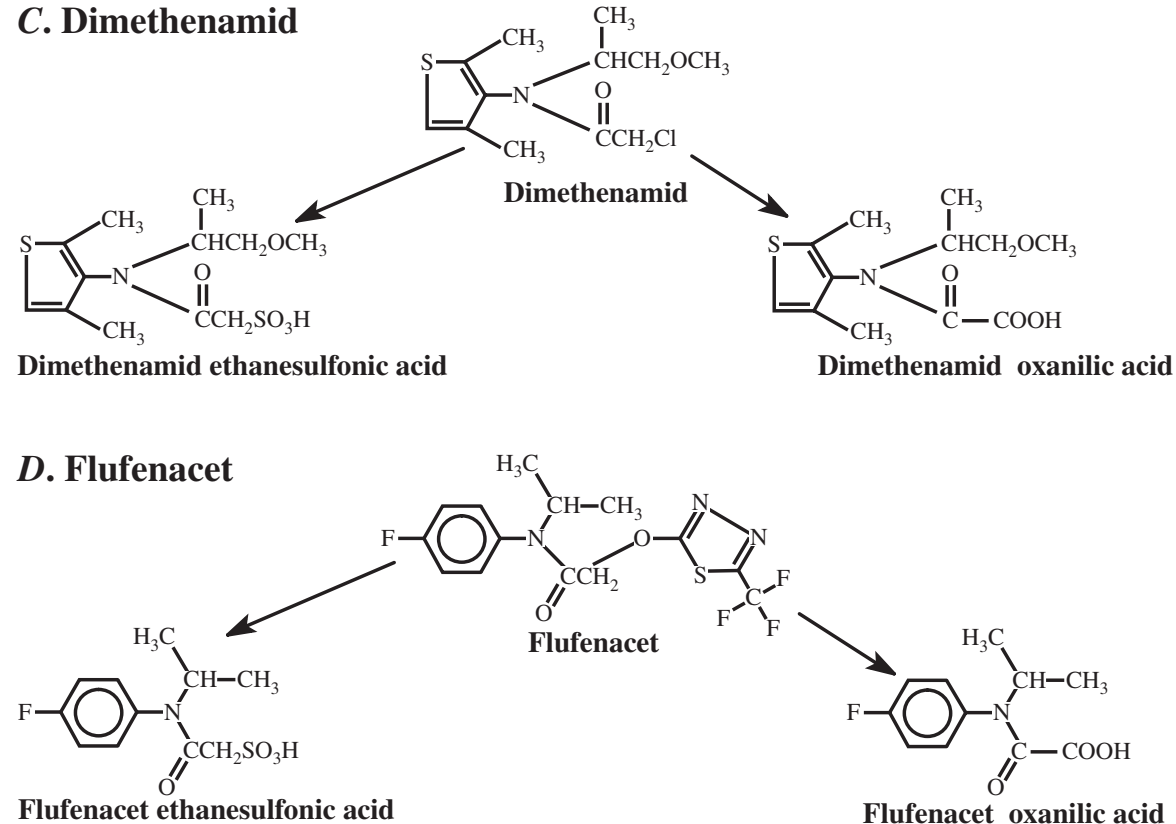

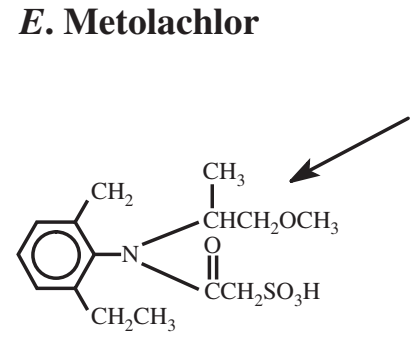

Metolachlor ethanesulfonic acid

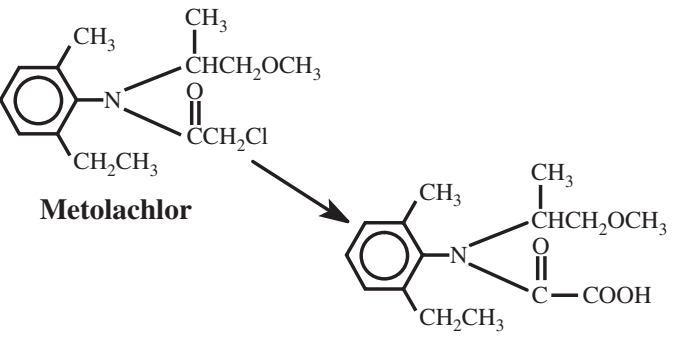

Metolachlor oxanilic acid

Figure 4. Chemical structures and pathways for degradation of acetochlor, alachlor, dimethenamid, flufenacet, and metolachlor. 


\section{Acknowledgments}

The authors wish to acknowledge the USGS scientists and field technicians who provided essential support to these projects by identifying candidate stream sites across the United States and in collecting and processing stream samples. In addition, thanks go to Mike Meyer, Kenneth Hostetler, and Edward Lee (current and former OGRL staff members) for their important contributions in developing the analytical methods used on samples in this report.

\section{Methods}

\section{Sampling Sites and Schedules}

Sampling sites were distributed geographically throughout each study area and represented ground water from wells of various sizes, depths, and open to various aquifers and surface water from various streams and reservoirs. Sampling schedules for the various studies ranged from short-term (about 2 months) synoptic to longer term (multi-year) assessments. Samples for the various studies were collected from 1993 to 2003.

\section{Sample Collection and Processing}

Sampling methods capable of collecting water samples that accurately represented the water-quality characteristics of the ground water or surface water at a given time or location were used. Descriptions of sampling methods used by the USGS to obtain representative ground-water samples are given in Hardy and others (1989) and Wilde and others (1998). Descriptions of sampling methods used for obtaining depthand width-integrated surface-water samples are given in Edwards and Glysson (1988), Ward and Harr (1990), and Wilde and others (1998).

Sample-collection equipment was made of Teflon or stainless-steel material and was free of materials that might leach interfering compounds into water samples or absorb the target analytical compounds from the water samples. Ground-water samples were discharged directly through the filtering device through tubing connected to sample taps at water-supply wells or the discharge outlet on sampling pumps. Water samples from each surface-water site were composited in a single container and filtered through a nominal $0.7-\mu \mathrm{m}$ glass-fiber filter using a peristaltic pump. Filters were preconditioned with about $200 \mathrm{~mL}$ of sample water prior to collection of the sample. The filtrate for analysis was collected in baked $125-\mathrm{mL}$ amber glass bottles with Teflon-lined lids. Samples were chilled immediately and shipped to the USGS OGRL in Lawrence, Kansas, and received within 3 days of collection. Herbicide bottles received at the laboratory were logged in, assigned identification numbers, and refrigerated at about $4{ }^{\circ} \mathrm{C}$ until extracted and analyzed.

\section{Laboratory Methods}

Analytical methods for the acetamide herbicide compounds were developed at the USGS OGRL in Lawrence (Scribner and others, 2000b). They include solid-phase extraction (SPE), with gas chromatography/mass spectrometry (GC/MS), high-performance liquid chromatography with diode-array detection (HPLC/DAD), and liquid chromatography/mass spectrometry (LC/MS). The HPLC/DAD method was replaced with the LC/MS method in 1999. Methods of analysis and chemical names are listed in table 2 .

\section{Gas Chromatography/Mass Spectrometry}

Acetamide parent herbicide samples analyzed by GC/MS were extracted from $125-\mathrm{mL}$ samples using two different instruments. From 1993 to 1999, SPE methods were prepared using the millilab workstation (Waters, Milford, Massachusetts). In 1999, the millilab workstation was replaced with the autotrace workstation (Tekmar-Dohrman, Cincinnati, Ohio) for SPE of GC/MS samples. For both SPE methods, compounds were extracted by passing water samples through a preconditioned C-18 cartridge (Waters, Milford, Massachusetts). The adsorbed compounds were removed from the C-18 cartridge with ethyl acetate. The eluate was evaporated further using a Turbovap (Zymark, Palo Alto, California). The eluate then was transferred to a labeled GC autosampler vial for GC/MS analysis (Meyer and others, 1993; Zimmerman and Thurman, 1999; Kish and others, 2000).

Sample eluates were analyzed using a Hewlett Packard model 5890 gas chromatograph (Wilmington, Delaware) and a 5970B mass-selective detector (Palo Alto, California). The sample components were separated, identified, and quantified by injecting an aliquot of the concentrated extract into a highresolution, fused-silica capillary column under selected-ion mode. Compounds eluting from the GC column were identified by comparing their measured ions and retention times to reference ions and retention times obtained by the measurement of control standards under the same conditions used for the water samples. The concentration of each identified compound was measured by relating the MS response of the quantitation ion produced by that compound to the MS response of the quantitation ion produced by a compound that was used as the internal standard. Surrogate compounds, whose concentrations are known in every sample, are measured with the same calibration procedure (Meyer and others, 1993; Zimmerman and Thurman, 1999; Kish and others, 2000).

The reporting limit for this method was $0.05 \mu \mathrm{g} / \mathrm{L}$. Concentrations of acetamide herbicides were reported from 0.05 to $5.0 \mu \mathrm{g} / \mathrm{L}$ without dilution. If the concentration was greater than $10 \mu \mathrm{g} / \mathrm{L}$, the sample was reextracted and reanalyzed (Zimmerman and others, 1999; Kish and others, 2000). 
Table 2. Methods of analysis for acetamide herbicides and their degradation products.

[GC/MS, gas chromatography/mass spectrometry; HPLC/DAD, high-performance liquid chromatography/diode array detection; LC/MS, liquid chromatography/mass spectrometry; X, method used; --, method not used]

\begin{tabular}{|c|c|c|c|c|}
\hline \multirow{2}{*}{ Common name } & \multirow{2}{*}{ Chemical name } & \multicolumn{3}{|c|}{ Method of analysis } \\
\hline & & GC/MS & HPLC/DAD & LC/MS \\
\hline \multicolumn{5}{|c|}{ Acetamide herbicides } \\
\hline Acetochlor & 2-chloro-N-(ethoxymethyl)-N-(2-ethyl-6-methylphenyl) acetamide & $\mathrm{X}$ & -- & $\mathrm{X}$ \\
\hline Alachlor & 2-chloro-N-(2,6-diethylphenyl)-N-(methoxymethyl) acetamide & $\mathrm{X}$ & -- & $\mathrm{X}$ \\
\hline Dimethenamid & 2-chloro-N-(2,4-dimethyl-3-thienyl))-N-(2-methoxy-1-methylethyl) acetamide & $\mathrm{X}$ & -- & $\mathrm{X}$ \\
\hline Flufenacet & $\mathrm{N}$-(4-fluorophenyl)-N-(1-methylethyl)-2-[[5-(trifluoromethyl)-1,3,4-thiadiazol-2-yl]oxy] acetamide & $\mathrm{X}$ & -- & $\mathrm{X}$ \\
\hline Metolachlor & 2-chloro-N-(2-ethyl-6-methylphenyl)-N-(2-methoxy-1-methylethyl) acetamide & $\mathrm{X}$ & -- & $\mathrm{X}$ \\
\hline \multicolumn{5}{|c|}{ Degradation products } \\
\hline Acetochlor ethanesulfonic acid & 2-[(2-ethyl-6-methylphenyl)(ethoxymethyl)-amino]-2-oxoethane sulfonic acid & -- & $\mathrm{X}$ & $\mathrm{X}$ \\
\hline Acetochlor oxanilic acid & 2-[(2-ethyl-6-methylphenyl)(ethoxymethyl)-amino]-2-oxoacetic acid & -- & $\mathrm{X}$ & $\mathrm{X}$ \\
\hline Acetochlor sulfinyl acetic acid & 2-(\{[N-(ethoxymethyl)-N-(6-ethyl-2-methylphenyl)carbamoyl]methyl $\}$ sulfinyl)acetic acid & -- & -- & $\mathrm{X}$ \\
\hline Alachlor ethanesulfonic acid & 2-[(2,6-diethylphenyl)(methoxymethyl)amino]-2-oxoethanesulfonic acid & -- & $\mathrm{X}$ & $\mathrm{X}$ \\
\hline Alachlor oxanilic acid & 2-[(2,6-diethylphenyl)(methoxymethyl)amino]- 2-oxoacetic acid & -- & $\mathrm{X}$ & $\mathrm{X}$ \\
\hline Alachlor sulfinyl acetic acid & 2-(\{[N-(2,6-diethylphenyl)-N-(methoxymethyl)carbamoyl]methyl $\}$ sulfinyl)acetic acid & -- & -- & $\mathrm{X}$ \\
\hline Dimethenamid ethanesulfonic acid & [(2,4-dimethyl-thiophen-3-yl)-(2-methoxy-1-methyl-ethyl)-carbamoyl]-methanesulfonic acid & -- & -- & $\mathrm{X}$ \\
\hline Dimethenamid oxanilic acid & N-(2,4-dimethyl-thiophen-3-yl)-N-(2-methoxy-1-methyl-ethyl)-oxalamic acid & -- & -- & $\mathrm{X}$ \\
\hline Flufenacet ethanesulfonic acid & [(4-fluoro-phenyl)-isopropyl-carbamoyl]-methanesulfonic acid & -- & -- & $\mathrm{X}$ \\
\hline Flufenacet oxanilic acid & $\mathrm{N}$-(4-fluro-phenyl)-N-isopropyl-oxalamic acid & -- & -- & $\mathrm{X}$ \\
\hline Metolachlor ethanesulfonic acid & 2-[(2-ethyl-6-methylphenyl)(2-methoxy-1-methylethyl)amino]-2-oxoethanesulfonic acid & -- & $\mathrm{X}$ & $\mathrm{X}$ \\
\hline Metolachlor oxanilic acid & 2-[(2-ethyl-6-methylphenyl)(2-methoxy-1-methylethyl)amino\}-2-oxoacetic acid & -- & $X$ & $X$ \\
\hline
\end{tabular}




\section{High-Performance Liquid Chromatography/Diode Array Detection}

Two automated SPE systems were used in the laboratory for HPLC/DAD analysis. As described in the GC/MS section of this report, one SPE method used the automated Waters millilab workstation, and the alternate SPE procedure used the Tekmar autotrace workstation. The same manufacturer of C-18 bonded-silica packing was used for both SPE procedures. The cartridge was rinsed with ethyl acetate. The adsorbed acetamide degradation products then were removed from the cartridge with methanol. The eluted solution was spiked with 2,4-dichlorophenoxyacetic acid (internal standard), and a Turbovap was used to evaporate the sample under a stream of nitrogen (Zimmerman and others, 2000).

The sample components were separated, identified, and measured by injecting $50 \mu \mathrm{L}$ of the sample extract into a Hewlett-Packard 1090 HPLC equipped with a diode array detector (Hewlett Packard, Palo Alto, California). The analytical columns consisted of Phenomenex (Torrance, California) 5$\mu \mathrm{m}, 250 \times 3$-mm C-18 column coupled to a Keystone (Bellefonte, Pennsylvania) 3- $\mu \mathrm{m}, 250$ X $4.6 \mathrm{~mm}$ C-18 column. Compounds eluting from the LC columns were identified by comparing their retention times obtained by the measurement of control samples under the same conditions used for the collected samples. The concentration of each identified compound was measured by relating the DAD response produced by that compound to the DAD response produced by the internal standard (Zimmerman and others, 2000).

The reporting limit for this method was $0.20 \mu \mathrm{g} / \mathrm{L}$. Concentrations of acetamide degradation products were reported in ranges from 0.20 to $3.0 \mu \mathrm{g} / \mathrm{L}$ without dilution. If the concentration was greater than $3.0 \mu \mathrm{g} / \mathrm{L}$, the sample was reextracted and reanalyzed (Hostetler and Thurman, 2000; Zimmerman and others, 2000).

\section{Liquid Chromatography/Mass Spectrometry}

Two automated SPE extraction systems were used in the laboratory for LC/MS analysis. One method used the Tekmar autotrace workstation. This method used vacuum C-18, 6-mL cartridges with bonded silica obtained from Waters Corporation (Milford, Massachusetts). The cartridge was rinsed with ethyl acetate. The adsorbed acetamide degradation products were removed from the cartridge with methanol. The methanolic extract was spiked with 2,4-dichlorophenoxyacetic acid (internal standard) solution, then evaporated in a Turbovap (Lee and others, 2001).

The alternate SPE method used the Prospekt automated online SPE instrument with the Triathlon autosampler (SparkHolland, The Netherlands). Filtered water samples were measured into $10-\mathrm{mL}$ vials and were spiked with the working surrogate compound. Each sample was acidified with acetic acid. The sample component then was isolated and concentrated using automated online SPE (Lee and Strahan, 2003).
The sample then was separated, identified, and measured by injecting an aliquot into the LC and MS detector operated in selected-ion monitoring mode. Compounds eluting from the LC were identified by comparing the retention times of massspectral signals against the retention times of standards analyzed under the same conditions used for the samples. Compounds were identified further by selected fragment ions or isotope ions that were characteristic for each compound. The concentration of each identified compound was calculated by determining the ratio of the MS response produced by that compound to the MS response produced by the internal standard, which was injected into the sample, to the ratio of the MS responses of the primary standard analyzed using the same method (Lee and others, 2001; Lee and Strahan, 2003).

The reporting limit for this method was $0.05 \mu \mathrm{g} / \mathrm{L}$ when extracted by the Tekmar autotrace workstation. The reporting limit for this method was $0.02 \mu \mathrm{g} / \mathrm{L}$ when using the Prospekt automated online SPE instrument with the Triathlon autosampler. The acetamide degradation products were reported in concentration ranges from 0.05 to $2.0 \mu \mathrm{g} / \mathrm{L}$. If the concentration was greater than $2.0 \mu \mathrm{g} / \mathrm{L}$, the sample was reextracted and reanalyzed (Lee and others, 2001; Lee and Strahan, 2003).

\section{Acetamide Herbicide and Degradation Product Results}

Analytical results for five acetamide herbicides and their degradation products in water-quality samples collected from various study areas throughout the United States are presented at the end of this report. Ground-water data from the Midcontinent Agricultural Chemical Research Project, NAWQA Program, and Federal-State cooperative studies are presented in table 5. Surface-water data collected from the Midcontinent Agricultural Chemical Research Project, NASQAN, NAWQA Program, and Federal-State studies are presented in table 6.

The percentage of detections in the acetamide herbicides and their degradation products in ground- and surface-water samples collected during these studies is shown in figures 5 and 6, respectively. A statistical summary of the analytical results, which includes the number of samples analyzed, number of samples at or above the method reporting limit (varied with each analytical method), maximum concentrations, median and percentage of detections for both ground- and surface-water samples is shown in tables 3 and 4, respectively. The median concentrations were calculated using only those samples in which the particular compound was detected.

About 2,420 samples were collected from 1994 through 2002 as part of various USGS ground-water studies. Figure 5 shows metolachlor (12 percent) was the most frequently detected acetamide parent compound, followed by acetochlor and alachlor ( 2 percent each), and dimethenamid ( 0.18 percent). The acetamide degradation products were detected more frequently than their parent compounds. Figure 5 also shows metolachlor ESA, the most frequently detected degradation 


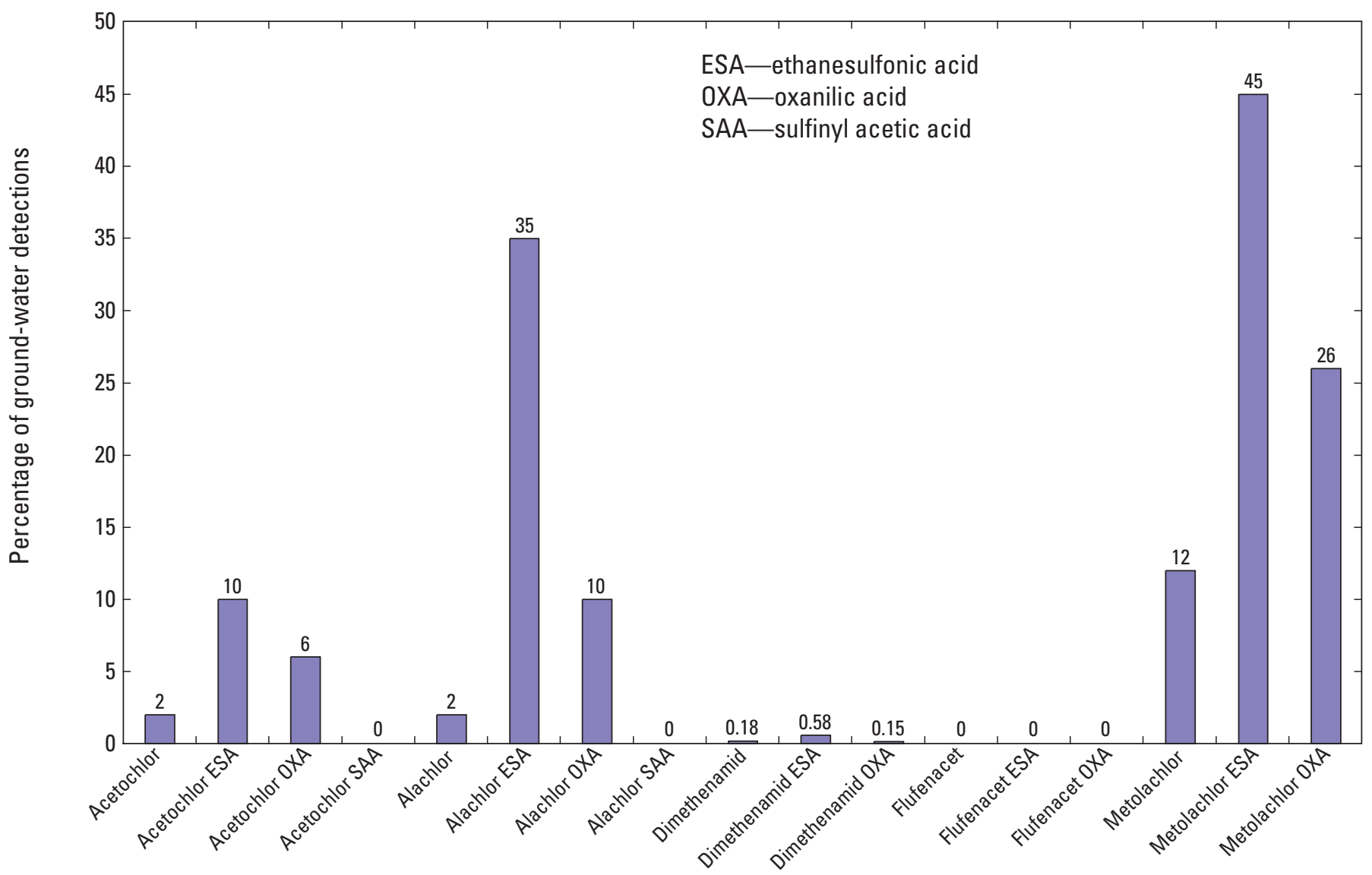

Acetamide herbicides or degradation product

Figure 5. Percentage of detections for acetamide herbicides and their degradation products determined by laboratory methods in ground-water samples collected for selected U.S. Geological Survey studies, 1994-2002.

product, was present in 45 percent of the samples analyzed, followed by alachlor ESA (35 percent) and metolachlor OXA (26 percent).

Overall, the median concentrations of detections for the acetochlor, alachlor, and metolachlor parent compounds were less than their ESA or OXA degradation products (table 3). For example, the median concentration for metolachlor detections in ground water $(0.17 \mu \mathrm{g} / \mathrm{L})$ was less than the median concentrations of detections for its degradation products, metolachlor ESA $(0.97 \mu \mathrm{g} / \mathrm{L})$ and metolachlor OXA $(0.70 \mu \mathrm{g} / \mathrm{L})$. However, the median concentration for the dimethenamid parent compound was greater than either its ESA or OXA degradation product. Flufenacet, its degradation products of ESA and OXA, and the SAAs were not detected in any of the ground-water samples.

About 2,756 samples were collected from 1993 through 2003 as part of various USGS surface-water studies. As shown in figure 6 , metolachlor (63 percent) was the most frequently detected acetamide parent compound, followed by acetochlor
(24 percent), dimethenamid (18 percent), alachlor (15 percent), and flufenacet ( 3 percent). Figure 6 also shows that the acetamide ESA and OXA degradation products were more frequently detected than their parent compounds with the exception of dimethenamid and flufenacet. Metolachlor ESA, the most frequently detected degradation product, was present in 82 percent of the samples analyzed, followed by metolachlor OXA (63 percent) and alachlor ESA (60 percent). In both the ground- and surface-water samples, alachlor ESA, metolachlor ESA, and metolachlor OXA were the most frequently detected compounds (figs. 5 and 6).

As shown in table 4, the median concentrations of detections for the acetochlor, alachlor, and metolachlor parent compounds were less than median concentrations for their ESA or OXA degradation products. The median concentration for the parent compound flufenacet was higher than either of its degradation products. Dimethenamid ESA was lower than dimethenamid, whereas dimethenamid OXA was higher than its parent. 


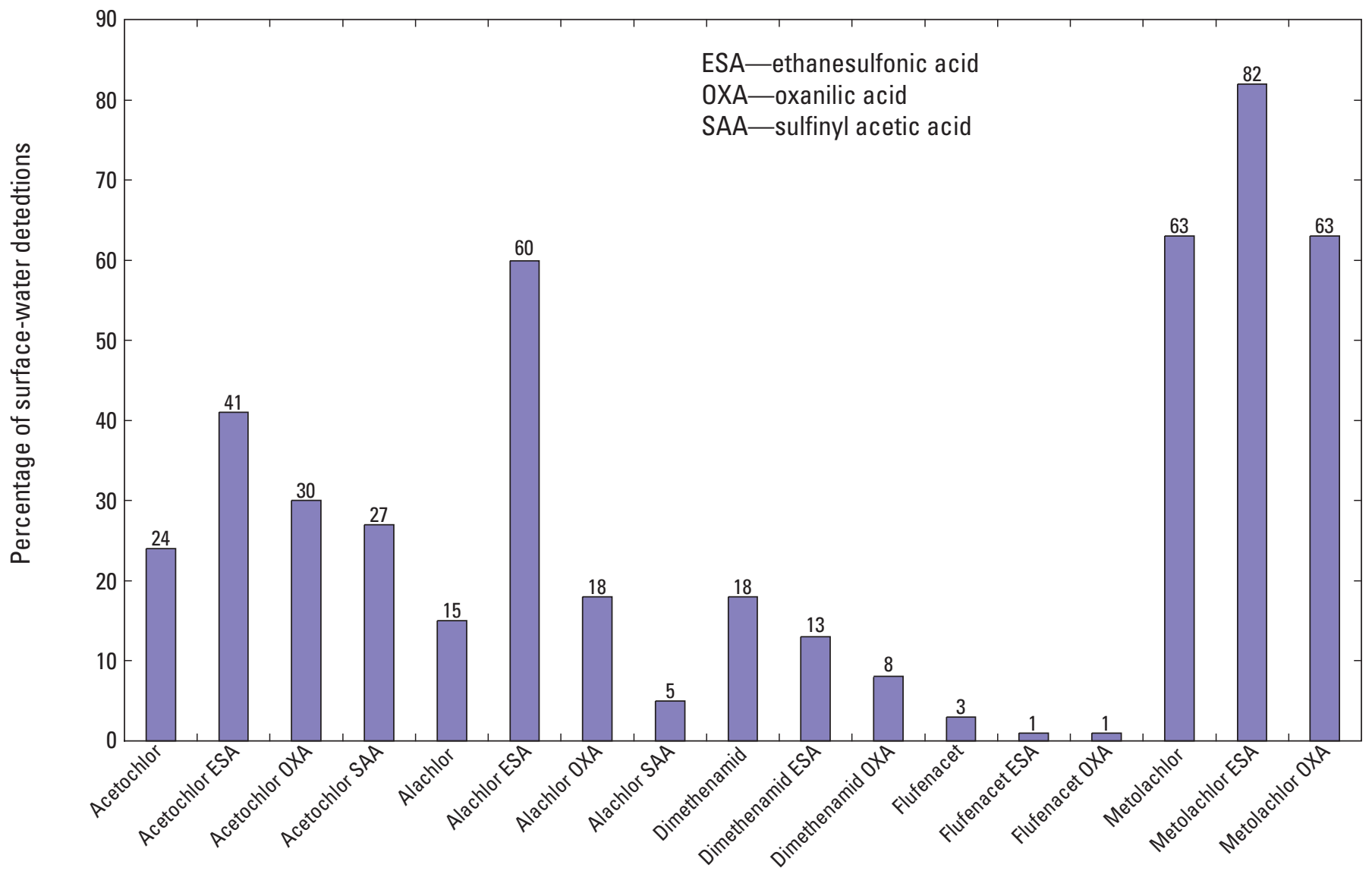

Acetamide herbicides or degradation product

Figure 6. Percentage of detections for acetamide herbicides and their degradation products determined by laboratory methods in surface-water samples collected for selected U.S. Geological Survey studies, 1993-2003.

\section{Summary}

Numerous studies have been conducted by the U.S. Geological Survey (USGS) throughout the 1990s and into the 2000s to investigate and document the occurrence, fate, and transport of herbicides and their degradation products in ground and surface water. Approximately 5,100 water samples were collected and analyzed for acetamide herbicides and their degradation products from 1993 through 2003 as part of various USGS studies throughout the United States, including the Midcontinent Agricultural Chemical Research Project, the National Stream Quality Accounting Network, several National WaterQuality Assessments, and individual Federal-State cooperative studies.

From 1993 through 2003, the USGS Organic Geochemistry Research Laboratory in Lawrence, Kansas, developed analytical methods to detect and measure concentrations of acetamide herbicides and their degradation products in ground and surface water. Gas chromatography/mass spectrometry was used for the analysis of the parent compounds acetochlor, alachlor, dimethenamid, flufenacet, and metolachlor. In December 2002, a liquid chromatography/mass spectrometry method became available for the analysis of the five parent compounds. The degradation products of these parent compounds, ethanesulfonic acid and oxanilic acid, were analyzed using two methods - high-performance liquid chromatography with diode-array detection and liquid chromatography/mass spectrometry. The acetochlor and alachlor degradation product of sulfinyl acetic acid was analyzed by liquid chromatography/ mass spectrometry.

Analytical results showed that: (1) the methods were valuable for acquiring information about the fate and transport of the parent acetamide herbicides in water; (2) degradation products of acetamide herbicides in ground water were detected more frequently and occurred at similar or higher concentrations than their parent compounds; (3) the acetamide herbicides and their degradation products were detected more frequently in surface water than in ground water, and in general, the parent compounds were detected at similar or greater frequencies than the degradation products in surface water; and (4) to more fully understand the occurrence, fate, and transport of herbicides and their degradation product, it is important to include both parent compounds and their degradation products in herbicide studies. 
Table 3. Statistical summary of concentrations of acetamide herbicides and their degradation products detected in ground-water samples collected as part of selected U.S. Geological Survey studies, 1994-2002.

[MRL, method reporting limit; $\mu \mathrm{g} / \mathrm{L}$, micrograms per liter]

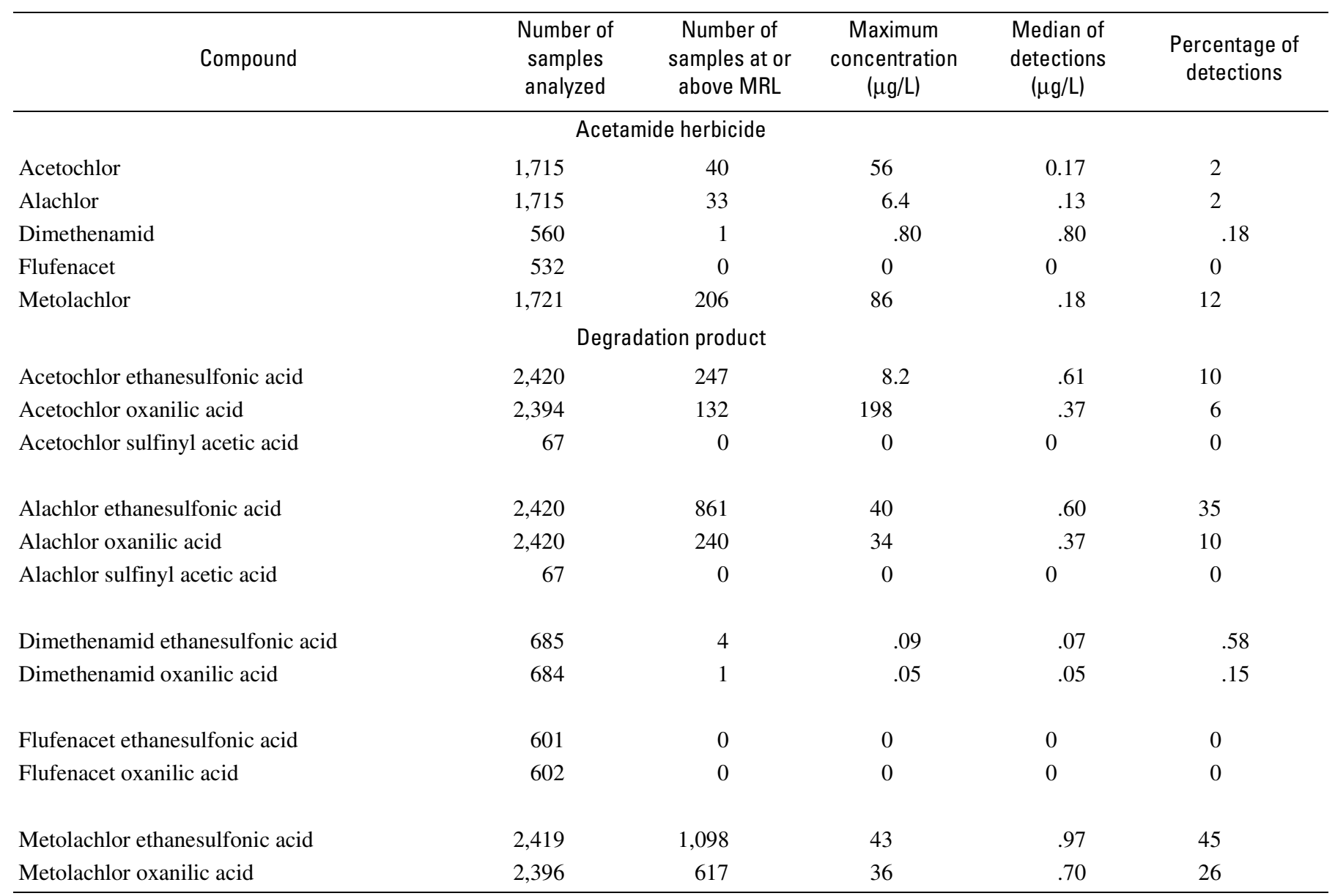

\section{References Cited}

Aga, D.S., and Thurman, E.M., 2001, Formation and transport of the sulfonic acid metabolites of alachlor and metolachlor in soil: Environmental Science \& Technology, v. 34, p. $2455-2460$.

Aga, D.S., Thurman, E.M., and Pomes, M.L., 1994, Determination of alachlor and its sulfonic acid degradation product in water by solid-phase extraction and enzyme-linked immunosorbent assay: Analytical Chemistry, v. 66, p. 1495-1499.

Aga, D.S., Thurman, E.M., Yockel, M.E., Zimmerman, L.R., and Williams, T.D., 1996, Identification of a new sulfonic acid degradation product of metolachlor in soil: Environmental Science \& Technology, v. 30, p. 592-597.

Anderson, M.T., Buxton, H.T., Rickert, D.A., and Morganwalp, D.W., 1995, Toxic Substances Hydrology Program: U.S. Geological Survey Fact Sheet 064-95, 4 p.

Battaglin, W.A., Thurman, E.M., Kalkhoff, S.J., and Porter, S.D., 2003, Herbicides and transformation products in surface water of the Midwestern United States: Journal of the American Water Resources Association, v. 39, p. 743-756.
Buxton, H.T., 2000, USGS Toxic Substances Hydrology Program, 2000: U.S. Geological Survey Fact Sheet 062-00, 4 p. Eckhardt, D.A.V., Hetcher, K.K., Phillips, P.J., and Miller, T.S., 2001, Pesticides and their metabolites in community water-supply wells of central and western New York, August 1999: U.S. Geological Survey Water-Resources Investigation Report 00-4128, 11 p.

Edwards, T.K., and Glysson, G.D., 1988, Field methods for measurement of fluvial sediment: U.S. Geological Survey Open-File Report 86-531, 118 p., revised July 1, 1992, p. 565-567.

Ferrer, Imma, Thurman, E.M., and Barcelo, Damia, 1997, Identification of ionic acetamide-herbicide degradation products in surface water and groundwater by HPLC/MS using negative ion spray: Journal of Analytical Chemistry, v. 69, p. 4547-4553.

Field, J.A., and Thurman, E.M., 1996, Glutathione conjugation and contaminant transformation: Environmental Science \& Technology, v. 30, no. 5, p. 1413-1417.

Gianessi, L.P., and Marcelli, M., 2000, Pesticide use in the U.S. crop production, 1997: Washington, D.C., National Summary Report, 101 p. 
Table 4. Statistical summary of concentrations of acetamide herbicides and degradation products detected in surface-water samples collected as part of selected U.S. Geological Survey studies, 1993-2003.

[MRL, method reporting limit; $\mu \mathrm{g} / \mathrm{L}$, micrograms per liter]

\begin{tabular}{|c|c|c|c|c|c|}
\hline Compound & $\begin{array}{c}\text { Number of } \\
\text { samples analyzed }\end{array}$ & $\begin{array}{c}\text { Number of } \\
\text { samples at or } \\
\text { above MRL }\end{array}$ & $\begin{array}{c}\text { Maximum } \\
\text { concentration } \\
(\mu \mathrm{g} / \mathrm{L})\end{array}$ & $\begin{array}{l}\text { Median of } \\
\text { detections } \\
(\mu \mathrm{g} / \mathrm{L})\end{array}$ & $\begin{array}{c}\text { Percentage of } \\
\text { detections }\end{array}$ \\
\hline \multicolumn{6}{|c|}{ Acetamide herbicide } \\
\hline Acetochlor & 2,262 & 545 & 21 & 0.24 & 24 \\
\hline Alachlor & 2,274 & 339 & 20 & .18 & 15 \\
\hline Dimethenamid & 767 & 141 & 4.7 & .10 & 18 \\
\hline Flufenacet & 681 & 22 & .93 & .18 & 3 \\
\hline Metolachlor & 2,274 & 1,445 & 124 & .23 & 63 \\
\hline \multicolumn{6}{|c|}{ Degradation product } \\
\hline Acetochlor ethanesulfonic acid & 2,756 & 1,153 & 22 & .45 & 41 \\
\hline Acetochlor oxanilic acid & 2,756 & 847 & 37 & .39 & 30 \\
\hline Acetochlor sulfinyl acetic acid & 322 & 86 & 1.2 & .20 & 27 \\
\hline Alachlor ethanesulfonic acid & 2,756 & 1,662 & 20 & .50 & 60 \\
\hline Alachlor oxanilic acid & 2,756 & 496 & 14 & .20 & 18 \\
\hline Alachlor sulfinyl acetic acid & 322 & 18 & .76 & .10 & 5 \\
\hline Dimethenamid ethanesulfonic acid & 989 & 132 & .95 & .09 & 13 \\
\hline Dimethenamid oxanilic acid & 989 & 80 & 1.7 & .12 & 8 \\
\hline Flufenacet ethanesulfonic acid & 867 & 9 & .29 & .08 & 1 \\
\hline Flufenacet oxanilic acid & 867 & 9 & .18 & .09 & 1 \\
\hline Metolachlor ethanesulfonic acid & 2,756 & 2,269 & 20 & .88 & 82 \\
\hline Metolachlor oxanilic acid & 2,756 & 1,748 & 17 & .41 & 63 \\
\hline
\end{tabular}

Graham, W.H., Graham, D.W., Denoyelles, F., Jr., Smith, V.H., Larive, C.K., and Thurman, E.M., 1999, Metolachlor and alachlor breakdown product formation patterns in aquatic field mesocosms: Environmental Science \& Technology, v. 33, no. 24, p. 4471-4476.

Hardy, M.A., Leahy, P.P., and Alley, W.M., 1989, Well installation documentation and ground-water sampling protocols for the pilot National Water-Quality Assessment Program: U.S. Geological Survey Open-File Report 89-396, 36 p.

Hooper, R.P., Goolsby, D.A., Rickert, D.A., and McKenzie, S.W., 1997, A river-basin perspective on monitoring water quality: U.S. Geological Survey Fact Sheet 055-97, 6 p.

Hostetler, K.A., and Thurman, E.M., 2000, Determination of chloroacetanilide herbicide metabolites in water using highperformance liquid chromatography-diode array detection and high-performance liquid chromatography/mass spectrometry: The Science of the Total Environment, v. 248, p. 147-156.

Kalkhoff, S.J., Kolpin, D.W., Thurman, E.M., Ferrer, I., and Barcelo, D., 1998, Degradation of chloroacetanilide herbicides - the prevalence of sulfonic and oxanilic acid metabolites in Iowa groundwaters and surface waters: Environmental Science \& Technology, v. 32, p. 1738-1740.

Kalkhoff, S.J., Lee, K.E., Porter, S.D., Terrio, P.J., and Thurman, E.M., 2003, Herbicides and herbicide degradation products in upper Midwest agricultural streams during August base-flow conditions: Journal of Environmental Quality, v. 32, p. 1025-1035.

Kish, J.L., Thurman, E.M., Scribner, E.A., and Zimmerman, L.R., 2000, Method of analysis by the U.S. Geological Survey Organic Geochemistry Research Group-determination of triazine and chloroacetanilide herbicides in water by solid-phase extraction and capillary-column gas chromatography/mass spectrometry with selected-ion monitoring: U.S. Geological Survey Open-File Report 00-385, 13 p.

Kolpin, D.W., Nations, B.K, Goolsby, D.A., and Thurman, E.M., 1996, Acetochlor in the hydrogeologic system in the midwestern United States, 1994: Environmental Science \& Technology, v. 30, p. 1459-1464.

Kolpin, D.W., Schnoebelen, D.J., and Thurman, E.M., in press, Degradates provide insight to spatial and temporal distribution of herbicides in ground water: Ground Water. 
Kolpin, D.W., Thurman, E.M., and Linhart, S.M., 1998, The environmental occurrence of herbicides-the importance of degradates in ground water: Archives of Environmental Contamination and Toxicology, v. 35, p. 385-390.

Kolpin, D.W., Thurman, E.M., and Linhart, S.M., 2000, Finding minimal herbicide concentrations in ground water? Try looking for degradates: The Science of the Total Environment, v. 248, p. 115-122.

Lee, E.A., Kish, J.L., Zimmerman, L.R., and Thurman, E.M., 2001, Methods of analysis by the U.S. Geological Survey Organic Geochemistry Research Group-update and additions to the determination of chloroacetanilide herbicide degradation compounds in water using high-performance liquid chromatography/ mass spectrometry: U.S. Geological Survey Open-File Report 01-10, 17 p.

Lee, E.A., and Strahan, A.P., 2003, Methods of analysis by the U.S. Geological Survey Organic Geochemistry Research Group-determination of acetamide herbicides and their degradation products in water using online solid-phase extraction and liquid chromatography/mass spectrometry: U.S. Geological Survey Open-File Report 03$173,17 \mathrm{p}$.

Meister, R.T., ed., 2000, Farm chemicals handbook 2000: Willoughby, Ohio, Meister Publishing Co., v. 86, 931 p.

Meyer, M.T., Mills, M.S., and Thurman, E.M., 1993, Automated solid-phase extraction of herbicides from water for gas chromatographic/mass spectrometric analysis: Journal of Chromatography, v. 629, p. 55-59.

Mills, P.C., and McMillan, W.D., 2004, Herbicides and their transformation products in source-water aquifers tapped by public-supply wells in Illinois, 2001-02: U.S. Geological Survey Water-Resources Investigations Report 03-4226, $57 \mathrm{p}$.

Phillips, P.J., Eckhardt, D.A., Smith, M.A., and Rosenmann, Larry, 2000, Pesticides and their metabolites in selected surface-water public supplies in New York State, 1999: U.S. Geological Survey Water-Resources Investigations Report 00-4119, 15 p.

Phillips, P.J., Wall, G.R., Thurman, E.M., Eckhardt, D.A, and Vanhoesen J., 1999, Metolachlor and its metabolites in tile drain and stream runoff in the Canajoharie Creek watershed: Environmental Science \& Technology, v. 33, no. 20, p. 3531-3535.

Schnoebelen, D.J., Kalkhoff, S.J., Becher, K.D., and Thurman, E.M., 2003, Water-quality assessment of the Eastern Iowa Basins - selected pesticides and pesticide degradates in streams, 1996-98: U.S. Geological Survey Water-Resources Investigations Report 03-4075, 62 p.

Scribner, E.A., Battaglin, W.A., Goolsby, D.A., and Thurman, E.M., 2000a, Changes in herbicide concentrations in Midwestern streams in relation to changes in use: The Science of the Total Environment, v. 248, p. 255-263.

Scribner, E.A., Battaglin, W.A., Dietze, J.E., and Thurman, E.M., 2003, Reconnaissance data for glyphosate, other selected herbicides, their degradation products, and antibiot- ics in 51 streams in nine Midwestern States, 2002: U.S. Geological Survey Open-File Report 03-217, 101 p.

Scribner, E.A, Goolsby, D.A., Thurman, E.M., and Battaglin, W.A., 1998, A reconnaissance for selected herbicides, metabolites, and nutrients in streams of nine Midwestern States, 1994-95: U.S. Geological Survey Open-File Report 98-181, 44 p.

Scribner, E.A., Thurman, E.M., and Zimmerman, L.R., 2000b, Analysis of selected herbicide metabolites in surface and ground water of the United States: The Science of the Total Environment, v. 248, p. 157-168.

SeedQuest, 2000, News release: accessed July 17, 2001, at URL http://www.seedquest.com/News/releases/usa/DowAgrol/ n3090.htm

Tauler, R., Barcelo, D., and Thurman, E.M., 2000, Multivariate correlation between concentrations of selected herbicides and derivatives in outflows from selected U.S. Midwestern reservoirs: Environmental Science \& Technology, v. 34, p. 3307-3314.

Thurman, E.M., and Cromwell, A.E., 2000, Atmospheric transport, deposition, and fate of triazine herbicides and their metabolites in pristine areas at Isle Royale National Park: Environmental Science \& Technology, v. 34, p. 3079-3085.

Thurman, E.M., Ferrer, Imma, and Parry, Rick, 2002, Accurate mass analysis of ethane sulfonic acid degradates of acetochlor and alachlor using high-performance liquid chromatography and time-of-flight mass spectrometry: Journal Chromatography A, v. 957, p. 3-9.

Thurman, E.M., Goolsby, D.A., Aga, D.S., Pomes, M.L., and Meyer, M.T., 1996, Occurrence of alachlor and its sulfonated degradation product in rivers and reservoirs of the Midwestern U.S.: Environmental Science \& Technology, v. 30, p. 569-574.

U.S. Environmental Protection Agency, Office of Prevention, 1998, Pesticides and toxics substances (7501C) April 1998: Washington, D.C., Pesticide Fact Sheet, 22 p.

U.S. Geological Survey, 2003, National Water-Quality Assessment Program (NAWQA): accessed November 4, 2003, at URL http://water.usgs.gov/nawqa/

Vargo, J.D., Lee, E.A., and Fuhrman, J.D., 2003, Interlaboratory comparison and validation of methods for chloroacetanilide and chloroacetamide solid degradates in environmental waters, in Ferrer, Imma, and Thurman, E.M., eds., Liquid chromatography/mass spectrometry, MS/MS and time of flight MS, analysis of emerging contaminants: Washington, D.C., American Chemical Society Symposium Series 850, p. 273-290.

Vencill, W.K., ed., 2002, Herbicide handbook (8th ed.): Lawrence, Kansas, Weed Science Society of America, 491 p. Ward, J.R., and Harr, C.A., 1990, Methods for collection and processing of surface-water and bed-material samples for physical and chemical analyses: U.S. Geological Survey Open-File Report 90-140, 71 p. 
Wilde, F.D., Radtke, D.B., Gibs, Jacob, and Iwatsubo, R.T., 1998, National field manual for the collection of water-quality data - preparations for water sampling: U.S. Geological Survey Techniques of Water-Resources Investigations, book 9, chap. A1-A5 [variously paged].

Yokley, R.A., Mayer, L.C., Huang, S.B., and Vargo, J.D., 2002, Analytical method for the determination of metolachlor, acetochlor, alachlor, dimethenamid, and their corresponding ethanesulfonic and oxanilic acid degradates in water using SPE and LC/ESI-MS/MS: Analytical Chemistry, v. 74, p. 3754-3759.

Zimmerman, L.R., Hostetler, K.A., and Thurman, E.M., 2000, Methods of analysis by the U.S. Geological Survey Organic Geochemistry Research Group-determination of chloroacetanilide herbicide metabolites in water using highperformance liquid chromatography-diode array detection and high-performance liquid chromatography/mass spectrometry: U.S. Geological Open-File Report 00-182, 30 p.
Zimmerman, L.R., Schneider, R.J., and Thurman, E.M., 2002, Analysis and detection of the herbicides dimethenamid and flufenacet and their sulfonic and oxanilic acid degradates in natural water: Journal of Agricultural and Food Chemistry, v. 50, no. 5, p. $1045-1052$.

Zimmerman, L.R., and Thurman, E.M., 1999, Method of analysis by the U.S. Geological Survey Organic Geochemistry Research Group-determination of triazine and chloroacetanilide herbicides in water by solid-phase extraction and capillary-column gas chromatography/mass spectrometry with selected-ion monitoring: U.S. Geological Survey Open-File Report 98-634, 21 p. 
Table 5. Analytical results for selected acetamide herbicides and their degradation products, ethanesulfonic acid, oxanilic acid, and sulfinyl acetic acid, in ground-water samples, 1994-2002.

[OGRL, U.S. Geological Survey Organic Geochemistry Research Laboratory, Lawrence, Kansas; ESA, ethanesulfonic acid; OXA, oxanilic acid; SAA, sulfinyl acetic acid; --, no data; <, less than]

\begin{tabular}{|c|c|c|c|c|c|c|c|c|c|c|c|c|c|c|c|c|c|c|c|}
\hline Site number & Site location & $\begin{array}{l}\text { Date of col- } \\
\text { lection } \\
\text { (month/day/ } \\
\text { year) }\end{array}$ & $\begin{array}{c}\text { Aceto- } \\
\text { chlor } \\
(\mu \mathrm{g} / \mathrm{L})\end{array}$ & $\begin{array}{c}\text { Aceto- } \\
\text { chlor } \\
\text { ESA } \\
(\mu \mathrm{g} / \mathrm{L})\end{array}$ & $\begin{array}{c}\text { Aceto- } \\
\text { chlor } \\
\text { OXA } \\
(\mu \mathrm{g} / \mathrm{L})\end{array}$ & $\begin{array}{l}\text { Aceto- } \\
\text { chlor } \\
\text { SAA } \\
(\mu \mathrm{g} / \mathrm{L})\end{array}$ & $\begin{array}{l}\text { Ala- } \\
\text { chlor } \\
(\mu \mathrm{g} / \mathrm{L})\end{array}$ & $\begin{array}{l}\text { Ala- } \\
\text { chlor } \\
\text { ESA } \\
(\mu \mathrm{g} / \mathrm{L})\end{array}$ & $\begin{array}{c}\text { Ala- } \\
\text { chlor } \\
\text { OXA } \\
(\mu \mathrm{g} / \mathrm{L})\end{array}$ & $\begin{array}{l}\text { Ala- } \\
\text { chlor } \\
\text { SAA } \\
(\mu \mathrm{g} / \mathrm{L})\end{array}$ & $\begin{array}{c}\text { Di- } \\
\text { meth- } \\
\text { enamid } \\
(\mu \mathrm{g} / \mathrm{L})\end{array}$ & $\begin{array}{c}\text { Di- } \\
\text { meth- } \\
\text { enamid } \\
\text { ESA } \\
(\mu \mathrm{g} / \mathrm{L})\end{array}$ & $\begin{array}{c}\text { Di- } \\
\text { meth- } \\
\text { enamid } \\
\text { OXA } \\
(\mu \mathrm{g} / \mathrm{L})\end{array}$ & $\begin{array}{c}\text { Flufen- } \\
\text { acet } \\
(\mu \mathrm{g} / \mathrm{L})\end{array}$ & $\begin{array}{c}\text { Flufen- } \\
\text { acet } \\
\text { ESA } \\
(\mu \mathrm{g} / \mathrm{L})\end{array}$ & $\begin{array}{c}\text { Flufen- } \\
\text { acet } \\
\text { OXA } \\
(\mu \mathrm{g} / \mathrm{L})\end{array}$ & $\begin{array}{c}\text { Meto- } \\
\text { lachlor } \\
(\mu \mathrm{g} / \mathrm{L})\end{array}$ & $\begin{array}{c}\text { Meto- } \\
\text { lachlor } \\
\text { ESA } \\
(\mu \mathrm{g} / \mathrm{L})\end{array}$ & $\begin{array}{c}\text { Meto- } \\
\text { lachlor } \\
\text { OXA } \\
(\mu \mathrm{g} / \mathrm{L})\end{array}$ \\
\hline \multicolumn{20}{|c|}{ Midcontinent Agricultural Chemical Research Herbicide Project (fig. 1) } \\
\hline \multicolumn{20}{|c|}{ Illinois monitoring wells (PMI) } \\
\hline 371512089110601 & Pulaski Co., IL & $11 / 02 / 00$ & $<0.05$ & $<0.05$ & $<0.05$ & -- & $<0.05$ & $<0.05$ & $<0.05$ & -- & $<0.05$ & $<0.05$ & $<0.05$ & $<0.05$ & $<0.05$ & $<0.05$ & $<0.05$ & $<0.05$ & $<0.05$ \\
\hline 371856089081801 & Pulaski Co., IL & $11 / 02 / 00$ & $<.05$ & $<.05$ & $<.05$ & -- & $<.05$ & $<.05$ & $<.05$ & -- & $<.05$ & $<.05$ & $<.05$ & $<.05$ & $<.05$ & $<.05$ & $<.05$ & $<.05$ & $<.05$ \\
\hline 375121088104401 & Gallatin Co., IL & $11 / 01 / 00$ & $<.05$ & $<.05$ & $<.05$ & -- & $<.05$ & .11 & $<.05$ & -- & $<.05$ & $<.05$ & $<.05$ & $<.05$ & $<.05$ & $<.05$ & $<.05$ & .66 & $<.05$ \\
\hline 375740089400001 & Randolph Co., IL & $10 / 31 / .00$ & $<.05$ & $<.05$ & $<.05$ & -- & $<.05$ & $<.05$ & $<.05$ & -- & $<.05$ & $<.05$ & $<.05$ & $<.05$ & $<.05$ & $<.05$ & $<.05$ & $<.05$ & $<.05$ \\
\hline 380020088523801 & Franklin Co, IL & $10 / 31 / 00$ & $<.05$ & $<.05$ & $<.05$ & -- & $<.05$ & $<.05$ & $<.05$ & -- & $<.05$ & $<.05$ & $<.05$ & $<.05$ & $<.05$ & $<.05$ & $<.05$ & $<.05$ & $<.05$ \\
\hline 382725089433001 & St. Clair Co., IL & $10 / 26 / 00$ & .41 & $<.05$ & .12 & -- & .07 & 3.4 & 5.9 & -- & $<.05$ & $<.05$ & $<.05$ & $<.05$ & $<.05$ & $<.05$ & .86 & .66 & .26 \\
\hline 382803089325201 & Washington Co., IL & $10 / 30 / 00$ & $<.05$ & $<.05$ & $<.05$ & -- & $<.05$ & $<.05$ & $<.05$ & -- & $<.05$ & $<.05$ & $<.05$ & $<.05$ & $<.05$ & $<.05$ & $<.05$ & $<.05$ & $<.05$ \\
\hline 383412088504701 & Marion Co., IL & $10 / 24 / 00$ & $<.05$ & $<.05$ & $<.05$ & -- & $<.05$ & $<.05$ & $<.05$ & -- & $<.05$ & $<.05$ & $<.05$ & $<.05$ & $<.05$ & $<.05$ & $<.05$ & $<.05$ & $<.05$ \\
\hline 383632089280101 & Clinton Co., IL & $10 / 26 / 00$ & $<.05$ & .14 & $<.05$ & -- & $<.05$ & $<.05$ & $<.05$ & -- & $<.05$ & $<.05$ & $<.05$ & $<.05$ & $<.05$ & $<.05$ & $<.05$ & .07 & $<.05$ \\
\hline 383742088334801 & Clay Co., IL & $10 / 25 / 00$ & $<.05$ & $<.05$ & $<.05$ & -- & $<.05$ & $<.05$ & $<.05$ & -- & $<.05$ & $<.05$ & $<.05$ & $<.05$ & $<.05$ & $<.05$ & $<.05$ & $<.05$ & $<.05$ \\
\hline 383958087453201 & Lawrence Co., IL & $10 / 23 / 00$ & $<.05$ & $<.05$ & $<.05$ & -- & $<.05$ & $<.05$ & $<.05$ & -- & $<.05$ & $<.05$ & $<.05$ & $<.05$ & $<.05$ & $<.05$ & $<.05$ & $<.05$ & $<.05$ \\
\hline 384049088374901 & Clay Co., IL & $10 / 25 / 00$ & $<.05$ & $<.05$ & $<.05$ & -- & $<.05$ & $<.05$ & $<.05$ & -- & $<.05$ & $<.05$ & $<.05$ & $<.05$ & $<.05$ & $<.05$ & $<.05$ & $<.05$ & $<.05$ \\
\hline 384533087364501 & Lawrence Co., IL & $10 / 23 / 00$ & $<.05$ & $<.05$ & $<.05$ & -- & $<.05$ & $<.05$ & $<.05$ & -- & $<.05$ & $<.05$ & $<.05$ & $<.05$ & $<.05$ & $<.05$ & $<.05$ & 1.4 & $<.05$ \\
\hline 384623088130001 & Richland Co., IL & $10 / 24 / 00$ & $<.05$ & $<.05$ & $<.05$ & -- & $<.05$ & $<.05$ & $<.05$ & -- & $<.05$ & $<.05$ & $<.05$ & $<.05$ & $<.05$ & $<.05$ & $<.05$ & $<.05$ & $<.05$ \\
\hline 390018087430701 & Crawford Co., IL & $10 / 24 / 00$ & $<.05$ & .12 & $<.05$ & -- & $<.05$ & $<.05$ & $<.05$ & -- & $<.05$ & $<.05$ & $<.05$ & $<.05$ & $<.05$ & $<.05$ & $<.05$ & $<.05$ & $<.05$ \\
\hline 390441090245401 & Jersey Co., IL & $10 / 30 / 00$ & $<.05$ & $<.05$ & $<.05$ & -- & $<.05$ & .10 & .06 & -- & $<.05$ & $<.05$ & $<.05$ & $<.05$ & $<.05$ & $<.05$ & $<.05$ & $<.05$ & $<.05$ \\
\hline 390910088243901 & Effingham Co., IL & $10 / 23 / 00$ & $<.05$ & $<.05$ & .07 & -- & $<.05$ & 1.5 & .52 & -- & $<.05$ & $<.05$ & $<.05$ & $<.05$ & $<.05$ & $<.05$ & $<.05$ & 2.3 & 1.0 \\
\hline 391028089280001 & Montgomery Co, IL & $10 / 23 / 00$ & $<.05$ & $<.05$ & $<.05$ & -- & $<.05$ & $<.05$ & $<.05$ & -- & $<.05$ & $<.05$ & $<.05$ & $<.05$ & $<.05$ & $<.05$ & $<.05$ & $<.05$ & $<.05$ \\
\hline 391545090304001 & Greene Co., IL & $10 / 30 / 00$ & $<.05$ & $<.05$ & $<.05$ & -- & $<.05$ & $<.05$ & $<.05$ & -- & $<.05$ & $<.05$ & $<.05$ & $<.05$ & $<.05$ & $<.05$ & $<.05$ & $<.05$ & $<.05$ \\
\hline 391820087535301 & Clark Co., IL & $10 / 24 / 00$ & $<.05$ & $<.05$ & $<.05$ & -- & $<.05$ & .08 & $<.05$ & -- & $<.05$ & $<.05$ & $<.05$ & $<.05$ & $<.05$ & $<.05$ & $<.05$ & .09 & $<.05$ \\
\hline 393635088312701 & Moultrie Co., IL & $10 / 27 / 00$ & $<.05$ & $<.05$ & $<.05$ & -- & $<.05$ & $<.05$ & $<.05$ & -- & $<.05$ & $<.05$ & $<.05$ & $<.05$ & $<.05$ & $<.05$ & $<.05$ & $<.05$ & $<.05$ \\
\hline 394750089044701 & Macon Co., IL & $10 / 25 / 00$ & $<.05$ & $<.05$ & $<.05$ & -- & $<.05$ & $<.05$ & $<.05$ & -- & $<.05$ & $<.05$ & $<.05$ & $<.05$ & $<.05$ & $<.05$ & $<.05$ & $<.05$ & $<.05$ \\
\hline 394926090335701 & Morgan Co., IL & $10 / 31 / 00$ & $<.05$ & $<.05$ & $<.05$ & -- & $<.05$ & $<.05$ & $<.05$ & -- & $<.05$ & $<.05$ & $<.05$ & $<.05$ & $<.05$ & $<.05$ & $<.05$ & $<.05$ & $<.05$ \\
\hline 395813091165001 & Adams Co., IL & $10 / 31 / 00$ & $<.05$ & $<.05$ & $<.05$ & -- & $<.05$ & $<.05$ & $<.05$ & -- & $<.05$ & $<.05$ & $<.05$ & $<.05$ & $<.05$ & $<.05$ & $<.05$ & $<.05$ & $<.05$ \\
\hline
\end{tabular}


Table 5. Analytical results for selected acetamide herbicides and their degradation products, ethanesulfonic acid, oxanilic acid, and sulfinyl acetic acid, in ground-water samples, 1994-2002.-Continued

[OGRL, U.S. Geological Survey Organic Geochemistry Research Laboratory, Lawrence, Kansas; ESA, ethanesulfonic acid; OXA, oxanilic acid; SAA, sulfinyl acetic acid; --, no data; <, less than]

\begin{tabular}{|c|c|c|c|c|c|c|c|c|c|c|c|c|c|c|c|c|c|c|c|}
\hline Site number & Site location & $\begin{array}{l}\text { Date of col- } \\
\text { lection } \\
\text { (month/day/ } \\
\text { year) }\end{array}$ & $\begin{array}{c}\text { Aceto- } \\
\text { chlor } \\
(\mu \mathrm{g} / \mathrm{L})\end{array}$ & $\begin{array}{l}\text { Aceto- } \\
\text { chlor } \\
\text { ESA } \\
(\mu \mathrm{g} / \mathrm{L})\end{array}$ & $\begin{array}{c}\text { Aceto- } \\
\text { chlor } \\
\text { OXA } \\
(\mu \mathrm{g} / \mathrm{L})\end{array}$ & $\begin{array}{l}\text { Aceto- } \\
\text { chlor } \\
\text { SAA } \\
(\mu \mathrm{g} / \mathrm{L})\end{array}$ & $\begin{array}{l}\text { Ala- } \\
\text { chlor } \\
(\mu \mathrm{g} / \mathrm{L})\end{array}$ & $\begin{array}{l}\text { Ala- } \\
\text { chlor } \\
\text { ESA } \\
(\mu \mathrm{g} / \mathrm{L})\end{array}$ & $\begin{array}{l}\text { Ala- } \\
\text { chlor } \\
\text { OXA } \\
(\mu \mathrm{g} / \mathrm{L})\end{array}$ & $\begin{array}{l}\text { Ala- } \\
\text { chlor } \\
\text { SAA } \\
(\mu \mathrm{g} / \mathrm{L})\end{array}$ & $\begin{array}{c}\text { Di- } \\
\text { meth- } \\
\text { enamid } \\
(\mu \mathrm{g} / \mathrm{L})\end{array}$ & $\begin{array}{c}\text { Di- } \\
\text { meth- } \\
\text { enamid } \\
\text { ESA } \\
(\mu \mathrm{g} / \mathrm{L})\end{array}$ & $\begin{array}{c}\text { Di- } \\
\text { meth- } \\
\text { enamid } \\
\text { OXA } \\
(\mu \mathrm{g} / \mathrm{L})\end{array}$ & $\begin{array}{c}\text { Flufen- } \\
\text { acet } \\
(\mu \mathrm{g} / \mathrm{L})\end{array}$ & $\begin{array}{c}\text { Flufen- } \\
\text { acet } \\
\text { ESA } \\
(\mu \mathrm{g} / \mathrm{L})\end{array}$ & $\begin{array}{c}\text { Flufen- } \\
\text { acet } \\
\text { OXA } \\
(\mu \mathrm{g} / \mathrm{L})\end{array}$ & $\begin{array}{l}\text { Meto- } \\
\text { lachlor } \\
(\mu \mathrm{g} / \mathrm{L})\end{array}$ & $\begin{array}{l}\text { Meto- } \\
\text { lachlor } \\
\text { ESA } \\
(\mu \mathrm{g} / \mathrm{L})\end{array}$ & $\begin{array}{c}\text { Meto- } \\
\text { lachlor } \\
\text { OXA } \\
(\mu \mathrm{g} / \mathrm{L})\end{array}$ \\
\hline \multicolumn{20}{|c|}{ Midcontinent Agricultural Chemical Research Herbicide Project (fig. 1)—Continued } \\
\hline \multicolumn{20}{|c|}{ Illinois monitoring wells (PMI)—Continued } \\
\hline 400312089165401 & Logan Co., IL & $10 / 26 / 00$ & $<0.05$ & $<0.05$ & 0.09 & -- & $<0.05$ & 0.26 & $<0.05$ & -- & $<0.05$ & $<0.05$ & $<0.05$ & $<0.05$ & $<0.05$ & $<0.05$ & $<0.05$ & 0.63 & 0.20 \\
\hline 401020089231201 & Logan Co., IL & $10 / 26 / 00$ & $<.05$ & $<.05$ & $<.05$ & -- & $<.05$ & .38 & .12 & -- & $<.05$ & $<.05$ & .05 & $<.05$ & $<.05$ & $<.05$ & $<.05$ & .94 & .46 \\
\hline 401256088545001 & Dewitt Co., IL & $11 / 02 / 00$ & $<.05$ & $<.05$ & $<.05$ & -- & $<.05$ & 4.7 & .27 & -- & $<.05$ & $<.05$ & $<.05$ & $<.05$ & $<.05$ & $<.05$ & $<.05$ & .59 & .17 \\
\hline 401509089540401 & Mason Co., IL & $10 / 31 / 00$ & $<.05$ & $<.05$ & $<.05$ & -- & $<.05$ & .75 & $<.05$ & -- & $<.05$ & $<.05$ & $<.05$ & $<.05$ & $<.05$ & $<.05$ & $<.05$ & 1.4 & .25 \\
\hline 401542090004701 & Mason Co., IL & $10 / 31 / 00$ & $<.05$ & $<.05$ & $<.05$ & -- & $<.05$ & $<.05$ & $<.05$ & -- & $<.05$ & $<.05$ & $<.05$ & $<.05$ & $<.05$ & $<.05$ & $<.05$ & .32 & .06 \\
\hline 402149090065601 & Fulton Co., IL & $11 / 02 / 00$ & $<.05$ & $<.05$ & $<.05$ & -- & $<.05$ & $<.05$ & $<.05$ & -- & $<.05$ & $<.05$ & $<.05$ & $<.05$ & $<.05$ & $<.05$ & $<.05$ & $<.05$ & $<.05$ \\
\hline 403029091180801 & Hancock Co., IL & $11 / 01 / 00$ & $<.05$ & $<.05$ & $<.05$ & -- & $<.05$ & $<.05$ & .05 & -- & $<.05$ & $<.05$ & $<.05$ & $<.05$ & $<.05$ & $<.05$ & $<.05$ & $<.05$ & $<.05$ \\
\hline 404453089314801 & Woodford Co., IL & $10 / 30 / 00$ & $<.05$ & $<.05$ & $<.05$ & -- & $<.05$ & .14 & $<.05$ & -- & $<.05$ & $<.05$ & $<.05$ & $<.05$ & $<.05$ & $<.05$ & $<.05$ & .06 & $<.05$ \\
\hline 405512090573601 & Henderson Co., IL & $02 / 21 / 01$ & $<.05$ & $<.05$ & .10 & -- & $<.05$ & 1.2 & $<.05$ & -- & $<.05$ & $<.05$ & $<.05$ & $<.05$ & $<.05$ & $<.05$ & $<.05$ & .49 & .11 \\
\hline 405536089300401 & Peoria Co., IL & $11 / 01 / 00$ & $<.05$ & $<.05$ & $<.05$ & -- & $<.05$ & $<.05$ & $<.05$ & -- & $<.05$ & $<.05$ & $<.05$ & $<.05$ & $<.05$ & $<.05$ & $<.05$ & .09 & $<.05$ \\
\hline 405755089283201 & Peoria Co., IL & $11 / 01 / 00$ & $<.05$ & .74 & .18 & -- & $<.05$ & $<.05$ & $<.05$ & -- & $<.05$ & $<.05$ & $<.05$ & $<.05$ & $<.05$ & $<.05$ & $<.05$ & 1.2 & .35 \\
\hline 410033089280901 & Marshall Co., IL & $11 / 01 / 00$ & $<.05$ & $<.05$ & $<.05$ & -- & $<.05$ & $<.05$ & $<.05$ & -- & $<.05$ & $<.05$ & $<.05$ & $<.05$ & $<.05$ & $<.05$ & $<.05$ & 3.0 & $<.05$ \\
\hline 410336087345101 & Kankakee Co., IL & $10 / 23 / 00$ & $<.05$ & .05 & $<.05$ & -- & $<.05$ & $<.05$ & $<.05$ & -- & $<.05$ & $<.05$ & $<.05$ & $<.05$ & $<.05$ & $<.05$ & $<.05$ & $<.05$ & $<.05$ \\
\hline 410408088054101 & Kankakee Co., IL & $10 / 23 / 00$ & $<.05$ & .05 & $<.05$ & -- & $<.05$ & $<.05$ & $<.05$ & -- & $<.05$ & $<.05$ & $<.05$ & $<.05$ & $<.05$ & $<.05$ & $<.05$ & $<.05$ & $<.05$ \\
\hline 411252087335901 & Kankakee Co., IL & $10 / 23 / 00$ & $<.05$ & $<.05$ & $<.05$ & -- & $<.05$ & $<.05$ & $<.05$ & -- & $<.05$ & $<.05$ & $<.05$ & $<.05$ & $<.05$ & $<.05$ & $<.05$ & $<.05$ & $<.05$ \\
\hline 411758089095801 & Putnam Co., IL & $10 / 30 / 00$ & $<.05$ & $<.05$ & .23 & -- & $<.05$ & .77 & .82 & -- & $<.05$ & $<.05$ & $<.05$ & $<.05$ & $<.05$ & $<.05$ & $<.05$ & 1.9 & 1.8 \\
\hline 413957088313001 & Kendall Co., IL & $10 / 24 / 00$ & $<.05$ & $<.05$ & $<.05$ & -- & $<.05$ & .11 & $<.05$ & -- & $<.05$ & $<.05$ & $<.05$ & $<.05$ & $<.05$ & $<.05$ & $<.05$ & .71 & $<.05$ \\
\hline 414128089243701 & Lee Co., IL & $10 / 27 / 00$ & $<.05$ & $<.05$ & $<.05$ & -- & $<.05$ & .92 & $<.05$ & -- & $<.05$ & $<.05$ & $<.05$ & $<.05$ & $<.05$ & $<.05$ & $<.05$ & 40 & 12 \\
\hline 414152090183701 & Rock Island Co., IL & $11 / 02 / 00$ & $<.05$ & $<.05$ & $<.05$ & -- & $<.05$ & 1.4 & $<.05$ & -- & $<.05$ & $<.05$ & $<.05$ & $<.05$ & $<.05$ & $<.05$ & $<.05$ & 6.8 & 1.1 \\
\hline 414529088264302 & Kane Co., IL & $10 / 24 / 00$ & $<.05$ & $<.05$ & $<.05$ & -- & $<.05$ & $<.05$ & $<.05$ & -- & $<.05$ & $<.05$ & $<.05$ & $<.05$ & $<.05$ & $<.05$ & $<.05$ & .26 & $<.05$ \\
\hline 420010090073501 & Carroll Co., IL & $11 / 03 / 00$ & $<.05$ & $<.05$ & $<.05$ & -- & $<.05$ & .98 & $<.05$ & -- & $<.05$ & $<.05$ & $<.05$ & $<.05$ & $<.05$ & $<.05$ & $<.05$ & 4.0 & 2.1 \\
\hline 420233089242601 & Ogle Co., IL & $10 / 27 / 00$ & $<.05$ & $<.05$ & $<.05$ & -- & $<.05$ & .55 & $<.05$ & -- & $<.05$ & $<.05$ & $<.05$ & $<.05$ & $<.05$ & $<.05$ & $<.05$ & .29 & $<.05$ \\
\hline 420602089185502 & Ogle Co., IL & $10 / 21 / 00$ & $<.05$ & $<.05$ & $<.05$ & -- & $<.05$ & .10 & $<.05$ & -- & $<.05$ & $<.05$ & $<.05$ & $<.05$ & $<.05$ & $<.05$ & $<.05$ & .06 & $<.05$ \\
\hline 420841089493901 & Carroll Co., IL & $11 / 03 / 00$ & $<.05$ & $<.05$ & $<.05$ & -- & $<.05$ & 1.2 & $<.05$ & -- & $<.05$ & .06 & $<.05$ & $<.05$ & $<.05$ & $<.05$ & $<.05$ & 14 & 7.2 \\
\hline 421555088520901 & Boone Co., IL & $10 / 20 / 00$ & $<.05$ & $<.05$ & $<.05$ & -- & $<.05$ & .22 & $<.05$ & -- & $<.05$ & $<.05$ & $<.05$ & $<.05$ & $<.05$ & $<.05$ & $<.05$ & 4.5 & .14 \\
\hline
\end{tabular}


Table 5. Analytical results for selected acetamide herbicides and their degradation products, ethanesulfonic acid, oxanilic acid, and sulfinyl acetic acid, in ground-water samples, 1994-2002.-Continued

[OGRL, U.S. Geological Survey Organic Geochemistry Research Laboratory, Lawrence, Kansas; ESA, ethanesulfonic acid; OXA, oxanilic acid; SAA, sulfinyl acetic acid; --, no data; <, less than]

\begin{tabular}{|c|c|c|c|c|c|c|c|c|c|c|c|c|c|c|c|c|c|c|c|}
\hline Site number & Site location & $\begin{array}{l}\text { Date of col- } \\
\text { lection } \\
\text { (month/day/ } \\
\text { year) }\end{array}$ & $\begin{array}{c}\text { Aceto- } \\
\text { chlor } \\
(\mu \mathrm{g} / \mathrm{L})\end{array}$ & $\begin{array}{c}\text { Aceto- } \\
\text { chlor } \\
\text { ESA } \\
(\mu \mathrm{g} / \mathrm{L})\end{array}$ & $\begin{array}{c}\text { Aceto- } \\
\text { chlor } \\
\text { OXA } \\
(\mu \mathrm{g} / \mathrm{L})\end{array}$ & $\begin{array}{l}\text { Aceto- } \\
\text { chlor } \\
\text { SAA } \\
(\mu \mathrm{g} / \mathrm{L})\end{array}$ & $\begin{array}{l}\text { Ala- } \\
\text { chlor } \\
(\mu \mathrm{g} / \mathrm{L})\end{array}$ & $\begin{array}{l}\text { Ala- } \\
\text { chlor } \\
\text { ESA } \\
(\mu \mathrm{g} / \mathrm{L})\end{array}$ & $\begin{array}{c}\text { Ala- } \\
\text { chlor } \\
\text { OXA } \\
(\mu \mathrm{g} / \mathrm{L})\end{array}$ & $\begin{array}{l}\text { Ala- } \\
\text { chlor } \\
\text { SAA } \\
(\mu \mathrm{g} / \mathrm{L})\end{array}$ & $\begin{array}{c}\text { Di- } \\
\text { meth- } \\
\text { enamid } \\
(\mu \mathrm{g} / \mathrm{L})\end{array}$ & $\begin{array}{c}\text { Di- } \\
\text { meth- } \\
\text { enamid } \\
\text { ESA } \\
(\mu \mathrm{g} / \mathrm{L})\end{array}$ & $\begin{array}{c}\text { Di- } \\
\text { meth- } \\
\text { enamid } \\
0 X A \\
(\mu \mathrm{g} / \mathrm{L})\end{array}$ & $\begin{array}{c}\text { Flufen- } \\
\text { acet } \\
(\mu \mathrm{g} / \mathrm{L})\end{array}$ & $\begin{array}{c}\text { Flufen- } \\
\text { acet } \\
\text { ESA } \\
(\mu \mathrm{g} / \mathrm{L})\end{array}$ & $\begin{array}{c}\text { Flufen- } \\
\text { acet } \\
\text { OXA } \\
(\mu \mathrm{g} / \mathrm{L})\end{array}$ & $\begin{array}{l}\text { Meto- } \\
\text { lachlor } \\
(\mu \mathrm{g} / \mathrm{L})\end{array}$ & $\begin{array}{c}\text { Meto- } \\
\text { lachlor } \\
\text { ESA } \\
(\mu \mathrm{g} / \mathrm{L})\end{array}$ & $\begin{array}{c}\text { Meto- } \\
\text { lachlor } \\
\text { OXA } \\
(\mu \mathrm{g} / \mathrm{L})\end{array}$ \\
\hline \multicolumn{20}{|c|}{ Midcontinent Agricultural Chemical Research Herbicide Project (fig. 1)—Continued } \\
\hline \multicolumn{20}{|c|}{ Illinois monitoring wells (PMI)—Continued } \\
\hline 421806089414401 & Stephenson Co., IL & $10 / 26 / 00$ & $<0.05$ & $<0.05$ & $<0.05$ & -- & $<0.05$ & $<0.05$ & $<0.05$ & -- & $<0.05$ & $<0.05$ & $<0.05$ & $<0.05$ & $<0.05$ & $<0.05$ & $<0.05$ & $<0.05$ & $<0.05$ \\
\hline 421826089374301 & Stephenson Co., IL & $11 / 03 / 00$ & $<.05$ & $<.05$ & .07 & -- & $<.05$ & .46 & .08 & -- & $<.05$ & $<.05$ & $<.05$ & $<.05$ & $<.05$ & $<.05$ & $<.05$ & .74 & .17 \\
\hline 421834089213501 & Winnebago Co., IL & $10 / 26 / 00$ & $<.05$ & $<.05$ & $<.05$ & -- & $<.05$ & $<.05$ & $<.05$ & -- & $<.05$ & $<.05$ & $<.05$ & $<.05$ & $<.05$ & $<.05$ & $<.05$ & $<.05$ & $<.05$ \\
\hline 422058090014401 & Jo Daviess Co., IL & $11 / 02 / 00$ & $<.05$ & $<.05$ & $<.05$ & -- & $<.05$ & $<.05$ & $<.05$ & -- & $<.05$ & $<.05$ & $<.05$ & $<.05$ & $<.05$ & $<.05$ & $<.05$ & $<.05$ & $<.05$ \\
\hline 422716088532001 & Boone Co., IL & $10 / 25 / 00$ & $<.05$ & $<.05$ & $<.05$ & -- & $<.05$ & 3.5 & $<.05$ & -- & $<.05$ & $<.05$ & $<.05$ & $<.05$ & $<.05$ & $<.05$ & $<.05$ & 2.1 & $<.05$ \\
\hline 422723089034001 & Winnebago Co., IL & $10 / 25 / 00$ & $<.05$ & $<.05$ & $<.05$ & -- & $<.05$ & .21 & $<.05$ & -- & $<.05$ & $<.05$ & $<.05$ & $<.05$ & $<.05$ & $<.05$ & $<.05$ & .77 & .05 \\
\hline \multicolumn{20}{|c|}{ lowa monitoring wells (DKE/DKW) } \\
\hline 402131095221101 & Crawford Co., IA & $07 / 24 / 96$ & $<.05$ & $<.20$ & $<.20$ & -- & $<.05$ & $<.20$ & $<.20$ & -- & -- & -- & -- & -- & -- & -- & $<.05$ & $<.20$ & $<.20$ \\
\hline \multirow[t]{5}{*}{403659094285301} & Taylor Co., IA & $08 / 15 / 95$ & $<.05$ & $<.20$ & $<.20$ & -- & $<.05$ & $<.20$ & $<.20$ & -- & -- & -- & -- & -- & -- & -- & $<.05$ & $<.20$ & $<.20$ \\
\hline & & 07/11/96 & $<.05$ & $<.20$ & $<.20$ & -- & $<.05$ & $<.20$ & $<.20$ & -- & -- & -- & -- & -- & -- & -- & $<.05$ & $<.20$ & $<.20$ \\
\hline & & $08 / 26 / 97$ & $<.05$ & $<.20$ & $<.20$ & -- & $<.05$ & $<.20$ & $<.20$ & -- & -- & -- & -- & -- & -- & -- & $<.05$ & $<.20$ & $<.20$ \\
\hline & & 07/14/99 & $<.05$ & $<.20$ & $<.20$ & -- & $<.05$ & $<.20$ & $<.20$ & -- & $<.05$ & -- & -- & $<.05$ & -- & -- & $<.05$ & $<.20$ & $<.20$ \\
\hline & & 08/14/01 & $<.05$ & $<.05$ & $<.05$ & -- & $<.05$ & $<.05$ & $<.05$ & -- & $<.05$ & $<.05$ & $<.05$ & $<.05$ & $<.05$ & $<.05$ & $<.05$ & $<.05$ & $<.05$ \\
\hline \multirow[t]{6}{*}{403745091174701} & Lee Co., IA & 08/01/95 & $<.05$ & $<.20$ & -- & -- & $<.05$ & 2.0 & $<.20$ & -- & -- & -- & -- & -- & -- & -- & $<.05$ & 1.2 & -- \\
\hline & & 07/18/96 & $<.05$ & $<.20$ & $<.20$ & -- & $<.05$ & .94 & .46 & -- & -- & -- & -- & -- & -- & -- & $<.05$ & .60 & $<.20$ \\
\hline & & $07 / 25 / 97$ & $<.05$ & $<.20$ & $<.20$ & -- & $<.05$ & 1.0 & .55 & -- & -- & -- & -- & -- & -- & -- & $<.05$ & .69 & .28 \\
\hline & & 07/22/98 & $<.05$ & $<.20$ & $<.20$ & -- & $<.05$ & 1.9 & .72 & -- & -- & -- & -- & -- & -- & -- & $<.05$ & 1.2 & .45 \\
\hline & & 06/16/99 & $<.05$ & $<.20$ & $<.20$ & -- & $<.05$ & 1.1 & .69 & -- & $<.05$ & -- & -- & $<.05$ & -- & -- & $<.05$ & .81 & .47 \\
\hline & & 09/06/01 & $<.05$ & $<.05$ & .13 & -- & $<.05$ & .22 & .12 & -- & $<.05$ & $<.05$ & $<.05$ & $<.05$ & $<.05$ & $<.05$ & $<.05$ & .19 & .37 \\
\hline \multirow[t]{5}{*}{403906095015001} & Page Co., IA & 08/16/95 & $<.05$ & $<.20$ & $<.20$ & -- & $<.05$ & $<.20$ & $<.20$ & -- & -- & -- & -- & -- & -- & -- & $<.05$ & $<.20$ & $<.20$ \\
\hline & & 07/16/96 & $<.05$ & $<.20$ & $<.20$ & -- & $<.05$ & $<.20$ & $<.20$ & -- & -- & -- & -- & -- & -- & -- & $<.05$ & $<.20$ & $<.20$ \\
\hline & & 08/26/97 & $<.05$ & $<.20$ & $<.20$ & -- & $<.05$ & $<.20$ & $<.20$ & -- & -- & -- & -- & -- & -- & -- & $<.05$ & $<.20$ & $<.20$ \\
\hline & & 07/14/99 & $<.05$ & $<.20$ & $<.20$ & -- & $<.05$ & $<.20$ & $<.20$ & -- & $<.05$ & -- & -- & $<.05$ & -- & -- & $<.05$ & $<.20$ & $<.20$ \\
\hline & & 08/14/01 & $<.05$ & $<.05$ & $<.05$ & -- & $<.05$ & .05 & $<.05$ & -- & $<.05$ & $<.05$ & $<.05$ & $<.05$ & $<.05$ & $<.05$ & $<.05$ & .09 & $<.05$ \\
\hline
\end{tabular}


Table 5. Analytical results for selected acetamide herbicides and their degradation products, ethanesulfonic acid, oxanilic acid, and sulfinyl acetic acid, in ground-water samples, 1994-2002.-Continued

[OGRL, U.S. Geological Survey Organic Geochemistry Research Laboratory, Lawrence, Kansas; ESA, ethanesulfonic acid; OXA, oxanilic acid; SAA, sulfinyl acetic acid; --, no data; <, less than]

\begin{tabular}{|c|c|c|c|c|c|c|c|c|c|c|c|c|c|c|c|c|c|c|c|c|}
\hline Site number & Site location & $\begin{array}{l}\text { Date of col- } \\
\text { lection } \\
\text { (month/day/ } \\
\text { year) }\end{array}$ & $\begin{array}{l}\text { Aceto- } \\
\text { chlor } \\
(\mu \mathrm{g} / \mathrm{L})\end{array}$ & $\begin{array}{c}\text { Aceto- } \\
\text { chlor } \\
\text { ESA } \\
(\mu \mathrm{g} / \mathrm{L})\end{array}$ & $\begin{array}{c}\text { Aceto- } \\
\text { chlor } \\
\text { OXA } \\
(\mu \mathrm{g} / \mathrm{L})\end{array}$ & $\begin{array}{c}\text { Aceto- } \\
\text { chlor } \\
\text { SAA } \\
(\mu \mathrm{g} / \mathrm{L})\end{array}$ & $\begin{array}{l}\text { Ala- } \\
\text { chlor } \\
(\mu \mathrm{g} / \mathrm{L})\end{array}$ & $\begin{array}{c}\text { Ala- } \\
\text { chlor } \\
\text { ESA } \\
(\mu \mathrm{g} / \mathrm{L})\end{array}$ & $\begin{array}{c}\text { Ala- } \\
\text { chlor } \\
\text { OXA } \\
(\mu \mathrm{g} / \mathrm{L})\end{array}$ & $\begin{array}{l}\text { Ala- } \\
\text { chlor } \\
\text { SAA } \\
(\mu \mathrm{g} / \mathrm{L})\end{array}$ & $\begin{array}{c}\text { Di- } \\
\text { meth- } \\
\text { enamid } \\
(\mu \mathrm{g} / \mathrm{L})\end{array}$ & $\begin{array}{c}\text { Di- } \\
\text { meth- } \\
\text { enamid } \\
\text { ESA } \\
(\mu \mathrm{g} / \mathrm{L})\end{array}$ & $\begin{array}{c}\text { Di- } \\
\text { meth- } \\
\text { enamid } \\
\text { OXA } \\
(\mu \mathrm{g} / \mathrm{L})\end{array}$ & $\begin{array}{l}\text { Flufen- } \\
\text { acet } \\
(\mu \mathrm{g} / \mathrm{L})\end{array}$ & $\begin{array}{c}\text { Flufen- } \\
\text { acet } \\
\text { ESA } \\
(\mu \mathrm{g} / \mathrm{L})\end{array}$ & $\begin{array}{c}\text { Flufen- } \\
\text { acet } \\
\text { OXA } \\
(\mu \mathrm{g} / \mathrm{L})\end{array}$ & $\begin{array}{l}\text { Meto- } \\
\text { lachlor } \\
(\mu \mathrm{g} / \mathrm{L})\end{array}$ & $\begin{array}{l}\text { Meto- } \\
\text { lachlor } \\
\text { ESA } \\
(\mu \mathrm{g} / \mathrm{L})\end{array}$ & $\begin{array}{c}\text { Meto- } \\
\text { lachlor } \\
\text { OXA } \\
(\mu \mathrm{g} / \mathrm{L})\end{array}$ & 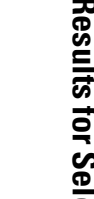 \\
\hline \multicolumn{20}{|c|}{ Midcontinent Agricultural Chemical Research Herbicide Project (fig. 1)—Continued } & \\
\hline \multicolumn{20}{|c|}{ lowa monitoring wells (DKE/DKW)—Continued } & \\
\hline \multirow[t]{6}{*}{404327095284801} & \multirow[t]{6}{*}{ Fremont Co., IA } & 08/16/95 & $<0.05$ & $<0.20$ & $<0.20$ & -- & $<0.05$ & $<0.20$ & $<0.20$ & -- & -- & -- & -- & -- & -- & -- & $<0.05$ & $<0.20$ & $<0.20$ & \\
\hline & & $07 / 11 / 96$ & $<.05$ & $<.20$ & $<.20$ & -- & $<.05$ & $<.20$ & $<.20$ & -- & -- & -- & -- & -- & -- & -- & $<.05$ & $<.20$ & $<.20$ & \\
\hline & & $08 / 26 / 97$ & $<.05$ & $<.20$ & $<.20$ & -- & $<.05$ & $<.20$ & $<.20$ & -- & -- & -- & -- & -- & -- & -- & $<.05$ & $<.20$ & $<.20$ & \\
\hline & & $07 / 24 / 98$ & $<.05$ & $<.20$ & $<.20$ & -- & $<.05$ & .20 & $<.20$ & -- & -- & -- & -- & -- & -- & -- & $<.05$ & $<.20$ & $<.20$ & \\
\hline & & $07 / 14 / 99$ & $<.05$ & $<.20$ & $<.20$ & -- & $<.05$ & $<.20$ & $<.20$ & -- & $<0.05$ & -- & -- & $<0.05$ & -- & -- & $<.05$ & $<.020$ & $<.20$ & \\
\hline & & 08/13/01 & $<.05$ & $<.05$ & $<.05$ & -- & $<.05$ & .14 & $<.05$ & -- & $<.05$ & $<0.05$ & $<0.05$ & $<.05$ & $<0.05$ & $<0.05$ & $<.05$ & $<.05$ & $<.05$ & \\
\hline 405500092121501 & Wapello Co., IA & $07 / 25 / 97$ & $<.05$ & $<.20$ & $<.20$ & -- & $<.05$ & $<.20$ & $<.20$ & -- & -- & -- & -- & -- & -- & -- & $<.05$ & $<.20$ & $<.20$ & \\
\hline \multirow[t]{2}{*}{405631094560802} & \multirow[t]{2}{*}{ Adams Co., IA } & $08 / 15 / 95$ & $<.05$ & $<.20$ & -- & -- & $<.05$ & .23 & $<.20$ & -- & -- & -- & -- & -- & -- & -- & $<.05$ & $<.20$ & -- & \\
\hline & & $07 / 11 / 96$ & $<.05$ & $<.20$ & $<.20$ & -- & $<.05$ & $<.20$ & $<.20$ & -- & -- & -- & -- & -- & -- & -- & $<.05$ & $<.20$ & $<.20$ & \\
\hline \multirow[t]{3}{*}{405632094534401} & \multirow[t]{3}{*}{ Adams Co., IA } & $08 / 25 / 97$ & $<.05$ & $<.20$ & $<.20$ & -- & $<.05$ & $<.20$ & $<.20$ & -- & -- & -- & -- & -- & -- & -- & $<.05$ & $<.20$ & $<.20$ & \\
\hline & & 07/13/99 & $<.05$ & $<.20$ & $<.20$ & -- & $<.05$ & $<.20$ & $<.20$ & -- & $<.05$ & -- & -- & $<.05$ & -- & -- & $<.05$ & $<.20$ & $<.20$ & 8 \\
\hline & & 08/13/01 & $<.05$ & $<.05$ & $<.05$ & -- & $<.05$ & $<.05$ & $<.05$ & -- & $<.05$ & $<.05$ & $<.05$ & $<.05$ & $<.05$ & $<.05$ & $<.05$ & $<.05$ & $<.05$ & \\
\hline \multirow[t]{5}{*}{405850095061701} & \multirow[t]{5}{*}{ Montgomery Co., IA } & 08/15/95 & $<.05$ & $<.20$ & $<.20$ & -- & $<.05$ & $<.20$ & $<.20$ & -- & -- & -- & -- & -- & -- & -- & $<.05$ & $<.20$ & $<.20$ & \\
\hline & & 07/10/96 & $<.05$ & $<.20$ & $<.20$ & -- & $<.05$ & $<.20$ & $<.20$ & -- & -- & -- & -- & -- & -- & -- & $<.05$ & $<.20$ & $<.20$ & \\
\hline & & $08 / 25 / 97$ & $<.05$ & $<.20$ & $<.20$ & -- & $<.05$ & $<.20$ & $<.20$ & -- & -- & -- & -- & -- & -- & -- & $<.05$ & $<.20$ & $<.20$ & \\
\hline & & 07/13/99 & $<.05$ & $<.20$ & $<.20$ & -- & $<.05$ & $<.20$ & $<.20$ & -- & $<.05$ & -- & -- & $<.05$ & -- & -- & $<.05$ & $<.20$ & $<.20$ & \\
\hline & & 08/13/01 & $<.05$ & $<.05$ & $<.05$ & -- & $<.05$ & $<.05$ & $<.05$ & -- & $<.05$ & $<.05$ & $<.05$ & $<.05$ & $<.05$ & $<.05$ & $<.05$ & $<.05$ & $<.05$ & \\
\hline \multirow[t]{2}{*}{405858093175701} & \multirow[t]{2}{*}{ Lucas Co., IA } & $07 / 21 / 97$ & $<.05$ & $<.20$ & $<.20$ & -- & $<.05$ & $<.20$ & $<.20$ & -- & -- & -- & -- & -- & -- & -- & $<.05$ & $<.20$ & $<.20$ & \\
\hline & & 08/15/01 & $<.05$ & $<.05$ & $<.05$ & -- & $<.05$ & $<.05$ & $<.05$ & -- & $<.05$ & $<.05$ & $<.05$ & $<.05$ & $<.05$ & $<.05$ & $<.05$ & $<.05$ & $<.05$ & \\
\hline 410046091555701 & Jefferson Co., IA & 09/06/01 & $<.05$ & $<.05$ & $<.05$ & -- & $<.05$ & $<.05$ & $<.05$ & -- & $<.05$ & $<.05$ & $<.05$ & $<.05$ & $<.05$ & $<.05$ & $<.05$ & $<.05$ & $<.05$ & \\
\hline \multirow[t]{2}{*}{410656095380201} & Mills Co., IA & 08/16/95 & $<.05$ & $<.20$ & $<.20$ & -- & $<.05$ & $<.20$ & $<.20$ & -- & -- & -- & -- & -- & -- & -- & $<.05$ & $<.20$ & $<.20$ & \\
\hline & & 07/09/96 & $<.05$ & $<.20$ & $<.20$ & -- & $<.05$ & $<.20$ & $<.20$ & -- & -- & -- & -- & -- & -- & -- & $<.05$ & $<.20$ & $<.20$ & \\
\hline
\end{tabular}


Table 5. Analytical results for selected acetamide herbicides and their degradation products, ethanesulfonic acid, oxanilic acid, and sulfinyl acetic acid, in ground-water samples, 1994-2002.-Continued

[OGRL, U.S. Geological Survey Organic Geochemistry Research Laboratory, Lawrence, Kansas; ESA, ethanesulfonic acid; OXA, oxanilic acid; SAA, sulfinyl acetic acid; --, no data; <, less than]

\begin{tabular}{|c|c|c|c|c|c|c|c|c|c|c|c|c|c|c|c|c|c|c|c|}
\hline Site number & Site location & $\begin{array}{l}\text { Date of col- } \\
\text { lection } \\
\text { (month/day/ } \\
\text { year) }\end{array}$ & $\begin{array}{c}\text { Aceto- } \\
\text { chlor } \\
(\mu \mathrm{g} / \mathrm{L})\end{array}$ & $\begin{array}{l}\text { Aceto- } \\
\text { chlor } \\
\text { ESA } \\
(\mu \mathrm{g} / \mathrm{L})\end{array}$ & $\begin{array}{c}\text { Aceto- } \\
\text { chlor } \\
\text { OXA } \\
(\mu \mathrm{g} / \mathrm{L})\end{array}$ & $\begin{array}{l}\text { Aceto- } \\
\text { chlor } \\
\text { SAA } \\
(\mu \mathrm{g} / \mathrm{L})\end{array}$ & $\begin{array}{l}\text { Ala- } \\
\text { chlor } \\
(\mu \mathrm{g} / \mathrm{L})\end{array}$ & $\begin{array}{l}\text { Ala- } \\
\text { chlor } \\
\text { ESA } \\
(\mu \mathrm{g} / \mathrm{L})\end{array}$ & $\begin{array}{c}\text { Ala- } \\
\text { chlor } \\
\text { OXA } \\
(\mu \mathrm{g} / \mathrm{L})\end{array}$ & $\begin{array}{l}\text { Ala- } \\
\text { chlor } \\
\text { SAA } \\
(\mu \mathrm{g} / \mathrm{L})\end{array}$ & $\begin{array}{c}\text { Di- } \\
\text { meth- } \\
\text { enamid } \\
(\mu \mathrm{g} / \mathrm{L})\end{array}$ & $\begin{array}{c}\text { Di- } \\
\text { meth- } \\
\text { enamid } \\
\text { ESA } \\
(\mu \mathrm{g} / \mathrm{L})\end{array}$ & $\begin{array}{c}\text { Di- } \\
\text { meth- } \\
\text { enamid } \\
\text { OXA } \\
(\mu \mathrm{g} / \mathrm{L})\end{array}$ & $\begin{array}{c}\text { Flufen- } \\
\text { acet } \\
(\mu \mathrm{g} / \mathrm{L})\end{array}$ & $\begin{array}{c}\text { Flufen- } \\
\text { acet } \\
\text { ESA } \\
(\mu \mathrm{g} / \mathrm{L})\end{array}$ & $\begin{array}{c}\text { Flufen- } \\
\text { acet } \\
\text { OXA } \\
(\mu \mathrm{g} / \mathrm{L})\end{array}$ & $\begin{array}{c}\text { Meto- } \\
\text { lachlor } \\
(\mu \mathrm{g} / \mathrm{L})\end{array}$ & $\begin{array}{c}\text { Meto- } \\
\text { lachlor } \\
\text { ESA } \\
(\mu \mathrm{g} / \mathrm{L})\end{array}$ & $\begin{array}{c}\text { Meto- } \\
\text { lachlor } \\
\text { OXA } \\
(\mu \mathrm{g} / \mathrm{L})\end{array}$ \\
\hline \multicolumn{20}{|c|}{ Midcontinent Agricultural Chemical Research Herbicide Project (fig. 1)—Continued } \\
\hline \multicolumn{20}{|c|}{ lowa monitoring wells (DKE/DKW)—Continued } \\
\hline \multirow[t]{3}{*}{410656095380201} & Mills Co., IA & 08/27/97 & $<0.05$ & $<0.20$ & $<0.20$ & -- & $<0.05$ & $<0.20$ & $<0.20$ & -- & -- & -- & -- & -- & -- & -- & $<0.05$ & $<0.20$ & $<0.20$ \\
\hline & & 07/16/99 & $<.05$ & $<.20$ & $<.20$ & -- & $<.05$ & $<.20$ & $<.20$ & -- & $<0.05$ & -- & -- & $<0.05$ & -- & -- & $<.05$ & .32 & $<.20$ \\
\hline & & 08/27/01 & $<.05$ & $<.05$ & $<.05$ & -- & $<.05$ & $<.05$ & $<.05$ & -- & $<.05$ & $<0.05$ & $<0.05$ & $<.05$ & $<0.05$ & $<0.05$ & $<.05$ & .31 & $<.05$ \\
\hline \multirow[t]{2}{*}{410905092375901} & Wapello Co., IA & 08/01/95 & $<.05$ & $<.20$ & -- & -- & $<.05$ & .20 & $<.20$ & -- & -- & -- & -- & -- & -- & -- & $<.05$ & .20 & -- \\
\hline & & 07/16/96 & $<.05$ & $<.20$ & $<.20$ & -- & $<.05$ & $<.20$ & $<.20$ & -- & -- & -- & -- & -- & -- & -- & $<.05$ & $<.20$ & $<.20$ \\
\hline \multirow[t]{4}{*}{410907092375301} & Wapello Co., IA & $07 / 24 / 97$ & $<.05$ & $<.20$ & $<.20$ & -- & $<.05$ & $<.20$ & $<.20$ & -- & -- & -- & -- & -- & -- & -- & $<.05$ & $<.20$ & $<.20$ \\
\hline & & 06/03/98 & $<.05$ & $<.20$ & $<.20$ & -- & $<.05$ & $<.20$ & $<.20$ & -- & -- & -- & -- & -- & -- & -- & $<.05$ & $<.20$ & $<.20$ \\
\hline & & 06/16/99 & $<.05$ & $<.20$ & $<.20$ & -- & $<.05$ & $<.20$ & $<.20$ & -- & $<.05$ & -- & -- & $<.05$ & -- & -- & $<.05$ & $<.20$ & $<.20$ \\
\hline & & 09/05/01 & $<.05$ & $<.05$ & $<.05$ & -- & $<.05$ & .06 & $<.05$ & -- & $<.05$ & $<.05$ & $<.05$ & $<.05$ & $<.05$ & $<.05$ & $<.05$ & .07 & $<.05$ \\
\hline \multirow[t]{5}{*}{411501095251301} & Pottawattamie Co., IA & 08/30/95 & $<.05$ & $<.20$ & -- & -- & $<.05$ & $<.20$ & $<.20$ & -- & -- & -- & -- & -- & -- & -- & $<.05$ & $<.20$ & -- \\
\hline & & 07/10/96 & $<.05$ & $<.20$ & $<.20$ & -- & $<.05$ & $<.20$ & $<.20$ & -- & -- & -- & -- & -- & -- & -- & $<.05$ & .38 & $<.20$ \\
\hline & & $08 / 28 / 97$ & $<.05$ & $<.20$ & $<.20$ & -- & $<.05$ & $<.20$ & $<.20$ & -- & -- & -- & -- & -- & -- & -- & $<.05$ & .50 & $<.20$ \\
\hline & & 07/15/99 & $<.05$ & $<.20$ & $<.20$ & -- & $<.05$ & $<.20$ & $<.20$ & -- & $<.05$ & -- & -- & $<.05$ & -- & -- & $<.05$ & .33 & $<.20$ \\
\hline & & 08/13/01 & $<.05$ & .10 & $<.05$ & -- & $<.05$ & $<.05$ & $<.05$ & -- & $<.05$ & $<.05$ & $<.05$ & $<.05$ & $<.05$ & $<.05$ & $<.05$ & .22 & $<.05$ \\
\hline \multirow[t]{5}{*}{411622094520901} & Cass Co., IA & $08 / 15 / 95$ & $<.05$ & $<.20$ & -- & -- & $<.05$ & $<.20$ & $<.20$ & -- & -- & -- & -- & -- & -- & -- & $<.05$ & $<.20$ & -- \\
\hline & & 07/10/96 & $<.05$ & $<.20$ & $<.20$ & -- & $<.05$ & $<.20$ & $<.20$ & -- & -- & -- & -- & -- & -- & -- & $<.05$ & $<.20$ & $<.20$ \\
\hline & & 08/27/97 & $<.05$ & $<.20$ & $<.20$ & -- & $<.05$ & $<.20$ & $<.20$ & -- & -- & -- & -- & -- & -- & -- & $<.05$ & $<.20$ & $<.20$ \\
\hline & & 07/13/99 & $<.05$ & $<.20$ & $<.20$ & -- & $<.05$ & $<.20$ & $<.20$ & -- & $<.05$ & -- & -- & $<.05$ & -- & -- & $<.05$ & $<.20$ & $<.20$ \\
\hline & & 08/14/01 & $<.05$ & $<.05$ & $<.05$ & -- & $<.05$ & $<.05$ & $<.05$ & -- & $<.05$ & $<.05$ & $<.05$ & $<.05$ & $<.05$ & $<.05$ & $<.05$ & $<.05$ & $<.05$ \\
\hline \multirow[t]{2}{*}{411639094521101} & Cass Co., IA & 08/27/97 & $<.05$ & $<.20$ & $<.20$ & -- & $<.05$ & $<.20$ & $<.20$ & -- & -- & -- & -- & -- & -- & -- & $<.05$ & $<.20$ & $<.20$ \\
\hline & & 08/14/01 & $<.05$ & $<.05$ & $<.05$ & -- & $<.05$ & $<.05$ & $<.05$ & -- & $<.05$ & $<.05$ & $<.05$ & $<.05$ & $<.05$ & $<.05$ & $<.05$ & $<.05$ & $<.05$ \\
\hline \multirow[t]{2}{*}{411644091110703} & Louisa Co., IA & 08/07/95 & $<.05$ & $<.20$ & -- & -- & $<.05$ & $<.20$ & $<.20$ & -- & -- & -- & -- & -- & -- & -- & $<.05$ & $<.20$ & -- \\
\hline & & $07 / 15 / 96$ & $<.05$ & $<.20$ & $<.20$ & -- & $<.05$ & $<.20$ & $<.20$ & -- & -- & -- & -- & -- & -- & -- & $<.05$ & $<.20$ & $<.20$ \\
\hline
\end{tabular}


Table 5. Analytical results for selected acetamide herbicides and their degradation products, ethanesulfonic acid, oxanilic acid, and sulfinyl acetic acid, in ground-water samples, 1994-2002.-Continued

[OGRL, U.S. Geological Survey Organic Geochemistry Research Laboratory, Lawrence, Kansas; ESA, ethanesulfonic acid; OXA, oxanilic acid; SAA, sulfinyl acetic acid; --, no data; <, less than]

\begin{tabular}{|c|c|c|c|c|c|c|c|c|c|c|c|c|c|c|c|c|c|c|c|}
\hline Site number & Site location & $\begin{array}{l}\text { Date of col- } \\
\text { lection } \\
\text { (month/day/ } \\
\text { year) }\end{array}$ & $\begin{array}{c}\text { Aceto- } \\
\text { chlor } \\
(\mu \mathrm{g} / \mathrm{L})\end{array}$ & $\begin{array}{l}\text { Aceto- } \\
\text { chlor } \\
\text { ESA } \\
(\mu \mathrm{g} / \mathrm{L})\end{array}$ & $\begin{array}{c}\text { Aceto- } \\
\text { chlor } \\
\text { OXA } \\
(\mu \mathrm{g} / \mathrm{L})\end{array}$ & $\begin{array}{l}\text { Aceto- } \\
\text { chlor } \\
\text { SAA } \\
(\mu \mathrm{g} / \mathrm{L})\end{array}$ & $\begin{array}{l}\text { Ala- } \\
\text { chlor } \\
(\mu \mathrm{g} / \mathrm{L})\end{array}$ & $\begin{array}{l}\text { Ala- } \\
\text { chlor } \\
\text { ESA } \\
(\mu \mathrm{g} / \mathrm{L})\end{array}$ & $\begin{array}{c}\text { Ala- } \\
\text { chlor } \\
\text { OXA } \\
(\mu \mathrm{g} / \mathrm{L})\end{array}$ & $\begin{array}{c}\text { Ala- } \\
\text { chlor } \\
\text { SAA } \\
(\mu \mathrm{g} / \mathrm{L})\end{array}$ & $\begin{array}{l}\text { Di- } \\
\text { meth- } \\
\text { enamid } \\
(\mu \mathrm{g} / \mathrm{L})\end{array}$ & $\begin{array}{c}\text { Di- } \\
\text { meth- } \\
\text { enamid } \\
\text { ESA } \\
(\mu \mathrm{g} / \mathrm{L})\end{array}$ & $\begin{array}{c}\text { Di- } \\
\text { meth- } \\
\text { enamid } \\
\text { OXA } \\
(\mu \mathrm{g} / \mathrm{L})\end{array}$ & $\begin{array}{c}\text { Flufen- } \\
\text { acet } \\
(\mu \mathrm{g} / \mathrm{L})\end{array}$ & $\begin{array}{c}\text { Flufen- } \\
\text { acet } \\
\text { ESA } \\
(\mu \mathrm{g} / \mathrm{L})\end{array}$ & $\begin{array}{c}\text { Flufen- } \\
\text { acet } \\
\text { OXA } \\
(\mu \mathrm{g} / \mathrm{L})\end{array}$ & $\begin{array}{l}\text { Meto- } \\
\text { lachlor } \\
(\mu \mathrm{g} / \mathrm{L})\end{array}$ & $\begin{array}{c}\text { Meto- } \\
\text { lachlor } \\
\text { ESA } \\
(\mu \mathrm{g} / \mathrm{L})\end{array}$ & $\begin{array}{c}\text { Meto- } \\
\text { lachlor } \\
\text { OXA } \\
(\mu \mathrm{g} / \mathrm{L})\end{array}$ \\
\hline \multicolumn{20}{|c|}{ Midcontinent Agricultural Chemical Research Herbicide Project (fig. 1)—Continued } \\
\hline & & & & Ion & va monit & toring $\mathrm{n}$ & vells (DK & KE/DKW & 1)-Con & inued & & & & & & & & & \\
\hline \multirow[t]{4}{*}{411644091110703} & Louisa Co., IA & 07/23/97 & $<0.05$ & $<0.20$ & $<0.20$ & -- & $<0.05$ & $<0.20$ & $<0.20$ & -- & -- & -- & -- & -- & -- & -- & $<0.05$ & $<0.20$ & $<0.20$ \\
\hline & & $07 / 22 / 98$ & $<.05$ & $<.20$ & $<.20$ & -- & $<.05$ & $<.20$ & $<.20$ & -- & -- & -- & -- & -- & -- & -- & $<.05$ & $<.20$ & $<.20$ \\
\hline & & 06/17/99 & $<.05$ & $<.05$ & $<.05$ & -- & $<.05$ & $<.05$ & $<.05$ & -- & $<0.05$ & -- & -- & $<0.05$ & -- & -- & $<.05$ & $<.05$ & $<.05$ \\
\hline & & 08/08/01 & $<.05$ & $<.05$ & $<.05$ & -- & $<.05$ & $<.05$ & $<.05$ & -- & $<.05$ & $<0.05$ & $<0.05$ & $<.05$ & $<0.05$ & $<0.05$ & $<.05$ & $<.05$ & $<.05$ \\
\hline 411652091213802 & Louisa Co., IA & 07/18/96 & $<.05$ & $<.20$ & $<.20$ & -- & $<.05$ & $<.20$ & $<.20$ & -- & -- & -- & -- & -- & -- & -- & $<.05$ & $<.20$ & $<.20$ \\
\hline \multirow[t]{3}{*}{411727094374001} & Adair Co., IA & 08/28/97 & $<.05$ & $<.20$ & $<.20$ & -- & $<.05$ & $<.20$ & $<.20$ & -- & -- & -- & -- & -- & -- & -- & .07 & .78 & .27 \\
\hline & & 07/13/99 & $<.05$ & $<.20$ & $<.20$ & -- & $<.05$ & $<.20$ & $<.20$ & -- & $<.05$ & -- & -- & $<.05$ & -- & -- & $<.05$ & .72 & .23 \\
\hline & & 08/14/01 & $<.05$ & $<.05$ & $<.05$ & -- & $<.05$ & $<.05$ & $<.05$ & -- & $<.05$ & $<.05$ & $<.05$ & $<.05$ & $<.05$ & $<.05$ & $<.05$ & .78 & .22 \\
\hline 411820093441201 & Warren Co., IA & $06 / 22 / 95$ & $<.05$ & $<.20$ & $<.20$ & -- & $<.05$ & $<.20$ & $<.20$ & -- & -- & -- & -- & -- & -- & -- & .05 & 1.2 & $<.20$ \\
\hline \multirow[t]{4}{*}{412013091485701} & Washington Co., IA & 07/19/96 & $<.05$ & $<.20$ & $<.20$ & -- & $<.05$ & $<.20$ & $<.20$ & -- & -- & -- & -- & -- & -- & -- & $<.05$ & $<.20$ & $<.20$ \\
\hline & & $07 / 23 / 97$ & $<.05$ & $<.20$ & $<.20$ & -- & $<.05$ & $<.20$ & $<.20$ & -- & -- & -- & -- & -- & -- & -- & $<.05$ & $<.20$ & $<.20$ \\
\hline & & 06/16/99 & $<.05$ & $<.20$ & $<.20$ & -- & $<.05$ & $<.20$ & $<.20$ & -- & $<.05$ & -- & -- & $<.05$ & -- & -- & $<.05$ & $<.20$ & $<.20$ \\
\hline & & 08/08/01 & $<.05$ & $<.05$ & $<.05$ & -- & $<.05$ & $<.05$ & $<.05$ & -- & $<.05$ & $<.05$ & $<.05$ & $<.05$ & $<.05$ & $<.05$ & $<.05$ & $<.05$ & $<.05$ \\
\hline \multirow[t]{2}{*}{412138091571501} & Keokuk Co., IA & 07/28/97 & $<.05$ & $<.20$ & $<.20$ & -- & $<.05$ & $<.20$ & $<.20$ & -- & -- & -- & -- & -- & -- & -- & $<.05$ & $<.20$ & $<.20$ \\
\hline & & 08/08/01 & $<.05$ & $<.05$ & $<.05$ & -- & $<.05$ & $<.05$ & $<.05$ & -- & $<.05$ & $<.05$ & $<.05$ & $<.05$ & $<.05$ & $<.05$ & $<.05$ & $<.05$ & $<.05$ \\
\hline \multirow[t]{2}{*}{412321091034801} & Muscatine Co., IA & 08/07/95 & $<.05$ & $<.20$ & $<.20$ & -- & $<.05$ & .80 & .27 & -- & -- & -- & -- & -- & -- & -- & $<.05$ & .70 & .33 \\
\hline & & $07 / 15 / 96$ & $<.05$ & $<.20$ & $<.20$ & -- & $<.05$ & .83 & .26 & -- & -- & -- & -- & -- & -- & -- & $<.05$ & .81 & .20 \\
\hline \multirow[t]{5}{*}{412850091342901} & Washington Co., IA & 07/16/96 & $<.05$ & $<.20$ & $<.20$ & -- & $<.05$ & $<.20$ & $<.20$ & -- & -- & -- & -- & -- & -- & -- & $<.05$ & $<.20$ & $<.20$ \\
\hline & & 07/23/97 & $<.05$ & $<.20$ & $<.20$ & -- & $<.05$ & $<.20$ & $<.20$ & -- & -- & -- & -- & -- & -- & -- & $<.05$ & $<.20$ & $<.20$ \\
\hline & & 08/25/98 & $<.05$ & $<.20$ & $<.20$ & -- & $<.05$ & $<.20$ & $<.20$ & -- & -- & -- & -- & -- & -- & -- & $<.05$ & $<.20$ & $<.20$ \\
\hline & & 06/16/99 & $<.05$ & $<.20$ & $<.20$ & -- & $<.05$ & $<.20$ & $<.20$ & -- & $<.05$ & -- & -- & $<.05$ & -- & -- & $<.05$ & $<.20$ & $<.20$ \\
\hline & & 08/02/01 & $<.05$ & $<.05$ & $<.05$ & -- & $<.05$ & $<.05$ & $<.05$ & -- & $<.05$ & $<.05$ & $<.05$ & $<.05$ & $<.05$ & $<.05$ & $<.05$ & $<.05$ & $<.05$ \\
\hline
\end{tabular}


Table 5. Analytical results for selected acetamide herbicides and their degradation products, ethanesulfonic acid, oxanilic acid, and sulfinyl acetic acid, in ground-water samples, 1994-2002.-Continued

[OGRL, U.S. Geological Survey Organic Geochemistry Research Laboratory, Lawrence, Kansas; ESA, ethanesulfonic acid; OXA, oxanilic acid; SAA, sulfinyl acetic acid; --, no data; <, less than]

\begin{tabular}{|c|c|c|c|c|c|c|c|c|c|c|c|c|c|c|c|c|c|c|c|}
\hline Site number & Site location & $\begin{array}{l}\text { Date of col- } \\
\text { lection } \\
\text { (month/day/ } \\
\text { year) }\end{array}$ & $\begin{array}{c}\text { Aceto- } \\
\text { chlor } \\
(\mu \mathrm{g} / \mathrm{L})\end{array}$ & $\begin{array}{c}\text { Aceto- } \\
\text { chlor } \\
\text { ESA } \\
(\mu \mathrm{g} / \mathrm{L})\end{array}$ & $\begin{array}{c}\text { Aceto- } \\
\text { chlor } \\
\text { OXA } \\
(\mu \mathrm{g} / \mathrm{L})\end{array}$ & $\begin{array}{c}\text { Aceto- } \\
\text { chlor } \\
\text { SAA } \\
(\mu \mathrm{g} / \mathrm{L})\end{array}$ & $\begin{array}{l}\text { Ala- } \\
\text { chlor } \\
(\mu \mathrm{g} / \mathrm{L})\end{array}$ & $\begin{array}{l}\text { Ala- } \\
\text { chlor } \\
\text { ESA } \\
(\mu \mathrm{g} / \mathrm{L})\end{array}$ & $\begin{array}{l}\text { Ala- } \\
\text { chlor } \\
\text { OXA } \\
(\mu \mathrm{g} / \mathrm{L})\end{array}$ & $\begin{array}{l}\text { Ala- } \\
\text { chlor } \\
\text { SAA } \\
(\mu \mathrm{g} / \mathrm{L})\end{array}$ & $\begin{array}{c}\text { Di- } \\
\text { meth- } \\
\text { enamid } \\
(\mu \mathrm{g} / \mathrm{L})\end{array}$ & $\begin{array}{c}\text { Di- } \\
\text { meth- } \\
\text { enamid } \\
\text { ESA } \\
(\mu \mathrm{g} / \mathrm{L})\end{array}$ & $\begin{array}{c}\text { Di- } \\
\text { meth- } \\
\text { enamid } \\
\text { OXA } \\
(\mu \mathrm{g} / \mathrm{L})\end{array}$ & $\begin{array}{c}\text { Flufen- } \\
\text { acet } \\
(\mu \mathrm{g} / \mathrm{L})\end{array}$ & $\begin{array}{c}\text { Flufen- } \\
\text { acet } \\
\text { ESA } \\
(\mu \mathrm{g} / \mathrm{L})\end{array}$ & $\begin{array}{c}\text { Flufen- } \\
\text { acet } \\
\text { OXA } \\
(\mu \mathrm{g} / \mathrm{L})\end{array}$ & $\begin{array}{c}\text { Meto- } \\
\text { lachlor } \\
(\mu \mathrm{g} / \mathrm{L})\end{array}$ & $\begin{array}{c}\text { Meto- } \\
\text { lachlor } \\
\text { ESA } \\
(\mu \mathrm{g} / \mathrm{L})\end{array}$ & $\begin{array}{c}\text { Meto- } \\
\text { lachlor } \\
\text { OXA } \\
(\mu \mathrm{g} / \mathrm{L})\end{array}$ \\
\hline \multicolumn{20}{|c|}{ Midcontinent Agricultural Chemical Research Herbicide Project (fig. 1)—Continued } \\
\hline \multicolumn{20}{|c|}{ lowa monitoring wells (DKE/DKW)—Continued } \\
\hline \multirow[t]{4}{*}{412852094275101} & Adair Co., IA & 08/30/95 & $<0.05$ & $<0.20$ & $<0.20$ & -- & $<0.05$ & 3.1 & $<0.20$ & -- & -- & -- & -- & -- & -- & -- & $<0.05$ & 1.2 & 0.75 \\
\hline & & 08/16/96 & $<.05$ & $<.20$ & $<.20$ & -- & $<.05$ & 3.3 & $<.20$ & -- & -- & -- & -- & -- & -- & -- & $<.05$ & .99 & $<.20$ \\
\hline & & 08/28/97 & $<.05$ & $<.20$ & $<.20$ & -- & $<.05$ & 1.3 & $<.20$ & -- & -- & -- & -- & -- & -- & -- & $<.05$ & .65 & $<.20$ \\
\hline & & 07/13/99 & $<.05$ & $<.20$ & $<.20$ & -- & $<.05$ & .91 & $<.20$ & -- & $<0.05$ & -- & -- & $<0.05$ & -- & -- & $<.05$ & .38 & $<.20$ \\
\hline \multirow[t]{2}{*}{412924094072203} & Dallas Co., IA & 06/20/95 & $<.05$ & $<.20$ & $<.20$ & -- & $<.05$ & $<.20$ & $<.20$ & -- & -- & -- & -- & -- & -- & -- & $<.05$ & 1.8 & $<.20$ \\
\hline & & 08/16/96 & $<.05$ & $<.20$ & $<.20$ & -- & $<.05$ & $<.20$ & $<.20$ & -- & -- & -- & -- & -- & -- & -- & $<.05$ & 2.0 & $<.20$ \\
\hline 412938082380201 & Mahaska Co., IA & 07/17/96 & $<.05$ & $<.20$ & $<.20$ & -- & $<.05$ & .23 & $<.20$ & -- & -- & -- & -- & -- & -- & -- & $<.05$ & $<.20$ & $<.20$ \\
\hline \multirow[t]{2}{*}{413037093290301} & Warren Co., IA & $06 / 22 / 95$ & $<.05$ & $<.20$ & $<.20$ & -- & $<.05$ & 3.5 & $<.20$ & -- & -- & -- & -- & -- & -- & -- & $<.05$ & 1.1 & $<.20$ \\
\hline & & 08/15/96 & $<.05$ & $<.20$ & $<.20$ & -- & $<.05$ & 2.5 & $<.20$ & -- & -- & -- & -- & -- & -- & -- & $<.05$ & 1.1 & $<.20$ \\
\hline \multirow[t]{4}{*}{413040090455001} & Scott Co., IA & 08/04/95 & $<.05$ & $<.20$ & $<.20$ & -- & $<.05$ & $<.20$ & $<.20$ & -- & -- & -- & -- & -- & -- & -- & $<.05$ & $<.20$ & $<.20$ \\
\hline & & $07 / 22 / 97$ & $<.05$ & $<.20$ & $<.20$ & -- & $<.05$ & $<.20$ & $<.20$ & -- & -- & -- & -- & -- & -- & -- & $<.05$ & $<.20$ & $<.20$ \\
\hline & & 06/15/99 & $<.05$ & $<.20$ & $<.20$ & -- & $<.05$ & $<.20$ & $<.20$ & -- & $<.05$ & -- & -- & $<.05$ & -- & -- & $<.05$ & $<.20$ & $<.20$ \\
\hline & & 08/07/01 & $<.05$ & $<.05$ & $<.05$ & -- & $<.05$ & $<.05$ & $<.05$ & -- & $<.05$ & $<0.05$ & $<0.05$ & $<.05$ & $<0.05$ & $<0.05$ & $<.05$ & $<.05$ & $<.05$ \\
\hline \multirow[t]{5}{*}{413040093290501} & Warren Co., IA & $06 / 22 / 95$ & $<.05$ & $<.20$ & -- & -- & $<.05$ & 2.3 & $<.20$ & -- & -- & -- & -- & -- & -- & -- & $<.05$ & .74 & -- \\
\hline & & 08/15/96 & $<.05$ & $<.20$ & $<.20$ & -- & $<.05$ & 2.4 & $<.20$ & -- & -- & -- & -- & -- & -- & -- & $<.05$ & 1.3 & $<.20$ \\
\hline & & 07/21/97 & $<.05$ & $<.20$ & $<.20$ & -- & $<.05$ & 1.7 & $<.20$ & -- & -- & -- & -- & -- & -- & -- & $<.05$ & .82 & $<.20$ \\
\hline & & 08/09/99 & $<.05$ & $<.20$ & $<.20$ & -- & $<.05$ & .56 & $<.20$ & -- & $<.05$ & -- & -- & $<.05$ & -- & -- & $<.05$ & .61 & $<.20$ \\
\hline & & 07/27/01 & $<.05$ & $<.05$ & $<.05$ & -- & $<.05$ & 1.6 & $<.05$ & -- & $<.05$ & $<.05$ & $<.05$ & $<.05$ & $<.05$ & $<.05$ & $<.05$ & .38 & $<.05$ \\
\hline \multirow[t]{2}{*}{413048093062101} & Jasper Co., IA & $07 / 21 / 97$ & $<.05$ & $<.20$ & $<.20$ & -- & $<.05$ & $<.20$ & $<.20$ & -- & -- & -- & -- & -- & -- & -- & $<.05$ & $<.20$ & $<.20$ \\
\hline & & 07/14/99 & $<.05$ & $<.20$ & $<.20$ & -- & $<.05$ & $<.20$ & $<.20$ & -- & $<.05$ & -- & -- & $<.05$ & -- & -- & $<.05$ & $<.20$ & $<.20$ \\
\hline \multirow[t]{2}{*}{413049095254501} & Shelby Co., IA & 08/18/95 & $<.05$ & $<.20$ & $<.20$ & -- & $<.05$ & $<.20$ & $<.20$ & -- & -- & -- & -- & -- & -- & -- & .11 & 2.3 & $<.20$ \\
\hline & & 07/09/96 & $<.05$ & $<.20$ & $<.20$ & -- & $<.05$ & $<.20$ & $<.20$ & -- & -- & -- & -- & -- & -- & -- & $<.05$ & 2.1 & $<.20$ \\
\hline
\end{tabular}


Table 5. Analytical results for selected acetamide herbicides and their degradation products, ethanesulfonic acid, oxanilic acid, and sulfinyl acetic acid, in ground-water samples, 1994-2002.-Continued

[OGRL, U.S. Geological Survey Organic Geochemistry Research Laboratory, Lawrence, Kansas; ESA, ethanesulfonic acid; OXA, oxanilic acid; SAA, sulfinyl acetic acid; --, no data; <, less than]

\begin{tabular}{|c|c|c|c|c|c|c|c|c|c|c|c|c|c|c|c|c|c|c|c|}
\hline Site number & Site location & $\begin{array}{c}\text { Date of col- } \\
\text { lection } \\
\text { (month/day/ } \\
\text { year) }\end{array}$ & $\begin{array}{c}\text { Aceto- } \\
\text { chlor } \\
(\mu \mathrm{g} / \mathrm{L})\end{array}$ & $\begin{array}{l}\text { Aceto- } \\
\text { chlor } \\
\text { ESA } \\
(\mu \mathrm{g} / \mathrm{L})\end{array}$ & $\begin{array}{c}\text { Aceto- } \\
\text { chlor } \\
\text { OXA } \\
(\mu \mathrm{g} / \mathrm{L})\end{array}$ & $\begin{array}{l}\text { Aceto- } \\
\text { chlor } \\
\text { SAA } \\
(\mu \mathrm{g} / \mathrm{L})\end{array}$ & $\begin{array}{l}\text { Ala- } \\
\text { chlor } \\
(\mu \mathrm{g} / \mathrm{L})\end{array}$ & $\begin{array}{c}\text { Ala- } \\
\text { chlor } \\
\text { ESA } \\
(\mu \mathrm{g} / \mathrm{L})\end{array}$ & $\begin{array}{c}\text { Ala- } \\
\text { chlor } \\
\text { OXA } \\
(\mu \mathrm{g} / \mathrm{L})\end{array}$ & $\begin{array}{l}\text { Ala- } \\
\text { chlor } \\
\text { SAA } \\
(\mu \mathrm{g} / \mathrm{L})\end{array}$ & $\begin{array}{c}\text { Di- } \\
\text { meth- } \\
\text { enamid } \\
(\mu \mathrm{g} / \mathrm{L})\end{array}$ & $\begin{array}{c}\text { Di- } \\
\text { meth- } \\
\text { enamid } \\
\text { ESA } \\
(\mu \mathrm{g} / \mathrm{L})\end{array}$ & $\begin{array}{c}\text { Di- } \\
\text { meth- } \\
\text { enamid } \\
\text { OXA } \\
(\mu \mathrm{g} / \mathrm{L})\end{array}$ & $\begin{array}{c}\text { Flufen- } \\
\text { acet } \\
(\mu \mathrm{g} / \mathrm{L})\end{array}$ & $\begin{array}{c}\text { Flufen- } \\
\text { acet } \\
\text { ESA } \\
(\mu \mathrm{g} / \mathrm{L})\end{array}$ & $\begin{array}{c}\text { Flufen- } \\
\text { acet } \\
\text { OXA } \\
(\mu \mathrm{g} / \mathrm{L})\end{array}$ & $\begin{array}{c}\text { Meto- } \\
\text { lachlor } \\
(\mu \mathrm{g} / \mathrm{L})\end{array}$ & $\begin{array}{c}\text { Meto- } \\
\text { lachlor } \\
\text { ESA } \\
(\mu \mathrm{g} / \mathrm{L})\end{array}$ & $\begin{array}{c}\text { Meto- } \\
\text { lachlor } \\
\text { OXA } \\
(\mu \mathrm{g} / \mathrm{L})\end{array}$ \\
\hline \multicolumn{20}{|c|}{ Midcontinent Agricultural Chemical Research Herbicide Project (fig. 1)—Continued } \\
\hline \multicolumn{20}{|c|}{ lowa monitoring wells (DKE/DKW)—Continued } \\
\hline \multirow[t]{4}{*}{413049095254501} & Shelby Co., IA & $08 / 25 / 97$ & $<0.05$ & $<0.20$ & $<0.20$ & -- & $<0.05$ & $<0.20$ & $<0.20$ & -- & -- & -- & -- & -- & -- & -- & $<0.05$ & 1.2 & $<0.20$ \\
\hline & & $07 / 22 / 98$ & $<.05$ & $<.20$ & $<.20$ & -- & $<.05$ & $<.20$ & $<.20$ & -- & -- & -- & -- & -- & -- & -- & $<.05$ & 3.5 & $<.20$ \\
\hline & & 07/12/99 & $<.05$ & $<.20$ & $<.20$ & -- & $<.05$ & $<.20$ & $<.20$ & -- & $<0.05$ & -- & -- & $<0.05$ & -- & -- & .05 & 2.1 & $<.20$ \\
\hline & & 08/17/01 & $<.05$ & $<.05$ & $<.05$ & -- & $<.05$ & $<.05$ & $<.05$ & -- & $<.05$ & $<0.05$ & $<0.05$ & $<.05$ & $<0.05$ & $<0.05$ & $<.05$ & 1.0 & $<.05$ \\
\hline \multirow[t]{6}{*}{413234094552401} & Audubon Co., IA & $08 / 16 / 95$ & $<.05$ & $<.20$ & $<.20$ & -- & $<.05$ & $<.20$ & $<.20$ & -- & -- & -- & -- & -- & -- & -- & $<.05$ & .60 & $<.20$ \\
\hline & & 07/10/96 & $<.05$ & $<.20$ & $<.20$ & -- & $<.05$ & $<.20$ & $<.20$ & -- & -- & -- & -- & -- & -- & -- & $<.05$ & .35 & $<.20$ \\
\hline & & $08 / 25 / 97$ & $<.05$ & $<.20$ & $<.20$ & -- & $<.05$ & $<.20$ & $<.20$ & -- & -- & -- & -- & -- & -- & -- & $<.05$ & .29 & $<.20$ \\
\hline & & $07 / 31 / 98$ & $<.05$ & $<.20$ & $<.20$ & -- & $<.05$ & $<.20$ & $<.20$ & -- & -- & -- & -- & -- & -- & -- & $<.05$ & .45 & $<.20$ \\
\hline & & 07/13/99 & $<.05$ & $<.20$ & $<.20$ & -- & $<.05$ & $<.20$ & $<.20$ & -- & $<.05$ & -- & -- & $<.05$ & -- & -- & $<.05$ & .32 & $<.20$ \\
\hline & & 08/17/01 & $<.05$ & $<.05$ & $<.05$ & -- & $<.05$ & .18 & $<.05$ & -- & $<.05$ & $<.05$ & $<.05$ & $<.05$ & $<.05$ & $<.05$ & $<.05$ & .63 & .18 \\
\hline \multirow[t]{2}{*}{413303094001001} & Dallas Co., IA & $06 / 20 / 95$ & $<.05$ & $<.20$ & -- & -- & $<.05$ & $<.20$ & $<.20$ & -- & -- & -- & -- & -- & -- & -- & .06 & $<.20$ & -- \\
\hline & & $08 / 16 / 96$ & $<.05$ & .45 & $<.20$ & -- & $<.05$ & $<.20$ & $<.20$ & -- & -- & -- & -- & -- & -- & -- & $<.05$ & $<.20$ & $<.20$ \\
\hline \multirow[t]{2}{*}{413418093432403} & Polk Co., IA & $06 / 20 / 95$ & $<.05$ & $<.20$ & -- & -- & $<.05$ & $<.20$ & $<.20$ & -- & -- & -- & -- & -- & -- & -- & $<.05$ & $<.20$ & -- \\
\hline & & $08 / 15 / 96$ & $<.05$ & $<.20$ & $<.20$ & -- & $<.05$ & $<.20$ & $<.20$ & -- & -- & -- & -- & -- & -- & -- & $<.05$ & $<.20$ & $<.20$ \\
\hline 413423092503601 & Jasper Co., IA & $07 / 24 / 97$ & $<.05$ & $<.20$ & $<.20$ & -- & $<.05$ & $<.20$ & $<.20$ & -- & -- & -- & -- & -- & -- & -- & $<.05$ & $<.20$ & $<.20$ \\
\hline \multirow[t]{4}{*}{413521090511001} & Muscatine Co., IA & $07 / 15 / 96$ & $<.05$ & $<.20$ & .39 & -- & $<.05$ & $<.20$ & $<.20$ & -- & -- & -- & -- & -- & -- & -- & $<.05$ & $<.20$ & $<.20$ \\
\hline & & $07 / 22 / 97$ & $<.05$ & $<.20$ & $<.20$ & -- & $<.05$ & $<.20$ & $<.20$ & -- & -- & -- & -- & -- & -- & -- & $<.05$ & $<.20$ & $<.20$ \\
\hline & & $06 / 14 / 99$ & $<.05$ & $<.20$ & $<.20$ & -- & $<.05$ & $<.20$ & $<.20$ & -- & $<.05$ & -- & -- & $<.05$ & -- & -- & $<.05$ & $<.20$ & $<.20$ \\
\hline & & 08/07/01 & $<.05$ & $<.05$ & $<.05$ & -- & $<.05$ & $<.05$ & $<.05$ & -- & $<.05$ & $<.05$ & $<.05$ & $<.05$ & $<.05$ & $<.05$ & $<.05$ & $<.05$ & $<.05$ \\
\hline \multirow[t]{2}{*}{413810095185401} & Shelby Co., IA & $08 / 18 / 95$ & $<.05$ & $<.20$ & $<.20$ & -- & $<.05$ & .20 & $<.20$ & -- & -- & -- & -- & -- & -- & -- & $<.05$ & .42 & .23 \\
\hline & & 07/09/96 & $<.05$ & $<.20$ & $<.20$ & -- & $<.05$ & 2.3 & .23 & -- & -- & -- & -- & -- & -- & -- & $<.05$ & .26 & $<.20$ \\
\hline
\end{tabular}


Table 5. Analytical results for selected acetamide herbicides and their degradation products, ethanesulfonic acid, oxanilic acid, and sulfinyl acetic acid, in ground-water samples, 1994-2002.-Continued

[OGRL, U.S. Geological Survey Organic Geochemistry Research Laboratory, Lawrence, Kansas; ESA, ethanesulfonic acid; OXA, oxanilic acid; SAA, sulfinyl acetic acid; --, no data; <, less than]

\begin{tabular}{|c|c|c|c|c|c|c|c|c|c|c|c|c|c|c|c|c|c|c|c|}
\hline Site number & Site location & $\begin{array}{l}\text { Date of col- } \\
\text { lection } \\
\text { (month/day/ } \\
\text { year) }\end{array}$ & $\begin{array}{c}\text { Aceto- } \\
\text { chlor } \\
(\mu \mathrm{g} / \mathrm{L})\end{array}$ & $\begin{array}{l}\text { Aceto- } \\
\text { chlor } \\
\text { ESA } \\
(\mu \mathrm{g} / \mathrm{L})\end{array}$ & $\begin{array}{c}\text { Aceto- } \\
\text { chlor } \\
\text { OXA } \\
(\mu \mathrm{g} / \mathrm{L})\end{array}$ & $\begin{array}{l}\text { Aceto- } \\
\text { chlor } \\
\text { SAA } \\
(\mu \mathrm{g} / \mathrm{L})\end{array}$ & $\begin{array}{l}\text { Ala- } \\
\text { chlor } \\
(\mu \mathrm{g} / \mathrm{L})\end{array}$ & $\begin{array}{c}\text { Ala- } \\
\text { chlor } \\
\text { ESA } \\
(\mu \mathrm{g} / \mathrm{L})\end{array}$ & $\begin{array}{c}\text { Ala- } \\
\text { chlor } \\
\text { OXA } \\
(\mu \mathrm{g} / \mathrm{L})\end{array}$ & $\begin{array}{c}\text { Ala- } \\
\text { chlor } \\
\text { SAA } \\
(\mu \mathrm{g} / \mathrm{L})\end{array}$ & $\begin{array}{c}\text { Di- } \\
\text { meth- } \\
\text { enamid } \\
(\mu \mathrm{g} / \mathrm{L})\end{array}$ & $\begin{array}{c}\text { Di- } \\
\text { meth- } \\
\text { enamid } \\
\text { ESA } \\
(\mu \mathrm{g} / \mathrm{L})\end{array}$ & $\begin{array}{c}\text { Di- } \\
\text { meth- } \\
\text { enamid } \\
\text { OXA } \\
(\mu \mathrm{g} / \mathrm{L})\end{array}$ & $\begin{array}{c}\text { Flufen- } \\
\text { acet } \\
(\mu \mathrm{g} / \mathrm{L})\end{array}$ & $\begin{array}{c}\text { Flufen- } \\
\text { acet } \\
\text { ESA } \\
(\mu \mathrm{g} / \mathrm{L})\end{array}$ & $\begin{array}{c}\text { Flufen- } \\
\text { acet } \\
\text { OXA } \\
(\mu \mathrm{g} / \mathrm{L})\end{array}$ & $\begin{array}{l}\text { Meto- } \\
\text { lachlor } \\
(\mu \mathrm{g} / \mathrm{L})\end{array}$ & $\begin{array}{c}\text { Meto- } \\
\text { lachlor } \\
\text { ESA } \\
(\mu \mathrm{g} / \mathrm{L})\end{array}$ & $\begin{array}{c}\text { Meto- } \\
\text { lachlor } \\
\text { OXA } \\
(\mu \mathrm{g} / \mathrm{L})\end{array}$ \\
\hline \multicolumn{20}{|c|}{ Midcontinent Agricultural Chemical Research Herbicide Project (fig. 1)—Continued } \\
\hline \multicolumn{20}{|c|}{ lowa monitoring wells (DKE/DKW)—Continued } \\
\hline \multirow[t]{2}{*}{413836094161701} & Dallas Co., IA & 08/28/97 & $<0.05$ & $<0.20$ & $<.20$ & -- & $<0.05$ & $<0.20$ & $<0.20$ & -- & -- & -- & -- & -- & -- & -- & $<0.05$ & $<0.20$ & $<0.20$ \\
\hline & & $08 / 15 / 01$ & $<.05$ & $<.05$ & $<.05$ & -- & $<.05$ & $<.05$ & $<.05$ & -- & $<0.05$ & $<0.05$ & $<0.05$ & $<0.05$ & $<0.05$ & $<0.05$ & $<.05$ & $<.05$ & $<.05$ \\
\hline \multirow[t]{5}{*}{413913093070001} & Jasper Co., IA & 07/16/96 & $<.05$ & $<.20$ & $<.20$ & -- & $<.05$ & $<.20$ & $<.20$ & -- & -- & -- & -- & -- & -- & -- & $<.05$ & .98 & $<.20$ \\
\hline & & $07 / 24 / 97$ & $<.05$ & $<.20$ & $<.20$ & -- & $<.05$ & $<.20$ & $<.20$ & -- & -- & -- & -- & -- & -- & -- & .07 & .83 & $<.20$ \\
\hline & & 06/03/98 & $<.05$ & $<.20$ & $<.20$ & -- & $<.05$ & $<.20$ & $<.20$ & -- & -- & -- & -- & -- & -- & -- & $<.05$ & 1.1 & $<.20$ \\
\hline & & 07/14/99 & $<.05$ & $<.20$ & $<.20$ & -- & $<.05$ & $<.20$ & $<.20$ & -- & $<.05$ & -- & -- & $<.05$ & -- & -- & $<.05$ & .97 & $<.20$ \\
\hline & & 09/05/01 & $<.05$ & $<.05$ & $<.05$ & -- & $<.05$ & .06 & $<.05$ & -- & $<.05$ & $<.05$ & $<.05$ & $<.05$ & $<.05$ & $<.05$ & $<.05$ & .20 & $<.05$ \\
\hline \multirow[t]{4}{*}{413923090350901} & Scott Co., IA & 08/03/95 & $<.05$ & $<.20$ & $<.20$ & -- & $<.05$ & $<.20$ & $<.20$ & -- & -- & -- & -- & -- & -- & -- & $<.05$ & $<.20$ & $<.20$ \\
\hline & & $07 / 22 / 97$ & $<.05$ & $<.20$ & $<.20$ & -- & $<.05$ & $<.20$ & $<.20$ & -- & -- & -- & -- & -- & -- & -- & $<.05$ & $<.20$ & $<.20$ \\
\hline & & $06 / 15 / 99$ & $<.05$ & $<.20$ & $<.20$ & -- & $<.05$ & $<.20$ & $<.20$ & -- & $<.05$ & -- & -- & $<.05$ & -- & -- & $<.05$ & $<.20$ & $<.20$ \\
\hline & & 08/06/01 & $<.05$ & $<.05$ & $<.05$ & -- & $<.05$ & $<.05$ & $<.05$ & -- & $<.05$ & $<.05$ & $<.05$ & $<.05$ & $<.05$ & $<.05$ & $<.05$ & $<.05$ & $<.05$ \\
\hline 414026091210201 & Cedar Co., IA & 06/27/96 & $<.05$ & $<.20$ & $<.20$ & -- & .11 & 8.5 & 33 & -- & -- & -- & -- & -- & -- & -- & 9.0 & 8.6 & 15 \\
\hline \multirow[t]{2}{*}{414032091210001} & Cedar Co., IA & 06/26/97 & $<.05$ & $<.20$ & $<.20$ & -- & $<.05$ & 2.0 & 7.4 & -- & -- & -- & -- & -- & -- & -- & 1.7 & 2.5 & 3.1 \\
\hline & & 09/19/01 & $<.05$ & $<.05$ & .16 & -- & $<.05$ & .41 & $<.05$ & -- & $<.05$ & $<.05$ & $<.05$ & $<.05$ & $<.05$ & $<.05$ & 3.2 & .54 & 3.6 \\
\hline \multirow[t]{6}{*}{414236096012501} & Harrison Co., IA & 08/29/95 & $<.05$ & $<.20$ & $<.20$ & -- & $<.05$ & $<.20$ & $<.20$ & -- & -- & -- & -- & -- & -- & -- & $<.05$ & $<.20$ & $<.20$ \\
\hline & & 08/12/96 & $<.05$ & $<.20$ & $<.20$ & -- & $<.05$ & $<.20$ & $<.20$ & -- & -- & -- & -- & -- & -- & -- & $<.05$ & $<.20$ & $<.20$ \\
\hline & & 06/27/97 & $<.05$ & $<.20$ & $<.20$ & -- & $<.05$ & $<.20$ & $<.20$ & -- & -- & -- & -- & -- & -- & -- & $<.05$ & $<.20$ & $<.20$ \\
\hline & & $07 / 22 / 98$ & $<.05$ & $<.20$ & $<.20$ & -- & $<.05$ & $<.20$ & $<.20$ & -- & -- & -- & -- & -- & -- & -- & $<.05$ & $<.20$ & $<.20$ \\
\hline & & 07/12/99 & $<.05$ & $<.20$ & $<.20$ & -- & $<.05$ & $<.20$ & $<.20$ & -- & $<.05$ & -- & -- & $<.05$ & -- & -- & $<.05$ & $<.20$ & $<.20$ \\
\hline & & 08/27/01 & $<.05$ & $<.05$ & $<.05$ & -- & $<.05$ & $<.05$ & $<.05$ & -- & $<.05$ & $<.05$ & $<.05$ & $<.05$ & $<.05$ & $<.05$ & $<.05$ & $<.05$ & $<.05$ \\
\hline 041429093145401 & Jasper Co., IA & 07/17/96 & $<.05$ & $<.20$ & $<.20$ & -- & $<.05$ & $<.20$ & .28 & -- & -- & -- & -- & -- & -- & -- & $<.05$ & $<.20$ & $<.20$ \\
\hline
\end{tabular}


Table 5. Analytical results for selected acetamide herbicides and their degradation products, ethanesulfonic acid, oxanilic acid, and sulfinyl acetic acid, in ground-water samples, 1994-2002.-Continued

[OGRL, U.S. Geological Survey Organic Geochemistry Research Laboratory, Lawrence, Kansas; ESA, ethanesulfonic acid; OXA, oxanilic acid; SAA, sulfinyl acetic acid; --, no data; <, less than]

\begin{tabular}{|c|c|c|c|c|c|c|c|c|c|c|c|c|c|c|c|c|c|c|c|}
\hline Site number & Site location & $\begin{array}{l}\text { Date of col- } \\
\text { lection } \\
\text { (month/day/ } \\
\text { year) }\end{array}$ & $\begin{array}{l}\text { Aceto- } \\
\text { chlor } \\
(\mu \mathrm{g} / \mathrm{L})\end{array}$ & $\begin{array}{l}\text { Aceto- } \\
\text { chlor } \\
\text { ESA } \\
(\mu \mathrm{g} / \mathrm{L})\end{array}$ & $\begin{array}{l}\text { Aceto- } \\
\text { chlor } \\
\text { OXA } \\
(\mu \mathrm{g} / \mathrm{L})\end{array}$ & $\begin{array}{l}\text { Aceto- } \\
\text { chlor } \\
\text { SAA } \\
(\mu \mathrm{g} / \mathrm{L})\end{array}$ & $\begin{array}{l}\text { Ala- } \\
\text { chlor } \\
(\mu \mathrm{g} / \mathrm{L})\end{array}$ & $\begin{array}{l}\text { Ala- } \\
\text { chlor } \\
\text { ESA } \\
(\mu \mathrm{g} / \mathrm{L})\end{array}$ & $\begin{array}{c}\text { Ala- } \\
\text { chlor } \\
\text { OXA } \\
(\mu \mathrm{g} / \mathrm{L})\end{array}$ & $\begin{array}{l}\text { Ala- } \\
\text { chlor } \\
\text { SAA } \\
(\mu \mathrm{g} / \mathrm{L})\end{array}$ & $\begin{array}{l}\text { Di- } \\
\text { meth- } \\
\text { enamid } \\
(\mu \mathrm{g} / \mathrm{L})\end{array}$ & $\begin{array}{c}\text { Di- } \\
\text { meth- } \\
\text { enamid } \\
\text { ESA } \\
(\mu \mathrm{g} / \mathrm{L})\end{array}$ & $\begin{array}{c}\text { Di- } \\
\text { meth- } \\
\text { enamid } \\
\text { OXA } \\
(\mu \mathrm{g} / \mathrm{L})\end{array}$ & $\begin{array}{c}\text { Flufen- } \\
\text { acet } \\
(\mu \mathrm{g} / \mathrm{L})\end{array}$ & $\begin{array}{c}\text { Flufen- } \\
\text { acet } \\
\text { ESA } \\
(\mu \mathrm{g} / \mathrm{L})\end{array}$ & $\begin{array}{c}\text { Flufen- } \\
\text { acet } \\
\text { OXA } \\
(\mu \mathrm{g} / \mathrm{L})\end{array}$ & $\begin{array}{l}\text { Meto- } \\
\text { lachlor } \\
(\mu \mathrm{g} / \mathrm{L})\end{array}$ & $\begin{array}{l}\text { Meto- } \\
\text { lachlor } \\
\text { ESA } \\
(\mu \mathrm{g} / \mathrm{L})\end{array}$ & $\begin{array}{c}\text { Meto- } \\
\text { lachlor } \\
\text { OXA } \\
(\mu \mathrm{g} / \mathrm{L})\end{array}$ \\
\hline \multicolumn{20}{|c|}{ Midcontinent Agricultural Chemical Research Herbicide Project (fig. 1)-Continued } \\
\hline \multicolumn{20}{|c|}{ lowa monitoring wells (DKE/DKW)—Continued } \\
\hline 414251092541701 & Jasper Co., IA & $07 / 17 / 96$ & $<0.05$ & $<0.20$ & $<0.20$ & -- & $<0.05$ & 0.39 & $<0.20$ & -- & -- & -- & -- & -- & -- & -- & $<0.05$ & 1.9 & $<0.20$ \\
\hline \multirow[t]{2}{*}{414409093241001} & Polk Co., IA & $06 / 21 / 95$ & $<.05$ & $<.20$ & $<.20$ & -- & $<.05$ & .21 & $<.20$ & -- & -- & -- & -- & -- & -- & -- & $<.05$ & 1.7 & .23 \\
\hline & & 08/15/96 & $<.05$ & $<.20$ & $<.20$ & -- & $<.05$ & $<.20$ & $<.20$ & -- & -- & -- & -- & -- & -- & -- & .07 & 2.1 & .21 \\
\hline 414430092433001 & Poweshiek Co., IA & 09/18/01 & $<.05$ & $<.05$ & $<.05$ & -- & $<.05$ & $<.05$ & $<.05$ & -- & $<0.05$ & $<0.05$ & $<0.05$ & $<0.05$ & $<0.05$ & $<0.05$ & $<.05$ & $<.05$ & $<.05$ \\
\hline \multirow[t]{4}{*}{414520092112001} & Iowa Co., IA & 08/28/97 & $<.05$ & $<.20$ & $<.20$ & -- & $<.05$ & $<.20$ & $<.20$ & -- & -- & -- & -- & -- & -- & -- & $<.05$ & $<.20$ & $<.20$ \\
\hline & & 07/23/98 & $<.05$ & $<.20$ & $<.20$ & -- & $<.05$ & $<.20$ & $<.20$ & -- & -- & -- & -- & -- & -- & -- & $<.05$ & $<.20$ & $<.20$ \\
\hline & & 08/09/99 & $<.05$ & $<.20$ & $<.20$ & -- & $<.05$ & $<.20$ & $<.20$ & -- & $<.05$ & -- & -- & $<.05$ & -- & -- & $<.05$ & $<.20$ & $<.20$ \\
\hline & & 09/20/01 & $<.05$ & $<.05$ & $<.05$ & -- & $<.05$ & $<.05$ & $<.05$ & -- & $<.05$ & $<.05$ & $<.05$ & $<.05$ & $<.05$ & $<.05$ & $<.05$ & $<.05$ & $<.05$ \\
\hline \multirow[t]{6}{*}{414652090153201} & Clinton Co., IA & $06 / 22 / 95$ & $<.05$ & $<.20$ & $<.20$ & -- & $<.05$ & $<.20$ & $<.20$ & -- & -- & -- & -- & -- & -- & -- & $<.05$ & $<.20$ & $<.20$ \\
\hline & & 06/20/96 & $<.05$ & $<.20$ & $<.20$ & -- & $<.05$ & .20 & $<.20$ & -- & -- & -- & -- & -- & -- & -- & $<.05$ & $<.20$ & $<.20$ \\
\hline & & $06 / 12 / 97$ & $<.05$ & $<.20$ & $<.20$ & -- & $<.05$ & $<.20$ & $<.20$ & -- & -- & -- & -- & -- & -- & -- & $<.05$ & $<.20$ & $<.20$ \\
\hline & & 07/21/98 & $<.05$ & $<.20$ & $<.20$ & -- & $<.05$ & $<.20$ & $<.20$ & -- & -- & -- & -- & -- & -- & -- & $<.05$ & $<.20$ & $<.20$ \\
\hline & & $06 / 14 / 99$ & $<.05$ & 1.1 & $<.20$ & -- & $<.05$ & $<.20$ & $<.20$ & -- & $<.05$ & -- & -- & $<.05$ & -- & -- & $<.05$ & $<.20$ & $<.20$ \\
\hline & & 08/06/01 & $<.05$ & $<.05$ & $<.05$ & -- & $<.05$ & $<.05$ & $<.05$ & -- & $<.05$ & $<.05$ & $<.05$ & $<.05$ & $<.05$ & $<.05$ & $<.05$ & $<.05$ & $<.05$ \\
\hline 414736091534501 & Iowa Co., IA & 07/19/96 & $<.05$ & $<.20$ & $<.20$ & -- & $<.05$ & .49 & $<.20$ & -- & -- & -- & -- & -- & -- & -- & $<.05$ & .97 & .31 \\
\hline \multirow[t]{2}{*}{414825091511201} & Iowa Co., IA & $07 / 28 / 97$ & $<.05$ & $<.20$ & $<.20$ & -- & $<.05$ & $<.20$ & $<.20$ & -- & -- & -- & -- & -- & -- & -- & $<.05$ & $<.20$ & $<.20$ \\
\hline & & 08/30/01 & $<.05$ & $<.05$ & $<.05$ & -- & $<.05$ & $<.05$ & $<.05$ & -- & $<.05$ & $<.05$ & $<.05$ & $<.05$ & $<.05$ & $<.05$ & $<.05$ & $<.05$ & $<.05$ \\
\hline \multirow[t]{4}{*}{414930090321601} & Clinton Co., IA & 08/03/95 & $<.05$ & $<.20$ & $<.20$ & -- & $<.05$ & $<.20$ & $<.20$ & -- & -- & -- & -- & -- & -- & -- & $<.05$ & $<.20$ & $<.20$ \\
\hline & & 06/12/97 & $<.05$ & $<.20$ & $<.20$ & -- & $<.05$ & $<.20$ & $<.20$ & -- & -- & -- & -- & -- & -- & -- & $<.05$ & $<.20$ & $<.20$ \\
\hline & & 06/14/99 & $<.05$ & $<.20$ & $<.20$ & -- & $<.05$ & $<.20$ & $<.20$ & -- & $<.05$ & -- & -- & $<.05$ & -- & -- & $<.05$ & $<.20$ & $<.20$ \\
\hline & & 08/06/01 & $<.05$ & $<.05$ & $<.05$ & -- & $<.05$ & $<.05$ & $<.05$ & -- & $<.05$ & $<.05$ & $<.05$ & $<.05$ & $<.05$ & $<.05$ & $<.05$ & $<.05$ & $<.05$ \\
\hline
\end{tabular}


Table 5. Analytical results for selected acetamide herbicides and their degradation products, ethanesulfonic acid, oxanilic acid, and sulfinyl acetic acid, in ground-water samples, 1994-2002.-Continued

[OGRL, U.S. Geological Survey Organic Geochemistry Research Laboratory, Lawrence, Kansas; ESA, ethanesulfonic acid; OXA, oxanilic acid; SAA, sulfinyl acetic acid; --, no data; <, less than]

\begin{tabular}{|c|c|c|c|c|c|c|c|c|c|c|c|c|c|c|c|c|c|c|c|}
\hline Site number & Site location & $\begin{array}{l}\text { Date of col- } \\
\text { lection } \\
\text { (month/day/ } \\
\text { year) }\end{array}$ & $\begin{array}{c}\text { Aceto- } \\
\text { chlor } \\
(\mu \mathrm{g} / \mathrm{L})\end{array}$ & $\begin{array}{l}\text { Aceto- } \\
\text { chlor } \\
\text { ESA } \\
(\mu \mathrm{g} / \mathrm{L})\end{array}$ & $\begin{array}{c}\text { Aceto- } \\
\text { chlor } \\
\text { OXA } \\
(\mu \mathrm{g} / \mathrm{L})\end{array}$ & $\begin{array}{c}\text { Aceto- } \\
\text { chlor } \\
\text { SAA } \\
(\mu \mathrm{g} / \mathrm{L})\end{array}$ & $\begin{array}{l}\text { Ala- } \\
\text { chlor } \\
(\mu \mathrm{g} / \mathrm{L})\end{array}$ & $\begin{array}{c}\text { Ala- } \\
\text { chlor } \\
\text { ESA } \\
(\mu \mathrm{g} / \mathrm{L})\end{array}$ & $\begin{array}{c}\text { Ala- } \\
\text { chlor } \\
\text { OXA } \\
(\mu \mathrm{g} / \mathrm{L})\end{array}$ & $\begin{array}{l}\text { Ala- } \\
\text { chlor } \\
\text { SAA } \\
(\mu \mathrm{g} / \mathrm{L})\end{array}$ & $\begin{array}{c}\text { Di- } \\
\text { meth- } \\
\text { enamid } \\
(\mu \mathrm{g} / \mathrm{L})\end{array}$ & $\begin{array}{c}\text { Di- } \\
\text { meth- } \\
\text { enamid } \\
\text { ESA } \\
(\mu \mathrm{g} / \mathrm{L})\end{array}$ & $\begin{array}{c}\text { Di- } \\
\text { meth- } \\
\text { enamid } \\
\text { OXA } \\
(\mu \mathrm{g} / \mathrm{L})\end{array}$ & $\begin{array}{c}\text { Flufen- } \\
\text { acet } \\
(\mu \mathrm{g} / \mathrm{L})\end{array}$ & $\begin{array}{c}\text { Flufen- } \\
\text { acet } \\
\text { ESA } \\
(\mu \mathrm{g} / \mathrm{L})\end{array}$ & $\begin{array}{c}\text { Flufen- } \\
\text { acet } \\
\text { OXA } \\
(\mu \mathrm{g} / \mathrm{L})\end{array}$ & $\begin{array}{l}\text { Meto- } \\
\text { lachlor } \\
(\mu \mathrm{g} / \mathrm{L})\end{array}$ & $\begin{array}{c}\text { Meto- } \\
\text { lachlor } \\
\text { ESA } \\
(\mu \mathrm{g} / \mathrm{L})\end{array}$ & $\begin{array}{c}\text { Meto- } \\
\text { lachlor } \\
\text { OXA } \\
(\mu \mathrm{g} / \mathrm{L})\end{array}$ \\
\hline \multicolumn{20}{|c|}{ Midcontinent Agricultural Chemical Research Herbicide Project (fig. 1)—Continued } \\
\hline & & & & lon & va moni & toring $n$ & vells (D) & KE/DKV & /)-Con & tinued & & & & & & & & & \\
\hline \multirow[t]{6}{*}{415057094065301} & Dallas Co., IA & $06 / 30 / 95$ & $<0.05$ & $<0.05$ & -- & -- & $<0.05$ & 1.5 & $<0.05$ & -- & -- & -- & -- & -- & -- & -- & $<0.05$ & 0.30 & -- \\
\hline & & $08 / 14 / 96$ & $<.05$ & $<.20$ & $<0.20$ & -- & $<.05$ & 1.0 & $<.20$ & -- & -- & -- & -- & -- & -- & -- & $<.05$ & 1.2 & 0.26 \\
\hline & & $07 / 22 / 97$ & $<.05$ & $<.05$ & $<.20$ & -- & $<.05$ & .55 & $<.20$ & -- & -- & -- & -- & -- & -- & -- & $<.05$ & .61 & $<.20$ \\
\hline & & $07 / 27 / 98$ & $<.05$ & .41 & $<.20$ & -- & $<.05$ & .94 & $<.20$ & -- & -- & -- & -- & -- & -- & -- & $<.05$ & 1.4 & .49 \\
\hline & & 07/14/99 & $<.05$ & .23 & .22 & -- & $<.05$ & .53 & $<.20$ & -- & $<0.05$ & -- & -- & $<0.05$ & -- & -- & .07 & .55 & $<.20$ \\
\hline & & $07 / 26 / 01$ & $<.05$ & $<.05$ & .05 & -- & $<.05$ & .70 & $<.05$ & -- & $<.05$ & $<0.05$ & $<0.05$ & $<.05$ & $<0.05$ & $<0.05$ & $<.05$ & 1.1 & .28 \\
\hline 415200921120012 & Iowa Co., IA & $07 / 19 / 96$ & $<.05$ & $<.20$ & $<.20$ & -- & $<.05$ & $<.20$ & $<.20$ & -- & -- & -- & -- & -- & -- & -- & $<.05$ & $<.20$ & $<.20$ \\
\hline \multirow[t]{4}{*}{415233094403201} & Carroll Co., IA & $06 / 30 / 95$ & $<.05$ & $<.20$ & $<.20$ & -- & $<.05$ & $<.20$ & $<.20$ & -- & -- & -- & -- & -- & -- & -- & $<.05$ & $<.20$ & $<.20$ \\
\hline & & $07 / 25 / 97$ & $<.05$ & $<.20$ & $<.20$ & -- & $<.05$ & $<.20$ & $<.20$ & -- & -- & -- & -- & -- & -- & -- & $<.05$ & $<.20$ & $<.20$ \\
\hline & & 07/14/99 & $<.05$ & $<.05$ & $<.05$ & -- & $<.05$ & $<.05$ & $<.05$ & -- & $<.05$ & -- & -- & $<.05$ & -- & -- & .08 & $<.05$ & $<.05$ \\
\hline & & 08/02/01 & $<.05$ & $<.05$ & $<.05$ & -- & $<.05$ & $<.05$ & $<.05$ & -- & $<.05$ & $<.05$ & $<.05$ & $<.05$ & $<.05$ & $<.05$ & $<.05$ & $<.05$ & $<.05$ \\
\hline \multirow[t]{6}{*}{415252093411401} & Story Co., IA & $06 / 21 / 95$ & $<.05$ & $<.20$ & $<.20$ & -- & $<.05$ & $<.20$ & $<.20$ & -- & -- & -- & -- & -- & -- & -- & $<.05$ & $<.20$ & $<.20$ \\
\hline & & 08/13/96 & $<.05$ & $<.20$ & $<.20$ & -- & $<.05$ & $<.20$ & $<.20$ & -- & -- & -- & -- & -- & -- & -- & $<.05$ & $<.20$ & $<.20$ \\
\hline & & $07 / 22 / 97$ & $<.05$ & $<.20$ & $<.20$ & -- & $<.05$ & $<.20$ & $<.20$ & -- & -- & -- & -- & -- & -- & -- & $<.05$ & $<.20$ & $<.20$ \\
\hline & & $07 / 28 / 98$ & $<.05$ & $<.20$ & $<.20$ & -- & $<.05$ & $<.20$ & $<.20$ & -- & -- & -- & -- & -- & -- & -- & $<.05$ & $<.20$ & $<.20$ \\
\hline & & 06/09/99 & $<.05$ & $<.05$ & $<.05$ & -- & $<.05$ & $<.05$ & $<.05$ & -- & $<.05$ & -- & -- & -- & -- & -- & $<.05$ & $<.05$ & $<.05$ \\
\hline & & $07 / 26 / 01$ & $<.05$ & $<.05$ & $<.05$ & -- & $<.05$ & $<.05$ & $<.05$ & -- & $<.05$ & $<.05$ & $<.05$ & $<.05$ & $<.05$ & $<.05$ & $<.05$ & $<.05$ & $<.05$ \\
\hline \multirow[t]{5}{*}{415417092180101} & Tama Co., IA & $06 / 27 / 95$ & $<.05$ & $<.20$ & -- & -- & $<.05$ & 2.5 & $<.20$ & -- & -- & -- & -- & -- & -- & -- & $<.05$ & 1.8 & -- \\
\hline & & $06 / 26 / 96$ & $<.05$ & $<.20$ & $<.20$ & -- & $<.05$ & 4.2 & 1.9 & -- & -- & -- & -- & -- & -- & -- & $<.05$ & 2.1 & .71 \\
\hline & & $06 / 13 / 97$ & $<.05$ & .32 & $<.20$ & -- & $<.05$ & 1.3 & $<.20$ & -- & -- & -- & -- & -- & -- & -- & $<.05$ & 3.9 & .31 \\
\hline & & $07 / 15 / 99$ & $<.05$ & $<.20$ & $<.20$ & -- & $<.05$ & 2.1 & .77 & -- & $<.05$ & -- & -- & $<.05$ & -- & -- & $<.05$ & 1.1 & .38 \\
\hline & & $08 / 29 / 01$ & $<.05$ & $<.05$ & $<.05$ & -- & $<.05$ & 5.7 & .44 & -- & $<.05$ & $<.05$ & $<.05$ & $<.05$ & $<.05$ & $<.05$ & $<.05$ & 1.6 & .09 \\
\hline
\end{tabular}


Table 5. Analytical results for selected acetamide herbicides and their degradation products, ethanesulfonic acid, oxanilic acid, and sulfinyl acetic acid, in ground-water samples, 1994-2002.-Continued

[OGRL, U.S. Geological Survey Organic Geochemistry Research Laboratory, Lawrence, Kansas; ESA, ethanesulfonic acid; OXA, oxanilic acid; SAA, sulfinyl acetic acid; --, no data; <, less than]

\begin{tabular}{|c|c|c|c|c|c|c|c|c|c|c|c|c|c|c|c|c|c|c|c|}
\hline Site number & Site location & $\begin{array}{l}\text { Date of col- } \\
\text { lection } \\
\text { (month/day/ } \\
\text { year) }\end{array}$ & $\begin{array}{c}\text { Aceto- } \\
\text { chlor } \\
(\mu \mathrm{g} / \mathrm{L})\end{array}$ & $\begin{array}{l}\text { Aceto- } \\
\text { chlor } \\
\text { ESA } \\
(\mu \mathrm{g} / \mathrm{L})\end{array}$ & $\begin{array}{c}\text { Aceto- } \\
\text { chlor } \\
\text { OXA } \\
(\mu \mathrm{g} / \mathrm{L})\end{array}$ & $\begin{array}{l}\text { Aceto- } \\
\text { chlor } \\
\text { SAA } \\
(\mu \mathrm{g} / \mathrm{L})\end{array}$ & $\begin{array}{l}\text { Ala- } \\
\text { chlor } \\
(\mu \mathrm{g} / \mathrm{L})\end{array}$ & $\begin{array}{l}\text { Ala- } \\
\text { chlor } \\
\text { ESA } \\
(\mu \mathrm{g} / \mathrm{L})\end{array}$ & $\begin{array}{c}\text { Ala- } \\
\text { chlor } \\
\text { OXA } \\
(\mu \mathrm{g} / \mathrm{L})\end{array}$ & $\begin{array}{l}\text { Ala- } \\
\text { chlor } \\
\text { SAA } \\
(\mu \mathrm{g} / \mathrm{L})\end{array}$ & $\begin{array}{c}\text { Di- } \\
\text { meth- } \\
\text { enamid } \\
(\mu \mathrm{g} / \mathrm{L})\end{array}$ & $\begin{array}{c}\text { Di- } \\
\text { meth- } \\
\text { enamid } \\
\text { ESA } \\
(\mu \mathrm{g} / \mathrm{L})\end{array}$ & $\begin{array}{c}\text { Di- } \\
\text { meth- } \\
\text { enamid } \\
\text { OXA } \\
(\mu \mathrm{g} / \mathrm{L})\end{array}$ & $\begin{array}{c}\text { Flufen- } \\
\text { acet } \\
(\mu \mathrm{g} / \mathrm{L})\end{array}$ & $\begin{array}{c}\text { Flufen- } \\
\text { acet } \\
\text { ESA } \\
(\mu \mathrm{g} / \mathrm{L})\end{array}$ & $\begin{array}{c}\text { Flufen- } \\
\text { acet } \\
\text { OXA } \\
(\mu \mathrm{g} / \mathrm{L})\end{array}$ & $\begin{array}{l}\text { Meto- } \\
\text { lachlor } \\
(\mu \mathrm{g} / \mathrm{L})\end{array}$ & $\begin{array}{c}\text { Meto- } \\
\text { lachlor } \\
\text { ESA } \\
(\mu \mathrm{g} / \mathrm{L})\end{array}$ & $\begin{array}{c}\text { Meto- } \\
\text { lachlor } \\
\text { OXA } \\
(\mu \mathrm{g} / \mathrm{L})\end{array}$ \\
\hline \multicolumn{20}{|c|}{ Midc ontinent Agricultural Chemical Research Herbicide Project (fig. 1)—Continued } \\
\hline \multicolumn{20}{|c|}{ lowa monitoring wells (DKE/DKW)—Continued } \\
\hline 415426092175901 & Tama Co., IA & $06 / 26 / 96$ & $<0.05$ & $<0.20$ & $<0.20$ & -- & $<0.05$ & 1.8 & $<0.20$ & -- & -- & -- & -- & -- & -- & -- & $<0.05$ & 3.5 & $<0.05$ \\
\hline 415752090485701 & Clinton Co., IA & 06/20/96 & $<.05$ & $<.20$ & $<.20$ & -- & $<.05$ & 2.0 & 2.5 & -- & -- & -- & -- & -- & -- & -- & $<.05$ & .26 & .60 \\
\hline \multirow[t]{6}{*}{415753092350201} & Tama Co., IA & $06 / 27 / 95$ & $<.05$ & $<.20$ & -- & -- & .15 & 2.5 & $<.20$ & -- & -- & -- & -- & -- & -- & -- & .12 & 1.8 & -- \\
\hline & & $06 / 24 / 96$ & $<.05$ & $<.20$ & $<.20$ & -- & $<.05$ & .71 & $<.20$ & -- & -- & -- & -- & -- & -- & -- & $<.05$ & $<.20$ & $<.20$ \\
\hline & & $06 / 24 / 97$ & $<.05$ & $<.20$ & $<.20$ & -- & $<.05$ & .54 & $<.20$ & -- & -- & -- & -- & -- & -- & -- & $<.05$ & $<.20$ & $<.20$ \\
\hline & & 06/02/98 & $<.05$ & $<.20$ & $<.20$ & -- & $<.05$ & .56 & $<.20$ & -- & -- & -- & -- & -- & -- & -- & $<.05$ & .31 & $<.20$ \\
\hline & & 07/15/99 & $<.05$ & $<.20$ & $<.20$ & -- & $<.05$ & .58 & $<.20$ & -- & $<0.05$ & -- & -- & $<0.05$ & -- & -- & .05 & .36 & $<.05$ \\
\hline & & 09/04/01 & $<.05$ & $<.05$ & $<.05$ & -- & $<.05$ & .33 & $<.05$ & -- & $<.05$ & $<0.05$ & $<0.05$ & $<.05$ & $<0.05$ & $<0.05$ & $<.05$ & .17 & $<.05$ \\
\hline \multirow[t]{5}{*}{420005091431201} & Linn Co., IA & 06/19/96 & $<.05$ & .20 & $<.20$ & -- & $<.05$ & 2.1 & .20 & -- & -- & -- & -- & -- & -- & -- & .08 & 3.6 & .89 \\
\hline & & $06 / 12 / 97$ & $<.05$ & .62 & .23 & -- & $<.05$ & 1.4 & $<.20$ & -- & -- & -- & -- & -- & -- & -- & .23 & 3.3 & .77 \\
\hline & & $08 / 25 / 98$ & $<.05$ & 1.2 & .28 & -- & $<.05$ & 1.6 & $<.20$ & -- & -- & -- & -- & -- & -- & -- & .07 & 4.0 & .66 \\
\hline & & 07/12/99 & $<.05$ & .93 & .48 & -- & $<.05$ & .69 & $<.20$ & -- & $<.05$ & -- & -- & $<.05$ & -- & -- & .14 & 2.6 & .68 \\
\hline & & 08/02/01 & $<.05$ & .71 & .25 & -- & $<.05$ & .77 & $<.05$ & -- & $<.05$ & $<.05$ & $<.05$ & $<.05$ & $<.05$ & $<.05$ & .05 & 2.0 & .32 \\
\hline 420020092465001 & Marshall Co., IA & $06 / 24 / 96$ & $<.05$ & .60 & $<.20$ & -- & $<.05$ & .38 & $<.20$ & -- & -- & -- & -- & -- & -- & -- & $<.05$ & .59 & $<.20$ \\
\hline 420131095221101 & Crawford Co., IA & $08 / 17 / 95$ & $<.05$ & $<.20$ & $<.20$ & -- & $<.05$ & $<.20$ & $<.20$ & -- & -- & -- & -- & -- & -- & -- & $<.05$ & $<.20$ & $<.20$ \\
\hline \multirow[t]{2}{*}{420156093562401} & Boone Co., IA & $06 / 27 / 95$ & $<.05$ & $<.20$ & $<.20$ & -- & $<.05$ & .20 & $<.20$ & -- & -- & -- & -- & -- & -- & -- & $<.05$ & .42 & $<.20$ \\
\hline & & 08/14/96 & $<.05$ & $<.20$ & $<.20$ & -- & $<.05$ & .70 & $<.20$ & -- & -- & -- & -- & -- & -- & -- & $<.05$ & .85 & $<.20$ \\
\hline \multirow[t]{4}{*}{420241095422001} & Monona Co., IA & 08/12/96 & $<.05$ & $<.20$ & $<.20$ & -- & $<.05$ & $<.20$ & $<.20$ & -- & -- & -- & -- & -- & -- & -- & $<.05$ & $<.20$ & $<.20$ \\
\hline & & 06/27/97 & $<.05$ & $<.20$ & $<.20$ & -- & $<.05$ & $<.20$ & $<.20$ & -- & -- & -- & -- & -- & -- & -- & $<.05$ & $<.20$ & $<.20$ \\
\hline & & 07/22/98 & $<.05$ & $<.20$ & $<.20$ & -- & $<.05$ & $<.20$ & $<.20$ & -- & -- & -- & -- & -- & -- & -- & $<.05$ & $<.20$ & $<.20$ \\
\hline & & 06/10/99 & $<.05$ & $<.05$ & $<.05$ & -- & $<.05$ & $<.05$ & $<.05$ & -- & $<.05$ & -- & -- & -- & -- & -- & $<.05$ & $<.05$ & $<.05$ \\
\hline
\end{tabular}


Table 5. Analytical results for selected acetamide herbicides and their degradation products, ethanesulfonic acid, oxanilic acid, and sulfinyl acetic acid, in ground-water samples, 1994-2002.-Continued

[OGRL, U.S. Geological Survey Organic Geochemistry Research Laboratory, Lawrence, Kansas; ESA, ethanesulfonic acid; OXA, oxanilic acid; SAA, sulfinyl acetic acid; --, no data; <, less than]

\begin{tabular}{|c|c|c|c|c|c|c|c|c|c|c|c|c|c|c|c|c|c|c|c|}
\hline Site number & Site location & $\begin{array}{l}\text { Date of col- } \\
\text { lection } \\
\text { (month/day/ } \\
\text { year) }\end{array}$ & $\begin{array}{l}\text { Aceto- } \\
\text { chlor } \\
(\mu \mathrm{g} / \mathrm{L})\end{array}$ & $\begin{array}{l}\text { Aceto- } \\
\text { chlor } \\
\text { ESA } \\
(\mu \mathrm{g} / \mathrm{L})\end{array}$ & $\begin{array}{c}\text { Aceto- } \\
\text { chlor } \\
\text { OXA } \\
(\mu \mathrm{g} / \mathrm{L})\end{array}$ & $\begin{array}{c}\text { Aceto- } \\
\text { chlor } \\
\text { SAA } \\
(\mu \mathrm{g} / \mathrm{L})\end{array}$ & $\begin{array}{l}\text { Ala- } \\
\text { chlor } \\
(\mu \mathrm{g} / \mathrm{L})\end{array}$ & $\begin{array}{c}\text { Ala- } \\
\text { chlor } \\
\text { ESA } \\
(\mu \mathrm{g} / \mathrm{L})\end{array}$ & $\begin{array}{c}\text { Ala- } \\
\text { chlor } \\
\text { OXA } \\
(\mu \mathrm{g} / \mathrm{L})\end{array}$ & $\begin{array}{l}\text { Ala- } \\
\text { chlor } \\
\text { SAA } \\
(\mu \mathrm{g} / \mathrm{L})\end{array}$ & $\begin{array}{c}\text { Di- } \\
\text { meth- } \\
\text { enamid } \\
(\mu \mathrm{g} / \mathrm{L})\end{array}$ & $\begin{array}{c}\text { Di- } \\
\text { meth- } \\
\text { enamid } \\
\text { ESA } \\
(\mu \mathrm{g} / \mathrm{L})\end{array}$ & $\begin{array}{c}\text { Di- } \\
\text { meth- } \\
\text { enamid } \\
0 X A \\
(\mu \mathrm{g} / \mathrm{L})\end{array}$ & $\begin{array}{c}\text { Flufen- } \\
\text { acet } \\
(\mu \mathrm{g} / \mathrm{L})\end{array}$ & $\begin{array}{c}\text { Flufen- } \\
\text { acet } \\
\text { ESA } \\
(\mu \mathrm{g} / \mathrm{L})\end{array}$ & $\begin{array}{c}\text { Flufen- } \\
\text { acet } \\
\text { OXA } \\
(\mu \mathrm{g} / \mathrm{L})\end{array}$ & $\begin{array}{c}\text { Meto- } \\
\text { lachlor } \\
(\mu \mathrm{g} / \mathrm{L})\end{array}$ & $\begin{array}{c}\text { Meto- } \\
\text { lachlor } \\
\text { ESA } \\
(\mu \mathrm{g} / \mathrm{L})\end{array}$ & $\begin{array}{c}\text { Meto- } \\
\text { lachlor } \\
\text { OXA } \\
(\mu \mathrm{g} / \mathrm{L})\end{array}$ \\
\hline \multicolumn{20}{|c|}{ Midcontinent Agricultural Chemical Research Herbicide Project (fig. 1)—Continued } \\
\hline \multicolumn{20}{|c|}{ lowa monitoring wells (DKE/DKW)_Continued } \\
\hline \multirow[t]{5}{*}{420336095115601} & Crawford Co., IA & 08/17/95 & $<0.05$ & $<0.20$ & -- & -- & $<0.05$ & $<0.20$ & $<0.20$ & -- & -- & -- & -- & -- & -- & -- & $<0.05$ & $<0.20$ & -- \\
\hline & & 07/24/96 & $<.05$ & $<.20$ & $<0.20$ & -- & $<.05$ & $<.20$ & $<.20$ & -- & -- & -- & -- & -- & -- & -- & $<.05$ & $<.20$ & $<0.20$ \\
\hline & & 06/30/97 & $<.05$ & $<.20$ & $<.20$ & -- & $<.05$ & $<.20$ & $<.20$ & -- & -- & -- & -- & -- & -- & -- & $<.05$ & $<.20$ & $<.20$ \\
\hline & & 07/14/99 & $<.05$ & $<.20$ & $<.20$ & -- & $<.05$ & $<.20$ & $<.20$ & -- & $<0.05$ & -- & -- & $<0.05$ & -- & -- & $<.05$ & $<.20$ & $<.20$ \\
\hline & & 08/02/01 & $<.05$ & $<.05$ & $<.05$ & -- & $<.05$ & $<.05$ & $<.05$ & -- & $<.05$ & $<0.05$ & $<0.05$ & $<.05$ & $<0.05$ & $<0.05$ & $<.05$ & $<.05$ & $<.05$ \\
\hline \multirow[t]{2}{*}{420352090552401} & Marshall Co., IA & 06/24/97 & $<.05$ & $<.20$ & $<.20$ & -- & $<.05$ & .56 & $<.20$ & -- & -- & -- & -- & -- & -- & -- & $<.05$ & 2.7 & .50 \\
\hline & & 09/04/01 & $<.05$ & .13 & .14 & -- & $<.05$ & .15 & .05 & -- & $<.05$ & $<.05$ & $<.05$ & $<.05$ & $<.05$ & $<.05$ & $<.05$ & .50 & .62 \\
\hline \multirow[t]{4}{*}{420405092545601} & Marshall Co., IA & $06 / 24 / 96$ & $<.05$ & $<.20$ & $<.20$ & -- & $<.05$ & 1.9 & .41 & -- & -- & -- & -- & -- & -- & -- & $<.05$ & 2.6 & .87 \\
\hline & & 06/24/97 & $<.05$ & $<.20$ & $<.20$ & -- & $<.05$ & 1.2 & .29 & -- & -- & -- & -- & -- & -- & -- & $<.05$ & 2.0 & .54 \\
\hline & & 07/13/99 & $<.05$ & $<.20$ & $<.20$ & -- & $<.05$ & .90 & $<.20$ & -- & $<.05$ & -- & -- & $<.05$ & -- & -- & $<.05$ & 1.6 & .45 \\
\hline & & 06/09/01 & $<.05$ & $<.05$ & $<.05$ & -- & $<.05$ & .24 & .09 & -- & $<.05$ & $<.05$ & $<.05$ & $<.05$ & $<.05$ & $<.05$ & $<.05$ & .28 & .52 \\
\hline \multirow[t]{4}{*}{420414090113201} & Jackson Co., IA & $06 / 22 / 95$ & $<.05$ & $<.20$ & $<.20$ & -- & $<.05$ & $<.20$ & $<.20$ & -- & -- & -- & -- & -- & -- & -- & $<.05$ & $<.20$ & $<.20$ \\
\hline & & 06/12/97 & $<.05$ & $<.20$ & $<.20$ & -- & $<.05$ & $<.20$ & $<.20$ & -- & -- & -- & -- & -- & -- & -- & $<.05$ & $<.20$ & $<.20$ \\
\hline & & 06/14/99 & $<.05$ & $<.20$ & $<.20$ & -- & $<.05$ & $<.20$ & $<.20$ & -- & $<.05$ & -- & -- & $<.05$ & -- & -- & $<.05$ & $<.20$ & $<.20$ \\
\hline & & 08/06/01 & $<.05$ & $<.05$ & $<.05$ & -- & $<.05$ & $<.05$ & $<.05$ & -- & $<.05$ & $<.05$ & $<.05$ & $<.05$ & $<.05$ & $<.05$ & $<.05$ & $<.05$ & $<.05$ \\
\hline \multirow[t]{5}{*}{420451093561301} & Boone Co., IA & $06 / 27 / 95$ & $<.05$ & 1.0 & -- & -- & $<.05$ & .91 & $<.20$ & -- & -- & -- & -- & -- & -- & -- & .15 & 2.9 & -- \\
\hline & & 08/14/96 & $<.05$ & 1.1 & .70 & -- & $<.05$ & .84 & $<.20$ & -- & -- & -- & -- & -- & -- & -- & .23 & 5.4 & 1.5 \\
\hline & & $07 / 22 / 97$ & $<.05$ & .83 & .48 & -- & $<.05$ & .48 & $<.20$ & -- & -- & -- & -- & -- & -- & -- & .33 & 3.8 & 1.0 \\
\hline & & 06/10/99 & $<.05$ & .54 & $<.05$ & -- & $<.05$ & .25 & $<.05$ & -- & $<.05$ & -- & -- & -- & -- & -- & .10 & 2.5 & .47 \\
\hline & & 07/26/01 & $<.05$ & 1.6 & .66 & -- & $<.05$ & $<.05$ & .06 & -- & $<.05$ & $<.05$ & $<.05$ & $<.05$ & $<.05$ & $<.05$ & .18 & 2.4 & .78 \\
\hline \multirow[t]{3}{*}{420535091524002} & Benton Co., IA & 06/19/96 & $<.05$ & $<.20$ & $<.20$ & -- & $<.05$ & $<.20$ & $<.20$ & -- & -- & -- & -- & -- & -- & -- & $<.05$ & .64 & $<.20$ \\
\hline & & 06/13/97 & $<.05$ & $<.20$ & $<.20$ & -- & $<.05$ & $<.20$ & $<.20$ & -- & -- & -- & -- & -- & -- & -- & $<.05$ & 1.3 & $<.20$ \\
\hline & & 08/02/01 & $<.05$ & $<.05$ & $<.05$ & -- & $<.05$ & $<.05$ & $<.05$ & -- & $<.05$ & $<.05$ & $<.05$ & $<.05$ & $<.05$ & $<.05$ & $<.05$ & 1.6 & $<.05$ \\
\hline
\end{tabular}


Table 5. Analytical results for selected acetamide herbicides and their degradation products, ethanesulfonic acid, oxanilic acid, and sulfinyl acetic acid, in ground-water samples, 1994-2002.-Continued

[OGRL, U.S. Geological Survey Organic Geochemistry Research Laboratory, Lawrence, Kansas; ESA, ethanesulfonic acid; OXA, oxanilic acid; SAA, sulfinyl acetic acid; --, no data; <, less than]

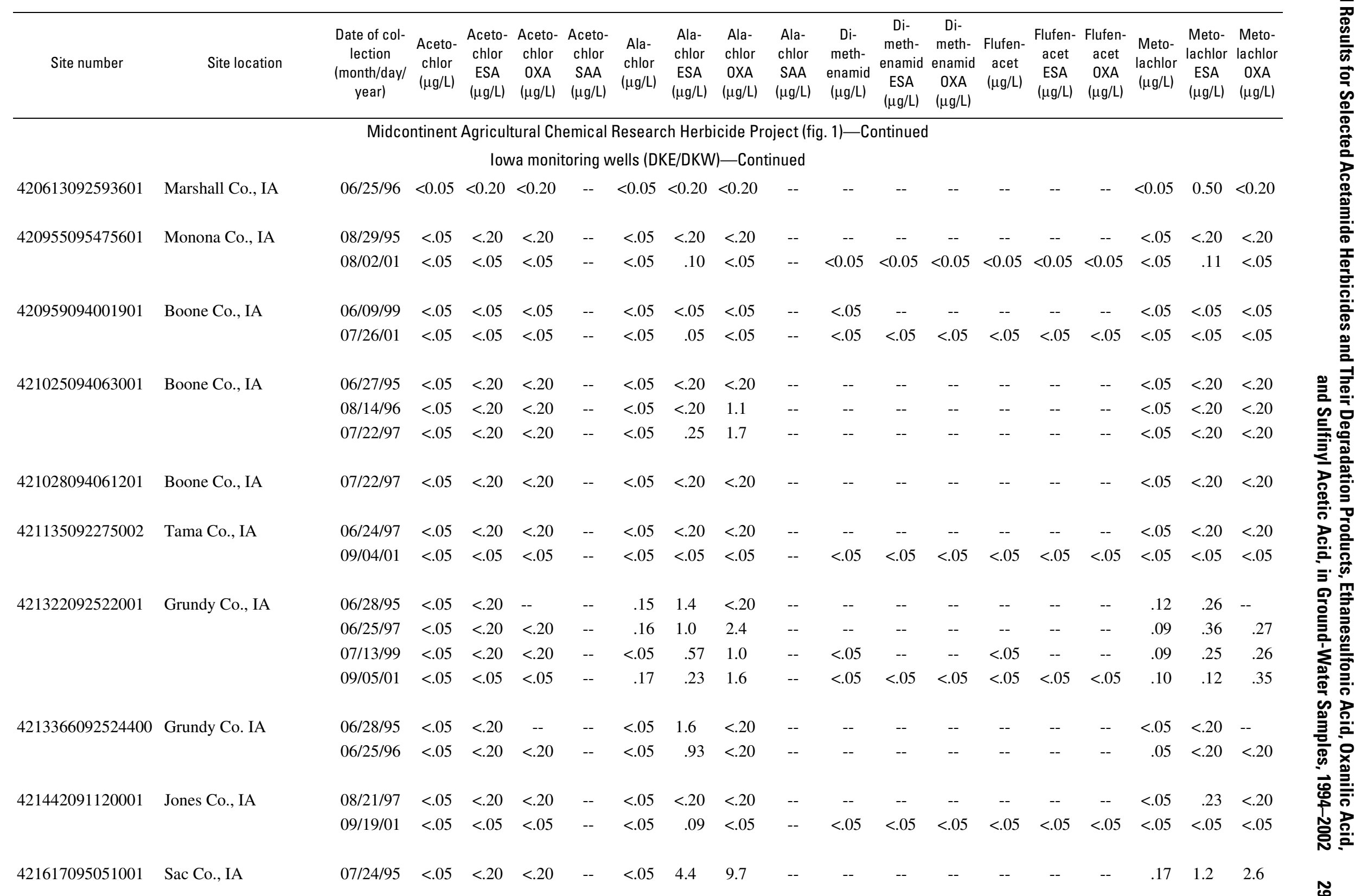


Table 5. Analytical results for selected acetamide herbicides and their degradation products, ethanesulfonic acid, oxanilic acid, and sulfinyl acetic acid, in ground-water samples, 1994-2002.-Continued

[OGRL, U.S. Geological Survey Organic Geochemistry Research Laboratory, Lawrence, Kansas; ESA, ethanesulfonic acid; OXA, oxanilic acid; SAA, sulfinyl acetic acid; --, no data; <, less than]

\begin{tabular}{|c|c|c|c|c|c|c|c|c|c|c|c|c|c|c|c|c|c|c|c|}
\hline Site number & Site location & $\begin{array}{l}\text { Date of col- } \\
\text { lection } \\
\text { (month/day/ } \\
\text { year) }\end{array}$ & $\begin{array}{l}\text { Aceto- } \\
\text { chlor } \\
(\mu \mathrm{g} / \mathrm{L})\end{array}$ & $\begin{array}{c}\text { Aceto- } \\
\text { chlor } \\
\text { ESA } \\
(\mu \mathrm{g} / \mathrm{L})\end{array}$ & $\begin{array}{c}\text { Aceto- } \\
\text { chlor } \\
\text { OXA } \\
(\mu \mathrm{g} / \mathrm{L})\end{array}$ & $\begin{array}{c}\text { Aceto- } \\
\text { chlor } \\
\text { SAA } \\
(\mu \mathrm{g} / \mathrm{L})\end{array}$ & $\begin{array}{l}\text { Ala- } \\
\text { chlor } \\
(\mu \mathrm{g} / \mathrm{L})\end{array}$ & $\begin{array}{l}\text { Ala- } \\
\text { chlor } \\
\text { ESA } \\
(\mu \mathrm{g} / \mathrm{L})\end{array}$ & $\begin{array}{c}\text { Ala- } \\
\text { chlor } \\
\text { OXA } \\
(\mu \mathrm{g} / \mathrm{L})\end{array}$ & $\begin{array}{c}\text { Ala- } \\
\text { chlor } \\
\text { SAA } \\
(\mu \mathrm{g} / \mathrm{L})\end{array}$ & $\begin{array}{c}\text { Di- } \\
\text { meth- } \\
\text { enamid } \\
(\mu \mathrm{g} / \mathrm{L})\end{array}$ & $\begin{array}{c}\text { Di- } \\
\text { meth- } \\
\text { enamid } \\
\text { ESA } \\
(\mu \mathrm{g} / \mathrm{L})\end{array}$ & $\begin{array}{c}\text { Di- } \\
\text { meth- } \\
\text { enamid } \\
\text { OXA } \\
(\mu \mathrm{g} / \mathrm{L})\end{array}$ & $\begin{array}{c}\text { Flufen- } \\
\text { acet } \\
(\mu \mathrm{g} / \mathrm{L})\end{array}$ & $\begin{array}{c}\text { Flufen- } \\
\text { acet } \\
\text { ESA } \\
(\mu \mathrm{g} / \mathrm{L})\end{array}$ & $\begin{array}{c}\text { Flufen- } \\
\text { acet } \\
0 X A \\
(\mu \mathrm{g} / \mathrm{L})\end{array}$ & $\begin{array}{l}\text { Meto- } \\
\text { lachlor } \\
(\mu \mathrm{g} / \mathrm{L})\end{array}$ & $\begin{array}{c}\text { Meto- } \\
\text { lachlor } \\
\text { ESA } \\
(\mu \mathrm{g} / \mathrm{L})\end{array}$ & $\begin{array}{c}\text { Meto- } \\
\text { lachlor } \\
\text { OXA } \\
(\mu \mathrm{g} / \mathrm{L})\end{array}$ \\
\hline \multicolumn{20}{|c|}{ Midcontinent Agricultural Chemical Research Herbicide Project (fig. 1)—Continued } \\
\hline \multicolumn{20}{|c|}{ lowa monitoring wells (DKE/DKW)—Continued } \\
\hline \multirow[t]{5}{*}{421617095051001} & Sac Co., IA & 08/12/96 & $<0.05$ & $<0.20$ & $<0.20$ & -- & $<0.05$ & 5.8 & 9.8 & -- & -- & -- & -- & -- & -- & -- & 0.17 & 1.7 & 2.3 \\
\hline & & 06/30/97 & $<.05$ & $<.20$ & .24 & -- & $<.05$ & 2.6 & 6.2 & -- & -- & -- & -- & -- & -- & -- & .27 & 1.0 & 2.1 \\
\hline & & 06/23/98 & $<.05$ & .49 & .25 & -- & $<.05$ & 5.0 & 9.5 & -- & -- & -- & -- & -- & -- & -- & .32 & 2.3 & 2.8 \\
\hline & & 06/07/99 & $<.05$ & .48 & .66 & -- & $<.05$ & 3.6 & 12 & -- & $<0.05$ & -- & -- & $<0.05$ & -- & -- & .36 & 1.6 & 3.0 \\
\hline & & 07/23/01 & $<.05$ & $<.05$ & .64 & -- & $<.05$ & 3.6 & 4.2 & -- & $<.05$ & $<0.05$ & $<0.05$ & $<.05$ & $<0.05$ & $<0.05$ & .41 & 2.0 & 2.5 \\
\hline \multirow[t]{2}{*}{421826095025101} & Sac Co., IA & $07 / 24 / 95$ & $<.05$ & $<.20$ & $<.20$ & -- & $<.05$ & .33 & $<.20$ & -- & -- & -- & -- & -- & -- & -- & $<.05$ & $<.20$ & $<.20$ \\
\hline & & $08 / 12 / 96$ & $<.05$ & $<.20$ & $<.20$ & -- & $<.05$ & .61 & $<.20$ & -- & -- & -- & -- & -- & -- & -- & $<.05$ & $<.20$ & $<.20$ \\
\hline \multirow[t]{5}{*}{422106095280201} & Ida Co., IA & $07 / 24 / 96$ & $<.05$ & $<.20$ & $<.20$ & -- & $<.05$ & $<.20$ & .36 & -- & -- & -- & -- & -- & -- & -- & $<.05$ & $<.20$ & $<.20$ \\
\hline & & 06/30/97 & $<.05$ & $<.20$ & $<.20$ & -- & $<.05$ & $<.20$ & $<.20$ & -- & -- & -- & -- & -- & -- & -- & $<.05$ & $<.20$ & $<.20$ \\
\hline & & $06 / 25 / 98$ & $<.05$ & $<.20$ & $<.20$ & -- & $<.05$ & $<.20$ & $<.20$ & -- & -- & -- & -- & -- & -- & -- & $<.05$ & $<.20$ & $<.20$ \\
\hline & & 06/10/99 & $<.05$ & $<.20$ & $<.20$ & -- & $<.05$ & $<.20$ & $<.20$ & -- & $<.05$ & -- & -- & -- & -- & -- & $<.05$ & $<.20$ & $<.20$ \\
\hline & & 08/01/01 & $<.05$ & $<.05$ & $<.05$ & -- & $<.05$ & .07 & $<.05$ & -- & $<.05$ & $<.05$ & $<.05$ & $<.05$ & $<.05$ & $<.05$ & $<.05$ & .16 & $<.05$ \\
\hline 422109095275401 & Ida Co., IA & $07 / 24 / 95$ & $<.05$ & $<.20$ & $<.20$ & -- & $<.05$ & $<.20$ & $<.20$ & -- & -- & -- & -- & -- & -- & -- & $<.05$ & .48 & $<.20$ \\
\hline \multirow[t]{2}{*}{422148092461801} & Grundy Co., IA & $06 / 28 / 95$ & $<.05$ & $<.20$ & $<.20$ & -- & $<.05$ & $<.20$ & $<.20$ & -- & -- & -- & -- & -- & -- & -- & $<.05$ & $<.20$ & $<.20$ \\
\hline & & $06 / 25 / 97$ & $<.05$ & $<.20$ & $<.20$ & -- & $<.05$ & $<.20$ & $<.20$ & -- & -- & -- & -- & -- & -- & -- & $<.05$ & $<.20$ & $<.20$ \\
\hline \multirow[t]{2}{*}{422611092552501} & Grundy Co., IA & $06 / 25 / 97$ & $<.05$ & $<.20$ & $<.20$ & -- & $<.05$ & $<.20$ & $<.20$ & -- & -- & -- & -- & -- & -- & -- & $<.05$ & $<.20$ & $<.20$ \\
\hline & & 09/18/01 & $<.05$ & $<.05$ & $<.05$ & -- & $<.05$ & $<.05$ & $<.05$ & -- & $<.05$ & $<.05$ & $<.05$ & $<.05$ & $<.05$ & $<.05$ & $<.05$ & $<.05$ & $<.05$ \\
\hline \multirow[t]{2}{*}{422819092212701} & Black Hawk Co., IA & 08/20/97 & $<.05$ & $<.20$ & $<.20$ & -- & $<.05$ & $<.20$ & $<.20$ & -- & -- & -- & -- & -- & -- & -- & $<.05$ & $<.20$ & $<.20$ \\
\hline & & 08/29/01 & $<.05$ & $<.05$ & $<.05$ & -- & $<.05$ & .28 & $<.05$ & -- & $<.05$ & $<.05$ & $<.05$ & $<.05$ & $<.05$ & $<.05$ & $<.05$ & .22 & $<.05$ \\
\hline \multirow[t]{3}{*}{422831095465102} & Woodbury Co., IA & $07 / 27 / 95$ & $<.05$ & $<.20$ & $<.20$ & -- & $<.05$ & $<.20$ & $<.20$ & -- & -- & -- & -- & -- & -- & -- & $<.05$ & 1.4 & $<.20$ \\
\hline & & $07 / 12 / 96$ & $<.05$ & $<.20$ & $<.20$ & -- & $<.05$ & $<.20$ & $<.20$ & -- & -- & -- & -- & -- & -- & -- & $<.05$ & .79 & $<.20$ \\
\hline & & 07/01/97 & $<.05$ & $<.20$ & $<.20$ & -- & $<.05$ & $<.20$ & $<.20$ & -- & -- & -- & -- & -- & -- & -- & $<.05$ & .43 & $<.20$ \\
\hline
\end{tabular}


Table 5. Analytical results for selected acetamide herbicides and their degradation products, ethanesulfonic acid, oxanilic acid, and sulfinyl acetic acid, in ground-water samples, 1994-2002.-Continued

[OGRL, U.S. Geological Survey Organic Geochemistry Research Laboratory, Lawrence, Kansas; ESA, ethanesulfonic acid; OXA, oxanilic acid; SAA, sulfinyl acetic acid; --, no data; <, less than]

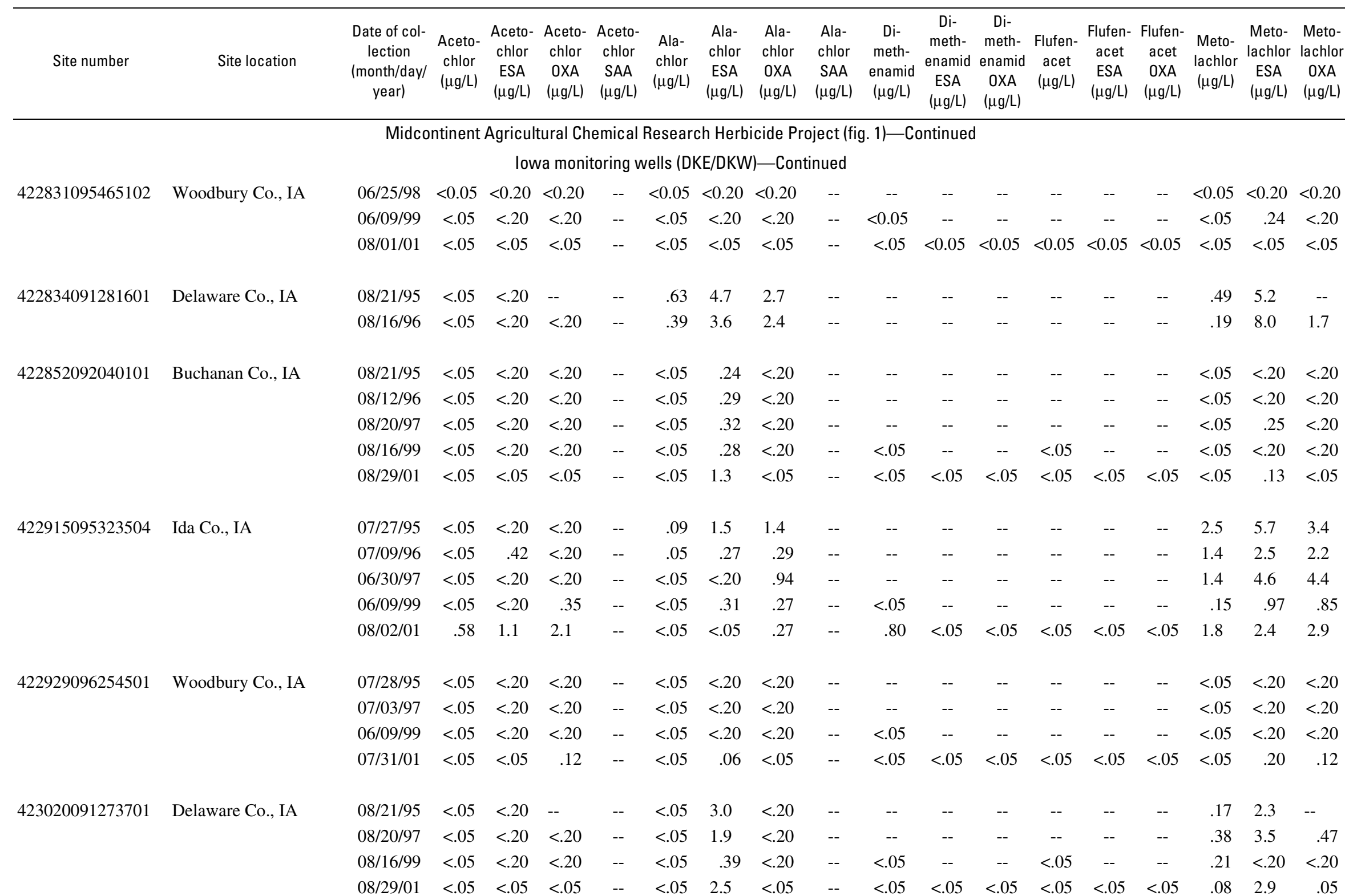


Table 5. Analytical results for selected acetamide herbicides and their degradation products, ethanesulfonic acid, oxanilic acid, and sulfinyl acetic acid, in ground-water samples, 1994-2002.-Continued

[OGRL, U.S. Geological Survey Organic Geochemistry Research Laboratory, Lawrence, Kansas; ESA, ethanesulfonic acid; OXA, oxanilic acid; SAA, sulfinyl acetic acid; --, no data; <, less than]

\begin{tabular}{|c|c|c|c|c|c|c|c|c|c|c|c|c|c|c|c|c|c|c|c|}
\hline Site number & Site location & $\begin{array}{l}\text { Date of col- } \\
\text { lection } \\
\text { (month/day/ } \\
\text { year) }\end{array}$ & $\begin{array}{l}\text { Aceto- } \\
\text { chlor } \\
(\mu \mathrm{g} / \mathrm{L})\end{array}$ & $\begin{array}{c}\text { Aceto- } \\
\text { chlor } \\
\text { ESA } \\
(\mu \mathrm{g} / \mathrm{L})\end{array}$ & $\begin{array}{c}\text { Aceto- } \\
\text { chlor } \\
\text { OXA } \\
(\mu \mathrm{g} / \mathrm{L})\end{array}$ & $\begin{array}{c}\text { Aceto- } \\
\text { chlor } \\
\text { SAA } \\
(\mu \mathrm{g} / \mathrm{L})\end{array}$ & $\begin{array}{l}\text { Ala- } \\
\text { chlor } \\
(\mu \mathrm{g} / \mathrm{L})\end{array}$ & $\begin{array}{c}\text { Ala- } \\
\text { chlor } \\
\text { ESA } \\
(\mu \mathrm{g} / \mathrm{L})\end{array}$ & $\begin{array}{c}\text { Ala- } \\
\text { chlor } \\
\text { OXA } \\
(\mu \mathrm{g} / \mathrm{L})\end{array}$ & $\begin{array}{l}\text { Ala- } \\
\text { chlor } \\
\text { SAA } \\
(\mu \mathrm{g} / \mathrm{L})\end{array}$ & $\begin{array}{c}\text { Di- } \\
\text { meth- } \\
\text { enamid } \\
(\mu \mathrm{g} / \mathrm{L})\end{array}$ & $\begin{array}{c}\text { Di- } \\
\text { meth- } \\
\text { enamid } \\
\text { ESA } \\
(\mu \mathrm{g} / \mathrm{L})\end{array}$ & $\begin{array}{c}\text { Di- } \\
\text { meth- } \\
\text { enamid } \\
\text { OXA } \\
(\mu \mathrm{g} / \mathrm{L})\end{array}$ & $\begin{array}{c}\text { Flufen- } \\
\text { acet } \\
(\mu \mathrm{g} / \mathrm{L})\end{array}$ & $\begin{array}{c}\text { Flufen- } \\
\text { acet } \\
\text { ESA } \\
(\mu \mathrm{g} / \mathrm{L})\end{array}$ & $\begin{array}{c}\text { Flufen- } \\
\text { acet } \\
\text { OXA } \\
(\mu \mathrm{g} / \mathrm{L})\end{array}$ & $\begin{array}{c}\text { Meto- } \\
\text { lachlor } \\
(\mu \mathrm{g} / \mathrm{L})\end{array}$ & $\begin{array}{c}\text { Meto- } \\
\text { lachlor } \\
\text { ESA } \\
(\mu \mathrm{g} / \mathrm{L})\end{array}$ & $\begin{array}{c}\text { Meto- } \\
\text { lachlor } \\
\text { OXA } \\
(\mu \mathrm{g} / \mathrm{L})\end{array}$ \\
\hline \multicolumn{20}{|c|}{ Midcontinent Agricultural Chemical Research Herbicide Project (fig. 1)—Continued } \\
\hline \multicolumn{20}{|c|}{ lowa monitoring wells (DKE/DKW)—Continued } \\
\hline \multirow[t]{4}{*}{423028094115101} & Webster Co., IA & $06 / 29 / 95$ & $<0.05$ & $<0.20$ & $<0.20$ & -- & $<0.05$ & $<0.20$ & $<0.20$ & -- & -- & -- & -- & -- & -- & -- & $<0.05$ & $<0.20$ & $<0.20$ \\
\hline & & $07 / 25 / 97$ & $<.05$ & $<.20$ & $<.20$ & -- & $<.05$ & $<.20$ & $<.20$ & -- & -- & -- & -- & -- & -- & -- & $<.05$ & $<.20$ & $<.20$ \\
\hline & & 06/10/99 & $<.05$ & $<.05$ & $<.05$ & -- & $<.05$ & $<.05$ & $<.05$ & -- & $<0.05$ & -- & -- & -- & -- & -- & $<.05$ & $<.05$ & $<.05$ \\
\hline & & $07 / 25 / 01$ & $<.05$ & $<.05$ & $<.05$ & -- & $<.05$ & $<.05$ & $<.05$ & -- & $<.05$ & $<0.05$ & $<0.05$ & $<0.05$ & $<0.05$ & $<0.05$ & $<.05$ & $<.05$ & $<.05$ \\
\hline \multirow[t]{2}{*}{423033095250501} & Ida Co., IA & $07 / 24 / 95$ & .77 & .25 & $<.20$ & -- & $<.05$ & .58 & .59 & -- & -- & -- & -- & -- & -- & -- & 3.0 & 3.0 & 2.5 \\
\hline & & 07/09/96 & .60 & .93 & .80 & -- & .08 & 1.4 & 2.9 & -- & -- & -- & -- & -- & -- & -- & 2.3 & 5.4 & 5.1 \\
\hline \multirow[t]{6}{*}{423135090383201} & Dubuque Co., IA & 09/06/95 & $<.05$ & $<.20$ & $<.20$ & -- & $<.05$ & .59 & $<.20$ & -- & -- & -- & -- & -- & -- & -- & $<.05$ & .52 & .22 \\
\hline & & 08/16/96 & $<.05$ & $<.20$ & $<.20$ & -- & $<.05$ & .60 & $<.20$ & -- & -- & -- & -- & -- & -- & -- & $<.05$ & .69 & $<.20$ \\
\hline & & $08 / 21 / 97$ & $<.05$ & $<.20$ & $<.20$ & -- & $<.05$ & .30 & $<.20$ & -- & -- & -- & -- & -- & -- & -- & $<.05$ & .33 & $<.20$ \\
\hline & & 08/26/98 & $<.05$ & $<.20$ & $<.20$ & -- & $<.05$ & .38 & $<.20$ & -- & -- & -- & -- & -- & -- & -- & $<.05$ & .37 & $<.20$ \\
\hline & & 08/10/99 & $<.05$ & $<.20$ & $<.20$ & -- & $<.05$ & .53 & $<.20$ & -- & $<.05$ & -- & -- & $<.05$ & -- & -- & $<.05$ & .34 & $<.20$ \\
\hline & & 09/11/01 & $<.05$ & .10 & .10 & -- & $<.05$ & .31 & $<.05$ & -- & $<.05$ & $<.05$ & $<.05$ & $<.05$ & $<.05$ & $<.05$ & $<.05$ & .34 & .10 \\
\hline \multirow[t]{5}{*}{423537095583901} & Plymouth Co., IA & $07 / 28 / 95$ & $<.05$ & $<.20$ & $<.20$ & -- & $<.05$ & $<.20$ & $<.20$ & -- & -- & -- & -- & -- & -- & -- & $<.05$ & .50 & $<.20$ \\
\hline & & 07/12/96 & $<.05$ & $<.20$ & $<.20$ & -- & $<.05$ & $<.20$ & $<.20$ & -- & -- & -- & -- & -- & -- & -- & $<.05$ & .92 & $<.20$ \\
\hline & & 07/01/97 & $<.05$ & $<.20$ & $<.20$ & -- & $<.05$ & $<.20$ & $<.20$ & -- & -- & -- & -- & -- & -- & -- & $<.05$ & .52 & $<.20$ \\
\hline & & 06/09/99 & $<.05$ & $<.20$ & $<.20$ & -- & $<.05$ & $<.20$ & $<.20$ & -- & $<.05$ & -- & -- & -- & -- & -- & $<.05$ & .48 & $<.20$ \\
\hline & & 07/31/01 & $<.05$ & .07 & $<.05$ & -- & $<.05$ & .05 & $<.05$ & -- & $<.05$ & $<.05$ & $<.05$ & $<.05$ & $<.05$ & $<.05$ & $<.05$ & .36 & .06 \\
\hline \multirow[t]{4}{*}{423602090595201} & Dubuque Co., IA & 09/06/95 & $<.05$ & $<.20$ & $<.20$ & -- & $<.05$ & $<.20$ & $<.20$ & -- & -- & -- & -- & -- & -- & -- & $<.05$ & $<.20$ & $<.20$ \\
\hline & & 08/21/97 & $<.05$ & $<.20$ & $<.20$ & -- & $<.05$ & $<.20$ & $<.20$ & -- & -- & -- & -- & -- & -- & -- & $<.05$ & $<.20$ & $<.20$ \\
\hline & & 08/10/99 & $<.05$ & $<.20$ & $<.20$ & -- & $<.05$ & $<.20$ & $<.20$ & -- & $<.05$ & -- & -- & $<.05$ & -- & -- & $<.05$ & $<.20$ & $<.20$ \\
\hline & & 09/11/01 & $<.05$ & $<.05$ & $<.05$ & -- & $<.05$ & $<.05$ & $<.05$ & -- & $<.05$ & $<.05$ & $<.05$ & $<.05$ & $<.05$ & $<.05$ & $<.05$ & $<.05$ & $<.05$ \\
\hline \multirow[t]{2}{*}{423744095383301} & Cherokee Co., IA & $07 / 27 / 95$ & $<.05$ & $<.20$ & $<.20$ & -- & $<.05$ & $<.20$ & $<.20$ & -- & -- & -- & -- & -- & -- & -- & $<.05$ & $<.20$ & $<.20$ \\
\hline & & 07/01/97 & $<.05$ & $<.20$ & $<.20$ & -- & $<.05$ & $<.20$ & $<.20$ & -- & -- & -- & -- & -- & -- & -- & $<.05$ & $<.20$ & $<.20$ \\
\hline
\end{tabular}


Table 5. Analytical results for selected acetamide herbicides and their degradation products, ethanesulfonic acid, oxanilic acid, and sulfinyl acetic acid, in ground-water samples, 1994-2002.-Continued

[OGRL, U.S. Geological Survey Organic Geochemistry Research Laboratory, Lawrence, Kansas; ESA, ethanesulfonic acid; OXA, oxanilic acid; SAA, sulfinyl acetic acid; --, no data; <, less than]

\begin{tabular}{|c|c|c|c|c|c|c|c|c|c|c|c|c|c|c|c|c|c|c|c|c|}
\hline Site number & Site location & $\begin{array}{l}\text { Date of col- } \\
\text { lection } \\
\text { (month/day/ } \\
\text { year) }\end{array}$ & $\begin{array}{c}\text { Aceto- } \\
\text { chlor } \\
(\mu \mathrm{g} / \mathrm{L})\end{array}$ & $\begin{array}{c}\text { Aceto- } \\
\text { chlor } \\
\text { ESA } \\
(\mu \mathrm{g} / \mathrm{L})\end{array}$ & $\begin{array}{c}\text { Aceto- } \\
\text { chlor } \\
\text { OXA } \\
(\mu \mathrm{g} / \mathrm{L})\end{array}$ & $\begin{array}{c}\text { Aceto- } \\
\text { chlor } \\
\text { SAA } \\
(\mu \mathrm{g} / \mathrm{L})\end{array}$ & $\begin{array}{l}\text { Ala- } \\
\text { chlor } \\
(\mu \mathrm{g} / \mathrm{L})\end{array}$ & $\begin{array}{c}\text { Ala- } \\
\text { chlor } \\
\text { ESA } \\
(\mu \mathrm{g} / \mathrm{L})\end{array}$ & $\begin{array}{c}\text { Ala- } \\
\text { chlor } \\
\text { OXA } \\
(\mu \mathrm{g} / \mathrm{L})\end{array}$ & $\begin{array}{l}\text { Ala- } \\
\text { chlor } \\
\text { SAA } \\
(\mu \mathrm{g} / \mathrm{L})\end{array}$ & $\begin{array}{c}\text { Di- } \\
\text { meth- } \\
\text { enamid } \\
(\mu \mathrm{g} / \mathrm{L})\end{array}$ & $\begin{array}{c}\text { Di- } \\
\text { meth- } \\
\text { enamid } \\
\text { ESA } \\
(\mu \mathrm{g} / \mathrm{L})\end{array}$ & $\begin{array}{c}\text { Di- } \\
\text { meth- } \\
\text { enamid } \\
0 X A \\
(\mu \mathrm{g} / \mathrm{L})\end{array}$ & $\begin{array}{c}\text { Flufen- } \\
\text { acet } \\
(\mu \mathrm{g} / \mathrm{L})\end{array}$ & $\begin{array}{l}\text { Flufen- } \\
\text { acet } \\
\text { ESA } \\
(\mu \mathrm{g} / \mathrm{L})\end{array}$ & $\begin{array}{c}\text { Flufen- } \\
\text { acet } \\
\text { OXA } \\
(\mu \mathrm{g} / \mathrm{L})\end{array}$ & $\begin{array}{c}\text { Meto- } \\
\text { lachlor } \\
(\mu \mathrm{g} / \mathrm{L})\end{array}$ & $\begin{array}{c}\text { Meto- } \\
\text { lachlor } \\
\text { ESA } \\
(\mu \mathrm{g} / \mathrm{L})\end{array}$ & $\begin{array}{c}\text { Meto- } \\
\text { lachlor } \\
\text { OXA } \\
(\mu \mathrm{g} / \mathrm{L})\end{array}$ & 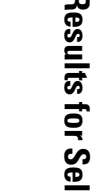 \\
\hline \multicolumn{20}{|c|}{ Midcontinent Agricultural Chemical Research Herbicide Project (fig. 1)—Continued } & \\
\hline \multicolumn{20}{|c|}{ lowa monitoring wells (DKE/DKW)—Continued } & \\
\hline \multirow[t]{2}{*}{423744095383301} & Cherokee Co., IA & 06/09/99 & $<0.05$ & $<0.20$ & $<0.20$ & -- & $<0.05$ & $<0.20$ & $<0.20$ & -- & $<0.05$ & -- & -- & -- & -- & -- & $<0.05$ & $<0.20$ & $<0.20$ & \\
\hline & & 08/01/01 & $<.05$ & $<.05$ & $<.05$ & -- & $<.05$ & $<.05$ & $<.05$ & -- & $<.05$ & $<0.05$ & $<0.05$ & $<0.05$ & $<0.05$ & $<0.05$ & $<.05$ & $<.05$ & $<.05$ & 兰. \\
\hline \multirow[t]{5}{*}{423954093535801} & Wright Co., IA & $06 / 29 / 95$ & $<.05$ & $<.20$ & $<.20$ & -- & $<.05$ & $<.20$ & $<.20$ & -- & -- & -- & -- & -- & -- & -- & $<.05$ & $<.20$ & $<.20$ & 퐁 \\
\hline & & 08/13/96 & $<.05$ & $<.20$ & $<.20$ & -- & $<.05$ & $<.20$ & $<.20$ & -- & -- & -- & -- & -- & -- & -- & $<.05$ & $<.20$ & $<.20$ & \\
\hline & & $07 / 23 / 97$ & $<.05$ & $<.20$ & $<.20$ & -- & $<.05$ & $<.20$ & $<.20$ & -- & -- & -- & -- & -- & -- & -- & $<.05$ & $<.20$ & $<.20$ & $\overline{\frac{1}{9}}$ \\
\hline & & $07 / 29 / 98$ & $<.05$ & $<.20$ & $<.20$ & -- & $<.05$ & $<.20$ & $<.20$ & -- & -- & -- & -- & -- & -- & -- & $<.05$ & $<.20$ & $<.20$ & \\
\hline & & 08/27/01 & $<.05$ & $<.05$ & $<.05$ & -- & $<.05$ & $<.05$ & $<.05$ & -- & $<.05$ & $<.05$ & $<.05$ & $<.05$ & $<.05$ & $<.05$ & $<.05$ & $<.05$ & $<.05$ & \\
\hline 423958093535701 & Wright Co, IA & 06/08/99 & $<.05$ & $<.20$ & $<.20$ & -- & $<.05$ & $<.20$ & $<.20$ & -- & $<.05$ & -- & -- & -- & -- & -- & $<.05$ & $<.20$ & $<.20$ & 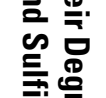 \\
\hline \multirow[t]{2}{*}{424026091321502} & Clayton Co., IA & $08 / 30 / 95$ & $<.05$ & $<.20$ & $<.20$ & -- & $<.05$ & 2.7 & 2.2 & -- & -- & -- & -- & -- & -- & -- & $<.05$ & 2.6 & .37 & \\
\hline & & $08 / 16 / 96$ & $<.05$ & $<.20$ & $<.20$ & -- & $<.05$ & 2.0 & $<.20$ & -- & & & & & & & $<.20$ & 2.2 & $<.20$ & \\
\hline 424319092283401 & Bremer Co., IA & $08 / 12 / 96$ & $<.05$ & $<.20$ & $<.20$ & -- & $<.05$ & 3.5 & $<.20$ & -- & -- & -- & -- & -- & -- & -- & $<.05$ & .33 & $<.20$ & \\
\hline \multirow[t]{2}{*}{424341095331301} & Cherokee Co., IA & 07/01/97 & $<.05$ & $<.20$ & $<.20$ & -- & $<.05$ & $<.20$ & $<.20$ & -- & -- & -- & -- & -- & -- & -- & $<.05$ & $<.20$ & $<.20$ & \\
\hline & & 08/01/01 & $<.05$ & $<.05$ & $<.05$ & -- & $<.05$ & $<.05$ & $<.05$ & -- & $<.05$ & $<.05$ & $<.05$ & $<.05$ & $<.05$ & $<.05$ & $<.05$ & $<.05$ & $<.05$ & \\
\hline 424705092320803 & Chickasaw Co., IA & 08/13/96 & $<.05$ & $<.20$ & $<.20$ & -- & $<.05$ & 2.6 & $<.20$ & -- & -- & -- & -- & -- & -- & -- & $<.05$ & 2.4 & .31 & \\
\hline \multirow[t]{6}{*}{424708094570801} & Buena Vista Co., IA & $07 / 25 / 95$ & $<.05$ & $<.20$ & $<.20$ & -- & $<.05$ & $<.20$ & $<.20$ & -- & -- & -- & -- & -- & -- & -- & $<.05$ & $<.20$ & $<.20$ & \\
\hline & & $07 / 10 / 96$ & $<.05$ & $<.20$ & $<.20$ & -- & $<.05$ & $<.20$ & $<.20$ & -- & -- & -- & -- & -- & -- & -- & $<.05$ & $<.20$ & $<.20$ & \\
\hline & & $07 / 02 / 97$ & $<.05$ & $<.20$ & $<.20$ & -- & $<.05$ & $<.20$ & $<.20$ & -- & -- & -- & -- & -- & -- & -- & $<.05$ & $<.20$ & $<.20$ & \\
\hline & & $06 / 23 / 98$ & $<.05$ & $<.20$ & $<.20$ & -- & $<.05$ & $<.20$ & $<.20$ & -- & -- & -- & -- & -- & -- & -- & $<.05$ & $<.20$ & $<.20$ & \\
\hline & & 06/07/99 & $<.05$ & $<.20$ & $<.20$ & -- & $<.05$ & $<.20$ & $<.20$ & -- & $<.05$ & -- & -- & -- & -- & -- & $<.05$ & $<.20$ & $<.20$ & 总 \\
\hline & & $07 / 23 / 01$ & $<.05$ & $<.05$ & $<.05$ & -- & $<.05$ & $<.05$ & $<.05$ & -- & $<.05$ & $<.05$ & $<.05$ & $<.05$ & $<.05$ & $<.05$ & $<.05$ & $<.05$ & $<.05$ & 冷韋 \\
\hline 424921095581501 & Plymouth Co., IA & $07 / 27 / 95$ & $<.05$ & $<.20$ & $<.20$ & -- & $<.05$ & .33 & $<.20$ & -- & -- & -- & -- & -- & -- & -- & $<.05$ & .55 & $<.20$ & \\
\hline
\end{tabular}


Table 5. Analytical results for selected acetamide herbicides and their degradation products, ethanesulfonic acid, oxanilic acid, and sulfinyl acetic acid, in ground-water samples, 1994-2002.-Continued

[OGRL, U.S. Geological Survey Organic Geochemistry Research Laboratory, Lawrence, Kansas; ESA, ethanesulfonic acid; OXA, oxanilic acid; SAA, sulfinyl acetic acid; --, no data; <, less than]

\begin{tabular}{|c|c|c|c|c|c|c|c|c|c|c|c|c|c|c|c|c|c|c|c|}
\hline Site number & Site location & $\begin{array}{l}\text { Date of col- } \\
\text { lection } \\
\text { (month/day/ } \\
\text { year) }\end{array}$ & $\begin{array}{c}\text { Aceto- } \\
\text { chlor } \\
(\mu \mathrm{g} / \mathrm{L})\end{array}$ & $\begin{array}{l}\text { Aceto- } \\
\text { chlor } \\
\text { ESA } \\
(\mu \mathrm{g} / \mathrm{L})\end{array}$ & $\begin{array}{c}\text { Aceto- } \\
\text { chlor } \\
\text { OXA } \\
(\mu \mathrm{g} / \mathrm{L})\end{array}$ & $\begin{array}{l}\text { Aceto- } \\
\text { chlor } \\
\text { SAA } \\
(\mu \mathrm{g} / \mathrm{L})\end{array}$ & $\begin{array}{l}\text { Ala- } \\
\text { chlor } \\
(\mu \mathrm{g} / \mathrm{L})\end{array}$ & $\begin{array}{l}\text { Ala- } \\
\text { chlor } \\
\text { ESA } \\
(\mu \mathrm{g} / \mathrm{L})\end{array}$ & $\begin{array}{l}\text { Ala- } \\
\text { chlor } \\
\text { OXA } \\
(\mu \mathrm{g} / \mathrm{L})\end{array}$ & $\begin{array}{l}\text { Ala- } \\
\text { chlor } \\
\text { SAA } \\
(\mu \mathrm{g} / \mathrm{L})\end{array}$ & $\begin{array}{c}\text { Di- } \\
\text { meth- } \\
\text { enamid } \\
(\mu \mathrm{g} / \mathrm{L})\end{array}$ & $\begin{array}{c}\text { Di- } \\
\text { meth- } \\
\text { enamid } \\
\text { ESA } \\
(\mu \mathrm{g} / \mathrm{L})\end{array}$ & $\begin{array}{c}\text { Di- } \\
\text { meth- } \\
\text { enamid } \\
\text { OXA } \\
(\mu \mathrm{g} / \mathrm{L})\end{array}$ & $\begin{array}{c}\text { Flufen- } \\
\text { acet } \\
(\mu \mathrm{g} / \mathrm{L})\end{array}$ & $\begin{array}{c}\text { Flufen- } \\
\text { acet } \\
\text { ESA } \\
(\mu \mathrm{g} / \mathrm{L})\end{array}$ & $\begin{array}{c}\text { Flufen- } \\
\text { acet } \\
\text { OXA } \\
(\mu \mathrm{g} / \mathrm{L})\end{array}$ & $\begin{array}{l}\text { Meto- } \\
\text { lachlor } \\
(\mu \mathrm{g} / \mathrm{L})\end{array}$ & $\begin{array}{c}\text { Meto- } \\
\text { lachlor } \\
\text { ESA } \\
(\mu \mathrm{g} / \mathrm{L})\end{array}$ & $\begin{array}{c}\text { Meto- } \\
\text { lachlor } \\
\text { OXA } \\
(\mu \mathrm{g} / \mathrm{L})\end{array}$ \\
\hline \multicolumn{20}{|c|}{ Midc ontinent Agricultural Chemical Research Herbicide Project (fig. 1)—Continued } \\
\hline \multicolumn{20}{|c|}{ lowa monitoring wells (DKE/DKW)—Continued } \\
\hline 424921095581501 & Plymouth Co., IA & 07/12/96 & $<0.05$ & $<0.20$ & $<0.20$ & -- & $<0.05$ & $<0.20$ & $<0.20$ & -- & -- & -- & -- & -- & -- & -- & $<0.05$ & 0.22 & $<0.20$ \\
\hline \multirow[t]{2}{*}{425330092483701} & Butler Co., IA & 08/27/97 & $<.05$ & $<.20$ & $<.20$ & -- & $<.05$ & .47 & $<.20$ & -- & -- & -- & -- & -- & -- & -- & $<.05$ & $<.20$ & $<.20$ \\
\hline & & 09/14/01 & $<.05$ & $<.05$ & $<.05$ & -- & $<.05$ & $<.05$ & $<.05$ & -- & $<0.05$ & $<0.05$ & $<0.05$ & $<0.05$ & $<0.05$ & $<0.05$ & $<.05$ & $<.05$ & $<.05$ \\
\hline \multirow[t]{4}{*}{425341093132501} & Franklin Co., IA & 08/13/96 & $<.05$ & $<.20$ & $<.20$ & -- & $<.05$ & $<.20$ & $<.20$ & -- & -- & -- & -- & -- & -- & -- & $<.05$ & 2.3 & $<.20$ \\
\hline & & 07/23/97 & $<.05$ & $<.20$ & $<.20$ & -- & $<.05$ & $<.20$ & $<.20$ & -- & -- & -- & -- & -- & -- & -- & $<.05$ & 1.2 & $<.20$ \\
\hline & & 08/12/99 & $<.05$ & $<.20$ & $<.20$ & -- & $<.05$ & $<.20$ & $<.20$ & -- & $<.05$ & -- & -- & $<.05$ & -- & -- & $<.05$ & .31 & $<.20$ \\
\hline & & $07 / 25 / 01$ & $<.05$ & .21 & $<.05$ & -- & $<.05$ & $<.05$ & $<.05$ & -- & $<.05$ & $<.05$ & $<.05$ & $<.05$ & $<.05$ & $<.05$ & $<.05$ & .36 & $<.05$ \\
\hline \multirow[t]{6}{*}{425344095090401} & Buena Vista Co., IA & $07 / 25 / 95$ & $<.05$ & $<.20$ & $<.20$ & -- & $<.05$ & 1.0 & .58 & -- & -- & -- & -- & -- & -- & -- & 2.3 & 2.9 & 2.0 \\
\hline & & 07/10/96 & $<.05$ & $<.20$ & $<.20$ & -- & $<.05$ & 1.1 & .59 & -- & -- & -- & -- & -- & -- & -- & 1.4 & 3.2 & 2.0 \\
\hline & & 07/02/97 & $<.05$ & $<.20$ & $<.20$ & -- & $<.05$ & .36 & .24 & -- & -- & -- & -- & -- & -- & -- & 1.4 & 1.2 & .87 \\
\hline & & 06/23/98 & $<.05$ & $<.20$ & $<.20$ & -- & $<.05$ & .41 & $<.20$ & -- & -- & -- & -- & -- & -- & -- & 1.1 & 1.4 & .56 \\
\hline & & 06/07/99 & $<.05$ & $<.20$ & $<.20$ & -- & $<.05$ & .60 & .93 & -- & $<.05$ & -- & -- & -- & -- & -- & 1.4 & 1.5 & 1.3 \\
\hline & & 07/23/01 & $<.05$ & $<.05$ & $<.05$ & -- & $<.05$ & .19 & .08 & -- & $<.05$ & $<.05$ & $<.05$ & $<.05$ & $<.05$ & $<.05$ & 2.6 & 1.1 & 1.3 \\
\hline \multirow[t]{2}{*}{425528093364501} & Hancock Co., IA & $07 / 23 / 97$ & $<.05$ & $<.20$ & $<.20$ & -- & $<.05$ & $<.20$ & $<.20$ & -- & -- & -- & -- & -- & -- & -- & $<.05$ & $<.20$ & $<.20$ \\
\hline & & 07/24/01 & $<.05$ & $<.05$ & $<.05$ & -- & $<.05$ & .09 & .10 & -- & $<.05$ & $<.05$ & $<.05$ & $<.05$ & $<.05$ & $<.05$ & $<.05$ & .05 & $<.05$ \\
\hline \multirow[t]{2}{*}{425606091565501} & Fayette Co., IA & 08/30/95 & $<.05$ & $<.20$ & $<.20$ & -- & $<.05$ & 1.3 & $<.20$ & -- & -- & -- & -- & -- & -- & -- & $<.05$ & .20 & $<.20$ \\
\hline & & $08 / 14 / 96$ & $<.05$ & $<.20$ & $<.20$ & -- & $<.05$ & 1.4 & $<.20$ & -- & -- & -- & -- & -- & -- & -- & $<.05$ & $<.20$ & $<.20$ \\
\hline \multirow[t]{5}{*}{425717091382602} & Fayette Co., IA & 08/30/95 & $<.05$ & $<.20$ & $<.20$ & -- & $<.05$ & .29 & $<.20$ & -- & -- & -- & -- & -- & -- & -- & $<.05$ & 2.3 & .30 \\
\hline & & 08/15/96 & $<.05$ & $<.20$ & $<.20$ & -- & $<.05$ & .21 & $<.20$ & -- & -- & -- & -- & -- & -- & -- & $<.05$ & 4.0 & $<.20$ \\
\hline & & $08 / 25 / 97$ & $<.05$ & $<.20$ & $<.20$ & -- & $<.05$ & .22 & $<.20$ & -- & -- & -- & -- & -- & -- & -- & $<.05$ & 2.8 & .20 \\
\hline & & 08/10/99 & $<.05$ & $<.20$ & $<.20$ & -- & $<.05$ & $<.20$ & $<.20$ & -- & $<.05$ & -- & -- & $<.05$ & -- & -- & $<.05$ & 1.7 & $<.20$ \\
\hline & & 09/12/01 & $<.05$ & $<.05$ & $<.05$ & -- & $<.05$ & .11 & $<.05$ & -- & $<.05$ & $<.05$ & $<.05$ & $<.05$ & $<.05$ & $<.05$ & $<.05$ & .95 & $<.05$ \\
\hline
\end{tabular}


Table 5. Analytical results for selected acetamide herbicides and their degradation products, ethanesulfonic acid, oxanilic acid, and sulfinyl acetic acid, in ground-water samples, 1994-2002.-Continued

[OGRL, U.S. Geological Survey Organic Geochemistry Research Laboratory, Lawrence, Kansas; ESA, ethanesulfonic acid; OXA, oxanilic acid; SAA, sulfinyl acetic acid; --, no data; <, less than]

\begin{tabular}{|c|c|c|c|c|c|c|c|c|c|c|c|c|c|c|c|c|c|c|c|}
\hline Site number & Site location & $\begin{array}{l}\text { Date of col- } \\
\text { lection } \\
\text { (month/day/ } \\
\text { year) }\end{array}$ & $\begin{array}{c}\text { Aceto- } \\
\text { chlor } \\
(\mu \mathrm{g} / \mathrm{L})\end{array}$ & $\begin{array}{l}\text { Aceto- } \\
\text { chlor } \\
\text { ESA } \\
(\mu \mathrm{g} / \mathrm{L})\end{array}$ & $\begin{array}{c}\text { Aceto- } \\
\text { chlor } \\
\text { OXA } \\
(\mu \mathrm{g} / \mathrm{L})\end{array}$ & $\begin{array}{l}\text { Aceto- } \\
\text { chlor } \\
\text { SAA } \\
(\mu \mathrm{g} / \mathrm{L})\end{array}$ & $\begin{array}{l}\text { Ala- } \\
\text { chlor } \\
(\mu \mathrm{g} / \mathrm{L})\end{array}$ & $\begin{array}{l}\text { Ala- } \\
\text { chlor } \\
\text { ESA } \\
(\mu \mathrm{g} / \mathrm{L})\end{array}$ & $\begin{array}{c}\text { Ala- } \\
\text { chlor } \\
\text { OXA } \\
(\mu \mathrm{g} / \mathrm{L})\end{array}$ & $\begin{array}{c}\text { Ala- } \\
\text { chlor } \\
\text { SAA } \\
(\mu \mathrm{g} / \mathrm{L})\end{array}$ & $\begin{array}{c}\text { Di- } \\
\text { meth- } \\
\text { enamid } \\
(\mu \mathrm{g} / \mathrm{L})\end{array}$ & $\begin{array}{c}\text { Di- } \\
\text { meth- } \\
\text { enamid } \\
\text { ESA } \\
(\mu \mathrm{g} / \mathrm{L})\end{array}$ & $\begin{array}{c}\text { Di- } \\
\text { meth- } \\
\text { enamid } \\
\text { OXA } \\
(\mu \mathrm{g} / \mathrm{L})\end{array}$ & $\begin{array}{c}\text { Flufen- } \\
\text { acet } \\
(\mu \mathrm{g} / \mathrm{L})\end{array}$ & $\begin{array}{c}\text { Flufen- } \\
\text { acet } \\
\text { ESA } \\
(\mu \mathrm{g} / \mathrm{L})\end{array}$ & $\begin{array}{c}\text { Flufen- } \\
\text { acet } \\
\text { OXA } \\
(\mu \mathrm{g} / \mathrm{L})\end{array}$ & $\begin{array}{l}\text { Meto- } \\
\text { lachlor } \\
(\mu \mathrm{g} / \mathrm{L})\end{array}$ & $\begin{array}{c}\text { Meto- } \\
\text { lachlor } \\
\text { ESA } \\
(\mu \mathrm{g} / \mathrm{L})\end{array}$ & $\begin{array}{c}\text { Meto- } \\
\text { lachlor } \\
\text { OXA } \\
(\mu \mathrm{g} / \mathrm{L})\end{array}$ \\
\hline \multicolumn{20}{|c|}{ Midcontinent Agricultural Chemical Research Herbicide Project (fig. 1)—Continued } \\
\hline \multicolumn{20}{|c|}{ lowa monitoring wells (DKE/DKW)—Continued } \\
\hline \multirow[t]{2}{*}{425719091483301} & Fayette Co., IA & $08 / 30 / 95$ & $<0.05$ & $<0.20$ & $<0.20$ & -- & $<0.05$ & 2.0 & $<0.20$ & -- & -- & -- & -- & -- & -- & -- & $<0.05$ & 2.3 & $<0.20$ \\
\hline & & $08 / 15 / 96$ & $<.05$ & $<.20$ & $<.20$ & -- & $<.05$ & 1.2 & $<.20$ & -- & -- & -- & -- & -- & -- & -- & $<.05$ & 2.1 & $<.20$ \\
\hline \multirow[t]{2}{*}{425731094270801} & Palo Alto Co., IA & $07 / 24 / 97$ & $<.05$ & $<.20$ & $<.20$ & -- & $<.05$ & $<.20$ & $<.20$ & -- & -- & -- & -- & -- & -- & -- & $<.05$ & $<.20$ & $<.20$ \\
\hline & & $07 / 23 / 01$ & $<.05$ & $<.05$ & $<.05$ & -- & $<.05$ & $<.05$ & $<.05$ & -- & $<0.05$ & $<0.05$ & $<0.05$ & $<0.05$ & $<0.05$ & $<0.05$ & $<.05$ & $<.05$ & $<.05$ \\
\hline \multirow[t]{3}{*}{425948096295401} & Sioux Co., IA & $07 / 26 / 95$ & $<.05$ & .20 & .20 & -- & $<.05$ & .43 & .25 & -- & -- & -- & -- & -- & -- & -- & $<.05$ & 1.5 & .45 \\
\hline & & $07 / 11 / 96$ & $<.05$ & $<.20$ & $<.20$ & -- & $<.05$ & $<.20$ & $<.20$ & -- & -- & -- & -- & -- & -- & -- & $<.05$ & 1.0 & .22 \\
\hline & & $07 / 11 / 96$ & $<.05$ & $<.20$ & $<.20$ & -- & $<.05$ & .25 & $<.20$ & -- & -- & -- & -- & -- & -- & -- & $<.05$ & 1.1 & .32 \\
\hline \multirow[t]{4}{*}{430015093360501} & Hancock Co., IA & 08/13/96 & $<.05$ & $<.20$ & $<.20$ & -- & $<.05$ & $<.20$ & $<.20$ & -- & -- & -- & -- & -- & -- & -- & $<.05$ & $<.20$ & $<.20$ \\
\hline & & $07 / 23 / 97$ & $<.05$ & $<.20$ & $<.20$ & -- & $<.05$ & $<.20$ & $<.20$ & -- & -- & -- & -- & -- & -- & -- & $<.05$ & $<.20$ & $<.20$ \\
\hline & & 06/08/99 & $<.05$ & $<.05$ & $<.05$ & -- & $<.05$ & $<.05$ & $<.05$ & -- & $<.05$ & -- & -- & -- & -- & -- & $<.05$ & $<.05$ & $<.05$ \\
\hline & & $07 / 24 / 01$ & $<.05$ & $<.05$ & $<.05$ & -- & $<.05$ & $<.05$ & $<.05$ & -- & $<.05$ & $<.05$ & $<.05$ & $<.05$ & $<.05$ & $<.05$ & $<.05$ & $<.05$ & .05 \\
\hline 430015093360502 & Hancock Co., IA & $08 / 27 / 01$ & $<.05$ & $<.05$ & $<.05$ & -- & $<.05$ & $<.05$ & $<.05$ & -- & $<.05$ & $<.05$ & $<.05$ & $<.05$ & $<.05$ & $<.05$ & $<.05$ & $<.05$ & $<.05$ \\
\hline \multirow[t]{4}{*}{430017096285301} & Sioux Co., IA & 07/03/97 & $<.05$ & $<.20$ & $<.20$ & -- & $<.05$ & $<.20$ & $<.20$ & -- & -- & -- & -- & -- & -- & -- & $<.05$ & $<.20$ & $<.20$ \\
\hline & & $06 / 24 / 98$ & $<.05$ & $<.20$ & $<.20$ & -- & $<.05$ & $<.20$ & $<.20$ & -- & -- & -- & -- & -- & -- & -- & $<.05$ & $<.20$ & $<.20$ \\
\hline & & 06/08/99 & $<.05$ & $<.20$ & $<.20$ & -- & $<.05$ & $<.20$ & $<.20$ & -- & $<.05$ & -- & -- & -- & -- & -- & $<.05$ & $<.20$ & $<.20$ \\
\hline & & $07 / 31 / 01$ & $<.05$ & $<.05$ & $<.05$ & -- & $<.05$ & $<.05$ & $<.05$ & -- & $<.05$ & $<.05$ & $<.05$ & $<.05$ & $<.05$ & $<.05$ & $<.05$ & .09 & $<.05$ \\
\hline \multirow[t]{2}{*}{430922095193501} & Clay Co., IA & $07 / 26 / 95$ & $<.05$ & $<.20$ & $<.20$ & -- & $<.05$ & 1.9 & 3.6 & -- & -- & -- & -- & -- & -- & -- & .05 & 1.2 & 1.7 \\
\hline & & $07 / 11 / 96$ & $<.05$ & $<.20$ & $<.20$ & -- & $<.05$ & 2.9 & 8.1 & -- & -- & -- & -- & -- & -- & -- & .10 & 1.7 & 2.7 \\
\hline 043112092213901 & Black Hawk Co., IA & $08 / 12 / 96$ & $<.05$ & $<.20$ & $<.20$ & -- & $<.05$ & .84 & $<.20$ & -- & -- & -- & -- & -- & -- & -- & $<.05$ & .43 & $<.20$ \\
\hline \multirow[t]{2}{*}{431151095505101} & O'Brien Co., IA & $07 / 26 / 95$ & $<.05$ & .53 & -- & -- & $<.05$ & .35 & $<.20$ & -- & -- & -- & -- & -- & -- & -- & $<.05$ & 2.7 & -- \\
\hline & & $07 / 11 / 96$ & $<.05$ & $<.20$ & $<.20$ & -- & $<.05$ & $<.20$ & $<.20$ & -- & -- & -- & -- & -- & -- & -- & $<.05$ & 3.0 & .21 \\
\hline
\end{tabular}


Table 5. Analytical results for selected acetamide herbicides and their degradation products, ethanesulfonic acid, oxanilic acid, and sulfinyl acetic acid, in ground-water samples, 1994-2002.-Continued

[OGRL, U.S. Geological Survey Organic Geochemistry Research Laboratory, Lawrence, Kansas; ESA, ethanesulfonic acid; OXA, oxanilic acid; SAA, sulfinyl acetic acid; --, no data; <, less than]

\begin{tabular}{|c|c|c|c|c|c|c|c|c|c|c|c|c|c|c|c|c|c|c|c|}
\hline Site number & Site location & $\begin{array}{l}\text { Date of col- } \\
\text { lection } \\
\text { (month/day/ } \\
\text { year) }\end{array}$ & $\begin{array}{c}\text { Aceto- } \\
\text { chlor } \\
(\mu \mathrm{g} / \mathrm{L})\end{array}$ & $\begin{array}{l}\text { Aceto- } \\
\text { chlor } \\
\text { ESA } \\
(\mu \mathrm{g} / \mathrm{L})\end{array}$ & $\begin{array}{c}\text { Aceto- } \\
\text { chlor } \\
\text { OXA } \\
(\mu \mathrm{g} / \mathrm{L})\end{array}$ & $\begin{array}{l}\text { Aceto- } \\
\text { chlor } \\
\text { SAA } \\
(\mu \mathrm{g} / \mathrm{L})\end{array}$ & $\begin{array}{l}\text { Ala- } \\
\text { chlor } \\
(\mu \mathrm{g} / \mathrm{L})\end{array}$ & $\begin{array}{l}\text { Ala- } \\
\text { chlor } \\
\text { ESA } \\
(\mu \mathrm{g} / \mathrm{L})\end{array}$ & $\begin{array}{l}\text { Ala- } \\
\text { chlor } \\
\text { OXA } \\
(\mu \mathrm{g} / \mathrm{L})\end{array}$ & $\begin{array}{l}\text { Ala- } \\
\text { chlor } \\
\text { SAA } \\
(\mu \mathrm{g} / \mathrm{L})\end{array}$ & $\begin{array}{c}\text { Di- } \\
\text { meth- } \\
\text { enamid } \\
(\mu \mathrm{g} / \mathrm{L})\end{array}$ & $\begin{array}{c}\text { Di- } \\
\text { meth- } \\
\text { enamid } \\
\text { ESA } \\
(\mu \mathrm{g} / \mathrm{L})\end{array}$ & $\begin{array}{c}\text { Di- } \\
\text { meth- } \\
\text { enamid } \\
\text { OXA } \\
(\mu \mathrm{g} / \mathrm{L})\end{array}$ & $\begin{array}{c}\text { Flufen- } \\
\text { acet } \\
(\mu \mathrm{g} / \mathrm{L})\end{array}$ & $\begin{array}{c}\text { Flufen- } \\
\text { acet } \\
\text { ESA } \\
(\mu \mathrm{g} / \mathrm{L})\end{array}$ & $\begin{array}{c}\text { Flufen- } \\
\text { acet } \\
\text { OXA } \\
(\mu \mathrm{g} / \mathrm{L})\end{array}$ & $\begin{array}{l}\text { Meto- } \\
\text { lachlor } \\
(\mu \mathrm{g} / \mathrm{L})\end{array}$ & $\begin{array}{l}\text { Meto- } \\
\text { lachlor } \\
\text { ESA } \\
(\mu \mathrm{g} / \mathrm{L})\end{array}$ & $\begin{array}{c}\text { Meto- } \\
\text { lachlor } \\
\text { OXA } \\
(\mu \mathrm{g} / \mathrm{L})\end{array}$ \\
\hline \multicolumn{20}{|c|}{ Midc ontinent Agricultural Chemical Research Herbicide Project (fig. 1)—Continued } \\
\hline \multicolumn{20}{|c|}{ lowa monitoring wells (DKE/DKW)—Continued } \\
\hline \multirow[t]{4}{*}{431151095505101} & O'Brien Co., IA & 07/02/97 & $<0.05$ & $<0.20$ & $<0.20$ & -- & $<0.05$ & $<0.20$ & $<0.20$ & -- & -- & -- & -- & -- & -- & -- & $<0.05$ & 2.2 & 0.52 \\
\hline & & 06/24/98 & $<.05$ & $<.20$ & $<.20$ & -- & $<.05$ & $<.20$ & $<.20$ & -- & -- & -- & -- & -- & -- & -- & $<.05$ & 2.8 & $<.20$ \\
\hline & & 06/08/99 & $<.05$ & $<.20$ & $<.20$ & -- & $<.05$ & $<.20$ & $<.20$ & -- & $<0.05$ & -- & -- & -- & -- & -- & $<.05$ & 2.0 & $<.20$ \\
\hline & & 07/31/01 & $<.05$ & .19 & .17 & -- & $<.05$ & .13 & $<.05$ & -- & $<.05$ & $<0.05$ & $<0.05$ & $<0.05$ & $<0.05$ & $<0.05$ & $<.05$ & 1.5 & .26 \\
\hline \multirow[t]{2}{*}{431303092052002} & Howard Co., IA & 09/05/95 & $<.05$ & $<.20$ & $<.20$ & -- & $<.05$ & 5.7 & $<.20$ & -- & -- & -- & -- & -- & -- & -- & $<.05$ & 1.2 & $<.20$ \\
\hline & & 08/14/96 & $<.05$ & .28 & $<.20$ & -- & $<.05$ & 6.4 & $<.20$ & -- & & & & & & & $<.05$ & 1.4 & $<.20$ \\
\hline \multirow[t]{2}{*}{431429094450601} & Palo Alto Co., IA & $07 / 25 / 95$ & $<.05$ & .83 & -- & -- & $<.05$ & .47 & $<.20$ & -- & -- & -- & -- & -- & -- & -- & .22 & 2.4 & -- \\
\hline & & 07/10/96 & $<.05$ & $<.20$ & $<.20$ & -- & $<.05$ & $<.20$ & $<.20$ & -- & -- & -- & -- & -- & -- & -- & $<.05$ & 1.9 & $<.20$ \\
\hline 431443092261401 & Howard Co., IA & 08/13/96 & $<.05$ & $<.20$ & $<.20$ & -- & $<.05$ & 1.7 & .54 & -- & -- & -- & -- & -- & -- & -- & .06 & 1.9 & $<.20$ \\
\hline \multirow[t]{2}{*}{431556093375401} & Winnebago Co., IA & 07/24/97 & $<.05$ & $<.20$ & $<.20$ & -- & $<.05$ & $<.20$ & $<.20$ & -- & -- & -- & -- & -- & -- & -- & $<.05$ & $<.20$ & $<.20$ \\
\hline & & $07 / 24 / 01$ & $<.05$ & $<.05$ & $<.05$ & -- & $<.05$ & $<.05$ & $<.05$ & -- & $<.05$ & $<.05$ & $<.05$ & $<.05$ & $<.05$ & $<.05$ & $<.05$ & $<.05$ & $<.05$ \\
\hline \multirow[t]{4}{*}{431638091282902} & Allamakee Co., IA & $08 / 25 / 95$ & $<.05$ & $<.20$ & $<.20$ & -- & $<.05$ & $<.20$ & $<.20$ & -- & -- & -- & -- & -- & -- & -- & $<.05$ & $<.20$ & $<.20$ \\
\hline & & $08 / 25 / 97$ & $<.05$ & $<.20$ & $<.20$ & -- & $<.05$ & $<.20$ & $<.20$ & -- & -- & -- & -- & -- & -- & -- & $<.05$ & $<.20$ & $<.20$ \\
\hline & & 08/11/99 & $<.05$ & $<.20$ & $<.20$ & -- & $<.05$ & $<.20$ & $<.20$ & -- & $<.05$ & -- & -- & $<.05$ & -- & -- & $<.05$ & $<.20$ & $<.20$ \\
\hline & & 09/12/01 & $<.05$ & $<.05$ & $<.05$ & -- & $<.05$ & .07 & $<.05$ & -- & $<.05$ & $<.05$ & $<.05$ & $<.05$ & $<.05$ & $<.05$ & $<.05$ & $<.05$ & $<.05$ \\
\hline \multirow[t]{2}{*}{431654092484501} & Mitchell Co., IA & 08/26/97 & $<.05$ & $<.20$ & $<.20$ & -- & $<.05$ & .54 & $<.20$ & -- & -- & -- & -- & -- & -- & -- & $<.05$ & .33 & $<.20$ \\
\hline & & 09/13/01 & $<.05$ & $<.05$ & $<.05$ & -- & $<.05$ & .45 & $<.05$ & -- & $<.05$ & $<.05$ & $<.05$ & $<.05$ & $<.05$ & $<.05$ & $<.05$ & .32 & .11 \\
\hline \multirow[t]{5}{*}{431828091473201} & Winneshiek Co., IA & $08 / 25 / 95$ & $<.05$ & $<.20$ & $<.20$ & -- & $<.05$ & .74 & $<.20$ & -- & -- & -- & -- & -- & -- & -- & $<.05$ & .44 & $<.20$ \\
\hline & & 08/14/96 & $<.05$ & .24 & $<.20$ & -- & $<.05$ & .66 & $<.20$ & -- & -- & -- & -- & -- & -- & -- & $<.05$ & .53 & $<.20$ \\
\hline & & $08 / 25 / 97$ & $<.05$ & $<.20$ & $<.20$ & -- & $<.05$ & .40 & $<.20$ & -- & -- & -- & -- & -- & -- & -- & $<.05$ & .52 & $<.20$ \\
\hline & & 08/26/98 & $<.05$ & .25 & $<.20$ & -- & $<.05$ & .44 & $<.20$ & -- & -- & -- & -- & -- & -- & -- & $<.05$ & .46 & $<.20$ \\
\hline & & 08/11/99 & $<.05$ & $<.20$ & $<.20$ & -- & $<.05$ & $<.20$ & $<.20$ & -- & $<.05$ & -- & -- & $<.05$ & -- & -- & $<.05$ & $<.20$ & $<.20$ \\
\hline
\end{tabular}


Table 5. Analytical results for selected acetamide herbicides and their degradation products, ethanesulfonic acid, oxanilic acid, and sulfinyl acetic acid, in ground-water samples, 1994-2002.-Continued

[OGRL, U.S. Geological Survey Organic Geochemistry Research Laboratory, Lawrence, Kansas; ESA, ethanesulfonic acid; OXA, oxanilic acid; SAA, sulfinyl acetic acid; --, no data; <, less than]

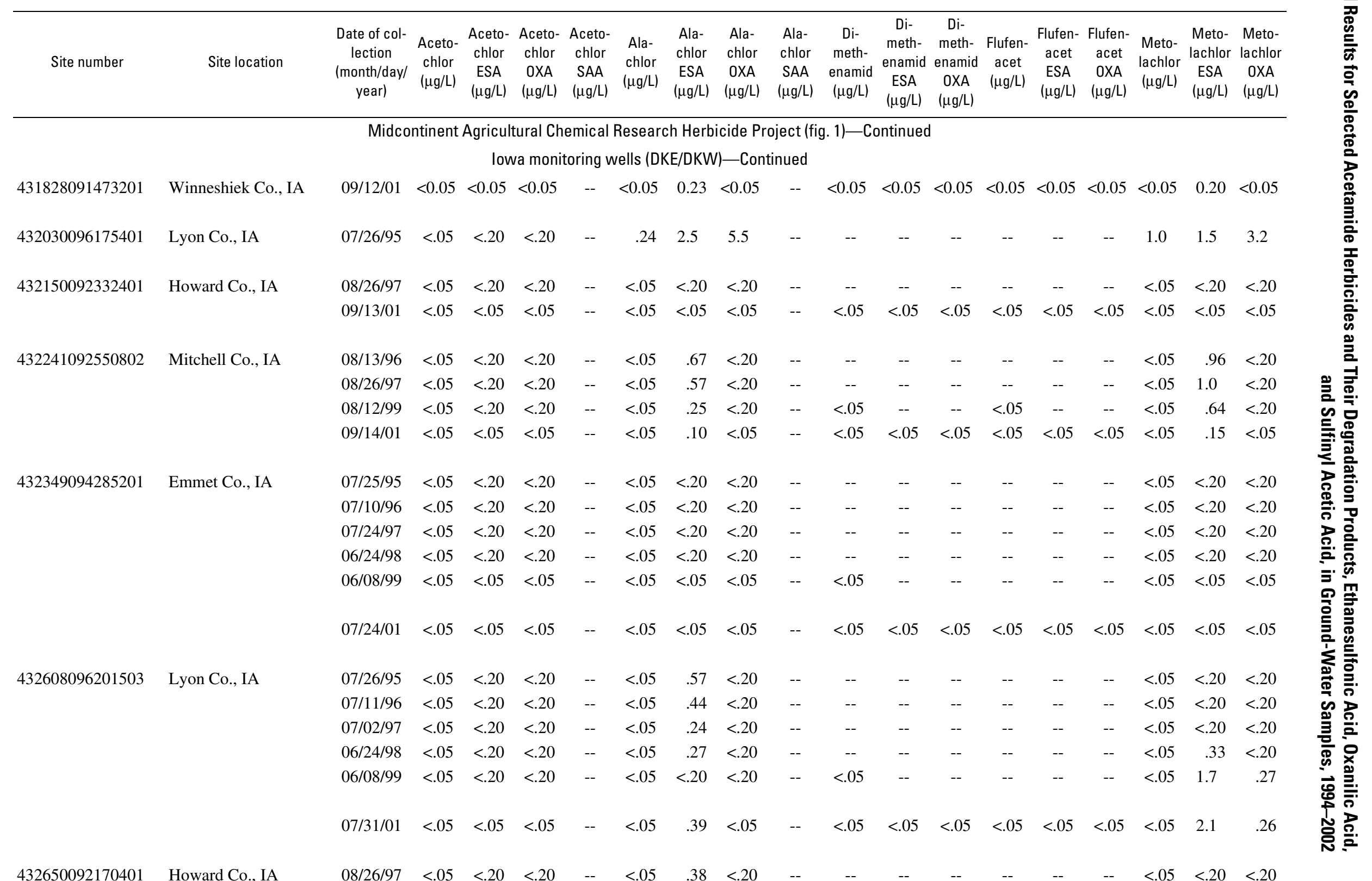


Table 5. Analytical results for selected acetamide herbicides and their degradation products, ethanesulfonic acid, oxanilic acid, and sulfinyl acetic acid, in ground-water samples, 1994-2002.-Continued

[OGRL, U.S. Geological Survey Organic Geochemistry Research Laboratory, Lawrence, Kansas; ESA, ethanesulfonic acid; OXA, oxanilic acid; SAA, sulfinyl acetic acid; --, no data; <, less than]

\begin{tabular}{|c|c|c|c|c|c|c|c|c|c|c|c|c|c|c|c|c|c|c|c|}
\hline Site number & Site location & $\begin{array}{l}\text { Date of col- } \\
\text { lection } \\
\text { (month/day/ } \\
\text { year) }\end{array}$ & $\begin{array}{l}\text { Aceto- } \\
\text { chlor } \\
(\mu \mathrm{g} / \mathrm{L})\end{array}$ & $\begin{array}{l}\text { Aceto- } \\
\text { chlor } \\
\text { ESA } \\
(\mu \mathrm{g} / \mathrm{L})\end{array}$ & $\begin{array}{c}\text { Aceto- } \\
\text { chlor } \\
\text { OXA } \\
(\mu \mathrm{g} / \mathrm{L})\end{array}$ & $\begin{array}{l}\text { Aceto- } \\
\text { chlor } \\
\text { SAA } \\
(\mu \mathrm{g} / \mathrm{L})\end{array}$ & $\begin{array}{l}\text { Ala- } \\
\text { chlor } \\
(\mu \mathrm{g} / \mathrm{L})\end{array}$ & $\begin{array}{c}\text { Ala- } \\
\text { chlor } \\
\text { ESA } \\
(\mu \mathrm{g} / \mathrm{L})\end{array}$ & $\begin{array}{l}\text { Ala- } \\
\text { chlor } \\
\text { OXA } \\
(\mu \mathrm{g} / \mathrm{L})\end{array}$ & $\begin{array}{l}\text { Ala- } \\
\text { chlor } \\
\text { SAA } \\
(\mu \mathrm{g} / \mathrm{L})\end{array}$ & $\begin{array}{c}\text { Di- } \\
\text { meth- } \\
\text { enamid } \\
(\mu \mathrm{g} / \mathrm{L})\end{array}$ & $\begin{array}{c}\text { Di- } \\
\text { meth- } \\
\text { enamid } \\
\text { ESA } \\
(\mu \mathrm{g} / \mathrm{L})\end{array}$ & $\begin{array}{c}\text { Di- } \\
\text { meth- } \\
\text { enamid } \\
\text { OXA } \\
(\mu \mathrm{g} / \mathrm{L})\end{array}$ & $\begin{array}{c}\text { Flufen- } \\
\text { acet } \\
(\mu \mathrm{g} / \mathrm{L})\end{array}$ & $\begin{array}{c}\text { Flufen- } \\
\text { acet } \\
\text { ESA } \\
(\mu \mathrm{g} / \mathrm{L})\end{array}$ & $\begin{array}{c}\text { Flufen- } \\
\text { acet } \\
0 X A \\
(\mu \mathrm{g} / \mathrm{L})\end{array}$ & $\begin{array}{l}\text { Meto- } \\
\text { lachlor } \\
(\mu \mathrm{g} / \mathrm{L})\end{array}$ & $\begin{array}{l}\text { Meto- } \\
\text { lachlor } \\
\text { ESA } \\
(\mu \mathrm{g} / \mathrm{L})\end{array}$ & $\begin{array}{c}\text { Meto- } \\
\text { lachlor } \\
\text { OXA } \\
(\mu \mathrm{g} / \mathrm{L})\end{array}$ \\
\hline \multicolumn{20}{|c|}{ Midcontinent Agricultural Chemical Research Herbicide Project (fig. 1)—Continued } \\
\hline \multicolumn{20}{|c|}{ lowa monitoring wells (DKE/DKW)—Continued } \\
\hline 432650092170401 & Howard Co., IA & 08/11/99 & $<0.05$ & $<0.20$ & $<0.20$ & -- & $<0.05$ & $<0.20$ & $<0.20$ & -- & $<0.05$ & -- & -- & $<0.05$ & -- & -- & $<0.05$ & $<0.20$ & $<0.20$ \\
\hline & & 09/13/01 & $<.05$ & $<.05$ & $<.05$ & -- & $<.05$ & .37 & $<.05$ & -- & $<.05$ & $<0.05$ & $<0.05$ & $<.05$ & $<0.05$ & $<0.05$ & $<.05$ & $<.05$ & $<.05$ \\
\hline 404553099341301 & Dawson Co., NE & 08/09/94 & $<.05$ & $<.20$ & -- & -- & $<.05$ & 2.3 & $<.20$ & -- & -- & -- & -- & -- & -- & -- & $<.05$ & 1.5 & -- \\
\hline 411501095251301 & Pottawattamie Co., IA & 07/01/94 & $<.05$ & -- & -- & -- & $<.05$ & -- & -- & -- & -- & -- & -- & -- & -- & -- & $<.05$ & -- & -- \\
\hline 412114092391001 & Mahaska Co., IA & 07/12/94 & $<.05$ & $<.20$ & -- & -- & $<.05$ & 2.3 & $<.20$ & -- & -- & -- & -- & -- & -- & -- & .19 & 2.8 & -- \\
\hline 430130096574401 & Clay Co., SD & 07/12/94 & $<.05$ & $<.20$ & -- & -- & $<.05$ & 1.8 & 1.8 & -- & -- & -- & -- & -- & -- & -- & $<.05$ & $<.20$ & -- \\
\hline 430546094411601 & Palo Alto Co., IA & 07/19/94 & $<.05$ & $<.20$ & -- & -- & $<.05$ & 4.6 & $<.20$ & -- & -- & -- & -- & -- & -- & -- & $<.05$ & 1.4 & -- \\
\hline 440037094372601 & Watowan Co., MN & 08/15/94 & $<.05$ & $<.20$ & -- & -- & $<.05$ & 3.3 & 4.6 & -- & -- & -- & -- & -- & -- & -- & $<.05$ & $<.20$ & -- \\
\hline 441753095160801 & Redwood Co., MN & 08/16/94 & $<.05$ & $<.20$ & -- & -- & $<.05$ & 7.7 & 9.5 & -- & -- & -- & -- & -- & -- & -- & $<.05$ & $<.20$ & -- \\
\hline \multicolumn{20}{|c|}{ National Water-Quality Assessment Program (NAWQA) (fig. 3) } \\
\hline \multicolumn{20}{|c|}{ Delaware River Basin (DELR/BET/DCG) } \\
\hline 400821075164001 & Montgomery Co., PA & 08/17/99 & -- & $<.05$ & $<.05$ & -- & -- & $<.05$ & $<.05$ & -- & -- & -- & -- & -- & -- & -- & -- & $<.05$ & $<0.05$ \\
\hline 400954075354501 & Chester Co., PA & 07/15/99 & -- & $<.05$ & $<.05$ & -- & -- & $<.05$ & $<.05$ & -- & -- & -- & -- & -- & -- & -- & -- & $<.05$ & $<.05$ \\
\hline 401154075055001 & Bucks Co., PA & 08/18/99 & -- & $<.05$ & $<.05$ & -- & -- & $<.05$ & $<.05$ & -- & -- & -- & -- & -- & -- & -- & -- & $<.05$ & $<.05$ \\
\hline 401304074552001 & Bucks Co., PA & $11 / 23 / 99$ & -- & $<.05$ & $<.05$ & -- & -- & $<.05$ & $<.05$ & -- & -- & -- & -- & -- & -- & -- & -- & $<.05$ & $<.05$ \\
\hline 401334075213801 & Montgomery Co., PA & $10 / 20 / 99$ & -- & $<.05$ & $<.05$ & -- & -- & .15 & $<.05$ & -- & -- & -- & -- & -- & -- & -- & -- & 1.1 & $<.05$ \\
\hline 401405075275101 & Berks Co., PA & 06/22/99 & -- & $<.05$ & $<.05$ & -- & -- & .05 & $<.05$ & -- & -- & -- & -- & -- & -- & -- & -- & .12 & $<.05$ \\
\hline 401416075445601 & Berks Co., PA & 06/29/99 & -- & $<.05$ & $<.05$ & -- & -- & .11 & $<.05$ & -- & -- & -- & -- & -- & -- & -- & -- & .44 & $<.05$ \\
\hline 401446075193701 & Montgomery Co., PA & $10 / 21 / 99$ & -- & $<.05$ & $<.05$ & -- & -- & .34 & $<.05$ & -- & -- & -- & -- & -- & -- & -- & -- & .10 & $<.05$ \\
\hline 401458079501101 & Mercer Co., NJ & 07/29/99 & -- & $<.05$ & $<.05$ & -- & -- & .12 & $<.05$ & -- & -- & -- & -- & -- & -- & -- & -- & .26 & $<.05$ \\
\hline 401620075055001 & Bucks Co., PA & $10 / 05 / 99$ & -- & $<.05$ & $<.05$ & -- & -- & .16 & $<.05$ & -- & -- & -- & -- & -- & -- & -- & -- & .06 & $<.05$ \\
\hline
\end{tabular}




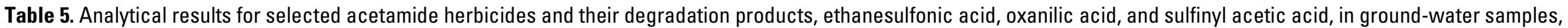
1994-2002.-Continued

[OGRL, U.S. Geological Survey Organic Geochemistry Research Laboratory, Lawrence, Kansas; ESA, ethanesulfonic acid; OXA, oxanilic acid; SAA, sulfinyl acetic acid; --, no data; <, less than]

\begin{tabular}{|c|c|c|c|c|c|c|c|c|c|c|c|c|c|c|c|c|c|c|c|c|}
\hline Site number & Site location & $\begin{array}{l}\text { Date of col- } \\
\text { lection } \\
\text { (month/day/ } \\
\text { year) }\end{array}$ & $\begin{array}{l}\text { Aceto- } \\
\text { chlor } \\
(\mu \mathrm{g} / \mathrm{L})\end{array}$ & $\begin{array}{l}\text { Aceto- } \\
\text { chlor } \\
\text { ESA } \\
(\mu \mathrm{g} / \mathrm{L})\end{array}$ & $\begin{array}{l}\text { Aceto- } \\
\text { chlor } \\
\text { OXA } \\
(\mu \mathrm{g} / \mathrm{L})\end{array}$ & $\begin{array}{l}\text { Aceto- } \\
\text { chlor } \\
\text { SAA } \\
(\mu \mathrm{g} / \mathrm{L})\end{array}$ & $\begin{array}{l}\text { Ala- } \\
\text { chlor } \\
(\mu \mathrm{g} / \mathrm{L})\end{array}$ & $\begin{array}{l}\text { Ala- } \\
\text { chlor } \\
\text { ESA } \\
(\mu \mathrm{g} / \mathrm{L})\end{array}$ & $\begin{array}{l}\text { Ala- } \\
\text { chlor } \\
\text { OXA } \\
(\mu \mathrm{g} / \mathrm{L})\end{array}$ & $\begin{array}{l}\text { Ala- } \\
\text { chlor } \\
\text { SAA } \\
(\mu \mathrm{g} / \mathrm{L})\end{array}$ & $\begin{array}{l}\text { Di- } \\
\text { meth- } \\
\text { enamid } \\
(\mu \mathrm{g} / \mathrm{L})\end{array}$ & $\begin{array}{c}\text { Di- } \\
\text { meth- } \\
\text { enamid } \\
\text { ESA } \\
(\mu \mathrm{g} / \mathrm{L})\end{array}$ & $\begin{array}{c}\text { Di- } \\
\text { meth- } \\
\text { enamid } \\
\text { OXA } \\
(\mu \mathrm{g} / \mathrm{L})\end{array}$ & $\begin{array}{l}\text { Flufen- } \\
\text { acet } \\
(\mu \mathrm{g} / \mathrm{L})\end{array}$ & $\begin{array}{l}\text { Flufen- } \\
\text { acet } \\
\text { ESA } \\
(\mu \mathrm{g} / \mathrm{L})\end{array}$ & $\begin{array}{c}\text { Flufen- } \\
\text { acet } \\
\text { OXA } \\
(\mu \mathrm{g} / \mathrm{L})\end{array}$ & $\begin{array}{l}\text { Meto- } \\
\text { lachlor } \\
(\mu \mathrm{g} / \mathrm{L})\end{array}$ & $\begin{array}{l}\text { Meto- } \\
\text { lachlor } \\
\text { ESA } \\
(\mu \mathrm{g} / \mathrm{L})\end{array}$ & $\begin{array}{l}\text { Meto- } \\
\text { lachlor } \\
\text { OXA } \\
(\mu \mathrm{g} / \mathrm{L})\end{array}$ & $\begin{array}{l}\bar{F} \\
\overrightarrow{0} \\
\frac{0}{a} \\
\frac{5}{D} \\
\frac{D}{9}\end{array}$ \\
\hline \multicolumn{20}{|c|}{ National Water-Quality Assessment Program (NAWQA) (fig. 3)—Continued } & \\
\hline \multicolumn{20}{|c|}{ Delaware River Basin (DELR/BET/DCG)-Continued } & \\
\hline 401705075313001 & Montgomery Co., PA & $11 / 15 / 99$ & -- & 0.15 & $<0.05$ & -- & -- & 0.06 & $<0.05$ & -- & -- & -- & -- & -- & -- & -- & -- & $<0.05$ & $<0.05$ & \\
\hline 401811075462501 & Berks Co., PA & 09/28/99 & -- & $<.05$ & $<.05$ & -- & -- & .14 & $<.05$ & -- & -- & -- & -- & -- & -- & -- & -- & 2.1 & 0.66 & \\
\hline 402027075072901 & Bucks Co., PA & 09/08/99 & -- & $<.05$ & $<.05$ & -- & -- & $<.05$ & $<.05$ & -- & -- & -- & -- & -- & -- & -- & -- & .08 & $<.05$ & \\
\hline 402119075173701 & Bucks Co., PA & 09/30/99 & -- & $<.05$ & $<.05$ & -- & -- & $<.05$ & $<.05$ & -- & -- & -- & -- & -- & -- & -- & -- & $<.05$ & $<.05$ & \\
\hline 402151074525301 & Hunterdon Co., NJ & 07/21/99 & -- & .43 & $<.05$ & -- & -- & 1.4 & $<.05$ & -- & -- & -- & -- & -- & -- & -- & -- & 5.5 & 1.1 & \\
\hline 402315075052701 & Bucks Co., PA & $10 / 13 / 99$ & -- & $<.05$ & $<.05$ & -- & -- & $<.05$ & $<.05$ & -- & -- & -- & -- & -- & -- & -- & -- & $<.05$ & $<.05$ & \\
\hline 402337075290001 & Montgomery Co., PA & 06/30/99 & -- & $<.05$ & $<.05$ & -- & -- & .60 & $<.05$ & -- & -- & -- & -- & -- & -- & -- & -- & 1.4 & .32 & \\
\hline 402555075114701 & Bucks Co., PA & 06/15/99 & -- & $<.05$ & $<.05$ & -- & -- & $<.05$ & $<.05$ & -- & -- & $<.05$ & $<.05$ & -- & -- & -- & -- & .90 & .13 & \\
\hline 402622076120201 & Berks Co., PA & 06/20/00 & -- & $<.05$ & $<.05$ & -- & -- & .21 & .21 & -- & -- & $<.05$ & $<.05$ & -- & -- & -- & -- & $<.05$ & $<.05$ & \\
\hline 402939076062701 & Berks Co., PA & 07/11/00 & -- & $<.05$ & $<.05$ & -- & -- & $<.05$ & $<.05$ & -- & -- & $<.05$ & $<.05$ & -- & $<0.05$ & $<0.05$ & -- & .08 & $<.05$ & \\
\hline 403002075250201 & LeHigh Co., PA & 09/21/99 & -- & $<.05$ & $<.05$ & -- & -- & $<.05$ & $<.05$ & -- & -- & -- & -- & -- & -- & -- & -- & $<.05$ & $<.05$ & \\
\hline 403003075000001 & Hunterdon Co., NJ & 08/19/99 & -- & $<.05$ & $<.05$ & -- & -- & .28 & $<.05$ & -- & -- & -- & -- & -- & -- & -- & -- & .20 & .10 & \\
\hline 403140075052901 & Bucks Co., PA & 07/01/99 & -- & $<.05$ & $<.05$ & -- & -- & $<.05$ & $<.05$ & -- & -- & -- & -- & -- & -- & -- & -- & $<.05$ & $<.05$ & \\
\hline 403156074583901 & Hunterdon Co., NJ & 06/17/99 & -- & $<.05$ & $<.05$ & -- & -- & .62 & $<.05$ & -- & -- & -- & -- & -- & -- & -- & -- & .09 & $<.05$ & 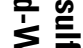 \\
\hline 403237075185301 & Bucks Co., PA & 06/16/99 & -- & $<.05$ & $<.05$ & -- & -- & $<.05$ & $<.05$ & -- & -- & -- & -- & -- & -- & -- & -- & $<.05$ & $<.05$ & \\
\hline 403342075485901 & Berks Co., PA & 05/08/00 & -- & $<.05$ & $<.05$ & -- & -- & .28 & $<.05$ & -- & -- & $<.05$ & $<.05$ & -- & -- & -- & -- & 1.4 & .08 & \\
\hline 040352907054201 & Schuylkill Co., PA & $04 / 25 / 00$ & -- & $<.05$ & $<.05$ & -- & -- & $<.05$ & $<.05$ & -- & -- & $<.05$ & $<.05$ & -- & -- & -- & -- & $<.05$ & $<.05$ & \\
\hline 403640075530001 & Berks Co., PA & $05 / 23 / 00$ & -- & $<.05$ & $<.05$ & -- & -- & $<.05$ & $<.05$ & -- & -- & $<.05$ & $<.05$ & -- & -- & -- & -- & $<.05$ & $<.05$ & \\
\hline 404150075371901 & Northamton Co., PA & 05/24/00 & -- & $<.05$ & $<.05$ & -- & -- & $<.05$ & $<.05$ & -- & -- & $<.05$ & $<.05$ & -- & -- & -- & -- & .42 & .12 & \\
\hline 404212075425401 & LeHigh Co., PA & $05 / 22 / 00$ & -- & $<.05$ & $<.05$ & -- & -- & $<.05$ & $<.05$ & -- & -- & $<.05$ & $<.05$ & -- & -- & -- & -- & .06 & $<.05$ & \\
\hline
\end{tabular}




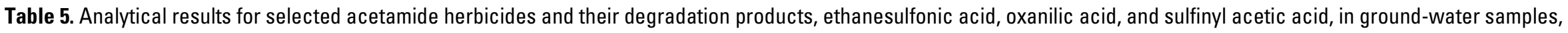
1994-2002.-Continued

[OGRL, U.S. Geological Survey Organic Geochemistry Research Laboratory, Lawrence, Kansas; ESA, ethanesulfonic acid; OXA, oxanilic acid; SAA, sulfinyl acetic acid; --, no data; <, less than]

\begin{tabular}{|c|c|c|c|c|c|c|c|c|c|c|c|c|c|c|c|c|c|c|c|}
\hline Site number & Site location & $\begin{array}{l}\text { Date of col- } \\
\text { lection } \\
\text { (month/day/ } \\
\text { year) }\end{array}$ & $\begin{array}{l}\text { Aceto- } \\
\text { chlor } \\
(\mu \mathrm{g} / \mathrm{L})\end{array}$ & $\begin{array}{l}\text { Aceto- } \\
\text { chlor } \\
\text { ESA } \\
(\mu \mathrm{g} / \mathrm{L})\end{array}$ & $\begin{array}{l}\text { Aceto- } \\
\text { chlor } \\
\text { OXA } \\
(\mu \mathrm{g} / \mathrm{L})\end{array}$ & $\begin{array}{l}\text { Aceto- } \\
\text { chlor } \\
\text { SAA } \\
(\mu \mathrm{g} / \mathrm{L})\end{array}$ & $\begin{array}{l}\text { Ala- } \\
\text { chlor } \\
(\mu \mathrm{g} / \mathrm{L})\end{array}$ & $\begin{array}{l}\text { Ala- } \\
\text { chlor } \\
\text { ESA } \\
(\mu \mathrm{g} / \mathrm{L})\end{array}$ & $\begin{array}{l}\text { Ala- } \\
\text { chlor } \\
\text { OXA } \\
(\mu \mathrm{g} / \mathrm{L})\end{array}$ & $\begin{array}{l}\text { Ala- } \\
\text { chlor } \\
\text { SAA } \\
(\mu \mathrm{g} / \mathrm{L})\end{array}$ & $\begin{array}{c}\text { Di- } \\
\text { meth- } \\
\text { enamid } \\
(\mu \mathrm{g} / \mathrm{L})\end{array}$ & $\begin{array}{c}\text { Di- } \\
\text { meth- } \\
\text { enamid } \\
\text { ESA } \\
(\mu \mathrm{g} / \mathrm{L})\end{array}$ & $\begin{array}{c}\text { Di- } \\
\text { meth- } \\
\text { enamid } \\
0 X A \\
(\mu \mathrm{g} / \mathrm{L})\end{array}$ & $\begin{array}{l}\text { Flufen- } \\
\text { acet } \\
(\mu \mathrm{g} / \mathrm{L})\end{array}$ & $\begin{array}{c}\text { Flufen- } \\
\text { acet } \\
\text { ESA } \\
(\mu \mathrm{g} / \mathrm{L})\end{array}$ & $\begin{array}{l}\text { Flufen- } \\
\text { acet } \\
\text { OXA } \\
(\mu \mathrm{g} / \mathrm{L})\end{array}$ & $\begin{array}{l}\text { Meto- } \\
\text { lachlor } \\
(\mu \mathrm{g} / \mathrm{L})\end{array}$ & $\begin{array}{c}\text { Meto- } \\
\text { lachlor } \\
\text { ESA } \\
(\mu \mathrm{g} / \mathrm{L})\end{array}$ & $\begin{array}{l}\text { Meto- } \\
\text { lachlor } \\
\text { OXA } \\
(\mu \mathrm{g} / \mathrm{L})\end{array}$ \\
\hline \multicolumn{20}{|c|}{ National Water-Quality Assessment Program (NAWQA) (fig. 3)—Continued } \\
\hline \multicolumn{20}{|c|}{ Delaware River Basin (DELR/BET/DCG)-Continued } \\
\hline 404306076031101 & Schuylkill Co., PA & $06 / 19 / 00$ & -- & $<0.05$ & $<0.05$ & -- & -- & $<0.05$ & $<0.05$ & -- & -- & $<0.05$ & $<0.05$ & -- & -- & -- & -- & $<0.05$ & $<0.05$ \\
\hline 404624075232001 & Northamton Co., PA & $05 / 09 / 00$ & -- & $<.05$ & $<.05$ & -- & -- & $<.05$ & $<.05$ & -- & -- & $<.05$ & $<.05$ & -- & -- & -- & -- & $<.05$ & $<.05$ \\
\hline 404708076070701 & Schuylkill Co., PA & $04 / 26 / 00$ & -- & $<.05$ & $<.05$ & -- & -- & $<.05$ & $<.05$ & -- & -- & $<.05$ & $<.05$ & -- & -- & -- & -- & .37 & $<.05$ \\
\hline 404734075491601 & Carbon Co., PA & 07/12/00 & -- & $<.05$ & $<.05$ & -- & -- & $<.05$ & $<.05$ & -- & -- & $<.05$ & $<.05$ & -- & $<0.05$ & $<0.05$ & -- & $<.05$ & $<.05$ \\
\hline 404740075562301 & Schuylkill Co., PA & $04 / 27 / 00$ & -- & $<.05$ & $<.05$ & -- & -- & $<.05$ & $<.05$ & -- & -- & $<.05$ & $<.05$ & -- & -- & -- & -- & $<.05$ & $<.05$ \\
\hline 404935075100201 & Northamton Co., PA & $06 / 14 / 00$ & -- & $<.05$ & $<.05$ & -- & -- & .26 & $<.05$ & -- & -- & $<.05$ & $<.05$ & -- & -- & -- & -- & $<.05$ & $<.05$ \\
\hline 405046075341901 & Carbon Co., PA & 05/10/00 & -- & $<.05$ & $<.05$ & -- & -- & .47 & $<.05$ & -- & -- & $<.05$ & $<.05$ & -- & -- & -- & -- & .59 & $<.05$ \\
\hline 405118075464101 & Carbon Co., PA & 06/13/00 & -- & $<.05$ & $<.05$ & -- & -- & $<.05$ & $<.05$ & -- & -- & $<.05$ & $<.05$ & -- & -- & -- & -- & $<.05$ & $<.05$ \\
\hline 405414075174001 & Monroe Co., PA & $06 / 12 / 00$ & -- & $<.05$ & $<.05$ & -- & -- & $<.05$ & $<.05$ & -- & -- & $<.05$ & $<.05$ & -- & -- & -- & -- & $<.05$ & $<.05$ \\
\hline 405453075522601 & Carbon Co., PA & 07/10/00 & -- & $<.05$ & $<.05$ & -- & -- & .15 & $<.05$ & -- & -- & $<.05$ & $<.05$ & -- & $<.05$ & $<.05$ & -- & 10 & 2.5 \\
\hline 405459074451201 & Sussex Co., NJ & 07/17/01 & -- & $<.05$ & $<.05$ & -- & -- & $<.05$ & $<.05$ & -- & -- & $<.05$ & $<.05$ & -- & $<.05$ & $<.05$ & -- & $<.05$ & $<.05$ \\
\hline 405501075155401 & Monroe Co., PA & 07/18/01 & -- & $<.05$ & $<.05$ & -- & -- & $<.05$ & $<.05$ & -- & -- & $<.05$ & $<.05$ & -- & $<.05$ & $<.05$ & -- & $<.05$ & $<.05$ \\
\hline 405552075091901 & Northampton Co., PA & $05 / 11 / 00$ & -- & $<.05$ & $<.05$ & -- & -- & $<.05$ & $<.05$ & -- & -- & $<.05$ & $<.05$ & -- & -- & -- & -- & $<.05$ & $<.05$ \\
\hline 405605075240901 & Monroe Co., PA & $05 / 15 / 00$ & -- & $<.05$ & $<.05$ & -- & -- & $<.05$ & $<.05$ & -- & -- & $<.05$ & $<.05$ & -- & -- & -- & -- & $<.05$ & $<.05$ \\
\hline 405702074585201 & Warren Co., NJ & $05 / 17 / 00$ & -- & $<.05$ & $<.05$ & -- & -- & .36 & $<.05$ & -- & -- & $<.05$ & $<.05$ & -- & -- & -- & -- & 1.4 & .60 \\
\hline 405754075470401 & Carbon Co., PA & $06 / 15 / 00$ & -- & $<.05$ & $<.05$ & -- & -- & $<.05$ & $<.05$ & -- & -- & $<.05$ & $<.05$ & -- & -- & -- & -- & $<.05$ & $<.05$ \\
\hline 405934075242001 & Monroe Co., PA & 05/16/00 & -- & $<.05$ & $<.05$ & -- & -- & .21 & $<.05$ & -- & -- & $<.05$ & $<.05$ & -- & -- & -- & -- & .22 & $<.05$ \\
\hline 410125074570701 & Warren Co., NJ & $05 / 18 / 00$ & -- & $<.05$ & $<.05$ & -- & -- & $<.05$ & $<.05$ & -- & -- & $<.05$ & $<.05$ & -- & -- & -- & -- & .48 & $<.05$ \\
\hline 410422074575101 & Warren Co., NJ & 06/07/00 & -- & $<.05$ & $<.05$ & -- & -- & $<.05$ & $<.05$ & -- & -- & $<.05$ & $<.05$ & -- & -- & -- & -- & $<.05$ & $<.05$ \\
\hline 410612074591501 & Pike Co., PA & $07 / 16 / 01$ & -- & $<.05$ & $<.05$ & -- & -- & $<.05$ & $<.05$ & -- & -- & $<.05$ & $<.05$ & -- & $<.05$ & $<.05$ & -- & $<.05$ & $<.05$ \\
\hline 411117074480401 & Sussex Co., NJ & 06/06/00 & -- & $<.05$ & $<.05$ & -- & -- & $<.05$ & $<.05$ & -- & -- & $<.05$ & $<.05$ & -- & -- & -- & -- & $<.05$ & $<.05$ \\
\hline 411252074522001 & Pike Co., PA & $07 / 12 / 01$ & -- & $<.05$ & $<.05$ & -- & -- & $<.05$ & $<.05$ & -- & -- & $<.05$ & $<.05$ & -- & $<.05$ & $<.05$ & -- & $<.05$ & $<.05$ \\
\hline 411401074452901 & Sussex Co., NJ & $06 / 05 / 00$ & -- & $<.05$ & $<.05$ & -- & -- & $<.05$ & $<.05$ & -- & -- & $<.05$ & $<.05$ & -- & -- & -- & -- & $<.05$ & $<.05$ \\
\hline 411930074431401 & Sussex Co., NJ & 07/19/00 & -- & $<.05$ & $<.05$ & -- & -- & $<.05$ & $<.05$ & -- & -- & $<.05$ & $<.05$ & -- & $<.05$ & $<.05$ & -- & $<.05$ & $<.05$ \\
\hline 411957074462601 & Pike Co., PA & 07/11/01 & -- & $<.05$ & $<.05$ & -- & -- & $<.05$ & $<.05$ & -- & -- & $<.05$ & $<.05$ & -- & $<.05$ & $<.05$ & -- & $<.05$ & $<.05$ \\
\hline
\end{tabular}




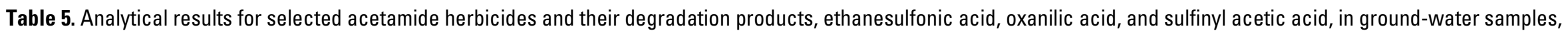
1994-2002.-Continued

[OGRL, U.S. Geological Survey Organic Geochemistry Research Laboratory, Lawrence, Kansas; ESA, ethanesulfonic acid; OXA, oxanilic acid; SAA, sulfinyl acetic acid; --, no data; <, less than]

\begin{tabular}{|c|c|c|c|c|c|c|c|c|c|c|c|c|c|c|c|c|c|c|c|c|}
\hline Site number & Site location & $\begin{array}{l}\text { Date of col- } \\
\text { lection } \\
\text { (month/day/ } \\
\text { year) }\end{array}$ & $\begin{array}{l}\text { Aceto- } \\
\text { chlor } \\
(\mu \mathrm{g} / \mathrm{L})\end{array}$ & $\begin{array}{l}\text { Aceto- } \\
\text { chlor } \\
\text { ESA } \\
(\mu \mathrm{g} / \mathrm{L})\end{array}$ & $\begin{array}{c}\text { Aceto- } \\
\text { chlor } \\
\text { OXA } \\
(\mu \mathrm{g} / \mathrm{L})\end{array}$ & $\begin{array}{c}\text { Aceto- } \\
\text { chlor } \\
\text { SAA } \\
(\mu \mathrm{g} / \mathrm{L})\end{array}$ & $\begin{array}{l}\text { Ala- } \\
\text { chlor } \\
(\mu \mathrm{g} / \mathrm{L})\end{array}$ & $\begin{array}{l}\text { Ala- } \\
\text { chlor } \\
\text { ESA } \\
(\mu \mathrm{g} / \mathrm{L})\end{array}$ & $\begin{array}{c}\text { Ala- } \\
\text { chlor } \\
\text { OXA } \\
(\mu \mathrm{g} / \mathrm{L})\end{array}$ & $\begin{array}{l}\text { Ala- } \\
\text { chlor } \\
\text { SAA } \\
(\mu \mathrm{g} / \mathrm{L})\end{array}$ & $\begin{array}{c}\text { Di- } \\
\text { meth- } \\
\text { enamid } \\
(\mu \mathrm{g} / \mathrm{L})\end{array}$ & $\begin{array}{c}\text { Di- } \\
\text { meth- } \\
\text { enamid } \\
\text { ESA } \\
(\mu \mathrm{g} / \mathrm{L})\end{array}$ & $\begin{array}{c}\text { Di- } \\
\text { meth- } \\
\text { enamid } \\
\text { OXA } \\
(\mu \mathrm{g} / \mathrm{L})\end{array}$ & $\begin{array}{c}\text { Flufen- } \\
\text { acet } \\
(\mu \mathrm{g} / \mathrm{L})\end{array}$ & $\begin{array}{c}\text { Flufen- } \\
\text { acet } \\
\text { ESA } \\
(\mu \mathrm{g} / \mathrm{L})\end{array}$ & $\begin{array}{c}\text { Flufen- } \\
\text { acet } \\
\text { OXA } \\
(\mu \mathrm{g} / \mathrm{L})\end{array}$ & $\begin{array}{c}\text { Meto- } \\
\text { lachlor } \\
(\mu \mathrm{g} / \mathrm{L})\end{array}$ & $\begin{array}{l}\text { Meto- } \\
\text { lachlor } \\
\text { ESA } \\
(\mu \mathrm{g} / \mathrm{L})\end{array}$ & $\begin{array}{l}\text { Meto- } \\
\text { lachlor } \\
\text { OXA } \\
(\mu \mathrm{g} / \mathrm{L})\end{array}$ & 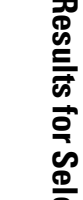 \\
\hline \multicolumn{20}{|c|}{ National Water-Quality Assessment Program (NAWOA) (fig. 3) -Continued } & \\
\hline \multicolumn{20}{|c|}{ Delaware River Basin (DELR/BET/DCG)-Continued } & \\
\hline 412522074441301 & Pike Co., PA & 07/10/01 & -- & $<0.05$ & $<0.05$ & -- & -- & $<0.05$ & $<0.05$ & -- & -- & $<0.05$ & $<0.05$ & -- & $<0.05$ & $<0.05$ & -- & $<0.05$ & $<0.05$ & \\
\hline 412722074354501 & Orange Co., NY & 08/09/01 & -- & $<.05$ & $<.05$ & -- & -- & $<.05$ & $<.05$ & -- & -- & $<.05$ & $<.05$ & -- & $<.05$ & $<.05$ & -- & $<.05$ & $<.05$ & \\
\hline 415040075070301 & Wayne, Co. PA & $07 / 25 / 01$ & -- & $<.05$ & $<.05$ & -- & -- & $<.05$ & $<.05$ & -- & -- & $<.05$ & $<.05$ & -- & $<.05$ & $<.05$ & -- & $<.05$ & $<.05$ & \\
\hline 415429074501101 & Sullivan Co., NY & $07 / 23 / 01$ & -- & $<.05$ & $<.05$ & -- & -- & $<.05$ & $<.05$ & -- & -- & $<.05$ & $<.05$ & -- & $<.05$ & $<.05$ & -- & $<.05$ & $<.05$ & \\
\hline 420125075064801 & Sullivan Co., NY & 08/01/01 & -- & $<.05$ & $<.05$ & -- & -- & $<.05$ & $<.05$ & -- & -- & $<.05$ & $<.05$ & -- & $<.05$ & $<.05$ & -- & $<.05$ & $<.05$ & \\
\hline 420207075020401 & Delaware Co., NY & 07/30/01 & -- & $<.05$ & $<.05$ & -- & -- & .08 & $<.05$ & -- & -- & $<.05$ & $<.05$ & -- & $<.05$ & $<.05$ & -- & .67 & $<.05$ & \\
\hline 420244075251101 & Broome Co., NY & $07 / 16 / 01$ & -- & $<.05$ & $<.05$ & -- & -- & $<.05$ & $<.05$ & -- & -- & $<.05$ & $<.05$ & -- & $<.05$ & $<.05$ & -- & .17 & $<.05$ & \\
\hline 421052075004501 & Delaware Co., NY & 08/11/01 & -- & $<.05$ & $<.05$ & -- & -- & $<.05$ & $<.05$ & -- & -- & $<.05$ & $<.05$ & -- & $<.05$ & $<.05$ & -- & $<.05$ & $<.05$ & \\
\hline 042140974355701 & Delaware Co., NY & $07 / 31 / 01$ & -- & $<.05$ & $<.05$ & -- & -- & $<.05$ & $<.05$ & -- & -- & $<.05$ & $<.05$ & -- & $<.05$ & $<.05$ & -- & .26 & $<.05$ & \\
\hline 421747074332301 & Delaware Co., NY & 08/14/01 & -- & $<.05$ & $<.05$ & -- & -- & $<.05$ & $<.05$ & -- & -- & $<.05$ & $<.05$ & -- & $<.05$ & $<.05$ & -- & $<.05$ & $<.05$ & \\
\hline 421758074524101 & Delaware Co., NY & 07/24/01 & -- & $<.05$ & $<.05$ & -- & -- & $<.05$ & $<.05$ & -- & -- & $<.05$ & $<.05$ & -- & $<.05$ & $<.05$ & -- & 1.3 & $<.05$ & \\
\hline \multicolumn{20}{|c|}{ Delmarva Peninsula (DLMV/JMD) } & \\
\hline 19-D-R & Sussex Co., DE & $10 / 29 / 01$ & -- & $<.05$ & $<.05$ & -- & -- & $<.05$ & $<.05$ & -- & -- & $<.05$ & $<.05$ & -- & $<.05$ & $<.05$ & -- & $<.05$ & $<.05$ & \\
\hline 22-D-R & Sussex Co., DE & $10 / 30 / 01$ & -- & $<.05$ & $<.05$ & -- & -- & .92 & .11 & -- & -- & $<.05$ & $<.05$ & -- & $<.05$ & $<.05$ & -- & 3.4 & 1.4 & \\
\hline 26D-R & Somerset Co., MD & $11 / 01 / 01$ & -- & $<.05$ & $<.05$ & -- & -- & .15 & $<.05$ & -- & -- & $<.05$ & $<.05$ & -- & $<.05$ & $<.05$ & -- & 6.4 & .06 & \\
\hline 63 F 28 & Northampton Co., VA & $10 / 19 / 99$ & -- & $<.05$ & $<.05$ & -- & -- & $<.05$ & $<.05$ & -- & -- & -- & -- & -- & -- & -- & -- & 2.4 & $<.05$ & \\
\hline 63 F 29 & Northampton Co., VA & $10 / 20 / 99$ & -- & $<.05$ & $<.05$ & -- & -- & $<.05$ & $<.05$ & -- & -- & -- & -- & -- & -- & -- & -- & 3.2 & $<.05$ & \\
\hline 63 F 30 & Northampton Co., VA & $10 / 18 / 99$ & -- & $<.05$ & $<.05$ & -- & -- & $<.05$ & $<.05$ & -- & -- & -- & -- & -- & -- & -- & -- & .64 & .35 & \\
\hline 63 F 44 & Northampton Co., VA & $10 / 19 / 99$ & -- & $<.05$ & $<.05$ & -- & -- & $<.05$ & $<.05$ & -- & -- & -- & -- & -- & -- & -- & -- & 3.2 & $<.05$ & \\
\hline 63 F 45 & Northampton Co., VA & $10 / 19 / 99$ & -- & $<.05$ & $<.05$ & -- & -- & $<.05$ & $<.05$ & -- & -- & -- & -- & -- & -- & -- & -- & 1.1 & $<.05$ & \\
\hline 63 F 47 & Northampton Co., VA & $10 / 19 / 99$ & -- & $<.05$ & $<.05$ & -- & -- & $<.05$ & $<.05$ & -- & -- & -- & -- & -- & -- & -- & -- & 1.0 & $<.05$ & \\
\hline 63 F 60 & Northampton Co., VA & $10 / 20 / 99$ & -- & $<.05$ & $<.05$ & -- & -- & $<.05$ & $<.05$ & -- & -- & -- & -- & -- & -- & -- & -- & .39 & $<.05$ & \\
\hline 63 F 81 & Northampton Co., VA & $10 / 21 / 99$ & -- & $<.05$ & $<.05$ & -- & -- & .24 & $<.05$ & -- & -- & -- & -- & -- & -- & -- & -- & 4.6 & .21 & \\
\hline 63 F 82 & Northampton Co., VA & $10 / 21 / 99$ & -- & $<.05$ & $<.05$ & -- & -- & .20 & $<.05$ & -- & -- & -- & -- & -- & -- & -- & -- & .51 & $<.05$ & \\
\hline
\end{tabular}




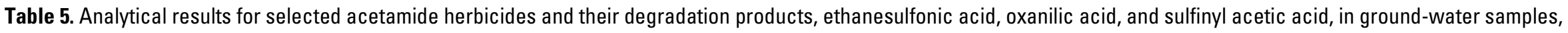
1994-2002.-Continued

[OGRL, U.S. Geological Survey Organic Geochemistry Research Laboratory, Lawrence, Kansas; ESA, ethanesulfonic acid; OXA, oxanilic acid; SAA, sulfinyl acetic acid; --, no data; <, less than]

\begin{tabular}{|c|c|c|c|c|c|c|c|c|c|c|c|c|c|c|c|c|c|c|c|}
\hline Site number & Site location & $\begin{array}{l}\text { Date of col- } \\
\text { lection } \\
\text { (month/day/ } \\
\text { year) }\end{array}$ & $\begin{array}{l}\text { Aceto- } \\
\text { chlor } \\
(\mu \mathrm{g} / \mathrm{L})\end{array}$ & $\begin{array}{l}\text { Aceto- } \\
\text { chlor } \\
\text { ESA } \\
(\mu \mathrm{g} / \mathrm{L})\end{array}$ & $\begin{array}{l}\text { Aceto- } \\
\text { chlor } \\
\text { OXA } \\
(\mu \mathrm{g} / \mathrm{L})\end{array}$ & $\begin{array}{l}\text { Aceto- } \\
\text { chlor } \\
\text { SAA } \\
(\mu \mathrm{g} / \mathrm{L})\end{array}$ & $\begin{array}{l}\text { Ala- } \\
\text { chlor } \\
(\mu \mathrm{g} / \mathrm{L})\end{array}$ & $\begin{array}{l}\text { Ala- } \\
\text { chlor } \\
\text { ESA } \\
(\mu \mathrm{g} / \mathrm{L})\end{array}$ & $\begin{array}{c}\text { Ala- } \\
\text { chlor } \\
\text { OXA } \\
(\mu \mathrm{g} / \mathrm{L})\end{array}$ & $\begin{array}{l}\text { Ala- } \\
\text { chlor } \\
\text { SAA } \\
(\mu \mathrm{g} / \mathrm{L})\end{array}$ & $\begin{array}{c}\text { Di- } \\
\text { meth- } \\
\text { enamid } \\
(\mu \mathrm{g} / \mathrm{L})\end{array}$ & $\begin{array}{c}\text { Di- } \\
\text { meth- } \\
\text { enamid } \\
\text { ESA } \\
(\mu \mathrm{g} / \mathrm{L})\end{array}$ & $\begin{array}{c}\text { Di- } \\
\text { meth- } \\
\text { enamid } \\
\text { OXA } \\
(\mu \mathrm{g} / \mathrm{L})\end{array}$ & $\begin{array}{c}\text { Flufen- } \\
\text { acet } \\
(\mu \mathrm{g} / \mathrm{L})\end{array}$ & $\begin{array}{c}\text { Flufen- } \\
\text { acet } \\
\text { ESA } \\
(\mu \mathrm{g} / \mathrm{L})\end{array}$ & $\begin{array}{c}\text { Flufen- } \\
\text { acet } \\
\text { OXA } \\
(\mu \mathrm{g} / \mathrm{L})\end{array}$ & $\begin{array}{l}\text { Meto- } \\
\text { lachlor } \\
(\mu \mathrm{g} / \mathrm{L})\end{array}$ & $\begin{array}{l}\text { Meto- } \\
\text { lachlor } \\
\text { ESA } \\
(\mu \mathrm{g} / \mathrm{L})\end{array}$ & $\begin{array}{c}\text { Meto- } \\
\text { lachlor } \\
\text { OXA } \\
(\mu \mathrm{g} / \mathrm{L})\end{array}$ \\
\hline \multicolumn{20}{|c|}{ National Water-Quality Assessment Program (NAWOA) (fig. 3)—Continued } \\
\hline \multicolumn{20}{|c|}{ Delmarva Peninsula (DLMV/JMD)—Continued } \\
\hline 63 F 86 & Northampton Co., VA & $10 / 20 / 99$ & -- & $<0.05$ & $<0.05$ & -- & -- & $<0.05$ & $<0.05$ & -- & -- & -- & -- & -- & -- & -- & -- & 2.1 & $<0.05$ \\
\hline $64 \mathrm{~J} 28$ & Accomack Co., VA & $07 / 31 / 01$ & -- & $<.05$ & $<.05$ & -- & -- & $<.05$ & $<.05$ & -- & -- & $<0.05$ & $<0.05$ & -- & $<0.05$ & $<0.05$ & -- & $<.05$ & $<.05$ \\
\hline $64 J 29$ & Accomack Co., VA & $07 / 31 / 01$ & -- & $<.05$ & $<.05$ & -- & -- & .47 & $<.05$ & -- & -- & $<.05$ & $<.05$ & -- & $<.05$ & $<.05$ & -- & 1.0 & .38 \\
\hline $64 \mathrm{~J} 30$ & Northampton Co., VA & 07/30/01 & -- & $<.05$ & $<.05$ & -- & -- & .11 & $<.05$ & -- & -- & $<.05$ & $<.05$ & -- & $<.05$ & $<.05$ & -- & 35 & 19 \\
\hline $64 \mathrm{~J} 31$ & Northampton Co., VA & 07/30/01 & -- & $<.05$ & $<.05$ & -- & -- & .26 & $<.05$ & -- & -- & $<.05$ & $<.05$ & -- & $<.05$ & $<.05$ & -- & 10 & 4.9 \\
\hline $66 \mathrm{~L} 11$ & Accomack Co., VA & 08/01/01 & -- & $<.05$ & $<.05$ & -- & -- & .33 & $<.05$ & -- & -- & $<.05$ & $<.05$ & -- & $<.05$ & $<.05$ & -- & .06 & $<.05$ \\
\hline 66L12 & Accomack Co., VA & 08/01/01 & -- & $<.05$ & $<.05$ & -- & -- & $<.05$ & $<.05$ & -- & -- & $<.05$ & $<.05$ & -- & $<.05$ & $<.05$ & -- & .07 & $<.05$ \\
\hline $\mathrm{Cd} 31-19$ & New Castle Co., DE & 08/06/01 & -- & $<.05$ & $<.05$ & -- & -- & $<.05$ & $<.05$ & -- & -- & $<.05$ & $<.05$ & -- & $<.05$ & $<.05$ & -- & $<.05$ & $<.05$ \\
\hline Cd52-15 & New Castle Co., DE & 08/08/00 & -- & $<.05$ & $<.05$ & -- & -- & $<.05$ & $<.05$ & -- & -- & $<.05$ & $<.05$ & -- & $<.05$ & $<.05$ & -- & $<.05$ & $<.05$ \\
\hline CECe86 & Cecil Co., MD & 07/19/01 & -- & $<.05$ & $<.05$ & -- & -- & $<.05$ & $<.05$ & -- & -- & $<.05$ & $<.05$ & -- & $<.05$ & $<.05$ & -- & .06 & $<.05$ \\
\hline CODe15 & Caroline Co., MD & $08 / 22 / 01$ & -- & $<.05$ & $<.05$ & -- & -- & 4.8 & $<.05$ & -- & -- & $<.05$ & $<.05$ & -- & $<.05$ & $<.05$ & -- & 3.2 & .17 \\
\hline COEc25 & Caroline Co., MD & $08 / 27 / 01$ & -- & $<.05$ & $<.05$ & -- & -- & 1.3 & $<.05$ & -- & -- & $<.05$ & $<.05$ & -- & $<.05$ & $<.05$ & -- & 2.9 & .41 \\
\hline Db11-27 & New Castle Co., DE & 08/07/00 & -- & $<.05$ & $<.05$ & -- & -- & .12 & $<.05$ & -- & -- & $<.05$ & $<.05$ & -- & $<.05$ & $<.05$ & -- & 1.3 & .18 \\
\hline Db11-28 & New Castle Co., DE & 08/07/00 & -- & $<.05$ & $<.05$ & -- & -- & .10 & $<.05$ & -- & -- & $<.05$ & $<.05$ & -- & $<.05$ & $<.05$ & -- & .13 & $<.05$ \\
\hline Db11-48 & New Castle Co., DE & 08/09/00 & -- & $<.05$ & $<.05$ & -- & -- & .05 & $<.05$ & -- & -- & $<.05$ & $<.05$ & -- & $<.05$ & $<.05$ & -- & $<.05$ & .06 \\
\hline DC31-15 & New Castle Co., DE & 08/08/00 & -- & $<.05$ & $<.05$ & -- & -- & .07 & $<.05$ & -- & -- & $<.05$ & $<.05$ & -- & $<.05$ & $<.05$ & -- & .41 & .05 \\
\hline DOCb8 & Dorchester Co., MD & 08/07/01 & -- & $<.05$ & $<.05$ & -- & -- & .60 & $<.05$ & -- & -- & $<.05$ & $<.05$ & -- & $<.05$ & $<.05$ & -- & .57 & $<.05$ \\
\hline Ec42-15 & New Castle Co., DE & 07/05/01 & -- & .74 & $<.05$ & -- & -- & .35 & .05 & -- & -- & $<.05$ & $<.05$ & -- & $<.05$ & $<.05$ & -- & 17 & 4.1 \\
\hline Gc14-03 & New Castle Co., DE & 07/17/01 & -- & $<.05$ & $<.05$ & -- & -- & .05 & $<.05$ & -- & -- & $<.05$ & $<.05$ & -- & $<.05$ & $<.05$ & -- & $<.05$ & $<.05$ \\
\hline Hc34-03 & New Castle Co., DE & $10 / 16 / 00$ & -- & $<.05$ & $<.05$ & -- & -- & $<.05$ & $<.05$ & -- & -- & $<.05$ & $<.05$ & -- & $<.05$ & $<.05$ & -- & $<.05$ & $<.05$ \\
\hline Ib32-05 & Kent Co., DE & $08 / 23 / 01$ & -- & $<.05$ & $<.05$ & -- & -- & .09 & $<.05$ & -- & -- & $<.05$ & $<.05$ & -- & $<.05$ & $<.05$ & -- & .09 & $<.05$ \\
\hline Ie42-03 & Kent Co., DE & $08 / 29 / 00$ & -- & $<.05$ & $<.05$ & -- & -- & .68 & $<.05$ & -- & -- & $<.05$ & $<.05$ & -- & $<.05$ & $<.05$ & -- & 2.9 & .09 \\
\hline JC 43-05 & Kent Co., DE & $10 / 26 / 00$ & -- & $<.05$ & $<.05$ & -- & -- & .44 & $<.05$ & -- & -- & $<.05$ & $<.05$ & -- & $<.05$ & $<.05$ & -- & .27 & $<.05$ \\
\hline Jc52-04 & Kent Co., DE & 07/02/01 & -- & $<.05$ & $<.05$ & -- & -- & $<.05$ & $<.05$ & -- & -- & $<.05$ & $<.05$ & -- & $<.05$ & $<.05$ & -- & .08 & $<.05$ \\
\hline Jc55-03 & Kent Co., DE & 06/28/01 & -- & $<.05$ & $<.05$ & -- & -- & .06 & $<.05$ & -- & -- & $<.05$ & $<.05$ & -- & $<.05$ & $<.05$ & -- & .07 & $<.05$ \\
\hline
\end{tabular}




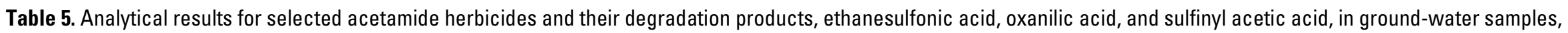
1994-2002.-Continued

[OGRL, U.S. Geological Survey Organic Geochemistry Research Laboratory, Lawrence, Kansas; ESA, ethanesulfonic acid; OXA, oxanilic acid; SAA, sulfinyl acetic acid; --, no data; <, less than]

\begin{tabular}{|c|c|c|c|c|c|c|c|c|c|c|c|c|c|c|c|c|c|c|c|c|}
\hline Site number & Site location & $\begin{array}{l}\text { Date of col- } \\
\text { lection } \\
\text { (month/day/ } \\
\text { year) }\end{array}$ & $\begin{array}{c}\text { Aceto- } \\
\text { chlor } \\
(\mu \mathrm{g} / \mathrm{L})\end{array}$ & $\begin{array}{l}\text { Aceto- } \\
\text { chlor } \\
\text { ESA } \\
(\mu \mathrm{g} / \mathrm{L})\end{array}$ & $\begin{array}{c}\text { Aceto- } \\
\text { chlor } \\
\text { OXA } \\
(\mu \mathrm{g} / \mathrm{L})\end{array}$ & $\begin{array}{c}\text { Aceto- } \\
\text { chlor } \\
\text { SAA } \\
(\mu \mathrm{g} / \mathrm{L})\end{array}$ & $\begin{array}{l}\text { Ala- } \\
\text { chlor } \\
(\mu \mathrm{g} / \mathrm{L})\end{array}$ & $\begin{array}{l}\text { Ala- } \\
\text { chlor } \\
\text { ESA } \\
(\mu \mathrm{g} / \mathrm{L})\end{array}$ & $\begin{array}{c}\text { Ala- } \\
\text { chlor } \\
\text { OXA } \\
(\mu \mathrm{g} / \mathrm{L})\end{array}$ & $\begin{array}{l}\text { Ala- } \\
\text { chlor } \\
\text { SAA } \\
(\mu \mathrm{g} / \mathrm{L})\end{array}$ & $\begin{array}{c}\text { Di- } \\
\text { meth- } \\
\text { enamid } \\
(\mu \mathrm{g} / \mathrm{L})\end{array}$ & $\begin{array}{c}\text { Di- } \\
\text { meth- } \\
\text { enamid } \\
\text { ESA } \\
(\mu \mathrm{g} / \mathrm{L})\end{array}$ & $\begin{array}{c}\text { Di- } \\
\text { meth- } \\
\text { enamid } \\
\text { OXA } \\
(\mu \mathrm{g} / \mathrm{L})\end{array}$ & $\begin{array}{c}\text { Flufen- } \\
\text { acet } \\
(\mu \mathrm{g} / \mathrm{L})\end{array}$ & $\begin{array}{l}\text { Flufen- } \\
\text { acet } \\
\text { ESA } \\
(\mu \mathrm{g} / \mathrm{L})\end{array}$ & $\begin{array}{c}\text { Flufen- } \\
\text { acet } \\
0 X A \\
(\mu \mathrm{g} / \mathrm{L})\end{array}$ & $\begin{array}{l}\text { Meto- } \\
\text { lachlor } \\
(\mu \mathrm{g} / \mathrm{L})\end{array}$ & $\begin{array}{c}\text { Meto- } \\
\text { lachlor } \\
\text { ESA } \\
(\mu \mathrm{g} / \mathrm{L})\end{array}$ & $\begin{array}{l}\text { Meto- } \\
\text { lachlor } \\
\text { OXA } \\
(\mu \mathrm{g} / \mathrm{L})\end{array}$ & 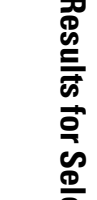 \\
\hline \multicolumn{20}{|c|}{ National Water-Quality Assessment Program (NAWOA) (fig. 3)—Continued } & \\
\hline \multicolumn{20}{|c|}{ Delmarva Peninsula (DLMV/JMD)_Continued } & \\
\hline Je41-08 & Kent Co., DE & $10 / 26 / 00$ & -- & $<0.05$ & $<0.05$ & -- & -- & 0.10 & $<0.05$ & -- & -- & $<0.05$ & $<0.05$ & -- & $<0.05$ & $<0.05$ & -- & 0.29 & $<0.05$ & \\
\hline Ke Bd 42 & Kent Co., MD & 07/03/01 & -- & $<.05$ & $<.05$ & -- & -- & .49 & $<.05$ & -- & -- & $<.05$ & $<.05$ & -- & $<.05$ & $<.05$ & -- & 14 & .78 & \\
\hline KEBe46 & Kent Co., DE & 07/18/01 & -- & $<.05$ & $<.05$ & -- & -- & $<.05$ & $<.05$ & -- & -- & $<.05$ & $<.05$ & -- & $<.05$ & $<.05$ & -- & $<.05$ & $<.05$ & \\
\hline Ke Be 52 & Kent Co., MD & 09/07/99 & -- & $<.05$ & $<.05$ & -- & -- & 1.4 & $<.05$ & -- & -- & -- & -- & -- & -- & -- & -- & 5.2 & .18 & $\frac{1}{7}$ \\
\hline Ke Be 59 & Kent Co., MD & 09/02/99 & -- & $<.05$ & $<.05$ & -- & -- & .51 & $<.05$ & -- & -- & -- & -- & -- & -- & -- & -- & $<.05$ & $<.05$ & \\
\hline KEBe 59 & Kent Co., DE & 07/12/01 & -- & .88 & $<.05$ & -- & -- & $<.05$ & $<.05$ & -- & -- & $<.05$ & $<.05$ & -- & $<.05$ & $<.05$ & -- & 3.3 & .14 & \\
\hline $\mathrm{Ke} \mathrm{Be} 61$ & Kent Co., MD & 09/07/99 & -- & $<.05$ & $<.05$ & -- & -- & 1.2 & $<.05$ & -- & -- & -- & -- & -- & -- & -- & -- & $<.05$ & $<.05$ & \\
\hline Ke Be 62 & Kent Co., MD & 09/08/99 & -- & $<.05$ & $<.05$ & -- & -- & $<.05$ & $<.05$ & -- & -- & -- & -- & -- & -- & -- & -- & 7.0 & .82 & $\stackrel{2}{=}$ \\
\hline Ke Be 159 & Kent Co., MD & 09/01/99 & -- & $<.05$ & $<.05$ & -- & -- & $<.05$ & $<.05$ & -- & -- & -- & -- & -- & -- & -- & -- & $<.05$ & $<.05$ & \\
\hline Ke Be 160 & Kent Co., MD & 09/01/99 & -- & $<.05$ & $<.05$ & -- & -- & $<.05$ & $<.05$ & -- & -- & -- & -- & -- & -- & -- & -- & $<.05$ & $<.05$ & \\
\hline Ke Be 161 & Kent Co., MD & 09/01/99 & -- & $<.05$ & $<.05$ & -- & -- & 1.2 & $<.05$ & -- & -- & -- & -- & -- & -- & -- & -- & 1.8 & $<.05$ & \\
\hline Ke Be 162 & Kent Co., MD & 09/08/99 & -- & $<.05$ & $<.05$ & -- & -- & .06 & $<.05$ & -- & -- & -- & -- & -- & -- & -- & -- & .39 & $<.05$ & $\bar{z}$ \\
\hline $\mathrm{Ke} \mathrm{Be} 163$ & Kent Co., MD & 09/08/99 & -- & $<.05$ & $<.05$ & -- & -- & .95 & $<.05$ & -- & -- & -- & -- & -- & -- & -- & -- & .87 & $<.05$ & \\
\hline Ke Be 164 & Kent Co., MD & 09/02/99 & -- & 1.6 & $<.05$ & -- & -- & $<.05$ & $<.05$ & -- & -- & -- & -- & -- & -- & -- & -- & 4.7 & .33 & \\
\hline Ke Be 170 & Kent Co., MD & 09/09/99 & -- & $<.05$ & $<.05$ & -- & -- & .92 & $<.05$ & -- & -- & -- & -- & -- & -- & -- & -- & 2.9 & .41 & \\
\hline Le24-11 & Kent Co., DE & $07 / 11 / 01$ & -- & $<.05$ & $<.05$ & -- & -- & $<.05$ & $<.05$ & -- & -- & $<.05$ & $<.05$ & -- & $<.05$ & $<.05$ & -- & $<.05$ & $<.05$ & \\
\hline Le35-11 & Kent Co., DE & 06/27/01 & -- & $<.05$ & $<.05$ & -- & -- & .52 & $<.05$ & -- & -- & $<.05$ & $<.05$ & -- & $<.05$ & $<.05$ & -- & 1.1 & $<.05$ & \\
\hline LE55-09 & Sussex Co., DE & 09/07/00 & -- & $<.05$ & $<.05$ & -- & -- & .22 & .27 & -- & -- & $<.05$ & $<.05$ & -- & $<.05$ & $<.05$ & -- & .87 & .06 & \\
\hline MD 11-04 & Kent Co., DE & 08/23/00 & -- & $<.05$ & $<.05$ & -- & -- & .49 & $<.05$ & -- & -- & $<.05$ & $<.05$ & -- & $<.05$ & $<.05$ & -- & .14 & $<.05$ & \\
\hline Nc $25-37$ & Sussex Co., DE & 09/12/00 & -- & $<.05$ & $<.05$ & -- & -- & .75 & .28 & -- & -- & $<.05$ & $<.05$ & -- & $<.05$ & $<.05$ & -- & 1.3 & .07 & \\
\hline Ng21-03 & Sussex Co., DE & 09/06/00 & -- & $<.05$ & $<.05$ & -- & -- & 1.6 & $<.05$ & -- & -- & $<.05$ & $<.05$ & -- & $<.05$ & $<.05$ & -- & .79 & $<.05$ & \\
\hline $\mathrm{Ng} 25-04$ & Sussex Co., DE & 09/06/00 & -- & $<.05$ & $<.05$ & -- & -- & $<.05$ & $<.05$ & -- & -- & $<.05$ & $<.05$ & -- & $<.05$ & $<.05$ & -- & $<.05$ & $<.05$ & \\
\hline Nh53-01 & Sussex Co., DE & $10 / 24 / 00$ & -- & $<.05$ & $<.05$ & -- & -- & .09 & $<.05$ & -- & -- & $<.05$ & $<.05$ & -- & $<.05$ & $<.05$ & -- & $<.05$ & $<.05$ & 놈 \\
\hline Ni 51-32 & Sussex Co., DE & 08/17/00 & -- & $<.05$ & $<.05$ & -- & -- & .09 & $<.05$ & -- & -- & $<.05$ & $<.05$ & -- & $<.05$ & $<.05$ & -- & .74 & .11 & \\
\hline
\end{tabular}




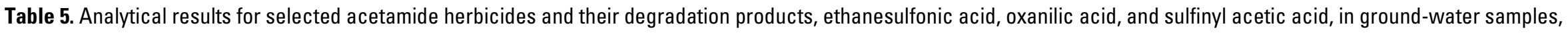
1994-2002.-Continued

[OGRL, U.S. Geological Survey Organic Geochemistry Research Laboratory, Lawrence, Kansas; ESA, ethanesulfonic acid; OXA, oxanilic acid; SAA, sulfinyl acetic acid; --, no data; <, less than]

\begin{tabular}{|c|c|c|c|c|c|c|c|c|c|c|c|c|c|c|c|c|c|c|c|}
\hline Site number & Site location & $\begin{array}{l}\text { Date of col- } \\
\text { lection } \\
\text { (month/day/ } \\
\text { year) }\end{array}$ & $\begin{array}{c}\text { Aceto- } \\
\text { chlor } \\
(\mu \mathrm{g} / \mathrm{L})\end{array}$ & $\begin{array}{l}\text { Aceto- } \\
\text { chlor } \\
\text { ESA } \\
(\mu \mathrm{g} / \mathrm{L})\end{array}$ & $\begin{array}{l}\text { Aceto- } \\
\text { chlor } \\
\text { OXA } \\
(\mu \mathrm{g} / \mathrm{L})\end{array}$ & $\begin{array}{l}\text { Aceto- } \\
\text { chlor } \\
\text { SAA } \\
(\mu \mathrm{g} / \mathrm{L})\end{array}$ & $\begin{array}{l}\text { Ala- } \\
\text { chlor } \\
(\mu \mathrm{g} / \mathrm{L})\end{array}$ & $\begin{array}{l}\text { Ala- } \\
\text { chlor } \\
\text { ESA } \\
(\mu \mathrm{g} / \mathrm{L})\end{array}$ & $\begin{array}{l}\text { Ala- } \\
\text { chlor } \\
\text { OXA } \\
(\mu \mathrm{g} / \mathrm{L})\end{array}$ & $\begin{array}{l}\text { Ala- } \\
\text { chlor } \\
\text { SAA } \\
(\mu \mathrm{g} / \mathrm{L})\end{array}$ & $\begin{array}{c}\text { Di- } \\
\text { meth- } \\
\text { enamid } \\
(\mu \mathrm{g} / \mathrm{L})\end{array}$ & $\begin{array}{c}\text { Di- } \\
\text { meth- } \\
\text { enamid } \\
\text { ESA } \\
(\mu \mathrm{g} / \mathrm{L})\end{array}$ & $\begin{array}{c}\text { Di- } \\
\text { meth- } \\
\text { enamid } \\
0 X A \\
(\mu \mathrm{g} / \mathrm{L})\end{array}$ & $\begin{array}{l}\text { Flufen- } \\
\text { acet } \\
(\mu \mathrm{g} / \mathrm{L})\end{array}$ & $\begin{array}{c}\text { Flufen- } \\
\text { acet } \\
\text { ESA } \\
(\mu \mathrm{g} / \mathrm{L})\end{array}$ & $\begin{array}{c}\text { Flufen- } \\
\text { acet } \\
\text { OXA } \\
(\mu \mathrm{g} / \mathrm{L})\end{array}$ & $\begin{array}{l}\text { Meto- } \\
\text { lachlor } \\
(\mu \mathrm{g} / \mathrm{L})\end{array}$ & $\begin{array}{l}\text { Meto- } \\
\text { lachlor } \\
\text { ESA } \\
(\mu \mathrm{g} / \mathrm{L})\end{array}$ & $\begin{array}{l}\text { Meto- } \\
\text { lachlor } \\
\text { OXA } \\
(\mu \mathrm{g} / \mathrm{L})\end{array}$ \\
\hline \multicolumn{20}{|c|}{ National Water-Quality Assessment Program (NAWQA) (fig. 3)—Continued } \\
\hline \multicolumn{20}{|c|}{ Delmarva Peninsula (DLMV/JMD)—Continued } \\
\hline Oc $15-11$ & Sussex Co., DE & $09 / 13 / 00$ & -- & $<0.05$ & $<0.05$ & -- & -- & 0.82 & $<0.05$ & -- & -- & $<0.05$ & $<0.05$ & -- & $<0.05$ & $<0.05$ & -- & 0.19 & $<0.05$ \\
\hline Oc21-02 & Sussex Co., DE & $07 / 25 / 01$ & -- & $<.05$ & $<.05$ & -- & -- & .33 & $<.05$ & -- & -- & $<.05$ & $<.05$ & -- & $<.05$ & $<.05$ & -- & 4.4 & $<.05$ \\
\hline OC21-03 & Sussex Co., DE & $07 / 25 / 01$ & -- & $<.05$ & $<.05$ & -- & -- & .16 & $<.05$ & -- & -- & $<.05$ & $<.05$ & -- & $<.05$ & $<.05$ & -- & 6.9 & 3.0 \\
\hline Oe44-01 & Sussex Co., DE & 07/24/01 & -- & $<.05$ & $<.05$ & -- & -- & $<.05$ & $<.05$ & -- & -- & $<.05$ & $<.05$ & -- & $<.05$ & $<.05$ & -- & 1.3 & .13 \\
\hline Oe44-02 & Sussex Co., DE & $07 / 24 / 01$ & -- & $<.05$ & $<.05$ & -- & -- & .34 & $<.05$ & -- & -- & $<.05$ & $<.05$ & -- & $<.05$ & $<.05$ & -- & .17 & $<.05$ \\
\hline Oi 25-18 & Sussex Co., DE & 09/13/00 & -- & $<.05$ & $<.05$ & -- & -- & $<.05$ & $<.05$ & -- & -- & $<.05$ & $<.05$ & -- & $<.05$ & $<.05$ & -- & $<.05$ & $<.05$ \\
\hline Oi 25-19 & Sussex Co., DE & $11 / 01 / 00$ & -- & $<.05$ & $<.05$ & -- & -- & $<.05$ & $<.05$ & -- & -- & $<.05$ & $<.05$ & -- & $<.05$ & $<.05$ & -- & .05 & $<.05$ \\
\hline Pc 33-44 & Sussex Co., DE & 09/12/00 & -- & $<.05$ & $<.05$ & -- & -- & .37 & $<.05$ & -- & -- & $<.05$ & $<.05$ & -- & $<.05$ & $<.05$ & -- & .32 & $<.05$ \\
\hline Pe 23-185 & Sussex Co., DE & 09/05/00 & -- & $<.05$ & $<.05$ & -- & -- & $<.05$ & $<.05$ & -- & -- & $<.05$ & $<.05$ & -- & $<.05$ & $<.05$ & -- & $<.05$ & $<.05$ \\
\hline $\mathrm{Ph} 13-03$ & Sussex Co., DE & 08/16/99 & -- & 2.4 & .56 & -- & -- & $<.05$ & $<.05$ & -- & -- & -- & -- & -- & -- & -- & -- & 10 & 5.3 \\
\hline $\mathrm{Ph} 13-04$ & Sussex Co., DE & 08/10/99 & -- & .22 & $<.05$ & -- & -- & $<.05$ & $<.05$ & -- & -- & -- & -- & -- & -- & -- & -- & 24 & 8.0 \\
\hline $\mathrm{Ph} 13-07$ & Sussex Co., DE & $11 / 05 / 99$ & -- & 3.5 & $<.05$ & -- & -- & .16 & $<.05$ & -- & -- & -- & -- & -- & -- & -- & -- & 31 & 11 \\
\hline $\mathrm{Ph} 13-13$ & Sussex Co., DE & 08/18/99 & -- & $<.05$ & $<.05$ & -- & -- & .18 & $<.05$ & -- & -- & -- & -- & -- & -- & -- & -- & $<.05$ & $<.05$ \\
\hline $\mathrm{Ph} 13-14$ & Sussex Co., DE & 08/18/99 & -- & $<.05$ & $<.05$ & -- & -- & .08 & $<.05$ & -- & -- & -- & -- & -- & -- & -- & -- & $<.05$ & $<.05$ \\
\hline $\mathrm{Ph} 13-15$ & Sussex Co., DE & 08/18/99 & -- & $<.05$ & $<.05$ & -- & -- & .06 & $<.05$ & -- & -- & -- & -- & -- & -- & -- & -- & .49 & .08 \\
\hline $\mathrm{Ph} 13-16$ & Sussex Co., DE & 11/04/99 & -- & 2.4 & $<.05$ & -- & -- & $<.05$ & $<.05$ & -- & -- & -- & -- & -- & -- & -- & -- & 12 & 1.8 \\
\hline $\mathrm{Ph} 13-17$ & Sussex Co., DE & 08/17/99 & -- & .61 & $<.05$ & -- & -- & $<.05$ & $<.05$ & -- & -- & -- & -- & -- & -- & -- & -- & 27 & 1.7 \\
\hline Ph 13-18 & Sussex Co., DE & 08/17/99 & -- & $<.05$ & $<.05$ & -- & -- & .23 & $<.05$ & -- & -- & -- & -- & -- & -- & -- & -- & 5.0 & .94 \\
\hline $\mathrm{Ph} 13-23$ & Sussex Co., DE & 08/11/99 & -- & $<.05$ & $<.05$ & -- & -- & 1.4 & $<.05$ & -- & -- & -- & -- & -- & -- & -- & -- & 19 & 4.9 \\
\hline $\mathrm{Ph} 13-24$ & Sussex Co., DE & 08/11/99 & -- & $<.05$ & $<.05$ & -- & -- & 1.5 & .08 & -- & -- & -- & -- & -- & -- & -- & -- & 15 & 4.0 \\
\hline $\mathrm{Ph} 13-25$ & Sussex Co., DE & 08/16/99 & -- & .85 & $<.05$ & -- & -- & $<.05$ & $<.05$ & -- & -- & -- & -- & -- & -- & -- & -- & .23 & .39 \\
\hline $\mathrm{Ph} 13-26$ & Sussex Co., DE & 08/11/99 & -- & $<.05$ & $<.05$ & -- & -- & 1.5 & $<.05$ & -- & -- & -- & -- & -- & -- & -- & -- & 1.5 & 1.3 \\
\hline $\mathrm{Ph} 13-28$ & Sussex Co., DE & $11 / 05 / 99$ & -- & $<.05$ & $<.05$ & -- & -- & .71 & $<.05$ & -- & -- & -- & -- & -- & -- & -- & -- & 17 & 1.9 \\
\hline $\mathrm{Ph} 13-30$ & Sussex Co., DE & 08/12/99 & -- & $<.05$ & $<.05$ & -- & -- & $<.05$ & $<.05$ & -- & -- & -- & -- & -- & -- & -- & -- & .32 & $<.05$ \\
\hline $\mathrm{Ph} 13-31$ & Sussex Co., DE & 08/12/99 & -- & $<.05$ & $<.05$ & -- & -- & $<.05$ & $<.05$ & -- & -- & -- & -- & -- & -- & -- & -- & .86 & $<.05$ \\
\hline
\end{tabular}




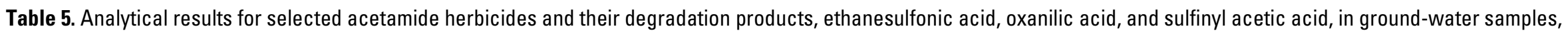
1994-2002.-Continued

[OGRL, U.S. Geological Survey Organic Geochemistry Research Laboratory, Lawrence, Kansas; ESA, ethanesulfonic acid; OXA, oxanilic acid; SAA, sulfinyl acetic acid; --, no data; <, less than]

\begin{tabular}{|c|c|c|c|c|c|c|c|c|c|c|c|c|c|c|c|c|c|c|c|c|}
\hline Site number & Site location & $\begin{array}{l}\text { Date of col- } \\
\text { lection } \\
\text { (month/day/ } \\
\text { year) }\end{array}$ & $\begin{array}{l}\text { Aceto- } \\
\text { chlor } \\
(\mu \mathrm{g} / \mathrm{L})\end{array}$ & $\begin{array}{l}\text { Aceto- } \\
\text { chlor } \\
\text { ESA } \\
(\mu \mathrm{g} / \mathrm{L})\end{array}$ & $\begin{array}{l}\text { Aceto- } \\
\text { chlor } \\
\text { OXA } \\
(\mu \mathrm{g} / \mathrm{L})\end{array}$ & $\begin{array}{l}\text { Aceto- } \\
\text { chlor } \\
\text { SAA } \\
(\mu \mathrm{g} / \mathrm{L})\end{array}$ & $\begin{array}{l}\text { Ala- } \\
\text { chlor } \\
(\mu \mathrm{g} / \mathrm{L})\end{array}$ & $\begin{array}{l}\text { Ala- } \\
\text { chlor } \\
\text { ESA } \\
(\mu \mathrm{g} / \mathrm{L})\end{array}$ & $\begin{array}{l}\text { Ala- } \\
\text { chlor } \\
\text { OXA } \\
(\mu \mathrm{g} / \mathrm{L})\end{array}$ & $\begin{array}{l}\text { Ala- } \\
\text { chlor } \\
\text { SAA } \\
(\mu \mathrm{g} / \mathrm{L})\end{array}$ & $\begin{array}{c}\text { Di- } \\
\text { meth- } \\
\text { enamid } \\
(\mu \mathrm{g} / \mathrm{L})\end{array}$ & $\begin{array}{c}\text { Di- } \\
\text { meth- } \\
\text { enamid } \\
\text { ESA } \\
(\mu \mathrm{g} / \mathrm{L})\end{array}$ & $\begin{array}{c}\text { Di- } \\
\text { meth- } \\
\text { enamid } \\
\text { OXA } \\
(\mu \mathrm{g} / \mathrm{L})\end{array}$ & $\begin{array}{l}\text { Flufen- } \\
\text { acet } \\
(\mu \mathrm{g} / \mathrm{L})\end{array}$ & $\begin{array}{l}\text { Flufen- } \\
\text { acet } \\
\text { ESA } \\
(\mu \mathrm{g} / \mathrm{L})\end{array}$ & $\begin{array}{c}\text { Flufen- } \\
\text { acet } \\
\text { OXA } \\
(\mu \mathrm{g} / \mathrm{L})\end{array}$ & $\begin{array}{c}\text { Meto- } \\
\text { lachlor } \\
(\mu \mathrm{g} / \mathrm{L})\end{array}$ & $\begin{array}{l}\text { Meto- } \\
\text { lachlor } \\
\text { ESA } \\
(\mu \mathrm{g} / \mathrm{L})\end{array}$ & $\begin{array}{c}\text { Meto- } \\
\text { lachlor } \\
\text { OXA } \\
(\mu \mathrm{g} / \mathrm{L})\end{array}$ & 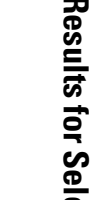 \\
\hline \multicolumn{20}{|c|}{ National Water-Quality Assessment Program (NAWOA) (fig. 3) -Continued } & \\
\hline \multicolumn{20}{|c|}{ Delmarva Peninsula (DLMV/JMD)—Continued } & \\
\hline $\mathrm{Ph} 14-13$ & Sussex Co., DE & $11 / 18 / 99$ & -- & $<0.05$ & $<0.05$ & -- & -- & 0.11 & $<0.05$ & -- & -- & -- & -- & -- & -- & -- & -- & 0.36 & $<0.05$ & \\
\hline $\mathrm{Ph} 23-08$ & Sussex Co., DE & 08/19/99 & -- & .90 & $<.05$ & -- & -- & $<.05$ & $<.05$ & -- & -- & -- & -- & -- & -- & -- & -- & 12 & 3.4 & \\
\hline $\mathrm{Ph} 23-10$ & Sussex Co., DE & 08/10/99 & -- & $<.05$ & $<.05$ & -- & -- & $<.05$ & $<.05$ & -- & -- & -- & -- & -- & -- & -- & -- & 35 & 19 & \\
\hline $\mathrm{Ph} 23-12$ & Sussex Co., DE & 08/10/99 & -- & $<.05$ & $<.05$ & -- & -- & .33 & .32 & -- & -- & -- & -- & -- & -- & -- & -- & 16 & 11 & \\
\hline $\mathrm{Ph} 23-18$ & Sussex Co., DE & 08/19/99 & -- & $<.05$ & $<.05$ & -- & -- & .06 & $<.05$ & -- & -- & -- & -- & -- & -- & -- & -- & 11 & 2.8 & \\
\hline $\mathrm{Ph} 23-19$ & Sussex Co., DE & 08/19/99 & -- & $<.05$ & $<.05$ & -- & -- & .17 & $<.05$ & -- & -- & -- & -- & -- & -- & -- & -- & .65 & .12 & \\
\hline Pi12-08 & Sussex Co., DE & $10 / 24 / 00$ & -- & $<.05$ & $<.05$ & -- & -- & $<.05$ & $<.05$ & -- & -- & $<0.05$ & $<0.05$ & -- & $<0.05$ & $<0.05$ & -- & $<.05$ & $<.05$ & \\
\hline Pi-31-02 & Sussex Co., DE & 08/17/00 & -- & $<.05$ & $<.05$ & -- & -- & .29 & $<.05$ & -- & -- & $<.05$ & $<.05$ & -- & $<.05$ & $<.05$ & -- & 1.5 & $<.05$ & \\
\hline Pot Nets\#1 & Sussex Co., DE & $10 / 11 / 00$ & -- & $<.05$ & $<.05$ & -- & -- & $<.05$ & $<.05$ & -- & -- & $<.05$ & $<.05$ & -- & $<.05$ & $<.05$ & -- & $<.05$ & $<.05$ & \\
\hline QADb-40 & Sussex Co., DE & 08/29/01 & -- & $<.05$ & $<.05$ & -- & -- & .08 & $<.05$ & -- & -- & $<.05$ & $<.05$ & -- & $<.05$ & $<.05$ & -- & .12 & $<.05$ & \\
\hline QADf54 & Queen Annes Co., MD & $07 / 26 / 01$ & -- & $<.05$ & $<.05$ & -- & -- & .94 & $<.05$ & -- & -- & $<.05$ & $<.05$ & -- & $<.05$ & $<.05$ & -- & 6.5 & 2.1 & \\
\hline QADf55 & Queen Annes Co., MD & $07 / 26 / 01$ & -- & $<.05$ & $<.05$ & -- & -- & .67 & $<.05$ & -- & -- & $<.05$ & $<.05$ & -- & $<.05$ & $<.05$ & -- & 17 & 4.8 & \\
\hline Qc22-04 & Queen Annes Co., MD & $08 / 28 / 01$ & -- & $<.05$ & $<.05$ & -- & -- & .08 & $<.05$ & -- & -- & $<.05$ & $<.05$ & -- & $<.05$ & $<.05$ & -- & .45 & $<.05$ & \\
\hline QD21-12 & Sussex Co., DE & $08 / 22 / 00$ & -- & $<.05$ & $<.05$ & -- & -- & .41 & $<.05$ & -- & -- & $<.05$ & $<.05$ & -- & $<.05$ & $<.05$ & -- & 1.8 & .70 & \\
\hline QD52-09 & Sussex Co., DE & $08 / 22 / 00$ & -- & $<.05$ & $<.05$ & -- & -- & $<.05$ & $<.05$ & -- & -- & $<.05$ & $<.05$ & -- & $<.05$ & $<.05$ & -- & .09 & $<.05$ & \\
\hline \multirow[t]{2}{*}{$\operatorname{Rd} 22-01$} & \multirow[t]{2}{*}{ Sussex Co., DE } & $08 / 31 / 00$ & -- & $<.05$ & $<.05$ & -- & -- & $<.05$ & $<.05$ & -- & -- & $<.05$ & $<.05$ & -- & $<.05$ & $<.05$ & -- & $<.05$ & $<.05$ & \multirow{5}{*}{ 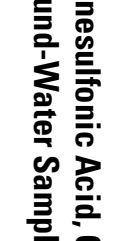 } \\
\hline & & $08 / 31 / 00$ & -- & $<.05$ & $<.05$ & -- & -- & $<.05$ & $<.05$ & -- & -- & $<.05$ & $<.05$ & -- & $<.05$ & $<.05$ & -- & $<.05$ & $<.05$ & \\
\hline Ri22-03 & Sussex Co., DE & $09 / 05 / 01$ & -- & $<.05$ & $<.05$ & -- & -- & .16 & $<.05$ & -- & -- & $<.05$ & $<.05$ & -- & $<.05$ & $<.05$ & -- & $<.05$ & $<.05$ & \\
\hline RI 23-04 & Sussex Co., DE & $08 / 21 / 00$ & -- & $<.05$ & $<.05$ & -- & -- & .42 & $<.05$ & -- & -- & $<.05$ & $<.05$ & -- & $<.05$ & $<.05$ & -- & .96 & .26 & \\
\hline SODe44 & Sussex Co., DE & $08 / 02 / 01$ & -- & $<.05$ & $<.05$ & -- & -- & $<.05$ & $<.05$ & -- & -- & $<.05$ & $<.05$ & -- & $<.05$ & $<.05$ & -- & $<.05$ & $<.05$ & \\
\hline Wi Bh 4 & Wicomico Co., MD & $10 / 06 / 99$ & -- & $<.05$ & $<.05$ & -- & -- & 6.9 & $<.05$ & -- & -- & -- & -- & -- & -- & -- & -- & 6.5 & 3.5 & \\
\hline Wi Bh 5 & Wicomico Co., MD & $10 / 06 / 99$ & -- & $<.05$ & $<.05$ & -- & -- & $<.05$ & $<.05$ & -- & -- & -- & -- & -- & -- & -- & -- & $<.05$ & $<.05$ & \\
\hline Wi Bh 8 & Wicomico Co., MD & $10 / 05 / 99$ & -- & $<.05$ & $<.05$ & -- & -- & .10 & $<.05$ & -- & -- & -- & -- & -- & -- & -- & -- & .38 & .16 & \\
\hline Wi Bh 9 & Wicomico Co., MD & $10 / 05 / 99$ & -- & $<.05$ & $<.05$ & -- & -- & $<.05$ & $<.05$ & -- & -- & -- & -- & -- & -- & -- & -- & $<.05$ & $<.05$ & \\
\hline
\end{tabular}




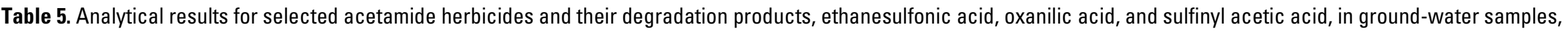
1994-2002.-Continued

[OGRL, U.S. Geological Survey Organic Geochemistry Research Laboratory, Lawrence, Kansas; ESA, ethanesulfonic acid; OXA, oxanilic acid; SAA, sulfinyl acetic acid; --, no data; <, less than]

\begin{tabular}{|c|c|c|c|c|c|c|c|c|c|c|c|c|c|c|c|c|c|c|c|}
\hline Site number & Site location & $\begin{array}{l}\text { Date of col- } \\
\text { lection } \\
\text { (month/day/ } \\
\text { year) }\end{array}$ & $\begin{array}{l}\text { Aceto- } \\
\text { chlor } \\
(\mu \mathrm{g} / \mathrm{L})\end{array}$ & $\begin{array}{c}\text { Aceto- } \\
\text { chlor } \\
\text { ESA } \\
(\mu \mathrm{g} / \mathrm{L})\end{array}$ & $\begin{array}{l}\text { Aceto- } \\
\text { chlor } \\
\text { OXA } \\
(\mu \mathrm{g} / \mathrm{L})\end{array}$ & $\begin{array}{l}\text { Aceto- } \\
\text { chlor } \\
\text { SAA } \\
(\mu \mathrm{g} / \mathrm{L})\end{array}$ & $\begin{array}{l}\text { Ala- } \\
\text { chlor } \\
(\mu \mathrm{g} / \mathrm{L})\end{array}$ & $\begin{array}{l}\text { Ala- } \\
\text { chlor } \\
\text { ESA } \\
(\mu \mathrm{g} / \mathrm{L})\end{array}$ & $\begin{array}{c}\text { Ala- } \\
\text { chlor } \\
\text { OXA } \\
(\mu \mathrm{g} / \mathrm{L})\end{array}$ & $\begin{array}{c}\text { Ala- } \\
\text { chlor } \\
\text { SAA } \\
(\mu \mathrm{g} / \mathrm{L})\end{array}$ & $\begin{array}{c}\text { Di- } \\
\text { meth- } \\
\text { enamid } \\
(\mu \mathrm{g} / \mathrm{L})\end{array}$ & $\begin{array}{c}\text { Di- } \\
\text { meth- } \\
\text { enamid } \\
\text { ESA } \\
(\mu \mathrm{g} / \mathrm{L})\end{array}$ & $\begin{array}{c}\text { Di- } \\
\text { meth- } \\
\text { enamid } \\
\text { OXA } \\
(\mu \mathrm{g} / \mathrm{L})\end{array}$ & $\begin{array}{c}\text { Flufen- } \\
\text { acet } \\
(\mu \mathrm{g} / \mathrm{L})\end{array}$ & $\begin{array}{c}\text { Flufen- } \\
\text { acet } \\
\text { ESA } \\
(\mu \mathrm{g} / \mathrm{L})\end{array}$ & $\begin{array}{c}\text { Flufen- } \\
\text { acet } \\
\text { OXA } \\
(\mu \mathrm{g} / \mathrm{L})\end{array}$ & $\begin{array}{l}\text { Meto- } \\
\text { lachlor } \\
(\mu \mathrm{g} / \mathrm{L})\end{array}$ & $\begin{array}{l}\text { Meto- } \\
\text { lachlor } \\
\text { ESA } \\
(\mu \mathrm{g} / \mathrm{L})\end{array}$ & $\begin{array}{l}\text { Meto- } \\
\text { lachlor } \\
\text { OXA } \\
(\mu \mathrm{g} / \mathrm{L})\end{array}$ \\
\hline \multicolumn{20}{|c|}{ National Water-Quality Assessment Program (NAWQA) (fig. 3)—Continued } \\
\hline \multicolumn{20}{|c|}{ Delmarva Peninsula (DLMV/JMD)—Continued } \\
\hline Wi Bh 12 & Wicomico Co., MD & $10 / 05 / 99$ & -- & $<0.05$ & $<0.05$ & -- & -- & 0.18 & $<0.05$ & -- & -- & -- & -- & -- & -- & -- & -- & .26 & .16 \\
\hline Wi Cg 56 & Wicomico Co., MD & 10/07/99 & -- & $<.05$ & $<.05$ & -- & -- & .21 & $<.05$ & -- & -- & -- & -- & -- & -- & -- & -- & 4.8 & .84 \\
\hline Wi Ch50 & Wicomico Co., MD & 09/24/01 & -- & 1.1 & $<.05$ & -- & -- & $<.05$ & 2.2 & -- & -- & $<0.05$ & $<0.05$ & -- & $<0.05$ & $<0.05$ & -- & $<.05$ & $<.05$ \\
\hline WI Ch 51 & Wicomico Co., MD & 08/21/01 & -- & $<.05$ & $<.05$ & -- & -- & 1.2 & 2.0 & -- & -- & $<.05$ & $<.05$ & -- & $<.05$ & $<.05$ & -- & .11 & $<.05$ \\
\hline WI Ch 55 & Wicomico Co., MD & $10 / 06 / 99$ & -- & $<.05$ & $<.05$ & -- & -- & $<.05$ & $<.05$ & -- & -- & -- & -- & -- & -- & -- & -- & $<.05$ & $<.05$ \\
\hline Wi Ch 57 & Wicomico Co., MD & $10 / 07 / 99$ & -- & $<.05$ & $<.05$ & -- & -- & 7.4 & 12 & -- & -- & -- & -- & -- & -- & -- & -- & 25 & 36 \\
\hline WOCc2 & Worcester Co., MD & 08/08/01 & -- & $<.05$ & $<.05$ & -- & -- & $<.05$ & $<.05$ & -- & -- & $<.05$ & $<.05$ & -- & $<.05$ & $<.05$ & -- & $<.05$ & $<.05$ \\
\hline WOCc3 & Worcester Co., MD & 08/08/01 & -- & $<.05$ & $<.05$ & -- & -- & $<.05$ & $<.05$ & -- & -- & $<.05$ & $<.05$ & -- & $<.05$ & $<.05$ & -- & $<.05$ & $<.05$ \\
\hline WOCg76 & Worcester Co., MD & 08/09/01 & -- & $<.05$ & $<.05$ & -- & -- & $<.05$ & $<.05$ & -- & -- & $<.05$ & $<.05$ & -- & $<.05$ & $<.05$ & -- & $<.05$ & $<.05$ \\
\hline WOCg78 & Worcester Co., MD & 08/09/01 & -- & $<.05$ & $<.05$ & -- & -- & $<.05$ & $<.05$ & -- & -- & $<.05$ & $<.05$ & -- & $<.05$ & $<.05$ & -- & $<.05$ & $<.05$ \\
\hline WOFc46 & Worcester Co., MD & 09/06/01 & -- & $<.05$ & $<.05$ & -- & -- & $<.05$ & $<.05$ & -- & -- & $<.05$ & $<.05$ & -- & $<.05$ & $<.05$ & -- & $<.05$ & $<.05$ \\
\hline \multicolumn{20}{|c|}{ Eastern lowa Basin (EIWA/SKI) } \\
\hline \multirow[t]{2}{*}{405405091335001} & Henry Co., IA & 07/02/97 & $<0.05$ & .28 & $<.20$ & -- & $<0.05$ & 4.5 & $<.20$ & -- & -- & -- & -- & -- & -- & -- & $<0.05$ & .56 & $<.20$ \\
\hline & & 08/02/99 & $<.05$ & $<.20$ & $<.20$ & -- & $<.05$ & .43 & $<.20$ & -- & $<0.05$ & -- & -- & -- & -- & -- & $<.05$ & 5.7 & $<.20$ \\
\hline 405601091551901 & Jefferson Co., IA & 07/10/97 & $<.05$ & $<.20$ & $<.20$ & -- & $<.05$ & .98 & $<.20$ & -- & -- & -- & -- & -- & -- & -- & $<.05$ & 1.4 & $<.20$ \\
\hline 410513091430401 & Jefferson Co., IA & 07/22/98 & $<.05$ & $<.20$ & $<.20$ & -- & $<.05$ & $<.20$ & $<.20$ & -- & -- & -- & -- & -- & -- & -- & $<.05$ & .60 & $<.20$ \\
\hline 410956091135601 & Louisa Co., IA & 06/24/98 & $<.05$ & $<.20$ & $<.20$ & -- & $<.05$ & $<.20$ & $<.20$ & -- & -- & -- & -- & -- & -- & -- & $<.05$ & $<.20$ & $<.20$ \\
\hline \multirow[t]{2}{*}{411511091155101} & Louisa Co., IA & 06/24/97 & $<.05$ & $<.20$ & $<.20$ & -- & $<.05$ & $<.20$ & $<.20$ & -- & -- & -- & -- & -- & -- & -- & $<.05$ & $<.20$ & $<.20$ \\
\hline & & 07/20/99 & $<.05$ & $<.20$ & $<.20$ & -- & $<.05$ & $<.20$ & $<.20$ & -- & $<.05$ & -- & -- & -- & -- & -- & $<.05$ & $<.20$ & $<.20$ \\
\hline 411622091193401 & Louisa Co., IA & $06 / 24 / 98$ & $<.05$ & $<.20$ & $<.20$ & -- & $<.05$ & $<.20$ & $<.20$ & -- & -- & -- & -- & -- & -- & -- & $<.05$ & 1.3 & $<.20$ \\
\hline 411843092105101 & Keokuk Co., IA & 07/14/97 & $<.05$ & $<.20$ & $<.20$ & -- & $<.05$ & .29 & $<.20$ & -- & -- & -- & -- & -- & -- & -- & $<.05$ & .26 & $<.20$ \\
\hline 412030091113801 & Muscatine Co., IA & 06/10/96 & $<.05$ & $<.20$ & $<.20$ & -- & $<.05$ & $<.20$ & $<.20$ & -- & -- & -- & -- & -- & -- & -- & $<.05$ & $<.20$ & $<.20$ \\
\hline 412711091122401 & Muscatine Co., IA & 06/15/98 & $<.05$ & $<.20$ & $<.20$ & -- & $<.05$ & $<.20$ & $<.20$ & -- & -- & -- & -- & -- & -- & -- & .05 & $<.20$ & $<.20$ \\
\hline 412748091285101 & Johnson Co., IA & 06/16/98 & $<.05$ & $<.20$ & $<.20$ & -- & $<.05$ & $<.20$ & $<.20$ & -- & -- & -- & -- & -- & -- & -- & $<.05$ & $<.20$ & $<.20$ \\
\hline \multirow[t]{2}{*}{412755091114101} & Muscatine Co., IA & $06 / 25 / 97$ & $<.05$ & $<.20$ & $<.20$ & -- & $<.05$ & 3.4 & .63 & -- & -- & -- & -- & -- & -- & -- & $<.05$ & 1.7 & $<.20$ \\
\hline & & 08/19/02 & -- & 2.0 & 1.3 & $<0.05$ & -- & .89 & $<.05$ & $<0.05$ & -- & $<.05$ & $<.05$ & -- & $<.05$ & $<.05$ & -- & 3.7 & .37 \\
\hline
\end{tabular}




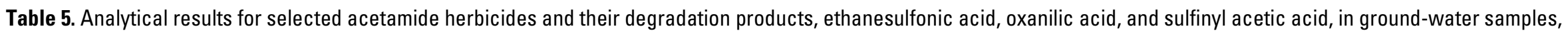
1994-2002.-Continued

[OGRL, U.S. Geological Survey Organic Geochemistry Research Laboratory, Lawrence, Kansas; ESA, ethanesulfonic acid; OXA, oxanilic acid; SAA, sulfinyl acetic acid; --, no data; <, less than]

\begin{tabular}{|c|c|c|c|c|c|c|c|c|c|c|c|c|c|c|c|c|c|c|c|c|}
\hline Site number & Site location & $\begin{array}{c}\text { Date of col- } \\
\text { lection } \\
\text { (month/day/ } \\
\text { year) }\end{array}$ & $\begin{array}{c}\text { Aceto- } \\
\text { chlor } \\
(\mu \mathrm{g} / \mathrm{L})\end{array}$ & $\begin{array}{l}\text { Aceto- } \\
\text { chlor } \\
\text { ESA } \\
(\mu \mathrm{g} / \mathrm{L})\end{array}$ & $\begin{array}{l}\text { Aceto- } \\
\text { chlor } \\
\text { OXA } \\
(\mu \mathrm{g} / \mathrm{L})\end{array}$ & $\begin{array}{l}\text { Aceto- } \\
\text { chlor } \\
\text { SAA } \\
(\mu \mathrm{g} / \mathrm{L})\end{array}$ & $\begin{array}{l}\text { Ala- } \\
\text { chlor } \\
(\mu \mathrm{g} / \mathrm{L})\end{array}$ & $\begin{array}{l}\text { Ala- } \\
\text { chlor } \\
\text { ESA } \\
(\mu \mathrm{g} / \mathrm{L})\end{array}$ & $\begin{array}{l}\text { Ala- } \\
\text { chlor } \\
\text { OXA } \\
(\mu \mathrm{g} / \mathrm{L})\end{array}$ & $\begin{array}{l}\text { Ala- } \\
\text { chlor } \\
\text { SAA } \\
(\mu \mathrm{g} / \mathrm{L})\end{array}$ & $\begin{array}{c}\text { Di- } \\
\text { meth- } \\
\text { enamid } \\
(\mu \mathrm{g} / \mathrm{L})\end{array}$ & $\begin{array}{c}\text { Di- } \\
\text { meth- } \\
\text { enamid } \\
\text { ESA } \\
(\mu \mathrm{g} / \mathrm{L})\end{array}$ & $\begin{array}{c}\text { Di- } \\
\text { meth- } \\
\text { enamid } \\
0 X A \\
(\mu \mathrm{g} / \mathrm{L})\end{array}$ & $\begin{array}{c}\text { Flufen- } \\
\text { acet } \\
(\mu \mathrm{g} / \mathrm{L})\end{array}$ & $\begin{array}{c}\text { Flufen- } \\
\text { acet } \\
\text { ESA } \\
(\mu \mathrm{g} / \mathrm{L})\end{array}$ & $\begin{array}{c}\text { Flufen- } \\
\text { acet } \\
\text { OXA } \\
(\mu \mathrm{g} / \mathrm{L})\end{array}$ & $\begin{array}{l}\text { Meto- } \\
\text { lachlor } \\
(\mu \mathrm{g} / \mathrm{L})\end{array}$ & $\begin{array}{c}\text { Meto- } \\
\text { lachlor } \\
\text { ESA } \\
(\mu \mathrm{g} / \mathrm{L})\end{array}$ & $\begin{array}{c}\text { Meto- } \\
\text { lachlor } \\
\text { OXA } \\
(\mu \mathrm{g} / \mathrm{L})\end{array}$ & 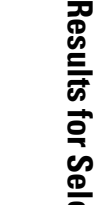 \\
\hline \multicolumn{20}{|c|}{ National Water-Quality Assessment Program (NAWQA) (fig. 3)—Continued } & \\
\hline \multicolumn{20}{|c|}{ Eastern lowa Basin (EIWA/SKI)—Continued } & \\
\hline 412808091345001 & Washington Co., IA & 06/17/98 & $<0.05$ & $<0.20$ & $<0.20$ & -- & $<0.05$ & 7.7 & $<0.20$ & -- & -- & -- & -- & -- & -- & -- & $<0.05$ & 20 & $<0.20$ & \\
\hline 412831091205601 & Muscatine Co., IA & $06 / 16 / 98$ & $<.05$ & .42 & $<.20$ & -- & $<.05$ & .90 & $<.20$ & -- & -- & -- & -- & -- & -- & -- & $<.05$ & .55 & $<.20$ & \\
\hline \multirow[t]{2}{*}{412855091421601} & Washington Co., IA & $06 / 26 / 97$ & $<.05$ & $<.20$ & $<.20$ & -- & $<.05$ & $<.20$ & $<.20$ & -- & -- & -- & -- & -- & -- & -- & $<.05$ & $<.20$ & $<.20$ & \\
\hline & & 07/10/97 & $<.05$ & $<.20$ & $<.20$ & -- & $<.05$ & $<.20$ & $<.20$ & -- & -- & -- & -- & -- & -- & -- & $<.05$ & $<.20$ & $<.20$ & \\
\hline 412916091405101 & Washington Co., IA & $06 / 17 / 98$ & $<.05$ & $<.20$ & $<.20$ & -- & $<.05$ & $<.20$ & $<.20$ & -- & -- & -- & -- & -- & -- & -- & $<.05$ & $<.20$ & $<.20$ & \\
\hline \multirow[t]{2}{*}{412927092575201} & Marion Co., IA & $07 / 14 / 97$ & $<.05$ & $<.20$ & $<.20$ & -- & .37 & $<.20$ & $<.20$ & -- & -- & -- & -- & -- & -- & -- & .15 & 1.6 & .20 & \\
\hline & & $07 / 19 / 99$ & $<.05$ & $<.20$ & $<.20$ & -- & $<.05$ & $<.20$ & $<.20$ & -- & $<0.05$ & -- & -- & $<0.05$ & -- & -- & $<.05$ & 1.4 & $<.20$ & \\
\hline \multirow[t]{2}{*}{413248092011301} & \multirow[t]{2}{*}{ Iowa Co., IA } & $07 / 07 / 97$ & $<.05$ & $<.20$ & $<.20$ & -- & $<.05$ & 7.6 & $<.20$ & -- & -- & -- & -- & -- & -- & -- & $<.05$ & $<.20$ & $<.20$ & \multirow{6}{*}{ 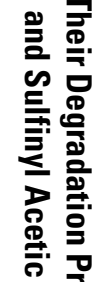 } \\
\hline & & $07 / 12 / 99$ & $<.05$ & $<.20$ & $<.20$ & -- & $<.05$ & 5.1 & $<.20$ & -- & $<.05$ & -- & -- & $<.05$ & -- & -- & $<.05$ & $<.20$ & $<.20$ & \\
\hline 413338091045601 & Muscatine Co., IA & $07 / 16 / 98$ & $<.05$ & $<.20$ & $<.20$ & -- & $<.05$ & $<.20$ & $<.20$ & -- & -- & -- & -- & -- & -- & -- & $<.05$ & .27 & $<.20$ & \\
\hline 413414091095501 & Muscatine Co., IA & $06 / 25 / 97$ & $<.05$ & $<.20$ & $<.20$ & -- & $<.05$ & $<.20$ & $<.20$ & -- & -- & -- & -- & -- & -- & -- & $<.05$ & 1.4 & $<.20$ & \\
\hline \multirow[t]{2}{*}{413438091341201} & \multirow[t]{2}{*}{ Johnson Co., IA } & 06/10/98 & $<.05$ & $<.20$ & $<.20$ & -- & $<.05$ & $<.20$ & $<.20$ & -- & -- & -- & -- & -- & -- & -- & $<.05$ & $<.20$ & $<.20$ & \\
\hline & & $08 / 21 / 02$ & -- & $<.05$ & $<.05$ & $<0.05$ & -- & $<.05$ & $<.05$ & $<0.05$ & -- & $<0.05$ & $<0.05$ & -- & $<0.05$ & $<0.05$ & -- & $<.05$ & $<.05$ & \\
\hline 413523092050501 & Iowa Co., IA & $06 / 22 / 98$ & $<.05$ & $<.20$ & $<.20$ & -- & $<.05$ & $<.20$ & $<.20$ & -- & -- & -- & -- & -- & -- & -- & $<.05$ & .85 & $<.20$ & \\
\hline 413560091341201 & Johnson Co., IA & 06/10/97 & $<.05$ & $<.20$ & $<.20$ & -- & $<.05$ & .79 & $<.20$ & -- & -- & -- & -- & -- & -- & -- & $<.05$ & .72 & $<.20$ & \\
\hline 413634091484301 & Johnson Co., IA & $06 / 18 / 98$ & $<.05$ & $<.20$ & $<.20$ & -- & $<.05$ & $<.20$ & $<.20$ & -- & -- & -- & -- & -- & -- & -- & $<.05$ & $<.20$ & $<.20$ & \\
\hline 413652092035801 & Iowa Co., IA & $06 / 12 / 96$ & $<.05$ & $<.20$ & $<.20$ & -- & $<.05$ & $<.20$ & $<.20$ & -- & -- & -- & -- & -- & -- & -- & $<.05$ & $<.20$ & $<.20$ & \\
\hline 413705091392701 & Johnson Co., IA & $06 / 11 / 98$ & $<.05$ & $<.20$ & $<.20$ & -- & $<.05$ & 2.1 & $<.20$ & -- & -- & -- & -- & -- & -- & -- & .05 & 2.6 & $<.20$ & \\
\hline 413823091322301 & Johnson Co., IA & 06/09/97 & $<.05$ & $<.20$ & $<.20$ & -- & $<.05$ & $<.20$ & $<.20$ & -- & -- & -- & -- & -- & -- & -- & $<.05$ & 1.1 & $<.20$ & \\
\hline 413933091304701 & Johnson Co., IA & $06 / 05 / 97$ & $<.05$ & $<.20$ & $<.20$ & -- & $<.05$ & $<.20$ & $<.20$ & -- & -- & -- & -- & -- & -- & -- & $<.05$ & $<.20$ & $<.20$ & 总 몽. \\
\hline 414036090460001 & Scott Co., IA & $06 / 23 / 98$ & $<.05$ & $<.20$ & $<.20$ & -- & $<.05$ & $<.20$ & $<.20$ & -- & -- & -- & -- & -- & -- & -- & $<.05$ & $<.20$ & $<.20$ & \\
\hline 414056091325201 & Johnson Co., IA & $06 / 05 / 96$ & $<.05$ & $<.20$ & $<.20$ & -- & $<.05$ & $<.20$ & $<.20$ & -- & -- & -- & -- & -- & -- & -- & $<.05$ & $<.20$ & $<.20$ & \\
\hline 414153090350801 & Scott Co., IA & $06 / 06 / 96$ & $<.05$ & $<.20$ & $<.20$ & -- & $<.05$ & $<.20$ & $<.20$ & -- & -- & -- & -- & -- & -- & -- & $<.05$ & $<.20$ & $<.20$ & \\
\hline 414208092312601 & Poweshiek Co., IA & $08 / 28 / 02$ & -- & $<.05$ & $<.05$ & $<.05$ & -- & $<.05$ & $<.05$ & $<.05$ & -- & $<.05$ & $<.05$ & -- & $<.05$ & $<.05$ & -- & .73 & $<.05$ & है \\
\hline 414430093220001 & Polk Co., IA & 07/09/97 & $<.05$ & $<.20$ & $<.20$ & -- & $<.05$ & $<.20$ & $<.20$ & -- & -- & -- & -- & -- & -- & -- & $<.05$ & 1.5 & $<.20$ & \\
\hline
\end{tabular}




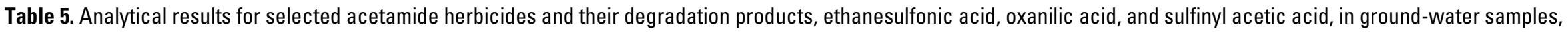
1994-2002.-Continued

[OGRL, U.S. Geological Survey Organic Geochemistry Research Laboratory, Lawrence, Kansas; ESA, ethanesulfonic acid; OXA, oxanilic acid; SAA, sulfinyl acetic acid; --, no data; <, less than]

\begin{tabular}{|c|c|c|c|c|c|c|c|c|c|c|c|c|c|c|c|c|c|c|c|}
\hline Site number & Site location & $\begin{array}{l}\text { Date of col- } \\
\text { lection } \\
\text { (month/day/ } \\
\text { year) }\end{array}$ & $\begin{array}{c}\text { Aceto- } \\
\text { chlor } \\
(\mu \mathrm{g} / \mathrm{L})\end{array}$ & $\begin{array}{l}\text { Aceto- } \\
\text { chlor } \\
\text { ESA } \\
(\mu \mathrm{g} / \mathrm{L})\end{array}$ & $\begin{array}{c}\text { Aceto- } \\
\text { chlor } \\
\text { OXA } \\
(\mu \mathrm{g} / \mathrm{L})\end{array}$ & $\begin{array}{l}\text { Aceto- } \\
\text { chlor } \\
\text { SAA } \\
(\mu \mathrm{g} / \mathrm{L})\end{array}$ & $\begin{array}{l}\text { Ala- } \\
\text { chlor } \\
(\mu \mathrm{g} / \mathrm{L})\end{array}$ & $\begin{array}{l}\text { Ala- } \\
\text { chlor } \\
\text { ESA } \\
(\mu \mathrm{g} / \mathrm{L})\end{array}$ & $\begin{array}{l}\text { Ala- } \\
\text { chlor } \\
\text { OXA } \\
(\mu \mathrm{g} / \mathrm{L})\end{array}$ & $\begin{array}{l}\text { Ala- } \\
\text { chlor } \\
\text { SAA } \\
(\mu \mathrm{g} / \mathrm{L})\end{array}$ & $\begin{array}{c}\text { Di- } \\
\text { meth- } \\
\text { enamid } \\
(\mu \mathrm{g} / \mathrm{L})\end{array}$ & $\begin{array}{c}\text { Di- } \\
\text { meth- } \\
\text { enamid } \\
\text { ESA } \\
(\mu \mathrm{g} / \mathrm{L})\end{array}$ & $\begin{array}{c}\text { Di- } \\
\text { meth- } \\
\text { enamid } \\
\text { OXA } \\
(\mu \mathrm{g} / \mathrm{L})\end{array}$ & $\begin{array}{c}\text { Flufen- } \\
\text { acet } \\
(\mu \mathrm{g} / \mathrm{L})\end{array}$ & $\begin{array}{c}\text { Flufen- } \\
\text { acet } \\
\text { ESA } \\
(\mu \mathrm{g} / \mathrm{L})\end{array}$ & $\begin{array}{c}\text { Flufen- } \\
\text { acet } \\
\text { OXA } \\
(\mu \mathrm{g} / \mathrm{L})\end{array}$ & $\begin{array}{l}\text { Meto- } \\
\text { lachlor } \\
(\mu \mathrm{g} / \mathrm{L})\end{array}$ & $\begin{array}{l}\text { Meto- } \\
\text { lachlor } \\
\text { ESA } \\
(\mu \mathrm{g} / \mathrm{L})\end{array}$ & $\begin{array}{c}\text { Meto- } \\
\text { lachlor } \\
\text { OXA } \\
(\mu \mathrm{g} / \mathrm{L})\end{array}$ \\
\hline \multicolumn{20}{|c|}{ National Water-Quality Assessment Program (NAWQA) (fig. 3)—Continued } \\
\hline \multicolumn{20}{|c|}{ Eastern lowa Basin (EIWA/SKI)—Continued } \\
\hline \multirow[t]{2}{*}{414430093220001} & Polk Co., IA & $07 / 14 / 98$ & $<0.05$ & 0.52 & $<0.20$ & -- & $<0.05$ & $<0.20$ & $<0.20$ & -- & -- & -- & -- & -- & -- & -- & $<0.05$ & 3.1 & 1.7 \\
\hline & & $08 / 28 / 02$ & -- & 1.2 & .45 & $<0.05$ & -- & .23 & $<.05$ & $<0.05$ & -- & $<0.05$ & $<0.05$ & -- & $<0.05$ & $<0.05$ & -- & 3.1 & 1.1 \\
\hline 414752092053201 & Iowa Co., IA & 08/19/98 & $<.05$ & $<.20$ & $<.20$ & -- & $<.05$ & 7.5 & $<.20$ & -- & -- & -- & -- & -- & -- & -- & $<.05$ & $<.20$ & $<.20$ \\
\hline 414752092053202 & Iowa Co., IA & 08/19/98 & $<.05$ & $<.20$ & $<.20$ & -- & $<.05$ & $<.20$ & $<.20$ & -- & -- & -- & -- & -- & -- & -- & $<.05$ & $<.20$ & $<.20$ \\
\hline 414752092053203 & Iowa Co., IA & 08/19/98 & $<.05$ & $<.20$ & $<.20$ & -- & $<.05$ & $<.20$ & $<.20$ & -- & -- & -- & -- & -- & -- & -- & $<.05$ & $<.20$ & $<.20$ \\
\hline \multirow[t]{2}{*}{414816092053401} & Iowa Co., IA & $07 / 31 / 96$ & $<.05$ & $<.20$ & $<.20$ & -- & $<.05$ & 1.4 & .26 & -- & -- & -- & -- & -- & -- & -- & $<.05$ & $<.20$ & $<.20$ \\
\hline & & 08/03/98 & $<.05$ & $<.20$ & $<.20$ & -- & $<.05$ & 2.1 & $<.20$ & -- & -- & -- & -- & -- & -- & -- & $<.05$ & $<.20$ & $<.20$ \\
\hline \multirow[t]{2}{*}{414816092053402} & Iowa Co., IA & $07 / 31 / 96$ & $<.05$ & .34 & .46 & -- & $<.05$ & .46 & .41 & -- & -- & -- & -- & -- & -- & -- & $<.05$ & .48 & $<.20$ \\
\hline & & 08/03/98 & $<.05$ & $<.20$ & $<.20$ & -- & $<.05$ & .37 & $<.20$ & -- & -- & -- & -- & -- & -- & -- & $<.05$ & $<.20$ & $<.20$ \\
\hline \multirow[t]{2}{*}{414816092053403} & Iowa Co., IA & $07 / 31 / 96$ & $<.05$ & $<.20$ & $<.20$ & -- & $<.05$ & $<.20$ & $<.20$ & -- & -- & -- & -- & -- & -- & -- & $<.05$ & .66 & .25 \\
\hline & & 08/03/98 & $<.05$ & .29 & .36 & -- & $<.05$ & $<.20$ & $<.20$ & -- & -- & -- & -- & -- & -- & -- & $<.05$ & .40 & $<.20$ \\
\hline \multirow[t]{2}{*}{414818092055401} & Iowa Co., IA & 08/06/96 & $<.05$ & $<.20$ & $<.20$ & -- & $<.05$ & $<.20$ & $<.20$ & -- & -- & -- & -- & -- & -- & -- & $<.05$ & $<.20$ & $<.20$ \\
\hline & & 08/20/98 & $<.05$ & $<.20$ & $<.20$ & -- & $<.05$ & $<.20$ & $<.20$ & -- & -- & -- & -- & -- & -- & -- & $<.05$ & .30 & $<.20$ \\
\hline \multirow[t]{2}{*}{414818092055402} & Iowa Co., IA & 08/06/96 & $<.05$ & $<.20$ & $<.20$ & -- & $<.05$ & .28 & $<.20$ & -- & -- & -- & -- & -- & -- & -- & $<.05$ & $<.20$ & $<.20$ \\
\hline & & 08/20/98 & $<.05$ & .57 & .34 & -- & $<.05$ & .86 & $<.20$ & -- & -- & -- & -- & -- & -- & -- & $<.05$ & 2.5 & .76 \\
\hline \multirow[t]{3}{*}{414818092055403} & Iowa Co., IA & $08 / 05 / 96$ & $<.05$ & $<.20$ & $<.20$ & -- & $<.05$ & .65 & .30 & -- & -- & -- & -- & -- & -- & -- & $<.05$ & .32 & $<.20$ \\
\hline & & 08/20/98 & $<.05$ & .53 & .51 & -- & $<.05$ & 1.1 & .28 & -- & -- & -- & -- & -- & -- & -- & $<.05$ & 2.8 & .82 \\
\hline & & 08/20/02 & -- & .12 & .07 & $<.05$ & -- & .18 & $<.05$ & $<.05$ & -- & $<.05$ & $<.05$ & -- & $<.05$ & $<.05$ & -- & .73 & .18 \\
\hline 414828092014201 & Iowa Co., IA & $08 / 14 / 96$ & $<.05$ & $<.20$ & $<.20$ & -- & $<.05$ & .24 & $<.20$ & -- & -- & -- & -- & -- & -- & -- & $<.05$ & $<.20$ & $<.20$ \\
\hline \multirow[t]{2}{*}{414860092073801} & Madison Co., IA & 08/01/96 & $<.05$ & $<.20$ & $<.20$ & -- & $<.05$ & 2.0 & $<.20$ & -- & -- & -- & -- & -- & -- & -- & $<.05$ & .27 & $<.20$ \\
\hline & & 08/13/98 & $<.05$ & $<.20$ & $<.20$ & -- & $<.05$ & 1.7 & $<.20$ & -- & -- & -- & -- & -- & -- & -- & $<.05$ & .83 & .25 \\
\hline \multirow[t]{2}{*}{414907092083001} & Iowa Co., IA & 07/30/96 & $<.05$ & $<.20$ & $<.20$ & -- & $<.05$ & $<.20$ & $<.20$ & -- & -- & -- & -- & -- & -- & -- & $<.05$ & $<.20$ & $<.20$ \\
\hline & & 08/06/98 & $<.05$ & $<.20$ & $<.20$ & -- & $<.05$ & .92 & 3.6 & -- & -- & -- & -- & -- & -- & -- & $<.05$ & $<.20$ & $<.20$ \\
\hline 414907092083002 & Iowa Co., IA & $07 / 30 / 96$ & $<.05$ & $<.20$ & $<.20$ & -- & $<.05$ & 34 & 14 & -- & -- & -- & -- & -- & -- & -- & $<.05$ & 4.8 & $<.20$ \\
\hline \multirow[t]{2}{*}{414907092083003} & Iowa Co., IA & 07/30/96 & $<.05$ & $<.20$ & $<.20$ & -- & $<.05$ & 5.3 & 1.8 & -- & -- & -- & -- & -- & -- & -- & $<.05$ & 1.8 & .71 \\
\hline & & 08/06/98 & $<.05$ & $<.20$ & $<.20$ & -- & $<.05$ & 6.5 & 2.7 & -- & -- & -- & -- & -- & -- & -- & $<.05$ & 1.2 & $<.20$ \\
\hline
\end{tabular}




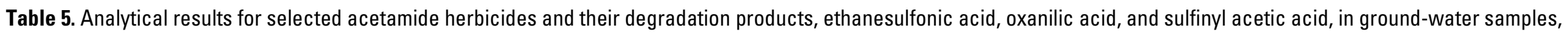
1994-2002.-Continued

[OGRL, U.S. Geological Survey Organic Geochemistry Research Laboratory, Lawrence, Kansas; ESA, ethanesulfonic acid; OXA, oxanilic acid; SAA, sulfinyl acetic acid; --, no data; <, less than]

\begin{tabular}{|c|c|c|c|c|c|c|c|c|c|c|c|c|c|c|c|c|c|c|c|c|}
\hline Site number & Site location & $\begin{array}{l}\text { Date of col- } \\
\text { lection } \\
\text { (month/day/ } \\
\text { year) }\end{array}$ & $\begin{array}{l}\text { Aceto- } \\
\text { chlor } \\
(\mu \mathrm{g} / \mathrm{L})\end{array}$ & $\begin{array}{l}\text { Aceto- } \\
\text { chlor } \\
\text { ESA } \\
(\mu \mathrm{g} / \mathrm{L})\end{array}$ & $\begin{array}{l}\text { Aceto- } \\
\text { chlor } \\
\text { OXA } \\
(\mu \mathrm{g} / \mathrm{L})\end{array}$ & $\begin{array}{l}\text { Aceto- } \\
\text { chlor } \\
\text { SAA } \\
(\mu \mathrm{g} / \mathrm{L})\end{array}$ & $\begin{array}{l}\text { Ala- } \\
\text { chlor } \\
(\mu \mathrm{g} / \mathrm{L})\end{array}$ & $\begin{array}{l}\text { Ala- } \\
\text { chlor } \\
\text { ESA } \\
(\mu \mathrm{g} / \mathrm{L})\end{array}$ & $\begin{array}{c}\text { Ala- } \\
\text { chlor } \\
\text { OXA } \\
(\mu \mathrm{g} / \mathrm{L})\end{array}$ & $\begin{array}{l}\text { Ala- } \\
\text { chlor } \\
\text { SAA } \\
(\mu \mathrm{g} / \mathrm{L})\end{array}$ & $\begin{array}{c}\text { Di- } \\
\text { meth- } \\
\text { enamid } \\
(\mu \mathrm{g} / \mathrm{L})\end{array}$ & $\begin{array}{c}\text { Di- } \\
\text { meth- } \\
\text { enamid } \\
\text { ESA } \\
(\mu \mathrm{g} / \mathrm{L})\end{array}$ & $\begin{array}{c}\text { Di- } \\
\text { meth- } \\
\text { enamid } \\
\text { OXA } \\
(\mu \mathrm{g} / \mathrm{L})\end{array}$ & $\begin{array}{c}\text { Flufen- } \\
\text { acet } \\
(\mu \mathrm{g} / \mathrm{L})\end{array}$ & $\begin{array}{l}\text { Flufen- } \\
\text { acet } \\
\text { ESA } \\
(\mu \mathrm{g} / \mathrm{L})\end{array}$ & $\begin{array}{l}\text { Flufen- } \\
\text { acet } \\
\text { OXA } \\
(\mu \mathrm{g} / \mathrm{L})\end{array}$ & $\begin{array}{c}\text { Meto- } \\
\text { lachlor } \\
(\mu \mathrm{g} / \mathrm{L})\end{array}$ & $\begin{array}{l}\text { Meto- } \\
\text { lachlor } \\
\text { ESA } \\
(\mu \mathrm{g} / \mathrm{L})\end{array}$ & $\begin{array}{l}\text { Meto- } \\
\text { lachlor } \\
\text { OXA } \\
(\mu \mathrm{g} / \mathrm{L})\end{array}$ & 葛 \\
\hline \multicolumn{20}{|c|}{ National Water-Quality Assessment Program (NAWQA) (fig. 3)—Continued } & \\
\hline \multicolumn{20}{|c|}{ Eastern lowa Basin (EIWA/SKI)—Continued } & \\
\hline 414907092083004 & Iowa Co., IA & 08/06/98 & $<0.05$ & 0.47 & $<0.20$ & -- & $<0.05$ & 1.5 & $<0.20$ & -- & -- & -- & -- & -- & -- & -- & $<0.05$ & 0.98 & 0.22 & \\
\hline 414914092024001 & Iowa Co., IA & $07 / 15 / 98$ & $<.05$ & $<.20$ & $<.20$ & -- & $<.05$ & $<.20$ & $<.20$ & -- & -- & -- & -- & -- & -- & -- & $<.05$ & $<.20$ & $<.20$ & \\
\hline \multirow[t]{2}{*}{414930092093801} & Iowa Co., IA & 08/01/96 & $<.05$ & $<.20$ & $<.20$ & -- & $<.05$ & 1.3 & $<.20$ & -- & -- & -- & -- & -- & -- & -- & $<.05$ & $<.20$ & $<.20$ & \\
\hline & & 08/13/98 & $<.05$ & $<.20$ & $<.20$ & -- & $<.05$ & 4.2 & $<.20$ & -- & -- & -- & -- & -- & -- & -- & $<.05$ & .20 & $<.20$ & \\
\hline 414944090470901 & Clinton Co., IA & 07/09/98 & $<.05$ & $<.20$ & $<.20$ & -- & $<.05$ & $<.20$ & $<.20$ & -- & -- & -- & -- & -- & -- & -- & $<.05$ & $<.20$ & $<.20$ & \\
\hline 414958090230301 & Clinton Co., IA & 06/12/97 & $<.05$ & $<.20$ & $<.20$ & -- & $<.05$ & 1.7 & $<.20$ & -- & -- & -- & -- & -- & -- & -- & $<.05$ & 43 & 5.1 & \\
\hline 415020092094001 & Iowa Co., IA & 08/04/98 & $<.05$ & $<.20$ & $<.20$ & -- & $<.05$ & 1.7 & $<.20$ & -- & -- & -- & -- & -- & -- & -- & $<.05$ & $<.20$ & $<.20$ & \multirow{4}{*}{ 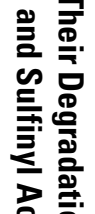 } \\
\hline 415020092094002 & Iowa Co., IA & 08/05/98 & $<.05$ & $<.20$ & $<.20$ & -- & $<.05$ & $<.20$ & $<.20$ & -- & -- & -- & -- & -- & -- & -- & $<.05$ & $<.20$ & $<.20$ & \\
\hline \multirow[t]{2}{*}{415020092094003} & Iowa Co., IA & 07/29/96 & $<.05$ & $<.20$ & $<.20$ & -- & $<.05$ & .26 & $<.20$ & -- & -- & -- & -- & -- & -- & -- & $<.05$ & $<.20$ & $<.20$ & \\
\hline & & 08/05/98 & $<.05$ & .83 & .36 & -- & $<.05$ & .26 & $<.20$ & -- & -- & -- & -- & -- & -- & -- & $<.05$ & .67 & .28 & \\
\hline 415020092094004 & Iowa Co., IA & 08/04/98 & $<.05$ & .74 & $<.20$ & -- & $<.05$ & $<.20$ & $<.20$ & -- & -- & -- & -- & -- & -- & -- & $<.05$ & .40 & $<.20$ & \multirow{2}{*}{ 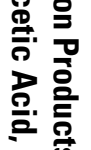 } \\
\hline 415020092094005 & Iowa Co., IA & 08/04/98 & $<.05$ & 1.0 & .21 & -- & $<.05$ & .20 & $<.20$ & -- & -- & -- & -- & -- & -- & -- & $<.05$ & .55 & $<.20$ & \\
\hline \multirow[t]{2}{*}{415020092094010} & Iowa Co., IA & 07/29/96 & $<.05$ & $<.20$ & $<.20$ & -- & $<.05$ & 2.4 & $<.20$ & -- & -- & -- & -- & -- & -- & -- & $<.05$ & $<.20$ & $<.20$ & \multirow{5}{*}{ 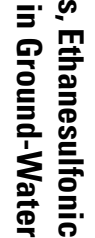 } \\
\hline & & 08/05/98 & $<.05$ & $<.20$ & $<.20$ & -- & $<.05$ & 1.3 & .48 & -- & -- & -- & -- & -- & -- & -- & $<.05$ & $<.20$ & $<.20$ & \\
\hline \multirow[t]{2}{*}{415039092164001} & Iowa Co., IA & 08/17/98 & $<.05$ & $<.20$ & $<.20$ & -- & $<.05$ & $<.20$ & $<.20$ & -- & -- & -- & -- & -- & -- & -- & $<.05$ & $<.20$ & $<.20$ & \\
\hline & & 08/13/96 & $<.05$ & $<.20$ & $<.20$ & -- & $<.05$ & .54 & $<.20$ & -- & -- & -- & -- & -- & -- & -- & $<.05$ & .60 & $<.20$ & \\
\hline \multirow[t]{2}{*}{415045092145601} & Iowa Co., IA & 08/08/96 & $<.05$ & $<.20$ & $<.20$ & -- & $<.05$ & 4.6 & $<.20$ & -- & -- & -- & -- & -- & -- & -- & $<.05$ & .57 & $<.20$ & \\
\hline & & 08/21/98 & $<.05$ & $<.20$ & $<.20$ & -- & $<.05$ & 6.1 & $<.20$ & -- & -- & -- & -- & -- & -- & -- & $<.05$ & 1.3 & $<.20$ & \\
\hline \multirow[t]{2}{*}{415052092120301} & Iowa Co., IA & 08/07/96 & $<.05$ & $<.20$ & $<.20$ & -- & $<.05$ & 2.6 & $<.20$ & -- & -- & -- & -- & -- & -- & -- & $<.05$ & .30 & $<.20$ & \multirow{3}{*}{ 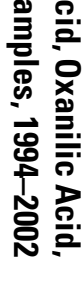 } \\
\hline & & 08/11/98 & $<.05$ & $<.20$ & $<.20$ & -- & $<.05$ & $<.20$ & $<.20$ & -- & -- & -- & -- & -- & -- & -- & $<.05$ & $<.20$ & $<.20$ & \\
\hline \multirow[t]{2}{*}{415105092132501} & Iowa Co., IA & 08/07/96 & $<.05$ & $<.20$ & $<.20$ & -- & $<.05$ & 7.5 & .35 & -- & -- & -- & -- & -- & -- & -- & $<.05$ & 3.2 & $<.20$ & \\
\hline & & 08/11/98 & $<.05$ & $<.20$ & $<.20$ & -- & $<.05$ & 7.1 & .35 & -- & -- & -- & -- & -- & -- & -- & $<.05$ & 2.2 & .36 & \\
\hline
\end{tabular}




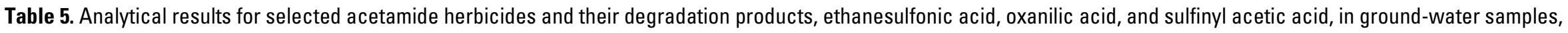
1994-2002.-Continued

[OGRL, U.S. Geological Survey Organic Geochemistry Research Laboratory, Lawrence, Kansas; ESA, ethanesulfonic acid; OXA, oxanilic acid; SAA, sulfinyl acetic acid; --, no data; <, less than]

\begin{tabular}{|c|c|c|c|c|c|c|c|c|c|c|c|c|c|c|c|c|c|c|c|}
\hline Site number & Site location & $\begin{array}{l}\text { Date of col- } \\
\text { lection } \\
\text { (month/day/ } \\
\text { year) }\end{array}$ & $\begin{array}{l}\text { Aceto- } \\
\text { chlor } \\
(\mu \mathrm{g} / \mathrm{L})\end{array}$ & $\begin{array}{l}\text { Aceto- } \\
\text { chlor } \\
\text { ESA } \\
(\mu \mathrm{g} / \mathrm{L})\end{array}$ & $\begin{array}{l}\text { Aceto- } \\
\text { chlor } \\
\text { OXA } \\
(\mu \mathrm{g} / \mathrm{L})\end{array}$ & $\begin{array}{l}\text { Aceto- } \\
\text { chlor } \\
\text { SAA } \\
(\mu \mathrm{g} / \mathrm{L})\end{array}$ & $\begin{array}{l}\text { Ala- } \\
\text { chlor } \\
(\mu \mathrm{g} / \mathrm{L})\end{array}$ & $\begin{array}{l}\text { Ala- } \\
\text { chlor } \\
\text { ESA } \\
(\mu \mathrm{g} / \mathrm{L})\end{array}$ & $\begin{array}{l}\text { Ala- } \\
\text { chlor } \\
\text { OXA } \\
(\mu \mathrm{g} / \mathrm{L})\end{array}$ & $\begin{array}{l}\text { Ala- } \\
\text { chlor } \\
\text { SAA } \\
(\mu \mathrm{g} / \mathrm{L})\end{array}$ & $\begin{array}{c}\text { Di- } \\
\text { meth- } \\
\text { enamid } \\
(\mu \mathrm{g} / \mathrm{L})\end{array}$ & $\begin{array}{c}\text { Di- } \\
\text { meth- } \\
\text { enamid } \\
\text { ESA } \\
(\mu \mathrm{g} / \mathrm{L})\end{array}$ & $\begin{array}{c}\text { Di- } \\
\text { meth- } \\
\text { enamid } \\
\text { OXA } \\
(\mu \mathrm{g} / \mathrm{L})\end{array}$ & $\begin{array}{c}\text { Flufen- } \\
\text { acet } \\
(\mu \mathrm{g} / \mathrm{L})\end{array}$ & $\begin{array}{c}\text { Flufen- } \\
\text { acet } \\
\text { ESA } \\
(\mu \mathrm{g} / \mathrm{L})\end{array}$ & $\begin{array}{c}\text { Flufen- } \\
\text { acet } \\
\text { OXA } \\
(\mu \mathrm{g} / \mathrm{L})\end{array}$ & $\begin{array}{l}\text { Meto- } \\
\text { lachlor } \\
(\mu \mathrm{g} / \mathrm{L})\end{array}$ & $\begin{array}{l}\text { Meto- } \\
\text { lachlor } \\
\text { ESA } \\
(\mu \mathrm{g} / \mathrm{L})\end{array}$ & $\begin{array}{l}\text { Meto- } \\
\text { lachlor } \\
\text { OXA } \\
(\mu \mathrm{g} / \mathrm{L})\end{array}$ \\
\hline \multicolumn{20}{|c|}{ National Water-Quality Assessment Program (NAWOA) (fig. 3)—Continued } \\
\hline \multicolumn{20}{|c|}{ Eastern lowa Basin (EIWA/SKI)—Continued } \\
\hline 415105092132501 & Iowa Co., IA & 08/18/98 & $<0.05$ & $<0.20$ & $<0.20$ & -- & $<0.05$ & 6.1 & $<0.20$ & -- & -- & -- & -- & -- & -- & -- & $<0.05$ & $<0.20$ & $<0.20$ \\
\hline 415139092190801 & Poweshiek Co., IA & 06/30/98 & $<.05$ & $<.20$ & $<.20$ & -- & $<.05$ & $<.20$ & $<.20$ & -- & -- & -- & -- & -- & -- & -- & $<.05$ & $<.20$ & $<.20$ \\
\hline 415147092115301 & Benton Co., IA & 06/30/98 & $<.05$ & .48 & $<.20$ & -- & $<.05$ & .31 & $<.20$ & -- & -- & -- & -- & -- & -- & -- & $<.05$ & .28 & $<.20$ \\
\hline 415147092115301 & Benton Co., IA & 08/20/02 & -- & $<.05$ & $<.05$ & $<0.05$ & -- & 1.4 & $<.05$ & $<0.05$ & -- & 0.05 & $<0.05$ & -- & $<0.05$ & $<0.05$ & -- & 1.0 & .37 \\
\hline \multirow[t]{2}{*}{415211092164101} & Benton Co., IA & $07 / 23 / 96$ & $<.05$ & $<.20$ & $<.20$ & -- & $<.05$ & 2.3 & .26 & -- & -- & -- & -- & -- & -- & -- & $<.05$ & $<.20$ & $<.20$ \\
\hline & & 08/17/98 & $<.05$ & $<.20$ & $<.20$ & -- & $<.05$ & 2.3 & $<.20$ & -- & -- & -- & -- & -- & -- & -- & $<.05$ & $<.20$ & $<.20$ \\
\hline \multirow[t]{2}{*}{415211092164102} & Benton Co., IA & $07 / 23 / 96$ & $<.05$ & $<.20$ & $<.20$ & -- & $<.05$ & 3.3 & .38 & -- & -- & -- & -- & -- & -- & -- & $<.05$ & .71 & $<.20$ \\
\hline & & 08/17/98 & $<.05$ & $<.20$ & $<.20$ & -- & $<.05$ & 7.1 & .36 & -- & -- & -- & -- & -- & -- & -- & $<.05$ & $<.20$ & $<.20$ \\
\hline \multirow[t]{2}{*}{415231092295301} & Tama Co., IA & 06/19/96 & $<.05$ & $<.20$ & $<.20$ & -- & $<.05$ & $<.20$ & $<.20$ & -- & -- & -- & -- & -- & -- & -- & $<.05$ & $<.20$ & $<.20$ \\
\hline & & $07 / 17 / 97$ & $<.05$ & $<.20$ & $<.20$ & -- & $<.05$ & $<.20$ & $<.20$ & -- & -- & -- & -- & -- & -- & -- & $<.05$ & $<.20$ & $<.20$ \\
\hline 415600091033701 & Cedar Co., IA & 06/17/96 & $<.05$ & $<.20$ & $<.20$ & -- & $<.05$ & $<.20$ & $<.20$ & -- & -- & -- & -- & -- & -- & -- & $<.05$ & $<.20$ & $<.20$ \\
\hline 415637091581001 & Benton Co., IA & $06 / 30 / 98$ & $<.05$ & $<.20$ & $<.20$ & -- & $<.05$ & 5.9 & 3.3 & -- & -- & -- & -- & -- & -- & -- & $<.05$ & $<.20$ & $<.20$ \\
\hline 415825091405601 & Linn Co., IA & 07/17/97 & $<.05$ & $<.20$ & $<.20$ & -- & $<.05$ & .52 & $<.20$ & -- & -- & -- & -- & -- & -- & -- & $<.05$ & .78 & .23 \\
\hline 415827091392401 & Linn Co., IA & 06/30/97 & $<.05$ & $<.20$ & $<.20$ & -- & $<.05$ & $<.20$ & $<.20$ & -- & -- & -- & -- & -- & -- & -- & $<.05$ & .33 & $<.20$ \\
\hline 415850090572201 & Jones Co., IA & $06 / 11 / 97$ & $<.05$ & $<.20$ & $<.20$ & -- & $<.05$ & .45 & .20 & -- & -- & -- & -- & -- & -- & -- & $<.05$ & 1.0 & .31 \\
\hline 415859090563901 & Jones Co., IA & 06/23/98 & $<.05$ & $<.20$ & $<.20$ & -- & $<.05$ & .98 & $<.20$ & -- & -- & -- & -- & -- & -- & -- & $<.05$ & .29 & $<.20$ \\
\hline 420117092505601 & Marshall Co., IA & 07/09/97 & $<.05$ & $<.20$ & $<.20$ & -- & $<.05$ & $<.20$ & $<.20$ & -- & -- & -- & -- & -- & -- & -- & $<.05$ & .41 & $<.20$ \\
\hline 420206091244901 & Linn Co., IA & $06 / 20 / 96$ & $<.05$ & $<.20$ & $<.20$ & -- & $<.05$ & $<.20$ & $<.20$ & -- & -- & -- & -- & -- & -- & -- & $<.05$ & $<.20$ & $<.20$ \\
\hline 420219093361301 & Story Co., IA & $07 / 15 / 97$ & $<.05$ & $<.20$ & $<.20$ & -- & $<.05$ & $<.20$ & $<.20$ & -- & -- & -- & -- & -- & -- & -- & $<.05$ & .33 & $<.20$ \\
\hline 420240092535001 & Marshall Co., IA & $07 / 16 / 97$ & $<.05$ & $<.20$ & $<.20$ & -- & $<.05$ & .93 & 1.5 & -- & -- & -- & -- & -- & -- & -- & $<.05$ & .70 & .58 \\
\hline 420347092541601 & Marshall Co., IA & 07/16/97 & $<.05$ & $<.20$ & $<.20$ & -- & $<.05$ & .84 & $<.20$ & -- & -- & -- & -- & -- & -- & -- & $<.05$ & 3.2 & .57 \\
\hline 420424092175101 & Benton Co., IA & 06/19/96 & $<.05$ & $<.20$ & $<.20$ & -- & $<.05$ & $<.20$ & $<.20$ & -- & -- & -- & -- & -- & -- & -- & $<.05$ & $<.20$ & $<.20$ \\
\hline 420936092005701 & Benton Co., IA & $07 / 21 / 97$ & $<.05$ & $<.20$ & $<.20$ & -- & $<.05$ & $<.20$ & $<.20$ & -- & -- & -- & -- & -- & -- & -- & $<.05$ & $<.20$ & $<.20$ \\
\hline 421012092020101 & Benton Co., IA & $07 / 21 / 97$ & $<.05$ & $<.20$ & $<.20$ & -- & .06 & 1.1 & 5.4 & -- & -- & -- & -- & -- & -- & -- & .54 & .72 & .54 \\
\hline 421030092340001 & Tama Co., IA & $06 / 25 / 96$ & $<.05$ & $<.20$ & $<.20$ & -- & $<.05$ & $<.20$ & $<.20$ & -- & -- & -- & -- & -- & -- & -- & $<.05$ & $<.20$ & $<.20$ \\
\hline
\end{tabular}




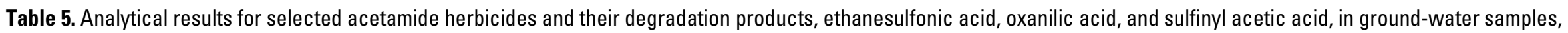
1994-2002.-Continued

[OGRL, U.S. Geological Survey Organic Geochemistry Research Laboratory, Lawrence, Kansas; ESA, ethanesulfonic acid; OXA, oxanilic acid; SAA, sulfinyl acetic acid; --, no data; <, less than]

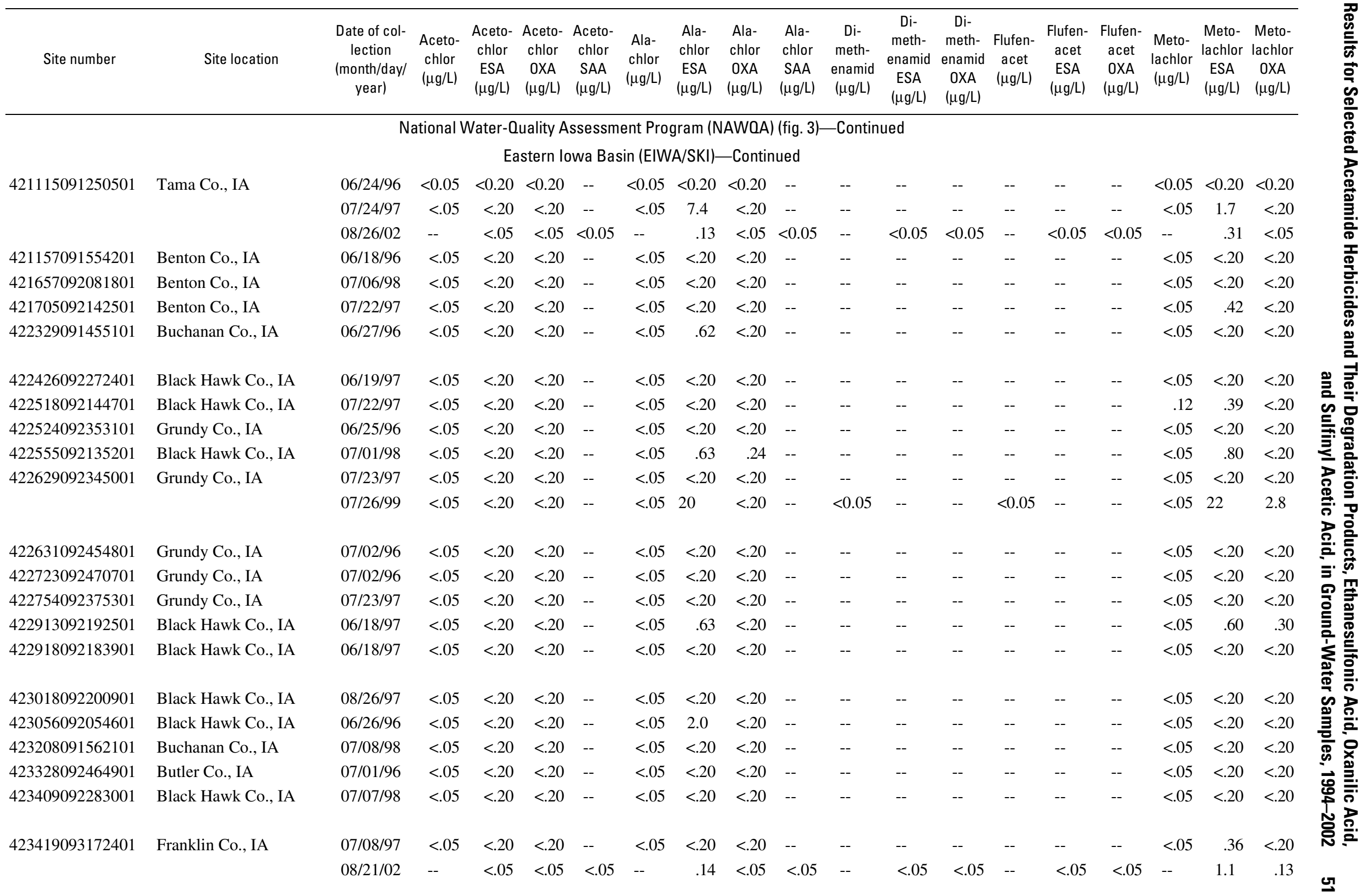




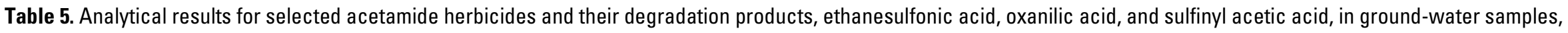
1994-2002.-Continued

[OGRL, U.S. Geological Survey Organic Geochemistry Research Laboratory, Lawrence, Kansas; ESA, ethanesulfonic acid; OXA, oxanilic acid; SAA, sulfinyl acetic acid; --, no data; <, less than]

\begin{tabular}{|c|c|c|c|c|c|c|c|c|c|c|c|c|c|c|c|c|c|c|c|}
\hline Site number & Site location & $\begin{array}{l}\text { Date of col- } \\
\text { lection } \\
\text { (month/day/ } \\
\text { year) }\end{array}$ & $\begin{array}{c}\text { Aceto- } \\
\text { chlor } \\
(\mu \mathrm{g} / \mathrm{L})\end{array}$ & $\begin{array}{l}\text { Aceto- } \\
\text { chlor } \\
\text { ESA } \\
(\mu \mathrm{g} / \mathrm{L})\end{array}$ & $\begin{array}{l}\text { Aceto- } \\
\text { chlor } \\
\text { OXA } \\
(\mu \mathrm{g} / \mathrm{L})\end{array}$ & $\begin{array}{l}\text { Aceto- } \\
\text { chlor } \\
\text { SAA } \\
(\mu \mathrm{g} / \mathrm{L})\end{array}$ & $\begin{array}{l}\text { Ala- } \\
\text { chlor } \\
(\mu \mathrm{g} / \mathrm{L})\end{array}$ & $\begin{array}{l}\text { Ala- } \\
\text { chlor } \\
\text { ESA } \\
(\mu \mathrm{g} / \mathrm{L})\end{array}$ & $\begin{array}{l}\text { Ala- } \\
\text { chlor } \\
\text { OXA } \\
(\mu \mathrm{g} / \mathrm{L})\end{array}$ & $\begin{array}{l}\text { Ala- } \\
\text { chlor } \\
\text { SAA } \\
(\mu \mathrm{g} / \mathrm{L})\end{array}$ & $\begin{array}{l}\text { Di- } \\
\text { meth- } \\
\text { enamid } \\
(\mu \mathrm{g} / \mathrm{L})\end{array}$ & $\begin{array}{c}\text { Di- } \\
\text { meth- } \\
\text { enamid } \\
\text { ESA } \\
(\mu \mathrm{g} / \mathrm{L})\end{array}$ & $\begin{array}{c}\text { Di- } \\
\text { meth- } \\
\text { enamid } \\
0 X A \\
(\mu \mathrm{g} / \mathrm{L})\end{array}$ & $\begin{array}{c}\text { Flufen- } \\
\text { acet } \\
(\mu \mathrm{g} / \mathrm{L})\end{array}$ & $\begin{array}{l}\text { Flufen- } \\
\text { acet } \\
\text { ESA } \\
(\mu \mathrm{g} / \mathrm{L})\end{array}$ & $\begin{array}{l}\text { Flufen- } \\
\text { acet } \\
\text { OXA } \\
(\mu \mathrm{g} / \mathrm{L})\end{array}$ & $\begin{array}{l}\text { Meto- } \\
\text { lachlor } \\
(\mu \mathrm{g} / \mathrm{L})\end{array}$ & $\begin{array}{l}\text { Meto- } \\
\text { lachlor } \\
\text { ESA } \\
(\mu \mathrm{g} / \mathrm{L})\end{array}$ & $\begin{array}{c}\text { Meto- } \\
\text { lachlor } \\
\text { OXA } \\
(\mu \mathrm{g} / \mathrm{L})\end{array}$ \\
\hline \multicolumn{20}{|c|}{ National Water-Quality Assessment Program (NAWOA) (fig. 3)—Continued } \\
\hline \multicolumn{20}{|c|}{ Eastern lowa Basin (EIWA/SKI)—Continued } \\
\hline 423459092523701 & Butler Co., IA & $07 / 28 / 97$ & $<0.05$ & $<0.20$ & $<0.20$ & -- & $<0.05$ & $<0.20$ & $<0.20$ & -- & -- & -- & -- & -- & -- & -- & $<0.05$ & $<0.20$ & $<0.20$ \\
\hline 423459092530501 & Butler Co., IA & $07 / 28 / 97$ & $<.05$ & $<.20$ & $<.20$ & -- & $<.05$ & $<.20$ & $<.20$ & -- & -- & -- & -- & -- & -- & -- & $<.05$ & $<.20$ & $<.20$ \\
\hline 423557091560501 & Buchanan Co., IA & $08 / 27 / 97$ & $<.05$ & $<.20$ & $<.20$ & -- & $<.05$ & $<.20$ & $<.20$ & -- & -- & -- & -- & -- & -- & -- & $<.05$ & $<.20$ & $<.20$ \\
\hline 423639092350901 & Butler Co., IA & $06 / 17 / 97$ & $<.05$ & $<.20$ & $<.20$ & -- & $<.05$ & $<.20$ & $<.20$ & -- & -- & -- & -- & -- & -- & -- & $<.05$ & .56 & $<.20$ \\
\hline \multirow[t]{2}{*}{423749092260801} & Black Hawk Co., IA & 07/07/98 & $<.05$ & $<.20$ & $<.20$ & -- & $<.05$ & 4.1 & $<.20$ & -- & -- & -- & -- & -- & -- & -- & $<.05$ & .32 & $<.20$ \\
\hline & & $08 / 27 / 02$ & -- & .38 & $<.05$ & $<0.05$ & -- & 2.6 & $<.05$ & $<0.05$ & -- & $<0.05$ & $<0.05$ & -- & $<0.05$ & $<0.05$ & -- & .33 & $<.05$ \\
\hline 423930093294901 & Franklin Co., IA & 07/08/97 & $<.05$ & $<.20$ & $<.20$ & -- & $<.05$ & 2.3 & $<.20$ & -- & -- & -- & -- & -- & -- & -- & $<.05$ & 2.9 & .49 \\
\hline 423940092102801 & Bremer Co., IA & $06 / 26 / 96$ & $<.05$ & $<.20$ & $<.20$ & -- & $<.05$ & $<.20$ & $<.20$ & -- & -- & -- & -- & -- & -- & -- & $<.05$ & $<.20$ & $<.20$ \\
\hline 424034091553401 & Fayette Co., IA & $08 / 27 / 97$ & $<.05$ & $<.20$ & $<.20$ & -- & $<.05$ & $<.20$ & $<.20$ & -- & -- & -- & -- & -- & -- & -- & $<.05$ & $<.20$ & $<.20$ \\
\hline 424203092551301 & Butler Co., IA & 07/31/97 & $<.05$ & $<.20$ & $<.20$ & -- & $<.05$ & .80 & $<.20$ & -- & -- & -- & -- & -- & -- & -- & $<.05$ & .20 & $<.20$ \\
\hline 424322092283901 & Bremer Co., IA & 06/17/97 & $<.05$ & $<.20$ & $<.20$ & -- & $<.05$ & .32 & $<.20$ & -- & -- & -- & -- & -- & -- & -- & $<.05$ & .24 & $<.20$ \\
\hline \multirow[t]{4}{*}{424539093372001} & Wright Co., IA & 05/05/98 & $<.05$ & .53 & $<.20$ & -- & $<.05$ & 1.7 & $<.20$ & -- & -- & -- & -- & -- & -- & -- & .21 & 7.2 & .63 \\
\hline & & 08/04/98 & $<.05$ & $<.20$ & $<.20$ & -- & $<.05$ & 4.1 & $<.20$ & -- & -- & -- & -- & -- & -- & -- & .88 & 9.2 & $<.20$ \\
\hline & & 09/01/98 & $<.05$ & $<.20$ & $<.20$ & -- & $<.05$ & .55 & $<.20$ & -- & -- & -- & -- & -- & -- & -- & .75 & 1.1 & .32 \\
\hline & & 08/07/01 & -- & $<.05$ & $<.05$ & $<.05$ & -- & 1.7 & $<.05$ & $<.05$ & -- & .09 & $<.05$ & -- & $<.05$ & $<.05$ & -- & 7.3 & 1.1 \\
\hline 424548092101701 & Bremer Co., IA & $08 / 26 / 97$ & $<.05$ & $<.20$ & $<.20$ & -- & $<.05$ & 6.0 & $<.20$ & -- & -- & -- & -- & -- & -- & -- & $<.05$ & .40 & $<.20$ \\
\hline 425158092402301 & Butler Co., IA & 07/03/96 & $<.05$ & $<.20$ & $<.20$ & -- & $<.05$ & $<.20$ & $<.20$ & -- & -- & -- & -- & -- & -- & -- & $<.05$ & $<.20$ & $<.20$ \\
\hline 425401093135201 & Franklin Co., IA & $07 / 31 / 97$ & $<.05$ & $<.20$ & $<.20$ & -- & $<.05$ & $<.20$ & $<.20$ & -- & -- & -- & -- & -- & -- & -- & $<.05$ & .90 & $<.20$ \\
\hline 425756092162401 & Chickasaw Co., IA & $08 / 21 / 97$ & $<.05$ & $<.20$ & $<.20$ & -- & $<.05$ & 18 & $<.20$ & -- & -- & -- & -- & -- & -- & -- & $<.05$ & $<.20$ & $<.20$ \\
\hline 425757092503001 & Butler Co., IA & 07/03/96 & $<.05$ & $<.20$ & $<.20$ & -- & $<.05$ & 2.1 & $<.20$ & -- & -- & -- & -- & -- & -- & -- & $<.05$ & .44 & .86 \\
\hline 430159093403201 & Hancock Co., IA & $07 / 29 / 97$ & $<.05$ & $<.20$ & $<.20$ & -- & $<.05$ & .66 & .31 & -- & -- & -- & -- & -- & -- & -- & $<.05$ & $<.20$ & $<.20$ \\
\hline 430216093142901 & Cerro Gordo Co., IA & 07/09/96 & $<.05$ & $<.20$ & $<.20$ & -- & $<.05$ & $<.20$ & $<.20$ & -- & -- & -- & -- & -- & -- & -- & $<.05$ & $<.20$ & $<.20$ \\
\hline 430255093083301 & Cerro Gordo Co., IA & $07 / 21 / 98$ & $<.05$ & $<.20$ & $<.20$ & -- & $<.05$ & $<.20$ & $<.20$ & -- & -- & -- & -- & -- & -- & -- & $<.05$ & $<.20$ & $<.20$ \\
\hline 430414092405801 & Floyd Co., IA & 08/21/98 & $<.05$ & $<.20$ & $<.20$ & -- & $<.05$ & $<.20$ & $<.20$ & -- & -- & -- & -- & -- & -- & -- & $<.05$ & $<.20$ & $<.20$ \\
\hline 430442092402201 & Floyd Co., IA & 08/19/97 & $<.05$ & $<.20$ & $<.20$ & -- & $<.05$ & 1.9 & .38 & -- & -- & -- & -- & -- & -- & -- & $<.05$ & 12 & 3.6 \\
\hline
\end{tabular}




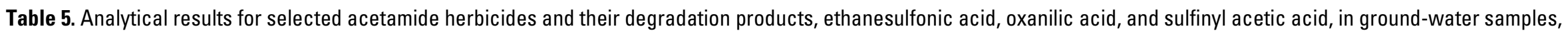
1994-2002.-Continued

[OGRL, U.S. Geological Survey Organic Geochemistry Research Laboratory, Lawrence, Kansas; ESA, ethanesulfonic acid; OXA, oxanilic acid; SAA, sulfinyl acetic acid; --, no data; <, less than]

\begin{tabular}{|c|c|c|c|c|c|c|c|c|c|c|c|c|c|c|c|c|c|c|c|c|}
\hline Site number & Site location & $\begin{array}{l}\text { Date of col- } \\
\text { lection } \\
\text { (month/day/ } \\
\text { year) }\end{array}$ & $\begin{array}{l}\text { Aceto- } \\
\text { chlor } \\
(\mu \mathrm{g} / \mathrm{L})\end{array}$ & $\begin{array}{l}\text { Aceto- } \\
\text { chlor } \\
\text { ESA } \\
(\mu \mathrm{g} / \mathrm{L})\end{array}$ & $\begin{array}{c}\text { Aceto- } \\
\text { chlor } \\
\text { OXA } \\
(\mu \mathrm{g} / \mathrm{L})\end{array}$ & $\begin{array}{l}\text { Aceto- } \\
\text { chlor } \\
\text { SAA } \\
(\mu \mathrm{g} / \mathrm{L})\end{array}$ & $\begin{array}{l}\text { Ala- } \\
\text { chlor } \\
(\mu \mathrm{g} / \mathrm{L})\end{array}$ & $\begin{array}{l}\text { Ala- } \\
\text { chlor } \\
\text { ESA } \\
(\mu \mathrm{g} / \mathrm{L})\end{array}$ & $\begin{array}{l}\text { Ala- } \\
\text { chlor } \\
\text { OXA } \\
(\mu \mathrm{g} / \mathrm{L})\end{array}$ & $\begin{array}{l}\text { Ala- } \\
\text { chlor } \\
\text { SAA } \\
(\mu \mathrm{g} / \mathrm{L})\end{array}$ & $\begin{array}{c}\text { Di- } \\
\text { meth- } \\
\text { enamid } \\
(\mu \mathrm{g} / \mathrm{L})\end{array}$ & $\begin{array}{c}\text { Di- } \\
\text { meth- } \\
\text { enamid } \\
\text { ESA } \\
(\mu \mathrm{g} / \mathrm{L})\end{array}$ & $\begin{array}{c}\text { Di- } \\
\text { meth- } \\
\text { enamid } \\
0 X A \\
(\mu \mathrm{g} / \mathrm{L})\end{array}$ & $\begin{array}{c}\text { Flufen- } \\
\text { acet } \\
(\mu \mathrm{g} / \mathrm{L})\end{array}$ & $\begin{array}{c}\text { Flufen- } \\
\text { acet } \\
\text { ESA } \\
(\mu \mathrm{g} / \mathrm{L})\end{array}$ & $\begin{array}{l}\text { Flufen- } \\
\text { acet } \\
\text { OXA } \\
(\mu \mathrm{g} / \mathrm{L})\end{array}$ & $\begin{array}{c}\text { Meto- } \\
\text { lachlor } \\
(\mu \mathrm{g} / \mathrm{L})\end{array}$ & $\begin{array}{c}\text { Meto- } \\
\text { lachlor } \\
\text { ESA } \\
(\mu \mathrm{g} / \mathrm{L})\end{array}$ & $\begin{array}{c}\text { Meto- } \\
\text { lachlor } \\
\text { OXA } \\
(\mu \mathrm{g} / \mathrm{L})\end{array}$ & 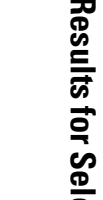 \\
\hline \multicolumn{20}{|c|}{ National Water-Quality Assessment Program (NAWQA) (fig. 3)—Continued } & \\
\hline \multicolumn{20}{|c|}{ Eastern lowa Basin (EIWA/SKI)—Continued } & \\
\hline 430525093023501 & Cerro Gordo & 07/30/97 & $<0.05$ & $<0.20$ & $<0.20$ & -- & $<0.05$ & $<0.20$ & $<0.20$ & -- & -- & -- & -- & -- & -- & -- & $<0.05$ & 3.3 & $<0.20$ & \\
\hline 430532092305401 & Chickasaw Co., IA & 07/08/96 & $<.05$ & $<.20$ & $<.20$ & -- & $<.05$ & $<.20$ & $<.20$ & -- & -- & -- & -- & -- & -- & -- & $<.05$ & $<.20$ & $<.20$ & \\
\hline 430549092272301 & Chickasaw Co., IA & $07 / 21 / 98$ & $<.05$ & $<.20$ & $<.20$ & -- & $<.05$ & $<.20$ & $<.20$ & -- & -- & -- & -- & -- & -- & -- & $<.05$ & $<.20$ & $<.20$ & $\frac{7}{0}$ \\
\hline 430549092272301 & Chickasaw Co., IA & $08 / 23 / 02$ & -- & $<.05$ & $<.05$ & $<0.05$ & -- & $<.05$ & $<.05$ & $<0.05$ & -- & $<0.05$ & $<0.05$ & -- & $<0.05$ & $<0.05$ & -- & $<.05$ & $<.05$ & D? \\
\hline 430947092590101 & Floyd Co., IA & 07/09/96 & $<.05$ & $<.20$ & $<.20$ & -- & $<.05$ & $<.20$ & $<.20$ & -- & -- & -- & -- & -- & -- & -- & $<.05$ & $<.20$ & $<.20$ & \\
\hline \multirow[t]{2}{*}{431222093313301} & Hancock Co., IA & $07 / 29 / 97$ & $<.05$ & 1.4 & $<.20$ & -- & $<.05$ & .31 & $<.20$ & -- & -- & -- & -- & -- & -- & -- & .07 & 11 & .47 & \\
\hline & & $07 / 28 / 99$ & $<.05$ & .87 & $<.20$ & -- & $<.05$ & $<.20$ & $<.20$ & -- & $<0.05$ & -- & -- & $<0.05$ & -- & -- & $<.05$ & 2.8 & $<.20$ & \\
\hline \multirow[t]{2}{*}{431339093155901} & Cerro Gordo Co., IA & 07/30/97 & $<.05$ & $<.20$ & $<.20$ & -- & $<.05$ & 1.3 & $<.20$ & -- & -- & -- & -- & -- & -- & -- & 15 & 12 & 2.5 & \\
\hline & & $08 / 21 / 02$ & -- & .48 & $<.05$ & $<.05$ & -- & .40 & $<.05$ & $<.05$ & -- & $<.05$ & $<.05$ & -- & & $<.05$ & -- & 11 & .11 & \\
\hline 431438092262201 & Howard Co., IA & 08/20/97 & $<.05$ & $<.20$ & $<.20$ & -- & $<.05$ & .39 & $<.20$ & -- & -- & -- & -- & -- & -- & -- & $<.05$ & .44 & $<.20$ & \\
\hline 431614092275401 & Howard Co., IA & $07 / 17 / 96$ & $<.05$ & $<.20$ & $<.20$ & -- & $<.05$ & $<.20$ & $<.20$ & -- & -- & -- & -- & -- & -- & -- & $<.05$ & $<.20$ & $<.20$ & \\
\hline 431725093310801 & Winnebago Co., IA & $07 / 10 / 96$ & $<.05$ & $<.20$ & $<.20$ & -- & $<.05$ & $<.20$ & $<.20$ & -- & -- & -- & -- & -- & -- & -- & $<.05$ & $<.20$ & $<.20$ & \\
\hline 432946093161901 & Worth Co., IA & 08/18/97 & $<.05$ & $<.20$ & $<.20$ & -- & $<.05$ & $<.20$ & $<.20$ & -- & -- & -- & -- & -- & -- & -- & $<.05$ & $<.20$ & $<.20$ & \\
\hline 433109093004001 & Mower Co., MN & 07/10/96 & $<.05$ & $<.20$ & $<.20$ & -- & $<.05$ & $<.20$ & $<.20$ & -- & -- & -- & -- & -- & -- & -- & $<.05$ & $<.20$ & $<.20$ & \\
\hline 433317093175601 & Freeborn Co., MN & $07 / 16 / 96$ & $<.05$ & $<.20$ & $<.20$ & -- & $<.05$ & $<.20$ & $<.20$ & -- & -- & -- & -- & -- & -- & -- & $<.05$ & $<.20$ & $<.20$ & \\
\hline 433323092383201 & Mower Co., MN & $07 / 11 / 96$ & $<.05$ & $<.20$ & $<.20$ & -- & $<.05$ & $<.20$ & $<.20$ & -- & -- & -- & -- & -- & -- & -- & $<.05$ & $<.20$ & $<.20$ & \\
\hline \multirow[t]{2}{*}{433815093000001} & Mower Co., MN & $08 / 04 / 97$ & $<.05$ & 1.2 & .63 & -- & $<.05$ & 3.8 & .43 & -- & -- & -- & -- & -- & -- & -- & .15 & 4.2 & 1.5 & \\
\hline & & $07 / 27 / 99$ & $<.05$ & 1.0 & .38 & -- & $<.05$ & 1.7 & .47 & -- & $<.05$ & -- & -- & $<.05$ & -- & -- & $<.05$ & 1.2 & .40 & \\
\hline 433855093222401 & Freeborn Co., MN & 08/06/97 & $<.05$ & $<.20$ & $<.20$ & -- & $<.05$ & $<.20$ & $<.20$ & -- & -- & -- & -- & -- & -- & -- & $<.05$ & $<.20$ & $<.20$ & \\
\hline 433944092583501 & Mower Co., MN & $08 / 05 / 97$ & $<.05$ & $<.20$ & $<.20$ & -- & $<.05$ & $<.20$ & $<.20$ & -- & -- & -- & -- & -- & -- & -- & $<.05$ & $<.20$ & $<.20$ & \\
\hline 434003092575401 & Mower Co., MN & 08/06/97 & $<.05$ & $<.20$ & $<.20$ & -- & $<.05$ & .45 & $<.20$ & -- & -- & -- & -- & -- & -- & -- & $<.05$ & $<.20$ & $<.20$ & \\
\hline 434012093243601 & Freeborn Co., MN & $07 / 16 / 96$ & $<.05$ & $<.20$ & $<.20$ & -- & $<.05$ & .92 & .62 & -- & -- & -- & -- & -- & -- & -- & $<.05$ & $<.20$ & $<.20$ & \\
\hline 434023093214201 & Freeborn Co., MN & 08/07/97 & $<.05$ & $<.20$ & $<.20$ & -- & $<.05$ & $<.20$ & $<.20$ & -- & -- & -- & -- & -- & -- & -- & $<.05$ & $<.20$ & $<.20$ & \\
\hline 434556093003501 & Mower Co., MN & $07 / 20 / 98$ & $<.05$ & $<.20$ & $<.20$ & -- & $<.05$ & $<.20$ & $<.20$ & -- & -- & -- & -- & -- & -- & -- & $<.05$ & $<.20$ & $<.20$ & \\
\hline 434732092545001 & Mower Co., MN & $07 / 15 / 96$ & $<.05$ & $<.20$ & $<.20$ & -- & $<.05$ & $<.20$ & $<.20$ & -- & -- & -- & -- & -- & -- & -- & $<.05$ & $<.20$ & $<.20$ & \\
\hline 435221093001901 & Dodge Co., MN & $08 / 05 / 97$ & $<.05$ & $<.20$ & $<.20$ & -- & $<.05$ & 7.0 & $<.20$ & -- & -- & -- & -- & -- & -- & -- & $<.05$ & .58 & $<.20$ & \\
\hline
\end{tabular}




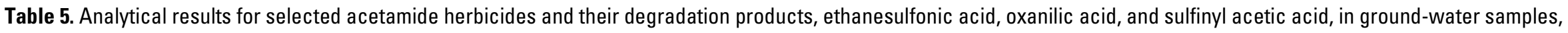
1994-2002.-Continued

[OGRL, U.S. Geological Survey Organic Geochemistry Research Laboratory, Lawrence, Kansas; ESA, ethanesulfonic acid; OXA, oxanilic acid; SAA, sulfinyl acetic acid; --, no data; <, less than]

\begin{tabular}{|c|c|c|c|c|c|c|c|c|c|c|c|c|c|c|c|c|c|c|c|}
\hline Site number & Site location & $\begin{array}{l}\text { Date of col- } \\
\text { lection } \\
\text { (month/day/ } \\
\text { year) }\end{array}$ & $\begin{array}{c}\text { Aceto- } \\
\text { chlor } \\
(\mu \mathrm{g} / \mathrm{L})\end{array}$ & $\begin{array}{c}\text { Aceto- } \\
\text { chlor } \\
\text { ESA } \\
(\mu \mathrm{g} / \mathrm{L})\end{array}$ & $\begin{array}{l}\text { Aceto- } \\
\text { chlor } \\
\text { OXA } \\
(\mu \mathrm{g} / \mathrm{L})\end{array}$ & $\begin{array}{l}\text { Aceto- } \\
\text { chlor } \\
\text { SAA } \\
(\mu \mathrm{g} / \mathrm{L})\end{array}$ & $\begin{array}{l}\text { Ala- } \\
\text { chlor } \\
(\mu \mathrm{g} / \mathrm{L})\end{array}$ & $\begin{array}{l}\text { Ala- } \\
\text { chlor } \\
\text { ESA } \\
(\mu \mathrm{g} / \mathrm{L})\end{array}$ & $\begin{array}{l}\text { Ala- } \\
\text { chlor } \\
\text { OXA } \\
(\mu \mathrm{g} / \mathrm{L})\end{array}$ & $\begin{array}{c}\text { Ala- } \\
\text { chlor } \\
\text { SAA } \\
(\mu \mathrm{g} / \mathrm{L})\end{array}$ & $\begin{array}{l}\text { Di- } \\
\text { meth- } \\
\text { enamid } \\
(\mu \mathrm{g} / \mathrm{L})\end{array}$ & $\begin{array}{c}\text { Di- } \\
\text { meth- } \\
\text { enamid } \\
\text { ESA } \\
(\mu \mathrm{g} / \mathrm{L})\end{array}$ & $\begin{array}{c}\text { Di- } \\
\text { meth- } \\
\text { enamid } \\
\text { OXA } \\
(\mu \mathrm{g} / \mathrm{L})\end{array}$ & $\begin{array}{c}\text { Flufen- } \\
\text { acet } \\
(\mu \mathrm{g} / \mathrm{L})\end{array}$ & $\begin{array}{c}\text { Flufen- } \\
\text { acet } \\
\text { ESA } \\
(\mu \mathrm{g} / \mathrm{L})\end{array}$ & $\begin{array}{c}\text { Flufen- } \\
\text { acet } \\
\text { OXA } \\
(\mu \mathrm{g} / \mathrm{L})\end{array}$ & $\begin{array}{l}\text { Meto- } \\
\text { lachlor } \\
(\mu \mathrm{g} / \mathrm{L})\end{array}$ & $\begin{array}{l}\text { Meto- } \\
\text { lachlor } \\
\text { ESA } \\
(\mu \mathrm{g} / \mathrm{L})\end{array}$ & $\begin{array}{l}\text { Meto- } \\
\text { lachlor } \\
\text { OXA } \\
(\mu \mathrm{g} / \mathrm{L})\end{array}$ \\
\hline \multicolumn{20}{|c|}{ National Water-Quality Assessment Program (NAWOA) (fig. 3)—Continued } \\
\hline \multicolumn{20}{|c|}{ Great and Little Miami River Basins (MIAM/BET/MIG) } \\
\hline 390832084133300 & Clermont Co., $\mathrm{OH}$ & 07/08/99 & -- & $<0.05$ & $<0.05$ & -- & -- & $<0.05$ & $<0.05$ & -- & -- & -- & -- & -- & -- & -- & -- & $<0.05$ & $<0.05$ \\
\hline 391444084474600 & Hamilton Co., $\mathrm{OH}$ & 07/01/99 & -- & $<.05$ & $<.05$ & -- & -- & $<.05$ & $<.05$ & -- & -- & -- & -- & -- & -- & -- & -- & $<.05$ & $<.05$ \\
\hline 391544084474200 & Hamilton Co., $\mathrm{OH}$ & 07/01/99 & -- & $<.05$ & $<.05$ & -- & -- & $<.05$ & $<.05$ & -- & -- & -- & -- & -- & -- & -- & -- & $<.05$ & $<.05$ \\
\hline 391636084452800 & Hamilton Co., $\mathrm{OH}$ & 08/03/00 & -- & $<.05$ & $<.05$ & -- & -- & $<.05$ & $<.05$ & -- & -- & $<0.05$ & $<0.05$ & -- & $<0.05$ & $<0.05$ & -- & $<.05$ & $<.05$ \\
\hline 391843084383200 & Butler Co., $\mathrm{OH}$ & 07/07/99 & -- & $<.05$ & $<.05$ & -- & -- & $<.05$ & $<.05$ & -- & -- & -- & -- & -- & -- & -- & -- & $<.05$ & $<.05$ \\
\hline 391858084364600 & Butler Co., OH & 06/08/99 & -- & .21 & .18 & -- & -- & .31 & .15 & -- & -- & -- & -- & -- & -- & -- & -- & .96 & .66 \\
\hline 392018084371800 & Clermont Co., $\mathrm{OH}$ & 08/02/00 & -- & $<.05$ & $<.05$ & -- & -- & $<.05$ & $<.05$ & -- & -- & $<.05$ & $<.05$ & -- & $<.05$ & $<.05$ & -- & $<.05$ & $<.05$ \\
\hline 392034084352700 & Butler Co., OH & 06/08/99 & -- & .44 & .37 & -- & -- & .50 & .20 & -- & -- & -- & -- & -- & -- & -- & -- & 1.3 & 1.1 \\
\hline 392134084563200 & Franklin Co., OH & 08/03/00 & -- & $<.05$ & $<.05$ & -- & -- & $<.05$ & $<.05$ & -- & -- & $<.05$ & $<.05$ & -- & $<.05$ & $<.05$ & -- & .07 & $<.05$ \\
\hline 392544084290300 & Butler Co., $\mathrm{OH}$ & 07/07/99 & -- & $<.05$ & $<.05$ & -- & -- & .11 & $<.05$ & -- & -- & -- & -- & -- & -- & -- & -- & .06 & .07 \\
\hline 392756084300900 & Butler Co., OH & 08/02/00 & -- & $<.05$ & $<.05$ & -- & -- & $<.05$ & $<.05$ & -- & -- & $<.05$ & $<.05$ & -- & $<.05$ & $<.05$ & -- & $<.05$ & $<.05$ \\
\hline 392756084330100 & Butler Co., OH & 06/07/99 & -- & $<.05$ & $<.05$ & -- & -- & $<.05$ & $<.05$ & -- & -- & -- & -- & -- & -- & -- & -- & $<.05$ & $<.05$ \\
\hline 392944084343000 & Butler Co., OH & 06/02/99 & -- & $<.05$ & $<.05$ & -- & -- & $<.05$ & $<.05$ & -- & -- & -- & -- & -- & -- & -- & -- & $<.05$ & $<.05$ \\
\hline 393206084240800 & Butler Co., OH & 06/23/99 & -- & .06 & $<.05$ & -- & -- & .05 & $<.05$ & -- & -- & -- & -- & -- & -- & -- & -- & .41 & .11 \\
\hline 393254084205500 & Warren Co., OH & 05/18/99 & -- & $<.05$ & $<.05$ & -- & -- & $<.05$ & .06 & -- & -- & -- & -- & -- & -- & -- & -- & .91 & $<.05$ \\
\hline 393330084204500 & Warren Co., OH & 06/23/99 & -- & $<.05$ & $<.05$ & -- & -- & $<.05$ & $<.05$ & -- & -- & -- & -- & -- & -- & -- & -- & .05 & $<.05$ \\
\hline 393421084003300 & Greene Co., OH & $07 / 26 / 00$ & -- & $<.05$ & $<.05$ & -- & -- & $<.05$ & $<.05$ & -- & -- & $<.05$ & $<.05$ & -- & $<.05$ & $<.05$ & -- & $<.05$ & $<.05$ \\
\hline 393421084003301 & Greene Co., OH & $07 / 26 / 00$ & -- & $<.05$ & $<.05$ & -- & -- & $<.05$ & $<.05$ & -- & -- & $<.05$ & $<.05$ & -- & $<.05$ & $<.05$ & -- & .13 & $<.05$ \\
\hline 393455084180600 & Warren Co., OH & 07/08/99 & -- & $<.05$ & $<.05$ & -- & -- & $<.05$ & $<.05$ & -- & -- & -- & -- & -- & -- & -- & -- & $<.05$ & $<.05$ \\
\hline 393505084173100 & Warren Co., OH & 05/18/99 & -- & $<.05$ & $<.05$ & -- & -- & .06 & .22 & -- & -- & -- & -- & -- & -- & -- & -- & .91 & $<.05$ \\
\hline 393512084594500 & Union Co., IN & 07/09/99 & -- & $<.05$ & $<.05$ & -- & -- & $<.05$ & $<.05$ & -- & -- & -- & -- & -- & -- & -- & -- & $<.05$ & $<.05$ \\
\hline 393605084013400 & Union Co., IN & $06 / 22 / 99$ & -- & $<.05$ & $<.05$ & -- & -- & $<.05$ & $<.05$ & -- & -- & -- & -- & -- & -- & -- & -- & $<.05$ & $<.05$ \\
\hline 393702084224200 & Montgomery Co., OH & 06/09/99 & -- & $<.05$ & $<.05$ & -- & -- & .12 & $<.05$ & -- & -- & -- & -- & -- & -- & -- & -- & .33 & $<.05$ \\
\hline 393815084174100 & Montgomery Co., $\mathrm{OH}$ & $05 / 26 / 99$ & -- & $<.05$ & $<.05$ & -- & -- & .20 & $<.05$ & -- & -- & -- & -- & -- & -- & -- & -- & .52 & .32 \\
\hline 393831085043500 & Fayette Co., IN & $08 / 09 / 00$ & -- & $<.05$ & $<.05$ & -- & -- & $<.05$ & $<.05$ & -- & -- & $<.05$ & $<.05$ & -- & $<.05$ & $<.05$ & -- & $<.05$ & $<.05$ \\
\hline
\end{tabular}




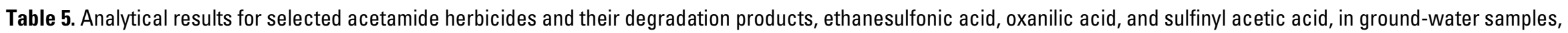
1994-2002.-Continued

[OGRL, U.S. Geological Survey Organic Geochemistry Research Laboratory, Lawrence, Kansas; ESA, ethanesulfonic acid; OXA, oxanilic acid; SAA, sulfinyl acetic acid; --, no data; <, less than]

\begin{tabular}{|c|c|c|c|c|c|c|c|c|c|c|c|c|c|c|c|c|c|c|c|c|}
\hline Site number & Site location & $\begin{array}{l}\text { Date of col- } \\
\text { lection } \\
\text { (month/day/ } \\
\text { year) }\end{array}$ & $\begin{array}{l}\text { Aceto- } \\
\text { chlor } \\
(\mu \mathrm{g} / \mathrm{L})\end{array}$ & $\begin{array}{l}\text { Aceto- } \\
\text { chlor } \\
\text { ESA } \\
(\mu \mathrm{g} / \mathrm{L})\end{array}$ & $\begin{array}{l}\text { Aceto- } \\
\text { chlor } \\
\text { OXA } \\
(\mu \mathrm{g} / \mathrm{L})\end{array}$ & $\begin{array}{l}\text { Aceto- } \\
\text { chlor } \\
\text { SAA } \\
(\mu \mathrm{g} / \mathrm{L})\end{array}$ & $\begin{array}{l}\text { Ala- } \\
\text { chlor } \\
(\mu \mathrm{g} / \mathrm{L})\end{array}$ & $\begin{array}{l}\text { Ala- } \\
\text { chlor } \\
\text { ESA } \\
(\mu \mathrm{g} / \mathrm{L})\end{array}$ & $\begin{array}{c}\text { Ala- } \\
\text { chlor } \\
\text { OXA } \\
(\mu \mathrm{g} / \mathrm{L})\end{array}$ & $\begin{array}{l}\text { Ala- } \\
\text { chlor } \\
\text { SAA } \\
(\mu \mathrm{g} / \mathrm{L})\end{array}$ & $\begin{array}{c}\text { Di- } \\
\text { meth- } \\
\text { enamid } \\
(\mu \mathrm{g} / \mathrm{L})\end{array}$ & $\begin{array}{c}\text { Di- } \\
\text { meth- } \\
\text { enamid } \\
\text { ESA } \\
(\mu \mathrm{g} / \mathrm{L})\end{array}$ & $\begin{array}{c}\text { Di- } \\
\text { meth- } \\
\text { enamid } \\
\text { OXA } \\
(\mu \mathrm{g} / \mathrm{L})\end{array}$ & $\begin{array}{c}\text { Flufen- } \\
\text { acet } \\
(\mu \mathrm{g} / \mathrm{L})\end{array}$ & $\begin{array}{c}\text { Flufen- } \\
\text { acet } \\
\text { ESA } \\
(\mu \mathrm{g} / \mathrm{L})\end{array}$ & $\begin{array}{c}\text { Flufen- } \\
\text { acet } \\
\text { OXA } \\
(\mu \mathrm{g} / \mathrm{L})\end{array}$ & $\begin{array}{l}\text { Meto- } \\
\text { lachlor } \\
(\mu \mathrm{g} / \mathrm{L})\end{array}$ & $\begin{array}{l}\text { Meto- } \\
\text { lachlor } \\
\text { ESA } \\
(\mu \mathrm{g} / \mathrm{L})\end{array}$ & $\begin{array}{c}\text { Meto- } \\
\text { lachlor } \\
\text { OXA } \\
(\mu \mathrm{g} / \mathrm{L})\end{array}$ & 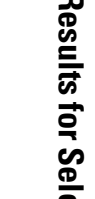 \\
\hline \multicolumn{20}{|c|}{ National Water-Quality Assessment Program (NAWOA) (fig. 3)—Continued } & \\
\hline \multicolumn{20}{|c|}{ Great and Little Miami River Basins (MIAM/BET/MIG)—Continued } & \\
\hline 394016084411300 & Preble Co., $\mathrm{OH}$ & 06/01/99 & -- & 0.58 & 0.96 & -- & -- & 0.18 & 0.35 & -- & -- & -- & -- & -- & -- & -- & -- & 0.92 & 0.44 & \\
\hline 394233083576000 & Greene Co., OH & $07 / 26 / 00$ & -- & $<.05$ & $<.05$ & -- & -- & $<.05$ & $<.05$ & -- & -- & $<0.05$ & $<0.05$ & -- & $<0.05$ & $<0.05$ & -- & .05 & $<.05$ & \\
\hline 394254084003000 & Greene Co., OH & 05/27/99 & -- & $<.05$ & $<.05$ & -- & -- & $<.05$ & $<.05$ & -- & -- & -- & -- & -- & -- & -- & -- & $<.05$ & $<.05$ & \\
\hline 394302084032700 & Greene Co., OH & 05/27/99 & -- & $<.05$ & $<.05$ & -- & -- & $<.05$ & $<.05$ & -- & -- & -- & -- & -- & -- & -- & -- & $<.05$ & $<.05$ & \\
\hline 394312084002600 & Greene Co., $\mathrm{OH}$ & 05/19/99 & -- & $<.05$ & $<.05$ & -- & -- & $<.05$ & $<.05$ & -- & -- & -- & -- & -- & -- & -- & -- & $<.05$ & $<.05$ & \\
\hline 394420084463001 & Preble Co., $\mathrm{OH}$ & 08/16/00 & -- & $<.05$ & $<.05$ & -- & -- & $<.05$ & $<.05$ & -- & -- & $<.05$ & $<.05$ & -- & $<.05$ & $<.05$ & -- & .06 & $<.05$ & \\
\hline 394650084320300 & Preble Co., OH & 07/17/00 & -- & .05 & .60 & -- & -- & $<.05$ & $<.05$ & -- & -- & $<.05$ & $<.05$ & -- & $<.05$ & $<.05$ & -- & .18 & .79 & \\
\hline 394654084160800 & Montgomery Co., OH & 05/20/99 & -- & $<.05$ & $<.05$ & -- & -- & $<.05$ & $<.05$ & -- & -- & -- & -- & -- & -- & -- & -- & .43 & $<.05$ & \\
\hline 394745085051300 & Wayne Co., IN & $08 / 25 / 00$ & -- & $<.05$ & $<.05$ & -- & -- & $<.05$ & $<.05$ & -- & -- & $<.05$ & $<.05$ & -- & $<.05$ & $<.05$ & -- & $<.05$ & $<.05$ & \\
\hline 394759085071000 & Wayne Co., IN & 08/10/00 & -- & $<.05$ & $<.05$ & -- & -- & 2.1 & 1.2 & -- & -- & $<.05$ & $<.05$ & -- & $<.05$ & $<.05$ & -- & .10 & .09 & \\
\hline 394759085071001 & Wayne Co., IN & 08/10/00 & -- & $<.05$ & $<.05$ & -- & -- & $<.05$ & $<.05$ & -- & -- & $<.05$ & $<.05$ & -- & $<.05$ & $<.05$ & -- & $<.05$ & $<.05$ & \\
\hline 394918084100100 & Montgomery Co., $\mathrm{OH}$ & 06/22/99 & -- & $<.05$ & $<.05$ & -- & -- & .37 & $<.05$ & -- & -- & -- & -- & -- & -- & -- & -- & .60 & .20 & D \\
\hline 395102084020900 & Clark Co., OH & 05/19/99 & -- & $<.05$ & $<.05$ & -- & -- & .20 & $<.05$ & -- & -- & -- & -- & -- & -- & -- & -- & 1.2 & $<.05$ & \\
\hline 395125084154800 & Montgomery Co., OH & 05/20/99 & -- & $<.05$ & $<.05$ & -- & -- & $<.05$ & $<.05$ & -- & -- & -- & -- & -- & -- & -- & -- & $<.05$ & $<.05$ & \\
\hline 395127083551300 & Clark Co., OH & 06/10/99 & -- & $<.05$ & $<.05$ & -- & -- & .32 & $<.05$ & -- & -- & -- & -- & -- & -- & -- & -- & .07 & .06 & \\
\hline 395134084160700 & Clark Co., $\mathrm{OH}$ & 05/26/99 & -- & $<.05$ & $<.05$ & -- & -- & .48 & $<.05$ & -- & -- & -- & -- & -- & -- & -- & -- & .52 & .40 & \\
\hline 395135085070000 & Wayne Co., IN & 08/09/00 & -- & $<.05$ & $<.05$ & -- & -- & .09 & $<.05$ & -- & -- & $<.05$ & $<.05$ & -- & $<.05$ & $<.05$ & -- & .24 & $<.05$ & \\
\hline 395135085070001 & Wayne Co., IN & $08 / 15 / 00$ & -- & $<.05$ & $<.05$ & -- & -- & $<.05$ & $<.05$ & -- & -- & $<.05$ & $<.05$ & -- & $<.05$ & $<.05$ & -- & $<.05$ & $<.05$ & \\
\hline 395213085052200 & Wayne Co., IN & 08/24/00 & -- & .06 & $<.05$ & -- & -- & $<.05$ & $<.05$ & -- & -- & $<.05$ & $<.05$ & -- & $<.05$ & $<.05$ & -- & 1.3 & .40 & \\
\hline 395149084592900 & Wayne Co., IN & 06/03/99 & -- & $<.05$ & $<.05$ & -- & -- & $<.05$ & $<.05$ & -- & -- & -- & -- & -- & -- & -- & -- & $<.05$ & $<.05$ & \\
\hline 395235083445700 & Clark Co., OH & $06 / 24 / 99$ & -- & $<.05$ & $<.05$ & -- & -- & .08 & $<.05$ & -- & -- & -- & -- & -- & -- & -- & -- & .16 & $<.05$ & \\
\hline 395248084010900 & Clark Co., $\mathrm{OH}$ & 06/29/99 & -- & $<.05$ & $<.05$ & -- & -- & .07 & $<.05$ & -- & -- & -- & -- & -- & -- & -- & -- & $<.05$ & $<.05$ & \\
\hline 395248084491600 & Wayne Co., IN & 08/16/00 & -- & $<.05$ & $<.05$ & -- & -- & $<.05$ & $<.05$ & -- & -- & $<.05$ & $<.05$ & -- & $<.05$ & $<.05$ & -- & .07 & $<.05$ & \\
\hline 395407083553400 & Greene Co., OH & 06/10/99 & -- & $<.05$ & $<.05$ & -- & -- & .06 & $<.05$ & -- & -- & -- & -- & -- & -- & -- & -- & $<.05$ & .22 & \\
\hline 395528083414400 & Clark Co., $\mathrm{OH}$ & 05/13/99 & & $<.05$ & $<.05$ & -- & & $<.05$ & $<.05$ & -- & -- & -- & -- & -- & -- & -- & & $<.05$ & $<.05$ & \\
\hline
\end{tabular}




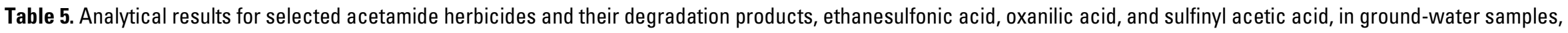
1994-2002.-Continued

[OGRL, U.S. Geological Survey Organic Geochemistry Research Laboratory, Lawrence, Kansas; ESA, ethanesulfonic acid; OXA, oxanilic acid; SAA, sulfinyl acetic acid; --, no data; <, less than]

\begin{tabular}{|c|c|c|c|c|c|c|c|c|c|c|c|c|c|c|c|c|c|c|c|}
\hline Site number & Site location & $\begin{array}{l}\text { Date of col- } \\
\text { lection } \\
\text { (month/day/ } \\
\text { year) }\end{array}$ & $\begin{array}{l}\text { Aceto- } \\
\text { chlor } \\
(\mu \mathrm{g} / \mathrm{L})\end{array}$ & $\begin{array}{l}\text { Aceto- } \\
\text { chlor } \\
\text { ESA } \\
(\mu \mathrm{g} / \mathrm{L})\end{array}$ & $\begin{array}{l}\text { Aceto- } \\
\text { chlor } \\
\text { OXA } \\
(\mu \mathrm{g} / \mathrm{L})\end{array}$ & $\begin{array}{l}\text { Aceto- } \\
\text { chlor } \\
\text { SAA } \\
(\mu \mathrm{g} / \mathrm{L})\end{array}$ & $\begin{array}{l}\text { Ala- } \\
\text { chlor } \\
(\mu \mathrm{g} / \mathrm{L})\end{array}$ & $\begin{array}{l}\text { Ala- } \\
\text { chlor } \\
\text { ESA } \\
(\mu \mathrm{g} / \mathrm{L})\end{array}$ & $\begin{array}{l}\text { Ala- } \\
\text { chlor } \\
\text { OXA } \\
(\mu \mathrm{g} / \mathrm{L})\end{array}$ & $\begin{array}{l}\text { Ala- } \\
\text { chlor } \\
\text { SAA } \\
(\mu \mathrm{g} / \mathrm{L})\end{array}$ & $\begin{array}{l}\text { Di- } \\
\text { meth- } \\
\text { enamid } \\
(\mu \mathrm{g} / \mathrm{L})\end{array}$ & $\begin{array}{c}\text { Di- } \\
\text { meth- } \\
\text { enamid } \\
\text { ESA } \\
(\mu \mathrm{g} / \mathrm{L})\end{array}$ & $\begin{array}{c}\text { Di- } \\
\text { meth- } \\
\text { enamid } \\
\text { OXA } \\
(\mu \mathrm{g} / \mathrm{L})\end{array}$ & $\begin{array}{c}\text { Flufen- } \\
\text { acet } \\
(\mu \mathrm{g} / \mathrm{L})\end{array}$ & $\begin{array}{c}\text { Flufen- } \\
\text { acet } \\
\text { ESA } \\
(\mu \mathrm{g} / \mathrm{L})\end{array}$ & $\begin{array}{c}\text { Flufen- } \\
\text { acet } \\
\text { OXA } \\
(\mu \mathrm{g} / \mathrm{L})\end{array}$ & $\begin{array}{l}\text { Meto- } \\
\text { lachlor } \\
(\mu \mathrm{g} / \mathrm{L})\end{array}$ & $\begin{array}{l}\text { Meto- } \\
\text { lachlor } \\
\text { ESA } \\
(\mu \mathrm{g} / \mathrm{L})\end{array}$ & $\begin{array}{c}\text { Meto- } \\
\text { lachlor } \\
\text { OXA } \\
(\mu \mathrm{g} / \mathrm{L})\end{array}$ \\
\hline \multicolumn{20}{|c|}{ National Water-Quality Assessment Program (NAWOA) (fig. 3)-Continued } \\
\hline \multicolumn{20}{|c|}{ Great and Little Miami River Basins (MIAM/BET/MIG)—Continued } \\
\hline 395635083445900 & Clark Co., OH & $06 / 24 / 99$ & -- & $<0.05$ & $<0.05$ & -- & -- & 0.06 & $<0.05$ & -- & -- & -- & -- & -- & -- & -- & -- & 0.16 & $<0.05$ \\
\hline 395706084035400 & Miami Co., OH & $06 / 28 / 99$ & -- & $<.05$ & $<.05$ & -- & -- & $<.05$ & $<.05$ & -- & -- & -- & -- & -- & -- & -- & -- & $<.05$ & $<.05$ \\
\hline 395734084085800 & Miami Co., OH & $05 / 25 / 99$ & -- & $<.05$ & $<.05$ & -- & -- & .52 & $<.05$ & -- & -- & -- & -- & -- & -- & -- & -- & .37 & .16 \\
\hline 395839083491500 & Clark Co., IN & 05/25/99 & -- & $<.05$ & $<.05$ & -- & -- & .51 & $<.05$ & -- & -- & -- & -- & -- & -- & -- & -- & .55 & .17 \\
\hline 395849083494501 & Clark Co., $\mathrm{OH}$ & $07 / 12 / 00$ & -- & $<.05$ & $<.05$ & -- & -- & .06 & $<.05$ & -- & -- & $<0.05$ & $<0.05$ & -- & $<0.05$ & $<0.05$ & -- & .33 & $<.05$ \\
\hline 395911084422700 & Darke Co., OH & 07/24/00 & -- & $<.05$ & $<.05$ & -- & -- & $<.05$ & $<.05$ & -- & -- & $<.05$ & $<.05$ & -- & $<.05$ & $<.05$ & -- & $<.05$ & $<.05$ \\
\hline 400019083412000 & Clark Co., $\mathrm{OH}$ & $07 / 19 / 00$ & -- & $<.05$ & $<.05$ & -- & -- & $<.05$ & $<.05$ & -- & -- & $<.05$ & $<.05$ & -- & $<.05$ & $<.05$ & -- & 1.3 & .24 \\
\hline 400049084125300 & Miami Co., $\mathrm{OH}$ & 06/23/99 & -- & $<.05$ & $<.05$ & -- & -- & .09 & $<.05$ & -- & -- & -- & -- & -- & -- & -- & -- & $<.05$ & $<.05$ \\
\hline 400113085051300 & Randolph Co., IN & 06/03/99 & -- & $<.05$ & $<.05$ & -- & -- & $<.05$ & $<.05$ & -- & -- & -- & -- & -- & -- & -- & -- & $<.05$ & $<.05$ \\
\hline 400142083423900 & Champaign Co., $\mathrm{OH}$ & $05 / 12 / 99$ & -- & $<.05$ & $<.05$ & -- & -- & $<.05$ & $<.05$ & -- & -- & -- & -- & -- & -- & -- & -- & .39 & $<.05$ \\
\hline 400145084106000 & Miami Co., OH & 08/01/00 & -- & $<.05$ & $<.05$ & -- & -- & .08 & $<.05$ & -- & -- & $<.05$ & $<.05$ & -- & $<.05$ & $<.05$ & -- & .84 & $<.05$ \\
\hline 400319084213100 & Miami Co., $\mathrm{OH}$ & 06/09/99 & -- & $<.05$ & $<.05$ & -- & -- & $<.05$ & $<.05$ & -- & -- & -- & -- & -- & -- & -- & -- & $<.05$ & $<.05$ \\
\hline 400320084402400 & Darke Co., $\mathrm{OH}$ & 08/08/00 & -- & $<.05$ & $<.05$ & -- & -- & $<.05$ & $<.05$ & -- & -- & $<.05$ & $<.05$ & -- & $<.05$ & $<.05$ & -- & $<.05$ & $<.05$ \\
\hline 400409083464500 & Champaign Co., OH & $06 / 29 / 99$ & -- & $<.05$ & $<.05$ & -- & -- & .16 & $<.05$ & -- & -- & -- & -- & -- & -- & -- & -- & $<.05$ & $<.05$ \\
\hline 400542083420900 & Champaign Co., $\mathrm{OH}$ & 06/30/99 & -- & $<.05$ & $<.05$ & -- & -- & $<.05$ & $<.05$ & -- & -- & -- & -- & -- & -- & -- & -- & $<.05$ & $<.05$ \\
\hline 400850083404700 & Champaign Co., OH & 07/13/00 & -- & $<.05$ & $<.05$ & -- & -- & $<.05$ & $<.05$ & -- & -- & $<.05$ & $<.05$ & -- & $<.05$ & $<.05$ & -- & $<.05$ & $<.05$ \\
\hline 400850083404701 & Champaign Co., OH & 07/13/00 & -- & $<.05$ & $<.05$ & -- & -- & $<.05$ & $<.05$ & -- & -- & $<.05$ & $<.05$ & -- & $<.05$ & $<.05$ & -- & $<.05$ & $<.05$ \\
\hline 401040084154000 & Miami Co., OH & 07/28/00 & -- & $<.05$ & $<.05$ & -- & -- & $<.05$ & $<.05$ & -- & -- & $<.05$ & $<.05$ & -- & $<.05$ & $<.05$ & -- & $<.05$ & $<.05$ \\
\hline 401200083443100 & Champaign Co., $\mathrm{OH}$ & 06/30/99 & -- & $<.05$ & $<.05$ & -- & -- & $<.05$ & $<.05$ & -- & -- & -- & -- & -- & -- & -- & -- & $<.05$ & $<.05$ \\
\hline 401237083485800 & Champaign Co., $\mathrm{OH}$ & 06/30/99 & -- & $<.05$ & $<.05$ & -- & -- & $<.05$ & $<.05$ & -- & -- & -- & -- & -- & -- & -- & -- & $<.05$ & $<.05$ \\
\hline 401238084144400 & Shelby Co., OH & 07/27/00 & -- & $<.05$ & $<.05$ & -- & -- & $<.05$ & $<.05$ & -- & -- & $<.05$ & $<.05$ & -- & $<.05$ & $<.05$ & -- & .05 & $<.05$ \\
\hline 401238084144401 & Shelby Co., OH & 07/27/00 & -- & $<.05$ & $<.05$ & -- & -- & $<.05$ & $<.05$ & -- & -- & $<.05$ & $<.05$ & -- & $<.05$ & $<.05$ & -- & $<.05$ & $<.05$ \\
\hline 401240083492600 & Champaign Co., OH & $07 / 19 / 00$ & -- & $<.05$ & $<.05$ & -- & -- & $<.05$ & $<.05$ & -- & -- & $<.05$ & $<.05$ & -- & $<.05$ & $<.05$ & -- & $<.05$ & $<.05$ \\
\hline 401240083492601 & Champaign Co., $\mathrm{OH}$ & 07/19/00 & -- & $<.05$ & $<.05$ & -- & -- & $<.05$ & $<.05$ & -- & -- & $<.05$ & $<.05$ & -- & $<.05$ & $<.05$ & -- & $<.05$ & $<.05$ \\
\hline 401307083450600 & Champaign Co., OH & $07 / 18 / 00$ & -- & $<.05$ & .06 & -- & -- & 3.6 & .06 & -- & -- & $<.05$ & $<.05$ & -- & $<.05$ & $<.05$ & -- & .21 & .10 \\
\hline
\end{tabular}




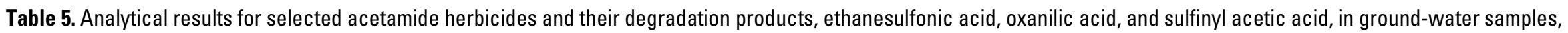
1994-2002.-Continued

[OGRL, U.S. Geological Survey Organic Geochemistry Research Laboratory, Lawrence, Kansas; ESA, ethanesulfonic acid; OXA, oxanilic acid; SAA, sulfinyl acetic acid; --, no data; <, less than]

\begin{tabular}{|c|c|c|c|c|c|c|c|c|c|c|c|c|c|c|c|c|c|c|c|}
\hline Site number & Site location & $\begin{array}{l}\text { Date of col- } \\
\text { lection } \\
\text { (month/day/ } \\
\text { year) }\end{array}$ & $\begin{array}{l}\text { Aceto- } \\
\text { chlor } \\
(\mu \mathrm{g} / \mathrm{L})\end{array}$ & $\begin{array}{c}\text { Aceto- } \\
\text { chlor } \\
\text { ESA } \\
(\mu \mathrm{g} / \mathrm{L})\end{array}$ & $\begin{array}{l}\text { Aceto- } \\
\text { chlor } \\
\text { OXA } \\
(\mu \mathrm{g} / \mathrm{L})\end{array}$ & $\begin{array}{l}\text { Aceto- } \\
\text { chlor } \\
\text { SAA } \\
(\mu \mathrm{g} / \mathrm{L})\end{array}$ & $\begin{array}{l}\text { Ala- } \\
\text { chlor } \\
(\mu \mathrm{g} / \mathrm{L})\end{array}$ & $\begin{array}{l}\text { Ala- } \\
\text { chlor } \\
\text { ESA } \\
(\mu \mathrm{g} / \mathrm{L})\end{array}$ & $\begin{array}{c}\text { Ala- } \\
\text { chlor } \\
\text { OXA } \\
(\mu \mathrm{g} / \mathrm{L})\end{array}$ & $\begin{array}{l}\text { Ala- } \\
\text { chlor } \\
\text { SAA } \\
(\mu \mathrm{g} / \mathrm{L})\end{array}$ & $\begin{array}{l}\text { Di- } \\
\text { meth- } \\
\text { enamid } \\
(\mu \mathrm{g} / \mathrm{L})\end{array}$ & $\begin{array}{c}\text { Di- } \\
\text { meth- } \\
\text { enamid } \\
\text { ESA } \\
(\mu \mathrm{g} / \mathrm{L})\end{array}$ & $\begin{array}{c}\text { Di- } \\
\text { meth- } \\
\text { enamid } \\
0 X A \\
(\mu \mathrm{g} / \mathrm{L})\end{array}$ & $\begin{array}{c}\text { Flufen- } \\
\text { acet } \\
(\mu \mathrm{g} / \mathrm{L})\end{array}$ & $\begin{array}{c}\text { Flufen- } \\
\text { acet } \\
\text { ESA } \\
(\mu \mathrm{g} / \mathrm{L})\end{array}$ & $\begin{array}{c}\text { Flufen- } \\
\text { acet } \\
\text { OXA } \\
(\mu \mathrm{g} / \mathrm{L})\end{array}$ & $\begin{array}{l}\text { Meto- } \\
\text { lachlor } \\
(\mu \mathrm{g} / \mathrm{L})\end{array}$ & $\begin{array}{c}\text { Meto- } \\
\text { lachlor } \\
\text { ESA } \\
(\mu \mathrm{g} / \mathrm{L})\end{array}$ & $\begin{array}{c}\text { Meto- } \\
\text { lachlor } \\
\text { OXA } \\
(\mu \mathrm{g} / \mathrm{L})\end{array}$ \\
\hline \multicolumn{20}{|c|}{ National Water-Quality Assessment Program (NAWOA) (fig. 3)—Continued } \\
\hline \multicolumn{20}{|c|}{ Great and Little Miami River Basins (MIAM/BET/MIG)—Continued } \\
\hline 401307083450601 & Champaign Co., $\mathrm{OH}$ & 07/18/00 & -- & $<0.05$ & 0.09 & -- & -- & 3.3 & .12 & -- & -- & $<0.05$ & $<0.05$ & -- & $<0.05$ & $<0.05$ & -- & $<0.05$ & $<0.05$ \\
\hline 401359083493100 & Champaign Co., $\mathrm{OH}$ & $07 / 25 / 00$ & -- & $<.05$ & $<.05$ & -- & -- & $<.05$ & $<.05$ & -- & -- & $<.05$ & $<.05$ & -- & $<.05$ & $<.05$ & -- & .06 & $<.05$ \\
\hline 401359083493101 & Champaign Co., OH & $07 / 25 / 00$ & -- & $<.05$ & $<.05$ & -- & -- & $<.05$ & $<.05$ & -- & -- & $<.05$ & $<.05$ & -- & $<.05$ & $<.05$ & -- & .07 & $<.05$ \\
\hline 401837083492900 & Logan Co., $\mathrm{OH}$ & 07/20/00 & -- & $<.05$ & $<.05$ & -- & -- & $<.05$ & $<.05$ & -- & -- & $<.05$ & $<.05$ & -- & $<.05$ & $<.05$ & -- & $<.05$ & $<.05$ \\
\hline \multicolumn{20}{|c|}{ High Plains Regional Ground Water Study (HPGW/HPC) } \\
\hline 344800101324901 & Armstrong Co., TX & 07/14/99 & -- & $<.05$ & $<.05$ & -- & -- & $<.05$ & $<.05$ & -- & -- & -- & -- & -- & -- & -- & -- & $<.05$ & $<.05$ \\
\hline 345826100580501 & Donley Co., TX & 06/28/99 & -- & $<.05$ & $<.05$ & -- & -- & $<.05$ & $<.05$ & -- & -- & -- & -- & -- & -- & -- & -- & $<.05$ & $<.05$ \\
\hline 351656101161101 & Carson Co., TX & 05/19/99 & -- & $<.05$ & $<.05$ & -- & -- & $<.05$ & $<.05$ & -- & -- & -- & -- & -- & -- & -- & -- & $<.05$ & $<.05$ \\
\hline 351914101204401 & Carson Co., TX & 07/01/99 & -- & $<.05$ & $<.05$ & -- & -- & $<.05$ & $<.05$ & -- & -- & -- & -- & -- & -- & -- & -- & $<.05$ & $<.05$ \\
\hline 352035100293301 & Wheeler Co., TX & 07/13/99 & -- & $<.05$ & $<.05$ & -- & -- & $<.05$ & $<.05$ & -- & -- & -- & -- & -- & -- & -- & -- & $<.05$ & $<.05$ \\
\hline 352249100425401 & Gray Co., TX & 06/30/99 & -- & $<.05$ & $<.05$ & -- & -- & $<.05$ & $<.05$ & -- & -- & -- & -- & -- & -- & -- & -- & $<.05$ & $<.05$ \\
\hline 352513101372101 & Potter Co., TX & 05/20/99 & -- & $<.05$ & $<.05$ & -- & -- & $<.05$ & $<.05$ & -- & -- & -- & -- & -- & -- & -- & -- & $<.05$ & $<.05$ \\
\hline 352909101084001 & Carson Co., TX & 07/01/99 & -- & $<.05$ & $<.05$ & -- & -- & $<.05$ & $<.05$ & -- & -- & -- & -- & -- & -- & -- & -- & $<.05$ & $<.05$ \\
\hline 353110100550401 & Gray Co., TX & 06/29/99 & -- & $<.05$ & $<.05$ & -- & -- & $<.05$ & $<.05$ & -- & -- & -- & -- & -- & -- & -- & -- & $<.05$ & $<.05$ \\
\hline 353941102350201 & Hartley Co., TX & 07/15/99 & -- & $<.05$ & $<.05$ & -- & -- & $<.05$ & $<.05$ & -- & -- & -- & -- & -- & -- & -- & -- & $<.05$ & $<.05$ \\
\hline 354248100501501 & Dallam Co., TX & 07/19/99 & -- & $<.05$ & $<.05$ & -- & -- & $<.05$ & $<.05$ & -- & -- & -- & -- & -- & -- & -- & -- & $<.05$ & $<.05$ \\
\hline 354331102183601 & Hartley Co., TX & 06/23/99 & -- & $<.05$ & $<.05$ & -- & -- & $<.05$ & $<.05$ & -- & -- & -- & -- & -- & -- & -- & -- & $<.05$ & $<.05$ \\
\hline 354348102543601 & Hartley Co., TX & 07/21/99 & -- & $<.05$ & $<.05$ & -- & -- & $<.05$ & $<.05$ & -- & -- & -- & -- & -- & -- & -- & -- & $<.05$ & $<.05$ \\
\hline 354656099480301 & Roger Mills Co., OK & 05/17/99 & -- & $<.05$ & $<.05$ & -- & -- & $<.05$ & $<.05$ & -- & -- & -- & -- & -- & -- & -- & -- & $<.05$ & $<.05$ \\
\hline 354728102295901 & Hartley Co., TX & 07/16/99 & -- & $<.05$ & $<.05$ & -- & -- & $<.05$ & $<.05$ & -- & -- & -- & -- & -- & -- & -- & -- & $<.05$ & $<.05$ \\
\hline 354918102020901 & Moore Co., TX & $06 / 23 / 99$ & -- & $<.05$ & $<.05$ & -- & -- & $<.05$ & $<.05$ & -- & -- & -- & -- & -- & -- & -- & -- & $<.05$ & $<.05$ \\
\hline 355701100313001 & Hamphill Co., TX & 05/18/99 & -- & $<.05$ & $<.05$ & -- & -- & $<.05$ & $<.05$ & -- & -- & -- & -- & -- & -- & -- & -- & $<.05$ & $<.05$ \\
\hline 355852101274101 & Hutchinson Co., TX & 07/02/99 & -- & $<.05$ & $<.05$ & -- & -- & $<.05$ & $<.05$ & -- & -- & -- & -- & -- & -- & -- & -- & $<.05$ & $<.05$ \\
\hline 355916103215201 & Union Co., NM & $06 / 25 / 99$ & -- & $<.05$ & $<.05$ & -- & -- & $<.05$ & $<.05$ & -- & -- & -- & -- & -- & -- & -- & -- & $<.05$ & $<.05$ \\
\hline 360159103044401 & Union Co., NM & $09 / 11 / 00$ & -- & $<.05$ & $<.05$ & -- & -- & $<.05$ & $<.05$ & -- & -- & $<.05$ & $<.05$ & -- & $<.05$ & $<.05$ & -- & $<.05$ & $<.05$ \\
\hline
\end{tabular}




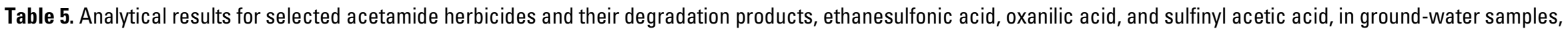
1994-2002.-Continued

[OGRL, U.S. Geological Survey Organic Geochemistry Research Laboratory, Lawrence, Kansas; ESA, ethanesulfonic acid; OXA, oxanilic acid; SAA, sulfinyl acetic acid; --, no data; <, less than]

\begin{tabular}{|c|c|c|c|c|c|c|c|c|c|c|c|c|c|c|c|c|c|c|c|}
\hline Site number & Site location & $\begin{array}{l}\text { Date of col- } \\
\text { lection } \\
\text { (month/day/ } \\
\text { year) }\end{array}$ & $\begin{array}{l}\text { Aceto- } \\
\text { chlor } \\
(\mu \mathrm{g} / \mathrm{L})\end{array}$ & $\begin{array}{l}\text { Aceto- } \\
\text { chlor } \\
\text { ESA } \\
(\mu \mathrm{g} / \mathrm{L})\end{array}$ & $\begin{array}{l}\text { Aceto- } \\
\text { chlor } \\
\text { OXA } \\
(\mu \mathrm{g} / \mathrm{L})\end{array}$ & $\begin{array}{l}\text { Aceto- } \\
\text { chlor } \\
\text { SAA } \\
(\mu \mathrm{g} / \mathrm{L})\end{array}$ & $\begin{array}{l}\text { Ala- } \\
\text { chlor } \\
(\mu \mathrm{g} / \mathrm{L})\end{array}$ & $\begin{array}{l}\text { Ala- } \\
\text { chlor } \\
\text { ESA } \\
(\mu \mathrm{g} / \mathrm{L})\end{array}$ & $\begin{array}{l}\text { Ala- } \\
\text { chlor } \\
\text { OXA } \\
(\mu \mathrm{g} / \mathrm{L})\end{array}$ & $\begin{array}{l}\text { Ala- } \\
\text { chlor } \\
\text { SAA } \\
(\mu \mathrm{g} / \mathrm{L})\end{array}$ & $\begin{array}{l}\text { Di- } \\
\text { meth- } \\
\text { enamid } \\
(\mu \mathrm{g} / \mathrm{L})\end{array}$ & $\begin{array}{c}\text { Di- } \\
\text { meth- } \\
\text { enamid } \\
\text { ESA } \\
(\mu \mathrm{g} / \mathrm{L})\end{array}$ & $\begin{array}{c}\text { Di- } \\
\text { meth- } \\
\text { enamid } \\
\text { OXA } \\
(\mu \mathrm{g} / \mathrm{L})\end{array}$ & $\begin{array}{c}\text { Flufen- } \\
\text { acet } \\
(\mu \mathrm{g} / \mathrm{L})\end{array}$ & $\begin{array}{c}\text { Flufen- } \\
\text { acet } \\
\text { ESA } \\
(\mu \mathrm{g} / \mathrm{L})\end{array}$ & $\begin{array}{c}\text { Flufen- } \\
\text { acet } \\
\text { OXA } \\
(\mu \mathrm{g} / \mathrm{L})\end{array}$ & $\begin{array}{l}\text { Meto- } \\
\text { lachlor } \\
(\mu \mathrm{g} / \mathrm{L})\end{array}$ & $\begin{array}{l}\text { Meto- } \\
\text { lachlor } \\
\text { ESA } \\
(\mu \mathrm{g} / \mathrm{L})\end{array}$ & $\begin{array}{c}\text { Meto- } \\
\text { lachlor } \\
\text { OXA } \\
(\mu \mathrm{g} / \mathrm{L})\end{array}$ \\
\hline \multicolumn{20}{|c|}{ National Water-Quality Assessment Program (NAWOA) (fig. 3)-Continued } \\
\hline \multicolumn{20}{|c|}{ High Plains Regional Ground Water Study (HPGW/HPC)—Continued } \\
\hline 360703102361901 & Dallam Co., TX & $06 / 22 / 99$ & -- & $<0.05$ & $<0.05$ & -- & -- & $<0.05$ & $<0.05$ & -- & -- & -- & -- & -- & -- & -- & -- & $<0.05$ & $<0.05$ \\
\hline 360838102560301 & Dallam Co., TX & $06 / 24 / 99$ & -- & $<.05$ & $<.05$ & -- & -- & $<.05$ & $<.05$ & -- & -- & -- & -- & -- & -- & -- & -- & $<.05$ & $<.05$ \\
\hline 360944100162101 & Lipscomb Co., TX & $04 / 12 / 99$ & -- & $<.05$ & $<.05$ & -- & -- & $<.05$ & $<.05$ & -- & -- & -- & -- & -- & -- & -- & -- & $<.05$ & $<.05$ \\
\hline 361014100221001 & Lipscomb Co., TX & 04/08/99 & -- & .07 & $<.05$ & -- & -- & .19 & $<.05$ & -- & -- & -- & -- & -- & -- & -- & -- & .14 & .07 \\
\hline 361049100564901 & Ochiltree Co., TX & $05 / 21 / 99$ & -- & $<.05$ & $<.05$ & -- & -- & $<.05$ & $<.05$ & -- & -- & -- & -- & -- & -- & -- & -- & $<.05$ & $<.05$ \\
\hline 361054101393601 & Sherman Co., TX & $06 / 21 / 99$ & -- & $<.05$ & $<.05$ & -- & -- & $<.05$ & $<.05$ & -- & -- & -- & -- & -- & -- & -- & -- & $<.05$ & $<.05$ \\
\hline 361114099420501 & Ellis Co., OK & $07 / 22 / 99$ & -- & $<.05$ & $<.05$ & -- & -- & $<.05$ & $<.05$ & -- & -- & -- & -- & -- & -- & -- & -- & $<.05$ & $<.05$ \\
\hline 361517102065301 & Sherman Co., TX & $05 / 26 / 99$ & -- & $<.05$ & $<.05$ & -- & -- & $<.05$ & $<.05$ & -- & -- & -- & -- & -- & -- & -- & -- & $<.05$ & $<.05$ \\
\hline 362115101173901 & Hansford Co., TX & 05/26/99 & -- & $<.05$ & $<.05$ & -- & -- & $<.05$ & $<.05$ & -- & -- & -- & -- & -- & -- & -- & -- & $<.05$ & $<.05$ \\
\hline 362134102035101 & Sherman Co., TX & $05 / 26 / 99$ & -- & $<.05$ & $<.05$ & -- & -- & $<.05$ & $<.05$ & -- & -- & -- & -- & -- & -- & -- & -- & $<.05$ & $<.05$ \\
\hline 362615100435801 & Ochiltree Co., TX & $05 / 24 / 99$ & -- & $<.05$ & $<.05$ & -- & -- & $<.05$ & $<.05$ & -- & -- & -- & -- & -- & -- & -- & -- & $<.05$ & $<.05$ \\
\hline 362744102334901 & Dallam Co., TX & 07/20/99 & -- & $<.05$ & $<.05$ & -- & -- & $<.05$ & $<.05$ & -- & -- & -- & -- & -- & -- & -- & -- & $<.05$ & $<.05$ \\
\hline 363057101384701 & Texas Co., OK & 05/27/99 & -- & $<.05$ & $<.05$ & -- & -- & $<.05$ & $<.05$ & -- & -- & -- & -- & -- & -- & -- & -- & $<.05$ & $<.05$ \\
\hline 363104100035501 & Beaver Co., OK & 04/09/99 & -- & $<.05$ & $<.05$ & -- & -- & $<.05$ & $<.05$ & -- & -- & -- & -- & -- & -- & -- & -- & $<.05$ & $<.05$ \\
\hline 363154099310401 & Woodward Co., OK & 07/22/99 & -- & $<.05$ & $<.05$ & -- & -- & $<.05$ & $<.05$ & -- & -- & -- & -- & -- & -- & -- & -- & $<.05$ & $<.05$ \\
\hline 363157102565601 & Cimarron Co., OK & 04/16/99 & -- & $<.05$ & $<.05$ & -- & -- & $<.05$ & $<.05$ & -- & -- & -- & -- & -- & -- & -- & -- & $<.05$ & $<.05$ \\
\hline 363239100301801 & Beaver Co., OK & 04/06/99 & -- & $<.05$ & $<.05$ & -- & -- & $<.05$ & $<.05$ & -- & -- & -- & -- & -- & -- & -- & -- & $<.05$ & $<.05$ \\
\hline 363427102440401 & Cimarron Co., OK & $04 / 15 / 99$ & -- & $<.05$ & $<.05$ & -- & -- & $<.05$ & $<.05$ & -- & -- & -- & -- & -- & -- & -- & -- & $<.05$ & $<.05$ \\
\hline \multirow[t]{2}{*}{363518101515101} & Texas Co., OK & 03/06/00 & -- & $<.05$ & $<.05$ & -- & -- & $<.05$ & $<.05$ & -- & -- & -- & -- & -- & -- & -- & -- & $<.05$ & $<.05$ \\
\hline & & $09 / 12 / 00$ & -- & $<.05$ & $<.05$ & -- & -- & $<.05$ & $<.05$ & -- & -- & $<0.05$ & $<0.05$ & -- & $<0.05$ & $<0.05$ & -- & $<.05$ & $<.05$ \\
\hline 364219101394101 & Texas Co., OK & 06/11/99 & -- & $<.05$ & $<.05$ & -- & -- & $<.05$ & $<.05$ & -- & -- & -- & -- & -- & -- & -- & -- & $<.05$ & $<.05$ \\
\hline 364411102321201 & Cimarron Co., OK & 06/10/99 & -- & $<.05$ & $<.05$ & -- & -- & $<.05$ & $<.05$ & -- & -- & -- & -- & -- & -- & -- & -- & $<.05$ & $<.05$ \\
\hline 364846102161201 & Cimarron Co., OK & 09/17/00 & -- & $<.05$ & $<.05$ & -- & -- & $<.05$ & $<.05$ & -- & -- & $<.05$ & $<.05$ & -- & $<.05$ & $<.05$ & -- & $<.05$ & $<.05$ \\
\hline 365223102113001 & Cimarron Co., OK & $04 / 14 / 99$ & -- & $<.05$ & $<.05$ & -- & -- & $<.05$ & $<.05$ & -- & -- & -- & -- & -- & -- & -- & -- & $<.05$ & $<.05$ \\
\hline 365422101083701 & Texas Co., OK & $06 / 11 / 99$ & -- & $<.05$ & $<.05$ & -- & -- & $<.05$ & $<.05$ & -- & -- & -- & -- & -- & -- & -- & -- & $<.05$ & $<.05$ \\
\hline
\end{tabular}




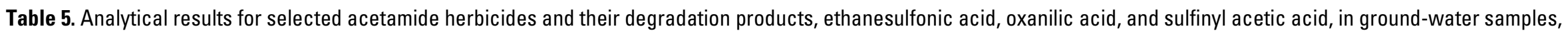
1994-2002.-Continued

[OGRL, U.S. Geological Survey Organic Geochemistry Research Laboratory, Lawrence, Kansas; ESA, ethanesulfonic acid; OXA, oxanilic acid; SAA, sulfinyl acetic acid; --, no data; <, less than]

\begin{tabular}{|c|c|c|c|c|c|c|c|c|c|c|c|c|c|c|c|c|c|c|c|c|}
\hline Site number & Site location & $\begin{array}{l}\text { Date of col- } \\
\text { lection } \\
\text { (month/day/ } \\
\text { year) }\end{array}$ & $\begin{array}{l}\text { Aceto- } \\
\text { chlor } \\
(\mu \mathrm{g} / \mathrm{L})\end{array}$ & $\begin{array}{l}\text { Aceto- } \\
\text { chlor } \\
\text { ESA } \\
(\mu \mathrm{g} / \mathrm{L})\end{array}$ & $\begin{array}{c}\text { Aceto- } \\
\text { chlor } \\
\text { OXA } \\
(\mu \mathrm{g} / \mathrm{L})\end{array}$ & $\begin{array}{l}\text { Aceto- } \\
\text { chlor } \\
\text { SAA } \\
(\mu \mathrm{g} / \mathrm{L})\end{array}$ & $\begin{array}{l}\text { Ala- } \\
\text { chlor } \\
(\mu \mathrm{g} / \mathrm{L})\end{array}$ & $\begin{array}{l}\text { Ala- } \\
\text { chlor } \\
\text { ESA } \\
(\mu \mathrm{g} / \mathrm{L})\end{array}$ & $\begin{array}{c}\text { Ala- } \\
\text { chlor } \\
\text { OXA } \\
(\mu \mathrm{g} / \mathrm{L})\end{array}$ & $\begin{array}{l}\text { Ala- } \\
\text { chlor } \\
\text { SAA } \\
(\mu \mathrm{g} / \mathrm{L})\end{array}$ & $\begin{array}{c}\text { Di- } \\
\text { meth- } \\
\text { enamid } \\
(\mu \mathrm{g} / \mathrm{L})\end{array}$ & $\begin{array}{c}\text { Di- } \\
\text { meth- } \\
\text { enamid } \\
\text { ESA } \\
(\mu \mathrm{g} / \mathrm{L})\end{array}$ & $\begin{array}{c}\text { Di- } \\
\text { meth- } \\
\text { enamid } \\
\text { OXA } \\
(\mu \mathrm{g} / \mathrm{L})\end{array}$ & $\begin{array}{c}\text { Flufen- } \\
\text { acet } \\
(\mu \mathrm{g} / \mathrm{L})\end{array}$ & $\begin{array}{c}\text { Flufen- } \\
\text { acet } \\
\text { ESA } \\
(\mu \mathrm{g} / \mathrm{L})\end{array}$ & $\begin{array}{c}\text { Flufen- } \\
\text { acet } \\
\text { OXA } \\
(\mu \mathrm{g} / \mathrm{L})\end{array}$ & $\begin{array}{l}\text { Meto- } \\
\text { lachlor } \\
(\mu \mathrm{g} / \mathrm{L})\end{array}$ & $\begin{array}{l}\text { Meto- } \\
\text { lachlor } \\
\text { ESA } \\
(\mu \mathrm{g} / \mathrm{L})\end{array}$ & $\begin{array}{c}\text { Meto- } \\
\text { lachlor } \\
\text { OXA } \\
(\mu \mathrm{g} / \mathrm{L})\end{array}$ & 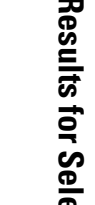 \\
\hline \multicolumn{20}{|c|}{ National Water-Quality Assessment Program (NAWOA) (fig. 3)—Continued } & \\
\hline \multicolumn{20}{|c|}{ High Plains Regional Ground Water Study (HPGW/HPC)_Continued } & \\
\hline 365821101375301 & Texas Co., OK & $05 / 28 / 99$ & -- & $<0.05$ & $<0.05$ & -- & -- & $<0.05$ & $<0.05$ & -- & -- & -- & -- & -- & -- & -- & -- & $<0.05$ & $<0.05$ & \\
\hline 365934101573001 & Texas Co., OK & 04/13/99 & -- & $<.05$ & $<.05$ & -- & -- & $<.05$ & $<.05$ & -- & -- & -- & -- & -- & -- & -- & -- & $<.05$ & $<.05$ & \\
\hline 370033100534204 & Seward Co., KS & 09/13/00 & -- & $<.05$ & $<.05$ & -- & -- & $<.05$ & $<.05$ & -- & -- & $<0.05$ & $<0.05$ & -- & $<0.05$ & $<0.05$ & -- & $<.05$ & $<.05$ & \\
\hline 370130101180904 & Stevens Co., KS & 09/14/00 & -- & $<.05$ & $<.05$ & -- & -- & $<.05$ & $<.05$ & -- & -- & $<.05$ & $<.05$ & -- & $<.05$ & $<.05$ & -- & $<.05$ & $<.05$ & \\
\hline 370358100510301 & Steward Co., KS & $04 / 15 / 99$ & -- & $<.05$ & $<.05$ & -- & -- & $<.05$ & $<.05$ & -- & -- & -- & -- & -- & -- & -- & -- & $<.05$ & $<.05$ & \\
\hline 370458101514401 & Morton Co., KS & $04 / 22 / 99$ & -- & $<.05$ & $<.05$ & -- & -- & $<.05$ & $<.05$ & -- & -- & -- & -- & -- & -- & -- & -- & $<.05$ & $<.05$ & \\
\hline 370724100281601 & Meade Co., KS & 04/23/99 & -- & $<.05$ & $<.05$ & -- & -- & $<.05$ & $<.05$ & -- & -- & -- & -- & -- & -- & -- & -- & $<.05$ & $<.05$ & \\
\hline 370941101540001 & Morton Co., KS & 09/16/00 & -- & $<.05$ & $<.05$ & -- & -- & $<.05$ & $<.05$ & -- & -- & $<.05$ & $<.05$ & -- & $<.05$ & $<.05$ & -- & $<.05$ & $<.05$ & \\
\hline 371303102003001 & Morton, KS & 09/14/00 & -- & $<.05$ & $<.05$ & -- & -- & $<.05$ & $<.05$ & -- & -- & $<.05$ & $<.05$ & -- & $<.05$ & $<.05$ & -- & $<.05$ & $<.05$ & \\
\hline 371728101311401 & Stevens Co., KS & 09/15/00 & -- & $<.05$ & $<.05$ & -- & -- & $<.05$ & $<.05$ & -- & -- & $<.05$ & $<.05$ & -- & $<.05$ & $<.05$ & -- & $<.05$ & $<.05$ & $\begin{array}{l}\mathbb{D} \\
\stackrel{\mathbb{2}}{2}\end{array}$ \\
\hline 371738101220301 & Stevens Co., KS & 04/20/99 & -- & $<.05$ & $<.05$ & -- & -- & $<.05$ & $<.05$ & -- & -- & -- & -- & -- & -- & -- & -- & $<.05$ & $<.05$ & \\
\hline 371909101413101 & Morton Co., KS & 09/16/00 & -- & $<.05$ & $<.05$ & -- & -- & $<.05$ & $<.05$ & -- & -- & $<.05$ & $<.05$ & -- & $<.05$ & $<.05$ & -- & $<.05$ & $<.05$ & D \\
\hline 372511100155601 & Meade Co., KS & 09/27/00 & -- & 1.8 & $<.05$ & -- & -- & $<.05$ & $<.05$ & -- & -- & $<.05$ & $<.05$ & -- & $<.05$ & $<.05$ & -- & .47 & $<.05$ & \\
\hline 372534100443501 & Haskell Co., KS & $04 / 16 / 99$ & -- & $<.05$ & $<.05$ & -- & -- & $<.05$ & $<.05$ & -- & -- & -- & -- & -- & -- & -- & -- & $<.05$ & $<.05$ & \\
\hline 372649101344701 & Stanton Co., KS & 04/19/99 & -- & $<.05$ & $<.05$ & -- & -- & $<.05$ & $<.05$ & -- & -- & -- & -- & -- & -- & -- & -- & $<.05$ & $<.05$ & \\
\hline 372956099512201 & Ford Co., KS & 05/26/99 & -- & $<.05$ & $<.05$ & -- & -- & $<.05$ & $<.05$ & -- & -- & -- & -- & -- & -- & -- & -- & $<.05$ & $<.05$ & \\
\hline 373229101193201 & Grant Co., KS & $04 / 21 / 99$ & -- & $<.05$ & $<.05$ & -- & -- & $<.05$ & $<.05$ & -- & -- & -- & -- & -- & -- & -- & -- & $<.05$ & $<.05$ & \\
\hline 373234099430201 & Ford Co., KS & $09 / 25 / 00$ & -- & $<.05$ & $<.05$ & -- & -- & $<.05$ & $<.05$ & -- & -- & $<.05$ & $<.05$ & -- & $<.05$ & $<.05$ & -- & $<.05$ & $<.05$ & \\
\hline 373345101070801 & Grant Co., KS & 04/06/99 & -- & $<.05$ & $<.05$ & -- & -- & $<.05$ & $<.05$ & -- & -- & -- & -- & -- & -- & -- & -- & $<.05$ & $<.05$ & i \\
\hline 373346100005701 & Ford Co., KS & 09/27/00 & -- & $<.05$ & $<.05$ & -- & -- & $<.05$ & $<.05$ & -- & -- & $<.05$ & $<.05$ & -- & $<.05$ & $<.05$ & -- & $<.05$ & $<.05$ & \\
\hline 373507102162001 & Baca Co., CO & 06/09/99 & -- & $<.05$ & $<.05$ & -- & -- & $<.05$ & $<.05$ & -- & -- & -- & -- & -- & -- & -- & -- & $<.05$ & $<.05$ & 氖 \\
\hline 373527100312701 & Gray Co., KS & 04/09/99 & -- & $<.05$ & $<.05$ & -- & -- & $<.05$ & $<.05$ & -- & -- & -- & -- & -- & -- & -- & -- & $<.05$ & $<.05$ & \\
\hline 373559102314901 & Baca Co., CO & 06/09/99 & -- & $<.05$ & $<.05$ & -- & -- & $<.05$ & $<.05$ & -- & -- & -- & -- & -- & -- & -- & -- & $<.05$ & $<.05$ & \\
\hline 373732099291201 & Kiowa Co., KS & 05/19/99 & -- & $<.05$ & $<.05$ & -- & -- & $<.05$ & $<.05$ & -- & -- & -- & -- & -- & -- & -- & -- & $<.05$ & $<.05$ & \\
\hline 373904099545701 & Ford Co., KS & $09 / 28 / 00$ & -- & $<.05$ & $<.05$ & -- & -- & .52 & .06 & -- & -- & $<.05$ & $<.05$ & -- & $<.05$ & $<.05$ & -- & .92 & .62 & \\
\hline
\end{tabular}




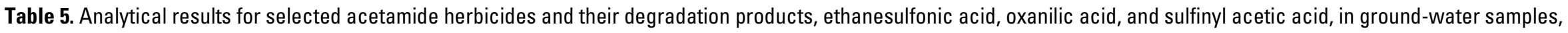
1994-2002.-Continued

[OGRL, U.S. Geological Survey Organic Geochemistry Research Laboratory, Lawrence, Kansas; ESA, ethanesulfonic acid; OXA, oxanilic acid; SAA, sulfinyl acetic acid; --, no data; <, less than]

\begin{tabular}{|c|c|c|c|c|c|c|c|c|c|c|c|c|c|c|c|c|c|c|c|}
\hline Site number & Site location & $\begin{array}{l}\text { Date of col- } \\
\text { lection } \\
\text { (month/day/ } \\
\text { year) }\end{array}$ & $\begin{array}{l}\text { Aceto- } \\
\text { chlor } \\
(\mu \mathrm{g} / \mathrm{L})\end{array}$ & $\begin{array}{l}\text { Aceto- } \\
\text { chlor } \\
\text { ESA } \\
(\mu \mathrm{g} / \mathrm{L})\end{array}$ & $\begin{array}{l}\text { Aceto- } \\
\text { chlor } \\
\text { OXA } \\
(\mu \mathrm{g} / \mathrm{L})\end{array}$ & $\begin{array}{l}\text { Aceto- } \\
\text { chlor } \\
\text { SAA } \\
(\mu \mathrm{g} / \mathrm{L})\end{array}$ & $\begin{array}{l}\text { Ala- } \\
\text { chlor } \\
(\mu \mathrm{g} / \mathrm{L})\end{array}$ & $\begin{array}{l}\text { Ala- } \\
\text { chlor } \\
\text { ESA } \\
(\mu \mathrm{g} / \mathrm{L})\end{array}$ & $\begin{array}{l}\text { Ala- } \\
\text { chlor } \\
\text { OXA } \\
(\mu \mathrm{g} / \mathrm{L})\end{array}$ & $\begin{array}{l}\text { Ala- } \\
\text { chlor } \\
\text { SAA } \\
(\mu \mathrm{g} / \mathrm{L})\end{array}$ & $\begin{array}{c}\text { Di- } \\
\text { meth- } \\
\text { enamid } \\
(\mu \mathrm{g} / \mathrm{L})\end{array}$ & $\begin{array}{c}\text { Di- } \\
\text { meth- } \\
\text { enamid } \\
\text { ESA } \\
(\mu \mathrm{g} / \mathrm{L})\end{array}$ & $\begin{array}{c}\text { Di- } \\
\text { meth- } \\
\text { enamid } \\
\text { OXA } \\
(\mu \mathrm{g} / \mathrm{L})\end{array}$ & $\begin{array}{l}\text { Flufen- } \\
\text { acet } \\
(\mu \mathrm{g} / \mathrm{L})\end{array}$ & $\begin{array}{c}\text { Flufen- } \\
\text { acet } \\
\text { ESA } \\
(\mu \mathrm{g} / \mathrm{L})\end{array}$ & $\begin{array}{l}\text { Flufen- } \\
\text { acet } \\
\text { OXA } \\
(\mu \mathrm{g} / \mathrm{L})\end{array}$ & $\begin{array}{l}\text { Meto- } \\
\text { lachlor } \\
(\mu \mathrm{g} / \mathrm{L})\end{array}$ & $\begin{array}{c}\text { Meto- } \\
\text { lachlor } \\
\text { ESA } \\
(\mu \mathrm{g} / \mathrm{L})\end{array}$ & $\begin{array}{l}\text { Meto- } \\
\text { lachlor } \\
\text { OXA } \\
(\mu \mathrm{g} / \mathrm{L})\end{array}$ \\
\hline \multicolumn{20}{|c|}{ National Water-Quality Assessment Program (NAWQA) (fig. 3)—Continued } \\
\hline \multicolumn{20}{|c|}{ High Plains Regional Ground Water Study (HPGW/HPC)—Continued } \\
\hline 374005101433501 & Stanton Co., KS & $08 / 08 / 00$ & -- & $<0.05$ & $<0.05$ & -- & -- & 0.07 & $<0.05$ & -- & -- & $<0.05$ & $<0.05$ & -- & $<0.05$ & $<0.05$ & -- & $<0.05$ & $<0.05$ \\
\hline 374220100422501 & Haskell Co., KS & $06 / 28 / 00$ & -- & .80 & $<.05$ & -- & -- & $<.05$ & $<.05$ & -- & -- & $<.05$ & $<.05$ & -- & $<.05$ & $<.05$ & -- & $<.05$ & $<.05$ \\
\hline 374412100453201 & Finney Co., KS & 07/10/00 & -- & $<.05$ & $<.05$ & -- & -- & $<.05$ & $<.05$ & -- & -- & $<.05$ & $<.05$ & -- & $<.05$ & $<.05$ & -- & $<.05$ & $<.05$ \\
\hline 374430100093801 & Ford Co., KS & $09 / 28 / 00$ & -- & $<.05$ & $<.05$ & -- & -- & .13 & $<.05$ & -- & -- & $<.05$ & $<.05$ & -- & $<.05$ & $<.05$ & -- & .08 & $<.05$ \\
\hline 374535102110301 & Baca Co., CO & 06/08/99 & -- & $<.05$ & $<.05$ & -- & -- & $<.05$ & $<.05$ & -- & -- & -- & -- & -- & -- & -- & -- & $<.05$ & $<.05$ \\
\hline 374625100490701 & Finney Co., KS & $06 / 29 / 00$ & -- & $<.05$ & $<.05$ & -- & -- & $<.05$ & $<.05$ & -- & -- & $<.05$ & $<.05$ & -- & $<.05$ & $<.05$ & -- & .24 & $<.05$ \\
\hline 374642100291701 & Gray Co., KS & 06/30/00 & -- & $<.05$ & $<.05$ & -- & -- & $<.05$ & $<.05$ & -- & -- & $<.05$ & $<.05$ & -- & $<.05$ & $<.05$ & -- & $<.05$ & $<.05$ \\
\hline 374650100181901 & Gray Co., KS & $04 / 14 / 99$ & -- & $<.05$ & $<.05$ & -- & -- & 4.8 & $<.05$ & -- & -- & -- & -- & -- & -- & -- & -- & 1.9 & .25 \\
\hline 374750101553501 & Hamilton Co., KS & $08 / 09 / 00$ & -- & $<.05$ & $<.05$ & -- & -- & $<.05$ & $<.05$ & -- & -- & $<.05$ & $<.05$ & -- & $<.05$ & $<.05$ & -- & $<.05$ & $<.05$ \\
\hline 375106100544301 & Finney Co., KS & $09 / 26 / 00$ & -- & $<.05$ & $<.05$ & -- & -- & .23 & $<.05$ & -- & -- & $<.05$ & $<.05$ & -- & $<.05$ & $<.05$ & -- & .09 & $<.05$ \\
\hline 375129100532301 & Finney Co., KS & $04 / 18 / 99$ & -- & $<.05$ & $<.05$ & -- & -- & $<.05$ & $<.05$ & -- & -- & -- & -- & -- & -- & -- & -- & $<.05$ & $<.05$ \\
\hline 375251101135301 & Kearny Co., KS & $08 / 11 / 00$ & -- & $<.05$ & $<.05$ & -- & -- & $<.05$ & $<.05$ & -- & -- & $<.05$ & $<.05$ & -- & $<.05$ & $<.05$ & -- & $<.05$ & $<.05$ \\
\hline 375447099245001 & Edwards Co., KS & 05/19/99 & -- & $<.05$ & $<.05$ & -- & -- & .15 & $<.05$ & -- & -- & -- & -- & -- & -- & -- & -- & .29 & $<.05$ \\
\hline 375502100363601 & Gray Co., KS & $09 / 26 / 00$ & -- & $<.05$ & $<.05$ & -- & -- & $<.05$ & $<.05$ & -- & -- & $<.05$ & $<.05$ & -- & $<.05$ & $<.05$ & -- & $<.05$ & $<.05$ \\
\hline 375828101111801 & Kearny Co., KS & 04/07/99 & -- & $<.05$ & $<.05$ & -- & -- & $<.05$ & $<.05$ & -- & -- & -- & -- & -- & -- & -- & -- & $<.05$ & $<.05$ \\
\hline 375855100484001 & Finney Co., KS & $06 / 27 / 00$ & -- & .47 & $<.05$ & -- & -- & $<.05$ & $<.05$ & -- & -- & $<.05$ & $<.05$ & -- & $<.05$ & $<.05$ & -- & $<.05$ & $<.05$ \\
\hline 380256101035901 & Finney Co., KS & 08/10/00 & -- & $<.05$ & $<.05$ & -- & -- & 1.2 & $<.05$ & -- & -- & $<.05$ & $<.05$ & -- & $<.05$ & $<.05$ & -- & $<.05$ & $<.05$ \\
\hline 380331100352801 & Finney Co., KS & $04 / 17 / 99$ & -- & $<.05$ & $<.05$ & -- & -- & $<.05$ & $<.05$ & -- & -- & -- & -- & -- & -- & -- & -- & $<.05$ & $<.05$ \\
\hline 381201100495101 & Finney Co., KS & $07 / 11 / 00$ & -- & $<.05$ & $<.05$ & -- & -- & $<.05$ & $<.05$ & -- & -- & $<.05$ & $<.05$ & -- & $<.05$ & $<.05$ & -- & .11 & $<.05$ \\
\hline 382947100443201 & Scott Co., KS & 04/08/99 & -- & $<.05$ & $<.05$ & -- & -- & $<.05$ & $<.05$ & -- & -- & -- & -- & -- & -- & -- & -- & $<.05$ & $<.05$ \\
\hline 383008101315601 & Wichita Co., KS & $04 / 28 / 99$ & -- & $<.05$ & $<.05$ & -- & -- & $<.05$ & $<.05$ & -- & -- & -- & -- & -- & -- & -- & -- & $<.05$ & $<.05$ \\
\hline 383334101131301 & Wichita Co., KS & $08 / 08 / 00$ & -- & $<.05$ & $<.05$ & -- & -- & $<.05$ & $<.05$ & -- & -- & $<.05$ & $<.05$ & -- & $<.05$ & $<.05$ & -- & $<.05$ & $<.05$ \\
\hline 383758101195801 & Wichita Co., KS & 05/22/99 & -- & $<.05$ & $<.05$ & -- & -- & $<.05$ & $<.05$ & -- & -- & -- & -- & -- & -- & -- & -- & $<.05$ & $<.05$ \\
\hline 383803101084701 & Wichita Co., KS & 07/13/00 & -- & $<.05$ & $<.05$ & -- & -- & .16 & $<.05$ & -- & -- & $<.05$ & $<.05$ & -- & $<.05$ & $<.05$ & -- & .26 & .09 \\
\hline 383919101040101 & Scott Co., KS & $04 / 29 / 99$ & -- & $<.05$ & $<.05$ & -- & -- & $<.05$ & $<.05$ & -- & -- & -- & -- & -- & -- & -- & -- & $<.05$ & $<.05$ \\
\hline
\end{tabular}




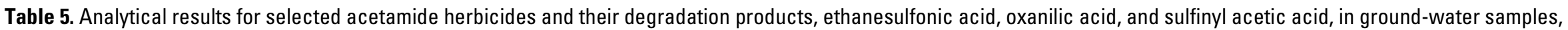
1994-2002.-Continued

[OGRL, U.S. Geological Survey Organic Geochemistry Research Laboratory, Lawrence, Kansas; ESA, ethanesulfonic acid; OXA, oxanilic acid; SAA, sulfinyl acetic acid; --, no data; <, less than]

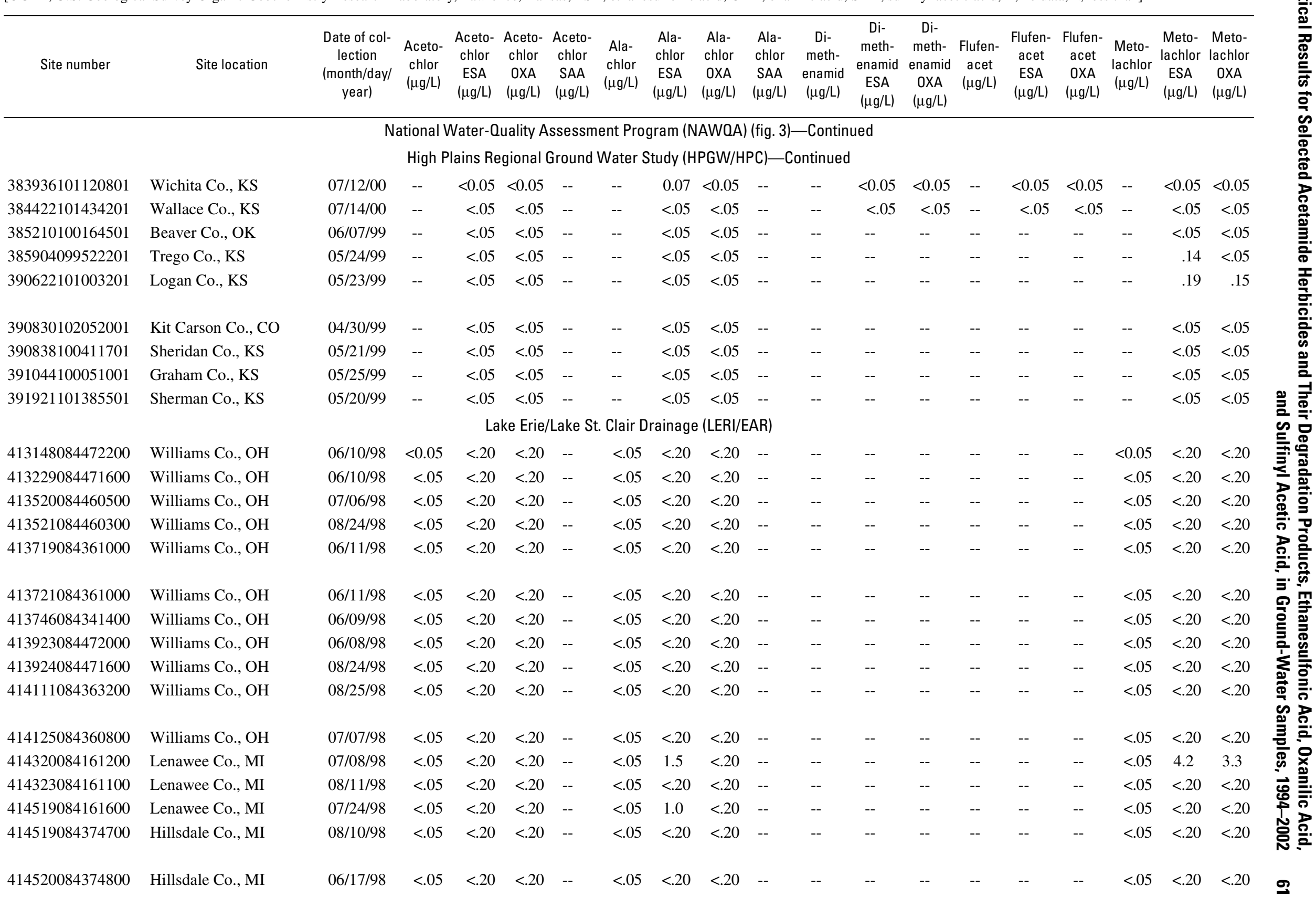




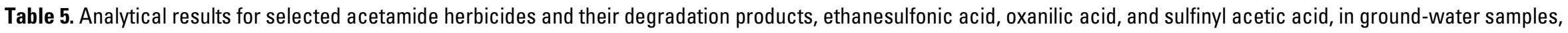
1994-2002.-Continued

[OGRL, U.S. Geological Survey Organic Geochemistry Research Laboratory, Lawrence, Kansas; ESA, ethanesulfonic acid; OXA, oxanilic acid; SAA, sulfinyl acetic acid; --, no data; <, less than]

\begin{tabular}{|c|c|c|c|c|c|c|c|c|c|c|c|c|c|c|c|c|c|c|c|}
\hline Site number & Site location & $\begin{array}{l}\text { Date of col- } \\
\text { lection } \\
\text { (month/day/ } \\
\text { year) }\end{array}$ & $\begin{array}{l}\text { Aceto- } \\
\text { chlor } \\
(\mu \mathrm{g} / \mathrm{L})\end{array}$ & $\begin{array}{l}\text { Aceto- } \\
\text { chlor } \\
\text { ESA } \\
(\mu \mathrm{g} / \mathrm{L})\end{array}$ & $\begin{array}{l}\text { Aceto- } \\
\text { chlor } \\
\text { OXA } \\
(\mu \mathrm{g} / \mathrm{L})\end{array}$ & $\begin{array}{l}\text { Aceto- } \\
\text { chlor } \\
\text { SAA } \\
(\mu \mathrm{g} / \mathrm{L})\end{array}$ & $\begin{array}{l}\text { Ala- } \\
\text { chlor } \\
\text { ( } \mu \mathrm{g} / \mathrm{L})\end{array}$ & $\begin{array}{l}\text { Ala- } \\
\text { chlor } \\
\text { ESA } \\
(\mu \mathrm{g} / \mathrm{L})\end{array}$ & $\begin{array}{l}\text { Ala- } \\
\text { chlor } \\
\text { OXA } \\
(\mu \mathrm{g} / \mathrm{L})\end{array}$ & $\begin{array}{l}\text { Ala- } \\
\text { chlor } \\
\text { SAA } \\
(\mu \mathrm{g} / \mathrm{L})\end{array}$ & $\begin{array}{c}\text { Di- } \\
\text { meth- } \\
\text { enamid } \\
(\mu \mathrm{g} / \mathrm{L})\end{array}$ & $\begin{array}{c}\text { Di- } \\
\text { meth- } \\
\text { enamid } \\
\text { ESA } \\
(\mu \mathrm{g} / \mathrm{L})\end{array}$ & $\begin{array}{c}\text { Di- } \\
\text { meth- } \\
\text { enamid } \\
\text { OXA } \\
(\mu \mathrm{g} / \mathrm{L})\end{array}$ & $\begin{array}{c}\text { Flufen- } \\
\text { acet } \\
(\mu \mathrm{g} / \mathrm{L})\end{array}$ & $\begin{array}{c}\text { Flufen- } \\
\text { acet } \\
\text { ESA } \\
(\mu \mathrm{g} / \mathrm{L})\end{array}$ & $\begin{array}{c}\text { Flufen- } \\
\text { acet } \\
\text { OXA } \\
(\mu \mathrm{g} / \mathrm{L})\end{array}$ & $\begin{array}{l}\text { Meto- } \\
\text { lachlor } \\
(\mu \mathrm{g} / \mathrm{L})\end{array}$ & $\begin{array}{l}\text { Meto- } \\
\text { lachlor } \\
\text { ESA } \\
(\mu \mathrm{g} / \mathrm{L})\end{array}$ & $\begin{array}{l}\text { Meto- } \\
\text { lachlor } \\
\text { OXA } \\
(\mu \mathrm{g} / \mathrm{L})\end{array}$ \\
\hline \multicolumn{20}{|c|}{ National Water-Quality Assessment Program (NAWOA) (fig. 3)—Continued } \\
\hline \multicolumn{20}{|c|}{ Lake Erie/Lake St. Clair Drainage (LERI/EAR)—Continued } \\
\hline 414604084264600 & Hillsdale Co., MI & $08 / 11 / 98$ & $<0.05$ & $<0.20$ & $<0.20$ & -- & $<0.05$ & $<0.20$ & $<0.20$ & -- & -- & -- & -- & -- & -- & -- & $<0.05$ & $<0.20$ & $<0.20$ \\
\hline 414611084262000 & Hillsdale Co., MI & $06 / 19 / 98$ & $<.05$ & $<.20$ & $<.20$ & -- & $<.05$ & .55 & $<.20$ & -- & -- & -- & -- & -- & -- & -- & $<.05$ & 1.1 & $<.20$ \\
\hline 414728084390400 & Hillsdale Co., MI & 06/16/98 & $<.05$ & $<.20$ & $<.20$ & -- & $<.05$ & .21 & $<.20$ & -- & -- & -- & -- & -- & -- & -- & $<.05$ & 1.5 & $<.20$ \\
\hline 414746084394800 & Hillsdale Co., MI & 08/10/98 & $<.05$ & $<.20$ & $<.20$ & -- & $<.05$ & $<.20$ & $<.20$ & -- & -- & -- & -- & -- & -- & -- & $<.05$ & $<.20$ & $<.20$ \\
\hline 414907084243100 & Hillsdale Co., MI & 07/10/98 & $<.05$ & $<.20$ & .81 & -- & $<.05$ & $<.20$ & .97 & -- & -- & -- & -- & -- & -- & -- & $<.05$ & $<.20$ & $<.20$ \\
\hline 415026084220000 & Hillsdale Co., MI & $07 / 22 / 98$ & $<.05$ & $<.20$ & $<.20$ & -- & $<.05$ & $<.20$ & $<.20$ & -- & -- & -- & -- & -- & -- & -- & $<.05$ & $<.20$ & $<.20$ \\
\hline 415028084220400 & Hillsdale Co., MI & 08/11/98 & $<.05$ & $<.20$ & $<.20$ & -- & $<.05$ & $<.20$ & $<.20$ & -- & -- & -- & -- & -- & -- & -- & $<.05$ & $<.20$ & $<.20$ \\
\hline 415456084095500 & Lenawee Co., MI & 07/20/98 & $<.05$ & $<.20$ & $<.20$ & -- & $<.05$ & $<.20$ & $<.20$ & -- & -- & -- & -- & -- & -- & -- & $<.05$ & $<.20$ & $<.20$ \\
\hline 415516084061200 & Lenawee Co., MI & 08/06/98 & $<.05$ & $<.20$ & $<.20$ & -- & $<.05$ & .27 & $<.20$ & -- & -- & -- & -- & -- & -- & -- & $<.05$ & .59 & $<.20$ \\
\hline 420022084024200 & Lenawee Co., MI & 08/13/98 & $<.05$ & $<.20$ & $<.20$ & -- & $<.05$ & $<.20$ & $<.20$ & -- & -- & -- & -- & -- & -- & -- & $<.05$ & $<.20$ & $<.20$ \\
\hline 420047084234900 & Hillsdale Co., MI & 07/09/98 & $<.05$ & 3.5 & 1.0 & -- & $<.05$ & 1.5 & $<.20$ & -- & -- & -- & -- & -- & -- & -- & $<.05$ & $<.20$ & $<.20$ \\
\hline 420049084235000 & Hillsdale Co., MI & 08/13/98 & $<.05$ & $<.20$ & $<.20$ & -- & $<.05$ & $<.20$ & $<.20$ & -- & -- & -- & -- & -- & -- & -- & $<.05$ & $<.20$ & $<.20$ \\
\hline 420054084024000 & Lenawee Co., MI & $07 / 23 / 98$ & $<.05$ & $<.20$ & $<.20$ & -- & $<.05$ & $<.20$ & $<.20$ & -- & -- & -- & -- & -- & -- & -- & $<.05$ & $<.20$ & $<.20$ \\
\hline 420303084040300 & Lenawee Co., MI & $07 / 21 / 98$ & $<.05$ & $<.20$ & $<.20$ & -- & $<.05$ & $<.20$ & $<.20$ & -- & -- & -- & -- & -- & -- & -- & $<.05$ & $<.20$ & $<.20$ \\
\hline 421124083534000 & Washtenaw Co., MI & 08/12/98 & $<.05$ & $<.20$ & $<.20$ & -- & $<.05$ & $<.20$ & $<.20$ & -- & -- & -- & -- & -- & -- & -- & $<.05$ & $<.20$ & $<.20$ \\
\hline 421125083533800 & Washtenaw Co., MI & $06 / 25 / 98$ & $<.05$ & $<.20$ & $<.20$ & -- & $<.05$ & $<.20$ & $<.20$ & -- & -- & -- & -- & -- & -- & -- & $<.05$ & $<.20$ & $<.20$ \\
\hline 421212083593000 & Washtenaw Co., MI & 08/06/98 & $<.05$ & $<.20$ & $<.20$ & -- & $<.05$ & $<.20$ & $<.20$ & -- & -- & -- & -- & -- & -- & -- & $<.05$ & $<.20$ & $<.20$ \\
\hline 421225083593300 & Washtenaw Co., MI & 06/26/98 & $<.05$ & $<.20$ & $<.20$ & -- & $<.05$ & .57 & $<.20$ & -- & -- & -- & -- & -- & -- & -- & $<.05$ & $<.20$ & $<.20$ \\
\hline 421237083523500 & Washtenaw Co., MI & 06/23/98 & $<.05$ & $<.20$ & $<.20$ & -- & $<.05$ & $<.20$ & $<.20$ & -- & -- & -- & -- & -- & -- & -- & $<.05$ & $<.20$ & $<.20$ \\
\hline 421244083492000 & Washtenaw Co., MI & $06 / 24 / 98$ & $<.05$ & $<.20$ & $<.20$ & -- & $<.05$ & $<.20$ & $<.20$ & -- & -- & -- & -- & -- & -- & -- & $<.05$ & $<.20$ & $<.20$ \\
\hline 421624083515600 & Washtenaw Co., MI & 08/06/98 & $<.05$ & $<.20$ & $<.20$ & -- & $<.05$ & $<.20$ & $<.20$ & -- & -- & -- & -- & -- & -- & -- & $<.05$ & $<.20$ & $<.20$ \\
\hline 423032083295700 & Oakland Co., MI & 09/03/98 & $<.05$ & $<.20$ & $<.20$ & -- & $<.05$ & $<.20$ & $<.20$ & -- & -- & -- & -- & -- & -- & -- & $<.05$ & $<.20$ & $<.20$ \\
\hline 423044083503600 & Livingston Co., MI & 08/02/98 & $<.05$ & $<.20$ & $<.20$ & -- & $<.05$ & $<.20$ & $<.20$ & -- & -- & -- & -- & -- & -- & -- & $<.05$ & $<.20$ & $<.20$ \\
\hline 423559083420700 & Livingston Co., MI & 08/26/98 & $<.05$ & $<.20$ & $<.20$ & -- & $<.05$ & $<.20$ & $<.20$ & -- & -- & -- & -- & -- & -- & -- & $<.05$ & $<.20$ & $<.20$ \\
\hline 424159082493200 & Macomb Co., MI & 09/02/98 & $<.05$ & $<.20$ & $<.20$ & -- & $<.05$ & $<.20$ & $<.20$ & -- & -- & -- & -- & -- & -- & -- & $<.05$ & $<.20$ & $<.20$ \\
\hline
\end{tabular}




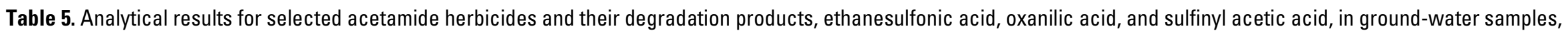
1994-2002.-Continued

[OGRL, U.S. Geological Survey Organic Geochemistry Research Laboratory, Lawrence, Kansas; ESA, ethanesulfonic acid; OXA, oxanilic acid; SAA, sulfinyl acetic acid; --, no data; <, less than]

\begin{tabular}{|c|c|c|c|c|c|c|c|c|c|c|c|c|c|c|c|c|c|c|c|}
\hline Site number & Site location & $\begin{array}{l}\text { Date of col- } \\
\text { lection } \\
\text { (month/day/ } \\
\text { year) }\end{array}$ & $\begin{array}{l}\text { Aceto- } \\
\text { chlor } \\
(\mu \mathrm{g} / \mathrm{L})\end{array}$ & $\begin{array}{l}\text { Aceto- } \\
\text { chlor } \\
\text { ESA } \\
(\mu \mathrm{g} / \mathrm{L})\end{array}$ & $\begin{array}{l}\text { Aceto- } \\
\text { chlor } \\
\text { OXA } \\
(\mu \mathrm{g} / \mathrm{L})\end{array}$ & $\begin{array}{l}\text { Aceto- } \\
\text { chlor } \\
\text { SAA } \\
(\mu \mathrm{g} / \mathrm{L})\end{array}$ & $\begin{array}{l}\text { Ala- } \\
\text { chlor } \\
(\mu \mathrm{g} / \mathrm{L})\end{array}$ & $\begin{array}{l}\text { Ala- } \\
\text { chlor } \\
\text { ESA } \\
(\mu \mathrm{g} / \mathrm{L})\end{array}$ & $\begin{array}{c}\text { Ala- } \\
\text { chlor } \\
\text { OXA } \\
(\mu \mathrm{g} / \mathrm{L})\end{array}$ & $\begin{array}{l}\text { Ala- } \\
\text { chlor } \\
\text { SAA } \\
(\mu \mathrm{g} / \mathrm{L})\end{array}$ & $\begin{array}{l}\text { Di- } \\
\text { meth- } \\
\text { enamid } \\
(\mu \mathrm{g} / \mathrm{L})\end{array}$ & $\begin{array}{c}\text { Di- } \\
\text { meth- } \\
\text { enamid } \\
\text { ESA } \\
(\mu \mathrm{g} / \mathrm{L})\end{array}$ & $\begin{array}{c}\text { Di- } \\
\text { meth- } \\
\text { enamid } \\
\text { OXA } \\
(\mu \mathrm{g} / \mathrm{L})\end{array}$ & $\begin{array}{c}\text { Flufen- } \\
\text { acet } \\
(\mu \mathrm{g} / \mathrm{L})\end{array}$ & $\begin{array}{c}\text { Flufen- } \\
\text { acet } \\
\text { ESA } \\
(\mu \mathrm{g} / \mathrm{L})\end{array}$ & $\begin{array}{c}\text { Flufen- } \\
\text { acet } \\
\text { OXA } \\
(\mu \mathrm{g} / \mathrm{L})\end{array}$ & $\begin{array}{l}\text { Meto- } \\
\text { lachlor } \\
(\mu \mathrm{g} / \mathrm{L})\end{array}$ & $\begin{array}{l}\text { Meto- } \\
\text { lachlor } \\
\text { ESA } \\
(\mu \mathrm{g} / \mathrm{L})\end{array}$ & $\begin{array}{l}\text { Meto- } \\
\text { lachlor } \\
\text { OXA } \\
(\mu \mathrm{g} / \mathrm{L})\end{array}$ \\
\hline \multicolumn{20}{|c|}{ National Water-Quality Assessment Program (NAWOA) (fig. 3)—Continued } \\
\hline \multicolumn{20}{|c|}{ Lake Erie/Lake St. Clair Drainage (LERI/EAR)—Continued } \\
\hline 424301083092400 & Oakland Co., MI & 09/02/98 & $<0.05$ & $<0.20$ & $<0.20$ & -- & $<0.05$ & $<0.20$ & $<0.20$ & -- & -- & -- & -- & -- & -- & -- & $<0.05$ & $<0.20$ & $<0.20$ \\
\hline 424658083270600 & Oakland Co., MI & 08/26/98 & $<.05$ & $<.20$ & $<.20$ & -- & $<.05$ & $<.20$ & $<.20$ & -- & -- & -- & -- & -- & -- & -- & $<.05$ & $<.20$ & $<.20$ \\
\hline 425033083080900 & Oakland Co., MI & 09/03/98 & $<.05$ & $<.20$ & $<.20$ & -- & $<.05$ & $<.20$ & $<.20$ & -- & -- & -- & -- & -- & -- & -- & $<.05$ & $<.20$ & $<.20$ \\
\hline 425152083001000 & Macomb Co., MI & 08/05/98 & $<.05$ & $<.20$ & $<.20$ & -- & $<.05$ & $<.20$ & $<.20$ & -- & -- & -- & -- & -- & -- & -- & $<.05$ & $<.20$ & $<.20$ \\
\hline 425204083011600 & Macomb Co., MI & 07/13/98 & $<.05$ & $<.20$ & $<.20$ & -- & $<.05$ & .58 & $<.20$ & -- & -- & -- & -- & -- & -- & -- & $<.05$ & 6.0 & 1.1 \\
\hline 425758083040100 & Lapeer Co., MI & 07/13/98 & $<.05$ & $<.20$ & $<.20$ & -- & $<.05$ & .37 & $<.20$ & -- & -- & -- & -- & -- & -- & -- & $<.05$ & $<.20$ & $<.20$ \\
\hline 425843083042400 & Lapeer Co., MI & 09/01/98 & $<.05$ & $<.20$ & $<.20$ & -- & $<.05$ & $<.20$ & $<.20$ & -- & -- & -- & -- & -- & -- & -- & $<.05$ & $<.20$ & $<.20$ \\
\hline 430313083085300 & Lapeer Co., MI & 08/04/98 & $<.05$ & $<.20$ & $<.20$ & -- & $<.05$ & $<.20$ & $<.20$ & -- & -- & -- & -- & -- & -- & -- & $<.05$ & $<.20$ & $<.20$ \\
\hline 430336083012700 & Lapeer Co., MI & $07 / 16 / 98$ & $<.05$ & $<.20$ & $<.20$ & -- & $<.05$ & $<.20$ & $<.20$ & -- & -- & -- & -- & -- & -- & -- & $<.05$ & $<.20$ & $<.20$ \\
\hline 430349083014700 & Lapeer Co., MI & $08 / 31 / 98$ & $<.05$ & $<.20$ & $<.20$ & -- & $<.05$ & $<.20$ & $<.20$ & -- & -- & -- & -- & -- & -- & -- & $<.05$ & $<.20$ & $<.20$ \\
\hline 430845082462500 & St.Clair Co., MI & 08/03/98 & $<.05$ & $<.20$ & $<.20$ & -- & $<.05$ & $<.20$ & $<.20$ & -- & -- & -- & -- & -- & -- & -- & $<.05$ & $<.20$ & $<.20$ \\
\hline 430847082462000 & St.Clair Co., MI & $07 / 15 / 98$ & $<.05$ & $<.20$ & $<.20$ & -- & $<.05$ & $<.20$ & $<.20$ & -- & -- & -- & -- & -- & -- & -- & $<.05$ & $<.20$ & $<.20$ \\
\hline 431240082511400 & Sanilac Co., MI & $08 / 31 / 98$ & $<.05$ & $<.20$ & $<.20$ & -- & $<.05$ & $<.20$ & $<.20$ & -- & -- & -- & -- & -- & -- & -- & $<.05$ & $<.20$ & $<.20$ \\
\hline 431730082492900 & Sanilac Co., MI & 07/14/98 & $<.05$ & $<.20$ & $<.20$ & -- & $<.05$ & $<.20$ & $<.20$ & -- & -- & -- & -- & -- & -- & -- & $<.05$ & $<.20$ & $<.20$ \\
\hline 433501082452500 & Sanilac Co., MI & 08/05/98 & $<.05$ & $<.20$ & $<.20$ & -- & $<.05$ & $<.20$ & $<.20$ & -- & -- & -- & -- & -- & -- & -- & $<.05$ & $<.20$ & $<.20$ \\
\hline \multicolumn{20}{|c|}{ Lower and Upper Illinois River Basin (LIRB/UIRB/KWI) } \\
\hline 395553088441401 & Piatt Co., IL & 05/05/98 & $<.05$ & $<.20$ & $<.20$ & -- & $<.05$ & $<.20$ & $<.20$ & -- & -- & -- & -- & -- & -- & -- & $<.05$ & $<.20$ & $<.20$ \\
\hline 395626089024901 & Macon Co., IL & $07 / 23 / 97$ & $<.05$ & $<.20$ & $<.20$ & -- & $<.05$ & $<.20$ & $<.20$ & -- & -- & -- & -- & -- & -- & -- & $<.05$ & $<.20$ & $<.20$ \\
\hline 395853088364401 & Piatt Co., IL & $09 / 22 / 97$ & $<.05$ & $<.20$ & $<.20$ & -- & $<.05$ & $<.20$ & $<.20$ & -- & -- & -- & -- & -- & -- & -- & .40 & 34 & 11 \\
\hline 400009088484601 & Macon Co., IL & 06/03/02 & -- & $<.05$ & $<.05$ & $<0.05$ & -- & $<.05$ & $<.05$ & $<0.05$ & -- & $<0.05$ & $<0.05$ & -- & $<0.05$ & $<0.05$ & -- & $<.05$ & $<.05$ \\
\hline 400033088300301 & Piatt Co., IL & 09/10/97 & $<.05$ & $<.05$ & $<.05$ & -- & $<.05$ & $<.20$ & $<.20$ & -- & -- & -- & -- & -- & -- & -- & $<.05$ & $<.20$ & $<.20$ \\
\hline 400057088550101 & Macon Co., IL & 06/04/02 & -- & $<.05$ & $<.05$ & $<.05$ & -- & $<.05$ & $<.05$ & $<.05$ & -- & $<.05$ & $<.05$ & -- & $<.05$ & $<.05$ & -- & $<.05$ & $<.05$ \\
\hline 400111088550501 & Macon Co., IL & 08/19/97 & $<.05$ & $<.20$ & $<.20$ & -- & $<.05$ & $<.20$ & $<.20$ & -- & -- & -- & -- & -- & -- & -- & $<.05$ & .22 & $<.20$ \\
\hline 400345088444901 & DeWitt Co., IL & $10 / 22 / 97$ & $<.05$ & $<.20$ & $<.20$ & -- & $<.05$ & $<.20$ & $<.20$ & -- & -- & -- & -- & -- & -- & -- & $<.05$ & $<.20$ & $<.20$ \\
\hline 040042208857101 & DeWitt Co., IL & $06 / 24 / 98$ & $<.05$ & $<.20$ & $<.20$ & -- & $<.05$ & $<.20$ & $<.20$ & -- & -- & -- & -- & -- & -- & -- & $<.05$ & $<.20$ & $<.20$ \\
\hline
\end{tabular}




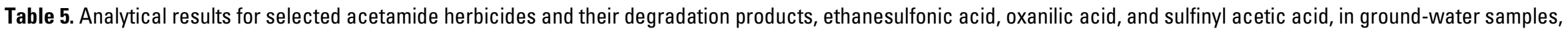
1994-2002.-Continued

[OGRL, U.S. Geological Survey Organic Geochemistry Research Laboratory, Lawrence, Kansas; ESA, ethanesulfonic acid; OXA, oxanilic acid; SAA, sulfinyl acetic acid; --, no data; <, less than]

\begin{tabular}{|c|c|c|c|c|c|c|c|c|c|c|c|c|c|c|c|c|c|c|c|}
\hline Site number & Site location & $\begin{array}{l}\text { Date of col- } \\
\text { lection } \\
\text { (month/day/ } \\
\text { year) }\end{array}$ & $\begin{array}{l}\text { Aceto- } \\
\text { chlor } \\
(\mu \mathrm{g} / \mathrm{L})\end{array}$ & $\begin{array}{l}\text { Aceto- } \\
\text { chlor } \\
\text { ESA } \\
(\mu \mathrm{g} / \mathrm{L})\end{array}$ & $\begin{array}{l}\text { Aceto- } \\
\text { chlor } \\
\text { OXA } \\
(\mu \mathrm{g} / \mathrm{L})\end{array}$ & $\begin{array}{l}\text { Aceto- } \\
\text { chlor } \\
\text { SAA } \\
(\mu \mathrm{g} / \mathrm{L})\end{array}$ & $\begin{array}{l}\text { Ala- } \\
\text { chlor } \\
(\mu \mathrm{g} / \mathrm{L})\end{array}$ & $\begin{array}{l}\text { Ala- } \\
\text { chlor } \\
\text { ESA } \\
(\mu \mathrm{g} / \mathrm{L})\end{array}$ & $\begin{array}{c}\text { Ala- } \\
\text { chlor } \\
\text { OXA } \\
(\mu \mathrm{g} / \mathrm{L})\end{array}$ & $\begin{array}{l}\text { Ala- } \\
\text { chlor } \\
\text { SAA } \\
(\mu \mathrm{g} / \mathrm{L})\end{array}$ & $\begin{array}{l}\text { Di- } \\
\text { meth- } \\
\text { enamid } \\
(\mu \mathrm{g} / \mathrm{L})\end{array}$ & $\begin{array}{c}\text { Di- } \\
\text { meth- } \\
\text { enamid } \\
\text { ESA } \\
(\mu \mathrm{g} / \mathrm{L})\end{array}$ & $\begin{array}{c}\text { Di- } \\
\text { meth- } \\
\text { enamid } \\
\text { OXA } \\
(\mu \mathrm{g} / \mathrm{L})\end{array}$ & $\begin{array}{c}\text { Flufen- } \\
\text { acet } \\
(\mu \mathrm{g} / \mathrm{L})\end{array}$ & $\begin{array}{l}\text { Flufen- } \\
\text { acet } \\
\text { ESA } \\
(\mu \mathrm{g} / \mathrm{L})\end{array}$ & $\begin{array}{c}\text { Flufen- } \\
\text { acet } \\
\text { OXA } \\
(\mu \mathrm{g} / \mathrm{L})\end{array}$ & $\begin{array}{l}\text { Meto- } \\
\text { lachlor } \\
(\mu \mathrm{g} / \mathrm{L})\end{array}$ & $\begin{array}{l}\text { Meto- } \\
\text { lachlor } \\
\text { ESA } \\
(\mu \mathrm{g} / \mathrm{L})\end{array}$ & $\begin{array}{l}\text { Meto- } \\
\text { lachlor } \\
\text { OXA } \\
(\mu \mathrm{g} / \mathrm{L})\end{array}$ \\
\hline \multicolumn{20}{|c|}{ National Water-Quality Assessment Program (NAWOA) (fig. 3)—Continued } \\
\hline \multicolumn{20}{|c|}{ Lower and Upper Illinois River Basin (LIRB/UIRB/KWI)_Continued } \\
\hline 400512089022601 & DeWitt Co., IL & 08/21/97 & $<0.05$ & $<0.20$ & $<0.20$ & -- & $<0.05$ & $<0.20$ & $<0.20$ & -- & -- & -- & -- & -- & -- & -- & $<0.05$ & $<0.20$ & $<0.20$ \\
\hline 400601088553401 & DeWitt Co., IL & $10 / 21 / 97$ & $<.05$ & $<.20$ & $<.20$ & -- & $<.05$ & $<.20$ & $<.20$ & -- & -- & -- & -- & -- & -- & -- & $<.05$ & .48 & $<.20$ \\
\hline 400603088300901 & Piatt Co., IL & 06/23/98 & $<.05$ & $<.20$ & $<.20$ & -- & $<.05$ & $<.20$ & $<.20$ & -- & -- & -- & -- & -- & -- & -- & $<.05$ & $<.20$ & $<.20$ \\
\hline 400736088344701 & Piatt Co., IL & 07/31/97 & $<.05$ & $<.20$ & $<.20$ & -- & $<.05$ & $<.20$ & $<.20$ & -- & -- & -- & -- & -- & -- & -- & $<.05$ & $<.20$ & $<.20$ \\
\hline 400805088531501 & Dewitt Co., IL & 05/30/02 & -- & $<.05$ & $<.05$ & $<0.05$ & -- & $<.05$ & $<.05$ & $<0.05$ & -- & $<0.05$ & $<0.05$ & -- & $<0.05$ & $<0.05$ & -- & $<.05$ & $<.05$ \\
\hline 400817088593401 & DeWitt Co., IL & 04/29/98 & $<.05$ & $<.20$ & $<.20$ & -- & $<.05$ & $<.20$ & $<.20$ & -- & -- & -- & -- & -- & -- & -- & $<.05$ & $<.20$ & $<.20$ \\
\hline 400846088491001 & DeWitt Co., IL & 08/25/97 & $<.05$ & $<.20$ & $<.20$ & -- & $<.05$ & .60 & $<.20$ & -- & -- & -- & -- & -- & -- & -- & $<.05$ & .99 & $<.20$ \\
\hline 400919088331501 & Piatt Co., IL & 05/12/98 & $<.05$ & $<.20$ & $<.20$ & -- & $<.05$ & $<.20$ & $<.20$ & -- & -- & -- & -- & -- & -- & -- & $<.05$ & $<.20$ & $<.20$ \\
\hline 401020089252801 & Logan Co., IL & $10 / 29 / 97$ & $<.05$ & $<.20$ & $<.20$ & -- & $<.05$ & $<.20$ & $<.20$ & -- & -- & -- & -- & -- & -- & -- & $<.05$ & $<.20$ & $<.20$ \\
\hline 401041088242901 & Champaign Co., IL & 06/09/98 & $<.05$ & $<.20$ & $<.20$ & -- & $<.05$ & $<.20$ & $<.20$ & -- & -- & -- & -- & -- & -- & -- & $<.05$ & $<.20$ & $<.20$ \\
\hline 401058088521001 & DeWitt Co., IL & 06/10/98 & $<.05$ & $<.20$ & $<.20$ & -- & $<.05$ & $<.20$ & $<.20$ & -- & -- & -- & -- & -- & -- & -- & $<.05$ & $<.20$ & $<.20$ \\
\hline 401100089004701 & DeWitt Co., IL & 05/06/98 & $<.05$ & $<.20$ & $<.20$ & -- & $<.05$ & $<.20$ & $<.20$ & -- & -- & -- & -- & -- & -- & -- & $<.05$ & $<.20$ & $<.20$ \\
\hline 401151088423401 & DeWitt Co., IL & $10 / 22 / 97$ & $<.05$ & $<.20$ & $<.20$ & -- & $<.05$ & $<.20$ & $<.20$ & -- & -- & -- & -- & -- & -- & -- & $<.05$ & $<.20$ & $<.20$ \\
\hline 401152088454801 & DeWitt Co., IL & 09/11/97 & $<.05$ & $<.20$ & $<.20$ & -- & $<.05$ & $<.20$ & $<.20$ & -- & -- & -- & -- & -- & -- & -- & $<.05$ & .86 & $<.20$ \\
\hline 401203089203201 & Logan Co., IL & $10 / 29 / 97$ & $<.05$ & $<.20$ & $<.20$ & -- & $<.05$ & $<.20$ & $<.20$ & -- & -- & -- & -- & -- & -- & -- & $<.05$ & $<.20$ & $<.20$ \\
\hline 401226088234101 & Champaign Co., IL & 09/25/97 & $<.05$ & $<.20$ & $<.20$ & -- & $<.05$ & $<.20$ & $<.20$ & -- & -- & -- & -- & -- & -- & -- & $<.05$ & $<.20$ & $<.20$ \\
\hline \multirow[t]{2}{*}{401246088585101} & DeWitt Co., IL & 06/30/98 & $<.05$ & $<.20$ & $<.20$ & -- & $<.05$ & $<.20$ & $<.20$ & -- & -- & -- & -- & -- & -- & -- & $<.05$ & $<.20$ & $<.20$ \\
\hline & & 05/28/02 & -- & $<.05$ & $<.05$ & $<.05$ & -- & $<.05$ & $<.05$ & $<.05$ & -- & $<.05$ & $<.05$ & -- & $<.05$ & $<.05$ & -- & $<.05$ & $<.05$ \\
\hline 401322089153901 & Logan Co., IL & 08/13/97 & $<.05$ & $<.20$ & $<.20$ & -- & $<.05$ & $<.20$ & $<.20$ & -- & -- & -- & -- & -- & -- & -- & $<.05$ & 11 & $<.20$ \\
\hline 401327089025201 & DeWitt Co., IL & 09/18/97 & $<.05$ & $<.20$ & $<.20$ & -- & $<.05$ & $<.20$ & $<.20$ & -- & -- & -- & -- & -- & -- & -- & $<.05$ & 2.5 & .39 \\
\hline 401508088293201 & Piatt Co., IL & 08/27/97 & $<.05$ & $<.20$ & $<.20$ & -- & $<.05$ & $<.20$ & $<.20$ & -- & -- & -- & -- & -- & -- & -- & $<.05$ & $<.20$ & $<.20$ \\
\hline 401601089154401 & Logan Co., IL & $10 / 28 / 97$ & $<.05$ & $<.20$ & $<.20$ & -- & $<.05$ & $<.20$ & $<.20$ & -- & -- & -- & -- & -- & -- & -- & $<.05$ & $<.20$ & $<.20$ \\
\hline 401751088214101 & Champaign, IL & 09/08/97 & $<.05$ & $<.20$ & $<.20$ & -- & $<.05$ & $<.20$ & $<.20$ & -- & -- & -- & -- & -- & -- & -- & $<.05$ & $<.20$ & $<.20$ \\
\hline 401805088361801 & McLean Co., IL & 08/27/97 & $<.05$ & $<.20$ & $<.20$ & -- & $<.05$ & $<.20$ & $<.20$ & -- & -- & -- & -- & -- & -- & -- & $<.05$ & $<.20$ & $<.20$ \\
\hline 401810089010101 & McLean Co., IL & 09/10/97 & $<.05$ & $<.20$ & $<.20$ & -- & $<.05$ & $<.20$ & $<.20$ & -- & -- & -- & -- & -- & -- & -- & $<.05$ & $<.20$ & $<.20$ \\
\hline
\end{tabular}




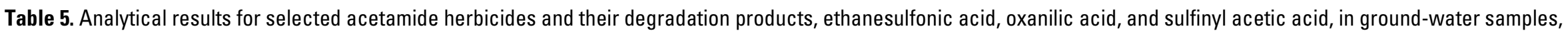
1994-2002.-Continued

[OGRL, U.S. Geological Survey Organic Geochemistry Research Laboratory, Lawrence, Kansas; ESA, ethanesulfonic acid; OXA, oxanilic acid; SAA, sulfinyl acetic acid; --, no data; <, less than]

\begin{tabular}{|c|c|c|c|c|c|c|c|c|c|c|c|c|c|c|c|c|c|c|c|c|}
\hline Site number & Site location & $\begin{array}{l}\text { Date of col- } \\
\text { lection } \\
\text { (month/day/ } \\
\text { year) }\end{array}$ & $\begin{array}{l}\text { Aceto- } \\
\text { chlor } \\
(\mu \mathrm{g} / \mathrm{L})\end{array}$ & $\begin{array}{l}\text { Aceto- } \\
\text { chlor } \\
\text { ESA } \\
(\mu \mathrm{g} / \mathrm{L})\end{array}$ & $\begin{array}{l}\text { Aceto- } \\
\text { chlor } \\
\text { OXA } \\
(\mu \mathrm{g} / \mathrm{L})\end{array}$ & $\begin{array}{l}\text { Aceto- } \\
\text { chlor } \\
\text { SAA } \\
(\mu \mathrm{g} / \mathrm{L})\end{array}$ & $\begin{array}{l}\text { Ala- } \\
\text { chlor } \\
(\mu \mathrm{g} / \mathrm{L})\end{array}$ & $\begin{array}{l}\text { Ala- } \\
\text { chlor } \\
\text { ESA } \\
(\mu \mathrm{g} / \mathrm{L})\end{array}$ & $\begin{array}{l}\text { Ala- } \\
\text { chlor } \\
\text { OXA } \\
(\mu \mathrm{g} / \mathrm{L})\end{array}$ & $\begin{array}{l}\text { Ala- } \\
\text { chlor } \\
\text { SAA } \\
(\mu \mathrm{g} / \mathrm{L})\end{array}$ & $\begin{array}{c}\text { Di- } \\
\text { meth- } \\
\text { enamid } \\
(\mu \mathrm{g} / \mathrm{L})\end{array}$ & $\begin{array}{c}\text { Di- } \\
\text { meth- } \\
\text { enamid } \\
\text { ESA } \\
(\mu \mathrm{g} / \mathrm{L})\end{array}$ & $\begin{array}{c}\text { Di- } \\
\text { meth- } \\
\text { enamid } \\
\text { OXA } \\
(\mu \mathrm{g} / \mathrm{L})\end{array}$ & $\begin{array}{c}\text { Flufen- } \\
\text { acet } \\
(\mu \mathrm{g} / \mathrm{L})\end{array}$ & $\begin{array}{c}\text { Flufen- } \\
\text { acet } \\
\text { ESA } \\
(\mu \mathrm{g} / \mathrm{L})\end{array}$ & $\begin{array}{c}\text { Flufen- } \\
\text { acet } \\
\text { OXA } \\
(\mu \mathrm{g} / \mathrm{L})\end{array}$ & $\begin{array}{l}\text { Meto- } \\
\text { lachlor } \\
(\mu \mathrm{g} / \mathrm{L})\end{array}$ & $\begin{array}{l}\text { Meto- } \\
\text { lachlor } \\
\text { ESA } \\
(\mu \mathrm{g} / \mathrm{L})\end{array}$ & $\begin{array}{l}\text { Meto- } \\
\text { lachlor } \\
\text { OXA } \\
(\mu \mathrm{g} / \mathrm{L})\end{array}$ & 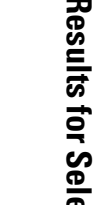 \\
\hline \multicolumn{20}{|c|}{ National Water-Quality Assessment Program (NAWOA) (fig. 3)—Continued } & \\
\hline \multicolumn{20}{|c|}{ Lower and Upper Illinois River Basin (LIRB/UIRB/KWI)—Continued } & \\
\hline 040181308916560 & Logan Co., IL & $10 / 28 / 97$ & $<0.05$ & $<0.20$ & $<0.20$ & -- & $<0.05$ & $<0.20$ & $<0.20$ & -- & -- & -- & -- & -- & -- & -- & $<0.05$ & $<0.20$ & $<0.20$ & \\
\hline \multirow[t]{2}{*}{402029088172001} & Champaign Co., IL & $06 / 22 / 98$ & $<.05$ & $<.20$ & $<.20$ & -- & $<.05$ & $<.20$ & $<.20$ & -- & -- & -- & -- & -- & -- & -- & $<.05$ & $<.20$ & $<.20$ & \\
\hline & & 06/05/02 & -- & $<.05$ & $<.05$ & $<0.05$ & -- & $<.05$ & $<.05$ & $<0.05$ & -- & $<0.05$ & $<0.05$ & -- & $<0.05$ & $<0.05$ & -- & $<.05$ & $<.05$ & \\
\hline 402059088571701 & McLean Co., IL & 09/17/97 & $<.05$ & $<.20$ & $<.20$ & -- & $<.05$ & $<.20$ & $<.20$ & -- & -- & -- & -- & -- & -- & -- & $<.05$ & $<.20$ & $<.20$ & \\
\hline 402122088161101 & Champaign Co., IL & 06/11/98 & $<.05$ & $<.20$ & $<.20$ & -- & $<.05$ & $<.20$ & $<.20$ & -- & -- & -- & -- & -- & -- & -- & $<.05$ & $<.20$ & $<.20$ & \\
\hline 402210088215701 & Champaign Co., IL & $09 / 15 / 97$ & $<.05$ & $<.20$ & $<.20$ & -- & $<.05$ & $<.20$ & $<.20$ & -- & -- & -- & -- & -- & -- & -- & $<.05$ & $<.20$ & $<.20$ & \\
\hline 402248088150501 & Champaign Co., IL & 05/13/98 & $<.05$ & $<.20$ & $<.20$ & -- & $<.05$ & $<.20$ & $<.20$ & -- & -- & -- & -- & -- & -- & -- & $<.05$ & $<.20$ & $<.20$ & \\
\hline 402250088551201 & McLean Co., IL & 08/27/97 & $<.05$ & $<.20$ & .21 & -- & $<.05$ & $<.20$ & $<.20$ & -- & -- & -- & -- & -- & -- & -- & $<.05$ & $<.20$ & $<.20$ & \multirow{7}{*}{ 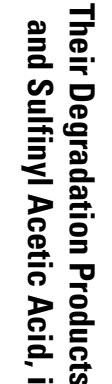 } \\
\hline 402304089032601 & McLean Co., IL & 08/20/97 & $<.05$ & $<.20$ & $<.20$ & -- & $<.05$ & $<.20$ & $<.20$ & -- & -- & -- & -- & -- & -- & -- & $<.05$ & 32 & 3.0 & \\
\hline 402727088464201 & McLean Co., IL & 08/05/97 & $<.05$ & $<.20$ & .65 & -- & $<.05$ & $<.20$ & $<.20$ & -- & -- & -- & -- & -- & -- & -- & $<.05$ & $<.20$ & $<.20$ & \\
\hline 404737089093201 & Woodford Co., IL & 08/06/97 & $<.05$ & $<.20$ & $<.20$ & -- & $<.05$ & $<.20$ & $<.20$ & -- & -- & -- & -- & -- & -- & -- & $<.05$ & $<.20$ & $<.20$ & \\
\hline 404849088281001 & Livingston Co., IL & 08/04/97 & $<.05$ & $<.20$ & $<.20$ & -- & $<.05$ & $<.20$ & $<.20$ & -- & -- & -- & -- & -- & -- & -- & $<.05$ & $<.20$ & $<.20$ & \\
\hline 405100089214701 & Woodford Co., IL & 07/30/97 & $<.05$ & $<.20$ & $<.20$ & -- & $<.05$ & $<.20$ & $<.20$ & -- & -- & -- & -- & -- & -- & -- & $<.05$ & $<.20$ & $<.20$ & \\
\hline 405145087044701 & Jasper Co., IN & 07/07/99 & $<.05$ & $<.20$ & $<.20$ & -- & $<.05$ & .51 & $<.20$ & -- & -- & -- & -- & -- & -- & -- & $<.05$ & .51 & $<.20$ & \\
\hline 405145087044702 & Jasper Co., IN & $08 / 29 / 00$ & -- & $<.05$ & $<.05$ & -- & -- & .07 & $<.05$ & -- & -- & $<.05$ & $<.05$ & -- & $<.05$ & $<.05$ & -- & .06 & $<.05$ & \\
\hline 405343087091501 & Jasper Co., IN & 09/09/99 & $<.05$ & .21 & $<.20$ & -- & $<.05$ & .23 & $<.20$ & -- & -- & -- & -- & -- & -- & -- & $<.05$ & .82 & .31 & \\
\hline 405345087100601 & Jasper Co., IN & $08 / 21 / 00$ & -- & $<.05$ & $<.05$ & -- & -- & $<.05$ & $<.05$ & -- & -- & $<.05$ & $<.05$ & -- & $<.05$ & $<.05$ & -- & $<.05$ & $<.05$ & \\
\hline 405357087495001 & Iroquois Co., IL & $09 / 28 / 00$ & -- & $<.05$ & $<.05$ & -- & -- & $<.05$ & $<.05$ & -- & -- & $<.05$ & $<.05$ & -- & $<.05$ & $<.05$ & -- & $<.05$ & $<.05$ & \\
\hline 405458087484601 & Iroquois Co., IL & 09/08/99 & $<.05$ & $<.20$ & $<.20$ & -- & $<.05$ & $<.20$ & $<.20$ & -- & -- & -- & -- & -- & -- & -- & $<.05$ & $<.20$ & $<.20$ & \\
\hline 405524087152701 & Jasper, IN & 07/08/99 & $<.05$ & $<.20$ & $<.20$ & -- & $<.05$ & $<.20$ & $<.20$ & -- & -- & -- & -- & -- & -- & -- & $<.05$ & $<.20$ & $<.20$ & 릉 믈 \\
\hline 405627087142101 & Jasper Co., IN & $09 / 25 / 00$ & -- & $<.05$ & $<.05$ & -- & -- & $<.05$ & $<.05$ & -- & -- & $<.05$ & $<.05$ & -- & $<.05$ & $<.05$ & -- & $<.05$ & $<.05$ & \\
\hline 405732087282301 & Newton Co., IN & 07/19/99 & $<.05$ & $<.20$ & $<.20$ & -- & $<.05$ & $<.20$ & $<.20$ & -- & -- & -- & -- & -- & -- & -- & $<.05$ & $<.20$ & $<.20$ & \\
\hline 405823087313101 & Newton Co., IN & $08 / 24 / 00$ & -- & $<.05$ & $<.05$ & -- & -- & $<.05$ & $<.05$ & -- & -- & $<.05$ & $<.05$ & -- & $<.05$ & $<.05$ & -- & $<.05$ & $<.05$ & \\
\hline 405832088592001 & LaSalle Co., IL & $07 / 24 / 97$ & $<.05$ & $<.20$ & $<.20$ & -- & $<.05$ & $<.20$ & $<.20$ & -- & -- & -- & -- & -- & -- & -- & $<.05$ & $<.20$ & $<.20$ & \\
\hline 405837089033701 & Marshall, IL & $05 / 20 / 98$ & $<.05$ & $<.20$ & $<.20$ & -- & $<.05$ & $<.20$ & $<.20$ & -- & -- & -- & -- & -- & -- & -- & $<.05$ & $<.20$ & $<.20$ & \\
\hline
\end{tabular}




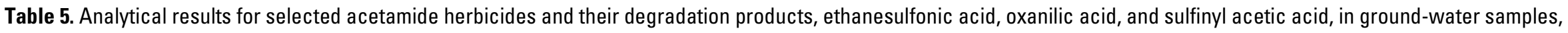
1994-2002.-Continued

[OGRL, U.S. Geological Survey Organic Geochemistry Research Laboratory, Lawrence, Kansas; ESA, ethanesulfonic acid; OXA, oxanilic acid; SAA, sulfinyl acetic acid; --, no data; <, less than]

\begin{tabular}{|c|c|c|c|c|c|c|c|c|c|c|c|c|c|c|c|c|c|c|c|}
\hline Site number & Site location & $\begin{array}{l}\text { Date of col- } \\
\text { lection } \\
\text { (month/day/ } \\
\text { year) }\end{array}$ & $\begin{array}{l}\text { Aceto- } \\
\text { chlor } \\
(\mu \mathrm{g} / \mathrm{L})\end{array}$ & $\begin{array}{l}\text { Aceto- } \\
\text { chlor } \\
\text { ESA } \\
(\mu \mathrm{g} / \mathrm{L})\end{array}$ & $\begin{array}{l}\text { Aceto- } \\
\text { chlor } \\
\text { OXA } \\
(\mu \mathrm{g} / \mathrm{L})\end{array}$ & $\begin{array}{l}\text { Aceto- } \\
\text { chlor } \\
\text { SAA } \\
(\mu \mathrm{g} / \mathrm{L})\end{array}$ & $\begin{array}{l}\text { Ala- } \\
\text { chlor } \\
(\mu \mathrm{g} / \mathrm{L})\end{array}$ & $\begin{array}{l}\text { Ala- } \\
\text { chlor } \\
\text { ESA } \\
(\mu \mathrm{g} / \mathrm{L})\end{array}$ & $\begin{array}{l}\text { Ala- } \\
\text { chlor } \\
\text { OXA } \\
(\mu \mathrm{g} / \mathrm{L})\end{array}$ & $\begin{array}{l}\text { Ala- } \\
\text { chlor } \\
\text { SAA } \\
(\mu \mathrm{g} / \mathrm{L})\end{array}$ & $\begin{array}{c}\text { Di- } \\
\text { meth- } \\
\text { enamid } \\
(\mu \mathrm{g} / \mathrm{L})\end{array}$ & $\begin{array}{c}\text { Di- } \\
\text { meth- } \\
\text { enamid } \\
\text { ESA } \\
(\mu \mathrm{g} / \mathrm{L})\end{array}$ & $\begin{array}{c}\text { Di- } \\
\text { meth- } \\
\text { enamid } \\
\text { OXA } \\
(\mu \mathrm{g} / \mathrm{L})\end{array}$ & $\begin{array}{c}\text { Flufen- } \\
\text { acet } \\
(\mu \mathrm{g} / \mathrm{L})\end{array}$ & $\begin{array}{c}\text { Flufen- } \\
\text { acet } \\
\text { ESA } \\
(\mu \mathrm{g} / \mathrm{L})\end{array}$ & $\begin{array}{c}\text { Flufen- } \\
\text { acet } \\
\text { OXA } \\
(\mu \mathrm{g} / \mathrm{L})\end{array}$ & $\begin{array}{l}\text { Meto- } \\
\text { lachlor } \\
(\mu \mathrm{g} / \mathrm{L})\end{array}$ & $\begin{array}{l}\text { Meto- } \\
\text { lachlor } \\
\text { ESA } \\
(\mu \mathrm{g} / \mathrm{L})\end{array}$ & $\begin{array}{l}\text { Meto- } \\
\text { lachlor } \\
\text { OXA } \\
(\mu \mathrm{g} / \mathrm{L})\end{array}$ \\
\hline \multicolumn{20}{|c|}{ National Water-Quality Assessment Program (NAWOA) (fig. 3)—Continued } \\
\hline \multicolumn{20}{|c|}{ Lower and Upper Illinois River Basin (LIRB/UIRB/KWI)—Continued } \\
\hline 410025087280701 & Newton Co., IN & $10 / 03 / 00$ & -- & $<0.05$ & $<0.05$ & -- & -- & $<0.05$ & $<0.05$ & -- & -- & $<0.05$ & $<0.05$ & -- & $<0.05$ & $<0.05$ & -- & $<0.05$ & $<0.05$ \\
\hline \multirow[t]{2}{*}{410030087263101} & Newton Co., IN & 07/19/99 & $<0.05$ & $<.20$ & $<.20$ & -- & $<0.05$ & .32 & $<.20$ & -- & -- & -- & -- & -- & -- & -- & $<0.05$ & .63 & $<.20$ \\
\hline & & 06/17/02 & -- & .05 & $<.05$ & $<0.05$ & -- & .10 & $<.05$ & $<0.05$ & -- & $<.05$ & $<.05$ & -- & $<.05$ & $<.05$ & -- & .44 & .06 \\
\hline 410030087263102 & Newton Co., IN & $09 / 11 / 00$ & -- & $<.05$ & $<.05$ & -- & -- & .09 & $<.05$ & -- & -- & $<.05$ & $<.05$ & -- & $<.05$ & $<.05$ & -- & 5.2 & 3.1 \\
\hline 410123087385601 & Kankakee Co., IL & $07 / 21 / 99$ & $<.05$ & $<.20$ & $<.20$ & -- & .92 & $<.20$ & $<.20$ & -- & -- & -- & -- & -- & -- & -- & .53 & $<.20$ & $<.20$ \\
\hline \multirow[t]{2}{*}{410253087280701} & Newton Co., IN & $07 / 22 / 99$ & $<.05$ & $<.20$ & $<.20$ & -- & $<.05$ & $<.20$ & $<.20$ & -- & -- & -- & -- & -- & -- & -- & $<.05$ & $<.20$ & $<.20$ \\
\hline & & 06/13/02 & -- & $<.05$ & $<.05$ & $<.05$ & -- & $<.05$ & $<.05$ & $<.05$ & -- & $<.05$ & $<.05$ & -- & $<.05$ & $<.05$ & -- & $<.05$ & $<.05$ \\
\hline 410309087381501 & Kankakee Co., IL & $07 / 14 / 99$ & $<.05$ & .30 & $<.20$ & -- & $<.05$ & $<.20$ & $<.20$ & -- & -- & -- & -- & -- & -- & -- & $<.05$ & $<.20$ & $<.20$ \\
\hline 410407087200201 & Newton Co., IN & $10 / 05 / 00$ & -- & $<.05$ & .06 & -- & -- & .96 & $<.05$ & -- & -- & $<.05$ & $<.05$ & -- & $<.05$ & $<.05$ & -- & 2.4 & .95 \\
\hline 410422088000001 & Kankakee., IL & 07/13/99 & $<.05$ & .23 & $<.20$ & -- & $<.05$ & $<.20$ & $<.20$ & -- & -- & -- & -- & -- & -- & -- & $<.05$ & $<.20$ & $<.20$ \\
\hline 410502089392501 & Stark Co., IL & $07 / 21 / 97$ & $<.05$ & $<.20$ & $<.20$ & -- & $<.05$ & $<.20$ & $<.20$ & -- & -- & -- & -- & -- & -- & -- & $<.05$ & $<.20$ & $<.20$ \\
\hline \multirow[t]{2}{*}{410505087201201} & Newton Co., IN & 07/20/99 & $<.05$ & $<.20$ & $<.20$ & -- & $<.05$ & $<.20$ & $<.20$ & -- & -- & -- & -- & -- & -- & -- & $<.05$ & $<.20$ & $<.20$ \\
\hline & & 07/20/99 & $<.05$ & $<.20$ & $<.20$ & -- & $<.05$ & $<.20$ & $<.20$ & -- & -- & -- & -- & -- & -- & -- & $<.05$ & $<.20$ & $<.20$ \\
\hline 410510087224201 & Newton Co., IN & 06/29/99 & $<.05$ & $<.20$ & $<.20$ & -- & $<.05$ & $<.20$ & $<.20$ & -- & -- & -- & -- & -- & -- & -- & $<.05$ & $<.20$ & $<.20$ \\
\hline 410511087295201 & Newton Co., IN & $08 / 24 / 99$ & $<.05$ & $<.20$ & $<.20$ & -- & $<.05$ & $<.20$ & $<.20$ & -- & -- & -- & -- & -- & -- & -- & $<.05$ & $<.20$ & $<.20$ \\
\hline 410533087232601 & Newton Co., IN & $10 / 04 / 00$ & -- & $<.05$ & $<.05$ & -- & -- & .27 & $<.05$ & -- & -- & $<.05$ & $<.05$ & -- & $<.05$ & $<.05$ & -- & $<.05$ & $<.05$ \\
\hline 410541087313701 & Newton Co., IN & $09 / 27 / 00$ & -- & $<.05$ & $<.05$ & -- & -- & 3.6 & $<.05$ & -- & -- & $<.05$ & $<.05$ & -- & $<.05$ & $<.05$ & -- & 5.2 & 2.6 \\
\hline 410600087113501 & Jasper Co., IN & 06/29/99 & $<.05$ & $<.20$ & $<.20$ & -- & $<.05$ & $<.20$ & $<.20$ & -- & -- & -- & -- & -- & -- & -- & $<.05$ & $<.20$ & $<.20$ \\
\hline 410625088443101 & Livingston Co., IL & 08/14/97 & $<.05$ & $<.20$ & .37 & -- & $<.05$ & $<.20$ & $<.20$ & -- & -- & -- & -- & -- & -- & -- & $<.05$ & $<.20$ & $<.20$ \\
\hline 410656087092901 & Jasper Co., IN & $10 / 10 / 00$ & -- & $<.05$ & $<.05$ & -- & -- & .37 & $<.05$ & -- & -- & $<.05$ & $<.05$ & -- & $<.05$ & $<.05$ & -- & .34 & .08 \\
\hline 410700087305801 & Newton Co., IN & $09 / 21 / 00$ & -- & $<.05$ & $<.05$ & -- & -- & $<.05$ & 1.0 & -- & -- & $<.05$ & $<.05$ & -- & $<.05$ & $<.05$ & -- & $<.05$ & .05 \\
\hline 410702087310201 & Newton Co., IN & $07 / 21 / 99$ & $<.05$ & $<.20$ & $<.20$ & -- & $<.05$ & $<.20$ & $<.20$ & -- & -- & -- & -- & -- & -- & -- & $<.05$ & $<.20$ & $<.20$ \\
\hline 410715087072001 & Jasper Co., IN & $10 / 16 / 00$ & -- & $<.05$ & $<.05$ & -- & -- & $<.05$ & $<.05$ & -- & -- & $<.05$ & $<.05$ & -- & $<.05$ & $<.05$ & -- & $<.05$ & $<.05$ \\
\hline 410715087072301 & Jasper Co., IN & 06/14/99 & $<.05$ & $<.20$ & $<.20$ & -- & $<.05$ & $<.20$ & $<.20$ & -- & -- & -- & -- & -- & -- & -- & $<.05$ & $<.20$ & $<.20$ \\
\hline 410748087060901 & Jasper Co., IN & 06/14/99 & $<.05$ & $<.20$ & $<.20$ & -- & $<.05$ & $<.20$ & $<.20$ & -- & -- & -- & -- & -- & -- & -- & $<.05$ & $<.20$ & $<.20$ \\
\hline
\end{tabular}




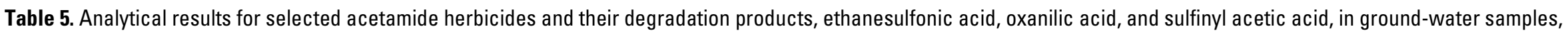
1994-2002.-Continued

[OGRL, U.S. Geological Survey Organic Geochemistry Research Laboratory, Lawrence, Kansas; ESA, ethanesulfonic acid; OXA, oxanilic acid; SAA, sulfinyl acetic acid; --, no data; <, less than]

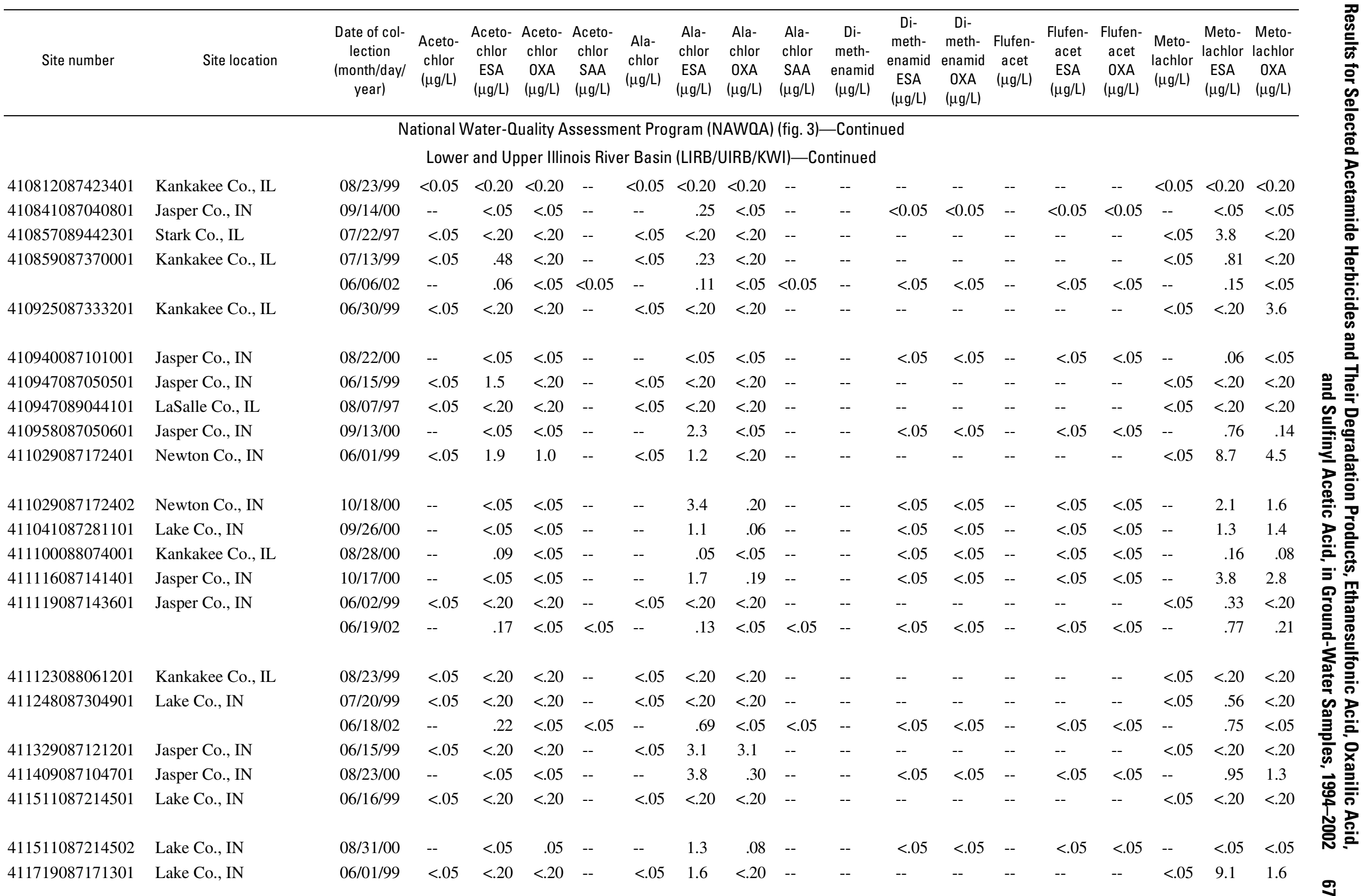




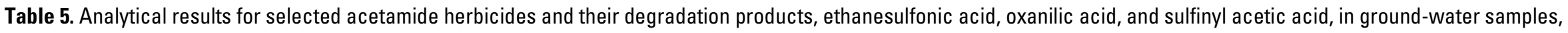
1994-2002.-Continued

[OGRL, U.S. Geological Survey Organic Geochemistry Research Laboratory, Lawrence, Kansas; ESA, ethanesulfonic acid; OXA, oxanilic acid; SAA, sulfinyl acetic acid; --, no data; <, less than]

\begin{tabular}{|c|c|c|c|c|c|c|c|c|c|c|c|c|c|c|c|c|c|c|c|}
\hline Site number & Site location & $\begin{array}{l}\text { Date of col- } \\
\text { lection } \\
\text { (month/day/ } \\
\text { year) }\end{array}$ & $\begin{array}{l}\text { Aceto- } \\
\text { chlor } \\
(\mu \mathrm{g} / \mathrm{L})\end{array}$ & $\begin{array}{l}\text { Aceto- } \\
\text { chlor } \\
\text { ESA } \\
(\mu \mathrm{g} / \mathrm{L})\end{array}$ & $\begin{array}{l}\text { Aceto- } \\
\text { chlor } \\
\text { OXA } \\
(\mu \mathrm{g} / \mathrm{L})\end{array}$ & $\begin{array}{l}\text { Aceto- } \\
\text { chlor } \\
\text { SAA } \\
(\mu \mathrm{g} / \mathrm{L})\end{array}$ & $\begin{array}{l}\text { Ala- } \\
\text { chlor } \\
(\mu \mathrm{g} / \mathrm{L})\end{array}$ & $\begin{array}{l}\text { Ala- } \\
\text { chlor } \\
\text { ESA } \\
(\mu \mathrm{g} / \mathrm{L})\end{array}$ & $\begin{array}{l}\text { Ala- } \\
\text { chlor } \\
\text { OXA } \\
(\mu \mathrm{g} / \mathrm{L})\end{array}$ & $\begin{array}{l}\text { Ala- } \\
\text { chlor } \\
\text { SAA } \\
(\mu \mathrm{g} / \mathrm{L})\end{array}$ & $\begin{array}{c}\text { Di- } \\
\text { meth- } \\
\text { enamid } \\
(\mu \mathrm{g} / \mathrm{L})\end{array}$ & $\begin{array}{c}\text { Di- } \\
\text { meth- } \\
\text { enamid } \\
\text { ESA } \\
(\mu \mathrm{g} / \mathrm{L})\end{array}$ & $\begin{array}{c}\text { Di- } \\
\text { meth- } \\
\text { enamid } \\
\text { OXA } \\
(\mu \mathrm{g} / \mathrm{L})\end{array}$ & $\begin{array}{c}\text { Flufen- } \\
\text { acet } \\
(\mu \mathrm{g} / \mathrm{L})\end{array}$ & $\begin{array}{c}\text { Flufen- } \\
\text { acet } \\
\text { ESA } \\
(\mu \mathrm{g} / \mathrm{L})\end{array}$ & $\begin{array}{c}\text { Flufen- } \\
\text { acet } \\
\text { OXA } \\
(\mu \mathrm{g} / \mathrm{L})\end{array}$ & $\begin{array}{l}\text { Meto- } \\
\text { lachlor } \\
(\mu \mathrm{g} / \mathrm{L})\end{array}$ & $\begin{array}{l}\text { Meto- } \\
\text { lachlor } \\
\text { ESA } \\
(\mu \mathrm{g} / \mathrm{L})\end{array}$ & $\begin{array}{l}\text { Meto- } \\
\text { lachlor } \\
\text { OXA } \\
(\mu \mathrm{g} / \mathrm{L})\end{array}$ \\
\hline \multicolumn{20}{|c|}{ National Water-Quality Assessment Program (NAWOA) (fig. 3)—Continued } \\
\hline \multicolumn{20}{|c|}{ Lower and Upper Illinois River Basin (LIRB/UIRB/KWI)—Continued } \\
\hline 411719087171302 & Lake Co., IN & $09 / 12 / 00$ & -- & $<0.05$ & $<0.05$ & -- & -- & $<0.05$ & $<0.05$ & -- & -- & $<0.05$ & $<0.05$ & -- & $<0.05$ & $<0.05$ & -- & $<0.05$ & $<0.05$ \\
\hline \multirow[t]{2}{*}{412136088571001} & LaSalle Co., IL & $07 / 17 / 97$ & $<0.05$ & $<.20$ & $<.20$ & -- & $<0.05$ & $<.20$ & $<.20$ & -- & -- & -- & -- & -- & -- & -- & $<0.05$ & $<.20$ & $<.20$ \\
\hline & & 07/12/99 & $<.05$ & .28 & $<.20$ & -- & $<.05$ & $<.20$ & $<.20$ & -- & -- & -- & -- & -- & -- & -- & $<.05$ & 2.3 & $<.20$ \\
\hline \multirow[t]{2}{*}{415752088244001} & Kane Co., IL & $05 / 24 / 00$ & -- & $<.05$ & $<.05$ & -- & -- & .22 & $<.05$ & -- & -- & $<.05$ & $<.05$ & -- & $<.05$ & $<.05$ & -- & .65 & $<.05$ \\
\hline & & $06 / 24 / 02$ & -- & $<.05$ & $<.05$ & $<0.05$ & -- & .06 & $<.05$ & $<0.05$ & -- & $<.05$ & $<.05$ & -- & $<.05$ & $<.05$ & -- & .43 & $<.05$ \\
\hline 420012088191101 & Kane Co., IL & $05 / 11 / 00$ & -- & $<.05$ & .36 & -- & -- & .53 & .43 & -- & -- & $<.05$ & $<.05$ & -- & $<.05$ & $<.05$ & -- & 1.3 & 1.7 \\
\hline 420114088141801 & Cook Co., IL & 06/20/00 & -- & $<.05$ & $<.05$ & -- & -- & $<.05$ & $<.05$ & -- & -- & $<.05$ & $<.05$ & -- & $<.05$ & $<.05$ & -- & .10 & $<.05$ \\
\hline 421052088184101 & McHenry Co., IL & $05 / 23 / 00$ & -- & $<.05$ & $<.05$ & -- & -- & $<.05$ & $<.05$ & -- & -- & $<.05$ & $<.05$ & -- & $<.05$ & $<.05$ & -- & $<.05$ & $<.05$ \\
\hline 421228088160701 & McHenry Co., IL & 04/18/00 & -- & $<.05$ & $<.05$ & -- & -- & .08 & $<.05$ & -- & -- & $<.05$ & $<.05$ & -- & $<.05$ & $<.05$ & -- & $<.05$ & $<.05$ \\
\hline 421301088191501 & McHenry Co., IL & 06/08/00 & -- & $<.05$ & $<.05$ & -- & -- & $<.05$ & $<.05$ & -- & -- & $<.05$ & $<.05$ & -- & $<.05$ & $<.05$ & -- & $<.05$ & $<.05$ \\
\hline 421324088105301 & McHenry Co., IL & 03/26/01 & -- & $<.05$ & $<.05$ & -- & -- & $<.05$ & $<.05$ & -- & -- & $<.05$ & $<.05$ & -- & $<.05$ & $<.05$ & -- & $<.05$ & $<.05$ \\
\hline 421402088173501 & McHenry Co., IL & $04 / 20 / 00$ & -- & $<.05$ & $<.05$ & -- & -- & $<.05$ & $<.05$ & -- & -- & $<.05$ & $<.05$ & -- & $<.05$ & $<.05$ & -- & $<.05$ & $<.05$ \\
\hline 421422088174001 & McHenry Co., IL & $05 / 15 / 01$ & -- & $<.05$ & $<.05$ & -- & -- & $<.05$ & $<.05$ & -- & -- & $<.05$ & $<.05$ & -- & $<.05$ & $<.05$ & -- & $<.05$ & $<.05$ \\
\hline 421545087564301 & Lake Co., IN & 05/10/00 & -- & $<.05$ & $<.05$ & -- & -- & $<.05$ & $<.05$ & -- & -- & $<.05$ & $<.05$ & -- & $<.05$ & $<.05$ & -- & $<.05$ & $<.05$ \\
\hline \multirow[t]{2}{*}{421633088125801} & McHenry Co., IL & 04/19/00 & -- & .06 & $<.05$ & -- & -- & .22 & $<.05$ & -- & -- & $<.05$ & $<.05$ & -- & $<.05$ & $<.05$ & -- & .31 & $<.05$ \\
\hline & & $06 / 25 / 02$ & -- & $<.05$ & $<.05$ & $<.05$ & -- & .23 & $<.05$ & $<.05$ & -- & $<.05$ & $<.05$ & -- & $<.05$ & $<.05$ & -- & .26 & $<.05$ \\
\hline 421651088190601 & McHenry Co., IL & 05/16/01 & -- & $<.05$ & $<.05$ & -- & -- & $<.05$ & $<.05$ & -- & -- & $<.05$ & $<.05$ & -- & $<.05$ & $<.05$ & -- & $<.05$ & $<.05$ \\
\hline 421850088252301 & McHenry Co., IL & 04/09/01 & -- & $<.05$ & $<.05$ & -- & -- & $<.05$ & $<.05$ & -- & -- & $<.05$ & $<.05$ & -- & $<.05$ & $<.05$ & -- & $<.05$ & $<.05$ \\
\hline 422002088263001 & McHenry Co., IL & $05 / 22 / 00$ & -- & $<.05$ & $<.05$ & -- & -- & $<.05$ & $<.05$ & -- & -- & $<.05$ & $<.05$ & -- & $<.05$ & $<.05$ & -- & $<.05$ & $<.05$ \\
\hline 422050088194001 & McHenry Co., IL & 03/29/01 & -- & $<.05$ & $<.05$ & -- & -- & $<.05$ & $<.05$ & -- & -- & $<.05$ & $<.05$ & -- & $<.05$ & $<.05$ & -- & $<.05$ & $<.05$ \\
\hline 422111088154901 & McHenry Co., IL & 05/23/00 & -- & $<.05$ & $<.05$ & -- & -- & .21 & $<.05$ & -- & -- & $<.05$ & $<.05$ & -- & $<.05$ & $<.05$ & -- & $<.05$ & $<.05$ \\
\hline 422146088124301 & McHenry Co., IL & $04 / 24 / 01$ & -- & $<.05$ & $<.05$ & -- & -- & $<.05$ & $<.05$ & -- & -- & $<.05$ & $<.05$ & -- & $<.05$ & $<.05$ & -- & .17 & $<.05$ \\
\hline 422252087553001 & Lake Co., IN & 05/10/00 & -- & $<.05$ & $<.05$ & -- & -- & $<.05$ & $<.05$ & -- & -- & $<.05$ & $<.05$ & -- & $<.05$ & $<.05$ & -- & $<.05$ & $<.05$ \\
\hline 422314088140001 & McHenry Co., IL & 05/09/00 & -- & $<.05$ & $<.05$ & -- & -- & $<.05$ & $<.05$ & -- & -- & $<.05$ & $<.05$ & -- & $<.05$ & $<.05$ & -- & $<.05$ & $<.05$ \\
\hline 422917088132901 & McHenry Co., IL & $04 / 26 / 01$ & -- & $<.05$ & $<.05$ & -- & -- & $<.05$ & $<.05$ & -- & -- & $<.05$ & $<.05$ & -- & $<.05$ & $<.05$ & -- & $<.05$ & $<.05$ \\
\hline
\end{tabular}




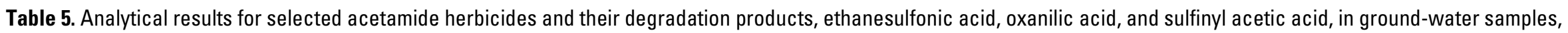
1994-2002.-Continued

[OGRL, U.S. Geological Survey Organic Geochemistry Research Laboratory, Lawrence, Kansas; ESA, ethanesulfonic acid; OXA, oxanilic acid; SAA, sulfinyl acetic acid; --, no data; <, less than]

\begin{tabular}{|c|c|c|c|c|c|c|c|c|c|c|c|c|c|c|c|c|c|c|c|c|}
\hline Site number & Site location & $\begin{array}{l}\text { Date of col- } \\
\text { lection } \\
\text { (month/day/ } \\
\text { year) }\end{array}$ & $\begin{array}{l}\text { Aceto- } \\
\text { chlor } \\
(\mu \mathrm{g} / \mathrm{L})\end{array}$ & $\begin{array}{l}\text { Aceto- } \\
\text { chlor } \\
\text { ESA } \\
(\mu \mathrm{g} / \mathrm{L})\end{array}$ & $\begin{array}{l}\text { Aceto- } \\
\text { chlor } \\
\text { OXA } \\
(\mu \mathrm{g} / \mathrm{L})\end{array}$ & $\begin{array}{l}\text { Aceto- } \\
\text { chlor } \\
\text { SAA } \\
(\mu \mathrm{g} / \mathrm{L})\end{array}$ & $\begin{array}{l}\text { Ala- } \\
\text { chlor } \\
(\mu \mathrm{g} / \mathrm{L})\end{array}$ & $\begin{array}{l}\text { Ala- } \\
\text { chlor } \\
\text { ESA } \\
(\mu \mathrm{g} / \mathrm{L})\end{array}$ & $\begin{array}{c}\text { Ala- } \\
\text { chlor } \\
\text { OXA } \\
(\mu \mathrm{g} / \mathrm{L})\end{array}$ & $\begin{array}{l}\text { Ala- } \\
\text { chlor } \\
\text { SAA } \\
(\mu \mathrm{g} / \mathrm{L})\end{array}$ & $\begin{array}{l}\text { Di- } \\
\text { meth- } \\
\text { enamid } \\
(\mu \mathrm{g} / \mathrm{L})\end{array}$ & $\begin{array}{c}\text { Di- } \\
\text { meth- } \\
\text { enamid } \\
\text { ESA } \\
(\mu \mathrm{g} / \mathrm{L})\end{array}$ & $\begin{array}{c}\text { Di- } \\
\text { meth- } \\
\text { enamid } \\
\text { OXA } \\
(\mu \mathrm{g} / \mathrm{L})\end{array}$ & $\begin{array}{l}\text { Flufen- } \\
\text { acet } \\
(\mu \mathrm{g} / \mathrm{L})\end{array}$ & $\begin{array}{l}\text { Flufen- } \\
\text { acet } \\
\text { ESA } \\
(\mu \mathrm{g} / \mathrm{L})\end{array}$ & $\begin{array}{l}\text { Flufen- } \\
\text { acet } \\
\text { OXA } \\
(\mu \mathrm{g} / \mathrm{L})\end{array}$ & $\begin{array}{l}\text { Meto- } \\
\text { lachlor } \\
(\mu \mathrm{g} / \mathrm{L})\end{array}$ & $\begin{array}{l}\text { Meto- } \\
\text { lachlor } \\
\text { ESA } \\
(\mu \mathrm{g} / \mathrm{L})\end{array}$ & $\begin{array}{l}\text { Meto- } \\
\text { lachlor } \\
\text { OXA } \\
(\mu \mathrm{g} / \mathrm{L})\end{array}$ & מי \\
\hline \multicolumn{20}{|c|}{ National Water-Quality Assessment Program (NAWQA) (fig. 3)—Continued } & \\
\hline \multicolumn{20}{|c|}{ Lower and Upper Illinois River Basin (LIRB/UIRB/KWI)—Continued } & \\
\hline 423007088305501 & Walworth Co., WI & 03/27/01 & -- & $<0.05$ & $<0.05$ & -- & -- & $<0.05$ & $<0.05$ & -- & -- & $<0.05$ & $<0.05$ & -- & $<0.05$ & $<0.05$ & -- & $<0.05$ & $<0.05$ & \\
\hline 423149088151101 & Kenosha Co., WI & $03 / 28 / 01$ & -- & $<.05$ & $<.05$ & -- & -- & $<.05$ & $<.05$ & -- & -- & $<.05$ & $<.05$ & -- & $<.05$ & $<.05$ & -- & $<.05$ & $<.05$ & \\
\hline 423423088233201 & Walworth Co., WI & $04 / 25 / 01$ & -- & $<.05$ & $<.05$ & -- & -- & $<.05$ & $<.05$ & -- & -- & $<.05$ & $<.05$ & -- & $<.05$ & $<.05$ & -- & $<.05$ & $<.05$ & \\
\hline 423454088303101 & Walworth Co., WI & 03/27/01 & -- & $<.05$ & $<.05$ & -- & -- & $<.05$ & $<.05$ & -- & -- & $<.05$ & $<.05$ & -- & $<.05$ & $<.05$ & -- & $<.05$ & $<.05$ & \\
\hline 423508088162701 & Kenosha Co., WI & 04/10/01 & -- & $<.05$ & $<.05$ & -- & -- & .18 & $<.05$ & -- & -- & $<.05$ & $<.05$ & -- & $<.05$ & $<.05$ & -- & $<.05$ & $<.05$ & \\
\hline 423508088162701 & Kenosha Co., WI & 04/10/01 & -- & $<.05$ & $<.05$ & -- & -- & .17 & $<.05$ & -- & -- & $<.05$ & $<.05$ & -- & $<.05$ & $<.05$ & -- & $<.05$ & $<.05$ & \\
\hline 423523088244901 & Walworth Co., WI & 05/09/00 & -- & $<.05$ & $<.05$ & -- & -- & .22 & .07 & -- & -- & $<.05$ & $<.05$ & -- & $<.05$ & $<.05$ & -- & .53 & .25 & \multirow{6}{*}{ 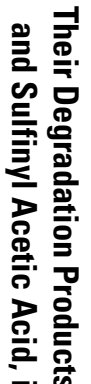 } \\
\hline 423741088252201 & Walworth Co., WI & 05/16/01 & -- & $<.05$ & $<.05$ & -- & -- & $<.05$ & $<.05$ & -- & -- & $<.05$ & $<.05$ & -- & $<.05$ & $<.05$ & -- & $<.05$ & $<.05$ & \\
\hline 423808088203801 & Walworth Co., WI & $04 / 25 / 01$ & -- & $<.05$ & $<.05$ & -- & -- & $<.05$ & $<.05$ & -- & -- & $<.05$ & $<.05$ & -- & $<.05$ & $<.05$ & -- & $<.05$ & $<.05$ & \\
\hline 424052088180101 & Racine Co., WI & 05/04/00 & -- & $<.05$ & $<.05$ & -- & -- & $<.05$ & $<.05$ & -- & -- & $<.05$ & $<.05$ & -- & $<.05$ & $<.05$ & -- & 2.9 & .62 & \\
\hline 424127088135601 & Racine Co., WI & 05/03/01 & -- & $<.05$ & $<.05$ & -- & -- & $<.05$ & $<.05$ & -- & -- & $<.05$ & $<.05$ & -- & $<.05$ & $<.05$ & -- & $<.05$ & $<.05$ & \\
\hline 424404088134601 & Racine Co., WI & 04/05/01 & -- & .13 & $<.05$ & -- & -- & $<.05$ & $<.05$ & -- & -- & $<.05$ & $<.05$ & -- & $<.05$ & $<.05$ & -- & $<.05$ & $<.05$ & \\
\hline \multirow[t]{2}{*}{424657088132001} & \multirow[t]{2}{*}{ Racine Co., WI } & 04/03/01 & -- & $<.05$ & $<.05$ & -- & -- & .05 & $<.05$ & -- & -- & $<.05$ & $<.05$ & -- & $<.05$ & $<.05$ & -- & .60 & $<.05$ & \multirow{4}{*}{ 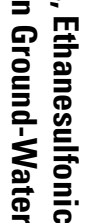 } \\
\hline & & 05/03/00 & -- & $<.05$ & $<.05$ & -- & -- & $<.05$ & $<.05$ & -- & -- & $<.05$ & $<.05$ & -- & $<.05$ & $<.05$ & -- & .44 & $<.05$ & \\
\hline 424756088194201 & Walworth Co., WI & 04/10/01 & -- & $<.05$ & $<.05$ & -- & -- & 1.3 & .06 & -- & -- & $<.05$ & $<.05$ & -- & $<.05$ & $<.05$ & -- & 3.2 & .64 & \\
\hline 424955088130901 & Racine Co., WI & 05/02/01 & -- & $<.05$ & $<.05$ & -- & -- & $<.05$ & $<.05$ & -- & -- & $<.05$ & $<.05$ & -- & $<.05$ & $<.05$ & -- & $<.05$ & $<.05$ & \\
\hline 425352088240101 & Waukesha Co., WI & 04/04/01 & -- & $<.05$ & $<.05$ & -- & -- & $<.05$ & $<.05$ & -- & -- & $<.05$ & $<.05$ & -- & $<.05$ & $<.05$ & -- & $<.05$ & $<.05$ & \\
\hline 425402088241601 & Waukesha Co., WI & 05/03/00 & -- & $<.05$ & $<.05$ & -- & -- & $<.05$ & $<.05$ & -- & -- & $<.05$ & $<.05$ & -- & $<.05$ & $<.05$ & -- & $<.05$ & $<.05$ & \\
\hline 425536088180901 & Waukesha Co., WI & $04 / 12 / 01$ & -- & $<.05$ & $<.05$ & -- & -- & $<.05$ & $<.05$ & -- & -- & $<.05$ & $<.05$ & -- & $<.05$ & $<.05$ & -- & $<.05$ & $<.05$ & \\
\hline 425656088124401 & Waukesha Co., WI & 05/02/01 & -- & $<.05$ & $<.05$ & -- & -- & $<.05$ & $<.05$ & -- & -- & $<.05$ & $<.05$ & -- & $<.05$ & $<.05$ & -- & .06 & $<.05$ & \\
\hline 425816088231401 & Waukesha Co., WI & 05/01/01 & -- & $<.05$ & $<.05$ & -- & -- & $<.05$ & $<.05$ & -- & -- & $<.05$ & $<.05$ & -- & $<.05$ & $<.05$ & -- & $<.05$ & $<.05$ & \\
\hline 425913088133801 & Waukesha Co., WI & 04/13/00 & -- & $<.05$ & $<.05$ & -- & -- & $<.05$ & $<.05$ & -- & -- & $<.05$ & $<.05$ & -- & $<.05$ & $<.05$ & -- & $<.05$ & $<.05$ & \\
\hline
\end{tabular}




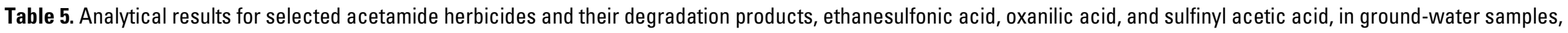
1994-2002.-Continued

[OGRL, U.S. Geological Survey Organic Geochemistry Research Laboratory, Lawrence, Kansas; ESA, ethanesulfonic acid; OXA, oxanilic acid; SAA, sulfinyl acetic acid; --, no data; <, less than]

\begin{tabular}{|c|c|c|c|c|c|c|c|c|c|c|c|c|c|c|c|c|c|c|c|}
\hline Site number & Site location & $\begin{array}{l}\text { Date of col- } \\
\text { lection } \\
\text { (month/day/ } \\
\text { year) }\end{array}$ & $\begin{array}{l}\text { Aceto- } \\
\text { chlor } \\
(\mu \mathrm{g} / \mathrm{L})\end{array}$ & $\begin{array}{l}\text { Aceto- } \\
\text { chlor } \\
\text { ESA } \\
(\mu \mathrm{g} / \mathrm{L})\end{array}$ & $\begin{array}{l}\text { Aceto- } \\
\text { chlor } \\
\text { OXA } \\
(\mu \mathrm{g} / \mathrm{L})\end{array}$ & $\begin{array}{l}\text { Aceto- } \\
\text { chlor } \\
\text { SAA } \\
(\mu \mathrm{g} / \mathrm{L})\end{array}$ & $\begin{array}{l}\text { Ala- } \\
\text { chlor } \\
(\mu \mathrm{g} / \mathrm{L})\end{array}$ & $\begin{array}{l}\text { Ala- } \\
\text { chlor } \\
\text { ESA } \\
(\mu \mathrm{g} / \mathrm{L})\end{array}$ & $\begin{array}{l}\text { Ala- } \\
\text { chlor } \\
\text { OXA } \\
(\mu \mathrm{g} / \mathrm{L})\end{array}$ & $\begin{array}{l}\text { Ala- } \\
\text { chlor } \\
\text { SAA } \\
(\mu \mathrm{g} / \mathrm{L})\end{array}$ & $\begin{array}{l}\text { Di- } \\
\text { meth- } \\
\text { enamid } \\
(\mu \mathrm{g} / \mathrm{L})\end{array}$ & $\begin{array}{c}\text { Di- } \\
\text { meth- } \\
\text { enamid } \\
\text { ESA } \\
(\mu \mathrm{g} / \mathrm{L})\end{array}$ & $\begin{array}{c}\text { Di- } \\
\text { meth- } \\
\text { enamid } \\
0 X A \\
(\mu \mathrm{g} / \mathrm{L})\end{array}$ & $\begin{array}{c}\text { Flufen- } \\
\text { acet } \\
(\mu \mathrm{g} / \mathrm{L})\end{array}$ & $\begin{array}{c}\text { Flufen- } \\
\text { acet } \\
\text { ESA } \\
(\mu \mathrm{g} / \mathrm{L})\end{array}$ & $\begin{array}{c}\text { Flufen- } \\
\text { acet } \\
\text { OXA } \\
(\mu \mathrm{g} / \mathrm{L})\end{array}$ & $\begin{array}{l}\text { Meto- } \\
\text { lachlor } \\
(\mu \mathrm{g} / \mathrm{L})\end{array}$ & $\begin{array}{c}\text { Meto- } \\
\text { lachlor } \\
\text { ESA } \\
(\mu \mathrm{g} / \mathrm{L})\end{array}$ & $\begin{array}{l}\text { Meto- } \\
\text { lachlor } \\
\text { OXA } \\
(\mu \mathrm{g} / \mathrm{L})\end{array}$ \\
\hline \multicolumn{20}{|c|}{ National Water-Quality Assessment Program (NAWQA) (fig. 3)—Continued } \\
\hline \multicolumn{20}{|c|}{ Lower and Upper Illinois River Basin (LIRB/UIRB/KWI)—Continued } \\
\hline 425913088133801 & Waukesha Co., WI & $06 / 26 / 02$ & -- & $<0.05$ & $<0.05$ & $<0.05$ & -- & $<0.05$ & $<0.05$ & $<0.05$ & -- & $<0.05$ & $<0.05$ & -- & $<0.05$ & $<0.05$ & -- & $<0.05$ & $<0.05$ \\
\hline 430003088164601 & Waukesha Co., WI & $04 / 12 / 00$ & -- & $<.05$ & $<.05$ & -- & -- & $<.05$ & $<.05$ & -- & -- & $<.05$ & $<.05$ & -- & $<.05$ & $<.05$ & -- & $<.05$ & $<.05$ \\
\hline 430155088114701 & Waukesha Co., WI & $05 / 08 / 00$ & -- & $<.05$ & $<.05$ & -- & -- & $<.05$ & $<.05$ & -- & -- & $<.05$ & $<.05$ & -- & $<.05$ & $<.05$ & -- & $<.05$ & $<.05$ \\
\hline 430239088164001 & Waukesha Co., WI & $04 / 12 / 00$ & -- & $<.05$ & $<.05$ & -- & -- & $<.05$ & $<.05$ & -- & -- & $<.05$ & $<.05$ & -- & $<.05$ & $<.05$ & -- & $<.05$ & $<.05$ \\
\hline 430353088095901 & Waukesha Co., WI & $05 / 02 / 00$ & -- & $<.05$ & $<.05$ & -- & -- & $<.05$ & $<.05$ & -- & -- & $<.05$ & $<.05$ & -- & $<.05$ & $<.05$ & -- & $<.05$ & $<.05$ \\
\hline 430407088075901 & Waukesha Co., WI & $04 / 11 / 01$ & -- & $<.05$ & $<.05$ & -- & -- & $<.05$ & $<.05$ & -- & -- & $<.05$ & $<.05$ & -- & $<.05$ & $<.05$ & -- & $<.05$ & $<.05$ \\
\hline \multirow[t]{2}{*}{430728088132801} & Waukehsa Co., WI & 05/01/00 & -- & $<.05$ & $<.05$ & -- & -- & $<.05$ & $<.05$ & -- & -- & $<.05$ & $<.05$ & -- & $<.05$ & $<.05$ & -- & .29 & $<.05$ \\
\hline & & $06 / 26 / 02$ & -- & $<.05$ & $<.05$ & $<.05$ & -- & $<.05$ & $<.05$ & $<.05$ & -- & $<.05$ & $<.05$ & -- & $<.05$ & $<.05$ & -- & .10 & $<.05$ \\
\hline 430846088123201 & Waukesha Co., WI & $04 / 11 / 00$ & -- & $<.05$ & $<.05$ & -- & -- & $<.05$ & $<.05$ & -- & -- & $<.05$ & $<.05$ & -- & $<.05$ & $<.05$ & -- & $<.05$ & $<.05$ \\
\hline 430923088141601 & Waukesha Co., WI & $04 / 11 / 01$ & -- & $<.05$ & $<.05$ & -- & -- & $<.05$ & $<.05$ & -- & -- & $<.05$ & $<.05$ & -- & $<.05$ & $<.05$ & -- & $<.05$ & $<.05$ \\
\hline \multicolumn{20}{|c|}{ Mississippi Embayment (MISE/GAG) } \\
\hline 320017091405501 & Franklin Parish, LA & 08/20/97 & $<0.05$ & $<.20$ & $<.20$ & -- & $<0.05$ & $<.20$ & $<.20$ & -- & -- & -- & -- & -- & -- & -- & $<0.05$ & $<.20$ & $<.20$ \\
\hline 320240091243701 & Tensas Parish, LA & 08/20/97 & $<.05$ & $<.20$ & $<.20$ & -- & $<.05$ & $<.20$ & $<.20$ & -- & -- & -- & -- & -- & -- & -- & $<.05$ & $<.20$ & $<.20$ \\
\hline \multirow[t]{2}{*}{321547091443401} & Franklin Parish, LA & 05/13/98 & $<.05$ & $<.20$ & $<.20$ & -- & $<.05$ & $<.20$ & $<.20$ & -- & -- & -- & -- & -- & -- & -- & $<.05$ & $<.20$ & $<.20$ \\
\hline & & $05 / 14 / 98$ & $<.05$ & $<.20$ & $<.20$ & -- & $<.05$ & $<.20$ & $<.20$ & -- & -- & -- & -- & -- & -- & -- & $<.05$ & $<.20$ & $<.20$ \\
\hline 322358091110701 & Madison Parish, LA & 05/11/98 & $<.05$ & $<.20$ & $<.20$ & -- & $<.05$ & $<.20$ & $<.20$ & -- & -- & -- & -- & -- & -- & -- & $<.05$ & $<.20$ & $<.20$ \\
\hline \multirow[t]{2}{*}{322644091420901} & Richland Parish, LA & 08/06/97 & $<.05$ & $<.20$ & $<.20$ & -- & $<.05$ & $<.20$ & $<.20$ & -- & -- & -- & -- & -- & -- & -- & $<.05$ & $<.20$ & $<.20$ \\
\hline & & 08/06/97 & $<.05$ & $<.20$ & $<.20$ & -- & $<.05$ & $<.20$ & $<.20$ & -- & -- & -- & -- & -- & -- & -- & $<.05$ & $<.20$ & $<.20$ \\
\hline 322934090493901 & Warren Co., MS & $05 / 20 / 98$ & $<.05$ & $<.20$ & $<.20$ & -- & $<.05$ & $<.20$ & $<.20$ & -- & -- & -- & -- & -- & -- & -- & $<.05$ & $<.20$ & $<.20$ \\
\hline 324355090391801 & Yazoo Co., MS & $06 / 11 / 98$ & $<.05$ & $<.20$ & $<.20$ & -- & $<.05$ & $<.20$ & $<.20$ & -- & -- & -- & -- & -- & -- & -- & $<.05$ & $<.20$ & $<.20$ \\
\hline 325355090513701 & Sharkey Co., MS & $08 / 05 / 97$ & $<.05$ & $<.20$ & $<.20$ & -- & $<.05$ & $<.20$ & $<.20$ & -- & -- & -- & -- & -- & -- & -- & $<.05$ & $<.20$ & $<.20$ \\
\hline 032572309100190 & Issaquena Co., MS & $05 / 21 / 98$ & $<.05$ & $<.20$ & $<.20$ & -- & $<.05$ & $<.20$ & $<.20$ & -- & -- & -- & -- & -- & -- & -- & $<.05$ & $<.20$ & $<.20$ \\
\hline 330142091000801 & Washington Co., MS & $06 / 24 / 98$ & $<.05$ & $<.20$ & $<.20$ & -- & $<.05$ & $<.20$ & $<.20$ & -- & -- & -- & -- & -- & -- & -- & $<.05$ & $<.20$ & $<.20$ \\
\hline 330309090394301 & Sharkey Co., MS & $07 / 22 / 97$ & $<.05$ & $<.20$ & $<.20$ & -- & $<.05$ & $<.20$ & $<.20$ & -- & -- & -- & -- & -- & -- & -- & $<.05$ & $<.20$ & $<.20$ \\
\hline 330657090190101 & Chicot Co., AR & 08/05/97 & $<.05$ & $<.20$ & $<.20$ & -- & $<.05$ & $<.20$ & $<.20$ & -- & -- & -- & -- & -- & -- & -- & $<.05$ & $<.20$ & $<.20$ \\
\hline 331536090281001 & Humphreys Co., MS & $07 / 22 / 97$ & $<.05$ & $<.20$ & $<.20$ & -- & $<.05$ & $<.20$ & $<.20$ & -- & -- & -- & -- & -- & -- & -- & $<.05$ & $<.20$ & $<.20$ \\
\hline
\end{tabular}




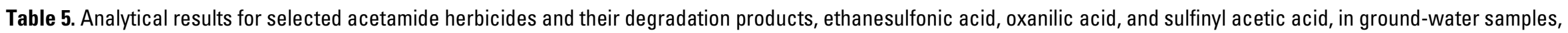
1994-2002.-Continued

[OGRL, U.S. Geological Survey Organic Geochemistry Research Laboratory, Lawrence, Kansas; ESA, ethanesulfonic acid; OXA, oxanilic acid; SAA, sulfinyl acetic acid; --, no data; <, less than]

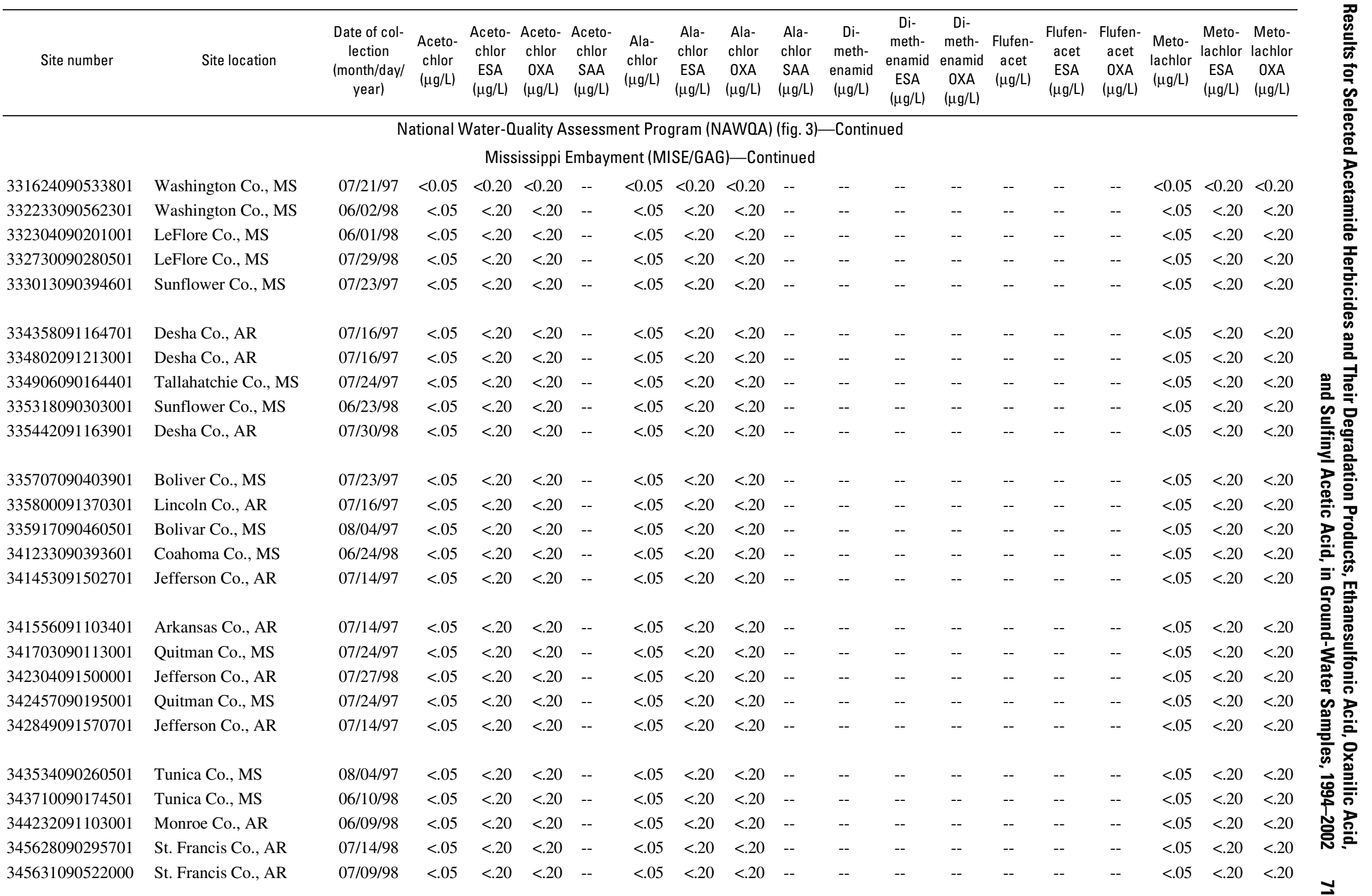




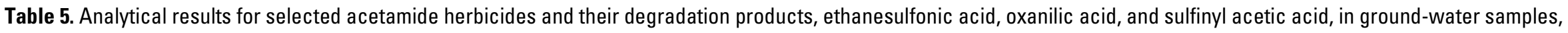
1994-2002.-Continued

[OGRL, U.S. Geological Survey Organic Geochemistry Research Laboratory, Lawrence, Kansas; ESA, ethanesulfonic acid; OXA, oxanilic acid; SAA, sulfinyl acetic acid; --, no data; <, less than]

\begin{tabular}{|c|c|c|c|c|c|c|c|c|c|c|c|c|c|c|c|c|c|c|c|}
\hline Site number & Site location & $\begin{array}{l}\text { Date of col- } \\
\text { lection } \\
\text { (month/day/ } \\
\text { year) }\end{array}$ & $\begin{array}{l}\text { Aceto- } \\
\text { chlor } \\
(\mu \mathrm{g} / \mathrm{L})\end{array}$ & $\begin{array}{l}\text { Aceto- } \\
\text { chlor } \\
\text { ESA } \\
(\mu \mathrm{g} / \mathrm{L})\end{array}$ & $\begin{array}{l}\text { Aceto- } \\
\text { chlor } \\
\text { OXA } \\
(\mu \mathrm{g} / \mathrm{L})\end{array}$ & $\begin{array}{l}\text { Aceto- } \\
\text { chlor } \\
\text { SAA } \\
(\mu \mathrm{g} / \mathrm{L})\end{array}$ & $\begin{array}{l}\text { Ala- } \\
\text { chlor } \\
(\mu \mathrm{g} / \mathrm{L})\end{array}$ & $\begin{array}{l}\text { Ala- } \\
\text { chlor } \\
\text { ESA } \\
(\mu \mathrm{g} / \mathrm{L})\end{array}$ & $\begin{array}{l}\text { Ala- } \\
\text { chlor } \\
\text { OXA } \\
(\mu \mathrm{g} / \mathrm{L})\end{array}$ & $\begin{array}{l}\text { Ala- } \\
\text { chlor } \\
\text { SAA } \\
(\mu \mathrm{g} / \mathrm{L})\end{array}$ & $\begin{array}{c}\text { Di- } \\
\text { meth- } \\
\text { enamid } \\
(\mu \mathrm{g} / \mathrm{L})\end{array}$ & $\begin{array}{c}\text { Di- } \\
\text { meth- } \\
\text { enamid } \\
\text { ESA } \\
(\mu \mathrm{g} / \mathrm{L})\end{array}$ & $\begin{array}{c}\text { Di- } \\
\text { meth- } \\
\text { enamid } \\
\text { OXA } \\
(\mu \mathrm{g} / \mathrm{L})\end{array}$ & $\begin{array}{c}\text { Flufen- } \\
\text { acet } \\
(\mu \mathrm{g} / \mathrm{L})\end{array}$ & $\begin{array}{c}\text { Flufen- } \\
\text { acet } \\
\text { ESA } \\
(\mu \mathrm{g} / \mathrm{L})\end{array}$ & $\begin{array}{c}\text { Flufen- } \\
\text { acet } \\
\text { OXA } \\
(\mu \mathrm{g} / \mathrm{L})\end{array}$ & $\begin{array}{l}\text { Meto- } \\
\text { lachlor } \\
(\mu \mathrm{g} / \mathrm{L})\end{array}$ & $\begin{array}{l}\text { Meto- } \\
\text { lachlor } \\
\text { ESA } \\
(\mu \mathrm{g} / \mathrm{L})\end{array}$ & $\begin{array}{l}\text { Meto- } \\
\text { lachlor } \\
\text { OXA } \\
(\mu \mathrm{g} / \mathrm{L})\end{array}$ \\
\hline \multicolumn{20}{|c|}{ National Water-Quality Assessment Program (NAWOA) (fig. 3)—Continued } \\
\hline \multicolumn{20}{|c|}{ Mississippi Embayment (MISE/GAG)—Continued } \\
\hline 345647091024500 & St. Francis Co., AR & 07/06/98 & $<0.05$ & $<0.20$ & $<0.20$ & -- & $<0.05$ & $<0.20$ & $<0.20$ & -- & -- & -- & -- & -- & -- & -- & $<0.05$ & $<0.20$ & $<0.20$ \\
\hline 350435090260901 & St. Francis Co., AR & $07 / 15 / 97$ & $<.05$ & $<.20$ & $<.20$ & -- & $<.05$ & $<.20$ & $<.20$ & -- & -- & -- & -- & -- & -- & -- & $<.05$ & $<.20$ & $<.20$ \\
\hline 352104090182301 & Crittenden Co., AR & $07 / 14 / 98$ & $<.05$ & $<.20$ & $<.20$ & -- & $<.05$ & $<.20$ & $<.20$ & -- & -- & -- & -- & -- & -- & -- & $<.05$ & $<.20$ & $<.20$ \\
\hline 352934090265401 & Poinsett Co., AR & 07/08/98 & $<.05$ & $<.20$ & $<.20$ & -- & $<.05$ & $<.20$ & $<.20$ & -- & -- & -- & -- & -- & -- & -- & $<.05$ & $<.20$ & $<.20$ \\
\hline 354341091120801 & Jackson Co., AR & 06/17/98 & $<.05$ & $<.20$ & $<.20$ & -- & $<.05$ & $<.20$ & $<.20$ & -- & -- & -- & -- & -- & -- & -- & $<.05$ & $<.20$ & $<.20$ \\
\hline \multirow[t]{2}{*}{354455091443401} & Jackson Co., AR & 06/16/98 & $<.05$ & $<.20$ & $<.20$ & -- & $<.05$ & $<.20$ & $<.20$ & -- & -- & -- & -- & -- & -- & -- & $<.05$ & $<.20$ & $<.20$ \\
\hline & & 06/18/98 & $<.05$ & $<.20$ & $<.20$ & -- & $<.05$ & $<.20$ & $<.20$ & -- & -- & -- & -- & -- & -- & -- & $<.05$ & $<.20$ & $<.20$ \\
\hline \multirow[t]{2}{*}{355516090285600} & Craighead Co., AR & 06/30/98 & $<.05$ & $<.20$ & $<.20$ & -- & $<.05$ & $<.20$ & $<.20$ & -- & -- & -- & -- & -- & -- & -- & $<.05$ & $<.20$ & $<.20$ \\
\hline & & 07/01/98 & $<.05$ & $<.20$ & $<.20$ & -- & $<.05$ & $<.20$ & $<.20$ & -- & -- & -- & -- & -- & -- & -- & $<.05$ & $<.20$ & $<.20$ \\
\hline 355839090515001 & Lawrence Co., AR & 07/01/98 & $<.05$ & $<.20$ & $<.20$ & -- & $<.05$ & $<.20$ & $<.20$ & -- & -- & -- & -- & -- & -- & -- & $<.05$ & $<.20$ & $<.20$ \\
\hline 360148090023801 & Dunklin Co., MO & 07/07/98 & $<.05$ & $<.20$ & $<.20$ & -- & $<.05$ & $<.20$ & $<.20$ & -- & -- & -- & -- & -- & -- & -- & $<.05$ & $<.20$ & $<.20$ \\
\hline 361412090150301 & Clay Co., AR & 06/30/98 & $<.05$ & $<.20$ & $<.20$ & -- & $<.05$ & .47 & $<.20$ & -- & -- & -- & -- & -- & -- & -- & $<.05$ & .94 & .20 \\
\hline 362501089290001 & Lake Co., TN & $07 / 23 / 98$ & $<.05$ & $<.20$ & $<.20$ & -- & $<.05$ & $<.20$ & $<.20$ & -- & -- & -- & -- & -- & -- & -- & $<.05$ & $<.20$ & $<.20$ \\
\hline 362858089440901 & New Madrid Co., MO & $07 / 22 / 98$ & $<.05$ & $<.20$ & $<.20$ & -- & $<.05$ & .29 & .45 & -- & -- & -- & -- & -- & -- & -- & $<.05$ & $<.20$ & $<.20$ \\
\hline 363956089584201 & Stoddard Co., MO & $07 / 21 / 98$ & $<.05$ & $<.20$ & $<.20$ & -- & $<.05$ & $<.20$ & $<.20$ & -- & -- & -- & -- & -- & -- & -- & $<.05$ & $<.20$ & $<.20$ \\
\hline 365430089265001 & Mississippi Co., MO & 07/20/98 & $<.05$ & $<.20$ & $<.20$ & -- & $<.05$ & $<.20$ & $<.20$ & -- & -- & -- & -- & -- & -- & -- & $<.05$ & $<.20$ & $<.20$ \\
\hline \multicolumn{20}{|c|}{ Santee Basin and Coastal Drainages (SANT/SCN) } \\
\hline 332310080423102 & Orangeburg, Co., SC & $11 / 03 / 97$ & $<.05$ & $<.20$ & $<.20$ & -- & $<.05$ & $<.20$ & $<.20$ & -- & -- & -- & -- & -- & -- & -- & $<.05$ & $<.20$ & $<.20$ \\
\hline 332310080423103 & Orangeburg, Co., SC & $11 / 04 / 97$ & $<.05$ & $<.20$ & $<.20$ & -- & $<.05$ & $<.20$ & $<.20$ & -- & -- & -- & -- & -- & -- & -- & .06 & $<.20$ & $<.20$ \\
\hline 332311080422902 & Orangeburg, Co., SC & $11 / 05 / 97$ & $<.05$ & $<.20$ & $<.20$ & -- & $<.05$ & $<.20$ & $<.20$ & -- & -- & -- & -- & -- & -- & -- & $<.05$ & $<.20$ & $<.20$ \\
\hline 332311080422903 & Orangeburg, Co., SC & $11 / 05 / 97$ & $<.05$ & $<.20$ & $<.20$ & -- & $<.05$ & $<.20$ & $<.20$ & & -- & -- & -- & -- & -- & -- & $<.05$ & $<.20$ & $<.20$ \\
\hline 332311080422904 & Orangeburg, Co., SC & $11 / 04 / 97$ & $<.05$ & $<.20$ & $<.20$ & -- & $<.05$ & $<.20$ & $<.20$ & -- & -- & -- & -- & -- & -- & -- & $<.05$ & $<.20$ & $<.20$ \\
\hline 332311080422905 & Orangeburg, Co., SC & $11 / 04 / 97$ & $<.05$ & $<.20$ & $<.20$ & -- & $<.05$ & $<.20$ & $<.20$ & -- & -- & -- & -- & -- & -- & -- & $<.05$ & $<.20$ & $<.20$ \\
\hline 332313080422602 & Orangeburg, Co., SC & $11 / 05 / 97$ & $<.05$ & $<.20$ & $<.20$ & -- & $<.05$ & $<.20$ & $<.20$ & -- & -- & -- & -- & -- & -- & -- & $<.05$ & $<.20$ & $<.20$ \\
\hline 332313080422603 & Orangeburg, Co., SC & $11 / 06 / 97$ & $<.05$ & $<.20$ & $<.20$ & -- & $<.05$ & $<.20$ & $<.20$ & -- & -- & -- & -- & -- & -- & -- & $<.05$ & $<.20$ & $<.20$ \\
\hline 332313080422604 & Orangeburg, Co., SC & $11 / 06 / 97$ & $<.05$ & $<.20$ & $<.20$ & -- & $<.05$ & $<.20$ & $<.20$ & -- & -- & -- & -- & -- & -- & -- & $<.05$ & $<.20$ & $<.20$ \\
\hline
\end{tabular}




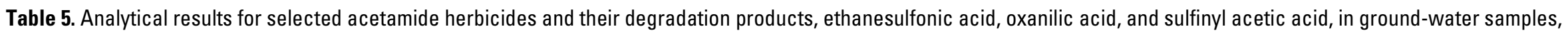
1994-2002.-Continued

[OGRL, U.S. Geological Survey Organic Geochemistry Research Laboratory, Lawrence, Kansas; ESA, ethanesulfonic acid; OXA, oxanilic acid; SAA, sulfinyl acetic acid; --, no data; <, less than]

\begin{tabular}{|c|c|c|c|c|c|c|c|c|c|c|c|c|c|c|c|c|c|c|c|c|}
\hline Site number & Site location & $\begin{array}{l}\text { Date of col- } \\
\text { lection } \\
\text { (month/day/ } \\
\text { year) }\end{array}$ & $\begin{array}{c}\text { Aceto- } \\
\text { chlor } \\
(\mu \mathrm{g} / \mathrm{L})\end{array}$ & $\begin{array}{l}\text { Aceto- } \\
\text { chlor } \\
\text { ESA } \\
(\mu \mathrm{g} / \mathrm{L})\end{array}$ & $\begin{array}{l}\text { Aceto- } \\
\text { chlor } \\
\text { OXA } \\
(\mu \mathrm{g} / \mathrm{L})\end{array}$ & $\begin{array}{l}\text { Aceto- } \\
\text { chlor } \\
\text { SAA } \\
(\mu \mathrm{g} / \mathrm{L})\end{array}$ & $\begin{array}{l}\text { Ala- } \\
\text { chlor } \\
(\mu \mathrm{g} / \mathrm{L})\end{array}$ & $\begin{array}{l}\text { Ala- } \\
\text { chlor } \\
\text { ESA } \\
(\mu \mathrm{g} / \mathrm{L})\end{array}$ & $\begin{array}{c}\text { Ala- } \\
\text { chlor } \\
\text { OXA } \\
(\mu \mathrm{g} / \mathrm{L})\end{array}$ & $\begin{array}{l}\text { Ala- } \\
\text { chlor } \\
\text { SAA } \\
(\mu \mathrm{g} / \mathrm{L})\end{array}$ & $\begin{array}{c}\text { Di- } \\
\text { meth- } \\
\text { enamid } \\
(\mu \mathrm{g} / \mathrm{L})\end{array}$ & $\begin{array}{c}\text { Di- } \\
\text { meth- } \\
\text { enamid } \\
\text { ESA } \\
(\mu \mathrm{g} / \mathrm{L})\end{array}$ & $\begin{array}{c}\text { Di- } \\
\text { meth- } \\
\text { enamid } \\
\text { OXA } \\
(\mu \mathrm{g} / \mathrm{L})\end{array}$ & $\begin{array}{c}\text { Flufen- } \\
\text { acet } \\
(\mu \mathrm{g} / \mathrm{L})\end{array}$ & $\begin{array}{c}\text { Flufen- } \\
\text { acet } \\
\text { ESA } \\
(\mu \mathrm{g} / \mathrm{L})\end{array}$ & $\begin{array}{c}\text { Flufen- } \\
\text { acet } \\
\text { OXA } \\
(\mu \mathrm{g} / \mathrm{L})\end{array}$ & $\begin{array}{l}\text { Meto- } \\
\text { lachlor } \\
(\mu \mathrm{g} / \mathrm{L})\end{array}$ & $\begin{array}{c}\text { Meto- } \\
\text { lachlor } \\
\text { ESA } \\
(\mu \mathrm{g} / \mathrm{L})\end{array}$ & $\begin{array}{c}\text { Meto- } \\
\text { lachlor } \\
\text { OXA } \\
(\mu \mathrm{g} / \mathrm{L})\end{array}$ & 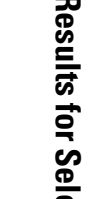 \\
\hline \multicolumn{20}{|c|}{ National Water-Quality Assessment Program (NAWQA) (fig. 3)-Continued } & \\
\hline \multicolumn{20}{|c|}{ Santee Basin and Coastal Drainages (SANT/SCN)—Continued } & \\
\hline 332320080422202 & Orangeburg, Co., SC & $11 / 06 / 97$ & $<0.05$ & $<0.20$ & $<0.20$ & -- & $<0.05$ & $<0.20$ & $<0.20$ & -- & -- & -- & -- & -- & -- & -- & $<0.05$ & $<0.20$ & $<0.20$ & \\
\hline 332320080422203 & Orangeburg, Co., SC & $11 / 12 / 97$ & $<.05$ & $<.20$ & $<.20$ & -- & $<.05$ & $<.20$ & $<.20$ & -- & -- & -- & -- & -- & -- & -- & $<.05$ & .24 & $<.20$ & \\
\hline 332324080421402 & Orangeburg, Co., SC & $11 / 17 / 97$ & $<.05$ & $<.20$ & $<.20$ & -- & $<.05$ & $<.20$ & $<.20$ & -- & -- & -- & -- & -- & -- & -- & $<.05$ & 14 & 1.0 & $\frac{2}{\Phi}$ \\
\hline 332324080421404 & Orangeburg, Co., SC & $11 / 17 / 97$ & $<.05$ & $<.20$ & $<.20$ & -- & $<.05$ & $<.20$ & $<.20$ & -- & -- & -- & -- & -- & -- & -- & $<.05$ & 6.9 & .20 & \\
\hline 332331080420701 & Orangeburg, Co., SC & $11 / 18 / 97$ & $<.05$ & $<.20$ & $<.20$ & -- & $<.05$ & $<.20$ & $<.20$ & -- & -- & -- & -- & -- & -- & -- & $<.05$ & 10 & .42 & \\
\hline 332331080420703 & Orangeburg, Co., SC & $11 / 18 / 97$ & $<.05$ & $<.20$ & $<.20$ & -- & $<.05$ & $<.20$ & $<.20$ & -- & -- & -- & -- & -- & -- & -- & $<.05$ & 11 & $<.20$ & \\
\hline 332331080420704 & Orangeburg, Co., SC & $11 / 18 / 97$ & $<.05$ & $<.20$ & $<.20$ & -- & $<.05$ & $<.20$ & $<.20$ & -- & -- & -- & -- & -- & -- & -- & $<.05$ & .88 & $<.20$ & \\
\hline 332335080420001 & Orangeburg, Co., SC & $11 / 20 / 97$ & $<.05$ & $<.20$ & $<.20$ & -- & $<.05$ & $<.20$ & $<.20$ & -- & -- & -- & -- & -- & -- & -- & $<.05$ & 2.8 & $<.20$ & \\
\hline 332335080420002 & Orangeburg, Co., SC & $11 / 19 / 97$ & $<.05$ & $<.20$ & $<.20$ & -- & $<.05$ & $<.20$ & $<.20$ & -- & -- & -- & -- & -- & -- & -- & .25 & 9.5 & .50 & \\
\hline 332335080420003 & Orangeburg, Co., SC & $11 / 19 / 97$ & $<.05$ & $<.20$ & $<.20$ & -- & $<.05$ & $<.20$ & $<.20$ & -- & -- & -- & -- & -- & -- & -- & $<.05$ & 2.1 & $<.20$ & 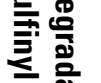 \\
\hline 332335080420004 & Orangeburg, Co., SC & $11 / 18 / 97$ & $<.05$ & $<.20$ & $<.20$ & -- & $<.05$ & $<.20$ & $<.20$ & -- & -- & -- & -- & -- & -- & -- & $<.05$ & $<.20$ & $<.20$ & \\
\hline 332335080420101 & Orangeburg, Co., SC & $11 / 20 / 97$ & $<.05$ & $<.20$ & $<.20$ & -- & $<.05$ & $<.20$ & $<.20$ & -- & -- & -- & -- & -- & -- & -- & $<.05$ & $<.20$ & $<.20$ & \\
\hline \multicolumn{20}{|c|}{ Federal-State Cooperative Studies } & \\
\hline \multicolumn{20}{|c|}{ lowa Cedar Rapids study (BBI) } & \\
\hline \multirow[t]{3}{*}{420012091442802} & \multirow[t]{3}{*}{ Linn Co., IA } & 06/03/98 & .17 & 2.1 & 1.7 & -- & $<.05$ & 1.4 & $<.20$ & -- & -- & -- & -- & -- & -- & -- & .33 & 5.5 & 1.2 & \\
\hline & & $07 / 22 / 98$ & $<.05$ & 2.9 & 1.1 & -- & $<.05$ & 2.4 & .20 & -- & -- & -- & -- & -- & -- & -- & .07 & 6.8 & 1.4 & \\
\hline & & 08/20/98 & $<.05$ & 1.6 & .26 & -- & $<.05$ & 2.2 & $<.20$ & -- & -- & -- & -- & -- & -- & -- & $<.05$ & 5.9 & .88 & \\
\hline \multirow[t]{3}{*}{420012091442803} & \multirow[t]{3}{*}{ Linn Co., IA } & 06/03/98 & $<.05$ & 2.0 & 1.2 & -- & $<.05$ & 1.3 & $<.20$ & -- & -- & -- & -- & -- & -- & -- & .11 & 5.2 & 1.2 & \\
\hline & & 07/22/98 & $<.05$ & 3.3 & 1.8 & -- & $<.05$ & 2.2 & .31 & -- & -- & -- & -- & -- & -- & -- & .16 & 6.5 & 1.7 & \\
\hline & & 08/20/98 & $<.05$ & 2.5 & .67 & -- & $<.05$ & 2.2 & .29 & -- & -- & -- & -- & -- & -- & -- & .08 & 6.5 & 1.0 & \\
\hline \multirow[t]{3}{*}{420012091442805} & \multirow[t]{3}{*}{ Linn Co., IA } & 06/03/98 & .07 & 1.7 & 1.0 & -- & $<.05$ & 1.4 & $<.20$ & -- & -- & -- & -- & -- & -- & -- & .14 & 5.3 & 1.2 & \\
\hline & & $07 / 22 / 98$ & $<.05$ & 2.5 & 1.3 & -- & $<.05$ & 2.0 & .25 & -- & -- & -- & -- & -- & -- & -- & .08 & 5.6 & 1.4 & \\
\hline & & 08/20/98 & $<.05$ & 1.1 & $<.20$ & -- & $<.05$ & 2.2 & $<.20$ & -- & -- & -- & -- & -- & -- & -- & .08 & 4.8 & .59 & \\
\hline
\end{tabular}


Table 5. Analytical results for selected acetamide herbicides and their degradation products, ethanesulfonic acid, oxanilic acid, and sulfinyl acetic acid, in ground-water samples, 1994-2002.-Continued

[OGRL, U.S. Geological Survey Organic Geochemistry Research Laboratory, Lawrence, Kansas; ESA, ethanesulfonic acid; OXA, oxanilic acid; SAA, sulfinyl acetic acid; --, no data; <, less than]

\begin{tabular}{|c|c|c|c|c|c|c|c|c|c|c|c|c|c|c|c|c|c|c|c|}
\hline Site number & Site location & $\begin{array}{c}\text { Date of } \\
\text { collection } \\
\text { (month/day/ } \\
\text { year) }\end{array}$ & $\begin{array}{c}\text { Aceto- } \\
\text { chlor } \\
(\mu \mathrm{g} / \mathrm{L})\end{array}$ & $\begin{array}{l}\text { Aceto- } \\
\text { chlor } \\
\text { ESA } \\
(\mu \mathrm{g} / \mathrm{L})\end{array}$ & $\begin{array}{c}\text { Aceto- } \\
\text { chlor } \\
\text { OXA } \\
(\mu \mathrm{g} / \mathrm{L})\end{array}$ & $\begin{array}{c}\text { Aceto- } \\
\text { chlor } \\
\text { SAA } \\
(\mu \mathrm{g} / \mathrm{L})\end{array}$ & $\begin{array}{l}\text { Ala- } \\
\text { chlor } \\
(\mu \mathrm{g} / \mathrm{L})\end{array}$ & $\begin{array}{l}\text { Ala- } \\
\text { chlor } \\
\text { ESA } \\
(\mu \mathrm{g} / \mathrm{L})\end{array}$ & $\begin{array}{l}\text { Ala- } \\
\text { chlor } \\
\text { OXA } \\
(\mu \mathrm{g} / \mathrm{L})\end{array}$ & $\begin{array}{l}\text { Ala- } \\
\text { chlor } \\
\text { SAA } \\
(\mu \mathrm{g} / \mathrm{L})\end{array}$ & $\begin{array}{c}\text { Di- } \\
\text { meth- } \\
\text { enamid } \\
(\mu \mathrm{g} / \mathrm{L})\end{array}$ & $\begin{array}{c}\text { Di- } \\
\text { meth- } \\
\text { enamid } \\
\text { ESA } \\
(\mu \mathrm{g} / \mathrm{L})\end{array}$ & $\begin{array}{c}\text { Di- } \\
\text { meth- } \\
\text { enamid } \\
0 X A \\
(\mu \mathrm{g} / \mathrm{L})\end{array}$ & $\begin{array}{c}\text { Flufen- } \\
\text { acet } \\
(\mu \mathrm{g} / \mathrm{L})\end{array}$ & $\begin{array}{c}\text { Flufen- } \\
\text { acet } \\
\text { ESA } \\
(\mu \mathrm{g} / \mathrm{L})\end{array}$ & $\begin{array}{c}\text { Flufen- } \\
\text { acet } \\
\text { OXA } \\
(\mu \mathrm{g} / \mathrm{L})\end{array}$ & $\begin{array}{c}\text { Meto- } \\
\text { lachlor } \\
(\mu \mathrm{g} / \mathrm{L})\end{array}$ & $\begin{array}{c}\text { Meto- } \\
\text { lachlor } \\
\text { ESA } \\
(\mu \mathrm{g} / \mathrm{L})\end{array}$ & $\begin{array}{c}\text { Meto- } \\
\text { lachlor } \\
\text { OXA } \\
(\mu \mathrm{g} / \mathrm{L})\end{array}$ \\
\hline \multicolumn{20}{|c|}{ Federal-State Cooperative Studies-Continued } \\
\hline \multicolumn{20}{|c|}{ lowa Cedar Rapids study (BBI)—Continued } \\
\hline \multirow[t]{2}{*}{420013091442801} & Linn Co., IA & 06/03/98 & $<0.05$ & 0.99 & 0.66 & -- & $<0.05$ & 1.4 & $<0.20$ & -- & -- & -- & -- & -- & -- & -- & 0.11 & 4.2 & 0.78 \\
\hline & & $07 / 22 / 98$ & .09 & 2.1 & 1.2 & -- & $<.05$ & 2.1 & $<.20$ & -- & -- & -- & -- & -- & -- & -- & .23 & 6.4 & 1.2 \\
\hline \multirow[t]{3}{*}{420014091442701} & Linn Co., IA & $06 / 11 / 98$ & $<.05$ & .71 & $<.20$ & -- & $<.05$ & 1.1 & $<.20$ & -- & -- & -- & -- & -- & -- & -- & $<.05$ & 3.1 & .77 \\
\hline & & $07 / 22 / 98$ & $<.05$ & 1.1 & $<.20$ & -- & $<.05$ & 2.2 & $<.20$ & -- & -- & -- & -- & -- & -- & -- & .18 & 5.6 & .89 \\
\hline & & 08/20/98 & $<.05$ & 1.4 & $<.20$ & -- & $<.05$ & 2.5 & $<.20$ & -- & -- & -- & -- & -- & -- & -- & .13 & 6.5 & .81 \\
\hline \multirow[t]{2}{*}{420014091442702} & Linn Co., IA & $07 / 22 / 98$ & $<.05$ & 1.4 & .69 & -- & $<.05$ & 1.7 & .29 & -- & -- & -- & -- & -- & -- & -- & .19 & 4.5 & 1.3 \\
\hline & & 08/20/98 & $<.05$ & 1.6 & .20 & -- & $<.05$ & 2.4 & $<.20$ & -- & -- & -- & -- & -- & -- & -- & .15 & 5.5 & .69 \\
\hline \multirow[t]{2}{*}{420019091442501} & Linn Co., IA & $06 / 02 / 98$ & $<.05$ & 1.3 & .85 & -- & $<.05$ & 1.6 & $<.20$ & -- & -- & -- & -- & -- & -- & -- & .10 & 4.8 & 1.2 \\
\hline & & 08/19/98 & $<.05$ & 1.5 & .61 & -- & $<.05$ & 1.3 & $<.20$ & -- & -- & -- & -- & -- & -- & -- & .06 & 4.1 & .87 \\
\hline \multirow[t]{3}{*}{420019091442502} & Linn Co., IA & 06/02/98 & $<.05$ & .39 & $<.20$ & -- & $<.05$ & 1.0 & $<.20$ & -- & -- & -- & -- & -- & -- & -- & $<.05$ & 2.3 & .45 \\
\hline & & $07 / 22 / 98$ & $<.05$ & .85 & .28 & -- & $<.05$ & 1.6 & $<.20$ & -- & -- & -- & -- & -- & -- & -- & $<.05$ & 3.9 & .76 \\
\hline & & 08/19/98 & $<.05$ & 1.1 & .35 & -- & $<.05$ & 1.2 & $<.20$ & -- & -- & -- & -- & -- & -- & -- & $<.05$ & 3.8 & .82 \\
\hline \multirow[t]{3}{*}{420031091441801} & Linn Co., IA & 06/02/98 & $<.05$ & $<.20$ & $<.20$ & -- & $<.05$ & $<.20$ & $<.20$ & -- & -- & -- & -- & -- & -- & -- & $<.05$ & $<.20$ & $<.20$ \\
\hline & & $07 / 22 / 98$ & $<.05$ & $<.20$ & $<.20$ & -- & $<.05$ & $<.20$ & $<.20$ & -- & -- & -- & -- & -- & -- & -- & $<.05$ & .38 & $<.20$ \\
\hline & & 08/19/98 & $<.05$ & $<.20$ & $<.20$ & -- & $<.05$ & $<.20$ & $<.20$ & -- & -- & -- & -- & -- & -- & -- & $<.05$ & .37 & $<.20$ \\
\hline \multicolumn{20}{|c|}{ lowa wells monitoring study (KIA) } \\
\hline 11415 & Floyd Co., IA & $04 / 17 / 00$ & -- & 1.2 & $<.05$ & -- & -- & .52 & $<.05$ & -- & -- & $<0.05$ & $<0.05$ & -- & -- & -- & -- & 2.0 & $<.05$ \\
\hline 11485 & Floyd Co., IA & 04/17/00 & -- & $<.05$ & $<.05$ & -- & -- & .53 & .24 & -- & -- & $<.05$ & $<.05$ & -- & -- & -- & -- & .26 & $<.05$ \\
\hline 11690 & Floyd Co., IA & $04 / 17 / 00$ & -- & $<.05$ & $<.05$ & -- & -- & .94 & .08 & -- & -- & $<.05$ & $<.05$ & -- & -- & -- & -- & 5.6 & 1.6 \\
\hline 11817 & Floyd Co., IA & $04 / 17 / 00$ & -- & $<.05$ & $<.05$ & -- & -- & .90 & $<.05$ & -- & -- & $<.05$ & $<.05$ & -- & -- & -- & -- & 2.1 & $<.05$ \\
\hline 20155 & Mitchell Co., IA & $04 / 17 / 00$ & -- & .51 & $<.05$ & -- & -- & .49 & $<.05$ & -- & -- & $<.05$ & $<.05$ & -- & -- & -- & -- & 4.8 & 1.2 \\
\hline 20318 & Mitchell Co., IA & $04 / 17 / 00$ & -- & $<.05$ & $<.05$ & -- & -- & .48 & .07 & -- & -- & $<.05$ & $<.05$ & -- & -- & -- & -- & 1.6 & .49 \\
\hline
\end{tabular}


Table 5. Analytical results for selected acetamide herbicides and their degradation products, ethanesulfonic acid, oxanilic acid, and sulfinyl acetic acid, in ground-water samples, 1994-2002.-Continued

[OGRL, U.S. Geological Survey Organic Geochemistry Research Laboratory, Lawrence, Kansas; ESA, ethanesulfonic acid; OXA, oxanilic acid; SAA, sulfinyl acetic acid; --, no data; <, less than]

\begin{tabular}{|c|c|c|c|c|c|c|c|c|c|c|c|c|c|c|c|c|c|c|c|c|}
\hline Site number & Site location & $\begin{array}{c}\text { Date of } \\
\text { collection } \\
\text { (month/day/ } \\
\text { year) }\end{array}$ & $\begin{array}{l}\text { Aceto- } \\
\text { chlor } \\
(\mu \mathrm{g} / \mathrm{L})\end{array}$ & $\begin{array}{l}\text { Aceto- } \\
\text { chlor } \\
\text { ESA } \\
(\mu \mathrm{g} / \mathrm{L})\end{array}$ & $\begin{array}{l}\text { Aceto- } \\
\text { chlor } \\
\text { OXA } \\
(\mu \mathrm{g} / \mathrm{L})\end{array}$ & $\begin{array}{l}\text { Aceto- } \\
\text { chlor } \\
\text { SAA } \\
(\mu \mathrm{g} / \mathrm{L})\end{array}$ & $\begin{array}{l}\text { Ala- } \\
\text { chlor } \\
(\mu \mathrm{g} / \mathrm{L})\end{array}$ & $\begin{array}{l}\text { Ala- } \\
\text { chlor } \\
\text { ESA } \\
(\mu \mathrm{g} / \mathrm{L})\end{array}$ & $\begin{array}{c}\text { Ala- } \\
\text { chlor } \\
\text { OXA } \\
(\mu \mathrm{g} / \mathrm{L})\end{array}$ & $\begin{array}{c}\text { Ala- } \\
\text { chlor } \\
\text { SAA } \\
(\mu \mathrm{g} / \mathrm{L})\end{array}$ & $\begin{array}{c}\text { Di- } \\
\text { meth- } \\
\text { enamid } \\
(\mu \mathrm{g} / \mathrm{L})\end{array}$ & $\begin{array}{c}\text { Di- } \\
\text { meth- } \\
\text { enamid } \\
\text { ESA } \\
(\mu \mathrm{g} / \mathrm{L})\end{array}$ & $\begin{array}{c}\text { Di- } \\
\text { meth- } \\
\text { enamid } \\
\text { OXA } \\
(\mu \mathrm{g} / \mathrm{L})\end{array}$ & $\begin{array}{c}\text { Flufen- } \\
\text { acet } \\
(\mu \mathrm{g} / \mathrm{L})\end{array}$ & $\begin{array}{c}\text { Flufen- } \\
\text { acet } \\
\text { ESA } \\
(\mu \mathrm{g} / \mathrm{L})\end{array}$ & $\begin{array}{c}\text { Flufen- } \\
\text { acet } \\
\text { OXA } \\
(\mu \mathrm{g} / \mathrm{L})\end{array}$ & $\begin{array}{c}\text { Meto- } \\
\text { lachlor } \\
(\mu \mathrm{g} / \mathrm{L})\end{array}$ & $\begin{array}{c}\text { Meto- } \\
\text { lachlor } \\
\text { ESA } \\
(\mu \mathrm{g} / \mathrm{L})\end{array}$ & $\begin{array}{c}\text { Meto- } \\
\text { lachlor } \\
\text { OXA } \\
(\mu \mathrm{g} / \mathrm{L})\end{array}$ & 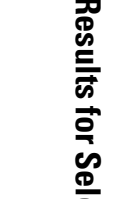 \\
\hline \multicolumn{20}{|c|}{ Federal-State Cooperative Studies-Continued } & \\
\hline \multicolumn{20}{|c|}{ lowa wells monitoring study $(\mathrm{KIA})$-Continued } & \\
\hline 20320 & Mitchell Co., IA & 04/17/00 & -- & $<0.05$ & $<0.05$ & -- & -- & 0.79 & $<0.05$ & -- & -- & $<0.05$ & $<0.05$ & -- & -- & -- & -- & 2.0 & 0.09 & \\
\hline 20334 & Mitchell Co., IA & 04/17/00 & -- & $<.05$ & $<.05$ & -- & -- & .40 & .06 & -- & -- & $<.05$ & $<.05$ & -- & -- & -- & -- & 1.2 & .93 & \\
\hline 20392 & Mitchell Co., IA & 04/17/00 & -- & 1.6 & 2.3 & -- & -- & .99 & .34 & -- & -- & $<.05$ & $<.05$ & -- & -- & -- & -- & 2.3 & .50 & \\
\hline 20428 & Mitchell Co., IA & 04/17/00 & -- & $<.05$ & $<.05$ & -- & -- & .06 & $<.05$ & -- & -- & $<.05$ & $<.05$ & -- & -- & -- & -- & .70 & $<.05$ & \\
\hline 20483 & Mitchell Co., IA & 04/17/00 & -- & $<.05$ & $<.05$ & -- & -- & $<.05$ & $<.05$ & -- & -- & $<.05$ & $<.05$ & -- & -- & -- & -- & .05 & $<.05$ & \\
\hline 20531 & Mitchell Co., IA & $04 / 17 / 00$ & -- & $<.05$ & $<.05$ & -- & -- & 8.1 & $<.05$ & -- & -- & $<.05$ & $<.05$ & -- & -- & -- & -- & 8.1 & .91 & \\
\hline 20581 & Mitchell Co., IA & 04/17/00 & -- & $<.05$ & $<.05$ & -- & -- & 2.0 & $<.05$ & -- & -- & $<.05$ & $<.05$ & -- & -- & -- & -- & 2.7 & .31 & \\
\hline 20651 & Mitchell Co., IA & 04/17/00 & -- & $<.05$ & $<.05$ & -- & -- & .68 & $<.05$ & -- & -- & $<.05$ & $<.05$ & -- & -- & -- & -- & .09 & $<.05$ & \\
\hline 20663 & Mitchell Co., IA & 04/17/00 & -- & .38 & $<.05$ & -- & -- & .34 & $<.05$ & -- & -- & $<.05$ & $<.05$ & -- & -- & -- & -- & 8.0 & .09 & \\
\hline 20696 & Mitchell Co., IA & $04 / 17 / 00$ & -- & $<.05$ & $<.05$ & -- & -- & 3.7 & $<.05$ & -- & -- & $<.05$ & $<.05$ & -- & -- & -- & -- & 3.2 & .47 & 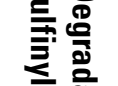 \\
\hline 20699 & Mitchell Co., IA & 04/17/00 & -- & $<.05$ & $<.05$ & -- & -- & .67 & $<.05$ & -- & -- & $<.05$ & $<.05$ & -- & -- & -- & -- & 4.2 & .40 & \\
\hline 20817 & Mitchell Co., IA & 04/17/00 & -- & $<.05$ & $<.05$ & -- & -- & $<.05$ & $<.05$ & -- & -- & $<.05$ & $<.05$ & -- & -- & -- & -- & $<.05$ & $<.05$ & \\
\hline 21068 & Mitchell Co., IA & $04 / 17 / 00$ & -- & .39 & $<.05$ & -- & -- & .37 & .51 & -- & -- & $<.05$ & $<.05$ & -- & -- & -- & -- & 19 & 14 & \\
\hline 21270 & Mitchell Co., IA & 04/17/00 & -- & $<.05$ & $<.05$ & -- & -- & $<.05$ & $<.05$ & -- & -- & $<.05$ & $<.05$ & -- & -- & -- & -- & $<.05$ & $<.05$ & \\
\hline 21305 & Sweeney Co., IA & 04/17/00 & -- & $<.05$ & $<.05$ & -- & -- & $<.05$ & $<.05$ & -- & -- & $<.05$ & $<.05$ & -- & -- & -- & -- & $<.05$ & $<.05$ & \\
\hline Fm 1-1 & Floyd Co., IA & 04/18/00 & -- & .54 & .24 & -- & -- & .84 & .25 & -- & -- & $<.05$ & $<.05$ & -- & -- & -- & -- & 5.6 & .99 & \\
\hline Fm 1-2 & Floyd Co., IA & 04/18/00 & -- & .40 & .22 & -- & -- & .46 & .24 & -- & -- & $<.05$ & $<.05$ & -- & -- & -- & -- & 1.8 & .40 & \\
\hline Fm 1-3 & Floyd Co., IA & 04/18/00 & -- & $<.05$ & $<.05$ & -- & -- & .79 & .25 & -- & -- & $<.05$ & $<.05$ & -- & -- & -- & -- & 3.2 & 1.1 & \\
\hline Fm 1-4 & Floyd Co., IA & 04/18/00 & -- & $<.05$ & $<.05$ & -- & -- & .45 & $<.05$ & -- & -- & $<.05$ & $<.05$ & -- & -- & -- & -- & .47 & .25 & \\
\hline Fm 3-1 & Floyd Co., IA & $04 / 18 / 00$ & -- & $<.05$ & $<.05$ & -- & -- & .97 & .25 & -- & -- & $<.05$ & $<.05$ & -- & -- & -- & -- & 4.8 & 3.7 & 흥 \\
\hline Fm 3-2 & Floyd Co., IA & 04/18/00 & -- & $<.05$ & $<.05$ & -- & -- & .28 & $<.05$ & -- & -- & $<.05$ & $<.05$ & -- & -- & -- & -- & 1.2 & .55 & 它 \\
\hline Fm 3-3 & Floyd Co., IA & $04 / 18 / 00$ & -- & $<.05$ & $<.05$ & -- & -- & .31 & $<.05$ & -- & -- & $<.05$ & $<.05$ & -- & -- & -- & -- & 1.3 & .61 & \\
\hline Fm 3-4 & Floyd Co., IA & $04 / 18 / 00$ & -- & $<.05$ & $<.05$ & -- & -- & .46 & .24 & -- & -- & $<.05$ & $<.05$ & -- & -- & -- & -- & 3.2 & 1.0 & 兑. \\
\hline Fm 3-T & Floyd Co., IA & 04/18/00 & -- & .66 & $<.05$ & -- & -- & $<.05$ & $<.05$ & -- & -- & $<.05$ & $<.05$ & -- & -- & -- & -- & .60 & $<.05$ & \\
\hline Olson well & Floyd Co., IA & 04/18/00 & -- & $<.05$ & $<.05$ & -- & -- & .29 & $<.05$ & -- & -- & $<.05$ & $<.05$ & -- & -- & -- & -- & 1.1 & .30 & \\
\hline
\end{tabular}




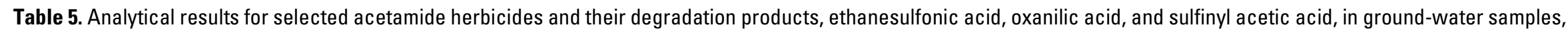
1994-2002.-Continued

[OGRL, U.S. Geological Survey Organic Geochemistry Research Laboratory, Lawrence, Kansas; ESA, ethanesulfonic acid; OXA, oxanilic acid; SAA, sulfinyl acetic acid; --, no data; <, less than]

\begin{tabular}{|c|c|c|c|c|c|c|c|c|c|c|c|c|c|c|c|c|c|c|c|}
\hline Site number & Site location & $\begin{array}{c}\text { Date of } \\
\text { collection } \\
\text { (month/day/ } \\
\text { year) }\end{array}$ & $\begin{array}{l}\text { Aceto- } \\
\text { chlor } \\
(\mu \mathrm{g} / \mathrm{L})\end{array}$ & $\begin{array}{l}\text { Aceto- } \\
\text { chlor } \\
\text { ESA } \\
(\mu \mathrm{g} / \mathrm{L})\end{array}$ & $\begin{array}{c}\text { Aceto- } \\
\text { chlor } \\
\text { OXA } \\
(\mu \mathrm{g} / \mathrm{L})\end{array}$ & $\begin{array}{c}\text { Aceto- } \\
\text { chlor } \\
\text { SAA } \\
(\mu \mathrm{g} / \mathrm{L})\end{array}$ & $\begin{array}{l}\text { Ala- } \\
\text { chlor } \\
(\mu \mathrm{g} / \mathrm{L})\end{array}$ & $\begin{array}{l}\text { Ala- } \\
\text { chlor } \\
\text { ESA } \\
(\mu \mathrm{g} / \mathrm{L})\end{array}$ & $\begin{array}{l}\text { Ala- } \\
\text { chlor } \\
\text { OXA } \\
(\mu \mathrm{g} / \mathrm{L})\end{array}$ & $\begin{array}{l}\text { Ala- } \\
\text { chlor } \\
\text { SAA } \\
(\mu \mathrm{g} / \mathrm{L})\end{array}$ & $\begin{array}{c}\text { Di- } \\
\text { meth- } \\
\text { enamid } \\
(\mu \mathrm{g} / \mathrm{L})\end{array}$ & $\begin{array}{c}\text { Di- } \\
\text { meth- } \\
\text { enamid } \\
\text { ESA } \\
(\mu \mathrm{g} / \mathrm{L})\end{array}$ & $\begin{array}{c}\text { Di- } \\
\text { meth- } \\
\text { enamid } \\
\text { OXA } \\
(\mu \mathrm{g} / \mathrm{L})\end{array}$ & $\begin{array}{c}\text { Flufen- } \\
\text { acet } \\
(\mu \mathrm{g} / \mathrm{L})\end{array}$ & $\begin{array}{c}\text { Flufen- } \\
\text { acet } \\
\text { ESA } \\
(\mu \mathrm{g} / \mathrm{L})\end{array}$ & $\begin{array}{c}\text { Flufen- } \\
\text { acet } \\
\text { OXA } \\
(\mu \mathrm{g} / \mathrm{L})\end{array}$ & $\begin{array}{c}\text { Meto- } \\
\text { lachlor } \\
(\mu \mathrm{g} / \mathrm{L})\end{array}$ & $\begin{array}{l}\text { Meto- } \\
\text { lachlor } \\
\text { ESA } \\
(\mu \mathrm{g} / \mathrm{L})\end{array}$ & $\begin{array}{c}\text { Meto- } \\
\text { Iachlor } \\
\text { OXA } \\
(\mu \mathrm{g} / \mathrm{L})\end{array}$ \\
\hline \multicolumn{20}{|c|}{ Federal-State Cooperative Studies-Continued } \\
\hline \multicolumn{20}{|c|}{ lowa wells monitoring study $(\mathrm{KIA})$ —Continued } \\
\hline Schmidt well & Floyd Co., IA & $04 / 18 / 00$ & -- & $<0.05$ & $<0.05$ & -- & -- & 0.51 & $<0.05$ & -- & -- & $<0.05$ & $<0.05$ & -- & -- & -- & -- & 4.0 & $<0.05$ \\
\hline \multicolumn{20}{|c|}{ Minnesota hydrologic study (FRG) } \\
\hline 441625093444201 & Le Seuer Co., MN & $11 / 04 / 97$ & $<0.05$ & $<.20$ & $<.20$ & -- & $<0.05$ & $<.20$ & $<.20$ & -- & -- & -- & -- & -- & -- & -- & $<0.05$ & $<.20$ & $<.20$ \\
\hline 443050093544601 & Sibley Co., MN & $11 / 04 / 97$ & $<.05$ & $<.20$ & $<.20$ & -- & $<.05$ & $<.20$ & $<.20$ & -- & -- & -- & -- & -- & -- & -- & $<.05$ & $<.20$ & $<.20$ \\
\hline \multirow[t]{5}{*}{443204093521401} & Le Seuer Co., MN & 06/04/98 & $<.05$ & 2.3 & .95 & -- & $<.05$ & .51 & $<.05$ & -- & -- & -- & -- & -- & -- & -- & $<.05$ & 1.2 & .52 \\
\hline & & 03/30/99 & $<.05$ & .87 & $<.20$ & -- & $<.05$ & $<.20$ & $<.20$ & -- & -- & -- & -- & -- & -- & -- & $<.05$ & .41 & $<.20$ \\
\hline & & 05/11/99 & $<.05$ & 2.5 & .24 & -- & $<.05$ & .26 & $<.20$ & -- & -- & -- & -- & -- & -- & -- & $<.05$ & .51 & $<.20$ \\
\hline & & $06 / 06 / 00$ & $<.05$ & .34 & $<.05$ & -- & $<.05$ & .05 & $<.05$ & -- & $<0.05$ & $<.05$ & $<.05$ & $<0.05$ & -- & -- & $<.05$ & .42 & $<.05$ \\
\hline & & $07 / 10 / 00$ & $<.05$ & .15 & $<.05$ & -- & $<.05$ & .06 & $<.05$ & -- & $<.05$ & $<.05$ & $<.05$ & $<.05$ & $<0.05$ & $<0.05$ & $<.05$ & .13 & $<.05$ \\
\hline \multirow[t]{2}{*}{443204093521404} & Le Seuer Co., MN & 04/09/98 & $<.05$ & .99 & $<.20$ & -- & $<.05$ & $<.20$ & $<.20$ & -- & -- & -- & -- & -- & -- & -- & $<.05$ & 1.4 & $<.20$ \\
\hline & & 04/09/98 & $<.05$ & .93 & $<.20$ & -- & $<.05$ & .22 & $<.20$ & -- & -- & -- & -- & -- & -- & -- & $<.05$ & .73 & $<.20$ \\
\hline 443205093525801 & Le Seuer Co., MN & $11 / 04 / 97$ & $<.05$ & $<.20$ & $<.20$ & -- & $<.05$ & $<.20$ & $<.20$ & -- & -- & -- & -- & -- & -- & -- & $<.05$ & .23 & $<.20$ \\
\hline \multirow[t]{8}{*}{443206093521101} & Le Seuer Co., MN & 04/09/98 & $<.05$ & .79 & $<.20$ & -- & $<.05$ & $<.20$ & $<.20$ & -- & -- & -- & -- & -- & -- & -- & $<.05$ & 1.6 & $<.20$ \\
\hline & & 06/04/98 & $<.05$ & 1.4 & $<.20$ & -- & $<.05$ & $<.20$ & $<.20$ & -- & -- & -- & -- & -- & -- & -- & .88 & 2.3 & $<.20$ \\
\hline & & $05 / 11 / 99$ & $<.05$ & 1.2 & $<.20$ & -- & $<.05$ & $<.20$ & $<.20$ & -- & -- & -- & -- & -- & -- & -- & $<.05$ & 1.2 & $<.20$ \\
\hline & & 06/17/99 & $<.05$ & 2.2 & $<.20$ & -- & $<.05$ & $<.20$ & $<.20$ & -- & $<.05$ & -- & -- & $<.05$ & -- & -- & $<.05$ & 1.4 & $<.20$ \\
\hline & & 06/06/00 & $<.05$ & .23 & $<.05$ & -- & $<.05$ & $<.05$ & $<.05$ & -- & $<.05$ & $<.05$ & $<.05$ & $<.05$ & $<.05$ & $<.05$ & $<.05$ & .66 & $<.05$ \\
\hline & & 07/09/99 & $<.05$ & 1.5 & $<.20$ & -- & $<.05$ & $<.20$ & $<.20$ & -- & $<.05$ & -- & -- & $<.05$ & -- & -- & $<.05$ & 1.2 & $<.20$ \\
\hline & & 07/09/99 & $<.05$ & .73 & $<.20$ & -- & $<.05$ & $<.20$ & $<.20$ & -- & $<.05$ & -- & -- & $<.05$ & -- & -- & $<.05$ & .58 & $<.20$ \\
\hline & & 07/10/00 & $<.05$ & .15 & $<.05$ & -- & $<.05$ & $<.05$ & $<.05$ & -- & $<.05$ & $<.05$ & $<.05$ & $<.05$ & $<.05$ & $<.05$ & $<.05$ & .61 & $<.05$ \\
\hline \multirow[t]{5}{*}{443206093521501} & Le Seuer Co., MN & 04/09/98 & $<.05$ & .77 & $<.20$ & -- & $<.05$ & 1.4 & $<.20$ & -- & -- & -- & -- & -- & -- & -- & 2.4 & 2.2 & 2.1 \\
\hline & & 04/01/99 & $<.05$ & .55 & $<.20$ & -- & $<.05$ & $<.20$ & $<.20$ & -- & -- & -- & -- & -- & -- & -- & $<.05$ & 1.0 & $<.20$ \\
\hline & & 04/01/99 & $<.05$ & $<.05$ & $<.05$ & -- & $<.05$ & $<.05$ & $<.05$ & -- & -- & -- & -- & -- & -- & -- & $<.05$ & .25 & $<.05$ \\
\hline & & $03 / 24 / 99$ & $<.05$ & .41 & .34 & -- & $<.05$ & 1.4 & $<.20$ & -- & $<.05$ & -- & -- & $<.05$ & -- & -- & $<.05$ & .67 & .25 \\
\hline & & 03/30/99 & $<.05$ & .21 & $<.20$ & -- & $<.05$ & .83 & $<.20$ & -- & -- & -- & -- & -- & -- & -- & $<.05$ & 1.4 & .23 \\
\hline
\end{tabular}


Table 5. Analytical results for selected acetamide herbicides and their degradation products, ethanesulfonic acid, oxanilic acid, and sulfinyl acetic acid, in ground-water samples, 1994-2002.-Continued

[OGRL, U.S. Geological Survey Organic Geochemistry Research Laboratory, Lawrence, Kansas; ESA, ethanesulfonic acid; OXA, oxanilic acid; SAA, sulfinyl acetic acid; --, no data; <, less than]

\begin{tabular}{|c|c|c|c|c|c|c|c|c|c|c|c|c|c|c|c|c|c|c|c|}
\hline Site number & Site location & $\begin{array}{c}\text { Date of } \\
\text { collection } \\
\text { (month/day/ } \\
\text { year) }\end{array}$ & $\begin{array}{c}\text { Aceto- } \\
\text { chlor } \\
(\mu \mathrm{g} / \mathrm{L})\end{array}$ & $\begin{array}{l}\text { Aceto- } \\
\text { chlor } \\
\text { ESA } \\
(\mu \mathrm{g} / \mathrm{L})\end{array}$ & $\begin{array}{l}\text { Aceto- } \\
\text { chlor } \\
\text { OXA } \\
(\mu \mathrm{g} / \mathrm{L})\end{array}$ & $\begin{array}{l}\text { Aceto- } \\
\text { chlor } \\
\text { SAA } \\
(\mu \mathrm{g} / \mathrm{L})\end{array}$ & $\begin{array}{l}\text { Ala- } \\
\text { chlor } \\
(\mu \mathrm{g} / \mathrm{L})\end{array}$ & $\begin{array}{l}\text { Ala- } \\
\text { chlor } \\
\text { ESA } \\
(\mu \mathrm{g} / \mathrm{L})\end{array}$ & $\begin{array}{l}\text { Ala- } \\
\text { chlor } \\
\text { OXA } \\
(\mu \mathrm{g} / \mathrm{L})\end{array}$ & $\begin{array}{l}\text { Ala- } \\
\text { chlor } \\
\text { SAA } \\
(\mu \mathrm{g} / \mathrm{L})\end{array}$ & $\begin{array}{c}\text { Di- } \\
\text { meth- } \\
\text { enamid } \\
(\mu \mathrm{g} / \mathrm{L})\end{array}$ & $\begin{array}{c}\text { Di- } \\
\text { meth- } \\
\text { enamid } \\
\text { ESA } \\
(\mu \mathrm{g} / \mathrm{L})\end{array}$ & $\begin{array}{c}\text { Di- } \\
\text { meth- } \\
\text { enamid } \\
\text { OXA } \\
(\mu \mathrm{g} / \mathrm{L})\end{array}$ & $\begin{array}{c}\text { Flufen- } \\
\text { acet } \\
(\mu \mathrm{g} / \mathrm{L})\end{array}$ & $\begin{array}{c}\text { Flufen- } \\
\text { acet } \\
\text { ESA } \\
(\mu \mathrm{g} / \mathrm{L})\end{array}$ & $\begin{array}{c}\text { Flufen- } \\
\text { acet } \\
\text { OXA } \\
(\mu \mathrm{g} / \mathrm{L})\end{array}$ & $\begin{array}{c}\text { Meto- } \\
\text { lachlor } \\
(\mu \mathrm{g} / \mathrm{L})\end{array}$ & $\begin{array}{c}\text { Meto- } \\
\text { lachlor } \\
\text { ESA } \\
(\mu \mathrm{g} / \mathrm{L})\end{array}$ & $\begin{array}{c}\text { Meto- } \\
\text { lachlor } \\
\text { OXA } \\
(\mu \mathrm{g} / \mathrm{L})\end{array}$ \\
\hline \multicolumn{20}{|c|}{ Federal-State Cooperative Studies-Continued } \\
\hline \multicolumn{20}{|c|}{ Minnesota hydrologic study (FRG)—Continued } \\
\hline \multirow[t]{9}{*}{443206093521501} & Le Seuer Co., MN & $05 / 11 / 99$ & $<0.05$ & 0.55 & $<0.20$ & -- & $<0.05$ & 1.4 & $<0.20$ & -- & $<0.05$ & -- & -- & $<0.05$ & -- & -- & 0.08 & 2.7 & 1.0 \\
\hline & & $06 / 17 / 99$ & $<.05$ & .80 & $<.20$ & -- & $<.05$ & 1.4 & $<.20$ & -- & $<.05$ & -- & -- & $<.05$ & -- & -- & .14 & 4.4 & 1.4 \\
\hline & & $06 / 17 / 99$ & $<.05$ & .85 & $<.20$ & -- & $<.05$ & 1.5 & $<.20$ & -- & $<.05$ & -- & -- & $<.05$ & -- & -- & .15 & 4.5 & 1.4 \\
\hline & & 07/09/99 & $<.05$ & .61 & $<.20$ & -- & $<.05$ & .79 & $<.20$ & -- & $<.05$ & -- & -- & $<.05$ & -- & -- & .12 & 3.3 & .62 \\
\hline & & 07/09/99 & $<.05$ & .62 & $<.20$ & -- & $<.05$ & .78 & $<.20$ & -- & $<.05$ & -- & -- & $<.05$ & -- & -- & .12 & 3.3 & .61 \\
\hline & & 07/09/99 & $<.05$ & .59 & $<.20$ & -- & $<.05$ & .79 & $<.20$ & -- & $<.05$ & -- & -- & $<.05$ & -- & -- & .10 & 3.4 & .58 \\
\hline & & 07/09/99 & $<.05$ & .61 & $<.20$ & -- & $<.05$ & .80 & $<.20$ & -- & $<.05$ & -- & -- & $<.05$ & -- & -- & .10 & 3.3 & .63 \\
\hline & & 06/06/00 & $<.05$ & .78 & 1.6 & -- & $<.05$ & .60 & .26 & -- & $<.05$ & $<0.05$ & $<0.05$ & $<.05$ & -- & -- & $<.05$ & 2.9 & .40 \\
\hline & & 07/06/00 & $<.05$ & .08 & $<.05$ & -- & $<.05$ & .54 & $<.05$ & -- & $<.05$ & $<.05$ & $<.05$ & $<.05$ & $<0.05$ & $<0.05$ & $<.05$ & .79 & .06 \\
\hline 443233093524701 & Le Seuer Co., MN & $11 / 04 / 97$ & $<.05$ & $<.20$ & $<.20$ & -- & $<.05$ & $<.20$ & $<.20$ & -- & -- & -- & -- & -- & -- & -- & $<.05$ & $<.20$ & $<.20$ \\
\hline 450305094262201 & Meeker Co., MN & $11 / 07 / 97$ & $<.05$ & $<.20$ & $<.20$ & -- & $<.05$ & $<.20$ & $<.20$ & -- & -- & -- & -- & -- & -- & -- & $<.05$ & $<.20$ & $<.20$ \\
\hline 450456094232901 & Meeker Co., MN & $11 / 07 / 97$ & $<.05$ & $<.20$ & $<.20$ & -- & $<.05$ & $<.20$ & $<.20$ & -- & -- & -- & -- & -- & -- & -- & $<.05$ & $<.20$ & $<.20$ \\
\hline 450457094233601 & Meeker Co., MN & $11 / 07 / 97$ & $<.05$ & $<.20$ & $<.20$ & -- & $<.05$ & $<.20$ & $<.20$ & -- & -- & -- & -- & -- & -- & -- & $<.05$ & $<.20$ & $<.20$ \\
\hline \multirow[t]{2}{*}{450459094233501} & Meeker Co., MN & 07/07/99 & $<.05$ & $<.20$ & $<.20$ & -- & $<.05$ & $<.20$ & $<.20$ & -- & -- & -- & -- & -- & -- & -- & $<.05$ & $<.20$ & $<.20$ \\
\hline & & 07/07/99 & $<.05$ & $<.20$ & $<.20$ & -- & $<.05$ & $<.20$ & $<.20$ & -- & -- & -- & -- & -- & -- & -- & $<.05$ & $<.20$ & $<.20$ \\
\hline 451316094271801 & Meeker Co., MN & $11 / 7 / 97$ & $<.05$ & $<.20$ & $<.20$ & -- & $<.05$ & $<.20$ & $<.20$ & -- & -- & -- & -- & -- & -- & -- & $<.05$ & $<.20$ & $<.20$ \\
\hline \multirow[t]{2}{*}{452046092513001} & Chisago Co., MN & $11 / 8 / 97$ & $<.05$ & $<.20$ & $<.20$ & -- & $<.05$ & .21 & $<.20$ & -- & -- & -- & -- & -- & -- & -- & $<.05$ & $<.20$ & $<.20$ \\
\hline & & $04 / 21 / 98$ & $<.05$ & $<.20$ & $<.20$ & -- & $<.05$ & .22 & $<.20$ & -- & -- & -- & -- & -- & -- & -- & $<.05$ & .33 & $<.20$ \\
\hline 452046092543001 & Chisago Co., MN & $11 / 8 / 97$ & $<.05$ & $<.20$ & $<.20$ & -- & $<.05$ & .23 & $<.20$ & -- & -- & -- & -- & -- & -- & -- & $<.05$ & $<.20$ & $<.20$ \\
\hline \multirow[t]{3}{*}{452901093002001} & Chisago Co., MN & $11 / 3 / 97$ & $<.05$ & $<.20$ & $<.20$ & -- & $<.05$ & .36 & $<.20$ & -- & -- & -- & -- & -- & -- & -- & $<.05$ & $<.20$ & $<.20$ \\
\hline & & $04 / 21 / 98$ & $<.05$ & $<.20$ & $<.20$ & -- & $<.05$ & .82 & $<.20$ & -- & -- & -- & -- & -- & -- & -- & $<.05$ & 1.7 & $<.20$ \\
\hline & & $04 / 21 / 98$ & $<.05$ & $<.20$ & $<.20$ & -- & $<.05$ & .48 & $<.20$ & -- & -- & -- & -- & -- & -- & -- & $<.05$ & $<.20$ & $<.20$ \\
\hline 452903093001701 & Chisago Co., MN & $11 / 03 / 97$ & $<.05$ & $<.20$ & $<.20$ & -- & $<.05$ & .40 & $<.20$ & -- & -- & -- & -- & -- & -- & -- & $<.05$ & 1.8 & $<.20$ \\
\hline 453139093372201 & Sherburne CO., MN & $01 / 08 / 98$ & $<.05$ & $<.20$ & $<.20$ & -- & .36 & 4.4 & .20 & -- & -- & -- & -- & -- & -- & -- & $<.05$ & $<.20$ & $<.20$ \\
\hline \multirow[t]{2}{*}{453144093370501} & Sherburne CO., MN & $01 / 08 / 98$ & $<.05$ & $<.20$ & $<.20$ & -- & $<.05$ & $<.20$ & $<.20$ & -- & -- & -- & -- & -- & -- & -- & $<.05$ & $<.20$ & $<.20$ \\
\hline & & $01 / 08 / 98$ & $<.05$ & $<.20$ & $<.20$ & -- & $<.05$ & $<.20$ & $<.20$ & -- & -- & -- & -- & -- & -- & -- & $<.05$ & $<.20$ & $<.20$ \\
\hline
\end{tabular}




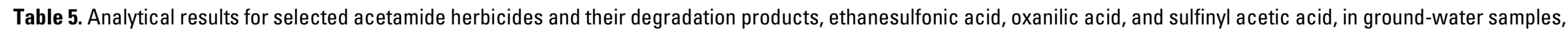
1994-2002.-Continued

[OGRL, U.S. Geological Survey Organic Geochemistry Research Laboratory, Lawrence, Kansas; ESA, ethanesulfonic acid; OXA, oxanilic acid; SAA, sulfinyl acetic acid; --, no data; <, less than]

\begin{tabular}{|c|c|c|c|c|c|c|c|c|c|c|c|c|c|c|c|c|c|c|c|}
\hline Site number & Site location & $\begin{array}{c}\text { Date of } \\
\text { collection } \\
\text { (month/day/ } \\
\text { year) }\end{array}$ & $\begin{array}{c}\text { Aceto- } \\
\text { chlor } \\
(\mu \mathrm{g} / \mathrm{L})\end{array}$ & $\begin{array}{l}\text { Aceto- } \\
\text { chlor } \\
\text { ESA } \\
(\mu \mathrm{g} / \mathrm{L})\end{array}$ & $\begin{array}{l}\text { Aceto- } \\
\text { chlor } \\
\text { OXA } \\
(\mu \mathrm{g} / \mathrm{L})\end{array}$ & $\begin{array}{l}\text { Aceto- } \\
\text { chlor } \\
\text { SAA } \\
(\mu \mathrm{g} / \mathrm{L})\end{array}$ & $\begin{array}{l}\text { Ala- } \\
\text { chlor } \\
(\mu \mathrm{g} / \mathrm{L})\end{array}$ & $\begin{array}{l}\text { Ala- } \\
\text { chlor } \\
\text { ESA } \\
(\mu \mathrm{g} / \mathrm{L})\end{array}$ & $\begin{array}{l}\text { Ala- } \\
\text { chlor } \\
\text { OXA } \\
(\mu \mathrm{g} / \mathrm{L})\end{array}$ & $\begin{array}{l}\text { Ala- } \\
\text { chlor } \\
\text { SAA } \\
(\mu \mathrm{g} / \mathrm{L})\end{array}$ & $\begin{array}{l}\text { Di- } \\
\text { meth- } \\
\text { enamid } \\
(\mu \mathrm{g} / \mathrm{L})\end{array}$ & $\begin{array}{c}\text { Di- } \\
\text { meth- } \\
\text { enamid } \\
\text { ESA } \\
(\mu \mathrm{g} / \mathrm{L})\end{array}$ & $\begin{array}{c}\text { Di- } \\
\text { meth- } \\
\text { enamid } \\
\text { OXA } \\
(\mu \mathrm{g} / \mathrm{L})\end{array}$ & $\begin{array}{l}\text { Flufen- } \\
\text { acet } \\
(\mu \mathrm{g} / \mathrm{L})\end{array}$ & $\begin{array}{c}\text { Flufen- } \\
\text { acet } \\
\text { ESA } \\
(\mu \mathrm{g} / \mathrm{L})\end{array}$ & $\begin{array}{l}\text { Flufen- } \\
\text { acet } \\
\text { OXA } \\
(\mu \mathrm{g} / \mathrm{L})\end{array}$ & $\begin{array}{l}\text { Meto- } \\
\text { lachlor } \\
(\mu \mathrm{g} / \mathrm{L})\end{array}$ & $\begin{array}{c}\text { Meto- } \\
\text { lachlor } \\
\text { ESA } \\
(\mu \mathrm{g} / \mathrm{L})\end{array}$ & $\begin{array}{c}\text { Meto- } \\
\text { lachlor } \\
\text { OXA } \\
(\mu \mathrm{g} / \mathrm{L})\end{array}$ \\
\hline \multicolumn{20}{|c|}{ Federal-State Cooperative Studies-Continued } \\
\hline \multicolumn{20}{|c|}{ Minnesota hydrologic study (FRG)—Continued } \\
\hline \multirow[t]{2}{*}{462151093552701} & Crow Wing Co., MN & 06/11/98 & $<0.05$ & $<0.20$ & $<0.20$ & -- & $<0.05$ & $<0.20$ & $<0.20$ & -- & -- & -- & -- & -- & -- & -- & $<0.05$ & $<0.20$ & $<0.20$ \\
\hline & & 06/11/98 & $<.05$ & $<.20$ & $<.20$ & -- & $<.05$ & $<.20$ & $<.20$ & -- & -- & -- & -- & -- & -- & -- & $<.05$ & $<.20$ & $<.20$ \\
\hline 462316093575401 & Crow Wing Co., MN & 06/29/98 & $<.05$ & $<.20$ & $<.20$ & -- & $<.05$ & $<.20$ & $<.20$ & -- & -- & -- & -- & -- & -- & -- & $<.05$ & $<.20$ & $<.20$ \\
\hline \multirow[t]{4}{*}{462318093575401} & Crow Wing Co., MN & 07/05/99 & $<.05$ & $<.20$ & $<.20$ & -- & $<.05$ & .34 & $<.20$ & -- & -- & -- & -- & -- & -- & -- & $<.05$ & $<.20$ & $<.20$ \\
\hline & & $06 / 12 / 00$ & $<.05$ & $<.05$ & $<.05$ & -- & $<.05$ & $<.05$ & $<.05$ & -- & -- & -- & -- & -- & -- & -- & $<.05$ & $<.05$ & $<.05$ \\
\hline & & 06/13/00 & $<.05$ & $<.05$ & $<.05$ & -- & $<.05$ & $<.05$ & $<.05$ & -- & -- & -- & -- & -- & -- & -- & $<.05$ & $<.05$ & $<.05$ \\
\hline & & 07/10/00 & $<.05$ & $<.05$ & $<.05$ & -- & $<.05$ & $<.05$ & $<.05$ & -- & -- & -- & -- & -- & -- & -- & $<.05$ & $<.05$ & $<.05$ \\
\hline 462326093574901 & Crow Wing Co., MN & 06/29/98 & $<.05$ & $<.20$ & $<.20$ & -- & $<.05$ & $<.20$ & $<.20$ & -- & -- & -- & -- & -- & -- & -- & $<.05$ & $<.20$ & $<.20$ \\
\hline \multirow[t]{3}{*}{462327093575801} & Crow Wing Co., MN & 06/07/01 & $<.05$ & $<.05$ & $<.05$ & -- & $<.05$ & $<.05$ & $<.05$ & -- & $<0.05$ & $<0.05$ & $<0.05$ & $<0.05$ & $<0.05$ & $<0.05$ & $<.05$ & $<.05$ & $<.05$ \\
\hline & & 08/13/01 & $<.05$ & $<.05$ & $<.05$ & -- & $<.05$ & $<.05$ & $<.05$ & -- & $<.05$ & $<.05$ & $<.05$ & $<.05$ & $<.05$ & $<.05$ & $<.05$ & $<.05$ & $<.05$ \\
\hline & & $10 / 09 / 01$ & $<.05$ & $<.05$ & $<.05$ & -- & $<.05$ & $<.05$ & $<.05$ & -- & $<.05$ & $<.05$ & $<.05$ & $<.05$ & $<.05$ & $<.05$ & $<.05$ & $<.05$ & $<.05$ \\
\hline 462327093580601 & Crow Wing Co., MN & $11 / 06 / 97$ & $<.05$ & $<.20$ & $<.20$ & -- & $<.05$ & $<.20$ & $<.20$ & -- & -- & -- & -- & -- & -- & -- & $<.05$ & $<.20$ & $<.20$ \\
\hline 462328093575101 & Crow Wing Co., MN & 07/05/99 & $<.05$ & $<.20$ & $<.20$ & -- & $<.05$ & $<.20$ & $<.20$ & -- & -- & -- & -- & -- & -- & -- & $<.05$ & $<.20$ & $<.20$ \\
\hline 462328093580601 & Crow Wing Co., MN & 06/29/98 & $<.05$ & $<.05$ & $<.05$ & -- & $<.05$ & $<.05$ & $<.05$ & -- & $<.05$ & -- & -- & $<.05$ & -- & -- & $<.05$ & $<.05$ & $<.05$ \\
\hline \multirow[t]{5}{*}{462328093575201} & Crow Wing Co., MN & 05/13/99 & $<.05$ & $<.20$ & $<.20$ & -- & $<.05$ & $<.20$ & $<.20$ & -- & -- & -- & -- & -- & -- & -- & $<.05$ & $<.20$ & $<.20$ \\
\hline & & 06/14/99 & $<.05$ & $<.20$ & $<.20$ & -- & $<.05$ & $<.20$ & $<.20$ & -- & -- & -- & -- & -- & -- & -- & $<.05$ & $<.20$ & $<.20$ \\
\hline & & 05/04/00 & $<.05$ & $<.20$ & $<.20$ & -- & $<.05$ & $<.20$ & $<.20$ & -- & -- & -- & -- & -- & -- & -- & $<.05$ & $<.20$ & $<.20$ \\
\hline & & $06 / 12 / 00$ & $<.05$ & $<.05$ & $<.05$ & -- & $<.05$ & $<.05$ & $<.05$ & -- & -- & -- & -- & -- & -- & -- & $<.05$ & $<.05$ & $<.05$ \\
\hline & & 07/10/00 & $<.05$ & $<.05$ & $<.05$ & -- & $<.05$ & $<.05$ & $<.05$ & -- & -- & -- & -- & -- & -- & -- & $<.05$ & $<.05$ & $<.05$ \\
\hline \multirow[t]{2}{*}{462330093580901} & Crow Wing Co., MN & $11 / 06 / 97$ & $<.05$ & $<.20$ & $<.20$ & -- & $<.05$ & $<.20$ & $<.20$ & -- & -- & -- & -- & -- & -- & -- & $<.05$ & $<.20$ & $<.20$ \\
\hline & & $06 / 12 / 00$ & $<.05$ & $<.05$ & $<.05$ & -- & $<.05$ & $<.05$ & $<.05$ & -- & -- & -- & -- & -- & -- & -- & $<.05$ & $<.05$ & $<.05$ \\
\hline \multirow[t]{4}{*}{462331093581301} & Crow Wing Co., MN & 07/05/99 & $<.05$ & $<.20$ & $<.20$ & -- & $<.05$ & $<.20$ & $<.20$ & -- & -- & -- & -- & -- & -- & -- & $<.05$ & $<.20$ & $<.20$ \\
\hline & & 05/04/00 & $<.05$ & $<.05$ & $<.05$ & -- & $<.05$ & $<.05$ & $<.05$ & -- & -- & -- & -- & -- & -- & -- & $<.05$ & $<.05$ & $<.05$ \\
\hline & & $06 / 12 / 00$ & $<.05$ & $<.05$ & $<.05$ & -- & $<.05$ & $<.05$ & $<.05$ & -- & -- & -- & -- & -- & -- & -- & $<.05$ & $<.05$ & $<.05$ \\
\hline & & $07 / 10 / 00$ & $<.05$ & $<.05$ & $<.05$ & -- & $<.05$ & $<.05$ & $<.05$ & -- & -- & -- & -- & -- & -- & -- & $<.05$ & $<.05$ & $<.05$ \\
\hline 462331093581901 & Crow Wing Co., MN & $11 / 06 / 97$ & $<.05$ & $<.20$ & $<.20$ & -- & $<.05$ & $<.20$ & $<.20$ & -- & -- & -- & -- & -- & -- & -- & $<.05$ & $<.20$ & $<.20$ \\
\hline 462335093575601 & Crow Wing Co., MN & 06/29/98 & $<.05$ & $<.20$ & $<.20$ & -- & $<.05$ & $<.20$ & $<.20$ & -- & -- & -- & -- & -- & -- & -- & $<.05$ & $<.20$ & $<.20$ \\
\hline
\end{tabular}


Table 5. Analytical results for selected acetamide herbicides and their degradation products, ethanesulfonic acid, oxanilic acid, and sulfinyl acetic acid, in ground-water samples, 1994-2002.-Continued

[OGRL, U.S. Geological Survey Organic Geochemistry Research Laboratory, Lawrence, Kansas; ESA, ethanesulfonic acid; OXA, oxanilic acid; SAA, sulfinyl acetic acid; --, no data; <, less than]

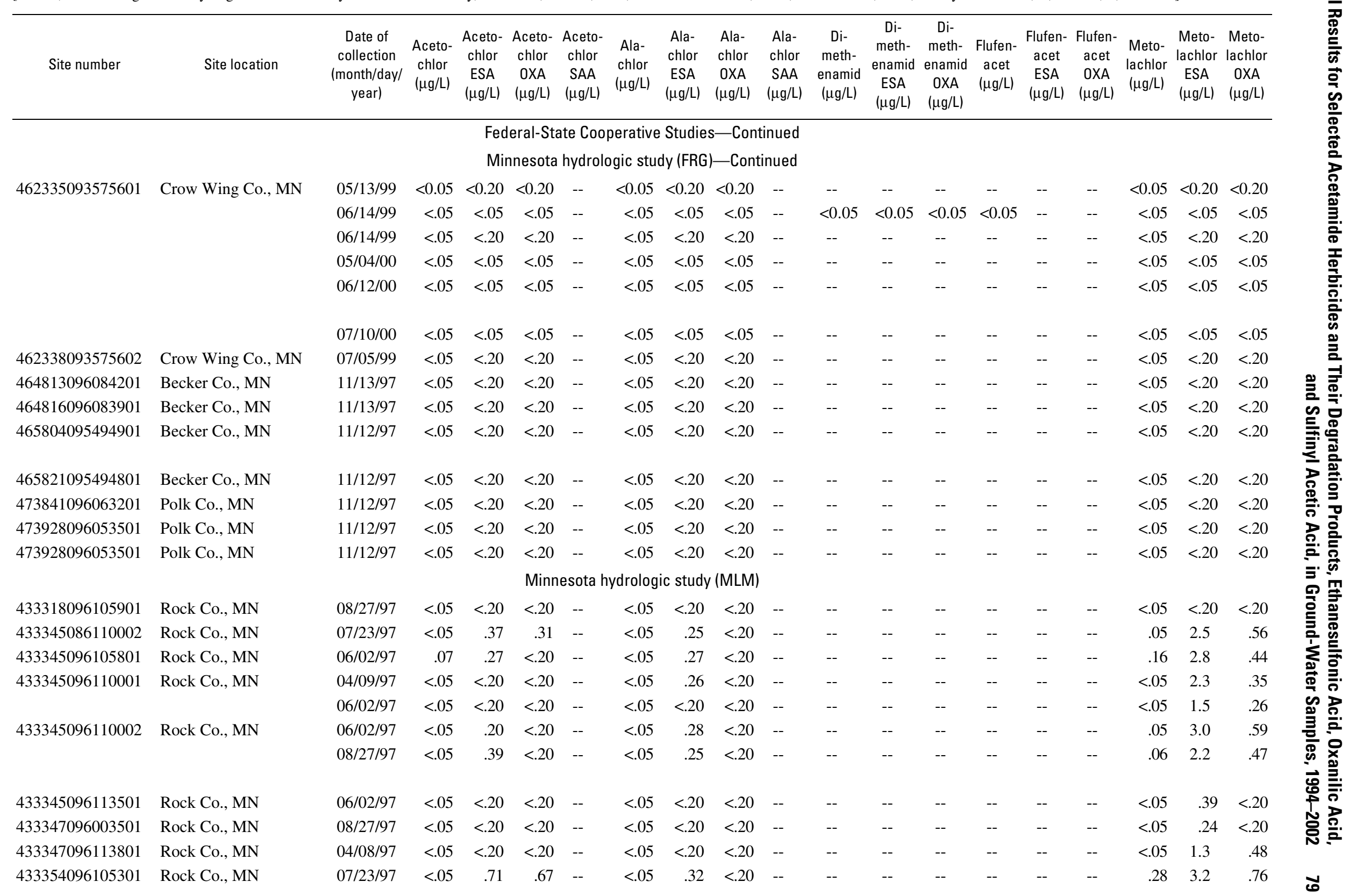




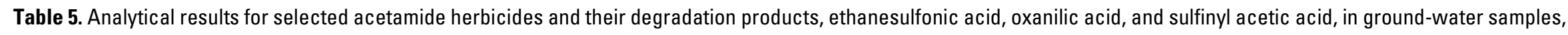
1994-2002.-Continued

[OGRL, U.S. Geological Survey Organic Geochemistry Research Laboratory, Lawrence, Kansas; ESA, ethanesulfonic acid; OXA, oxanilic acid; SAA, sulfinyl acetic acid; --, no data; <, less than]

\begin{tabular}{|c|c|c|c|c|c|c|c|c|c|c|c|c|c|c|c|c|c|c|c|}
\hline Site number & Site location & $\begin{array}{c}\text { Date of } \\
\text { collection } \\
\text { (month/day/ } \\
\text { year) }\end{array}$ & $\begin{array}{c}\text { Aceto- } \\
\text { chlor } \\
(\mu \mathrm{g} / \mathrm{L})\end{array}$ & $\begin{array}{l}\text { Aceto- } \\
\text { chlor } \\
\text { ESA } \\
(\mu \mathrm{g} / \mathrm{L})\end{array}$ & $\begin{array}{l}\text { Aceto- } \\
\text { chlor } \\
\text { OXA } \\
(\mu \mathrm{g} / \mathrm{L})\end{array}$ & $\begin{array}{l}\text { Aceto- } \\
\text { chlor } \\
\text { SAA } \\
(\mu \mathrm{g} / \mathrm{L})\end{array}$ & $\begin{array}{l}\text { Ala- } \\
\text { chlor } \\
(\mu \mathrm{g} / \mathrm{L})\end{array}$ & $\begin{array}{l}\text { Ala- } \\
\text { chlor } \\
\text { ESA } \\
(\mu \mathrm{g} / \mathrm{L})\end{array}$ & $\begin{array}{l}\text { Ala- } \\
\text { chlor } \\
\text { OXA } \\
(\mu \mathrm{g} / \mathrm{L})\end{array}$ & $\begin{array}{l}\text { Ala- } \\
\text { chlor } \\
\text { SAA } \\
(\mu \mathrm{g} / \mathrm{L})\end{array}$ & $\begin{array}{c}\text { Di- } \\
\text { meth- } \\
\text { enamid } \\
(\mu \mathrm{g} / \mathrm{L})\end{array}$ & $\begin{array}{c}\text { Di- } \\
\text { meth- } \\
\text { enamid } \\
\text { ESA } \\
(\mu \mathrm{g} / \mathrm{L})\end{array}$ & $\begin{array}{c}\text { Di- } \\
\text { meth- } \\
\text { enamid } \\
\text { OXA } \\
(\mu \mathrm{g} / \mathrm{L})\end{array}$ & $\begin{array}{l}\text { Flufen- } \\
\text { acet } \\
(\mu \mathrm{g} / \mathrm{L})\end{array}$ & $\begin{array}{c}\text { Flufen- } \\
\text { acet } \\
\text { ESA } \\
(\mu \mathrm{g} / \mathrm{L})\end{array}$ & $\begin{array}{c}\text { Flufen- } \\
\text { acet } \\
\text { OXA } \\
(\mu \mathrm{g} / \mathrm{L})\end{array}$ & $\begin{array}{l}\text { Meto- } \\
\text { lachlor } \\
(\mu \mathrm{g} / \mathrm{L})\end{array}$ & $\begin{array}{c}\text { Meto- } \\
\text { lachlor } \\
\text { ESA } \\
(\mu \mathrm{g} / \mathrm{L})\end{array}$ & $\begin{array}{c}\text { Meto- } \\
\text { lachlor } \\
\text { OXA } \\
(\mu \mathrm{g} / \mathrm{L})\end{array}$ \\
\hline \multicolumn{20}{|c|}{ Federal-State Cooperative Studies-Continued } \\
\hline \multicolumn{20}{|c|}{ Minnesota hydrologic study (MLM) —Continued } \\
\hline \multirow[t]{2}{*}{433354096105801} & Rock Co., MN & 04/09/97 & 0.13 & 0.39 & 0.26 & -- & $<0.05$ & 0.29 & $<0.20$ & -- & -- & -- & -- & -- & -- & -- & 0.32 & 2.4 & 0.64 \\
\hline & & 08/28/97 & $<.05$ & $<.20$ & $<.20$ & -- & $<.05$ & $<.20$ & $<.20$ & -- & -- & -- & -- & -- & -- & -- & .14 & 1.9 & $<.20$ \\
\hline 433413096111701 & Rock Co., MN & 08/28/97 & $<.05$ & $<.20$ & $<.20$ & -- & $<.05$ & .24 & $<.20$ & -- & -- & -- & -- & -- & -- & -- & $<.05$ & 1.9 & .20 \\
\hline 433422096120801 & Rock Co., MN & 06/02/97 & $<.05$ & $<.20$ & $<.20$ & -- & $<.05$ & $<.20$ & $<.20$ & -- & -- & -- & -- & -- & -- & -- & .07 & 3.4 & .32 \\
\hline 433424096120901 & Rock Co., MN & 08/27/97 & $<.05$ & $<.20$ & $<.20$ & -- & $<.05$ & $<.20$ & $<.20$ & -- & -- & -- & -- & -- & -- & -- & $<.05$ & 2.6 & .36 \\
\hline 433750096123201 & Rock Co., MN & 06/02/97 & $<.05$ & $<.20$ & $<.20$ & -- & $<.05$ & $<.20$ & $<.20$ & -- & -- & -- & -- & -- & -- & -- & $<.05$ & .69 & $<.20$ \\
\hline 433812096115601 & Rock Co., MN & 08/28/97 & $<.05$ & $<.20$ & $<.20$ & -- & $<.05$ & $<.20$ & $<.20$ & -- & -- & -- & -- & -- & -- & -- & $<.05$ & $<.20$ & $<.20$ \\
\hline 433912096113501 & Rock Co., MN & 04/08/97 & $<.05$ & $<.20$ & $<.20$ & -- & $<.05$ & $<.20$ & $<.20$ & -- & -- & -- & -- & -- & -- & -- & $<.05$ & $<.20$ & $<.20$ \\
\hline \multirow[t]{4}{*}{433920096114901} & Rock Co., MN & 04/08/97 & $<.05$ & $<.20$ & $<.20$ & -- & $<.05$ & $<.20$ & $<.20$ & -- & -- & -- & -- & -- & -- & -- & .05 & 1.3 & .23 \\
\hline & & 06/03/97 & $<.05$ & $<.20$ & $<.20$ & -- & $<.05$ & .24 & $<.20$ & -- & -- & -- & -- & -- & -- & -- & .08 & 2.5 & .28 \\
\hline & & $07 / 23 / 97$ & $<.05$ & .38 & .53 & -- & $<.05$ & .26 & $<.20$ & -- & -- & -- & -- & -- & -- & -- & .11 & 2.1 & .43 \\
\hline & & $08 / 27 / 97$ & $<.05$ & .27 & $<.20$ & -- & $<.05$ & .25 & $<.20$ & -- & -- & -- & -- & -- & -- & -- & .07 & 1.7 & .29 \\
\hline 433928096120801 & Rock Co., MN & 08/28/97 & $<.05$ & $<.20$ & $<.20$ & -- & $<.05$ & $<.20$ & $<.20$ & -- & -- & -- & -- & -- & -- & -- & $<.05$ & $<.20$ & $<.20$ \\
\hline \multirow[t]{2}{*}{433929096113601} & Rock Co., MN & 04/08/97 & $<.05$ & $<.20$ & $<.20$ & -- & $<.05$ & .34 & $<.20$ & -- & -- & -- & -- & -- & -- & -- & $<.05$ & .80 & $<.20$ \\
\hline & & 08/27/97 & $<.05$ & $<.20$ & $<.20$ & -- & $<.05$ & $<.20$ & $<.20$ & -- & -- & -- & -- & -- & -- & -- & $<.05$ & $<.20$ & $<.20$ \\
\hline \multirow[t]{2}{*}{433929096114701} & Rock Co., MN & 06/02/97 & $<.05$ & $<.20$ & $<.20$ & -- & $<.05$ & $<.20$ & $<.20$ & -- & -- & -- & -- & -- & -- & -- & $<.05$ & .71 & $<.20$ \\
\hline & & 08/28/97 & $<.05$ & $<.20$ & $<.20$ & -- & $<.05$ & $<.20$ & $<.20$ & -- & -- & -- & -- & -- & -- & -- & $<.05$ & .76 & $<.20$ \\
\hline \multirow[t]{2}{*}{433929096114702} & Rock Co., MN & 06/02/97 & $<.05$ & .50 & $<.20$ & -- & $<.05$ & .30 & $<.20$ & -- & -- & -- & -- & -- & -- & -- & $<.05$ & 3.7 & .52 \\
\hline & & 08/28/97 & $<.05$ & $<.20$ & $<.20$ & -- & $<.05$ & $<.20$ & $<.20$ & -- & -- & -- & -- & -- & -- & -- & $<.05$ & .33 & $<.20$ \\
\hline \multirow[t]{2}{*}{433934096113501} & Rock Co., MN & 04/08/97 & $<.05$ & $<.20$ & $<.20$ & -- & $<.05$ & .25 & $<.20$ & -- & -- & -- & -- & -- & -- & -- & $<.05$ & .84 & $<.20$ \\
\hline & & 08/27/97 & $<.05$ & $<.20$ & $<.20$ & -- & $<.05$ & .42 & $<.20$ & -- & -- & -- & -- & -- & -- & -- & $<.05$ & 1.2 & $<.20$ \\
\hline \multirow[t]{2}{*}{433952096113101} & Rock Co., MN & 06/03/97 & $<.05$ & $<.20$ & $<.20$ & -- & $<.05$ & $<.20$ & $<.20$ & -- & -- & -- & -- & -- & -- & -- & .06 & $<.20$ & $<.20$ \\
\hline & & 08/28/97 & $<.05$ & .21 & $<.20$ & -- & $<.05$ & $<.20$ & $<.20$ & -- & -- & -- & -- & -- & -- & -- & $<.05$ & 2.9 & .32 \\
\hline Gene Fusco & Benton Co., MN & 04/30/97 & $<.05$ & $<.20$ & $<.20$ & -- & $<.05$ & 2.9 & $<.20$ & -- & -- & -- & -- & -- & -- & -- & $<.05$ & 1.5 & .32 \\
\hline Jeffers \#1 & Cottonwood Co., MN & 05/07/97 & $<.05$ & $<.20$ & $<.20$ & -- & $<.05$ & $<.20$ & $<.20$ & -- & -- & -- & -- & -- & -- & -- & $<.05$ & $<.20$ & $<.20$ \\
\hline Nick Weis & Benton Co., MN & $04 / 30 / 97$ & $<.05$ & $<.20$ & $<.20$ & -- & $<.05$ & 7.4 & $<.20$ & -- & -- & -- & -- & -- & -- & -- & $<.05$ & 1.5 & .43 \\
\hline
\end{tabular}


Table 5. Analytical results for selected acetamide herbicides and their degradation products, ethanesulfonic acid, oxanilic acid, and sulfinyl acetic acid, in ground-water samples, 1994-2002.-Continued

[OGRL, U.S. Geological Survey Organic Geochemistry Research Laboratory, Lawrence, Kansas; ESA, ethanesulfonic acid; OXA, oxanilic acid; SAA, sulfinyl acetic acid; --, no data; <, less than]

\begin{tabular}{|c|c|c|c|c|c|c|c|c|c|c|c|c|c|c|c|c|c|c|c|}
\hline Site number & Site location & $\begin{array}{c}\text { Date of } \\
\text { collection } \\
\text { (month/day/ } \\
\text { year) }\end{array}$ & $\begin{array}{c}\text { Aceto- } \\
\text { chlor } \\
(\mu \mathrm{g} / \mathrm{L})\end{array}$ & $\begin{array}{l}\text { Aceto- } \\
\text { chlor } \\
\text { ESA } \\
(\mu \mathrm{g} / \mathrm{L})\end{array}$ & $\begin{array}{c}\text { Aceto- } \\
\text { chlor } \\
\text { OXA } \\
(\mu \mathrm{g} / \mathrm{L})\end{array}$ & $\begin{array}{l}\text { Aceto- } \\
\text { chlor } \\
\text { SAA } \\
(\mu \mathrm{g} / \mathrm{L})\end{array}$ & $\begin{array}{l}\text { Ala- } \\
\text { chlor } \\
(\mu \mathrm{g} / \mathrm{L})\end{array}$ & $\begin{array}{l}\text { Ala- } \\
\text { chlor } \\
\text { ESA } \\
(\mu \mathrm{g} / \mathrm{L})\end{array}$ & $\begin{array}{l}\text { Ala- } \\
\text { chlor } \\
\text { OXA } \\
(\mu \mathrm{g} / \mathrm{L})\end{array}$ & $\begin{array}{l}\text { Ala- } \\
\text { chlor } \\
\text { SAA } \\
(\mu \mathrm{g} / \mathrm{L})\end{array}$ & $\begin{array}{c}\text { Di- } \\
\text { meth- } \\
\text { enamid } \\
(\mu \mathrm{g} / \mathrm{L})\end{array}$ & $\begin{array}{c}\text { Di- } \\
\text { meth- } \\
\text { enamid } \\
\text { ESA } \\
(\mu \mathrm{g} / \mathrm{L})\end{array}$ & $\begin{array}{c}\text { Di- } \\
\text { meth- } \\
\text { enamid } \\
\text { OXA } \\
(\mu \mathrm{g} / \mathrm{L})\end{array}$ & $\begin{array}{c}\text { Flufen- } \\
\text { acet } \\
(\mu \mathrm{g} / \mathrm{L})\end{array}$ & $\begin{array}{c}\text { Flufen- } \\
\text { acet } \\
\text { ESA } \\
(\mu \mathrm{g} / \mathrm{L})\end{array}$ & $\begin{array}{c}\text { Flufen- } \\
\text { acet } \\
\text { OXA } \\
(\mu \mathrm{g} / \mathrm{L})\end{array}$ & $\begin{array}{c}\text { Meto- } \\
\text { lachlor } \\
(\mu \mathrm{g} / \mathrm{L})\end{array}$ & $\begin{array}{c}\text { Meto- } \\
\text { lachlor } \\
\text { ESA } \\
(\mu \mathrm{g} / \mathrm{L})\end{array}$ & $\begin{array}{c}\text { Meto- } \\
\text { lachlor } \\
\text { OXA } \\
(\mu \mathrm{g} / \mathrm{L})\end{array}$ \\
\hline \multicolumn{20}{|c|}{ Federal-State Cooperative Studies-Continued } \\
\hline \multicolumn{20}{|c|}{ Minnesota hydrologic study (MLM) —Continued } \\
\hline Perham \#6 & Otter Tail Co., MN & $05 / 06 / 97$ & $<0.05$ & $<0.20$ & $<0.20$ & -- & $<0.05$ & $<0.20$ & $<0.20$ & -- & -- & -- & -- & -- & -- & -- & $<0.05$ & $<0.20$ & $<0.20$ \\
\hline Sutton & Benton Co., MN & $04 / 30 / 97$ & $<.05$ & $<.20$ & $<.20$ & -- & $<.05$ & .53 & $<.20$ & -- & -- & -- & -- & -- & -- & -- & $<.05$ & .37 & $<.20$ \\
\hline Windom \#81 & Cottonwood Co., MN & 05/08/97 & $<.05$ & $<.20$ & $<.20$ & -- & $<.05$ & $<.20$ & $<.20$ & -- & -- & -- & -- & -- & -- & -- & $<.05$ & $<.20$ & $<.20$ \\
\hline \multicolumn{20}{|c|}{ Minnesota hydrologic study (TCM) } \\
\hline \multirow[t]{3}{*}{435008095060301} & Jackson Co., MN & 08/31/99 & $<.05$ & $<.20$ & $<.20$ & -- & $<.05$ & $<.20$ & $<.20$ & -- & $<0.05$ & -- & -- & $<0.05$ & -- & -- & $<.05$ & $<.20$ & $<.20$ \\
\hline & & $05 / 09 / 00$ & $<.05$ & $<.05$ & $<.05$ & -- & $<.05$ & $<.05$ & $<.05$ & -- & $<.05$ & $<0.05$ & $<0.05$ & $<.05$ & $<0.05$ & $<0.05$ & $<.05$ & $<.05$ & $<.05$ \\
\hline & & $06 / 27 / 00$ & $<.05$ & $<.05$ & $<.05$ & -- & $<.05$ & $<.05$ & $<.05$ & -- & $<.05$ & $<.05$ & $<.05$ & $<.05$ & $<.05$ & $<.05$ & $<.05$ & $<.05$ & $<.05$ \\
\hline 435014095060201 & Jackson Co., MN & $06 / 27 / 00$ & $<.05$ & $<.05$ & $<.05$ & -- & $<.05$ & $<.05$ & $<.05$ & -- & $<.05$ & $<.05$ & $<.05$ & $<.05$ & $<.05$ & $<.05$ & $<.05$ & $<.05$ & $<.05$ \\
\hline 435054095045801 & Cottonwood Co., MN & $06 / 28 / 00$ & $<.05$ & $<.05$ & $<.05$ & -- & $<.05$ & $<.05$ & $<.05$ & -- & $<.05$ & $<.05$ & $<.05$ & $<.05$ & $<.05$ & $<.05$ & $<.05$ & $<.05$ & $<.05$ \\
\hline 435145095051701 & Cottonwood Co., MN & 07/09/99 & $<.05$ & $<.05$ & $<.05$ & -- & $<.05$ & $<.05$ & $<.05$ & -- & $<.05$ & -- & -- & $<.05$ & -- & -- & $<.05$ & .19 & $<.05$ \\
\hline 435026095055601 & Cottonwood Co., MN & 07/14/99 & $<.05$ & $<.20$ & $<.20$ & -- & $<.05$ & $<.20$ & $<.20$ & -- & $<.05$ & -- & -- & $<.05$ & -- & -- & $<.05$ & $<.20$ & $<.20$ \\
\hline 435203095070101 & Cottonwood Co., MN & $06 / 28 / 00$ & $<.05$ & $<.05$ & $<.05$ & -- & $<.05$ & $<.05$ & $<.05$ & -- & $<.05$ & $<.05$ & $<.05$ & $<.05$ & $<.05$ & $<.05$ & $<.05$ & $<.05$ & $<.05$ \\
\hline 435205095065302 & Cottonwood Co., MN & $06 / 29 / 00$ & $<.05$ & $<.05$ & $<.05$ & -- & $<.05$ & $<.05$ & $<.05$ & -- & $<.05$ & $<.05$ & $<.05$ & $<.05$ & $<.05$ & $<.05$ & $<.05$ & $<.05$ & $<.05$ \\
\hline 435211095063701 & Cottonwood Co., MN & $07 / 12 / 99$ & $<.05$ & $<.05$ & $<.05$ & -- & $<.05$ & $<.05$ & $<.05$ & -- & $<.05$ & -- & -- & $<.05$ & -- & -- & $<.05$ & $<.05$ & $<.05$ \\
\hline 435217095063401 & Cottonwood Co., MN & $07 / 26 / 00$ & $<.05$ & $<.05$ & $<.05$ & -- & $<.05$ & .52 & .30 & -- & $<.05$ & $<.05$ & $<.05$ & $<.05$ & $<.05$ & $<.05$ & $<.05$ & .28 & .14 \\
\hline \multirow[t]{4}{*}{435218095062801} & Cottonwood Co., MN & 07/08/99 & $<.05$ & $<.05$ & $<.05$ & -- & $<.05$ & $<.05$ & $<.05$ & -- & $<.05$ & -- & -- & $<.05$ & -- & -- & $<.05$ & $<.05$ & $<.05$ \\
\hline & & 09/01/99 & $<.05$ & $<.20$ & $<.20$ & -- & $<.05$ & $<.20$ & $<.20$ & -- & $<.05$ & -- & -- & $<.05$ & -- & -- & $<.05$ & $<.20$ & $<.20$ \\
\hline & & $05 / 09 / 00$ & $<.05$ & $<.05$ & $<.05$ & -- & $<.05$ & $<.05$ & $<.05$ & -- & $<.05$ & $<.05$ & $<.05$ & $<.05$ & $<.05$ & $<.05$ & $<.05$ & $<.05$ & $<.05$ \\
\hline & & $07 / 26 / 00$ & $<.05$ & $<.05$ & $<.05$ & -- & $<.05$ & $<.05$ & $<.05$ & -- & $<.05$ & $<.05$ & $<.05$ & $<.05$ & $<.05$ & $<.05$ & $<.05$ & $<.05$ & $<.05$ \\
\hline 435239095074801 & Cottonwood Co., MN & $07 / 14 / 99$ & $<.05$ & $<.20$ & $<.20$ & -- & $<.05$ & $<.20$ & $<.20$ & -- & $<.05$ & -- & -- & $<.05$ & -- & -- & $<.05$ & $<.20$ & $<.20$ \\
\hline 435310095082501 & Cottonwood Co., MN & 07/08/99 & $<.05$ & $<.05$ & $<.05$ & -- & $<.05$ & $<.05$ & $<.05$ & -- & $<.05$ & -- & -- & $<.05$ & -- & -- & $<.05$ & $<.05$ & $<.05$ \\
\hline 435311095061801 & Cottonwood Co., MN & $06 / 27 / 00$ & $<.05$ & $<.05$ & $<.05$ & -- & $<.05$ & $<.05$ & $<.05$ & -- & $<.05$ & $<.05$ & $<.05$ & $<.05$ & $<.05$ & $<.05$ & $<.05$ & $<.05$ & $<.05$ \\
\hline 435315095091601 & Cottonwood Co., MN & $07 / 26 / 00$ & $<.05$ & $<.05$ & $<.05$ & -- & $<.05$ & $<.05$ & $<.05$ & -- & $<.05$ & $<.05$ & $<.05$ & $<.05$ & $<.05$ & $<.05$ & $<.05$ & .16 & $<.05$ \\
\hline \multirow[t]{3}{*}{435320095094901} & Cottonwood Co., MN & 07/06/99 & $<.05$ & $<.05$ & $<.05$ & -- & $<.05$ & $<.05$ & $<.05$ & -- & $<.05$ & -- & -- & $<.05$ & -- & -- & $<.05$ & $<.05$ & $<.05$ \\
\hline & & $08 / 31 / 99$ & 56 & $<.20$ & $<.20$ & -- & -- & $<.20$ & $<.20$ & -- & -- & -- & -- & -- & -- & -- & -- & $<.20$ & $<.20$ \\
\hline & & $05 / 08 / 00$ & $<.05$ & $<.05$ & $<.05$ & -- & $<.05$ & $<.05$ & $<.05$ & -- & $<.05$ & $<.05$ & $<.05$ & $<.05$ & -- & -- & $<.05$ & $<.05$ & $<.05$ \\
\hline
\end{tabular}




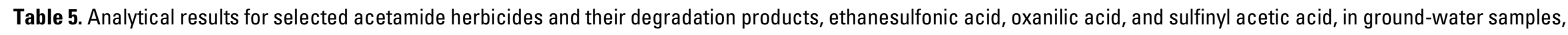
1994-2002.-Continued

[OGRL, U.S. Geological Survey Organic Geochemistry Research Laboratory, Lawrence, Kansas; ESA, ethanesulfonic acid; OXA, oxanilic acid; SAA, sulfinyl acetic acid; --, no data; <, less than]

\begin{tabular}{|c|c|c|c|c|c|c|c|c|c|c|c|c|c|c|c|c|c|c|c|}
\hline Site number & Site location & $\begin{array}{c}\text { Date of } \\
\text { collection } \\
\text { (month/day/ } \\
\text { year) }\end{array}$ & $\begin{array}{c}\text { Aceto- } \\
\text { chlor } \\
(\mu \mathrm{g} / \mathrm{L})\end{array}$ & $\begin{array}{l}\text { Aceto- } \\
\text { chlor } \\
\text { ESA } \\
(\mu \mathrm{g} / \mathrm{L})\end{array}$ & $\begin{array}{c}\text { Aceto- } \\
\text { chlor } \\
\text { OXA } \\
(\mu \mathrm{g} / \mathrm{L})\end{array}$ & $\begin{array}{l}\text { Aceto- } \\
\text { chlor } \\
\text { SAA } \\
(\mu \mathrm{g} / \mathrm{L})\end{array}$ & $\begin{array}{l}\text { Ala- } \\
\text { chlor } \\
(\mu \mathrm{g} / \mathrm{L})\end{array}$ & $\begin{array}{l}\text { Ala- } \\
\text { chlor } \\
\text { ESA } \\
(\mu \mathrm{g} / \mathrm{L})\end{array}$ & $\begin{array}{l}\text { Ala- } \\
\text { chlor } \\
\text { OXA } \\
(\mu \mathrm{g} / \mathrm{L})\end{array}$ & $\begin{array}{l}\text { Ala- } \\
\text { chlor } \\
\text { SAA } \\
(\mu \mathrm{g} / \mathrm{L})\end{array}$ & $\begin{array}{l}\text { Di- } \\
\text { meth- } \\
\text { enamid } \\
(\mu \mathrm{g} / \mathrm{L})\end{array}$ & $\begin{array}{c}\text { Di- } \\
\text { meth- } \\
\text { enamid } \\
\text { ESA } \\
(\mu \mathrm{g} / \mathrm{L})\end{array}$ & $\begin{array}{c}\text { Di- } \\
\text { meth- } \\
\text { enamid } \\
\text { OXA } \\
(\mu \mathrm{g} / \mathrm{L})\end{array}$ & $\begin{array}{c}\text { Flufen- } \\
\text { acet } \\
(\mu \mathrm{g} / \mathrm{L})\end{array}$ & $\begin{array}{l}\text { Flufen- } \\
\text { acet } \\
\text { ESA } \\
(\mu \mathrm{g} / \mathrm{L})\end{array}$ & $\begin{array}{c}\text { Flufen- } \\
\text { acet } \\
\text { OXA } \\
(\mu \mathrm{g} / \mathrm{L})\end{array}$ & $\begin{array}{l}\text { Meto- } \\
\text { lachlor } \\
(\mu \mathrm{g} / \mathrm{L})\end{array}$ & $\begin{array}{l}\text { Meto- } \\
\text { lachlor } \\
\text { ESA } \\
(\mu \mathrm{g} / \mathrm{L})\end{array}$ & $\begin{array}{c}\text { Meto- } \\
\text { lachlor } \\
\text { OXA } \\
(\mu \mathrm{g} / \mathrm{L})\end{array}$ \\
\hline \multicolumn{20}{|c|}{ Federal-State Cooperative Studies-Continued } \\
\hline \multicolumn{20}{|c|}{ Minnesota hydrologic study (TCM) —Continued } \\
\hline 435320095094901 & Cottonwood Co., MN & $04 / 27 / 99$ & $<0.05$ & $<0.20$ & $<0.20$ & -- & $<0.05$ & $<0.20$ & $<0.20$ & -- & -- & -- & -- & -- & -- & -- & $<0.05$ & $<0.20$ & $<0.20$ \\
\hline & & $06 / 29 / 00$ & $<.05$ & $<.05$ & $<.05$ & -- & $<.05$ & $<.05$ & $<.05$ & -- & $<0.05$ & $<0.05$ & $<0.05$ & $<0.05$ & $<0.05$ & $<0.05$ & $<.05$ & $<.05$ & $<.05$ \\
\hline 435417095101001 & Cottonwood Co., MN & $06 / 28 / 00$ & $<.05$ & .42 & .06 & -- & $<.05$ & .07 & $<.05$ & -- & $<.05$ & $<.05$ & $<.05$ & $<.05$ & $<.05$ & $<.05$ & $<.05$ & .53 & .06 \\
\hline 435500095105701 & Cottonwood Co., MN & 07/13/99 & $<.05$ & .27 & .19 & -- & $<.05$ & .19 & .08 & -- & $<.05$ & -- & -- & $<.05$ & -- & -- & $<.05$ & 2.8 & .48 \\
\hline 435500095112401 & Cottonwood Co., MN & 07/13/99 & $<.05$ & $<.20$ & $<.20$ & -- & $<.05$ & $<.20$ & $<.20$ & -- & $<.05$ & -- & -- & $<.05$ & -- & -- & $<.05$ & $<.20$ & $<.20$ \\
\hline 435514095112201 & Cottonwood Co., MN & $07 / 27 / 00$ & $<.05$ & $<.05$ & .07 & -- & $<.05$ & 1.2 & .16 & -- & $<.05$ & .08 & $<.05$ & $<.05$ & $<.05$ & $<.05$ & $<.05$ & .55 & .06 \\
\hline 435515095093601 & Cottonwood Co., MN & 07/07/99 & $<.05$ & $<.05$ & $<.05$ & -- & $<.05$ & $<.05$ & $<.05$ & -- & $<.05$ & -- & -- & $<.05$ & -- & -- & $<.05$ & $<.05$ & $<.05$ \\
\hline 435533095111601 & Cottonwood Co., MN & $06 / 27 / 00$ & $<.05$ & $<.05$ & $<.05$ & -- & $<.05$ & $<.05$ & $<.05$ & -- & $<.05$ & $<.05$ & $<.05$ & $<.05$ & $<.05$ & $<.05$ & $<.05$ & $<.05$ & $<.05$ \\
\hline 435605095121201 & Cottonwood Co., MN & $07 / 26 / 00$ & $<.05$ & $<.05$ & $<.05$ & -- & $<.05$ & .09 & 1.3 & -- & $<.05$ & $<.05$ & $<.05$ & $<.05$ & $<.05$ & $<.05$ & $<.05$ & $<.05$ & $<.05$ \\
\hline 435647095122201 & Cottonwood Co., MN & $07 / 27 / 00$ & $<.05$ & $<.05$ & $<.05$ & -- & $<.05$ & $<.05$ & $<.05$ & -- & $<.05$ & $<.05$ & $<.05$ & $<.05$ & $<.05$ & $<.05$ & $<.05$ & .17 & $<.05$ \\
\hline \multirow[t]{4}{*}{435748095122901} & Cottonwood Co., MN & 07/07/99 & $<.05$ & $<.05$ & $<.05$ & -- & $<.05$ & $<.05$ & $<.05$ & -- & $<.05$ & -- & -- & $<.05$ & -- & -- & $<.05$ & .19 & $<.05$ \\
\hline & & 09/01/99 & $<.05$ & $<.20$ & $<.20$ & -- & $<.05$ & $<.20$ & $<.20$ & -- & $<.05$ & -- & -- & $<.05$ & -- & -- & $<.05$ & $<.20$ & $<.20$ \\
\hline & & $05 / 09 / 00$ & $<.05$ & .21 & $<.05$ & -- & $<.05$ & $<.05$ & $<.05$ & -- & $<.05$ & $<.05$ & $<.05$ & $<.05$ & -- & -- & $<.05$ & .25 & $<.05$ \\
\hline & & $06 / 29 / 00$ & $<.05$ & $<.05$ & $<.05$ & -- & $<.05$ & $<.05$ & $<.05$ & -- & $<.05$ & $<.05$ & $<.05$ & $<.05$ & $<.05$ & $<.05$ & $<.05$ & .08 & $<.05$ \\
\hline 435842095132001 & Cottonwood Co., MN & $06 / 28 / 00$ & $<.05$ & .06 & .31 & -- & $<.05$ & .69 & .38 & -- & $<.05$ & $<.05$ & $<.05$ & $<.05$ & $<.05$ & $<.05$ & $<.05$ & $<.05$ & $<.05$ \\
\hline 435934095134701 & Cottonwood Co., MN & $07 / 13 / 99$ & $<.05$ & $<.05$ & $<.05$ & -- & $<.05$ & .13 & $<.05$ & -- & $<.05$ & -- & -- & $<.05$ & -- & -- & $<.05$ & 1.1 & $<.05$ \\
\hline 440426095112401 & Cottonwood Co., MN & $07 / 25 / 99$ & $<.05$ & $<.05$ & $<.05$ & -- & $<.05$ & $<.05$ & $<.05$ & -- & $<.05$ & $<.05$ & $<.05$ & $<.05$ & $<.05$ & $<.05$ & $<.05$ & .19 & $<.05$ \\
\hline \multicolumn{20}{|c|}{ Minnesota Pollution Control Agency (JMM) } \\
\hline 0010 & Stearns Co., MN & $11 / 00 / 99$ & $<.05$ & $<.20$ & $<.20$ & -- & $<.05$ & .38 & $<.20$ & -- & $<.05$ & -- & -- & $<.05$ & -- & -- & $<.05$ & .47 & $<.20$ \\
\hline 0017 & Stearns Co., MN & $11 / 00 / 99$ & $<.05$ & .74 & .61 & -- & $<.05$ & .80 & .55 & -- & $<.05$ & -- & -- & $<.05$ & -- & -- & $<.05$ & .43 & .37 \\
\hline 0021 & Stearns Co., MN & 07/00/99 & $<.05$ & $<.20$ & $<.20$ & -- & $<.05$ & .20 & $<.20$ & -- & $<.05$ & -- & -- & $<.05$ & -- & -- & $<.05$ & $<.20$ & $<.20$ \\
\hline 0024 & Stearns Co., MN & 07/00/99 & $<.05$ & $<.20$ & $<.20$ & -- & $<.05$ & $<.20$ & .34 & -- & $<.05$ & -- & -- & $<.05$ & -- & -- & $<.05$ & $<.20$ & $<.20$ \\
\hline 0026 & Stearns Co., MN & 07/00/99 & $<.05$ & $<.20$ & $<.20$ & -- & $<.05$ & $<.20$ & $<.20$ & -- & $<.05$ & -- & -- & $<.05$ & -- & -- & $<.05$ & $<.20$ & $<.20$ \\
\hline 0030 & Stearns Co., MN & 07/00/99 & $<.05$ & $<.20$ & $<.20$ & -- & $<.05$ & 3.1 & .27 & -- & $<.05$ & -- & -- & $<.05$ & -- & -- & $<.05$ & .66 & $<.20$ \\
\hline 0034 & Stearns Co., MN & 07/00/99 & $<.05$ & $<.20$ & $<.20$ & -- & $<.05$ & 1.2 & $<.20$ & -- & $<.05$ & -- & -- & $<.05$ & -- & -- & $<.05$ & .34 & .26 \\
\hline 0036 & Stearns Co., MN & 07/00/99 & $<.05$ & $<.20$ & $<.20$ & -- & $<.05$ & 2.6 & .38 & -- & $<.05$ & -- & -- & $<.05$ & -- & -- & $<.05$ & $<.20$ & $<.20$ \\
\hline
\end{tabular}


Table 5. Analytical results for selected acetamide herbicides and their degradation products, ethanesulfonic acid, oxanilic acid, and sulfinyl acetic acid, in ground-water samples, 1994-2002.-Continued

[OGRL, U.S. Geological Survey Organic Geochemistry Research Laboratory, Lawrence, Kansas; ESA, ethanesulfonic acid; OXA, oxanilic acid; SAA, sulfinyl acetic acid; --, no data; <, less than]

\begin{tabular}{|c|c|c|c|c|c|c|c|c|c|c|c|c|c|c|c|c|c|c|c|c|}
\hline Site number & Site location & $\begin{array}{c}\text { Date of } \\
\text { collection } \\
\text { (month/day/ } \\
\text { year) }\end{array}$ & $\begin{array}{l}\text { Aceto- } \\
\text { chlor } \\
(\mu \mathrm{g} / \mathrm{L})\end{array}$ & $\begin{array}{c}\text { Aceto- } \\
\text { chlor } \\
\text { ESA } \\
(\mu \mathrm{g} / \mathrm{L})\end{array}$ & $\begin{array}{l}\text { Aceto- } \\
\text { chlor } \\
\text { OXA } \\
(\mu \mathrm{g} / \mathrm{L})\end{array}$ & $\begin{array}{l}\text { Aceto- } \\
\text { chlor } \\
\text { SAA } \\
(\mu \mathrm{g} / \mathrm{L})\end{array}$ & $\begin{array}{l}\text { Ala- } \\
\text { chlor } \\
(\mu \mathrm{g} / \mathrm{L})\end{array}$ & $\begin{array}{l}\text { Ala- } \\
\text { chlor } \\
\text { ESA } \\
(\mu \mathrm{g} / \mathrm{L})\end{array}$ & $\begin{array}{l}\text { Ala- } \\
\text { chlor } \\
\text { OXA } \\
(\mu \mathrm{g} / \mathrm{L})\end{array}$ & $\begin{array}{c}\text { Ala- } \\
\text { chlor } \\
\text { SAA } \\
(\mu \mathrm{g} / \mathrm{L})\end{array}$ & $\begin{array}{c}\text { Di- } \\
\text { meth- } \\
\text { enamid } \\
(\mu \mathrm{g} / \mathrm{L})\end{array}$ & $\begin{array}{c}\text { Di- } \\
\text { meth- } \\
\text { enamid } \\
\text { ESA } \\
(\mu \mathrm{g} / \mathrm{L})\end{array}$ & $\begin{array}{c}\text { Di- } \\
\text { meth- } \\
\text { enamid } \\
\text { OXA } \\
(\mu \mathrm{g} / \mathrm{L})\end{array}$ & $\begin{array}{l}\text { Flufen- } \\
\text { acet } \\
(\mu \mathrm{g} / \mathrm{L})\end{array}$ & $\begin{array}{c}\text { Flufen- } \\
\text { acet } \\
\text { ESA } \\
(\mu \mathrm{g} / \mathrm{L})\end{array}$ & $\begin{array}{c}\text { Flufen- } \\
\text { acet } \\
\text { OXA } \\
(\mu \mathrm{g} / \mathrm{L})\end{array}$ & $\begin{array}{c}\text { Meto- } \\
\text { lachlor } \\
(\mu \mathrm{g} / \mathrm{L})\end{array}$ & $\begin{array}{c}\text { Meto- } \\
\text { lachlor } \\
\text { ESA } \\
(\mu \mathrm{g} / \mathrm{L})\end{array}$ & $\begin{array}{c}\text { Meto- } \\
\text { lachlor } \\
\text { OXA } \\
(\mu \mathrm{g} / \mathrm{L})\end{array}$ & 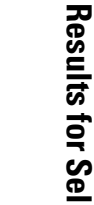 \\
\hline \multicolumn{20}{|c|}{ Federal-State Cooperative Studies-Continued } & \\
\hline \multicolumn{20}{|c|}{ Minnesota Pollution Control Agency (JMM)—Continued } & \\
\hline 0039 & Stearns Co., MN & 07/00/99 & $<0.05$ & $<0.20$ & $<0.20$ & -- & $<0.05$ & 2.0 & 0.37 & -- & $<0.05$ & -- & -- & $<0.05$ & -- & -- & $<0.05$ & $<0.20$ & $<0.20$ & \\
\hline 0040 & Stearns Co., MN & 07/00/99 & $<.05$ & $<.20$ & $<.20$ & -- & $<.05$ & 2.2 & .40 & -- & $<.05$ & -- & -- & $<.05$ & -- & -- & $<.05$ & $<.20$ & $<.20$ & \\
\hline 0042 & Stearns Co., MN & 07/00/99 & $<.05$ & $<.20$ & $<.20$ & -- & $<.05$ & $<.20$ & $<.20$ & -- & $<.05$ & -- & -- & $<.05$ & -- & -- & $<.05$ & $<.20$ & $<.20$ & \\
\hline 0043 & Stearns Co., MN & 07/00/99 & $<.05$ & $<.20$ & $<.20$ & -- & $<.05$ & 7.5 & 1.2 & -- & $<.05$ & -- & -- & $<.05$ & -- & -- & $<.05$ & $<.20$ & $<.20$ & \\
\hline 0048 & Stearns Co., MN & $06 / 00 / 99$ & $<.05$ & $<.20$ & $<.20$ & -- & $<.05$ & 2.2 & $<.20$ & -- & $<.05$ & -- & -- & $<.05$ & -- & -- & $<.05$ & 1.6 & 1.3 & \\
\hline 0050 & Stearns Co., MN & 06/00/99 & $<.05$ & $<.20$ & $<.20$ & -- & $<.05$ & .31 & $<.20$ & -- & $<.05$ & -- & -- & $<.05$ & -- & -- & $<.05$ & $<.20$ & $<.20$ & \\
\hline 0052 & Stearns Co., MN & 06/00/99 & $<.05$ & $<.20$ & $<.20$ & -- & $<.05$ & 1.1 & $<.20$ & -- & $<.05$ & -- & -- & $<.05$ & -- & -- & $<.05$ & .34 & .26 & \\
\hline 0054 & Stearns Co., MN & 06/00/99 & $<.05$ & $<.20$ & $<.20$ & -- & $<.05$ & $<.20$ & $<.20$ & -- & $<.05$ & -- & -- & $<.05$ & -- & -- & $<.05$ & $<.20$ & $<.20$ & \\
\hline 0055 & Stearns Co., MN & 06/00/99 & $<.05$ & $<.20$ & $<.20$ & -- & $<.05$ & .20 & $<.20$ & -- & $<.05$ & -- & -- & $<.05$ & -- & -- & $<.05$ & $<.20$ & $<.20$ & \\
\hline 0059 & Stearns Co., MN & 06/00/99 & $<.05$ & $<.20$ & $<.20$ & -- & $<.05$ & 1.3 & .41 & -- & $<.05$ & -- & -- & $<.05$ & -- & -- & .06 & .65 & .69 & $\overline{\underline{B}}$ \\
\hline 0062 & Stearns Co., MN & 06/00/99 & $<.05$ & $<.20$ & $<.20$ & -- & $<.05$ & 1.3 & .43 & -- & $<.05$ & -- & -- & $<.05$ & -- & -- & .06 & .67 & .75 & \\
\hline 0064 & Stearns Co., MN & 06/00/99 & $<.05$ & $<.20$ & $<.20$ & -- & .18 & 1.2 & .65 & -- & $<.05$ & -- & -- & $<.05$ & -- & -- & $<.05$ & .60 & .47 & \\
\hline 0066 & Stearns Co., MN & 06/00/99 & $<.05$ & $<.20$ & $<.20$ & -- & $<.05$ & $<.20$ & $<.20$ & -- & $<.05$ & -- & -- & $<.05$ & -- & -- & $<.05$ & $<.20$ & $<.20$ & 을 \\
\hline 0069 & Stearns Co., MN & 06/00/99 & $<.05$ & $<.20$ & $<.20$ & -- & $<.05$ & 1.4 & .29 & -- & $<.05$ & -- & -- & $<.05$ & -- & -- & $<.05$ & $<.20$ & $<.20$ & 를 \\
\hline 0071 & Stearns Co., MN & 06/00/99 & $<.05$ & $<.20$ & $<.20$ & -- & $<.05$ & $<.20$ & $<.20$ & -- & $<.05$ & -- & -- & $<.05$ & -- & -- & $<.05$ & $<.20$ & $<.20$ & 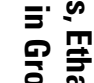 \\
\hline 0073 & Stearns Co., MN & 06/00/99 & $<.05$ & $<.20$ & $<.20$ & -- & $<.05$ & $<.20$ & $<.20$ & -- & $<.05$ & -- & -- & $<.05$ & -- & -- & $<.20$ & $<.20$ & $<.20$ & \\
\hline 0075 & Stearns Co., MN & 06/00/99 & $<.05$ & $<.20$ & $<.20$ & -- & $<.05$ & $<.20$ & $<.20$ & -- & $<.05$ & -- & -- & $<.05$ & -- & -- & $<.05$ & $<.20$ & $<.20$ & \\
\hline 0076 & Stearns Co., MN & 06/00/99 & $<.05$ & $<.20$ & $<.20$ & -- & $<.05$ & $<.20$ & $<.20$ & -- & $<.05$ & -- & -- & $<.05$ & -- & -- & $<.05$ & $<.20$ & $<.20$ & \\
\hline 0080 & Stearns Co., MN & 07/00/99 & $<.05$ & $<.20$ & $<.20$ & -- & $<.05$ & 1.8 & 1.2 & -- & $<.05$ & -- & -- & $<.05$ & -- & -- & $<.05$ & $<.20$ & $<.20$ & \\
\hline 0083 & Stearns Co., MN & 07/00/99 & $<.05$ & $<.20$ & $<.20$ & -- & $<.05$ & 2.8 & $<.20$ & -- & $<.05$ & -- & -- & $<.05$ & -- & -- & $<.05$ & $<.20$ & $<.20$ & \\
\hline 0089 & Stearns Co., MN & 07/00/99 & $<.05$ & $<.20$ & $<.20$ & -- & $<.05$ & 3.1 & .30 & -- & $<.05$ & -- & -- & $<.05$ & -- & -- & $<.05$ & .36 & $<.20$ & 氧. \\
\hline 0096 & Stearns Co., MN & 06/00/99 & $<.05$ & $<.20$ & $<.20$ & -- & $<.05$ & $<.20$ & $<.20$ & -- & $<.05$ & -- & -- & $<.05$ & -- & -- & $<.05$ & $<.20$ & $<.20$ & क्ष \\
\hline 0098 & Stearns Co., MN & 06/00/99 & $<.05$ & $<.20$ & $<.20$ & -- & $<.05$ & 1.9 & $<.20$ & -- & $<.05$ & -- & -- & $<.05$ & -- & -- & $<.05$ & 1.7 & 1.2 & 疍. \\
\hline 0099 & Stearns Co., MN & 06/00/99 & $<.05$ & $<.20$ & $<.20$ & -- & $<.05$ & 1.1 & $<.20$ & -- & $<.05$ & -- & -- & $<.05$ & -- & -- & $<.05$ & .81 & $<.20$ & \\
\hline 0102 & Stearns Co., MN & $04 / 18 / 99$ & $<.05$ & $<.20$ & $<.20$ & -- & $<.05$ & 1.9 & $<.20$ & -- & -- & -- & -- & -- & -- & -- & $<.05$ & $<.20$ & $<.20$ & \\
\hline
\end{tabular}




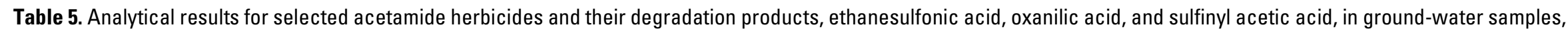
1994-2002.-Continued

[OGRL, U.S. Geological Survey Organic Geochemistry Research Laboratory, Lawrence, Kansas; ESA, ethanesulfonic acid; OXA, oxanilic acid; SAA, sulfinyl acetic acid; --, no data; <, less than]

\begin{tabular}{|c|c|c|c|c|c|c|c|c|c|c|c|c|c|c|c|c|c|c|c|}
\hline Site number & Site location & $\begin{array}{c}\text { Date of } \\
\text { collection } \\
\text { (month/day/ } \\
\text { year) }\end{array}$ & $\begin{array}{l}\text { Aceto- } \\
\text { chlor } \\
(\mu \mathrm{g} / \mathrm{L})\end{array}$ & $\begin{array}{l}\text { Aceto- } \\
\text { chlor } \\
\text { ESA } \\
(\mu \mathrm{g} / \mathrm{L})\end{array}$ & $\begin{array}{l}\text { Aceto- } \\
\text { chlor } \\
\text { OXA } \\
(\mu \mathrm{g} / \mathrm{L})\end{array}$ & $\begin{array}{c}\text { Aceto- } \\
\text { chlor } \\
\text { SAA } \\
(\mu \mathrm{g} / \mathrm{L})\end{array}$ & $\begin{array}{l}\text { Ala- } \\
\text { chlor } \\
(\mu \mathrm{g} / \mathrm{L})\end{array}$ & $\begin{array}{l}\text { Ala- } \\
\text { chlor } \\
\text { ESA } \\
(\mu \mathrm{g} / \mathrm{L})\end{array}$ & $\begin{array}{l}\text { Ala- } \\
\text { chlor } \\
\text { OXA } \\
(\mu \mathrm{g} / \mathrm{L})\end{array}$ & $\begin{array}{l}\text { Ala- } \\
\text { chlor } \\
\text { SAA } \\
(\mu \mathrm{g} / \mathrm{L})\end{array}$ & $\begin{array}{c}\text { Di- } \\
\text { meth- } \\
\text { enamid } \\
(\mu \mathrm{g} / \mathrm{L})\end{array}$ & $\begin{array}{c}\text { Di- } \\
\text { meth- } \\
\text { enamid } \\
\text { ESA } \\
(\mu \mathrm{g} / \mathrm{L})\end{array}$ & $\begin{array}{c}\text { Di- } \\
\text { meth- } \\
\text { enamid } \\
\text { OXA } \\
(\mu \mathrm{g} / \mathrm{L})\end{array}$ & $\begin{array}{l}\text { Flufen- } \\
\text { acet } \\
(\mu \mathrm{g} / \mathrm{L})\end{array}$ & $\begin{array}{c}\text { Flufen- } \\
\text { acet } \\
\text { ESA } \\
(\mu \mathrm{g} / \mathrm{L})\end{array}$ & $\begin{array}{c}\text { Flufen- } \\
\text { acet } \\
\text { OXA } \\
(\mu \mathrm{g} / \mathrm{L})\end{array}$ & $\begin{array}{l}\text { Meto- } \\
\text { lachlor } \\
(\mu \mathrm{g} / \mathrm{L})\end{array}$ & $\begin{array}{c}\text { Meto- } \\
\text { lachlor } \\
\text { ESA } \\
(\mu \mathrm{g} / \mathrm{L})\end{array}$ & $\begin{array}{c}\text { Meto- } \\
\text { lachlor } \\
\text { OXA } \\
(\mu \mathrm{g} / \mathrm{L})\end{array}$ \\
\hline \multicolumn{20}{|c|}{ Federal-State Cooperative Studies-Continued } \\
\hline \multicolumn{20}{|c|}{ Minnesota Pollution Control Agency (JMM)—Continued } \\
\hline 0103 & Stearns Co., MN & $04 / 14 / 99$ & $<0.05$ & $<0.20$ & $<0.20$ & -- & $<0.05$ & 2.0 & $<0.20$ & -- & -- & -- & -- & -- & -- & -- & $<0.05$ & $<0.20$ & $<0.20$ \\
\hline 0104 & Stearns Co., MN & 04/08/99 & $<.05$ & $<.20$ & $<.20$ & -- & $<.05$ & 2.1 & $<.20$ & -- & -- & -- & -- & -- & -- & -- & $<.05$ & $<.20$ & $<.20$ \\
\hline 0105 & Stearns Co., MN & 04/11/99 & $<.05$ & $<.20$ & $<.20$ & -- & $<.05$ & 2.2 & $<.20$ & -- & -- & -- & -- & -- & -- & -- & $<.05$ & $<.20$ & $<.20$ \\
\hline 0106 & Stearns Co., MN & 04/05/99 & $<.05$ & $<.20$ & $<.20$ & -- & $<.05$ & 2.3 & $<.20$ & -- & -- & -- & -- & -- & -- & -- & $<.05$ & $<.20$ & $<.20$ \\
\hline 0107 & Stearns Co., MN & $04 / 02 / 99$ & $<.05$ & $<.20$ & $<.20$ & -- & $<.05$ & 2.3 & $<.20$ & -- & -- & -- & -- & -- & -- & -- & $<.05$ & $<.20$ & $<.20$ \\
\hline 0108 & Stearns Co., MN & 03/29/99 & $<.05$ & $<.20$ & $<.20$ & -- & $<.05$ & 2.1 & $<.20$ & -- & -- & -- & -- & -- & -- & -- & $<.05$ & $<.20$ & $<.20$ \\
\hline 0109 & Stearns Co., MN & 03/22/99 & $<.05$ & $<.20$ & $<.20$ & -- & $<.05$ & 2.2 & $<.20$ & -- & -- & -- & -- & -- & -- & -- & $<.05$ & $<.20$ & $<.20$ \\
\hline 0211 & Stearns Co., MN & 08/00/99 & $<.05$ & $<.20$ & $<.20$ & -- & $<.05$ & .29 & $<.20$ & -- & $<0.05$ & -- & -- & $<0.05$ & -- & -- & $<.05$ & .44 & $<.20$ \\
\hline 0214 & Stearns Co., MN & 08/00/99 & $<.05$ & $<.20$ & $<.20$ & -- & $<.05$ & $<.20$ & $<.20$ & -- & $<.05$ & -- & -- & $<.05$ & -- & -- & $<.05$ & $<.20$ & $<.20$ \\
\hline 0226 & Stearns Co., MN & 08/00/99 & $<.05$ & $<.20$ & $<.20$ & -- & $<.05$ & 3.0 & $<.20$ & -- & $<.05$ & -- & -- & $<.05$ & -- & -- & $<.05$ & $<.20$ & $<.20$ \\
\hline 0230 & Stearns Co., MN & 08/00/99 & $<.05$ & $<.20$ & $<.20$ & -- & $<.05$ & $<.20$ & $<.20$ & -- & $<.05$ & -- & -- & $<.05$ & -- & -- & $<.05$ & $<.20$ & $<.20$ \\
\hline 0233 & Stearns Co., MN & 08/00/99 & $<.05$ & .82 & $<.20$ & -- & $<.05$ & .53 & $<.20$ & -- & $<.05$ & -- & -- & $<.05$ & -- & -- & $<.05$ & .47 & $<.20$ \\
\hline 0237 & Stearns Co., MN & 08/00/99 & $<.05$ & 1.0 & $<.20$ & -- & $<.05$ & .63 & $<.20$ & -- & $<.05$ & -- & -- & $<.05$ & -- & -- & $<.05$ & .85 & .32 \\
\hline 0239 & Stearns Co., MN & 08/00/99 & $<.05$ & 2.9 & 1.3 & -- & $<.05$ & .48 & $<.20$ & -- & $<.05$ & -- & -- & $<.05$ & -- & -- & $<.05$ & 5.0 & 1.5 \\
\hline 0244 & Stearns Co., MN & 06/00/99 & $<.05$ & $<.20$ & $<.20$ & -- & $<.05$ & 2.0 & .45 & -- & $<.05$ & -- & -- & $<.05$ & -- & -- & $<.05$ & $<.20$ & .31 \\
\hline 0245 & Stearns Co., MN & 06/00/99 & $<.05$ & $<.20$ & $<.20$ & -- & $<.05$ & $<.20$ & $<.20$ & -- & $<.05$ & -- & -- & $<.05$ & -- & -- & $<.05$ & $<.20$ & $<.20$ \\
\hline 0290 & Stearns Co., MN & 06/00/99 & $<.05$ & $<.20$ & $<.20$ & -- & $<.05$ & $<.20$ & $<.20$ & -- & $<.05$ & -- & -- & -- & -- & -- & $<.05$ & $<.20$ & $<.20$ \\
\hline 0291 & Stearns Co., MN & 06/00/99 & $<.05$ & $<.20$ & $<.20$ & -- & $<.05$ & .73 & .30 & -- & $<.05$ & -- & -- & -- & -- & -- & $<.05$ & $<.20$ & .20 \\
\hline 0294 & Stearns Co., MN & 06/00/99 & $<.05$ & $<.20$ & $<.20$ & -- & $<.05$ & 1.3 & .76 & -- & $<.05$ & -- & -- & -- & -- & -- & $<.05$ & .28 & .51 \\
\hline 0296 & Stearns Co., MN & 06/00/99 & $<.05$ & $<.20$ & $<.20$ & -- & $<.05$ & 2.9 & .91 & -- & $<.05$ & -- & -- & -- & -- & -- & $<.05$ & $<.20$ & $<.20$ \\
\hline 0300 & Stearns Co., MN & 06/00/99 & $<.05$ & $<.20$ & $<.20$ & -- & $<.05$ & 6.2 & $<.20$ & -- & $<.05$ & -- & -- & -- & -- & -- & $<.05$ & $<.20$ & $<.20$ \\
\hline 0301 & Stearns Co., MN & 05/24/99 & $<.05$ & $<.20$ & $<.20$ & -- & $<.05$ & 4.3 & $<.20$ & -- & -- & -- & -- & -- & -- & -- & $<.05$ & $<.20$ & $<.20$ \\
\hline 0302 & Stearns Co., MN & 05/21/99 & $<.05$ & $<.20$ & $<.20$ & -- & $<.05$ & 2.4 & $<.20$ & -- & -- & -- & -- & -- & -- & -- & $<.05$ & $<.20$ & $<.20$ \\
\hline 0303 & Stearns Co., MN & 05/14/99 & $<.05$ & $<.20$ & $<.20$ & -- & $<.05$ & 1.6 & $<.20$ & -- & -- & -- & -- & -- & -- & -- & $<.05$ & $<.20$ & $<.20$ \\
\hline 0304 & Stearns Co., MN & 05/17/99 & $<.05$ & $<.20$ & $<.20$ & -- & $<.05$ & 1.6 & $<.20$ & -- & -- & -- & -- & -- & -- & -- & $<.05$ & $<.20$ & $<.20$ \\
\hline
\end{tabular}


Table 5. Analytical results for selected acetamide herbicides and their degradation products, ethanesulfonic acid, oxanilic acid, and sulfinyl acetic acid, in ground-water samples, 1994-2002.-Continued

[OGRL, U.S. Geological Survey Organic Geochemistry Research Laboratory, Lawrence, Kansas; ESA, ethanesulfonic acid; OXA, oxanilic acid; SAA, sulfinyl acetic acid; --, no data; <, less than]

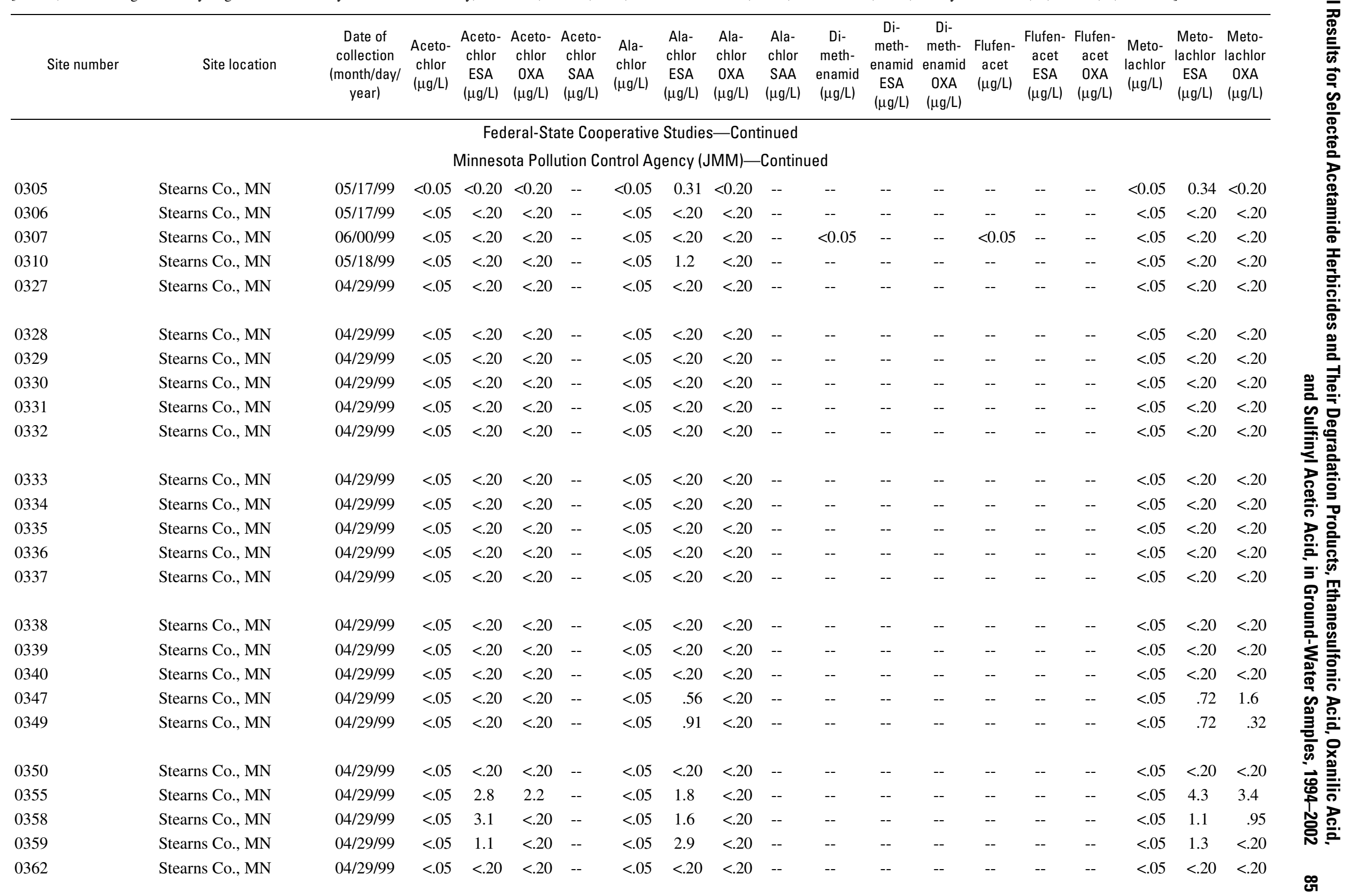




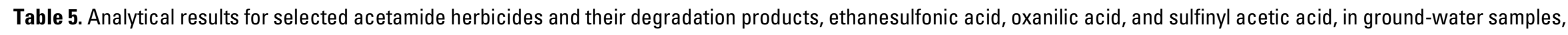
1994-2002.-Continued

[OGRL, U.S. Geological Survey Organic Geochemistry Research Laboratory, Lawrence, Kansas; ESA, ethanesulfonic acid; OXA, oxanilic acid; SAA, sulfinyl acetic acid; --, no data; <, less than]

\begin{tabular}{|c|c|c|c|c|c|c|c|c|c|c|c|c|c|c|c|c|c|c|c|}
\hline Site number & Site location & $\begin{array}{c}\text { Date of } \\
\text { collection } \\
\text { (month/day/ } \\
\text { year) }\end{array}$ & $\begin{array}{l}\text { Aceto- } \\
\text { chlor } \\
(\mu \mathrm{g} / \mathrm{L})\end{array}$ & $\begin{array}{l}\text { Aceto- } \\
\text { chlor } \\
\text { ESA } \\
(\mu \mathrm{g} / \mathrm{L})\end{array}$ & $\begin{array}{l}\text { Aceto- } \\
\text { chlor } \\
\text { OXA } \\
(\mu \mathrm{g} / \mathrm{L})\end{array}$ & $\begin{array}{c}\text { Aceto- } \\
\text { chlor } \\
\text { SAA } \\
(\mu \mathrm{g} / \mathrm{L})\end{array}$ & $\begin{array}{l}\text { Ala- } \\
\text { chlor } \\
(\mu \mathrm{g} / \mathrm{L})\end{array}$ & $\begin{array}{l}\text { Ala- } \\
\text { chlor } \\
\text { ESA } \\
(\mu \mathrm{g} / \mathrm{L})\end{array}$ & $\begin{array}{l}\text { Ala- } \\
\text { chlor } \\
\text { OXA } \\
(\mu \mathrm{g} / \mathrm{L})\end{array}$ & $\begin{array}{l}\text { Ala- } \\
\text { chlor } \\
\text { SAA } \\
(\mu \mathrm{g} / \mathrm{L})\end{array}$ & $\begin{array}{c}\text { Di- } \\
\text { meth- } \\
\text { enamid } \\
(\mu \mathrm{g} / \mathrm{L})\end{array}$ & $\begin{array}{c}\text { Di- } \\
\text { meth- } \\
\text { enamid } \\
\text { ESA } \\
(\mu \mathrm{g} / \mathrm{L})\end{array}$ & $\begin{array}{c}\text { Di- } \\
\text { meth- } \\
\text { enamid } \\
\text { OXA } \\
(\mu \mathrm{g} / \mathrm{L})\end{array}$ & $\begin{array}{l}\text { Flufen- } \\
\text { acet } \\
(\mu \mathrm{g} / \mathrm{L})\end{array}$ & $\begin{array}{c}\text { Flufen- } \\
\text { acet } \\
\text { ESA } \\
(\mu \mathrm{g} / \mathrm{L})\end{array}$ & $\begin{array}{c}\text { Flufen- } \\
\text { acet } \\
\text { OXA } \\
(\mu \mathrm{g} / \mathrm{L})\end{array}$ & $\begin{array}{l}\text { Meto- } \\
\text { lachlor } \\
(\mu \mathrm{g} / \mathrm{L})\end{array}$ & $\begin{array}{c}\text { Meto- } \\
\text { lachlor } \\
\text { ESA } \\
(\mu \mathrm{g} / \mathrm{L})\end{array}$ & $\begin{array}{c}\text { Meto- } \\
\text { lachlor } \\
\text { OXA } \\
(\mu \mathrm{g} / \mathrm{L})\end{array}$ \\
\hline \multicolumn{20}{|c|}{ Federal-State Cooperative Studies-Continued } \\
\hline \multicolumn{20}{|c|}{ Minnesota Pollution Control Agency (JMM) —Continued } \\
\hline 0365 & Stearns Co., MN & $04 / 29 / 99$ & $<0.05$ & $<0.20$ & $<0.20$ & -- & $<0.05$ & $<0.20$ & $<0.20$ & -- & -- & -- & -- & -- & -- & -- & $<0.05$ & $<0.20$ & $<0.20$ \\
\hline 0366 & Stearns Co., MN & $04 / 29 / 99$ & $<.05$ & $<.20$ & $<.20$ & -- & $<.05$ & $<.20$ & $<.20$ & -- & -- & -- & -- & -- & -- & -- & $<.05$ & $<.20$ & $<.20$ \\
\hline 0378 & Stearns Co., MN & 04/29/99 & $<.05$ & $<.20$ & $<.20$ & -- & $<.05$ & $<.20$ & $<.20$ & -- & -- & -- & -- & -- & -- & -- & $<.05$ & $<.20$ & $<.20$ \\
\hline 0380 & Stearns Co., MN & 04/29/99 & $<.05$ & $<.20$ & $<.20$ & -- & $<.05$ & $<.20$ & $<.20$ & -- & -- & -- & -- & -- & -- & -- & $<.05$ & $<.20$ & $<.20$ \\
\hline 0382 & Stearns Co., MN & $04 / 29 / 99$ & $<.05$ & $<.20$ & $<.20$ & -- & $<.05$ & 1.8 & $<.20$ & -- & -- & -- & -- & -- & -- & -- & $<.05$ & $<.20$ & $<.20$ \\
\hline 0390 & Stearns Co., MN & 03/03/99 & $<.05$ & 2.8 & $<.20$ & -- & $<.05$ & 1.4 & $<.20$ & -- & -- & -- & -- & -- & -- & -- & $<.05$ & 1.1 & .63 \\
\hline 0391 & Stearns Co., MN & 03/03/99 & $<.05$ & 1.4 & $<.20$ & -- & $<.05$ & 1.3 & $<.20$ & -- & -- & -- & -- & -- & -- & -- & $<.05$ & .56 & $<.20$ \\
\hline 0395 & Stearns Co., MN & 03/03/99 & $<.05$ & $<.20$ & $<.20$ & -- & $<.05$ & $<.20$ & $<.20$ & -- & -- & -- & -- & -- & -- & -- & $<.05$ & $<.20$ & $<.20$ \\
\hline 0401 & Stearns Co., MN & 03/02/99 & $<.05$ & $<.20$ & $<.20$ & -- & $<.05$ & $<.20$ & $<.20$ & -- & -- & -- & -- & -- & -- & -- & $<.05$ & $<.20$ & $<.20$ \\
\hline 0406 & Stearns Co., MN & 03/02/99 & $<.05$ & 2.2 & .39 & -- & $<.05$ & 1.5 & $<.20$ & -- & -- & -- & -- & -- & -- & -- & $<.05$ & 7.3 & 2.8 \\
\hline 0408 & Stearns Co., MN & 03/02/99 & $<.05$ & $<.20$ & $<.20$ & -- & $<.05$ & $<.20$ & $<.20$ & -- & -- & -- & -- & -- & -- & -- & $<.05$ & $<.20$ & $<.20$ \\
\hline 0409 & Stearns Co., MN & 03/02/99 & $<.05$ & $<.20$ & $<.20$ & -- & $<.05$ & $<.20$ & $<.20$ & -- & -- & -- & -- & -- & -- & -- & $<.05$ & $<.20$ & .23 \\
\hline 0414 & Stearns Co., MN & 03/01/99 & $<.05$ & $<.20$ & $<.20$ & -- & $<.05$ & 3.1 & $<.20$ & -- & -- & -- & -- & -- & -- & -- & $<.05$ & $<.20$ & $<.20$ \\
\hline 0416 & Stearns Co., MN & 03/01/99 & $<.05$ & $<.20$ & $<.20$ & -- & $<.05$ & $<.20$ & $<.20$ & -- & -- & -- & -- & -- & -- & -- & $<.05$ & $<.20$ & $<.20$ \\
\hline 0420 & Stearns Co., MN & 03/01/99 & $<.05$ & $<.20$ & $<.20$ & -- & $<.05$ & $<.20$ & $<.20$ & -- & -- & -- & -- & -- & -- & -- & $<.05$ & $<.20$ & $<.20$ \\
\hline 0451 & Stearns Co., MN & $11 / 00 / 99$ & $<.05$ & $<.20$ & $<.20$ & -- & $<.05$ & $<.20$ & $<.20$ & -- & $<0.05$ & -- & -- & $<0.05$ & -- & -- & $<.05$ & $<.20$ & $<.20$ \\
\hline 0454 & Stearns Co., MN & $11 / 00 / 99$ & $<.05$ & $<.20$ & $<.20$ & -- & $<.05$ & 2.5 & $<.20$ & -- & $<.05$ & -- & -- & $<.05$ & -- & -- & $<.05$ & $<.20$ & $<.20$ \\
\hline 0461 & Stearns Co., MN & $11 / 00 / 99$ & $<.05$ & $<.20$ & $<.20$ & -- & $<.05$ & $<.20$ & $<.20$ & -- & $<.05$ & -- & -- & $<.05$ & -- & -- & $<.05$ & $<.20$ & $<.20$ \\
\hline 0472 & Stearns Co., MN & $11 / 00 / 99$ & $<.05$ & $<.20$ & $<.20$ & -- & $<.05$ & .29 & $<.20$ & -- & $<.05$ & -- & -- & $<.05$ & -- & -- & $<.05$ & .40 & $<.20$ \\
\hline 0473 & Stearns Co., MN & $11 / 00 / 99$ & $<.05$ & $<.20$ & $<.20$ & -- & $<.05$ & $<.20$ & $<.20$ & -- & $<.05$ & -- & -- & $<.05$ & -- & -- & $<.05$ & $<.20$ & $<.20$ \\
\hline 0474 & Stearns Co., MN & $11 / 00 / 99$ & $<.05$ & $<.20$ & $<.20$ & -- & $<.05$ & .25 & $<.20$ & -- & $<.05$ & -- & -- & $<.05$ & -- & -- & $<.05$ & .40 & $<.20$ \\
\hline 0480 & Stearns Co., MN & $11 / 00 / 99$ & $<.05$ & $<.20$ & $<.20$ & -- & $<.05$ & $<.20$ & $<.20$ & -- & $<.05$ & -- & -- & $<.05$ & -- & -- & $<.05$ & $<.20$ & $<.20$ \\
\hline 0481 & Stearns Co., MN & $11 / 00 / 99$ & $<.05$ & $<.20$ & $<.20$ & -- & $<.05$ & .33 & $<.20$ & -- & $<.05$ & -- & -- & $<.05$ & -- & -- & $<.05$ & $<.20$ & $<.20$ \\
\hline 0482 & Stearns Co., MN & $11 / 00 / 99$ & $<.05$ & .77 & .97 & -- & $<.05$ & .82 & .75 & -- & $<.05$ & -- & -- & $<.05$ & -- & -- & $<.05$ & .45 & .35 \\
\hline 0483 & Stearns Co., MN & $11 / 00 / 99$ & $<.05$ & $<.20$ & $<.20$ & -- & $<.05$ & $<.20$ & $<.20$ & -- & $<.05$ & -- & -- & $<.05$ & -- & -- & $<.05$ & $<.20$ & $<.20$ \\
\hline
\end{tabular}


Table 5. Analytical results for selected acetamide herbicides and their degradation products, ethanesulfonic acid, oxanilic acid, and sulfinyl acetic acid, in ground-water samples, 1994-2002.-Continued

[OGRL, U.S. Geological Survey Organic Geochemistry Research Laboratory, Lawrence, Kansas; ESA, ethanesulfonic acid; OXA, oxanilic acid; SAA, sulfinyl acetic acid; --, no data; <, less than]

\begin{tabular}{|c|c|c|c|c|c|c|c|c|c|c|c|c|c|c|c|c|c|c|c|c|}
\hline Site number & Site location & $\begin{array}{c}\text { Date of } \\
\text { collection } \\
\text { (month/day/ } \\
\text { year) }\end{array}$ & $\begin{array}{l}\text { Aceto- } \\
\text { chlor } \\
(\mu \mathrm{g} / \mathrm{L})\end{array}$ & $\begin{array}{l}\text { Aceto- } \\
\text { chlor } \\
\text { ESA } \\
(\mu \mathrm{g} / \mathrm{L})\end{array}$ & $\begin{array}{c}\text { Aceto- } \\
\text { chlor } \\
\text { OXA } \\
(\mu \mathrm{g} / \mathrm{L})\end{array}$ & $\begin{array}{l}\text { Aceto- } \\
\text { chlor } \\
\text { SAA } \\
(\mu \mathrm{g} / \mathrm{L})\end{array}$ & $\begin{array}{l}\text { Ala- } \\
\text { chlor } \\
(\mu \mathrm{g} / \mathrm{L})\end{array}$ & $\begin{array}{l}\text { Ala- } \\
\text { chlor } \\
\text { ESA } \\
(\mu \mathrm{g} / \mathrm{L})\end{array}$ & $\begin{array}{l}\text { Ala- } \\
\text { chlor } \\
\text { OXA } \\
(\mu \mathrm{g} / \mathrm{L})\end{array}$ & $\begin{array}{c}\text { Ala- } \\
\text { chlor } \\
\text { SAA } \\
(\mu \mathrm{g} / \mathrm{L})\end{array}$ & $\begin{array}{c}\mathrm{Di}- \\
\text { meth- } \\
\text { enamid } \\
(\mu \mathrm{g} / \mathrm{L})\end{array}$ & $\begin{array}{c}\text { Di- } \\
\text { meth- } \\
\text { enamid } \\
\text { ESA } \\
(\mu \mathrm{g} / \mathrm{L})\end{array}$ & $\begin{array}{c}\text { Di- } \\
\text { meth- } \\
\text { enamid } \\
\text { OXA } \\
(\mu \mathrm{g} / \mathrm{L})\end{array}$ & $\begin{array}{l}\text { Flufen- } \\
\text { acet } \\
(\mu \mathrm{g} / \mathrm{L})\end{array}$ & $\begin{array}{c}\text { Flufen- } \\
\text { acet } \\
\text { ESA } \\
(\mu \mathrm{g} / \mathrm{L})\end{array}$ & $\begin{array}{c}\text { Flufen- } \\
\text { acet } \\
\text { OXA } \\
(\mu \mathrm{g} / \mathrm{L})\end{array}$ & $\begin{array}{c}\text { Meto- } \\
\text { lachlor } \\
(\mu \mathrm{g} / \mathrm{L})\end{array}$ & $\begin{array}{c}\text { Meto- } \\
\text { lachlor } \\
\text { ESA } \\
(\mu \mathrm{g} / \mathrm{L})\end{array}$ & $\begin{array}{c}\text { Meto- } \\
\text { lachlor } \\
\text { OXA } \\
(\mu \mathrm{g} / \mathrm{L})\end{array}$ & 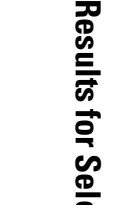 \\
\hline \multicolumn{20}{|c|}{ Federal-State Cooperative Studies-Continued } & \\
\hline \multicolumn{20}{|c|}{ Minnesota Pollution Control Agency (JMM) —Continued } & \\
\hline 0484 & Stearns Co., MN & $05 / 02 / 00$ & $<0.05$ & $<0.20$ & $<0.20$ & -- & $<0.05$ & $<0.20$ & $<0.20$ & -- & $<0.05$ & -- & -- & $<0.05$ & -- & -- & $<0.05$ & $<0.20$ & $<0.20$ & \\
\hline 0485 & Stearns Co., MN & $05 / 02 / 00$ & $<.05$ & $<.20$ & $<.20$ & -- & $<.05$ & .47 & $<.20$ & -- & $<.05$ & -- & -- & $<.05$ & -- & -- & $<.05$ & $<.20$ & $<.20$ & \\
\hline 0486 & Stearns Co., MN & $05 / 02 / 00$ & $<.05$ & $<.20$ & .46 & -- & $<.05$ & .21 & .34 & -- & $<.05$ & -- & -- & $<.05$ & -- & -- & $<.05$ & $<.20$ & $<.20$ & \\
\hline 0487 & Stearns Co., MN & $05 / 02 / 00$ & $<.05$ & $<.20$ & $<.20$ & -- & $<.05$ & .31 & $<.20$ & -- & $<.05$ & -- & -- & $<.05$ & -- & -- & $<.05$ & .44 & $<.20$ & 䄈 \\
\hline 0488 & Stearns Co., MN & $05 / 02 / 00$ & $<.05$ & $<.20$ & $<.20$ & -- & $<.05$ & $<.20$ & $<.20$ & -- & $<.05$ & -- & -- & $<.05$ & -- & -- & $<.05$ & $<.20$ & $<.20$ & \\
\hline 0489 & Stearns Co., MN & $05 / 02 / 00$ & $<.05$ & $<.20$ & $<.20$ & -- & $<.05$ & $<.20$ & $<.20$ & -- & $<.05$ & -- & -- & $<.05$ & -- & -- & $<.05$ & $<.20$ & $<.20$ & \\
\hline 0490 & Stearns Co., MN & 05/02/00 & $<.05$ & $<.20$ & $<.20$ & -- & $<.05$ & $<.20$ & $<.20$ & -- & $<.05$ & -- & -- & $<.05$ & -- & -- & $<.05$ & $<.20$ & $<.20$ & \\
\hline 0491 & Stearns Co., MN & $05 / 18 / 00$ & $<.05$ & $<.20$ & $<.20$ & -- & $<.05$ & $<.20$ & $<.20$ & -- & $<.05$ & -- & -- & $<.05$ & -- & -- & $<.05$ & $<.20$ & $<.20$ & $\stackrel{2}{2}$ \\
\hline 0492 & Stearns Co., MN & 06/08/00 & .05 & .93 & 1.4 & -- & $<.05$ & $<.20$ & $<.20$ & -- & $<.05$ & -- & -- & $<.05$ & -- & -- & $<.05$ & $<.20$ & .32 & \\
\hline 0493 & Stearns Co., MN & 06/08/00 & .09 & 1.0 & 1.4 & -- & $<.05$ & $<.20$ & $<.20$ & -- & $<.05$ & -- & -- & $<.05$ & -- & -- & $<.05$ & .64 & $<.20$ & 总 \\
\hline 0495 & Stearns Co., MN & 06/08/00 & .06 & 1.4 & 2.1 & -- & $<.05$ & .20 & $<.20$ & -- & $<.05$ & -- & -- & $<.05$ & -- & -- & $<.05$ & 1.1 & $<.20$ & \\
\hline 0496 & Stearns Co., MN & $06 / 08 / 00$ & .12 & .33 & 1.3 & -- & $<.05$ & .20 & $<.20$ & -- & $<.05$ & -- & -- & $<.05$ & -- & -- & $<.05$ & $<.20$ & $<.20$ & $\overline{0}$ \\
\hline 0505 & Stearns Co., MN & 05/18/00 & $<.05$ & $<.20$ & $<.20$ & -- & $<.05$ & $<.20$ & $<.20$ & -- & $<.05$ & -- & -- & $<.05$ & -- & -- & $<.05$ & $<.20$ & $<.20$ & 응 \\
\hline 0506 & Stearns Co., MN & $11 / 00 / 99$ & $<.05$ & $<.20$ & $<.20$ & -- & $<.05$ & $<.20$ & $<.20$ & -- & $<.05$ & -- & -- & $<.05$ & -- & -- & $<.05$ & $<.20$ & .72 & 高 \\
\hline 0507 & Stearns Co., MN & $11 / 00 / 99$ & $<.05$ & $<.20$ & $<.20$ & -- & $<.05$ & $<.20$ & $<.20$ & -- & $<.05$ & -- & -- & $<.05$ & -- & -- & $<.05$ & $<.20$ & $<.20$ & 䍃 \\
\hline 0508 & Stearns Co., MN & $11 / 00 / 99$ & $<.05$ & $<.20$ & $<.20$ & -- & $<.05$ & $<.20$ & $<.20$ & -- & $<.05$ & -- & -- & $<.05$ & -- & -- & $<.05$ & $<.20$ & 2.0 & \\
\hline 0509 & Stearns Co., MN & $11 / 00 / 99$ & $<.05$ & $<.20$ & $<.20$ & -- & $<.05$ & $<.20$ & $<.20$ & -- & $<.05$ & -- & -- & $<.05$ & -- & -- & $<.05$ & $<.20$ & $<.20$ & \\
\hline 0510 & Stearns Co., MN & $11 / 00 / 99$ & $<.05$ & $<.20$ & $<.20$ & -- & $<.05$ & $<.20$ & $<.20$ & -- & $<.05$ & -- & -- & $<.05$ & -- & -- & $<.05$ & $<.20$ & $<.20$ & \\
\hline 0511 & Stearns Co., MN & $11 / 00 / 99$ & $<.05$ & $<.20$ & $<.20$ & -- & $<.05$ & $<.20$ & $<.20$ & -- & $<.05$ & -- & -- & $<.05$ & -- & -- & $<.05$ & .40 & 2.0 & \\
\hline 0512 & Stearns Co., MN & $11 / 00 / 99$ & $<.05$ & $<.20$ & $<.20$ & -- & $<.05$ & $<.20$ & $<.20$ & -- & $<.05$ & -- & -- & $<.05$ & -- & -- & $<.05$ & $<.20$ & $<.20$ & 总 $\frac{0}{2}$ \\
\hline 0513 & Stearns Co., MN & $11 / 00 / 99$ & $<.05$ & $<.20$ & $<.20$ & -- & $<.05$ & $<.20$ & $<.20$ & -- & $<.05$ & -- & -- & $<.05$ & -- & -- & $<.05$ & $<.20$ & $<.20$ & 气. \\
\hline 0514 & Stearns Co., MN & $11 / 00 / 99$ & $<.05$ & $<.20$ & $<.20$ & -- & $<.05$ & $<.20$ & $<.20$ & -- & $<.05$ & -- & -- & $<.05$ & -- & -- & $<.05$ & $<.20$ & $<.20$ & \\
\hline 0524 & Stearns Co., MN & $11 / 00 / 99$ & $<.05$ & $<.20$ & $<.20$ & -- & $<.05$ & $<.20$ & $<.20$ & -- & $<.05$ & -- & -- & $<.05$ & -- & -- & $<.05$ & .29 & $<.20$ & 疍. \\
\hline 0525 & Stearns Co., MN & $11 / 00 / 99$ & $<.05$ & $<.20$ & $<.20$ & -- & $<.05$ & $<.20$ & $<.20$ & -- & $<.05$ & -- & -- & $<.05$ & -- & -- & $<.05$ & .31 & $<.20$ & \\
\hline 0529 & Stearns Co., MN & $11 / 00 / 99$ & $<.05$ & $<.20$ & $<.20$ & -- & $<.05$ & $<.20$ & $<.20$ & -- & $<.05$ & -- & -- & $<.05$ & -- & -- & $<.05$ & $<.20$ & $<.20$ & \\
\hline
\end{tabular}




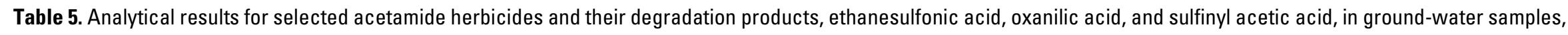
1994-2002.-Continued

[OGRL, U.S. Geological Survey Organic Geochemistry Research Laboratory, Lawrence, Kansas; ESA, ethanesulfonic acid; OXA, oxanilic acid; SAA, sulfinyl acetic acid; --, no data; <, less than]

\begin{tabular}{|c|c|c|c|c|c|c|c|c|c|c|c|c|c|c|c|c|c|c|c|}
\hline Site number & Site location & $\begin{array}{c}\text { Date of } \\
\text { collection } \\
\text { (month/day/ } \\
\text { year) }\end{array}$ & $\begin{array}{l}\text { Aceto- } \\
\text { chlor } \\
(\mu \mathrm{g} / \mathrm{L})\end{array}$ & $\begin{array}{l}\text { Aceto- } \\
\text { chlor } \\
\text { ESA } \\
(\mu \mathrm{g} / \mathrm{L})\end{array}$ & $\begin{array}{c}\text { Aceto- } \\
\text { chlor } \\
0 X A \\
(\mu \mathrm{g} / \mathrm{L})\end{array}$ & $\begin{array}{c}\text { Aceto- } \\
\text { chlor } \\
\text { SAA } \\
(\mu \mathrm{g} / \mathrm{L})\end{array}$ & $\begin{array}{l}\text { Ala- } \\
\text { chlor } \\
(\mu \mathrm{g} / \mathrm{L})\end{array}$ & $\begin{array}{l}\text { Ala- } \\
\text { chlor } \\
\text { ESA } \\
(\mu \mathrm{g} / \mathrm{L})\end{array}$ & $\begin{array}{l}\text { Ala- } \\
\text { chlor } \\
\text { OXA } \\
(\mu \mathrm{g} / \mathrm{L})\end{array}$ & $\begin{array}{l}\text { Ala- } \\
\text { chlor } \\
\text { SAA } \\
(\mu \mathrm{g} / \mathrm{L})\end{array}$ & $\begin{array}{c}\text { Di- } \\
\text { meth- } \\
\text { enamid } \\
(\mu \mathrm{g} / \mathrm{L})\end{array}$ & $\begin{array}{c}\text { Di- } \\
\text { meth- } \\
\text { enamid } \\
\text { ESA } \\
(\mu \mathrm{g} / \mathrm{L})\end{array}$ & $\begin{array}{c}\text { Di- } \\
\text { meth- } \\
\text { enamid } \\
\text { OXA } \\
(\mu \mathrm{g} / \mathrm{L})\end{array}$ & $\begin{array}{l}\text { Flufen- } \\
\text { acet } \\
(\mu \mathrm{g} / \mathrm{L})\end{array}$ & $\begin{array}{c}\text { Flufen- } \\
\text { acet } \\
\text { ESA } \\
(\mu \mathrm{g} / \mathrm{L})\end{array}$ & $\begin{array}{c}\text { Flufen- } \\
\text { acet } \\
\text { OXA } \\
(\mu \mathrm{g} / \mathrm{L})\end{array}$ & $\begin{array}{l}\text { Meto- } \\
\text { lachlor } \\
(\mu \mathrm{g} / \mathrm{L})\end{array}$ & $\begin{array}{c}\text { Meto- } \\
\text { lachlor } \\
\text { ESA } \\
(\mu \mathrm{g} / \mathrm{L})\end{array}$ & $\begin{array}{c}\text { Meto- } \\
\text { lachlor } \\
\text { OXA } \\
(\mu \mathrm{g} / \mathrm{L})\end{array}$ \\
\hline \multicolumn{20}{|c|}{ Federal-State Cooperative Studies-Continued } \\
\hline \multicolumn{20}{|c|}{ Minnesota Pollution Control Agency (JMM) —Continued } \\
\hline 0530 & Stearns Co., MN & $11 / 00 / 99$ & $<0.05$ & $<0.20$ & $<0.20$ & -- & $<0.05$ & 0.46 & $<0.20$ & -- & $<0.05$ & -- & -- & $<0.05$ & -- & -- & $<0.05$ & 0.43 & 0.86 \\
\hline 0531 & Stearns Co., MN & $11 / 00 / 99$ & $<.05$ & $<.20$ & $<.20$ & -- & $<.05$ & $<.20$ & $<.20$ & -- & $<.05$ & -- & -- & $<.05$ & -- & -- & $<.05$ & 2.6 & $<.20$ \\
\hline 0532 & Stearns Co., MN & $11 / 00 / 99$ & $<.05$ & .91 & $<.20$ & -- & $<.05$ & .46 & $<.20$ & -- & $<.05$ & -- & -- & $<.05$ & -- & -- & $<.05$ & .62 & $<.20$ \\
\hline 0533 & Stearns Co., MN & $11 / 00 / 99$ & $<.05$ & .80 & $<.20$ & -- & $<.05$ & .56 & $<.20$ & -- & $<.05$ & -- & -- & $<.05$ & -- & -- & $<.05$ & .61 & .22 \\
\hline 0537 & Stearns Co., MN & $11 / 00 / 99$ & $<.05$ & $<.20$ & $<.20$ & -- & $<.05$ & $<.20$ & $<.20$ & -- & $<.05$ & -- & -- & $<.05$ & -- & -- & $<.05$ & $<.20$ & $<.20$ \\
\hline 0544 & Stearns Co., MN & $11 / 00 / 99$ & $<.05$ & $<.20$ & $<.20$ & -- & $<.05$ & 1.3 & $<.20$ & -- & $<.05$ & -- & -- & $<.05$ & -- & -- & $<.05$ & $<.20$ & $<.20$ \\
\hline 0545 & Stearns Co., MN & $11 / 00 / 99$ & $<.05$ & $<.20$ & $<.20$ & -- & $<.05$ & $<.20$ & $<.20$ & -- & $<.05$ & -- & -- & $<.05$ & -- & -- & $<.05$ & $<.20$ & $<.20$ \\
\hline 0549 & Stearns Co., MN & $11 / 00 / 99$ & $<.05$ & $<.20$ & $<.20$ & -- & $<.05$ & $<.20$ & $<.20$ & -- & $<.05$ & -- & -- & $<.05$ & -- & -- & $<.05$ & $<.20$ & $<.20$ \\
\hline 0555 & Stearns Co., MN & $11 / 00 / 99$ & $<.05$ & $<.20$ & .33 & -- & $<.05$ & $<.20$ & .32 & -- & $<.05$ & -- & -- & $<.05$ & -- & -- & $<.05$ & 3.0 & $<.20$ \\
\hline 0556 & Stearns Co., MN & $11 / 00 / 99$ & $<.05$ & $<.20$ & $<.20$ & -- & $<.05$ & $<.20$ & $<.20$ & -- & $<.05$ & -- & -- & $<.05$ & -- & -- & $<.05$ & 3.0 & $<.20$ \\
\hline 0557 & Stearns Co., MN & $11 / 00 / 99$ & $<.05$ & .99 & $<.20$ & -- & $<.05$ & .65 & $<.20$ & -- & $<.05$ & -- & -- & $<.05$ & -- & -- & $<.05$ & .84 & .28 \\
\hline 0558 & Stearns Co., MN & $11 / 00 / 99$ & $<.05$ & $<.20$ & $<.20$ & -- & $<.05$ & $<.20$ & $<.20$ & -- & $<.05$ & -- & -- & $<.05$ & -- & -- & $<.05$ & $<.20$ & $<.20$ \\
\hline 0560 & Stearns Co., MN & $11 / 00 / 99$ & $<.05$ & 1.5 & $<.20$ & -- & $<.05$ & .55 & $<.20$ & -- & $<.05$ & -- & -- & $<.05$ & -- & -- & $<.05$ & .41 & $<.20$ \\
\hline 0563 & Stearns Co., MN & $11 / 00 / 99$ & $<.05$ & $<.20$ & $<.20$ & -- & $<.05$ & .22 & .22 & -- & $<.05$ & -- & -- & $<.05$ & -- & -- & $<.05$ & .26 & $<.20$ \\
\hline 0564 & Stearns Co., MN & $11 / 00 / 99$ & $<.05$ & $<.20$ & $<.20$ & -- & $<.05$ & .24 & $<.20$ & -- & $<.05$ & -- & -- & $<.05$ & -- & -- & $<.05$ & .45 & $<.20$ \\
\hline 0570 & Stearns Co., MN & $11 / 00 / 99$ & $<.05$ & $<.05$ & .10 & -- & $<.05$ & .39 & .07 & -- & $<.05$ & -- & -- & $<.05$ & -- & -- & $<.05$ & .47 & .09 \\
\hline 0571 & Stearns Co., MN & 06/08/00 & $<.05$ & $<.20$ & $<.20$ & -- & $<.05$ & .92 & $<.20$ & -- & $<.05$ & -- & -- & $<.05$ & -- & -- & $<.05$ & $<.20$ & .71 \\
\hline 0572 & Stearns Co., MN & 06/08/00 & $<.05$ & $<.20$ & $<.20$ & -- & $<.05$ & .24 & $<.20$ & -- & $<.05$ & -- & -- & $<.05$ & -- & -- & $<.05$ & $<.20$ & $<.20$ \\
\hline 0573 & Stearns Co., MN & 06/08/00 & $<.05$ & $<.20$ & $<.20$ & -- & $<.05$ & $<.20$ & $<.20$ & -- & $<.05$ & -- & -- & $<.05$ & -- & -- & $<.05$ & .74 & $<.20$ \\
\hline 0574 & Stearns Co., MN & 08/00/00 & $<.05$ & 1.2 & $<.20$ & -- & $<.05$ & .46 & $<.20$ & -- & $<.05$ & -- & -- & $<.05$ & -- & -- & $<.05$ & .43 & $<.20$ \\
\hline 0575 & Stearns Co., MN & 08/00/00 & $<.05$ & .88 & $<.20$ & -- & $<.05$ & .61 & $<.20$ & -- & $<.05$ & -- & -- & $<.05$ & -- & -- & $<.05$ & .66 & $<.20$ \\
\hline 0578 & Stearns Co., MN & 06/08/00 & $<.05$ & $<.20$ & $<.20$ & -- & $<.05$ & $<.20$ & $<.20$ & -- & $<.05$ & -- & -- & $<.05$ & -- & -- & $<.05$ & $<.20$ & $<.20$ \\
\hline 0579 & Stearns Co., MN & 06/08/00 & $<.05$ & $<.20$ & $<.20$ & -- & $<.05$ & $<.20$ & $<.20$ & -- & $<.05$ & -- & -- & $<.05$ & -- & -- & $<.05$ & $<.20$ & $<.20$ \\
\hline 0580 & Stearns Co., MN & 06/08/00 & $<.05$ & $<.20$ & $<.20$ & -- & $<.05$ & $<.20$ & $<.20$ & -- & $<.05$ & -- & -- & $<.05$ & -- & -- & $<.05$ & $<.20$ & $<.20$ \\
\hline 0581 & Stearns Co., MN & 06/08/00 & $<.05$ & .35 & $<.20$ & -- & $<.05$ & $<.20$ & $<.20$ & -- & $<.05$ & -- & -- & $<.05$ & -- & -- & $<.05$ & .42 & $<.20$ \\
\hline
\end{tabular}


Table 5. Analytical results for selected acetamide herbicides and their degradation products, ethanesulfonic acid, oxanilic acid, and sulfinyl acetic acid, in ground-water samples, 1994-2002.-Continued

[OGRL, U.S. Geological Survey Organic Geochemistry Research Laboratory, Lawrence, Kansas; ESA, ethanesulfonic acid; OXA, oxanilic acid; SAA, sulfinyl acetic acid; --, no data; <, less than]

\begin{tabular}{|c|c|c|c|c|c|c|c|c|c|c|c|c|c|c|c|c|c|c|c|c|}
\hline Site number & Site location & $\begin{array}{c}\text { Date of } \\
\text { collection } \\
\text { (month/day/ } \\
\text { year) }\end{array}$ & $\begin{array}{l}\text { Aceto- } \\
\text { chlor } \\
(\mu \mathrm{g} / \mathrm{L})\end{array}$ & $\begin{array}{l}\text { Aceto- } \\
\text { chlor } \\
\text { ESA } \\
(\mu \mathrm{g} / \mathrm{L})\end{array}$ & $\begin{array}{l}\text { Aceto- } \\
\text { chlor } \\
\text { OXA } \\
(\mu \mathrm{g} / \mathrm{L})\end{array}$ & $\begin{array}{l}\text { Aceto- } \\
\text { chlor } \\
\text { SAA } \\
(\mu \mathrm{g} / \mathrm{L})\end{array}$ & $\begin{array}{l}\text { Ala- } \\
\text { chlor } \\
(\mu \mathrm{g} / \mathrm{L})\end{array}$ & $\begin{array}{l}\text { Ala- } \\
\text { chlor } \\
\text { ESA } \\
(\mu \mathrm{g} / \mathrm{L})\end{array}$ & $\begin{array}{l}\text { Ala- } \\
\text { chlor } \\
\text { OXA } \\
(\mu \mathrm{g} / \mathrm{L})\end{array}$ & $\begin{array}{c}\text { Ala- } \\
\text { chlor } \\
\text { SAA } \\
(\mu \mathrm{g} / \mathrm{L})\end{array}$ & $\begin{array}{c}\text { Di- } \\
\text { meth- } \\
\text { enamid } \\
(\mu \mathrm{g} / \mathrm{L})\end{array}$ & $\begin{array}{c}\text { Di- } \\
\text { meth- } \\
\text { enamid } \\
\text { ESA } \\
(\mu \mathrm{g} / \mathrm{L})\end{array}$ & $\begin{array}{c}\text { Di- } \\
\text { meth- } \\
\text { enamid } \\
\text { OXA } \\
(\mu \mathrm{g} / \mathrm{L})\end{array}$ & $\begin{array}{c}\text { Flufen- } \\
\text { acet } \\
(\mu \mathrm{g} / \mathrm{L})\end{array}$ & $\begin{array}{c}\text { Flufen- } \\
\text { acet } \\
\text { ESA } \\
(\mu \mathrm{g} / \mathrm{L})\end{array}$ & $\begin{array}{c}\text { Flufen- } \\
\text { acet } \\
\text { OXA } \\
(\mu \mathrm{g} / \mathrm{L})\end{array}$ & $\begin{array}{l}\text { Meto- } \\
\text { lachlor } \\
(\mu \mathrm{g} / \mathrm{L})\end{array}$ & $\begin{array}{c}\text { Meto- } \\
\text { lachlor } \\
\text { ESA } \\
(\mu \mathrm{g} / \mathrm{L})\end{array}$ & $\begin{array}{c}\text { Meto- } \\
\text { lachlor } \\
\text { OXA } \\
(\mu \mathrm{g} / \mathrm{L})\end{array}$ & 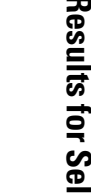 \\
\hline \multicolumn{20}{|c|}{ Federal-State Cooperative Studies-Continued } & \\
\hline \multicolumn{20}{|c|}{ Minnesota Pollution Control Agency (JMM) —Continued } & \\
\hline 0583 & Stearns Co., MN & $11 / 00 / 00$ & $<0.05$ & $<0.05$ & 0.09 & -- & $<0.05$ & 0.33 & 0.08 & -- & $<0.05$ & $<0.05$ & $<0.05$ & $<0.05$ & $<0.05$ & $<0.05$ & $<0.05$ & 0.44 & 0.09 & \\
\hline 0593 & Stearns Co., MN & 08/00/00 & $<.05$ & $<.20$ & $<.20$ & -- & $<.05$ & $<.20$ & $<.20$ & -- & $<.05$ & -- & -- & $<.05$ & -- & -- & $<.05$ & $<.20$ & $<.20$ & \\
\hline 0606 & Stearns Co., MN & 08/00/00 & $<.05$ & $<.20$ & $<.20$ & -- & $<.05$ & .20 & $<.20$ & -- & $<.05$ & -- & -- & $<.05$ & -- & -- & $<.05$ & .27 & $<.20$ & \\
\hline 0620 & Stearns Co., MN & 08/00/00 & $<.05$ & $<.20$ & $<.20$ & -- & $<.05$ & $<.20$ & $<.20$ & -- & $<.05$ & -- & -- & $<.05$ & -- & -- & $<.05$ & $<.20$ & $<.20$ & \\
\hline 0625 & Stearns Co., MN & 08/00/00 & $<.05$ & $<.20$ & $<.20$ & -- & $<.05$ & $<.20$ & $<.20$ & -- & $<.05$ & -- & -- & $<.05$ & -- & -- & $<.05$ & $<.20$ & $<.20$ & \\
\hline 0626 & Stearns Co., MN & 08/00/00 & $<.05$ & $<.20$ & $<.20$ & -- & $<.05$ & .93 & $<.20$ & -- & $<.05$ & -- & -- & $<.05$ & -- & -- & $<.05$ & $<.20$ & $<.20$ & \multirow{9}{*}{ 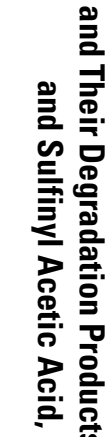 } \\
\hline 0817 & Stearns Co., MN & $11 / 00 / 00$ & $<.05$ & $<.05$ & $<.05$ & -- & $<.05$ & $<.05$ & $<.05$ & -- & $<.05$ & $<.05$ & $<.05$ & $<.05$ & $<.05$ & $<.05$ & $<.05$ & .05 & $<.05$ & \\
\hline 0818 & Stearns Co., MN & $11 / 00 / 00$ & $<.05$ & $<.05$ & $<.05$ & -- & $<.05$ & $<.05$ & $<.05$ & -- & $<.05$ & $<.05$ & $<.05$ & $<.05$ & $<.05$ & $<.05$ & $<.05$ & $<.05$ & $<.05$ & \\
\hline 0819 & Stearns Co., MN & $11 / 00 / 00$ & $<.05$ & $<.05$ & $<.05$ & -- & $<.05$ & $<.05$ & $<.05$ & -- & $<.05$ & $<.05$ & $<.05$ & $<.05$ & $<.05$ & $<.05$ & $<.05$ & $<.05$ & $<.05$ & \\
\hline 0820 & Stearns Co., MN & $11 / 00 / 00$ & $<.05$ & $<.05$ & $<.05$ & -- & $<.05$ & 2.8 & $<.05$ & -- & $<.05$ & $<.05$ & $<.05$ & $<.05$ & $<.05$ & $<.05$ & $<.05$ & .17 & $<.05$ & \\
\hline 0824 & Stearns Co., MN & $11 / 00 / 00$ & $<.05$ & 1.1 & $<.05$ & -- & $<.05$ & .59 & $<.05$ & -- & $<.05$ & $<.05$ & $<.05$ & $<.05$ & $<.05$ & $<.05$ & $<.05$ & .66 & $<.05$ & \\
\hline 0892 & Stearns Co., MN & 03/03/99 & $<.05$ & .88 & $<.20$ & -- & $<.05$ & .71 & .34 & -- & -- & -- & -- & -- & -- & -- & $<.05$ & .42 & .28 & \\
\hline 0997 & Stearns Co., MN & 03/03/99 & $<.05$ & .21 & $<.20$ & -- & $<.05$ & $<.20$ & $<.20$ & -- & -- & -- & -- & -- & -- & -- & $<.05$ & $<.20$ & $<.20$ & \\
\hline 0998 & Stearns Co., MN & 03/03/99 & $<.05$ & $<.20$ & $<.20$ & -- & $<.05$ & .59 & $<.20$ & -- & -- & -- & -- & -- & -- & -- & $<.05$ & .44 & $<.20$ & \\
\hline 9000 & Stearns Co., MN & $04 / 23 / 01$ & $<.05$ & $<.05$ & $<.05$ & -- & $<.05$ & 1.8 & $<.05$ & -- & $<.05$ & $<.05$ & $<.05$ & $<.05$ & $<.05$ & $<.05$ & $<.05$ & .13 & $<.05$ & \\
\hline 9001 & Stearns Co., MN & $04 / 23 / 01$ & $<.05$ & $<.05$ & $<.05$ & -- & $<.05$ & $<.05$ & $<.05$ & -- & $<.05$ & $<.05$ & $<.05$ & $<.05$ & $<.05$ & $<.05$ & $<.05$ & $<.05$ & $<.05$ & \\
\hline 9002 & Stearns Co., MN & $04 / 23 / 01$ & $<.05$ & $<.05$ & $<.05$ & -- & $<.05$ & $<.05$ & $<.05$ & -- & $<.05$ & $<.05$ & $<.05$ & $<.05$ & $<.05$ & $<.05$ & $<.05$ & $<.05$ & $<.05$ & \\
\hline 9003 & Stearns Co., MN & $04 / 23 / 01$ & $<.05$ & $<.05$ & $<.05$ & -- & $<.05$ & $<.05$ & $<.05$ & -- & $<.05$ & $<.05$ & $<.05$ & $<.05$ & $<.05$ & $<.05$ & $<.05$ & $<.05$ & $<.05$ & \\
\hline 9004 & Stearns Co., MN & $04 / 24 / 01$ & $<.05$ & .19 & .15 & -- & $<.05$ & .24 & $<.05$ & -- & $<.05$ & $<.05$ & $<.05$ & $<.05$ & $<.05$ & $<.05$ & $<.05$ & .24 & .09 & \\
\hline 9006 & Stearns Co., MN & $04 / 24 / 01$ & $<.05$ & $<.05$ & $<.05$ & -- & $<.05$ & $<.05$ & $<.05$ & -- & $<.05$ & $<.05$ & $<.05$ & $<.05$ & $<.05$ & $<.05$ & $<.05$ & $<.05$ & $<.05$ & \\
\hline 9007 & Stearns Co., MN & $04 / 24 / 01$ & $<.05$ & .45 & $<.05$ & -- & $<.05$ & .32 & $<.05$ & -- & $<.05$ & $<.05$ & $<.05$ & $<.05$ & $<.05$ & $<.05$ & $<.05$ & .23 & $<.05$ & \\
\hline 9008 & Stearns Co., MN & $04 / 24 / 01$ & $<.05$ & $<.05$ & $<.05$ & -- & $<.05$ & $<.05$ & $<.05$ & -- & $<.05$ & $<.05$ & $<.05$ & $<.05$ & $<.05$ & $<.05$ & $<.05$ & $<.05$ & $<.05$ & \\
\hline 9009 & Stearns Co., MN & $04 / 24 / 01$ & $<.05$ & $<.05$ & $<.05$ & -- & $<.05$ & .08 & $<.05$ & -- & $<.05$ & $<.05$ & $<.05$ & $<.05$ & $<.05$ & $<.05$ & $<.05$ & .54 & .06 & \\
\hline 9010 & Stearns Co., MN & $04 / 24 / 01$ & $<.05$ & .76 & $<.05$ & -- & $<.05$ & .31 & $<.05$ & -- & $<.05$ & $<.05$ & $<.05$ & $<.05$ & $<.05$ & $<.05$ & $<.05$ & .34 & .11 & \\
\hline 9011 & Stearns Co., MN & $04 / 24 / 01$ & $<.05$ & .19 & .14 & -- & $<.05$ & .26 & $<.05$ & -- & $<.05$ & $<.05$ & $<.05$ & $<.05$ & $<.05$ & $<.05$ & $<.05$ & .26 & .10 & \\
\hline
\end{tabular}




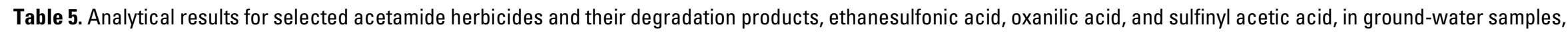
1994-2002.-Continued

[OGRL, U.S. Geological Survey Organic Geochemistry Research Laboratory, Lawrence, Kansas; ESA, ethanesulfonic acid; OXA, oxanilic acid; SAA, sulfinyl acetic acid; --, no data; <, less than]

\begin{tabular}{|c|c|c|c|c|c|c|c|c|c|c|c|c|c|c|c|c|c|c|c|}
\hline Site number & Site location & $\begin{array}{c}\text { Date of } \\
\text { collection } \\
\text { (month/day/ } \\
\text { year) }\end{array}$ & $\begin{array}{l}\text { Aceto- } \\
\text { chlor } \\
(\mu \mathrm{g} / \mathrm{L})\end{array}$ & $\begin{array}{l}\text { Aceto- } \\
\text { chlor } \\
\text { ESA } \\
(\mu \mathrm{g} / \mathrm{L})\end{array}$ & $\begin{array}{c}\text { Aceto- } \\
\text { chlor } \\
0 X A \\
(\mu \mathrm{g} / \mathrm{L})\end{array}$ & $\begin{array}{c}\text { Aceto- } \\
\text { chlor } \\
\text { SAA } \\
(\mu \mathrm{g} / \mathrm{L})\end{array}$ & $\begin{array}{l}\text { Ala- } \\
\text { chlor } \\
(\mu \mathrm{g} / \mathrm{L})\end{array}$ & $\begin{array}{l}\text { Ala- } \\
\text { chlor } \\
\text { ESA } \\
(\mu \mathrm{g} / \mathrm{L})\end{array}$ & $\begin{array}{l}\text { Ala- } \\
\text { chlor } \\
\text { OXA } \\
(\mu \mathrm{g} / \mathrm{L})\end{array}$ & $\begin{array}{l}\text { Ala- } \\
\text { chlor } \\
\text { SAA } \\
(\mu \mathrm{g} / \mathrm{L})\end{array}$ & $\begin{array}{c}\text { Di- } \\
\text { meth- } \\
\text { enamid } \\
(\mu \mathrm{g} / \mathrm{L})\end{array}$ & $\begin{array}{c}\text { Di- } \\
\text { meth- } \\
\text { enamid } \\
\text { ESA } \\
(\mu \mathrm{g} / \mathrm{L})\end{array}$ & $\begin{array}{c}\text { Di- } \\
\text { meth- } \\
\text { enamid } \\
\text { OXA } \\
(\mu \mathrm{g} / \mathrm{L})\end{array}$ & $\begin{array}{c}\text { Flufen- } \\
\text { acet } \\
(\mu \mathrm{g} / \mathrm{L})\end{array}$ & $\begin{array}{c}\text { Flufen- } \\
\text { acet } \\
\text { ESA } \\
(\mu \mathrm{g} / \mathrm{L})\end{array}$ & $\begin{array}{c}\text { Flufen- } \\
\text { acet } \\
\text { OXA } \\
(\mu \mathrm{g} / \mathrm{L})\end{array}$ & $\begin{array}{l}\text { Meto- } \\
\text { lachlor } \\
(\mu \mathrm{g} / \mathrm{L})\end{array}$ & $\begin{array}{l}\text { Meto- } \\
\text { lachlor } \\
\text { ESA } \\
(\mu \mathrm{g} / \mathrm{L})\end{array}$ & $\begin{array}{c}\text { Meto- } \\
\text { lachlor } \\
\text { OXA } \\
(\mu \mathrm{g} / \mathrm{L})\end{array}$ \\
\hline \multicolumn{20}{|c|}{ Federal-State Cooperative Studies-Continued } \\
\hline \multicolumn{20}{|c|}{ Minnesota Pollution Control Agency (JMM)—Continued } \\
\hline 9012 & Stearns Co., MN & 05/30/01 & $<0.05$ & 0.46 & 0.13 & -- & $<0.05$ & 0.16 & $<0.05$ & -- & $<0.05$ & $<0.05$ & $<0.05$ & $<0.05$ & $<0.05$ & $<0.05$ & $<0.05$ & 0.21 & $<0.05$ \\
\hline 9013 & Stearns Co., MN & $05 / 30 / 01$ & $<.05$ & .64 & .23 & -- & $<.05$ & .11 & $<.05$ & -- & $<.05$ & $<.05$ & $<.05$ & $<.05$ & $<.05$ & $<.05$ & $<.05$ & .55 & $<.05$ \\
\hline 9014 & Stearns Co., MN & 05/30/01 & $<.05$ & .35 & .14 & -- & $<.05$ & .07 & $<.05$ & -- & $<.05$ & $<.05$ & $<.05$ & $<.05$ & $<.05$ & $<.05$ & $<.05$ & .21 & $<.05$ \\
\hline 9016 & Lincoln Co., MN & 05/30/01 & $<.05$ & $<.05$ & $<.05$ & -- & $<.05$ & .09 & $<.05$ & -- & $<.05$ & $<.05$ & $<.05$ & $<.05$ & $<.05$ & $<.05$ & $<.05$ & $<.05$ & $<.05$ \\
\hline 9019 & Lincoln Co., MN & $05 / 30 / 01$ & $<.05$ & .05 & $<.05$ & -- & $<.05$ & .06 & $<.05$ & -- & $<.05$ & $<.05$ & $<.05$ & $<.05$ & $<.05$ & $<.05$ & $<.05$ & .48 & $<.05$ \\
\hline 9020 & Lincoln Co., MN & $05 / 30 / 01$ & $<.05$ & $<.05$ & $<.05$ & -- & $<.05$ & .15 & $<.05$ & -- & $<.05$ & $<.05$ & $<.05$ & $<.05$ & $<.05$ & $<.05$ & $<.05$ & .14 & $<.05$ \\
\hline 9021 & Lincoln Co., MN & $05 / 30 / 01$ & $<.05$ & $<.05$ & $<.05$ & -- & $<.05$ & .06 & $<.05$ & -- & $<.05$ & $<.05$ & $<.05$ & $<.05$ & $<.05$ & $<.05$ & $<.05$ & .72 & $<.05$ \\
\hline 9022 & Lincoln Co., MN & $05 / 30 / 01$ & $<.05$ & $<.05$ & $<.05$ & -- & $<.05$ & $<.05$ & $<.05$ & -- & $<.05$ & $<.05$ & $<.05$ & $<.05$ & $<.05$ & $<.05$ & $<.05$ & .16 & $<.05$ \\
\hline 9024 & Hubbard Co., MN & 06/12/01 & $<.05$ & $<.05$ & $<.05$ & -- & $<.05$ & $<.05$ & $<.05$ & -- & $<.05$ & $<.05$ & $<.05$ & $<.05$ & $<.05$ & $<.05$ & $<.05$ & $<.05$ & $<.05$ \\
\hline 9025 & Hubbard Co., MN & $06 / 12 / 01$ & $<.05$ & $<.05$ & $<.05$ & -- & $<.05$ & $<.05$ & $<.05$ & -- & $<.05$ & $<.05$ & $<.05$ & $<.05$ & $<.05$ & $<.05$ & $<.05$ & $<.05$ & $<.05$ \\
\hline 9026 & Hubbard Co., MN & 06/12/01 & $<.05$ & $<.05$ & $<.05$ & -- & $<.05$ & $<.05$ & $<.05$ & -- & $<.05$ & $<.05$ & $<.05$ & $<.05$ & $<.05$ & $<.05$ & $<.05$ & $<.05$ & $<.05$ \\
\hline 9027 & Hubbard Co., MN & $06 / 12 / 01$ & $<.05$ & $<.05$ & $<.05$ & -- & $<.05$ & $<.05$ & $<.05$ & -- & $<.05$ & $<.05$ & $<.05$ & $<.05$ & $<.05$ & $<.05$ & $<.05$ & $<.05$ & $<.05$ \\
\hline 9028 & Ottertail Co., MN & $06 / 12 / 01$ & $<.05$ & $<.05$ & $<.05$ & -- & $<.05$ & $<.05$ & $<.05$ & -- & $<.05$ & $<.05$ & $<.05$ & $<.05$ & $<.05$ & $<.05$ & $<.05$ & .28 & $<.05$ \\
\hline 9029 & Ottertail Co., MN & $06 / 12 / 01$ & $<.05$ & $<.05$ & $<.05$ & -- & $<.05$ & $<.05$ & $<.05$ & -- & $<.05$ & $<.05$ & $<.05$ & $<.05$ & $<.05$ & $<.05$ & $<.05$ & .13 & $<.05$ \\
\hline 9030 & Ottertail Co., MN & $06 / 12 / 01$ & $<.05$ & $<.05$ & $<.05$ & -- & $<.05$ & .36 & .06 & -- & $<.05$ & $<.05$ & $<.05$ & $<.05$ & $<.05$ & $<.05$ & $<.05$ & .13 & $<.05$ \\
\hline 9031 & Ottertail Co., MN & 06/12/01 & $<.05$ & $<.05$ & $<.05$ & -- & $<.05$ & .35 & .06 & -- & $<.05$ & $<.05$ & $<.05$ & $<.05$ & $<.05$ & $<.05$ & $<.05$ & .11 & $<.05$ \\
\hline 9032 & Ottertail Co., MN & 06/12/01 & $<.05$ & $<.05$ & $<.05$ & -- & $<.05$ & .38 & $<.05$ & -- & $<.05$ & $<.05$ & $<.05$ & $<.05$ & $<.05$ & $<.05$ & $<.05$ & 4.7 & .31 \\
\hline 9033 & Ottertail Co., MN & 06/12/01 & $<.05$ & $<.05$ & $<.05$ & -- & $<.05$ & $<.05$ & $<.05$ & -- & $<.05$ & $<.05$ & $<.05$ & $<.05$ & $<.05$ & $<.05$ & $<.05$ & .96 & $<.05$ \\
\hline 9034 & Ottertail Co., MN & 06/12/01 & $<.05$ & .13 & .11 & -- & $<.05$ & .06 & $<.05$ & -- & $<.05$ & $<.05$ & $<.05$ & $<.05$ & $<.05$ & $<.05$ & $<.05$ & .85 & $<.05$ \\
\hline 9035 & Grant Co., MN & $06 / 12 / 01$ & $<.05$ & $<.05$ & $<.05$ & -- & $<.05$ & $<.05$ & $<.05$ & -- & $<.05$ & $<.05$ & $<.05$ & $<.05$ & $<.05$ & $<.05$ & $<.05$ & $<.05$ & $<.05$ \\
\hline 9036 & Grant Co., MN & 06/12/01 & $<.05$ & $<.05$ & $<.05$ & -- & $<.05$ & $<.05$ & $<.05$ & -- & $<.05$ & $<.05$ & $<.05$ & $<.05$ & $<.05$ & $<.05$ & $<.05$ & $<.05$ & $<.05$ \\
\hline 9037 & Grant Co., MN & $06 / 12 / 01$ & $<.05$ & $<.05$ & $<.05$ & -- & $<.05$ & $<.05$ & $<.05$ & -- & $<.05$ & $<.05$ & $<.05$ & $<.05$ & $<.05$ & $<.05$ & $<.05$ & $<.05$ & $<.05$ \\
\hline 9038 & Todd Co., MN & $06 / 12 / 01$ & $<.05$ & $<.05$ & $<.05$ & -- & $<.05$ & 1.1 & $<.05$ & -- & $<.05$ & $<.05$ & $<.05$ & $<.05$ & $<.05$ & $<.05$ & $<.05$ & 2.0 & $<.05$ \\
\hline 9039 & Todd Co., MN & $06 / 12 / 01$ & $<.05$ & $<.05$ & $<.05$ & -- & $<.05$ & $<.05$ & $<.05$ & -- & $<.05$ & $<.05$ & $<.05$ & $<.05$ & $<.05$ & $<.05$ & $<.05$ & $<.05$ & $<.05$ \\
\hline 9040 & Todd Co., MN & $06 / 12 / 01$ & $<.05$ & $<.05$ & $<.05$ & -- & $<.05$ & $<.05$ & $<.05$ & -- & $<.05$ & $<.05$ & $<.05$ & $<.05$ & $<.05$ & $<.05$ & $<.05$ & .06 & $<.05$ \\
\hline
\end{tabular}


Table 5. Analytical results for selected acetamide herbicides and their degradation products, ethanesulfonic acid, oxanilic acid, and sulfinyl acetic acid, in ground-water samples, 1994-2002.-Continued

[OGRL, U.S. Geological Survey Organic Geochemistry Research Laboratory, Lawrence, Kansas; ESA, ethanesulfonic acid; OXA, oxanilic acid; SAA, sulfinyl acetic acid; --, no data; <, less than]

\begin{tabular}{|c|c|c|c|c|c|c|c|c|c|c|c|c|c|c|c|c|c|c|c|c|}
\hline Site number & Site location & $\begin{array}{c}\text { Date of } \\
\text { collection } \\
\text { (month/day/ } \\
\text { year) }\end{array}$ & $\begin{array}{l}\text { Aceto- } \\
\text { chlor } \\
(\mu \mathrm{g} / \mathrm{L})\end{array}$ & $\begin{array}{c}\text { Aceto- } \\
\text { chlor } \\
\text { ESA } \\
(\mu \mathrm{g} / \mathrm{L})\end{array}$ & $\begin{array}{c}\text { Aceto- } \\
\text { chlor } \\
\text { OXA } \\
(\mu \mathrm{g} / \mathrm{L})\end{array}$ & $\begin{array}{c}\text { Aceto- } \\
\text { chlor } \\
\text { SAA } \\
(\mu \mathrm{g} / \mathrm{L})\end{array}$ & $\begin{array}{l}\text { Ala- } \\
\text { chlor } \\
(\mu \mathrm{g} / \mathrm{L})\end{array}$ & $\begin{array}{l}\text { Ala- } \\
\text { chlor } \\
\text { ESA } \\
(\mu \mathrm{g} / \mathrm{L})\end{array}$ & $\begin{array}{l}\text { Ala- } \\
\text { chlor } \\
\text { OXA } \\
(\mu \mathrm{g} / \mathrm{L})\end{array}$ & $\begin{array}{l}\text { Ala- } \\
\text { chlor } \\
\text { SAA } \\
(\mu \mathrm{g} / \mathrm{L})\end{array}$ & $\begin{array}{c}\text { Di- } \\
\text { meth- } \\
\text { enamid } \\
(\mu \mathrm{g} / \mathrm{L})\end{array}$ & $\begin{array}{c}\text { Di- } \\
\text { meth- } \\
\text { enamid } \\
\text { ESA } \\
(\mu \mathrm{g} / \mathrm{L})\end{array}$ & $\begin{array}{c}\text { Di- } \\
\text { meth- } \\
\text { enamid } \\
\text { OXA } \\
(\mu \mathrm{g} / \mathrm{L})\end{array}$ & $\begin{array}{c}\text { Flufen- } \\
\text { acet } \\
(\mu \mathrm{g} / \mathrm{L})\end{array}$ & $\begin{array}{c}\text { Flufen- } \\
\text { acet } \\
\text { ESA } \\
(\mu \mathrm{g} / \mathrm{L})\end{array}$ & $\begin{array}{c}\text { Flufen- } \\
\text { acet } \\
\text { OXA } \\
(\mu \mathrm{g} / \mathrm{L})\end{array}$ & $\begin{array}{l}\text { Meto- } \\
\text { lachlor } \\
(\mu \mathrm{g} / \mathrm{L})\end{array}$ & $\begin{array}{c}\text { Meto- } \\
\text { lachlor } \\
\text { ESA } \\
(\mu \mathrm{g} / \mathrm{L})\end{array}$ & $\begin{array}{l}\text { Meto- } \\
\text { lachlor } \\
\text { OXA } \\
(\mu \mathrm{g} / \mathrm{L})\end{array}$ & 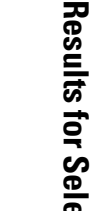 \\
\hline \multicolumn{20}{|c|}{ Federal-State Cooperative Studies-Continued } & \\
\hline \multicolumn{20}{|c|}{ Minnesota Pollution Control Agency (JMM)—Continued } & \\
\hline 9041 & Todd Co., MN & $06 / 19 / 01$ & $<0.05$ & $<0.05$ & $<0.05$ & -- & $<0.05$ & 0.57 & $<0.05$ & -- & $<0.05$ & $<0.05$ & $<0.05$ & $<0.05$ & $<0.05$ & $<0.05$ & $<0.05$ & 0.81 & 0.17 & \\
\hline 9042 & Todd Co., MN & $06 / 19 / 01$ & $<.05$ & $<.05$ & $<.05$ & -- & $<.05$ & .66 & $<.05$ & -- & $<.05$ & $<.05$ & $<.05$ & $<.05$ & $<.05$ & $<.05$ & $<.05$ & .99 & .19 & \\
\hline 9043 & Morrison Co., MN & 06/19/01 & $<.05$ & $<.05$ & $<.05$ & -- & $<.05$ & .07 & $<.05$ & -- & $<.05$ & $<.05$ & $<.05$ & $<.05$ & $<.05$ & $<.05$ & $<.05$ & $<.05$ & $<.05$ & \\
\hline 9044 & Morrison Co., MN & 06/19/01 & $<.05$ & .07 & $<.05$ & -- & $<.05$ & .43 & $<.05$ & -- & $<.05$ & $<.05$ & $<.05$ & $<.05$ & $<.05$ & $<.05$ & $<.05$ & .14 & $<.05$ & \\
\hline 9045 & Isanti Co., MN & $06 / 19 / 01$ & $<.05$ & $<.05$ & $<.05$ & -- & $<.05$ & .24 & $<.05$ & -- & $<.05$ & $<.05$ & $<.05$ & $<.05$ & $<.05$ & $<.05$ & $<.05$ & .30 & .14 & \\
\hline 9046 & Wabasha Co., MN & $06 / 27 / 01$ & $<.05$ & $<.05$ & $<.05$ & -- & $<.05$ & 1.9 & $<.05$ & -- & $<.05$ & $<.05$ & $<.05$ & $<.05$ & $<.05$ & $<.05$ & $<.05$ & .35 & $<.05$ & \\
\hline 9047 & Wabasha Co., MN & 06/27/01 & $<.05$ & $<.05$ & .05 & -- & $<.05$ & 4.9 & .24 & -- & $<.05$ & $<.05$ & $<.05$ & $<.05$ & $<.05$ & $<.05$ & $<.05$ & 3.1 & .81 & \\
\hline 9048 & Fillmore Co., MN & 06/27/01 & $<.05$ & $<.05$ & $<.05$ & -- & $<.05$ & 1.1 & .06 & -- & $<.05$ & $<.05$ & $<.05$ & $<.05$ & $<.05$ & $<.05$ & $<.05$ & .99 & .24 & $\cong$ \\
\hline 9049 & Fillmore Co., MN & 06/27/01 & $<.05$ & $<.05$ & $<.05$ & -- & $<.05$ & $<.05$ & $<.05$ & -- & $<.05$ & $<.05$ & $<.05$ & $<.05$ & $<.05$ & $<.05$ & $<.05$ & $<.05$ & $<.05$ & \\
\hline 9050 & Fillmore Co., MN & $06 / 27 / 01$ & $<.05$ & $<.05$ & $<.05$ & -- & $<.05$ & $<.05$ & $<.05$ & -- & $<.05$ & $<.05$ & $<.05$ & $<.05$ & $<.05$ & $<.05$ & $<.05$ & $<.05$ & $<.05$ & $\frac{\Phi}{\mathbb{\Phi}}$ \\
\hline 9051 & Nobles Co., MN & $06 / 27 / 01$ & $<.05$ & .07 & .06 & -- & $<.05$ & .09 & $<.05$ & -- & $<.05$ & $<.05$ & $<.05$ & $<.05$ & $<.05$ & $<.05$ & .07 & 3.7 & 1.7 & \\
\hline 9052 & Pipestone Co., MN & 06/27/01 & $<.05$ & $<.05$ & $<.05$ & -- & $<.05$ & .06 & $<.05$ & -- & $<.05$ & $<.05$ & $<.05$ & $<.05$ & $<.05$ & $<.05$ & $<.05$ & .31 & $<.05$ & \\
\hline 9053 & Redwood Co., MN & $06 / 27 / 01$ & $<.05$ & $<.05$ & $<.05$ & -- & $<.05$ & $<.05$ & $<.05$ & -- & $<.05$ & $<.05$ & $<.05$ & $<.05$ & $<.05$ & $<.05$ & $<.05$ & $<.05$ & $<.05$ & \\
\hline 5124 & Stearns Co., MN & 06/00/99 & $<.05$ & $<.20$ & $<.20$ & -- & $<.05$ & 2.0 & .68 & -- & $<.05$ & -- & -- & $<.05$ & -- & -- & $<.05$ & .21 & .21 & 를 \\
\hline 5164 & Stearns Co., MN & 03/02/98 & $<.05$ & $<.20$ & $<.20$ & -- & $<.05$ & $<.20$ & $<.20$ & -- & -- & -- & -- & -- & -- & -- & $<.05$ & $<.20$ & $<.20$ & \\
\hline 5165 & Stearns Co., MN & 03/02/98 & $<.05$ & $<.20$ & $<.20$ & -- & $<.05$ & 1.1 & $<.20$ & -- & -- & -- & -- & -- & -- & -- & $<.05$ & $<.20$ & $<.20$ & \\
\hline 5166 & Stearns Co., MN & 03/02/98 & $<.05$ & $<.20$ & $<.20$ & -- & $<.05$ & $<.20$ & $<.20$ & -- & -- & -- & -- & -- & -- & -- & $<.05$ & $<.20$ & $<.20$ & \\
\hline 5167 & Stearns Co., MN & 03/03/98 & $<.05$ & $<.20$ & $<.20$ & -- & $<.05$ & $<.20$ & $<.20$ & -- & -- & -- & -- & -- & -- & -- & $<.05$ & $<.20$ & $<.20$ & \\
\hline 5168 & Stearns Co., MN & 03/03/98 & $<.05$ & $<.20$ & $<.20$ & -- & $<.05$ & $<.20$ & $<.20$ & -- & -- & -- & -- & -- & -- & -- & $<.05$ & $<.20$ & $<.20$ & \\
\hline 5169 & Stearns Co., MN & 03/03/98 & $<.05$ & $<.20$ & $<.20$ & -- & $<.05$ & $<.20$ & $<.20$ & -- & -- & -- & -- & -- & -- & -- & $<.05$ & $<.20$ & $<.20$ & 을 음 \\
\hline 5181 & Stearns Co., MN & 03/04/98 & $<.05$ & $<.20$ & $<.20$ & -- & $<.05$ & $<.20$ & $<.20$ & -- & -- & -- & -- & -- & -- & -- & $<.05$ & $<.20$ & $<.20$ & 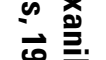 \\
\hline 5182 & Stearns Co., MN & 03/04/98 & $<.05$ & $<.20$ & $<.20$ & -- & $<.05$ & $<.20$ & $<.20$ & -- & -- & -- & -- & -- & -- & -- & $<.05$ & $<.20$ & $<.20$ & \\
\hline 5183 & Stearns Co., MN & 03/04/98 & $<.05$ & 1.4 & .25 & -- & $<.05$ & .81 & $<.20$ & -- & -- & -- & -- & -- & -- & -- & $<.05$ & 6.3 & 2.0 & \\
\hline 5184 & Stearns Co., MN & 03/04/98 & $<.05$ & 2.5 & $<.20$ & -- & $<.05$ & 1.0 & $<.20$ & -- & -- & -- & -- & -- & -- & -- & $<.05$ & .85 & $<.20$ & \\
\hline 5185 & Stearns Co., MN & 03/05/98 & $<.05$ & $<.20$ & $<.20$ & -- & $<.05$ & $<.20$ & $<.20$ & -- & -- & -- & -- & -- & -- & -- & $<.05$ & $<.20$ & $<.20$ & \\
\hline
\end{tabular}




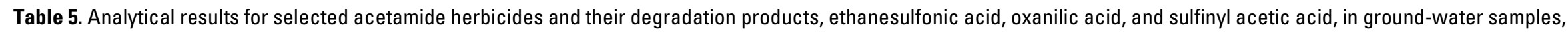
1994-2002.-Continued

[OGRL, U.S. Geological Survey Organic Geochemistry Research Laboratory, Lawrence, Kansas; ESA, ethanesulfonic acid; OXA, oxanilic acid; SAA, sulfinyl acetic acid; --, no data; <, less than]

\begin{tabular}{|c|c|c|c|c|c|c|c|c|c|c|c|c|c|c|c|c|c|c|c|}
\hline Site number & Site location & $\begin{array}{c}\text { Date of } \\
\text { collection } \\
\text { (month/day/ } \\
\text { year) }\end{array}$ & $\begin{array}{l}\text { Aceto- } \\
\text { chlor } \\
(\mu \mathrm{g} / \mathrm{L})\end{array}$ & $\begin{array}{l}\text { Aceto- } \\
\text { chlor } \\
\text { ESA } \\
(\mu \mathrm{g} / \mathrm{L})\end{array}$ & $\begin{array}{c}\text { Aceto- } \\
\text { chlor } \\
0 X A \\
(\mu \mathrm{g} / \mathrm{L})\end{array}$ & $\begin{array}{c}\text { Aceto- } \\
\text { chlor } \\
\text { SAA } \\
(\mu \mathrm{g} / \mathrm{L})\end{array}$ & $\begin{array}{l}\text { Ala- } \\
\text { chlor } \\
(\mu \mathrm{g} / \mathrm{L})\end{array}$ & $\begin{array}{l}\text { Ala- } \\
\text { chlor } \\
\text { ESA } \\
(\mu \mathrm{g} / \mathrm{L})\end{array}$ & $\begin{array}{l}\text { Ala- } \\
\text { chlor } \\
\text { OXA } \\
(\mu \mathrm{g} / \mathrm{L})\end{array}$ & $\begin{array}{l}\text { Ala- } \\
\text { chlor } \\
\text { SAA } \\
(\mu \mathrm{g} / \mathrm{L})\end{array}$ & $\begin{array}{c}\text { Di- } \\
\text { meth- } \\
\text { enamid } \\
(\mu \mathrm{g} / \mathrm{L})\end{array}$ & $\begin{array}{c}\text { Di- } \\
\text { meth- } \\
\text { enamid } \\
\text { ESA } \\
(\mu \mathrm{g} / \mathrm{L})\end{array}$ & $\begin{array}{c}\text { Di- } \\
\text { meth- } \\
\text { enamid } \\
\text { OXA } \\
(\mu \mathrm{g} / \mathrm{L})\end{array}$ & $\begin{array}{c}\text { Flufen- } \\
\text { acet } \\
(\mu \mathrm{g} / \mathrm{L})\end{array}$ & $\begin{array}{c}\text { Flufen- } \\
\text { acet } \\
\text { ESA } \\
(\mu \mathrm{g} / \mathrm{L})\end{array}$ & $\begin{array}{c}\text { Flufen- } \\
\text { acet } \\
\text { OXA } \\
(\mu \mathrm{g} / \mathrm{L})\end{array}$ & $\begin{array}{l}\text { Meto- } \\
\text { lachlor } \\
(\mu \mathrm{g} / \mathrm{L})\end{array}$ & $\begin{array}{l}\text { Meto- } \\
\text { lachlor } \\
\text { ESA } \\
(\mu \mathrm{g} / \mathrm{L})\end{array}$ & $\begin{array}{c}\text { Meto- } \\
\text { lachlor } \\
\text { OXA } \\
(\mu \mathrm{g} / \mathrm{L})\end{array}$ \\
\hline \multicolumn{20}{|c|}{ Federal-State Cooperative Studies-Continued } \\
\hline \multicolumn{20}{|c|}{ Minnesota Pollution Control Agency (JMM)—Continued } \\
\hline 5186 & Stearns Co., MN & 03/05/98 & $<0.05$ & $<0.20$ & $<0.20$ & -- & $<0.05$ & $<0.20$ & $<0.20$ & -- & -- & -- & -- & -- & -- & -- & $<0.05$ & $<0.20$ & $<0.20$ \\
\hline 5187 & Stearns Co., MN & 03/05/98 & $<.05$ & .91 & $<.20$ & -- & $<.05$ & .47 & $<.20$ & -- & -- & -- & -- & -- & -- & -- & $<.05$ & .46 & .22 \\
\hline 5407 & Stearns Co., MN & $05 / 12 / 98$ & $<.05$ & $<.20$ & $<.20$ & -- & $<.05$ & $<.20$ & $<.20$ & -- & -- & -- & -- & -- & -- & -- & $<.05$ & $<.20$ & $<.20$ \\
\hline 5409 & Stearns Co., MN & 05/12/98 & $<.05$ & 6.1 & $<.20$ & -- & $<.05$ & 1.7 & $<.20$ & -- & -- & -- & -- & -- & -- & -- & $<.05$ & 1.2 & $<.20$ \\
\hline 5417 & Stearns Co., MN & $05 / 13 / 98$ & $<.05$ & $<.20$ & $<.20$ & -- & $<.05$ & $<.20$ & $<.20$ & -- & -- & -- & -- & -- & -- & -- & $<.05$ & $<.20$ & $<.20$ \\
\hline 5527 & Stearns Co., MN & 09/29/98 & $<.05$ & .87 & $<.20$ & -- & $<.05$ & .80 & $<.20$ & -- & -- & -- & -- & -- & -- & -- & $<.05$ & 11 & 1.3 \\
\hline 5528 & Stearns Co., MN & 09/09/98 & $<.05$ & 1.7 & $<.20$ & -- & $<.05$ & 1.1 & $<.20$ & -- & -- & -- & -- & -- & -- & -- & $<.05$ & 12 & 1.6 \\
\hline 5531 & Stearns Co., MN & 06/00/99 & $<.05$ & $<.20$ & $<.20$ & -- & $<.05$ & $<.20$ & $<.20$ & -- & $<0.05$ & -- & -- & $<0.05$ & -- & -- & $<.05$ & $<.20$ & $<.20$ \\
\hline 5537 & Stearns Co., MN & 06/00/99 & $<.05$ & $<.20$ & $<.20$ & -- & $<.05$ & 3.6 & 1.8 & -- & $<.05$ & -- & -- & $<.05$ & -- & -- & $<.05$ & 1.1 & 1.1 \\
\hline 5542 & Stearns Co., MN & $10 / 28 / 98$ & $<.05$ & $<.20$ & $<.20$ & -- & $<.05$ & $<.20$ & $<.20$ & -- & -- & -- & -- & -- & -- & -- & $<.05$ & $<.20$ & $<.20$ \\
\hline 5543 & Stearns Co., MN & $10 / 28 / 98$ & $<.05$ & $<.20$ & $<.20$ & -- & $<.05$ & $<.20$ & $<.20$ & -- & -- & -- & -- & -- & -- & -- & $<.05$ & $<.20$ & $<.20$ \\
\hline 5546 & Stearns Co., MN & $10 / 28 / 98$ & $<.05$ & $<.20$ & $<.20$ & -- & $<.05$ & 3.3 & .20 & -- & -- & -- & -- & -- & -- & -- & $<.05$ & .24 & .33 \\
\hline 5547 & Stearns Co., MN & $10 / 00 / 98$ & $<.05$ & $<.20$ & $<.20$ & -- & $<.05$ & $<.20$ & $<.20$ & -- & -- & -- & -- & -- & -- & -- & $<.05$ & $<.20$ & $<.20$ \\
\hline 5553 & Stearns Co., MN & $10 / 28 / 98$ & $<.05$ & $<.20$ & $<.20$ & -- & $<.05$ & $<.20$ & $<.20$ & -- & -- & -- & -- & -- & -- & -- & $<.05$ & $<.20$ & $<.20$ \\
\hline 5554 & Stearns Co., MN & $10 / 28 / 98$ & $<.05$ & $<.20$ & $<.20$ & -- & $<.05$ & .66 & $<.20$ & -- & -- & -- & -- & -- & -- & -- & $<.05$ & .78 & $<.20$ \\
\hline 5555 & Stearns Co., MN & $10 / 28 / 98$ & $<.05$ & 5.6 & $<.20$ & -- & $<.05$ & 1.9 & $<.20$ & -- & -- & -- & -- & -- & -- & -- & $<.05$ & 2.1 & .91 \\
\hline 5556 & Stearns Co., MN & $10 / 28 / 98$ & $<.05$ & .42 & $<.20$ & -- & $<.05$ & 1.2 & $<.20$ & -- & -- & -- & -- & -- & -- & -- & $<.05$ & 15 & 1.6 \\
\hline 5558 & Stearns Co., MN & $10 / 28 / 98$ & $<.05$ & $<.20$ & $<.20$ & -- & $<.05$ & .32 & $<.20$ & -- & -- & -- & -- & -- & -- & -- & $<.05$ & $<.20$ & $<.20$ \\
\hline 5596 & Stearns Co., MN & 09/29/98 & $<.05$ & $<.20$ & $<.20$ & -- & $<.05$ & .61 & $<.20$ & -- & -- & -- & -- & -- & -- & -- & $<.05$ & .68 & $<.20$ \\
\hline 5784 & Stearns Co., MN & 03/04/98 & $<.05$ & $<.20$ & $<.20$ & -- & .35 & $<.20$ & $<.20$ & -- & -- & -- & -- & -- & -- & -- & .33 & $<.20$ & $<.20$ \\
\hline 5785 & Stearns Co., MN & 03/04/98 & $<.05$ & $<.20$ & $<.20$ & -- & 6.4 & $<.20$ & $<.20$ & -- & -- & -- & -- & -- & -- & -- & 5.9 & $<.20$ & $<.20$ \\
\hline 5954 & Stearns Co., MN & 09/09/98 & $<.05$ & $<.20$ & $<.20$ & -- & $<.05$ & .83 & $<.20$ & -- & -- & -- & -- & -- & -- & -- & $<.05$ & .49 & $<.20$ \\
\hline 5955 & Stearns Co., MN & 08/04/98 & $<.05$ & .27 & .24 & -- & $<.05$ & .65 & $<.20$ & -- & -- & -- & -- & -- & -- & -- & $<.05$ & .60 & $<.20$ \\
\hline 5956 & Stearns Co., MN & 08/04/98 & $<.05$ & .45 & .20 & -- & $<.05$ & .67 & $<.20$ & -- & -- & -- & -- & -- & -- & -- & $<.05$ & .80 & $<.20$ \\
\hline 5995 & Stearns Co., MN & $10 / 28 / 98$ & $<.05$ & $<.20$ & $<.20$ & -- & $<.05$ & $<.20$ & $<.20$ & -- & -- & -- & -- & -- & -- & -- & $<.05$ & $<.20$ & $<.20$ \\
\hline
\end{tabular}


Table 5. Analytical results for selected acetamide herbicides and their degradation products, ethanesulfonic acid, oxanilic acid, and sulfinyl acetic acid, in ground-water samples, 1994-2002.-Continued

[OGRL, U.S. Geological Survey Organic Geochemistry Research Laboratory, Lawrence, Kansas; ESA, ethanesulfonic acid; OXA, oxanilic acid; SAA, sulfinyl acetic acid; --, no data; <, less than]

\begin{tabular}{|c|c|c|c|c|c|c|c|c|c|c|c|c|c|c|c|c|c|c|c|c|}
\hline Site number & Site location & $\begin{array}{c}\text { Date of } \\
\text { collection } \\
\text { (month/day/ } \\
\text { year) }\end{array}$ & $\begin{array}{l}\text { Aceto- } \\
\text { chlor } \\
(\mu \mathrm{g} / \mathrm{L})\end{array}$ & $\begin{array}{c}\text { Aceto- } \\
\text { chlor } \\
\text { ESA } \\
(\mu \mathrm{g} / \mathrm{L})\end{array}$ & $\begin{array}{c}\text { Aceto- } \\
\text { chlor } \\
\text { OXA } \\
(\mu \mathrm{g} / \mathrm{L})\end{array}$ & $\begin{array}{c}\text { Aceto- } \\
\text { chlor } \\
\text { SAA } \\
(\mu \mathrm{g} / \mathrm{L})\end{array}$ & $\begin{array}{l}\text { Ala- } \\
\text { chlor } \\
(\mu \mathrm{g} / \mathrm{L})\end{array}$ & $\begin{array}{l}\text { Ala- } \\
\text { chlor } \\
\text { ESA } \\
(\mu \mathrm{g} / \mathrm{L})\end{array}$ & $\begin{array}{l}\text { Ala- } \\
\text { chlor } \\
\text { OXA } \\
(\mu \mathrm{g} / \mathrm{L})\end{array}$ & $\begin{array}{l}\text { Ala- } \\
\text { chlor } \\
\text { SAA } \\
(\mu \mathrm{g} / \mathrm{L})\end{array}$ & $\begin{array}{c}\text { Di- } \\
\text { meth- } \\
\text { enamid } \\
(\mu \mathrm{g} / \mathrm{L})\end{array}$ & $\begin{array}{c}\text { Di- } \\
\text { meth- } \\
\text { enamid } \\
\text { ESA } \\
(\mu \mathrm{g} / \mathrm{L})\end{array}$ & $\begin{array}{c}\text { Di- } \\
\text { meth- } \\
\text { enamid } \\
\text { OXA } \\
(\mu \mathrm{g} / \mathrm{L})\end{array}$ & $\begin{array}{c}\text { Flufen- } \\
\text { acet } \\
(\mu \mathrm{g} / \mathrm{L})\end{array}$ & $\begin{array}{c}\text { Flufen- } \\
\text { acet } \\
\text { ESA } \\
(\mu \mathrm{g} / \mathrm{L})\end{array}$ & $\begin{array}{c}\text { Flufen- } \\
\text { acet } \\
\text { OXA } \\
(\mu \mathrm{g} / \mathrm{L})\end{array}$ & $\begin{array}{l}\text { Meto- } \\
\text { lachlor } \\
(\mu \mathrm{g} / \mathrm{L})\end{array}$ & $\begin{array}{c}\text { Meto- } \\
\text { lachlor } \\
\text { ESA } \\
(\mu \mathrm{g} / \mathrm{L})\end{array}$ & $\begin{array}{l}\text { Meto- } \\
\text { lachlor } \\
\text { OXA } \\
(\mu \mathrm{g} / \mathrm{L})\end{array}$ & 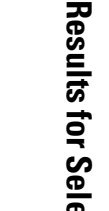 \\
\hline \multicolumn{20}{|c|}{ Federal-State Cooperative Studies-Continued } & \\
\hline \multicolumn{20}{|c|}{ Minnesota Pollution Control Agency (JMM) - Continued } & \\
\hline 5997 & Stearns Co., MN & $10 / 28 / 98$ & $<0.05$ & 0.26 & $<0.20$ & -- & $<0.05$ & 0.70 & $<0.20$ & -- & -- & -- & -- & -- & -- & -- & $<0.05$ & 0.60 & 0.29 & \\
\hline 6283 & Stearns Co., MN & $11 / 00 / 99$ & $<.05$ & $<.20$ & $<.20$ & -- & $<.05$ & $<.20$ & $<.20$ & -- & $<0.05$ & -- & -- & $<0.05$ & -- & -- & $<.05$ & $<.20$ & $<.20$ & \\
\hline 6287 & Stearns Co., MN & $11 / 00 / 99$ & $<.05$ & $<.20$ & $<.20$ & -- & $<.05$ & $<.20$ & $<.20$ & -- & $<.05$ & -- & -- & $<.05$ & -- & -- & $<.05$ & $<.20$ & $<.20$ & \\
\hline 6291 & Stearns Co., MN & $11 / 00 / 99$ & $<.05$ & .77 & $<.20$ & -- & $<.05$ & .60 & $<.20$ & -- & $<.05$ & -- & -- & $<.05$ & -- & -- & $<.05$ & .75 & .25 & \\
\hline 6292 & Stearns Co., MN & $11 / 00 / 99$ & $<.05$ & $<.20$ & $<.20$ & -- & $<.05$ & .64 & $<.20$ & -- & $<.05$ & -- & -- & $<.05$ & -- & -- & $<.05$ & 5.3 & .64 & \\
\hline 6605 & Stearns Co., MN & 08/04/98 & $<.05$ & $<.20$ & $<.20$ & -- & $<.05$ & $<.20$ & $<.20$ & -- & -- & -- & -- & -- & -- & -- & $<.05$ & $<.20$ & $<.20$ & \\
\hline 6606 & Stearns Co., MN & 08/04/98 & $<.05$ & $<.20$ & $<.20$ & -- & $<.05$ & 5.7 & .25 & -- & -- & -- & -- & -- & -- & -- & $<.05$ & .40 & $<.20$ & \\
\hline 6607 & Stearns Co., MN & 08/04/98 & $<.05$ & $<.20$ & $<.20$ & -- & $<.05$ & .44 & .46 & -- & -- & -- & -- & -- & -- & -- & $<.05$ & $<.20$ & $<.20$ & $\cong$ \\
\hline 6608 & Stearns Co., MN & 08/04/98 & $<.05$ & $<.20$ & $<.20$ & -- & $<.05$ & $<.20$ & $<.20$ & -- & -- & -- & -- & -- & -- & -- & $<.05$ & $<.20$ & $<.20$ & \\
\hline 6609 & Stearns Co., MN & 08/04/98 & $<.05$ & 2.5 & $<.20$ & -- & $<.05$ & 1.5 & $<.20$ & -- & -- & -- & -- & -- & -- & -- & $<.05$ & 15 & 1.5 & \\
\hline 6610 & Stearns Co., MN & 08/04/98 & $<.05$ & $<.20$ & $<.20$ & -- & $<.05$ & $<.20$ & $<.20$ & -- & -- & -- & -- & -- & -- & -- & $<.05$ & $<.20$ & $<.20$ & \\
\hline 6611 & Stearns Co., MN & 08/04/98 & $<.05$ & $<.20$ & $<.20$ & -- & $<.05$ & $<.20$ & $<.20$ & -- & -- & -- & -- & -- & -- & -- & $<.05$ & $<.20$ & $<.20$ & \\
\hline 6612 & Stearns Co., MN & 08/04/98 & $<.05$ & 8.2 & $<.20$ & -- & $<.05$ & 2.0 & $<.20$ & -- & -- & -- & -- & -- & -- & -- & $<.05$ & 1.1 & $<.20$ & \\
\hline 6627 & Stearns Co., MN & 06/00/99 & $<.05$ & $<.20$ & $<.20$ & -- & $<.05$ & 1.3 & .93 & -- & $<.05$ & -- & -- & $<.05$ & -- & -- & $<.05$ & $<.20$ & $<.20$ & 를 \\
\hline 7185 & Stearns Co., MN & 03/05/98 & $<.05$ & $<.20$ & $<.20$ & -- & $<.05$ & $<.20$ & $<.20$ & -- & -- & -- & -- & -- & -- & -- & $<.05$ & $<.20$ & $<.20$ & \\
\hline 7313 & Stearns Co., MN & $05 / 12 / 98$ & $<.05$ & $<.20$ & $<.20$ & -- & $<.05$ & $<.20$ & $<.20$ & -- & -- & -- & -- & -- & -- & -- & $<.05$ & $<.20$ & $<.20$ & \\
\hline 7314 & Stearns Co., MN & $05 / 12 / 98$ & $<.05$ & 3.0 & $<.20$ & -- & $<.05$ & 1.8 & $<.20$ & -- & -- & -- & -- & -- & -- & -- & $<.05$ & 9.5 & 3.6 & \\
\hline 7315 & Stearns Co., MN & $05 / 12 / 98$ & $<.05$ & 1.2 & $<.20$ & -- & $<.05$ & .49 & $<.20$ & -- & -- & -- & -- & -- & -- & -- & $<.05$ & .68 & .36 & \\
\hline 7318 & Stearns Co., MN & $05 / 12 / 98$ & $<.05$ & $<.20$ & $<.20$ & -- & $<.05$ & $<.20$ & $<.20$ & -- & -- & -- & -- & -- & -- & -- & $<.05$ & $<.20$ & $<.20$ & \\
\hline 7319 & Stearns Co., MN & $05 / 12 / 98$ & $<.05$ & $<.20$ & $<.20$ & -- & $<.05$ & $<.20$ & $<.20$ & -- & -- & -- & -- & -- & -- & -- & $<.05$ & $<.20$ & $<.20$ & 总 $\frac{\Omega}{2}$ \\
\hline 7320 & Stearns Co., MN & $05 / 12 / 98$ & $<.05$ & $<.20$ & $<.20$ & -- & $<.05$ & $<.20$ & $<.20$ & -- & -- & -- & -- & -- & -- & -- & $<.05$ & $<.20$ & $<.20$ & 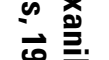 \\
\hline 7321 & Stearns Co., MN & 05/12/98 & $<.05$ & $<.20$ & $<.20$ & -- & $<.05$ & $<.20$ & $<.20$ & -- & -- & -- & -- & -- & -- & -- & $<.05$ & .64 & $<.20$ & \\
\hline 7322 & Stearns Co., MN & $05 / 12 / 98$ & $<.05$ & $<.20$ & $<.20$ & -- & $<.05$ & .60 & $<.20$ & -- & -- & -- & -- & -- & -- & -- & $<.05$ & .77 & $<.20$ & \\
\hline 7368 & Stearns Co., MN & 06/00/99 & $<.05$ & $<.20$ & $<.20$ & -- & $<.05$ & 1.6 & .28 & -- & $<.05$ & -- & -- & $<.05$ & -- & -- & $<.05$ & $<.20$ & $<.20$ & \\
\hline 7371 & Stearns Co., MN & 06/00/99 & $<.05$ & $<.20$ & $<.20$ & -- & $<.05$ & 1.9 & 1.1 & -- & $<.05$ & -- & -- & $<.05$ & -- & -- & $<.05$ & $<.20$ & .35 & \\
\hline
\end{tabular}




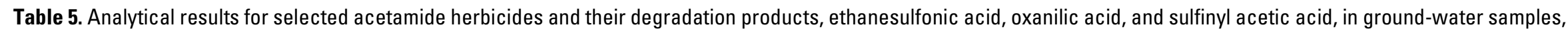
1994-2002.-Continued

[OGRL, U.S. Geological Survey Organic Geochemistry Research Laboratory, Lawrence, Kansas; ESA, ethanesulfonic acid; OXA, oxanilic acid; SAA, sulfinyl acetic acid; --, no data; <, less than]

\begin{tabular}{|c|c|c|c|c|c|c|c|c|c|c|c|c|c|c|c|c|c|c|c|}
\hline Site number & Site location & $\begin{array}{c}\text { Date of } \\
\text { collection } \\
\text { (month/day/ } \\
\text { year) }\end{array}$ & $\begin{array}{l}\text { Aceto- } \\
\text { chlor } \\
(\mu \mathrm{g} / \mathrm{L})\end{array}$ & $\begin{array}{l}\text { Aceto- } \\
\text { chlor } \\
\text { ESA } \\
(\mu \mathrm{g} / \mathrm{L})\end{array}$ & $\begin{array}{l}\text { Aceto- } \\
\text { chlor } \\
\text { OXA } \\
(\mu \mathrm{g} / \mathrm{L})\end{array}$ & $\begin{array}{c}\text { Aceto- } \\
\text { chlor } \\
\text { SAA } \\
(\mu \mathrm{g} / \mathrm{L})\end{array}$ & $\begin{array}{l}\text { Ala- } \\
\text { chlor } \\
(\mu \mathrm{g} / \mathrm{L})\end{array}$ & $\begin{array}{l}\text { Ala- } \\
\text { chlor } \\
\text { ESA } \\
(\mu \mathrm{g} / \mathrm{L})\end{array}$ & $\begin{array}{l}\text { Ala- } \\
\text { chlor } \\
\text { OXA } \\
(\mu \mathrm{g} / \mathrm{L})\end{array}$ & $\begin{array}{l}\text { Ala- } \\
\text { chlor } \\
\text { SAA } \\
(\mu \mathrm{g} / \mathrm{L})\end{array}$ & $\begin{array}{c}\text { Di- } \\
\text { meth- } \\
\text { enamid } \\
(\mu \mathrm{g} / \mathrm{L})\end{array}$ & $\begin{array}{c}\text { Di- } \\
\text { meth- } \\
\text { enamid } \\
\text { ESA } \\
(\mu \mathrm{g} / \mathrm{L})\end{array}$ & $\begin{array}{c}\text { Di- } \\
\text { meth- } \\
\text { enamid } \\
\text { OXA } \\
(\mu \mathrm{g} / \mathrm{L})\end{array}$ & $\begin{array}{l}\text { Flufen- } \\
\text { acet } \\
(\mu \mathrm{g} / \mathrm{L})\end{array}$ & $\begin{array}{c}\text { Flufen- } \\
\text { acet } \\
\text { ESA } \\
(\mu \mathrm{g} / \mathrm{L})\end{array}$ & $\begin{array}{c}\text { Flufen- } \\
\text { acet } \\
\text { OXA } \\
(\mu \mathrm{g} / \mathrm{L})\end{array}$ & $\begin{array}{l}\text { Meto- } \\
\text { lachlor } \\
(\mu \mathrm{g} / \mathrm{L})\end{array}$ & $\begin{array}{l}\text { Meto- } \\
\text { lachlor } \\
\text { ESA } \\
(\mu \mathrm{g} / \mathrm{L})\end{array}$ & $\begin{array}{c}\text { Meto- } \\
\text { lachlor } \\
\text { OXA } \\
(\mu \mathrm{g} / \mathrm{L})\end{array}$ \\
\hline \multicolumn{20}{|c|}{ Federal-State Cooperative Studies-Continued } \\
\hline \multicolumn{20}{|c|}{ Minnesota Pollution Control Agency (JMM) —Continued } \\
\hline 7373 & Stearns Co., MN & 06/00/99 & $<0.05$ & $<0.20$ & $<0.20$ & -- & $<0.05$ & 0.64 & 0.37 & -- & $<0.05$ & -- & -- & $<0.05$ & -- & -- & $<0.05$ & 0.21 & 0.28 \\
\hline 7376 & Stearns Co., MN & 06/00/99 & $<.05$ & $<.20$ & $<.20$ & -- & $<.05$ & 1.7 & $<.20$ & -- & $<.05$ & -- & -- & $<.05$ & -- & -- & $<.05$ & $<.20$ & $<.20$ \\
\hline 7378 & Stearns Co., MN & 06/00/99 & $<.05$ & $<.20$ & $<.20$ & -- & $<.05$ & 7.1 & 1.7 & -- & $<.05$ & -- & -- & $<.05$ & -- & -- & $<.05$ & $<.20$ & $<.20$ \\
\hline 7381 & Stearns Co., MN & 06/00/99 & $<.05$ & $<.20$ & $<.20$ & -- & $<.05$ & $<.20$ & $<.20$ & -- & $<.05$ & -- & -- & $<.05$ & -- & -- & $<.05$ & $<.20$ & $<.20$ \\
\hline 7382 & Stearns Co., MN & 06/00/99 & $<.05$ & $<.20$ & $<.20$ & -- & $<.05$ & 3.6 & 2.1 & -- & $<.05$ & -- & -- & $<.05$ & -- & -- & $<.05$ & 1.6 & 1.9 \\
\hline 7385 & Stearns Co., MN & 06/00/99 & $<.05$ & $<.20$ & $<.20$ & -- & .06 & 1.1 & .81 & -- & $<.05$ & -- & -- & $<.05$ & -- & -- & $<.05$ & .66 & .63 \\
\hline 7390 & Stearns Co., MN & 06/00/99 & $<.05$ & $<.20$ & $<.20$ & -- & $<.05$ & 1.1 & $<.20$ & -- & $<.05$ & -- & -- & $<.05$ & -- & -- & $<.05$ & .35 & .26 \\
\hline 7391 & Stearns Co., MN & 06/00/99 & $<.05$ & $<.20$ & $<.20$ & -- & $<.05$ & $<.20$ & $<.20$ & -- & $<.05$ & -- & -- & $<.05$ & -- & -- & .06 & $<.20$ & $<.20$ \\
\hline 7393 & Stearns Co., MN & 06/00/99 & $<.05$ & $<.20$ & $<.20$ & -- & $<.05$ & .21 & $<.20$ & -- & $<.05$ & -- & -- & $<.05$ & -- & -- & $<.05$ & $<.20$ & $<.20$ \\
\hline 7399 & Stearns Co., MN & 06/00/99 & $<.05$ & $<.20$ & $<.20$ & -- & $<.05$ & $<.20$ & $<.20$ & -- & $<.05$ & -- & -- & $<.05$ & -- & -- & $<.05$ & $<.20$ & $<.20$ \\
\hline 7401 & Stearns Co., MN & 06/00/99 & $<.05$ & $<.20$ & $<.20$ & -- & $<.05$ & .50 & $<.20$ & -- & $<.05$ & -- & -- & $<.05$ & -- & -- & $<.05$ & $<.20$ & $<.20$ \\
\hline 7402 & Stearns Co., MN & 06/00/99 & $<.05$ & $<.20$ & $<.20$ & -- & $<.05$ & 1.4 & $<.20$ & -- & $<.05$ & -- & -- & $<.05$ & -- & -- & $<.05$ & $<.20$ & $<.20$ \\
\hline 7404 & Stearns Co., MN & 06/00/99 & $<.05$ & $<.20$ & $<.20$ & -- & $<.05$ & 2.0 & $<.20$ & -- & $<.05$ & -- & -- & $<.05$ & -- & -- & $<.05$ & $<.20$ & .27 \\
\hline 7406 & Stearns Co., MN & 06/00/99 & $<.05$ & $<.20$ & $<.20$ & -- & $<.05$ & $<.20$ & $<.20$ & -- & $<.05$ & -- & -- & $<.05$ & -- & -- & $<.05$ & $<.20$ & $<.20$ \\
\hline 7409 & Stearns Co., MN & 06/00/99 & $<.05$ & $<.20$ & $<.20$ & -- & $<.05$ & 2.5 & .48 & -- & $<.05$ & -- & -- & $<.05$ & -- & -- & $<.05$ & .39 & .47 \\
\hline 7411 & Stearns Co., MN & 06/00/99 & $<.05$ & $<.20$ & $<.20$ & -- & $<.05$ & $<.20$ & $<.20$ & -- & $<.05$ & -- & -- & $<.05$ & -- & -- & $<.05$ & $<.20$ & $<.20$ \\
\hline 7412 & Stearns Co., MN & 06/00/99 & $<.05$ & $<.20$ & $<.20$ & -- & $<.05$ & .20 & $<.20$ & -- & $<.05$ & -- & -- & $<.05$ & -- & -- & $<.05$ & $<.20$ & $<.20$ \\
\hline 7415 & Stearns Co., MN & 06/00/99 & $<.05$ & $<.20$ & $<.20$ & -- & $<.05$ & $<.20$ & $<.20$ & -- & $<.05$ & -- & -- & $<.05$ & -- & -- & $<.05$ & $<.20$ & $<.20$ \\
\hline 7417 & Stearns Co., MN & 06/00/99 & $<.05$ & $<.20$ & $<.20$ & -- & $<.05$ & .31 & $<.20$ & -- & $<.05$ & -- & -- & $<.05$ & -- & -- & $<.05$ & $<.20$ & $<.20$ \\
\hline 7418 & Stearns Co., MN & 06/00/99 & $<.05$ & $<.20$ & $<.20$ & -- & $<.05$ & 1.1 & $<.20$ & -- & $<.05$ & -- & -- & $<.05$ & -- & -- & $<.05$ & .34 & .25 \\
\hline 7421 & Stearns Co., MN & 06/00/99 & $<.05$ & $<.20$ & $<.20$ & -- & $<.05$ & .67 & $<.20$ & -- & $<.05$ & -- & -- & $<.05$ & -- & -- & $<.05$ & .36 & .25 \\
\hline 7423 & Stearns Co., MN & 06/00/99 & $<.05$ & $<.20$ & $<.20$ & -- & $<.05$ & 1.3 & $<.20$ & -- & $<.05$ & -- & -- & $<.05$ & -- & -- & $<.05$ & $<.20$ & $<.20$ \\
\hline 7424 & Stearns Co., MN & 06/00/99 & $<.05$ & $<.20$ & $<.20$ & -- & $<.05$ & .28 & $<.20$ & -- & $<.05$ & -- & -- & $<.05$ & -- & -- & $<.05$ & $<.20$ & $<.20$ \\
\hline 7427 & Stearns Co., MN & 06/00/99 & $<.05$ & $<.20$ & $<.20$ & -- & $<.05$ & .26 & $<.20$ & -- & $<.05$ & -- & -- & $<.05$ & -- & -- & $<.05$ & $<.20$ & $<.20$ \\
\hline 7428 & Stearns Co., MN & 06/00/99 & $<.05$ & $<.20$ & $<.20$ & -- & $<.05$ & .35 & $<.20$ & -- & $<.05$ & -- & -- & $<.05$ & -- & -- & $<.05$ & $<.20$ & $<.20$ \\
\hline
\end{tabular}


Table 5. Analytical results for selected acetamide herbicides and their degradation products, ethanesulfonic acid, oxanilic acid, and sulfinyl acetic acid, in ground-water samples, 1994-2002.-Continued

[OGRL, U.S. Geological Survey Organic Geochemistry Research Laboratory, Lawrence, Kansas; ESA, ethanesulfonic acid; OXA, oxanilic acid; SAA, sulfinyl acetic acid; --, no data; <, less than]

\begin{tabular}{|c|c|c|c|c|c|c|c|c|c|c|c|c|c|c|c|c|c|c|c|}
\hline Site number & Site location & $\begin{array}{c}\text { Date of } \\
\text { collection } \\
\text { (month/day/ } \\
\text { year) }\end{array}$ & $\begin{array}{c}\text { Aceto- } \\
\text { chlor } \\
(\mu \mathrm{g} / \mathrm{L})\end{array}$ & $\begin{array}{l}\text { Aceto- } \\
\text { chlor } \\
\text { ESA } \\
(\mu \mathrm{g} / \mathrm{L})\end{array}$ & $\begin{array}{l}\text { Aceto- } \\
\text { chlor } \\
\text { OXA } \\
(\mu \mathrm{g} / \mathrm{L})\end{array}$ & $\begin{array}{l}\text { Aceto- } \\
\text { chlor } \\
\text { SAA } \\
(\mu \mathrm{g} / \mathrm{L})\end{array}$ & $\begin{array}{l}\text { Ala- } \\
\text { chlor } \\
(\mu \mathrm{g} / \mathrm{L})\end{array}$ & $\begin{array}{l}\text { Ala- } \\
\text { chlor } \\
\text { ESA } \\
(\mu \mathrm{g} / \mathrm{L})\end{array}$ & $\begin{array}{c}\text { Ala- } \\
\text { chlor } \\
\text { OXA } \\
(\mu \mathrm{g} / \mathrm{L})\end{array}$ & $\begin{array}{l}\text { Ala- } \\
\text { chlor } \\
\text { SAA } \\
(\mu \mathrm{g} / \mathrm{L})\end{array}$ & $\begin{array}{l}\text { Di- } \\
\text { meth- } \\
\text { enamid } \\
(\mu \mathrm{g} / \mathrm{L})\end{array}$ & $\begin{array}{c}\text { Di- } \\
\text { meth- } \\
\text { enamid } \\
\text { ESA } \\
(\mu \mathrm{g} / \mathrm{L})\end{array}$ & $\begin{array}{c}\text { Di- } \\
\text { meth- } \\
\text { enamid } \\
\text { OXA } \\
(\mu \mathrm{g} / \mathrm{L})\end{array}$ & $\begin{array}{c}\text { Flufen- } \\
\text { acet } \\
(\mu \mathrm{g} / \mathrm{L})\end{array}$ & $\begin{array}{l}\text { Flufen- } \\
\text { acet } \\
\text { ESA } \\
(\mu \mathrm{g} / \mathrm{L})\end{array}$ & $\begin{array}{c}\text { Flufen- } \\
\text { acet } \\
\text { OXA } \\
(\mu \mathrm{g} / \mathrm{L})\end{array}$ & $\begin{array}{l}\text { Meto- } \\
\text { lachlor } \\
(\mu \mathrm{g} / \mathrm{L})\end{array}$ & $\begin{array}{c}\text { Meto- } \\
\text { lachlor } \\
\text { ESA } \\
(\mu \mathrm{g} / \mathrm{L})\end{array}$ & $\begin{array}{c}\text { Meto- } \\
\text { lachlor } \\
\text { OXA } \\
(\mu \mathrm{g} / \mathrm{L})\end{array}$ \\
\hline \multicolumn{20}{|c|}{ Federal-State Cooperative Studies-Continued } \\
\hline \multicolumn{20}{|c|}{ Minnesota Pollution Control Agency (JMM) —Continued } \\
\hline 7431 & Stearns Co., MN & 06/00/99 & $<0.05$ & $<0.20$ & $<0.20$ & -- & $<0.05$ & $<0.20$ & 0.29 & -- & $<0.05$ & -- & -- & $<0.05$ & -- & -- & $<0.05$ & $<0.20$ & 3.5 \\
\hline 7433 & Stearns Co., MN & 06/00/99 & $<.05$ & $<.20$ & $<.20$ & -- & $<.05$ & $<.20$ & .56 & -- & $<.05$ & -- & -- & $<.05$ & -- & -- & $<.05$ & .63 & 6.9 \\
\hline \multicolumn{20}{|c|}{ Nebraska Platte River study (IVL) } \\
\hline \multirow[t]{10}{*}{410322096191701} & Sarpy Co., NE & 05/27/96 & $<.05$ & $<.20$ & $<.20$ & -- & $<.05$ & $<.20$ & $<.20$ & -- & -- & -- & -- & -- & -- & -- & .07 & $<.20$ & $<.20$ \\
\hline & & $05 / 28 / 96$ & .17 & $<.20$ & $<.20$ & -- & .05 & $<.20$ & $<.20$ & -- & -- & -- & -- & -- & -- & -- & .36 & $<.20$ & $<.20$ \\
\hline & & 06/03/96 & 1.2 & $<.20$ & $<.20$ & -- & .48 & $<.20$ & $<.20$ & -- & -- & -- & -- & -- & -- & -- & 2.8 & $<.20$ & $<.20$ \\
\hline & & $04 / 14 / 97$ & $<.05$ & $<.20$ & $<.20$ & -- & $<.05$ & $<.20$ & $<.20$ & -- & -- & -- & -- & -- & -- & -- & $<.05$ & .26 & $<.20$ \\
\hline & & 06/01/97 & .19 & $<.20$ & $<.20$ & -- & $<.05$ & $<.20$ & $<.20$ & -- & -- & -- & -- & -- & -- & -- & .41 & .26 & $<.20$ \\
\hline & & $06 / 26 / 97$ & .17 & .34 & .34 & -- & $<.05$ & $<.20$ & $<.20$ & -- & -- & -- & -- & -- & -- & -- & .59 & .28 & .23 \\
\hline & & 08/04/97 & $<.05$ & $<.20$ & $<.20$ & -- & $<.05$ & $<.20$ & $<.20$ & -- & -- & -- & -- & -- & -- & -- & .12 & .24 & $<.20$ \\
\hline & & 05/04/98 & $<.05$ & $<.20$ & $<.20$ & -- & $<.05$ & .22 & $<.20$ & -- & -- & -- & -- & -- & -- & -- & .08 & .42 & $<.20$ \\
\hline & & $05 / 20 / 98$ & .20 & $<.20$ & $<.20$ & -- & $<.05$ & .25 & $<.20$ & -- & -- & -- & -- & -- & -- & -- & 69 & .39 & $<.20$ \\
\hline & & $05 / 21 / 98$ & .19 & $<.20$ & $<.20$ & -- & $<.05$ & .25 & $<.20$ & -- & -- & -- & -- & -- & -- & -- & .52 & .39 & $<.20$ \\
\hline \multirow[t]{5}{*}{410322096191701} & Sarpy Co., NE & 05/30/98 & .37 & .38 & .49 & -- & .09 & .34 & .25 & -- & -- & -- & -- & -- & -- & -- & 1.7 & .44 & .27 \\
\hline & & $05 / 31 / 98$ & .33 & .30 & .39 & -- & .09 & .34 & .21 & -- & -- & -- & -- & -- & -- & -- & 1.8 & .45 & .29 \\
\hline & & 06/01/98 & .09 & $<.20$ & $<.20$ & -- & $<.05$ & $<.20$ & $<.20$ & -- & -- & -- & -- & -- & -- & -- & .33 & $<.20$ & $<.20$ \\
\hline & & 06/02/98 & $<.05$ & .42 & .48 & -- & $<.05$ & .43 & .26 & -- & -- & -- & -- & -- & -- & -- & $<.05$ & .60 & .47 \\
\hline & & 06/03/98 & .18 & .46 & .51 & -- & $<.05$ & .48 & .29 & -- & -- & -- & -- & -- & -- & -- & 1.4 & .62 & .48 \\
\hline \multirow[t]{4}{*}{410254096185501} & Sarpy Co., NE & 06/16/95 & .13 & $<.20$ & $<.20$ & -- & .08 & $<.20$ & $<.20$ & -- & -- & -- & -- & -- & -- & -- & .59 & $<.20$ & $<.20$ \\
\hline & & $04 / 14 / 97$ & $<.05$ & $<.20$ & $<.20$ & -- & $<.05$ & .20 & $<.20$ & -- & -- & -- & -- & -- & -- & -- & .10 & .27 & $<.20$ \\
\hline & & 06/01/97 & .46 & $<.20$ & .37 & -- & .11 & .24 & .21 & -- & -- & -- & -- & -- & -- & -- & 1.2 & .32 & .24 \\
\hline & & $06 / 02 / 97$ & $<.05$ & $<.20$ & $<.20$ & -- & $<.05$ & $<.20$ & $<.20$ & -- & -- & -- & -- & -- & -- & -- & .14 & $<.20$ & .35 \\
\hline
\end{tabular}




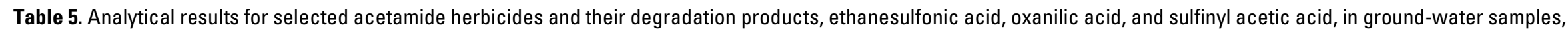
1994-2002.-Continued

[OGRL, U.S. Geological Survey Organic Geochemistry Research Laboratory, Lawrence, Kansas; ESA, ethanesulfonic acid; OXA, oxanilic acid; SAA, sulfinyl acetic acid; --, no data; <, less than]

\begin{tabular}{|c|c|c|c|c|c|c|c|c|c|c|c|c|c|c|c|c|c|c|c|}
\hline Site number & Site location & $\begin{array}{c}\text { Date of } \\
\text { collection } \\
\text { (month/day/ } \\
\text { year) }\end{array}$ & $\begin{array}{c}\text { Aceto- } \\
\text { chlor } \\
(\mu \mathrm{g} / \mathrm{L})\end{array}$ & $\begin{array}{l}\text { Aceto- } \\
\text { chlor } \\
\text { ESA } \\
(\mu \mathrm{g} / \mathrm{L})\end{array}$ & $\begin{array}{l}\text { Aceto- } \\
\text { chlor } \\
\text { OXA } \\
(\mu \mathrm{g} / \mathrm{L})\end{array}$ & $\begin{array}{l}\text { Aceto- } \\
\text { chlor } \\
\text { SAA } \\
(\mu \mathrm{g} / \mathrm{L})\end{array}$ & $\begin{array}{l}\text { Ala- } \\
\text { chlor } \\
(\mu \mathrm{g} / \mathrm{L})\end{array}$ & $\begin{array}{l}\text { Ala- } \\
\text { chlor } \\
\text { ESA } \\
(\mu \mathrm{g} / \mathrm{L})\end{array}$ & $\begin{array}{l}\text { Ala- } \\
\text { chlor } \\
\text { OXA } \\
(\mu \mathrm{g} / \mathrm{L})\end{array}$ & $\begin{array}{l}\text { Ala- } \\
\text { chlor } \\
\text { SAA } \\
(\mu \mathrm{g} / \mathrm{L})\end{array}$ & $\begin{array}{c}\text { Di- } \\
\text { meth- } \\
\text { enamid } \\
(\mu \mathrm{g} / \mathrm{L})\end{array}$ & $\begin{array}{c}\text { Di- } \\
\text { meth- } \\
\text { enamid } \\
\text { ESA } \\
(\mu \mathrm{g} / \mathrm{L})\end{array}$ & $\begin{array}{c}\text { Di- } \\
\text { meth- } \\
\text { enamid } \\
\text { OXA } \\
(\mu \mathrm{g} / \mathrm{L})\end{array}$ & $\begin{array}{c}\text { Flufen- } \\
\text { acet } \\
(\mu \mathrm{g} / \mathrm{L})\end{array}$ & $\begin{array}{c}\text { Flufen- } \\
\text { acet } \\
\text { ESA } \\
(\mu \mathrm{g} / \mathrm{L})\end{array}$ & $\begin{array}{c}\text { Flufen- } \\
\text { acet } \\
\text { OXA } \\
(\mu \mathrm{g} / \mathrm{L})\end{array}$ & $\begin{array}{l}\text { Meto- } \\
\text { lachlor } \\
(\mu \mathrm{g} / \mathrm{L})\end{array}$ & $\begin{array}{l}\text { Meto- } \\
\text { lachlor } \\
\text { ESA } \\
(\mu \mathrm{g} / \mathrm{L})\end{array}$ & $\begin{array}{c}\text { Meto- } \\
\text { lachlor } \\
\text { OXA } \\
(\mu \mathrm{g} / \mathrm{L})\end{array}$ \\
\hline \multicolumn{20}{|c|}{ Federal-State Cooperative Studies-Continued } \\
\hline \multicolumn{20}{|c|}{ Nebraska Platte River study (IVL)_Continued } \\
\hline \multirow[t]{15}{*}{410254096185501} & \multirow[t]{14}{*}{ Sarpy Co., NE } & $06 / 26 / 97$ & 0.49 & $<0.20$ & $<0.20$ & -- & 0.10 & $<0.20$ & $<0.20$ & -- & -- & -- & -- & -- & -- & -- & 1.3 & $<0.20$ & $<0.20$ \\
\hline & & 08/04/97 & .06 & $<.20$ & .39 & -- & $<.05$ & $<.20$ & .23 & -- & -- & -- & -- & -- & -- & -- & .50 & .22 & .27 \\
\hline & & $05 / 04 / 98$ & $<.05$ & $<.20$ & $<.20$ & -- & $<.05$ & .21 & $<.20$ & -- & -- & -- & -- & -- & -- & -- & .11 & .46 & $<.20$ \\
\hline & & $05 / 20 / 98$ & .19 & $<.20$ & $<.20$ & -- & $<.05$ & .24 & $<.20$ & -- & -- & -- & -- & -- & -- & -- & .69 & .41 & $<.20$ \\
\hline & & $05 / 21 / 98$ & .16 & $<.20$ & $<.20$ & -- & $<.05$ & .29 & $<.20$ & -- & -- & -- & -- & -- & -- & -- & .48 & .51 & $<.20$ \\
\hline & & $05 / 21 / 98$ & .05 & $<.20$ & $<.20$ & -- & $<.05$ & $<.20$ & $<.20$ & -- & -- & -- & -- & -- & -- & -- & .13 & $<.20$ & $<.20$ \\
\hline & & $05 / 22 / 98$ & .23 & $<.20$ & $<.20$ & -- & $<.05$ & .21 & $<.20$ & -- & -- & -- & -- & -- & -- & -- & .64 & .33 & $<.20$ \\
\hline & & $05 / 23 / 98$ & .15 & $<.20$ & $<.20$ & -- & $<.05$ & .20 & $<.20$ & -- & -- & -- & -- & -- & -- & -- & .64 & .29 & $<.20$ \\
\hline & & $05 / 24 / 98$ & .14 & $<.20$ & $<.20$ & -- & $<.05$ & .21 & $<.20$ & -- & -- & -- & -- & -- & -- & -- & .70 & .35 & $<.20$ \\
\hline & & $05 / 30 / 98$ & .35 & .25 & .43 & -- & .08 & .32 & .21 & -- & -- & -- & -- & -- & -- & -- & 1.6 & .39 & .24 \\
\hline & & $05 / 31 / 98$ & .06 & $<.20$ & $<.20$ & -- & $<.05$ & $<.20$ & $<.20$ & -- & -- & -- & -- & -- & -- & -- & .29 & $<.20$ & $<.20$ \\
\hline & & $06 / 01 / 98$ & .29 & $<.20$ & $<.20$ & -- & $<.05$ & $<.20$ & $<.20$ & -- & -- & -- & -- & -- & -- & -- & .28 & $<.20$ & $<.20$ \\
\hline & & 06/03/98 & .16 & $<.20$ & $<.20$ & -- & $<.05$ & $<.20$ & $<.20$ & -- & -- & -- & -- & -- & -- & -- & 1.2 & $<.20$ & $<.20$ \\
\hline & & 06/09/99 & 1.0 & .51 & 1.3 & -- & .24 & .27 & .39 & -- & -- & -- & -- & -- & -- & -- & 2.1 & .43 & .42 \\
\hline & \multicolumn{19}{|c|}{ New Jersey public supply wells (ABN/PSG) } \\
\hline \multirow[t]{5}{*}{393104075122201} & \multirow[t]{5}{*}{ Cumberland Co., NJ } & $06 / 12 / 97$ & $<.05$ & $<.20$ & $<.20$ & -- & $<.05$ & .45 & $<.20$ & -- & -- & -- & -- & -- & -- & -- & .99 & 2.6 & .70 \\
\hline & & $06 / 12 / 97$ & $<.05$ & $<.20$ & $<.20$ & -- & $<.05$ & .53 & $<.20$ & -- & -- & -- & -- & -- & -- & -- & .98 & 3.0 & .81 \\
\hline & & $06 / 24 / 97$ & $<.05$ & $<.20$ & $<.20$ & -- & $<.05$ & .40 & $<.20$ & -- & -- & -- & -- & -- & -- & -- & .79 & 3.4 & .86 \\
\hline & & $06 / 25 / 97$ & $<.05$ & $<.20$ & $<.20$ & -- & $<.05$ & $<.20$ & $<.20$ & -- & -- & -- & -- & -- & -- & -- & $<.05$ & $<.20$ & $<.20$ \\
\hline & & $09 / 04 / 97$ & $<.05$ & $<.20$ & $<.20$ & -- & $<.05$ & .99 & .37 & -- & -- & -- & -- & -- & -- & -- & .08 & 5.7 & 1.6 \\
\hline 393104075122204 & Cumberland Co., NJ & $09 / 04 / 97$ & $<.05$ & $<.20$ & $<.20$ & -- & $<.05$ & .65 & $<.20$ & -- & -- & -- & -- & -- & -- & -- & .89 & 9.4 & 2.0 \\
\hline 393532075101201 & Salem Co., NJ & $06 / 10 / 97$ & $<.05$ & $<.20$ & $<.20$ & -- & $<.05$ & $<.20$ & $<.20$ & -- & -- & -- & -- & -- & -- & -- & $<.05$ & $<.20$ & $<.20$ \\
\hline 393946074582901 & Gloucester Co., NJ & $05 / 20 / 97$ & $<.05$ & $<.20$ & $<.20$ & -- & $<.05$ & $<.20$ & $<.20$ & -- & -- & -- & -- & -- & -- & -- & $<.05$ & $<.20$ & $<.20$ \\
\hline \multirow[t]{2}{*}{394243075051401} & Cumberland Co., NJ & $05 / 28 / 97$ & $<.05$ & $<.20$ & $<.20$ & -- & $<.05$ & $<.20$ & $<.20$ & -- & -- & -- & -- & -- & -- & -- & $<.05$ & $<.20$ & $<.20$ \\
\hline & & $05 / 28 / 97$ & $<.05$ & $<.20$ & $<.20$ & -- & $<.05$ & $<.20$ & $<.20$ & -- & -- & -- & -- & -- & -- & -- & $<.05$ & $<.20$ & $<.20$ \\
\hline
\end{tabular}


Table 5. Analytical results for selected acetamide herbicides and their degradation products, ethanesulfonic acid, oxanilic acid, and sulfinyl acetic acid, in ground-water samples, 1994-2002.-Continued

[OGRL, U.S. Geological Survey Organic Geochemistry Research Laboratory, Lawrence, Kansas; ESA, ethanesulfonic acid; OXA, oxanilic acid; SAA, sulfinyl acetic acid; --, no data; <, less than]

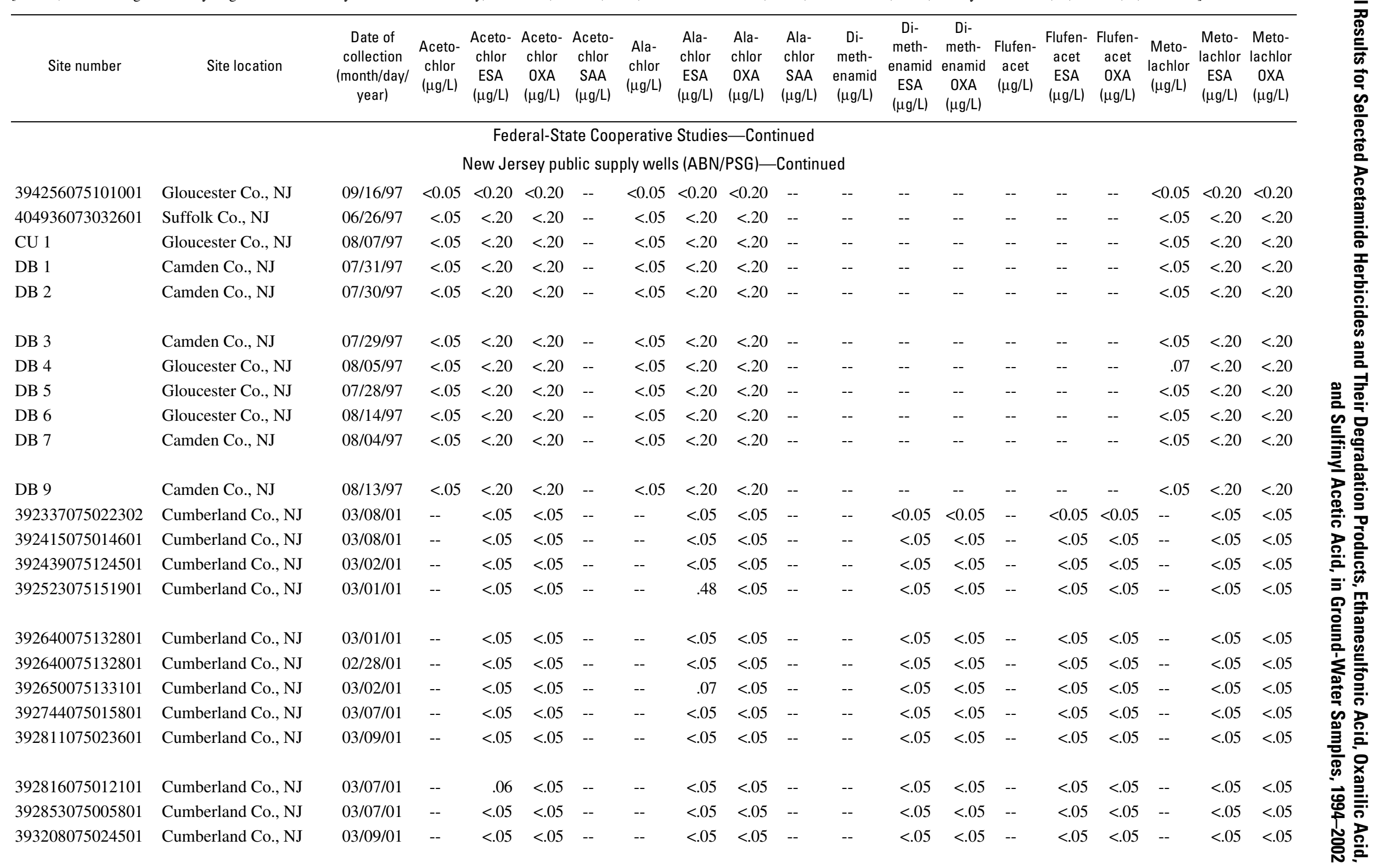




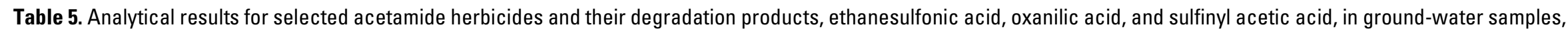
1994-2002.-Continued

[OGRL, U.S. Geological Survey Organic Geochemistry Research Laboratory, Lawrence, Kansas; ESA, ethanesulfonic acid; OXA, oxanilic acid; SAA, sulfinyl acetic acid; --, no data; <, less than]

\begin{tabular}{|c|c|c|c|c|c|c|c|c|c|c|c|c|c|c|c|c|c|c|c|}
\hline Site number & Site location & $\begin{array}{c}\text { Date of } \\
\text { collection } \\
\text { (month/day/ } \\
\text { year) }\end{array}$ & $\begin{array}{c}\text { Aceto- } \\
\text { chlor } \\
(\mu \mathrm{g} / \mathrm{L})\end{array}$ & $\begin{array}{c}\text { Aceto- } \\
\text { chlor } \\
\text { ESA } \\
(\mu \mathrm{g} / \mathrm{L})\end{array}$ & $\begin{array}{c}\text { Aceto- } \\
\text { chlor } \\
\text { OXA } \\
(\mu \mathrm{g} / \mathrm{L})\end{array}$ & $\begin{array}{l}\text { Aceto- } \\
\text { chlor } \\
\text { SAA } \\
(\mu \mathrm{g} / \mathrm{L})\end{array}$ & $\begin{array}{l}\text { Ala- } \\
\text { chlor } \\
(\mu \mathrm{g} / \mathrm{L})\end{array}$ & $\begin{array}{l}\text { Ala- } \\
\text { chlor } \\
\text { ESA } \\
(\mu \mathrm{g} / \mathrm{L})\end{array}$ & $\begin{array}{l}\text { Ala- } \\
\text { chlor } \\
\text { OXA } \\
(\mu \mathrm{g} / \mathrm{L})\end{array}$ & $\begin{array}{c}\text { Ala- } \\
\text { chlor } \\
\text { SAA } \\
(\mu \mathrm{g} / \mathrm{L})\end{array}$ & $\begin{array}{l}\text { Di- } \\
\text { meth- } \\
\text { enamid } \\
(\mu \mathrm{g} / \mathrm{L})\end{array}$ & $\begin{array}{c}\text { Di- } \\
\text { meth- } \\
\text { enamid } \\
\text { ESA } \\
(\mu \mathrm{g} / \mathrm{L})\end{array}$ & $\begin{array}{c}\text { Di- } \\
\text { meth- } \\
\text { enamid } \\
\text { OXA } \\
(\mu \mathrm{g} / \mathrm{L})\end{array}$ & $\begin{array}{l}\text { Flufen- } \\
\text { acet } \\
(\mu \mathrm{g} / \mathrm{L})\end{array}$ & $\begin{array}{c}\text { Flufen- } \\
\text { acet } \\
\text { ESA } \\
(\mu \mathrm{g} / \mathrm{L})\end{array}$ & $\begin{array}{c}\text { Flufen- } \\
\text { acet } \\
\text { OXA } \\
(\mu \mathrm{g} / \mathrm{L})\end{array}$ & $\begin{array}{l}\text { Meto- } \\
\text { lachlor } \\
(\mu \mathrm{g} / \mathrm{L})\end{array}$ & $\begin{array}{l}\text { Meto- } \\
\text { lachlor } \\
\text { ESA } \\
(\mu \mathrm{g} / \mathrm{L})\end{array}$ & $\begin{array}{c}\text { Meto- } \\
\text { lachlor } \\
\text { OXA } \\
(\mu \mathrm{g} / \mathrm{L})\end{array}$ \\
\hline \multicolumn{20}{|c|}{ Federal-State Cooperative Studies-Continued } \\
\hline \multicolumn{20}{|c|}{ Long Island, New York hydrologic study (LIN) } \\
\hline 403920073353001 & Nassau Co., NY & 08/02/99 & $<0.05$ & $<0.20$ & $<0.20$ & -- & $<0.05$ & $<0.20$ & $<0.20$ & -- & $<0.05$ & -- & -- & $<0.05$ & -- & -- & $<0.05$ & $<0.20$ & $<0.20$ \\
\hline 404016073371901 & Nassau Co., NY & 07/26/99 & $<.05$ & $<.20$ & $<.20$ & -- & $<.05$ & $<.20$ & $<.20$ & -- & $<.05$ & -- & -- & $<.05$ & -- & -- & $<.05$ & $<.20$ & $<.20$ \\
\hline 404131073211301 & Suffolk Co., NY & 06/11/98 & $<.05$ & $<.20$ & $<.20$ & -- & $<.05$ & $<.20$ & $<.20$ & -- & -- & -- & -- & -- & -- & -- & $<.05$ & $<.20$ & $<.20$ \\
\hline 404132073383302 & Nassau Co., NY & 08/05/99 & $<.05$ & $<.20$ & $<.20$ & -- & $<.05$ & $<.20$ & $<.20$ & -- & $<.05$ & -- & -- & $<.05$ & -- & -- & $<.05$ & $<.20$ & $<.20$ \\
\hline 404149073383201 & Nassau Co., NY & 07/26/99 & $<.05$ & $<.20$ & $<.20$ & -- & $<.05$ & $<.20$ & $<.20$ & -- & $<.05$ & -- & -- & $<.05$ & -- & -- & $<.05$ & $<.20$ & $<.20$ \\
\hline 404158073225802 & Suffolk Co., NY & 06/11/98 & $<.05$ & $<.20$ & $<.20$ & -- & $<.05$ & $<.20$ & $<.20$ & -- & -- & -- & -- & -- & -- & -- & $<.05$ & $<.20$ & $<.20$ \\
\hline 404237073420401 & Nassau Co., NY & 08/12/99 & $<.05$ & $<.20$ & $<.20$ & -- & $<.05$ & $<.20$ & $<.20$ & -- & $<.05$ & -- & -- & $<.05$ & -- & -- & $<.05$ & $<.20$ & $<.20$ \\
\hline 404318073055001 & Suffolk Co., NY & 07/22/99 & $<.05$ & $<.20$ & $<.20$ & -- & $<.05$ & $<.20$ & $<.20$ & -- & $<.05$ & -- & -- & $<.05$ & -- & -- & $<.05$ & $<.20$ & $<.20$ \\
\hline 404319073055001 & Suffolk Co., NY & $07 / 22 / 99$ & $<.05$ & $<.20$ & $<.20$ & -- & $<.05$ & $<.20$ & $<.20$ & -- & $<.05$ & -- & -- & $<.05$ & -- & -- & $<.05$ & $<.20$ & $<.20$ \\
\hline \multirow[t]{2}{*}{404319073055101} & Suffolk Co., NY & $06 / 23 / 98$ & $<.05$ & $<.20$ & $<.20$ & -- & $<.05$ & $<.20$ & $<.20$ & -- & -- & -- & -- & -- & -- & -- & $<.05$ & $<.20$ & $<.20$ \\
\hline & & 07/22/99 & $<.05$ & $<.20$ & $<.20$ & -- & $<.05$ & $<.20$ & $<.20$ & -- & $<.05$ & -- & -- & $<.05$ & -- & -- & $<.05$ & $<.20$ & $<.20$ \\
\hline 404319073055102 & Suffolk Co., NY & 07/22/99 & $<.05$ & $<.20$ & $<.20$ & -- & $<.05$ & $<.20$ & $<.20$ & -- & $<.05$ & -- & -- & $<.05$ & -- & -- & $<.05$ & $<.20$ & $<.20$ \\
\hline 404320073305602 & Nassau Co., NY & 07/26/99 & $<.05$ & $<.20$ & $<.20$ & -- & $<.05$ & $<.20$ & $<.20$ & -- & $<.05$ & -- & -- & $<.05$ & -- & -- & $<.05$ & $<.20$ & $<.20$ \\
\hline 404339073090501 & Suffolk Co., NY & 07/29/99 & $<.05$ & $<.20$ & $<.20$ & -- & $<.05$ & $<.20$ & $<.20$ & -- & $<.05$ & -- & -- & $<.05$ & -- & -- & $<.05$ & $<.20$ & $<.20$ \\
\hline 404339073090502 & Suffolk Co., NY & 07/29/99 & $<.05$ & $<.20$ & $<.20$ & -- & $<.05$ & $<.20$ & $<.20$ & -- & $<.05$ & -- & -- & $<.05$ & -- & -- & $<.05$ & $<.20$ & $<.20$ \\
\hline 404339073090601 & Suffolk Co., NY & 07/29/99 & $<.05$ & $<.20$ & $<.20$ & -- & $<.05$ & $<.20$ & $<.20$ & -- & $<.05$ & -- & -- & $<.05$ & -- & -- & $<.05$ & $<.20$ & $<.20$ \\
\hline 404339073090602 & Suffolk Co., NY & $11 / 30 / 99$ & $<.05$ & $<.20$ & $<.20$ & -- & $<.05$ & $<.20$ & $<.20$ & -- & $<.05$ & -- & -- & $<.05$ & -- & -- & $<.05$ & $<.20$ & .27 \\
\hline 404339073090901 & Suffolk Co., NY & $06 / 23 / 98$ & $<.05$ & $<.20$ & $<.20$ & -- & $<.05$ & $<.20$ & $<.20$ & -- & -- & -- & -- & -- & -- & -- & $<.05$ & $<.20$ & $<.20$ \\
\hline 404340073090401 & Suffolk Co., NY & 07/29/99 & $<.05$ & $<.20$ & $<.20$ & -- & $<.05$ & $<.20$ & $<.20$ & -- & $<.05$ & -- & -- & $<.05$ & -- & -- & $<.05$ & $<.20$ & $<.20$ \\
\hline \multirow[t]{2}{*}{404345073273101} & Nassau Co., NY & 07/20/99 & $<.05$ & .31 & .59 & -- & $<.05$ & .26 & $<.20$ & -- & $<.05$ & -- & -- & $<.05$ & -- & -- & $<.05$ & .56 & .46 \\
\hline & & $11 / 01 / 99$ & $<.05$ & $<.20$ & $<.20$ & -- & $<.05$ & $<.20$ & $<.20$ & -- & $<.05$ & -- & -- & $<.05$ & -- & -- & $<.05$ & $<.20$ & $<.20$ \\
\hline \multirow[t]{2}{*}{404418073095001} & Suffolk Co., NY & $06 / 23 / 98$ & $<.05$ & $<.20$ & $<.20$ & -- & $<.05$ & $<.20$ & $<.20$ & -- & -- & -- & -- & -- & -- & -- & $<.05$ & $<.20$ & $<.20$ \\
\hline & & 09/23/99 & $<.05$ & $<.20$ & $<.20$ & -- & $<.05$ & $<.20$ & $<.20$ & -- & $<.05$ & -- & -- & $<.05$ & -- & -- & $<.05$ & $<.20$ & $<.20$ \\
\hline 404433073244905 & Suffolk Co., NY & $06 / 11 / 98$ & $<.05$ & $<.20$ & $<.20$ & -- & $<.05$ & $<.20$ & $<.20$ & -- & -- & -- & -- & -- & -- & -- & $<.05$ & $<.20$ & $<.20$ \\
\hline 404442073240503 & Suffolk Co., NY & $12 / 02 / 99$ & $<.05$ & $<.20$ & $<.20$ & -- & $<.05$ & $<.20$ & 2.5 & -- & $<.05$ & -- & -- & $<.05$ & -- & -- & $<.05$ & $<.20$ & $<.20$ \\
\hline
\end{tabular}


Table 5. Analytical results for selected acetamide herbicides and their degradation products, ethanesulfonic acid, oxanilic acid, and sulfinyl acetic acid, in ground-water samples, 1994-2002.-Continued

[OGRL, U.S. Geological Survey Organic Geochemistry Research Laboratory, Lawrence, Kansas; ESA, ethanesulfonic acid; OXA, oxanilic acid; SAA, sulfinyl acetic acid; --, no data; <, less than]

\begin{tabular}{|c|c|c|c|c|c|c|c|c|c|c|c|c|c|c|c|c|c|c|c|}
\hline Site number & Site location & $\begin{array}{c}\text { Date of } \\
\text { collection } \\
\text { (month/day/ } \\
\text { year) }\end{array}$ & $\begin{array}{c}\text { Aceto- } \\
\text { chlor } \\
(\mu \mathrm{g} / \mathrm{L})\end{array}$ & $\begin{array}{l}\text { Aceto- } \\
\text { chlor } \\
\text { ESA } \\
(\mu \mathrm{g} / \mathrm{L})\end{array}$ & $\begin{array}{c}\text { Aceto- } \\
\text { chlor } \\
\text { OXA } \\
(\mu \mathrm{g} / \mathrm{L})\end{array}$ & $\begin{array}{c}\text { Aceto- } \\
\text { chlor } \\
\text { SAA } \\
(\mu \mathrm{g} / \mathrm{L})\end{array}$ & $\begin{array}{l}\text { Ala- } \\
\text { chlor } \\
(\mu \mathrm{g} / \mathrm{L})\end{array}$ & $\begin{array}{l}\text { Ala- } \\
\text { chlor } \\
\text { ESA } \\
(\mu \mathrm{g} / \mathrm{L})\end{array}$ & $\begin{array}{c}\text { Ala- } \\
\text { chlor } \\
\text { OXA } \\
(\mu \mathrm{g} / \mathrm{L})\end{array}$ & $\begin{array}{l}\text { Ala- } \\
\text { chlor } \\
\text { SAA } \\
(\mu \mathrm{g} / \mathrm{L})\end{array}$ & $\begin{array}{c}\text { Di- } \\
\text { meth- } \\
\text { enamid } \\
(\mu \mathrm{g} / \mathrm{L})\end{array}$ & $\begin{array}{c}\text { Di- } \\
\text { meth- } \\
\text { enamid } \\
\text { ESA } \\
(\mu \mathrm{g} / \mathrm{L})\end{array}$ & $\begin{array}{c}\text { Di- } \\
\text { meth- } \\
\text { enamid } \\
\text { OXA } \\
(\mu \mathrm{g} / \mathrm{L})\end{array}$ & $\begin{array}{c}\text { Flufen- } \\
\text { acet } \\
(\mu \mathrm{g} / \mathrm{L})\end{array}$ & $\begin{array}{c}\text { Flufen- } \\
\text { acet } \\
\text { ESA } \\
(\mu \mathrm{g} / \mathrm{L})\end{array}$ & $\begin{array}{c}\text { Flufen- } \\
\text { acet } \\
\text { OXA } \\
(\mu \mathrm{g} / \mathrm{L})\end{array}$ & $\begin{array}{c}\text { Meto- } \\
\text { lachlor } \\
(\mu \mathrm{g} / \mathrm{L})\end{array}$ & $\begin{array}{c}\text { Meto- } \\
\text { lachlor } \\
\text { ESA } \\
(\mu \mathrm{g} / \mathrm{L})\end{array}$ & $\begin{array}{c}\text { Meto- } \\
\text { lachlor } \\
\text { OXA } \\
(\mu \mathrm{g} / \mathrm{L})\end{array}$ \\
\hline
\end{tabular}

\section{Federal-State Cooperative Studies-Continued}

404532073420901 Nassau Co., NY 404534073163101 Suffolk Co., NY 404555073240501 Suffolk Co., NY 404620073383401 Nassau Co., NY 404707073234201 Suffolk Co., NY

404716073131602 Suffolk Co., NY 404717073201301 Suffolk Co., NY 404719073205701 Suffolk Co., NY 404739073392101 Nassau Co., NY 404804073411301 Nassau Co., NY

404832073333203 Nassau Co., NY 404900072451701 Suffolk Co., NY

404908073278102 Suffolk Co., NY 404922072451701 Suffolk Co., NY 404922073292501 Nassau Co., NY

404936073032601 Suffolk Co., NY 404945073174501 Suffolk Co., NY 404953073170501 Suffolk Co., NY

040495307317050 Suffolk Co., NY 404953073170503 Suffolk Co., NY

404955073170401 Suffolk Co., NY 404955073170403 Suffolk Co., NY

\section{Long Island, New York hydrologic study (LIN) —Continued}

\begin{tabular}{|c|c|c|c|c|c|c|c|c|c|c|c|c|c|c|c|c|c|}
\hline 07/28/99 & $<0.05$ & $<0.20$ & $<0.20$ & -- & $<0.05$ & $<0.20$ & $<0.20$ & -- & $<0.05$ & -- & -- & $<0.05$ & -- & -- & $<0.05$ & $<0.20$ & $<0.20$ \\
\hline 09/14/99 & $<.05$ & $<.20$ & $<.20$ & -- & $<.05$ & $<.20$ & $<.20$ & -- & $<.05$ & -- & -- & $<.05$ & -- & -- & $<.05$ & $<.20$ & $<.20$ \\
\hline 07/06/98 & $<.05$ & $<.20$ & $<.20$ & -- & $<.05$ & $<.20$ & $<.20$ & -- & -- & -- & -- & -- & -- & -- & $<.05$ & $<.20$ & $<.20$ \\
\hline 07/27/99 & $<.05$ & $<.20$ & $<.20$ & -- & $<.05$ & $<.20$ & $<.20$ & -- & $<.05$ & -- & -- & $<.05$ & -- & -- & $<.05$ & $<.20$ & $<.20$ \\
\hline 05/19/98 & $<.05$ & $<.20$ & $<.20$ & -- & $<.05$ & $<.20$ & $<.20$ & -- & -- & -- & -- & -- & -- & -- & $<.05$ & $<.20$ & $<.20$ \\
\hline 06/16/98 & $<.05$ & $<.20$ & $<.20$ & -- & $<.05$ & $<.20$ & $<.20$ & -- & -- & -- & -- & -- & -- & -- & $<.05$ & $<.20$ & $<.20$ \\
\hline 05/28/98 & $<.05$ & $<.20$ & $<.20$ & -- & $<.05$ & $<.20$ & $<.20$ & -- & -- & -- & -- & -- & -- & -- & $<.05$ & $<.20$ & $<.20$ \\
\hline 06/03/98 & $<.05$ & $<.20$ & $<.20$ & -- & $<.05$ & .46 & $<.20$ & -- & -- & -- & -- & -- & -- & -- & $<.05$ & $<.20$ & $<.20$ \\
\hline 08/11/99 & $<.05$ & $<.20$ & $<.20$ & -- & $<.05$ & $<.20$ & $<.20$ & -- & $<.05$ & -- & -- & $<.05$ & -- & -- & $<.05$ & $<.20$ & $<.20$ \\
\hline 07/28/99 & $<.05$ & $<.20$ & $<.20$ & -- & $<.05$ & $<.20$ & $<.20$ & -- & $<.05$ & -- & -- & $<.05$ & -- & -- & $<.05$ & $<.20$ & $<.20$ \\
\hline 08/09/99 & $<.05$ & $<.20$ & $<.20$ & -- & $<.05$ & .28 & $<.20$ & -- & $<.05$ & -- & -- & $<.05$ & -- & -- & $<.05$ & $<.20$ & $<.20$ \\
\hline 06/29/98 & $<.05$ & $<.20$ & $<.20$ & -- & .13 & 40 & 23 & -- & -- & -- & -- & -- & -- & -- & $<.05$ & $<.20$ & $<.20$ \\
\hline 09/21/99 & $<.05$ & $<.20$ & $<.20$ & -- & $<.05$ & $<.20$ & $<.20$ & -- & $<.05$ & -- & -- & $<.05$ & -- & -- & $<.05$ & $<.20$ & $<.20$ \\
\hline 07/23/99 & $<.05$ & $<.20$ & $<.20$ & -- & $<.05$ & $<.20$ & $<.20$ & -- & $<.05$ & -- & -- & $<.05$ & -- & -- & $<.05$ & $<.20$ & $<.20$ \\
\hline 06/29/98 & $<.05$ & $<.20$ & $<.20$ & -- & $<.05$ & $<.20$ & $<.20$ & -- & -- & -- & -- & -- & -- & -- & $<.05$ & 2.5 & 1.7 \\
\hline 07/23/99 & $<.05$ & $<.20$ & $<.20$ & -- & $<.05$ & $<.20$ & $<.20$ & -- & $<.05$ & -- & -- & $<.05$ & -- & -- & $<.05$ & $<.20$ & $<.20$ \\
\hline 06/30/98 & $<.05$ & $<.20$ & $<.20$ & -- & $<.05$ & $<.20$ & $<.20$ & -- & -- & -- & -- & -- & -- & -- & $<.05$ & $<.20$ & $<.20$ \\
\hline 06/03/98 & $<.05$ & $<.20$ & $<.20$ & -- & $<.05$ & $<.20$ & $<.20$ & -- & -- & -- & -- & -- & -- & -- & $<.05$ & $<.20$ & $<.20$ \\
\hline 06/18/98 & $<.05$ & $<.20$ & $<.20$ & -- & $<.05$ & $<.20$ & $<.20$ & -- & -- & -- & -- & -- & -- & -- & $<.05$ & $<.20$ & $<.20$ \\
\hline 09/14/99 & $<.05$ & $<.20$ & $<.20$ & -- & $<.05$ & $<.20$ & $<.20$ & -- & $<.05$ & -- & -- & $<.05$ & -- & -- & $<.05$ & $<.20$ & $<.20$ \\
\hline 06/18/98 & $<.05$ & $<.20$ & $<.20$ & -- & $<.05$ & $<.20$ & $<.20$ & -- & -- & -- & -- & -- & -- & -- & $<.05$ & $<.20$ & $<.20$ \\
\hline 06/18/98 & $<.05$ & $<.20$ & $<.20$ & -- & $<.05$ & $<.20$ & $<.20$ & -- & -- & -- & -- & -- & -- & -- & $<.05$ & $<.20$ & $<.20$ \\
\hline 08/24/99 & $<.05$ & $<.20$ & $<.20$ & -- & $<.05$ & $<.20$ & $<.20$ & -- & $<.05$ & -- & -- & $<.05$ & -- & -- & $<.05$ & $<.20$ & .22 \\
\hline 02/02/99 & $<.05$ & $<.20$ & $<.20$ & -- & $<.05$ & $<.20$ & $<.20$ & -- & -- & -- & -- & -- & -- & -- & $<.05$ & $<.20$ & $<.20$ \\
\hline 08/24/99 & $<.05$ & $<.20$ & $<.20$ & -- & $<.05$ & $<.20$ & $<.20$ & -- & $<.05$ & -- & -- & $<.05$ & -- & -- & $<.05$ & $<.20$ & $<.20$ \\
\hline
\end{tabular}




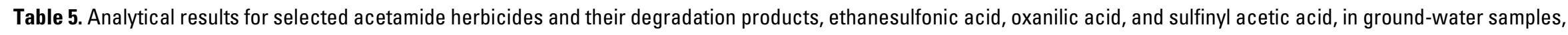
1994-2002.-Continued

[OGRL, U.S. Geological Survey Organic Geochemistry Research Laboratory, Lawrence, Kansas; ESA, ethanesulfonic acid; OXA, oxanilic acid; SAA, sulfinyl acetic acid; --, no data; <, less than]

\begin{tabular}{|c|c|c|c|c|c|c|c|c|c|c|c|c|c|c|c|c|c|c|c|}
\hline Site number & Site location & $\begin{array}{c}\text { Date of } \\
\text { collection } \\
\text { (month/day/ } \\
\text { year) }\end{array}$ & $\begin{array}{l}\text { Aceto- } \\
\text { chlor } \\
(\mu \mathrm{g} / \mathrm{L})\end{array}$ & $\begin{array}{l}\text { Aceto- } \\
\text { chlor } \\
\text { ESA } \\
(\mu \mathrm{g} / \mathrm{L})\end{array}$ & $\begin{array}{l}\text { Aceto- } \\
\text { chlor } \\
\text { OXA } \\
(\mu \mathrm{g} / \mathrm{L})\end{array}$ & $\begin{array}{l}\text { Aceto- } \\
\text { chlor } \\
\text { SAA } \\
(\mu \mathrm{g} / \mathrm{L})\end{array}$ & $\begin{array}{l}\text { Ala- } \\
\text { chlor } \\
(\mu \mathrm{g} / \mathrm{L})\end{array}$ & $\begin{array}{l}\text { Ala- } \\
\text { chlor } \\
\text { ESA } \\
(\mu \mathrm{g} / \mathrm{L})\end{array}$ & $\begin{array}{c}\text { Ala- } \\
\text { chlor } \\
\text { OXA } \\
(\mu \mathrm{g} / \mathrm{L})\end{array}$ & $\begin{array}{l}\text { Ala- } \\
\text { chlor } \\
\text { SAA } \\
(\mu \mathrm{g} / \mathrm{L})\end{array}$ & $\begin{array}{c}\text { Di- } \\
\text { meth- } \\
\text { enamid } \\
(\mu \mathrm{g} / \mathrm{L})\end{array}$ & $\begin{array}{c}\text { Di- } \\
\text { meth- } \\
\text { enamid } \\
\text { ESA } \\
(\mu \mathrm{g} / \mathrm{L})\end{array}$ & $\begin{array}{c}\text { Di- } \\
\text { meth- } \\
\text { enamid } \\
\text { OXA } \\
(\mu \mathrm{g} / \mathrm{L})\end{array}$ & $\begin{array}{c}\text { Flufen- } \\
\text { acet } \\
(\mu \mathrm{g} / \mathrm{L})\end{array}$ & $\begin{array}{c}\text { Flufen- } \\
\text { acet } \\
\text { ESA } \\
(\mu \mathrm{g} / \mathrm{L})\end{array}$ & $\begin{array}{c}\text { Flufen- } \\
\text { acet } \\
\text { OXA } \\
(\mu \mathrm{g} / \mathrm{L})\end{array}$ & $\begin{array}{l}\text { Meto- } \\
\text { lachlor } \\
(\mu \mathrm{g} / \mathrm{L})\end{array}$ & $\begin{array}{c}\text { Meto- } \\
\text { lachlor } \\
\text { ESA } \\
(\mu \mathrm{g} / \mathrm{L})\end{array}$ & $\begin{array}{c}\text { Meto- } \\
\text { lachlor } \\
\text { OXA } \\
(\mu \mathrm{g} / \mathrm{L})\end{array}$ \\
\hline \multicolumn{20}{|c|}{ Federal-State Cooperative Studies-Continued } \\
\hline \multicolumn{20}{|c|}{ Long Island, New York hydrologic study (LIN)—Continued } \\
\hline 404955073170901 & Suffolk Co., NY & $02 / 02 / 99$ & $<0.05$ & $<0.20$ & $<0.20$ & -- & $<0.05$ & $<0.20$ & $<0.20$ & -- & -- & -- & -- & -- & -- & -- & $<0.05$ & $<0.20$ & $<0.20$ \\
\hline \multirow[t]{2}{*}{405005073233701} & Suffolk Co., NY & 06/16/98 & $<.05$ & $<.20$ & $<.20$ & -- & $<.05$ & $<.20$ & $<.20$ & -- & -- & -- & -- & -- & -- & -- & $<.05$ & $<.20$ & $<.20$ \\
\hline & & $09 / 14 / 99$ & $<.05$ & $<.20$ & $<.20$ & -- & $<.05$ & $<.20$ & $<.20$ & -- & $<0.05$ & -- & -- & $<0.05$ & -- & -- & $<.05$ & $<.20$ & $<.20$ \\
\hline 405010073414401 & Nassau Co., NY & 07/28/99 & $<.05$ & $<.20$ & $<.20$ & -- & $<.05$ & $<.20$ & $<.20$ & -- & $<.05$ & -- & -- & $<.05$ & -- & -- & $<.05$ & $<.20$ & $<.20$ \\
\hline 405011073355901 & Nassau Co., NY & 07/27/99 & $<.05$ & $<.20$ & $<.20$ & -- & $<.05$ & $<.20$ & $<.20$ & -- & $<.05$ & -- & -- & $<.05$ & -- & -- & $<.05$ & $<.20$ & $<.20$ \\
\hline 405030073180601 & Suffolk Co., NY & $05 / 19 / 98$ & $<.05$ & $<.20$ & $<.20$ & -- & $<.05$ & $<.20$ & $<.20$ & -- & -- & -- & -- & -- & -- & -- & $<.05$ & $<.20$ & $<.20$ \\
\hline 405111072485401 & Suffolk Co., NY & $06 / 24 / 98$ & $<.05$ & $<.20$ & $<.20$ & -- & $<.05$ & $<.20$ & $<.20$ & -- & -- & -- & -- & -- & -- & -- & $<.05$ & $<.20$ & $<.20$ \\
\hline 405111073065801 & Suffolk Co., NY & 06/17/98 & $<.05$ & $<.20$ & $<.20$ & -- & $<.05$ & $<.20$ & $<.20$ & -- & -- & -- & -- & -- & -- & -- & $<.05$ & $<.20$ & $<.20$ \\
\hline 405122073420401 & Nassau Co., NY & 07/27/99 & $<.05$ & $<.20$ & $<.20$ & -- & $<.05$ & $<.20$ & $<.20$ & -- & $<.05$ & -- & -- & $<.05$ & -- & -- & $<.05$ & $<.20$ & $<.20$ \\
\hline \multirow[t]{2}{*}{405124072353603} & Suffolk Co., NY & $01 / 27 / 99$ & $<.05$ & $<.20$ & $<.20$ & -- & $<.05$ & $<.20$ & $<.20$ & -- & -- & -- & -- & $<.05$ & -- & -- & $<.05$ & $<.20$ & $<.20$ \\
\hline & & $08 / 25 / 99$ & $<.05$ & $<.20$ & $<.20$ & -- & $<.05$ & $<.20$ & $<.20$ & -- & $<.05$ & -- & -- & $<.05$ & -- & -- & $<.05$ & .80 & .28 \\
\hline 405128073370504 & Nassau Co., NY & 07/30/99 & $<.05$ & $<.20$ & $<.20$ & -- & $<.05$ & $<.20$ & $<.20$ & -- & $<.05$ & -- & -- & $<.05$ & -- & -- & $<.05$ & $<.20$ & $<.20$ \\
\hline 405205073363401 & Nassau Co., NY & 07/30/99 & $<.05$ & $<.20$ & $<.20$ & -- & $<.05$ & $<.20$ & $<.20$ & -- & $<.05$ & -- & -- & $<.05$ & -- & -- & $<.05$ & $<.20$ & $<.20$ \\
\hline 405243073102301 & Suffolk Co., NY & 06/25/98 & $<.05$ & $<.20$ & $<.20$ & -- & $<.05$ & $<.20$ & $<.20$ & -- & -- & -- & -- & -- & -- & -- & $<.05$ & $<.20$ & $<.20$ \\
\hline 405259073010301 & Suffolk Co., NY & 06/17/98 & $<.05$ & $<.20$ & $<.20$ & -- & $<.05$ & $<.20$ & $<.20$ & -- & -- & -- & -- & -- & -- & -- & $<.05$ & $<.20$ & $<.20$ \\
\hline 405311072263001 & Suffolk Co., NY & $12 / 08 / 99$ & $<.05$ & $<.20$ & $<.20$ & -- & $<.05$ & $<.20$ & $<.20$ & -- & $<.05$ & -- & -- & $<.05$ & -- & -- & $<.05$ & $<.20$ & $<.20$ \\
\hline 405349072234801 & Suffolk Co., NY & $06 / 04 / 98$ & $<.05$ & $<.20$ & $<.20$ & -- & $<.05$ & $<.20$ & $<.20$ & -- & -- & -- & -- & -- & -- & -- & $<.05$ & $<.20$ & $<.20$ \\
\hline 405400072263901 & Suffolk Co., NY & $12 / 08 / 99$ & $<.05$ & $<.20$ & $<.20$ & -- & $<.05$ & $<.20$ & $<.20$ & -- & $<.05$ & -- & -- & $<.05$ & -- & -- & $<.05$ & $<.20$ & $<.20$ \\
\hline 405411072232701 & Suffolk Co., NY & $01 / 25 / 99$ & $<.05$ & $<.20$ & $<.20$ & -- & $<.05$ & $<.20$ & $<.20$ & -- & -- & -- & -- & -- & -- & -- & $<.05$ & 4.0 & 3.5 \\
\hline 405413072232901 & Suffolk Co., NY & $08 / 25 / 99$ & $<.05$ & $<.20$ & $<.20$ & -- & $<.05$ & $<.20$ & $<.20$ & -- & $<.05$ & -- & -- & $<.05$ & -- & -- & .43 & 3.0 & 2.2 \\
\hline \multirow[t]{2}{*}{405413072232902} & Suffolk Co., NY & $01 / 25 / 99$ & $<.05$ & $<.20$ & $<.20$ & -- & $<.05$ & $<.20$ & $<.20$ & -- & -- & -- & -- & -- & -- & -- & $<.05$ & $<.20$ & $<.20$ \\
\hline & & $08 / 25 / 99$ & $<.05$ & $<.20$ & $<.20$ & -- & $<.05$ & $<.20$ & $<.20$ & -- & $<.05$ & -- & -- & $<.05$ & -- & -- & $<.05$ & $<.20$ & $<.20$ \\
\hline 405419072232901 & Suffolk Co., NY & $02 / 05 / 99$ & $<.05$ & $<.20$ & $<.20$ & -- & $<.05$ & $<.20$ & $<.20$ & -- & -- & -- & -- & -- & -- & -- & $<.05$ & $<.20$ & $<.20$ \\
\hline 405507072382101 & Suffolk Co., NY & 08/03/99 & $<.05$ & $<.20$ & $<.20$ & -- & $<.05$ & .27 & $<.20$ & -- & $<.05$ & -- & -- & $<.05$ & -- & -- & $<.05$ & 1.3 & $<.20$ \\
\hline 405512072395202 & Suffolk Co., NY & $06 / 24 / 98$ & $<.05$ & $<.20$ & $<.20$ & -- & $<.05$ & $<.20$ & $<.20$ & -- & -- & -- & -- & -- & -- & -- & $<.05$ & $<.20$ & $<.20$ \\
\hline
\end{tabular}


Table 5. Analytical results for selected acetamide herbicides and their degradation products, ethanesulfonic acid, oxanilic acid, and sulfinyl acetic acid, in ground-water samples, 1994-2002.-Continued

[OGRL, U.S. Geological Survey Organic Geochemistry Research Laboratory, Lawrence, Kansas; ESA, ethanesulfonic acid; OXA, oxanilic acid; SAA, sulfinyl acetic acid; --, no data; <, less than]

\begin{tabular}{|c|c|c|c|c|c|c|c|c|c|c|c|c|c|c|c|c|c|c|c|}
\hline Site number & Site location & $\begin{array}{c}\text { Date of } \\
\text { collection } \\
\text { (month/day/ } \\
\text { year) }\end{array}$ & $\begin{array}{l}\text { Aceto- } \\
\text { chlor } \\
(\mu \mathrm{g} / \mathrm{L})\end{array}$ & $\begin{array}{c}\text { Aceto- } \\
\text { chlor } \\
\text { ESA } \\
(\mu \mathrm{g} / \mathrm{L})\end{array}$ & $\begin{array}{c}\text { Aceto- } \\
\text { chlor } \\
\text { OXA } \\
(\mu \mathrm{g} / \mathrm{L})\end{array}$ & $\begin{array}{c}\text { Aceto- } \\
\text { chlor } \\
\text { SAA } \\
(\mu \mathrm{g} / \mathrm{L})\end{array}$ & $\begin{array}{l}\text { Ala- } \\
\text { chlor } \\
(\mu \mathrm{g} / \mathrm{L})\end{array}$ & $\begin{array}{l}\text { Ala- } \\
\text { chlor } \\
\text { ESA } \\
(\mu \mathrm{g} / \mathrm{L})\end{array}$ & $\begin{array}{l}\text { Ala- } \\
\text { chlor } \\
\text { OXA } \\
(\mu \mathrm{g} / \mathrm{L})\end{array}$ & $\begin{array}{l}\text { Ala- } \\
\text { chlor } \\
\text { SAA } \\
(\mu \mathrm{g} / \mathrm{L})\end{array}$ & $\begin{array}{l}\text { Di- } \\
\text { meth- } \\
\text { enamid } \\
(\mu \mathrm{g} / \mathrm{L})\end{array}$ & $\begin{array}{c}\text { Di- } \\
\text { meth- } \\
\text { enamid } \\
\text { ESA } \\
(\mu \mathrm{g} / \mathrm{L})\end{array}$ & $\begin{array}{c}\text { Di- } \\
\text { meth- } \\
\text { enamid } \\
\text { OXA } \\
(\mu \mathrm{g} / \mathrm{L})\end{array}$ & $\begin{array}{c}\text { Flufen- } \\
\text { acet } \\
(\mu \mathrm{g} / \mathrm{L})\end{array}$ & $\begin{array}{c}\text { Flufen- } \\
\text { acet } \\
\text { ESA } \\
(\mu \mathrm{g} / \mathrm{L})\end{array}$ & $\begin{array}{c}\text { Flufen- } \\
\text { acet } \\
\text { OXA } \\
(\mu \mathrm{g} / \mathrm{L})\end{array}$ & $\begin{array}{l}\text { Meto- } \\
\text { lachlor } \\
(\mu \mathrm{g} / \mathrm{L})\end{array}$ & $\begin{array}{c}\text { Meto- } \\
\text { lachlor } \\
\text { ESA } \\
(\mu \mathrm{g} / \mathrm{L})\end{array}$ & $\begin{array}{c}\text { Meto- } \\
\text { lachlor } \\
\text { OXA } \\
(\mu \mathrm{g} / \mathrm{L})\end{array}$ \\
\hline
\end{tabular}

\section{Federal-State Cooperative Studies-Continued}

405516072183401 Suffolk Co., NY 405519073190701 Suffolk Co., NY 405520072380001 Suffolk Co., NY 405522072381001 Suffolk Co., NY 405522072381002 Suffolk Co., NY

405535072000002 Suffolk Co., NY 405535072000003 Suffolk Co., NY 405535072000004 Suffolk Co., NY

405547072365001 Suffolk Co., NY 405554072352201 Suffolk Co., NY

405607073021301 Suffolk Co., NY 405626072442701 Suffolk Co., NY 405640072200501 Suffolk Co., NY 405652072590001 Suffolk Co., NY 405655072334702 Suffolk Co., NY

405656072443201 Suffolk Co., NY 405701072531101 Suffolk Co., NY

405701072531102 Suffolk Co., NY

$405715072360201 \quad$ Suffolk Co., NY

405715072360202 Suffolk Co., NY

\section{Long Island, New York hydrologic study (LIN) —Continued}

\begin{tabular}{|c|c|c|c|c|c|c|c|c|c|c|c|c|c|c|c|c|c|}
\hline 07/02/98 & $<0.05$ & $<0.20$ & $<0.20$ & -- & $<0.05$ & $<0.20$ & $<0.20$ & -- & -- & -- & -- & -- & -- & -- & $<0.05$ & $<0.20$ & $<0.20$ \\
\hline 02/03/00 & $<.05$ & $<.20$ & $<.20$ & -- & $<.05$ & $<.20$ & $<.20$ & -- & $<0.05$ & -- & -- & $<0.05$ & -- & -- & $<.05$ & .65 & $<.20$ \\
\hline 08/25/99 & $<.05$ & .23 & .36 & -- & $<.05$ & $<.20$ & .60 & -- & $<.05$ & -- & -- & $<.05$ & -- & -- & $<.05$ & .81 & .49 \\
\hline 08/03/99 & $<.05$ & $<.20$ & $<.20$ & -- & $<.05$ & $<.20$ & $<.20$ & -- & $<.05$ & -- & -- & $<.05$ & -- & -- & $<.05$ & .44 & $<.20$ \\
\hline 08/03/99 & $<.05$ & $<.20$ & $<.20$ & -- & $<.05$ & $<.20$ & $<.20$ & -- & $<.05$ & -- & -- & $<.05$ & -- & -- & $<.05$ & $<.20$ & $<.20$ \\
\hline 06/09/98 & $<.05$ & $<.20$ & $<.20$ & -- & $<.05$ & $<.20$ & $<.20$ & -- & -- & -- & -- & -- & -- & -- & $<.05$ & $<.20$ & $<.20$ \\
\hline 06/09/98 & $<.05$ & $<.20$ & $<.20$ & -- & $<.05$ & $<.20$ & $<.20$ & -- & -- & -- & -- & -- & -- & -- & $<.05$ & 2.3 & 2.1 \\
\hline 06/10/98 & $<.05$ & $<.20$ & $<.20$ & -- & $<.05$ & $<.20$ & $<.20$ & -- & -- & -- & -- & -- & -- & -- & .75 & 6.2 & 5.2 \\
\hline 09/08/99 & $<.05$ & $<.20$ & $<.20$ & -- & $<.05$ & 1.0 & $<.20$ & -- & $<.05$ & -- & -- & $<.05$ & -- & -- & 86 & $<.20$ & $<.20$ \\
\hline 07/01/98 & $<.05$ & $<.20$ & $<.20$ & -- & $<.05$ & $<.20$ & $<.20$ & -- & -- & -- & -- & -- & -- & -- & $<.05$ & $<.20$ & $<.20$ \\
\hline 07/01/98 & $<.05$ & $<.20$ & $<.20$ & -- & $<.05$ & $<.20$ & $<.20$ & -- & -- & -- & -- & -- & -- & -- & $<.05$ & $<.20$ & $<.20$ \\
\hline 08/24/99 & $<.05$ & $<.20$ & $<.20$ & -- & $<.05$ & $<.20$ & $<.20$ & -- & $<.05$ & -- & -- & $<.05$ & -- & -- & $<.05$ & $<.20$ & $<.20$ \\
\hline 05/14/98 & $<.05$ & $<.20$ & $<.20$ & -- & $<.05$ & $<.20$ & $<.20$ & -- & -- & -- & -- & -- & -- & -- & $<.05$ & $<.20$ & $<.20$ \\
\hline 08/24/98 & $<.05$ & $<.20$ & $<.20$ & -- & $<.05$ & $<.20$ & $<.20$ & -- & -- & -- & -- & -- & -- & -- & $<.05$ & 2.9 & .90 \\
\hline 08/24/99 & $<.05$ & $<.20$ & $<.20$ & -- & .55 & 3.8 & 5.1 & -- & $<.05$ & -- & -- & $<.05$ & -- & -- & $<.05$ & $<.20$ & $<.20$ \\
\hline 05/13/98 & $<.05$ & $<.20$ & $<.20$ & -- & $<.05$ & $<.20$ & $<.20$ & -- & -- & -- & -- & -- & -- & -- & .86 & 30 & 25 \\
\hline 08/31/99 & $<.05$ & $<.20$ & $<.20$ & -- & $<.05$ & 20 & $<.20$ & -- & $<.05$ & -- & -- & $<.05$ & -- & -- & .64 & 13 & $<.20$ \\
\hline 05/14/98 & $<.05$ & $<.20$ & $<.20$ & -- & $<.05$ & $<.20$ & $<.20$ & -- & -- & -- & -- & -- & -- & -- & $<.05$ & 1.0 & .91 \\
\hline 02/01/99 & $<.05$ & $<.20$ & $<.20$ & -- & $<.05$ & $<.20$ & $<.20$ & -- & -- & -- & -- & -- & -- & -- & $<.05$ & $<.20$ & $<.20$ \\
\hline 08/24/99 & $<.05$ & .24 & $<.20$ & -- & $<.05$ & $<.20$ & $<.20$ & -- & $<.05$ & -- & -- & $<.05$ & -- & -- & $<.05$ & .84 & $<.20$ \\
\hline 02/01/99 & $<.05$ & $<.20$ & $<.20$ & -- & $<.05$ & $<.20$ & $<.20$ & -- & -- & -- & -- & -- & -- & -- & $<.05$ & $<.20$ & $<.20$ \\
\hline 08/24/99 & $<.05$ & $<.20$ & $<.20$ & -- & $<.05$ & $<.20$ & $<.20$ & -- & $<.05$ & -- & -- & $<.05$ & -- & -- & $<.05$ & $<.20$ & $<.20$ \\
\hline 05/27/98 & $<.05$ & $<.20$ & $<.20$ & -- & $<.05$ & $<.20$ & $<.20$ & -- & -- & -- & -- & -- & -- & -- & $<.05$ & $<.20$ & $<.20$ \\
\hline 08/12/99 & $<.05$ & $<.20$ & $<.20$ & -- & $<.05$ & $<.20$ & $<.20$ & -- & $<.05$ & -- & -- & $<.05$ & -- & -- & $<.05$ & $<.20$ & $<.20$ \\
\hline 08/18/99 & $<.05$ & $<.20$ & $<.20$ & -- & $<.05$ & $<.20$ & $<.20$ & -- & $<.05$ & -- & -- & $<.05$ & -- & -- & $<.05$ & $<.20$ & $<.20$ \\
\hline
\end{tabular}


Table 5. Analytical results for selected acetamide herbicides and their degradation products, ethanesulfonic acid, oxanilic acid, and sulfinyl acetic acid, in ground-water samples, 1994-2002.-Continued

[OGRL, U.S. Geological Survey Organic Geochemistry Research Laboratory, Lawrence, Kansas; ESA, ethanesulfonic acid; OXA, oxanilic acid; SAA, sulfinyl acetic acid; --, no data; <, less than]

\begin{tabular}{|c|c|c|c|c|c|c|c|c|c|c|c|c|c|c|c|c|c|c|c|}
\hline Site number & Site location & $\begin{array}{c}\text { Date of } \\
\text { collection } \\
\text { (month/day/ } \\
\text { year) }\end{array}$ & $\begin{array}{c}\text { Aceto- } \\
\text { chlor } \\
(\mu \mathrm{g} / \mathrm{L})\end{array}$ & $\begin{array}{l}\text { Aceto- } \\
\text { chlor } \\
\text { ESA } \\
(\mu \mathrm{g} / \mathrm{L})\end{array}$ & $\begin{array}{l}\text { Aceto- } \\
\text { chlor } \\
\text { OXA } \\
(\mu \mathrm{g} / \mathrm{L})\end{array}$ & $\begin{array}{l}\text { Aceto- } \\
\text { chlor } \\
\text { SAA } \\
(\mu \mathrm{g} / \mathrm{L})\end{array}$ & $\begin{array}{l}\text { Ala- } \\
\text { chlor } \\
(\mu \mathrm{g} / \mathrm{L})\end{array}$ & $\begin{array}{l}\text { Ala- } \\
\text { chlor } \\
\text { ESA } \\
(\mu \mathrm{g} / \mathrm{L})\end{array}$ & $\begin{array}{c}\text { Ala- } \\
\text { chlor } \\
\text { OXA } \\
(\mu \mathrm{g} / \mathrm{L})\end{array}$ & $\begin{array}{c}\text { Ala- } \\
\text { chlor } \\
\text { SAA } \\
(\mu \mathrm{g} / \mathrm{L})\end{array}$ & $\begin{array}{l}\text { Di- } \\
\text { meth- } \\
\text { enamid } \\
(\mu \mathrm{g} / \mathrm{L})\end{array}$ & $\begin{array}{c}\text { Di- } \\
\text { meth- } \\
\text { enamid } \\
\text { ESA } \\
(\mu \mathrm{g} / \mathrm{L})\end{array}$ & $\begin{array}{c}\text { Di- } \\
\text { meth- } \\
\text { enamid } \\
\text { OXA } \\
(\mu \mathrm{g} / \mathrm{L})\end{array}$ & $\begin{array}{c}\text { Flufen- } \\
\text { acet } \\
(\mu \mathrm{g} / \mathrm{L})\end{array}$ & $\begin{array}{c}\text { Flufen- } \\
\text { acet } \\
\text { ESA } \\
(\mu \mathrm{g} / \mathrm{L})\end{array}$ & $\begin{array}{c}\text { Flufen- } \\
\text { acet } \\
\text { OXA } \\
(\mu \mathrm{g} / \mathrm{L})\end{array}$ & $\begin{array}{c}\text { Meto- } \\
\text { lachlor } \\
(\mu \mathrm{g} / \mathrm{L})\end{array}$ & $\begin{array}{l}\text { Meto- } \\
\text { lachlor } \\
\text { ESA } \\
(\mu \mathrm{g} / \mathrm{L})\end{array}$ & $\begin{array}{c}\text { Meto- } \\
\text { lachlor } \\
\text { OXA } \\
(\mu \mathrm{g} / \mathrm{L})\end{array}$ \\
\hline \multicolumn{20}{|c|}{ Federal-State Cooperative Studies-Continued } \\
\hline \multicolumn{20}{|c|}{ Long Island, New York hydrologic study (LIN)—Continued } \\
\hline 405715072360203 & Suffolk Co., NY & 08/18/99 & $<0.05$ & $<0.20$ & $<0.20$ & -- & $<0.05$ & $<0.20$ & $<0.20$ & -- & $<0.05$ & -- & -- & $<0.05$ & -- & -- & $<0.05$ & $<0.20$ & $<0.20$ \\
\hline \multirow[t]{2}{*}{405716072413301} & Suffolk Co., NY & 06/10/98 & $<.05$ & $<.20$ & $<.20$ & -- & $<.05$ & 1.8 & $<.20$ & -- & -- & -- & -- & -- & -- & -- & .10 & 6.0 & 3.7 \\
\hline & & $08 / 31 / 99$ & $<.05$ & $<.20$ & $<.20$ & -- & $<.05$ & .72 & $<.20$ & -- & $<.05$ & -- & -- & $<.05$ & -- & -- & 1.6 & 2.5 & 2.6 \\
\hline 405720072103001 & Suffolk Co., NY & $12 / 08 / 99$ & $<.05$ & $<.20$ & $<.20$ & -- & $<.05$ & $<.20$ & $<.20$ & -- & $<.05$ & -- & -- & $<.05$ & -- & -- & $<.05$ & $<.20$ & $<.20$ \\
\hline 405720072122701 & Suffolk Co., NY & $01 / 25 / 99$ & $<.05$ & $<.20$ & $<.20$ & -- & $<.05$ & $<.20$ & $<.20$ & -- & -- & -- & -- & -- & -- & -- & $<.05$ & $<.20$ & $<.20$ \\
\hline 405720072122702 & Suffolk Co., NY & $02 / 05 / 99$ & $<.05$ & $<.20$ & $<.20$ & -- & $<.05$ & $<.20$ & $<.20$ & -- & -- & -- & -- & -- & -- & -- & $<.05$ & $<.20$ & $<.20$ \\
\hline 405720072122703 & Suffolk Co., NY & 02/05/99 & $<.05$ & $<.20$ & $<.20$ & -- & $<.05$ & $<.20$ & $<.20$ & -- & -- & -- & -- & -- & -- & -- & $<.05$ & $<.20$ & $<.20$ \\
\hline \multirow[t]{3}{*}{405720072122704} & Suffolk Co., NY & $07 / 02 / 98$ & $<.05$ & $<.20$ & $<.20$ & -- & $<.05$ & $<.20$ & $<.20$ & -- & -- & -- & -- & -- & -- & -- & $<.05$ & $<.20$ & $<.20$ \\
\hline & & $01 / 25 / 99$ & $<.05$ & $<.20$ & $<.20$ & -- & $<.05$ & $<.20$ & $<.20$ & -- & -- & -- & -- & -- & -- & -- & $<.05$ & $<.20$ & $<.20$ \\
\hline & & $08 / 25 / 99$ & $<.05$ & $<.20$ & $<.20$ & -- & $<.05$ & $<.20$ & $<.20$ & -- & $<.05$ & -- & -- & $<.05$ & -- & -- & $<.05$ & $<.20$ & $<.20$ \\
\hline \multirow[t]{2}{*}{4057200721227050} & Suffolk Co., NY & $01 / 25 / 99$ & $<.05$ & $<.20$ & $<.20$ & -- & $<.05$ & $<.20$ & $<.20$ & -- & -- & -- & -- & -- & -- & -- & $<.05$ & $<.20$ & $<.20$ \\
\hline & & $08 / 25 / 99$ & $<.05$ & $<.20$ & $<.20$ & -- & $<.05$ & $<.20$ & $<.20$ & -- & $<.05$ & -- & -- & $<.05$ & -- & -- & $<.05$ & $<.20$ & $<.20$ \\
\hline \multirow[t]{2}{*}{405730072364101} & Suffolk Co., NY & $06 / 25 / 98$ & $<.05$ & $<.20$ & $<.20$ & -- & $<.05$ & .32 & $<.20$ & -- & -- & -- & -- & -- & -- & -- & $<.05$ & 1.4 & .53 \\
\hline & & $09 / 21 / 99$ & $<.05$ & $<.20$ & $<.20$ & -- & $<.05$ & $<.20$ & $<.20$ & -- & $<.05$ & -- & -- & $<.05$ & -- & -- & $<.05$ & $<.20$ & $<.20$ \\
\hline \multirow[t]{2}{*}{405805072403701} & Suffolk Co., NY & 06/04/98 & $<.05$ & $<.20$ & $<.20$ & -- & $<.05$ & $<.20$ & $<.20$ & -- & -- & -- & -- & -- & -- & -- & $<.05$ & 7.4 & 6.4 \\
\hline & & $06 / 10 / 98$ & $<.05$ & $<.20$ & $<.20$ & -- & $<.05$ & $<.20$ & $<.20$ & -- & -- & -- & -- & -- & -- & -- & .17 & 8.6 & 7.6 \\
\hline 405807072121001 & Suffolk Co., NY & 09/08/99 & $<.05$ & $<.20$ & $<.20$ & -- & $<.05$ & $<.20$ & $<.20$ & -- & $<.05$ & -- & -- & $<.05$ & -- & -- & $<.05$ & .22 & $<.20$ \\
\hline 405924072303401 & Suffolk Co., NY & $08 / 25 / 98$ & $<.05$ & $<.20$ & $<.20$ & -- & $<.05$ & $<.20$ & $<.20$ & -- & -- & -- & -- & -- & -- & -- & .24 & 17 & 13 \\
\hline 405935072305601 & Suffolk Co., NY & 08/13/98 & $<.05$ & $<.20$ & $<.20$ & -- & $<.05$ & $<.20$ & $<.20$ & -- & -- & -- & -- & -- & -- & -- & .66 & 7.1 & 5.8 \\
\hline 405937072342801 & Suffolk Co., NY & $12 / 07 / 99$ & $<.05$ & $<.20$ & $<.20$ & -- & $<.05$ & 1.0 & $<.20$ & -- & $<.05$ & -- & -- & $<.05$ & -- & -- & $<.05$ & 1.3 & $<.20$ \\
\hline 405942072341001 & Suffolk Co., NY & $12 / 07 / 99$ & $<.05$ & $<.20$ & $<.20$ & -- & $<.05$ & $<.20$ & $<.20$ & -- & $<.05$ & -- & -- & $<.05$ & -- & -- & $<.05$ & 2.4 & 2.4 \\
\hline 405944072340701 & Suffolk Co., NY & $12 / 07 / 99$ & $<.05$ & $<.20$ & $<.20$ & -- & $<.05$ & .78 & $<.20$ & -- & $<.05$ & -- & -- & $<.05$ & -- & -- & $<.05$ & 1.9 & 3.0 \\
\hline 410126072273301 & Suffolk Co., NY & $10 / 07 / 99$ & $<.05$ & $<.20$ & $<.20$ & -- & $<.05$ & $<.20$ & $<.20$ & -- & $<.05$ & -- & -- & $<.05$ & -- & -- & $<.05$ & $<.20$ & $<.20$ \\
\hline 410126072273601 & Suffolk Co., NY & $10 / 06 / 99$ & $<.05$ & $<.20$ & $<.20$ & -- & $<.05$ & $<.20$ & $<.20$ & -- & $<.05$ & -- & -- & $<.05$ & -- & -- & $<.05$ & $<.20$ & $<.20$ \\
\hline 410127072273501 & Suffolk Co., NY & $10 / 06 / 99$ & $<.05$ & $<.20$ & $<.20$ & -- & $<.05$ & $<.20$ & $<.20$ & -- & $<.05$ & -- & -- & $<.05$ & -- & -- & $<.05$ & $<.20$ & $<.20$ \\
\hline 410150072285101 & Suffolk Co., NY & $11 / 22 / 99$ & $<.05$ & $<.20$ & $<.20$ & -- & $<.05$ & $<.20$ & $<.20$ & -- & $<.05$ & -- & -- & $<.05$ & -- & -- & $<.05$ & $<.20$ & $<.20$ \\
\hline
\end{tabular}


Table 5. Analytical results for selected acetamide herbicides and their degradation products, ethanesulfonic acid, oxanilic acid, and sulfinyl acetic acid, in ground-water samples, 1994-2002.-Continued

[OGRL, U.S. Geological Survey Organic Geochemistry Research Laboratory, Lawrence, Kansas; ESA, ethanesulfonic acid; OXA, oxanilic acid; SAA, sulfinyl acetic acid; --, no data; <, less than]

\begin{tabular}{|c|c|c|c|c|c|c|c|c|c|c|c|c|c|c|c|c|c|c|c|}
\hline Site number & Site location & $\begin{array}{c}\text { Date of } \\
\text { collection } \\
\text { (month/day/ } \\
\text { year) }\end{array}$ & $\begin{array}{c}\text { Aceto- } \\
\text { chlor } \\
(\mu \mathrm{g} / \mathrm{L})\end{array}$ & $\begin{array}{l}\text { Aceto- } \\
\text { chlor } \\
\text { ESA } \\
(\mu \mathrm{g} / \mathrm{L})\end{array}$ & $\begin{array}{c}\text { Aceto- } \\
\text { chlor } \\
\text { OXA } \\
(\mu \mathrm{g} / \mathrm{L})\end{array}$ & $\begin{array}{c}\text { Aceto- } \\
\text { chlor } \\
\text { SAA } \\
(\mu \mathrm{g} / \mathrm{L})\end{array}$ & $\begin{array}{l}\text { Ala- } \\
\text { chlor } \\
(\mu \mathrm{g} / \mathrm{L})\end{array}$ & $\begin{array}{l}\text { Ala- } \\
\text { chlor } \\
\text { ESA } \\
(\mu \mathrm{g} / \mathrm{L})\end{array}$ & $\begin{array}{c}\text { Ala- } \\
\text { chlor } \\
\text { OXA } \\
(\mu \mathrm{g} / \mathrm{L})\end{array}$ & $\begin{array}{l}\text { Ala- } \\
\text { chlor } \\
\text { SAA } \\
(\mu \mathrm{g} / \mathrm{L})\end{array}$ & $\begin{array}{c}\text { Di- } \\
\text { meth- } \\
\text { enamid } \\
(\mu \mathrm{g} / \mathrm{L})\end{array}$ & $\begin{array}{c}\text { Di- } \\
\text { meth- } \\
\text { enamid } \\
\text { ESA } \\
(\mu \mathrm{g} / \mathrm{L})\end{array}$ & $\begin{array}{c}\text { Di- } \\
\text { meth- } \\
\text { enamid } \\
\text { OXA } \\
(\mu \mathrm{g} / \mathrm{L})\end{array}$ & $\begin{array}{c}\text { Flufen- } \\
\text { acet } \\
(\mu \mathrm{g} / \mathrm{L})\end{array}$ & $\begin{array}{c}\text { Flufen- } \\
\text { acet } \\
\text { ESA } \\
(\mu \mathrm{g} / \mathrm{L})\end{array}$ & $\begin{array}{c}\text { Flufen- } \\
\text { acet } \\
\text { OXA } \\
(\mu \mathrm{g} / \mathrm{L})\end{array}$ & $\begin{array}{c}\text { Meto- } \\
\text { lachlor } \\
(\mu \mathrm{g} / \mathrm{L})\end{array}$ & $\begin{array}{c}\text { Meto- } \\
\text { lachlor } \\
\text { ESA } \\
(\mu \mathrm{g} / \mathrm{L})\end{array}$ & $\begin{array}{c}\text { Meto- } \\
\text { lachlor } \\
\text { OXA } \\
(\mu \mathrm{g} / \mathrm{L})\end{array}$ \\
\hline
\end{tabular}

\section{Federal-State Cooperative Studies-Continued}

410152072284901 Suffolk Co., NY 410208072242501 Suffolk Co., NY 410222072310001 Suffolk Co., NY 410226072295901 Suffolk Co., NY 410226072295902 Suffolk Co., NY

410226072295903 Suffolk Co., NY 410252072275001 Suffolk Co., NY 410337072264401 Suffolk Co., NY

410337072264402 Suffolk Co., NY 410338072264401 Suffolk Co., NY

410403072263701 Suffolk Co., NY 410414072265001 Suffolk Co., NY 410415072260701 Suffolk Co., NY

410415072260702 Suffolk Co., NY

410430072260901 Suffolk Co., NY

410728072210601 Suffolk Co., NY 410730072213401 Suffolk Co., NY 410904072171201 Suffolk Co., NY

\section{Long Island, New York hydrologic study (LIN) — Continued}

\begin{tabular}{|c|c|c|c|c|c|c|c|c|c|c|c|c|c|c|c|c|c|}
\hline $11 / 22 / 99$ & $<0.05$ & $<0.20$ & $<0.20$ & -- & $<0.05$ & $<0.20$ & $<0.20$ & -- & $<0.05$ & -- & -- & $<0.05$ & -- & -- & $<0.05$ & $<0.20$ & $<0.20$ \\
\hline $01 / 27 / 99$ & $<.05$ & $<.20$ & $<.20$ & -- & $<.05$ & $<.20$ & $<.20$ & -- & -- & -- & -- & -- & -- & -- & $<.05$ & $<.20$ & $<.20$ \\
\hline $08 / 25 / 98$ & $<.05$ & $<.20$ & $<.20$ & -- & $<.05$ & $<.20$ & $<.20$ & -- & -- & -- & -- & -- & -- & -- & 2.1 & 14 & 10 \\
\hline 08/18/99 & $<.05$ & $<.20$ & $<.20$ & -- & $<.05$ & $<.20$ & $<.20$ & -- & $<.05$ & -- & -- & $<.05$ & -- & -- & $<.05$ & $<.20$ & $<.20$ \\
\hline 08/18/99 & $<.05$ & $<.20$ & $<.20$ & -- & $<.05$ & $<.20$ & $<.20$ & -- & $<.05$ & -- & -- & $<.05$ & -- & -- & $<.05$ & $<.20$ & $<.20$ \\
\hline 08/18/99 & $<.05$ & $<.20$ & $<.20$ & -- & $<.05$ & $<.20$ & $<.20$ & -- & $<.05$ & -- & -- & $<.05$ & -- & -- & .16 & .55 & .46 \\
\hline 08/13/98 & $<.05$ & $<.20$ & $<.20$ & -- & $<.05$ & $<.20$ & $<.20$ & -- & $<.05$ & -- & -- & -- & -- & -- & $<.05$ & $<.20$ & $<.20$ \\
\hline $05 / 21 / 98$ & $<.05$ & $<.20$ & $<.20$ & -- & .11 & $<.20$ & $<.20$ & -- & $<.05$ & -- & -- & -- & -- & -- & .79 & 3.2 & 2.9 \\
\hline $01 / 27 / 99$ & $<.05$ & $<.20$ & $<.20$ & -- & $<.05$ & $<.20$ & $<.20$ & -- & -- & -- & -- & -- & -- & -- & .08 & 3.8 & 2.2 \\
\hline 08/26/99 & $<.05$ & $<.20$ & $<.20$ & -- & $<.05$ & 1.0 & $<.20$ & -- & $<.05$ & -- & -- & $<.05$ & -- & -- & .60 & 4.6 & $<.20$ \\
\hline 01/27/99 & $<.05$ & $<.20$ & $<.20$ & -- & .08 & $<.20$ & $<.20$ & -- & -- & -- & -- & -- & -- & -- & .55 & 2.9 & 2.2 \\
\hline 01/27/99 & $<.05$ & $<.20$ & $<.20$ & -- & $<.05$ & $<.20$ & $<.20$ & -- & -- & -- & -- & -- & -- & -- & $<.05$ & 2.1 & .89 \\
\hline 08/26/99 & $<.05$ & $<.20$ & $<.20$ & -- & $<.05$ & $<.20$ & $<.20$ & -- & $<.05$ & -- & -- & $<.05$ & -- & -- & $<.05$ & $<.20$ & $<.20$ \\
\hline 01/26/99 & $<.05$ & $<.20$ & $<.20$ & -- & $<.05$ & $<.20$ & $<.20$ & -- & -- & -- & -- & -- & -- & -- & .07 & 2.0 & 1.6 \\
\hline 01/26/99 & $<.05$ & $<.20$ & $<.20$ & -- & $<.05$ & $<.20$ & $<.20$ & -- & -- & -- & -- & -- & -- & -- & $<.05$ & $<.20$ & $<.20$ \\
\hline $05 / 21 / 98$ & $<.05$ & $<.20$ & $<.20$ & -- & $<.05$ & $<.20$ & $<.20$ & -- & -- & -- & -- & -- & -- & -- & .36 & 19 & 15 \\
\hline 01/26/99 & $<.05$ & $<.20$ & $<.20$ & -- & $<.05$ & $<.20$ & $<.20$ & -- & -- & -- & -- & -- & -- & -- & .26 & 13 & 15 \\
\hline 08/26/99 & $<.05$ & $<.20$ & $<.20$ & -- & $<.05$ & $<.20$ & .33 & -- & $<.05$ & -- & -- & $<.05$ & -- & -- & .35 & 8.9 & 16 \\
\hline $01 / 26 / 99$ & $<.05$ & $<.20$ & $<.20$ & & $<.05$ & $<.20$ & $<.20$ & & & & & & & & $<.05$ & $<.20$ & $<.20$ \\
\hline 08/26/99 & $<.05$ & $<.20$ & $<.20$ & -- & $<.05$ & $<.20$ & $<.20$ & -- & $<.05$ & -- & -- & $<.05$ & -- & -- & $<.05$ & .31 & $<.20$ \\
\hline 01/27/99 & $<.05$ & $<.20$ & $<.20$ & -- & $<.05$ & $<.20$ & $<.20$ & -- & -- & -- & -- & -- & -- & -- & .16 & 3.8 & 2.4 \\
\hline 01/26/99 & $<.05$ & $<.20$ & $<.20$ & -- & $<.05$ & $<.20$ & $<.20$ & -- & -- & -- & -- & -- & -- & -- & $<.05$ & $<.20$ & $<.20$ \\
\hline 01/06/00 & $<.05$ & $<.20$ & 198 & -- & $<.05$ & $<.20$ & $<.20$ & -- & $<.05$ & -- & -- & -- & -- & -- & $<.05$ & 1.5 & 3.4 \\
\hline 01/26/99 & $<.05$ & $<.20$ & $<.20$ & -- & $<.05$ & 1.8 & $<.20$ & -- & -- & -- & -- & -- & -- & -- & $<.05$ & 2.8 & 2.0 \\
\hline 08/26/99 & $<.05$ & $<.20$ & $<.20$ & -- & $<.05$ & .91 & $<.20$ & -- & $<.05$ & -- & -- & $<.05$ & -- & -- & $<.05$ & 1.8 & 1.9 \\
\hline
\end{tabular}




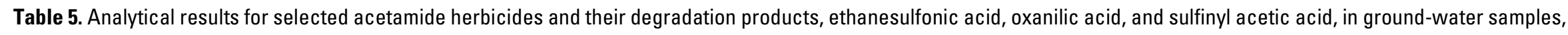
1994-2002.-Continued

[OGRL, U.S. Geological Survey Organic Geochemistry Research Laboratory, Lawrence, Kansas; ESA, ethanesulfonic acid; OXA, oxanilic acid; SAA, sulfinyl acetic acid; --, no data; <, less than]

\begin{tabular}{|c|c|c|c|c|c|c|c|c|c|c|c|c|c|c|c|c|c|c|c|}
\hline Site number & Site location & $\begin{array}{c}\text { Date of } \\
\text { collection } \\
\text { (month/day/ } \\
\text { year) }\end{array}$ & $\begin{array}{l}\text { Aceto- } \\
\text { chlor } \\
(\mu \mathrm{g} / \mathrm{L})\end{array}$ & $\begin{array}{l}\text { Aceto- } \\
\text { chlor } \\
\text { ESA } \\
(\mu \mathrm{g} / \mathrm{L})\end{array}$ & $\begin{array}{c}\text { Aceto- } \\
\text { chlor } \\
\text { OXA } \\
(\mu \mathrm{g} / \mathrm{L})\end{array}$ & $\begin{array}{c}\text { Aceto- } \\
\text { chlor } \\
\text { SAA } \\
(\mu \mathrm{g} / \mathrm{L})\end{array}$ & $\begin{array}{l}\text { Ala- } \\
\text { chlor } \\
(\mu \mathrm{g} / \mathrm{L})\end{array}$ & $\begin{array}{l}\text { Ala- } \\
\text { chlor } \\
\text { ESA } \\
(\mu \mathrm{g} / \mathrm{L})\end{array}$ & $\begin{array}{l}\text { Ala- } \\
\text { chlor } \\
\text { OXA } \\
(\mu \mathrm{g} / \mathrm{L})\end{array}$ & $\begin{array}{l}\text { Ala- } \\
\text { chlor } \\
\text { SAA } \\
(\mu \mathrm{g} / \mathrm{L})\end{array}$ & $\begin{array}{l}\text { Di- } \\
\text { meth- } \\
\text { enamid } \\
(\mu \mathrm{g} / \mathrm{L})\end{array}$ & $\begin{array}{c}\text { Di- } \\
\text { meth- } \\
\text { enamid } \\
\text { ESA } \\
(\mu \mathrm{g} / \mathrm{L})\end{array}$ & $\begin{array}{c}\text { Di- } \\
\text { meth- } \\
\text { enamid } \\
\text { OXA } \\
(\mu \mathrm{g} / \mathrm{L})\end{array}$ & $\begin{array}{c}\text { Flufen- } \\
\text { acet } \\
(\mu \mathrm{g} / \mathrm{L})\end{array}$ & $\begin{array}{c}\text { Flufen- } \\
\text { acet } \\
\text { ESA } \\
(\mu \mathrm{g} / \mathrm{L})\end{array}$ & $\begin{array}{c}\text { Flufen- } \\
\text { acet } \\
\text { OXA } \\
(\mu \mathrm{g} / \mathrm{L})\end{array}$ & $\begin{array}{l}\text { Meto- } \\
\text { lachlor } \\
(\mu \mathrm{g} / \mathrm{L})\end{array}$ & $\begin{array}{c}\text { Meto- } \\
\text { lachlor } \\
\text { ESA } \\
(\mu \mathrm{g} / \mathrm{L})\end{array}$ & $\begin{array}{c}\text { Meto- } \\
\text { lachlor } \\
\text { OXA } \\
(\mu \mathrm{g} / \mathrm{L})\end{array}$ \\
\hline \multicolumn{20}{|c|}{ Federal-State Cooperative Studies-Continued } \\
\hline \multicolumn{20}{|c|}{ Long Island, New York hydrologic study (LIN)_Continued } \\
\hline 410904072171202 & Suffolk Co., NY & $01 / 26 / 99$ & $<0.05$ & $<0.20$ & $<0.20$ & -- & $<0.05$ & $<0.20$ & $<0.20$ & -- & -- & -- & -- & -- & -- & -- & $<0.05$ & $<0.20$ & $<0.20$ \\
\hline & & 08/26/99 & $<.05$ & $<.20$ & $<.20$ & -- & $<.05$ & $<.20$ & $<.20$ & -- & $<0.05$ & -- & -- & $<0.05$ & -- & -- & $<.05$ & $<.20$ & $<.20$ \\
\hline 410918072143001 & Suffolk Co., NY & $08 / 24 / 98$ & $<.05$ & $<.20$ & $<.20$ & -- & $<.05$ & $<.20$ & $<.20$ & -- & -- & -- & -- & -- & -- & -- & $<.05$ & $<.20$ & $<.20$ \\
\hline \multicolumn{20}{|c|}{ New York hydrologic study (PPN) } \\
\hline 400000076000002 & Orange Co., NY & 08/03/99 & $<.05$ & $<.05$ & $<.05$ & -- & $<.05$ & $<.05$ & $<.05$ & -- & -- & -- & -- & -- & -- & -- & $<.05$ & $<.05$ & $<.05$ \\
\hline 400000076000003 & Sullivan Co., NY & 08/03/99 & $<.05$ & $<.05$ & $<.05$ & -- & $<.05$ & $<.05$ & $<.05$ & -- & -- & -- & -- & -- & -- & -- & $<.05$ & 1.5 & $<.05$ \\
\hline 410659074093701 & Rockland Co., NY & 08/04/99 & $<.05$ & $<.05$ & $<.05$ & -- & $<.05$ & $<.05$ & $<.05$ & -- & -- & -- & -- & -- & -- & -- & $<.05$ & $<.05$ & $<.05$ \\
\hline 411301073515401 & Westchester Co., NY & 08/04/99 & $<.05$ & $<.05$ & $<.05$ & -- & $<.05$ & $<.05$ & $<.05$ & -- & -- & -- & -- & -- & -- & -- & $<.05$ & $<.05$ & $<.05$ \\
\hline \multirow[t]{5}{*}{412815175235901} & Wayne Co., NY & 08/02/99 & $<.05$ & $<.05$ & $<.05$ & -- & $<.05$ & $<.05$ & $<.05$ & -- & -- & -- & -- & -- & -- & -- & .15 & 3.6 & 1.4 \\
\hline & & 02/09/00 & $<.05$ & $<.05$ & $<.05$ & -- & $<.05$ & $<.05$ & $<.05$ & -- & -- & -- & -- & -- & -- & -- & .46 & 3.0 & 1.0 \\
\hline & & 02/07/01 & -- & $<.05$ & $<.05$ & -- & -- & .06 & $<.05$ & -- & -- & $<0.05$ & $<0.05$ & -- & $<0.05$ & $<0.05$ & -- & 2.9 & 1.0 \\
\hline & & $04 / 24 / 01$ & -- & $<.05$ & $<.05$ & -- & -- & .09 & $<.05$ & -- & -- & $<.05$ & $<.05$ & -- & $<.05$ & $<.05$ & -- & 3.2 & 1.0 \\
\hline & & $07 / 31 / 01$ & -- & $<.05$ & $<.05$ & -- & -- & $<.05$ & $<.05$ & -- & -- & $<.05$ & $<.05$ & -- & $<.05$ & $<.05$ & -- & 3.1 & 1.1 \\
\hline 413520073511201 & Dutchess Co., NY & 08/04/99 & $<.05$ & $<.05$ & $<.05$ & -- & $<.05$ & $<.05$ & $<.05$ & -- & -- & -- & -- & -- & -- & -- & $<.05$ & $<.05$ & $<.05$ \\
\hline 420406078241701 & Cattaraugus Co., NY & 08/02/99 & $<.05$ & $<.05$ & $<.05$ & -- & $<.05$ & $<.05$ & $<.05$ & -- & -- & -- & -- & -- & -- & -- & $<.05$ & .38 & $<.05$ \\
\hline 420647075584201 & Broome Co., NY & 08/03/99 & $<.05$ & $<.05$ & $<.05$ & -- & $<.05$ & $<.05$ & $<.05$ & -- & -- & -- & -- & -- & -- & -- & $<.05$ & $<.05$ & $<.05$ \\
\hline 420850077030701 & Steuben Co., NY & 08/03/99 & $<.05$ & $<.05$ & $<.05$ & -- & $<.05$ & $<.05$ & $<.05$ & -- & -- & -- & -- & -- & -- & -- & $<.05$ & .24 & $<.05$ \\
\hline 420926079360001 & Chautaqua Co., NY & 08/02/99 & $<.05$ & $<.05$ & $<.05$ & -- & $<.05$ & $<.05$ & $<.05$ & -- & -- & -- & -- & -- & -- & -- & $<.05$ & $<.05$ & $<.05$ \\
\hline 421820075235901 & Delaware Co., NY & 08/03/99 & $<.05$ & $<.05$ & $<.05$ & -- & $<.05$ & $<.05$ & $<.05$ & -- & -- & -- & -- & -- & -- & -- & $<.05$ & $<.05$ & $<.05$ \\
\hline 422328073413304 & Columbia Co., NY & 08/04/99 & $<.05$ & $<.05$ & $<.05$ & -- & $<.05$ & 1.4 & $<.05$ & -- & -- & -- & -- & -- & -- & -- & $<.05$ & 2.3 & .85 \\
\hline 422633076020801 & Cortland Co., NY & 08/02/99 & $<.05$ & $<.05$ & $<.05$ & -- & $<.05$ & $<.05$ & $<.05$ & -- & -- & -- & -- & -- & -- & -- & $<.05$ & $<.05$ & $<.05$ \\
\hline 422937076531401 & Schyuler Co., NY & 08/04/99 & $<.05$ & $<.05$ & $<.05$ & -- & $<.05$ & $<.05$ & $<.05$ & -- & -- & -- & -- & -- & -- & -- & $<.05$ & $<.05$ & $<.05$ \\
\hline \multirow[t]{5}{*}{423001077300801} & Steuben Co., NY & 08/03/99 & $<.05$ & $<.05$ & $<.05$ & -- & $<.05$ & $<.05$ & .31 & -- & -- & -- & -- & -- & -- & -- & $<.05$ & .63 & 1.9 \\
\hline & & 02/06/01 & -- & $<.05$ & $<.05$ & -- & -- & .12 & .40 & -- & -- & $<.05$ & $<.05$ & -- & $<.05$ & $<.05$ & $<.05$ & 1.0 & 2.7 \\
\hline & & $04 / 24 / 01$ & -- & $<.05$ & $<.05$ & -- & -- & .14 & .30 & -- & -- & $<.05$ & $<.05$ & -- & $<.05$ & $<.05$ & $<.05$ & 1.0 & 3.0 \\
\hline & & 07/30/01 & -- & $<.05$ & $<.05$ & -- & -- & $<.05$ & .12 & -- & -- & $<.05$ & $<.05$ & -- & $<.05$ & $<.05$ & $<.05$ & .72 & 2.2 \\
\hline & & $10 / 30 / 01$ & -- & $<.05$ & $<.05$ & $<.05$ & -- & .13 & .24 & $<.05$ & -- & $<.05$ & $<.05$ & -- & $<.05$ & $<.05$ & -- & .83 & 3.1 \\
\hline
\end{tabular}


Table 5. Analytical results for selected acetamide herbicides and their degradation products, ethanesulfonic acid, oxanilic acid, and sulfinyl acetic acid, in ground-water samples, 1994-2002.-Continued

[OGRL, U.S. Geological Survey Organic Geochemistry Research Laboratory, Lawrence, Kansas; ESA, ethanesulfonic acid; OXA, oxanilic acid; SAA, sulfinyl acetic acid; --, no data; <, less than]

\begin{tabular}{|c|c|c|c|c|c|c|c|c|c|c|c|c|c|c|c|c|c|c|c|c|}
\hline Site number & Site location & $\begin{array}{c}\text { Date of } \\
\text { collection } \\
\text { (month/day/ } \\
\text { year) }\end{array}$ & $\begin{array}{l}\text { Aceto- } \\
\text { chlor } \\
(\mu \mathrm{g} / \mathrm{L})\end{array}$ & $\begin{array}{l}\text { Aceto- } \\
\text { chlor } \\
\text { ESA } \\
(\mu \mathrm{g} / \mathrm{L})\end{array}$ & $\begin{array}{l}\text { Aceto- } \\
\text { chlor } \\
\text { OXA } \\
(\mu \mathrm{g} / \mathrm{L})\end{array}$ & $\begin{array}{l}\text { Aceto- } \\
\text { chlor } \\
\text { SAA } \\
(\mu \mathrm{g} / \mathrm{L})\end{array}$ & $\begin{array}{l}\text { Ala- } \\
\text { chlor } \\
(\mu \mathrm{g} / \mathrm{L})\end{array}$ & $\begin{array}{l}\text { Ala- } \\
\text { chlor } \\
\text { ESA } \\
(\mu \mathrm{g} / \mathrm{L})\end{array}$ & $\begin{array}{l}\text { Ala- } \\
\text { chlor } \\
\text { OXA } \\
(\mu \mathrm{g} / \mathrm{L})\end{array}$ & $\begin{array}{l}\text { Ala- } \\
\text { chlor } \\
\text { SAA } \\
(\mu \mathrm{g} / \mathrm{L})\end{array}$ & $\begin{array}{c}\text { Di- } \\
\text { meth- } \\
\text { enamid } \\
(\mu \mathrm{g} / \mathrm{L})\end{array}$ & $\begin{array}{c}\text { Di- } \\
\text { meth- } \\
\text { enamid } \\
\text { ESA } \\
(\mu \mathrm{g} / \mathrm{L})\end{array}$ & $\begin{array}{c}\text { Di- } \\
\text { meth- } \\
\text { enamid } \\
\text { OXA } \\
(\mu \mathrm{g} / \mathrm{L})\end{array}$ & $\begin{array}{c}\text { Flufen- } \\
\text { acet } \\
(\mu \mathrm{g} / \mathrm{L})\end{array}$ & $\begin{array}{c}\text { Flufen- } \\
\text { acet } \\
\text { ESA } \\
(\mu \mathrm{g} / \mathrm{L})\end{array}$ & $\begin{array}{c}\text { Flufen- } \\
\text { acet } \\
\text { OXA } \\
(\mu \mathrm{g} / \mathrm{L})\end{array}$ & $\begin{array}{l}\text { Meto- } \\
\text { lachlor } \\
(\mu \mathrm{g} / \mathrm{L})\end{array}$ & $\begin{array}{l}\text { Meto- } \\
\text { lachlor } \\
\text { ESA } \\
(\mu \mathrm{g} / \mathrm{L})\end{array}$ & $\begin{array}{c}\text { Meto- } \\
\text { lachlor } \\
\text { OXA } \\
(\mu \mathrm{g} / \mathrm{L})\end{array}$ & 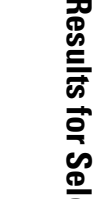 \\
\hline \multicolumn{20}{|c|}{ Federal-State Cooperative Studies-Continued } & \\
\hline \multicolumn{20}{|c|}{ New York hydrologic study (PPN)—Continued } & \\
\hline 423001077300801 & Steuben Co., NY & $01 / 29 / 02$ & -- & $<0.05$ & $<0.05$ & $<0.05$ & -- & 0.10 & 0.23 & $<0.05$ & -- & $<0.05$ & $<0.05$ & -- & $<0.05$ & $<0.05$ & -- & 0.80 & 2.7 & \\
\hline 423001078565401 & Erie Co., NY & 08/03/99 & $<0.05$ & $<.05$ & $<.05$ & -- & $<0.05$ & $<.05$ & $<.05$ & -- & -- & -- & -- & -- & -- & -- & $<0.05$ & $<.05$ & $<.05$ & \\
\hline 423331076358501 & Cayuga Co., NY & $07 / 28 / 98$ & $<.05$ & $<.05$ & $<.05$ & -- & $<.05$ & .52 & $<.05$ & -- & -- & -- & -- & -- & -- & -- & $<.05$ & .35 & .34 & $\frac{5}{6}$ \\
\hline 423454076124001 & Cortland Co., NY & 08/02/99 & $<.05$ & $<.05$ & $<.05$ & -- & $<.05$ & $<.05$ & $<.05$ & -- & -- & -- & -- & -- & -- & -- & $<.05$ & $<.05$ & $<.05$ & \\
\hline 423919073553801 & Albany Co., NY & 08/02/99 & $<.05$ & $<.05$ & $<.05$ & -- & $<.05$ & $<.05$ & $<.05$ & -- & -- & -- & -- & -- & -- & -- & $<.05$ & $<.05$ & $<.05$ & \\
\hline \multirow[t]{4}{*}{424025076321001} & \multirow[t]{4}{*}{ Cayuga Co., NY } & $01 / 11 / 00$ & $<.05$ & .28 & $<.05$ & -- & $<.05$ & $<.05$ & $<.05$ & -- & -- & -- & -- & -- & -- & -- & $<.05$ & 1.6 & $<.05$ & \\
\hline & & 02/07/01 & -- & $<.05$ & $<.05$ & -- & -- & $<.05$ & $<.05$ & -- & -- & $<.05$ & $<.05$ & -- & $<.05$ & $<.05$ & -- & .78 & .08 & \\
\hline & & $04 / 23 / 01$ & -- & $<.05$ & $<.05$ & -- & -- & $<.05$ & $<.05$ & -- & -- & $<.05$ & $<.05$ & -- & $<.05$ & $<.05$ & -- & .22 & $<.05$ & \\
\hline & & $07 / 31 / 01$ & -- & $<.05$ & $<.05$ & -- & -- & $<.05$ & $<.05$ & -- & -- & $<.05$ & $<.05$ & -- & $<.05$ & $<.05$ & -- & 1.8 & .35 & \\
\hline \multirow[t]{5}{*}{424710073473801} & \multirow[t]{5}{*}{ Saratoga Co., NY } & 07/08/99 & $<.05$ & $<.05$ & $<.05$ & -- & $<.05$ & $<.05$ & $<.05$ & -- & -- & -- & -- & -- & -- & -- & $<.05$ & $<.05$ & $<.05$ & \\
\hline & & $08 / 02 / 99$ & $<.05$ & $<.05$ & $<.05$ & -- & $<.05$ & $<.05$ & $<.05$ & -- & -- & -- & -- & -- & -- & -- & $<.05$ & $<.05$ & $<.05$ & \\
\hline & & 09/13/99 & $<.05$ & $<.05$ & $<.05$ & -- & $<.05$ & $<.05$ & $<.05$ & -- & -- & -- & -- & -- & -- & -- & $<.05$ & $<.05$ & $<.05$ & \\
\hline & & $10 / 21 / 99$ & $<.05$ & $<.05$ & $<.05$ & -- & $<.05$ & $<.05$ & $<.05$ & -- & -- & -- & -- & -- & -- & -- & $<.05$ & $<.05$ & $<.05$ & \\
\hline & & $02 / 05 / 01$ & -- & $<.05$ & $<.05$ & -- & -- & $<.05$ & $<.05$ & -- & -- & $<.05$ & $<.05$ & -- & $<.05$ & $<.05$ & -- & $<.05$ & $<.05$ & 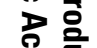 \\
\hline \multirow[t]{6}{*}{424724073472301} & \multirow[t]{6}{*}{ Saratoga Co., NY } & 06/10/99 & $<.05$ & $<.05$ & $<.05$ & -- & $<.05$ & $<.05$ & $<.05$ & -- & -- & -- & -- & -- & -- & -- & $<.05$ & $<.05$ & $<.05$ & \\
\hline & & 08/02/99 & $<.05$ & $<.05$ & $<.05$ & -- & $<.05$ & $<.05$ & $<.05$ & -- & -- & -- & -- & -- & -- & -- & $<.05$ & $<.05$ & $<.05$ & \\
\hline & & 09/13/99 & $<.05$ & $<.05$ & $<.05$ & -- & $<.05$ & $<.05$ & $<.05$ & -- & -- & -- & -- & -- & -- & -- & $<.05$ & $<.05$ & $<.05$ & \\
\hline & & $10 / 21 / 99$ & $<.05$ & $<.05$ & $<.05$ & -- & $<.05$ & $<.05$ & $<.05$ & -- & -- & -- & -- & -- & -- & -- & $<.05$ & $<.05$ & $<.05$ & \\
\hline & & $02 / 05 / 01$ & -- & $<.05$ & $<.05$ & -- & -- & .06 & $<.05$ & -- & -- & $<.05$ & $<.05$ & -- & $<.05$ & $<.05$ & $<.05$ & $<.05$ & $<.05$ & 竞 \\
\hline & & $04 / 24 / 01$ & -- & $<.05$ & $<.05$ & -- & -- & .08 & $<.05$ & -- & -- & $<.05$ & $<.05$ & -- & $<.05$ & $<.05$ & $<.05$ & .06 & $<.05$ & \\
\hline 424742074453201 & Ostego Co., NY & 08/03/99 & $<.05$ & $<.05$ & $<.05$ & -- & $<.05$ & $<.05$ & $<.05$ & -- & -- & -- & -- & -- & -- & -- & $<.05$ & .35 & $<.05$ & 岂 몽. \\
\hline 424909073591601 & Saratoga Co., NY & $02 / 05 / 01$ & -- & $<.05$ & $<.05$ & -- & -- & $<.05$ & $<.05$ & -- & -- & $<.05$ & $<.05$ & -- & $<.05$ & $<.05$ & -- & .09 & $<.05$ & \\
\hline 424912073591801 & Schenectady Co., NY & 08/03/99 & $<.05$ & $<.05$ & $<.05$ & -- & $<.05$ & $<.05$ & $<.05$ & -- & -- & -- & -- & -- & -- & -- & $<.05$ & $<.05$ & $<.05$ & \\
\hline \multirow[t]{3}{*}{425030074393901} & \multirow[t]{3}{*}{ Montgomery Co., NY } & $04 / 24 / 97$ & $<.05$ & $<.05$ & $<.05$ & -- & $<.05$ & $<.05$ & $<.05$ & -- & -- & -- & -- & -- & -- & -- & $<.05$ & 10 & 3.1 & مُ \\
\hline & & $05 / 22 / 97$ & $<.05$ & $<.05$ & $<.05$ & -- & $<.05$ & $<.05$ & $<.05$ & -- & -- & -- & -- & -- & -- & -- & $<.05$ & 9.5 & 2.8 & \\
\hline & & 05/28/97 & $<.05$ & $<.05$ & $<.05$ & -- & $<.05$ & $<.05$ & $<.05$ & -- & -- & -- & -- & -- & -- & -- & $<.05$ & 8.8 & 2.6 & \\
\hline
\end{tabular}


Table 5. Analytical results for selected acetamide herbicides and their degradation products, ethanesulfonic acid, oxanilic acid, and sulfinyl acetic acid, in ground-water samples, 1994-2002.-Continued

[OGRL, U.S. Geological Survey Organic Geochemistry Research Laboratory, Lawrence, Kansas; ESA, ethanesulfonic acid; OXA, oxanilic acid; SAA, sulfinyl acetic acid; --, no data; <, less than]

\begin{tabular}{|c|c|c|c|c|c|c|c|c|c|c|c|c|c|c|c|c|c|c|c|}
\hline Site number & Site location & $\begin{array}{c}\text { Date of } \\
\text { collection } \\
\text { (month/day/ } \\
\text { year) }\end{array}$ & $\begin{array}{l}\text { Aceto- } \\
\text { chlor } \\
(\mu \mathrm{g} / \mathrm{L})\end{array}$ & $\begin{array}{c}\text { Aceto- } \\
\text { chlor } \\
\text { ESA } \\
(\mu \mathrm{g} / \mathrm{L})\end{array}$ & $\begin{array}{c}\text { Aceto- } \\
\text { chlor } \\
\text { OXA } \\
(\mu \mathrm{g} / \mathrm{L})\end{array}$ & $\begin{array}{c}\text { Aceto- } \\
\text { chlor } \\
\text { SAA } \\
(\mu \mathrm{g} / \mathrm{L})\end{array}$ & $\begin{array}{l}\text { Ala- } \\
\text { chlor } \\
(\mu \mathrm{g} / \mathrm{L})\end{array}$ & $\begin{array}{l}\text { Ala- } \\
\text { chlor } \\
\text { ESA } \\
(\mu \mathrm{g} / \mathrm{L})\end{array}$ & $\begin{array}{l}\text { Ala- } \\
\text { chlor } \\
\text { OXA } \\
(\mu \mathrm{g} / \mathrm{L})\end{array}$ & $\begin{array}{l}\text { Ala- } \\
\text { chlor } \\
\text { SAA } \\
(\mu \mathrm{g} / \mathrm{L})\end{array}$ & $\begin{array}{c}\mathrm{Di}- \\
\text { meth- } \\
\text { enamid } \\
(\mu \mathrm{g} / \mathrm{L})\end{array}$ & $\begin{array}{c}\text { Di- } \\
\text { meth- } \\
\text { enamid } \\
\text { ESA } \\
(\mu \mathrm{g} / \mathrm{L})\end{array}$ & $\begin{array}{c}\text { Di- } \\
\text { meth- } \\
\text { enamid } \\
\text { OXA } \\
(\mu \mathrm{g} / \mathrm{L})\end{array}$ & $\begin{array}{c}\text { Flufen- } \\
\text { acet } \\
(\mu \mathrm{g} / \mathrm{L})\end{array}$ & $\begin{array}{c}\text { Flufen- } \\
\text { acet } \\
\text { ESA } \\
(\mu \mathrm{g} / \mathrm{L})\end{array}$ & $\begin{array}{c}\text { Flufen- } \\
\text { acet } \\
\text { OXA } \\
(\mu \mathrm{g} / \mathrm{L})\end{array}$ & $\begin{array}{c}\text { Meto- } \\
\text { lachlor } \\
(\mu \mathrm{g} / \mathrm{L})\end{array}$ & $\begin{array}{c}\text { Meto- } \\
\text { lachlor } \\
\text { ESA } \\
(\mu \mathrm{g} / \mathrm{L})\end{array}$ & $\begin{array}{c}\text { Meto- } \\
\text { lachlor } \\
\text { OXA } \\
(\mu \mathrm{g} / \mathrm{L})\end{array}$ \\
\hline \multicolumn{20}{|c|}{ Federal-State Cooperative Studies-Continued } \\
\hline \multicolumn{20}{|c|}{ New York hydrologic study (PPN)_Continued } \\
\hline 425030074393901 & Montgomery Co., NY & $06 / 04 / 97$ & $<0.05$ & $<0.05$ & $<0.05$ & -- & $<0.05$ & $<0.05$ & $<0.05$ & -- & -- & -- & -- & -- & -- & -- & $<0.05$ & 11 & 3.6 \\
\hline & & $06 / 11 / 97$ & $<.05$ & $<.05$ & $<.05$ & -- & $<.05$ & $<.05$ & $<.05$ & -- & -- & -- & -- & -- & -- & -- & $<.05$ & 11 & 3.4 \\
\hline & & $06 / 11 / 97$ & $<.05$ & $<.05$ & $<.05$ & -- & $<.05$ & $<.05$ & $<.05$ & -- & -- & -- & -- & -- & -- & -- & $<.05$ & 12 & 3.9 \\
\hline & & 06/18/97 & $<.05$ & $<.05$ & $<.05$ & -- & $<.05$ & $<.05$ & $<.05$ & -- & -- & -- & -- & -- & -- & -- & $<.05$ & 13 & 4.1 \\
\hline & & $06 / 19 / 97$ & $<.05$ & $<.05$ & $<.05$ & -- & $<.05$ & $<.05$ & $<.05$ & -- & -- & -- & -- & -- & -- & -- & $<.05$ & 10 & 2.9 \\
\hline & & 07/01/97 & $<.05$ & $<.05$ & $<.05$ & -- & $<.05$ & $<.05$ & $<.05$ & -- & -- & -- & -- & -- & -- & -- & $<.05$ & 17 & 4.2 \\
\hline & & $07 / 16 / 97$ & $<.05$ & $<.05$ & $<.05$ & -- & $<.05$ & $<.05$ & $<.05$ & -- & -- & -- & -- & -- & -- & -- & .08 & 17 & 5.6 \\
\hline & & $11 / 09 / 97$ & $<.05$ & $<.05$ & $<.05$ & -- & $<.05$ & $<.05$ & $<.05$ & -- & -- & -- & -- & -- & -- & -- & $<.05$ & 23 & 13 \\
\hline & & $11 / 10 / 97$ & $<.05$ & $<.05$ & $<.05$ & -- & $<.05$ & $<.05$ & $<.05$ & -- & -- & -- & -- & -- & -- & -- & $<.05$ & 13 & 3.3 \\
\hline & & $02 / 11 / 98$ & $<.05$ & $<.05$ & $<.05$ & -- & $<.05$ & $<.05$ & $<.05$ & -- & -- & -- & -- & -- & -- & -- & $<.05$ & 7.0 & 2.5 \\
\hline & & 03/05/98 & $<.05$ & $<.05$ & $<.05$ & -- & $<.05$ & $<.05$ & $<.05$ & -- & -- & -- & -- & -- & -- & -- & $<.05$ & 8.2 & 3.7 \\
\hline & & 04/09/98 & $<.05$ & $<.05$ & $<.05$ & -- & $<.05$ & $<.05$ & $<.05$ & -- & -- & -- & -- & -- & -- & -- & $<.05$ & 8.5 & 3.4 \\
\hline & & $05 / 14 / 98$ & $<.05$ & $<.05$ & $<.05$ & -- & $<.05$ & $<.05$ & $<.05$ & -- & -- & -- & -- & -- & -- & -- & $<.05$ & 7.7 & 2.7 \\
\hline & & 05/20/98 & $<.05$ & $<.05$ & $<.05$ & -- & $<.05$ & $<.05$ & $<.05$ & -- & -- & -- & -- & -- & -- & -- & $<.05$ & 11 & 3.7 \\
\hline & & 05/26/98 & $<.05$ & $<.05$ & $<.05$ & -- & $<.05$ & $<.05$ & $<.05$ & -- & -- & -- & -- & -- & -- & -- & $<.05$ & 8.2 & 4.2 \\
\hline & & 06/01/98 & $<.05$ & $<.05$ & $<.05$ & -- & $<.05$ & $<.05$ & $<.05$ & -- & -- & -- & -- & -- & -- & -- & .21 & 9.2 & 4.1 \\
\hline & & $06 / 02 / 98$ & $<.05$ & $<.05$ & $<.05$ & -- & $<.05$ & $<.05$ & $<.05$ & -- & -- & -- & -- & -- & -- & -- & .05 & 16 & 3.8 \\
\hline & & 06/03/98 & $<.05$ & $<.05$ & $<.05$ & -- & $<.05$ & $<.05$ & $<.05$ & -- & -- & -- & -- & -- & -- & -- & .63 & 17 & 5.8 \\
\hline & & 06/08/98 & $<.05$ & $<.05$ & $<.05$ & -- & $<.05$ & $<.05$ & $<.05$ & -- & -- & -- & -- & -- & -- & -- & $<.05$ & 10 & 3.1 \\
\hline & & $06 / 12 / 98$ & $<.05$ & $<.05$ & $<.05$ & -- & $<.05$ & $<.05$ & $<.05$ & -- & -- & -- & -- & -- & -- & -- & $<.05$ & 15 & 3.8 \\
\hline & & $06 / 14 / 98$ & $<.05$ & $<.05$ & $<.05$ & -- & $<.05$ & $<.05$ & $<.05$ & -- & -- & -- & -- & -- & -- & -- & .08 & 12 & 4.1 \\
\hline & & $06 / 15 / 98$ & $<.05$ & $<.05$ & $<.05$ & -- & $<.05$ & $<.05$ & $<.05$ & -- & -- & -- & -- & -- & -- & -- & .09 & 13 & 4.5 \\
\hline & & $06 / 23 / 98$ & $<.05$ & $<.05$ & $<.05$ & -- & $<.05$ & $<.05$ & $<.05$ & -- & -- & -- & -- & -- & -- & -- & $<.05$ & 8.8 & 2.5 \\
\hline & & 06/30/98 & $<.05$ & $<.05$ & $<.05$ & -- & $<.05$ & $<.05$ & $<.05$ & -- & -- & -- & -- & -- & -- & -- & $<.05$ & 15 & 3.9 \\
\hline & & 07/01/98 & $<.05$ & $<.05$ & $<.05$ & -- & $<.05$ & $<.05$ & $<.05$ & -- & -- & -- & -- & -- & -- & -- & $<.05$ & 19 & 6.7 \\
\hline
\end{tabular}


Table 5. Analytical results for selected acetamide herbicides and their degradation products, ethanesulfonic acid, oxanilic acid, and sulfinyl acetic acid, in ground-water samples, 1994-2002.-Continued

[OGRL, U.S. Geological Survey Organic Geochemistry Research Laboratory, Lawrence, Kansas; ESA, ethanesulfonic acid; OXA, oxanilic acid; SAA, sulfinyl acetic acid; --, no data; <, less than]

\begin{tabular}{|c|c|c|c|c|c|c|c|c|c|c|c|c|c|c|c|c|c|c|c|}
\hline Site number & Site location & $\begin{array}{c}\text { Date of } \\
\text { collection } \\
\text { (month/day/ } \\
\text { year) }\end{array}$ & $\begin{array}{l}\text { Aceto- } \\
\text { chlor } \\
(\mu \mathrm{g} / \mathrm{L})\end{array}$ & $\begin{array}{l}\text { Aceto- } \\
\text { chlor } \\
\text { ESA } \\
(\mu \mathrm{g} / \mathrm{L})\end{array}$ & $\begin{array}{l}\text { Aceto- } \\
\text { chlor } \\
\text { OXA } \\
(\mu \mathrm{g} / \mathrm{L})\end{array}$ & $\begin{array}{l}\text { Aceto- } \\
\text { chlor } \\
\text { SAA } \\
(\mu \mathrm{g} / \mathrm{L})\end{array}$ & $\begin{array}{l}\text { Ala- } \\
\text { chlor } \\
(\mu \mathrm{g} / \mathrm{L})\end{array}$ & $\begin{array}{l}\text { Ala- } \\
\text { chlor } \\
\text { ESA } \\
(\mu \mathrm{g} / \mathrm{L})\end{array}$ & $\begin{array}{l}\text { Ala- } \\
\text { chlor } \\
\text { OXA } \\
(\mu \mathrm{g} / \mathrm{L})\end{array}$ & $\begin{array}{l}\text { Ala- } \\
\text { chlor } \\
\text { SAA } \\
(\mu \mathrm{g} / \mathrm{L})\end{array}$ & $\begin{array}{l}\text { Di- } \\
\text { meth- } \\
\text { enamid } \\
(\mu \mathrm{g} / \mathrm{L})\end{array}$ & $\begin{array}{c}\text { Di- } \\
\text { meth- } \\
\text { enamid } \\
\text { ESA } \\
(\mu \mathrm{g} / \mathrm{L})\end{array}$ & $\begin{array}{c}\text { Di- } \\
\text { meth- } \\
\text { enamid } \\
\text { OXA } \\
(\mu \mathrm{g} / \mathrm{L})\end{array}$ & $\begin{array}{c}\text { Flufen- } \\
\text { acet } \\
(\mu \mathrm{g} / \mathrm{L})\end{array}$ & $\begin{array}{c}\text { Flufen- } \\
\text { acet } \\
\text { ESA } \\
(\mu \mathrm{g} / \mathrm{L})\end{array}$ & $\begin{array}{c}\text { Flufen- } \\
\text { acet } \\
\text { OXA } \\
(\mu \mathrm{g} / \mathrm{L})\end{array}$ & $\begin{array}{l}\text { Meto- } \\
\text { lachlor } \\
(\mu \mathrm{g} / \mathrm{L})\end{array}$ & $\begin{array}{l}\text { Meto- } \\
\text { lachlor } \\
\text { ESA } \\
(\mu \mathrm{g} / \mathrm{L})\end{array}$ & $\begin{array}{c}\text { Meto- } \\
\text { lachlor } \\
\text { OXA } \\
(\mu \mathrm{g} / \mathrm{L})\end{array}$ \\
\hline \multicolumn{20}{|c|}{ Federal-State Cooperative Studies-Continued } \\
\hline \multicolumn{20}{|c|}{ New York hydrologic study (PPN)_Continued } \\
\hline \multirow[t]{12}{*}{425030074393901} & Montgomery Co., NY & 07/07/98 & $<0.05$ & $<0.05$ & $<0.05$ & -- & $<0.05$ & $<0.05$ & $<0.05$ & -- & -- & -- & -- & -- & -- & -- & $<0.05$ & 14 & 3.8 \\
\hline & & $10 / 15 / 98$ & $<.05$ & $<.05$ & $<.05$ & -- & $<.05$ & $<.05$ & $<.05$ & -- & -- & -- & -- & -- & -- & -- & .05 & 1.7 & .53 \\
\hline & & 04/06/99 & $<.05$ & $<.05$ & $<.05$ & -- & $<.05$ & $<.05$ & $<.05$ & -- & -- & -- & -- & -- & -- & -- & $<.05$ & 5.2 & 2.3 \\
\hline & & 07/04/99 & $<.05$ & $<.05$ & $<.05$ & -- & $<.05$ & $<.05$ & $<.05$ & -- & -- & -- & -- & -- & -- & -- & .24 & 8.0 & 3.7 \\
\hline & & 07/06/99 & $<.05$ & $<.05$ & $<.05$ & -- & $<.05$ & $<.05$ & $<.05$ & -- & -- & -- & -- & -- & -- & -- & .05 & 4.3 & 4.0 \\
\hline & & 07/07/99 & $<.05$ & $<.05$ & $<.05$ & -- & $<.05$ & $<.05$ & $<.05$ & -- & -- & -- & -- & -- & -- & -- & .10 & 6.5 & 1.8 \\
\hline & & 09/17/99 & $<.05$ & $<.05$ & $<.05$ & -- & $<.05$ & .72 & $<.05$ & -- & -- & -- & -- & -- & -- & -- & .06 & 9.5 & 7.7 \\
\hline & & 09/18/99 & $<.05$ & $<.05$ & $<.05$ & -- & $<.05$ & $<.05$ & $<.05$ & -- & -- & -- & -- & -- & -- & -- & $<.05$ & 8.6 & 3.0 \\
\hline & & 09/19/99 & $<.05$ & $<.05$ & $<.05$ & -- & $<.05$ & $<.05$ & $<.05$ & -- & -- & -- & -- & -- & -- & -- & $<.05$ & 8.1 & 2.8 \\
\hline & & 09/20/99 & $<.05$ & $<.05$ & $<.05$ & -- & $<.05$ & $<.05$ & $<.05$ & -- & -- & -- & -- & -- & -- & -- & $<.05$ & 8.0 & 2.4 \\
\hline & & 09/28/99 & $<.05$ & $<.05$ & $<.05$ & -- & $<.05$ & $<.05$ & $<.05$ & -- & -- & -- & -- & -- & -- & -- & $<.05$ & 8.6 & 2.8 \\
\hline & & $10 / 01 / 99$ & $<.05$ & $<.05$ & $<.05$ & -- & $<.05$ & $<.05$ & $<.05$ & -- & -- & -- & -- & -- & -- & -- & $<.05$ & 9.1 & 2.8 \\
\hline 425031074402302 & Montgomery Co., NY & 07/09/97 & $<.05$ & $<.05$ & $<.05$ & -- & $<.05$ & $<.05$ & $<.05$ & -- & -- & -- & -- & -- & -- & -- & $<.05$ & 15 & 5.8 \\
\hline 425033074400001 & Montgomery Co., NY & 07/09/97 & $<.05$ & $<.05$ & $<.05$ & -- & $<.05$ & $<.05$ & $<.05$ & -- & -- & -- & -- & -- & -- & -- & $<.05$ & .57 & $<.05$ \\
\hline \multirow[t]{12}{*}{425041074393201} & Montgomery Co., NY & $04 / 24 / 97$ & $<.05$ & $<.05$ & $<.05$ & -- & $<.05$ & $<.05$ & $<.05$ & -- & -- & -- & -- & -- & -- & -- & $<.05$ & 6.0 & 1.4 \\
\hline & & $05 / 22 / 97$ & $<.05$ & $<.05$ & $<.05$ & -- & $<.05$ & $<.05$ & $<.05$ & -- & -- & -- & -- & -- & -- & -- & $<.05$ & 5.0 & 1.1 \\
\hline & & 05/28/97 & $<.05$ & $<.05$ & $<.05$ & -- & $<.05$ & $<.05$ & $<.05$ & -- & -- & -- & -- & -- & -- & -- & $<.05$ & 5.7 & 1.2 \\
\hline & & 06/04/97 & $<.05$ & $<.05$ & $<.05$ & -- & $<.05$ & $<.05$ & $<.05$ & -- & -- & -- & -- & -- & -- & -- & $<.05$ & 8.2 & 2.0 \\
\hline & & $06 / 11 / 97$ & $<.05$ & $<.05$ & $<.05$ & -- & $<.05$ & $<.05$ & $<.05$ & -- & -- & -- & -- & -- & -- & -- & $<.05$ & 5.9 & 1.3 \\
\hline & & 06/18/97 & $<.05$ & $<.05$ & $<.05$ & -- & $<.05$ & $<.05$ & $<.05$ & -- & -- & -- & -- & -- & -- & -- & $<.05$ & 8.6 & 1.9 \\
\hline & & 06/19/97 & $<.05$ & $<.05$ & $<.05$ & -- & $<.05$ & $<.05$ & $<.05$ & -- & -- & -- & -- & -- & -- & -- & $<.05$ & 10 & 2.1 \\
\hline & & 07/01/97 & $<.05$ & $<.05$ & $<.05$ & -- & $<.05$ & $<.05$ & $<.05$ & -- & -- & -- & -- & -- & -- & -- & $<.05$ & 10 & 2.9 \\
\hline & & $11 / 09 / 97$ & $<.05$ & $<.05$ & $<.05$ & -- & $<.05$ & $<.05$ & $<.05$ & -- & -- & -- & -- & -- & -- & -- & $<.05$ & 3.3 & 1.1 \\
\hline & & 11/10/97 & $<.05$ & $<.05$ & $<.05$ & -- & $<.05$ & $<.05$ & $<.05$ & -- & -- & -- & -- & -- & -- & -- & $<.05$ & 6.4 & 1.6 \\
\hline & & 02/06/98 & $<.05$ & $<.05$ & $<.05$ & -- & $<.05$ & $<.05$ & $<.05$ & -- & -- & -- & -- & -- & -- & -- & $<.05$ & 4.6 & 1.0 \\
\hline & & 02/11/98 & $<.05$ & $<.05$ & $<.05$ & -- & $<.05$ & .22 & $<.05$ & -- & -- & -- & -- & -- & -- & -- & $<.05$ & 3.6 & .78 \\
\hline
\end{tabular}


Table 5. Analytical results for selected acetamide herbicides and their degradation products, ethanesulfonic acid, oxanilic acid, and sulfinyl acetic acid, in ground-water samples, 1994-2002.-Continued

[OGRL, U.S. Geological Survey Organic Geochemistry Research Laboratory, Lawrence, Kansas; ESA, ethanesulfonic acid; OXA, oxanilic acid; SAA, sulfinyl acetic acid; --, no data; <, less than]

\begin{tabular}{|c|c|c|c|c|c|c|c|c|c|c|c|c|c|c|c|c|c|c|c|}
\hline Site number & Site location & $\begin{array}{c}\text { Date of } \\
\text { collection } \\
\text { (month/day/ } \\
\text { year) }\end{array}$ & $\begin{array}{c}\text { Aceto- } \\
\text { chlor } \\
(\mu \mathrm{g} / \mathrm{L})\end{array}$ & $\begin{array}{l}\text { Aceto- } \\
\text { chlor } \\
\text { ESA } \\
(\mu \mathrm{g} / \mathrm{L})\end{array}$ & $\begin{array}{l}\text { Aceto- } \\
\text { chlor } \\
\text { OXA } \\
(\mu \mathrm{g} / \mathrm{L})\end{array}$ & $\begin{array}{l}\text { Aceto- } \\
\text { chlor } \\
\text { SAA } \\
(\mu \mathrm{g} / \mathrm{L})\end{array}$ & $\begin{array}{l}\text { Ala- } \\
\text { chlor } \\
(\mu \mathrm{g} / \mathrm{L})\end{array}$ & $\begin{array}{l}\text { Ala- } \\
\text { chlor } \\
\text { ESA } \\
(\mu \mathrm{g} / \mathrm{L})\end{array}$ & $\begin{array}{c}\text { Ala- } \\
\text { chlor } \\
\text { OXA } \\
(\mu \mathrm{g} / \mathrm{L})\end{array}$ & $\begin{array}{l}\text { Ala- } \\
\text { chlor } \\
\text { SAA } \\
(\mu \mathrm{g} / \mathrm{L})\end{array}$ & $\begin{array}{l}\text { Di- } \\
\text { meth- } \\
\text { enamid } \\
(\mu \mathrm{g} / \mathrm{L})\end{array}$ & $\begin{array}{c}\text { Di- } \\
\text { meth- } \\
\text { enamid } \\
\text { ESA } \\
(\mu \mathrm{g} / \mathrm{L})\end{array}$ & $\begin{array}{c}\text { Di- } \\
\text { meth- } \\
\text { enamid } \\
\text { OXA } \\
(\mu \mathrm{g} / \mathrm{L})\end{array}$ & $\begin{array}{c}\text { Flufen- } \\
\text { acet } \\
(\mu \mathrm{g} / \mathrm{L})\end{array}$ & $\begin{array}{l}\text { Flufen- } \\
\text { acet } \\
\text { ESA } \\
(\mu \mathrm{g} / \mathrm{L})\end{array}$ & $\begin{array}{c}\text { Flufen- } \\
\text { acet } \\
\text { OXA } \\
(\mu \mathrm{g} / \mathrm{L})\end{array}$ & $\begin{array}{l}\text { Meto- } \\
\text { lachlor } \\
(\mu \mathrm{g} / \mathrm{L})\end{array}$ & $\begin{array}{l}\text { Meto- } \\
\text { lachlor } \\
\text { ESA } \\
(\mu \mathrm{g} / \mathrm{L})\end{array}$ & $\begin{array}{c}\text { Meto- } \\
\text { lachlor } \\
\text { OXA } \\
(\mu \mathrm{g} / \mathrm{L})\end{array}$ \\
\hline \multicolumn{20}{|c|}{ Federal-State Cooperative Studies-Continued } \\
\hline \multicolumn{20}{|c|}{ New York hydrologic study (PPN)_Continued } \\
\hline 425041074393201 & Montgomery Co., NY & 03/05/98 & $<0.05$ & $<0.05$ & $<0.05$ & -- & $<0.50$ & $<0.05$ & $<0.05$ & -- & -- & -- & -- & -- & -- & -- & $<0.05$ & 2.5 & 0.72 \\
\hline & & 04/09/98 & $<.05$ & $<.05$ & $<.05$ & -- & $<.05$ & $<.05$ & $<.05$ & -- & -- & -- & -- & -- & -- & -- & $<.05$ & 4.2 & 1.0 \\
\hline & & 05/14/98 & $<.05$ & $<.05$ & $<.05$ & -- & $<.05$ & $<.05$ & $<.05$ & -- & -- & -- & -- & -- & -- & -- & $<.05$ & 4.5 & 1.0 \\
\hline & & $05 / 20 / 98$ & $<.05$ & $<.05$ & $<.05$ & -- & $<.05$ & $<.05$ & $<.05$ & -- & -- & -- & -- & -- & -- & -- & $<.05$ & 6.6 & 1.4 \\
\hline & & $05 / 26 / 98$ & $<.05$ & $<.05$ & $<.05$ & -- & $<.05$ & $<.05$ & $<.05$ & -- & -- & -- & -- & -- & -- & -- & $<.05$ & 5.2 & 1.8 \\
\hline & & 06/01/98 & $<.05$ & $<.05$ & $<.05$ & -- & $<.05$ & $<.05$ & $<.05$ & -- & -- & -- & -- & -- & -- & -- & .15 & 6.4 & 1.6 \\
\hline & & 06/02/98 & $<.05$ & $<.05$ & $<.05$ & -- & $<.05$ & $<.05$ & $<.05$ & -- & -- & -- & -- & -- & -- & -- & .05 & 5.5 & 1.1 \\
\hline & & 06/03/98 & $<.05$ & $<.05$ & $<.05$ & -- & $<.05$ & $<.05$ & $<.05$ & -- & -- & -- & -- & -- & -- & -- & 7.5 & 6.3 & 3.9 \\
\hline & & 06/08/98 & $<.05$ & $<.05$ & $<.05$ & -- & $<.05$ & $<.05$ & $<.05$ & -- & -- & -- & -- & -- & -- & -- & .14 & 5.5 & 1.1 \\
\hline & & $06 / 15 / 98$ & $<.05$ & $<.05$ & $<.05$ & -- & $<.05$ & $<.05$ & $<.05$ & -- & -- & -- & -- & -- & -- & -- & 21 & 17 & 11 \\
\hline & & $06 / 23 / 98$ & $<.05$ & $<.05$ & $<.05$ & -- & $<.05$ & $<.05$ & $<.05$ & -- & -- & -- & -- & -- & -- & -- & .23 & 6.3 & 2.0 \\
\hline & & 06/30/98 & $<.05$ & $<.05$ & $<.05$ & -- & $<.05$ & $<.05$ & $<.05$ & -- & -- & -- & -- & -- & -- & -- & .52 & .74 & $<.05$ \\
\hline & & 07/01/98 & $<.05$ & $<.05$ & $<.05$ & -- & $<.05$ & $<.05$ & $<.05$ & -- & -- & -- & -- & -- & -- & -- & .08 & 10 & 2.9 \\
\hline & & $07 / 22 / 98$ & $<.05$ & $<.05$ & $<.05$ & -- & $<.05$ & $<.05$ & $<.05$ & -- & -- & -- & -- & -- & -- & -- & .09 & 12 & 2.2 \\
\hline & & 04/06/99 & $<.05$ & $<.05$ & $<.05$ & -- & $<.05$ & $<.05$ & $<.05$ & -- & -- & -- & -- & -- & -- & -- & $<.05$ & 3.9 & 1.4 \\
\hline & & 07/04/99 & $<.05$ & $<.05$ & $<.05$ & -- & $<.05$ & $<.05$ & $<.05$ & -- & -- & -- & -- & -- & -- & -- & .26 & 5.9 & .30 \\
\hline & & 09/17/99 & $<.05$ & $<.05$ & $<.05$ & -- & $<.05$ & $<.05$ & $<.05$ & -- & -- & -- & -- & -- & -- & -- & .33 & 6.1 & 2.5 \\
\hline & & 09/18/99 & $<.05$ & $<.05$ & $<.05$ & -- & $<.05$ & $<.05$ & $<.05$ & -- & -- & -- & -- & -- & -- & -- & .09 & 6.2 & 2.7 \\
\hline & & 09/19/99 & $<.05$ & $<.05$ & $<.05$ & -- & $<.05$ & $<.05$ & $<.05$ & -- & -- & -- & -- & -- & -- & -- & $<.05$ & 5.6 & 1.9 \\
\hline 425045074394401 & Montgomery Co., NY & 07/09/97 & $<.05$ & $<.05$ & $<.05$ & -- & $<.05$ & $<.05$ & $<.05$ & -- & -- & -- & -- & -- & -- & -- & .09 & 11 & 3.2 \\
\hline 425048074393401 & Montgomery Co., NY & 07/09/97 & $<.05$ & $<.05$ & $<.05$ & -- & $<.05$ & $<.05$ & $<.05$ & -- & -- & -- & -- & -- & -- & -- & .19 & .75 & $<.05$ \\
\hline 425056076412201 & Cayuga Co., NY & 08/03/99 & $<.05$ & $<.05$ & $<.05$ & -- & $<.05$ & $<.05$ & $<.05$ & -- & -- & -- & -- & -- & -- & -- & $<.05$ & .67 & $<.05$ \\
\hline 425142078020802 & Genesee Co., NY & 08/03/99 & $<.05$ & $<.05$ & $<.05$ & -- & $<.05$ & $<.05$ & $<.05$ & -- & -- & -- & -- & -- & -- & -- & $<.05$ & 1.5 & .30 \\
\hline 430503078104201 & Genesee Co., NY & 08/03/99 & $<.05$ & $<.05$ & $<.05$ & -- & $<.05$ & .44 & $<.05$ & -- & -- & -- & -- & -- & -- & -- & $<.05$ & 1.3 & .61 \\
\hline
\end{tabular}


Table 5. Analytical results for selected acetamide herbicides and their degradation products, ethanesulfonic acid, oxanilic acid, and sulfinyl acetic acid, in ground-water samples, 1994-2002.-Continued

[OGRL, U.S. Geological Survey Organic Geochemistry Research Laboratory, Lawrence, Kansas; ESA, ethanesulfonic acid; OXA, oxanilic acid; SAA, sulfinyl acetic acid; --, no data; <, less than]

\begin{tabular}{|c|c|c|c|c|c|c|c|c|c|c|c|c|c|c|c|c|c|c|c|c|}
\hline Site number & Site location & $\begin{array}{c}\text { Date of } \\
\text { collection } \\
\text { (month/day/ } \\
\text { year) }\end{array}$ & $\begin{array}{l}\text { Aceto- } \\
\text { chlor } \\
(\mu \mathrm{g} / \mathrm{L})\end{array}$ & $\begin{array}{l}\text { Aceto- } \\
\text { chlor } \\
\text { ESA } \\
(\mu \mathrm{g} / \mathrm{L})\end{array}$ & $\begin{array}{l}\text { Aceto- } \\
\text { chlor } \\
\text { OXA } \\
(\mu \mathrm{g} / \mathrm{L})\end{array}$ & $\begin{array}{l}\text { Aceto- } \\
\text { chlor } \\
\text { SAA } \\
(\mu \mathrm{g} / \mathrm{L})\end{array}$ & $\begin{array}{l}\text { Ala- } \\
\text { chlor } \\
(\mu \mathrm{g} / \mathrm{L})\end{array}$ & $\begin{array}{l}\text { Ala- } \\
\text { chlor } \\
\text { ESA } \\
(\mu \mathrm{g} / \mathrm{L})\end{array}$ & $\begin{array}{l}\text { Ala- } \\
\text { chlor } \\
\text { OXA } \\
(\mu \mathrm{g} / \mathrm{L})\end{array}$ & $\begin{array}{l}\text { Ala- } \\
\text { chlor } \\
\text { SAA } \\
(\mu \mathrm{g} / \mathrm{L})\end{array}$ & $\begin{array}{l}\text { Di- } \\
\text { meth- } \\
\text { enamid } \\
(\mu \mathrm{g} / \mathrm{L})\end{array}$ & $\begin{array}{c}\text { Di- } \\
\text { meth- } \\
\text { enamid } \\
\text { ESA } \\
(\mu \mathrm{g} / \mathrm{L})\end{array}$ & $\begin{array}{c}\mathrm{Di}- \\
\text { meth- } \\
\text { enamid } \\
\text { OXA } \\
(\mu \mathrm{g} / \mathrm{L})\end{array}$ & $\begin{array}{c}\text { Flufen- } \\
\text { acet } \\
(\mu \mathrm{g} / \mathrm{L})\end{array}$ & $\begin{array}{c}\text { Flufen- } \\
\text { acet } \\
\text { ESA } \\
(\mu \mathrm{g} / \mathrm{L})\end{array}$ & $\begin{array}{c}\text { Flufen- } \\
\text { acet } \\
\text { OXA } \\
(\mu \mathrm{g} / \mathrm{L})\end{array}$ & $\begin{array}{l}\text { Meto- } \\
\text { lachlor } \\
(\mu \mathrm{g} / \mathrm{L})\end{array}$ & $\begin{array}{l}\text { Meto- } \\
\text { lachlor } \\
\text { ESA } \\
(\mu \mathrm{g} / \mathrm{L})\end{array}$ & $\begin{array}{l}\text { Meto- } \\
\text { lachlor } \\
\text { OXA } \\
(\mu \mathrm{g} / \mathrm{L})\end{array}$ & 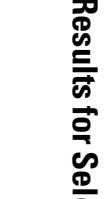 \\
\hline \multicolumn{20}{|c|}{ Federal-State Cooperative Studies-Continued } & \\
\hline \multicolumn{20}{|c|}{ New York hydrologic study (PPN)_Continued } & \\
\hline 430527076453401 & Wayne Co., NY & 08/02/99 & $<0.05$ & $<0.05$ & $<0.05$ & -- & $<0.05$ & 0.75 & $<0.05$ & -- & -- & -- & -- & -- & -- & -- & $<0.05$ & 0.50 & $<0.05$ & \\
\hline \multirow[t]{3}{*}{430647076552901} & \multirow{3}{*}{$\begin{array}{l}\text { Village of Clyde public } \\
\text { supply well, Wayne } \\
\text { Co., NY }\end{array}$} & $10 / 29 / 01$ & -- & $<.05$ & $<.05$ & $<0.05$ & -- & $<.05$ & $<.05$ & $<0.05$ & -- & $<0.05$ & $<0.05$ & -- & $<0.05$ & $<0.05$ & -- & $<.05$ & $<.05$ & \\
\hline & & 10/30/01 & -- & $<.05$ & $<.05$ & $<.05$ & -- & .07 & $<.05$ & $<.05$ & -- & $<.05$ & $<.05$ & -- & $<.05$ & $<.05$ & -- & 2.9 & 1.0 & 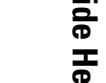 \\
\hline & & $01 / 29 / 02$ & -- & $<.05$ & $<.05$ & $<.05$ & -- & .08 & $<.05$ & $<.05$ & -- & $<.05$ & $<.05$ & -- & $<.05$ & $<.05$ & -- & 2.8 & .96 & \\
\hline \multirow[t]{7}{*}{431815076234001} & \multirow[t]{7}{*}{ Fulton Co., NY } & 06/08/99 & $<.05$ & $<.05$ & $<.05$ & -- & $<.05$ & $<.05$ & $<.05$ & -- & -- & -- & -- & -- & -- & -- & $<.05$ & $<.05$ & $<.05$ & \\
\hline & & $07 / 12 / 99$ & $<.05$ & -- & -- & -- & $<.05$ & -- & -- & -- & -- & -- & -- & -- & -- & -- & $<.05$ & -- & -- & \\
\hline & & 08/02/99 & $<.05$ & $<.05$ & $<.05$ & -- & $<.05$ & $<.05$ & $<.05$ & -- & -- & -- & -- & -- & -- & -- & $<.05$ & $<.05$ & $<.05$ & \\
\hline & & $09 / 14 / 99$ & $<.05$ & $<.05$ & $<.05$ & -- & $<.05$ & $<.05$ & $<.05$ & -- & -- & -- & -- & -- & -- & -- & $<.05$ & $<.05$ & $<.05$ & \\
\hline & & $10 / 13 / 99$ & $<.05$ & $<.05$ & $<.05$ & -- & $<.05$ & $<.05$ & $<.05$ & -- & -- & -- & -- & -- & -- & -- & $<.05$ & $<.05$ & $<.05$ & \\
\hline & & $10 / 18 / 99$ & $<.05$ & $<.05$ & $<.05$ & -- & $<.05$ & $<.05$ & $<.05$ & -- & -- & -- & -- & -- & -- & -- & $<.05$ & $<.05$ & $<.05$ & \\
\hline & & $01 / 11 / 00$ & $<.05$ & $<.05$ & $<.05$ & -- & $<.05$ & $<.05$ & $<.05$ & -- & -- & -- & -- & -- & -- & -- & $<.05$ & $<.05$ & $<.05$ & 堂. \\
\hline \multirow[t]{2}{*}{433418077353401} & Steuben Co., NY & 08/03/99 & $<.05$ & $<.05$ & $<.05$ & -- & $<.05$ & $<.05$ & $<.05$ & -- & -- & -- & -- & -- & -- & -- & $<.05$ & $<.05$ & $<.05$ & \\
\hline & \multicolumn{19}{|c|}{ New York ground-water hydrologic study (PPG) } & \\
\hline \multirow[t]{2}{*}{404237073420401} & \multirow[t]{2}{*}{ Nassau Co., NY } & $05 / 29 / 02$ & -- & $<.05$ & $<.05$ & $<.05$ & -- & $<.05$ & $<.05$ & $<.05$ & -- & $<.05$ & $<.05$ & -- & $<.05$ & $<.05$ & -- & $<.05$ & $<.05$ & \\
\hline & & $09 / 03 / 02$ & -- & $<.05$ & $<.05$ & $<.05$ & -- & $<.05$ & $<.05$ & $<.05$ & -- & $<.05$ & $<.05$ & -- & $<.05$ & $<.05$ & -- & $<.05$ & $<.05$ & \\
\hline 410659074093701 & Rockland Co., NY & $06 / 27 / 00$ & -- & $<.05$ & $<.05$ & -- & -- & $<.05$ & $<.05$ & -- & -- & $<.05$ & $<.05$ & -- & $<.05$ & $<.05$ & -- & $<.05$ & $<.05$ & \\
\hline 411301073515401 & Westchester Co., NY & $06 / 27 / 00$ & -- & $<.05$ & $<.05$ & -- & -- & $<.05$ & $<.05$ & -- & -- & $<.05$ & $<.05$ & -- & $<.05$ & $<.05$ & -- & $<.05$ & $<.05$ & \\
\hline 411736074275101 & Orange Co., NY & $06 / 27 / 00$ & -- & $<.05$ & $<.05$ & -- & -- & $<.05$ & $<.05$ & -- & -- & $<.05$ & $<.05$ & -- & $<.05$ & $<.05$ & -- & .30 & $<.05$ & \\
\hline 413119074150801 & Orange Co., NY & $06 / 27 / 00$ & -- & $<.05$ & $<.05$ & -- & -- & .35 & $<.05$ & -- & -- & $<.05$ & $<.05$ & -- & $<.05$ & $<.05$ & -- & 1.1 & .48 & \\
\hline 413520073511201 & Dutchess Co., NY & $06 / 27 / 00$ & -- & .31 & $<.05$ & -- & -- & $<.05$ & $<.05$ & -- & -- & -- & -- & -- & $<.05$ & $<.05$ & -- & .30 & $<.05$ & 을 을. \\
\hline 414504074355301 & Sullivan Co., NY & $07 / 26 / 00$ & -- & $<.05$ & $<.05$ & -- & -- & $<.05$ & $<.05$ & -- & -- & $<.05$ & $<.05$ & -- & $<.05$ & $<.05$ & -- & .62 & .06 & \\
\hline 420406078241701 & Cattaraugus Co., NY & $06 / 26 / 00$ & -- & $<.05$ & $<.05$ & -- & -- & .23 & $<.05$ & -- & -- & $<.05$ & $<.05$ & -- & $<.05$ & $<.05$ & -- & .42 & $<.05$ & \\
\hline 420647075584201 & Broome Co., NY & $06 / 26 / 00$ & -- & $<.05$ & $<.05$ & -- & -- & .22 & $<.05$ & -- & -- & $<.05$ & $<.05$ & -- & $<.05$ & $<.05$ & -- & .33 & $<.05$ & \\
\hline 420850077030701 & Steuben Co., NY & $06 / 27 / 00$ & -- & $<.05$ & $<.05$ & -- & -- & $<.05$ & $<.05$ & -- & -- & $<.05$ & $<.05$ & -- & $<.05$ & $<.05$ & -- & .26 & .06 & 옹 \\
\hline 420926079360001 & Steuben Co., NY & $06 / 26 / 00$ & -- & $<.05$ & $<.05$ & -- & -- & $<.05$ & $<.05$ & -- & -- & $<.05$ & $<.05$ & -- & $<.05$ & $<.05$ & -- & .27 & $<.05$ & \\
\hline
\end{tabular}


Table 5. Analytical results for selected acetamide herbicides and their degradation products, ethanesulfonic acid, oxanilic acid, and sulfinyl acetic acid, in ground-water samples, 1994-2002.-Continued

[OGRL, U.S. Geological Survey Organic Geochemistry Research Laboratory, Lawrence, Kansas; ESA, ethanesulfonic acid; OXA, oxanilic acid; SAA, sulfinyl acetic acid; --, no data; <, less than]

\begin{tabular}{|c|c|c|c|c|c|c|c|c|c|c|c|c|c|c|c|c|c|c|c|}
\hline Site number & Site location & $\begin{array}{c}\text { Date of } \\
\text { collection } \\
\text { (month/day/ } \\
\text { year) }\end{array}$ & $\begin{array}{l}\text { Aceto- } \\
\text { chlor } \\
(\mu \mathrm{g} / \mathrm{L})\end{array}$ & $\begin{array}{l}\text { Aceto- } \\
\text { chlor } \\
\text { ESA } \\
(\mu \mathrm{g} / \mathrm{L})\end{array}$ & $\begin{array}{c}\text { Aceto- } \\
\text { chlor } \\
\text { OXA } \\
(\mu \mathrm{g} / \mathrm{L})\end{array}$ & $\begin{array}{l}\text { Aceto- } \\
\text { chlor } \\
\text { SAA } \\
(\mu \mathrm{g} / \mathrm{L})\end{array}$ & $\begin{array}{l}\text { Ala- } \\
\text { chlor } \\
(\mu \mathrm{g} / \mathrm{L})\end{array}$ & $\begin{array}{l}\text { Ala- } \\
\text { chlor } \\
\text { ESA } \\
(\mu \mathrm{g} / \mathrm{L})\end{array}$ & $\begin{array}{l}\text { Ala- } \\
\text { chlor } \\
\text { OXA } \\
(\mu \mathrm{g} / \mathrm{L})\end{array}$ & $\begin{array}{l}\text { Ala- } \\
\text { chlor } \\
\text { SAA } \\
(\mu \mathrm{g} / \mathrm{L})\end{array}$ & $\begin{array}{l}\text { Di- } \\
\text { meth- } \\
\text { enamid } \\
(\mu \mathrm{g} / \mathrm{L})\end{array}$ & $\begin{array}{c}\mathrm{Di}- \\
\text { meth- } \\
\text { enamid } \\
\mathrm{ESA} \\
(\mu \mathrm{g} / \mathrm{L})\end{array}$ & $\begin{array}{c}\text { Di- } \\
\text { meth- } \\
\text { enamid } \\
\text { OXA } \\
(\mu \mathrm{g} / \mathrm{L})\end{array}$ & $\begin{array}{l}\text { Flufen- } \\
\text { acet } \\
(\mu \mathrm{g} / \mathrm{L})\end{array}$ & $\begin{array}{l}\text { Flufen- } \\
\text { acet } \\
\text { ESA } \\
(\mu \mathrm{g} / \mathrm{L})\end{array}$ & $\begin{array}{l}\text { Flufen- } \\
\text { acet } \\
\text { OXA } \\
(\mu \mathrm{g} / \mathrm{L})\end{array}$ & $\begin{array}{l}\text { Meto- } \\
\text { lachlor } \\
(\mu \mathrm{g} / \mathrm{L})\end{array}$ & $\begin{array}{l}\text { Meto- } \\
\text { lachlor } \\
\text { ESA } \\
(\mu \mathrm{g} / \mathrm{L})\end{array}$ & $\begin{array}{c}\text { Meto- } \\
\text { lachlor } \\
\text { OXA } \\
(\mu \mathrm{g} / \mathrm{L})\end{array}$ \\
\hline \multicolumn{20}{|c|}{ Federal-State Cooperative Studies-Continued } \\
\hline \multicolumn{20}{|c|}{ New York ground-water hydrologic study (PPG)—Continued } \\
\hline 421404078023001 & Allegheny Co., NY & 06/26/00 & -- & $<0.05$ & $<0.05$ & -- & -- & 0.29 & $<0.05$ & -- & -- & $<0.05$ & $<0.05$ & -- & $<0.05$ & $<0.05$ & -- & 2.9 & 0.17 \\
\hline 421851075235901 & Delaware Co., NY & $06 / 26 / 00$ & -- & $<.05$ & $<.05$ & -- & -- & $<.05$ & $<.05$ & -- & -- & $<.05$ & $<.05$ & -- & $<.05$ & $<.05$ & -- & .26 & $<.05$ \\
\hline 422328073413301 & Columbia Co., NY & 06/27/00 & -- & $<.05$ & $<.05$ & -- & -- & 1.5 & $<.05$ & -- & -- & $<.05$ & $<.05$ & -- & $<.05$ & $<.05$ & -- & 2.4 & .82 \\
\hline 422633076020801 & Cortland Co., NY & 06/26/00 & -- & $<.05$ & $<.05$ & -- & -- & .22 & $<.05$ & -- & -- & $<.05$ & $<.05$ & -- & $<.05$ & $<.05$ & -- & .37 & .06 \\
\hline 422937076531401 & Schuyler Co., NY & 06/28/00 & -- & $<.05$ & $<.05$ & -- & -- & $<.05$ & $<.05$ & -- & -- & $<.05$ & $<.05$ & -- & $<.05$ & $<.05$ & -- & $<.05$ & $<.05$ \\
\hline \multirow[t]{4}{*}{423001077300801} & Steuben Co., NY & 06/27/00 & -- & $<.05$ & $<.05$ & -- & -- & .22 & .26 & -- & -- & $<.05$ & $<.05$ & -- & $<.05$ & $<.05$ & -- & .62 & 1.7 \\
\hline & & 05/07/02 & -- & $<.05$ & $<.05$ & $<0.05$ & -- & .13 & .38 & $<0.05$ & -- & $<.05$ & $<.05$ & -- & $<.05$ & $<.05$ & -- & .95 & 3.6 \\
\hline & & 09/04/02 & -- & $<.05$ & $<.05$ & $<.05$ & -- & .10 & .14 & $<.05$ & -- & $<.05$ & $<.05$ & -- & $<.05$ & $<.05$ & -- & .69 & 1.9 \\
\hline & & $12 / 10 / 02$ & -- & $<.05$ & $<.05$ & $<.05$ & -- & .10 & .20 & $<.05$ & -- & $<.05$ & $<.05$ & -- & $<.05$ & $<.05$ & -- & .92 & .56 \\
\hline 423001078565401 & Erie Co., NY & $06 / 27 / 00$ & -- & $<.05$ & $<.05$ & -- & -- & $<.05$ & $<.05$ & -- & -- & $<.05$ & $<.05$ & -- & $<.05$ & $<.05$ & -- & $<.05$ & $<.05$ \\
\hline 423418077353401 & Steuben Co., NY & 06/27/00 & -- & $<.05$ & $<.05$ & $<.05$ & -- & $<.05$ & $<.05$ & $<.05$ & -- & $<.05$ & $<.05$ & -- & $<.05$ & $<.05$ & -- & .31 & $<.05$ \\
\hline 423454076124001 & Cortland Co., NY & 06/26/00 & -- & $<.05$ & $<.05$ & $<.05$ & -- & $<.05$ & $<.05$ & $<.05$ & -- & $<.05$ & $<.05$ & -- & $<.05$ & $<.05$ & -- & .34 & $<.05$ \\
\hline 423919073553801 & Albany Co., NY & 06/28/00 & -- & $<.05$ & $<.05$ & -- & -- & $<.05$ & $<.05$ & -- & -- & $<.05$ & $<.05$ & -- & $<.05$ & $<.05$ & -- & $<.05$ & $<.05$ \\
\hline \multirow[t]{8}{*}{424025076321001} & Cayuga Co., NY & 05/15/00 & -- & $<.05$ & $<.05$ & -- & -- & $<.05$ & $<.05$ & -- & -- & -- & -- & -- & $<.05$ & $<.05$ & -- & .72 & .22 \\
\hline & & 06/26/00 & -- & $<.05$ & $<.05$ & -- & -- & $<.05$ & $<.05$ & -- & -- & $<.05$ & $<.05$ & -- & $<.05$ & $<.05$ & -- & .40 & $<.05$ \\
\hline & & 07/26/00 & -- & $<.05$ & $<.05$ & -- & -- & $<.05$ & $<.05$ & -- & -- & $<.05$ & $<.05$ & -- & $<.05$ & $<.05$ & -- & .89 & .25 \\
\hline & & 08/31/00 & -- & $<.05$ & $<.05$ & -- & -- & $<.05$ & $<.05$ & -- & -- & $<.05$ & $<.05$ & -- & $<.05$ & $<.05$ & -- & 2.2 & .52 \\
\hline & & 09/27/00 & -- & $<.05$ & $<.05$ & -- & -- & .05 & $<.05$ & -- & -- & $<.05$ & $<.05$ & -- & $<.05$ & $<.05$ & -- & 2.5 & .53 \\
\hline & & 05/08/02 & -- & $<.05$ & $<.05$ & $<.05$ & -- & $<.05$ & $<.05$ & $<.05$ & -- & $<.05$ & $<.05$ & -- & $<.05$ & $<.05$ & -- & .24 & $<.05$ \\
\hline & & 09/04/02 & -- & $<.05$ & $<.05$ & $<.05$ & -- & $<.05$ & $<.05$ & $<.05$ & -- & $<.05$ & $<.05$ & -- & $<.05$ & $<.05$ & -- & 1.1 & .23 \\
\hline & & $12 / 10 / 02$ & -- & $<.05$ & $<.05$ & $<.05$ & -- & $<.05$ & $<.05$ & $<.05$ & -- & $<.05$ & $<.05$ & -- & $<.05$ & $<.05$ & -- & 1.3 & .12 \\
\hline \multirow[t]{4}{*}{424710073473801} & Saratoga Co., NY & 06/26/00 & -- & $<.05$ & $<.05$ & -- & -- & $<.05$ & $<.05$ & -- & -- & -- & -- & -- & $<.05$ & $<.05$ & -- & .56 & $<.05$ \\
\hline & & 08/29/00 & -- & $<.05$ & $<.05$ & -- & -- & $<.05$ & $<.05$ & -- & -- & $<.05$ & $<.05$ & -- & $<.05$ & $<.05$ & -- & .07 & $<.05$ \\
\hline & & $07 / 25 / 00$ & -- & $<.05$ & $<.05$ & -- & -- & $<.05$ & $<.05$ & -- & -- & $<.05$ & $<.05$ & -- & $<.05$ & $<.05$ & -- & $<.05$ & $<.05$ \\
\hline & & 08/29/00 & -- & $<.05$ & $<.05$ & -- & -- & $<.05$ & $<.05$ & -- & -- & $<.05$ & $<.05$ & -- & $<.05$ & $<.05$ & -- & .07 & $<.05$ \\
\hline
\end{tabular}


Table 5. Analytical results for selected acetamide herbicides and their degradation products, ethanesulfonic acid, oxanilic acid, and sulfinyl acetic acid, in ground-water samples, 1994-2002.-Continued

[OGRL, U.S. Geological Survey Organic Geochemistry Research Laboratory, Lawrence, Kansas; ESA, ethanesulfonic acid; OXA, oxanilic acid; SAA, sulfinyl acetic acid; --, no data; <, less than]

\begin{tabular}{|c|c|c|c|c|c|c|c|c|c|c|c|c|c|c|c|c|c|c|c|}
\hline Site number & Site location & $\begin{array}{c}\text { Date of } \\
\text { collection } \\
\text { (month/day/ } \\
\text { year) }\end{array}$ & $\begin{array}{l}\text { Aceto- } \\
\text { chlor } \\
(\mu \mathrm{g} / \mathrm{L})\end{array}$ & $\begin{array}{c}\text { Aceto- } \\
\text { chlor } \\
\text { ESA } \\
(\mu \mathrm{g} / \mathrm{L})\end{array}$ & $\begin{array}{c}\text { Aceto- } \\
\text { chlor } \\
\text { OXA } \\
(\mu \mathrm{g} / \mathrm{L})\end{array}$ & $\begin{array}{c}\text { Aceto- } \\
\text { chlor } \\
\text { SAA } \\
(\mu \mathrm{g} / \mathrm{L})\end{array}$ & $\begin{array}{l}\text { Ala- } \\
\text { chlor } \\
(\mu \mathrm{g} / \mathrm{L})\end{array}$ & $\begin{array}{l}\text { Ala- } \\
\text { chlor } \\
\text { ESA } \\
(\mu \mathrm{g} / \mathrm{L})\end{array}$ & $\begin{array}{l}\text { Ala- } \\
\text { chlor } \\
\text { OXA } \\
(\mu \mathrm{g} / \mathrm{L})\end{array}$ & $\begin{array}{l}\text { Ala- } \\
\text { chlor } \\
\text { SAA } \\
(\mu \mathrm{g} / \mathrm{L})\end{array}$ & $\begin{array}{c}\text { Di- } \\
\text { meth- } \\
\text { enamid } \\
(\mu \mathrm{g} / \mathrm{L})\end{array}$ & $\begin{array}{c}\text { Di- } \\
\text { meth- } \\
\text { enamid } \\
\text { ESA } \\
(\mu \mathrm{g} / \mathrm{L})\end{array}$ & $\begin{array}{c}\text { Di- } \\
\text { meth- } \\
\text { enamid } \\
\text { OXA } \\
(\mu \mathrm{g} / \mathrm{L})\end{array}$ & $\begin{array}{c}\text { Flufen- } \\
\text { acet } \\
(\mu \mathrm{g} / \mathrm{L})\end{array}$ & $\begin{array}{c}\text { Flufen- } \\
\text { acet } \\
\text { ESA } \\
(\mu \mathrm{g} / \mathrm{L})\end{array}$ & $\begin{array}{c}\text { Flufen- } \\
\text { acet } \\
\text { OXA } \\
(\mu \mathrm{g} / \mathrm{L})\end{array}$ & $\begin{array}{c}\text { Meto- } \\
\text { lachlor } \\
(\mu \mathrm{g} / \mathrm{L})\end{array}$ & $\begin{array}{c}\text { Meto- } \\
\text { lachlor } \\
\text { ESA } \\
(\mu \mathrm{g} / \mathrm{L})\end{array}$ & $\begin{array}{c}\text { Meto- } \\
\text { lachlor } \\
\text { OXA } \\
(\mu \mathrm{g} / \mathrm{L})\end{array}$ \\
\hline
\end{tabular}

$$
\text { Federal-State Cooperative Studies-Continued }
$$

$424710073473801 \quad$ Saratoga Co., NY

$424724073472301 \quad$ Saratoga Co., NY

424742074453001 Otsego Co., NY 424748073503401 Schenectady Co., NY

424836074005501 Schenectady Co., NY

$424909073591601 \quad$ Schenectady Co., NY

$424909073591603 \quad$ Schenectady Co., NY

\section{New York ground-water hydrologic study (PPG) —Continued}

\begin{tabular}{|c|c|c|c|c|c|c|c|c|c|c|c|c|c|c|c|c|c|}
\hline $09 / 26 / 00$ & -- & $<0.05$ & $<0.05$ & -- & -- & $<0.05$ & $<0.05$ & -- & -- & $<0.05$ & $<0.05$ & -- & $<0.05$ & $<0.05$ & -- & 0.08 & $<0.05$ \\
\hline 07/31/01 & -- & $<.05$ & $<.05$ & -- & -- & $<.05$ & $<.05$ & -- & -- & $<.05$ & $<.05$ & -- & $<.05$ & $<.05$ & -- & $<.05$ & $<.05$ \\
\hline $10 / 31 / 01$ & -- & $<.05$ & $<.05$ & -- & -- & $<.05$ & $<.05$ & -- & -- & $<.05$ & $<.05$ & -- & $<.05$ & $<.05$ & -- & .06 & $<.05$ \\
\hline $02 / 12 / 02$ & -- & $<.05$ & $<.05$ & $<0.05$ & -- & $<.05$ & $<.05$ & $<0.05$ & -- & $<.05$ & $<.05$ & -- & $<.05$ & $<.05$ & -- & $<.05$ & $<.05$ \\
\hline 08/28/02 & -- & $<.05$ & $<.05$ & $<.05$ & -- & $<.05$ & $<.05$ & $<.05$ & -- & $<.05$ & $<.05$ & -- & $<.05$ & $<.05$ & -- & .05 & $<.05$ \\
\hline 06/26/00 & -- & $<.05$ & $<.05$ & -- & -- & $<.05$ & $<.05$ & -- & -- & -- & -- & -- & $<.05$ & $<.05$ & -- & $<.05$ & $<.05$ \\
\hline $07 / 25 / 00$ & -- & $<.05$ & $<.05$ & -- & -- & $<.05$ & $<.05$ & -- & -- & $<.05$ & $<.05$ & -- & $<.05$ & $<.05$ & -- & $<.05$ & $<.05$ \\
\hline 08/29/00 & -- & $<.05$ & $<.05$ & -- & -- & .05 & $<.05$ & -- & -- & $<.05$ & $<.05$ & -- & $<.05$ & $<.05$ & -- & $<.05$ & $<.05$ \\
\hline 09/26/00 & -- & $<.05$ & $<.05$ & -- & -- & .09 & $<.05$ & -- & -- & $<.05$ & $<.05$ & -- & $<.05$ & $<.05$ & -- & $<.05$ & $<.05$ \\
\hline 07/31/01 & -- & $<.05$ & $<.05$ & -- & -- & $<.05$ & $<.05$ & -- & -- & $<.05$ & $<.05$ & -- & $<.05$ & $<.05$ & -- & $<.05$ & $<.05$ \\
\hline $10 / 31 / 01$ & -- & $<.05$ & $<.05$ & -- & -- & $<.05$ & $<.05$ & -- & -- & $<.05$ & $<.05$ & -- & $<.05$ & $<.05$ & -- & .09 & $<.05$ \\
\hline $02 / 12 / 02$ & -- & $<.05$ & $<.05$ & $<.05$ & -- & $<.05$ & $<.05$ & $<.05$ & -- & $<.05$ & $<.05$ & -- & $<.05$ & $<.05$ & -- & .06 & $<.05$ \\
\hline $06 / 26 / 00$ & -- & $<.05$ & $<.05$ & -- & -- & $<.05$ & $<.05$ & -- & -- & $<.05$ & $<.05$ & -- & $<.05$ & $<.05$ & -- & .36 & $<.05$ \\
\hline 08/26/02 & -- & $<.05$ & $<.05$ & $<.05$ & -- & $<.05$ & $<.05$ & $<.05$ & -- & $<.05$ & $<.05$ & -- & $<.05$ & $<.05$ & -- & $<.05$ & $<.05$ \\
\hline 09/04/02 & -- & $<.05$ & $<.05$ & $<.05$ & -- & $<.05$ & $<.05$ & $<.05$ & -- & $<.05$ & $<.05$ & -- & $<.05$ & $<.05$ & -- & $<.05$ & $<.05$ \\
\hline $05 / 17 / 00$ & -- & $<.05$ & $<.05$ & -- & -- & $<.05$ & $<.05$ & -- & -- & -- & -- & -- & -- & -- & -- & .31 & $<.05$ \\
\hline $07 / 25 / 00$ & -- & $<.05$ & $<.05$ & -- & -- & $<.05$ & $<.05$ & -- & -- & $<.05$ & $<.05$ & -- & $<.05$ & $<.05$ & -- & .07 & $<.05$ \\
\hline 08/29/00 & -- & $<.05$ & $<.05$ & -- & -- & $<.05$ & $<.05$ & -- & -- & $<.05$ & $<.05$ & -- & $<.05$ & $<.05$ & -- & .17 & $<.05$ \\
\hline 09/26/00 & -- & $<.05$ & $<.05$ & -- & -- & $<.05$ & $<.05$ & -- & -- & $<.05$ & $<.05$ & -- & $<.05$ & $<.05$ & -- & .16 & .05 \\
\hline 07/31/01 & -- & $<.05$ & $<.05$ & -- & -- & $<.05$ & $<.05$ & -- & -- & $<.05$ & $<.05$ & -- & $<.05$ & $<.05$ & -- & .09 & $<.05$ \\
\hline $11 / 26 / 01$ & -- & $<.05$ & $<.05$ & -- & -- & $<.05$ & $<.05$ & -- & -- & $<.05$ & $<.05$ & -- & $<.05$ & $<.05$ & -- & .08 & $<.05$ \\
\hline $02 / 12 / 02$ & -- & $<.05$ & $<.05$ & $<.05$ & -- & $<.05$ & $<.05$ & $<.05$ & -- & $<.05$ & $<.05$ & -- & $<.05$ & $<.05$ & -- & .06 & $<.05$ \\
\hline $05 / 21 / 02$ & -- & $<.05$ & $<.05$ & $<.05$ & -- & $<.05$ & $<.05$ & $<.05$ & -- & $<.05$ & $<.05$ & -- & $<.05$ & $<.05$ & -- & .16 & $<.05$ \\
\hline $08 / 21 / 02$ & -- & $<.05$ & $<.05$ & $<.05$ & -- & $<.05$ & $<.05$ & $<.05$ & -- & $<.05$ & $<.05$ & -- & $<.05$ & $<.05$ & -- & .10 & $<.05$ \\
\hline 05/17/00 & -- & $<.05$ & $<.05$ & -- & -- & .21 & $<.05$ & -- & -- & -- & -- & -- & -- & -- & -- & .29 & .05 \\
\hline $06 / 26 / 00$ & -- & $<.05$ & $<.05$ & -- & -- & $<.05$ & $<.05$ & -- & -- & -- & -- & -- & $<.05$ & $<.05$ & -- & .09 & $<.05$ \\
\hline
\end{tabular}


Table 5. Analytical results for selected acetamide herbicides and their degradation products, ethanesulfonic acid, oxanilic acid, and sulfinyl acetic acid, in ground-water samples, 1994-2002.-Continued

[OGRL, U.S. Geological Survey Organic Geochemistry Research Laboratory, Lawrence, Kansas; ESA, ethanesulfonic acid; OXA, oxanilic acid; SAA, sulfinyl acetic acid; --, no data; <, less than]

\begin{tabular}{|c|c|c|c|c|c|c|c|c|c|c|c|c|c|c|c|c|c|c|c|}
\hline Site number & Site location & $\begin{array}{c}\text { Date of } \\
\text { collection } \\
\text { (month/day/ } \\
\text { year) }\end{array}$ & $\begin{array}{c}\text { Aceto- } \\
\text { chlor } \\
(\mu \mathrm{g} / \mathrm{L})\end{array}$ & $\begin{array}{l}\text { Aceto- } \\
\text { chlor } \\
\text { ESA } \\
(\mu \mathrm{g} / \mathrm{L})\end{array}$ & $\begin{array}{l}\text { Aceto- } \\
\text { chlor } \\
\text { OXA } \\
(\mu \mathrm{g} / \mathrm{L})\end{array}$ & $\begin{array}{l}\text { Aceto- } \\
\text { chlor } \\
\text { SAA } \\
(\mu \mathrm{g} / \mathrm{L})\end{array}$ & $\begin{array}{l}\text { Ala- } \\
\text { chlor } \\
(\mu \mathrm{g} / \mathrm{L})\end{array}$ & $\begin{array}{l}\text { Ala- } \\
\text { chlor } \\
\text { ESA } \\
(\mu \mathrm{g} / \mathrm{L})\end{array}$ & $\begin{array}{c}\text { Ala- } \\
\text { chlor } \\
\text { OXA } \\
(\mu \mathrm{g} / \mathrm{L})\end{array}$ & $\begin{array}{c}\text { Ala- } \\
\text { chlor } \\
\text { SAA } \\
(\mu \mathrm{g} / \mathrm{L})\end{array}$ & $\begin{array}{l}\text { Di- } \\
\text { meth- } \\
\text { enamid } \\
(\mu \mathrm{g} / \mathrm{L})\end{array}$ & $\begin{array}{c}\text { Di- } \\
\text { meth- } \\
\text { enamid } \\
\text { ESA } \\
(\mu \mathrm{g} / \mathrm{L})\end{array}$ & $\begin{array}{c}\text { Di- } \\
\text { meth- } \\
\text { enamid } \\
\text { OXA } \\
(\mu \mathrm{g} / \mathrm{L})\end{array}$ & $\begin{array}{c}\text { Flufen- } \\
\text { acet } \\
(\mu \mathrm{g} / \mathrm{L})\end{array}$ & $\begin{array}{c}\text { Flufen- } \\
\text { acet } \\
\text { ESA } \\
(\mu \mathrm{g} / \mathrm{L})\end{array}$ & $\begin{array}{c}\text { Flufen- } \\
\text { acet } \\
\text { OXA } \\
(\mu \mathrm{g} / \mathrm{L})\end{array}$ & $\begin{array}{c}\text { Meto- } \\
\text { lachlor } \\
(\mu \mathrm{g} / \mathrm{L})\end{array}$ & $\begin{array}{c}\text { Meto- } \\
\text { lachlor } \\
\text { ESA } \\
(\mu \mathrm{g} / \mathrm{L})\end{array}$ & $\begin{array}{c}\text { Meto- } \\
\text { lachlor } \\
\text { OXA } \\
(\mu \mathrm{g} / \mathrm{L})\end{array}$ \\
\hline \multicolumn{20}{|c|}{ Federal-State Cooperative Studies-Continued } \\
\hline \multicolumn{20}{|c|}{ New York ground-water hydrologic study (PPG)—Continued } \\
\hline 424909073591603 & Schenectady Co., NY & $06 / 26 / 00$ & -- & $<0.05$ & $<0.05$ & -- & -- & $<0.05$ & $<0.05$ & -- & -- & -- & -- & -- & $<0.05$ & $<0.05$ & -- & 0.35 & $<0.05$ \\
\hline 424918073591001 & Schenectady Co., NY & $08 / 21 / 02$ & -- & $<.05$ & $<.05$ & $<0.05$ & -- & $<.05$ & $<.05$ & $<0.05$ & -- & $<0.05$ & $<0.05$ & -- & $<.05$ & $<.05$ & -- & .09 & $<.05$ \\
\hline 425052073585102 & Schenectady Co., NY & $08 / 26 / 02$ & -- & $<.05$ & $<.05$ & $<.05$ & -- & $<.05$ & $<.05$ & $<.05$ & -- & $<.05$ & $<.05$ & -- & $<.05$ & $<.05$ & -- & $<.05$ & $<.05$ \\
\hline 425056076412201 & Cayuga Co., NY & $06 / 26 / 00$ & -- & $<.05$ & $<.05$ & -- & -- & $<.05$ & $<.05$ & -- & -- & $<.05$ & $<.05$ & -- & $<.05$ & $<.05$ & -- & .71 & .06 \\
\hline 425111074010501 & Schenectady Co., NY & $08 / 26 / 02$ & -- & $<.05$ & $<.05$ & $<.05$ & -- & $<.05$ & $<.05$ & $<.05$ & -- & $<.05$ & $<.05$ & -- & $<.05$ & $<.05$ & -- & .06 & $<.05$ \\
\hline 425142078020802 & Genesee Co., NY & $06 / 27 / 00$ & -- & $<.05$ & $<.05$ & -- & -- & $<.05$ & $<.05$ & -- & -- & $<.05$ & $<.05$ & -- & $<.05$ & $<.05$ & -- & .88 & .10 \\
\hline 425211074021605 & Schenectady Co., NY & $08 / 21 / 02$ & -- & $<.05$ & $<.05$ & $<.05$ & -- & $<.05$ & $<.05$ & $<.05$ & -- & $<.05$ & $<.05$ & -- & $<.05$ & $<.05$ & -- & .06 & $<.05$ \\
\hline 425342075382601 & Madison Co., NY & $06 / 27 / 00$ & -- & $<.05$ & $<.05$ & -- & -- & $<.05$ & $<.05$ & -- & -- & $<.05$ & $<.05$ & -- & $<.05$ & $<.05$ & -- & .21 & $<.05$ \\
\hline 425550074330701 & Mongomery Co., NY & 09/09/02 & -- & $<.05$ & $<.05$ & $<.05$ & -- & .12 & $<.05$ & $<.05$ & -- & $<.05$ & $<.05$ & -- & $<.05$ & $<.05$ & -- & .93 & .92 \\
\hline 425553075283601 & Madison Co., NY & $09 / 25 / 02$ & -- & $<.05$ & $<.05$ & $<.05$ & -- & $<.05$ & $<.05$ & $<.05$ & -- & $<.05$ & $<.05$ & -- & $<.05$ & $<.05$ & -- & $<.05$ & $<.05$ \\
\hline 425747075144501 & Oneida Co., NY & $09 / 25 / 02$ & -- & $<.05$ & $<.05$ & $<.05$ & -- & $<.05$ & $<.05$ & $<.05$ & -- & $<.05$ & $<.05$ & -- & $<.05$ & $<.05$ & -- & $<.05$ & $<.05$ \\
\hline 425824075140101 & Oneida Co., NY & $09 / 25 / 02$ & -- & $<.05$ & $<.05$ & $<.05$ & -- & $<.05$ & $<.05$ & $<.05$ & -- & $<.05$ & $<.05$ & -- & $<.05$ & $<.05$ & -- & .07 & $<.05$ \\
\hline 425853077240801 & Ontario Co., NY & $06 / 26 / 00$ & -- & $<.05$ & $<.05$ & -- & -- & .22 & $<.05$ & -- & -- & $<.05$ & $<.05$ & -- & $<.05$ & $<.05$ & -- & .27 & $<.05$ \\
\hline 425916074175101 & Montgomery Co., NY & $09 / 04 / 02$ & -- & $<.05$ & $<.05$ & $<.05$ & -- & $<.05$ & $<.05$ & $<.05$ & -- & $<.05$ & $<.05$ & -- & $<.05$ & $<.05$ & -- & $<.05$ & $<.05$ \\
\hline 425957074412101 & Montgomery Co., NY & $09 / 09 / 02$ & -- & $<.05$ & $<.05$ & $<.05$ & -- & $<.05$ & $<.05$ & $<.05$ & -- & $<.05$ & $<.05$ & -- & $<.05$ & $<.05$ & -- & $<.05$ & $<.05$ \\
\hline 430047075003001 & Herkimer Co., NY & $09 / 11 / 02$ & -- & $<.05$ & $<.05$ & $<.05$ & -- & $<.05$ & $<.05$ & $<.05$ & -- & $<.05$ & $<.05$ & -- & $<.05$ & $<.05$ & -- & $<.05$ & $<.05$ \\
\hline 430107074591301 & Herkimer Co., NY & $09 / 11 / 02$ & -- & $<.05$ & $<.05$ & $<.05$ & -- & $<.05$ & $<.05$ & $<.05$ & -- & $<.05$ & $<.05$ & -- & $<.05$ & $<.05$ & -- & .08 & .10 \\
\hline 430503078104201 & Genesse Co., NY & $06 / 27 / 00$ & -- & $<.05$ & $<.05$ & -- & -- & .61 & $<.05$ & -- & -- & $<.05$ & $<.05$ & -- & $<.05$ & $<.05$ & -- & 1.3 & .56 \\
\hline 430212075034901 & Herkimer Co., NY & $09 / 11 / 02$ & -- & $<.05$ & $<.05$ & $<.05$ & -- & $<.05$ & $<.05$ & $<.05$ & -- & $<.05$ & $<.05$ & -- & $<.05$ & $<.05$ & -- & $<.05$ & $<.05$ \\
\hline 430527076453401 & Wayne Co., NY & $06 / 27 / 00$ & -- & $<.05$ & $<.05$ & -- & -- & .72 & $<.05$ & -- & -- & $<.05$ & $<.05$ & -- & $<.05$ & $<.05$ & -- & .46 & .05 \\
\hline \multirow[t]{5}{*}{430647076552901} & Wayne Co., NY & $05 / 15 / 00$ & -- & $<.05$ & $<.05$ & -- & -- & .24 & $<.05$ & -- & -- & -- & -- & -- & -- & -- & -- & 2.6 & 1.0 \\
\hline & & $06 / 30 / 00$ & -- & $<.05$ & $<.05$ & -- & -- & $<.05$ & $<.05$ & -- & -- & $<.05$ & $<.05$ & -- & $<.05$ & $<.05$ & -- & 2.5 & .89 \\
\hline & & $07 / 25 / 00$ & -- & $<.05$ & $<.05$ & -- & -- & .07 & $<.05$ & -- & -- & $<.05$ & $<.05$ & -- & $<.05$ & $<.05$ & -- & 2.6 & 1.1 \\
\hline & & $08 / 31 / 00$ & -- & $<.05$ & $<.05$ & -- & -- & $<.05$ & $<.05$ & -- & -- & $<.05$ & $<.05$ & -- & $<.05$ & $<.05$ & -- & 3.1 & 1.1 \\
\hline & & $09 / 28 / 00$ & -- & $<.05$ & $<.05$ & -- & -- & $<.05$ & $<.05$ & -- & -- & $<.05$ & $<.05$ & -- & $<.05$ & $<.05$ & -- & 3.0 & 1.1 \\
\hline
\end{tabular}


Table 5. Analytical results for selected acetamide herbicides and their degradation products, ethanesulfonic acid, oxanilic acid, and sulfinyl acetic acid, in ground-water samples, 1994-2002.-Continued

[OGRL, U.S. Geological Survey Organic Geochemistry Research Laboratory, Lawrence, Kansas; ESA, ethanesulfonic acid; OXA, oxanilic acid; SAA, sulfinyl acetic acid; --, no data; <, less than]

\begin{tabular}{|c|c|c|c|c|c|c|c|c|c|c|c|c|c|c|c|c|c|c|c|c|}
\hline Site number & Site location & $\begin{array}{c}\text { Date of } \\
\text { collection } \\
\text { (month/day/ } \\
\text { year) }\end{array}$ & $\begin{array}{c}\text { Aceto- } \\
\text { chlor } \\
(\mu \mathrm{g} / \mathrm{L})\end{array}$ & $\begin{array}{l}\text { Aceto- } \\
\text { chlor } \\
\text { ESA } \\
(\mu \mathrm{g} / \mathrm{L})\end{array}$ & $\begin{array}{l}\text { Aceto- } \\
\text { chlor } \\
\text { OXA } \\
(\mu \mathrm{g} / \mathrm{L})\end{array}$ & $\begin{array}{l}\text { Aceto- } \\
\text { chlor } \\
\text { SAA } \\
(\mu \mathrm{g} / \mathrm{L})\end{array}$ & $\begin{array}{l}\text { Ala- } \\
\text { chlor } \\
(\mu \mathrm{g} / \mathrm{L})\end{array}$ & $\begin{array}{l}\text { Ala- } \\
\text { chlor } \\
\text { ESA } \\
(\mu \mathrm{g} / \mathrm{L})\end{array}$ & $\begin{array}{l}\text { Ala- } \\
\text { chlor } \\
\text { OXA } \\
(\mu \mathrm{g} / \mathrm{L})\end{array}$ & $\begin{array}{l}\text { Ala- } \\
\text { chlor } \\
\text { SAA } \\
(\mu \mathrm{g} / \mathrm{L})\end{array}$ & $\begin{array}{c}\text { Di- } \\
\text { meth- } \\
\text { enamid } \\
(\mu \mathrm{g} / \mathrm{L})\end{array}$ & $\begin{array}{c}\text { Di- } \\
\text { meth- } \\
\text { enamid } \\
\text { ESA } \\
(\mu \mathrm{g} / \mathrm{L})\end{array}$ & $\begin{array}{c}\text { Di- } \\
\text { meth- } \\
\text { enamid } \\
\text { OXA } \\
(\mu \mathrm{g} / \mathrm{L})\end{array}$ & $\begin{array}{c}\text { Flufen- } \\
\text { acet } \\
(\mu \mathrm{g} / \mathrm{L})\end{array}$ & $\begin{array}{c}\text { Flufen- } \\
\text { acet } \\
\text { ESA } \\
(\mu \mathrm{g} / \mathrm{L})\end{array}$ & $\begin{array}{c}\text { Flufen- } \\
\text { acet } \\
\text { OXA } \\
(\mu \mathrm{g} / \mathrm{L})\end{array}$ & $\begin{array}{c}\text { Meto- } \\
\text { lachlor } \\
(\mu \mathrm{g} / \mathrm{L})\end{array}$ & $\begin{array}{c}\text { Meto- } \\
\text { lachlor } \\
\text { ESA } \\
(\mu \mathrm{g} / \mathrm{L})\end{array}$ & $\begin{array}{c}\text { Meto- } \\
\text { lachlor } \\
\text { OXA } \\
(\mu \mathrm{g} / \mathrm{L})\end{array}$ & 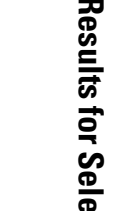 \\
\hline \multicolumn{20}{|c|}{ Federal-State Cooperative Studies-Continued } & \\
\hline \multicolumn{20}{|c|}{ New York ground-water hydrologic study (PPG) —Continued } & \\
\hline 430655075240001 & Oneida Co., NY & 09/04/02 & -- & $<0.05$ & $<0.05$ & $<0.05$ & -- & $<0.05$ & $<0.05$ & $<0.05$ & -- & $<0.05$ & $<0.05$ & -- & $<0.05$ & $<0.05$ & -- & $<0.05$ & $<0.05$ & \\
\hline & & 09/23/02 & -- & $<.05$ & $<.05$ & $<.05$ & -- & $<.05$ & $<.05$ & $<.05$ & -- & $<.05$ & $<.05$ & -- & $<.05$ & $<.05$ & -- & $<.05$ & $<.05$ & \\
\hline 430848074581301 & Herkimer Co., NY & $09 / 16 / 02$ & -- & $<.05$ & $<.05$ & $<.05$ & -- & $<.05$ & $<.05$ & $<.05$ & -- & $<.05$ & $<.05$ & -- & $<.05$ & $<.05$ & -- & $<.05$ & $<.05$ & \\
\hline 431353075041801 & Herkimer Co., NY & $09 / 16 / 02$ & -- & $<.05$ & $<.05$ & $<.05$ & -- & $<.05$ & $<.05$ & $<.05$ & -- & $<.05$ & $<.05$ & -- & $<.05$ & $<.05$ & -- & $<.05$ & $<.05$ & \\
\hline 431815076234001 & Oswego Co., NY & $06 / 27 / 00$ & -- & $<.05$ & $<.05$ & -- & -- & $<.05$ & $<.05$ & -- & -- & $<.05$ & $<.05$ & -- & $<.05$ & $<.05$ & -- & .23 & $<.05$ & \\
\hline 431832075083501 & Oneida Co., NY & 09/18/02 & -- & $<.05$ & $<.05$ & $<.05$ & -- & $<.05$ & $<.05$ & $<.05$ & -- & $<.05$ & $<.05$ & -- & $<.05$ & $<.05$ & -- & $<.05$ & $<.05$ & \\
\hline 432004075105101 & Oneida Co., NY & 09/18/02 & -- & $<.05$ & $<.05$ & $<.05$ & -- & $<.05$ & $<.05$ & $<.05$ & -- & $<.05$ & $<.05$ & -- & $<.05$ & $<.05$ & -- & $<.05$ & $<.05$ & \\
\hline \multicolumn{20}{|c|}{ Wisconsin Department of Agriculture (WDA) } & \\
\hline $13 \mathrm{C}-1$ & Dane Co., WI & $10 / 18 / 99$ & -- & 6.4 & $<.05$ & -- & -- & 7.2 & .55 & -- & -- & -- & -- & -- & -- & -- & -- & 17 & 13 & \\
\hline $13 \mathrm{~J}-1$ & Dane Co., WI & 10/06/99 & -- & 3.0 & $<.05$ & -- & -- & $<.05$ & $<.05$ & -- & -- & -- & -- & -- & -- & -- & -- & 2.0 & $<.05$ & \\
\hline $23 \mathrm{I}-3$ & Green Co., WI & $10 / 07 / 99$ & -- & $<.05$ & .52 & -- & -- & $<.05$ & .14 & -- & -- & -- & -- & -- & -- & -- & -- & 40 & 22 & \\
\hline 27B2 & Juneau Co., WI & $10 / 11 / 99$ & -- & 2.5 & 1.0 & -- & -- & .75 & $<.05$ & -- & -- & -- & -- & -- & -- & -- & -- & 14 & .24 & \\
\hline 29L1 & Juneau Co., WI & $10 / 14 / 99$ & -- & 2.8 & $<.05$ & -- & -- & 1.2 & $<.05$ & -- & -- & -- & -- & -- & -- & -- & -- & 25 & 12 & \\
\hline 29L2 & Juneau Co., WI & $10 / 14 / 99$ & -- & 3.6 & 1.3 & -- & -- & 9.6 & 1.5 & -- & -- & -- & -- & -- & -- & -- & -- & 21 & 14 & \\
\hline 29L3 & Juneau Co., WI & $10 / 14 / 99$ & -- & 5.6 & $<.05$ & -- & -- & 13 & $<.05$ & -- & -- & -- & -- & -- & -- & -- & -- & 19 & 11 & \\
\hline 70G-1 & Waushara Co., WI & $10 / 20 / 99$ & -- & $<.05$ & $<.05$ & -- & -- & 33 & 15 & -- & -- & -- & -- & -- & -- & -- & -- & .55 & .28 & \\
\hline AP729 PW & Juneau Co., WI & $12 / 20 / 99$ & -- & $<.05$ & $<.05$ & -- & -- & 4.7 & 34 & -- & -- & -- & -- & -- & -- & -- & -- & 18 & 23 & \\
\hline AP938 PW & Iowa Co., WI & $11 / 10 / 99$ & -- & $<.05$ & $<.05$ & -- & -- & 3.5 & .74 & -- & -- & -- & -- & -- & & & -- & 7.4 & 4.7 & \\
\hline AR650, Well \#10 & Marathon Co., WI & $04 / 18 / 00$ & -- & $<.05$ & $<.05$ & -- & -- & $<.05$ & $<.05$ & -- & -- & $<.05$ & $<.05$ & -- & -- & -- & -- & $<.05$ & $<.05$ & \\
\hline $\begin{array}{l}\text { AY376, Ellesworth } \\
\text { Well \#2 }\end{array}$ & Pierce Co., WI & $04 / 20 / 00$ & -- & $<.05$ & $<.05$ & -- & -- & .55 & 1.3 & -- & -- & $<.05$ & $<.05$ & -- & -- & -- & -- & .67 & .57 & 름 름 \\
\hline AY379 & Fond Du La Co., WI & $04 / 12 / 00$ & -- & $<.05$ & $<.05$ & -- & -- & $<.05$ & $<.05$ & -- & -- & $<.05$ & $<.05$ & -- & -- & -- & -- & $<.05$ & $<.05$ & 总. \\
\hline BF366 Lodi & Columbia Co., WI & 04/13/00 & -- & $<.05$ & $<.05$ & -- & -- & 1.8 & 1.4 & -- & -- & $<.05$ & $<.05$ & -- & -- & -- & -- & $<.05$ & $<.05$ & \\
\hline BF522 Madison & Dane Co., WI & 04/05/00 & -- & $<.05$ & $<.05$ & -- & -- & $<.05$ & $<.05$ & -- & -- & $<.05$ & $<.05$ & -- & -- & -- & -- & $<.05$ & $<.05$ & 客 \\
\hline BF597 Beaver Dam & Dodge Co., WI & $04 / 12 / 00$ & -- & $<.05$ & $<.05$ & -- & -- & $<.05$ & $<.05$ & -- & -- & $<.05$ & $<.05$ & -- & -- & -- & -- & $<.05$ & $<.05$ & $=$ \\
\hline
\end{tabular}


Table 5. Analytical results for selected acetamide herbicides and their degradation products, ethanesulfonic acid, oxanilic acid, and sulfinyl acetic acid, in ground-water samples, 1994-2002.-Continued

[OGRL, U.S. Geological Survey Organic Geochemistry Research Laboratory, Lawrence, Kansas; ESA, ethanesulfonic acid; OXA, oxanilic acid; SAA, sulfinyl acetic acid; --, no data; <, less than]

\begin{tabular}{|c|c|c|c|c|c|c|c|c|c|c|c|c|c|c|c|c|c|c|c|}
\hline Site number & Site location & $\begin{array}{c}\text { Date of } \\
\text { collection } \\
\text { (month/day/ } \\
\text { year) }\end{array}$ & $\begin{array}{c}\text { Aceto- } \\
\text { chlor } \\
(\mu \mathrm{g} / \mathrm{L})\end{array}$ & $\begin{array}{l}\text { Aceto- } \\
\text { chlor } \\
\text { ESA } \\
(\mu \mathrm{g} / \mathrm{L})\end{array}$ & $\begin{array}{l}\text { Aceto- } \\
\text { chlor } \\
\text { OXA } \\
(\mu \mathrm{g} / \mathrm{L})\end{array}$ & $\begin{array}{l}\text { Aceto- } \\
\text { chlor } \\
\text { SAA } \\
(\mu \mathrm{g} / \mathrm{L})\end{array}$ & $\begin{array}{l}\text { Ala- } \\
\text { chlor } \\
(\mu \mathrm{g} / \mathrm{L})\end{array}$ & $\begin{array}{l}\text { Ala- } \\
\text { chlor } \\
\text { ESA } \\
(\mu \mathrm{g} / \mathrm{L})\end{array}$ & $\begin{array}{l}\text { Ala- } \\
\text { chlor } \\
\text { OXA } \\
(\mu \mathrm{g} / \mathrm{L})\end{array}$ & $\begin{array}{l}\text { Ala- } \\
\text { chlor } \\
\text { SAA } \\
(\mu \mathrm{g} / \mathrm{L})\end{array}$ & $\begin{array}{c}\text { Di- } \\
\text { meth- } \\
\text { enamid } \\
(\mu \mathrm{g} / \mathrm{L})\end{array}$ & $\begin{array}{c}\text { Di- } \\
\text { meth- } \\
\text { enamid } \\
\text { ESA } \\
(\mu \mathrm{g} / \mathrm{L})\end{array}$ & $\begin{array}{c}\text { Di- } \\
\text { meth- } \\
\text { enamid } \\
\text { OXA } \\
(\mu \mathrm{g} / \mathrm{L})\end{array}$ & $\begin{array}{c}\text { Flufen- } \\
\text { acet } \\
(\mu \mathrm{g} / \mathrm{L})\end{array}$ & $\begin{array}{c}\text { Flufen- } \\
\text { acet } \\
\text { ESA } \\
(\mu \mathrm{g} / \mathrm{L})\end{array}$ & $\begin{array}{c}\text { Flufen- } \\
\text { acet } \\
\text { OXA } \\
(\mu \mathrm{g} / \mathrm{L})\end{array}$ & $\begin{array}{c}\text { Meto- } \\
\text { lachlor } \\
(\mu \mathrm{g} / \mathrm{L})\end{array}$ & $\begin{array}{l}\text { Meto- } \\
\text { lachlor } \\
\text { ESA } \\
(\mu \mathrm{g} / \mathrm{L})\end{array}$ & $\begin{array}{c}\text { Meto- } \\
\text { lachlor } \\
\text { OXA } \\
(\mu \mathrm{g} / \mathrm{L})\end{array}$ \\
\hline \multicolumn{20}{|c|}{ Federal-State Cooperative Studies-Continued } \\
\hline \multicolumn{20}{|c|}{ Wisconsin Department of Agriculture (WDA)—Continued } \\
\hline $\begin{array}{l}\text { BF842 Melrose } \\
\text { Well \#3 }\end{array}$ & Jackson Co., WI & $04 / 19 / 00$ & -- & $<0.05$ & $<0.05$ & -- & -- & 1.5 & $<0.05$ & -- & -- & $<0.05$ & $<0.05$ & -- & -- & -- & -- & $<0.05$ & $<0.05$ \\
\hline $\begin{array}{l}\text { BF879 Platteville } \\
\text { Well \#3 }\end{array}$ & Grant Co., WI & $04 / 11 / 00$ & -- & $<.05$ & $<.05$ & -- & -- & $<.05$ & $<.05$ & -- & -- & $<.05$ & $<.05$ & -- & -- & -- & -- & $<.05$ & $<.05$ \\
\hline $\begin{array}{l}\text { BF900 Monroe } \\
\text { Well \#3 }\end{array}$ & Green Co., WI & $04 / 11 / 00$ & -- & $<.05$ & $<.05$ & -- & -- & $<.05$ & $<.05$ & -- & -- & $<.05$ & $<.05$ & -- & -- & -- & -- & $<.05$ & $<.05$ \\
\hline $\begin{array}{l}\text { BG178 Lacrosse } \\
\text { Well \#25 }\end{array}$ & La Crosse Co., WI & $04 / 19 / 00$ & -- & $<.05$ & $<.05$ & -- & -- & $<.05$ & $<.05$ & -- & -- & $<.05$ & $<.05$ & -- & -- & -- & -- & $<.05$ & $<.05$ \\
\hline BG796 Beloit & Rock Co., WI & $04 / 06 / 00$ & -- & $<.05$ & $<.05$ & -- & -- & 1.4 & $<.05$ & -- & -- & $<.05$ & $<.05$ & -- & -- & -- & -- & 60 & .22 \\
\hline $\begin{array}{l}\text { BG865 Wilson } \\
\text { Well \#1 }\end{array}$ & St Croix Co., WI & 04/06/00 & -- & $<.05$ & $<.05$ & -- & -- & $<.05$ & $<.05$ & -- & -- & $<.05$ & $<.05$ & -- & -- & -- & -- & $<.05$ & $<.05$ \\
\hline $\begin{array}{l}\text { BH098 Viroqua } \\
\text { Well \#2 }\end{array}$ & Vernon Co., WI & $04 / 18 / 00$ & -- & $<.05$ & $<.05$ & -- & -- & 3.6 & .14 & -- & -- & $<.05$ & $<.05$ & -- & -- & -- & -- & $<.05$ & $<.05$ \\
\hline BH470 Well \#9 & Waupaca Co., WI & $04 / 24 / 00$ & -- & $<.05$ & $<.05$ & -- & -- & 3.3 & .47 & -- & -- & $<.05$ & $<.05$ & -- & -- & -- & -- & 3.0 & 2.7 \\
\hline $\begin{array}{l}\text { BH562 Marshfield } \\
\text { Well \#20 }\end{array}$ & Wood Co., WI & $04 / 18 / 00$ & -- & $<.05$ & $<.05$ & -- & -- & .20 & $<.05$ & -- & -- & $<.05$ & $<.05$ & -- & -- & -- & -- & .38 & .10 \\
\hline BI079 PW & Adams Co., WI & $01 / 26 / 00$ & -- & 1.3 & $<.05$ & -- & -- & 3.8 & 1.9 & -- & -- & -- & -- & -- & -- & -- & -- & 10 & 7.0 \\
\hline CK495 PW & Outagamie Co., WI & $11 / 9 / 99$ & -- & $<.05$ & $<.05$ & -- & -- & 2.8 & .61 & -- & -- & -- & -- & -- & -- & -- & -- & 10 & .55 \\
\hline DH294 ES & Columbia Co., WI & $11 / 4 / 99$ & -- & $<.05$ & $<.05$ & -- & -- & 8.8 & $<.05$ & -- & -- & -- & -- & -- & -- & -- & -- & 3.5 & .44 \\
\hline DH522 PW & Rock Co., WI & $11 / 16 / 99$ & -- & $<.05$ & $<.05$ & -- & -- & 5.3 & 2.2 & -- & -- & -- & -- & -- & -- & -- & -- & 1.8 & 6.1 \\
\hline DH908 PW & Iowa Co., WI & $11 / 23 / 99$ & -- & $<.05$ & $<.05$ & -- & -- & 1.2 & .13 & -- & -- & -- & -- & -- & -- & -- & -- & .64 & .22 \\
\hline DJ531 PW & Richland Co., WI & $11 / 23 / 99$ & -- & $<.05$ & $<.05$ & -- & -- & $<.05$ & $<.05$ & -- & -- & -- & -- & -- & -- & -- & -- & 15 & 8.3 \\
\hline DN1-2 MW & Dane Co., WI & $10 / 26 / 99$ & -- & $<.05$ & $<.05$ & -- & -- & .10 & .25 & -- & -- & -- & -- & -- & -- & -- & -- & 35 & 21 \\
\hline DT804 Well \#19 & Eau Claire Co., WI & $04 / 20 / 00$ & -- & $<.05$ & $<.05$ & -- & -- & $<.05$ & $<.05$ & -- & -- & $<.05$ & $<.05$ & -- & -- & -- & -- & $<.05$ & $<.05$ \\
\hline ED914 PW & Wood Co., WI & $02 / 01 / 00$ & -- & $<.05$ & $<.05$ & -- & -- & 2.4 & 1.6 & -- & -- & -- & -- & -- & -- & -- & -- & 3.0 & 3.4 \\
\hline EZ981 PW & Portage Co., WI & $02 / 01 / 00$ & -- & .77 & $<.05$ & -- & -- & 2.8 & .38 & -- & -- & -- & -- & -- & -- & -- & -- & .32 & .26 \\
\hline FB374 PW & Juneau Co., WI & $01 / 13 / 00$ & -- & $<.05$ & $<.05$ & -- & -- & 2.5 & 1.4 & -- & -- & -- & -- & -- & -- & -- & -- & .63 & .56 \\
\hline
\end{tabular}


Table 5. Analytical results for selected acetamide herbicides and their degradation products, ethanesulfonic acid, oxanilic acid, and sulfinyl acetic acid, in ground-water samples, 1994-2002.-Continued

[OGRL, U.S. Geological Survey Organic Geochemistry Research Laboratory, Lawrence, Kansas; ESA, ethanesulfonic acid; OXA, oxanilic acid; SAA, sulfinyl acetic acid; --, no data; <, less than]

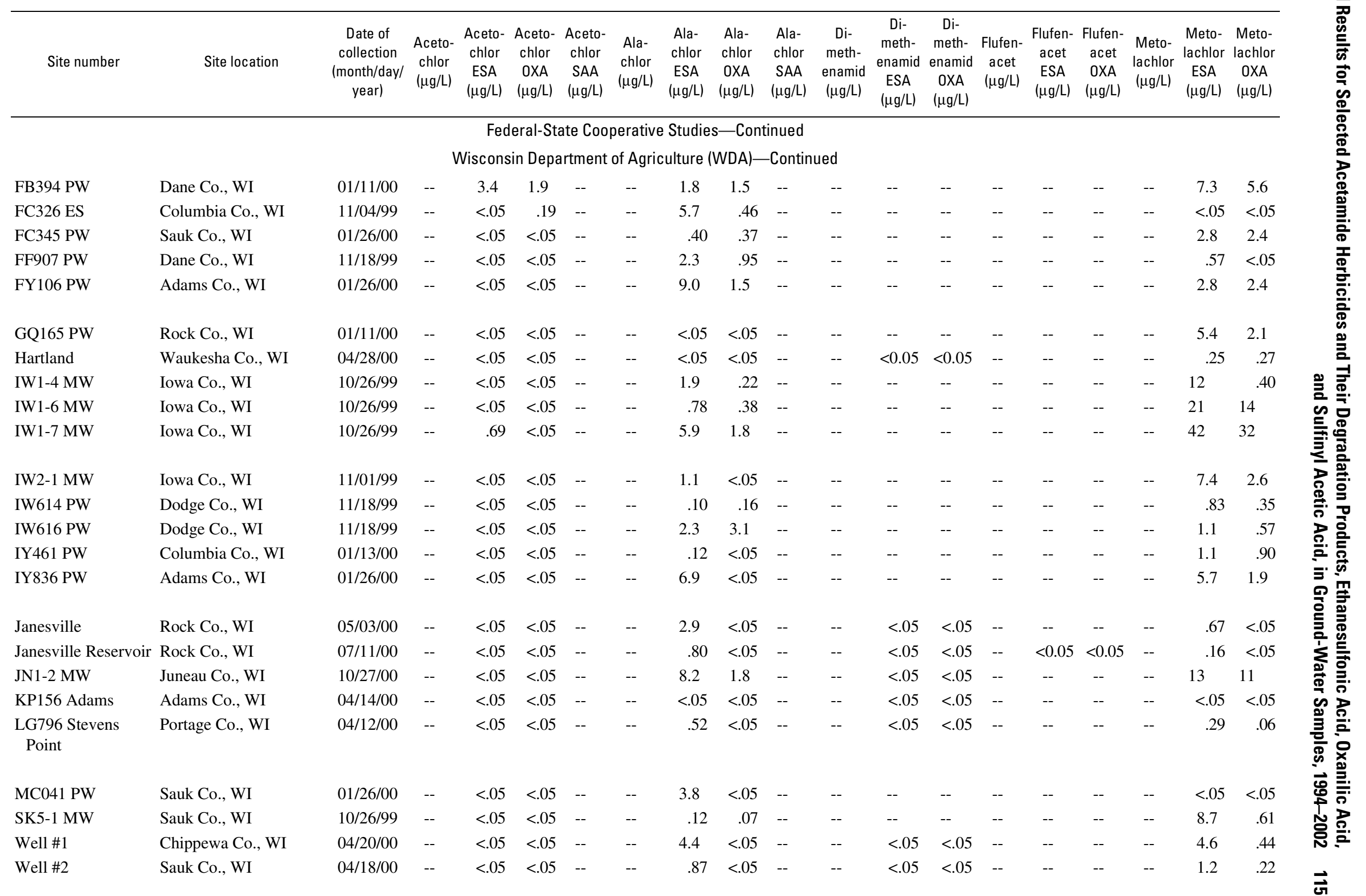


Table 5. Analytical results for selected acetamide herbicides and their degradation products, ethanesulfonic acid, oxanilic acid, and sulfinyl acetic acid, in ground-water samples, 1994-2002.-Continued

[OGRL, U.S. Geological Survey Organic Geochemistry Research Laboratory, Lawrence, Kansas; ESA, ethanesulfonic acid; OXA, oxanilic acid; SAA, sulfinyl acetic acid; --, no data; <, less than]

\begin{tabular}{|c|c|c|c|c|c|c|c|c|c|c|c|c|c|c|c|c|c|c|c|}
\hline Site number & Site location & $\begin{array}{c}\text { Date of } \\
\text { collection } \\
\text { (month/day/ } \\
\text { year) }\end{array}$ & $\begin{array}{l}\text { Aceto- } \\
\text { chlor } \\
(\mu \mathrm{g} / \mathrm{L})\end{array}$ & $\begin{array}{l}\text { Aceto- } \\
\text { chlor } \\
\text { ESA } \\
(\mu \mathrm{g} / \mathrm{L})\end{array}$ & $\begin{array}{l}\text { Aceto- } \\
\text { chlor } \\
\text { OXA } \\
(\mu \mathrm{g} / \mathrm{L})\end{array}$ & $\begin{array}{l}\text { Aceto- } \\
\text { chlor } \\
\text { SAA } \\
(\mu \mathrm{g} / \mathrm{L})\end{array}$ & $\begin{array}{l}\text { Ala- } \\
\text { chlor } \\
(\mu \mathrm{g} / \mathrm{L})\end{array}$ & $\begin{array}{l}\text { Ala- } \\
\text { chlor } \\
\text { ESA } \\
(\mu \mathrm{g} / \mathrm{L})\end{array}$ & $\begin{array}{c}\text { Ala- } \\
\text { chlor } \\
\text { OXA } \\
(\mu \mathrm{g} / \mathrm{L})\end{array}$ & $\begin{array}{l}\text { Ala- } \\
\text { chlor } \\
\text { SAA } \\
(\mu \mathrm{g} / \mathrm{L})\end{array}$ & $\begin{array}{l}\text { Di- } \\
\text { meth- } \\
\text { enamid } \\
(\mu \mathrm{g} / \mathrm{L})\end{array}$ & $\begin{array}{c}\text { Di- } \\
\text { meth- } \\
\text { enamid } \\
\text { ESA } \\
(\mu \mathrm{g} / \mathrm{L})\end{array}$ & $\begin{array}{c}\text { Di- } \\
\text { meth- } \\
\text { enamid } \\
\text { OXA } \\
(\mu \mathrm{g} / \mathrm{L})\end{array}$ & $\begin{array}{c}\text { Flufen- } \\
\text { acet } \\
(\mu \mathrm{g} / \mathrm{L})\end{array}$ & $\begin{array}{l}\text { Flufen- } \\
\text { acet } \\
\text { ESA } \\
(\mu \mathrm{g} / \mathrm{L})\end{array}$ & $\begin{array}{c}\text { Flufen- } \\
\text { acet } \\
\text { OXA } \\
(\mu \mathrm{g} / \mathrm{L})\end{array}$ & $\begin{array}{l}\text { Meto- } \\
\text { lachlor } \\
(\mu \mathrm{g} / \mathrm{L})\end{array}$ & $\begin{array}{c}\text { Meto- } \\
\text { lachlor } \\
\text { ESA } \\
(\mu \mathrm{g} / \mathrm{L})\end{array}$ & $\begin{array}{c}\text { Meto- } \\
\text { lachlor } \\
\text { OXA } \\
(\mu \mathrm{g} / \mathrm{L})\end{array}$ \\
\hline \multicolumn{20}{|c|}{ Federal-State Cooperative Studies-Continued } \\
\hline \multicolumn{20}{|c|}{ Wisconsin Department of Agriculture (WDA) —Continued } \\
\hline Whitewater Well \#9 & Walworth Co., WI & $04 / 26 / 00$ & -- & $<0.05$ & $<0.05$ & -- & -- & $<0.05$ & $<0.05$ & -- & -- & $<0.05$ & $<0.05$ & -- & -- & -- & -- & $<0.05$ & $<0.05$ \\
\hline WI03 ARP & Portage Co., WI & $10 / 19 / 99$ & -- & $<.05$ & $<.05$ & -- & -- & 3.2 & $<.05$ & -- & -- & -- & -- & -- & -- & -- & -- & 5.9 & .07 \\
\hline WI-04 ARP & Portage Co., WI & $10 / 19 / 99$ & -- & $<.05$ & .11 & -- & -- & .44 & $<.05$ & -- & -- & -- & -- & -- & -- & -- & -- & 5.8 & .09 \\
\hline WI-05 ARP & Portage Co., WI & $10 / 19 / 99$ & -- & $<.05$ & $<.05$ & -- & -- & 4.6 & .16 & -- & -- & -- & -- & -- & -- & -- & -- & $<.05$ & $<.05$ \\
\hline WI-06 ARP & Portage Co., WI & $10 / 19 / 99$ & -- & 1.5 & $<.05$ & -- & -- & .87 & $<.05$ & -- & -- & -- & -- & -- & -- & -- & -- & .13 & $<.05$ \\
\hline WI-07 ARI & Waupaca Co., WI & $10 / 19 / 99$ & -- & $<.05$ & $<.05$ & -- & -- & .08 & $<.05$ & -- & -- & -- & -- & -- & -- & -- & -- & $<.05$ & $<.05$ \\
\hline WI-09 ARP & Waupaca Co., WI & $10 / 19 / 99$ & -- & $<.05$ & $<.05$ & -- & -- & 2.9 & $<.05$ & -- & -- & -- & -- & -- & -- & -- & -- & .09 & $<.05$ \\
\hline WI-10 ARP & Waupaca Co., WI & $10 / 19 / 99$ & -- & $<.05$ & $<.05$ & -- & -- & .57 & $<.05$ & -- & -- & -- & -- & -- & -- & -- & -- & .05 & $<.05$ \\
\hline WI-12 ARP & Dodge Co., WI & $10 / 20 / 99$ & -- & .12 & 1.8 & -- & -- & $<.05$ & $<.05$ & -- & -- & -- & -- & -- & -- & -- & -- & $<.05$ & $<.05$ \\
\hline WI-14 ARP & Dodge Co., WI & $10 / 20 / 99$ & -- & $<.05$ & $<.05$ & -- & -- & $<.05$ & $<.05$ & -- & -- & -- & -- & -- & -- & -- & -- & $<.05$ & $<.05$ \\
\hline WI-17 ARP & Jefferson Co., WI & $10 / 20 / 99$ & -- & $<.05$ & $<.05$ & -- & -- & $<.05$ & $<.05$ & -- & -- & -- & -- & -- & -- & -- & -- & $<.05$ & $<.05$ \\
\hline WI-18 ARP & Jefferson Co., WI & $10 / 20 / 99$ & -- & $<.05$ & $<.05$ & -- & -- & 3.8 & $<.05$ & -- & -- & -- & -- & -- & -- & -- & -- & 8.9 & 3.4 \\
\hline WI-24 ARP & Rock Co., WI & $10 / 20 / 99$ & -- & $<.05$ & $<.05$ & -- & -- & 5.0 & $<.05$ & -- & -- & -- & -- & -- & -- & -- & -- & $<.05$ & $<.05$ \\
\hline
\end{tabular}




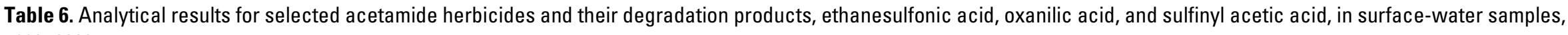
1993-2003.

[OGRL, U.S. Geological Survey Organic Geochemistry Research Laboratory, Lawrence, Kansas; ESA, ethanesulfonic acid; OXA, oxanilic acid; SAA, sulfinyl acetic acid; --, no data; <, less than]

\begin{tabular}{|c|c|c|c|c|c|c|c|c|c|c|c|c|c|c|c|c|c|c|c|}
\hline \multirow[b]{2}{*}{ Site number } & \multirow[b]{2}{*}{ Site location } & \multirow[b]{2}{*}{$\begin{array}{c}\text { Date of } \\
\text { collection } \\
\text { (month/day/ } \\
\text { year) }\end{array}$} & \multicolumn{17}{|c|}{ Concentrations, in micrograms per liter } \\
\hline & & & $\begin{array}{l}\text { Aceto- } \\
\text { chlor } \\
(\mu \mathrm{g} / \mathrm{L})\end{array}$ & $\begin{array}{l}\text { Aceto- } \\
\text { chlor } \\
\text { ESA } \\
(\mu \mathrm{g} / \mathrm{L})\end{array}$ & $\begin{array}{l}\text { Aceto- } \\
\text { chlor } \\
\text { OXA } \\
(\mu \mathrm{g} / \mathrm{L})\end{array}$ & $\begin{array}{l}\text { Aceto- } \\
\text { chlor } \\
\text { SAA } \\
(\mu \mathrm{g} / \mathrm{L})\end{array}$ & $\begin{array}{l}\text { Ala- } \\
\text { chlor } \\
\text { ( } \mu \mathrm{g} / \mathrm{L})\end{array}$ & $\begin{array}{l}\text { Ala- } \\
\text { chlor } \\
\text { ESA } \\
(\mu \mathrm{g} / \mathrm{L})\end{array}$ & $\begin{array}{l}\text { Ala- } \\
\text { chlor } \\
\text { OXA } \\
(\mu \mathrm{g} / \mathrm{L})\end{array}$ & $\begin{array}{l}\text { Ala- } \\
\text { chlor } \\
\text { SAA } \\
(\mu \mathrm{g} / \mathrm{L})\end{array}$ & $\begin{array}{c}\text { Di- } \\
\text { meth- } \\
\text { enamid } \\
(\mu \mathrm{g} / \mathrm{L})\end{array}$ & $\begin{array}{c}\text { Di- } \\
\text { meth- } \\
\text { enamid } \\
\text { ESA } \\
(\mu \mathrm{g} / \mathrm{L})\end{array}$ & $\begin{array}{c}\text { Di- } \\
\text { meth- } \\
\text { enamid } \\
\text { OXA } \\
(\mu \mathrm{g} / \mathrm{L})\end{array}$ & $\begin{array}{c}\text { Flufen- } \\
\text { acet } \\
(\mu \mathrm{g} / \mathrm{L})\end{array}$ & $\begin{array}{c}\text { Flufen- } \\
\text { acet } \\
\text { ESA } \\
(\mu \mathrm{g} / \mathrm{L})\end{array}$ & $\begin{array}{c}\text { Flufen- } \\
\text { acet } \\
0 X A \\
(\mu \mathrm{g} / \mathrm{L})\end{array}$ & $\begin{array}{c}\text { Meto- } \\
\text { lachlor } \\
(\mu \mathrm{g} / \mathrm{L})\end{array}$ & $\begin{array}{c}\text { Meto- } \\
\text { lachlor } \\
\text { ESA } \\
(\mu \mathrm{g} / \mathrm{L})\end{array}$ & $\begin{array}{c}\text { Meto- } \\
\text { lachlor } \\
\text { OXA } \\
(\mu \mathrm{g} / \mathrm{L})\end{array}$ \\
\hline \multicolumn{20}{|c|}{ Midcontinent Agricultural Chemical Research Project (fig. 1) } \\
\hline \multicolumn{20}{|c|}{ Sulfonylurea reconnaissance study (DBD) } \\
\hline \multirow[t]{5}{*}{04186500} & \multirow{5}{*}{$\begin{array}{l}\text { Auglaize River near } \\
\text { Fort Jennings, OH }\end{array}$} & $06 / 17 / 98$ & 1.3 & 4.0 & 4.2 & -- & 0.14 & 2.2 & $<0.20$ & -- & -- & -- & -- & -- & -- & -- & 4.7 & 6.2 & 3.8 \\
\hline & & 07/08/98 & .19 & 1.9 & 1.9 & -- & $<.05$ & 2.0 & $<.20$ & -- & -- & -- & -- & -- & -- & -- & 1.5 & 5.0 & 3.5 \\
\hline & & $05 / 28 / 02$ & .24 & .44 & .30 & $<0.05$ & $<.05$ & .13 & $<.05$ & $<0.05$ & 0.08 & 0.06 & $<0.05$ & $<0.05$ & $<0.05$ & $<0.05$ & .75 & 1.2 & .33 \\
\hline & & $07 / 23 / 02$ & $<.05$ & 2.2 & 2.9 & .57 & $<.05$ & .66 & .71 & .27 & $<.05$ & .14 & .13 & $<.05$ & $<.05$ & $<.05$ & .17 & 1.3 & .83 \\
\hline & & $10 / 30 / 02$ & $<.05$ & .08 & .08 & $<.05$ & $<.05$ & .13 & .05 & $<.05$ & $<.05$ & $<.05$ & $<.05$ & $<.05$ & $<.05$ & $<.05$ & .11 & .25 & .23 \\
\hline \multirow[t]{5}{*}{06882000} & \multirow{5}{*}{$\begin{array}{l}\text { Big Blue River at } \\
\text { Barneston, NE }\end{array}$} & $05 / 15 / 98$ & 1.0 & $<.20$ & $<.20$ & -- & 2.8 & .26 & $<.20$ & -- & -- & -- & -- & -- & -- & -- & 3.8 & .38 & .21 \\
\hline & & 06/09/98 & .57 & .61 & 1.2 & -- & 8.0 & 2.7 & 4.3 & -- & -- & -- & -- & -- & -- & -- & 10 & 1.6 & 1.8 \\
\hline & & 05/07/02 & 4.2 & .44 & .54 & .27 & .38 & .17 & .10 & $<.05$ & .72 & $<.05$ & $<.05$ & .80 & $<.05$ & $<.05$ & 4.3 & .40 & .31 \\
\hline & & $06 / 15 / 02$ & .20 & .23 & .50 & .19 & .11 & .29 & .28 & .07 & .16 & $<.05$ & $<.05$ & .08 & $<.05$ & .13 & 1.6 & .34 & .35 \\
\hline & & $10 / 04 / 02$ & $<.05$ & .08 & .08 & $<.05$ & $<.05$ & .16 & .05 & $<.05$ & $<.05$ & $<.05$ & $<.05$ & $<.05$ & $<.05$ & $<.05$ & $<.05$ & .16 & .10 \\
\hline \multirow[t]{2}{*}{06887000} & \multirow{2}{*}{$\begin{array}{l}\text { Big Blue River near } \\
\text { Manhattan, KS }\end{array}$} & $06 / 04 / 98$ & .16 & $<.20$ & $<.20$ & -- & .35 & .36 & $<.20$ & -- & -- & -- & -- & -- & -- & -- & .74 & .52 & .33 \\
\hline & & $07 / 29 / 98$ & .27 & .56 & .79 & -- & .65 & 1.3 & 2.0 & -- & -- & -- & -- & -- & -- & -- & 2.4 & .96 & 1.4 \\
\hline \multirow[t]{3}{*}{03230500} & \multirow{3}{*}{$\begin{array}{l}\text { Big Darby Creek at } \\
\text { Darbyville, } \mathrm{OH}\end{array}$} & 06/03/02 & .83 & .26 & .48 & .22 & $<.05$ & .05 & $<.05$ & $<.05$ & .48 & .10 & .09 & $<.05$ & $<.05$ & $<.05$ & 3.8 & .91 & .46 \\
\hline & & 08/01/02 & .05 & .71 & .92 & .29 & $<.05$ & .11 & $<.05$ & $<.05$ & $<.05$ & .10 & .06 & $<.05$ & $<.05$ & $<.05$ & .24 & 1.4 & .68 \\
\hline & & $10 / 01 / 02$ & $<.05$ & .57 & .76 & .28 & $<.05$ & .13 & .07 & $<.05$ & $<.05$ & .15 & .14 & $<.05$ & $<.05$ & $<.05$ & .16 & 1.4 & .90 \\
\hline \multirow[t]{5}{*}{06815000} & \multirow{5}{*}{$\begin{array}{l}\text { Big Nemaha River at } \\
\text { Falls City, NE }\end{array}$} & $05 / 26 / 98$ & .36 & $<.20$ & $<.20$ & -- & .20 & .30 & $<.20$ & -- & -- & -- & -- & -- & -- & -- & 1.5 & .52 & .42 \\
\hline & & 06/08/98 & 1.3 & .46 & .69 & -- & .85 & .42 & .42 & -- & -- & -- & -- & -- & -- & -- & 3.1 & .58 & .43 \\
\hline & & $05 / 06 / 02$ & 7.6 & 1.1 & 1.9 & .77 & .98 & .19 & .16 & $<.05$ & .29 & .20 & .23 & .47 & .06 & .05 & .98 & .10 & .07 \\
\hline & & $07 / 12 / 02$ & .07 & .47 & 1.1 & $<.05$ & $<.05$ & .13 & .14 & $<.05$ & $<.05$ & $<.05$ & $<.05$ & $<.05$ & $<.05$ & $<.05$ & .14 & .26 & .25 \\
\hline & & $10 / 04 / 02$ & $<.05$ & .11 & .14 & $<.05$ & $<.05$ & .09 & .05 & $<.05$ & $<.05$ & $<.05$ & $<.05$ & $<.05$ & $<.05$ & $<.05$ & $<.05$ & .09 & .08 \\
\hline \multirow[t]{5}{*}{06885500} & \multirow{5}{*}{$\begin{array}{l}\text { Black Vermillion River } \\
\text { near Frankfort, KS }\end{array}$} & $05 / 05 / 98$ & $<.05$ & $<.20$ & $<.20$ & -- & .05 & .22 & $<.20$ & -- & -- & -- & -- & -- & -- & -- & .11 & .35 & .21 \\
\hline & & 06/09/98 & $<.05$ & $<.20$ & $<.20$ & -- & 1.1 & 1.2 & 1.4 & -- & -- & -- & -- & -- & -- & -- & 8.8 & 1.2 & 1.3 \\
\hline & & 05/07/02 & 1.0 & .10 & .12 & .07 & 1.8 & .24 & .17 & .10 & $<.05$ & $<.05$ & $<.05$ & $<.05$ & $<.05$ & $<.05$ & 2.8 & .41 & .31 \\
\hline & & 08/17/02 & $<.05$ & $<.05$ & $<.05$ & $<.05$ & $<.05$ & .07 & $<.05$ & $<.05$ & $<.05$ & $<.05$ & $<.05$ & $<.05$ & $<.05$ & $<.05$ & $<.05$ & .19 & .14 \\
\hline & & $10 / 03 / 02$ & .28 & .13 & .25 & $<.05$ & .71 & .29 & .49 & $<.05$ & .08 & $<.05$ & $<.05$ & $<.05$ & $<.05$ & $<.05$ & .11 & .10 & .16 \\
\hline
\end{tabular}




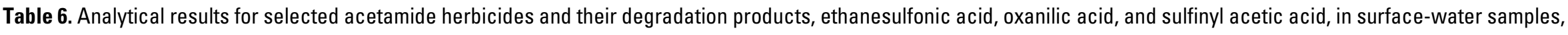
1993-2003-Continued

[OGRL, U.S. Geological Survey Organic Geochemistry Research Laboratory, Lawrence, Kansas; ESA, ethanesulfonic acid; OXA, oxanilic acid; SAA, sulfinyl acetic acid; --, no data; <, less than]

\begin{tabular}{|c|c|c|c|c|c|c|c|c|c|c|c|c|c|c|c|c|c|c|c|}
\hline \multirow[b]{2}{*}{ Site number } & \multirow[b]{2}{*}{ Site location } & \multirow[b]{2}{*}{$\begin{array}{c}\text { Date of } \\
\text { collection } \\
\text { (month/day/ } \\
\text { year) }\end{array}$} & \multicolumn{17}{|c|}{ Concentrations, in micrograms per liter } \\
\hline & & & $\begin{array}{c}\text { Aceto- } \\
\text { chlor } \\
(\mu \mathrm{g} / \mathrm{L})\end{array}$ & $\begin{array}{l}\text { Aceto- } \\
\text { chlor } \\
\text { ESA } \\
(\mu \mathrm{g} / \mathrm{L})\end{array}$ & $\begin{array}{c}\text { Aceto- } \\
\text { chlor } \\
\text { OXA } \\
(\mu \mathrm{g} / \mathrm{L})\end{array}$ & $\begin{array}{l}\text { Aceto- } \\
\text { chlor } \\
\text { SAA } \\
(\mu \mathrm{g} / \mathrm{L})\end{array}$ & $\begin{array}{l}\text { Ala- } \\
\text { chlor } \\
(\mu \mathrm{g} / \mathrm{L})\end{array}$ & $\begin{array}{l}\text { Ala- } \\
\text { chlor } \\
\text { ESA } \\
(\mu \mathrm{g} / \mathrm{L})\end{array}$ & $\begin{array}{l}\text { Ala- } \\
\text { chlor } \\
\text { OXA } \\
(\mu \mathrm{g} / \mathrm{L})\end{array}$ & $\begin{array}{l}\text { Ala- } \\
\text { chlor } \\
\text { SAA } \\
(\mu \mathrm{g} / \mathrm{L})\end{array}$ & $\begin{array}{c}\text { Di- } \\
\text { meth- } \\
\text { enamid } \\
(\mu \mathrm{g} / \mathrm{L})\end{array}$ & $\begin{array}{c}\text { Di- } \\
\text { meth- } \\
\text { enamid } \\
\text { ESA } \\
(\mu \mathrm{g} / \mathrm{L})\end{array}$ & $\begin{array}{c}\text { Di- } \\
\text { meth- } \\
\text { enamid } \\
0 X A \\
(\mu \mathrm{g} / \mathrm{L})\end{array}$ & $\begin{array}{c}\text { Flufen- } \\
\text { acet } \\
(\mu \mathrm{g} / \mathrm{L})\end{array}$ & $\begin{array}{c}\text { Flufen- } \\
\text { acet } \\
\text { ESA } \\
(\mu \mathrm{g} / \mathrm{L})\end{array}$ & $\begin{array}{c}\text { Flufen- } \\
\text { acet } \\
\text { OXA } \\
(\mu \mathrm{g} / \mathrm{L})\end{array}$ & $\begin{array}{l}\text { Meto- } \\
\text { lachlor } \\
(\mu \mathrm{g} / \mathrm{L})\end{array}$ & $\begin{array}{c}\text { Meto- } \\
\text { lachlor } \\
\text { ESA } \\
(\mu \mathrm{g} / \mathrm{L})\end{array}$ & $\begin{array}{c}\text { Meto- } \\
\text { lachlor } \\
\text { OXA } \\
(\mu \mathrm{g} / \mathrm{L})\end{array}$ \\
\hline \multicolumn{20}{|c|}{ Midcontinent Agricultural Chemical Research Project (fig. 1)—Continued } \\
\hline \multirow{6}{*}{03302800} & \multirow{6}{*}{$\begin{array}{l}\text { Blue River at } \\
\text { Fredricksburg, IN }\end{array}$} & & & Sulfon & nylurea & reconne & aissanc & study 1 & (DBD) - & -Contin & & & & & & & & & \\
\hline & & $05 / 27 / 98$ & 0.40 & 0.49 & 0.28 & -- & $<0.05$ & 0.24 & $<0.20$ & -- & -- & -- & -- & -- & -- & -- & 1.1 & 1.0 & 0.26 \\
\hline & & 07/08/98 & .05 & $<.20$ & $<.20$ & -- & $<.05$ & $<.20$ & $<.20$ & -- & -- & -- & -- & -- & -- & -- & .12 & .59 & $<.20$ \\
\hline & & $06 / 06 / 02$ & .82 & .37 & .69 & 0.26 & $<.05$ & $<.05$ & $<.05$ & $<0.05$ & $<0.05$ & $<0.05$ & $<0.05$ & $<0.05$ & $<0.05$ & $<0.05$ & 2.1 & .57 & .32 \\
\hline & & $08 / 21 / 02$ & $<.05$ & $<.05$ & $<.05$ & $<.05$ & $<.05$ & $<.05$ & $<.05$ & $<.05$ & $<.05$ & $<.05$ & $<.05$ & $<.05$ & $<.05$ & $<.05$ & $<.05$ & .22 & .07 \\
\hline & & 09/27/02 & $<.05$ & .06 & .09 & $<.05$ & $<.05$ & .06 & $<.05$ & $<.05$ & $<.05$ & $<.05$ & $<.05$ & $<.05$ & $<.05$ & $<.05$ & .08 & .16 & .11 \\
\hline \multirow[t]{5}{*}{03378000} & \multirow[t]{5}{*}{ Bonpas Creek at Browns, IL } & $05 / 23 / 98$ & 8.4 & 1.5 & 2.3 & -- & .39 & .50 & $<.20$ & -- & -- & -- & -- & -- & -- & -- & 14 & 1.2 & 1.1 \\
\hline & & 07/07/98 & .08 & 1.2 & .92 & -- & $<.05$ & .91 & $<.20$ & -- & -- & -- & -- & -- & -- & -- & .93 & 2.6 & 1.9 \\
\hline & & $06 / 06 / 02$ & .12 & .09 & .06 & $<.05$ & $<.05$ & .12 & $<.05$ & $<.05$ & $<.05$ & $<.05$ & $<.05$ & $<.05$ & $<.05$ & $<.05$ & .24 & 1.1 & .19 \\
\hline & & $06 / 25 / 02$ & 1.5 & .87 & 1.6 & .59 & $<.05$ & .05 & $<.05$ & $<.05$ & .07 & $<.05$ & $<.05$ & .77 & .08 & .18 & 5.4 & 1.5 & 1.2 \\
\hline & & $09 / 25 / 02$ & .07 & .29 & .45 & .16 & $<.05$ & .06 & $<.05$ & $<.05$ & $<.05$ & .06 & $<.05$ & .13 & .08 & .13 & .28 & .47 & .58 \\
\hline \multirow[t]{5}{*}{06609500} & \multirow[t]{5}{*}{ Boyer River at Logan, IA } & $05 / 22 / 98$ & 3.9 & 2.1 & 2.6 & -- & .41 & .70 & $<.20$ & -- & -- & -- & -- & -- & -- & -- & 10 & 1.7 & 1.9 \\
\hline & & 06/09/98 & .64 & .68 & .82 & -- & $<.05$ & .20 & $<.20$ & -- & -- & -- & -- & -- & -- & -- & 2.0 & .76 & .53 \\
\hline & & $05 / 29 / 02$ & 4.5 & .56 & .92 & $<.05$ & $<.05$ & .06 & $<.05$ & $<.05$ & 3.6 & .07 & .08 & .08 & $<.05$ & $<.05$ & 3.0 & .48 & .28 \\
\hline & & $06 / 11 / 02$ & 1.3 & .41 & .86 & .20 & $<.05$ & $<.05$ & $<.05$ & $<.05$ & .75 & .09 & .09 & .25 & $<.05$ & $<.05$ & 9.5 & .87 & .80 \\
\hline & & $11 / 01 / 02$ & $<.05$ & .06 & .06 & $<.05$ & $<.05$ & .09 & .05 & $<.05$ & $<.05$ & $<.05$ & $<.05$ & $<.05$ & $<.05$ & $<.05$ & .16 & .59 & .17 \\
\hline \multirow[t]{2}{*}{06904010} & \multirow{2}{*}{$\begin{array}{l}\text { Chariton River near } \\
\text { Rathburn, IA }\end{array}$} & $06 / 04 / 98$ & .13 & $<.20$ & $<.20$ & -- & $<.05$ & .26 & $<.20$ & -- & -- & -- & -- & -- & -- & -- & .15 & .81 & .38 \\
\hline & & $06 / 29 / 98$ & .23 & .33 & .25 & -- & $<.05$ & .22 & $<.20$ & -- & -- & -- & -- & -- & -- & -- & .34 & .76 & .51 \\
\hline \multirow[t]{5}{*}{03157000} & \multirow{5}{*}{$\begin{array}{l}\text { Clear Creek near } \\
\text { Rockbridge, } \mathrm{OH}\end{array}$} & 06/10/98 & $<.05$ & .50 & $<.20$ & -- & $<.05$ & $<.20$ & $<.20$ & -- & -- & -- & -- & -- & -- & -- & .16 & .91 & .28 \\
\hline & & $06 / 30 / 98$ & .19 & 4.2 & 3.9 & -- & $<.05$ & .81 & $<.20$ & -- & -- & -- & -- & -- & -- & -- & 1.1 & 3.7 & 2.3 \\
\hline & & 06/05/02 & .83 & .87 & .93 & .40 & $<.05$ & $<.05$ & $<.05$ & $<.05$ & .05 & .14 & .06 & $<.05$ & $<.05$ & $<.05$ & .24 & .81 & .24 \\
\hline & & 07/29/02 & $<.05$ & .13 & .07 & $<.05$ & $<.05$ & .05 & $<.05$ & $<.05$ & $<.05$ & $<.05$ & $<.05$ & $<.05$ & $<.05$ & $<.05$ & $<.05$ & .38 & .07 \\
\hline & & $10 / 26 / 02$ & $<.05$ & .11 & .07 & $<.05$ & $<.05$ & .08 & $<.05$ & $<.05$ & $<.05$ & $<.05$ & $<.05$ & $<.05$ & $<.05$ & $<.05$ & $<.05$ & .49 & .12 \\
\hline \multirow[t]{5}{*}{05317000} & \multirow{5}{*}{$\begin{array}{l}\text { Cottonwood River near } \\
\text { New Ulm, MN }\end{array}$} & $05 / 26 / 98$ & .09 & .40 & $<.20$ & -- & $<.05$ & .48 & $<.20$ & -- & -- & -- & -- & -- & -- & -- & .07 & 1.3 & .36 \\
\hline & & $07 / 21 / 98$ & $<.05$ & .65 & .28 & -- & $<.05$ & .79 & $<.20$ & -- & -- & -- & -- & -- & -- & -- & .20 & 1.6 & .46 \\
\hline & & $07 / 21 / 98$ & $<.05$ & .63 & $<.20$ & -- & $<.05$ & .78 & $<.20$ & -- & -- & -- & -- & -- & -- & -- & .20 & 1.4 & .43 \\
\hline & & 05/30/02 & .06 & .21 & .11 & $<.05$ & $<.05$ & .17 & $<.05$ & $<.05$ & $<.05$ & $<.05$ & $<.05$ & $<.05$ & $<.05$ & $<.05$ & $<.05$ & .83 & .09 \\
\hline & & $06 / 25 / 02$ & .24 & 1.3 & 1.1 & .32 & $<.05$ & .15 & $<.05$ & $<.05$ & .18 & .22 & .11 & $<.05$ & $<.05$ & $<.05$ & .13 & .93 & .22 \\
\hline
\end{tabular}




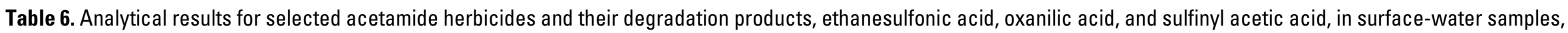
1993-2003.-Continued

[OGRL, U.S. Geological Survey Organic Geochemistry Research Laboratory, Lawrence, Kansas; ESA, ethanesulfonic acid; OXA, oxanilic acid; SAA, sulfinyl acetic acid; --, no data; <, less than]

\begin{tabular}{|c|c|c|c|c|c|c|c|c|c|c|c|c|c|c|c|c|c|c|c|}
\hline \multirow[b]{2}{*}{ Site number } & \multirow[b]{2}{*}{ Site location } & \multirow[b]{2}{*}{$\begin{array}{c}\text { Date of } \\
\text { collection } \\
\text { (month/day/ } \\
\text { year) }\end{array}$} & \multicolumn{17}{|c|}{ Concentrations, in micrograms per liter } \\
\hline & & & $\begin{array}{l}\text { Aceto- } \\
\text { chlor } \\
(\mu \mathrm{g} / \mathrm{L})\end{array}$ & $\begin{array}{l}\text { Aceto- } \\
\text { chlor } \\
\text { ESA } \\
(\mu \mathrm{g} / \mathrm{L})\end{array}$ & $\begin{array}{c}\text { Aceto- } \\
\text { chlor } \\
\text { OXA } \\
(\mu \mathrm{g} / \mathrm{L})\end{array}$ & $\begin{array}{l}\text { Aceto- } \\
\text { chlor } \\
\text { SAA } \\
(\mu \mathrm{g} / \mathrm{L})\end{array}$ & $\begin{array}{l}\text { Ala- } \\
\text { chlor } \\
(\mu \mathrm{g} / \mathrm{L})\end{array}$ & $\begin{array}{l}\text { Ala- } \\
\text { chlor } \\
\text { ESA } \\
(\mu \mathrm{g} / \mathrm{L})\end{array}$ & $\begin{array}{l}\text { Ala- } \\
\text { chlor } \\
\text { OXA } \\
(\mu \mathrm{g} / \mathrm{L})\end{array}$ & $\begin{array}{l}\text { Ala- } \\
\text { chlor } \\
\text { SAA } \\
(\mu \mathrm{g} / \mathrm{L})\end{array}$ & $\begin{array}{c}\text { Di- } \\
\text { meth- } \\
\text { enamid } \\
(\mu \mathrm{g} / \mathrm{L})\end{array}$ & $\begin{array}{c}\text { Di- } \\
\text { meth- } \\
\text { enamid } \\
\text { ESA } \\
(\mu \mathrm{g} / \mathrm{L})\end{array}$ & $\begin{array}{c}\text { Di- } \\
\text { meth- } \\
\text { enamid } \\
\text { OXA } \\
(\mu \mathrm{g} / \mathrm{L})\end{array}$ & $\begin{array}{c}\text { Flufen- } \\
\text { acet } \\
(\mu \mathrm{g} / \mathrm{L})\end{array}$ & $\begin{array}{l}\text { Flufen- } \\
\text { acet } \\
\text { ESA } \\
(\mu \mathrm{g} / \mathrm{L})\end{array}$ & $\begin{array}{c}\text { Flufen- } \\
\text { acet } \\
\text { OXA } \\
(\mu \mathrm{g} / \mathrm{L})\end{array}$ & $\begin{array}{l}\text { Meto- } \\
\text { lachlor } \\
(\mu \mathrm{g} / \mathrm{L})\end{array}$ & $\begin{array}{c}\text { Meto- } \\
\text { lachlor } \\
\text { ESA } \\
(\mu \mathrm{g} / \mathrm{L})\end{array}$ & $\begin{array}{c}\text { Meto- } \\
\text { lachlor } \\
\text { OXA } \\
(\mu \mathrm{g} / \mathrm{L})\end{array}$ \\
\hline \multicolumn{20}{|c|}{ Midcontinent Agricultural Chemical Research Project (fig. 1)—Continued } \\
\hline \multicolumn{20}{|c|}{ Sulfonylurea reconnaissance study (DBD)—Continued } \\
\hline 05317000 & $\begin{array}{l}\text { Cottonwood River near } \\
\text { New Ulm, MN }\end{array}$ & $10 / 04 / 02$ & $<0.05$ & 0.12 & 0.08 & $<0.05$ & $<0.05$ & 0.16 & $<0.05$ & $<0.05$ & $<0.05$ & $<0.05$ & $<0.05$ & $<0.05$ & $<0.05$ & $<0.05$ & $<0.05$ & 0.21 & 0.06 \\
\hline \multirow[t]{5}{*}{06890100} & \multirow{5}{*}{$\begin{array}{l}\text { Delaware River near } \\
\text { Muscotah, KS }\end{array}$} & 05/05/98 & $<.05$ & $<.20$ & $<.20$ & -- & $<.05$ & $<.20$ & $<.20$ & -- & -- & -- & -- & -- & -- & -- & .09 & .38 & $<.20$ \\
\hline & & 06/10/98 & .09 & .36 & $<.20$ & -- & .41 & .37 & $<.20$ & -- & -- & -- & -- & -- & -- & -- & 1.5 & .83 & .61 \\
\hline & & 05/07/02 & 2.2 & .83 & 1.1 & .52 & .51 & .27 & .32 & .21 & .70 & .09 & $<.05$ & .25 & $<.05$ & $<.05$ & 2.5 & .59 & .51 \\
\hline & & $06 / 27 / 02$ & .84 & 1.1 & 1.9 & .85 & .20 & .30 & .30 & .23 & .27 & .16 & .17 & .16 & .13 & .18 & .85 & .64 & .59 \\
\hline & & 09/19/02 & .28 & .08 & .13 & $<.05$ & $<.05$ & .08 & .07 & $<.05$ & $<.05$ & .06 & $<.05$ & $<.05$ & $<.05$ & $<.05$ & $<.05$ & .15 & .14 \\
\hline \multirow[t]{5}{*}{05480500} & \multirow{5}{*}{$\begin{array}{l}\text { Des Moines River at } \\
\text { Fort Dodge, IA }\end{array}$} & 05/16/98 & 12 & 1.8 & $<.20$ & -- & $<.05$ & .52 & $<.20$ & -- & -- & -- & -- & -- & -- & -- & 5.1 & 2.1 & 1.3 \\
\hline & & $06 / 12 / 98$ & .11 & 1.5 & .65 & -- & $<.05$ & .58 & $<.20$ & -- & -- & -- & -- & -- & -- & -- & .22 & 4.4 & .86 \\
\hline & & $05 / 17 / 02$ & .29 & .28 & .21 & $<.05$ & $<.05$ & .11 & $<.05$ & $<.05$ & $<.05$ & $<.05$ & $<.05$ & $<.05$ & $<.05$ & $<.05$ & .11 & 1.8 & .16 \\
\hline & & $06 / 13 / 02$ & .47 & .66 & .95 & .22 & $<.05$ & .08 & $<.05$ & $<.05$ & .05 & $<.05$ & $<.05$ & .06 & $<.05$ & $<.05$ & .52 & 1.8 & .35 \\
\hline & & $10 / 30 / 02$ & $<.05$ & .27 & .15 & $<.05$ & $<.05$ & .21 & $<.05$ & $<.05$ & $<.05$ & $<.05$ & $<.05$ & $<.05$ & $<.05$ & $<.05$ & .05 & 2.0 & .20 \\
\hline \multirow[t]{5}{*}{05476000} & \multirow{5}{*}{$\begin{array}{l}\text { Des Moines River at } \\
\text { Jackson, MN }\end{array}$} & $05 / 26 / 98$ & .39 & .71 & .66 & -- & $<.05$ & .37 & $<.20$ & -- & -- & -- & -- & -- & -- & -- & .07 & 2.0 & .49 \\
\hline & & 08/01/98 & $<.05$ & 1.5 & 1.2 & -- & $<.05$ & .93 & $<.20$ & -- & -- & -- & -- & -- & -- & -- & .13 & 5.0 & 1.2 \\
\hline & & $05 / 31 / 02$ & .05 & .20 & .19 & $<.05$ & $<.05$ & .13 & $<.05$ & $<.05$ & $<.05$ & $<.05$ & $<.05$ & $<.05$ & $<.05$ & $<.05$ & $<.05$ & 1.6 & .14 \\
\hline & & $06 / 25 / 02$ & .08 & .35 & .34 & .10 & $<.05$ & .09 & $<.05$ & $<.05$ & $<.05$ & $<.05$ & $<.05$ & $<.05$ & $<.05$ & $<.05$ & $<.05$ & 1.6 & .14 \\
\hline & & $10 / 08 / 02$ & $<.05$ & .14 & .10 & $<.05$ & $<.05$ & .25 & .05 & $<.05$ & $<.05$ & $<.05$ & $<.05$ & $<.05$ & $<.05$ & $<.05$ & $<.05$ & .55 & .09 \\
\hline \multirow[t]{5}{*}{05540500} & \multirow{5}{*}{$\begin{array}{l}\text { Dupage River near } \\
\text { Shorwood, IL }\end{array}$} & 06/09/98 & .06 & $<.20$ & $<.20$ & -- & $<.05$ & $<.20$ & $<.20$ & -- & -- & -- & -- & -- & -- & -- & .19 & $<.20$ & $<.20$ \\
\hline & & $07 / 29 / 98$ & $<.05$ & 1.4 & $<.20$ & -- & $<.05$ & 1.2 & $<.20$ & -- & -- & -- & -- & -- & -- & -- & $<.05$ & .44 & .36 \\
\hline & & 05/15/02 & 1.3 & .21 & .20 & $<.05$ & $<.05$ & .05 & $<.05$ & $<.05$ & .06 & $<.05$ & $<.05$ & $<.05$ & $<.05$ & $<.05$ & .43 & .35 & .14 \\
\hline & & $06 / 27 / 02$ & $<.05$ & .38 & .34 & .08 & $<.05$ & .06 & $<.05$ & $<.05$ & $<.05$ & $<.05$ & $<.05$ & $<.05$ & $<.05$ & $<.05$ & 1.4 & .61 & .30 \\
\hline & & $09 / 25 / 02$ & $<.05$ & .07 & $<.05$ & $<.05$ & $<.05$ & .10 & $<.05$ & $<.05$ & $<.05$ & $<.05$ & $<.05$ & $<.05$ & $<.05$ & $<.05$ & $<.05$ & .11 & .06 \\
\hline \multirow[t]{5}{*}{03371500} & \multirow{5}{*}{$\begin{array}{l}\text { East Fork White River near } \\
\text { Bedford, IN }\end{array}$} & $05 / 28 / 98$ & 4.4 & 1.7 & 1.9 & -- & .73 & .67 & .40 & -- & -- & -- & -- & -- & -- & -- & 4.7 & 1.5 & 1.0 \\
\hline & & 07/06/98 & .15 & .88 & .60 & -- & .16 & .51 & $<.20$ & -- & -- & -- & -- & -- & -- & -- & .74 & 1.2 & .82 \\
\hline & & 06/06/02 & 1.2 & .33 & .66 & .20 & .18 & .17 & .10 & .07 & $<.05$ & $<.05$ & $<.05$ & $<.05$ & $<.05$ & $<.05$ & 3.0 & .50 & .28 \\
\hline & & $07 / 23 / 02$ & $<.05$ & .27 & .24 & $<.05$ & $<.05$ & .11 & .09 & $<.05$ & $<.05$ & $<.05$ & $<.05$ & $<.05$ & $<.05$ & $<.05$ & .22 & .43 & .28 \\
\hline & & $10 / 01 / 02$ & $<.05$ & .11 & .11 & .05 & $<.05$ & .09 & .05 & $<.05$ & $<.05$ & $<.05$ & $<.05$ & $<.05$ & $<.05$ & $<.05$ & .13 & .29 & .22 \\
\hline
\end{tabular}




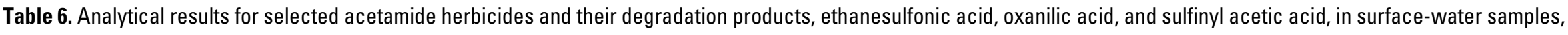
1993-2003-Continued

[OGRL, U.S. Geological Survey Organic Geochemistry Research Laboratory, Lawrence, Kansas; ESA, ethanesulfonic acid; OXA, oxanilic acid; SAA, sulfinyl acetic acid; --, no data; <, less than]

\begin{tabular}{|c|c|c|c|c|c|c|c|c|c|c|c|c|c|c|c|c|c|c|c|}
\hline \multirow[b]{2}{*}{ Site number } & \multirow[b]{2}{*}{ Site location } & \multirow[b]{2}{*}{$\begin{array}{c}\text { Date of } \\
\text { collection } \\
\text { (month/day/ } \\
\text { year) }\end{array}$} & \multicolumn{17}{|c|}{ Concentrations, in micrograms per liter } \\
\hline & & & $\begin{array}{l}\text { Aceto- } \\
\text { chlor } \\
(\mu \mathrm{g} / \mathrm{L})\end{array}$ & $\begin{array}{l}\text { Aceto- } \\
\text { chlor } \\
\text { ESA } \\
(\mu \mathrm{g} / \mathrm{L})\end{array}$ & $\begin{array}{l}\text { Aceto- } \\
\text { chlor } \\
\text { OXA } \\
(\mu \mathrm{g} / \mathrm{L})\end{array}$ & $\begin{array}{l}\text { Aceto- } \\
\text { chlor } \\
\text { SAA } \\
(\mu \mathrm{g} / \mathrm{L})\end{array}$ & $\begin{array}{l}\text { Ala- } \\
\text { chlor } \\
(\mu \mathrm{g} / \mathrm{L})\end{array}$ & $\begin{array}{l}\text { Ala- } \\
\text { chlor } \\
\text { ESA } \\
(\mu \mathrm{g} / \mathrm{L})\end{array}$ & $\begin{array}{l}\text { Ala- } \\
\text { chlor } \\
\text { OXA } \\
(\mu \mathrm{g} / \mathrm{L})\end{array}$ & $\begin{array}{l}\text { Ala- } \\
\text { chlor } \\
\text { SAA } \\
(\mu \mathrm{g} / \mathrm{L})\end{array}$ & $\begin{array}{c}\text { Di- } \\
\text { meth- } \\
\text { enamid } \\
(\mu \mathrm{g} / \mathrm{L})\end{array}$ & $\begin{array}{c}\text { Di- } \\
\text { meth- } \\
\text { enamid } \\
\text { ESA } \\
(\mu \mathrm{g} / \mathrm{L})\end{array}$ & $\begin{array}{c}\text { Di- } \\
\text { meth- } \\
\text { enamid } \\
\text { OXA } \\
(\mu \mathrm{g} / \mathrm{L})\end{array}$ & $\begin{array}{c}\text { Flufen- } \\
\text { acet } \\
(\mu \mathrm{g} / \mathrm{L})\end{array}$ & $\begin{array}{c}\text { Flufen- } \\
\text { acet } \\
\text { ESA } \\
(\mu \mathrm{g} / \mathrm{L})\end{array}$ & $\begin{array}{c}\text { Flufen- } \\
\text { acet } \\
\text { OXA } \\
(\mu \mathrm{g} / \mathrm{L})\end{array}$ & $\begin{array}{l}\text { Meto- } \\
\text { lachlor } \\
\text { ( } \mu \mathrm{g} / \mathrm{L})\end{array}$ & $\begin{array}{l}\text { Meto- } \\
\text { lachlor } \\
\text { ESA } \\
(\mu \mathrm{g} / \mathrm{L})\end{array}$ & $\begin{array}{c}\text { Meto- } \\
\text { lachlor } \\
\text { OXA } \\
(\mu \mathrm{g} / \mathrm{L})\end{array}$ \\
\hline \multicolumn{20}{|c|}{ Midcontinent Agricultural Chemical Research Project (fig. 1)—Continued } \\
\hline \multicolumn{20}{|c|}{ Sulfonylurea reconnaissance study (DBD)—Continued } \\
\hline \multirow[t]{5}{*}{03328500} & \multirow{5}{*}{$\begin{array}{l}\text { Eel River near } \\
\text { Logansport, IN }\end{array}$} & $05 / 21 / 98$ & 2.8 & $<0.20$ & $<0.20$ & -- & 0.06 & 0.53 & $<0.20$ & -- & -- & -- & -- & -- & -- & -- & 2.5 & 1.3 & 1.3 \\
\hline & & 06/30/98 & .22 & 2.2 & 2.1 & -- & .86 & 1.2 & .61 & -- & -- & -- & -- & -- & -- & -- & .52 & 1.4 & .96 \\
\hline & & 06/05/02 & .13 & .16 & .10 & $<0.05$ & $<.05$ & .21 & .06 & $<0.05$ & $<0.05$ & $<0.05$ & $<0.05$ & $<0.05$ & $<0.05$ & $<0.05$ & .10 & .42 & .11 \\
\hline & & 07/30/02 & $<.05$ & .09 & .08 & $<.05$ & $<.05$ & .19 & .05 & $<.05$ & $<.05$ & $<.05$ & $<.05$ & $<.05$ & $<.05$ & $<.05$ & $<.05$ & .26 & .07 \\
\hline & & $12 / 10 / 02$ & $<.05$ & $<.05$ & $<.05$ & $<.05$ & $<.05$ & .11 & $<.05$ & $<.05$ & $<.05$ & $<.05$ & $<.05$ & $<.05$ & $<.05$ & $<.05$ & $<.05$ & .23 & .06 \\
\hline 05461390 & $\begin{array}{l}\text { Flood Creek near } \\
\text { Powersville, MO }\end{array}$ & 06/03/98 & $<.05$ & 1.6 & $<.20$ & -- & $<.05$ & 2.2 & $<.20$ & -- & -- & -- & -- & -- & -- & -- & .18 & 9.9 & 1.7 \\
\hline \multirow[t]{2}{*}{05543500} & \multirow{2}{*}{$\begin{array}{l}\text { Illinois River at } \\
\text { Marseilles, IL }\end{array}$} & $06 / 12 / 98$ & .98 & 1.7 & 1.6 & -- & .05 & 1.0 & .61 & -- & -- & -- & -- & -- & -- & -- & 1.2 & 1.8 & 1.5 \\
\hline & & 07/09/98 & .13 & 1.6 & 1.5 & & $<.05$ & 1.0 & $<.20$ & & -- & -- & -- & -- & -- & -- & .50 & 2.1 & 1.1 \\
\hline \multirow[t]{2}{*}{05586100} & \multirow{2}{*}{$\begin{array}{l}\text { Illinois River at Valley } \\
\text { City, IL }\end{array}$} & 06/18/98 & .98 & 2.3 & 2.2 & -- & .06 & .74 & $<.20$ & -- & -- & -- & -- & -- & -- & -- & 1.6 & 1.9 & 1.4 \\
\hline & & 08/12/98 & $<.05$ & .47 & .30 & -- & $<.05$ & .96 & $<.20$ & -- & -- & -- & -- & -- & -- & -- & .09 & 1.5 & .57 \\
\hline \multirow[t]{2}{*}{05465500} & \multirow[t]{2}{*}{ Iowa River at Wapello, IA } & $05 / 27 / 98$ & .43 & .88 & .43 & -- & .05 & 1.1 & $<.20$ & -- & -- & -- & -- & -- & -- & -- & .65 & 4.0 & .85 \\
\hline & & $06 / 19 / 98$ & .38 & 1.7 & 1.3 & -- & $<.05$ & .98 & $<.20$ & -- & -- & -- & -- & -- & -- & -- & 1.4 & 2.7 & 1.2 \\
\hline \multirow[t]{2}{*}{05449500} & \multirow[t]{2}{*}{ Iowa River near Rowan, IA } & 06/02/98 & .05 & .76 & $<.20$ & -- & $<.05$ & 1.6 & $<.20$ & -- & -- & -- & -- & -- & -- & -- & .15 & 7.0 & 1.1 \\
\hline & & 06/23/98 & .63 & 1.5 & 1.3 & -- & $<.05$ & .88 & $<.20$ & -- & -- & -- & -- & -- & -- & -- & 1.6 & 3.2 & 1.4 \\
\hline \multirow[t]{5}{*}{05526000} & \multirow{5}{*}{$\begin{array}{l}\text { Iroquois River near } \\
\text { Chebanse, IL }\end{array}$} & 06/10/98 & .22 & .90 & .55 & -- & $<.05$ & 1.6 & $<.20$ & -- & -- & -- & -- & -- & -- & -- & .75 & 4.6 & 1.4 \\
\hline & & 07/08/98 & .09 & 1.2 & .89 & -- & $<.05$ & 1.0 & $<.20$ & -- & -- & -- & -- & -- & -- & -- & .91 & 2.9 & 1.5 \\
\hline & & 06/06/02 & .15 & .54 & .21 & .06 & $<.05$ & .97 & .09 & .10 & .05 & .07 & $<.05$ & $<.05$ & $<.05$ & $<.05$ & .09 & 2.4 & .56 \\
\hline & & 06/28/02 & .17 & 1.0 & .94 & .29 & $<.05$ & .42 & .05 & $<.05$ & .21 & .21 & .15 & $<.05$ & $<.05$ & $<.05$ & .25 & 2.1 & .64 \\
\hline & & $09 / 25 / 02$ & $<.05$ & .63 & .25 & $<.05$ & $<.05$ & 1.8 & .17 & $<.05$ & $<.05$ & $<.05$ & $<.05$ & $<.05$ & $<.05$ & $<.05$ & .06 & 2.3 & .83 \\
\hline \multirow[t]{5}{*}{05592100} & \multirow{5}{*}{$\begin{array}{l}\text { Kaskaskia River near } \\
\text { Cowden, IL }\end{array}$} & $05 / 20 / 98$ & .33 & 1.1 & .34 & -- & $<.05$ & .36 & $<.20$ & -- & -- & -- & -- & -- & -- & -- & .41 & 2.5 & .79 \\
\hline & & 07/08/98 & .77 & 1.7 & 1.5 & -- & $<.05$ & .63 & $<.20$ & -- & -- & -- & -- & -- & -- & -- & 1.2 & 2.5 & 1.2 \\
\hline & & $05 / 29 / 02$ & 5.3 & .58 & .57 & .43 & $<.05$ & $<.05$ & $<.05$ & $<.05$ & .37 & $<.05$ & $<.05$ & $<.05$ & $<.05$ & $<.05$ & 4.6 & .75 & .30 \\
\hline & & 06/25/02 & .89 & .64 & .66 & .32 & $<.05$ & .05 & $<.05$ & $<.05$ & .08 & $<.05$ & $<.05$ & $<.05$ & $<.05$ & $<.05$ & 1.1 & .92 & .39 \\
\hline & & $09 / 25 / 02$ & .23 & .37 & .54 & .18 & $<.05$ & .05 & .05 & $<.05$ & .05 & .07 & $<.05$ & $<.05$ & $<.05$ & $<.05$ & .44 & .47 & .30 \\
\hline
\end{tabular}




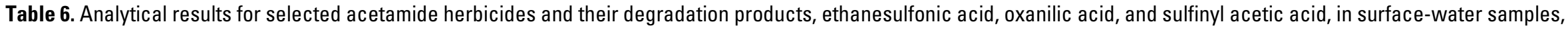
1993-2003.-Continued

[OGRL, U.S. Geological Survey Organic Geochemistry Research Laboratory, Lawrence, Kansas; ESA, ethanesulfonic acid; OXA, oxanilic acid; SAA, sulfinyl acetic acid; --, no data; <, less than]

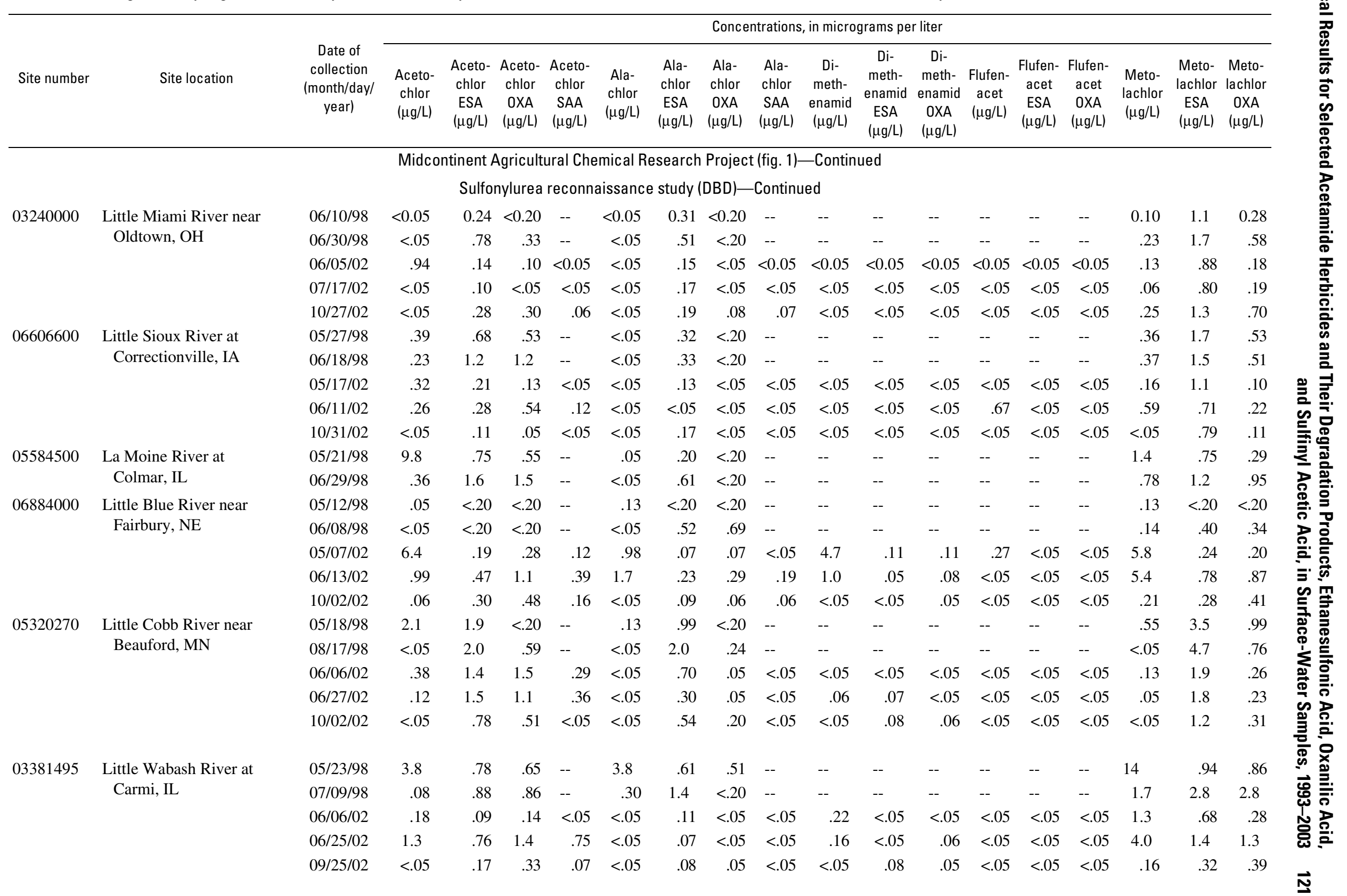




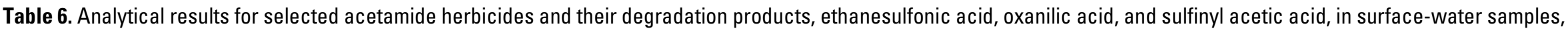
1993-2003-Continued

[OGRL, U.S. Geological Survey Organic Geochemistry Research Laboratory, Lawrence, Kansas; ESA, ethanesulfonic acid; OXA, oxanilic acid; SAA, sulfinyl acetic acid; --, no data; <, less than]

\begin{tabular}{|c|c|c|c|c|c|c|c|c|c|c|c|c|c|c|c|c|c|c|c|}
\hline \multirow[b]{2}{*}{ Site number } & \multirow[b]{2}{*}{ Site location } & \multirow[b]{2}{*}{$\begin{array}{c}\text { Date of } \\
\text { collection } \\
\text { (month/day/ } \\
\text { year) }\end{array}$} & \multicolumn{17}{|c|}{ Concentrations, in micrograms per liter } \\
\hline & & & $\begin{array}{l}\text { Aceto- } \\
\text { chlor } \\
(\mu \mathrm{g} / \mathrm{L})\end{array}$ & $\begin{array}{l}\text { Aceto- } \\
\text { chlor } \\
\text { ESA } \\
(\mu \mathrm{g} / \mathrm{L})\end{array}$ & $\begin{array}{l}\text { Aceto- } \\
\text { chlor } \\
\text { OXA } \\
(\mu \mathrm{g} / \mathrm{L})\end{array}$ & $\begin{array}{l}\text { Aceto- } \\
\text { chlor } \\
\text { SAA } \\
(\mu \mathrm{g} / \mathrm{L})\end{array}$ & $\begin{array}{l}\text { Ala- } \\
\text { chlor } \\
(\mu \mathrm{g} / \mathrm{L})\end{array}$ & $\begin{array}{l}\text { Ala- } \\
\text { chlor } \\
\text { ESA } \\
(\mu \mathrm{g} / \mathrm{L})\end{array}$ & $\begin{array}{c}\text { Ala- } \\
\text { chlor } \\
\text { OXA } \\
(\mu \mathrm{g} / \mathrm{L})\end{array}$ & $\begin{array}{l}\text { Ala- } \\
\text { chlor } \\
\text { SAA } \\
(\mu \mathrm{g} / \mathrm{L})\end{array}$ & $\begin{array}{l}\text { Di- } \\
\text { meth- } \\
\text { enamid } \\
(\mu \mathrm{g} / \mathrm{L})\end{array}$ & $\begin{array}{c}\text { Di- } \\
\text { meth- } \\
\text { enamid } \\
\text { ESA } \\
(\mu \mathrm{g} / \mathrm{L})\end{array}$ & $\begin{array}{c}\text { Di- } \\
\text { meth- } \\
\text { enamid } \\
\text { OXA } \\
(\mu \mathrm{g} / \mathrm{L})\end{array}$ & $\begin{array}{c}\text { Flufen- } \\
\text { acet } \\
(\mu \mathrm{g} / \mathrm{L})\end{array}$ & $\begin{array}{c}\text { Flufen- } \\
\text { acet } \\
\text { ESA } \\
(\mu \mathrm{g} / \mathrm{L})\end{array}$ & $\begin{array}{c}\text { Flufen- } \\
\text { acet } \\
\text { OXA } \\
(\mu \mathrm{g} / \mathrm{L})\end{array}$ & $\begin{array}{l}\text { Meto- } \\
\text { lachlor } \\
(\mu \mathrm{g} / \mathrm{L})\end{array}$ & $\begin{array}{l}\text { Meto- } \\
\text { lachlor } \\
\text { ESA } \\
(\mu \mathrm{g} / \mathrm{L})\end{array}$ & $\begin{array}{l}\text { Meto- } \\
\text { lachlor } \\
\text { OXA } \\
(\mu \mathrm{g} / \mathrm{L})\end{array}$ \\
\hline \multicolumn{20}{|c|}{ Midcontinent Agricultural Chemical Research Project (fig. 1)—Continued } \\
\hline \multicolumn{20}{|c|}{ Sulfonylurea reconnaissance study (DBD)—Continued } \\
\hline \multirow[t]{3}{*}{05587000} & \multirow{3}{*}{$\begin{array}{l}\text { Macoupin Creek near } \\
\text { Kane, IL }\end{array}$} & 06/06/02 & 0.36 & 0.37 & 0.26 & 0.13 & $<0.05$ & 0.05 & $<0.05$ & $<0.05$ & 0.31 & 0.09 & 0.05 & $<0.05$ & $<0.05$ & $<0.05$ & 0.27 & 0.76 & 0.16 \\
\hline & & 07/29/02 & .14 & .54 & .98 & .30 & $<.05$ & .05 & $<.05$ & $<.05$ & .12 & .08 & .08 & $<.05$ & $<.05$ & $<.05$ & .74 & .61 & .50 \\
\hline & & 09/24/02 & .05 & .28 & .49 & .13 & $<.05$ & .05 & $<.05$ & $<.05$ & .05 & .10 & .06 & $<.05$ & $<.05$ & $<.05$ & .27 & .42 & .44 \\
\hline \multirow[t]{5}{*}{03267900} & \multirow{5}{*}{$\begin{array}{l}\text { Mad River at Eagle } \\
\text { City, OH }\end{array}$} & 06/10/98 & $<.05$ & $<.20$ & $<.20$ & -- & $<.05$ & .31 & $<.20$ & -- & -- & -- & -- & -- & -- & -- & $<.05$ & .63 & $<.20$ \\
\hline & & 06/30/98 & $<.05$ & .44 & .22 & -- & $<.05$ & .44 & $<.20$ & -- & -- & -- & -- & -- & -- & -- & $<.05$ & 1.3 & .50 \\
\hline & & 06/05/02 & $<.05$ & .10 & .07 & $<.05$ & $<.05$ & .18 & $<.05$ & $<.05$ & $<.05$ & $<.05$ & $<.05$ & $<.05$ & $<.05$ & $<.05$ & .10 & .41 & .10 \\
\hline & & 07/17/02 & $<.05$ & $<.05$ & $<.05$ & $<.05$ & $<.05$ & .18 & $<.05$ & $<.05$ & $<.05$ & $<.05$ & $<.05$ & $<.05$ & $<.05$ & $<.05$ & $<.05$ & .31 & .06 \\
\hline & & $10 / 27 / 02$ & $<.05$ & .06 & $<.05$ & $<.05$ & $<.05$ & .17 & $<.05$ & $<.05$ & $<.05$ & $<.05$ & $<.05$ & $<.05$ & $<.05$ & $<.05$ & $<.05$ & .39 & .12 \\
\hline \multirow[t]{2}{*}{06800000} & \multirow{2}{*}{$\begin{array}{l}\text { Maple Creek near } \\
\text { Nickerson, NE }\end{array}$} & 05/21/98 & 21 & 1.2 & 1.7 & -- & 8.8 & .38 & .50 & -- & -- & -- & -- & -- & -- & -- & 15 & .50 & .40 \\
\hline & & 06/08/98 & 2.1 & 1.7 & 2.6 & -- & .05 & .47 & $<.20$ & -- & -- & -- & -- & -- & -- & -- & 4.6 & .59 & .67 \\
\hline \multirow[t]{5}{*}{06607200} & \multirow{5}{*}{$\begin{array}{r}\text { Maple River at } \\
\text { Mapleton, IA }\end{array}$} & 05/29/98 & 2.4 & .45 & .51 & -- & $<.05$ & .24 & $<.20$ & -- & -- & -- & -- & -- & -- & -- & 1.9 & .85 & .67 \\
\hline & & 06/09/98 & .19 & .34 & $<.20$ & -- & $<.05$ & .30 & $<.20$ & -- & -- & -- & -- & -- & -- & -- & 2.3 & 1.4 & .39 \\
\hline & & 05/29/02 & $<.05$ & .07 & .05 & $<.05$ & $<.05$ & .05 & $<.05$ & $<.05$ & $<.05$ & $<.05$ & $<.05$ & $<.05$ & $<.05$ & $<.05$ & .29 & .51 & .12 \\
\hline & & 06/11/02 & .17 & .09 & .18 & $<.05$ & $<.05$ & $<.05$ & $<.05$ & $<.05$ & $<.05$ & $<.05$ & $<.05$ & $<.05$ & $<.05$ & $<.05$ & 1.1 & .54 & .22 \\
\hline & & $10 / 31 / 02$ & $<.05$ & .07 & .05 & $<.05$ & $<.05$ & .06 & $<.05$ & $<.05$ & $<.05$ & $<.05$ & $<.05$ & $<.05$ & $<.05$ & $<.05$ & $<.05$ & .64 & .16 \\
\hline \multirow[t]{2}{*}{05330000} & \multirow{2}{*}{$\begin{array}{l}\text { Minnesota River near } \\
\text { Jordan, MN }\end{array}$} & 05/19/98 & 1.2 & .95 & .65 & -- & .07 & .70 & $<.20$ & -- & -- & -- & -- & -- & -- & -- & .36 & 1.8 & .62 \\
\hline & & 06/26/98 & .24 & 1.8 & 1.3 & -- & .09 & 1.5 & .43 & -- & & & & & & & .41 & 2.7 & .83 \\
\hline \multirow[t]{2}{*}{03327000} & \multirow{2}{*}{$\begin{array}{l}\text { Mississinewa River at } \\
\text { Peoria, IN }\end{array}$} & 06/09/98 & .45 & .61 & .50 & -- & $<.05$ & .51 & $<.20$ & -- & -- & -- & -- & -- & -- & -- & 1.2 & 2.1 & .82 \\
\hline & & 07/09/98 & .74 & 1.6 & 1.6 & -- & .08 & .70 & $<.20$ & -- & -- & -- & -- & -- & -- & -- & 2.5 & 2.2 & 1.5 \\
\hline \multirow[t]{2}{*}{05420500} & \multirow{2}{*}{$\begin{array}{l}\text { Mississippi River at } \\
\text { Clinton, IA }\end{array}$} & 05/27/98 & .25 & .31 & $<.20$ & -- & $<.05$ & .42 & $<.20$ & -- & -- & -- & -- & -- & -- & -- & .12 & .73 & .29 \\
\hline & & 07/01/98 & .10 & .65 & .43 & -- & $<.05$ & .66 & $<.20$ & -- & -- & -- & -- & -- & -- & -- & .20 & .86 & .31 \\
\hline \multirow[t]{3}{*}{05331580} & \multirow{3}{*}{$\begin{array}{l}\text { Mississippi River at } \\
\text { Hastings, MN }\end{array}$} & 05/20/98 & .32 & $<.20$ & $<.20$ & -- & .05 & .45 & $<.20$ & -- & -- & -- & -- & -- & -- & -- & .14 & .99 & .34 \\
\hline & & 05/20/98 & .38 & $<.20$ & $<.20$ & -- & .06 & .46 & $<.20$ & -- & -- & -- & -- & -- & -- & -- & .17 & 1.0 & .32 \\
\hline & & 08/11/98 & $<.05$ & $<.20$ & $<.20$ & -- & $<.05$ & .62 & $<.20$ & -- & -- & -- & -- & -- & -- & -- & $<.05$ & .53 & $<.20$ \\
\hline \multirow[t]{2}{*}{05587455} & \multirow{2}{*}{$\begin{array}{l}\text { Mississippi River below } \\
\text { Grafton, IL }\end{array}$} & 06/02/98 & .79 & .82 & .71 & -- & $<.05$ & .64 & $<.20$ & -- & -- & -- & -- & -- & -- & -- & 1.1 & 2.0 & .73 \\
\hline & & 06/15/98 & .44 & 1.9 & 1.3 & -- & $<.05$ & 1.0 & $<.20$ & -- & -- & -- & -- & -- & -- & -- & .84 & 2.6 & 1.0 \\
\hline
\end{tabular}




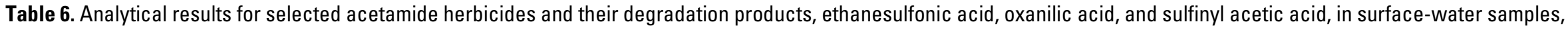
1993-2003.-Continued

[OGRL, U.S. Geological Survey Organic Geochemistry Research Laboratory, Lawrence, Kansas; ESA, ethanesulfonic acid; OXA, oxanilic acid; SAA, sulfinyl acetic acid; --, no data; <, less than]

\begin{tabular}{|c|c|c|c|c|c|c|c|c|c|c|c|c|c|c|c|c|c|c|c|}
\hline \multirow[b]{2}{*}{ Site number } & \multirow[b]{2}{*}{ Site location } & \multirow[b]{2}{*}{$\begin{array}{c}\text { Date of } \\
\text { collection } \\
\text { (month/day/ } \\
\text { year) }\end{array}$} & \multicolumn{17}{|c|}{ Concentrations, in micrograms per liter } \\
\hline & & & $\begin{array}{l}\text { Aceto- } \\
\text { chlor } \\
(\mu \mathrm{g} / \mathrm{L})\end{array}$ & $\begin{array}{l}\text { Aceto- } \\
\text { chlor } \\
\text { ESA } \\
(\mu \mathrm{g} / \mathrm{L})\end{array}$ & $\begin{array}{l}\text { Aceto- } \\
\text { chlor } \\
\text { OXA } \\
(\mu \mathrm{g} / \mathrm{L})\end{array}$ & $\begin{array}{l}\text { Aceto- } \\
\text { chlor } \\
\text { SAA } \\
(\mu \mathrm{g} / \mathrm{L})\end{array}$ & $\begin{array}{l}\text { Ala- } \\
\text { chlor } \\
(\mu \mathrm{g} / \mathrm{L})\end{array}$ & $\begin{array}{l}\text { Ala- } \\
\text { chlor } \\
\text { ESA } \\
(\mu \mathrm{g} / \mathrm{L})\end{array}$ & $\begin{array}{l}\text { Ala- } \\
\text { chlor } \\
\text { OXA } \\
(\mu \mathrm{g} / \mathrm{L})\end{array}$ & $\begin{array}{l}\text { Ala- } \\
\text { chlor } \\
\text { SAA } \\
(\mu \mathrm{g} / \mathrm{L})\end{array}$ & $\begin{array}{l}\text { Di- } \\
\text { meth- } \\
\text { enamid } \\
(\mu \mathrm{g} / \mathrm{L})\end{array}$ & $\begin{array}{c}\text { Di- } \\
\text { meth- } \\
\text { enamid } \\
\text { ESA } \\
(\mu \mathrm{g} / \mathrm{L})\end{array}$ & $\begin{array}{c}\text { Di- } \\
\text { meth- } \\
\text { enamid } \\
\text { OXA } \\
(\mu \mathrm{g} / \mathrm{L})\end{array}$ & $\begin{array}{l}\text { Flufen- } \\
\text { acet } \\
(\mu \mathrm{g} / \mathrm{L})\end{array}$ & $\begin{array}{l}\text { Flufen- } \\
\text { acet } \\
\text { ESA } \\
(\mu \mathrm{g} / \mathrm{L})\end{array}$ & $\begin{array}{c}\text { Flufen- } \\
\text { acet } \\
\text { OXA } \\
(\mu \mathrm{g} / \mathrm{L})\end{array}$ & $\begin{array}{l}\text { Meto- } \\
\text { lachlor } \\
(\mu \mathrm{g} / \mathrm{L})\end{array}$ & $\begin{array}{c}\text { Meto- } \\
\text { lachlor } \\
\text { ESA } \\
(\mu \mathrm{g} / \mathrm{L})\end{array}$ & $\begin{array}{l}\text { Meto- } \\
\text { lachlor } \\
\text { OXA } \\
(\mu \mathrm{g} / \mathrm{L})\end{array}$ \\
\hline \multicolumn{20}{|c|}{ Midcontinent Agricultural Chemical Research Project (fig. 1)—Continued } \\
\hline \multicolumn{20}{|c|}{ Sulfonylurea reconnaissance study (DBD)—Continued } \\
\hline \multirow[t]{2}{*}{06770195} & \multirow{2}{*}{$\begin{array}{l}\text { North Dry Creek at } \\
\text { Kearney, NE }\end{array}$} & $05 / 22 / 98$ & 5.6 & 0.65 & 0.59 & -- & 0.14 & 0.28 & $<0.20$ & -- & -- & -- & -- & -- & -- & -- & 3.0 & 0.51 & 0.34 \\
\hline & & 06/08/98 & .18 & 1.3 & 1.5 & -- & .05 & .53 & $<.20$ & -- & -- & -- & -- & -- & -- & -- & .82 & .56 & .56 \\
\hline \multirow[t]{4}{*}{05472500} & \multirow{4}{*}{$\begin{array}{l}\text { North Skunk River near } \\
\text { Sigourney, IA }\end{array}$} & $05 / 21 / 98$ & 15 & 1.5 & .95 & -- & $<.05$ & .52 & $<.20$ & -- & -- & -- & -- & -- & -- & -- & 2.4 & 2.9 & 1.0 \\
\hline & & 05/13/02 & 3.1 & .90 & .94 & 0.62 & .05 & .19 & .06 & $<0.05$ & 1.5 & 0.31 & 0.26 & $<0.05$ & $<0.05$ & $<0.05$ & 8.7 & 2.0 & 1.1 \\
\hline & & 07/08/02 & .07 & .63 & .59 & $<.05$ & $<.05$ & .07 & $<.05$ & $<.05$ & .09 & .12 & .08 & $<.05$ & $<.05$ & $<.05$ & .39 & .79 & .28 \\
\hline & & $10 / 29 / 02$ & $<.05$ & .22 & .13 & $<.05$ & $<.05$ & .32 & $<.05$ & $<.05$ & $<.05$ & $<.05$ & $<.05$ & $<.05$ & $<.05$ & $<.05$ & .06 & 1.8 & .27 \\
\hline \multirow[t]{4}{*}{06817700} & \multirow{4}{*}{$\begin{array}{l}\text { Nodaway River near } \\
\text { Graham, MO }\end{array}$} & 06/17/98 & .12 & .95 & .63 & -- & $<.05$ & .38 & $<.20$ & -- & -- & -- & -- & -- & -- & -- & .93 & 1.6 & .75 \\
\hline & & 08/19/98 & $<.05$ & $<.20$ & $<.20$ & -- & $<.05$ & .26 & $<.20$ & -- & -- & -- & -- & -- & -- & -- & .05 & .91 & $<.20$ \\
\hline & & 07/29/02 & .08 & .11 & .17 & $<.05$ & $<.05$ & .11 & $<.05$ & $<.05$ & $<.05$ & $<.05$ & $<.05$ & $<.05$ & $<.05$ & $<.05$ & .25 & .34 & .18 \\
\hline & & $10 / 04 / 02$ & .06 & .12 & .14 & $<.05$ & $<.05$ & .12 & .08 & $<.05$ & $<.05$ & $<.05$ & $<.05$ & $<.05$ & $<.05$ & $<.05$ & .06 & .25 & .12 \\
\hline \multirow[t]{4}{*}{05472500} & \multirow{4}{*}{$\begin{array}{l}\text { North Skunk River near } \\
\text { Sigourney, IA }\end{array}$} & 06/10/98 & .35 & 1.4 & 1.3 & -- & .10 & .91 & $<.20$ & -- & -- & -- & -- & -- & -- & -- & 2.4 & 3.9 & 1.6 \\
\hline & & 05/13/02 & 3.1 & .90 & .94 & .62 & .05 & .19 & .06 & $<.05$ & 1.5 & .31 & .26 & .06 & $<.05$ & $<.05$ & 8.7 & 2.0 & 1.1 \\
\hline & & 07/08/02 & .07 & .59 & .63 & .13 & $<.05$ & .07 & $<.05$ & $<.05$ & .09 & .12 & .08 & $<.05$ & $<.05$ & $<.05$ & .39 & .79 & .28 \\
\hline & & $10 / 29 / 02$ & $<.05$ & .17 & .11 & $<.05$ & $<.05$ & .23 & $<.05$ & $<.05$ & $<.05$ & $<.05$ & $<.05$ & $<.05$ & $<.05$ & $<.05$ & $<.05$ & 1.5 & .33 \\
\hline \multirow[t]{2}{*}{03303280} & \multirow{2}{*}{$\begin{array}{l}\text { Ohio River at Cannelton } \\
\text { Dam, OH }\end{array}$} & 06/04/98 & .14 & $<.20$ & $<.20$ & -- & $<.05$ & $<.20$ & $<.20$ & -- & -- & -- & -- & -- & -- & -- & .23 & $<.20$ & $<.20$ \\
\hline & & 07/07/98 & .06 & $<.20$ & $<.20$ & -- & $<.05$ & .25 & $<.20$ & -- & -- & -- & -- & -- & -- & -- & .30 & $<.20$ & .38 \\
\hline \multirow[t]{5}{*}{05455100} & \multirow{5}{*}{$\begin{array}{l}\text { Old Man's Creek near } \\
\text { Iowa City, IA }\end{array}$} & 06/10/98 & .57 & 2.0 & 1.5 & -- & .21 & 1.1 & $<.20$ & -- & -- & -- & -- & -- & -- & -- & 1.2 & 3.2 & 1.4 \\
\hline & & 06/30/98 & .28 & 2.0 & 1.4 & -- & $<.05$ & .80 & $<.20$ & -- & -- & -- & -- & -- & -- & -- & .98 & 2.7 & 1.4 \\
\hline & & 05/13/02 & 2.2 & 1.0 & .86 & .44 & .17 & .33 & $<.05$ & $<.05$ & .53 & .10 & .10 & $<.05$ & $<.05$ & $<.05$ & 1.6 & 1.5 & .33 \\
\hline & & 06/27/02 & .05 & .54 & .32 & .11 & $<.05$ & .29 & $<.05$ & $<.05$ & $<.05$ & .10 & $<.05$ & $<.05$ & $<.05$ & $<.05$ & .25 & 1.4 & .28 \\
\hline & & $10 / 30 / 02$ & $<.05$ & .25 & .06 & $<.05$ & $<.05$ & .55 & .05 & $<.05$ & $<.05$ & $<.05$ & $<.05$ & $<.05$ & $<.05$ & $<.05$ & $<.05$ & 1.4 & .15 \\
\hline \multirow{3}{*}{03223000} & \multirow{3}{*}{$\begin{array}{l}\text { Olentangy River at } \\
\text { Claridon, } \mathrm{OH}\end{array}$} & 06/04/02 & .67 & .59 & .55 & .17 & $<.05$ & .24 & $<.05$ & $<.05$ & .07 & .05 & $<.05$ & $<.05$ & $<.05$ & $<.05$ & 1.1 & 2.8 & .72 \\
\hline & & 07/31/02 & $<.05$ & .27 & .32 & $<.05$ & $<.05$ & .23 & $<.05$ & $<.05$ & $<.05$ & $<.05$ & $<.05$ & $<.05$ & $<.05$ & $<.05$ & .19 & .86 & .30 \\
\hline & & $10 / 26 / 02$ & $<.05$ & .18 & .18 & $<.05$ & $<.05$ & .20 & .05 & $<.05$ & $<.05$ & .05 & $<.05$ & $<.05$ & $<.05$ & $<.05$ & .06 & .69 & .33 \\
\hline
\end{tabular}




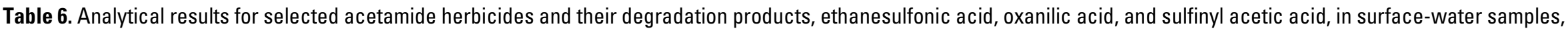
1993-2003-Continued

[OGRL, U.S. Geological Survey Organic Geochemistry Research Laboratory, Lawrence, Kansas; ESA, ethanesulfonic acid; OXA, oxanilic acid; SAA, sulfinyl acetic acid; --, no data; <, less than]

\begin{tabular}{|c|c|c|c|c|c|c|c|c|c|c|c|c|c|c|c|c|c|c|c|}
\hline \multirow[b]{2}{*}{ Site number } & \multirow[b]{2}{*}{ Site location } & \multirow[b]{2}{*}{$\begin{array}{c}\text { Date of } \\
\text { collection } \\
\text { (month/day/ } \\
\text { year) }\end{array}$} & \multicolumn{17}{|c|}{ Concentrations, in micrograms per liter } \\
\hline & & & $\begin{array}{l}\text { Aceto- } \\
\text { chlor } \\
(\mu \mathrm{g} / \mathrm{L})\end{array}$ & $\begin{array}{l}\text { Aceto- } \\
\text { chlor } \\
\text { ESA } \\
(\mu \mathrm{g} / \mathrm{L})\end{array}$ & $\begin{array}{c}\text { Aceto- } \\
\text { chlor } \\
\text { OXA } \\
(\mu \mathrm{g} / \mathrm{L})\end{array}$ & $\begin{array}{l}\text { Aceto- } \\
\text { chlor } \\
\text { SAA } \\
(\mu \mathrm{g} / \mathrm{L})\end{array}$ & $\begin{array}{l}\text { Ala- } \\
\text { chlor } \\
(\mu \mathrm{g} / \mathrm{L})\end{array}$ & $\begin{array}{l}\text { Ala- } \\
\text { chlor } \\
\text { ESA } \\
(\mu \mathrm{g} / \mathrm{L})\end{array}$ & $\begin{array}{l}\text { Ala- } \\
\text { chlor } \\
\text { OXA } \\
(\mu \mathrm{g} / \mathrm{L})\end{array}$ & $\begin{array}{l}\text { Ala- } \\
\text { chlor } \\
\text { SAA } \\
(\mu \mathrm{g} / \mathrm{L})\end{array}$ & $\begin{array}{l}\text { Di- } \\
\text { meth- } \\
\text { enamid } \\
(\mu \mathrm{g} / \mathrm{L})\end{array}$ & $\begin{array}{c}\text { Di- } \\
\text { meth- } \\
\text { enamid } \\
\text { ESA } \\
(\mu \mathrm{g} / \mathrm{L})\end{array}$ & $\begin{array}{c}\text { Di- } \\
\text { meth- } \\
\text { enamid } \\
O X A \\
(\mu \mathrm{g} / \mathrm{L})\end{array}$ & $\begin{array}{c}\text { Flufen- } \\
\text { acet } \\
(\mu \mathrm{g} / \mathrm{L})\end{array}$ & $\begin{array}{c}\text { Flufen- } \\
\text { acet } \\
\text { ESA } \\
(\mu \mathrm{g} / \mathrm{L})\end{array}$ & $\begin{array}{c}\text { Flufen- } \\
\text { acet } \\
\text { OXA } \\
(\mu \mathrm{g} / \mathrm{L})\end{array}$ & $\begin{array}{l}\text { Meto- } \\
\text { lachlor } \\
(\mu \mathrm{g} / \mathrm{L})\end{array}$ & $\begin{array}{l}\text { Meto- } \\
\text { lachlor } \\
\text { ESA } \\
(\mu \mathrm{g} / \mathrm{L})\end{array}$ & $\begin{array}{l}\text { Meto- } \\
\text { lachlor } \\
\text { OXA } \\
(\mu \mathrm{g} / \mathrm{L})\end{array}$ \\
\hline \multicolumn{20}{|c|}{ Midcontinent Agricultural Chemical Research Project (fig. 1)—Continued } \\
\hline \multicolumn{20}{|c|}{ Sulfonylurea reconnaissance study (DBD)—Continued } \\
\hline \multirow[t]{3}{*}{03225500} & Olentangy River near & 06/09/98 & 0.20 & 0.58 & $<0.20$ & -- & $<0.05$ & 0.75 & $<0.20$ & -- & -- & -- & -- & -- & -- & -- & 1.5 & 3.2 & 1.2 \\
\hline & Delaware, $\mathrm{OH}$ & 07/01/98 & .53 & 2.9 & 3.2 & -- & $<.05$ & 1.3 & $<.20$ & -- & -- & -- & -- & -- & -- & -- & 4.1 & 5.3 & 4.8 \\
\hline & & 07/01/98 & .41 & 2.4 & 2.9 & -- & $<.05$ & 1.1 & .25 & -- & -- & -- & -- & -- & -- & -- & 3.3 & 4.7 & 4.3 \\
\hline \multirow[t]{2}{*}{06805500} & Platte River at & 05/22/98 & 2.8 & 1.2 & 1.6 & -- & 2.2 & .83 & 1.1 & -- & -- & -- & -- & -- & -- & -- & 6.8 & .92 & 1.1 \\
\hline & Louisville, NE & 06/09/98 & .65 & 1.1 & 1.5 & -- & .74 & .72 & .61 & -- & -- & -- & -- & -- & -- & -- & 2.7 & .92 & 1.1 \\
\hline \multirow[t]{5}{*}{05484500} & Raccoon River at & 05/17/98 & .94 & $<.20$ & $<.20$ & -- & $<.05$ & .49 & $<.20$ & -- & -- & -- & -- & -- & -- & -- & 2.9 & 2.3 & 1.2 \\
\hline & Van Meter, IA & 06/10/98 & .32 & 1.5 & 1.2 & -- & $<.05$ & .60 & $<.20$ & -- & -- & -- & -- & -- & -- & -- & 1.2 & 3.4 & 1.3 \\
\hline & & 05/17/02 & .38 & .47 & .43 & $<0.05$ & $<.05$ & .07 & $<.05$ & $<0.05$ & 0.13 & 0.06 & $<0.05$ & $<0.05$ & $<0.05$ & $<0.05$ & .61 & 2.4 & .47 \\
\hline & & $06 / 12 / 02$ & .27 & .49 & .65 & .17 & $<.05$ & $<.05$ & $<.05$ & $<.05$ & .19 & .07 & .06 & .20 & $<.05$ & $<.05$ & .92 & 1.4 & .35 \\
\hline & & $10 / 30 / 02$ & $<.05$ & .20 & .05 & $<.05$ & $<.05$ & .10 & $<.05$ & $<.05$ & $<.05$ & $<.05$ & $<.05$ & $<.05$ & $<.05$ & $<.05$ & .05 & 2.5 & .23 \\
\hline \multirow[t]{5}{*}{05430500} & Rock River at Afton, WI & 06/17/98 & .11 & .60 & $<.20$ & -- & $<.05$ & .85 & $<.20$ & -- & -- & -- & -- & -- & -- & -- & .07 & .93 & .41 \\
\hline & & $07 / 21 / 98$ & $<.05$ & .76 & .74 & -- & $<.05$ & 1.2 & .25 & -- & -- & -- & -- & -- & -- & -- & $<.05$ & .76 & .42 \\
\hline & & 06/04/02 & .08 & .11 & .08 & $<.05$ & $<.05$ & .43 & $<.05$ & $<.05$ & .05 & $<.05$ & $<.05$ & $<.05$ & $<.05$ & $<.05$ & .80 & .48 & .23 \\
\hline & & 07/11/02 & $<.05$ & .36 & .41 & $<.05$ & $<.05$ & .70 & $<.05$ & $<.05$ & $<.05$ & $<.05$ & $<.05$ & $<.05$ & $<.05$ & $<.05$ & $<.05$ & .59 & .24 \\
\hline & & 09/20/02 & $<.05$ & .10 & .10 & $<.05$ & $<.05$ & .67 & $<.05$ & $<.05$ & $<.05$ & .07 & $<.05$ & $<.05$ & $<.05$ & $<.05$ & $<.05$ & .37 & .10 \\
\hline \multirow[t]{2}{*}{06483000} & Rock River at Luverne, MN & 06/24/98 & $<.05$ & $<.20$ & $<.20$ & -- & $<.05$ & $<.20$ & $<.20$ & -- & -- & -- & -- & -- & -- & -- & .05 & 1.7 & $<.20$ \\
\hline & & $06 / 25 / 98$ & .05 & $<.20$ & .50 & -- & $<.05$ & $<.20$ & $<.20$ & -- & -- & -- & -- & -- & -- & -- & .24 & 1.3 & $<.20$ \\
\hline \multirow[t]{5}{*}{04087240} & Root River at Racine,WI & 06/01/98 & .14 & $<.20$ & $<.20$ & -- & $<.05$ & $<.20$ & .38 & -- & -- & -- & -- & -- & -- & -- & 1.8 & .69 & .30 \\
\hline & & 08/04/98 & $<.05$ & $<.20$ & $<.20$ & -- & $<.05$ & .37 & $<.20$ & -- & -- & -- & -- & -- & -- & -- & $<.05$ & 1.4 & $<.20$ \\
\hline & & 06/03/02 & $<.05$ & $<.05$ & $<.05$ & $<.05$ & $<.05$ & .10 & $<.05$ & $<.05$ & $<.05$ & .06 & $<.05$ & $<.05$ & $<.05$ & $<.05$ & .06 & .64 & .13 \\
\hline & & 07/26/02 & $<.05$ & .07 & .05 & $<.05$ & $<.05$ & .08 & $<.05$ & $<.05$ & $<.05$ & .06 & $<.05$ & $<.05$ & $<.05$ & $<.05$ & $<.05$ & .51 & .20 \\
\hline & & 09/03/02 & $<.05$ & $<.05$ & $<.05$ & $<.05$ & $<.05$ & $<.05$ & $<.05$ & $<.05$ & $<.05$ & .05 & $<.05$ & $<.05$ & $<.05$ & $<.05$ & $<.05$ & .26 & .11 \\
\hline \multirow[t]{2}{*}{06803000} & Salt Creek at Roca, NE & 05/15/98 & .18 & $<.20$ & $<.20$ & -- & 18 & .59 & $<.20$ & -- & -- & -- & -- & -- & -- & -- & 124 & 1.5 & 1.0 \\
\hline & & 06/10/98 & .81 & 1.3 & 2.2 & -- & 1.6 & 2.8 & 1.0 & -- & -- & -- & -- & -- & -- & -- & 12 & 5.2 & 6.4 \\
\hline
\end{tabular}




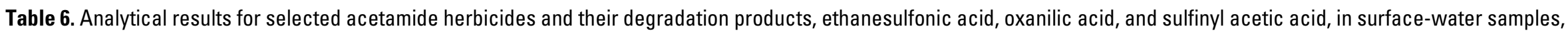
1993-2003.-Continued

[OGRL, U.S. Geological Survey Organic Geochemistry Research Laboratory, Lawrence, Kansas; ESA, ethanesulfonic acid; OXA, oxanilic acid; SAA, sulfinyl acetic acid; --, no data; <, less than]

\begin{tabular}{|c|c|c|c|c|c|c|c|c|c|c|c|c|c|c|c|c|c|c|c|}
\hline \multirow[b]{2}{*}{ Site number } & \multirow[b]{2}{*}{ Site location } & \multirow[b]{2}{*}{$\begin{array}{c}\text { Date of } \\
\text { collection } \\
\text { (month/day/ } \\
\text { year) }\end{array}$} & \multicolumn{17}{|c|}{ Concentrations, in micrograms per liter } \\
\hline & & & $\begin{array}{l}\text { Aceto- } \\
\text { chlor } \\
(\mu \mathrm{g} / \mathrm{L})\end{array}$ & $\begin{array}{l}\text { Aceto- } \\
\text { chlor } \\
\text { ESA } \\
(\mu \mathrm{g} / \mathrm{L})\end{array}$ & $\begin{array}{l}\text { Aceto- } \\
\text { chlor } \\
\text { OXA } \\
(\mu \mathrm{g} / \mathrm{L})\end{array}$ & $\begin{array}{l}\text { Aceto- } \\
\text { chlor } \\
\text { SAA } \\
(\mu \mathrm{g} / \mathrm{L})\end{array}$ & $\begin{array}{l}\text { Ala- } \\
\text { chlor } \\
(\mu \mathrm{g} / \mathrm{L})\end{array}$ & $\begin{array}{l}\text { Ala- } \\
\text { chlor } \\
\text { ESA } \\
(\mu \mathrm{g} / \mathrm{L})\end{array}$ & $\begin{array}{l}\text { Ala- } \\
\text { chlor } \\
\text { OXA } \\
(\mu \mathrm{g} / \mathrm{L})\end{array}$ & $\begin{array}{l}\text { Ala- } \\
\text { chlor } \\
\text { SAA } \\
(\mu \mathrm{g} / \mathrm{L})\end{array}$ & $\begin{array}{c}\text { Di- } \\
\text { meth- } \\
\text { enamid } \\
(\mu \mathrm{g} / \mathrm{L})\end{array}$ & $\begin{array}{c}\text { Di- } \\
\text { meth- } \\
\text { enamid } \\
\text { ESA } \\
(\mu \mathrm{g} / \mathrm{L})\end{array}$ & $\begin{array}{c}\text { Di- } \\
\text { meth- } \\
\text { enamid } \\
\text { OXA } \\
(\mu \mathrm{g} / \mathrm{L})\end{array}$ & $\begin{array}{c}\text { Flufen- } \\
\text { acet } \\
(\mu \mathrm{g} / \mathrm{L})\end{array}$ & $\begin{array}{c}\text { Flufen- } \\
\text { acet } \\
\text { ESA } \\
(\mu \mathrm{g} / \mathrm{L})\end{array}$ & $\begin{array}{c}\text { Flufen- } \\
\text { acet } \\
\text { OXA } \\
(\mu \mathrm{g} / \mathrm{L})\end{array}$ & $\begin{array}{l}\text { Meto- } \\
\text { lachlor } \\
(\mu \mathrm{g} / \mathrm{L})\end{array}$ & $\begin{array}{l}\text { Meto- } \\
\text { lachlor } \\
\text { ESA } \\
(\mu \mathrm{g} / \mathrm{L})\end{array}$ & $\begin{array}{l}\text { Meto- } \\
\text { lachlor } \\
\text { OXA } \\
(\mu \mathrm{g} / \mathrm{L})\end{array}$ \\
\hline \multicolumn{20}{|c|}{ Midcontinent Agricultural Chemical Research Project (fig. 1)—Continued } \\
\hline \multicolumn{20}{|c|}{ Sulfonylurea reconnaissance study (DBD)—Continued } \\
\hline \multirow[t]{2}{*}{05572000} & \multirow{2}{*}{$\begin{array}{l}\text { Sangamon River at } \\
\text { Monticello, IL }\end{array}$} & 05/23/98 & 0.54 & 1.6 & 0.91 & -- & $<0.05$ & 0.72 & $<0.20$ & & -- & -- & -- & -- & -- & -- & 2.6 & 4.0 & 1.8 \\
\hline & & 08/05/98 & $<.05$ & 1.6 & $<.20$ & -- & $<.05$ & .58 & $<.20$ & & -- & -- & -- & -- & -- & -- & .11 & 3.9 & .71 \\
\hline \multirow[t]{5}{*}{05576500} & \multirow{5}{*}{$\begin{array}{l}\text { Sangamon River at } \\
\text { Riverton, IL }\end{array}$} & $05 / 26 / 98$ & 1.5 & 1.2 & .95 & -- & .13 & .46 & $<.20$ & & -- & -- & -- & -- & -- & -- & 1.7 & 1.6 & 1.0 \\
\hline & & 07/09/98 & .07 & $<.20$ & $<.20$ & -- & $<.05$ & $<.20$ & $<.20$ & & -- & -- & -- & -- & -- & -- & .33 & $<.20$ & $<.20$ \\
\hline & & $05 / 29 / 02$ & 1.1 & .66 & .49 & 0.33 & .13 & .19 & .08 & $<0.05$ & 1.8 & 0.34 & 0.19 & $<0.05$ & $<0.05$ & $<0.05$ & .91 & .91 & .31 \\
\hline & & 07/17/02 & $<.05$ & .60 & .26 & $<.05$ & $<.05$ & .16 & .10 & $<.05$ & .08 & .10 & .06 & $<.05$ & $<.05$ & $<.05$ & .13 & .84 & .14 \\
\hline & & $09 / 25 / 02$ & $<.05$ & .23 & .25 & .06 & $<.05$ & .16 & .09 & $<.05$ & $<.05$ & .13 & .05 & $<.05$ & $<.05$ & $<.05$ & .06 & .43 & .23 \\
\hline \multirow[t]{5}{*}{03234500} & \multirow[t]{5}{*}{ Scioto River at Higby, OH } & 06/04/98 & .90 & .57 & .49 & -- & .05 & .04 & $<.20$ & -- & -- & -- & -- & -- & -- & -- & 2.0 & 1.6 & .59 \\
\hline & & 07/08/98 & .22 & 1.1 & 1.1 & -- & $<.05$ & .99 & $<.20$ & -- & -- & -- & -- & -- & -- & -- & 1.7 & 2.5 & 1.6 \\
\hline & & 06/04/02 & .49 & .48 & .57 & .24 & $<.05$ & .09 & $<.05$ & $<.05$ & .18 & .06 & .05 & $<.05$ & $<.05$ & $<.05$ & .97 & .86 & .43 \\
\hline & & $07 / 30 / 02$ & .17 & .58 & .78 & .25 & $<.05$ & .09 & $<.05$ & $<.05$ & $<.05$ & $<.05$ & $<.05$ & $<.05$ & $<.05$ & $<.05$ & .35 & .67 & .34 \\
\hline & & $10 / 28 / 02$ & $<.05$ & .19 & .22 & .05 & $<.05$ & .09 & .05 & $<.05$ & $<.05$ & .05 & $<.05$ & $<.05$ & $<.05$ & $<.05$ & .59 & .37 & .25 \\
\hline \multirow[t]{5}{*}{03219500} & \multirow{5}{*}{$\begin{array}{l}\text { Scioto River near } \\
\text { Prospect, OH }\end{array}$} & 06/02/98 & .83 & 2.4 & .49 & -- & $<.05$ & 4.5 & $<.20$ & -- & -- & -- & -- & -- & -- & -- & 2.8 & 2.4 & 3.2 \\
\hline & & $06 / 29 / 98$ & .20 & 1.5 & 1.1 & -- & $<.05$ & 2.1 & .21 & -- & -- & -- & -- & -- & -- & -- & 4.2 & 5.1 & 3.3 \\
\hline & & 06/04/02 & 1.4 & 1.3 & 1.6 & .80 & $<.05$ & .17 & .06 & $<.05$ & .25 & .13 & .11 & .09 & $<.05$ & $<.05$ & 2.6 & 2.2 & .89 \\
\hline & & $07 / 31 / 02$ & $<.05$ & .33 & .25 & $<.05$ & $<.05$ & .30 & .06 & $<.05$ & $<.05$ & $<.05$ & $<.05$ & $<.05$ & $<.05$ & $<.05$ & .07 & .55 & .22 \\
\hline & & $10 / 01 / 02$ & .05 & 1.2 & 1.7 & .67 & $<.05$ & .29 & .17 & .10 & $<.05$ & .10 & .11 & $<.05$ & .07 & .05 & .44 & 2.3 & 1.8 \\
\hline \multirow[t]{5}{*}{05594000} & \multirow[t]{5}{*}{ Shoal Creek near Breese, IL } & $05 / 22 / 98$ & 9.5 & .77 & .75 & -- & .21 & .41 & .33 & -- & -- & -- & -- & -- & -- & -- & 7.6 & .87 & .66 \\
\hline & & 07/08/98 & 1.1 & 1.5 & .80 & -- & $<.05$ & .83 & $<.20$ & -- & -- & -- & -- & -- & -- & -- & 1.7 & 2.6 & .95 \\
\hline & & 06/06/02 & .52 & .05 & .08 & $<.05$ & .06 & .13 & $<.05$ & $<.05$ & .23 & $<.05$ & $<.05$ & $<.05$ & $<.05$ & $<.05$ & .97 & .82 & .27 \\
\hline & & $07 / 29 / 02$ & $<.05$ & .10 & .14 & $<.05$ & $<.05$ & .20 & .07 & $<.05$ & $<.05$ & $<.05$ & .05 & $<.05$ & $<.05$ & $<.05$ & .39 & .83 & .41 \\
\hline & & $09 / 24 / 02$ & $<.05$ & .09 & .11 & $<.05$ & $<.05$ & .09 & .05 & $<.05$ & $<.05$ & .08 & $<.05$ & $<.05$ & $<.05$ & $<.05$ & .21 & .48 & .38 \\
\hline \multirow[t]{4}{*}{05474000} & \multirow[t]{4}{*}{ Skunk River at Augusta, IA } & $06 / 18 / 98$ & .90 & 2.9 & 2.4 & -- & .06 & 1.0 & $<.20$ & -- & -- & -- & -- & -- & -- & -- & 2.9 & 4.6 & 2.1 \\
\hline & & 05/13/02 & 4.8 & 1.7 & 2.2 & 1.2 & $<.05$ & $<.05$ & $<.05$ & $<.05$ & 3.2 & .45 & .48 & $<.05$ & .09 & .09 & 4.6 & 1.3 & .93 \\
\hline & & 06/27/02 & .32 & .77 & .90 & .27 & $<.05$ & .19 & $<.05$ & $<.05$ & .08 & .08 & .05 & $<.05$ & $<.05$ & $<.05$ & .20 & 1.3 & .27 \\
\hline & & $10 / 29 / 02$ & $<.05$ & .22 & .13 & $<.05$ & $<.05$ & .32 & $<.05$ & $<.05$ & $<.05$ & $<.05$ & $<.05$ & $<.05$ & $<.05$ & $<.05$ & .06 & 1.8 & .27 \\
\hline
\end{tabular}




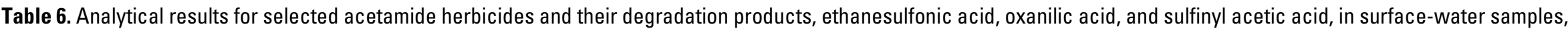
1993-2003-Continued

[OGRL, U.S. Geological Survey Organic Geochemistry Research Laboratory, Lawrence, Kansas; ESA, ethanesulfonic acid; OXA, oxanilic acid; SAA, sulfinyl acetic acid; --, no data; <, less than]

\begin{tabular}{|c|c|c|c|c|c|c|c|c|c|c|c|c|c|c|c|c|c|c|c|}
\hline \multirow[b]{2}{*}{ Site number } & \multirow[b]{2}{*}{ Site location } & \multirow[b]{2}{*}{$\begin{array}{c}\text { Date of } \\
\text { collection } \\
\text { (month/day/ } \\
\text { year) }\end{array}$} & \multicolumn{17}{|c|}{ Concentrations, in micrograms per liter } \\
\hline & & & $\begin{array}{l}\text { Aceto- } \\
\text { chlor } \\
(\mu \mathrm{g} / \mathrm{L})\end{array}$ & $\begin{array}{l}\text { Aceto- } \\
\text { chlor } \\
\text { ESA } \\
(\mu \mathrm{g} / \mathrm{L})\end{array}$ & $\begin{array}{l}\text { Aceto- } \\
\text { chlor } \\
\text { OXA } \\
(\mu \mathrm{g} / \mathrm{L})\end{array}$ & $\begin{array}{c}\text { Aceto- } \\
\text { chlor } \\
\text { SAA } \\
(\mu \mathrm{g} / \mathrm{L})\end{array}$ & $\begin{array}{l}\text { Ala- } \\
\text { chlor } \\
(\mu \mathrm{g} / \mathrm{L})\end{array}$ & $\begin{array}{l}\text { Ala- } \\
\text { chlor } \\
\text { ESA } \\
(\mu \mathrm{g} / \mathrm{L})\end{array}$ & $\begin{array}{l}\text { Ala- } \\
\text { chlor } \\
\text { OXA } \\
(\mu \mathrm{g} / \mathrm{L})\end{array}$ & $\begin{array}{l}\text { Ala- } \\
\text { chlor } \\
\text { SAA } \\
(\mu \mathrm{g} / \mathrm{L})\end{array}$ & $\begin{array}{c}\text { Di- } \\
\text { meth- } \\
\text { enamid } \\
(\mu \mathrm{g} / \mathrm{L})\end{array}$ & $\begin{array}{c}\text { Di- } \\
\text { meth- } \\
\text { enamid } \\
\text { ESA } \\
(\mu \mathrm{g} / \mathrm{L})\end{array}$ & $\begin{array}{c}\text { Di- } \\
\text { meth- } \\
\text { enamid } \\
\text { OXA } \\
(\mu \mathrm{g} / \mathrm{L})\end{array}$ & $\begin{array}{c}\text { Flufen- } \\
\text { acet } \\
(\mu \mathrm{g} / \mathrm{L})\end{array}$ & $\begin{array}{l}\text { Flufen- } \\
\text { acet } \\
\text { ESA } \\
(\mu \mathrm{g} / \mathrm{L})\end{array}$ & $\begin{array}{c}\text { Flufen- } \\
\text { acet } \\
\text { OXA } \\
(\mu \mathrm{g} / \mathrm{L})\end{array}$ & $\begin{array}{l}\text { Meto- } \\
\text { lachlor } \\
(\mu \mathrm{g} / \mathrm{L})\end{array}$ & $\begin{array}{l}\text { Meto- } \\
\text { lachlor } \\
\text { ESA } \\
(\mu \mathrm{g} / \mathrm{L})\end{array}$ & $\begin{array}{l}\text { Meto- } \\
\text { lachlor } \\
\text { OXA } \\
(\mu \mathrm{g} / \mathrm{L})\end{array}$ \\
\hline \multicolumn{20}{|c|}{ Midcontinent Agricultural Chemical Research Project (fig. 1)—Continued } \\
\hline \multicolumn{20}{|c|}{ Sulfonylurea reconnaissance study (DBD)—Continued } \\
\hline \multirow[t]{5}{*}{05439500} & \multirow{5}{*}{$\begin{array}{l}\text { South Branch Kishwaukee } \\
\text { at Fairdale, IL }\end{array}$} & 05/20/98 & 13 & 2.8 & 1.1 & -- & 0.37 & 1.2 & $<0.20$ & -- & -- & -- & -- & -- & -- & -- & 9.6 & 5.0 & 2.1 \\
\hline & & 07/29/98 & $<.05$ & 1.5 & .39 & -- & $<.05$ & 3.0 & $<.20$ & -- & -- & -- & -- & -- & -- & -- & .05 & 5.6 & .81 \\
\hline & & $05 / 14 / 02$ & 2.4 & 1.9 & 1.2 & $<0.05$ & $<.05$ & .34 & .11 & $<0.05$ & 1.8 & 0.13 & 0.09 & $<0.05$ & $<0.05$ & $<0.05$ & 4.1 & 3.1 & .99 \\
\hline & & 08/02/02 & $<.05$ & .33 & .16 & $<.05$ & $<.05$ & .71 & .08 & $<.05$ & $<.05$ & $<.05$ & $<.05$ & $<.05$ & $<.05$ & $<.05$ & .05 & 1.5 & .13 \\
\hline & & $09 / 25 / 02$ & $<.05$ & .25 & .12 & $<.05$ & $<.05$ & .56 & .07 & $<.05$ & $<.05$ & $<.05$ & $<.05$ & $<.05$ & $<.05$ & $<.05$ & $<.05$ & 1.1 & .15 \\
\hline \multirow[t]{5}{*}{05569500} & \multirow{5}{*}{$\begin{array}{l}\text { Spoon River at London } \\
\text { Mills, IL }\end{array}$} & 05/20/98 & 4.1 & 1.5 & 1.4 & -- & .20 & .32 & .27 & -- & -- & -- & -- & -- & -- & -- & 4.3 & 1.9 & .58 \\
\hline & & 07/01/98 & .14 & .86 & .51 & -- & $<.05$ & .35 & $<.20$ & -- & -- & -- & -- & -- & -- & -- & .68 & 2.9 & .79 \\
\hline & & 05/14/02 & 2.8 & .78 & .86 & .54 & $<.05$ & $<.05$ & $<.05$ & $<.05$ & 2.4 & .33 & .28 & .16 & $<.05$ & $<.05$ & 10 & 2.2 & 1.7 \\
\hline & & 07/30/02 & $<.05$ & .27 & .26 & .08 & $<.05$ & $<.05$ & $<.05$ & $<.05$ & $<.05$ & .05 & $<.05$ & $<.05$ & $<.05$ & $<.05$ & .17 & 1.4 & .27 \\
\hline & & 09/24/02 & $<.05$ & .09 & .05 & $<.05$ & $<.05$ & .21 & $<.05$ & $<.05$ & $<.05$ & $<.05$ & $<.05$ & $<.05$ & $<.05$ & $<.05$ & .05 & 1.4 & .15 \\
\hline \multirow[t]{5}{*}{05340500} & \multirow{5}{*}{$\begin{array}{l}\text { St. Croix River at St Croix } \\
\text { Falls, WI }\end{array}$} & 06/03/98 & $<.05$ & $<.20$ & $<.20$ & -- & $<.05$ & $<.20$ & $<.20$ & -- & -- & -- & -- & -- & -- & -- & $<.05$ & $<.20$ & $<.20$ \\
\hline & & 08/05/98 & $<.05$ & $<.20$ & $<.20$ & -- & $<.05$ & $<.20$ & $<.20$ & -- & -- & -- & -- & -- & -- & -- & $<.05$ & $<.20$ & $<.20$ \\
\hline & & $06 / 20 / 02$ & $<.05$ & $<.05$ & $<.05$ & $<.05$ & $<.05$ & $<.05$ & $<.05$ & $<.05$ & $<.05$ & $<.05$ & $<.05$ & $<.05$ & $<.05$ & $<.05$ & $<.05$ & $<.05$ & $<.05$ \\
\hline & & 06/27/02 & $<.05$ & $<.05$ & .06 & $<.05$ & $<.05$ & $<.05$ & $<.05$ & $<.05$ & $<.05$ & $<.05$ & $<.05$ & $<.05$ & $<.05$ & $<.05$ & $<.05$ & $<.05$ & $<.05$ \\
\hline & & 09/03/02 & $<.05$ & $<.05$ & $<.05$ & $<.05$ & $<.05$ & $<.05$ & $<.05$ & $<.05$ & $<.05$ & $<.05$ & $<.05$ & $<.05$ & $<.05$ & $<.05$ & $<.05$ & $<.05$ & $<.05$ \\
\hline \multirow[t]{5}{*}{03362500} & \multirow{5}{*}{$\begin{array}{l}\text { Sugar Creek near } \\
\text { Edinburgh, IN }\end{array}$} & 05/26/98 & 3.4 & 1.7 & 1.6 & -- & .12 & .63 & $<.20$ & -- & -- & -- & -- & -- & -- & -- & 4.0 & 2.6 & 1.3 \\
\hline & & 07/20/98 & .07 & .64 & .43 & -- & $<.05$ & .39 & $<.20$ & -- & -- & -- & -- & -- & -- & -- & 4.1 & 1.5 & 1.0 \\
\hline & & 06/07/02 & 1.4 & .30 & .50 & .15 & $<.05$ & .08 & $<.05$ & $<.05$ & .63 & .09 & .08 & $<.05$ & $<.05$ & $<.05$ & 1.3 & .74 & .29 \\
\hline & & $06 / 26 / 02$ & 2.1 & .41 & .48 & .16 & $<.05$ & .05 & $<.05$ & $<.05$ & .09 & $<.05$ & $<.05$ & $<.05$ & $<.05$ & $<.05$ & 6.7 & .84 & .66 \\
\hline & & $10 / 26 / 02$ & $<.05$ & .19 & .26 & $<.05$ & $<.05$ & .11 & .07 & $<.05$ & $<.05$ & $<.05$ & $<.05$ & $<.05$ & $<.05$ & $<.05$ & $<.05$ & .29 & .17 \\
\hline \multirow[t]{5}{*}{04185000} & \multirow[t]{5}{*}{ Tiffin River at Stryker, OH } & 06/01/98 & .24 & 1.3 & 1.0 & -- & .13 & .70 & $<.20$ & -- & -- & -- & -- & -- & -- & -- & .41 & 2.2 & .79 \\
\hline & & 07/07/98 & $<.05$ & .32 & $<.20$ & -- & $<.05$ & .54 & $<.20$ & -- & -- & -- & -- & -- & -- & -- & .10 & .66 & .33 \\
\hline & & $05 / 29 / 02$ & .33 & .35 & .24 & $<.05$ & $<.05$ & .30 & .05 & $<.05$ & .11 & .05 & $<.05$ & $<.05$ & $<.05$ & $<.05$ & .32 & .60 & .20 \\
\hline & & $07 / 23 / 02$ & $<.05$ & .19 & .17 & $<.05$ & $<.05$ & .54 & .06 & $<.05$ & $<.05$ & $<.05$ & $<.05$ & $<.05$ & $<.05$ & $<.05$ & $<.05$ & .79 & .24 \\
\hline & & $11 / 06 / 02$ & $<.05$ & .06 & $<.05$ & $<.05$ & $<.05$ & .23 & $<.05$ & $<.05$ & $<.05$ & $<.05$ & $<.05$ & $<.05$ & $<.05$ & $<.05$ & $<.05$ & .24 & .08 \\
\hline
\end{tabular}




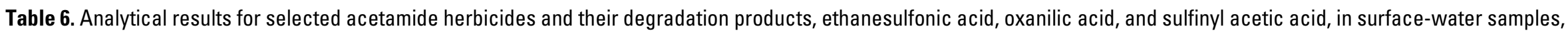
1993-2003.-Continued

[OGRL, U.S. Geological Survey Organic Geochemistry Research Laboratory, Lawrence, Kansas; ESA, ethanesulfonic acid; OXA, oxanilic acid; SAA, sulfinyl acetic acid; --, no data; <, less than]

\begin{tabular}{|c|c|c|c|c|c|c|c|c|c|c|c|c|c|c|c|c|c|c|c|}
\hline \multirow[b]{2}{*}{ Site number } & \multirow[b]{2}{*}{ Site location } & \multirow[b]{2}{*}{$\begin{array}{c}\text { Date of } \\
\text { collection } \\
\text { (month/day/ } \\
\text { year) }\end{array}$} & \multicolumn{17}{|c|}{ Concentrations, in micrograms per liter } \\
\hline & & & $\begin{array}{c}\text { Aceto- } \\
\text { chlor } \\
(\mu \mathrm{g} / \mathrm{L})\end{array}$ & $\begin{array}{l}\text { Aceto- } \\
\text { chlor } \\
\text { ESA } \\
(\mu \mathrm{g} / \mathrm{L})\end{array}$ & $\begin{array}{c}\text { Aceto- } \\
\text { chlor } \\
\text { OXA } \\
(\mu \mathrm{g} / \mathrm{L})\end{array}$ & $\begin{array}{l}\text { Aceto- } \\
\text { chlor } \\
\text { SAA } \\
(\mu \mathrm{g} / \mathrm{L})\end{array}$ & $\begin{array}{l}\text { Ala- } \\
\text { chlor } \\
(\mu \mathrm{g} / \mathrm{L})\end{array}$ & $\begin{array}{l}\text { Ala- } \\
\text { chlor } \\
\text { ESA } \\
(\mu \mathrm{g} / \mathrm{L})\end{array}$ & $\begin{array}{c}\text { Ala- } \\
\text { chlor } \\
\text { OXA } \\
(\mu \mathrm{g} / \mathrm{L})\end{array}$ & $\begin{array}{l}\text { Ala- } \\
\text { chlor } \\
\text { SAA } \\
(\mu \mathrm{g} / \mathrm{L})\end{array}$ & $\begin{array}{c}\text { Di- } \\
\text { meth- } \\
\text { enamid } \\
(\mu \mathrm{g} / \mathrm{L})\end{array}$ & $\begin{array}{c}\text { Di- } \\
\text { meth- } \\
\text { enamid } \\
\text { ESA } \\
(\mu \mathrm{g} / \mathrm{L})\end{array}$ & $\begin{array}{c}\text { Di- } \\
\text { meth- } \\
\text { enamid } \\
\text { OXA } \\
(\mu \mathrm{g} / \mathrm{L})\end{array}$ & $\begin{array}{c}\text { Flufen- } \\
\text { acet } \\
(\mu \mathrm{g} / \mathrm{L})\end{array}$ & $\begin{array}{c}\text { Flufen- } \\
\text { acet } \\
\text { ESA } \\
(\mu \mathrm{g} / \mathrm{L})\end{array}$ & $\begin{array}{c}\text { Flufen- } \\
\text { acet } \\
\text { OXA } \\
(\mu \mathrm{g} / \mathrm{L})\end{array}$ & $\begin{array}{c}\text { Meto- } \\
\text { lachlor } \\
(\mu \mathrm{g} / \mathrm{L})\end{array}$ & $\begin{array}{c}\text { Meto- } \\
\text { lachlor } \\
\text { ESA } \\
(\mu \mathrm{g} / \mathrm{L})\end{array}$ & $\begin{array}{c}\text { Meto- } \\
\text { lachlor } \\
\text { OXA } \\
(\mu \mathrm{g} / \mathrm{L})\end{array}$ \\
\hline \multicolumn{20}{|c|}{ Midcontinent Agricultural Chemical Research Project (fig. 1)—Continued } \\
\hline \multicolumn{20}{|c|}{ Sulfonylurea reconnaissance study (DBD)—Continued } \\
\hline \multirow[t]{3}{*}{05411850} & \multirow{3}{*}{$\begin{array}{l}\text { Turkey River near } \\
\text { Eldorado, IA }\end{array}$} & $05 / 14 / 02$ & 0.27 & 0.75 & 0.17 & $<0.05$ & $<0.05$ & 1.1 & $<0.05$ & $<0.05$ & 0.11 & 0.05 & $<0.05$ & $<0.05$ & $<0.05$ & $<0.05$ & $<0.05$ & 1.1 & 0.08 \\
\hline & & $06 / 24 / 02$ & .11 & 1.6 & .76 & .46 & $<.05$ & .99 & $<.05$ & $<.05$ & .21 & .22 & .09 & $<.05$ & $<.05$ & $<.05$ & .06 & 1.2 & .20 \\
\hline & & $10 / 29 / 02$ & $<.05$ & .27 & $<.05$ & $<.05$ & $<.05$ & 1.3 & $<.05$ & $<.05$ & $<.05$ & $<.05$ & $<.05$ & $<.05$ & $<.05$ & $<.05$ & $<.05$ & .80 & .06 \\
\hline \multirow[t]{2}{*}{05411600} & \multirow{2}{*}{$\begin{array}{l}\text { Turkey River at } \\
\text { Spillville, IA }\end{array}$} & $06 / 02 / 98$ & .24 & 1.2 & .40 & -- & $<.05$ & 2.1 & $<.20$ & -- & -- & -- & -- & -- & -- & -- & .10 & 2.1 & 1.1 \\
\hline & & $06 / 12 / 98$ & 1.3 & 5.0 & 4.3 & -- & .25 & 3.0 & .96 & -- & -- & -- & -- & -- & -- & -- & .14 & 1.6 & $<.20$ \\
\hline \multirow[t]{2}{*}{03338890} & \multirow{2}{*}{$\begin{array}{l}\text { Vermilion River at } \\
\text { Danville, IL }\end{array}$} & 06/10/98 & .36 & 1.3 & .62 & -- & $<.05$ & .59 & $<.20$ & -- & -- & -- & -- & -- & -- & -- & .97 & 4.2 & 1.2 \\
\hline & & $07 / 16 / 98$ & $<.05$ & .45 & $<.20$ & -- & $<.05$ & .66 & $<.20$ & -- & -- & -- & -- & -- & -- & -- & .48 & 3.3 & 1.3 \\
\hline \multirow[t]{5}{*}{06880800} & \multirow{5}{*}{$\begin{array}{l}\text { West Fork Big Blue River } \\
\text { at Dorchester, NE }\end{array}$} & $05 / 23 / 98$ & 2.2 & $<.20$ & $<.20$ & -- & 4.2 & .58 & $<.20$ & -- & -- & -- & -- & -- & -- & -- & 12 & .59 & .42 \\
\hline & & 06/10/98 & .83 & .69 & 1.1 & -- & .57 & .69 & .67 & -- & -- & -- & -- & -- & -- & -- & 3.9 & .58 & .72 \\
\hline & & 05/07/02 & 5.4 & .39 & .54 & .18 & .38 & .12 & .09 & $<.05$ & 4.1 & .15 & .20 & .39 & $<.05$ & $<.05$ & $<.05$ & .63 & .65 \\
\hline & & $07 / 26 / 02$ & .05 & .16 & .27 & $<.05$ & .05 & .27 & .18 & $<.05$ & $<.05$ & $<.05$ & $<.05$ & .10 & $<.05$ & $<.05$ & .21 & .28 & .33 \\
\hline & & $10 / 04 / 02$ & $<.05$ & .13 & .17 & .07 & $<.05$ & .15 & .08 & .06 & $<.05$ & $<.05$ & $<.05$ & $<.05$ & $<.05$ & $<.05$ & .13 & .35 & .44 \\
\hline \multirow[t]{2}{*}{03378500} & \multirow{2}{*}{$\begin{array}{l}\text { Wabash River at New } \\
\text { Harmony, IN }\end{array}$} & $05 / 27 / 98$ & 2.0 & .78 & .80 & -- & .14 & .35 & $<.20$ & -- & -- & -- & -- & -- & -- & -- & 1.9 & .86 & .65 \\
\hline & & $06 / 23 / 98$ & .98 & 2.3 & 2.0 & -- & .17 & 1.4 & .26 & -- & -- & -- & -- & -- & -- & -- & 4.2 & 3.3 & 2.0 \\
\hline \multirow[t]{5}{*}{06804000} & \multirow[t]{5}{*}{ Wahoo Creek at Ithaca, NE } & $05 / 15 / 98$ & 1.8 & $<.20$ & $<.20$ & -- & 4.9 & .55 & .66 & -- & -- & -- & -- & -- & -- & -- & 16 & .55 & .76 \\
\hline & & 06/08/98 & .33 & .36 & .47 & -- & .22 & .41 & .41 & -- & -- & -- & -- & -- & -- & -- & .80 & .34 & .41 \\
\hline & & $05 / 13 / 02$ & 18 & .65 & 1.3 & .57 & .34 & .11 & .05 & .07 & .53 & $<.05$ & $<.05$ & .93 & .07 & $<.05$ & 1.5 & .12 & .11 \\
\hline & & $06 / 12 / 02$ & 10 & 1.0 & 1.8 & .79 & .77 & .15 & .19 & .07 & 1.6 & .09 & .10 & 1.6 & .09 & .08 & 3.0 & .08 & .13 \\
\hline & & $10 / 04 / 02$ & $<.05$ & .20 & .22 & .08 & $<.05$ & .09 & $<.05$ & $<.05$ & $<.05$ & .05 & $<.05$ & $<.05$ & $<.05$ & $<.05$ & .12 & .07 & .07 \\
\hline \multirow[t]{5}{*}{05421000} & \multirow{5}{*}{$\begin{array}{l}\text { Wapsipinicon River near } \\
\text { Independence, IA }\end{array}$} & $05 / 29 / 98$ & 1.5 & 1.7 & .85 & -- & .32 & 1.4 & $<.20$ & -- & -- & -- & -- & -- & -- & -- & 1.8 & 4.4 & 1.0 \\
\hline & & $06 / 12 / 98$ & 1.2 & 3.0 & 2.4 & -- & .08 & 1.3 & $<.20$ & -- & -- & -- & -- & -- & -- & -- & 2.2 & 3.7 & 1.5 \\
\hline & & $05 / 14 / 02$ & .62 & .83 & .21 & $<.05$ & .08 & 1.3 & .06 & $<.05$ & .33 & $<.05$ & $<.05$ & $<.05$ & $<.05$ & $<.05$ & .26 & 3.2 & .29 \\
\hline & & $06 / 24 / 02$ & .42 & 1.8 & 1.2 & .38 & $<.05$ & 1.1 & .10 & $<.05$ & .24 & .25 & .15 & $<.05$ & $<.05$ & $<.05$ & .39 & 3.4 & .67 \\
\hline & & $10 / 29 / 02$ & $<.05$ & .44 & .15 & $<.05$ & $<.05$ & 2.2 & .12 & $<.05$ & $<.05$ & .07 & $<.05$ & $<.05$ & $<.05$ & $<.05$ & .07 & 3.0 & .35 \\
\hline \multirow[t]{2}{*}{05420680} & \multirow{2}{*}{$\begin{array}{l}\text { Wapsipinicon River near } \\
\text { Tripoli, IA }\end{array}$} & $05 / 27 / 98$ & .33 & 2.8 & 1.1 & -- & .08 & 3.2 & $<.20$ & -- & -- & -- & -- & -- & -- & -- & .54 & 12 & 2.5 \\
\hline & & $06 / 20 / 98$ & .36 & 2.2 & 1.3 & -- & .12 & 1.9 & .39 & -- & -- & -- & -- & -- & -- & -- & 2.0 & 4.2 & 2.0 \\
\hline
\end{tabular}




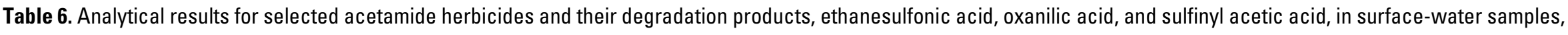
1993-2003-Continued

[OGRL, U.S. Geological Survey Organic Geochemistry Research Laboratory, Lawrence, Kansas; ESA, ethanesulfonic acid; OXA, oxanilic acid; SAA, sulfinyl acetic acid; --, no data; <, less than]

\begin{tabular}{|c|c|c|c|c|c|c|c|c|c|c|c|c|c|c|c|c|c|c|c|}
\hline \multirow[b]{2}{*}{ Site number } & \multirow[b]{2}{*}{ Site location } & \multirow[b]{2}{*}{$\begin{array}{c}\text { Date of } \\
\text { collection } \\
\text { (month/day/ } \\
\text { year) }\end{array}$} & \multicolumn{17}{|c|}{ Concentrations, in micrograms per liter } \\
\hline & & & $\begin{array}{l}\text { Aceto- } \\
\text { chlor } \\
(\mu \mathrm{g} / \mathrm{L})\end{array}$ & $\begin{array}{l}\text { Aceto- } \\
\text { chlor } \\
\text { ESA } \\
(\mu \mathrm{g} / \mathrm{L})\end{array}$ & $\begin{array}{l}\text { Aceto- } \\
\text { chlor } \\
\text { OXA } \\
(\mu \mathrm{g} / \mathrm{L})\end{array}$ & $\begin{array}{c}\text { Aceto- } \\
\text { chlor } \\
\text { SAA } \\
(\mu \mathrm{g} / \mathrm{L})\end{array}$ & $\begin{array}{l}\text { Ala- } \\
\text { chlor } \\
(\mu \mathrm{g} / \mathrm{L})\end{array}$ & $\begin{array}{l}\text { Ala- } \\
\text { chlor } \\
\text { ESA } \\
(\mu \mathrm{g} / \mathrm{L})\end{array}$ & $\begin{array}{c}\text { Ala- } \\
\text { chlor } \\
\text { OXA } \\
(\mu \mathrm{g} / \mathrm{L})\end{array}$ & $\begin{array}{l}\text { Ala- } \\
\text { chlor } \\
\text { SAA } \\
(\mu \mathrm{g} / \mathrm{L})\end{array}$ & $\begin{array}{c}\text { Di- } \\
\text { meth- } \\
\text { enamid } \\
(\mu \mathrm{g} / \mathrm{L})\end{array}$ & $\begin{array}{c}\text { Di- } \\
\text { meth- } \\
\text { enamid } \\
\text { ESA } \\
(\mu \mathrm{g} / \mathrm{L})\end{array}$ & $\begin{array}{c}\text { Di- } \\
\text { meth- } \\
\text { enamid } \\
\text { OXA } \\
(\mu \mathrm{g} / \mathrm{L})\end{array}$ & $\begin{array}{c}\text { Flufen- } \\
\text { acet } \\
(\mu \mathrm{g} / \mathrm{L})\end{array}$ & $\begin{array}{c}\text { Flufen- } \\
\text { acet } \\
\text { ESA } \\
(\mu \mathrm{g} / \mathrm{L})\end{array}$ & $\begin{array}{c}\text { Flufen- } \\
\text { acet } \\
\text { OXA } \\
(\mu \mathrm{g} / \mathrm{L})\end{array}$ & $\begin{array}{l}\text { Meto- } \\
\text { lachlor } \\
(\mu \mathrm{g} / \mathrm{L})\end{array}$ & $\begin{array}{c}\text { Meto- } \\
\text { lachlor } \\
\text { ESA } \\
(\mu \mathrm{g} / \mathrm{L})\end{array}$ & $\begin{array}{l}\text { Meto- } \\
\text { lachlor } \\
\text { OXA } \\
(\mu \mathrm{g} / \mathrm{L})\end{array}$ \\
\hline \multicolumn{20}{|c|}{ Midcontinent Agricultural Chemical Research Project (fig. 1)—Continued } \\
\hline \multicolumn{20}{|c|}{ Sulfonylurea reconnaissance study (DBD)—Continued } \\
\hline \multirow[t]{2}{*}{03351000} & \multirow[t]{2}{*}{ White River near Nora, IN } & 05/28/98 & 1.4 & 0.97 & 0.80 & -- & $<0.05$ & 0.62 & 0.22 & -- & -- & -- & -- & -- & -- & -- & 3.8 & 3.1 & 1.3 \\
\hline & & 07/01/98 & .37 & 1.5 & 1.2 & -- & .09 & .76 & .20 & -- & -- & -- & -- & -- & -- & -- & 2.3 & 3.5 & 2.0 \\
\hline \multirow[t]{5}{*}{03275000} & \multirow{5}{*}{$\begin{array}{l}\text { Whitewater River near } \\
\text { Alpine, IN }\end{array}$} & 05/26/98 & 1.7 & 1.2 & .76 & -- & 1.1 & .50 & .55 & -- & -- & -- & -- & -- & -- & -- & 1.4 & .69 & .47 \\
\hline & & 07/08/98 & .13 & .48 & .35 & -- & .06 & .58 & .40 & -- & -- & -- & -- & -- & -- & -- & .71 & .99 & .50 \\
\hline & & 06/14/02 & .98 & .65 & .94 & 0.51 & 6.0 & .86 & 1.1 & 0.76 & 0.59 & 0.07 & $<0.05$ & $<0.05$ & $<0.05$ & $<0.05$ & 2.2 & .41 & .37 \\
\hline & & 06/28/02 & .61 & .93 & 1.2 & .43 & 1.3 & .79 & .84 & .36 & .06 & $<.05$ & $<.05$ & $<.05$ & $<.05$ & $<.05$ & .92 & .50 & .43 \\
\hline & & $10 / 29 / 02$ & $<.05$ & $<.05$ & $<.05$ & $<.05$ & $<.05$ & .05 & $<.05$ & $<.05$ & $<.05$ & $<.05$ & $<.05$ & $<.05$ & $<.05$ & $<.05$ & $<.05$ & .07 & $<.05$ \\
\hline \multirow[t]{5}{*}{03333450} & \multirow{5}{*}{$\begin{array}{l}\text { Wildcat Creek near Jerome, } \\
\text { IN }\end{array}$} & 05/21/98 & 1.3 & .99 & .65 & -- & $<.05$ & .53 & $<.20$ & -- & -- & -- & -- & -- & -- & -- & 1.0 & 4.8 & 1.0 \\
\hline & & 06/30/98 & .21 & 1.9 & 1.1 & -- & .22 & 1.2 & .44 & -- & -- & -- & -- & -- & -- & -- & 2.1 & 6.4 & 2.4 \\
\hline & & 05/30/02 & 1.6 & .52 & .50 & .31 & .05 & .15 & $<.05$ & $<.05$ & .15 & .05 & $<.05$ & $<.05$ & $<.05$ & $<.05$ & .60 & 2.5 & .37 \\
\hline & & 08/19/02 & $<.05$ & .09 & .13 & $<.05$ & $<.05$ & .16 & $<.05$ & $<.05$ & $<.05$ & $<.05$ & $<.05$ & $<.05$ & $<.05$ & $<.05$ & .05 & .99 & .23 \\
\hline & & 09/20/02 & $<.05$ & .16 & .23 & .05 & $<.05$ & .15 & .09 & $<.05$ & $<.05$ & .08 & $<.05$ & $<.05$ & $<.05$ & .05 & .09 & .80 & .37 \\
\hline \multirow[t]{5}{*}{03335000} & \multirow{5}{*}{$\begin{array}{l}\text { Wildcat Creek near } \\
\text { Lafayette, IN }\end{array}$} & 05/20/98 & .78 & .85 & .46 & -- & $<.05$ & .44 & $<.20$ & -- & -- & -- & -- & -- & -- & -- & 2.1 & 3.1 & .91 \\
\hline & & 07/01/98 & .14 & 1.6 & 1.0 & -- & $<.05$ & .72 & $<.20$ & -- & -- & -- & -- & -- & -- & -- & 1.2 & 3.7 & 1.8 \\
\hline & & 05/30/02 & .38 & .26 & .19 & .10 & $<.05$ & .14 & .05 & $<.05$ & .10 & .05 & $<.05$ & $<.05$ & $<.05$ & $<.05$ & .30 & 1.4 & .28 \\
\hline & & 07/23/02 & $<.05$ & .12 & .09 & $<.05$ & $<.05$ & .11 & $<.05$ & $<.05$ & $<.05$ & $<.05$ & $<.05$ & $<.05$ & $<.05$ & $<.05$ & .07 & .52 & .11 \\
\hline & & $11 / 12 / 02$ & $<.05$ & .38 & .45 & $<.05$ & $<.05$ & .13 & .08 & $<.05$ & $<.05$ & .07 & $<.05$ & $<.05$ & $<.05$ & $<.05$ & .07 & .87 & .48 \\
\hline \multirow[t]{5}{*}{05407000} & \multirow{5}{*}{$\begin{array}{l}\text { Wisconsin River at } \\
\text { Muscoda,WI }\end{array}$} & 06/16/98 & .05 & $<.20$ & $<.20$ & -- & $<.05$ & .29 & $<.20$ & -- & -- & -- & -- & -- & -- & -- & .15 & $<.20$ & $<.20$ \\
\hline & & 08/07/98 & $<.05$ & .26 & $<.20$ & -- & $<.05$ & .56 & $<.20$ & -- & -- & -- & -- & -- & -- & -- & $<.05$ & .56 & .21 \\
\hline & & 05/28/02 & $<.05$ & $<.05$ & $<.05$ & $<.05$ & $<.05$ & .23 & $<.05$ & $<.05$ & $<.05$ & $<.05$ & $<.05$ & $<.05$ & $<.05$ & $<.05$ & $<.05$ & .17 & .07 \\
\hline & & 06/27/02 & .06 & .05 & .05 & $<.05$ & $<.05$ & .18 & $<.05$ & $<.05$ & $<.05$ & $<.05$ & $<.05$ & $<.05$ & $<.05$ & $<.05$ & .05 & .16 & .07 \\
\hline & & $09 / 23 / 02$ & $<.05$ & .07 & .07 & $<.05$ & $<.05$ & .22 & .05 & $<.05$ & $<.05$ & .06 & $<.05$ & $<.05$ & $<.05$ & $<.05$ & $<.05$ & .15 & .09 \\
\hline \multirow[t]{2}{*}{05464220} & \multirow[t]{2}{*}{ Wolf Creek near Dysart, IA } & 06/10/98 & .64 & 1.9 & 1.6 & -- & $<.05$ & 1.2 & $<.20$ & -- & -- & -- & -- & -- & -- & -- & 1.8 & 6.3 & 1.9 \\
\hline & & $06 / 22 / 98$ & .68 & 1.3 & 1.1 & -- & $<.05$ & .63 & $<.20$ & -- & -- & -- & -- & -- & -- & -- & 2.0 & 2.4 & 1.1 \\
\hline
\end{tabular}




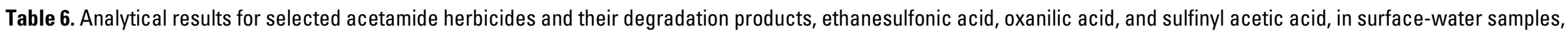
1993-2003.-Continued

[OGRL, U.S. Geological Survey Organic Geochemistry Research Laboratory, Lawrence, Kansas; ESA, ethanesulfonic acid; OXA, oxanilic acid; SAA, sulfinyl acetic acid; --, no data; <, less than]

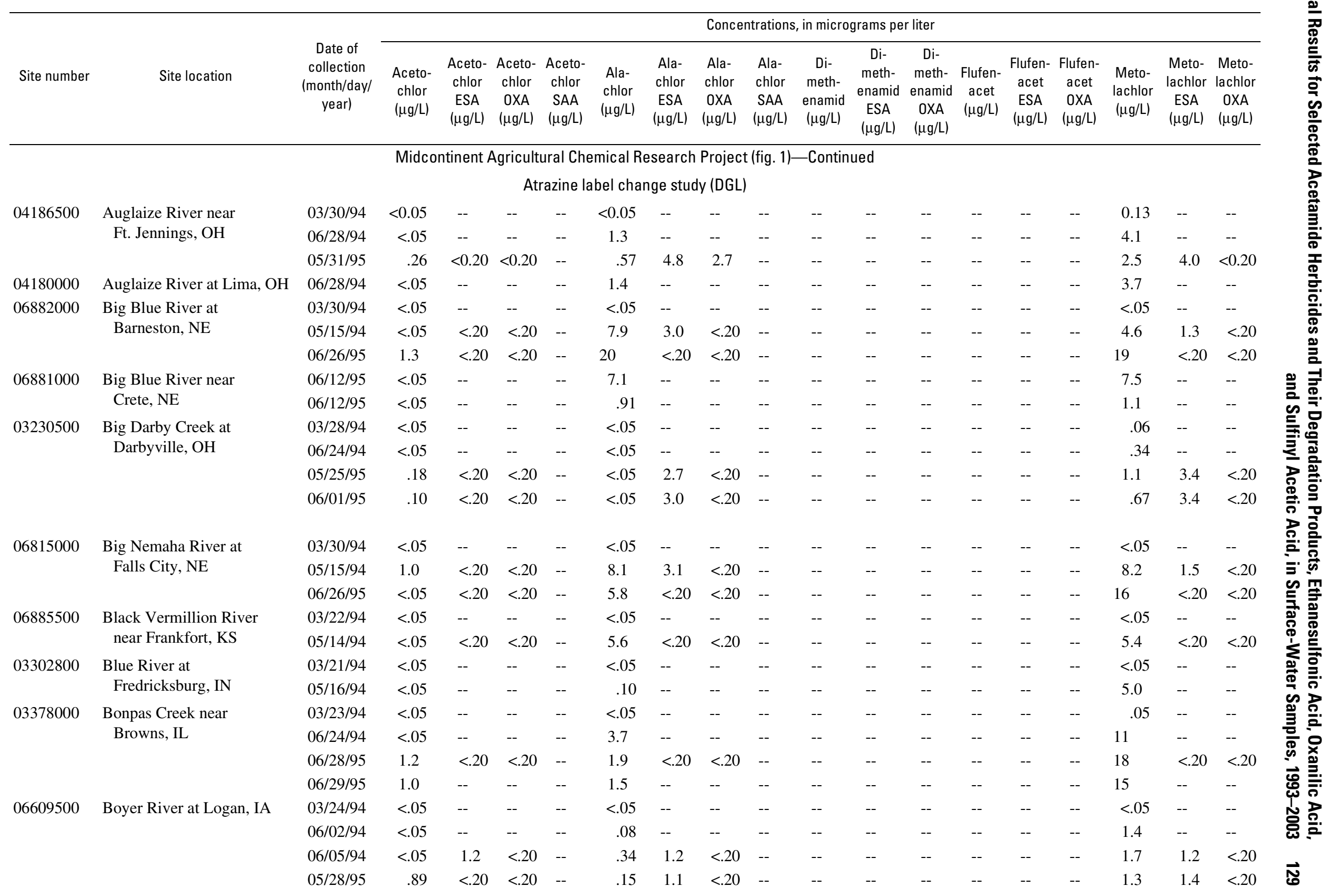




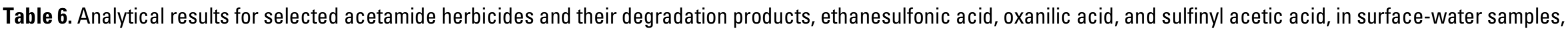
1993-2003-Continued

[OGRL, U.S. Geological Survey Organic Geochemistry Research Laboratory, Lawrence, Kansas; ESA, ethanesulfonic acid; OXA, oxanilic acid; SAA, sulfinyl acetic acid; --, no data; <, less than]

\begin{tabular}{|c|c|c|c|c|c|c|c|c|c|c|c|c|c|c|c|c|c|c|c|}
\hline \multirow[b]{2}{*}{ Site number } & \multirow[b]{2}{*}{ Site location } & \multirow[b]{2}{*}{$\begin{array}{c}\text { Date of } \\
\text { collection } \\
\text { (month/day/ } \\
\text { year) }\end{array}$} & \multicolumn{17}{|c|}{ Concentrations, in micrograms per liter } \\
\hline & & & $\begin{array}{c}\text { Aceto- } \\
\text { chlor } \\
(\mu \mathrm{g} / \mathrm{L})\end{array}$ & $\begin{array}{l}\text { Aceto- } \\
\text { chlor } \\
\text { ESA } \\
(\mu \mathrm{g} / \mathrm{L})\end{array}$ & $\begin{array}{c}\text { Aceto- } \\
\text { chlor } \\
\text { OXA } \\
(\mu \mathrm{g} / \mathrm{L})\end{array}$ & $\begin{array}{l}\text { Aceto- } \\
\text { chlor } \\
\text { SAA } \\
(\mu \mathrm{g} / \mathrm{L})\end{array}$ & $\begin{array}{l}\text { Ala- } \\
\text { chlor } \\
(\mu \mathrm{g} / \mathrm{L})\end{array}$ & $\begin{array}{l}\text { Ala- } \\
\text { chlor } \\
\text { ESA } \\
(\mu \mathrm{g} / \mathrm{L})\end{array}$ & $\begin{array}{l}\text { Ala- } \\
\text { chlor } \\
\text { OXA } \\
(\mu \mathrm{g} / \mathrm{L})\end{array}$ & $\begin{array}{l}\text { Ala- } \\
\text { chlor } \\
\text { SAA } \\
(\mu \mathrm{g} / \mathrm{L})\end{array}$ & $\begin{array}{c}\text { Di- } \\
\text { meth- } \\
\text { enamid } \\
(\mu \mathrm{g} / \mathrm{L})\end{array}$ & $\begin{array}{c}\text { Di- } \\
\text { meth- } \\
\text { enamid } \\
\text { ESA } \\
(\mu \mathrm{g} / \mathrm{L})\end{array}$ & $\begin{array}{c}\text { Di- } \\
\text { meth- } \\
\text { enamid } \\
\text { OXA } \\
(\mu \mathrm{g} / \mathrm{L})\end{array}$ & $\begin{array}{c}\text { Flufen- } \\
\text { acet } \\
(\mu \mathrm{g} / \mathrm{L})\end{array}$ & $\begin{array}{c}\text { Flufen- } \\
\text { acet } \\
\text { ESA } \\
(\mu \mathrm{g} / \mathrm{L})\end{array}$ & $\begin{array}{l}\text { Flufen- } \\
\text { acet } \\
\text { OXA } \\
(\mu \mathrm{g} / \mathrm{L})\end{array}$ & $\begin{array}{l}\text { Meto- } \\
\text { lachlor } \\
(\mu \mathrm{g} / \mathrm{L})\end{array}$ & $\begin{array}{l}\text { Meto- } \\
\text { lachlor } \\
\text { ESA } \\
(\mu \mathrm{g} / \mathrm{L})\end{array}$ & $\begin{array}{c}\text { Meto- } \\
\text { lachlor } \\
\text { OXA } \\
(\mu \mathrm{g} / \mathrm{L})\end{array}$ \\
\hline \multicolumn{20}{|c|}{ Midcontinent Agricultural Chemical Research Project (fig. 1)—Continued } \\
\hline \multicolumn{20}{|c|}{ Atrazine label change study (DGL) — Continued } \\
\hline \multirow[t]{2}{*}{05587770} & \multirow{2}{*}{$\begin{array}{l}\text { Cahokia Creek tributary } \\
\text { near Worden, IL }\end{array}$} & $03 / 30 / 94$ & $<0.05$ & -- & -- & -- & 0.20 & -- & -- & -- & -- & -- & -- & -- & -- & -- & 0.20 & -- & -- \\
\hline & & $06 / 15 / 94$ & $<.05$ & -- & -- & -- & 7.0 & -- & -- & -- & -- & -- & -- & -- & -- & -- & 7.2 & -- & -- \\
\hline \multirow[t]{3}{*}{05576005} & \multirow{3}{*}{$\begin{array}{l}\text { Canoe Creek above } \\
\text { Rochester, IL }\end{array}$} & $03 / 25 / 94$ & $<.05$ & -- & -- & -- & .19 & -- & -- & -- & -- & -- & -- & -- & -- & -- & .18 & -- & -- \\
\hline & & $06 / 29 / 94$ & $<.05$ & -- & -- & -- & 7.8 & -- & -- & -- & -- & -- & -- & -- & -- & -- & 7.0 & -- & -- \\
\hline & & $06 / 30 / 95$ & $<.05$ & -- & -- & -- & 7.8 & -- & -- & -- & -- & -- & -- & -- & -- & -- & 9.7 & -- & -- \\
\hline \multirow[t]{3}{*}{05491200} & \multirow[t]{3}{*}{ Cedar Creek near Tracy, IA } & $05 / 21 / 94$ & $<.05$ & -- & -- & -- & $<.05$ & -- & -- & -- & -- & -- & -- & -- & -- & -- & .33 & -- & -- \\
\hline & & $05 / 26 / 94$ & $<.05$ & -- & -- & -- & $<.05$ & -- & -- & -- & -- & -- & -- & -- & -- & -- & .12 & -- & -- \\
\hline & & $05 / 30 / 95$ & .34 & $<0.20$ & $<0.20$ & -- & .09 & 7.5 & 3.8 & -- & -- & -- & -- & -- & -- & -- & .48 & 4.7 & $<0.20$ \\
\hline \multirow[t]{2}{*}{03157000} & \multirow{2}{*}{$\begin{array}{l}\text { Clear Creek at } \\
\text { Rockbridge, } \mathrm{OH}\end{array}$} & $03 / 28 / 94$ & $<.05$ & -- & -- & -- & $<.05$ & -- & -- & -- & -- & -- & -- & -- & -- & -- & .20 & -- & -- \\
\hline & & $06 / 01 / 95$ & .07 & 1.2 & $<.20$ & -- & $<.05$ & 1.2 & $<.20$ & -- & -- & -- & -- & -- & -- & -- & .29 & 2.1 & $<.20$ \\
\hline 04170000 & $\begin{array}{l}\text { Clear Creek near Grand } \\
\text { Blanc, } \mathrm{OH}\end{array}$ & $06 / 27 / 94$ & $<.05$ & -- & -- & -- & $<.05$ & -- & -- & -- & -- & -- & -- & -- & -- & -- & $<.05$ & -- & -- \\
\hline \multirow[t]{3}{*}{05317000} & \multirow{3}{*}{$\begin{array}{l}\text { Cottonwood River near } \\
\text { New Ulm, MN }\end{array}$} & $04 / 05 / 94$ & $<.05$ & -- & -- & -- & $<.05$ & -- & -- & -- & -- & -- & -- & -- & -- & -- & .05 & -- & -- \\
\hline & & $05 / 25 / 94$ & $<.05$ & 2.6 & $<.20$ & -- & 4.5 & 2.1 & 1.8 & -- & -- & -- & -- & -- & -- & -- & 1.2 & 1.4 & $<.20$ \\
\hline & & $05 / 28 / 95$ & .07 & $<.20$ & $<.20$ & -- & .06 & 1.6 & $<.20$ & -- & -- & -- & -- & -- & -- & -- & .28 & 1.9 & $<.20$ \\
\hline \multirow[t]{2}{*}{03612020} & \multirow{2}{*}{$\begin{array}{l}\text { Day Creek above } \\
\text { Forman, IL }\end{array}$} & $03 / 30 / 94$ & $<.05$ & -- & -- & -- & .27 & -- & -- & -- & -- & -- & -- & -- & -- & -- & .29 & -- & -- \\
\hline & & $06 / 14 / 94$ & $<.05$ & -- & -- & -- & 7.2 & -- & -- & -- & -- & -- & -- & -- & -- & -- & 7.8 & -- & -- \\
\hline \multirow[t]{3}{*}{06890100} & \multirow{3}{*}{$\begin{array}{l}\text { Delaware River near } \\
\text { Muscotah, KS }\end{array}$} & $03 / 21 / 94$ & $<.05$ & -- & -- & -- & $<.05$ & -- & -- & -- & -- & -- & -- & -- & -- & -- & $<.05$ & -- & -- \\
\hline & & $05 / 15 / 94$ & $<.05$ & $<.20$ & $<.20$ & -- & 3.0 & 3.5 & $<.20$ & -- & -- & -- & -- & -- & -- & -- & 7.2 & 2.2 & $<.20$ \\
\hline & & $06 / 25 / 95$ & .19 & $<.20$ & $<.20$ & -- & 5.3 & 2.4 & $<.20$ & -- & -- & -- & -- & -- & -- & -- & 9.3 & 1.6 & $<.20$ \\
\hline \multirow[t]{3}{*}{05480500} & \multirow{3}{*}{$\begin{array}{l}\text { Des Moines River at } \\
\text { Fort Dodge, IA }\end{array}$} & $03 / 23 / 94$ & $<.05$ & -- & -- & -- & .05 & -- & -- & -- & -- & -- & -- & -- & -- & -- & .19 & -- & -- \\
\hline & & $06 / 06 / 94$ & $<.05$ & -- & -- & -- & .12 & -- & -- & -- & -- & -- & -- & -- & -- & -- & .26 & -- & -- \\
\hline & & $05 / 29 / 95$ & 1.4 & $<.20$ & $<.20$ & -- & .32 & 2.9 & $<.20$ & -- & -- & -- & -- & -- & -- & -- & 2.6 & 4.1 & $<.20$ \\
\hline \multirow[t]{3}{*}{05476000} & \multirow{3}{*}{$\begin{array}{l}\text { Des Moines River at } \\
\text { Jackson, MN }\end{array}$} & $04 / 05 / 94$ & $<.05$ & -- & -- & -- & $<.05$ & -- & -- & -- & -- & -- & -- & -- & -- & -- & .08 & -- & -- \\
\hline & & $06 / 06 / 94$ & $<.05$ & $<.20$ & $<.20$ & -- & .33 & 2.4 & $<.20$ & -- & -- & -- & -- & -- & -- & -- & .12 & 2.9 & $<.20$ \\
\hline & & $05 / 28 / 95$ & .28 & $<.20$ & $<.20$ & -- & .05 & 2.6 & $<.20$ & -- & -- & -- & -- & -- & -- & -- & .22 & 3.4 & $<.20$ \\
\hline
\end{tabular}




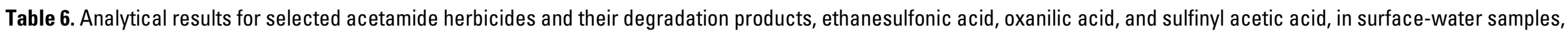
1993-2003.-Continued

[OGRL, U.S. Geological Survey Organic Geochemistry Research Laboratory, Lawrence, Kansas; ESA, ethanesulfonic acid; OXA, oxanilic acid; SAA, sulfinyl acetic acid; --, no data; <, less than]

\begin{tabular}{|c|c|c|c|c|c|c|c|c|c|c|c|c|c|c|c|c|c|c|c|}
\hline \multirow[b]{2}{*}{ Site number } & \multirow[b]{2}{*}{ Site location } & \multirow[b]{2}{*}{$\begin{array}{c}\text { Date of } \\
\text { collection } \\
\text { (month/day/ } \\
\text { year) }\end{array}$} & \multicolumn{17}{|c|}{ Concentrations, in micrograms per liter } \\
\hline & & & $\begin{array}{l}\text { Aceto- } \\
\text { chlor } \\
(\mu \mathrm{g} / \mathrm{L})\end{array}$ & $\begin{array}{l}\text { Aceto- } \\
\text { chlor } \\
\text { ESA } \\
(\mu \mathrm{g} / \mathrm{L})\end{array}$ & $\begin{array}{c}\text { Aceto- } \\
\text { chlor } \\
\text { OXA } \\
(\mu \mathrm{g} / \mathrm{L})\end{array}$ & $\begin{array}{l}\text { Aceto- } \\
\text { chlor } \\
\text { SAA } \\
(\mu \mathrm{g} / \mathrm{L})\end{array}$ & $\begin{array}{c}\text { Ala- } \\
\text { chlor } \\
(\mu \mathrm{g} / \mathrm{L})\end{array}$ & $\begin{array}{l}\text { Ala- } \\
\text { chlor } \\
\text { ESA } \\
(\mu \mathrm{g} / \mathrm{L})\end{array}$ & $\begin{array}{c}\text { Ala- } \\
\text { chlor } \\
\text { OXA } \\
(\mu \mathrm{g} / \mathrm{L})\end{array}$ & $\begin{array}{l}\text { Ala- } \\
\text { chlor } \\
\text { SAA } \\
(\mu \mathrm{g} / \mathrm{L})\end{array}$ & $\begin{array}{c}\text { Di- } \\
\text { meth- } \\
\text { enamid } \\
(\mu \mathrm{g} / \mathrm{L})\end{array}$ & $\begin{array}{c}\text { Di- } \\
\text { meth- } \\
\text { enamid } \\
\text { ESA } \\
(\mu \mathrm{g} / \mathrm{L})\end{array}$ & $\begin{array}{c}\text { Di- } \\
\text { meth- } \\
\text { enamid } \\
\text { OXA } \\
(\mu \mathrm{g} / \mathrm{L})\end{array}$ & $\begin{array}{c}\text { Flufen- } \\
\text { acet } \\
(\mu \mathrm{g} / \mathrm{L})\end{array}$ & $\begin{array}{l}\text { Flufen- } \\
\text { acet } \\
\text { ESA } \\
(\mu \mathrm{g} / \mathrm{L})\end{array}$ & $\begin{array}{l}\text { Flufen- } \\
\text { acet } \\
\text { OXA } \\
(\mu \mathrm{g} / \mathrm{L})\end{array}$ & $\begin{array}{l}\text { Meto- } \\
\text { lachlor } \\
(\mu \mathrm{g} / \mathrm{L})\end{array}$ & $\begin{array}{c}\text { Meto- } \\
\text { lachlor } \\
\text { ESA } \\
(\mu \mathrm{g} / \mathrm{L})\end{array}$ & $\begin{array}{c}\text { Meto- } \\
\text { lachlor } \\
\text { OXA } \\
(\mu \mathrm{g} / \mathrm{L})\end{array}$ \\
\hline \multicolumn{20}{|c|}{ Midcontinent Agricultural Chemical Research Project (fig. 1)—Continued } \\
\hline \multicolumn{20}{|c|}{ Atrazine label change study (DGL)—Continued } \\
\hline \multirow[t]{3}{*}{05540500} & \multirow{3}{*}{$\begin{array}{l}\text { Dupage River near } \\
\text { Shorewood, IL }\end{array}$} & $03 / 30 / 94$ & $<0.05$ & -- & -- & -- & $<0.05$ & -- & -- & -- & -- & -- & -- & -- & -- & -- & $<0.05$ & -- & -- \\
\hline & & $06 / 14 / 94$ & $<.05$ & -- & -- & -- & .52 & -- & -- & -- & -- & -- & -- & -- & -- & -- & 1.1 & -- & -- \\
\hline & & -- & $<.05$ & -- & -- & -- & $<.05$ & -- & -- & -- & -- & -- & -- & -- & -- & -- & .07 & -- & -- \\
\hline \multirow[t]{3}{*}{03371500} & \multirow{3}{*}{$\begin{array}{l}\text { East Fork White River at } \\
\text { Bedford, IN }\end{array}$} & $03 / 22 / 94$ & $<.05$ & -- & -- & -- & $<.05$ & -- & -- & -- & -- & -- & -- & -- & -- & -- & $<.05$ & -- & -- \\
\hline & & $05 / 16 / 94$ & $<.05$ & $<0.20$ & $<0.20$ & -- & .91 & 2.2 & $<0.20$ & -- & -- & -- & -- & -- & -- & -- & 1.8 & 1.5 & $<0.20$ \\
\hline & & $06 / 24 / 95$ & .57 & $<.20$ & $<.20$ & -- & .94 & 2.1 & $<.20$ & -- & -- & -- & -- & -- & -- & -- & .84 & 1.5 & $<.20$ \\
\hline \multirow[t]{4}{*}{03328500} & \multirow{4}{*}{$\begin{array}{l}\text { Eel River near } \\
\text { Logansport, IN }\end{array}$} & $03 / 23 / 94$ & $<.05$ & -- & -- & -- & $<.05$ & -- & -- & -- & -- & -- & -- & -- & -- & -- & $<.05$ & -- & -- \\
\hline & & $06 / 08 / 94$ & $<.05$ & -- & -- & -- & .11 & -- & -- & -- & -- & -- & -- & -- & -- & -- & .15 & -- & -- \\
\hline & & $06 / 14 / 94$ & $<.05$ & 1.0 & $<.20$ & -- & 4.4 & 3.9 & $<.20$ & -- & -- & -- & -- & -- & -- & -- & 1.9 & 1.5 & $<.20$ \\
\hline & & $06 / 08 / 95$ & .11 & $<.20$ & $<.20$ & -- & .46 & 2.3 & 1.4 & -- & -- & -- & -- & -- & -- & -- & .68 & 1.7 & $<.20$ \\
\hline 03302500 & Flat Creek near English, IN & $06 / 26 / 95$ & $<.05$ & $<.20$ & $<.20$ & -- & .96 & $<.20$ & $<.20$ & -- & -- & -- & -- & -- & -- & -- & 1.1 & $<.20$ & $<.20$ \\
\hline \multirow[t]{2}{*}{03353800} & \multirow{2}{*}{$\begin{array}{l}\text { Indian Creek near } \\
\text { Elizabeth, IN }\end{array}$} & $03 / 24 / 94$ & $<.05$ & -- & -- & -- & .27 & -- & -- & -- & -- & -- & -- & -- & -- & -- & .29 & -- & -- \\
\hline & & $05 / 24 / 94$ & $<.05$ & -- & -- & -- & 1.2 & -- & -- & -- & -- & -- & -- & -- & -- & -- & 1.1 & -- & -- \\
\hline \multirow[t]{3}{*}{05526000} & \multirow{3}{*}{$\begin{array}{l}\text { Iroquis River near } \\
\text { Chebanse, IL }\end{array}$} & $03 / 30 / 94$ & $<.05$ & -- & -- & -- & .05 & -- & -- & -- & -- & -- & -- & -- & -- & -- & .08 & -- & -- \\
\hline & & $06 / 16 / 94$ & $<.05$ & -- & -- & -- & 1.1 & -- & -- & -- & -- & -- & -- & -- & -- & -- & 1.1 & -- & -- \\
\hline & & $07 / 03 / 95$ & .42 & -- & -- & -- & .13 & -- & -- & -- & -- & -- & -- & -- & -- & -- & 1.3 & -- & -- \\
\hline \multirow[t]{3}{*}{05592100} & \multirow{3}{*}{$\begin{array}{l}\text { Kaskaskia River near } \\
\text { Cowden, IL }\end{array}$} & $03 / 23 / 94$ & $<.05$ & -- & -- & -- & $<.05$ & -- & -- & -- & -- & -- & -- & -- & -- & -- & .09 & -- & -- \\
\hline & & $06 / 13 / 94$ & $<.05$ & $<.20$ & $<.20$ & -- & 2.1 & 5.7 & $<.20$ & -- & -- & -- & -- & -- & -- & -- & 5.6 & 3.2 & $<.20$ \\
\hline & & $06 / 21 / 95$ & $<.05$ & $<.20$ & $<.20$ & -- & $<.05$ & 2.0 & $<.20$ & -- & -- & -- & -- & -- & -- & -- & .30 & 2.4 & $<.20$ \\
\hline 03352000 & $\begin{array}{l}\text { Kitchel Creek near } \\
\text { Fishers, IN }\end{array}$ & $06 / 26 / 95$ & $<.05$ & $<.20$ & $<.20$ & -- & 5.5 & $<.20$ & $<.20$ & -- & -- & -- & -- & -- & -- & -- & 6.3 & $<.20$ & $<.20$ \\
\hline \multirow[t]{3}{*}{06884000} & \multirow{3}{*}{$\begin{array}{l}\text { Little Blue River near } \\
\text { Fairbury, NE }\end{array}$} & $03 / 30 / 94$ & $<.05$ & -- & -- & -- & $<.05$ & -- & -- & -- & -- & -- & -- & -- & -- & -- & $<.05$ & -- & -- \\
\hline & & $06 / 02 / 94$ & $<.05$ & -- & -- & -- & 6.6 & -- & -- & -- & -- & -- & -- & -- & -- & -- & 5.1 & -- & -- \\
\hline & & $06 / 26 / 95$ & 2.4 & 1.3 & $<.20$ & -- & 5.3 & 2.6 & $<.20$ & -- & -- & -- & -- & -- & -- & -- & 5.6 & 1.4 & $<.20$ \\
\hline 03234700 & $\begin{array}{l}\text { Little Miami River at } \\
\text { Columbus, } \mathrm{OH}\end{array}$ & $05 / 30 / 95$ & .32 & 2.2 & $<.20$ & -- & .12 & 4.1 & 1.6 & -- & -- & -- & -- & -- & -- & -- & 1.0 & 3.1 & $<.20$ \\
\hline
\end{tabular}




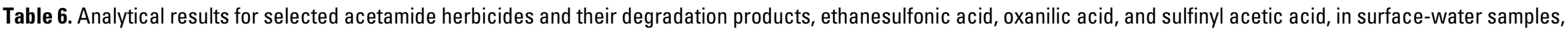
1993-2003-Continued

[OGRL, U.S. Geological Survey Organic Geochemistry Research Laboratory, Lawrence, Kansas; ESA, ethanesulfonic acid; OXA, oxanilic acid; SAA, sulfinyl acetic acid; --, no data; <, less than]

\begin{tabular}{|c|c|c|c|c|c|c|c|c|c|c|c|c|c|c|c|c|c|c|c|}
\hline \multirow[b]{2}{*}{ Site number } & \multirow[b]{2}{*}{ Site location } & \multirow[b]{2}{*}{$\begin{array}{c}\text { Date of } \\
\text { collection } \\
\text { (month/day/ } \\
\text { year) }\end{array}$} & \multicolumn{17}{|c|}{ Concentrations, in micrograms per liter } \\
\hline & & & $\begin{array}{l}\text { Aceto- } \\
\text { chlor } \\
(\mu \mathrm{g} / \mathrm{L})\end{array}$ & $\begin{array}{l}\text { Aceto- } \\
\text { chlor } \\
\text { ESA } \\
(\mu \mathrm{g} / \mathrm{L})\end{array}$ & $\begin{array}{l}\text { Aceto- } \\
\text { chlor } \\
\text { OXA } \\
(\mu \mathrm{g} / \mathrm{L})\end{array}$ & $\begin{array}{l}\text { Aceto- } \\
\text { chlor } \\
\text { SAA } \\
(\mu \mathrm{g} / \mathrm{L})\end{array}$ & $\begin{array}{l}\text { Ala- } \\
\text { chlor } \\
(\mu \mathrm{g} / \mathrm{L})\end{array}$ & $\begin{array}{c}\text { Ala- } \\
\text { chlor } \\
\text { ESA } \\
(\mu \mathrm{g} / \mathrm{L})\end{array}$ & $\begin{array}{l}\text { Ala- } \\
\text { chlor } \\
\text { OXA } \\
(\mu \mathrm{g} / \mathrm{L})\end{array}$ & $\begin{array}{l}\text { Ala- } \\
\text { chlor } \\
\text { SAA } \\
(\mu \mathrm{g} / \mathrm{L})\end{array}$ & $\begin{array}{c}\text { Di- } \\
\text { meth- } \\
\text { enamid } \\
(\mu \mathrm{g} / \mathrm{L})\end{array}$ & $\begin{array}{c}\text { Di- } \\
\text { meth- } \\
\text { enamid } \\
\text { ESA } \\
(\mu \mathrm{g} / \mathrm{L})\end{array}$ & $\begin{array}{c}\text { Di- } \\
\text { meth- } \\
\text { enamid } \\
\text { OXA } \\
(\mu \mathrm{g} / \mathrm{L})\end{array}$ & $\begin{array}{c}\text { Flufen- } \\
\text { acet } \\
(\mu \mathrm{g} / \mathrm{L})\end{array}$ & $\begin{array}{c}\text { Flufen- } \\
\text { acet } \\
\text { ESA } \\
(\mu \mathrm{g} / \mathrm{L})\end{array}$ & $\begin{array}{c}\text { Flufen- } \\
\text { acet } \\
\text { OXA } \\
(\mu \mathrm{g} / \mathrm{L})\end{array}$ & $\begin{array}{l}\text { Meto- } \\
\text { lachlor } \\
(\mu \mathrm{g} / \mathrm{L})\end{array}$ & $\begin{array}{l}\text { Meto- } \\
\text { lachlor } \\
\text { ESA } \\
(\mu \mathrm{g} / \mathrm{L})\end{array}$ & $\begin{array}{c}\text { Meto- } \\
\text { lachlor } \\
\text { OXA } \\
(\mu \mathrm{g} / \mathrm{L})\end{array}$ \\
\hline \multicolumn{20}{|c|}{ Midcontinent Agricultural Chemical Research Project (fig. 1)—Continued } \\
\hline \multicolumn{20}{|c|}{ Atrazine label change study (DGL)—Continued } \\
\hline \multirow[t]{3}{*}{03240000} & \multirow{3}{*}{$\begin{array}{l}\text { Little Miami River at } \\
\text { Oldtown, OH }\end{array}$} & $03 / 29 / 94$ & $<0.05$ & -- & -- & -- & 0.07 & -- & -- & -- & -- & -- & -- & -- & -- & -- & 0.31 & -- & -- \\
\hline & & $06 / 29 / 94$ & $<.05$ & -- & -- & -- & .13 & -- & -- & -- & -- & -- & -- & -- & -- & -- & 1.2 & -- & -- \\
\hline & & $05 / 30 / 95$ & .33 & 2.2 & $<0.20$ & -- & .13 & 4.1 & 1.5 & -- & -- & -- & -- & -- & -- & -- & 1.0 & 3.0 & $<0.20$ \\
\hline \multirow[t]{3}{*}{06606600} & \multirow{3}{*}{$\begin{array}{r}\text { Little Sioux River at } \\
\text { Correctionville, IA }\end{array}$} & $03 / 24 / 94$ & $<.05$ & -- & -- & -- & $<.05$ & -- & -- & -- & -- & -- & -- & -- & -- & -- & .09 & -- & -- \\
\hline & & 06/06/94 & $<.05$ & $<.20$ & $<.20$ & -- & $<.05$ & $<.20$ & $<.20$ & -- & -- & -- & -- & -- & -- & -- & .10 & $<.20$ & $<.20$ \\
\hline & & $05 / 28 / 95$ & .88 & $<.20$ & $<.20$ & -- & .10 & $<.20$ & $<.20$ & -- & -- & -- & -- & -- & -- & -- & 3.3 & $<.20$ & $<.20$ \\
\hline \multirow[t]{3}{*}{03381495} & \multirow{3}{*}{$\begin{array}{l}\text { Little Wabash River at } \\
\text { Carmi, IL }\end{array}$} & $03 / 23 / 94$ & $<.05$ & -- & -- & -- & $<.05$ & -- & -- & -- & -- & -- & -- & -- & -- & -- & .05 & -- & -- \\
\hline & & $06 / 28 / 94$ & $<.05$ & $<.20$ & $<.20$ & -- & 2.9 & 7.6 & $<.20$ & -- & -- & -- & -- & -- & -- & -- & 2.8 & 1.9 & $<.20$ \\
\hline & & 06/29/95 & .80 & $<.20$ & $<.20$ & -- & 1.1 & 4.7 & $<.20$ & -- & -- & -- & -- & -- & -- & -- & 6.4 & 2.5 & $<.20$ \\
\hline \multirow[t]{4}{*}{05549510} & \multirow{4}{*}{$\begin{array}{l}\text { Lucky Creek at } \\
\text { McHenry, IL }\end{array}$} & $03 / 25 / 94$ & $<.05$ & -- & -- & -- & .26 & -- & -- & -- & -- & -- & -- & -- & -- & -- & .27 & -- & -- \\
\hline & & $05 / 07 / 94$ & .16 & $<.20$ & $<.20$ & -- & $<.05$ & 1.9 & $<.20$ & -- & -- & -- & -- & -- & -- & -- & 1.3 & 3.3 & $<.20$ \\
\hline & & $06 / 28 / 94$ & $<.05$ & -- & -- & -- & 7.1 & -- & -- & -- & -- & -- & -- & -- & -- & -- & 7.3 & -- & -- \\
\hline & & 06/30/95 & $<.05$ & -- & -- & -- & .94 & -- & -- & -- & -- & -- & -- & -- & -- & -- & 1.1 & -- & -- \\
\hline 05587000 & $\begin{array}{l}\text { Macoupin Creek near } \\
\text { Kane, IL }\end{array}$ & $03 / 24 / 94$ & $<.05$ & -- & -- & -- & $<.05$ & -- & -- & -- & -- & -- & -- & -- & -- & -- & $<.05$ & -- & -- \\
\hline \multirow[t]{3}{*}{03267900} & \multirow{3}{*}{$\begin{array}{l}\text { Mad River at Eagle City, } \\
\text { OH }\end{array}$} & 03/29/94 & $<.05$ & -- & -- & -- & $<.05$ & -- & -- & -- & -- & -- & -- & -- & -- & -- & $<.05$ & -- & -- \\
\hline & & $06 / 24 / 94$ & $<.05$ & -- & -- & -- & .90 & -- & -- & -- & -- & -- & -- & -- & -- & -- & 3.0 & -- & -- \\
\hline & & $05 / 30 / 95$ & .09 & $<.20$ & $<.20$ & -- & $<.05$ & 1.9 & $<.20$ & -- & -- & -- & -- & -- & -- & -- & .64 & 1.8 & $<.20$ \\
\hline \multirow[t]{3}{*}{06607200} & \multirow{3}{*}{$\begin{array}{l}\text { Maple River at } \\
\text { Mapleton, IA }\end{array}$} & $03 / 24 / 94$ & $<.05$ & -- & -- & -- & $<.05$ & -- & -- & -- & -- & -- & -- & -- & -- & -- & .08 & -- & -- \\
\hline & & 06/05/94 & $<.05$ & $<.20$ & $<.20$ & -- & $<.05$ & 1.1 & $<.20$ & -- & -- & -- & -- & -- & -- & -- & .54 & 1.6 & $<.20$ \\
\hline & & $05 / 28 / 95$ & 2.7 & $<.20$ & $<.20$ & -- & .06 & 2.3 & $<.20$ & -- & -- & -- & -- & -- & -- & -- & 11 & 1.9 & $<.20$ \\
\hline 06817700 & $\begin{array}{l}\text { Nodaway River at } \\
\text { Graham, MO }\end{array}$ & 06/02/94 & $<.05$ & -- & -- & -- & 3.4 & -- & -- & -- & -- & -- & -- & -- & -- & -- & 3.1 & -- & -- \\
\hline
\end{tabular}




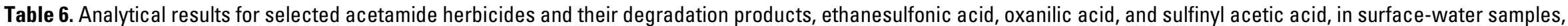
1993-2003.-Continued

[OGRL, U.S. Geological Survey Organic Geochemistry Research Laboratory, Lawrence, Kansas; ESA, ethanesulfonic acid; OXA, oxanilic acid; SAA, sulfinyl acetic acid; --, no data; <, less than]

\begin{tabular}{|c|c|c|c|c|c|c|c|c|c|c|c|c|c|c|c|c|c|c|c|}
\hline \multirow[b]{2}{*}{ Site number } & \multirow[b]{2}{*}{ Site location } & \multirow[b]{2}{*}{$\begin{array}{c}\text { Date of } \\
\text { collection } \\
\text { (month/day/ } \\
\text { year) }\end{array}$} & \multicolumn{17}{|c|}{ Concentrations, in micrograms per liter } \\
\hline & & & $\begin{array}{l}\text { Aceto- } \\
\text { chlor } \\
(\mu \mathrm{g} / \mathrm{L})\end{array}$ & $\begin{array}{l}\text { Aceto- } \\
\text { chlor } \\
\text { ESA } \\
(\mu \mathrm{g} / \mathrm{L})\end{array}$ & $\begin{array}{l}\text { Aceto- } \\
\text { chlor } \\
\text { OXA } \\
(\mu \mathrm{g} / \mathrm{L})\end{array}$ & $\begin{array}{l}\text { Aceto- } \\
\text { chlor } \\
\text { SAA } \\
(\mu \mathrm{g} / \mathrm{L})\end{array}$ & $\begin{array}{l}\text { Ala- } \\
\text { chlor } \\
(\mu \mathrm{g} / \mathrm{L})\end{array}$ & $\begin{array}{l}\text { Ala- } \\
\text { chlor } \\
\text { ESA } \\
(\mu \mathrm{g} / \mathrm{L})\end{array}$ & $\begin{array}{l}\text { Ala- } \\
\text { chlor } \\
\text { OXA } \\
(\mu \mathrm{g} / \mathrm{L})\end{array}$ & $\begin{array}{l}\text { Ala- } \\
\text { chlor } \\
\text { SAA } \\
(\mu \mathrm{g} / \mathrm{L})\end{array}$ & $\begin{array}{l}\text { Di- } \\
\text { meth- } \\
\text { enamid } \\
(\mu \mathrm{g} / \mathrm{L})\end{array}$ & $\begin{array}{c}\text { Di- } \\
\text { meth- } \\
\text { enamid } \\
\text { ESA } \\
(\mu \mathrm{g} / \mathrm{L})\end{array}$ & $\begin{array}{c}\text { Di- } \\
\text { meth- } \\
\text { enamid } \\
\text { OXA } \\
(\mu \mathrm{g} / \mathrm{L})\end{array}$ & $\begin{array}{c}\text { Flufen- } \\
\text { acet } \\
(\mu \mathrm{g} / \mathrm{L})\end{array}$ & $\begin{array}{c}\text { Flufen- } \\
\text { acet } \\
\text { ESA } \\
(\mu \mathrm{g} / \mathrm{L})\end{array}$ & $\begin{array}{l}\text { Flufen- } \\
\text { acet } \\
\text { OXA } \\
(\mu \mathrm{g} / \mathrm{L})\end{array}$ & $\begin{array}{l}\text { Meto- } \\
\text { lachlor } \\
(\mu \mathrm{g} / \mathrm{L})\end{array}$ & $\begin{array}{l}\text { Meto- } \\
\text { lachlor } \\
\text { ESA } \\
(\mu \mathrm{g} / \mathrm{L})\end{array}$ & $\begin{array}{l}\text { Meto- } \\
\text { lachlor } \\
\text { OXA } \\
(\mu \mathrm{g} / \mathrm{L})\end{array}$ \\
\hline \multicolumn{20}{|c|}{ Midcontinent Agricultural Chemical Research Project (fig. 1)—Continued } \\
\hline \multicolumn{20}{|c|}{ Atrazine label change study (DGL)—Continued } \\
\hline \multirow[t]{2}{*}{05472500} & \multirow{2}{*}{$\begin{array}{l}\text { North Skunk River near } \\
\text { Sigourny, IA }\end{array}$} & $03 / 24 / 94$ & $<0.05$ & -- & -- & -- & $<0.05$ & -- & -- & -- & -- & -- & -- & -- & -- & -- & $<0.05$ & -- & -- \\
\hline & & $05 / 25 / 94$ & $<.05$ & -- & -- & -- & $<.05$ & -- & -- & -- & -- & -- & -- & -- & -- & -- & .12 & -- & -- \\
\hline \multirow[t]{8}{*}{05455100} & \multirow{8}{*}{$\begin{array}{l}\text { Old Man's Creek near Iowa } \\
\text { City, IA }\end{array}$} & $03 / 21 / 94$ & $<.05$ & -- & -- & -- & $<.05$ & -- & -- & -- & -- & -- & -- & -- & -- & -- & $<.05$ & -- & -- \\
\hline & & $05 / 24 / 94$ & $<.05$ & -- & -- & -- & $<.05$ & -- & -- & -- & -- & -- & -- & -- & -- & -- & .07 & -- & -- \\
\hline & & 06/02/95 & 2.0 & 2.7 & $<0.20$ & -- & .54 & 4.3 & $<0.20$ & -- & -- & -- & -- & -- & -- & -- & 2.9 & 2.8 & $<0.20$ \\
\hline & & $06 / 14 / 95$ & $<.05$ & $<.20$ & $<.20$ & -- & .07 & 3.5 & $<.20$ & -- & -- & -- & -- & -- & -- & -- & .17 & 2.5 & $<.20$ \\
\hline & & $07 / 05 / 95$ & .12 & -- & -- & -- & .05 & -- & -- & -- & -- & -- & -- & -- & -- & -- & 1.2 & -- & -- \\
\hline & & 07/19/95 & $<.05$ & -- & -- & -- & $<.05$ & -- & -- & -- & -- & -- & -- & -- & -- & -- & .05 & -- & -- \\
\hline & & 08/09/95 & $<.05$ & -- & -- & -- & $<.05$ & -- & -- & -- & -- & -- & -- & -- & -- & -- & .05 & -- & -- \\
\hline & & 08/14/95 & $<.05$ & -- & -- & -- & $<.05$ & -- & -- & -- & -- & -- & -- & -- & -- & -- & $<.05$ & -- & -- \\
\hline 03234990 & $\begin{array}{l}\text { Olentangy River at Athens, } \\
\text { OH }\end{array}$ & $05 / 26 / 95$ & .90 & $<.20$ & $<.20$ & -- & $<.05$ & $<.20$ & $<.20$ & -- & -- & -- & -- & -- & -- & -- & 1.2 & $<.20$ & $<.20$ \\
\hline \multirow[t]{3}{*}{03223000} & \multirow{3}{*}{$\begin{array}{l}\text { Olentangy River at } \\
\text { Claridon, } \mathrm{OH}\end{array}$} & $03 / 25 / 94$ & $<.05$ & -- & -- & -- & .05 & -- & -- & -- & -- & -- & -- & -- & -- & -- & .18 & -- & -- \\
\hline & & $06 / 24 / 94$ & 2.0 & $<.20$ & $<.20$ & -- & $<.05$ & 14 & $<.20$ & -- & -- & -- & -- & -- & -- & -- & 3.9 & 5.1 & $<.20$ \\
\hline & & 05/30/95 & 1.7 & 2.7 & $<.20$ & -- & .27 & 5.2 & $<.20$ & -- & -- & -- & -- & -- & -- & -- & 5.0 & 4.3 & $<.20$ \\
\hline \multirow[t]{2}{*}{03363500} & \multirow{2}{*}{$\begin{array}{l}\text { Pigeon River near } \\
\text { Hanna, IN }\end{array}$} & $03 / 23 / 94$ & $<.05$ & -- & -- & -- & .22 & -- & -- & -- & -- & -- & -- & -- & -- & -- & .25 & -- & -- \\
\hline & & 06/08/94 & $<.05$ & -- & -- & -- & 1.1 & -- & -- & -- & -- & -- & -- & -- & -- & -- & 1.0 & -- & -- \\
\hline \multirow[t]{3}{*}{03327520} & \multirow{3}{*}{$\begin{array}{l}\text { Pipe Creek near Bunker } \\
\text { Hill, IN }\end{array}$} & 03/23/94 & $<.05$ & -- & -- & -- & $<.05$ & -- & -- & -- & -- & -- & -- & -- & -- & -- & .06 & -- & -- \\
\hline & & 05/16/94 & $<.05$ & -- & -- & -- & 2.2 & -- & -- & -- & -- & -- & -- & -- & -- & -- & 3.4 & -- & -- \\
\hline & & 06/08/95 & .10 & -- & -- & -- & .42 & -- & -- & -- & -- & -- & -- & -- & -- & -- & .61 & -- & -- \\
\hline 05484500 & $\begin{array}{l}\text { Raccoon River at Van } \\
\text { Meter, IA }\end{array}$ & $05 / 29 / 95$ & 4.5 & 1.5 & $<.20$ & -- & .10 & 2.2 & $<.20$ & -- & -- & -- & -- & -- & -- & -- & 6.7 & 2.4 & $<.20$ \\
\hline \multirow[t]{3}{*}{05430500} & \multirow[t]{3}{*}{ Rock River at Afton, WI } & $04 / 05 / 94$ & $<.05$ & -- & -- & -- & $<.05$ & -- & -- & -- & -- & -- & -- & -- & -- & -- & $<.05$ & -- & -- \\
\hline & & 06/30/94 & $<.05$ & -- & -- & -- & $<.05$ & -- & -- & -- & -- & -- & -- & -- & -- & -- & $<.05$ & -- & -- \\
\hline & & 06/08/95 & $<.05$ & $<.20$ & $<.20$ & -- & $<.05$ & 1.7 & $<.20$ & -- & -- & -- & -- & -- & -- & -- & .18 & $<.20$ & $<.20$ \\
\hline
\end{tabular}




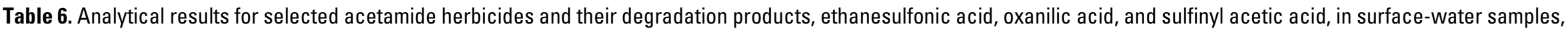
1993-2003-Continued

[OGRL, U.S. Geological Survey Organic Geochemistry Research Laboratory, Lawrence, Kansas; ESA, ethanesulfonic acid; OXA, oxanilic acid; SAA, sulfinyl acetic acid; --, no data; <, less than]

\begin{tabular}{|c|c|c|c|c|c|c|c|c|c|c|c|c|c|c|c|c|c|c|c|}
\hline \multirow[b]{2}{*}{ Site number } & \multirow[b]{2}{*}{ Site location } & \multirow[b]{2}{*}{$\begin{array}{c}\text { Date of } \\
\text { collection } \\
\text { (month/day/ } \\
\text { year) }\end{array}$} & \multicolumn{17}{|c|}{ Concentrations, in micrograms per liter } \\
\hline & & & $\begin{array}{l}\text { Aceto- } \\
\text { chlor } \\
(\mu \mathrm{g} / \mathrm{L})\end{array}$ & $\begin{array}{l}\text { Aceto- } \\
\text { chlor } \\
\text { ESA } \\
(\mu \mathrm{g} / \mathrm{L})\end{array}$ & $\begin{array}{c}\text { Aceto- } \\
\text { chlor } \\
\text { OXA } \\
(\mu \mathrm{g} / \mathrm{L})\end{array}$ & $\begin{array}{c}\text { Aceto- } \\
\text { chlor } \\
\text { SAA } \\
(\mu \mathrm{g} / \mathrm{L})\end{array}$ & $\begin{array}{l}\text { Ala- } \\
\text { chlor } \\
(\mu \mathrm{g} / \mathrm{L})\end{array}$ & $\begin{array}{l}\text { Ala- } \\
\text { chlor } \\
\text { ESA } \\
(\mu \mathrm{g} / \mathrm{L})\end{array}$ & $\begin{array}{l}\text { Ala- } \\
\text { chlor } \\
\text { OXA } \\
(\mu \mathrm{g} / \mathrm{L})\end{array}$ & $\begin{array}{l}\text { Ala- } \\
\text { chlor } \\
\text { SAA } \\
(\mu \mathrm{g} / \mathrm{L})\end{array}$ & $\begin{array}{c}\text { Di- } \\
\text { meth- } \\
\text { enamid } \\
(\mu \mathrm{g} / \mathrm{L})\end{array}$ & $\begin{array}{c}\text { Di- } \\
\text { meth- } \\
\text { enamid } \\
\text { ESA } \\
(\mu \mathrm{g} / \mathrm{L})\end{array}$ & $\begin{array}{c}\text { Di- } \\
\text { meth- } \\
\text { enamid } \\
\text { OXA } \\
(\mu \mathrm{g} / \mathrm{L})\end{array}$ & $\begin{array}{c}\text { Flufen- } \\
\text { acet } \\
(\mu \mathrm{g} / \mathrm{L})\end{array}$ & $\begin{array}{c}\text { Flufen- } \\
\text { acet } \\
\text { ESA } \\
(\mu \mathrm{g} / \mathrm{L})\end{array}$ & $\begin{array}{c}\text { Flufen- } \\
\text { acet } \\
\text { OXA } \\
(\mu \mathrm{g} / \mathrm{L})\end{array}$ & $\begin{array}{l}\text { Meto- } \\
\text { lachlor } \\
(\mu \mathrm{g} / \mathrm{L})\end{array}$ & $\begin{array}{l}\text { Meto- } \\
\text { lachlor } \\
\text { ESA } \\
(\mu \mathrm{g} / \mathrm{L})\end{array}$ & $\begin{array}{c}\text { Meto- } \\
\text { lachlor } \\
\text { OXA } \\
(\mu \mathrm{g} / \mathrm{L})\end{array}$ \\
\hline \multicolumn{20}{|c|}{ Midcontinent Agricultural Chemical Research Project (fig. 1)—Continued } \\
\hline \multicolumn{20}{|c|}{ Atrazine label change study (DGL)—Continued } \\
\hline \multirow[t]{3}{*}{06483000} & Rock River at Luverne, $\mathrm{MN}$ & $04 / 04 / 94$ & $<0.05$ & -- & -- & -- & $<0.05$ & -- & -- & -- & -- & -- & -- & -- & -- & -- & 0.05 & -- & -- \\
\hline & & 06/06/94 & 1.0 & $<0.20$ & $<0.20$ & -- & 1.9 & 3.9 & $<0.20$ & -- & -- & -- & -- & -- & -- & -- & 5.3 & 3.4 & $<0.20$ \\
\hline & & $05 / 28 / 95$ & 5.6 & 1.7 & $<.20$ & -- & .10 & 3.9 & $<.20$ & -- & -- & -- & -- & -- & -- & -- & 11 & 4.4 & $<.20$ \\
\hline \multirow[t]{3}{*}{04087240} & Root River at Racine, WI & $04 / 15 / 94$ & $<.05$ & $<.20$ & $<.20$ & -- & $<.05$ & $<.20$ & $<.20$ & -- & -- & -- & -- & -- & -- & -- & $<.05$ & $<.20$ & $<.20$ \\
\hline & & $06 / 24 / 94$ & $<.05$ & -- & -- & -- & $<.05$ & -- & -- & -- & -- & -- & -- & -- & -- & -- & $<.05$ & -- & -- \\
\hline & & $05 / 25 / 95$ & $<.05$ & -- & -- & -- & $<.05$ & -- & -- & -- & -- & -- & -- & -- & -- & -- & .10 & -- & -- \\
\hline \multirow[t]{2}{*}{05439500} & South Branch Kishwaukee & $03 / 29 / 94$ & $<.05$ & -- & -- & -- & $<.05$ & -- & -- & -- & -- & -- & -- & -- & -- & -- & .05 & -- & -- \\
\hline & River at Fairdale, IL & $06 / 25 / 94$ & $<.05$ & 1.5 & $<.20$ & -- & 1.9 & 8.2 & 9.6 & -- & -- & -- & -- & -- & -- & -- & 3.5 & 4.7 & $<.20$ \\
\hline \multirow[t]{8}{*}{06803000} & Salt Creek at Roca, NE & $03 / 29 / 94$ & $<.05$ & -- & -- & -- & $<.05$ & -- & -- & -- & -- & -- & -- & -- & -- & -- & $<.05$ & -- & -- \\
\hline & & $03 / 29 / 94$ & $<.05$ & -- & -- & -- & $<.05$ & -- & -- & -- & -- & -- & -- & -- & -- & -- & $<.05$ & -- & -- \\
\hline & & $05 / 15 / 94$ & 1.0 & $<.20$ & $<.20$ & -- & 6.5 & $<.20$ & $<.20$ & -- & -- & -- & -- & -- & -- & -- & 6.3 & $<.20$ & $<.20$ \\
\hline & & $07 / 04 / 95$ & .08 & -- & -- & -- & 1.4 & -- & -- & -- & -- & -- & -- & -- & -- & -- & 19 & -- & -- \\
\hline & & $07 / 25 / 95$ & $<.05$ & -- & -- & -- & $<.05$ & -- & -- & -- & -- & -- & -- & -- & -- & -- & .22 & -- & -- \\
\hline & & 08/08/95 & $<.05$ & $<.20$ & $<.20$ & -- & .54 & 3.2 & 2.0 & -- & -- & -- & -- & -- & -- & -- & .52 & 7.4 & 1.9 \\
\hline & & 09/02/95 & $<.05$ & -- & -- & -- & $<.05$ & -- & -- & -- & -- & -- & -- & -- & -- & -- & .07 & -- & -- \\
\hline & & $09 / 02 / 95$ & $<.05$ & -- & -- & -- & $<.05$ & -- & -- & -- & -- & -- & -- & -- & -- & -- & .05 & -- & -- \\
\hline \multirow[t]{3}{*}{05576500} & Sangamon River at & $03 / 25 / 94$ & $<.05$ & -- & -- & -- & $<.05$ & -- & -- & -- & -- & -- & -- & -- & -- & -- & .22 & -- & -- \\
\hline & Riverton, IL & 05/10/94 & 1.0 & $<.20$ & $<.20$ & -- & .84 & 3.4 & $<.20$ & -- & -- & -- & -- & -- & -- & -- & 3.3 & 3.4 & $<.20$ \\
\hline & & 06/23/95 & .29 & $<.20$ & $<.20$ & -- & .27 & 3.0 & $<.20$ & -- & -- & -- & -- & -- & -- & -- & 1.6 & 2.8 & $<.20$ \\
\hline 03230100 & $\begin{array}{l}\text { Scioto River at } \\
\text { Harrisburg, } \mathrm{OH}\end{array}$ & 04/06/94 & $<.05$ & -- & -- & -- & .89 & -- & -- & -- & -- & -- & -- & -- & -- & -- & 1.1 & -- & -- \\
\hline \multirow[t]{3}{*}{03234500} & Scioto River at Higby, $\mathrm{OH}$ & 04/06/94 & $<.05$ & -- & -- & -- & $<.05$ & -- & -- & -- & -- & -- & -- & -- & -- & -- & .22 & -- & -- \\
\hline & & 07/01/94 & $<.05$ & -- & -- & -- & .35 & -- & -- & -- & -- & -- & -- & -- & -- & -- & 2.7 & -- & -- \\
\hline & & $05 / 26 / 95$ & 1.0 & $<.20$ & $<.20$ & -- & .37 & $<.20$ & $<.20$ & -- & -- & -- & -- & -- & -- & -- & 3.4 & $<.20$ & $<.20$ \\
\hline 03234888 & $\begin{array}{l}\text { Scioto River at } \\
\text { Westerville, } \mathrm{OH}\end{array}$ & $05 / 31 / 95$ & $<.05$ & -- & -- & -- & 7.1 & -- & -- & -- & -- & -- & -- & -- & -- & -- & 8.1 & -- & -- \\
\hline
\end{tabular}




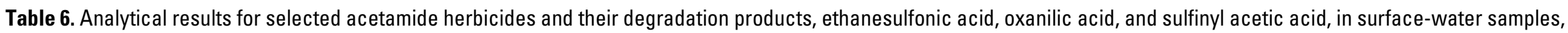
1993-2003.-Continued

[OGRL, U.S. Geological Survey Organic Geochemistry Research Laboratory, Lawrence, Kansas; ESA, ethanesulfonic acid; OXA, oxanilic acid; SAA, sulfinyl acetic acid; --, no data; <, less than]

\begin{tabular}{|c|c|c|c|c|c|c|c|c|c|c|c|c|c|c|c|c|c|c|c|}
\hline \multirow[b]{2}{*}{ Site number } & \multirow[b]{2}{*}{ Site location } & \multirow[b]{2}{*}{$\begin{array}{c}\text { Date of } \\
\text { collection } \\
\text { (month/day/ } \\
\text { year) }\end{array}$} & \multicolumn{17}{|c|}{ Concentrations, in micrograms per liter } \\
\hline & & & $\begin{array}{l}\text { Aceto- } \\
\text { chlor } \\
(\mu \mathrm{g} / \mathrm{L})\end{array}$ & $\begin{array}{l}\text { Aceto- } \\
\text { chlor } \\
\text { ESA } \\
(\mu \mathrm{g} / \mathrm{L})\end{array}$ & $\begin{array}{l}\text { Aceto- } \\
\text { chlor } \\
\text { OXA } \\
(\mu \mathrm{g} / \mathrm{L})\end{array}$ & $\begin{array}{l}\text { Aceto- } \\
\text { chlor } \\
\text { SAA } \\
(\mu \mathrm{g} / \mathrm{L})\end{array}$ & $\begin{array}{l}\text { Ala- } \\
\text { chlor } \\
(\mu \mathrm{g} / \mathrm{L})\end{array}$ & $\begin{array}{l}\text { Ala- } \\
\text { chlor } \\
\text { ESA } \\
(\mu \mathrm{g} / \mathrm{L})\end{array}$ & $\begin{array}{l}\text { Ala- } \\
\text { chlor } \\
\text { OXA } \\
(\mu \mathrm{g} / \mathrm{L})\end{array}$ & $\begin{array}{c}\text { Ala- } \\
\text { chlor } \\
\text { SAA } \\
(\mu \mathrm{g} / \mathrm{L})\end{array}$ & $\begin{array}{l}\text { Di- } \\
\text { meth- } \\
\text { enamid } \\
(\mu \mathrm{g} / \mathrm{L})\end{array}$ & $\begin{array}{c}\text { Di- } \\
\text { meth- } \\
\text { enamid } \\
\text { ESA } \\
(\mu \mathrm{g} / \mathrm{L})\end{array}$ & $\begin{array}{c}\text { Di- } \\
\text { meth- } \\
\text { enamid } \\
\text { OXA } \\
(\mu \mathrm{g} / \mathrm{L})\end{array}$ & $\begin{array}{l}\text { Flufen- } \\
\text { acet } \\
(\mu \mathrm{g} / \mathrm{L})\end{array}$ & $\begin{array}{c}\text { Flufen- } \\
\text { acet } \\
\text { ESA } \\
(\mu \mathrm{g} / \mathrm{L})\end{array}$ & $\begin{array}{c}\text { Flufen- } \\
\text { acet } \\
\text { OXA } \\
(\mu \mathrm{g} / \mathrm{L})\end{array}$ & $\begin{array}{l}\text { Meto- } \\
\text { lachlor } \\
(\mu \mathrm{g} / \mathrm{L})\end{array}$ & $\begin{array}{c}\text { Meto- } \\
\text { lachlor } \\
\text { ESA } \\
(\mu \mathrm{g} / \mathrm{L})\end{array}$ & $\begin{array}{l}\text { Meto- } \\
\text { lachlor } \\
\text { OXA } \\
(\mu \mathrm{g} / \mathrm{L})\end{array}$ \\
\hline \multicolumn{20}{|c|}{ Midcontinent Agricultural Chemical Research Project (fig. 1)—Continued } \\
\hline \multicolumn{20}{|c|}{ Atrazine label change study (DGL)—Continued } \\
\hline 03230500 & $\begin{array}{c}\text { Scioto River near } \\
\text { Columbus, } \mathrm{OH}\end{array}$ & $04 / 06 / 94$ & $<0.05$ & -- & -- & -- & 0.97 & -- & -- & -- & -- & -- & -- & -- & -- & -- & 1.0 & -- & -- \\
\hline \multirow[t]{3}{*}{03219500} & \multirow{3}{*}{$\begin{array}{l}\text { Scioto River near } \\
\text { Prospect, } \mathrm{OH}\end{array}$} & $03 / 25 / 94$ & $<.05$ & -- & -- & -- & .05 & -- & -- & -- & -- & -- & -- & -- & -- & -- & .18 & -- & -- \\
\hline & & $06 / 15 / 94$ & $<.05$ & -- & -- & -- & 3.8 & -- & -- & -- & -- & -- & -- & -- & -- & -- & 4.0 & -- & -- \\
\hline & & 05/30/95 & 1.1 & $<0.20$ & $<0.20$ & -- & .25 & 5.2 & $<0.20$ & -- & -- & -- & -- & -- & -- & -- & 6.6 & 3.8 & $<0.20$ \\
\hline 03157000 & $\begin{array}{l}\text { Scioto River near } \\
\text { Rockbridge, } \mathrm{OH}\end{array}$ & $05 / 25 / 95$ & .96 & 1.7 & $<.20$ & -- & .06 & 1.4 & $<.20$ & -- & -- & -- & -- & -- & -- & -- & 1.7 & 1.9 & $<.20$ \\
\hline 03220000 & $\begin{array}{l}\text { Scioto River near } \\
\text { Scioto, } \mathrm{OH}\end{array}$ & 04/01/94 & $<.05$ & -- & -- & -- & .90 & -- & -- & -- & -- & -- & -- & -- & -- & -- & 1.0 & -- & -- \\
\hline \multirow[t]{3}{*}{05594000} & \multirow[t]{3}{*}{ Shoal Creek near Breese, IL } & $03 / 24 / 94$ & $<.05$ & -- & -- & -- & .24 & -- & -- & -- & -- & -- & -- & -- & -- & -- & .83 & -- & -- \\
\hline & & 06/26/95 & .63 & $<.20$ & $<.20$ & -- & 1.2 & 3.5 & $<.20$ & -- & -- & -- & -- & -- & -- & -- & 3.7 & 2.0 & $<.20$ \\
\hline & & $06 / 30 / 95$ & .42 & -- & -- & -- & .65 & -- & -- & -- & -- & -- & -- & -- & -- & -- & 7.0 & -- & -- \\
\hline \multirow[t]{2}{*}{03380530} & \multirow{2}{*}{$\begin{array}{l}\text { Skillet Fork tributary near } \\
\text { Wayne City, IL }\end{array}$} & 03/29/94 & $<.05$ & -- & -- & -- & $<.05$ & -- & -- & -- & -- & -- & -- & -- & -- & -- & .23 & -- & -- \\
\hline & & 06/29/95 & .82 & $<.20$ & $<.20$ & -- & 1.1 & 4.7 & $<.20$ & -- & -- & -- & -- & -- & -- & -- & 6.6 & 2.4 & $<.20$ \\
\hline \multirow[t]{3}{*}{05474000} & \multirow{3}{*}{ Skunk River at Augusta, IA } & $03 / 24 / 94$ & $<.05$ & -- & -- & -- & $<.05$ & -- & -- & -- & -- & -- & -- & -- & -- & -- & .05 & -- & -- \\
\hline & & 06/03/94 & $<.05$ & -- & -- & -- & .09 & -- & -- & -- & -- & -- & -- & -- & -- & -- & .40 & -- & -- \\
\hline & & 06/08/95 & 1.7 & $<.20$ & $<.20$ & -- & .06 & $<.20$ & $<.20$ & -- & -- & -- & -- & -- & -- & -- & 2.4 & $<.20$ & $<.20$ \\
\hline 05472500 & $\begin{array}{l}\text { Skunk River near } \\
\text { Sigourney, IA }\end{array}$ & 06/06/95 & 2.7 & 2.4 & $<.20$ & -- & $<.05$ & 3.5 & $<.20$ & -- & -- & -- & -- & -- & -- & -- & 3.0 & 3.4 & $<.20$ \\
\hline \multirow[t]{3}{*}{05569500} & \multirow{3}{*}{$\begin{array}{l}\text { Spoon River at London } \\
\text { Mills, IL }\end{array}$} & 03/29/94 & $<.05$ & -- & -- & -- & $<.05$ & -- & -- & -- & -- & -- & -- & -- & -- & -- & .24 & -- & -- \\
\hline & & 05/07/94 & $<.05$ & $<.20$ & $<.20$ & -- & .15 & 2.0 & $<.20$ & -- & -- & -- & -- & -- & -- & -- & 1.3 & 3.4 & $<.20$ \\
\hline & & -- & .48 & -- & -- & -- & .06 & -- & -- & -- & -- & -- & -- & -- & -- & -- & .74 & -- & -- \\
\hline \multirow[t]{3}{*}{05340500} & \multirow{3}{*}{$\begin{array}{l}\text { St. Croix River at St. Croix } \\
\text { Falls, WI }\end{array}$} & $04 / 13 / 94$ & $<.05$ & -- & -- & -- & $<.05$ & -- & -- & -- & -- & -- & -- & -- & -- & -- & $<.05$ & -- & -- \\
\hline & & 06/20/94 & $<.05$ & -- & -- & -- & $<.05$ & -- & -- & -- & -- & -- & -- & -- & -- & -- & $<.05$ & -- & -- \\
\hline & & 06/12/95 & $<.05$ & $<.20$ & $<.20$ & -- & $<.05$ & $<.20$ & $<.20$ & -- & -- & -- & -- & -- & -- & -- & .16 & $<.20$ & $<.20$ \\
\hline
\end{tabular}




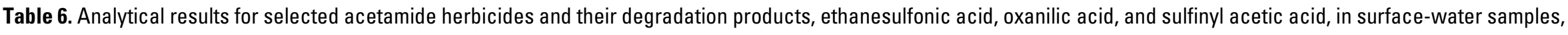
1993-2003-Continued

[OGRL, U.S. Geological Survey Organic Geochemistry Research Laboratory, Lawrence, Kansas; ESA, ethanesulfonic acid; OXA, oxanilic acid; SAA, sulfinyl acetic acid; --, no data; <, less than]

\begin{tabular}{|c|c|c|c|c|c|c|c|c|c|c|c|c|c|c|c|c|c|c|c|}
\hline \multirow[b]{2}{*}{ Site number } & \multirow[b]{2}{*}{ Site location } & \multirow[b]{2}{*}{$\begin{array}{c}\text { Date of } \\
\text { collection } \\
\text { (month/day/ } \\
\text { year) }\end{array}$} & \multicolumn{17}{|c|}{ Concentrations, in micrograms per liter } \\
\hline & & & $\begin{array}{c}\text { Aceto- } \\
\text { chlor } \\
(\mu \mathrm{g} / \mathrm{L})\end{array}$ & $\begin{array}{l}\text { Aceto- } \\
\text { chlor } \\
\text { ESA } \\
(\mu \mathrm{g} / \mathrm{L})\end{array}$ & $\begin{array}{l}\text { Aceto- } \\
\text { chlor } \\
\text { OXA } \\
(\mu \mathrm{g} / \mathrm{L})\end{array}$ & $\begin{array}{c}\text { Aceto- } \\
\text { chlor } \\
\text { SAA } \\
(\mu \mathrm{g} / \mathrm{L})\end{array}$ & $\begin{array}{c}\text { Ala- } \\
\text { chlor } \\
(\mu \mathrm{g} / \mathrm{L})\end{array}$ & $\begin{array}{l}\text { Ala- } \\
\text { chlor } \\
\text { ESA } \\
(\mu \mathrm{g} / \mathrm{L})\end{array}$ & $\begin{array}{l}\text { Ala- } \\
\text { chlor } \\
\text { OXA } \\
(\mu \mathrm{g} / \mathrm{L})\end{array}$ & $\begin{array}{l}\text { Ala- } \\
\text { chlor } \\
\text { SAA } \\
(\mu \mathrm{g} / \mathrm{L})\end{array}$ & $\begin{array}{c}\text { Di- } \\
\text { meth- } \\
\text { enamid } \\
(\mu \mathrm{g} / \mathrm{L})\end{array}$ & $\begin{array}{c}\text { Di- } \\
\text { meth- } \\
\text { enamid } \\
\text { ESA } \\
(\mu \mathrm{g} / \mathrm{L})\end{array}$ & $\begin{array}{c}\text { Di- } \\
\text { meth- } \\
\text { enamid } \\
\text { OXA } \\
(\mu \mathrm{g} / \mathrm{L})\end{array}$ & $\begin{array}{c}\text { Flufen- } \\
\text { acet } \\
(\mu \mathrm{g} / \mathrm{L})\end{array}$ & $\begin{array}{c}\text { Flufen- } \\
\text { acet } \\
\text { ESA } \\
(\mu \mathrm{g} / \mathrm{L})\end{array}$ & $\begin{array}{c}\text { Flufen- } \\
\text { acet } \\
\text { OXA } \\
(\mu \mathrm{g} / \mathrm{L})\end{array}$ & $\begin{array}{l}\text { Meto- } \\
\text { lachlor } \\
(\mu \mathrm{g} / \mathrm{L})\end{array}$ & $\begin{array}{c}\text { Meto- } \\
\text { lachlor } \\
\text { ESA } \\
(\mu \mathrm{g} / \mathrm{L})\end{array}$ & $\begin{array}{c}\text { Meto- } \\
\text { lachlor } \\
\text { OXA } \\
(\mu \mathrm{g} / \mathrm{L})\end{array}$ \\
\hline \multicolumn{20}{|c|}{ Midcontinent Agricultural Chemical Research Project (fig. 1)—Continued } \\
\hline \multicolumn{20}{|c|}{ Atrazine label change study (DGL) — Continued } \\
\hline \multirow[t]{3}{*}{03362500} & \multirow{3}{*}{$\begin{array}{l}\text { Sugar Creek near } \\
\text { Edinburgh, IN }\end{array}$} & $03 / 21 / 94$ & $<0.05$ & -- & -- & -- & $<0.05$ & -- & -- & -- & -- & -- & -- & -- & -- & -- & $<0.05$ & -- & -- \\
\hline & & $05 / 16 / 94$ & $<.05$ & -- & -- & -- & 2.0 & -- & -- & -- & -- & -- & -- & -- & -- & -- & 3.1 & -- & -- \\
\hline & & $06 / 03 / 95$ & .97 & 1.9 & $<0.20$ & -- & 2.3 & 3.3 & $<0.20$ & -- & -- & -- & -- & -- & -- & -- & 1.4 & 2.9 & $<0.20$ \\
\hline \multirow[t]{4}{*}{04185000} & \multirow[t]{4}{*}{ Tiffin River at Stryker, OH } & $03 / 30 / 94$ & $<.05$ & -- & -- & -- & .05 & -- & -- & -- & -- & -- & -- & -- & -- & -- & .07 & -- & -- \\
\hline & & $06 / 28 / 94$ & $<.05$ & -- & -- & -- & .38 & -- & -- & -- & -- & -- & -- & -- & -- & -- & 1.4 & -- & -- \\
\hline & & 07/07/94 & $<.05$ & -- & -- & -- & 5.6 & -- & -- & -- & -- & -- & -- & -- & -- & -- & 6.1 & -- & -- \\
\hline & & $05 / 31 / 95$ & .84 & $<.20$ & $<.20$ & -- & .70 & 3.9 & $<.20$ & -- & -- & -- & -- & -- & -- & -- & 1.7 & 3.0 & $<.20$ \\
\hline 04123400 & Tim Creek near Tim, OH & $03 / 30 / 94$ & $<.05$ & -- & -- & -- & $<.05$ & -- & -- & -- & -- & -- & -- & -- & -- & -- & .14 & -- & -- \\
\hline \multirow[t]{2}{*}{03336420} & \multirow[t]{2}{*}{ Trail Creek near Deputy, IN } & $03 / 29 / 94$ & $<.05$ & -- & -- & -- & .20 & -- & -- & -- & -- & -- & -- & -- & -- & -- & .20 & -- & -- \\
\hline & & $06 / 14 / 94$ & $<.05$ & -- & -- & -- & 1.2 & -- & -- & -- & -- & -- & -- & -- & -- & -- & 1.0 & -- & -- \\
\hline \multirow[t]{3}{*}{05411600} & \multirow{3}{*}{$\begin{array}{l}\text { Turkey River at } \\
\text { Spillville, IA }\end{array}$} & $03 / 22 / 94$ & $<.05$ & -- & -- & -- & .08 & -- & -- & -- & -- & -- & -- & -- & -- & -- & .11 & -- & -- \\
\hline & & $05 / 24 / 94$ & $<.05$ & -- & -- & -- & .14 & -- & -- & -- & -- & -- & -- & -- & -- & -- & .05 & -- & -- \\
\hline & & 06/08/95 & .62 & $<.20$ & $<.20$ & -- & 1.9 & 3.9 & 4.0 & -- & -- & -- & -- & -- & -- & -- & .38 & 1.8 & $<.20$ \\
\hline \multirow[t]{3}{*}{06880800} & \multirow{3}{*}{$\begin{array}{l}\text { West Fork Big Blue River } \\
\text { at Dorchester, NE }\end{array}$} & $03 / 29 / 94$ & $<.05$ & -- & -- & -- & $<.05$ & -- & -- & -- & -- & -- & -- & -- & -- & -- & $<.05$ & -- & -- \\
\hline & & $06 / 27 / 94$ & $<.05$ & -- & -- & -- & 3.1 & -- & -- & -- & -- & -- & -- & -- & -- & -- & 3.1 & -- & -- \\
\hline & & 06/09/95 & 5.6 & 3.3 & $<.20$ & -- & 2.0 & 3.1 & $<.20$ & -- & -- & -- & -- & -- & -- & -- & 7.9 & 1.5 & $<.20$ \\
\hline 05411100 & $\begin{array}{l}\text { West Fork Iowa River near } \\
\text { Tiffin, IA }\end{array}$ & $03 / 24 / 94$ & $<.05$ & -- & -- & -- & $<.05$ & -- & -- & -- & -- & -- & -- & -- & -- & -- & .06 & -- & -- \\
\hline \multirow[t]{3}{*}{06804000} & \multirow[t]{3}{*}{ Wahoo Creek at Itica, NE } & $03 / 29 / 94$ & $<.05$ & -- & -- & -- & $<.05$ & -- & -- & -- & -- & -- & -- & -- & -- & -- & $<.05$ & -- & -- \\
\hline & & $06 / 02 / 94$ & $<.05$ & -- & -- & -- & 10 & -- & -- & -- & -- & -- & -- & -- & -- & -- & 6.6 & -- & -- \\
\hline & & $07 / 05 / 95$ & $<.05$ & -- & -- & -- & .07 & -- & -- & -- & -- & -- & -- & -- & -- & -- & .24 & -- & -- \\
\hline \multirow[t]{3}{*}{05421000} & \multirow{3}{*}{$\begin{array}{l}\text { Wapsipinicon River at } \\
\text { Independence, IA }\end{array}$} & $03 / 22 / 94$ & $<.05$ & -- & -- & -- & .09 & -- & -- & -- & -- & -- & -- & -- & -- & -- & .15 & -- & -- \\
\hline & & $05 / 24 / 94$ & $<.05$ & -- & -- & -- & .08 & -- & -- & -- & -- & -- & -- & -- & -- & -- & .11 & -- & -- \\
\hline & & $05 / 30 / 95$ & .36 & $<.20$ & $<.20$ & -- & .10 & 5.9 & $<.20$ & -- & -- & -- & -- & -- & -- & -- & .50 & 4.3 & $<.20$ \\
\hline \multirow[t]{3}{*}{03351000} & \multirow[t]{3}{*}{ White River near Nora, IN } & $03 / 22 / 94$ & $<.05$ & -- & -- & -- & $<.05$ & -- & -- & -- & -- & -- & -- & -- & -- & -- & $<.05$ & -- & -- \\
\hline & & $05 / 26 / 94$ & $<.05$ & -- & -- & -- & 2.3 & -- & -- & -- & -- & -- & -- & -- & -- & -- & 2.0 & -- & -- \\
\hline & & $06 / 25 / 95$ & .07 & 2.0 & $<.20$ & -- & .05 & 3.8 & $<.20$ & -- & -- & -- & -- & -- & -- & -- & .90 & 2.8 & $<.20$ \\
\hline
\end{tabular}




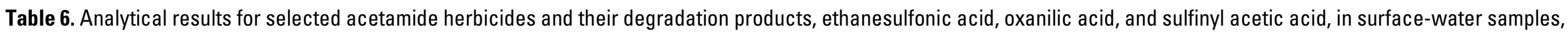
1993-2003.-Continued

[OGRL, U.S. Geological Survey Organic Geochemistry Research Laboratory, Lawrence, Kansas; ESA, ethanesulfonic acid; OXA, oxanilic acid; SAA, sulfinyl acetic acid; --, no data; <, less than]

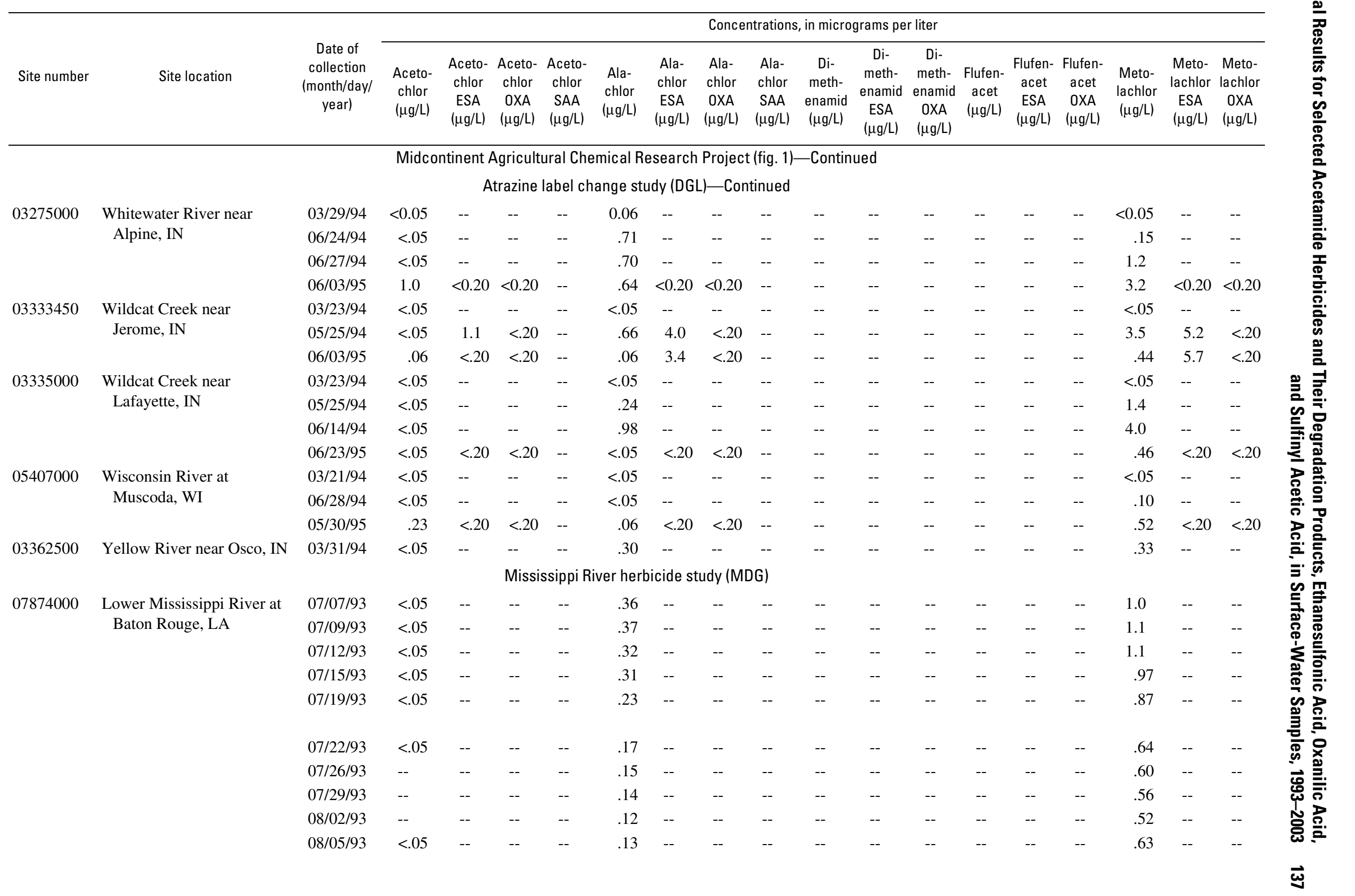




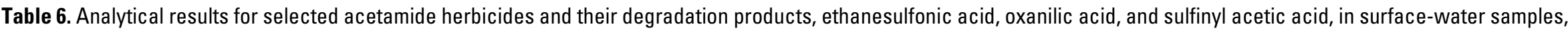
1993-2003-Continued

[OGRL, U.S. Geological Survey Organic Geochemistry Research Laboratory, Lawrence, Kansas; ESA, ethanesulfonic acid; OXA, oxanilic acid; SAA, sulfinyl acetic acid; --, no data; <, less than]

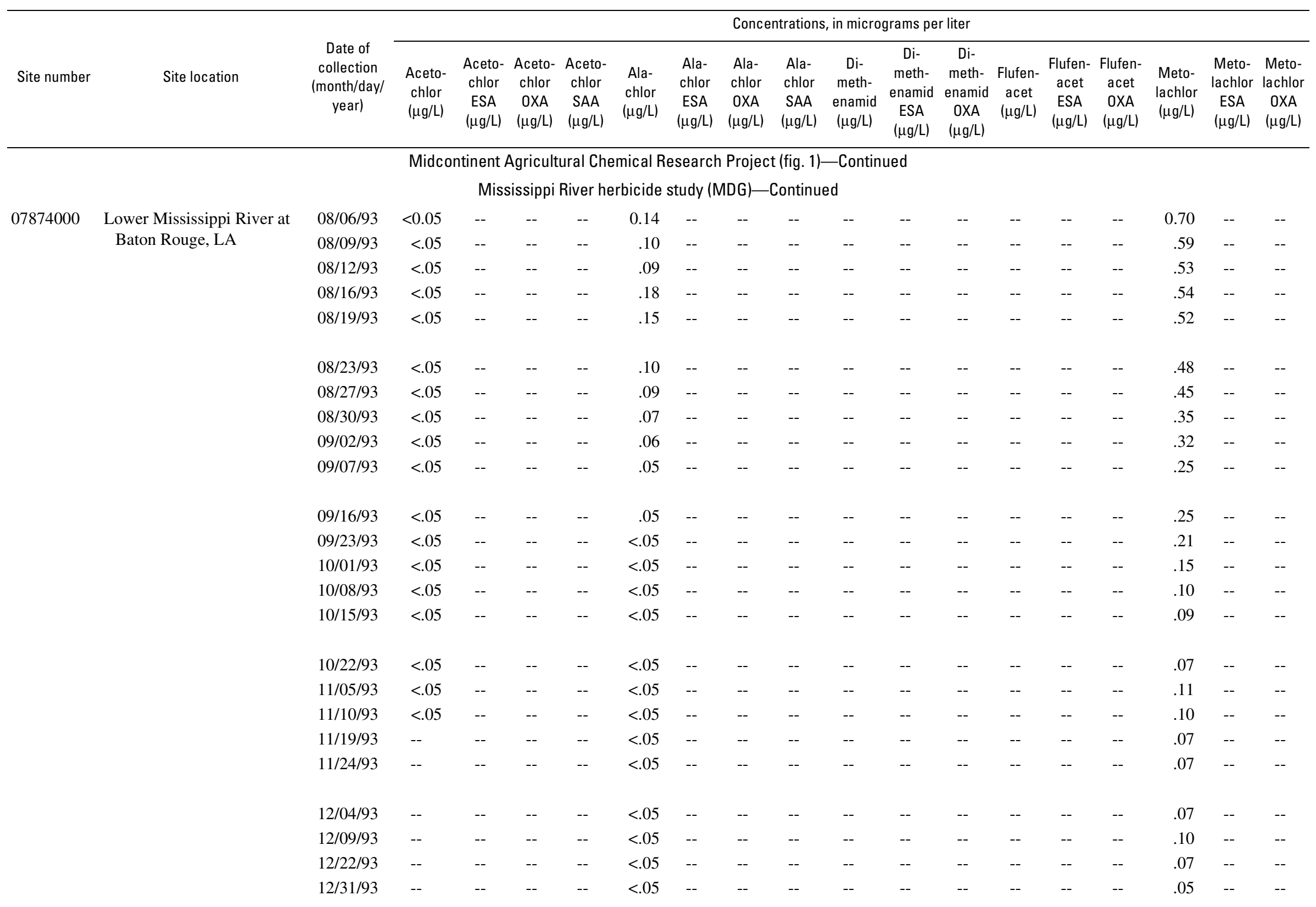




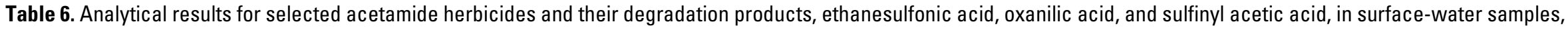
1993-2003.-Continued

[OGRL, U.S. Geological Survey Organic Geochemistry Research Laboratory, Lawrence, Kansas; ESA, ethanesulfonic acid; OXA, oxanilic acid; SAA, sulfinyl acetic acid; --, no data; <, less than]

\begin{tabular}{|c|c|c|c|c|c|c|c|c|c|c|c|c|c|c|c|c|c|c|c|}
\hline \multirow[b]{2}{*}{ Site number } & \multirow[b]{2}{*}{ Site location } & \multirow[b]{2}{*}{$\begin{array}{c}\text { Date of } \\
\text { collection } \\
\text { (month/day/ } \\
\text { year) }\end{array}$} & \multicolumn{17}{|c|}{ Concentrations, in micrograms per liter } \\
\hline & & & $\begin{array}{l}\text { Aceto- } \\
\text { chlor } \\
(\mu \mathrm{g} / \mathrm{L})\end{array}$ & $\begin{array}{l}\text { Aceto- } \\
\text { chlor } \\
\text { ESA } \\
(\mu \mathrm{g} / \mathrm{L})\end{array}$ & $\begin{array}{c}\text { Aceto- } \\
\text { chlor } \\
\text { OXA } \\
(\mu \mathrm{g} / \mathrm{L})\end{array}$ & $\begin{array}{c}\text { Aceto- } \\
\text { chlor } \\
\text { SAA } \\
(\mu \mathrm{g} / \mathrm{L})\end{array}$ & $\begin{array}{l}\text { Ala- } \\
\text { chlor } \\
(\mu \mathrm{g} / \mathrm{L})\end{array}$ & $\begin{array}{l}\text { Ala- } \\
\text { chlor } \\
\text { ESA } \\
(\mu \mathrm{g} / \mathrm{L})\end{array}$ & $\begin{array}{l}\text { Ala- } \\
\text { chlor } \\
\text { OXA } \\
(\mu \mathrm{g} / \mathrm{L})\end{array}$ & $\begin{array}{l}\text { Ala- } \\
\text { chlor } \\
\text { SAA } \\
(\mu \mathrm{g} / \mathrm{L})\end{array}$ & $\begin{array}{l}\text { Di- } \\
\text { meth- } \\
\text { enamid } \\
(\mu \mathrm{g} / \mathrm{L})\end{array}$ & $\begin{array}{c}\text { Di- } \\
\text { meth- } \\
\text { enamid } \\
\text { ESA } \\
(\mu \mathrm{g} / \mathrm{L})\end{array}$ & $\begin{array}{c}\text { Di- } \\
\text { meth- } \\
\text { enamid } \\
\text { OXA } \\
(\mu \mathrm{g} / \mathrm{L})\end{array}$ & $\begin{array}{c}\text { Flufen- } \\
\text { acet } \\
(\mu \mathrm{g} / \mathrm{L})\end{array}$ & $\begin{array}{c}\text { Flufen- } \\
\text { acet } \\
\text { ESA } \\
(\mu \mathrm{g} / \mathrm{L})\end{array}$ & $\begin{array}{c}\text { Flufen- } \\
\text { acet } \\
\text { OXA } \\
(\mu \mathrm{g} / \mathrm{L})\end{array}$ & $\begin{array}{l}\text { Meto- } \\
\text { lachlor } \\
(\mu \mathrm{g} / \mathrm{L})\end{array}$ & $\begin{array}{c}\text { Meto- } \\
\text { lachlor } \\
\text { ESA } \\
(\mu \mathrm{g} / \mathrm{L})\end{array}$ & $\begin{array}{c}\text { Meto- } \\
\text { lachlor } \\
\text { OXA } \\
(\mu \mathrm{g} / \mathrm{L})\end{array}$ \\
\hline
\end{tabular}

Midcontinent Agricultural Chemical Research Project (fig. 1)—Continued Baton Rouge, LA

Mississippi River herbicide study (MDG)—Continued

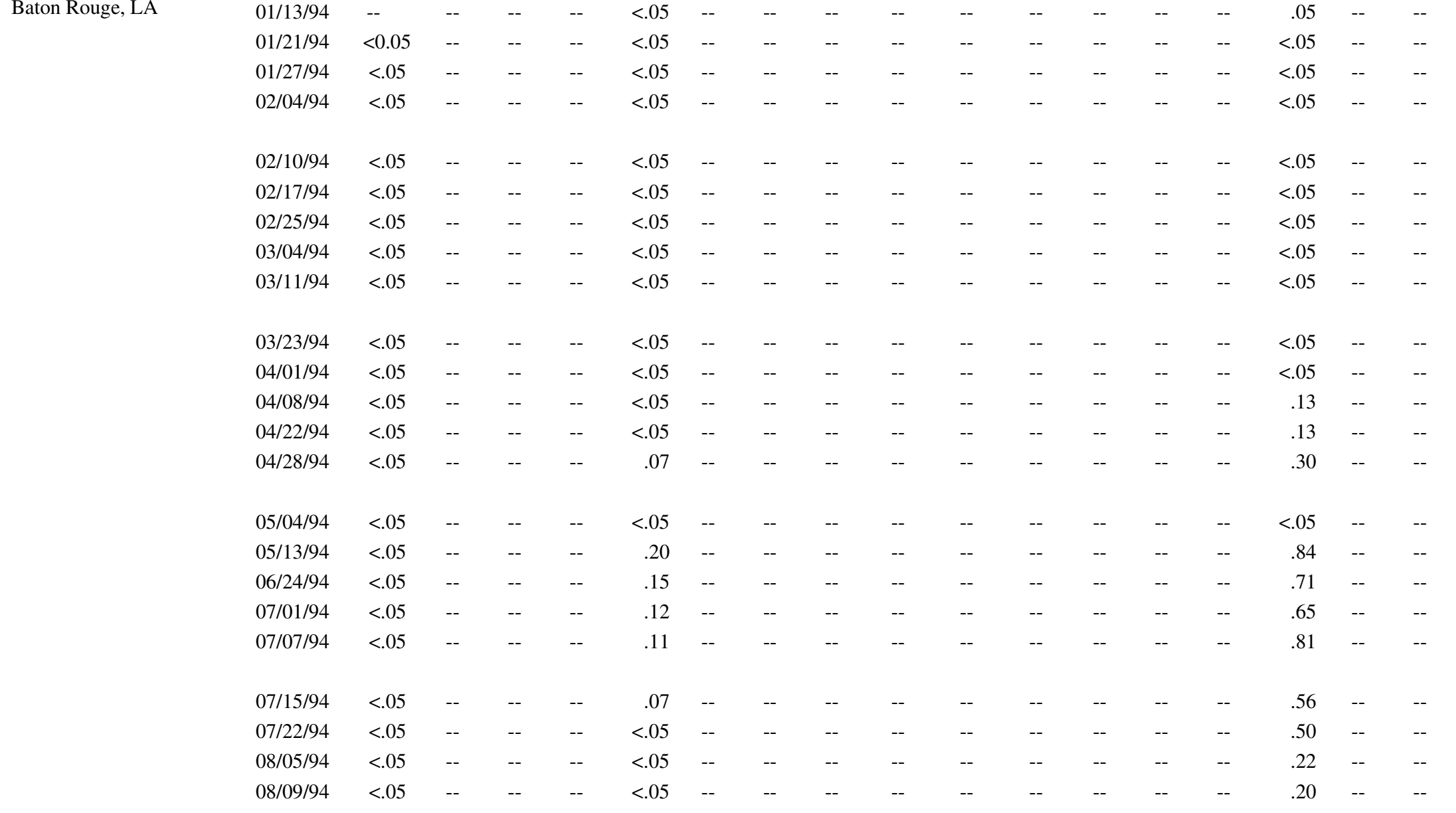




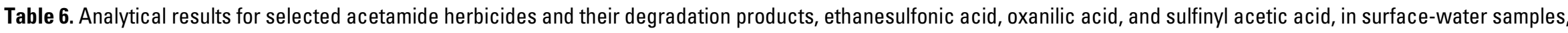
1993-2003-Continued

[OGRL, U.S. Geological Survey Organic Geochemistry Research Laboratory, Lawrence, Kansas; ESA, ethanesulfonic acid; OXA, oxanilic acid; SAA, sulfinyl acetic acid; --, no data; <, less than]

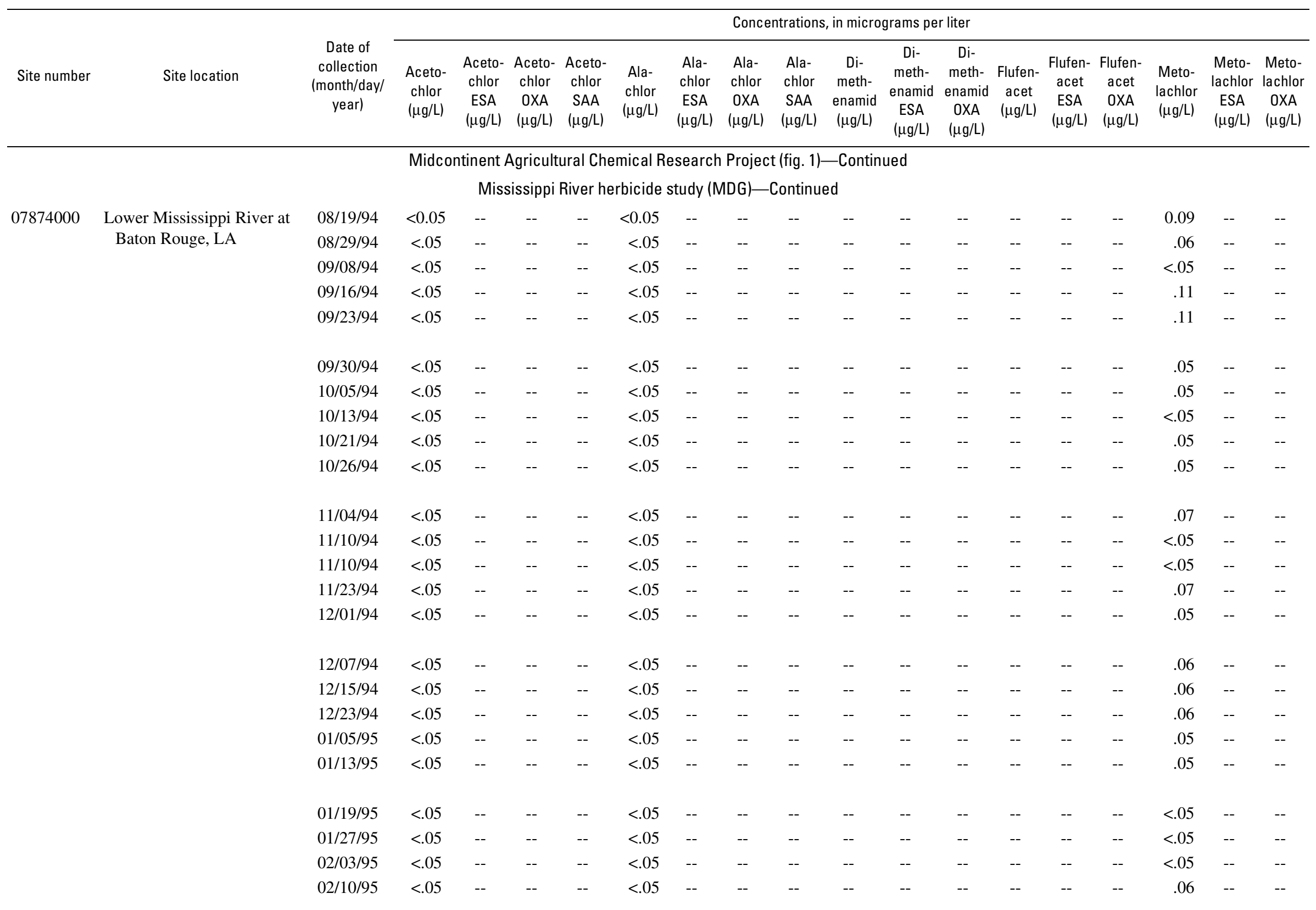




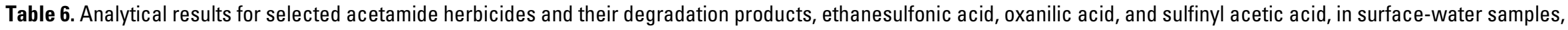
1993-2003.-Continued

[OGRL, U.S. Geological Survey Organic Geochemistry Research Laboratory, Lawrence, Kansas; ESA, ethanesulfonic acid; OXA, oxanilic acid; SAA, sulfinyl acetic acid; --, no data; <, less than]

\begin{tabular}{|c|c|c|c|c|c|c|c|c|c|c|c|c|c|c|c|c|c|c|c|}
\hline \multirow[b]{2}{*}{ Site number } & \multirow[b]{2}{*}{ Site location } & \multirow[b]{2}{*}{$\begin{array}{c}\text { Date of } \\
\text { collection } \\
\text { (month/day/ } \\
\text { year) }\end{array}$} & \multicolumn{17}{|c|}{ Concentrations, in micrograms per liter } \\
\hline & & & $\begin{array}{l}\text { Aceto- } \\
\text { chlor } \\
(\mu \mathrm{g} / \mathrm{L})\end{array}$ & $\begin{array}{l}\text { Aceto- } \\
\text { chlor } \\
\text { ESA } \\
(\mu \mathrm{g} / \mathrm{L})\end{array}$ & $\begin{array}{c}\text { Aceto- } \\
\text { chlor } \\
\text { OXA } \\
(\mu \mathrm{g} / \mathrm{L})\end{array}$ & $\begin{array}{c}\text { Aceto- } \\
\text { chlor } \\
\text { SAA } \\
(\mu \mathrm{g} / \mathrm{L})\end{array}$ & $\begin{array}{l}\text { Ala- } \\
\text { chlor } \\
(\mu \mathrm{g} / \mathrm{L})\end{array}$ & $\begin{array}{l}\text { Ala- } \\
\text { chlor } \\
\text { ESA } \\
(\mu \mathrm{g} / \mathrm{L})\end{array}$ & $\begin{array}{l}\text { Ala- } \\
\text { chlor } \\
\text { OXA } \\
(\mu \mathrm{g} / \mathrm{L})\end{array}$ & $\begin{array}{l}\text { Ala- } \\
\text { chlor } \\
\text { SAA } \\
(\mu \mathrm{g} / \mathrm{L})\end{array}$ & $\begin{array}{l}\text { Di- } \\
\text { meth- } \\
\text { enamid } \\
(\mu \mathrm{g} / \mathrm{L})\end{array}$ & $\begin{array}{c}\text { Di- } \\
\text { meth- } \\
\text { enamid } \\
\text { ESA } \\
(\mu \mathrm{g} / \mathrm{L})\end{array}$ & $\begin{array}{c}\text { Di- } \\
\text { meth- } \\
\text { enamid } \\
\text { OXA } \\
(\mu \mathrm{g} / \mathrm{L})\end{array}$ & $\begin{array}{c}\text { Flufen- } \\
\text { acet } \\
(\mu \mathrm{g} / \mathrm{L})\end{array}$ & $\begin{array}{c}\text { Flufen- } \\
\text { acet } \\
\text { ESA } \\
(\mu \mathrm{g} / \mathrm{L})\end{array}$ & $\begin{array}{c}\text { Flufen- } \\
\text { acet } \\
\text { OXA } \\
(\mu \mathrm{g} / \mathrm{L})\end{array}$ & $\begin{array}{l}\text { Meto- } \\
\text { lachlor } \\
(\mu \mathrm{g} / \mathrm{L})\end{array}$ & $\begin{array}{c}\text { Meto- } \\
\text { lachlor } \\
\text { ESA } \\
(\mu \mathrm{g} / \mathrm{L})\end{array}$ & $\begin{array}{c}\text { Meto- } \\
\text { lachlor } \\
\text { OXA } \\
(\mu \mathrm{g} / \mathrm{L})\end{array}$ \\
\hline
\end{tabular}

Midcontinent Agricultural Chemical Research Project (fig. 1)—Continued

Mississippi River herbicide study (MDG)—Continued

07874000 Lower Mississippi River at Baton Rouge, LA

$\begin{array}{lccccc}02 / 17 / 95 & <0.05 & -- & -- & -- & <0.05 \\ 02 / 24 / 95 & <.05 & -- & -- & -- & <.05 \\ 03 / 02 / 95 & <.05 & -- & -- & -- & <.05 \\ 03 / 10 / 95 & <.05 & -- & -- & -- & <.05 \\ 03 / 15 / 95 & <.05 & -- & -- & -- & <.05 \\ & & & & & \\ 03 / 29 / 95 & .07 & -- & -- & -- & <.05 \\ 04 / 05 / 95 & <.05 & -- & -- & -- & <.05 \\ 04 / 14 / 95 & <.05 & -- & -- & -- & <.05 \\ 04 / 21 / 95 & <.05 & -- & -- & -- & <.05 \\ 04 / 28 / 95 & .06 & -- & -- & -- & <.05 \\ & & & & & \\ 05 / 05 / 95 & .08 & -- & -- & -- & <.05 \\ 05 / 15 / 95 & .09 & -- & -- & -- & <.05 \\ 05 / 19 / 95 & .09 & -- & -- & -- & <.05 \\ 05 / 26 / 95 & .19 & -- & -- & -- & <.05 \\ 06 / 07 / 95 & .20 & -- & -- & -- & .07 \\ 07 / 15 / 95 & .28 & -- & -- & -- & .07 \\ 06 / 23 / 95 & .21 & -- & -- & -- & .07 \\ 07 / 03 / 95 & .10 & -- & -- & -- & .05 \\ 07 / 06 / 95 & .08 & -- & -- & -- & .05 \\ 07 / 20 / 95 & <.05 & -- & -- & -- & <.05 \\ 07 / 27 / 95 & <.05 & -- & -- & -- & <.05 \\ 08 / 02 / 95 & <.05 & -- & -- & -- & <.05 \\ 08 / 18 / 95 & <.05 & -- & -- & -- & <.05 \\ & <.05 & -- & -- & -- & <.05 \\ 055 & & & & & \end{array}$




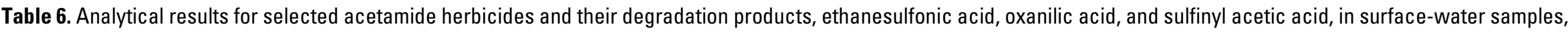
1993-2003-Continued

[OGRL, U.S. Geological Survey Organic Geochemistry Research Laboratory, Lawrence, Kansas; ESA, ethanesulfonic acid; OXA, oxanilic acid; SAA, sulfinyl acetic acid; --, no data; <, less than]

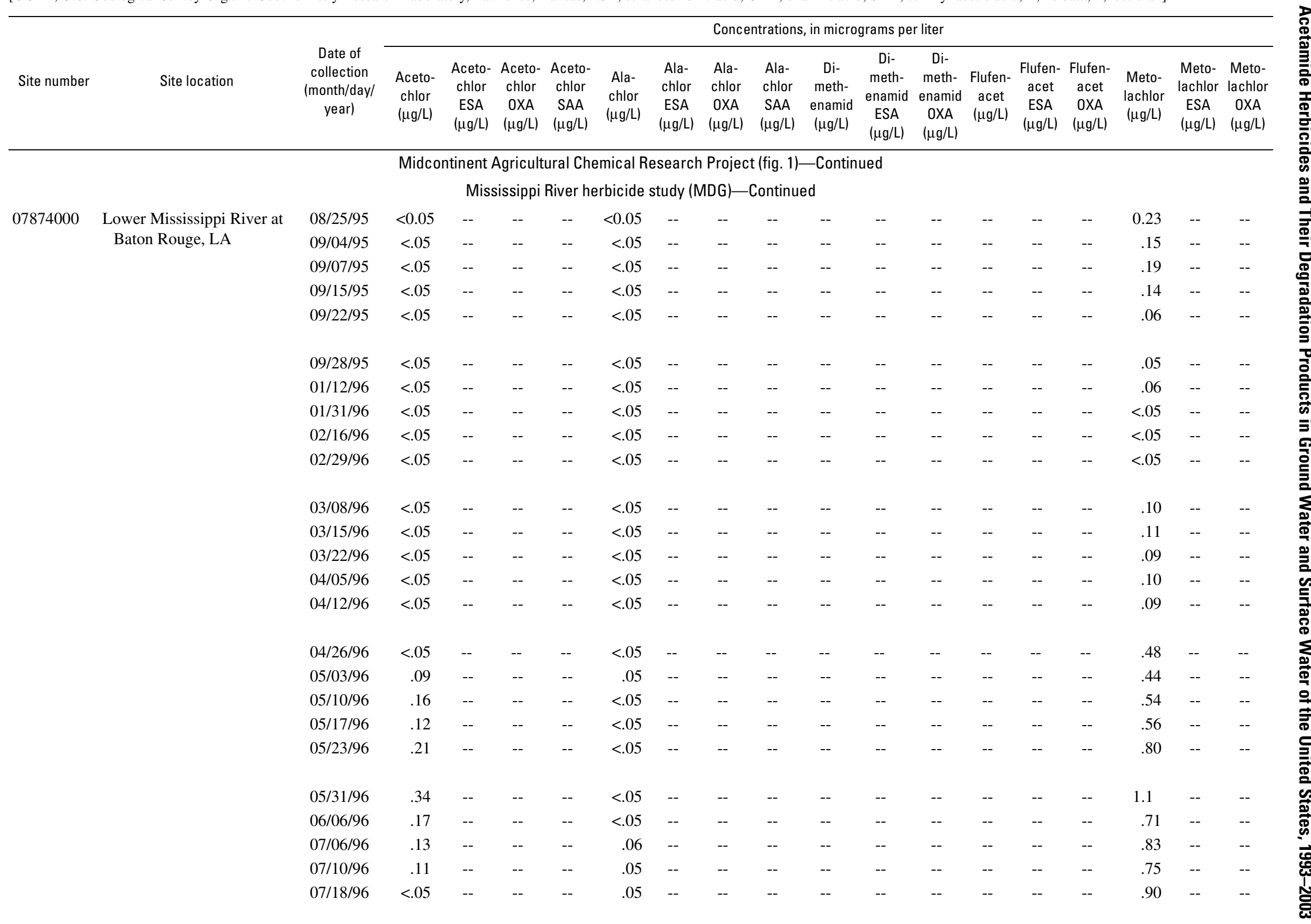




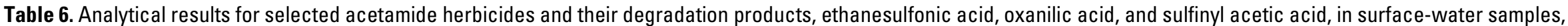
1993-2003. - Continued

[OGRL, U.S. Geological Survey Organic Geochemistry Research Laboratory, Lawrence, Kansas; ESA, ethanesulfonic acid; OXA, oxanilic acid; SAA, sulfinyl acetic acid; --, no data; <, less than]

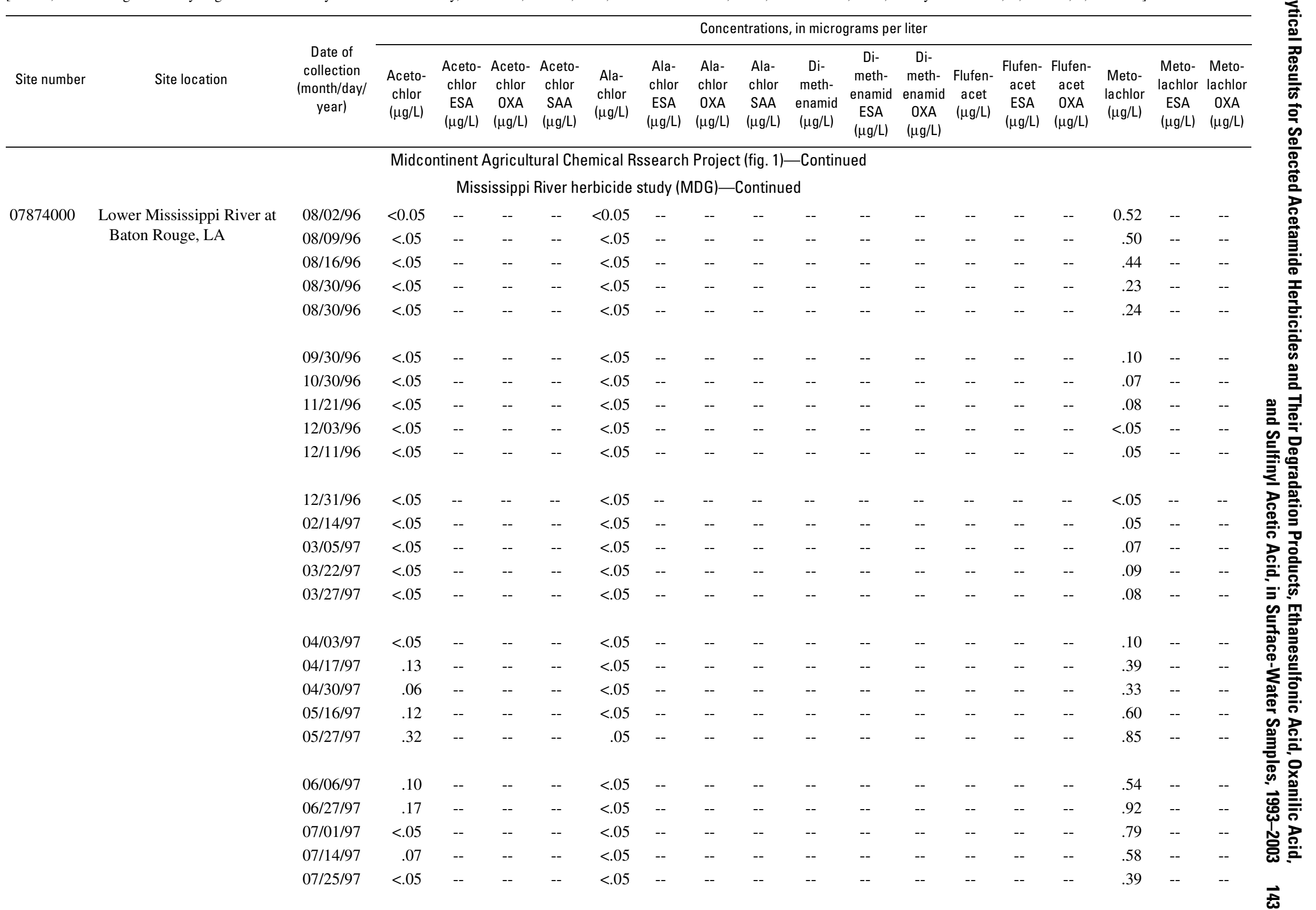




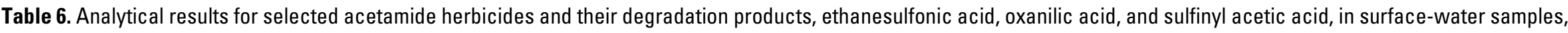
1993-2003-Continued

[OGRL, U.S. Geological Survey Organic Geochemistry Research Laboratory, Lawrence, Kansas; ESA, ethanesulfonic acid; OXA, oxanilic acid; SAA, sulfinyl acetic acid; --, no data; <, less than]

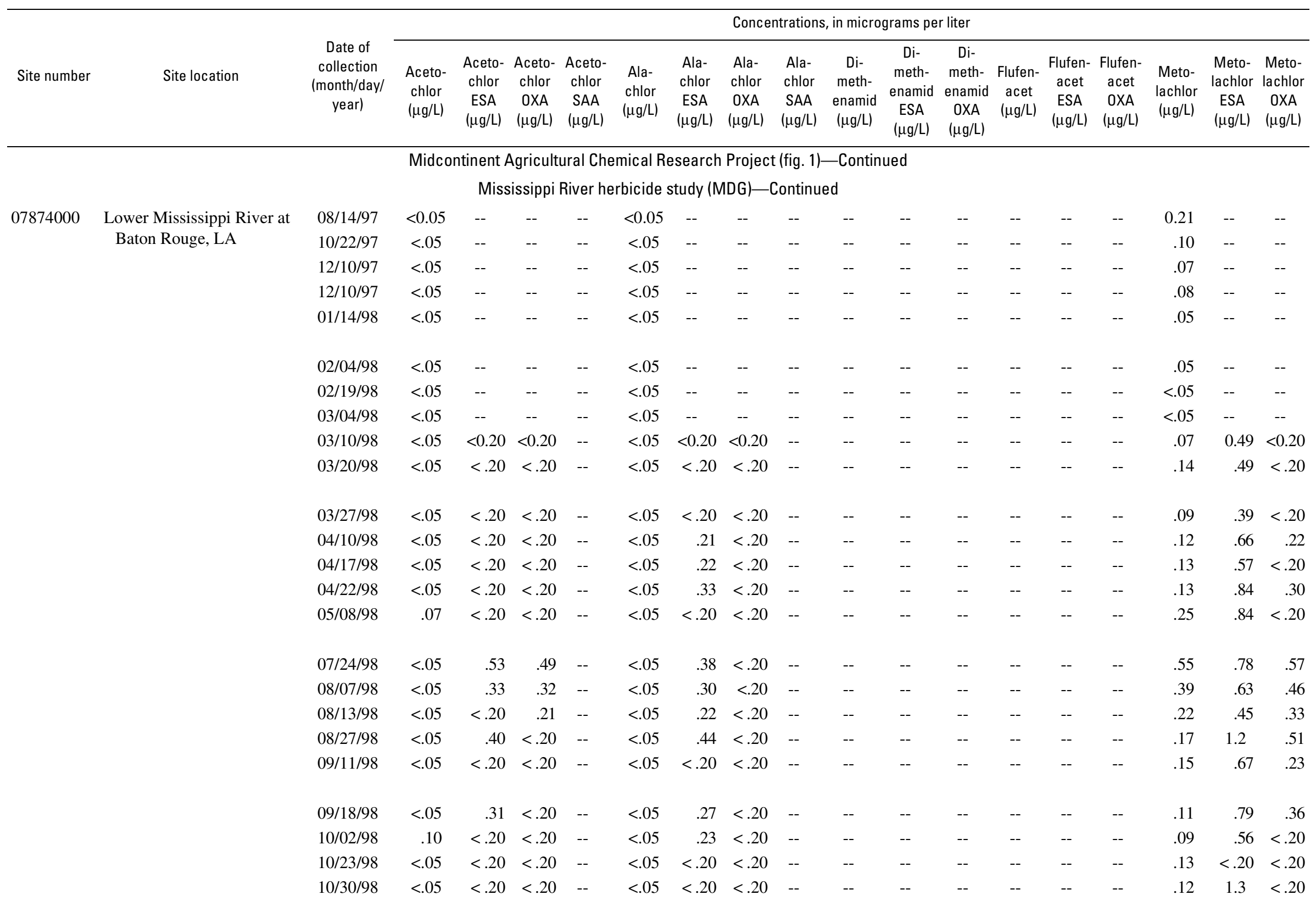




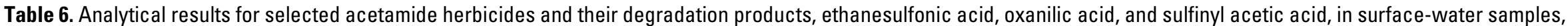
1993-2003.-Continued

[OGRL, U.S. Geological Survey Organic Geochemistry Research Laboratory, Lawrence, Kansas; ESA, ethanesulfonic acid; OXA, oxanilic acid; SAA, sulfinyl acetic acid; --, no data; <, less than]

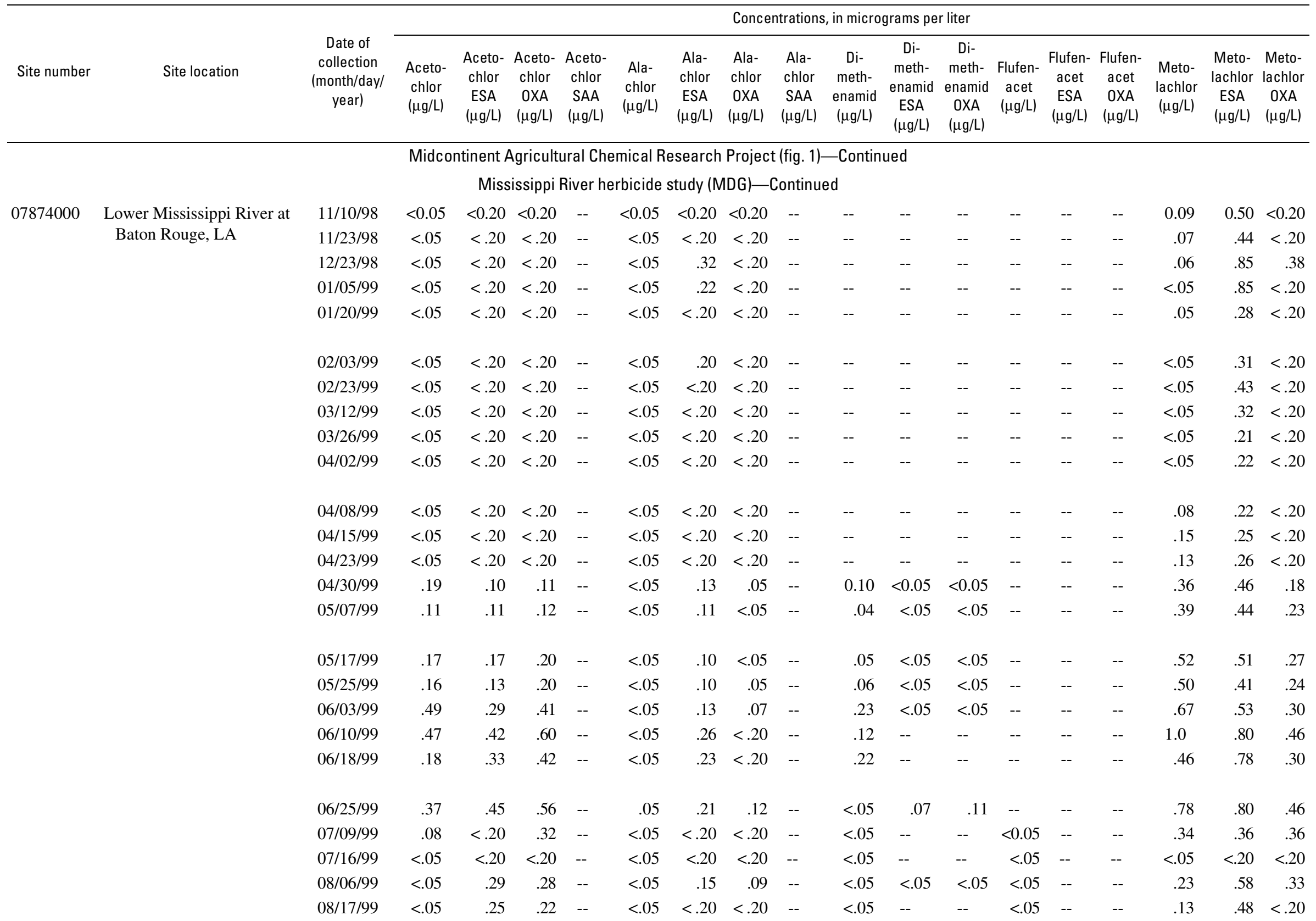




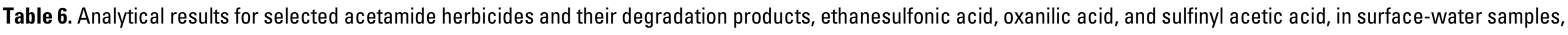
1993-2003-Continued

[OGRL, U.S. Geological Survey Organic Geochemistry Research Laboratory, Lawrence, Kansas; ESA, ethanesulfonic acid; OXA, oxanilic acid; SAA, sulfinyl acetic acid; --, no data; <, less than]

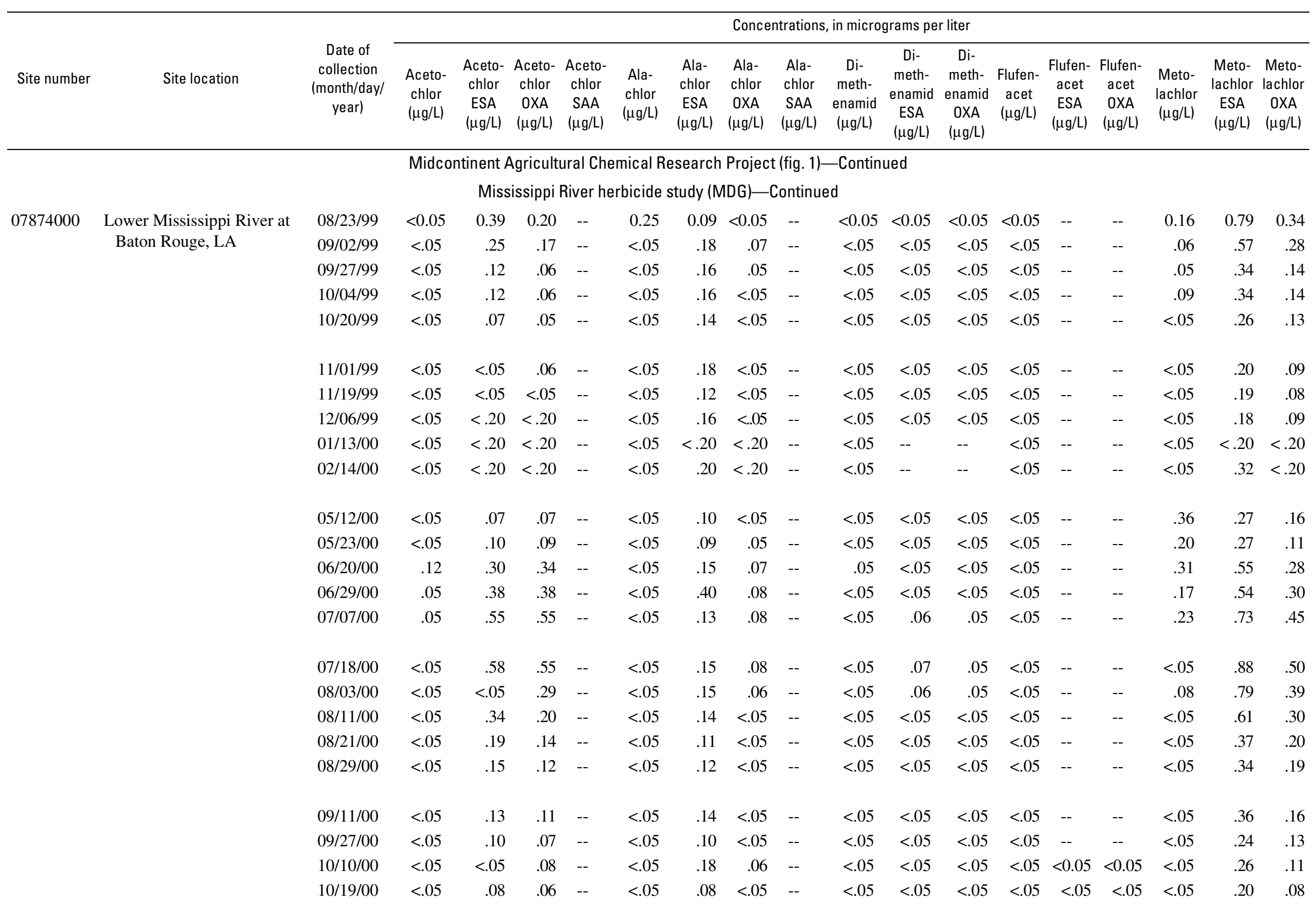




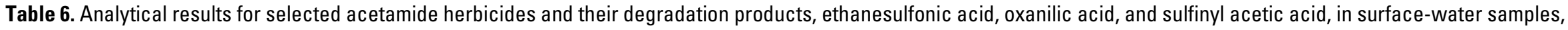
1993-2003. - Continued

[OGRL, U.S. Geological Survey Organic Geochemistry Research Laboratory, Lawrence, Kansas; ESA, ethanesulfonic acid; OXA, oxanilic acid; SAA, sulfinyl acetic acid; --, no data; <, less than]

\begin{tabular}{|c|c|c|c|c|c|c|c|c|c|c|c|c|c|c|c|c|c|c|c|}
\hline \multirow[b]{2}{*}{ Site number } & \multirow[b]{2}{*}{ Site location } & \multirow[b]{2}{*}{$\begin{array}{c}\text { Date of } \\
\text { collection } \\
\text { (month/day/ } \\
\text { year) }\end{array}$} & \multicolumn{17}{|c|}{ Concentrations, in micrograms per liter } \\
\hline & & & $\begin{array}{l}\text { Aceto- } \\
\text { chlor } \\
(\mu \mathrm{g} / \mathrm{L})\end{array}$ & $\begin{array}{l}\text { Aceto- } \\
\text { chlor } \\
\text { ESA } \\
(\mu \mathrm{g} / \mathrm{L})\end{array}$ & $\begin{array}{c}\text { Aceto- } \\
\text { chlor } \\
\text { OXA } \\
(\mu \mathrm{g} / \mathrm{L})\end{array}$ & $\begin{array}{c}\text { Aceto- } \\
\text { chlor } \\
\text { SAA } \\
(\mu \mathrm{g} / \mathrm{L})\end{array}$ & $\begin{array}{l}\text { Ala- } \\
\text { chlor } \\
(\mu \mathrm{g} / \mathrm{L})\end{array}$ & $\begin{array}{l}\text { Ala- } \\
\text { chlor } \\
\text { ESA } \\
(\mu \mathrm{g} / \mathrm{L})\end{array}$ & $\begin{array}{l}\text { Ala- } \\
\text { chlor } \\
\text { OXA } \\
(\mu \mathrm{g} / \mathrm{L})\end{array}$ & $\begin{array}{l}\text { Ala- } \\
\text { chlor } \\
\text { SAA } \\
(\mu \mathrm{g} / \mathrm{L})\end{array}$ & $\begin{array}{l}\text { Di- } \\
\text { meth- } \\
\text { enamid } \\
(\mu \mathrm{g} / \mathrm{L})\end{array}$ & $\begin{array}{c}\text { Di- } \\
\text { meth- } \\
\text { enamid } \\
\text { ESA } \\
(\mu \mathrm{g} / \mathrm{L})\end{array}$ & $\begin{array}{c}\text { Di- } \\
\text { meth- } \\
\text { enamid } \\
\text { OXA } \\
(\mu \mathrm{g} / \mathrm{L})\end{array}$ & $\begin{array}{c}\text { Flufen- } \\
\text { acet } \\
(\mu \mathrm{g} / \mathrm{L})\end{array}$ & $\begin{array}{c}\text { Flufen- } \\
\text { acet } \\
\text { ESA } \\
(\mu \mathrm{g} / \mathrm{L})\end{array}$ & $\begin{array}{c}\text { Flufen- } \\
\text { acet } \\
\text { OXA } \\
(\mu \mathrm{g} / \mathrm{L})\end{array}$ & $\begin{array}{l}\text { Meto- } \\
\text { lachlor } \\
(\mu \mathrm{g} / \mathrm{L})\end{array}$ & $\begin{array}{c}\text { Meto- } \\
\text { lachlor } \\
\text { ESA } \\
(\mu \mathrm{g} / \mathrm{L})\end{array}$ & $\begin{array}{c}\text { Meto- } \\
\text { lachlor } \\
\text { OXA } \\
(\mu \mathrm{g} / \mathrm{L})\end{array}$ \\
\hline
\end{tabular}

Midcontinent Agricultural Chemical Research Project (fig. 1)—Continued

\section{Mississippi River herbicide study (MDG)—Continued}

07874000 Lower Mississippi River at Baton Rouge, LA

\begin{tabular}{|c|c|c|c|c|c|c|c|c|c|c|c|c|c|c|c|c|c|}
\hline $11 / 06 / 00$ & $<0.05$ & 0.10 & 0.08 & -- & $<0.05$ & 0.08 & $<0.05$ & -- & $<0.05$ & $<0.05$ & $<0.05$ & $<0.05$ & $<0.05$ & $<0.05$ & $<0.05$ & 0.28 & 0.12 \\
\hline $11 / 20 / 00$ & $<.05$ & $<.05$ & $<.05$ & -- & $<.05$ & .15 & $<.05$ & -- & $<.05$ & $<.05$ & $<.05$ & $<.05$ & $<.05$ & $<.05$ & $<.05$ & .22 & .11 \\
\hline $12 / 04 / 00$ & $<.05$ & .08 & $<.05$ & -- & $<.05$ & .06 & $<.05$ & -- & $<.05$ & $<.05$ & $<.05$ & $<.05$ & $<.05$ & $<.05$ & $<.05$ & .26 & .11 \\
\hline $12 / 18 / 00$ & $<.05$ & .11 & .07 & -- & $<.05$ & .10 & $<.05$ & -- & $<.05$ & $<.05$ & $<.05$ & $<.05$ & $<.05$ & $<.05$ & $<.05$ & .34 & .09 \\
\hline 01/05/01 & $<.05$ & $<.05$ & $<.05$ & -- & $<.05$ & $<.05$ & $<.05$ & -- & $<.05$ & $<.05$ & $<.05$ & $<.05$ & $<.05$ & $<.05$ & $<.05$ & .13 & .09 \\
\hline 02/08/01 & $<.05$ & $<.05$ & $<.05$ & -- & $<.05$ & .09 & $<.05$ & -- & $<.05$ & $<.05$ & $<.05$ & $<.05$ & $<.05$ & $<.05$ & $<.05$ & .19 & .08 \\
\hline 02/23/01 & $<.05$ & .06 & $<.05$ & -- & $<.05$ & .10 & $<.05$ & -- & $<.05$ & $<.05$ & $<.05$ & $<.05$ & $<.05$ & $<.05$ & $<.05$ & .26 & .11 \\
\hline 03/06/01 & $<.05$ & .06 & $<.05$ & -- & $<.05$ & .06 & $<.05$ & -- & $<.05$ & $<.05$ & $<.05$ & $<.05$ & $<.05$ & $<.05$ & $<.05$ & .23 & .10 \\
\hline $03 / 23 / 01$ & $<.05$ & .08 & .06 & -- & $<.05$ & .08 & $<.05$ & -- & $<.05$ & $<.05$ & $<.05$ & $<.05$ & $<.05$ & $<.05$ & .06 & .24 & .11 \\
\hline 04/06/01 & $<.05$ & .08 & .06 & -- & $<.05$ & .11 & .05 & -- & $<.05$ & $<.05$ & $<.05$ & $<.05$ & $<.05$ & $<.05$ & .05 & .30 & .14 \\
\hline $05 / 24 / 01$ & .31 & .25 & .29 & -- & $<.05$ & .17 & $<.05$ & -- & .06 & $<.05$ & $<.05$ & $<.05$ & $<.05$ & $<.05$ & .47 & .53 & .22 \\
\hline $05 / 31 / 01$ & .19 & .25 & .32 & -- & $<.05$ & .13 & $<.05$ & -- & .07 & $<.05$ & $<.05$ & $<.05$ & $<.05$ & $<.05$ & .41 & .53 & .25 \\
\hline 06/08/01 & .16 & .20 & .26 & -- & $<.05$ & .11 & $<.05$ & -- & $<.05$ & .05 & $<.05$ & $<.05$ & $<.05$ & $<.05$ & .38 & .37 & .21 \\
\hline 07/02/01 & .14 & .45 & .49 & -- & $<.05$ & .18 & .07 & -- & .06 & .05 & .05 & $<.05$ & $<.05$ & $<.05$ & .40 & .64 & .37 \\
\hline 07/16/01 & $<.05$ & .33 & .31 & -- & $<.05$ & .17 & .05 & -- & $<.05$ & $<.05$ & $<.05$ & $<.05$ & $<.05$ & $<.05$ & .20 & .57 & .26 \\
\hline 07/30/01 & $<.05$ & .25 & .26 & -- & $<.05$ & .13 & $<.05$ & -- & $<.05$ & $<.05$ & $<.05$ & $<.05$ & $<.05$ & $<.05$ & .11 & .40 & .21 \\
\hline 08/13/01 & $<.05$ & .20 & .20 & -- & $<.05$ & .14 & $<.05$ & -- & $<.05$ & $<.05$ & $<.05$ & $<.05$ & $<.05$ & $<.05$ & .07 & .37 & .21 \\
\hline 08/28/01 & $<.05$ & .08 & .11 & -- & $<.05$ & .07 & $<.05$ & -- & $<.05$ & $<.05$ & $<.05$ & $<.05$ & $<.05$ & $<.05$ & $<.05$ & .10 & .13 \\
\hline 09/18/01 & $<.05$ & .07 & .08 & -- & $<.05$ & .07 & .05 & -- & $<.05$ & $<.05$ & $<.05$ & $<.05$ & $<.05$ & $<.05$ & $<.05$ & .08 & .10 \\
\hline 10/01/01 & $<.05$ & $<.05$ & .06 & -- & $<.05$ & .11 & $<.05$ & -- & $<.05$ & $<.05$ & $<.05$ & $<.05$ & $<.05$ & $<.05$ & $<.05$ & .22 & .10 \\
\hline $10 / 16 / 01$ & $<.05$ & .05 & .07 & -- & $<.05$ & .11 & $<.05$ & -- & $<.05$ & $<.05$ & $<.05$ & $<.05$ & $<.05$ & $<.05$ & $<.05$ & .22 & .12 \\
\hline $10 / 31 / 01$ & $<.05$ & .05 & .07 & -- & $<.05$ & .09 & $<.05$ & -- & $<.05$ & $<.05$ & $<.05$ & $<.05$ & $<.05$ & $<.05$ & $<.05$ & .23 & .13 \\
\hline $11 / 14 / 01$ & $<.05$ & .16 & .10 & -- & $<.05$ & .13 & $<.05$ & -- & $<.05$ & $<.05$ & $<.05$ & $<.05$ & $<.05$ & $<.05$ & $<.05$ & .43 & .17 \\
\hline $11 / 28 / 01$ & $<.05$ & .19 & .10 & -- & $<.05$ & .15 & $<.05$ & -- & $<.05$ & $<.05$ & $<.05$ & $<.05$ & $<.05$ & $<.05$ & $<.05$ & .51 & .19 \\
\hline
\end{tabular}




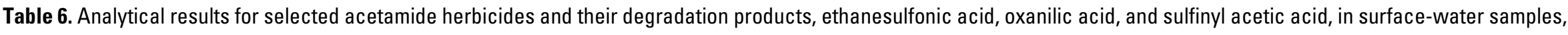
1993-2003-Continued

[OGRL, U.S. Geological Survey Organic Geochemistry Research Laboratory, Lawrence, Kansas; ESA, ethanesulfonic acid; OXA, oxanilic acid; SAA, sulfinyl acetic acid; --, no data; <, less than]

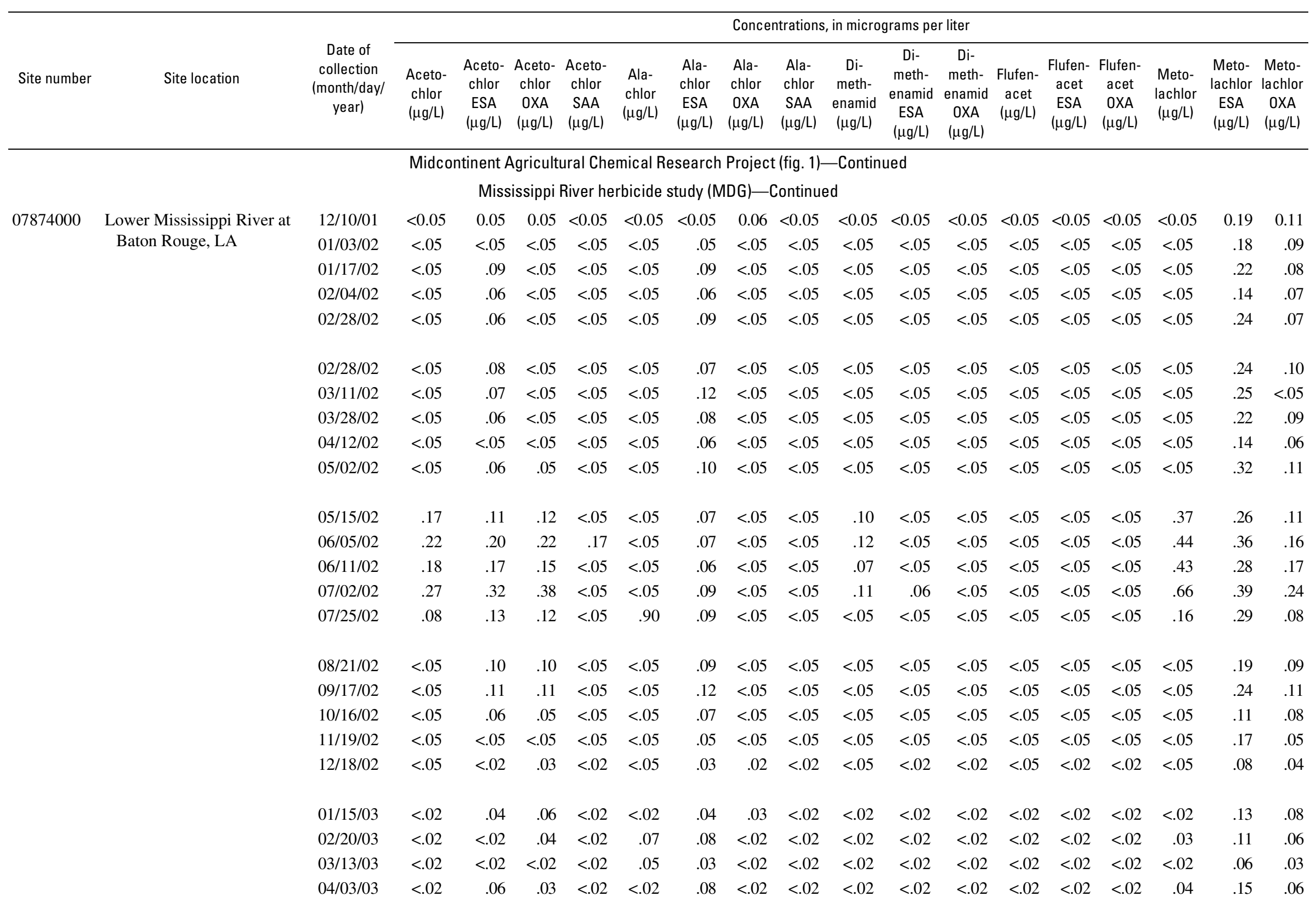


Table 6. Analytical results for selected acetamide herbicides and their degradation products, ethanesulfonic acid, oxanilic acid, and sulfinyl acetic acid, in surface-water samples, 1993-2003. - Continued

[OGRL, U.S. Geological Survey Organic Geochemistry Research Laboratory, Lawrence, Kansas; ESA, ethanesulfonic acid; OXA, oxanilic acid; SAA, sulfinyl acetic acid; --, no data; <, less than]

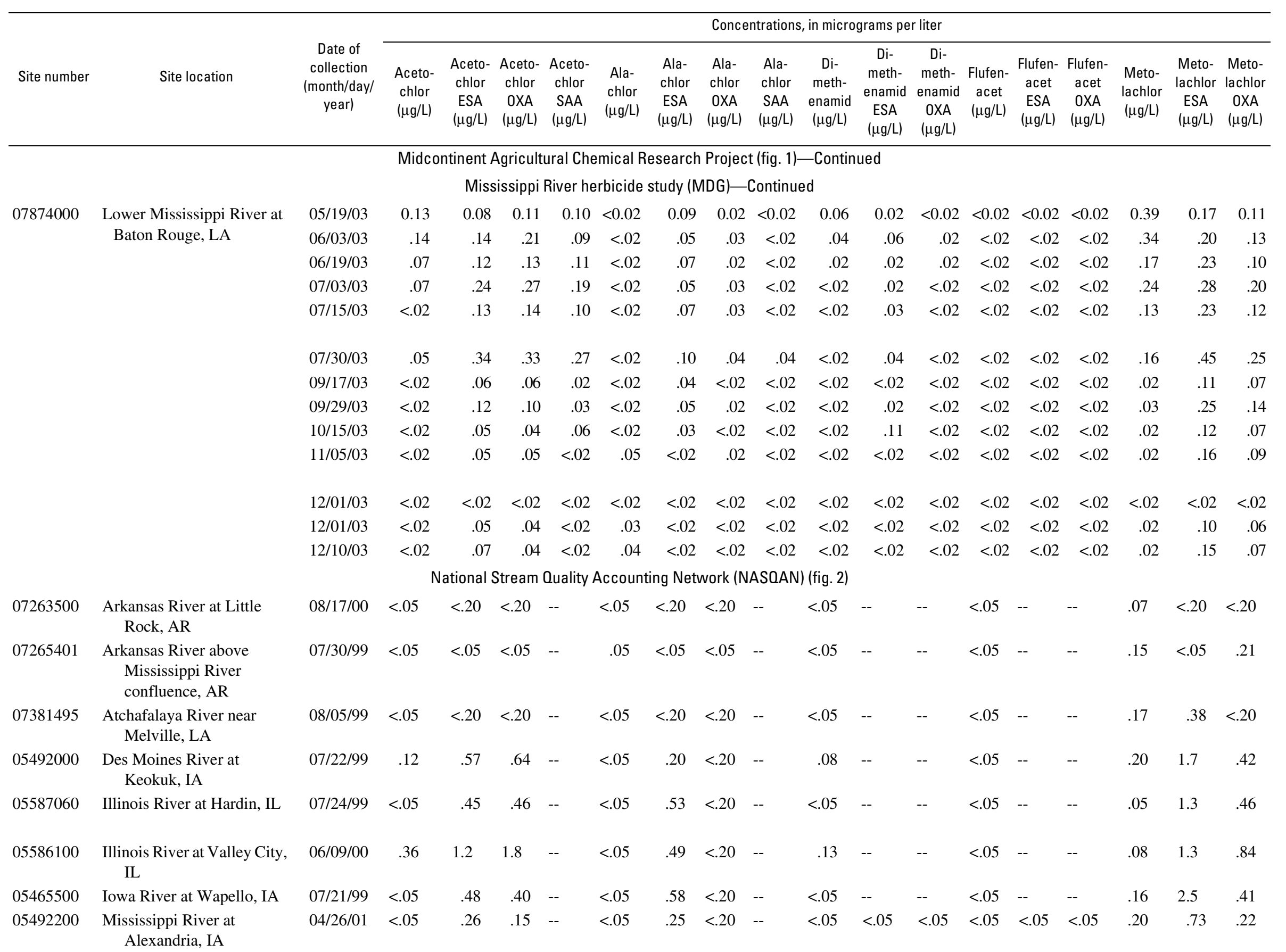




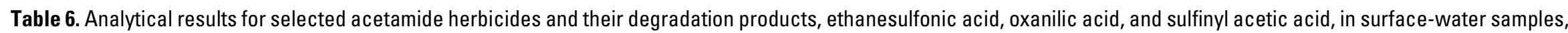
1993-2003.-Continued

[OGRL, U.S. Geological Survey Organic Geochemistry Research Laboratory, Lawrence, Kansas; ESA, ethanesulfonic acid; OXA, oxanilic acid; SAA, sulfinyl acetic acid; --, no data; <, less than]

\begin{tabular}{|c|c|c|c|c|c|c|c|c|c|c|c|c|c|c|c|c|c|c|c|}
\hline \multirow[b]{2}{*}{ Site number } & \multirow[b]{2}{*}{ Site location } & \multirow[b]{2}{*}{$\begin{array}{l}\text { Date of } \\
\text { collection } \\
\text { (month/ } \\
\text { day/year) }\end{array}$} & \multicolumn{17}{|c|}{ Concentrations, in micrograms per liter } \\
\hline & & & $\begin{array}{l}\text { Aceto- } \\
\text { chlor } \\
(\mu \mathrm{g} / \mathrm{L})\end{array}$ & $\begin{array}{l}\text { Aceto- } \\
\text { chlor } \\
\text { ESA } \\
(\mu \mathrm{g} / \mathrm{L})\end{array}$ & $\begin{array}{l}\text { Aceto- } \\
\text { chlor } \\
\text { OXA } \\
(\mu \mathrm{g} / \mathrm{L})\end{array}$ & $\begin{array}{l}\text { Aceto- } \\
\text { chlor } \\
\text { SAA } \\
(\mu \mathrm{g} / \mathrm{L})\end{array}$ & $\begin{array}{l}\text { Ala- } \\
\text { chlor } \\
(\mu \mathrm{g} / \mathrm{L})\end{array}$ & $\begin{array}{l}\text { Ala- } \\
\text { chlor } \\
\text { ESA } \\
(\mu \mathrm{g} / \mathrm{L})\end{array}$ & $\begin{array}{l}\text { Ala- } \\
\text { chlor } \\
\text { OXA } \\
(\mu \mathrm{g} / \mathrm{L})\end{array}$ & $\begin{array}{l}\text { Ala- } \\
\text { chlor } \\
\text { SAA } \\
(\mu \mathrm{g} / \mathrm{L})\end{array}$ & $\begin{array}{c}\text { Di- } \\
\text { meth- } \\
\text { enamid } \\
(\mu \mathrm{g} / \mathrm{L})\end{array}$ & $\begin{array}{c}\text { Di- } \\
\text { meth- } \\
\text { enamid } \\
\text { ESA } \\
(\mu \mathrm{g} / \mathrm{L})\end{array}$ & $\begin{array}{c}\text { Di- } \\
\text { meth- } \\
\text { enamid } \\
\text { OXA } \\
(\mu \mathrm{g} / \mathrm{L})\end{array}$ & $\begin{array}{l}\text { Flufen- } \\
\text { acet } \\
(\mu \mathrm{g} / \mathrm{L})\end{array}$ & $\begin{array}{l}\text { Flufen- } \\
\text { acet } \\
\text { ESA } \\
(\mu \mathrm{g} / \mathrm{L})\end{array}$ & $\begin{array}{c}\text { Flufen- } \\
\text { acet } \\
\text { OXA } \\
(\mu \mathrm{g} / \mathrm{L})\end{array}$ & $\begin{array}{l}\text { Meto- } \\
\text { lachlor } \\
(\mu \mathrm{g} / \mathrm{L})\end{array}$ & $\begin{array}{l}\text { Meto- } \\
\text { lachlor } \\
\text { ESA } \\
(\mu \mathrm{g} / \mathrm{L})\end{array}$ & $\begin{array}{c}\text { Meto- } \\
\text { lachlor } \\
\text { OXA } \\
(\mu \mathrm{g} / \mathrm{L})\end{array}$ \\
\hline \multicolumn{20}{|c|}{ National Stream Quality Accounting Network (NASOAN) (fig. 2)-Continued } \\
\hline \multirow[t]{2}{*}{07374000} & Mississippi River at Baton & $05 / 02 / 00$ & $<0.05$ & $<0.20$ & $<0.20$ & -- & $<0.05$ & $<0.20$ & $<0.20$ & -- & $<0.05$ & $<0.05$ & $<0.05$ & $<0.05$ & -- & -- & 0.07 & 0.28 & $<0.20$ \\
\hline & Rouge, LA & 05/03/01 & $<.05$ & .11 & .06 & -- & $<.05$ & .13 & $<.05$ & -- & $<.05$ & $<.05$ & $<.05$ & $<.05$ & $<0.05$ & $<0.05$ & .20 & .45 & .16 \\
\hline 07374525 & $\begin{array}{l}\text { Mississippi River at Bell } \\
\text { Chasse, LA }\end{array}$ & 08/09/99 & $<.05$ & $<.20$ & $<.20$ & -- & $<.05$ & $<.20$ & $<.20$ & -- & $<.05$ & $<.05$ & $<.05$ & $<.05$ & $<.05$ & $<.05$ & .20 & .37 & $<.20$ \\
\hline 05448095 & $\begin{array}{l}\text { Mississippi River at } \\
\text { Buffalo, IA }\end{array}$ & $04 / 25 / 01$ & $<.05$ & .26 & .16 & -- & $<.05$ & .21 & $<.05$ & -- & $<.05$ & $<.05$ & $<.05$ & $<.05$ & $<.05$ & $<.05$ & .22 & .57 & .20 \\
\hline \multirow[t]{5}{*}{05420500} & Mississippi River at Clinton, IA & 07/20/99 & $<.05$ & $<.20$ & $<.20$ & -- & $<.05$ & .28 & $<.20$ & -- & $<.05$ & -- & -- & $<.05$ & -- & -- & $<.05$ & .48 & $<.20$ \\
\hline & & $06 / 06 / 00$ & .37 & .24 & .44 & -- & .06 & $<.20$ & $<.20$ & -- & .09 & -- & -- & $<.05$ & -- & -- & .31 & .37 & $<.20$ \\
\hline & & 08/08/00 & $<.05$ & .24 & $<.20$ & -- & $<.05$ & .29 & $<.20$ & -- & $<.05$ & -- & -- & $<.05$ & -- & -- & $<.05$ & .59 & $<.20$ \\
\hline & & $04 / 24 / 01$ & $<.05$ & .24 & .16 & -- & $<.05$ & .19 & $<.05$ & -- & $<.05$ & $<.05$ & $<.05$ & $<.05$ & $<.05$ & $<.05$ & .32 & .50 & .19 \\
\hline & & 05/09/01 & .07 & .27 & .18 & -- & $<.05$ & .24 & $<.05$ & -- & $<.05$ & $<.05$ & $<.05$ & $<.05$ & $<.05$ & $<.05$ & .18 & .51 & .17 \\
\hline 07265402 & $\begin{array}{l}\text { Mississippi River above } \\
\text { Arkansas River confluence, } \\
\text { AR }\end{array}$ & $07 / 30 / 99$ & .06 & .36 & .26 & -- & $<.05$ & $<.05$ & .06 & -- & $<.05$ & -- & -- & $<.05$ & -- & -- & .08 & .63 & .35 \\
\hline 05466000 & $\begin{array}{l}\text { Mississippi River at } \\
\text { Keithsburg, IA }\end{array}$ & $04 / 25 / 01$ & $<.05$ & .28 & .16 & -- & $<.05$ & .29 & $<.05$ & -- & $<.05$ & $<.05$ & $<.05$ & $<.05$ & $<.05$ & $<.05$ & .82 & .24 & .27 \\
\hline 00703200 & $\begin{array}{l}\text { Mississippi River at } \\
\text { Memphis, TN }\end{array}$ & $07 / 28 / 99$ & .06 & .46 & .34 & -- & $<.05$ & .08 & .11 & -- & $<.05$ & -- & -- & $<.05$ & -- & -- & .09 & 1.0 & .38 \\
\hline \multirow[t]{10}{*}{07373420} & Mississippi River at St. & $03 / 30 / 99$ & $<.05$ & $<.20$ & $<.20$ & -- & $<.05$ & $<.20$ & $<.20$ & -- & -- & -- & -- & -- & -- & -- & $<.05$ & .20 & $<.20$ \\
\hline & Francisville, LA & $04 / 19 / 99$ & $<.05$ & $<.20$ & $<.20$ & -- & $<.05$ & $<.20$ & $<.20$ & -- & -- & -- & -- & -- & -- & -- & .14 & .29 & $<.20$ \\
\hline & & $04 / 28 / 99$ & .05 & .07 & .07 & -- & $<.05$ & .25 & .05 & -- & $<.05$ & -- & -- & -- & -- & -- & .14 & .41 & .19 \\
\hline & & $05 / 26 / 99$ & .17 & $<.20$ & $<.20$ & -- & $<.05$ & $<.20$ & $<.20$ & -- & .07 & -- & -- & $<.05$ & -- & -- & .44 & .37 & $<.20$ \\
\hline & & $06 / 10 / 99$ & .51 & .29 & .50 & -- & $<.05$ & $<.20$ & $<.20$ & -- & .19 & -- & -- & -- & & -- & .73 & .67 & .24 \\
\hline & & $06 / 24 / 99$ & .35 & .32 & .48 & -- & $<.05$ & .23 & $<.20$ & -- & .15 & -- & -- & $<.05$ & -- & -- & .69 & .60 & .27 \\
\hline & & $07 / 21 / 99$ & $<.05$ & $<.20$ & $<.20$ & -- & $<.05$ & $<.20$ & $<.20$ & -- & $<.05$ & -- & -- & $<.05$ & -- & -- & .12 & .39 & $<.20$ \\
\hline & & 08/05/99 & $<.05$ & $<.20$ & $<.20$ & -- & $<.05$ & $<.20$ & $<.20$ & -- & $<.05$ & -- & -- & $<.05$ & -- & -- & .09 & .37 & $<.20$ \\
\hline & & 09/14/99 & $<.05$ & $<.20$ & $<.20$ & -- & $<.05$ & $<.20$ & $<.20$ & -- & $<.05$ & -- & -- & $<.05$ & -- & -- & $<.05$ & .30 & $<.20$ \\
\hline & & $11 / 11 / 99$ & $<.05$ & $<.20$ & $<.20$ & -- & $<.05$ & $<.20$ & $<.20$ & -- & $<.05$ & -- & -- & $<.05$ & -- & -- & $<.05$ & $<.20$ & $<.20$ \\
\hline
\end{tabular}


Table 6. Analytical results for selected acetamide herbicides and their degradation products, ethanesulfonic acid, oxanilic acid, and sulfinyl acetic acid, in surface-water samples, 1993-2003.-Continued

[OGRL, U.S. Geological Survey Organic Geochemistry Research Laboratory, Lawrence, Kansas; ESA, ethanesulfonic acid; OXA, oxanilic acid; SAA, sulfinyl acetic acid; --, no data; <, less than]

\begin{tabular}{|c|c|c|c|c|c|c|c|c|c|c|c|c|c|c|c|c|c|c|c|}
\hline \multirow[b]{2}{*}{ Site number } & \multirow[b]{2}{*}{ Site location } & \multirow[b]{2}{*}{$\begin{array}{l}\text { Date of } \\
\text { collection } \\
\text { (month/ } \\
\text { day/year) }\end{array}$} & \multicolumn{17}{|c|}{ Concentrations, in micrograms per liter } \\
\hline & & & $\begin{array}{l}\text { Aceto- } \\
\text { chlor } \\
(\mu \mathrm{g} / \mathrm{L})\end{array}$ & $\begin{array}{c}\text { Aceto- } \\
\text { chlor } \\
\text { ESA } \\
(\mu \mathrm{g} / \mathrm{L})\end{array}$ & $\begin{array}{l}\text { Aceto- } \\
\text { chlor } \\
\text { OXA } \\
(\mu \mathrm{g} / \mathrm{L})\end{array}$ & $\begin{array}{l}\text { Aceto- } \\
\text { chlor } \\
\text { SAA } \\
(\mu \mathrm{g} / \mathrm{L})\end{array}$ & $\begin{array}{l}\text { Ala- } \\
\text { chlor } \\
(\mu \mathrm{g} / \mathrm{L})\end{array}$ & $\begin{array}{l}\text { Ala- } \\
\text { chlor } \\
\text { ESA } \\
(\mu \mathrm{g} / \mathrm{L})\end{array}$ & $\begin{array}{l}\text { Ala- } \\
\text { chlor } \\
\text { OXA } \\
(\mu \mathrm{g} / \mathrm{L})\end{array}$ & $\begin{array}{l}\text { Ala- } \\
\text { chlor } \\
\text { SAA } \\
(\mu \mathrm{g} / \mathrm{L})\end{array}$ & $\begin{array}{l}\text { Di- } \\
\text { meth- } \\
\text { enamid } \\
(\mu \mathrm{g} / \mathrm{L})\end{array}$ & $\begin{array}{c}\text { Di- } \\
\text { meth- } \\
\text { enamid } \\
\text { ESA } \\
(\mu \mathrm{g} / \mathrm{L})\end{array}$ & $\begin{array}{c}\text { Di- } \\
\text { meth- } \\
\text { enamid } \\
\text { OXA } \\
(\mu \mathrm{g} / \mathrm{L})\end{array}$ & $\begin{array}{l}\text { Flufen- } \\
\text { acet } \\
(\mu \mathrm{g} / \mathrm{L})\end{array}$ & $\begin{array}{c}\text { Flufen- } \\
\text { acet } \\
\text { ESA } \\
(\mu \mathrm{g} / \mathrm{L})\end{array}$ & $\begin{array}{c}\text { Flufen- } \\
\text { acet } \\
\text { OXA } \\
(\mu \mathrm{g} / \mathrm{L})\end{array}$ & $\begin{array}{l}\text { Meto- } \\
\text { lachlor } \\
(\mu \mathrm{g} / \mathrm{L})\end{array}$ & $\begin{array}{l}\text { Meto- } \\
\text { lachlor } \\
\text { ESA } \\
(\mu \mathrm{g} / \mathrm{L})\end{array}$ & $\begin{array}{c}\text { Meto- } \\
\text { lachlor } \\
\text { OXA } \\
(\mu \mathrm{g} / \mathrm{L})\end{array}$ \\
\hline
\end{tabular}

\section{National Stream Quality Accounting Network (NASQAN) (fig. 2)—Continued}

$07373420 \quad$ Mississippi River at St. Francisville, LA

\begin{tabular}{|c|c|c|c|c|c|c|c|c|c|c|c|c|c|c|c|c|c|}
\hline $12 / 01 / 99$ & $<0.05$ & 0.27 & $<0.20$ & -- & $<0.05$ & 0.22 & $<0.20$ & -- & $<0.05$ & -- & -- & $<0.05$ & -- & -- & $<0.05$ & $<0.20$ & 0.35 \\
\hline $01 / 27 / 00$ & $<.05$ & $<.20$ & $<.20$ & -- & $<.05$ & $<.20$ & $<.20$ & -- & $<.05$ & -- & -- & $<.05$ & -- & -- & $<.05$ & $<.20$ & $<.20$ \\
\hline $02 / 17 / 00$ & $<.05$ & $<.20$ & $<.20$ & -- & $<.05$ & $<.20$ & $<.20$ & -- & $<.05$ & -- & -- & $<.05$ & -- & -- & $<.05$ & $<.20$ & $<.20$ \\
\hline 03/14/00 & $<.05$ & $<.20$ & $<.20$ & -- & $<.05$ & $<.20$ & $<.20$ & -- & $<.05$ & -- & -- & $<.05$ & -- & -- & $<.05$ & $<.20$ & $<.20$ \\
\hline 03/29/00 & $<.05$ & $<.20$ & $<.20$ & -- & $<.05$ & $<.20$ & $<.20$ & -- & $<.05$ & -- & -- & $<.05$ & -- & -- & .06 & $<.20$ & $<.20$ \\
\hline $04 / 12 / 00$ & $<.05$ & $<.20$ & .31 & -- & $<.05$ & $<.20$ & $<.20$ & -- & $<.05$ & $<0.05$ & $<0.05$ & $<.05$ & & & .09 & $<.20$ & $<.20$ \\
\hline 05/10/00 & $<.05$ & $<.20$ & $<.20$ & -- & $<.05$ & $<.20$ & $<.20$ & -- & $<.05$ & $<.05$ & $<.05$ & $<.05$ & & & .16 & $<.20$ & $<.20$ \\
\hline 05/23/00 & $<.05$ & $<.20$ & $<.20$ & -- & $<.05$ & $<.20$ & $<.20$ & -- & $<.05$ & $<.05$ & $<.05$ & $<.05$ & -- & -- & .20 & $<.20$ & $<.20$ \\
\hline 06/06/00 & .08 & $<.20$ & $<.20$ & -- & $<.05$ & $<.20$ & $<.20$ & -- & $<.05$ & $<.05$ & $<.05$ & $<.05$ & -- & -- & .30 & .35 & $<.20$ \\
\hline 06/23/00 & .13 & .33 & .47 & -- & $<.05$ & .21 & $<.20$ & -- & .06 & $<.05$ & $<.05$ & $<.05$ & -- & -- & .35 & .53 & $<.20$ \\
\hline 07/18/00 & $<.05$ & .57 & .42 & -- & $<.05$ & .26 & $<.20$ & -- & $<.05$ & $<.05$ & $<.05$ & $<.05$ & $<0.05$ & $<0.05$ & .17 & .84 & .38 \\
\hline 08/25/00 & $<.05$ & $<.20$ & .34 & -- & $<.05$ & $<.20$ & $<.20$ & -- & $<.05$ & $<.05$ & $<.05$ & $<.05$ & $<.05$ & $<.05$ & $<.05$ & .31 & .21 \\
\hline 09/21/00 & $<.05$ & $<.20$ & $<.20$ & -- & $<.05$ & $<.20$ & $<.20$ & -- & $<.05$ & $<.05$ & $<.05$ & $<.05$ & $<.05$ & $<.05$ & $<.05$ & .24 & $<.20$ \\
\hline $11 / 28 / 00$ & $<.05$ & .08 & .05 & -- & .09 & .09 & $<.05$ & -- & $<.05$ & $<.05$ & $<.05$ & $<.05$ & $<.05$ & $<.05$ & .09 & .27 & .12 \\
\hline $12 / 12 / 00$ & $<.05$ & .08 & .06 & -- & $<.05$ & .10 & $<.05$ & -- & $<.05$ & $<.05$ & $<.05$ & $<.05$ & $<.05$ & $<.05$ & $<.05$ & .30 & .12 \\
\hline 01/24/01 & $<.05$ & $<.05$ & $<.05$ & -- & $<.05$ & .12 & $<.05$ & -- & $<.05$ & $<.05$ & $<.05$ & $<.05$ & $<.05$ & $<.05$ & $<.05$ & .20 & .11 \\
\hline 02/27/01 & $<.05$ & .07 & .05 & -- & $<.05$ & .06 & $<.05$ & -- & $<.05$ & $<.05$ & $<.05$ & $<.05$ & $<.05$ & $<.05$ & $<.05$ & .23 & .10 \\
\hline 03/14/01 & $<.05$ & .06 & .05 & -- & $<.05$ & .06 & $<.05$ & -- & $<.05$ & $<.05$ & $<.05$ & $<.05$ & $<.05$ & $<.05$ & .06 & .20 & .10 \\
\hline 04/02/01 & $<.05$ & .08 & .07 & -- & $<.05$ & .10 & $<.05$ & -- & $<.05$ & $<.05$ & $<.05$ & $<.05$ & $<.05$ & $<.05$ & .05 & .28 & .12 \\
\hline 04/16/01 & $<.05$ & .08 & $<.05$ & -- & $<.05$ & .11 & $<.05$ & -- & $<.05$ & $<.05$ & $<.05$ & $<.05$ & $<.05$ & $<.05$ & .26 & .39 & .16 \\
\hline 05/07/01 & $<.05$ & .14 & .09 & -- & $<.05$ & .15 & $<.05$ & -- & $<.05$ & $<.05$ & $<.05$ & $<.05$ & $<.05$ & $<.05$ & .13 & .52 & .19 \\
\hline 05/21/01 & .12 & .22 & .19 & -- & $<.05$ & .19 & $<.05$ & -- & $<.05$ & $<.05$ & $<.05$ & $<.05$ & $<.05$ & $<.05$ & .26 & .54 & .21 \\
\hline 05/23/01 & .07 & .06 & $<.05$ & -- & $<.05$ & $<.05$ & $<.05$ & -- & $<.05$ & $<.05$ & $<.05$ & $<.05$ & $<.05$ & $<.05$ & .24 & .24 & .10 \\
\hline 06/25/01 & .10 & .41 & .49 & -- & $<.05$ & .13 & .07 & -- & .05 & .05 & $<.05$ & $<.05$ & $<.05$ & $<.05$ & .33 & .57 & .35 \\
\hline 07/10/01 & .09 & .42 & .45 & -- & $<.05$ & .16 & .05 & -- & $<.05$ & .05 & $<.05$ & $<.05$ & $<.05$ & $<.05$ & .23 & .60 & .31 \\
\hline
\end{tabular}


Table 6. Analytical results for selected acetamide herbicides and their degradation products, ethanesulfonic acid, oxanilic acid, and sulfinyl acetic acid, in surface-water samples, 1993-2003.-Continued

[OGRL, U.S. Geological Survey Organic Geochemistry Research Laboratory, Lawrence, Kansas; ESA, ethanesulfonic acid; OXA, oxanilic acid; SAA, sulfinyl acetic acid; --, no data; <, less than]

\begin{tabular}{|c|c|c|c|c|c|c|c|c|c|c|c|c|c|c|c|c|c|c|c|}
\hline \multirow[b]{2}{*}{ Site number } & \multirow[b]{2}{*}{ Site location } & \multirow[b]{2}{*}{$\begin{array}{l}\text { Date of } \\
\text { collection } \\
\text { (month/ } \\
\text { day/year) }\end{array}$} & \multicolumn{17}{|c|}{ Concentrations, in micrograms per liter } \\
\hline & & & $\begin{array}{c} \\
\text { Aceto- } \\
\text { chlor } \\
(\mu \mathrm{g} / \mathrm{L})\end{array}$ & $\begin{array}{l}\text { Aceto- } \\
\text { chlor } \\
\text { ESA } \\
(\mu \mathrm{g} / \mathrm{L})\end{array}$ & $\begin{array}{l}\text { Aceto- } \\
\text { chlor } \\
\text { OXA } \\
(\mu \mathrm{g} / \mathrm{L})\end{array}$ & $\begin{array}{l}\text { Aceto- } \\
\text { chlor } \\
\text { SAA } \\
(\mu \mathrm{g} / \mathrm{L})\end{array}$ & $\begin{array}{c}\text { Ala- } \\
\text { chlor } \\
(\mu \mathrm{g} / \mathrm{L})\end{array}$ & $\begin{array}{l}\text { Ala- } \\
\text { chlor } \\
\text { ESA } \\
(\mu \mathrm{g} / \mathrm{L})\end{array}$ & $\begin{array}{c}\text { Ala- } \\
\text { chlor } \\
\text { OXA } \\
(\mu \mathrm{g} / \mathrm{L})\end{array}$ & $\begin{array}{l}\text { Ala- } \\
\text { chlor } \\
\text { SAA } \\
(\mu \mathrm{g} / \mathrm{L})\end{array}$ & $\begin{array}{c}\text { Di- } \\
\text { meth- } \\
\text { enamid } \\
(\mu \mathrm{g} / \mathrm{L})\end{array}$ & $\begin{array}{c}\text { Di- } \\
\text { meth- } \\
\text { enamid } \\
\text { ESA } \\
(\mu \mathrm{g} / \mathrm{L})\end{array}$ & $\begin{array}{c}\text { Di- } \\
\text { meth- } \\
\text { enamid } \\
\text { OXA } \\
(\mu \mathrm{g} / \mathrm{L})\end{array}$ & $\begin{array}{c}\text { Flufen- } \\
\text { acet } \\
(\mu \mathrm{g} / \mathrm{L})\end{array}$ & $\begin{array}{c}\text { Flufen- } \\
\text { acet } \\
\text { ESA } \\
(\mu \mathrm{g} / \mathrm{L})\end{array}$ & $\begin{array}{c}\text { Flufen- } \\
\text { acet } \\
0 X A \\
(\mu \mathrm{g} / \mathrm{L})\end{array}$ & $\begin{array}{l}\text { Meto- } \\
\text { lachlor } \\
(\mu \mathrm{g} / \mathrm{L})\end{array}$ & $\begin{array}{l}\text { Meto- } \\
\text { lachlor } \\
\text { ESA } \\
(\mu \mathrm{g} / \mathrm{L})\end{array}$ & $\begin{array}{c}\text { Meto- } \\
\text { lachlor } \\
\text { OXA } \\
(\mu \mathrm{g} / \mathrm{L})\end{array}$ \\
\hline \multicolumn{20}{|c|}{ National Stream Quality Accounting Network (NASQAN) (fig. 2)—Continued } \\
\hline \multirow[t]{9}{*}{07373420} & \multirow{9}{*}{$\begin{array}{l}\text { Mississippi River at St. } \\
\text { Francisville, LA }\end{array}$} & $07 / 18 / 01$ & $<0.05$ & 0.31 & 0.28 & -- & $<0.05$ & 0.15 & 0.05 & -- & $<0.05$ & $<0.05$ & $<0.05$ & $<0.05$ & $<0.05$ & $<0.05$ & 0.19 & 0.55 & 0.26 \\
\hline & & 08/27/01 & $<.05$ & .07 & .11 & -- & $<.05$ & .07 & .05 & -- & $<.05$ & $<.05$ & $<.05$ & $<.05$ & $<.05$ & $<.05$ & $<.05$ & .10 & .12 \\
\hline & & 09/19/01 & $<.05$ & .07 & .07 & -- & $<.05$ & .12 & $<.05$ & -- & $<.05$ & $<.05$ & $<.05$ & $<.05$ & $<.05$ & $<.05$ & $<.05$ & .18 & .10 \\
\hline & & $10 / 30 / 01$ & $<.05$ & .06 & .07 & -- & $<.05$ & .10 & $<.05$ & -- & $<.05$ & $<.05$ & $<.05$ & $<.05$ & $<.05$ & $<.05$ & $<.05$ & .24 & .13 \\
\hline & & $12 / 18 / 01$ & $<.05$ & .05 & $<.05$ & -- & $<.05$ & .07 & $<.05$ & -- & $<.05$ & $<.05$ & $<.05$ & $<.05$ & $<.05$ & $<.05$ & $<.05$ & .20 & .10 \\
\hline & & $01 / 22 / 02$ & $<.05$ & .07 & .05 & $<0.05$ & $<.05$ & .10 & $<.05$ & $<0.05$ & $<.05$ & $<.05$ & $<.05$ & $<.05$ & $<.05$ & $<.05$ & $<.05$ & .22 & .09 \\
\hline & & $04 / 22 / 02$ & $<.05$ & $<.05$ & $<.05$ & $<.05$ & $<.05$ & .08 & $<.05$ & $<.05$ & $<.05$ & $<.05$ & $<.05$ & $<.05$ & $<.05$ & $<.05$ & .05 & .25 & .07 \\
\hline & & 05/07/02 & .14 & .09 & .08 & $<.05$ & $<.05$ & .10 & $<.05$ & $<.05$ & .06 & $<.05$ & $<.05$ & $<.05$ & $<.05$ & $<.05$ & .36 & .29 & .12 \\
\hline & & $05 / 28 / 02$ & .26 & .17 & .21 & .14 & $<.05$ & .08 & $<.05$ & $<.05$ & .10 & $<.05$ & $<.05$ & $<.05$ & $<.05$ & $<.05$ & .43 & .27 & .14 \\
\hline \multirow[t]{9}{*}{07022000} & \multirow[t]{5}{*}{ Mississippi River at Thebes, IL } & $04 / 21 / 99$ & .57 & $<.20$ & $<.20$ & -- & .06 & .25 & $<.20$ & -- & .31 & -- & -- & $<.05$ & -- & -- & .73 & .53 & .23 \\
\hline & & $04 / 29 / 99$ & .32 & $<.20$ & $<.20$ & -- & $<.05$ & $<.20$ & $<.20$ & -- & $<.05$ & -- & -- & $<.05$ & -- & -- & .43 & .58 & .43 \\
\hline & & 07/26/99 & $<.05$ & .43 & .27 & -- & $<.05$ & .31 & $<.20$ & -- & $<.05$ & -- & -- & $<.05$ & -- & -- & .12 & .95 & .37 \\
\hline & & $06 / 12 / 00$ & .31 & .39 & .53 & -- & $<.05$ & .31 & $<.20$ & -- & .09 & $<.05$ & $<.05$ & $<.05$ & -- & -- & .48 & .73 & .28 \\
\hline & & $08 / 14 / 00$ & $<.05$ & $<.20$ & $<.20$ & -- & $<.05$ & $<.20$ & $<.20$ & -- & $<.05$ & $<.05$ & $<.05$ & $<.05$ & $<.05$ & $<.05$ & $<.05$ & $<.20$ & $<.20$ \\
\hline & & $04 / 29 / 02$ & .41 & .13 & .13 & $<.05$ & $<.05$ & .17 & $<.05$ & $<.05$ & .25 & $<.05$ & $<.05$ & $<.05$ & $<.05$ & $<.05$ & .68 & .40 & .13 \\
\hline & & 05/09/02 & .27 & .18 & .19 & $<.05$ & $<.05$ & .15 & $<.05$ & $<.05$ & .15 & $<.05$ & $<.05$ & $<.05$ & $<.05$ & $<.05$ & .42 & .41 & .16 \\
\hline & & $05 / 14 / 02$ & .53 & .24 & .31 & .18 & .06 & .12 & .05 & $<.05$ & .16 & $<.05$ & $<.05$ & $<.05$ & $<.05$ & $<.05$ & .82 & .33 & .17 \\
\hline & & $06 / 12 / 02$ & .44 & .45 & .50 & .18 & $<.05$ & .19 & $<.05$ & $<.05$ & .12 & .05 & $<.05$ & $<.05$ & $<.05$ & $<.05$ & .60 & .70 & .25 \\
\hline 05474500 & $\begin{array}{l}\text { Mississippi River at } \\
\text { Keokuk, IA }\end{array}$ & $07 / 22 / 99$ & $<.05$ & .26 & .34 & -- & $<.05$ & .36 & $<.20$ & -- & $<.05$ & -- & -- & $<.05$ & -- & -- & .05 & .95 & .20 \\
\hline \multirow[t]{7}{*}{05587455} & \multirow{7}{*}{$\begin{array}{l}\text { Mississippi River below } \\
\text { Grafton, IL }\end{array}$} & 03/17/99 & $<.05$ & $<.20$ & $<.20$ & -- & $<.05$ & .50 & $<.20$ & -- & -- & -- & -- & -- & -- & -- & .08 & .80 & .26 \\
\hline & & $04 / 12 / 99$ & .14 & $<.20$ & $<.20$ & -- & $<.05$ & .43 & $<.20$ & -- & -- & -- & -- & -- & -- & -- & .22 & .66 & .23 \\
\hline & & $04 / 20 / 99$ & .65 & .61 & .23 & -- & $<.05$ & $<.20$ & $<.20$ & -- & .46 & -- & -- & $<.05$ & -- & -- & .59 & 1.1 & .50 \\
\hline & & 05/10/99 & .24 & .35 & $<.20$ & -- & $<.05$ & .47 & $<.20$ & -- & .12 & -- & -- & $<.05$ & -- & -- & .40 & 1.2 & .40 \\
\hline & & $05 / 24 / 99$ & 1.2 & .75 & .64 & -- & .05 & .70 & $<.20$ & -- & .47 & -- & -- & -- & -- & -- & 1.3 & 1.4 & .52 \\
\hline & & 06/07/99 & .68 & .57 & .71 & -- & $<.05$ & .39 & $<.20$ & -- & .43 & -- & -- & -- & -- & -- & .59 & 1.3 & .37 \\
\hline & & 06/21/99 & 1.7 & 1.2 & 1.3 & -- & .10 & .50 & $<.20$ & -- & .53 & -- & -- & $<.05$ & -- & -- & 1.9 & 2.1 & .73 \\
\hline
\end{tabular}


Table 6. Analytical results for selected acetamide herbicides and their degradation products, ethanesulfonic acid, oxanilic acid, and sulfinyl acetic acid, in surface-water samples, 1993-2003.-Continued

[OGRL, U.S. Geological Survey Organic Geochemistry Research Laboratory, Lawrence, Kansas; ESA, ethanesulfonic acid; OXA, oxanilic acid; SAA, sulfinyl acetic acid; --, no data; <, less than]

\begin{tabular}{|c|c|c|c|c|c|c|c|c|c|c|c|c|c|c|c|c|c|c|c|}
\hline \multirow[b]{2}{*}{ Site number } & \multirow[b]{2}{*}{ Site location } & \multirow[b]{2}{*}{$\begin{array}{l}\text { Date of } \\
\text { collection } \\
\text { (month/ } \\
\text { day/year) }\end{array}$} & \multicolumn{17}{|c|}{ Concentrations, in micrograms per liter } \\
\hline & & & $\begin{array}{l}\text { Aceto- } \\
\text { chlor } \\
(\mu \mathrm{g} / \mathrm{L})\end{array}$ & $\begin{array}{c}\text { Aceto- } \\
\text { chlor } \\
\text { ESA } \\
(\mu \mathrm{g} / \mathrm{L})\end{array}$ & $\begin{array}{l}\text { Aceto- } \\
\text { chlor } \\
\text { OXA } \\
(\mu \mathrm{g} / \mathrm{L})\end{array}$ & $\begin{array}{l}\text { Aceto- } \\
\text { chlor } \\
\text { SAA } \\
(\mu \mathrm{g} / \mathrm{L})\end{array}$ & $\begin{array}{l}\text { Ala- } \\
\text { chlor } \\
(\mu \mathrm{g} / \mathrm{L})\end{array}$ & $\begin{array}{l}\text { Ala- } \\
\text { chlor } \\
\text { ESA } \\
(\mu \mathrm{g} / \mathrm{L})\end{array}$ & $\begin{array}{l}\text { Ala- } \\
\text { chlor } \\
\text { OXA } \\
(\mu \mathrm{g} / \mathrm{L})\end{array}$ & $\begin{array}{l}\text { Ala- } \\
\text { chlor } \\
\text { SAA } \\
(\mu \mathrm{g} / \mathrm{L})\end{array}$ & $\begin{array}{l}\text { Di- } \\
\text { meth- } \\
\text { enamid } \\
(\mu \mathrm{g} / \mathrm{L})\end{array}$ & $\begin{array}{c}\text { Di- } \\
\text { meth- } \\
\text { enamid } \\
\text { ESA } \\
(\mu \mathrm{g} / \mathrm{L})\end{array}$ & $\begin{array}{c}\text { Di- } \\
\text { meth- } \\
\text { enamid } \\
\text { OXA } \\
(\mu \mathrm{g} / \mathrm{L})\end{array}$ & $\begin{array}{l}\text { Flufen- } \\
\text { acet } \\
(\mu \mathrm{g} / \mathrm{L})\end{array}$ & $\begin{array}{c}\text { Flufen- } \\
\text { acet } \\
\text { ESA } \\
(\mu \mathrm{g} / \mathrm{L})\end{array}$ & $\begin{array}{c}\text { Flufen- } \\
\text { acet } \\
\text { OXA } \\
(\mu \mathrm{g} / \mathrm{L})\end{array}$ & $\begin{array}{l}\text { Meto- } \\
\text { lachlor } \\
(\mu \mathrm{g} / \mathrm{L})\end{array}$ & $\begin{array}{l}\text { Meto- } \\
\text { lachlor } \\
\text { ESA } \\
(\mu \mathrm{g} / \mathrm{L})\end{array}$ & $\begin{array}{c}\text { Meto- } \\
\text { lachlor } \\
\text { OXA } \\
(\mu \mathrm{g} / \mathrm{L})\end{array}$ \\
\hline
\end{tabular}

05587455 Mississippi River below Grafton, IL

\section{National Stream Quality Accounting Network (NASQAN) (fig. 2)—Continued}

\begin{tabular}{|c|c|c|c|c|c|c|c|c|c|c|c|c|c|c|c|c|c|}
\hline $07 / 24 / 99$ & 0.05 & 0.47 & 0.42 & -- & $<0.05$ & 0.39 & $<0.20$ & -- & $<0.05$ & -- & -- & $<0.05$ & -- & -- & 0.11 & 0.28 & 0.35 \\
\hline 08/09/99 & $<.05$ & .44 & .30 & -- & $<.05$ & .34 & $<.20$ & -- & $<.05$ & -- & -- & $<.05$ & -- & -- & .06 & 1.1 & .28 \\
\hline 09/13/99 & $<.05$ & $<.20$ & $<.20$ & -- & $<.05$ & .28 & $<.20$ & -- & $<.05$ & -- & -- & $<.05$ & -- & -- & $<.05$ & .59 & $<.20$ \\
\hline $11 / 22 / 99$ & $<.05$ & $<.20$ & $<.20$ & -- & $<.05$ & .33 & $<.20$ & -- & $<.05$ & -- & -- & $<.05$ & -- & -- & $<.05$ & .40 & $<.20$ \\
\hline $12 / 07 / 99$ & $<.05$ & $<.20$ & $<.20$ & -- & $<.05$ & .37 & $<.20$ & -- & $<.05$ & -- & -- & $<.05$ & -- & -- & $<.05$ & .38 & .21 \\
\hline $01 / 19 / 00$ & $<.05$ & $<.20$ & $<.20$ & -- & $<.05$ & .44 & $<.20$ & -- & $<.05$ & -- & -- & $<.05$ & -- & -- & $<.05$ & .46 & .25 \\
\hline $02 / 15 / 00$ & .05 & $<.20$ & $<.20$ & -- & $<.05$ & .51 & $<.20$ & -- & $<.05$ & -- & -- & $<.05$ & -- & -- & $<.05$ & .54 & $<.20$ \\
\hline 03/13/00 & $<.05$ & $<.20$ & $<.20$ & -- & $<.05$ & .23 & $<.20$ & -- & $<.05$ & -- & -- & $<.05$ & -- & -- & .05 & .69 & $<.20$ \\
\hline $05 / 04 / 00$ & .17 & .54 & .28 & -- & $<.05$ & .66 & $<.20$ & -- & .08 & -- & -- & $<.05$ & -- & -- & .43 & 2.5 & .64 \\
\hline 06/09/00 & .46 & .57 & .88 & -- & $<.05$ & .38 & $<.20$ & -- & .15 & -- & -- & $<.05$ & -- & -- & .63 & .96 & $<.20$ \\
\hline $06 / 26 / 00$ & .12 & .90 & 1.1 & -- & $<.05$ & .46 & $<.20$ & -- & $<.05$ & -- & -- & $<.05$ & -- & -- & .28 & 1.3 & .57 \\
\hline 07/10/00 & $<.05$ & .68 & .49 & -- & $<.05$ & .41 & $<.20$ & -- & $<.05$ & -- & -- & $<.05$ & -- & -- & .11 & .94 & .36 \\
\hline $08 / 11 / 00$ & $<.05$ & .32 & $<.20$ & -- & $<.05$ & .36 & $<.20$ & -- & $<.05$ & -- & -- & $<.05$ & -- & -- & $<.05$ & .97 & .28 \\
\hline $09 / 11 / 00$ & $<.05$ & $<.20$ & $<.20$ & -- & $<.05$ & .39 & $<.20$ & -- & $<.05$ & -- & -- & $<.05$ & -- & -- & $<.05$ & .77 & $<.20$ \\
\hline $10 / 02 / 00$ & $<.05$ & $<.05$ & .09 & -- & $<.05$ & .38 & .07 & -- & $<.05$ & $<0.05$ & $<0.05$ & $<.05$ & $<0.05$ & $<0.05$ & $<.05$ & .54 & .12 \\
\hline $10 / 19 / 00$ & $<.05$ & $<.20$ & $<.20$ & -- & $<.05$ & .33 & $<.20$ & -- & $<.05$ & $<.05$ & $<.05$ & $<.05$ & $<.05$ & $<.05$ & $<.05$ & .60 & $<.20$ \\
\hline $11 / 07 / 00$ & $<.05$ & $<.05$ & .06 & -- & $<.05$ & .41 & .06 & -- & $<.05$ & $<.05$ & $<.05$ & $<.05$ & $<.05$ & $<.05$ & $<.05$ & .64 & .15 \\
\hline 02/06/01 & $<.05$ & .13 & .09 & -- & $<.05$ & .26 & .07 & -- & $<.05$ & $<.05$ & $<.05$ & $<.05$ & $<.05$ & $<.05$ & .10 & .53 & .21 \\
\hline $02 / 21 / 01$ & $<.05$ & .29 & .19 & -- & $<.05$ & .34 & .08 & -- & $<.05$ & $<.05$ & $<.05$ & $<.05$ & $<.05$ & $<.05$ & .10 & .94 & .36 \\
\hline 03/01/01 & $<.05$ & .19 & .13 & -- & $<.05$ & .29 & $<.05$ & -- & $<.05$ & $<.05$ & $<.05$ & $<.05$ & $<.05$ & $<.05$ & .11 & .68 & .22 \\
\hline 03/07/01 & $<.05$ & .29 & .18 & -- & $<.05$ & .31 & .09 & -- & $<.05$ & $<.05$ & $<.05$ & $<.05$ & $<.05$ & $<.05$ & .25 & .90 & .36 \\
\hline $03 / 21 / 01$ & .08 & .20 & .13 & -- & $<.05$ & .29 & .07 & -- & $<.05$ & $<.05$ & $<.05$ & $<.05$ & $<.05$ & $<.05$ & .12 & .79 & .27 \\
\hline $04 / 02 / 01$ & $<.05$ & .21 & .13 & -- & $<.05$ & .31 & .07 & -- & $<.05$ & $<.05$ & $<.05$ & $<.05$ & $<.05$ & $<.05$ & .87 & 1.0 & .32 \\
\hline $04 / 16 / 01$ & .11 & .22 & .11 & -- & $<.05$ & .35 & $<.05$ & -- & .13 & $<.05$ & $<.05$ & $<.05$ & $<.05$ & $<.05$ & .32 & .93 & .25 \\
\hline 04/30/01 & $<.05$ & .27 & .16 & -- & $<.05$ & .26 & $<.05$ & -- & $<.05$ & $<.05$ & $<.05$ & $<.05$ & $<.05$ & $<.05$ & .22 & .77 & .25 \\
\hline
\end{tabular}


Table 6. Analytical results for selected acetamide herbicides and their degradation products, ethanesulfonic acid, oxanilic acid, and sulfinyl acetic acid, in surface-water samples, 1993-2003.-Continued

[OGRL, U.S. Geological Survey Organic Geochemistry Research Laboratory, Lawrence, Kansas; ESA, ethanesulfonic acid; OXA, oxanilic acid; SAA, sulfinyl acetic acid; --, no data; <, less than]

\begin{tabular}{|c|c|c|c|c|c|c|c|c|c|c|c|c|c|c|c|c|c|c|c|}
\hline \multirow[b]{2}{*}{ Site number } & \multirow[b]{2}{*}{ Site location } & \multirow[b]{2}{*}{$\begin{array}{c}\text { Date of } \\
\text { collection } \\
\text { (month/ } \\
\text { day/year) }\end{array}$} & \multicolumn{17}{|c|}{ Concentrations, in micrograms per liter } \\
\hline & & & $\begin{array}{l}\text { Aceto- } \\
\text { chlor } \\
(\mu \mathrm{g} / \mathrm{L})\end{array}$ & $\begin{array}{l}\text { Aceto- } \\
\text { chlor } \\
\text { ESA } \\
(\mu \mathrm{g} / \mathrm{L})\end{array}$ & $\begin{array}{l}\text { Aceto- } \\
\text { chlor } \\
\text { OXA } \\
(\mu \mathrm{g} / \mathrm{L})\end{array}$ & $\begin{array}{c}\text { Aceto- } \\
\text { chlor } \\
\text { SAA } \\
(\mu \mathrm{g} / \mathrm{L})\end{array}$ & $\begin{array}{l}\text { Ala- } \\
\text { chlor } \\
(\mu \mathrm{g} / \mathrm{L})\end{array}$ & $\begin{array}{l}\text { Ala- } \\
\text { chlor } \\
\text { ESA } \\
(\mu \mathrm{g} / \mathrm{L})\end{array}$ & $\begin{array}{c}\text { Ala- } \\
\text { chlor } \\
\text { OXA } \\
(\mu \mathrm{g} / \mathrm{L})\end{array}$ & $\begin{array}{l}\text { Ala- } \\
\text { chlor } \\
\text { SAA } \\
(\mu \mathrm{g} / \mathrm{L})\end{array}$ & $\begin{array}{l}\text { Di- } \\
\text { meth- } \\
\text { enamid } \\
(\mu \mathrm{g} / \mathrm{L})\end{array}$ & $\begin{array}{c}\text { Di- } \\
\text { meth- } \\
\text { enamid } \\
\text { ESA } \\
(\mu \mathrm{g} / \mathrm{L})\end{array}$ & $\begin{array}{c}\text { Di- } \\
\text { meth- } \\
\text { enamid } \\
\text { OXA } \\
(\mu \mathrm{g} / \mathrm{L})\end{array}$ & $\begin{array}{c}\text { Flufen- } \\
\text { acet } \\
(\mu \mathrm{g} / \mathrm{L})\end{array}$ & $\begin{array}{c}\text { Flufen- } \\
\text { acet } \\
\text { ESA } \\
(\mu \mathrm{g} / \mathrm{L})\end{array}$ & $\begin{array}{c}\text { Flufen- } \\
\text { acet } \\
\text { OXA } \\
(\mu \mathrm{g} / \mathrm{L})\end{array}$ & $\begin{array}{l}\text { Meto- } \\
\text { lachlor } \\
(\mu \mathrm{g} / \mathrm{L})\end{array}$ & $\begin{array}{l}\text { Meto- } \\
\text { lachlor } \\
\text { ESA } \\
(\mu \mathrm{g} / \mathrm{L})\end{array}$ & $\begin{array}{c}\text { Meto- } \\
\text { lachlor } \\
\text { OXA } \\
(\mu \mathrm{g} / \mathrm{L})\end{array}$ \\
\hline \multicolumn{20}{|c|}{ National Stream Quality Accounting Network (NASQAN) (fig. 2)—Continued } \\
\hline \multirow[t]{6}{*}{05587455} & Mississippi River below & 05/14/01 & 0.23 & 0.30 & 0.25 & -- & $<0.05$ & 0.30 & $<0.05$ & -- & 0.06 & $<0.05$ & $<0.05$ & $<0.05$ & $<0.05$ & $<0.05$ & 0.29 & 0.84 & 0.26 \\
\hline & Grafton, IL & 06/06/01 & .42 & .77 & 1.1 & -- & $<.05$ & .33 & .10 & -- & .17 & .14 & .13 & $<.05$ & $<.05$ & $<.05$ & .88 & 1.2 & .80 \\
\hline & & $06 / 11 / 01$ & .30 & .66 & .82 & -- & $<.05$ & .24 & $<.05$ & -- & .13 & .12 & .11 & $<.05$ & $<.05$ & $<.05$ & .63 & 1.1 & .58 \\
\hline & & $07 / 16 / 01$ & .08 & .54 & .50 & -- & $<.05$ & .31 & $<.05$ & -- & $<.05$ & .05 & .05 & $<.05$ & $<.05$ & $<.05$ & .16 & .88 & .30 \\
\hline & & 08/06/01 & .05 & .45 & .43 & -- & $<.05$ & .38 & .07 & -- & $<.05$ & $<.05$ & $<.05$ & $<.05$ & $<.05$ & $<.05$ & .11 & .99 & .31 \\
\hline & & 09/12/01 & $<.05$ & .09 & .14 & -- & $<.05$ & .12 & .05 & -- & $<.05$ & $<.05$ & $<.05$ & $<.05$ & $<.05$ & $<.05$ & $<.05$ & .16 & .16 \\
\hline \multirow[t]{2}{*}{06610000} & Missouri River at Omaha, NE & $06 / 02 / 00$ & $<.05$ & $<.20$ & $<.20$ & -- & $<.05$ & $<.20$ & $<.20$ & -- & $<.05$ & -- & -- & $<.05$ & -- & -- & $<.05$ & $<.20$ & $<.20$ \\
\hline & & 08/04/00 & $<.05$ & $<.20$ & $<.20$ & -- & $<.05$ & $<.20$ & $<.20$ & -- & $<.05$ & -- & -- & $<.05$ & -- & -- & $<.05$ & $<.20$ & $<.20$ \\
\hline \multirow[t]{17}{*}{06934500} & Missouri River near & 03/18/99 & $<.05$ & .20 & $<.20$ & -- & $<.05$ & $<.20$ & $<.20$ & -- & -- & -- & -- & -- & -- & -- & .05 & $<.20$ & $<.20$ \\
\hline & Hermann, MO & 04/13/99 & .09 & $<.20$ & $<.20$ & -- & $<.05$ & $<.20$ & $<.20$ & -- & -- & -- & -- & -- & -- & -- & .20 & $<.20$ & $<.20$ \\
\hline & & 04/19/99 & .64 & $<.20$ & $<.20$ & -- & .09 & $<.20$ & $<.20$ & -- & -- & -- & -- & -- & -- & -- & .93 & .31 & $<.20$ \\
\hline & & $04 / 28 / 99$ & .37 & $<.20$ & $<.20$ & -- & $<.05$ & $<.20$ & $<.20$ & -- & $<.05$ & -- & -- & -- & -- & -- & .66 & .22 & $<.20$ \\
\hline & & $05 / 11 / 99$ & .39 & $<.20$ & $<.20$ & -- & $<.05$ & $<.20$ & $<.20$ & -- & .08 & -- & -- & -- & -- & -- & .62 & .24 & $<.20$ \\
\hline & & 05/19/99 & .44 & $<.20$ & $<.20$ & -- & .07 & $<.20$ & $<.20$ & -- & .12 & -- & -- & -- & -- & -- & .85 & .28 & $<.20$ \\
\hline & & 06/08/99 & .97 & .30 & .55 & -- & .18 & $<.20$ & $<.20$ & -- & .32 & -- & -- & -- & -- & -- & 2.0 & .31 & .26 \\
\hline & & $06 / 22 / 99$ & .13 & .30 & .45 & -- & .16 & $<.20$ & .26 & -- & .18 & -- & -- & $<.05$ & -- & -- & 1.0 & .45 & .29 \\
\hline & & 07/23/99 & $<.05$ & $<.20$ & $<.20$ & -- & $<.05$ & $<.20$ & $<.20$ & -- & $<.05$ & -- & -- & $<.05$ & -- & -- & .18 & .28 & $<.20$ \\
\hline & & 08/10/99 & $<.05$ & $<.20$ & $<.20$ & & .08 & $<.20$ & $<.20$ & & $<.05$ & -- & -- & $<.05$ & -- & -- & .34 & .24 & .22 \\
\hline & & 09/14/99 & $<.05$ & $<.20$ & $<.20$ & -- & $<.05$ & $<.20$ & $<.20$ & -- & $<.05$ & -- & -- & $<.05$ & -- & -- & $<.05$ & $<.20$ & $<.20$ \\
\hline & & $10 / 18 / 99$ & $<.05$ & $<.20$ & $<.20$ & -- & $<.05$ & $<.20$ & $<.20$ & -- & $<.05$ & -- & -- & $<.05$ & -- & -- & $<.05$ & $<.20$ & $<.20$ \\
\hline & & $12 / 08 / 99$ & $<.05$ & $<.20$ & $<.20$ & -- & $<.05$ & $<.20$ & $<.20$ & -- & $<.05$ & -- & -- & $<.05$ & -- & -- & $<.05$ & $<.20$ & $<.20$ \\
\hline & & $02 / 15 / 00$ & $<.05$ & $<.20$ & $<.20$ & -- & $<.05$ & $<.20$ & $<.20$ & -- & $<.05$ & -- & -- & $<.05$ & -- & -- & $<.05$ & $<.20$ & $<.20$ \\
\hline & & 03/08/00 & $<.05$ & $<.20$ & $<.20$ & -- & $<.05$ & $<.20$ & $<.20$ & -- & $<.05$ & -- & -- & $<.05$ & -- & -- & $<.05$ & $<.20$ & $<.20$ \\
\hline & & $04 / 04 / 00$ & $<.05$ & $<.20$ & $<.20$ & -- & $<.05$ & $<.20$ & $<.20$ & -- & $<.05$ & -- & -- & $<.05$ & -- & -- & $<.05$ & $<.20$ & $<.20$ \\
\hline & & 05/05/00 & $<.05$ & $<.20$ & $<.20$ & -- & $<.05$ & $<.20$ & $<.20$ & -- & $<.05$ & -- & -- & $<.05$ & -- & -- & .06 & $<.20$ & $<.20$ \\
\hline
\end{tabular}


Table 6. Analytical results for selected acetamide herbicides and their degradation products, ethanesulfonic acid, oxanilic acid, and sulfinyl acetic acid, in surface-water samples, 1993-2003.-Continued

[OGRL, U.S. Geological Survey Organic Geochemistry Research Laboratory, Lawrence, Kansas; ESA, ethanesulfonic acid; OXA, oxanilic acid; SAA, sulfinyl acetic acid; --, no data; <, less than]

\begin{tabular}{|c|c|c|c|c|c|c|c|c|c|c|c|c|c|c|c|c|c|c|c|}
\hline \multirow[b]{2}{*}{ Site number } & \multirow[b]{2}{*}{ Site location } & \multirow[b]{2}{*}{$\begin{array}{c}\text { Date of } \\
\text { collection } \\
\text { (month/ } \\
\text { day/year) }\end{array}$} & \multicolumn{17}{|c|}{ Concentrations, in micrograms per liter } \\
\hline & & & $\begin{array}{l}\text { Aceto- } \\
\text { chlor } \\
(\mu \mathrm{g} / \mathrm{L})\end{array}$ & $\begin{array}{l}\text { Aceto- } \\
\text { chlor } \\
\text { ESA } \\
(\mu \mathrm{g} / \mathrm{L})\end{array}$ & $\begin{array}{c}\text { Aceto- } \\
\text { chlor } \\
\text { OXA } \\
(\mu \mathrm{g} / \mathrm{L})\end{array}$ & $\begin{array}{c}\text { Aceto- } \\
\text { chlor } \\
\text { SAA } \\
(\mu \mathrm{g} / \mathrm{L})\end{array}$ & $\begin{array}{l}\text { Ala- } \\
\text { chlor } \\
(\mu \mathrm{g} / \mathrm{L})\end{array}$ & $\begin{array}{c}\text { Ala- } \\
\text { chlor } \\
\text { ESA } \\
(\mu \mathrm{g} / \mathrm{L})\end{array}$ & $\begin{array}{c}\text { Ala- } \\
\text { chlor } \\
\text { OXA } \\
(\mu \mathrm{g} / \mathrm{L})\end{array}$ & $\begin{array}{l}\text { Ala- } \\
\text { chlor } \\
\text { SAA } \\
(\mu \mathrm{g} / \mathrm{L})\end{array}$ & $\begin{array}{c}\text { Di- } \\
\text { meth- } \\
\text { enamid } \\
(\mu \mathrm{g} / \mathrm{L})\end{array}$ & $\begin{array}{c}\text { Di- } \\
\text { meth- } \\
\text { enamid } \\
\text { ESA } \\
(\mu \mathrm{g} / \mathrm{L})\end{array}$ & $\begin{array}{c}\text { Di- } \\
\text { meth- } \\
\text { enamid } \\
\text { OXA } \\
(\mu \mathrm{g} / \mathrm{L})\end{array}$ & $\begin{array}{c}\text { Flufen- } \\
\text { acet } \\
(\mu \mathrm{g} / \mathrm{L})\end{array}$ & $\begin{array}{c}\text { Flufen- } \\
\text { acet } \\
\text { ESA } \\
(\mu \mathrm{g} / \mathrm{L})\end{array}$ & $\begin{array}{c}\text { Flufen- } \\
\text { acet } \\
\text { OXA } \\
(\mu \mathrm{g} / \mathrm{L})\end{array}$ & $\begin{array}{c}\text { Meto- } \\
\text { lachlor } \\
(\mu \mathrm{g} / \mathrm{L})\end{array}$ & $\begin{array}{c}\text { Meto- } \\
\text { lachlor } \\
\text { ESA } \\
(\mu \mathrm{g} / \mathrm{L})\end{array}$ & $\begin{array}{c}\text { Meto- } \\
\text { lachlor } \\
\text { OXA } \\
(\mu \mathrm{g} / \mathrm{L})\end{array}$ \\
\hline
\end{tabular}

National Stream Quality Accounting Network (NASQAN) (fig. 2)—Continued

06934500 Missouri River near Hermann, MO

\begin{tabular}{|c|c|c|c|c|c|c|c|c|c|c|c|c|c|c|c|c|c|}
\hline 05/30/00 & 0.24 & $<0.20$ & $<0.20$ & -- & 0.11 & $<0.20$ & $<0.20$ & -- & 0.17 & -- & -- & $<0.05$ & -- & -- & 0.75 & $<0.20$ & $<0.20$ \\
\hline 06/08/00 & $<.05$ & .09 & .11 & -- & $<.05$ & .06 & .06 & -- & $<.05$ & $<0.05$ & $<0.05$ & $<.05$ & & & .10 & .11 & .11 \\
\hline $06 / 23 / 00$ & $<.05$ & $<.20$ & $<.20$ & -- & $<.05$ & $<.20$ & $<.20$ & -- & $<.05$ & -- & -- & $<.05$ & -- & -- & .18 & .23 & .21 \\
\hline 06/28/00 & .16 & 1.5 & 2.0 & -- & $<.05$ & .74 & $<.20$ & -- & $<.05$ & -- & -- & $<.05$ & -- & -- & .52 & 1.7 & 1.2 \\
\hline 07/11/00 & $<.05$ & $<.20$ & $<.20$ & -- & $<.05$ & $<.20$ & $<.20$ & -- & $<.05$ & -- & -- & $<.05$ & -- & -- & .16 & .20 & $<.20$ \\
\hline 08/10/00 & $<.05$ & $<.20$ & $<.20$ & -- & $<.05$ & $<.20$ & $<.20$ & -- & $<.05$ & -- & -- & $<.05$ & -- & -- & $<.05$ & $<.20$ & $<.20$ \\
\hline 08/13/00 & $<.05$ & .29 & .32 & -- & $<.05$ & $<.20$ & $<.20$ & -- & $<.05$ & -- & -- & $<.05$ & -- & -- & $<.05$ & .48 & .28 \\
\hline 08/25/00 & $<.05$ & $<.20$ & $<.20$ & -- & $<.05$ & $<.20$ & $<.20$ & -- & $<.05$ & -- & -- & $<.05$ & -- & -- & .10 & $<.20$ & $<.20$ \\
\hline 09/12/00 & $<.05$ & $<.20$ & $<.20$ & -- & $<.05$ & $<.20$ & $<.20$ & -- & $<.05$ & -- & -- & $<.05$ & -- & -- & $<.05$ & $<.20$ & $<.20$ \\
\hline $10 / 03 / 00$ & $<.05$ & .06 & .06 & -- & $<.05$ & $<.05$ & .07 & -- & $<.05$ & $<.05$ & $<.05$ & $<.05$ & $<0.05$ & $<0.05$ & $<.05$ & .11 & .09 \\
\hline $11 / 29 / 00$ & $<.05$ & $<.05$ & $<.05$ & -- & $<.05$ & $<.05$ & $<.05$ & -- & $<.05$ & $<.05$ & $<.05$ & $<.05$ & $<.05$ & $<.05$ & $<.05$ & $<.05$ & $<.05$ \\
\hline 01/22/01 & $<.05$ & $<.05$ & $<.05$ & -- & $<.05$ & .05 & $<.05$ & -- & $<.05$ & $<.05$ & $<.05$ & $<.05$ & $<.05$ & $<.05$ & $<.05$ & .11 & .10 \\
\hline 02/05/01 & $<.05$ & $<.05$ & $<.05$ & -- & $<.05$ & .06 & $<.05$ & -- & $<.05$ & $<.05$ & $<.05$ & $<.05$ & $<.05$ & $<.05$ & $<.05$ & .12 & .09 \\
\hline 02/28/01 & $<.05$ & $<.05$ & $<.05$ & -- & $<.05$ & .05 & $<.05$ & -- & $<.05$ & $<.05$ & $<.05$ & $<.05$ & $<.05$ & $<.05$ & $<.05$ & .09 & .09 \\
\hline 03/08/01 & $<.05$ & .05 & .08 & -- & $<.05$ & .09 & .08 & -- & $<.05$ & $<.05$ & $<.05$ & $<.05$ & $<.05$ & $<.05$ & .07 & .16 & .15 \\
\hline 04/03/01 & $<.05$ & .10 & .13 & -- & $<.05$ & .07 & .07 & -- & $<.05$ & $<.05$ & $<.05$ & $<.05$ & $<.05$ & $<.05$ & .13 & .22 & .19 \\
\hline 05/01/01 & .16 & .12 & .11 & -- & $<.05$ & .06 & $<.05$ & -- & $<.05$ & $<.05$ & $<.05$ & $<.05$ & $<.05$ & $<.05$ & .21 & .25 & .11 \\
\hline 05/10/01 & 1.6 & .33 & .60 & -- & .08 & .05 & $<.05$ & -- & .20 & $<.05$ & $<.05$ & .12 & $<.05$ & $<.05$ & 1.6 & .38 & .29 \\
\hline 06/07/01 & .28 & .31 & .56 & -- & .13 & .12 & .07 & -- & .08 & .06 & .05 & .05 & $<.05$ & $<.05$ & .65 & .30 & .31 \\
\hline 06/12/01 & .19 & .36 & .55 & -- & .08 & .11 & .10 & -- & .06 & .06 & .05 & $<.05$ & $<.05$ & $<.05$ & .58 & .32 & .35 \\
\hline $06 / 25 / 01$ & .13 & .46 & .60 & -- & .06 & .16 & .12 & -- & .06 & $<.05$ & $<.05$ & $<.05$ & $<.05$ & $<.05$ & .44 & .39 & .36 \\
\hline 07/17/01 & $<.05$ & .14 & .17 & -- & $<.05$ & .05 & $<.05$ & -- & $<.05$ & $<.05$ & $<.05$ & $<.05$ & $<.05$ & $<.05$ & .12 & .19 & .13 \\
\hline 08/07/01 & $<.05$ & .19 & .19 & -- & $<.05$ & .10 & .07 & -- & $<.05$ & $<.05$ & $<.05$ & $<.05$ & $<.05$ & $<.05$ & .10 & .28 & .27 \\
\hline 09/13/01 & $<.05$ & .07 & .06 & -- & $<.05$ & .07 & .05 & -- & $<.05$ & $<.05$ & $<.05$ & $<.05$ & $<.05$ & $<.05$ & .06 & .08 & .16 \\
\hline
\end{tabular}


Table 6. Analytical results for selected acetamide herbicides and their degradation products, ethanesulfonic acid, oxanilic acid, and sulfinyl acetic acid, in surface-water samples, 1993-2003.-Continued

[OGRL, U.S. Geological Survey Organic Geochemistry Research Laboratory, Lawrence, Kansas; ESA, ethanesulfonic acid; OXA, oxanilic acid; SAA, sulfinyl acetic acid; --, no data; <, less than]

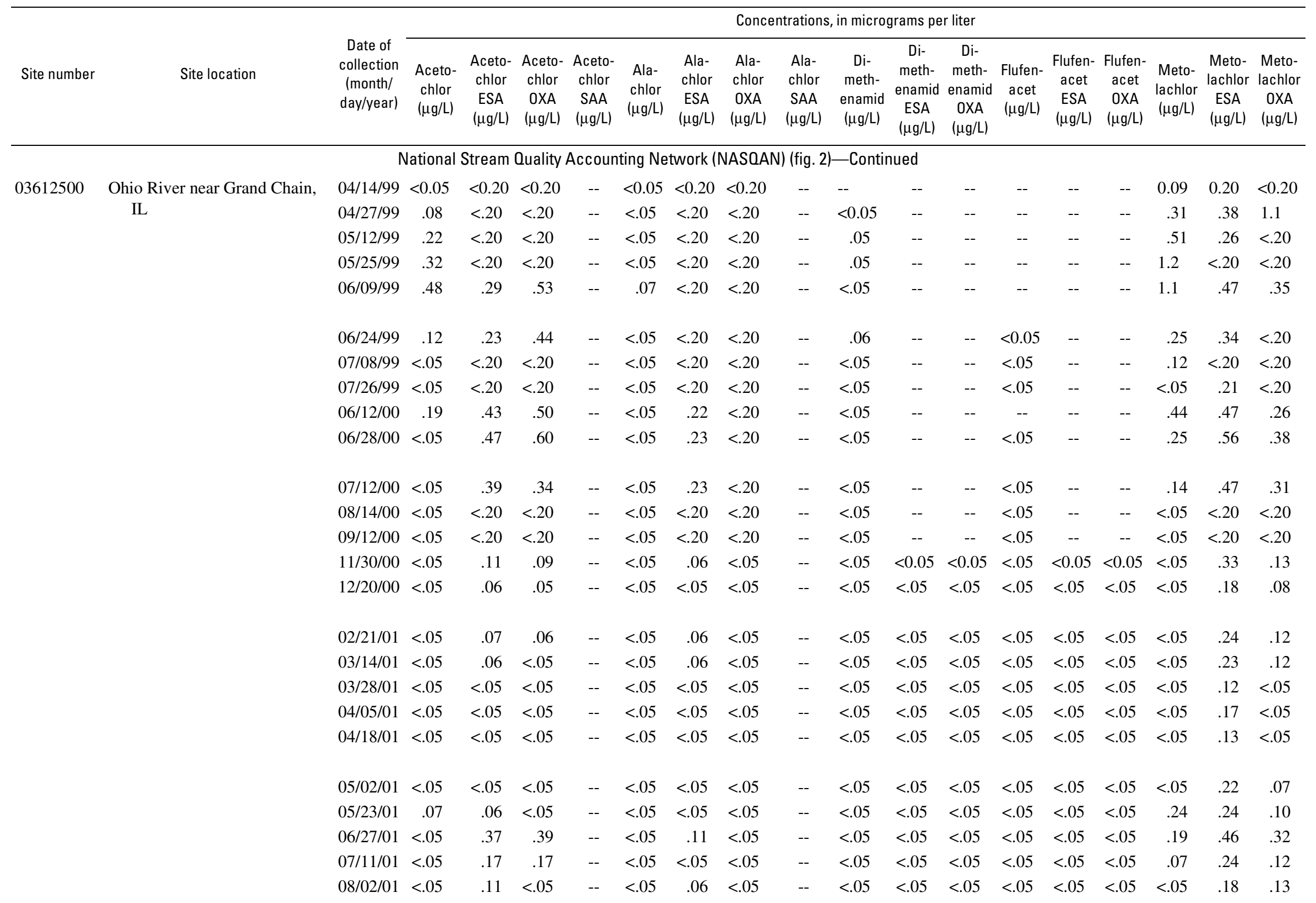


Table 6. Analytical results for selected acetamide herbicides and their degradation products, ethanesulfonic acid, oxanilic acid, and sulfinyl acetic acid, in surface-water samples, 1993-2003.-Continued

[OGRL, U.S. Geological Survey Organic Geochemistry Research Laboratory, Lawrence, Kansas; ESA, ethanesulfonic acid; OXA, oxanilic acid; SAA, sulfinyl acetic acid; --, no data; <, less than]

\begin{tabular}{|c|c|c|c|c|c|c|c|c|c|c|c|c|c|c|c|c|c|c|c|}
\hline \multirow[b]{2}{*}{ Site number } & \multirow[b]{2}{*}{ Site location } & \multirow[b]{2}{*}{$\begin{array}{l}\text { Date of } \\
\text { collection } \\
\text { (month/ } \\
\text { day/year) }\end{array}$} & \multicolumn{17}{|c|}{ Concentrations, in micrograms per liter } \\
\hline & & & $\begin{array}{l}\text { Aceto- } \\
\text { chlor } \\
(\mu \mathrm{g} / \mathrm{L})\end{array}$ & $\begin{array}{l}\text { Aceto- } \\
\text { chlor } \\
\text { ESA } \\
(\mu \mathrm{g} / \mathrm{L})\end{array}$ & $\begin{array}{c}\text { Aceto- } \\
\text { chlor } \\
\text { OXA } \\
(\mu \mathrm{g} / \mathrm{L})\end{array}$ & $\begin{array}{c}\text { Aceto- } \\
\text { chlor } \\
\text { SAA } \\
(\mu \mathrm{g} / \mathrm{L})\end{array}$ & $\begin{array}{l}\text { Ala- } \\
\text { chlor } \\
(\mu \mathrm{g} / \mathrm{L})\end{array}$ & $\begin{array}{c}\text { Ala- } \\
\text { chlor } \\
\text { ESA } \\
(\mu \mathrm{g} / \mathrm{L})\end{array}$ & $\begin{array}{c}\text { Ala- } \\
\text { chlor } \\
\text { OXA } \\
(\mu \mathrm{g} / \mathrm{L})\end{array}$ & $\begin{array}{c}\text { Ala- } \\
\text { chlor } \\
\text { SAA } \\
(\mu \mathrm{g} / \mathrm{L})\end{array}$ & $\begin{array}{c}\text { Di- } \\
\text { meth- } \\
\text { enamid } \\
(\mu \mathrm{g} / \mathrm{L})\end{array}$ & $\begin{array}{c}\text { Di- } \\
\text { meth- } \\
\text { enamid } \\
\text { ESA } \\
(\mu \mathrm{g} / \mathrm{L})\end{array}$ & $\begin{array}{c}\mathrm{Di}- \\
\text { meth- } \\
\text { enamid } \\
\text { OXA } \\
(\mu \mathrm{g} / \mathrm{L})\end{array}$ & $\begin{array}{l}\text { Flufen- } \\
\text { acet } \\
(\mu \mathrm{g} / \mathrm{L})\end{array}$ & $\begin{array}{c}\text { Flufen- } \\
\text { acet } \\
\text { ESA } \\
(\mu \mathrm{g} / \mathrm{L})\end{array}$ & $\begin{array}{c}\text { Flufen- } \\
\text { acet } \\
\text { OXA } \\
(\mu \mathrm{g} / \mathrm{L})\end{array}$ & $\begin{array}{l}\text { Meto- } \\
\text { lachlor } \\
(\mu \mathrm{g} / \mathrm{L})\end{array}$ & $\begin{array}{c}\text { Meto- } \\
\text { lachlor } \\
\text { ESA } \\
(\mu \mathrm{g} / \mathrm{L})\end{array}$ & $\begin{array}{c}\text { Meto- } \\
\text { lachlor } \\
\text { OXA } \\
(\mu \mathrm{g} / \mathrm{L})\end{array}$ \\
\hline \multicolumn{20}{|c|}{ National Stream Quality Accounting Network (NASOAN) (fig. 2)—Continued } \\
\hline \multirow[t]{5}{*}{03612500} & \multirow{5}{*}{$\begin{array}{l}\text { Ohio River near Grand Chain, } \\
\text { IL }\end{array}$} & $08 / 22 / 01$ & $<0.05$ & $<0.05$ & $<0.05$ & -- & $<0.05$ & $<0.05$ & $<0.05$ & -- & $<0.05$ & $<0.05$ & $<0.05$ & $<0.05$ & $<0.05$ & $<0.05$ & $<0.05$ & 0.06 & 0.08 \\
\hline & & $04 / 25 / 02$ & $<.05$ & $<.05$ & $<.05$ & $<0.05$ & $<.05$ & $<.05$ & $<.05$ & $<0.05$ & $<.05$ & $<.05$ & $<.05$ & $<.05$ & $<.05$ & $<.05$ & .07 & .32 & .09 \\
\hline & & 05/07/02 & .10 & .07 & .05 & $<.05$ & $<.05$ & $<.05$ & $<.05$ & $<.05$ & $<.05$ & $<.05$ & $<.05$ & $<.05$ & $<.05$ & $<.05$ & .31 & .22 & .10 \\
\hline & & $05 / 29 / 02$ & .12 & .14 & .12 & .07 & $<.05$ & .05 & $<.05$ & $<.05$ & $<.05$ & $<.05$ & $<.05$ & $<.05$ & $<.05$ & $<.05$ & .28 & .31 & .13 \\
\hline & & $06 / 12 / 02$ & .20 & .10 & .12 & $<.05$ & $<.05$ & $<.05$ & $<.05$ & $<.05$ & $<.05$ & $<.05$ & $<.05$ & $<.05$ & $<.05$ & $<.05$ & .35 & .21 & .08 \\
\hline \multirow[t]{2}{*}{06805500} & \multirow{2}{*}{$\begin{array}{l}\text { Platte River near } \\
\text { Louisville, NE }\end{array}$} & 06/02/00 & .07 & $<.20$ & $<.20$ & -- & $<.05$ & $<.20$ & $<.20$ & -- & .05 & -- & -- & $<.05$ & -- & -- & .29 & $<.20$ & $<.20$ \\
\hline & & $08 / 04 / 00$ & $<.05$ & $<.20$ & $<.20$ & -- & $<.05$ & $<.20$ & $<.20$ & -- & $<.05$ & -- & -- & $<.05$ & -- & -- & $<.05$ & .27 & $<.20$ \\
\hline \multirow[t]{5}{*}{03609750} & \multirow{5}{*}{$\begin{array}{l}\text { Tennessee River near } \\
\text { Paducah, KY }\end{array}$} & 07/13/00 & $<.05$ & $<.20$ & $<.20$ & -- & $<.05$ & $<.20$ & $<.20$ & -- & $<.05$ & -- & -- & $<.05$ & -- & -- & $<.05$ & $<.20$ & $<.20$ \\
\hline & & $08 / 14 / 00$ & $<.05$ & $<.20$ & $<.20$ & -- & $<.05$ & $<.20$ & $<.20$ & -- & $<.05$ & -- & -- & $<.05$ & -- & -- & $<.05$ & $<.20$ & $<.20$ \\
\hline & & $09 / 11 / 00$ & $<.05$ & $<.20$ & $<.20$ & -- & $<.05$ & $<.20$ & $<.20$ & -- & $<.05$ & -- & -- & $<.05$ & -- & -- & $<.05$ & $<.20$ & $<.20$ \\
\hline & & $11 / 29 / 00$ & $<.05$ & $<.05$ & $<.05$ & -- & $<.05$ & $<.05$ & $<.05$ & -- & $<.05$ & $<.05$ & $<.05$ & $<.05$ & $<.05$ & $<.05$ & $<.05$ & $<.05$ & $<.05$ \\
\hline & & $12 / 19 / 00$ & $<.05$ & $<.05$ & $<.05$ & -- & $<.05$ & $<.05$ & $<.05$ & -- & $<.05$ & $<.05$ & $<.05$ & $<.05$ & $<.05$ & $<.05$ & $<.05$ & $<.05$ & $<.05$ \\
\hline \multirow[t]{5}{*}{03375800} & \multirow{5}{*}{$\begin{array}{l}\text { Wabash River at New } \\
\text { Harmony, IN }\end{array}$} & $04 / 13 / 99$ & $<.05$ & $<.20$ & $<.20$ & -- & $<.05$ & .27 & $<.20$ & -- & -- & -- & -- & -- & -- & -- & .12 & .48 & .22 \\
\hline & & $04 / 26 / 99$ & .31 & .31 & .20 & -- & $<.05$ & .38 & $<.20$ & -- & .09 & -- & -- & -- & -- & -- & .73 & 1.2 & .59 \\
\hline & & $05 / 11 / 99$ & .24 & .31 & $<.20$ & -- & $<.05$ & .53 & $<.20$ & -- & $<.05$ & -- & -- & -- & -- & -- & .42 & .90 & .38 \\
\hline & & $05 / 26 / 99$ & .88 & .39 & .58 & -- & .05 & .29 & $<.20$ & -- & .24 & -- & -- & -- & -- & -- & .88 & .74 & .41 \\
\hline & & 06/08/99 & 1.7 & 1.7 & 3.0 & -- & .11 & .45 & .27 & -- & .43 & -- & -- & -- & -- & -- & 4.0 & 1.8 & 1.3 \\
\hline \multirow[t]{5}{*}{03378500} & \multirow{5}{*}{$\begin{array}{l}\text { Wabash River at New } \\
\text { Harmony, IN }\end{array}$} & $08 / 24 / 99$ & $<.05$ & $<.20$ & $<.20$ & -- & $<.05$ & $<.20$ & $<.20$ & -- & $<.05$ & -- & -- & $<.05$ & -- & -- & $<.05$ & .29 & $<.20$ \\
\hline & & $06 / 11 / 00$ & .19 & 1.1 & 1.2 & -- & $<.05$ & .62 & $<.20$ & -- & .09 & -- & -- & $<.05$ & -- & -- & .63 & 1.4 & .72 \\
\hline & & $06 / 27 / 00$ & $<.05$ & .12 & $<.20$ & -- & $<.05$ & .16 & $<.20$ & -- & $<.05$ & -- & -- & $<.05$ & -- & -- & .15 & .24 & .20 \\
\hline & & $07 / 11 / 00$ & $<.05$ & .96 & .66 & -- & $<.05$ & .52 & $<.20$ & -- & $<.05$ & -- & -- & $<.05$ & -- & -- & .18 & 1.2 & .65 \\
\hline & & $11 / 28 / 00$ & $<.05$ & .43 & .27 & -- & $<.05$ & .12 & .07 & -- & $<.05$ & $<.05$ & $<.05$ & $<.05$ & $<.05$ & $<.05$ & .06 & .74 & .30 \\
\hline \multirow[t]{5}{*}{07288955} & \multirow{5}{*}{$\begin{array}{l}\text { Yazoo River below Steele } \\
\text { Bayou, MS }\end{array}$} & 08/02/99 & $<.05$ & $<.20$ & $<.20$ & -- & $<.05$ & $<.20$ & $<.20$ & -- & $<.05$ & -- & -- & $<.05$ & -- & -- & .19 & $<.20$ & $<.20$ \\
\hline & & 06/19/00 & $<.05$ & $<.20$ & $<.20$ & -- & $<.05$ & .55 & $<.20$ & -- & .13 & -- & -- & .05 & -- & -- & 3.0 & .54 & $<.20$ \\
\hline & & $08 / 22 / 00$ & $<.05$ & $<.20$ & $<.20$ & -- & $<.05$ & $<.20$ & $<.20$ & -- & $<.05$ & -- & -- & $<.05$ & -- & -- & .10 & $<.20$ & $<.20$ \\
\hline & & 05/10/02 & $<.05$ & $<.05$ & $<.05$ & $<.05$ & $<.05$ & $<.05$ & $<.05$ & $<.05$ & .09 & $<.05$ & $<.05$ & $<.05$ & $<.05$ & $<.05$ & 1.1 & .09 & .10 \\
\hline & & $06 / 27 / 02$ & $<.05$ & $<.05$ & $<.05$ & $<.05$ & $<.05$ & $<.05$ & $<.05$ & $<.05$ & $<.05$ & $<.05$ & $<.05$ & $<.05$ & $<.05$ & $<.05$ & .34 & .07 & .09 \\
\hline
\end{tabular}


Table 6. Analytical results for selected acetamide herbicides and their degradation products, ethanesulfonic acid, oxanilic acid, and sulfinyl acetic acid, in surface-water samples, 1993-2003.-Continued

[OGRL, U.S. Geological Survey Organic Geochemistry Research Laboratory, Lawrence, Kansas; ESA, ethanesulfonic acid; OXA, oxanilic acid; SAA, sulfinyl acetic acid; --, no data; <, less than]

\begin{tabular}{|c|c|c|c|c|c|c|c|c|c|c|c|c|c|c|c|c|c|c|c|}
\hline \multirow[b]{2}{*}{ Site number } & \multirow[b]{2}{*}{ Site location } & \multirow[b]{2}{*}{$\begin{array}{c}\text { Date of } \\
\text { collection } \\
\text { (month/day/ } \\
\text { year) }\end{array}$} & \multicolumn{17}{|c|}{ Concentrations, in micrograms per liter } \\
\hline & & & $\begin{array}{l}\text { Aceto- } \\
\text { chlor } \\
(\mu \mathrm{g} / \mathrm{L})\end{array}$ & $\begin{array}{l}\text { Aceto- } \\
\text { chlor } \\
\text { ESA } \\
(\mu \mathrm{g} / \mathrm{L})\end{array}$ & $\begin{array}{l}\text { Aceto- } \\
\text { chlor } \\
\text { OXA } \\
(\mu \mathrm{g} / \mathrm{L})\end{array}$ & $\begin{array}{l}\text { Aceto- } \\
\text { chlor } \\
\text { SAA } \\
(\mu \mathrm{g} / \mathrm{L})\end{array}$ & $\begin{array}{l}\text { Ala- } \\
\text { chlor } \\
(\mu \mathrm{g} / \mathrm{L})\end{array}$ & $\begin{array}{l}\text { Ala- } \\
\text { chlor } \\
\text { ESA } \\
(\mu \mathrm{g} / \mathrm{L})\end{array}$ & $\begin{array}{l}\text { Ala- } \\
\text { chlor } \\
\text { OXA } \\
(\mu \mathrm{g} / \mathrm{L})\end{array}$ & $\begin{array}{l}\text { Ala- } \\
\text { chlor } \\
\text { SAA } \\
(\mu \mathrm{g} / \mathrm{L})\end{array}$ & $\begin{array}{c}\text { Di- } \\
\text { meth- } \\
\text { enamid } \\
(\mu \mathrm{g} / \mathrm{L})\end{array}$ & $\begin{array}{l}\text { Dimeth- } \\
\text { enamid } \\
\text { ESA } \\
(\mu \mathrm{g} / \mathrm{L})\end{array}$ & $\begin{array}{l}\text { Dimeth- } \\
\text { enamid } \\
\text { OXA } \\
(\mu \mathrm{g} / \mathrm{L})\end{array}$ & $\begin{array}{l}\text { Flufen- } \\
\text { acet } \\
(\mu \mathrm{g} / \mathrm{L})\end{array}$ & $\begin{array}{l}\text { Flufen- } \\
\text { acet } \\
\text { ESA } \\
(\mu \mathrm{g} / \mathrm{L})\end{array}$ & $\begin{array}{l}\text { Flufen- } \\
\text { acet } \\
\text { OXA } \\
(\mu \mathrm{g} / \mathrm{L})\end{array}$ & $\begin{array}{l}\text { Meto- } \\
\text { lachlor } \\
(\mu \mathrm{g} / \mathrm{L})\end{array}$ & $\begin{array}{l}\text { Meto- } \\
\text { lachlor } \\
\text { ESA } \\
(\mu \mathrm{g} / \mathrm{L})\end{array}$ & $\begin{array}{l}\text { Meto- } \\
\text { lachlor } \\
\text { OXA } \\
(\mu \mathrm{g} / \mathrm{L})\end{array}$ \\
\hline \multicolumn{20}{|c|}{ National Water-Quality Assessment Program (NAWOA) (fig. 3) } \\
\hline \multicolumn{20}{|c|}{ Delaware River Basin (DELR/BET) } \\
\hline 01464907 & $\begin{array}{l}\text { Little Neshamny } \\
\text { Creek near Valley } \\
\text { Road, PA }\end{array}$ & $06 / 23 / 99$ & -- & 0.05 & $<0.05$ & -- & -- & 0.11 & $<0.05$ & -- & -- & -- & -- & -- & -- & -- & -- & 0.47 & 0.11 \\
\hline \multirow[t]{21}{*}{01474500} & Schuylkill River at & $03 / 24 / 99$ & -- & $<.05$ & $<.05$ & -- & -- & .16 & $<.05$ & -- & -- & -- & -- & -- & -- & -- & -- & .60 & .13 \\
\hline & Philadelphia, PA & $04 / 21 / 99$ & -- & .23 & $<.05$ & -- & -- & .23 & $<.05$ & -- & -- & -- & -- & -- & -- & -- & -- & .23 & .11 \\
\hline & & 05/06/99 & -- & $<.05$ & $<.05$ & -- & -- & .26 & $<.05$ & -- & -- & -- & -- & -- & -- & -- & -- & .40 & $<.05$ \\
\hline & & $05 / 20 / 99$ & -- & $<.05$ & $<.05$ & -- & -- & .11 & $<.05$ & -- & -- & -- & -- & -- & -- & -- & -- & .43 & $<.05$ \\
\hline & & 06/03/99 & -- & $<.05$ & $<.05$ & -- & -- & .05 & $<.05$ & -- & -- & -- & -- & -- & -- & -- & -- & .19 & $<.05$ \\
\hline & & 06/15/99 & -- & $<.05$ & $<.05$ & -- & -- & .09 & $<.05$ & -- & -- & -- & -- & -- & -- & -- & -- & .53 & $<.05$ \\
\hline & & 07/01/99 & -- & $<.05$ & $<.05$ & -- & -- & .06 & $<.05$ & -- & -- & -- & -- & -- & -- & -- & -- & .42 & $<.05$ \\
\hline & & 07/14/99 & -- & $<.05$ & $<.05$ & -- & -- & $<.05$ & $<.05$ & -- & -- & -- & -- & -- & -- & -- & -- & .14 & $<.05$ \\
\hline & & 07/28/99 & -- & $<.05$ & $<.05$ & -- & -- & $<.05$ & $<.05$ & -- & -- & -- & -- & -- & -- & -- & -- & .13 & $<.05$ \\
\hline & & 08/04/99 & -- & $<.05$ & $<.05$ & -- & -- & $<.05$ & $<.05$ & -- & -- & -- & -- & -- & -- & -- & -- & .14 & $<.05$ \\
\hline & & 08/18/99 & -- & $<.05$ & $<.05$ & -- & -- & $<.05$ & $<.05$ & -- & -- & -- & -- & -- & -- & -- & -- & .14 & $<.05$ \\
\hline & & 09/07/99 & -- & $<.05$ & $<.05$ & -- & -- & $<.05$ & $<.05$ & -- & -- & -- & -- & -- & -- & -- & -- & .23 & $<.05$ \\
\hline & & 09/13/99 & -- & $<.05$ & $<.05$ & -- & -- & $<.05$ & $<.05$ & -- & -- & -- & -- & -- & -- & -- & -- & .05 & $<.05$ \\
\hline & & 09/17/99 & -- & $<.05$ & .06 & -- & -- & $<.05$ & $<.05$ & -- & -- & -- & -- & -- & -- & -- & -- & .26 & .33 \\
\hline & & $10 / 06 / 99$ & -- & $<.05$ & $<.05$ & -- & -- & .07 & $<.05$ & -- & -- & -- & -- & -- & -- & -- & -- & .65 & .16 \\
\hline & & $11 / 08 / 99$ & & $<.05$ & $<.05$ & -- & -- & .18 & $<.05$ & -- & -- & -- & -- & -- & -- & -- & -- & .61 & .14 \\
\hline & & 11/08/99 & -- & $<.05$ & $<.05$ & -- & -- & .16 & $<.05$ & -- & -- & -- & -- & -- & -- & -- & -- & .62 & .11 \\
\hline & & 12/01/99 & -- & $<.05$ & $<.05$ & -- & -- & .10 & $<.05$ & -- & -- & -- & -- & -- & -- & -- & -- & .51 & .09 \\
\hline & & $12 / 15 / 99$ & -- & $<.05$ & $<.05$ & -- & -- & .16 & $<.05$ & -- & -- & -- & -- & -- & -- & -- & -- & .97 & .37 \\
\hline & & 01/11/00 & -- & $<.05$ & $<.05$ & -- & -- & $<.05$ & $<.05$ & -- & -- & -- & -- & -- & -- & -- & -- & .38 & .06 \\
\hline & & $02 / 17 / 00$ & -- & $<.05$ & $<.05$ & -- & -- & $<.05$ & $<.05$ & -- & -- & -- & -- & -- & -- & -- & -- & .45 & .07 \\
\hline
\end{tabular}


Table 6. Analytical results for selected acetamide herbicides and their degradation products, ethanesulfonic acid, oxanilic acid, and sulfinyl acetic acid, in surface-water samples, 1993-2003.-Continued

[OGRL, U.S. Geological Survey Organic Geochemistry Research Laboratory, Lawrence, Kansas; ESA, ethanesulfonic acid; OXA, oxanilic acid; SAA, sulfinyl acetic acid; --, no data; <, less than]

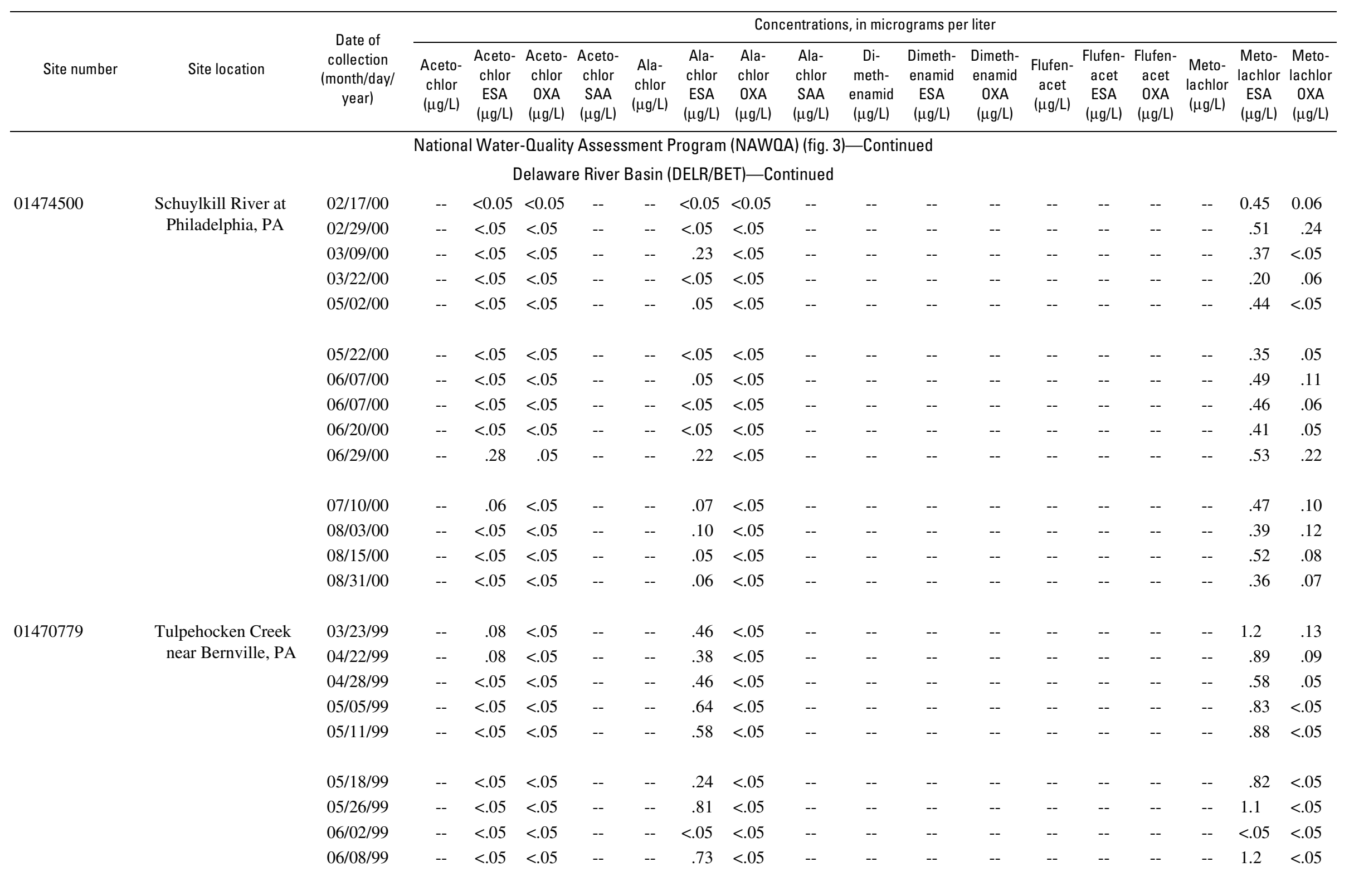


Table 6. Analytical results for selected acetamide herbicides and their degradation products, ethanesulfonic acid, oxanilic acid, and sulfinyl acetic acid, in surface-water samples, 1993-2003.-Continued

[OGRL, U.S. Geological Survey Organic Geochemistry Research Laboratory, Lawrence, Kansas; ESA, ethanesulfonic acid; OXA, oxanilic acid; SAA, sulfinyl acetic acid; --, no data; <, less than]

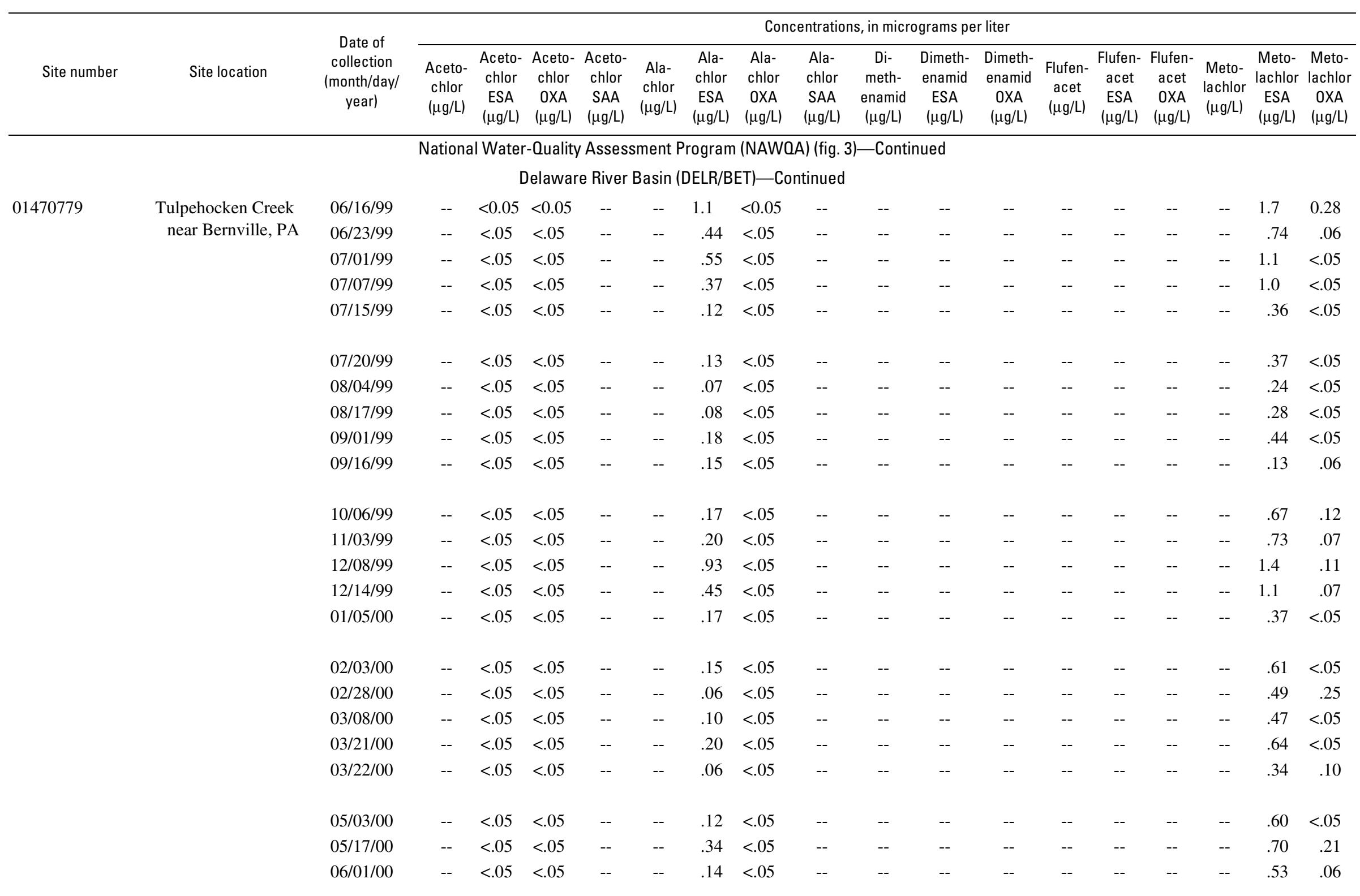


Table 6. Analytical results for selected acetamide herbicides and their degradation products, ethanesulfonic acid, oxanilic acid, and sulfinyl acetic acid, in surface-water samples, 1993-2003.-Continued

[OGRL, U.S. Geological Survey Organic Geochemistry Research Laboratory, Lawrence, Kansas; ESA, ethanesulfonic acid; OXA, oxanilic acid; SAA, sulfinyl acetic acid; --, no data; <, less than]

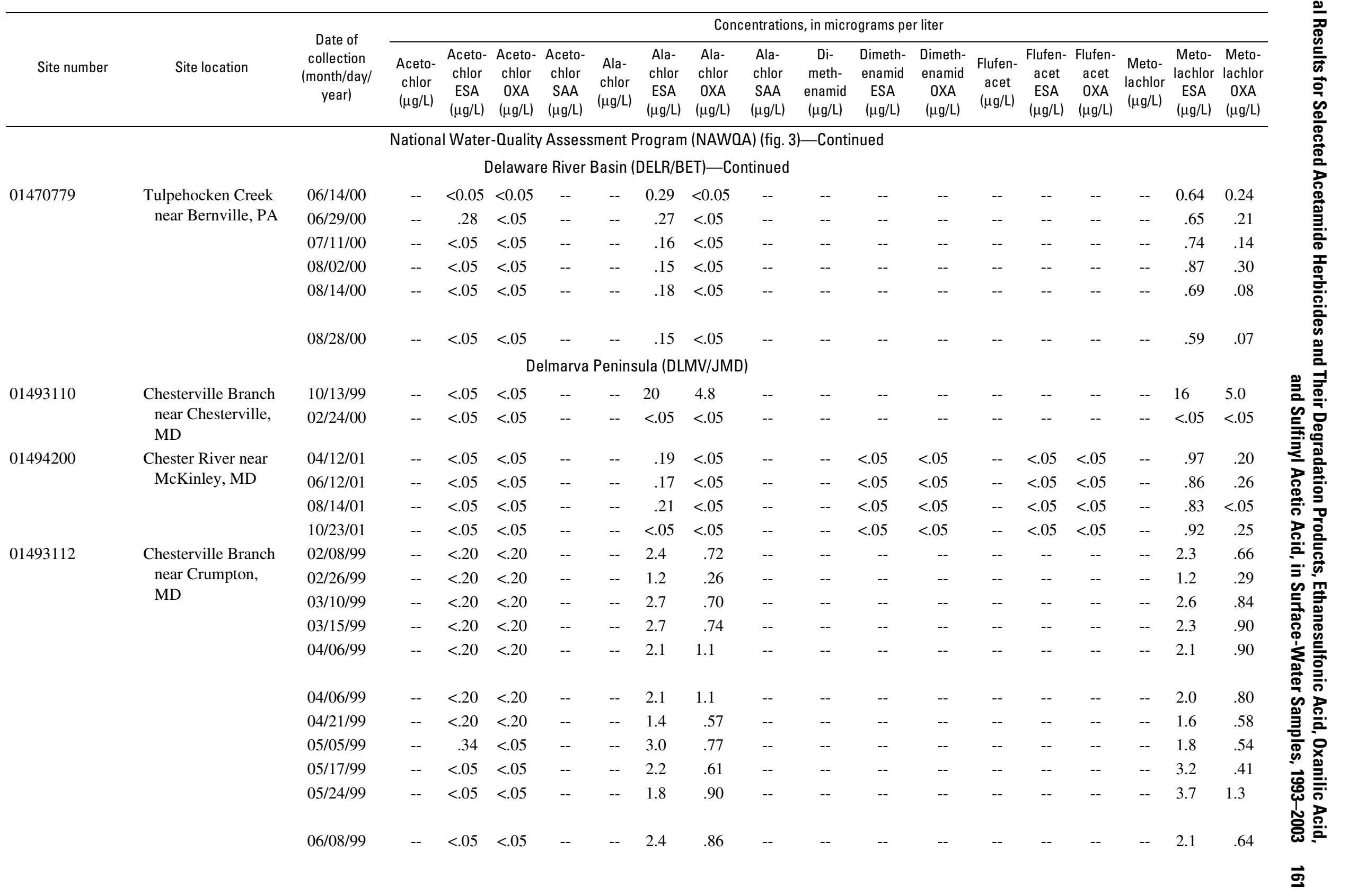


Table 6. Analytical results for selected acetamide herbicides and their degradation products, ethanesulfonic acid, oxanilic acid, and sulfinyl acetic acid, in surface-water samples, 1993-2003.-Continued

[OGRL, U.S. Geological Survey Organic Geochemistry Research Laboratory, Lawrence, Kansas; ESA, ethanesulfonic acid; OXA, oxanilic acid; SAA, sulfinyl acetic acid; --, no data; <, less than]

\begin{tabular}{|c|c|c|c|c|c|c|c|c|c|c|c|c|c|c|c|c|c|c|c|}
\hline \multirow[b]{2}{*}{ Site number } & \multirow[b]{2}{*}{ Site location } & \multirow[b]{2}{*}{$\begin{array}{c}\text { Date of } \\
\text { collection } \\
\text { (month/day/ } \\
\text { year) }\end{array}$} & \multicolumn{17}{|c|}{ Concentrations, in micrograms per liter } \\
\hline & & & $\begin{array}{l}\text { Aceto- } \\
\text { chlor } \\
(\mu \mathrm{g} / \mathrm{L})\end{array}$ & $\begin{array}{l}\text { Aceto- } \\
\text { chlor } \\
\text { ESA } \\
(\mu \mathrm{g} / \mathrm{L})\end{array}$ & $\begin{array}{c}\text { Aceto- } \\
\text { chlor } \\
\text { OXA } \\
(\mu \mathrm{g} / \mathrm{L})\end{array}$ & $\begin{array}{l}\text { Aceto- } \\
\text { chlor } \\
\text { SAA } \\
(\mu \mathrm{g} / \mathrm{L})\end{array}$ & $\begin{array}{l}\text { Ala- } \\
\text { chlor } \\
(\mu \mathrm{g} / \mathrm{L})\end{array}$ & $\begin{array}{l}\text { Ala- } \\
\text { chlor } \\
\text { ESA } \\
(\mu \mathrm{g} / \mathrm{L})\end{array}$ & $\begin{array}{l}\text { Ala- } \\
\text { chlor } \\
\text { OXA } \\
(\mu \mathrm{g} / \mathrm{L})\end{array}$ & $\begin{array}{l}\text { Ala- } \\
\text { chlor } \\
\text { SAA } \\
(\mu \mathrm{g} / \mathrm{L})\end{array}$ & $\begin{array}{l}\text { Di- } \\
\text { meth- } \\
\text { enamid } \\
(\mu \mathrm{g} / \mathrm{L})\end{array}$ & $\begin{array}{c}\text { Dimeth- } \\
\text { enamid } \\
\text { ESA } \\
(\mu \mathrm{g} / \mathrm{L})\end{array}$ & $\begin{array}{c}\text { Dimeth- } \\
\text { enamid } \\
\text { OXA } \\
(\mu \mathrm{g} / \mathrm{L})\end{array}$ & $\begin{array}{c}\text { Flufen- } \\
\text { acet } \\
(\mu \mathrm{g} / \mathrm{L})\end{array}$ & $\begin{array}{l}\text { Flufen- } \\
\text { acet } \\
\text { ESA } \\
(\mu \mathrm{g} / \mathrm{L})\end{array}$ & $\begin{array}{l}\text { Flufen- } \\
\text { acet } \\
\text { OXA } \\
(\mu \mathrm{g} / \mathrm{L})\end{array}$ & $\begin{array}{l}\text { Meto- } \\
\text { lachlor } \\
(\mu \mathrm{g} / \mathrm{L})\end{array}$ & $\begin{array}{l}\text { Meto- } \\
\text { lachlor } \\
\text { ESA } \\
(\mu \mathrm{g} / \mathrm{L})\end{array}$ & $\begin{array}{l}\text { Meto- } \\
\text { lachlor } \\
\text { OXA } \\
(\mu \mathrm{g} / \mathrm{L})\end{array}$ \\
\hline \multicolumn{20}{|c|}{ National Water-Quality Assessment Program (NAWQA) (fig. 3)—Continued } \\
\hline \multicolumn{20}{|c|}{ Delmarva Peninsula (DLMV/JMD)—Continued } \\
\hline \multirow[t]{12}{*}{01493112} & \multirow{12}{*}{$\begin{array}{l}\text { Chesterville Branch } \\
\text { near Crumpton, } \\
\text { MD }\end{array}$} & 06/20/99 & -- & $<0.05$ & $<0.05$ & -- & -- & 2.2 & 2.2 & -- & -- & -- & -- & -- & -- & -- & -- & 1.7 & 1.4 \\
\hline & & $06 / 21 / 99$ & -- & $<.05$ & $<.05$ & -- & -- & 3.3 & 4.1 & -- & -- & -- & -- & -- & -- & -- & -- & 2.1 & 2.4 \\
\hline & & 07/06/99 & -- & $<.05$ & $<.05$ & -- & -- & .76 & .19 & -- & -- & -- & -- & -- & -- & -- & -- & 1.3 & .38 \\
\hline & & 07/21/99 & -- & $<.05$ & $<.05$ & -- & -- & 1.6 & .30 & -- & -- & -- & -- & -- & -- & -- & -- & 1.5 & .32 \\
\hline & & 08/03/99 & -- & $<.05$ & $<.05$ & -- & -- & 1.4 & .29 & -- & -- & -- & -- & -- & -- & -- & -- & 1.7 & .35 \\
\hline & & $01 / 04 / 00$ & -- & $<.05$ & $<.05$ & -- & -- & 1.4 & 1.5 & -- & -- & -- & -- & -- & -- & -- & -- & 1.8 & 1.4 \\
\hline & & 06/12/01 & -- & $<.05$ & $<.05$ & -- & -- & 2.5 & .67 & -- & -- & $<0.05$ & $<0.05$ & & $<0.05$ & $<0.05$ & -- & 3.0 & 1.1 \\
\hline & & 07/16/01 & -- & $<.05$ & $<.05$ & -- & -- & 2.5 & .63 & -- & -- & $<.05$ & $<.05$ & -- & $<.05$ & $<.05$ & -- & 3.0 & 1.0 \\
\hline & & 08/14/01 & -- & $<.05$ & $<.05$ & -- & -- & 2.1 & .71 & -- & -- & $<.05$ & $<.05$ & -- & $<.05$ & $<.05$ & -- & 2.5 & .93 \\
\hline & & 09/10/01 & -- & $<.05$ & $<.05$ & -- & -- & .46 & .32 & -- & -- & $<.05$ & $<.05$ & -- & $<.05$ & $<.05$ & -- & .53 & 1.1 \\
\hline & & $11 / 15 / 01$ & -- & $<.05$ & $<.05$ & -- & -- & 2.3 & .54 & -- & -- & $<.05$ & $<.05$ & -- & $<.05$ & $<.05$ & -- & 2.9 & .75 \\
\hline & & $12 / 03 / 01$ & -- & $<.05$ & $<.05$ & $<0.05$ & -- & 1.8 & .39 & $<0.05$ & -- & $<.05$ & $<.05$ & -- & $<.05$ & $<.05$ & -- & 2.3 & .53 \\
\hline \multirow[t]{4}{*}{01493500} & \multirow{4}{*}{$\begin{array}{l}\text { Morgan Creek near } \\
\text { Kennedyville, MD }\end{array}$} & $02 / 22 / 00$ & -- & .40 & .06 & -- & -- & .20 & .21 & -- & -- & -- & -- & -- & -- & -- & -- & 2.2 & .55 \\
\hline & & $02 / 23 / 00$ & -- & .46 & $<.05$ & -- & -- & .23 & $<.05$ & -- & -- & -- & -- & -- & -- & -- & -- & 1.4 & .30 \\
\hline & & 03/06/02 & -- & $<.05$ & $<.05$ & $<.05$ & -- & .67 & .15 & $<.05$ & -- & $<.05$ & $<.05$ & -- & $<.05$ & $<.05$ & -- & 3.8 & .42 \\
\hline & & 03/27/02 & -- & .05 & $<.05$ & $<.05$ & -- & .40 & .11 & $<.05$ & -- & $<.05$ & $<.05$ & -- & $<.05$ & $<.05$ & -- & 2.5 & .42 \\
\hline \multirow[t]{6}{*}{01485719} & \multirow{6}{*}{$\begin{array}{l}\text { Pocomoke River at } \\
\text { Shelltown, MD }\end{array}$} & 04/09/01 & -- & .11 & .07 & -- & -- & .42 & .05 & -- & -- & $<.05$ & $<.05$ & -- & $<.05$ & $<.05$ & -- & 2.4 & .55 \\
\hline & & 06/11/01 & -- & .08 & $<.05$ & -- & -- & .38 & $<.05$ & -- & -- & $<.05$ & $<.05$ & -- & $<.05$ & $<.05$ & -- & 2.0 & .67 \\
\hline & & 08/13/01 & -- & $<.05$ & $<.05$ & -- & -- & .36 & $<.05$ & -- & -- & $<.05$ & $<.05$ & -- & $<.05$ & $<.05$ & -- & 1.3 & $<.05$ \\
\hline & & $10 / 22 / 01$ & -- & .16 & .08 & -- & -- & .30 & $<.05$ & -- & -- & $<.05$ & $<.05$ & -- & $<.05$ & $<.05$ & -- & 1.8 & .60 \\
\hline & & $03 / 25 / 02$ & -- & .06 & $<.05$ & $<.05$ & -- & .29 & $<.05$ & $<.05$ & -- & $<.05$ & $<.05$ & -- & $<.05$ & $<.05$ & -- & 1.3 & .33 \\
\hline & & $04 / 29 / 02$ & -- & .15 & $<.05$ & $<.05$ & -- & .46 & .05 & $<.05$ & -- & $<.05$ & $<.05$ & -- & $<.05$ & $<.05$ & -- & 2.2 & .63 \\
\hline
\end{tabular}


Table 6. Analytical results for selected acetamide herbicides and their degradation products, ethanesulfonic acid, oxanilic acid, and sulfinyl acetic acid, in surface-water samples, 1993-2003.-Continued

[OGRL, U.S. Geological Survey Organic Geochemistry Research Laboratory, Lawrence, Kansas; ESA, ethanesulfonic acid; OXA, oxanilic acid; SAA, sulfinyl acetic acid; --, no data; <, less than]

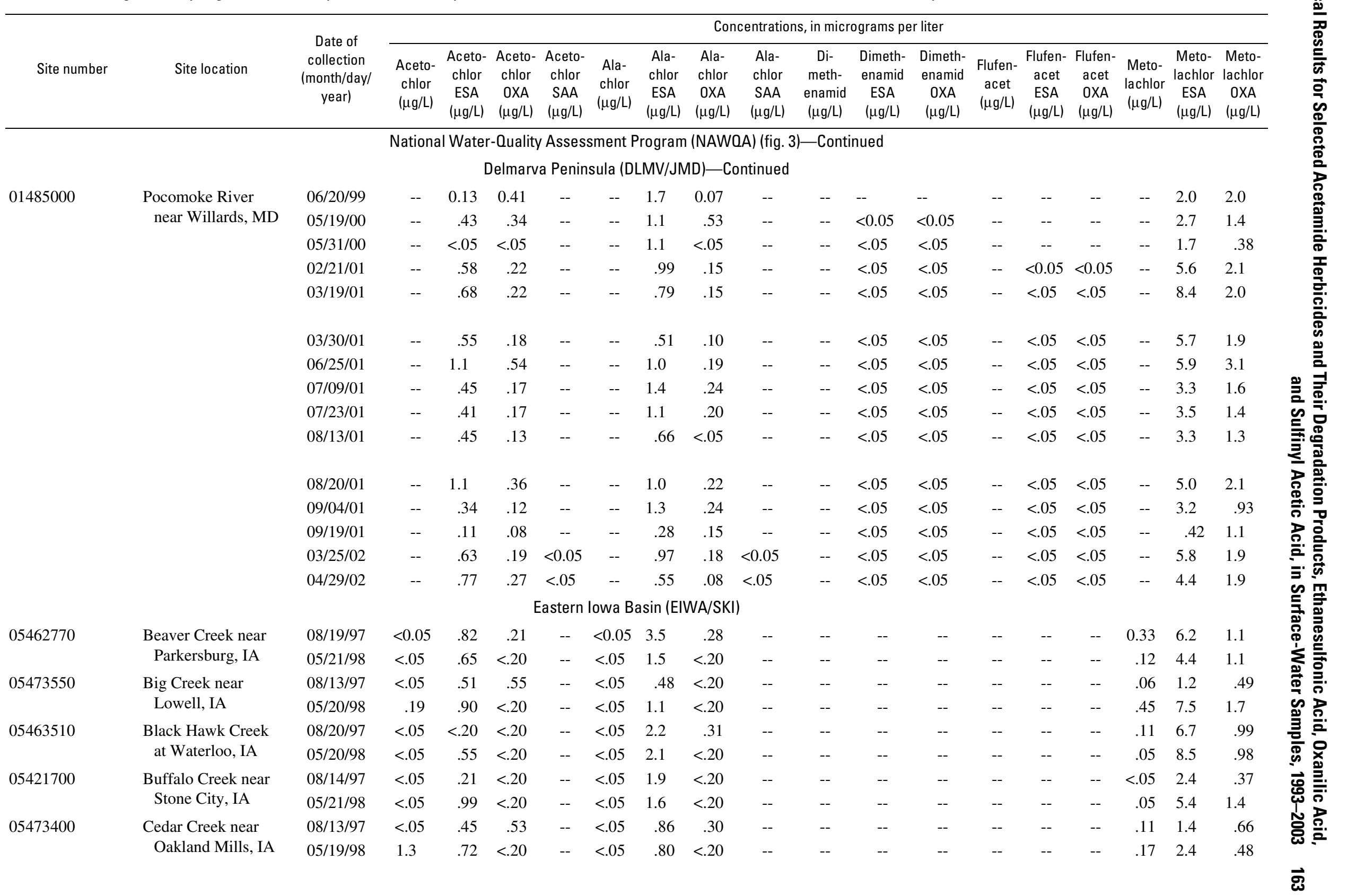


Table 6. Analytical results for selected acetamide herbicides and their degradation products, ethanesulfonic acid, oxanilic acid, and sulfinyl acetic acid, in surface-water samples, 1993-2003.-Continued

[OGRL, U.S. Geological Survey Organic Geochemistry Research Laboratory, Lawrence, Kansas; ESA, ethanesulfonic acid; OXA, oxanilic acid; SAA, sulfinyl acetic acid; --, no data; <, less than]

\begin{tabular}{|c|c|c|c|c|c|c|c|c|c|c|c|c|c|c|c|c|c|c|c|}
\hline \multirow[b]{2}{*}{ Site number } & \multirow[b]{2}{*}{ Site location } & \multirow[b]{2}{*}{$\begin{array}{c}\text { Date of } \\
\text { collection } \\
\text { (month/day/ } \\
\text { year) }\end{array}$} & \multicolumn{17}{|c|}{ Concentrations, in micrograms per liter } \\
\hline & & & $\begin{array}{l}\text { Aceto- } \\
\text { chlor } \\
(\mu \mathrm{g} / \mathrm{L})\end{array}$ & $\begin{array}{l}\text { Aceto- } \\
\text { chlor } \\
\text { ESA } \\
(\mu \mathrm{g} / \mathrm{L})\end{array}$ & $\begin{array}{l}\text { Aceto- } \\
\text { chlor } \\
\text { OXA } \\
(\mu \mathrm{g} / \mathrm{L})\end{array}$ & $\begin{array}{l}\text { Aceto- } \\
\text { chlor } \\
\text { SAA } \\
(\mu \mathrm{g} / \mathrm{L})\end{array}$ & $\begin{array}{l}\text { Ala- } \\
\text { chlor } \\
(\mu \mathrm{g} / \mathrm{L})\end{array}$ & $\begin{array}{l}\text { Ala- } \\
\text { chlor } \\
\text { ESA } \\
(\mu \mathrm{g} / \mathrm{L})\end{array}$ & $\begin{array}{l}\text { Ala- } \\
\text { chlor } \\
\text { OXA } \\
(\mu \mathrm{g} / \mathrm{L})\end{array}$ & $\begin{array}{l}\text { Ala- } \\
\text { chlor } \\
\text { SAA } \\
(\mu \mathrm{g} / \mathrm{L})\end{array}$ & $\begin{array}{l}\text { Di- } \\
\text { meth- } \\
\text { enamid } \\
(\mu \mathrm{g} / \mathrm{L})\end{array}$ & $\begin{array}{c}\text { Dimeth- } \\
\text { enamid } \\
\text { ESA } \\
(\mu \mathrm{g} / \mathrm{L})\end{array}$ & $\begin{array}{c}\text { Dimeth- } \\
\text { enamid } \\
\text { OXA } \\
(\mu \mathrm{g} / \mathrm{L})\end{array}$ & $\begin{array}{c}\text { Flufen- } \\
\text { acet } \\
(\mu \mathrm{g} / \mathrm{L})\end{array}$ & $\begin{array}{c}\text { Flufen- } \\
\text { acet } \\
\text { ESA } \\
(\mu \mathrm{g} / \mathrm{L})\end{array}$ & $\begin{array}{l}\text { Flufen- } \\
\text { acet } \\
\text { OXA } \\
(\mu \mathrm{g} / \mathrm{L})\end{array}$ & $\begin{array}{l}\text { Meto- } \\
\text { lachlor } \\
(\mu \mathrm{g} / \mathrm{L})\end{array}$ & $\begin{array}{c}\text { Meto- } \\
\text { lachlor } \\
\text { ESA } \\
(\mu \mathrm{g} / \mathrm{L})\end{array}$ & $\begin{array}{c}\text { Meto- } \\
\text { lachlor } \\
\text { OXA } \\
(\mu \mathrm{g} / \mathrm{L})\end{array}$ \\
\hline \multicolumn{20}{|c|}{ National Water-Quality Assessment Program (NAWQA) (fig. 3)—Continued } \\
\hline \multicolumn{20}{|c|}{ Eastern lowa Basin (EIWA/SKI)—Continued } \\
\hline \multirow[t]{5}{*}{05465000} & \multirow{5}{*}{$\begin{array}{l}\text { Cedar River at } \\
\text { Conesville, IA }\end{array}$} & 03/11/96 & $<0.05$ & 0.31 & $<0.20$ & -- & $<0.05$ & 1.4 & $<0.20$ & -- & -- & -- & -- & -- & -- & -- & 1.2 & 2.1 & 0.50 \\
\hline & & 04/10/96 & .12 & $<.20$ & $<.20$ & -- & $<.05$ & 1.3 & $<.20$ & -- & -- & -- & -- & -- & -- & -- & .83 & 2.6 & .95 \\
\hline & & 06/13/96 & .19 & 2.7 & 2.5 & -- & $<.05$ & 3.2 & $<.20$ & -- & -- & -- & -- & -- & -- & -- & .48 & 8.5 & 3.2 \\
\hline & & 07/08/96 & $<.05$ & .96 & .36 & -- & $<.05$ & 2.5 & $<.20$ & -- & -- & -- & -- & -- & -- & -- & .10 & 4.8 & .60 \\
\hline & & 08/05/96 & $<.05$ & .21 & $<.20$ & -- & $<.05$ & 1.8 & $<.20$ & -- & -- & -- & -- & -- & -- & -- & .05 & 3.3 & .67 \\
\hline \multirow{8}{*}{05464020} & \multirow{8}{*}{$\begin{array}{l}\text { Cedar River at } \\
\text { Gilbertville, IA }\end{array}$} & 09/10/96 & $<.05$ & .33 & $<.20$ & -- & $<.05$ & 2.1 & $<.20$ & -- & -- & -- & -- & -- & -- & -- & $<.05$ & 1.6 & .50 \\
\hline & & $10 / 10 / 96$ & $<.05$ & $<.20$ & $<.20$ & -- & $<.05$ & 1.6 & $<.20$ & -- & -- & -- & -- & -- & -- & -- & $<.05$ & 2.3 & .49 \\
\hline & & $10 / 29 / 96$ & $<.05$ & $<.20$ & $<.20$ & -- & $<.05$ & 1.3 & $<.20$ & -- & -- & -- & -- & -- & -- & -- & .06 & 2.0 & .38 \\
\hline & & 03/26/96 & $<.05$ & $<.20$ & $<.20$ & -- & $<.05$ & 1.6 & $<.20$ & -- & -- & -- & -- & -- & -- & -- & .37 & 2.6 & $<.20$ \\
\hline & & $06 / 25 / 96$ & .11 & 1.7 & 1.3 & -- & $<.05$ & 2.3 & .32 & -- & -- & -- & -- & -- & -- & -- & .47 & 5.4 & 1.2 \\
\hline & & 07/23/96 & $<.05$ & $<.20$ & $<.20$ & -- & $<.05$ & 2.0 & $<.20$ & -- & -- & -- & -- & -- & -- & -- & .07 & 3.4 & .56 \\
\hline & & 08/29/96 & $<.05$ & .48 & $<.20$ & -- & $<.05$ & 1.9 & $<.20$ & -- & -- & -- & -- & -- & -- & -- & .09 & 4.2 & 1.5 \\
\hline & & 09/24/96 & $<.05$ & $<.20$ & $<.20$ & -- & $<.05$ & 1.2 & $<.20$ & -- & -- & -- & -- & -- & -- & -- & $<.05$ & 1.8 & .36 \\
\hline \multirow{13}{*}{05464935} & \multirow{13}{*}{$\begin{array}{l}\text { Cedar River near } \\
\text { Nichols, IA }\end{array}$} & $10 / 15 / 96$ & $<.05$ & $<.20$ & $<.20$ & -- & $<.05$ & 2.6 & .23 & -- & -- & -- & -- & -- & -- & -- & $<.05$ & 3.4 & .51 \\
\hline & & $11 / 12 / 96$ & $<.05$ & $<.20$ & .21 & -- & $<.05$ & 1.4 & $<.20$ & -- & -- & -- & -- & -- & -- & -- & .07 & 2.7 & .30 \\
\hline & & $12 / 12 / 96$ & $<.05$ & .41 & $<.20$ & -- & $<.05$ & 2.0 & .30 & -- & -- & -- & -- & -- & -- & -- & .07 & 4.0 & .48 \\
\hline & & 01/15/97 & $<.05$ & $<.20$ & $<.20$ & -- & $<.05$ & 2.0 & $<.20$ & -- & -- & -- & -- & -- & -- & -- & .40 & 4.3 & .66 \\
\hline & & 02/10/97 & $<.05$ & $<.20$ & $<.20$ & -- & $<.05$ & 2.4 & $<.20$ & -- & -- & -- & -- & -- & -- & -- & .19 & 2.9 & .67 \\
\hline & & $10 / 29 / 96$ & $<.05$ & .22 & $<.20$ & -- & $<.05$ & 1.3 & $<.20$ & -- & -- & -- & -- & -- & -- & -- & .06 & 2.1 & .25 \\
\hline & & $11 / 13 / 96$ & $<.05$ & $<.20$ & .20 & -- & $<.05$ & 1.3 & $<.20$ & -- & -- & -- & -- & -- & -- & -- & .08 & 2.9 & .33 \\
\hline & & $12 / 04 / 96$ & $<.05$ & .38 & $<.20$ & -- & $<.05$ & 1.8 & $<.20$ & -- & -- & -- & -- & -- & -- & -- & .13 & 4.2 & .71 \\
\hline & & $01 / 22 / 97$ & $<.05$ & .28 & $<.20$ & -- & $<.05$ & 1.7 & $<.20$ & -- & -- & -- & -- & -- & -- & -- & .37 & 3.2 & .47 \\
\hline & & 02/03/97 & $<.05$ & .30 & $<.20$ & -- & $<.05$ & 1.4 & $<.20$ & -- & -- & -- & -- & -- & -- & -- & .36 & 2.3 & .35 \\
\hline & & 03/13/97 & $<.05$ & .97 & $<.20$ & -- & $<.05$ & 1.3 & $<.20$ & -- & -- & -- & -- & -- & -- & -- & .90 & 3.2 & 1.1 \\
\hline & & 05/07/97 & .80 & 1.1 & .49 & -- & $<.05$ & 1.6 & $<.20$ & -- & -- & -- & -- & -- & -- & -- & .78 & 5.0 & 1.0 \\
\hline & & 04/03/98 & $<.05$ & .57 & $<.20$ & -- & $<.05$ & .73 & $<.20$ & -- & -- & -- & -- & -- & -- & -- & .21 & 2.9 & .71 \\
\hline
\end{tabular}


Table 6. Analytical results for selected acetamide herbicides and their degradation products, ethanesulfonic acid, oxanilic acid, and sulfinyl acetic acid, in surface-water samples, 1993-2003.-Continued

[OGRL, U.S. Geological Survey Organic Geochemistry Research Laboratory, Lawrence, Kansas; ESA, ethanesulfonic acid; OXA, oxanilic acid; SAA, sulfinyl acetic acid; --, no data; <, less than]

\begin{tabular}{|c|c|c|c|c|c|c|c|c|c|c|c|c|c|c|c|c|c|c|c|}
\hline \multirow[b]{2}{*}{ Site number } & \multirow[b]{2}{*}{ Site location } & \multirow[b]{2}{*}{$\begin{array}{l}\text { Date of } \\
\text { collection } \\
\text { (month/day/ } \\
\text { year) }\end{array}$} & \multicolumn{17}{|c|}{ Concentrations, in micrograms per liter } \\
\hline & & & $\begin{array}{l}\text { Aceto- } \\
\text { chlor } \\
(\mu \mathrm{g} / \mathrm{L})\end{array}$ & $\begin{array}{l}\text { Aceto- } \\
\text { chlor } \\
\text { ESA } \\
(\mu \mathrm{g} / \mathrm{L})\end{array}$ & $\begin{array}{l}\text { Aceto- } \\
\text { chlor } \\
\text { OXA } \\
(\mu \mathrm{g} / \mathrm{L})\end{array}$ & $\begin{array}{l}\text { Aceto- } \\
\text { chlor } \\
\text { SAA } \\
(\mu \mathrm{g} / \mathrm{L})\end{array}$ & $\begin{array}{l}\text { Ala- } \\
\text { chlor } \\
(\mu \mathrm{g} / \mathrm{L})\end{array}$ & $\begin{array}{l}\text { Ala- } \\
\text { chlor } \\
\text { ESA } \\
(\mu \mathrm{g} / \mathrm{L})\end{array}$ & $\begin{array}{l}\text { Ala- } \\
\text { chlor } \\
\text { OXA } \\
(\mu \mathrm{g} / \mathrm{L})\end{array}$ & $\begin{array}{c}\text { Ala- } \\
\text { chlor } \\
\text { SAA } \\
(\mu \mathrm{g} / \mathrm{L})\end{array}$ & $\begin{array}{l}\text { Di- } \\
\text { meth- } \\
\text { enamid } \\
(\mu \mathrm{g} / \mathrm{L})\end{array}$ & $\begin{array}{l}\text { Dimeth- } \\
\text { enamid } \\
\text { ESA } \\
(\mu \mathrm{g} / \mathrm{L})\end{array}$ & $\begin{array}{l}\text { Dimeth- } \\
\text { enamid } \\
\text { OXA } \\
(\mu \mathrm{g} / \mathrm{L})\end{array}$ & $\begin{array}{l}\text { Flufen- } \\
\text { acet } \\
(\mu \mathrm{g} / \mathrm{L})\end{array}$ & $\begin{array}{l}\text { Flufen- } \\
\text { acet } \\
\text { ESA } \\
(\mu \mathrm{g} / \mathrm{L})\end{array}$ & $\begin{array}{l}\text { Flufen- } \\
\text { acet } \\
\text { OXA } \\
(\mu \mathrm{g} / \mathrm{L})\end{array}$ & $\begin{array}{l}\text { Meto- } \\
\text { lachlor } \\
(\mu \mathrm{g} / \mathrm{L})\end{array}$ & $\begin{array}{l}\text { Meto- } \\
\text { lachlor } \\
\text { ESA } \\
(\mu \mathrm{g} / \mathrm{L})\end{array}$ & $\begin{array}{l}\text { Meto- } \\
\text { lachlor } \\
\text { OXA } \\
(\mu \mathrm{g} / \mathrm{L})\end{array}$ \\
\hline \multicolumn{20}{|c|}{ National Water-Quality Assessment Program (NAWQA) (fig. 3)—Continued } \\
\hline \multicolumn{20}{|c|}{ Eastern lowa Basin (EIWA/SKI)—Continued } \\
\hline \multirow[t]{6}{*}{05464935} & \multirow{6}{*}{$\begin{array}{l}\text { Cedar River near } \\
\text { Nichols, IA }\end{array}$} & 05/07/98 & 0.14 & 0.58 & $<0.20$ & -- & $<0.05$ & 1.2 & $<0.20$ & -- & -- & -- & -- & -- & -- & -- & 0.28 & 3.5 & 0.73 \\
\hline & & 06/05/98 & .29 & 1.3 & .70 & -- & $<.05$ & 1.4 & $<.20$ & -- & -- & -- & -- & -- & -- & -- & .28 & 4.9 & .73 \\
\hline & & 07/06/98 & .09 & 2.1 & 1.2 & -- & $<.05$ & 1.4 & $<.20$ & -- & -- & -- & -- & -- & -- & -- & .39 & 4.1 & 1.2 \\
\hline & & $08 / 04 / 98$ & $<.05$ & .64 & $<.20$ & -- & $<.05$ & 1.8 & $<.20$ & -- & -- & -- & -- & -- & -- & -- & $<.05$ & 3.7 & .40 \\
\hline & & 09/03/98 & $<.05$ & 1.1 & $<.20$ & -- & $<.05$ & 2.2 & $<.20$ & -- & -- & -- & -- & -- & -- & -- & .05 & 3.6 & .65 \\
\hline & & 07/28/99 & .07 & 3.0 & 1.8 & -- & $<.05$ & 1.3 & $<.20$ & -- & 0.06 & -- & -- & $<0.05$ & -- & -- & .28 & 4.9 & 1.7 \\
\hline \multirow[t]{2}{*}{05473060} & \multirow{2}{*}{$\begin{array}{l}\text { Crooked Creek at } \\
\text { Coppock, IA }\end{array}$} & 08/12/97 & $<.05$ & .69 & .87 & -- & $<.05$ & .66 & .24 & -- & -- & -- & -- & -- & -- & -- & .18 & 2.8 & .87 \\
\hline & & 05/19/98 & .12 & .76 & $<.20$ & -- & $<.05$ & 1.1 & $<.20$ & -- & -- & -- & -- & -- & -- & -- & .49 & 5.4 & 1.1 \\
\hline \multirow[t]{2}{*}{05471120} & \multirow{2}{*}{$\begin{array}{l}\text { East Branch Indian } \\
\text { Creek near Iowa } \\
\text { Center, IA }\end{array}$} & 08/18/97 & $<.05$ & .59 & $<.20$ & -- & $<.05$ & .23 & $<.20$ & -- & -- & -- & -- & -- & -- & -- & .06 & 3.3 & .56 \\
\hline & & $05 / 21 / 98$ & .30 & .91 & $<.20$ & -- & $<.05$ & .38 & $<.20$ & -- & -- & -- & -- & -- & -- & -- & .60 & 4.4 & .78 \\
\hline \multirow[t]{2}{*}{05449200} & \multirow{2}{*}{$\begin{array}{l}\text { East Branch Iowa } \\
\text { River at Belmond, } \\
\text { IA }\end{array}$} & 08/26/97 & $<.05$ & .21 & $<.20$ & -- & $<.05$ & 3.0 & $<.20$ & -- & -- & -- & -- & -- & -- & -- & $<.05$ & 4.1 & .66 \\
\hline & & 05/20/98 & $<.05$ & .74 & $<.20$ & -- & $<.05$ & 1.9 & $<.20$ & -- & -- & -- & -- & -- & -- & -- & .06 & 7.3 & 1.8 \\
\hline 05420720 & $\begin{array}{l}\text { East Fork Wapsipin- } \\
\text { icon near Tripoli, } \\
\text { IA }\end{array}$ & 08/28/97 & $<.05$ & .31 & $<.20$ & -- & $<.05$ & 2.3 & .54 & -- & -- & -- & -- & -- & -- & -- & .06 & 2.3 & .50 \\
\hline \multirow[t]{2}{*}{05455500} & \multirow{2}{*}{$\begin{array}{l}\text { English River at } \\
\text { Kalona, IA }\end{array}$} & 08/11/97 & $<.05$ & $<.20$ & .35 & -- & $<.05$ & 1.0 & $<.20$ & -- & -- & -- & -- & -- & -- & -- & .06 & 1.3 & .29 \\
\hline & & 05/18/98 & .07 & .61 & $<.20$ & -- & $<.05$ & .65 & $<.20$ & -- & -- & -- & -- & -- & -- & -- & .19 & 2.8 & .58 \\
\hline \multirow[t]{7}{*}{05461390} & \multirow{7}{*}{$\begin{array}{l}\text { Flood Creek near } \\
\text { Powersville, IA }\end{array}$} & 03/28/96 & $<.05$ & $<.20$ & $<.20$ & -- & $<.05$ & 2.0 & $<.20$ & -- & -- & -- & -- & -- & -- & -- & .22 & 2.8 & $<.20$ \\
\hline & & 06/04/96 & .05 & 1.9 & $<.20$ & -- & $<.05$ & 2.7 & $<.20$ & -- & -- & -- & -- & -- & -- & -- & .46 & 12 & 3.3 \\
\hline & & 06/07/96 & .36 & 2.0 & 1.7 & -- & .13 & 3.3 & $<.20$ & -- & -- & -- & -- & -- & -- & -- & 1.2 & 12 & 2.7 \\
\hline & & 06/19/96 & .08 & 2.1 & 1.7 & -- & $<.05$ & 3.3 & .45 & -- & -- & -- & -- & -- & -- & -- & .75 & 11 & 1.9 \\
\hline & & 07/17/96 & $<.05$ & $<.20$ & $<.20$ & -- & $<.05$ & 1.6 & $<.20$ & -- & -- & -- & -- & -- & -- & -- & $<.05$ & 3.8 & .20 \\
\hline & & $08 / 14 / 96$ & $<.05$ & $<.20$ & $<.20$ & -- & $<.05$ & 1.8 & $<.20$ & -- & -- & -- & -- & -- & -- & -- & $<.05$ & 4.0 & $<.20$ \\
\hline & & 09/18/96 & $<.05$ & $<.20$ & $<.20$ & -- & $<.05$ & 1.2 & $<.20$ & -- & -- & -- & -- & -- & -- & -- & $<.05$ & 2.0 & $<.20$ \\
\hline
\end{tabular}


Table 6. Analytical results for selected acetamide herbicides and their degradation products, ethanesulfonic acid, oxanilic acid, and sulfinyl acetic acid, in surface-water samples, 1993-2003.-Continued

[OGRL, U.S. Geological Survey Organic Geochemistry Research Laboratory, Lawrence, Kansas; ESA, ethanesulfonic acid; OXA, oxanilic acid; SAA, sulfinyl acetic acid; --, no data; <, less than]

\begin{tabular}{|c|c|c|c|c|c|c|c|c|c|c|c|c|c|c|c|c|c|c|c|}
\hline \multirow[b]{2}{*}{ Site number } & \multirow[b]{2}{*}{ Site location } & \multirow[b]{2}{*}{$\begin{array}{c}\text { Date of } \\
\text { collection } \\
\text { (month/day/ } \\
\text { year) }\end{array}$} & \multicolumn{17}{|c|}{ Concentrations, in micrograms per liter } \\
\hline & & & $\begin{array}{c}\text { Aceto- } \\
\text { chlor } \\
(\mu \mathrm{g} / \mathrm{L})\end{array}$ & $\begin{array}{c}\text { Aceto- } \\
\text { chlor } \\
\text { ESA } \\
(\mu \mathrm{g} / \mathrm{L})\end{array}$ & $\begin{array}{c}\text { Aceto- } \\
\text { chlor } \\
\text { OXA } \\
(\mu \mathrm{g} / \mathrm{L})\end{array}$ & $\begin{array}{c}\text { Aceto- } \\
\text { chlor } \\
\text { SAA } \\
(\mu \mathrm{g} / \mathrm{L})\end{array}$ & $\begin{array}{l}\text { Ala- } \\
\text { chlor } \\
(\mu \mathrm{g} / \mathrm{L})\end{array}$ & $\begin{array}{c}\text { Ala- } \\
\text { chlor } \\
\text { ESA } \\
(\mu \mathrm{g} / \mathrm{L})\end{array}$ & $\begin{array}{c}\text { Ala- } \\
\text { chlor } \\
\text { OXA } \\
(\mu \mathrm{g} / \mathrm{L})\end{array}$ & $\begin{array}{l}\text { Ala- } \\
\text { chlor } \\
\text { SAA } \\
(\mu \mathrm{g} / \mathrm{L})\end{array}$ & $\begin{array}{c}\text { Di- } \\
\text { meth- } \\
\text { enamid } \\
(\mu \mathrm{g} / \mathrm{L})\end{array}$ & $\begin{array}{c}\text { Dimeth- } \\
\text { enamid } \\
\text { ESA } \\
(\mu \mathrm{g} / \mathrm{L})\end{array}$ & $\begin{array}{c}\text { Dimeth- } \\
\text { enamid } \\
\text { OXA } \\
(\mu \mathrm{g} / \mathrm{L})\end{array}$ & $\begin{array}{c}\text { Flufen- } \\
\text { acet } \\
(\mu \mathrm{g} / \mathrm{L})\end{array}$ & $\begin{array}{c}\text { Flufen- } \\
\text { acet } \\
\text { ESA } \\
(\mu \mathrm{g} / \mathrm{L})\end{array}$ & $\begin{array}{c}\text { Flufen- } \\
\text { acet } \\
\text { OXA } \\
(\mu \mathrm{g} / \mathrm{L})\end{array}$ & $\begin{array}{l}\text { Meto- } \\
\text { lachlor } \\
(\mu \mathrm{g} / \mathrm{L})\end{array}$ & $\begin{array}{c}\text { Meto- } \\
\text { lachlor } \\
\text { ESA } \\
(\mu \mathrm{g} / \mathrm{L})\end{array}$ & $\begin{array}{c}\text { Meto- } \\
\text { lachlor } \\
\text { OXA } \\
(\mu \mathrm{g} / \mathrm{L})\end{array}$ \\
\hline \multicolumn{20}{|c|}{ National Water-Quality Assessment Program (NAWQA) (fig. 3)—Continued } \\
\hline \multicolumn{20}{|c|}{ Eastern lowa Basin (EIWA/SKI)—Continued } \\
\hline \multirow[t]{15}{*}{05461390} & \multirow{10}{*}{$\begin{array}{c}\text { Flood Creek near } \\
\text { Powersville, IA }\end{array}$} & 09/18/96 & $<0.05$ & $<0.20$ & $<0.20$ & -- & $<0.05$ & 1.2 & $0<.20$ & -- & -- & -- & -- & -- & -- & -- & $<0.05$ & 1.9 & $<0.20$ \\
\hline & & $10 / 23 / 96$ & $<.05$ & $<.20$ & $<.20$ & -- & $<.05$ & 1.3 & $<.20$ & -- & -- & -- & -- & -- & -- & -- & $<.05$ & 1.7 & $<.20$ \\
\hline & & $12 / 11 / 96$ & $<.05$ & $<.20$ & $<.20$ & -- & $<.05$ & 1.4 & $<.20$ & -- & -- & -- & -- & -- & -- & -- & $<.05$ & 3.0 & $<.20$ \\
\hline & & $01 / 14 / 97$ & $<.05$ & $<.20$ & $<.20$ & -- & $<.05$ & 1.7 & $<.20$ & -- & -- & -- & -- & -- & -- & -- & $<.05$ & 4.6 & $<.20$ \\
\hline & & $02 / 19 / 97$ & $<.05$ & $<.20$ & $<.20$ & -- & $<.05$ & 1.4 & $<.20$ & -- & -- & -- & -- & -- & -- & -- & $<.05$ & 2.5 & $<.20$ \\
\hline & & 03/18/97 & $<.05$ & 1.8 & .77 & -- & .08 & .91 & $<.20$ & -- & -- & -- & -- & -- & -- & -- & 2.4 & 4.7 & 2.1 \\
\hline & & 05/03/97 & 2.3 & 1.9 & .93 & -- & $<.05$ & 2.6 & $<.20$ & -- & -- & -- & -- & -- & -- & -- & 2.3 & 12 & 2.6 \\
\hline & & $08 / 25 / 97$ & $<.05$ & .37 & $<.20$ & -- & $<.05$ & 1.1 & $<.20$ & -- & -- & -- & -- & -- & -- & -- & $<.05$ & 3.4 & .28 \\
\hline & & $04 / 02 / 98$ & $<.05$ & 1.4 & $<.20$ & -- & $<.05$ & 1.7 & $<.20$ & -- & -- & -- & -- & -- & -- & -- & 1.4 & 9.8 & 2.1 \\
\hline & & $05 / 06 / 98$ & $<.05$ & .48 & $<.20$ & -- & $<.05$ & 1.7 & $<.20$ & -- & -- & -- & -- & -- & -- & -- & .15 & 8.0 & .40 \\
\hline & & 05/18/98 & .05 & .51 & $<.20$ & -- & $<.05$ & 1.0 & $<.20$ & -- & -- & -- & -- & -- & -- & -- & .11 & 3.8 & .50 \\
\hline & & 07/08/98 & $<.05$ & 1.7 & $<.20$ & -- & $<.05$ & 2.4 & $<.20$ & -- & -- & -- & -- & -- & -- & -- & .07 & 7.8 & 1.1 \\
\hline & & 08/05/98 & $<.05$ & .33 & $<.20$ & -- & $<.05$ & 1.2 & $<.20$ & -- & -- & -- & -- & -- & -- & -- & $<.05$ & 3.6 & .15 \\
\hline & & $09 / 02 / 98$ & $<.05$ & .48 & $<.20$ & -- & $<.05$ & 2.0 & $<.20$ & -- & -- & -- & -- & -- & -- & -- & $<.05$ & 5.4 & .35 \\
\hline & & $07 / 22 / 99$ & .05 & 1.1 & .60 & -- & .06 & 1.0 & .46 & -- & 0.05 & -- & -- & $<0.05$ & -- & -- & .54 & 2.9 & .99 \\
\hline \multirow[t]{3}{*}{05464137} & \multirow{3}{*}{$\begin{array}{c}\text { Four Mile Creek } \\
\text { near Trear, IA }\end{array}$} & $04 / 15 / 98$ & $<.05$ & .49 & $<.20$ & -- & $<.05$ & .85 & $<.20$ & -- & -- & -- & -- & -- & -- & -- & $<.05$ & 2.6 & .57 \\
\hline & & $07 / 16 / 98$ & $<.05$ & 1.2 & $<.20$ & -- & $<.05$ & 1.0 & $<.20$ & -- & -- & -- & -- & -- & -- & -- & $<.05$ & 3.8 & .65 \\
\hline & & $08 / 12 / 98$ & $<.05$ & 1.0 & $<.20$ & -- & $<.05$ & 1.2 & $<.20$ & -- & -- & -- & -- & -- & -- & -- & $<.05$ & 4.7 & .37 \\
\hline \multirow[t]{7}{*}{05453100} & \multirow{7}{*}{$\begin{array}{l}\text { Iowa River at } \\
\text { Marengo, IA }\end{array}$} & $03 / 15 / 96$ & $<.05$ & $<.20$ & $<.20$ & & $<.05$ & 1.1 & $<.20$ & & & & & & & & .25 & 1.9 & $<.20$ \\
\hline & & $04 / 25 / 96$ & $<.05$ & $<.20$ & $<.20$ & -- & $<.05$ & 1.1 & $<.20$ & -- & -- & -- & -- & -- & -- & -- & .14 & 2.8 & .49 \\
\hline & & $05 / 23 / 96$ & 1.1 & .50 & .77 & -- & $<.05$ & .80 & $<.20$ & -- & -- & -- & -- & -- & -- & -- & .71 & 2.6 & .49 \\
\hline & & $05 / 31 / 96$ & .19 & .98 & .75 & -- & $<.05$ & 1.1 & .26 & -- & -- & -- & -- & -- & -- & -- & .62 & 4.2 & 1.0 \\
\hline & & $06 / 27 / 96$ & $<.05$ & .70 & .67 & -- & $<.05$ & .90 & .32 & -- & -- & -- & -- & -- & -- & -- & .48 & 4.6 & 1.3 \\
\hline & & $07 / 25 / 96$ & $<.05$ & .38 & $<.20$ & -- & $<.05$ & 1.1 & $<.20$ & -- & -- & -- & -- & -- & -- & -- & .32 & 3.8 & .89 \\
\hline & & $08 / 26 / 96$ & $<.05$ & $<.20$ & $<.20$ & -- & $<.05$ & .89 & $<.20$ & -- & -- & -- & -- & -- & -- & -- & .05 & 2.2 & $<.20$ \\
\hline
\end{tabular}


Table 6. Analytical results for selected acetamide herbicides and their degradation products, ethanesulfonic acid, oxanilic acid, and sulfinyl acetic acid, in surface-water samples, 1993-2003.-Continued

[OGRL, U.S. Geological Survey Organic Geochemistry Research Laboratory, Lawrence, Kansas; ESA, ethanesulfonic acid; OXA, oxanilic acid; SAA, sulfinyl acetic acid; --, no data; <, less than]

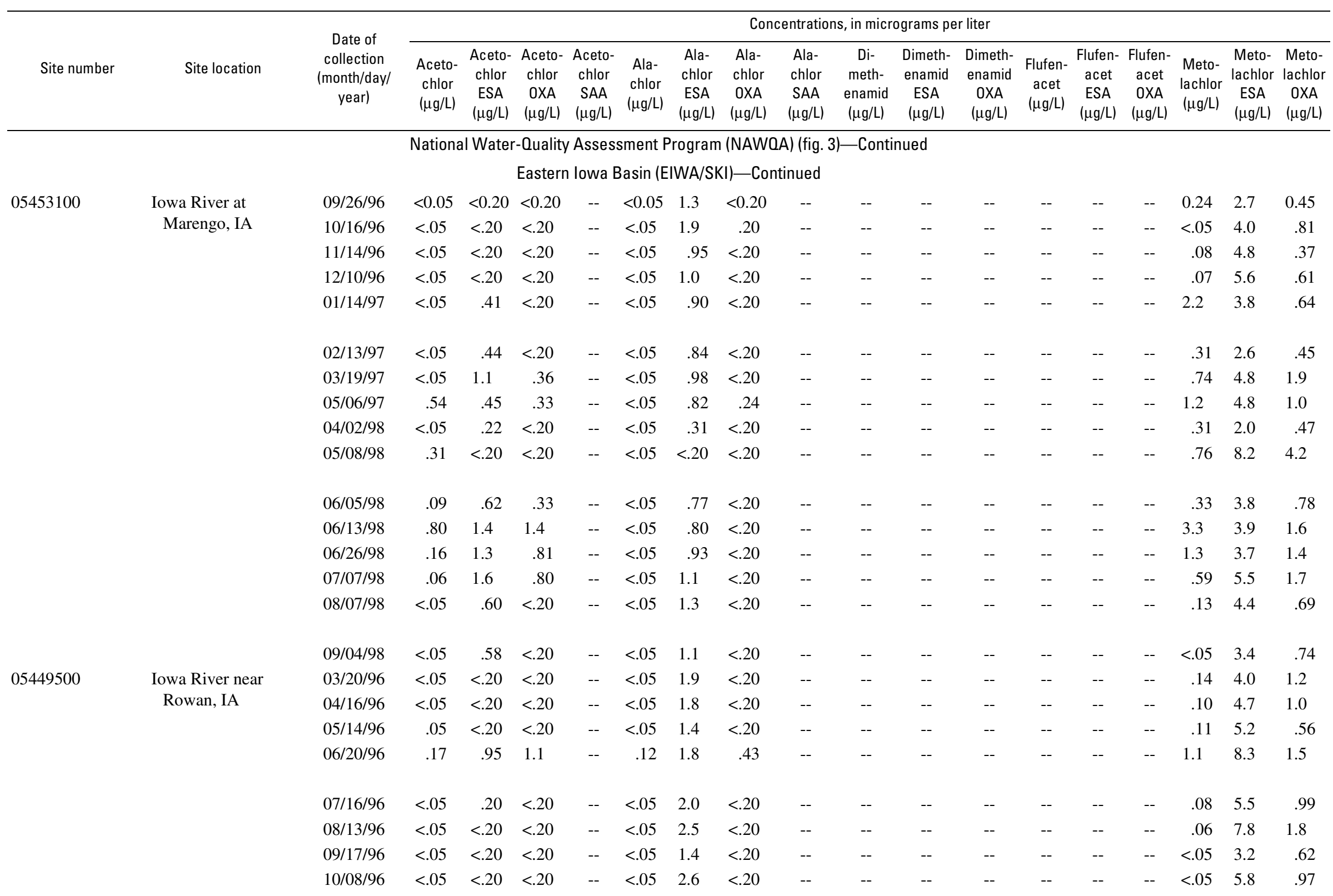


Table 6. Analytical results for selected acetamide herbicides and their degradation products, ethanesulfonic acid, oxanilic acid, and sulfinyl acetic acid, in surface-water samples, 1993-2003.-Continued

[OGRL, U.S. Geological Survey Organic Geochemistry Research Laboratory, Lawrence, Kansas; ESA, ethanesulfonic acid; OXA, oxanilic acid; SAA, sulfinyl acetic acid; --, no data; <, less than]

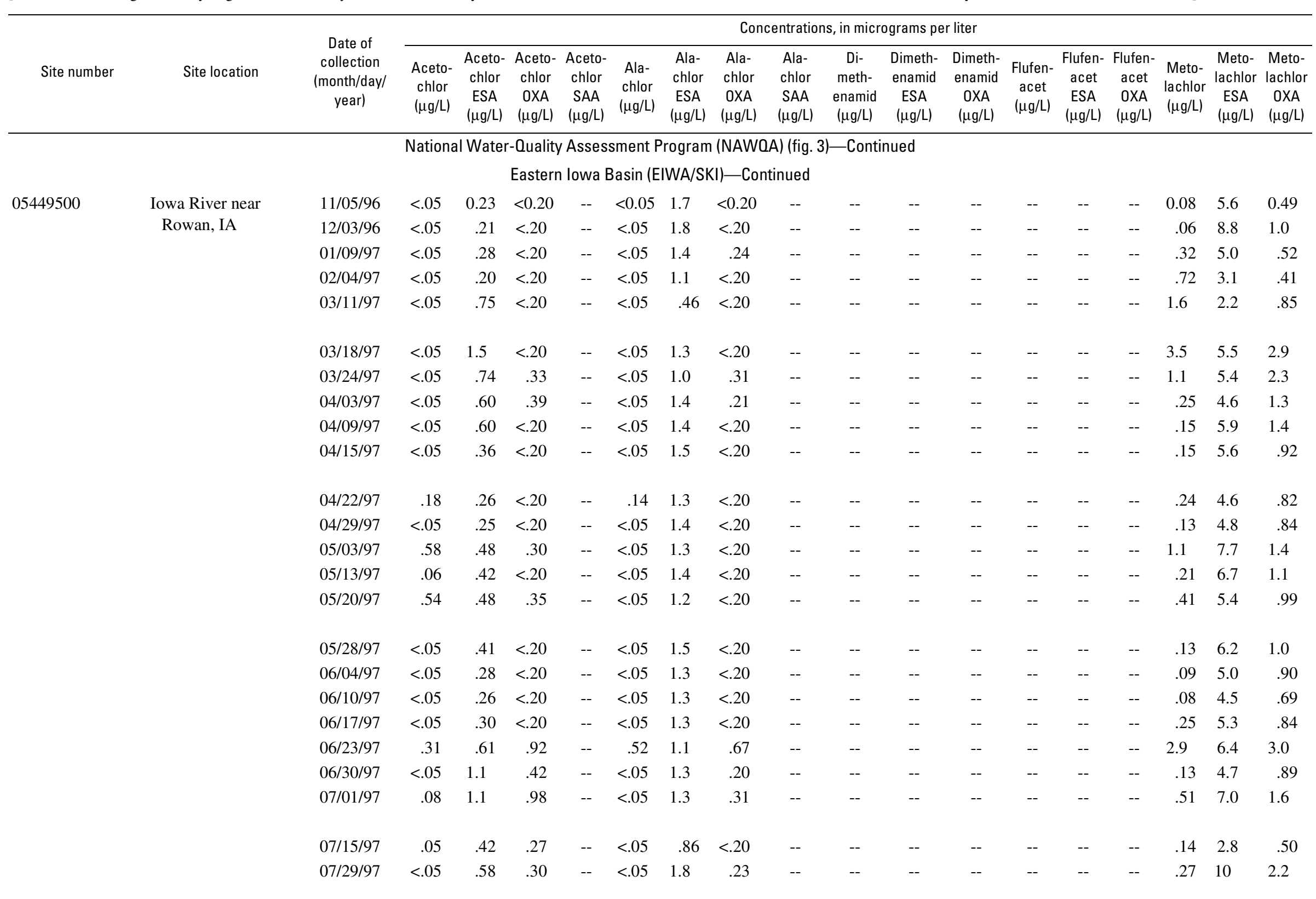


Table 6. Analytical results for selected acetamide herbicides and their degradation products, ethanesulfonic acid, oxanilic acid, and sulfinyl acetic acid, in surface-water samples, 1993-2003.-Continued

[OGRL, U.S. Geological Survey Organic Geochemistry Research Laboratory, Lawrence, Kansas; ESA, ethanesulfonic acid; OXA, oxanilic acid; SAA, sulfinyl acetic acid; --, no data; <, less than]

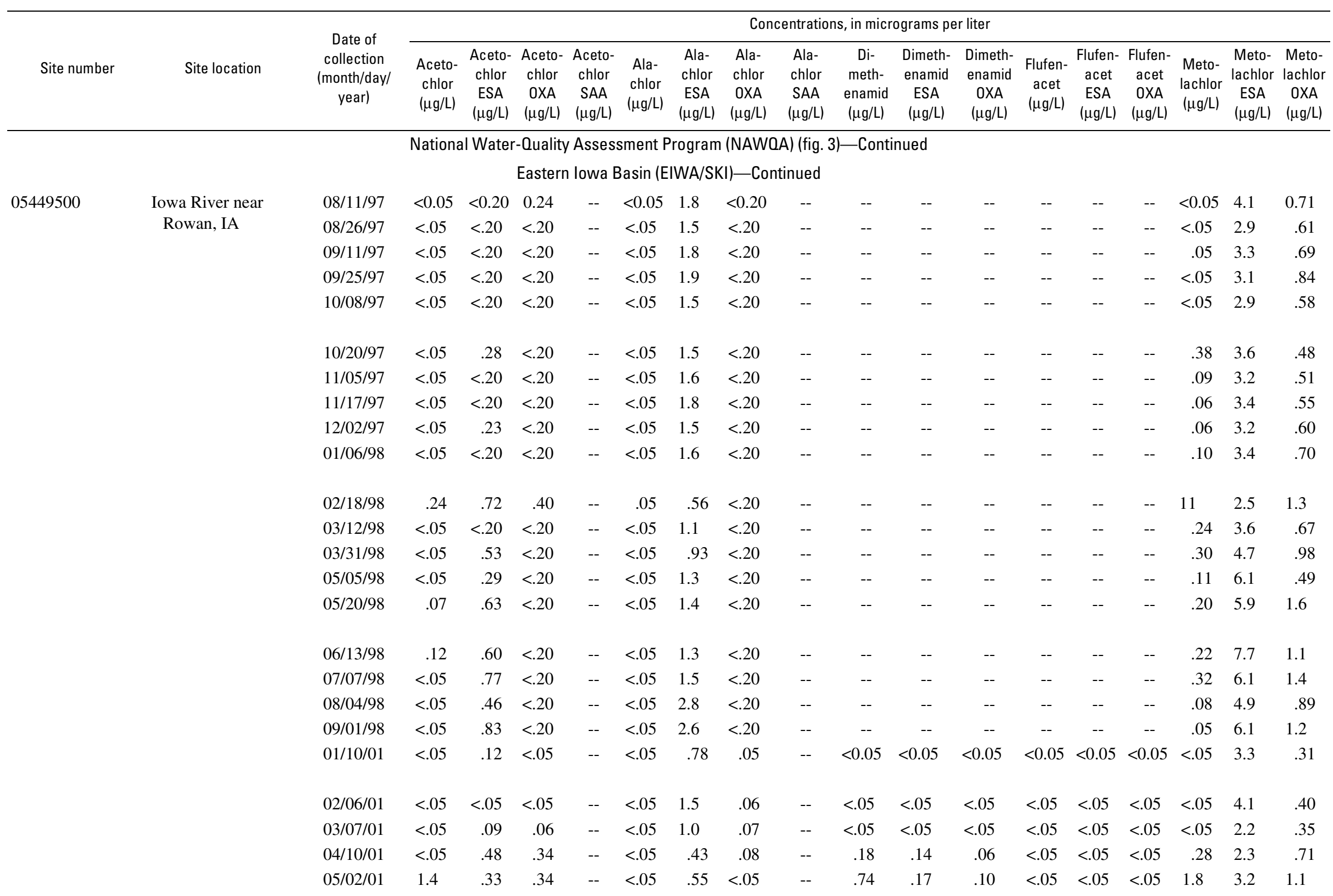


Table 6. Analytical results for selected acetamide herbicides and their degradation products, ethanesulfonic acid, oxanilic acid, and sulfinyl acetic acid, in surface-water samples, 1993-2003.-Continued

[OGRL, U.S. Geological Survey Organic Geochemistry Research Laboratory, Lawrence, Kansas; ESA, ethanesulfonic acid; OXA, oxanilic acid; SAA, sulfinyl acetic acid; --, no data; <, less than]

\begin{tabular}{|c|c|c|c|c|c|c|c|c|c|c|c|c|c|c|c|c|c|c|c|}
\hline \multirow[b]{2}{*}{ Site number } & \multirow[b]{2}{*}{ Site location } & \multirow[b]{2}{*}{$\begin{array}{c}\text { Date of } \\
\text { collection } \\
\text { (month/day/ } \\
\text { year) }\end{array}$} & \multicolumn{17}{|c|}{ Concentrations, in micrograms per liter } \\
\hline & & & $\begin{array}{l}\text { Aceto- } \\
\text { chlor } \\
(\mu \mathrm{g} / \mathrm{L})\end{array}$ & $\begin{array}{c}\text { Aceto- } \\
\text { chlor } \\
\text { ESA } \\
(\mu \mathrm{g} / \mathrm{L})\end{array}$ & $\begin{array}{l}\text { Aceto- } \\
\text { chlor } \\
\text { OXA } \\
(\mu \mathrm{g} / \mathrm{L})\end{array}$ & $\begin{array}{c}\text { Aceto- } \\
\text { chlor } \\
\text { SAA } \\
(\mu \mathrm{g} / \mathrm{L})\end{array}$ & $\begin{array}{l}\text { Ala- } \\
\text { chlor } \\
(\mu \mathrm{g} / \mathrm{L})\end{array}$ & $\begin{array}{l}\text { Ala- } \\
\text { chlor } \\
\text { ESA } \\
(\mu \mathrm{g} / \mathrm{L})\end{array}$ & $\begin{array}{c}\text { Ala- } \\
\text { chlor } \\
\text { OXA } \\
(\mu \mathrm{g} / \mathrm{L})\end{array}$ & $\begin{array}{l}\text { Ala- } \\
\text { chlor } \\
\text { SAA } \\
(\mu \mathrm{g} / \mathrm{L})\end{array}$ & $\begin{array}{c}\text { Di- } \\
\text { meth- } \\
\text { enamid } \\
(\mu \mathrm{g} / \mathrm{L})\end{array}$ & $\begin{array}{c}\text { Dimeth- } \\
\text { enamid } \\
\text { ESA } \\
(\mu \mathrm{g} / \mathrm{L})\end{array}$ & $\begin{array}{c}\text { Dimeth- } \\
\text { enamid } \\
\text { OXA } \\
(\mu \mathrm{g} / \mathrm{L})\end{array}$ & $\begin{array}{c}\text { Flufen- } \\
\text { acet } \\
(\mu \mathrm{g} / \mathrm{L})\end{array}$ & 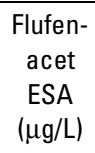 & $\begin{array}{c}\text { Flufen- } \\
\text { acet } \\
\text { OXA } \\
(\mu \mathrm{g} / \mathrm{L})\end{array}$ & $\begin{array}{l}\text { Meto- } \\
\text { lachlor } \\
(\mu \mathrm{g} / \mathrm{L})\end{array}$ & $\begin{array}{l}\text { Meto- } \\
\text { lachlor } \\
\text { ESA } \\
(\mu \mathrm{g} / \mathrm{L})\end{array}$ & $\begin{array}{c}\text { Meto- } \\
\text { lachlor } \\
\text { OXA } \\
(\mu \mathrm{g} / \mathrm{L})\end{array}$ \\
\hline \multicolumn{20}{|c|}{ National Water-Quality Assessment Program (NAWQA) (fig. 3)—Continued } \\
\hline \multicolumn{20}{|c|}{ Eastern lowa Basin (EIWA/SKI)—Continued } \\
\hline \multirow[t]{4}{*}{05449500} & \multirow{4}{*}{$\begin{array}{l}\text { Iowa River near } \\
\text { Rowan, IA }\end{array}$} & 06/08/01 & 0.12 & 0.92 & 0.47 & -- & $<0.05$ & 0.63 & $<0.05$ & -- & 0.11 & 0.18 & 0.06 & $<0.05$ & $<0.05$ & $<0.05$ & 0.09 & 4.5 & 0.82 \\
\hline & & $07 / 12 / 01$ & $<.05$ & .52 & .13 & -- & $<.05$ & .88 & $<.05$ & -- & $<.05$ & .13 & $<.05$ & $<.05$ & $<.05$ & $<.05$ & .06 & 3.4 & .52 \\
\hline & & 08/07/01 & $<.05$ & $<.05$ & $<.05$ & -- & $<.05$ & 1.5 & $<.05$ & -- & $<.05$ & $<.05$ & $<.05$ & $<.05$ & $<.05$ & $<.05$ & .06 & 3.9 & .49 \\
\hline & & 09/06/01 & $<.05$ & $<.05$ & .05 & -- & $<.05$ & .25 & $<.05$ & -- & $<.05$ & $<.05$ & $<.05$ & $<.05$ & $<.05$ & $<.05$ & $<.05$ & .45 & .44 \\
\hline 05451810 & $\begin{array}{l}\text { Iowa River at Tama, } \\
\text { IA }\end{array}$ & 07/25/96 & $<.05$ & .39 & $<.20$ & -- & $<.05$ & 1.1 & $<.20$ & -- & -- & -- & -- & -- & -- & -- & .10 & 4.2 & .80 \\
\hline \multirow[t]{18}{*}{05465500} & \multirow{18}{*}{$\begin{array}{l}\text { Iowa River at } \\
\text { Wapello, IA }\end{array}$} & 03/13/96 & $<.05$ & .35 & $<.20$ & -- & $<.05$ & 1.3 & .19 & -- & -- & -- & -- & -- & -- & -- & .91 & 2.3 & .51 \\
\hline & & 04/08/96 & $<.05$ & 1.4 & $<.20$ & -- & $<.05$ & 1.8 & $<.20$ & -- & -- & -- & -- & -- & -- & -- & 1.1 & 2.6 & $<.20$ \\
\hline & & 04/08/96 & $<.05$ & 1.7 & $<.20$ & -- & $<.05$ & 2.3 & $<.20$ & -- & -- & -- & -- & -- & -- & -- & 1.2 & 3.1 & $<.20$ \\
\hline & & 05/08/96 & .14 & $<.20$ & $<.20$ & -- & $<.05$ & 1.7 & $<.20$ & -- & -- & -- & -- & -- & -- & -- & .29 & 2.8 & .51 \\
\hline & & 06/11/96 & .28 & 2.6 & 2.1 & -- & $<.05$ & 2.6 & $<.20$ & -- & -- & -- & -- & -- & -- & -- & .64 & 6.7 & 2.5 \\
\hline & & 07/09/96 & $<.05$ & .82 & .61 & -- & $<.05$ & 2.0 & .20 & -- & -- & -- & -- & -- & -- & -- & .25 & 4.8 & 1.6 \\
\hline & & 08/06/96 & $<.05$ & .27 & $<.20$ & -- & $<.05$ & 1.7 & $<.20$ & -- & -- & -- & -- & -- & -- & -- & .07 & 3.1 & .73 \\
\hline & & 09/11/96 & $<.05$ & $<.20$ & $<.20$ & -- & $<.05$ & 1.6 & $<.20$ & -- & -- & -- & -- & -- & -- & -- & $<.05$ & 2.7 & .72 \\
\hline & & $10 / 08 / 96$ & $<.05$ & $<.20$ & $<.20$ & -- & $<.05$ & 1.6 & $<.20$ & -- & -- & -- & -- & -- & -- & -- & $<.05$ & 2.5 & .59 \\
\hline & & $11 / 06 / 96$ & $<.05$ & $<.20$ & .43 & -- & $<.05$ & 1.6 & $<.20$ & -- & -- & -- & -- & -- & -- & -- & .07 & 3.5 & 1.0 \\
\hline & & $12 / 03 / 96$ & $<.05$ & .28 & .27 & -- & $<.05$ & 1.6 & $<.20$ & -- & -- & -- & -- & -- & -- & -- & .12 & 4.4 & .51 \\
\hline & & 01/07/97 & $<.05$ & .37 & $<.20$ & -- & $<.05$ & 1.4 & $<.20$ & -- & -- & -- & -- & -- & -- & -- & .08 & 3.3 & .40 \\
\hline & & $02 / 05 / 97$ & $<.05$ & .47 & $<.20$ & -- & $<.05$ & 1.0 & $<.20$ & -- & -- & -- & -- & -- & -- & -- & .43 & 2.2 & .39 \\
\hline & & 03/11/97 & $<.05$ & .47 & $<.20$ & -- & $<.05$ & .58 & $<.20$ & -- & -- & -- & -- & -- & -- & -- & .68 & 1.8 & .41 \\
\hline & & 03/20/97 & $<.05$ & 1.0 & .40 & -- & $<.05$ & .91 & $<.20$ & -- & -- & -- & -- & -- & -- & -- & 1.1 & 3.1 & 1.3 \\
\hline & & 03/27/97 & $<.05$ & .29 & .33 & -- & $<.05$ & .74 & $<.20$ & -- & -- & -- & -- & -- & -- & -- & 1.0 & 2.6 & .79 \\
\hline & & 03/31/97 & $<.05$ & .43 & $<.20$ & -- & $<.05$ & .86 & $<.20$ & -- & -- & -- & -- & -- & -- & -- & .75 & 2.9 & .75 \\
\hline & & 04/10/97 & $<.05$ & .69 & .31 & -- & $<.05$ & 1.4 & $<.20$ & -- & -- & -- & -- & -- & -- & -- & .33 & 3.5 & .90 \\
\hline
\end{tabular}


Table 6. Analytical results for selected acetamide herbicides and their degradation products, ethanesulfonic acid, oxanilic acid, and sulfinyl acetic acid, in surface-water samples, 1993-2003.-Continued

[OGRL, U.S. Geological Survey Organic Geochemistry Research Laboratory, Lawrence, Kansas; ESA, ethanesulfonic acid; OXA, oxanilic acid; SAA, sulfinyl acetic acid; --, no data; <, less than]

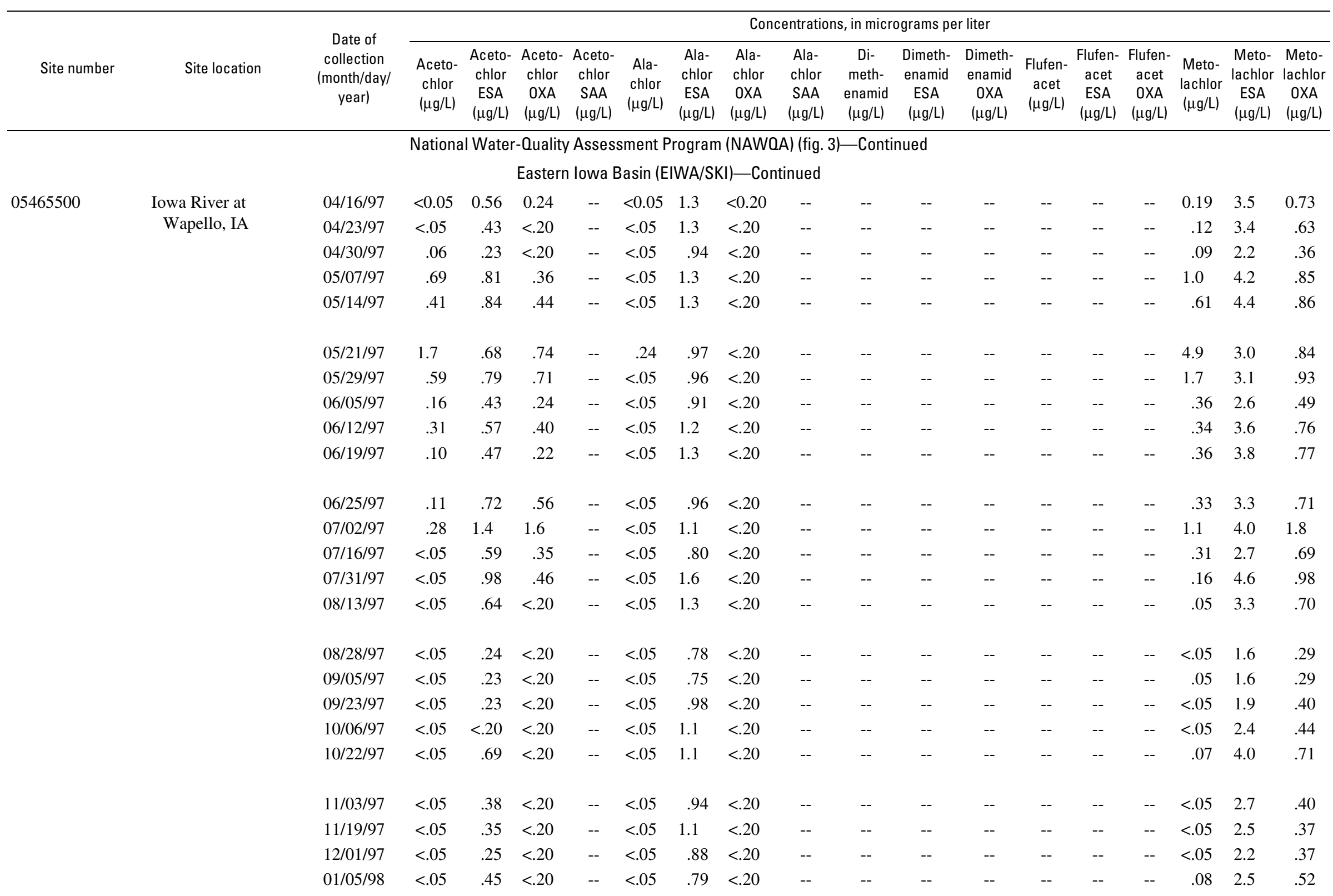


Table 6. Analytical results for selected acetamide herbicides and their degradation products, ethanesulfonic acid, oxanilic acid, and sulfinyl acetic acid, in surface-water samples, 1993-2003.-Continued

[OGRL, U.S. Geological Survey Organic Geochemistry Research Laboratory, Lawrence, Kansas; ESA, ethanesulfonic acid; OXA, oxanilic acid; SAA, sulfinyl acetic acid; --, no data; <, less than]

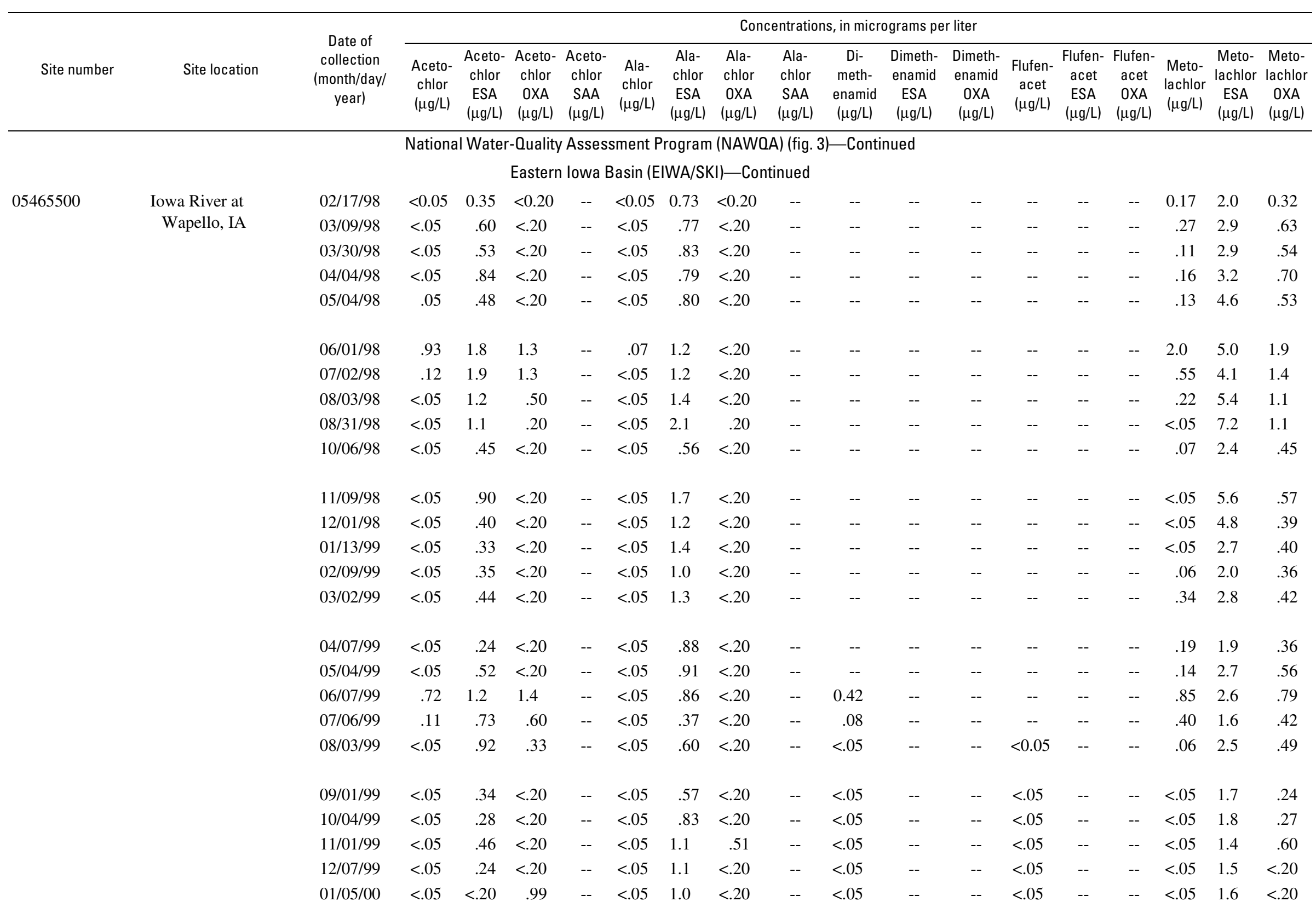


Table 6. Analytical results for selected acetamide herbicides and their degradation products, ethanesulfonic acid, oxanilic acid, and sulfinyl acetic acid, in surface-water samples, 1993-2003.-Continued

[OGRL, U.S. Geological Survey Organic Geochemistry Research Laboratory, Lawrence, Kansas; ESA, ethanesulfonic acid; OXA, oxanilic acid; SAA, sulfinyl acetic acid; --, no data; <, less than]

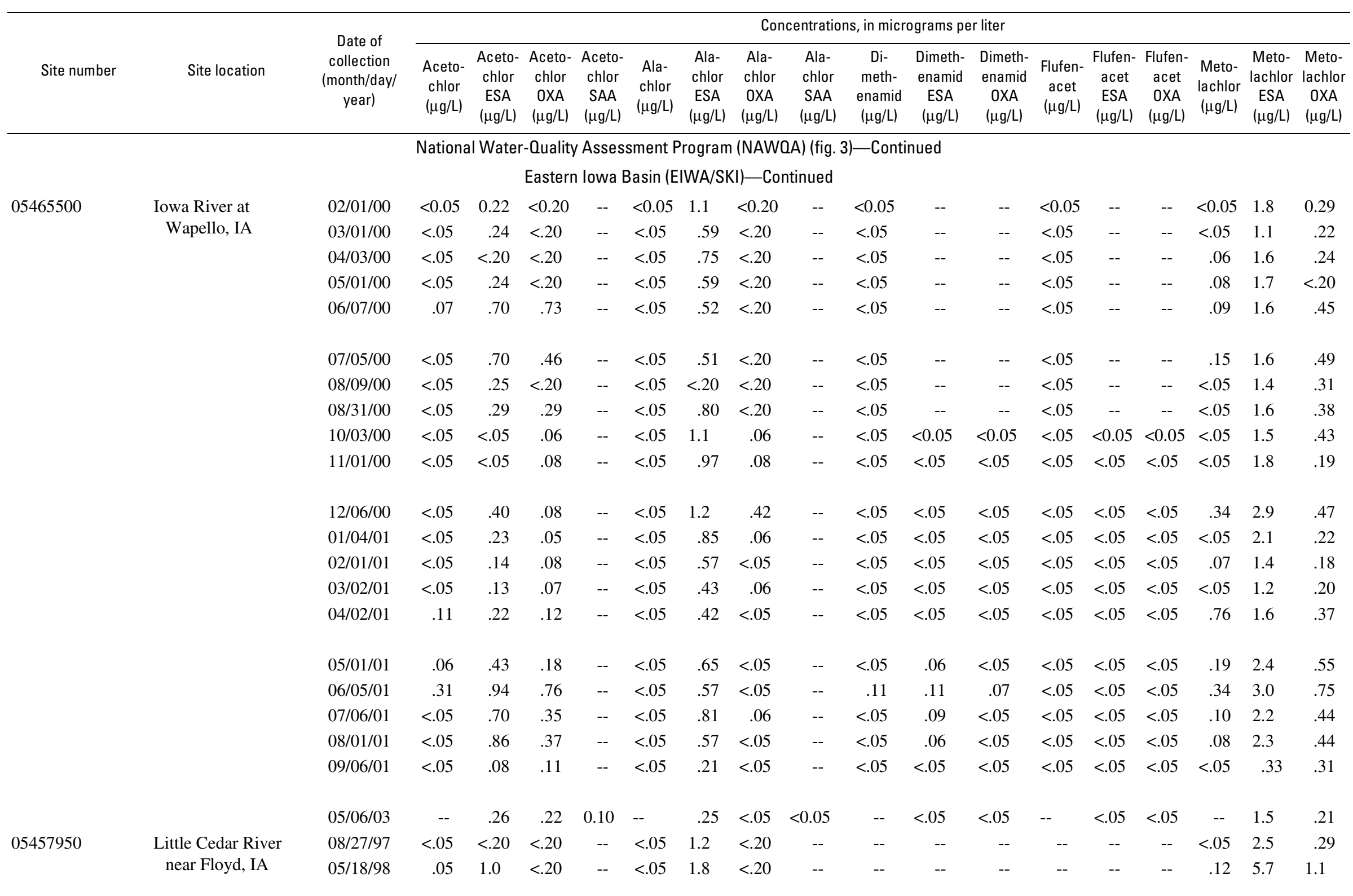


Table 6. Analytical results for selected acetamide herbicides and their degradation products, ethanesulfonic acid, oxanilic acid, and sulfinyl acetic acid, in surface-water samples, 1993-2003.-Continued

[OGRL, U.S. Geological Survey Organic Geochemistry Research Laboratory, Lawrence, Kansas; ESA, ethanesulfonic acid; OXA, oxanilic acid; SAA, sulfinyl acetic acid; --, no data; <, less than]

\begin{tabular}{|c|c|c|c|c|c|c|c|c|c|c|c|c|c|c|c|c|c|c|c|}
\hline \multirow[b]{2}{*}{ Site number } & \multirow[b]{2}{*}{ Site location } & \multirow[b]{2}{*}{$\begin{array}{c}\text { Date of } \\
\text { collection } \\
\text { (month/day/ } \\
\text { year) }\end{array}$} & \multicolumn{17}{|c|}{ Concentrations, in micrograms per liter } \\
\hline & & & $\begin{array}{l}\text { Aceto- } \\
\text { chlor } \\
(\mu \mathrm{g} / \mathrm{L})\end{array}$ & $\begin{array}{l}\text { Aceto- } \\
\text { chlor } \\
\text { ESA } \\
(\mu \mathrm{g} / \mathrm{L})\end{array}$ & $\begin{array}{c}\text { Aceto- } \\
\text { chlor } \\
\text { OXA } \\
(\mu \mathrm{g} / \mathrm{L})\end{array}$ & $\begin{array}{l}\text { Aceto- } \\
\text { chlor } \\
\text { SAA } \\
(\mu \mathrm{g} / \mathrm{L})\end{array}$ & $\begin{array}{l}\text { Ala- } \\
\text { chlor } \\
(\mu \mathrm{g} / \mathrm{L})\end{array}$ & $\begin{array}{l}\text { Ala- } \\
\text { chlor } \\
\text { ESA } \\
(\mu \mathrm{g} / \mathrm{L})\end{array}$ & $\begin{array}{l}\text { Ala- } \\
\text { chlor } \\
\text { OXA } \\
(\mu \mathrm{g} / \mathrm{L})\end{array}$ & $\begin{array}{l}\text { Ala- } \\
\text { chlor } \\
\text { SAA } \\
(\mu \mathrm{g} / \mathrm{L})\end{array}$ & $\begin{array}{c}\text { Di- } \\
\text { meth- } \\
\text { enamid } \\
(\mu \mathrm{g} / \mathrm{L})\end{array}$ & $\begin{array}{c}\text { Dimeth- } \\
\text { enamid } \\
\text { ESA } \\
(\mu \mathrm{g} / \mathrm{L})\end{array}$ & $\begin{array}{c}\text { Dimeth- } \\
\text { enamid } \\
\text { OXA } \\
(\mu \mathrm{g} / \mathrm{L})\end{array}$ & $\begin{array}{c}\text { Flufen- } \\
\text { acet } \\
(\mu \mathrm{g} / \mathrm{L})\end{array}$ & $\begin{array}{c}\text { Flufen- } \\
\text { acet } \\
\text { ESA } \\
(\mu \mathrm{g} / \mathrm{L})\end{array}$ & $\begin{array}{c}\text { Flufen- } \\
\text { acet } \\
\text { OXA } \\
(\mu \mathrm{g} / \mathrm{L})\end{array}$ & $\begin{array}{l}\text { Meto- } \\
\text { lachlor } \\
(\mu \mathrm{g} / \mathrm{L})\end{array}$ & $\begin{array}{l}\text { Meto- } \\
\text { lachlor } \\
\text { ESA } \\
(\mu \mathrm{g} / \mathrm{L})\end{array}$ & $\begin{array}{c}\text { Meto- } \\
\text { lachlor } \\
\text { OXA } \\
(\mu \mathrm{g} / \mathrm{L})\end{array}$ \\
\hline \multicolumn{20}{|c|}{ National Water-Quality Assessment Program (NAWQA) (fig. 3)—Continued } \\
\hline \multicolumn{20}{|c|}{ Eastern lowa Basin (EIWA/SKI)—Continued } \\
\hline \multirow[t]{2}{*}{05420900} & Little Wapsipinicon & $08 / 20 / 97$ & 0.14 & 0.55 & $<0.20$ & -- & $<0.05$ & 3.2 & $<0.20$ & -- & -- & -- & -- & -- & -- & -- & 0.12 & 2.1 & 0.70 \\
\hline & $\begin{array}{l}\text { River at Littleton, } \\
\text { IA }\end{array}$ & 05/20/98 & $<.05$ & 2.0 & $<.20$ & -- & $<.05$ & 4.0 & $<.20$ & -- & -- & -- & -- & -- & -- & -- & $<.05$ & 4.8 & .76 \\
\hline \multirow[t]{2}{*}{05465310} & Long Creek near & 08/12/97 & $<.05$ & .80 & .61 & -- & $<.05$ & .90 & $<.20$ & -- & -- & -- & -- & -- & -- & -- & .08 & 1.0 & .40 \\
\hline & $\begin{array}{l}\text { Columbus Junc- } \\
\text { tion, IA }\end{array}$ & 05/20/98 & .15 & .94 & $<.20$ & -- & $<.05$ & .55 & $<.20$ & -- & -- & -- & -- & -- & -- & -- & .07 & 2.5 & .56 \\
\hline \multirow[t]{3}{*}{05455870} & Maynes Creek near & $08 / 25 / 97$ & $<.05$ & .37 & $<.20$ & -- & $<.05$ & 1.3 & $<.20$ & -- & -- & -- & -- & -- & -- & -- & $<.05$ & 3.1 & .46 \\
\hline & Kesley, IA & $05 / 21 / 98$ & $<.05$ & $<.20$ & $<.20$ & -- & $<.05$ & .89 & $<.20$ & -- & -- & -- & -- & -- & -- & -- & .08 & 3.4 & .67 \\
\hline & & 09/09/98 & $<.05$ & $<.20$ & $<.20$ & -- & $<.05$ & .44 & $<.20$ & -- & -- & -- & -- & -- & -- & -- & $<.05$ & 1.4 & $<.20$ \\
\hline \multirow[t]{2}{*}{05421870} & Mud Creek near & 08/14/97 & $<.05$ & .20 & $<.20$ & -- & $<.05$ & 1.4 & $<.20$ & -- & -- & -- & -- & -- & -- & -- & .07 & 3.1 & .60 \\
\hline & Donahue, IA & 05/21/98 & .07 & .68 & $<.20$ & -- & $<.05$ & 1.1 & $<.20$ & -- & -- & -- & -- & -- & -- & -- & .10 & 6.2 & 1.0 \\
\hline \multirow[t]{15}{*}{05455100} & Old Man's Creek & 03/18/96 & $<.05$ & .22 & .14 & -- & $<.05$ & 1.0 & .25 & -- & -- & -- & -- & -- & -- & -- & .05 & .94 & .27 \\
\hline & near Iowa City, IA & $04 / 11 / 96$ & $<.05$ & $<.20$ & $<.20$ & -- & $<.05$ & 1.5 & $<.20$ & -- & -- & -- & -- & -- & -- & -- & $<.05$ & 1.2 & $<.20$ \\
\hline & & 05/07/96 & .19 & $<.20$ & $<.20$ & -- & $<.05$ & 1.3 & $<.20$ & -- & -- & -- & -- & -- & -- & -- & .32 & 1.4 & $<.20$ \\
\hline & & 05/10/96 & 2.5 & $<.20$ & .92 & -- & $<.05$ & $<.20$ & $<.20$ & -- & -- & -- & -- & -- & -- & -- & 9.7 & .77 & .28 \\
\hline & & $05 / 21 / 96$ & 3.2 & 1.4 & 2.1 & -- & $<.05$ & .74 & $<.20$ & -- & -- & -- & -- & -- & -- & -- & 3.6 & 1.7 & .88 \\
\hline & & $06 / 14 / 96$ & .06 & 1.3 & .24 & -- & $<.05$ & 1.1 & $<.20$ & -- & -- & -- & -- & -- & -- & -- & .19 & 1.7 & .53 \\
\hline & & $06 / 14 / 96$ & .05 & 1.7 & .39 & & $<.05$ & 1.6 & $<.20$ & -- & -- & -- & -- & -- & -- & -- & .21 & 2.6 & .60 \\
\hline & & $07 / 11 / 96$ & $<.05$ & 1.3 & .23 & -- & $<.05$ & 1.7 & $<.20$ & -- & -- & -- & -- & -- & -- & -- & .09 & 2.6 & .74 \\
\hline & & 08/08/96 & $<.05$ & .70 & $<.20$ & -- & $<.05$ & 1.9 & $<.20$ & -- & -- & -- & -- & -- & -- & -- & .08 & 1.9 & .59 \\
\hline & & 09/09/96 & $<.05$ & .29 & $<.20$ & -- & $<.05$ & 1.6 & $<.20$ & -- & -- & -- & -- & -- & -- & -- & .08 & 3.2 & .78 \\
\hline & & $10 / 07 / 96$ & $<.05$ & 1.9 & 1.7 & -- & $<.05$ & 2.4 & .24 & -- & -- & -- & -- & -- & -- & -- & .10 & 6.4 & 1.6 \\
\hline & & $10 / 24 / 96$ & .10 & .28 & $<.20$ & -- & $<.05$ & 1.3 & .79 & -- & -- & -- & -- & -- & -- & -- & .10 & .48 & .36 \\
\hline & & $11 / 12 / 96$ & $<.05$ & .45 & $<.20$ & -- & $<.05$ & 1.9 & .38 & -- & -- & -- & -- & -- & -- & -- & .07 & 1.4 & .39 \\
\hline & & $12 / 02 / 96$ & $<.05$ & .25 & $<.20$ & -- & $<.05$ & 1.5 & .36 & -- & -- & -- & -- & -- & -- & -- & .08 & .92 & .31 \\
\hline & & 01/06/97 & $<.05$ & .39 & .26 & -- & $<.05$ & 1.5 & .33 & -- & -- & -- & -- & -- & -- & -- & .09 & 1.2 & .26 \\
\hline
\end{tabular}


Table 6. Analytical results for selected acetamide herbicides and their degradation products, ethanesulfonic acid, oxanilic acid, and sulfinyl acetic acid, in surface-water samples, 1993-2003.-Continued

[OGRL, U.S. Geological Survey Organic Geochemistry Research Laboratory, Lawrence, Kansas; ESA, ethanesulfonic acid; OXA, oxanilic acid; SAA, sulfinyl acetic acid; --, no data; <, less than]

\begin{tabular}{|c|c|c|c|c|c|c|c|c|c|c|c|c|c|c|c|c|c|c|c|}
\hline \multirow[b]{2}{*}{ Site number } & \multirow[b]{2}{*}{ Site location } & \multirow[b]{2}{*}{$\begin{array}{c}\text { Date of } \\
\text { collection } \\
\text { (month/day/ } \\
\text { year) }\end{array}$} & \multicolumn{17}{|c|}{ Concentrations, in micrograms per liter } \\
\hline & & & $\begin{array}{l}\text { Aceto- } \\
\text { chlor } \\
(\mu \mathrm{g} / \mathrm{L})\end{array}$ & $\begin{array}{c}\text { Aceto- } \\
\text { chlor } \\
\text { ESA } \\
(\mu \mathrm{g} / \mathrm{L})\end{array}$ & $\begin{array}{c}\text { Aceto- } \\
\text { chlor } \\
\text { OXA } \\
(\mu \mathrm{g} / \mathrm{L})\end{array}$ & $\begin{array}{c}\text { Aceto- } \\
\text { chlor } \\
\text { SAA } \\
(\mu \mathrm{g} / \mathrm{L})\end{array}$ & $\begin{array}{l}\text { Ala- } \\
\text { chlor } \\
(\mu \mathrm{g} / \mathrm{L})\end{array}$ & $\begin{array}{c}\text { Ala- } \\
\text { chlor } \\
\text { ESA } \\
(\mu \mathrm{g} / \mathrm{L})\end{array}$ & $\begin{array}{c}\text { Ala- } \\
\text { chlor } \\
\text { OXA } \\
(\mu \mathrm{g} / \mathrm{L})\end{array}$ & $\begin{array}{l}\text { Ala- } \\
\text { chlor } \\
\text { SAA } \\
(\mu \mathrm{g} / \mathrm{L})\end{array}$ & $\begin{array}{c}\text { Di- } \\
\text { meth- } \\
\text { enamid } \\
(\mu \mathrm{g} / \mathrm{L})\end{array}$ & $\begin{array}{c}\text { Dimeth- } \\
\text { enamid } \\
\text { ESA } \\
(\mu \mathrm{g} / \mathrm{L})\end{array}$ & $\begin{array}{c}\text { Dimeth- } \\
\text { enamid } \\
\text { OXA } \\
(\mu \mathrm{g} / \mathrm{L})\end{array}$ & $\begin{array}{c}\text { Flufen- } \\
\text { acet } \\
(\mu \mathrm{g} / \mathrm{L})\end{array}$ & $\begin{array}{c}\text { Flufen- } \\
\text { acet } \\
\text { ESA } \\
(\mu \mathrm{g} / \mathrm{L})\end{array}$ & $\begin{array}{c}\text { Flufen- } \\
\text { acet } \\
\text { OXA } \\
(\mu \mathrm{g} / \mathrm{L})\end{array}$ & $\begin{array}{c}\text { Meto- } \\
\text { lachlor } \\
(\mu \mathrm{g} / \mathrm{L})\end{array}$ & $\begin{array}{l}\text { Meto- } \\
\text { lachlor } \\
\text { ESA } \\
(\mu \mathrm{g} / \mathrm{L})\end{array}$ & $\begin{array}{c}\text { Meto- } \\
\text { lachlor } \\
\text { OXA } \\
(\mu \mathrm{g} / \mathrm{L})\end{array}$ \\
\hline \multicolumn{20}{|c|}{ National Water-Quality Assessment Program (NAWOA) (fig. 3)—Continued } \\
\hline \multicolumn{20}{|c|}{ Eastern lowa Basin (EIWA/SKI)—Continued } \\
\hline \multirow[t]{14}{*}{05455100} & \multirow{5}{*}{$\begin{array}{l}\text { Old Man's Creek } \\
\text { near Iowa City, IA }\end{array}$} & 02/04/97 & 0.14 & 0.58 & 0.49 & -- & $<0.05$ & 0.28 & $<0.20$ & -- & -- & -- & -- & -- & -- & -- & 0.34 & 0.84 & 0.45 \\
\hline & & 03/10/97 & $<.05$ & 1.6 & 1.6 & -- & $<.05$ & .62 & .51 & -- & -- & -- & -- & -- & -- & -- & .34 & 1.9 & 1.3 \\
\hline & & $05 / 01 / 97$ & 1.6 & .28 & .30 & -- & $<.05$ & .44 & $<.20$ & -- & -- & -- & -- & -- & -- & -- & 1.7 & 1.4 & .39 \\
\hline & & 05/08/97 & 4.4 & .81 & .84 & -- & $<.05$ & .61 & $<.20$ & -- & -- & -- & -- & -- & -- & -- & 2.1 & 1.7 & .67 \\
\hline & & 08/11/97 & $<.05$ & .34 & .40 & -- & $<.05$ & 1.3 & .29 & -- & -- & -- & -- & -- & -- & -- & $<.05$ & 1.6 & .36 \\
\hline & & $03 / 31 / 98$ & $<.05$ & $<.20$ & $<.20$ & -- & $<.05$ & .25 & $<.20$ & -- & -- & -- & -- & -- & -- & -- & .09 & 1.2 & $<.20$ \\
\hline & & $05 / 05 / 98$ & $<.05$ & .63 & $<.20$ & -- & $<.05$ & .71 & $<.20$ & -- & -- & -- & -- & -- & -- & -- & .05 & 3.0 & .59 \\
\hline & & $05 / 18 / 98$ & .09 & 1.1 & $<.20$ & -- & $<.05$ & 1.1 & $<.20$ & -- & -- & -- & -- & -- & -- & -- & .09 & 2.6 & .81 \\
\hline & & 05/18/98 & .10 & 1.2 & $<.20$ & -- & $<.05$ & 1.1 & $<.20$ & -- & -- & -- & -- & -- & -- & -- & .10 & 2.6 & .86 \\
\hline & & $06 / 02 / 98$ & .05 & 1.3 & $<.20$ & -- & $<.05$ & 1.2 & $<.20$ & -- & -- & -- & -- & -- & -- & -- & .09 & 3.0 & .70 \\
\hline & & 07/10/98 & $<.05$ & .97 & $<.20$ & -- & $<.05$ & 1.0 & $<.20$ & -- & -- & -- & -- & -- & -- & -- & .06 & 2.9 & .65 \\
\hline & & 08/05/98 & $<.05$ & .48 & $<.20$ & -- & $<.05$ & 1.0 & $<.20$ & -- & -- & -- & -- & -- & -- & -- & $<.05$ & 1.9 & .31 \\
\hline & & $09 / 01 / 98$ & $<.05$ & .49 & $<.20$ & -- & $<.05$ & .91 & $<.20$ & -- & -- & -- & -- & -- & -- & -- & $<.05$ & 3.2 & .42 \\
\hline & & 11/10/99 & $<.05$ & $<.20$ & $<.20$ & -- & $<.05$ & 1.2 & .20 & -- & -- & -- & -- & -- & -- & -- & $<.05$ & 1.1 & .25 \\
\hline \multirow[t]{2}{*}{05452020} & \multirow{2}{*}{$\begin{array}{l}\text { Salt Creek at Belle } \\
\text { Plaine, IA }\end{array}$} & $08 / 21 / 97$ & $<.05$ & $<.20$ & $<.20$ & -- & $<.05$ & 1.2 & $<.20$ & -- & -- & -- & -- & -- & -- & -- & $<.05$ & 2.2 & .21 \\
\hline & & 05/18/98 & $<.05$ & .24 & $<.20$ & -- & $<.05$ & 1.2 & $<.20$ & -- & -- & -- & -- & -- & -- & -- & $<.05$ & 3.9 & .39 \\
\hline \multirow[t]{9}{*}{05474000} & \multirow{9}{*}{$\begin{array}{l}\text { Skunk River at } \\
\text { Augusta, IA }\end{array}$} & $03 / 12 / 96$ & $<.05$ & .25 & .23 & -- & $<.05$ & .51 & .34 & -- & -- & -- & -- & -- & -- & -- & .35 & 1.4 & .45 \\
\hline & & $04 / 09 / 96$ & $<.05$ & .73 & $<.20$ & -- & $<.05$ & .94 & $<.20$ & -- & -- & -- & -- & -- & -- & -- & .14 & 1.8 & $<.20$ \\
\hline & & $05 / 09 / 96$ & 3.7 & 1.3 & 2.7 & -- & .42 & .64 & .50 & -- & -- & -- & -- & -- & -- & -- & 3.0 & 1.5 & .97 \\
\hline & & $06 / 12 / 96$ & .13 & .91 & .68 & -- & $<.05$ & .56 & $<.20$ & -- & -- & -- & -- & -- & -- & -- & .62 & 2.4 & .67 \\
\hline & & $07 / 10 / 96$ & 1.6 & .86 & .77 & -- & $<.05$ & .60 & $<.20$ & -- & -- & -- & -- & -- & -- & -- & .32 & 3.8 & 1.1 \\
\hline & & 08/07/96 & $<.05$ & .40 & $<.20$ & -- & $<.05$ & .42 & $<.20$ & -- & -- & -- & -- & -- & -- & -- & .16 & 2.1 & .61 \\
\hline & & $09 / 12 / 96$ & $<.05$ & $<.20$ & $<.20$ & -- & $<.05$ & .43 & $<.20$ & -- & -- & -- & -- & -- & -- & -- & .09 & .81 & .50 \\
\hline & & $10 / 09 / 96$ & $<.05$ & $<.20$ & $<.20$ & -- & $<.05$ & .41 & $<.20$ & -- & -- & -- & -- & -- & -- & -- & .12 & 2.3 & .59 \\
\hline & & $11 / 07 / 96$ & $<.05$ & $<.20$ & $<.20$ & -- & $<.05$ & .32 & $<.20$ & -- & -- & -- & -- & -- & -- & -- & .13 & 2.7 & $<.20$ \\
\hline
\end{tabular}


Table 6. Analytical results for selected acetamide herbicides and their degradation products, ethanesulfonic acid, oxanilic acid, and sulfinyl acetic acid, in surface-water samples, 1993-2003.-Continued

[OGRL, U.S. Geological Survey Organic Geochemistry Research Laboratory, Lawrence, Kansas; ESA, ethanesulfonic acid; OXA, oxanilic acid; SAA, sulfinyl acetic acid; --, no data; <, less than]

\begin{tabular}{|c|c|c|c|c|c|c|c|c|c|c|c|c|c|c|c|c|c|c|c|}
\hline \multirow[b]{2}{*}{ Site number } & \multirow[b]{2}{*}{ Site location } & \multirow[b]{2}{*}{$\begin{array}{c}\text { Date of } \\
\text { collection } \\
\text { (month/day/ } \\
\text { year) }\end{array}$} & \multicolumn{17}{|c|}{ Concentrations, in micrograms per liter } \\
\hline & & & $\begin{array}{l}\text { Aceto- } \\
\text { chlor } \\
(\mu \mathrm{g} / \mathrm{L})\end{array}$ & $\begin{array}{l}\text { Aceto- } \\
\text { chlor } \\
\text { ESA } \\
(\mu \mathrm{g} / \mathrm{L})\end{array}$ & $\begin{array}{c}\text { Aceto- } \\
\text { chlor } \\
\text { OXA } \\
(\mu \mathrm{g} / \mathrm{L})\end{array}$ & $\begin{array}{l}\text { Aceto- } \\
\text { chlor } \\
\text { SAA } \\
(\mu \mathrm{g} / \mathrm{L})\end{array}$ & $\begin{array}{l}\text { Ala- } \\
\text { chlor } \\
(\mu \mathrm{g} / \mathrm{L})\end{array}$ & $\begin{array}{l}\text { Ala- } \\
\text { chlor } \\
\text { ESA } \\
(\mu \mathrm{g} / \mathrm{L})\end{array}$ & $\begin{array}{l}\text { Ala- } \\
\text { chlor } \\
\text { OXA } \\
(\mu \mathrm{g} / \mathrm{L})\end{array}$ & $\begin{array}{l}\text { Ala- } \\
\text { chlor } \\
\text { SAA } \\
(\mu \mathrm{g} / \mathrm{L})\end{array}$ & $\begin{array}{c}\text { Di- } \\
\text { meth- } \\
\text { enamid } \\
(\mu \mathrm{g} / \mathrm{L})\end{array}$ & $\begin{array}{c}\text { Dimeth- } \\
\text { enamid } \\
\text { ESA } \\
(\mu \mathrm{g} / \mathrm{L})\end{array}$ & $\begin{array}{c}\text { Dimeth- } \\
\text { enamid } \\
\text { OXA } \\
(\mu \mathrm{g} / \mathrm{L})\end{array}$ & $\begin{array}{l}\text { Flufen- } \\
\text { acet } \\
(\mu \mathrm{g} / \mathrm{L})\end{array}$ & $\begin{array}{l}\text { Flufen- } \\
\text { acet } \\
\text { ESA } \\
(\mu \mathrm{g} / \mathrm{L})\end{array}$ & $\begin{array}{c}\text { Flufen- } \\
\text { acet } \\
\text { OXA } \\
(\mu \mathrm{g} / \mathrm{L})\end{array}$ & $\begin{array}{l}\text { Meto- } \\
\text { lachlor } \\
(\mu \mathrm{g} / \mathrm{L})\end{array}$ & $\begin{array}{l}\text { Meto- } \\
\text { lachlor } \\
\text { ESA } \\
(\mu \mathrm{g} / \mathrm{L})\end{array}$ & $\begin{array}{c}\text { Meto- } \\
\text { lachlor } \\
\text { OXA } \\
(\mu \mathrm{g} / \mathrm{L})\end{array}$ \\
\hline \multicolumn{20}{|c|}{ National Water-Quality Assessment Program (NAWQA) (fig. 3)—Continued } \\
\hline \multicolumn{20}{|c|}{ Eastern lowa Basin (EIWA/SKI)_Continued } \\
\hline \multirow[t]{11}{*}{05474000} & \multirow{11}{*}{$\begin{array}{c}\text { Skunk River at } \\
\text { Augusta, IA }\end{array}$} & $12 / 04 / 96$ & $<0.05$ & 0.21 & 0.27 & -- & $<0.05$ & 0.34 & $<0.20$ & -- & -- & -- & -- & -- & -- & -- & 0.09 & 4.7 & 0.52 \\
\hline & & $01 / 08 / 97$ & $<.05$ & .29 & $<.20$ & -- & $<.05$ & .40 & $<.20$ & -- & -- & -- & -- & -- & -- & -- & $<.05$ & 3.1 & .39 \\
\hline & & $02 / 06 / 97$ & $<.05$ & .63 & $<.20$ & -- & $<.05$ & .31 & $<.20$ & -- & -- & -- & -- & -- & -- & -- & .29 & 1.4 & .41 \\
\hline & & $03 / 12 / 97$ & $<.05$ & .51 & $<.20$ & -- & $<.05$ & .56 & $<.20$ & -- & -- & -- & -- & -- & -- & -- & .21 & 2.4 & .81 \\
\hline & & $03 / 30 / 98$ & $<.05$ & $<.20$ & $<.20$ & -- & $<.05$ & .21 & $<.20$ & -- & -- & -- & -- & -- & -- & -- & .08 & 1.1 & $<.20$ \\
\hline & & $05 / 05 / 98$ & .14 & .30 & $<.20$ & -- & $<.05$ & .39 & $<.20$ & -- & -- & -- & -- & -- & -- & -- & .64 & 3.5 & .39 \\
\hline & & $05 / 26 / 98$ & 7.6 & 2.7 & 4.0 & -- & .10 & .87 & $<.20$ & -- & -- & -- & -- & -- & -- & -- & 6.5 & 2.9 & 1.6 \\
\hline & & $06 / 02 / 98$ & .62 & 1.2 & 1.3 & -- & $<.05$ & .77 & $<.20$ & -- & -- & -- & -- & -- & -- & -- & 1.5 & 3.5 & 1.3 \\
\hline & & 07/08/98 & .09 & 2.1 & 1.6 & -- & $<.05$ & .96 & .20 & -- & -- & -- & -- & -- & -- & -- & .82 & 3.9 & 1.9 \\
\hline & & 08/04/98 & $<.05$ & .61 & .20 & -- & $<.05$ & .60 & $<.20$ & -- & -- & -- & -- & -- & -- & -- & .06 & 3.4 & .56 \\
\hline & & 09/01/98 & $<.05$ & .63 & $<.20$ & -- & $<.05$ & .44 & $<.20$ & -- & -- & -- & -- & -- & -- & -- & .13 & 2.6 & .83 \\
\hline \multirow[t]{12}{*}{05451210} & \multirow{12}{*}{$\begin{array}{c}\text { South Fork Iowa } \\
\text { River near New } \\
\text { Providence, IA }\end{array}$} & $03 / 19 / 96$ & $<.05$ & $<.20$ & $<.20$ & -- & $<.05$ & .91 & $<.20$ & -- & -- & -- & -- & -- & -- & -- & .87 & 3.3 & 1.0 \\
\hline & & $04 / 15 / 96$ & $<.05$ & $<.20$ & $<.20$ & -- & $<.05$ & .94 & $<.20$ & -- & -- & -- & -- & -- & -- & -- & .39 & 4.8 & .86 \\
\hline & & 05/13/96 & .43 & $<.20$ & $<.20$ & -- & $<.05$ & .79 & $<.20$ & -- & -- & -- & -- & -- & -- & -- & .57 & 4.7 & .86 \\
\hline & & 06/17/96 & .34 & 1.9 & 2.6 & -- & $<.05$ & 1.3 & $<.20$ & -- & -- & -- & -- & -- & -- & -- & 2.7 & 9.5 & 5.3 \\
\hline & & $07 / 15 / 96$ & $<.05$ & $<.20$ & .23 & -- & $<.05$ & .77 & $<.20$ & -- & -- & -- & -- & -- & -- & -- & .09 & 5.2 & .90 \\
\hline & & $08 / 12 / 96$ & $<.05$ & .27 & $<.20$ & -- & $<.05$ & 1.3 & $<.20$ & -- & -- & -- & -- & -- & -- & -- & .14 & 6.4 & 1.9 \\
\hline & & $09 / 16 / 96$ & $<.05$ & $<.20$ & $<.20$ & -- & $<.05$ & .67 & $<.20$ & -- & -- & -- & -- & -- & -- & -- & .05 & 2.5 & .96 \\
\hline & & $10 / 07 / 96$ & $<.05$ & $<.20$ & $<.20$ & -- & $<.05$ & .70 & .27 & -- & -- & -- & -- & -- & -- & -- & .30 & 3.6 & .83 \\
\hline & & $11 / 04 / 96$ & $<.05$ & .22 & $<.20$ & -- & $<.05$ & .67 & $<.20$ & -- & -- & -- & -- & -- & -- & -- & .21 & 5.1 & .56 \\
\hline & & $12 / 02 / 96$ & $<.05$ & $<.20$ & $<.20$ & -- & $<.05$ & .76 & $<.20$ & -- & -- & -- & -- & -- & -- & -- & .32 & 7.7 & .74 \\
\hline & & $01 / 06 / 97$ & .05 & .78 & $<.20$ & -- & $<.05$ & .22 & $<.20$ & -- & -- & -- & -- & -- & -- & -- & 5.5 & 2.1 & .44 \\
\hline & & $02 / 03 / 97$ & $<.05$ & .35 & $<.20$ & -- & $<.05$ & .23 & $<.20$ & -- & -- & -- & -- & -- & -- & -- & 1.1 & 1.8 & .31 \\
\hline
\end{tabular}


Table 6. Analytical results for selected acetamide herbicides and their degradation products, ethanesulfonic acid, oxanilic acid, and sulfinyl acetic acid, in surface-water samples, 1993-2003.-Continued

[OGRL, U.S. Geological Survey Organic Geochemistry Research Laboratory, Lawrence, Kansas; ESA, ethanesulfonic acid; OXA, oxanilic acid; SAA, sulfinyl acetic acid; --, no data; <, less than]

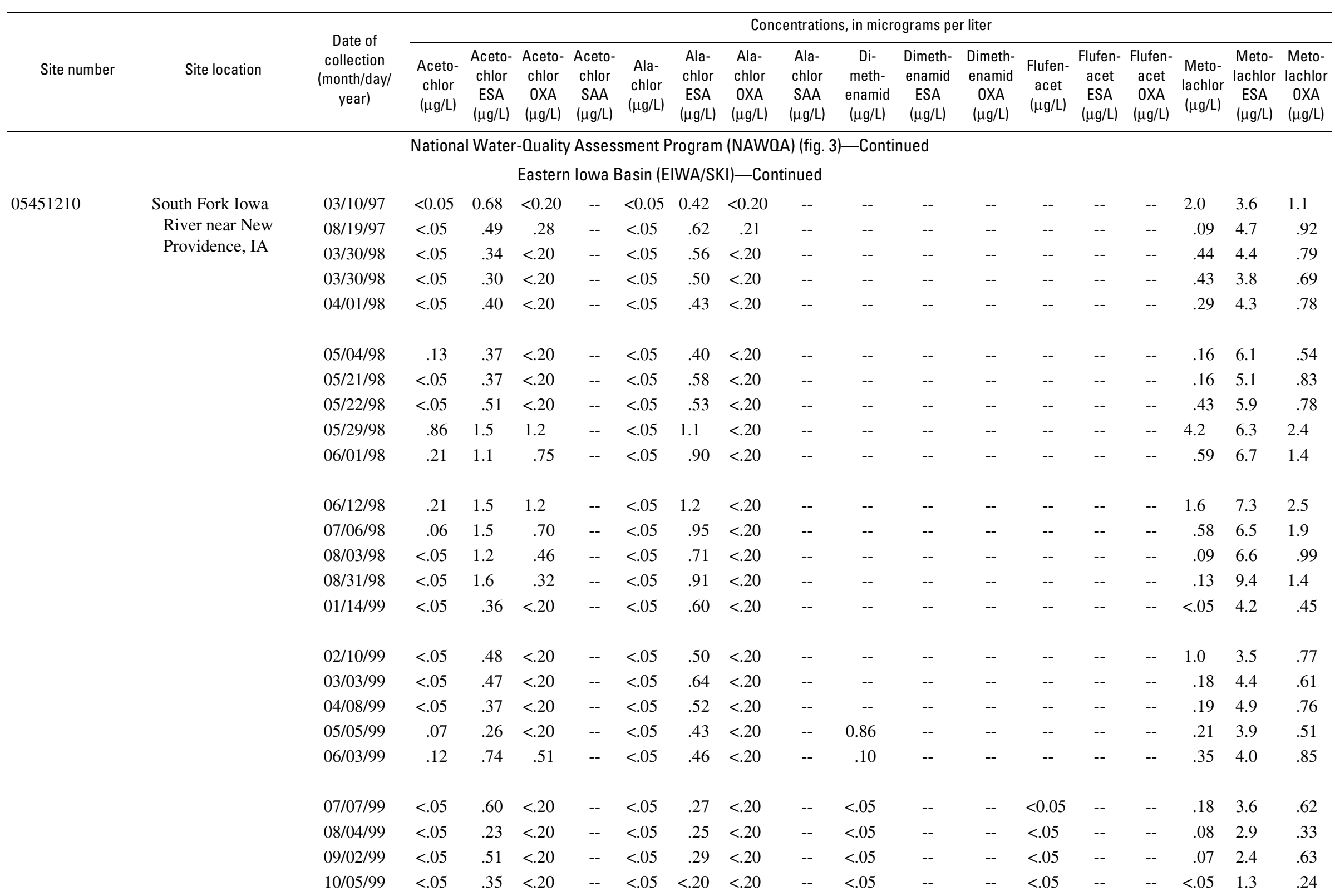


Table 6. Analytical results for selected acetamide herbicides and their degradation products, ethanesulfonic acid, oxanilic acid, and sulfinyl acetic acid, in surface-water samples, 1993-2003.-Continued

[OGRL, U.S. Geological Survey Organic Geochemistry Research Laboratory, Lawrence, Kansas; ESA, ethanesulfonic acid; OXA, oxanilic acid; SAA, sulfinyl acetic acid; --, no data; <, less than]

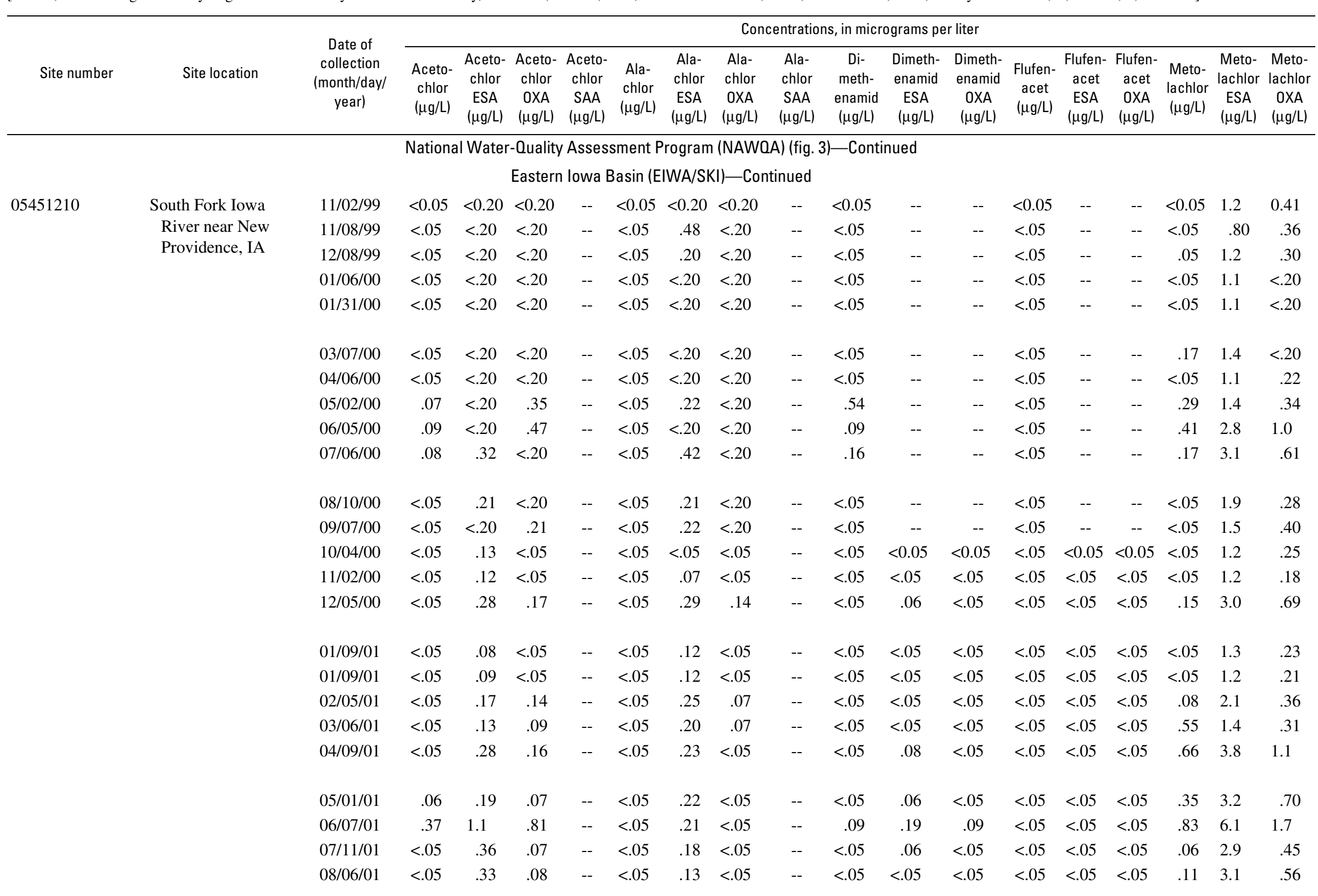


Table 6. Analytical results for selected acetamide herbicides and their degradation products, ethanesulfonic acid, oxanilic acid, and sulfinyl acetic acid, in surface-water samples, 1993-2003.-Continued

[OGRL, U.S. Geological Survey Organic Geochemistry Research Laboratory, Lawrence, Kansas; ESA, ethanesulfonic acid; OXA, oxanilic acid; SAA, sulfinyl acetic acid; --, no data; <, less than]

\begin{tabular}{|c|c|c|c|c|c|c|c|c|c|c|c|c|c|c|c|c|c|c|c|}
\hline \multirow[b]{2}{*}{ Site number } & \multirow[b]{2}{*}{ Site location } & \multirow[b]{2}{*}{$\begin{array}{c}\text { Date of } \\
\text { collection } \\
\text { (month/day/ } \\
\text { year) }\end{array}$} & \multicolumn{17}{|c|}{ Concentrations, in micrograms per liter } \\
\hline & & & $\begin{array}{l}\text { Aceto- } \\
\text { chlor } \\
(\mu \mathrm{g} / \mathrm{L})\end{array}$ & $\begin{array}{l}\text { Aceto- } \\
\text { chlor } \\
\text { ESA } \\
(\mu \mathrm{g} / \mathrm{L})\end{array}$ & $\begin{array}{l}\text { Aceto- } \\
\text { chlor } \\
\text { OXA } \\
(\mu \mathrm{g} / \mathrm{L})\end{array}$ & $\begin{array}{l}\text { Aceto- } \\
\text { chlor } \\
\text { SAA } \\
(\mu \mathrm{g} / \mathrm{L})\end{array}$ & $\begin{array}{l}\text { Ala- } \\
\text { chlor } \\
(\mu \mathrm{g} / \mathrm{L})\end{array}$ & $\begin{array}{l}\text { Ala- } \\
\text { chlor } \\
\text { ESA } \\
(\mu \mathrm{g} / \mathrm{L})\end{array}$ & $\begin{array}{l}\text { Ala- } \\
\text { chlor } \\
\text { OXA } \\
(\mu \mathrm{g} / \mathrm{L})\end{array}$ & $\begin{array}{l}\text { Ala- } \\
\text { chlor } \\
\text { SAA } \\
(\mu \mathrm{g} / \mathrm{L})\end{array}$ & $\begin{array}{l}\text { Di- } \\
\text { meth- } \\
\text { enamid } \\
(\mu \mathrm{g} / \mathrm{L})\end{array}$ & $\begin{array}{c}\text { Dimeth- } \\
\text { enamid } \\
\text { ESA } \\
(\mu \mathrm{g} / \mathrm{L})\end{array}$ & $\begin{array}{c}\text { Dimeth- } \\
\text { enamid } \\
\text { OXA } \\
(\mu \mathrm{g} / \mathrm{L})\end{array}$ & $\begin{array}{l}\text { Flufen- } \\
\text { acet } \\
(\mu \mathrm{g} / \mathrm{L})\end{array}$ & $\begin{array}{l}\text { Flufen- } \\
\text { acet } \\
\text { ESA } \\
(\mu \mathrm{g} / \mathrm{L})\end{array}$ & $\begin{array}{c}\text { Flufen- } \\
\text { acet } \\
\text { OXA } \\
(\mu \mathrm{g} / \mathrm{L})\end{array}$ & $\begin{array}{l}\text { Meto- } \\
\text { lachlor } \\
(\mu \mathrm{g} / \mathrm{L})\end{array}$ & $\begin{array}{l}\text { Meto- } \\
\text { lachlor } \\
\text { ESA } \\
(\mu \mathrm{g} / \mathrm{L})\end{array}$ & $\begin{array}{l}\text { Meto- } \\
\text { lachlor } \\
\text { OXA } \\
(\mu \mathrm{g} / \mathrm{L})\end{array}$ \\
\hline \multicolumn{20}{|c|}{ National Water-Quality Assessment Program (NAWQA) (fig. 3)—Continued } \\
\hline \multicolumn{20}{|c|}{ Eastern lowa Basin (EIWA/SKI)—Continued } \\
\hline 05451210 & $\begin{array}{c}\text { South Fork Iowa } \\
\text { River near New } \\
\text { Providence, IA }\end{array}$ & 09/05/01 & $<0.05$ & 0.08 & 0.10 & -- & $<0.05$ & 0.08 & 0.05 & -- & $<0.05$ & $<0.05$ & $<0.05$ & $<0.05$ & $<0.05$ & $<0.05$ & $<0.05$ & 0.33 & 0.49 \\
\hline \multirow[t]{2}{*}{05456510} & \multirow{2}{*}{$\begin{array}{c}\text { Turtle Creek at } \\
\text { Austin, IA }\end{array}$} & $08 / 27 / 97$ & $<.05$ & .38 & $<.20$ & -- & $<.05$ & 1.4 & $<.20$ & -- & -- & -- & -- & -- & -- & -- & .06 & 1.8 & .76 \\
\hline & & 05/19/98 & .06 & $<.20$ & $<.20$ & -- & $<.05$ & 1.1 & $<.20$ & -- & -- & -- & -- & -- & -- & -- & .09 & 2.6 & $<.20$ \\
\hline \multirow[t]{20}{*}{05422000} & \multirow{20}{*}{$\begin{array}{l}\text { Wapsipinicon River } \\
\text { near Dewitt, IA }\end{array}$} & $03 / 14 / 96$ & $<.05$ & .36 & $<.20$ & -- & $<.05$ & 1.8 & .26 & -- & -- & -- & -- & -- & -- & -- & .67 & 2.1 & .47 \\
\hline & & $04 / 24 / 96$ & $<.05$ & $<.20$ & $<.20$ & -- & $<.05$ & 2.4 & $<.20$ & -- & -- & -- & -- & -- & -- & -- & .19 & 2.6 & .49 \\
\hline & & $05 / 22 / 96$ & 1.6 & 2.6 & 2.9 & -- & .06 & 2.4 & .46 & -- & -- & -- & -- & -- & -- & -- & 2.8 & 5.5 & 2.4 \\
\hline & & $06 / 26 / 96$ & .15 & 2.3 & 1.9 & -- & .09 & 2.4 & .46 & -- & -- & -- & -- & -- & -- & -- & .69 & 5.9 & 2.3 \\
\hline & & 07/24/96 & $<.05$ & .51 & .37 & -- & $<.05$ & 2.4 & $<.20$ & -- & -- & -- & -- & -- & -- & -- & .15 & 3.5 & 1.0 \\
\hline & & 08/27/96 & $<.05$ & $<.20$ & $<.20$ & -- & $<.05$ & 2.2 & $<.20$ & -- & -- & -- & -- & -- & -- & -- & $<.05$ & 2.5 & $<.20$ \\
\hline & & $09 / 25 / 96$ & $<.05$ & $<.20$ & $<.20$ & -- & $<.05$ & 2.2 & $<.20$ & -- & -- & -- & -- & -- & -- & -- & $<.05$ & 2.2 & .34 \\
\hline & & $10 / 15 / 96$ & $<.05$ & $<.20$ & $<.20$ & -- & $<.05$ & 2.0 & $<.20$ & -- & -- & -- & -- & -- & -- & -- & .09 & 2.0 & .45 \\
\hline & & $11 / 13 / 96$ & $<.05$ & .83 & $<.20$ & -- & $<.05$ & 3.1 & .24 & -- & -- & -- & -- & -- & -- & -- & .13 & 4.1 & .69 \\
\hline & & $12 / 09 / 96$ & $<.05$ & 1.0 & $<.20$ & -- & $<.05$ & 3.4 & $<.20$ & -- & -- & -- & -- & -- & -- & -- & .08 & 5.1 & .80 \\
\hline & & 01/13/97 & $<.05$ & 1.0 & $<.20$ & -- & $<.05$ & 2.4 & $<.20$ & -- & -- & -- & -- & -- & -- & -- & 1.0 & 4.0 & .77 \\
\hline & & 02/11/97 & $<.05$ & .42 & $<.20$ & -- & $<.05$ & 2.7 & $<.20$ & -- & -- & -- & -- & -- & -- & -- & .21 & 3.2 & .67 \\
\hline & & 03/20/97 & $<.05$ & .51 & $<.20$ & -- & $<.05$ & .80 & $<.20$ & -- & -- & -- & -- & -- & -- & -- & .63 & 1.9 & .62 \\
\hline & & $05 / 06 / 97$ & 1.3 & 1.4 & .42 & -- & $<.05$ & 2.2 & .22 & -- & -- & -- & -- & -- & -- & -- & .84 & 4.9 & 1.1 \\
\hline & & 04/02/98 & $<.05$ & .73 & $<.20$ & -- & $<.05$ & .62 & $<.20$ & -- & -- & -- & -- & -- & -- & -- & .22 & 3.3 & .90 \\
\hline & & 05/06/98 & .08 & .96 & $<.20$ & -- & $<.05$ & 2.2 & $<.20$ & -- & -- & -- & -- & -- & -- & -- & .08 & 6.1 & .79 \\
\hline & & 06/03/98 & 1.1 & 2.6 & 1.9 & -- & .07 & 2.0 & $<.20$ & -- & -- & -- & -- & -- & -- & -- & 1.4 & 5.6 & 1.6 \\
\hline & & 06/17/98 & .53 & 2.7 & 2.1 & -- & .05 & 1.7 & $<.20$ & -- & -- & -- & -- & -- & -- & -- & 1.1 & 4.6 & 1.7 \\
\hline & & 07/09/98 & .05 & 2.3 & 1.2 & -- & $<.05$ & 1.8 & .32 & -- & -- & -- & -- & -- & -- & -- & .36 & 4.9 & 1.7 \\
\hline & & 08/06/98 & $<.05$ & 1.1 & .20 & -- & $<.05$ & 3.6 & $<.20$ & -- & -- & -- & -- & -- & -- & -- & $<.05$ & 4.6 & .78 \\
\hline
\end{tabular}


Table 6. Analytical results for selected acetamide herbicides and their degradation products, ethanesulfonic acid, oxanilic acid, and sulfinyl acetic acid, in surface-water samples, 1993-2003.-Continued

[OGRL, U.S. Geological Survey Organic Geochemistry Research Laboratory, Lawrence, Kansas; ESA, ethanesulfonic acid; OXA, oxanilic acid; SAA, sulfinyl acetic acid; --, no data; <, less than]

\begin{tabular}{|c|c|c|c|c|c|c|c|c|c|c|c|c|c|c|c|c|c|c|c|}
\hline \multirow[b]{2}{*}{ Site number } & \multirow[b]{2}{*}{ Site location } & \multirow[b]{2}{*}{$\begin{array}{c}\text { Date of } \\
\text { collection } \\
\text { (month/day/ } \\
\text { year) }\end{array}$} & \multicolumn{17}{|c|}{ Concentrations, in micrograms per liter } \\
\hline & & & $\begin{array}{l}\text { Aceto- } \\
\text { chlor } \\
(\mu \mathrm{g} / \mathrm{L})\end{array}$ & $\begin{array}{l}\text { Aceto- } \\
\text { chlor } \\
\text { ESA } \\
(\mu \mathrm{g} / \mathrm{L})\end{array}$ & $\begin{array}{c}\text { Aceto- } \\
\text { chlor } \\
\text { OXA } \\
(\mu \mathrm{g} / \mathrm{L})\end{array}$ & $\begin{array}{c}\text { Aceto- } \\
\text { chlor } \\
\text { SAA } \\
(\mu \mathrm{g} / \mathrm{L})\end{array}$ & $\begin{array}{l}\text { Ala- } \\
\text { chlor } \\
(\mu \mathrm{g} / \mathrm{L})\end{array}$ & $\begin{array}{l}\text { Ala- } \\
\text { chlor } \\
\text { ESA } \\
(\mu \mathrm{g} / \mathrm{L})\end{array}$ & $\begin{array}{c}\text { Ala- } \\
\text { chlor } \\
\text { OXA } \\
(\mu \mathrm{g} / \mathrm{L})\end{array}$ & $\begin{array}{l}\text { Ala- } \\
\text { chlor } \\
\text { SAA } \\
(\mu \mathrm{g} / \mathrm{L})\end{array}$ & $\begin{array}{l}\text { Di- } \\
\text { meth- } \\
\text { enamid } \\
(\mu \mathrm{g} / \mathrm{L})\end{array}$ & $\begin{array}{c}\text { Dimeth- } \\
\text { enamid } \\
\text { ESA } \\
(\mu \mathrm{g} / \mathrm{L})\end{array}$ & $\begin{array}{c}\text { Dimeth- } \\
\text { enamid } \\
\text { OXA } \\
(\mu \mathrm{g} / \mathrm{L})\end{array}$ & $\begin{array}{l}\text { Flufen- } \\
\text { acet } \\
(\mu \mathrm{g} / \mathrm{L})\end{array}$ & $\begin{array}{c}\text { Flufen- } \\
\text { acet } \\
\text { ESA } \\
(\mu \mathrm{g} / \mathrm{L})\end{array}$ & $\begin{array}{c}\text { Flufen- } \\
\text { acet } \\
\text { OXA } \\
(\mu \mathrm{g} / \mathrm{L})\end{array}$ & $\begin{array}{l}\text { Meto- } \\
\text { lachlor } \\
(\mu \mathrm{g} / \mathrm{L})\end{array}$ & $\begin{array}{l}\text { Meto- } \\
\text { lachlor } \\
\text { ESA } \\
(\mu \mathrm{g} / \mathrm{L})\end{array}$ & $\begin{array}{c}\text { Meto- } \\
\text { lachlor } \\
\text { OXA } \\
(\mu \mathrm{g} / \mathrm{L})\end{array}$ \\
\hline \multicolumn{20}{|c|}{ National Water-Quality Assessment Program (NAWQA) (fig. 3)—Continued } \\
\hline \multicolumn{20}{|c|}{ Eastern lowa Basin (EIWA/SKI)—Continued } \\
\hline 05422000 & $\begin{array}{l}\text { Wapsipinicon River } \\
\text { near Dewitt, IA }\end{array}$ & 09/02/98 & $<0.05$ & 2.1 & 0.39 & -- & $<0.05$ & 2.3 & $<0.20$ & -- & -- & -- & -- & -- & -- & -- & 0.11 & 5.6 & 1.1 \\
\hline \multirow[t]{23}{*}{05420680} & Wapsipinicon River & $03 / 27 / 96$ & $<.05$ & .87 & .92 & -- & $<.05$ & 1.3 & .78 & -- & -- & -- & -- & -- & -- & -- & 4.7 & 3.5 & 1.6 \\
\hline & near Tripoli, IA & $04 / 18 / 96$ & $<.05$ & $<.20$ & $<.20$ & -- & $<.05$ & 2.9 & $<.20$ & -- & -- & -- & -- & -- & -- & -- & .42 & 4.0 & .82 \\
\hline & & 05/16/96 & .14 & $<.20$ & $<.20$ & -- & $<.05$ & $<.20$ & $<.20$ & -- & -- & -- & -- & -- & -- & -- & .22 & $<.20$ & $<.20$ \\
\hline & & $06 / 24 / 96$ & .09 & 3.1 & 2.4 & -- & .07 & 4.1 & .49 & -- & -- & -- & -- & -- & -- & -- & .44 & 10 & 3.2 \\
\hline & & $07 / 18 / 96$ & $<.05$ & .70 & $<.20$ & -- & $<.05$ & 3.7 & .26 & -- & -- & -- & -- & -- & -- & -- & .09 & 4.7 & 1.1 \\
\hline & & $08 / 15 / 96$ & $<.05$ & $<.20$ & $<.20$ & -- & $<.05$ & .35 & .29 & -- & -- & -- & -- & -- & -- & -- & .08 & 2.4 & .24 \\
\hline & & 09/19/96 & $<.05$ & $<.20$ & $<.20$ & -- & $<.05$ & 2.3 & $<.20$ & -- & -- & -- & -- & -- & -- & -- & $<.05$ & 1.7 & $<.20$ \\
\hline & & $10 / 09 / 96$ & $<.05$ & $<.20$ & $<.20$ & -- & $<.05$ & 3.9 & .32 & -- & -- & -- & -- & -- & -- & -- & .05 & 2.8 & .73 \\
\hline & & $11 / 06 / 96$ & $<.05$ & $<.20$ & $<.20$ & -- & $<.05$ & 3.1 & .28 & -- & -- & -- & -- & -- & -- & -- & .13 & 2.4 & .42 \\
\hline & & $12 / 04 / 96$ & $<.05$ & .93 & .30 & -- & $<.05$ & 2.8 & .29 & -- & -- & -- & -- & -- & -- & -- & .12 & 5.8 & 1.0 \\
\hline & & 01/08/97 & $<.05$ & .77 & .23 & -- & $<.05$ & 2.5 & .24 & & -- & -- & -- & -- & -- & -- & .15 & 5.6 & .92 \\
\hline & & $02 / 05 / 97$ & $<.05$ & .20 & $<.20$ & -- & $<.05$ & 3.1 & .32 & -- & -- & -- & -- & -- & -- & -- & .08 & 2.9 & .50 \\
\hline & & 03/17/97 & $<.05$ & 1.4 & .29 & -- & $<.05$ & 1.8 & $<.20$ & -- & -- & -- & -- & -- & -- & -- & .57 & 4.9 & 2.0 \\
\hline & & 05/05/97 & 1.4 & 2.3 & .96 & -- & .20 & 3.3 & .34 & -- & -- & -- & -- & -- & -- & -- & 1.7 & 10 & 3.1 \\
\hline & & $06 / 22 / 97$ & .45 & 3.0 & 2.3 & -- & $<.05$ & 3.8 & .78 & -- & -- & -- & -- & -- & -- & -- & .53 & 11 & 3.5 \\
\hline & & $08 / 28 / 97$ & $<.05$ & .27 & $<.20$ & -- & $<.05$ & 2.1 & $<.20$ & -- & -- & -- & -- & -- & -- & -- & .11 & 2.3 & .45 \\
\hline & & 04/02/98 & $<.05$ & 1.1 & .28 & -- & $<.05$ & 1.2 & $<.20$ & -- & -- & -- & -- & -- & -- & -- & $<.05$ & 5.1 & 1.3 \\
\hline & & 05/06/98 & .14 & 1.5 & .25 & -- & $<.05$ & 3.5 & .25 & -- & -- & -- & -- & -- & -- & -- & .24 & 8.2 & 2.1 \\
\hline & & 05/19/98 & .10 & 1.7 & .21 & -- & $<.05$ & 4.1 & $<.20$ & -- & -- & -- & -- & -- & -- & -- & .07 & 7.6 & 1.4 \\
\hline & & 05/19/98 & .15 & 1.7 & .21 & -- & $<.05$ & 4.0 & $<.20$ & -- & -- & -- & -- & -- & -- & -- & .11 & 7.4 & 1.4 \\
\hline & & 05/19/98 & $<.05$ & 1.9 & $<.20$ & -- & $<.05$ & 4.0 & $<.20$ & -- & -- & -- & -- & -- & -- & -- & .07 & 6.5 & 1.1 \\
\hline & & $05 / 29 / 96$ & 1.5 & 3.9 & 4.9 & -- & .12 & 2.2 & $<.20$ & -- & -- & -- & -- & -- & -- & -- & 3.7 & 4.9 & 3.5 \\
\hline & & 07/08/98 & $<.05$ & 2.5 & .61 & -- & $<.05$ & 3.2 & .32 & -- & -- & -- & -- & -- & -- & -- & .16 & 7.5 & 2.1 \\
\hline
\end{tabular}


Table 6. Analytical results for selected acetamide herbicides and their degradation products, ethanesulfonic acid, oxanilic acid, and sulfinyl acetic acid, in surface-water samples, 1993-2003.-Continued

[OGRL, U.S. Geological Survey Organic Geochemistry Research Laboratory, Lawrence, Kansas; ESA, ethanesulfonic acid; OXA, oxanilic acid; SAA, sulfinyl acetic acid; --, no data; <, less than]

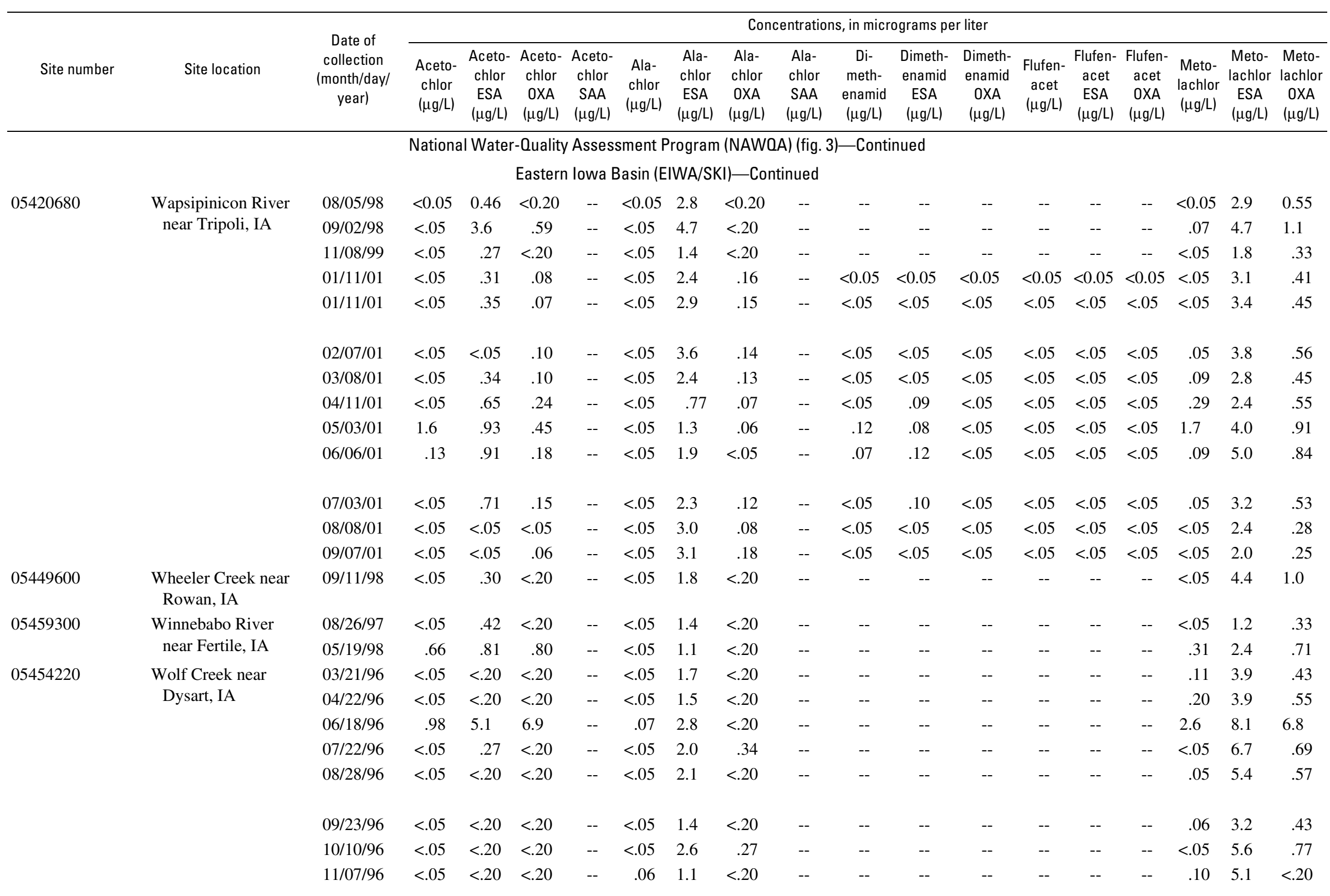


Table 6. Analytical results for selected acetamide herbicides and their degradation products, ethanesulfonic acid, oxanilic acid, and sulfinyl acetic acid, in surface-water samples, 1993-2003.-Continued

[OGRL, U.S. Geological Survey Organic Geochemistry Research Laboratory, Lawrence, Kansas; ESA, ethanesulfonic acid; OXA, oxanilic acid; SAA, sulfinyl acetic acid; --, no data; <, less than]

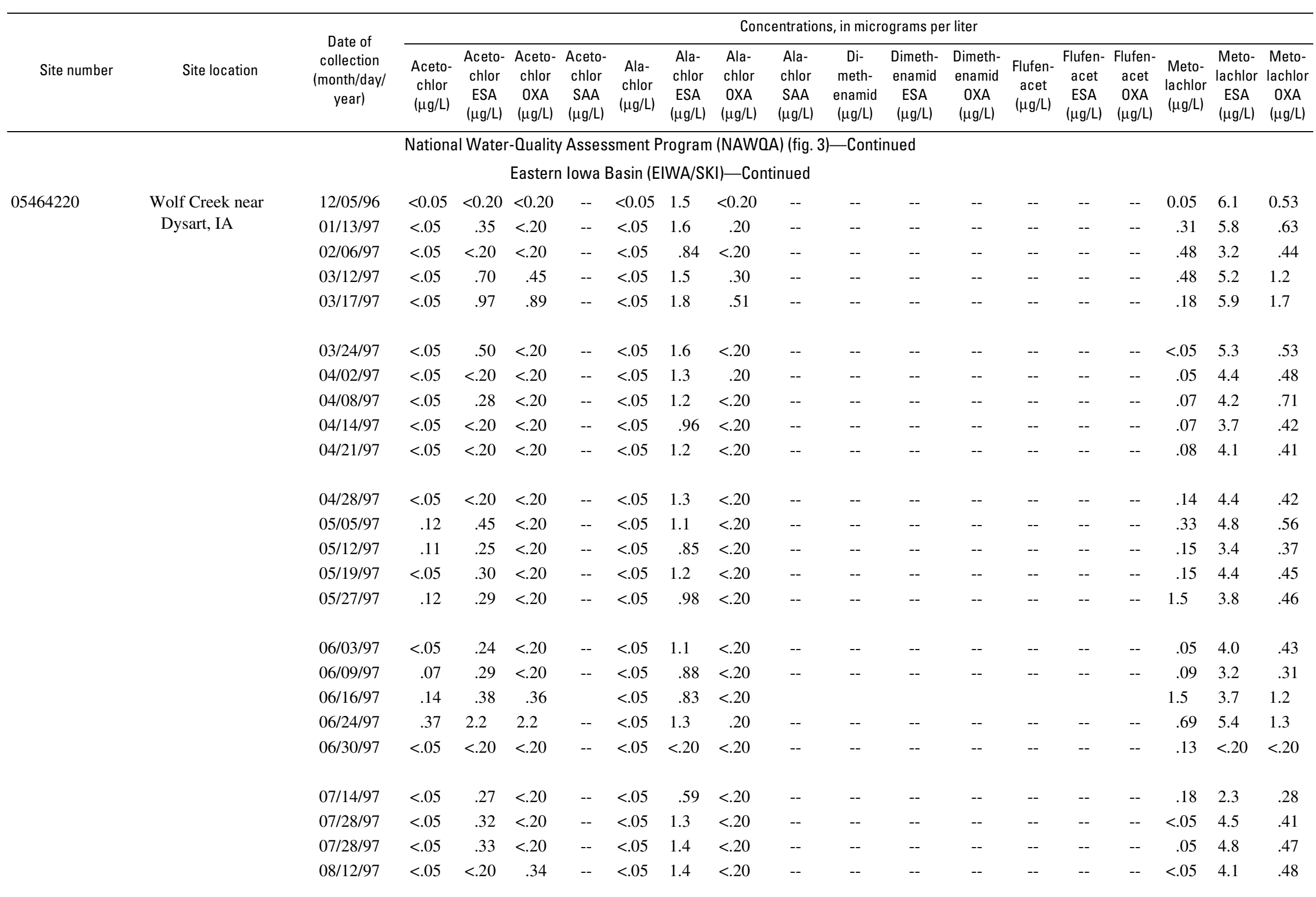


Table 6. Analytical results for selected acetamide herbicides and their degradation products, ethanesulfonic acid, oxanilic acid, and sulfinyl acetic acid, in surface-water samples, 1993-2003.-Continued

[OGRL, U.S. Geological Survey Organic Geochemistry Research Laboratory, Lawrence, Kansas; ESA, ethanesulfonic acid; OXA, oxanilic acid; SAA, sulfinyl acetic acid; --, no data; <, less than]

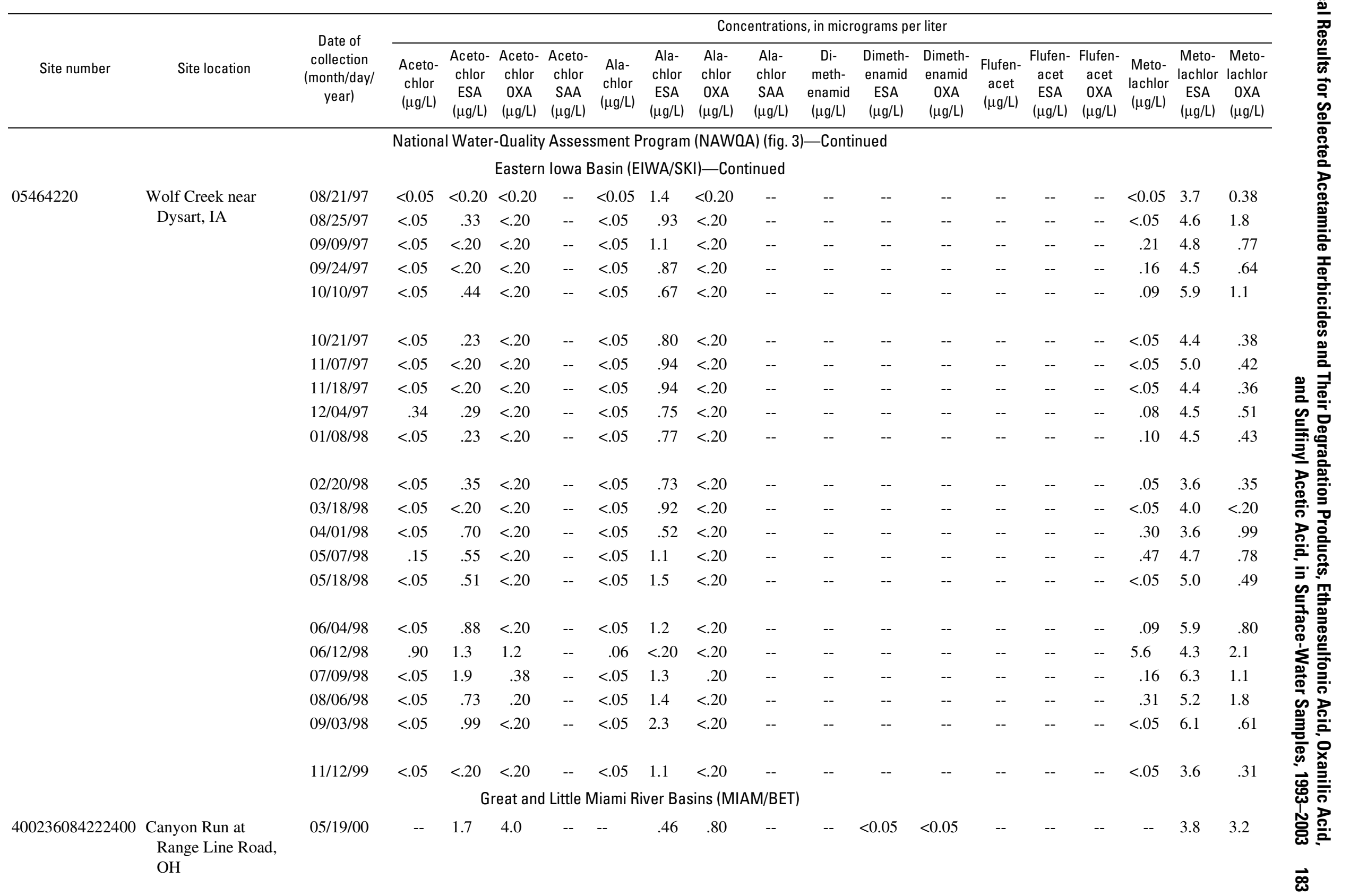


Table 6. Analytical results for selected acetamide herbicides and their degradation products, ethanesulfonic acid, oxanilic acid, and sulfinyl acetic acid, in surface-water samples, 1993-2003.-Continued

[OGRL, U.S. Geological Survey Organic Geochemistry Research Laboratory, Lawrence, Kansas; ESA, ethanesulfonic acid; OXA, oxanilic acid; SAA, sulfinyl acetic acid; --, no data; <, less than]

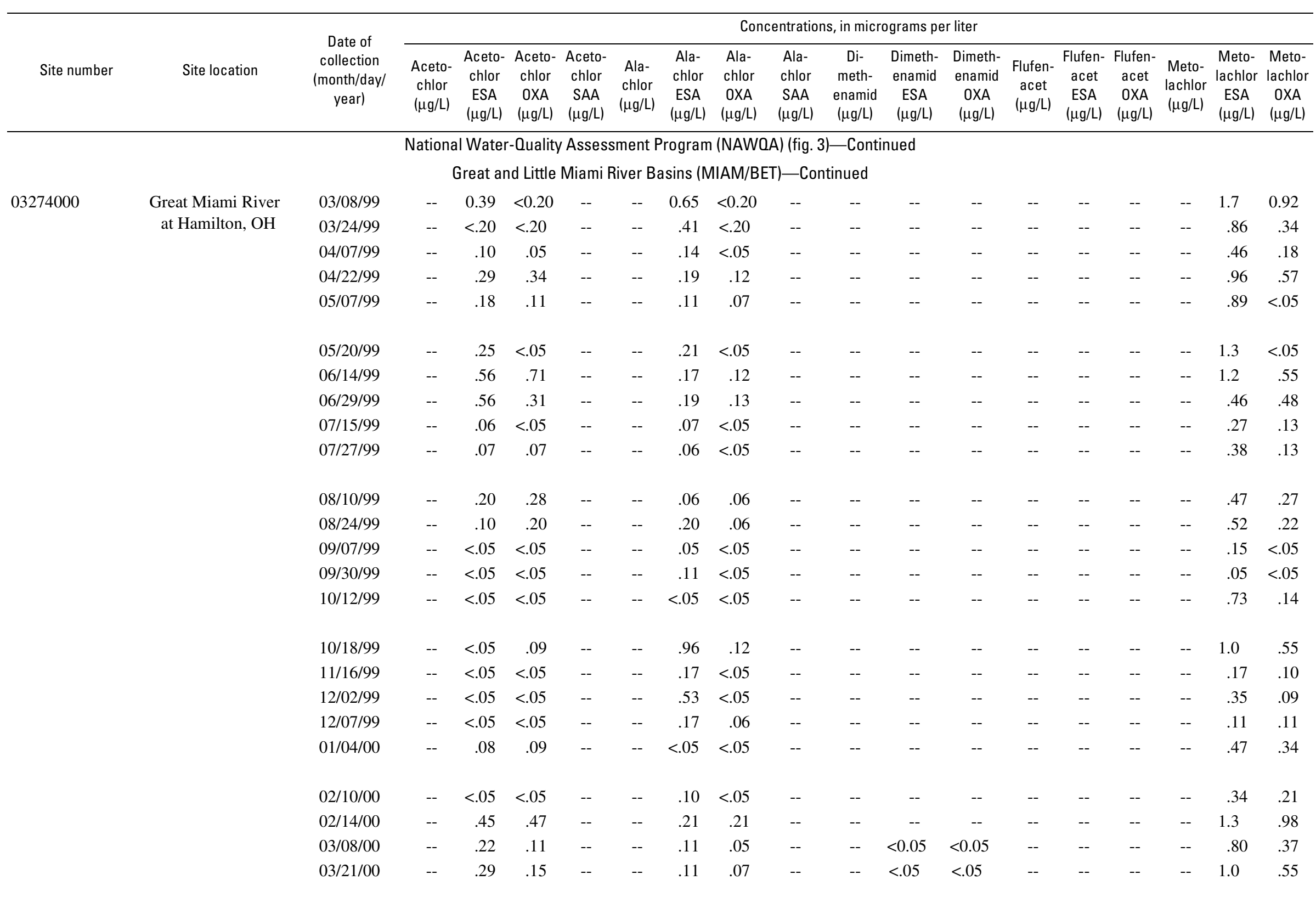


Table 6. Analytical results for selected acetamide herbicides and their degradation products, ethanesulfonic acid, oxanilic acid, and sulfinyl acetic acid, in surface-water samples, 1993-2003.-Continued

[OGRL, U.S. Geological Survey Organic Geochemistry Research Laboratory, Lawrence, Kansas; ESA, ethanesulfonic acid; OXA, oxanilic acid; SAA, sulfinyl acetic acid; --, no data; <, less than]

\begin{tabular}{|c|c|c|c|c|c|c|c|c|c|c|c|c|c|c|c|c|c|c|c|}
\hline \multirow[b]{2}{*}{ Site number } & \multirow[b]{2}{*}{ Site location } & \multirow[b]{2}{*}{$\begin{array}{c}\text { Date of } \\
\text { collection } \\
\text { (month/day/ } \\
\text { year) }\end{array}$} & \multicolumn{17}{|c|}{ Concentrations, in micrograms per liter } \\
\hline & & & $\begin{array}{l}\text { Aceto- } \\
\text { chlor } \\
(\mu \mathrm{g} / \mathrm{L})\end{array}$ & $\begin{array}{c}\text { Aceto- } \\
\text { chlor } \\
\text { ESA } \\
(\mu \mathrm{g} / \mathrm{L})\end{array}$ & $\begin{array}{c}\text { Aceto- } \\
\text { chlor } \\
\text { OXA } \\
(\mu \mathrm{g} / \mathrm{L})\end{array}$ & $\begin{array}{c}\text { Aceto- } \\
\text { chlor } \\
\text { SAA } \\
(\mu \mathrm{g} / \mathrm{L})\end{array}$ & $\begin{array}{l}\text { Ala- } \\
\text { chlor } \\
(\mu \mathrm{g} / \mathrm{L})\end{array}$ & $\begin{array}{l}\text { Ala- } \\
\text { chlor } \\
\text { ESA } \\
(\mu \mathrm{g} / \mathrm{L})\end{array}$ & $\begin{array}{c}\text { Ala- } \\
\text { chlor } \\
\text { OXA } \\
(\mu \mathrm{g} / \mathrm{L})\end{array}$ & $\begin{array}{l}\text { Ala- } \\
\text { chlor } \\
\text { SAA } \\
(\mu \mathrm{g} / \mathrm{L})\end{array}$ & $\begin{array}{c}\text { Di- } \\
\text { meth- } \\
\text { enamid } \\
(\mu \mathrm{g} / \mathrm{L})\end{array}$ & $\begin{array}{c}\text { Dimeth- } \\
\text { enamid } \\
\text { ESA } \\
(\mu \mathrm{g} / \mathrm{L})\end{array}$ & $\begin{array}{c}\text { Dimeth- } \\
\text { enamid } \\
\text { OXA } \\
(\mu \mathrm{g} / \mathrm{L})\end{array}$ & $\begin{array}{c}\text { Flufen- } \\
\text { acet } \\
(\mu \mathrm{g} / \mathrm{L})\end{array}$ & $\begin{array}{c}\text { Flufen- } \\
\text { acet } \\
\text { ESA } \\
(\mu \mathrm{g} / \mathrm{L})\end{array}$ & $\begin{array}{c}\text { Flufen- } \\
\text { acet } \\
\text { OXA } \\
(\mu \mathrm{g} / \mathrm{L})\end{array}$ & $\begin{array}{l}\text { Meto- } \\
\text { lachlor } \\
(\mu \mathrm{g} / \mathrm{L})\end{array}$ & $\begin{array}{l}\text { Meto- } \\
\text { lachlor } \\
\text { ESA } \\
(\mu \mathrm{g} / \mathrm{L})\end{array}$ & $\begin{array}{c}\text { Meto- } \\
\text { lachlor } \\
\text { OXA } \\
(\mu \mathrm{g} / \mathrm{L})\end{array}$ \\
\hline \multicolumn{20}{|c|}{ National Water-Quality Assessment Program (NAWQA) (fig. 3)—Continued } \\
\hline \multicolumn{20}{|c|}{ Great and Little Miami River Basins (MIAM/BET)—Continued } \\
\hline \multirow[t]{5}{*}{03274000} & \multirow{5}{*}{$\begin{array}{c}\text { Great Miami River } \\
\text { at Hamilton, } \mathrm{OH}\end{array}$} & $04 / 08 / 00$ & -- & 0.21 & 0.10 & -- & -- & 0.11 & 0.06 & -- & -- & $<0.05$ & $<0.05$ & -- & -- & -- & -- & 0.79 & 0.48 \\
\hline & & $07 / 13 / 00$ & -- & .54 & .39 & -- & -- & .16 & .10 & -- & -- & $<.05$ & $<.05$ & -- & $<0.05$ & $<0.05$ & -- & 1.1 & .57 \\
\hline & & $07 / 19 / 00$ & -- & .28 & .21 & -- & -- & .14 & .07 & -- & -- & $<.05$ & $<.05$ & -- & $<.05$ & $<.05$ & -- & .62 & .28 \\
\hline & & $05 / 17 / 01$ & -- & .08 & .51 & -- & -- & .13 & $<.05$ & -- & -- & $<.05$ & $<.05$ & -- & $<.05$ & $<.05$ & -- & .47 & .14 \\
\hline & & 06/13/01 & -- & .45 & .40 & -- & -- & .10 & $<.05$ & -- & -- & $<.05$ & $<.05$ & -- & $<.05$ & $<.05$ & -- & .69 & .40 \\
\hline \multirow{16}{*}{03274500} & \multirow{8}{*}{$\begin{array}{l}\text { Great Miami River } \\
\text { at Venice, } \mathrm{OH}\end{array}$} & $07 / 26 / 01$ & -- & .33 & .12 & -- & -- & $<.05$ & $<.05$ & -- & -- & $<.05$ & $<.05$ & -- & $<.05$ & $<.05$ & -- & .44 & .11 \\
\hline & & 08/15/01 & -- & .30 & .14 & -- & -- & .08 & $<.05$ & -- & -- & $<.05$ & $<.05$ & -- & $<.05$ & $<.05$ & -- & .52 & .27 \\
\hline & & 09/18/01 & -- & .31 & .22 & -- & -- & .14 & $<.05$ & -- & -- & $<.05$ & $<.05$ & -- & $<.05$ & $<.05$ & -- & .85 & .38 \\
\hline & & $04 / 26 / 00$ & -- & .55 & .33 & -- & -- & .41 & .27 & -- & -- & $<.05$ & $<.05$ & -- & -- & -- & -- & 1.3 & .69 \\
\hline & & 05/03/00 & -- & .24 & $<.05$ & -- & -- & .23 & .05 & -- & -- & $<.05$ & $<.05$ & -- & -- & -- & -- & .63 & .34 \\
\hline & & 06/01/00 & -- & .68 & .59 & -- & -- & .23 & .13 & -- & -- & $<.05$ & $<.05$ & -- & -- & -- & -- & 1.1 & .51 \\
\hline & & $06 / 14 / 00$ & -- & .81 & 1.1 & -- & -- & .12 & .10 & -- & -- & .07 & .08 & -- & $<.05$ & $<.05$ & -- & 1.1 & .60 \\
\hline & & $06 / 29 / 00$ & -- & 1.1 & 1.1 & -- & -- & .25 & .15 & -- & -- & .09 & .06 & -- & $<.05$ & $<.05$ & -- & 1.8 & 1.1 \\
\hline & & $08 / 16 / 00$ & -- & .55 & .47 & -- & -- & .16 & .10 & -- & -- & $<.05$ & $<.05$ & -- & $<.05$ & $<.05$ & -- & 1.6 & 1.0 \\
\hline & & 09/13/00 & -- & .15 & .10 & -- & -- & .14 & .06 & -- & -- & $<.05$ & $<.05$ & -- & $<.05$ & $<.05$ & -- & .52 & .30 \\
\hline & & $09 / 25 / 00$ & -- & $<.05$ & $<.05$ & -- & -- & $<.05$ & $<.05$ & -- & -- & $<.05$ & $<.05$ & -- & $<.05$ & $<.05$ & -- & .36 & .07 \\
\hline & & $11 / 16 / 00$ & -- & .46 & .35 & -- & -- & .13 & .07 & -- & -- & $<.05$ & $<.05$ & -- & $<.05$ & $<.05$ & -- & 1.2 & .55 \\
\hline & & $12 / 20 / 00$ & -- & .56 & .42 & -- & -- & .17 & .10 & -- & -- & $<.05$ & $<.05$ & -- & $<.05$ & $<.05$ & -- & 1.7 & .85 \\
\hline & & $04 / 11 / 01$ & -- & .09 & .05 & -- & -- & .07 & $<.05$ & -- & -- & $<.05$ & $<.05$ & -- & $<.05$ & $<.05$ & -- & .30 & .13 \\
\hline & & $04 / 11 / 01$ & -- & .09 & .05 & -- & -- & .08 & $<.05$ & -- & -- & $<.05$ & $<.05$ & -- & $<.05$ & $<.05$ & -- & .32 & .13 \\
\hline & & $04 / 12 / 01$ & -- & .19 & .14 & -- & -- & .09 & .05 & -- & -- & $<.05$ & $<.05$ & -- & $<.05$ & $<.05$ & -- & .79 & .42 \\
\hline 400616084355700 & $\begin{array}{l}\text { Greenville Creek at } \\
\text { Jaysville, } \mathrm{OH}\end{array}$ & $05 / 20 / 00$ & -- & 1.3 & 2.3 & -- & -- & .57 & .64 & -- & -- & .47 & .68 & -- & -- & -- & -- & 1.6 & 1.3 \\
\hline 400919084450300 & $\begin{array}{l}\text { Greenville Creek at } \\
\text { Palestine, } \mathrm{OH}\end{array}$ & $05 / 20 / 00$ & -- & 1.5 & 2.4 & -- & -- & $<.05$ & .51 & -- & -- & .53 & .75 & -- & -- & -- & -- & 2.4 & 1.7 \\
\hline
\end{tabular}


Table 6. Analytical results for selected acetamide herbicides and their degradation products, ethanesulfonic acid, oxanilic acid, and sulfinyl acetic acid, in surface-water samples, 1993-2003.-Continued

[OGRL, U.S. Geological Survey Organic Geochemistry Research Laboratory, Lawrence, Kansas; ESA, ethanesulfonic acid; OXA, oxanilic acid; SAA, sulfinyl acetic acid; --, no data; <, less than]

\begin{tabular}{|c|c|c|c|c|c|c|c|c|c|c|c|c|c|c|c|c|c|c|c|}
\hline \multirow[b]{2}{*}{ Site number } & \multirow[b]{2}{*}{ Site location } & \multirow[b]{2}{*}{$\begin{array}{l}\text { Date of } \\
\text { collection } \\
\text { (month/day/ } \\
\text { year) }\end{array}$} & \multicolumn{17}{|c|}{ Concentrations, in micrograms per liter } \\
\hline & & & $\begin{array}{l}\text { Aceto- } \\
\text { chlor } \\
(\mu \mathrm{g} / \mathrm{L})\end{array}$ & $\begin{array}{l}\text { Aceto- } \\
\text { chlor } \\
\text { ESA } \\
(\mu \mathrm{g} / \mathrm{L})\end{array}$ & $\begin{array}{l}\text { Aceto- } \\
\text { chlor } \\
\text { OXA } \\
(\mu \mathrm{g} / \mathrm{L})\end{array}$ & $\begin{array}{l}\text { Aceto- } \\
\text { chlor } \\
\text { SAA } \\
(\mu \mathrm{g} / \mathrm{L})\end{array}$ & $\begin{array}{l}\text { Ala- } \\
\text { chlor } \\
(\mu \mathrm{g} / \mathrm{L})\end{array}$ & $\begin{array}{l}\text { Ala- } \\
\text { chlor } \\
\text { ESA } \\
(\mu \mathrm{g} / \mathrm{L})\end{array}$ & $\begin{array}{l}\text { Ala- } \\
\text { chlor } \\
\text { OXA } \\
(\mu \mathrm{g} / \mathrm{L})\end{array}$ & $\begin{array}{l}\text { Ala- } \\
\text { chlor } \\
\text { SAA } \\
(\mu \mathrm{g} / \mathrm{L})\end{array}$ & $\begin{array}{l}\text { Di- } \\
\text { meth- } \\
\text { enamid } \\
(\mu \mathrm{g} / \mathrm{L})\end{array}$ & $\begin{array}{c}\text { Dimeth- } \\
\text { enamid } \\
\text { ESA } \\
(\mu \mathrm{g} / \mathrm{L})\end{array}$ & $\begin{array}{l}\text { Dimeth- } \\
\text { enamid } \\
\text { OXA } \\
(\mu \mathrm{g} / \mathrm{L})\end{array}$ & $\begin{array}{l}\text { Flufen- } \\
\text { acet } \\
(\mu \mathrm{g} / \mathrm{L})\end{array}$ & $\begin{array}{l}\text { Flufen- } \\
\text { acet } \\
\text { ESA } \\
(\mu \mathrm{g} / \mathrm{L})\end{array}$ & $\begin{array}{l}\text { Flufen- } \\
\text { acet } \\
\text { OXA } \\
(\mu \mathrm{g} / \mathrm{L})\end{array}$ & $\begin{array}{l}\text { Meto- } \\
\text { lachlor } \\
(\mu \mathrm{g} / \mathrm{L})\end{array}$ & $\begin{array}{l}\text { Meto- } \\
\text { lachlor } \\
\text { ESA } \\
(\mu \mathrm{g} / \mathrm{L})\end{array}$ & $\begin{array}{l}\text { Meto- } \\
\text { lachlor } \\
\text { OXA } \\
(\mu \mathrm{g} / \mathrm{L})\end{array}$ \\
\hline \multicolumn{20}{|c|}{ National Water-Quality Assessment Program (NAWOA) (fig. 3)—Continued } \\
\hline \multicolumn{20}{|c|}{ Great and Little Miami River Basins (MIAM/BET)_Continued } \\
\hline 400627084271400 & $\begin{array}{c}\text { Greenville Creek at } \\
\text { Smith Road, OH }\end{array}$ & $05 / 20 / 00$ & -- & 1.5 & 2.5 & -- & -- & $<0.05$ & 0.65 & -- & -- & 0.46 & 0.67 & -- & -- & -- & -- & 1.5 & 1.2 \\
\hline 400906084255300 & $\begin{array}{l}\text { Harris Creek at } \\
\text { State Route } 721 \text {, } \\
\text { OH }\end{array}$ & $05 / 20 / 00$ & -- & .91 & 1.5 & -- & -- & .49 & .48 & -- & -- & $<.05$ & $<.05$ & -- & -- & -- & -- & 1.9 & 1.3 \\
\hline 400039084250900 & $\begin{array}{l}\text { Hog Run at Jones } \\
\text { Road, OH }\end{array}$ & 05/19/00 & -- & 1.7 & 3.4 & -- & -- & .25 & .31 & -- & -- & $<.05$ & $<.05$ & -- & -- & -- & -- & 2.7 & 2.1 \\
\hline \multirow[t]{2}{*}{393944084120700} & \multirow{2}{*}{$\begin{array}{l}\text { Holes Creek, Huff- } \\
\text { man Park near } \\
\text { Kettering, OH }\end{array}$} & $07 / 11 / 00$ & -- & .24 & .34 & -- & -- & $<.05$ & $<.05$ & -- & -- & $<.05$ & $<.05$ & -- & $<0.05$ & $<0.05$ & -- & .11 & .10 \\
\hline & & $04 / 24 / 01$ & -- & $<.05$ & $<.05$ & -- & -- & $<.05$ & $<.05$ & -- & -- & $<.05$ & $<.05$ & -- & $<.05$ & $<.05$ & -- & $<.05$ & $<.05$ \\
\hline 401401084304800 & $\begin{array}{l}\text { Indian Creek at } \\
\text { Conover Road, } \\
\text { OH }\end{array}$ & $05 / 19 / 00$ & -- & 3.6 & 5.6 & -- & -- & .27 & .30 & -- & -- & $<.05$ & $<.05$ & -- & -- & -- & -- & 3.3 & 2.7 \\
\hline 400834084445500 & $\begin{array}{l}\text { Kraut Creek at } \\
\text { Bickel Road, OH }\end{array}$ & $05 / 20 / 00$ & -- & 1.5 & 2.7 & -- & -- & $<.05$ & .47 & -- & -- & .49 & .72 & -- & -- & -- & -- & 1.5 & 1.4 \\
\hline 400119084403700 & $\begin{array}{l}\text { Lake Branch Ditch } \\
\text { at Weaver-Ft. Jeff } \\
\text { Road, OH }\end{array}$ & 05/19/00 & -- & 2.3 & 4.2 & -- & -- & $<.05$ & .44 & -- & -- & .30 & .62 & -- & -- & -- & -- & .61 & .31 \\
\hline 395929084210500 & $\begin{array}{l}\text { Ludlow Creek at } \\
\text { Davis Road, OH }\end{array}$ & 05/20/00 & -- & 1.6 & 2.5 & -- & -- & $<.05$ & .10 & -- & -- & $<.05$ & $<.05$ & -- & $<.05$ & $<.05$ & -- & 1.8 & .88 \\
\hline \multirow[t]{8}{*}{03267900} & \multirow{8}{*}{$\begin{array}{l}\text { Mad River at St. } \\
\text { Paris at Eagle } \\
\text { City, OH }\end{array}$} & 03/03/99 & -- & $<.20$ & $<.20$ & -- & -- & .47 & $<.20$ & -- & -- & -- & -- & -- & -- & -- & -- & 1.3 & .43 \\
\hline & & 03/10/99 & -- & $<.20$ & $<.20$ & -- & -- & .47 & $<.20$ & -- & -- & -- & -- & -- & -- & -- & -- & .72 & .25 \\
\hline & & 03/17/99 & -- & $<.20$ & $<.20$ & -- & -- & .33 & $<.20$ & -- & -- & -- & -- & -- & -- & -- & -- & .67 & .26 \\
\hline & & 03/25/99 & -- & $<.20$ & $<.20$ & -- & -- & .41 & $<.20$ & -- & -- & -- & -- & -- & -- & -- & -- & .45 & $<.20$ \\
\hline & & 04/05/99 & -- & $<.05$ & $<.05$ & -- & -- & .46 & $<.05$ & -- & -- & -- & -- & -- & -- & -- & -- & .40 & .10 \\
\hline & & 04/09/99 & -- & .06 & $<.05$ & -- & -- & .27 & $<.05$ & -- & -- & -- & -- & -- & -- & -- & -- & .24 & .07 \\
\hline & & 04/20/99 & -- & $<.05$ & .05 & -- & -- & .75 & $<.05$ & -- & -- & -- & -- & -- & -- & -- & -- & 1.0 & .41 \\
\hline & & $04 / 21 / 99$ & -- & $<.05$ & .06 & -- & -- & .36 & $<.05$ & -- & -- & -- & -- & -- & -- & -- & -- & 1.3 & .97 \\
\hline
\end{tabular}


Table 6. Analytical results for selected acetamide herbicides and their degradation products, ethanesulfonic acid, oxanilic acid, and sulfinyl acetic acid, in surface-water samples, 1993-2003.-Continued

[OGRL, U.S. Geological Survey Organic Geochemistry Research Laboratory, Lawrence, Kansas; ESA, ethanesulfonic acid; OXA, oxanilic acid; SAA, sulfinyl acetic acid; --, no data; <, less than]

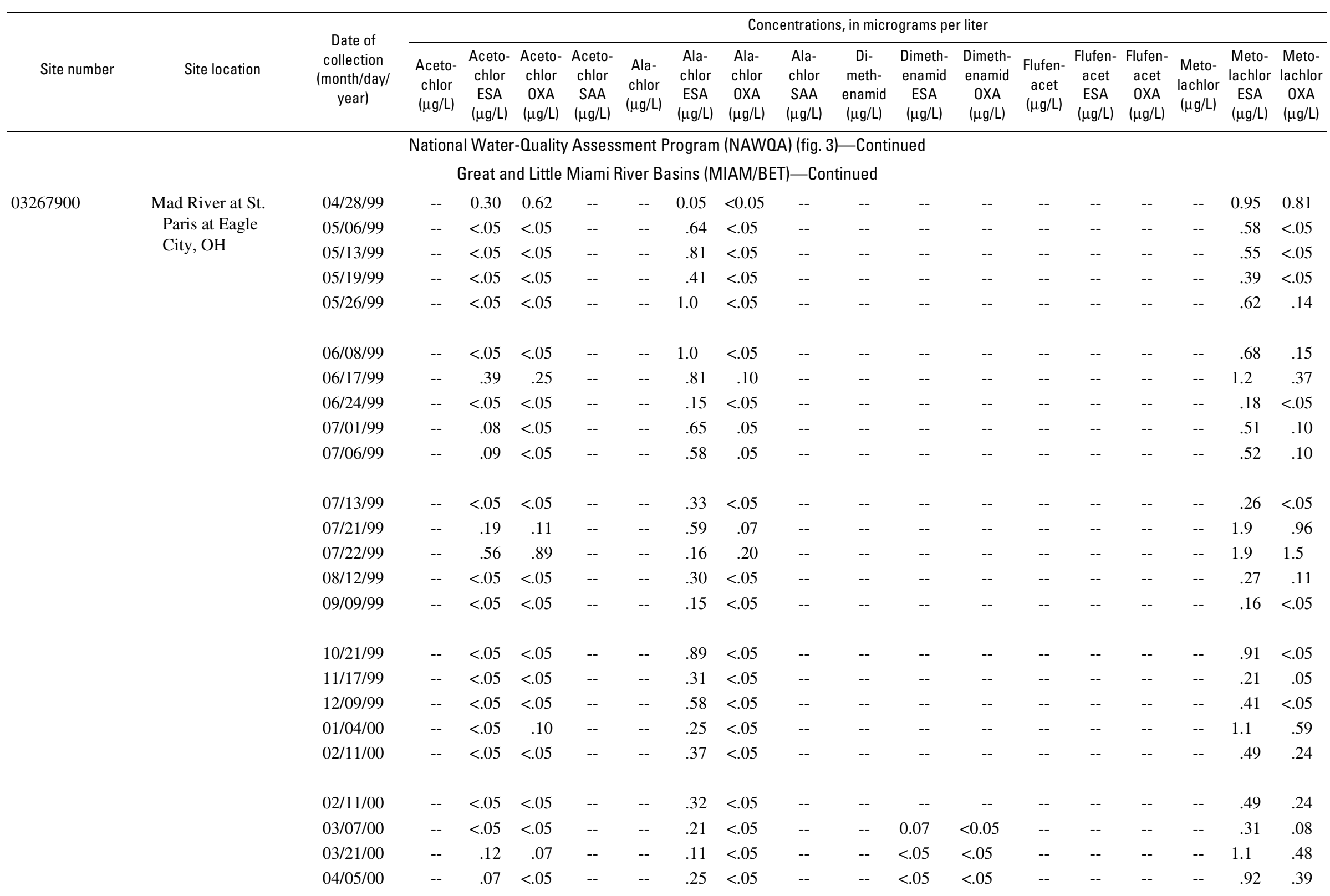


Table 6. Analytical results for selected acetamide herbicides and their degradation products, ethanesulfonic acid, oxanilic acid, and sulfinyl acetic acid, in surface-water samples, 1993-2003.-Continued

[OGRL, U.S. Geological Survey Organic Geochemistry Research Laboratory, Lawrence, Kansas; ESA, ethanesulfonic acid; OXA, oxanilic acid; SAA, sulfinyl acetic acid; --, no data; <, less than]

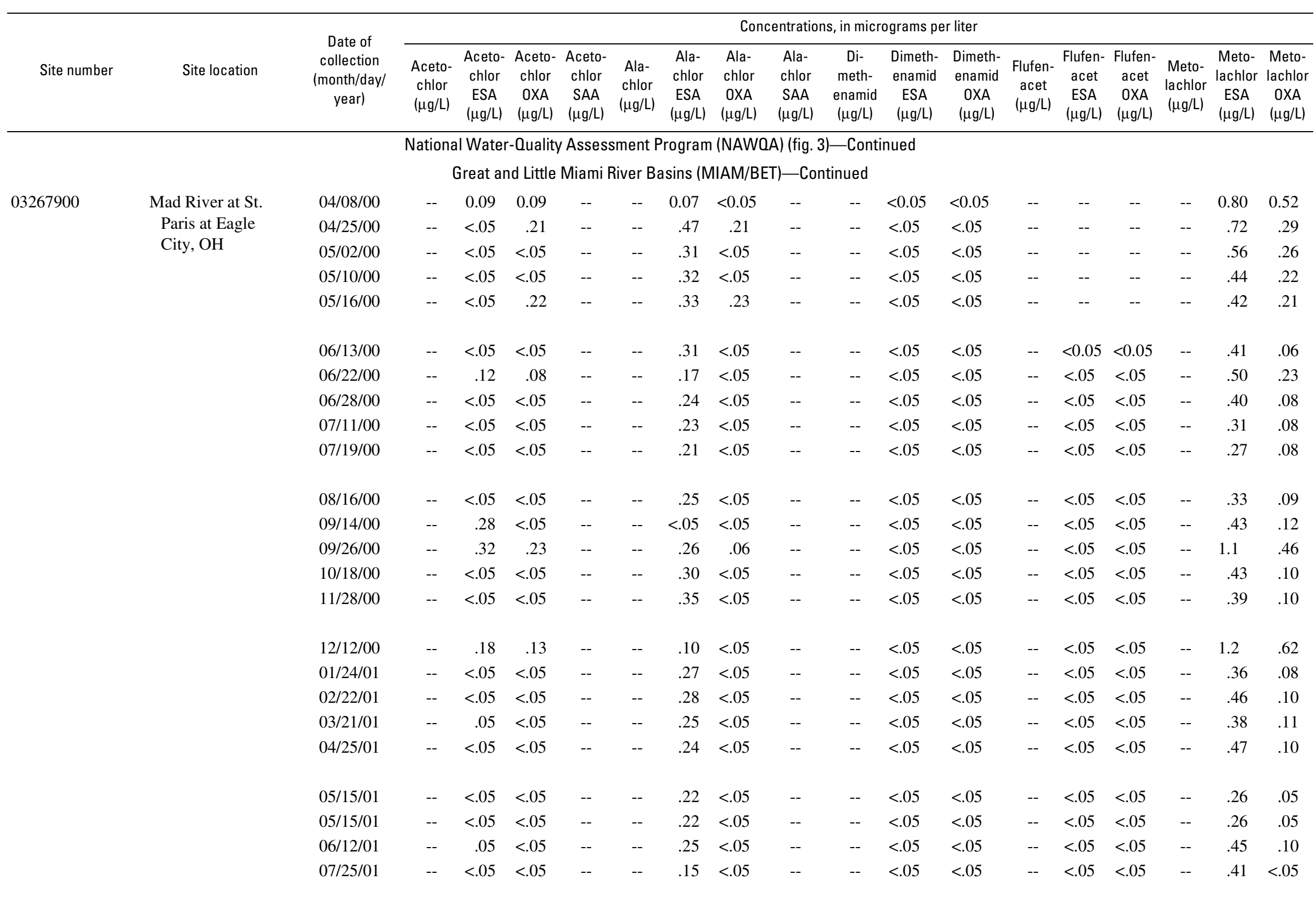


Table 6. Analytical results for selected acetamide herbicides and their degradation products, ethanesulfonic acid, oxanilic acid, and sulfinyl acetic acid, in surface-water samples, 1993-2003.-Continued

[OGRL, U.S. Geological Survey Organic Geochemistry Research Laboratory, Lawrence, Kansas; ESA, ethanesulfonic acid; OXA, oxanilic acid; SAA, sulfinyl acetic acid; --, no data; <, less than]

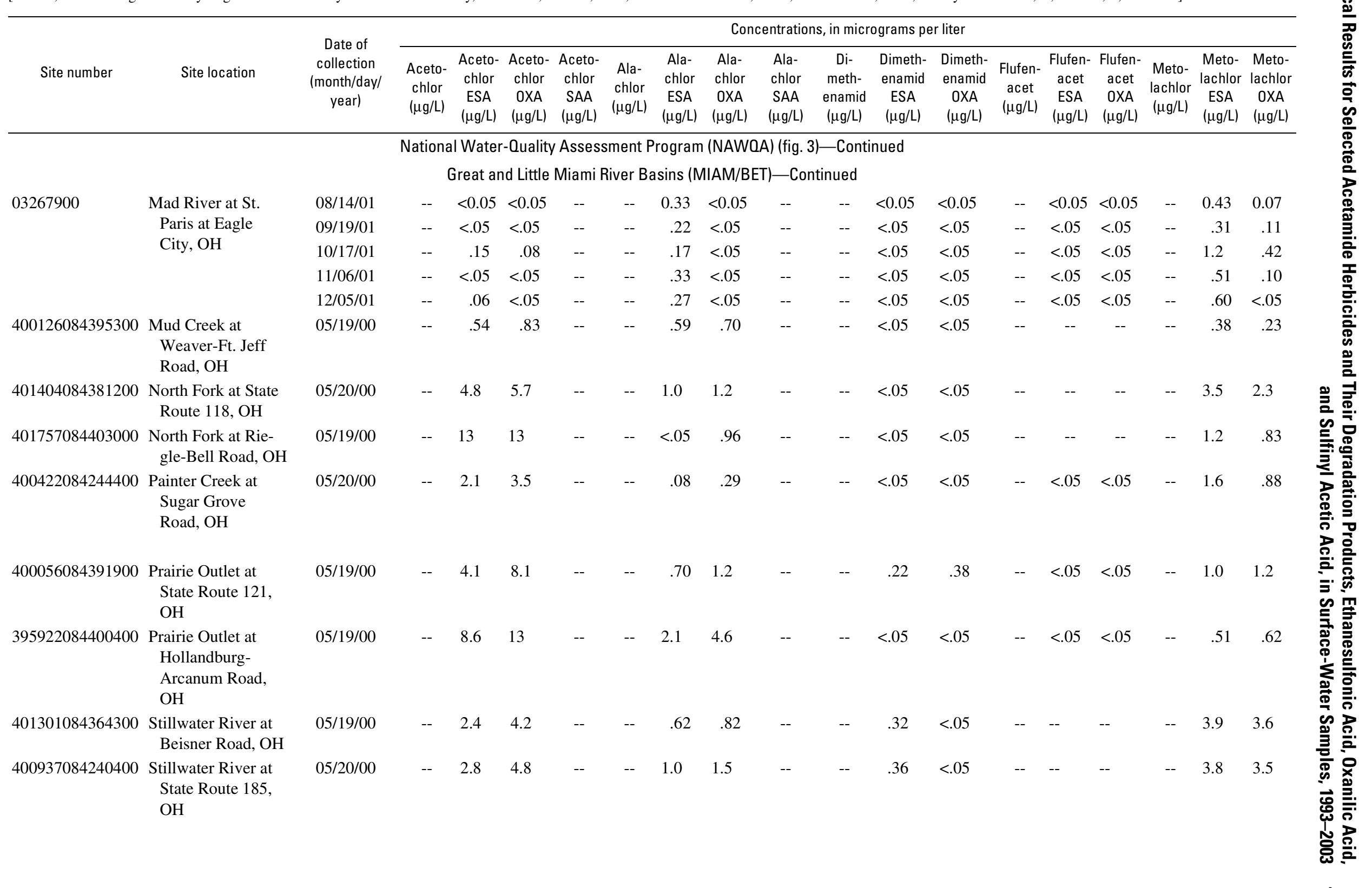


Table 6. Analytical results for selected acetamide herbicides and their degradation products, ethanesulfonic acid, oxanilic acid, and sulfinyl acetic acid, in surface-water samples, 1993-2003.-Continued

[OGRL, U.S. Geological Survey Organic Geochemistry Research Laboratory, Lawrence, Kansas; ESA, ethanesulfonic acid; OXA, oxanilic acid; SAA, sulfinyl acetic acid; --, no data; <, less than]

\begin{tabular}{|c|c|c|c|c|c|c|c|c|c|c|c|c|c|c|c|c|c|c|c|}
\hline \multirow[b]{2}{*}{ Site number } & \multirow[b]{2}{*}{ Site location } & \multirow[b]{2}{*}{$\begin{array}{c}\text { Date of } \\
\text { collection } \\
\text { (month/day/ } \\
\text { year) }\end{array}$} & \multicolumn{17}{|c|}{ Concentrations, in micrograms per liter } \\
\hline & & & $\begin{array}{l}\text { Aceto- } \\
\text { chlor } \\
(\mu \mathrm{g} / \mathrm{L})\end{array}$ & $\begin{array}{l}\text { Aceto- } \\
\text { chlor } \\
\text { ESA } \\
(\mu \mathrm{g} / \mathrm{L})\end{array}$ & $\begin{array}{l}\text { Aceto- } \\
\text { chlor } \\
\text { OXA } \\
(\mu \mathrm{g} / \mathrm{L})\end{array}$ & $\begin{array}{l}\text { Aceto- } \\
\text { chlor } \\
\text { SAA } \\
(\mu \mathrm{g} / \mathrm{L})\end{array}$ & $\begin{array}{l}\text { Ala- } \\
\text { chlor } \\
(\mu \mathrm{g} / \mathrm{L})\end{array}$ & $\begin{array}{l}\text { Ala- } \\
\text { chlor } \\
\text { ESA } \\
(\mu \mathrm{g} / \mathrm{L})\end{array}$ & $\begin{array}{l}\text { Ala- } \\
\text { chlor } \\
\text { OXA } \\
(\mu \mathrm{g} / \mathrm{L})\end{array}$ & $\begin{array}{l}\text { Ala- } \\
\text { chlor } \\
\text { SAA } \\
(\mu \mathrm{g} / \mathrm{L})\end{array}$ & $\begin{array}{l}\text { Di- } \\
\text { meth- } \\
\text { enamid } \\
(\mu \mathrm{g} / \mathrm{L})\end{array}$ & $\begin{array}{c}\text { Dimeth- } \\
\text { enamid } \\
\text { ESA } \\
(\mu \mathrm{g} / \mathrm{L})\end{array}$ & $\begin{array}{c}\text { Dimeth- } \\
\text { enamid } \\
\text { OXA } \\
(\mu \mathrm{g} / \mathrm{L})\end{array}$ & $\begin{array}{c}\text { Flufen- } \\
\text { acet } \\
(\mu \mathrm{g} / \mathrm{L})\end{array}$ & $\begin{array}{l}\text { Flufen- } \\
\text { acet } \\
\text { ESA } \\
(\mu \mathrm{g} / \mathrm{L})\end{array}$ & $\begin{array}{l}\text { Flufen- } \\
\text { acet } \\
\text { OXA } \\
(\mu \mathrm{g} / \mathrm{L})\end{array}$ & $\begin{array}{l}\text { Meto- } \\
\text { lachlor } \\
(\mu \mathrm{g} / \mathrm{L})\end{array}$ & $\begin{array}{l}\text { Meto- } \\
\text { lachlor } \\
\text { ESA } \\
(\mu \mathrm{g} / \mathrm{L})\end{array}$ & $\begin{array}{c}\text { Meto- } \\
\text { lachlor } \\
\text { OXA } \\
(\mu \mathrm{g} / \mathrm{L})\end{array}$ \\
\hline \multicolumn{20}{|c|}{ National Water-Quality Assessment Program (NAWOA) (fig. 3)—Continued } \\
\hline \multicolumn{20}{|c|}{ Great and Little Miami River Basins (MIAM/BET)—Continued } \\
\hline \multirow[t]{3}{*}{395355084173600} & \multirow{3}{*}{$\begin{array}{l}\text { Stillwater River on } \\
\text { Marindale Road, } \\
\text { OH }\end{array}$} & $03 / 09 / 00$ & -- & 0.18 & 0.21 & -- & -- & 0.25 & 0.23 & -- & -- & $<0.05$ & $<0.05$ & -- & -- & -- & -- & 1.1 & 0.55 \\
\hline & & $03 / 20 / 00$ & -- & .15 & $<.05$ & -- & -- & .13 & .06 & -- & -- & $<.05$ & $<.05$ & -- & -- & -- & -- & 1.1 & .39 \\
\hline & & $04 / 04 / 00$ & -- & .27 & .07 & -- & -- & .29 & .09 & -- & -- & $<.05$ & $<.05$ & -- & -- & -- & -- & 1.4 & .54 \\
\hline \multirow[t]{9}{*}{395611084180600} & \multirow{9}{*}{$\begin{array}{l}\text { Stillwater River at } \\
\text { Fred-Garland } \\
\text { Road, OH }\end{array}$} & 04/08/00 & -- & .36 & .34 & -- & -- & .22 & .11 & -- & -- & $<.05$ & $<.05$ & -- & -- & -- & -- & 1.6 & 1.0 \\
\hline & & $04 / 21 / 00$ & -- & .58 & .31 & -- & -- & .45 & .29 & -- & -- & $<.05$ & $<.05$ & -- & -- & -- & -- & 1.6 & .75 \\
\hline & & $05 / 02 / 00$ & -- & .26 & $<.05$ & -- & -- & .27 & .08 & -- & -- & $<.05$ & $<.05$ & -- & -- & -- & -- & .90 & .33 \\
\hline & & $05 / 20 / 00$ & -- & 2.2 & 4.1 & -- & -- & .31 & .45 & -- & -- & .10 & .25 & -- & $<0.05$ & $<0.05$ & -- & 2.3 & 1.9 \\
\hline & & 06/15/00 & -- & .39 & .34 & -- & -- & .11 & .10 & -- & -- & $<.05$ & $<.05$ & -- & $<.05$ & $<.05$ & -- & .99 & .47 \\
\hline & & 07/20/00 & -- & .27 & .15 & -- & -- & .16 & .10 & -- & -- & $<.05$ & $<.05$ & -- & $<.05$ & $<.05$ & -- & .86 & .32 \\
\hline & & $08 / 21 / 00$ & -- & .15 & .12 & -- & -- & .19 & .13 & -- & -- & $<.05$ & $<.05$ & -- & $<.05$ & $<.05$ & -- & .90 & .48 \\
\hline & & 08/23/00 & -- & .11 & .08 & -- & -- & .19 & .12 & -- & -- & $<.05$ & $<.05$ & -- & $<.05$ & $<.05$ & -- & .64 & .33 \\
\hline & & 09/12/00 & -- & .16 & .11 & -- & -- & .16 & .10 & -- & -- & $<.05$ & $<.05$ & -- & $<.05$ & $<.05$ & -- & .75 & .38 \\
\hline 401502084421500 & $\begin{array}{l}\text { South Fork at Wash- } \\
\text { ington Road, OH }\end{array}$ & $05 / 19 / 00$ & -- & 1.0 & 1.2 & -- & -- & $<.05$ & .42 & -- & -- & .34 & $<.05$ & -- & -- & -- & -- & 3.1 & 2.7 \\
\hline 401436084404800 & $\begin{array}{l}\text { Stillwater River at } \\
\text { Zumbrum Road, } \\
\text { OH }\end{array}$ & $05 / 20 / 00$ & -- & 1.0 & 1.2 & -- & -- & $<.05$ & .28 & -- & -- & .33 & .59 & -- & -- & -- & -- & 3.6 & 1.9 \\
\hline 401612084280200 & $\begin{array}{l}\text { Swamp Creek at } \\
\text { Pitsburg Road, } \\
\text { OH }\end{array}$ & $05 / 20 / 00$ & -- & 3.8 & 6.3 & -- & -- & 5.8 & 5.6 & -- & -- & $<.05$ & $<.05$ & -- & -- & -- & -- & 4.5 & 2.7 \\
\hline 401247084295500 & $\begin{array}{c}\text { Swamp Creek State } \\
\text { Route } 121, \mathrm{OH}\end{array}$ & $05 / 19 / 00$ & -- & 4.3 & 7.3 & -- & -- & 1.3 & 1.4 & -- & -- & $<.05$ & $<.05$ & -- & -- & -- & -- & 4.2 & 3.2 \\
\hline 401723084325900 & $\begin{array}{c}\text { Swamp Creek at } \\
\text { Greenville, St } \\
\text { Mary's, OH }\end{array}$ & $05 / 19 / 00$ & -- & 5.1 & 9.0 & -- & -- & 19 & 14 & -- & -- & $<.05$ & $<.05$ & -- & -- & -- & -- & 2.6 & 2.5 \\
\hline 400850084204500 & $\begin{array}{l}\text { Trotters Creek at } \\
\text { Piqua-Clay Road, } \\
\text { OH }\end{array}$ & $05 / 19 / 00$ & -- & .70 & 1.2 & -- & -- & .33 & .35 & -- & -- & $<.05$ & $<.05$ & -- & -- & -- & -- & 3.4 & 3.7 \\
\hline
\end{tabular}


Table 6. Analytical results for selected acetamide herbicides and their degradation products, ethanesulfonic acid, oxanilic acid, and sulfinyl acetic acid, in surface-water samples, 1993-2003.-Continued

[OGRL, U.S. Geological Survey Organic Geochemistry Research Laboratory, Lawrence, Kansas; ESA, ethanesulfonic acid; OXA, oxanilic acid; SAA, sulfinyl acetic acid; --, no data; <, less than]

\begin{tabular}{|c|c|c|c|c|c|c|c|c|c|c|c|c|c|c|c|c|c|c|c|}
\hline \multirow[b]{2}{*}{ Site number } & \multirow[b]{2}{*}{ Site location } & \multirow[b]{2}{*}{$\begin{array}{l}\text { Date of } \\
\text { collection } \\
\text { (month/day/ } \\
\text { year) }\end{array}$} & \multicolumn{17}{|c|}{ Concentrations, in micrograms per liter } \\
\hline & & & $\begin{array}{l}\text { Aceto- } \\
\text { chlor } \\
(\mu \mathrm{g} / \mathrm{L})\end{array}$ & $\begin{array}{l}\text { Aceto- } \\
\text { chlor } \\
\text { ESA } \\
(\mu \mathrm{g} / \mathrm{L})\end{array}$ & $\begin{array}{l}\text { Aceto- } \\
\text { chlor } \\
\text { OXA } \\
(\mu \mathrm{g} / \mathrm{L})\end{array}$ & $\begin{array}{l}\text { Aceto- } \\
\text { chlor } \\
\text { SAA } \\
(\mu \mathrm{g} / \mathrm{L})\end{array}$ & $\begin{array}{l}\text { Ala- } \\
\text { chlor } \\
(\mu \mathrm{g} / \mathrm{L})\end{array}$ & $\begin{array}{l}\text { Ala- } \\
\text { chlor } \\
\text { ESA } \\
(\mu \mathrm{g} / \mathrm{L})\end{array}$ & $\begin{array}{l}\text { Ala- } \\
\text { chlor } \\
\text { OXA } \\
(\mu \mathrm{g} / \mathrm{L})\end{array}$ & $\begin{array}{l}\text { Ala- } \\
\text { chlor } \\
\text { SAA } \\
(\mu \mathrm{g} / \mathrm{L})\end{array}$ & $\begin{array}{l}\text { Di- } \\
\text { meth- } \\
\text { enamid } \\
(\mu \mathrm{g} / \mathrm{L})\end{array}$ & $\begin{array}{c}\text { Dimeth- } \\
\text { enamid } \\
\text { ESA } \\
(\mu \mathrm{g} / \mathrm{L})\end{array}$ & $\begin{array}{c}\text { Dimeth- } \\
\text { enamid } \\
\text { OXA } \\
(\mu \mathrm{g} / \mathrm{L})\end{array}$ & $\begin{array}{c}\text { Flufen- } \\
\text { acet } \\
(\mu \mathrm{g} / \mathrm{L})\end{array}$ & $\begin{array}{l}\text { Flufen- } \\
\text { acet } \\
\text { ESA } \\
(\mu \mathrm{g} / \mathrm{L})\end{array}$ & $\begin{array}{c}\text { Flufen- } \\
\text { acet } \\
\text { OXA } \\
(\mu \mathrm{g} / \mathrm{L})\end{array}$ & $\begin{array}{l}\text { Meto- } \\
\text { lachlor } \\
(\mu \mathrm{g} / \mathrm{L})\end{array}$ & $\begin{array}{l}\text { Meto- } \\
\text { lachlor } \\
\text { ESA } \\
(\mu \mathrm{g} / \mathrm{L})\end{array}$ & $\begin{array}{l}\text { Meto- } \\
\text { lachlor } \\
\text { OXA } \\
(\mu \mathrm{g} / \mathrm{L})\end{array}$ \\
\hline \multicolumn{20}{|c|}{ National Water-Quality Assessment Program (NAWQA) (fig. 3)—Continued } \\
\hline \multicolumn{20}{|c|}{ Great and Little Miami River Basins (MIAM/BET)—Continued } \\
\hline 401340084275400 & $\begin{array}{l}\text { Tributary at State } \\
\text { Route } 47, \mathrm{OH}\end{array}$ & $05 / 19 / 00$ & -- & 5.9 & 9.9 & -- & -- & $<0.05$ & 1.1 & -- & -- & $<0.05$ & $<0.05$ & -- & -- & -- & -- & 6.0 & 4.5 \\
\hline 401208084325700 & $\begin{array}{l}\text { Tributary at State } \\
\text { Route } 242, \mathrm{OH}\end{array}$ & $05 / 20 / 00$ & -- & .58 & .55 & -- & -- & .59 & .49 & -- & -- & $<.05$ & $<.05$ & -- & -- & -- & -- & 2.8 & 1.4 \\
\hline 400958084330900 & $\begin{array}{c}\text { Tributary at State } \\
\text { Route } 121, \mathrm{OH}\end{array}$ & $05 / 20 / 00$ & -- & 1.2 & 2.0 & -- & -- & $<.05$ & .25 & -- & -- & $<.05$ & $<.05$ & -- & -- & -- & -- & 1.4 & .84 \\
\hline 401522084423400 & $\begin{array}{l}\text { West Fork at Cole- } \\
\text { town Road, OH }\end{array}$ & $05 / 19 / 00$ & -- & 2.6 & 4.6 & -- & -- & $<.05$ & .35 & -- & -- & $<.05$ & $<.05$ & -- & -- & -- & -- & 4.8 & 4.0 \\
\hline 400701084405100 & $\begin{array}{l}\text { West Branch } \\
\text { Greenville Creek } \\
\text { at Heller Road, } \\
\text { OH }\end{array}$ & $05 / 20 / 00$ & -- & 1.1 & 1.9 & -- & -- & .56 & .65 & -- & -- & .43 & .59 & -- & -- & -- & -- & .97 & .65 \\
\hline \multicolumn{20}{|c|}{ Upper Illinois River Basin (UIRB/BET) } \\
\hline \multirow[t]{14}{*}{05532500} & \multirow{14}{*}{$\begin{array}{l}\text { Des Plaines River at } \\
\text { Riverside, IL }\end{array}$} & 03/02/99 & -- & $<.05$ & $<.05$ & -- & -- & $<.05$ & $<.05$ & -- & -- & -- & -- & -- & -- & -- & -- & $<.05$ & $<.05$ \\
\hline & & 04/07/99 & -- & .05 & $<.05$ & -- & -- & .18 & $<.05$ & -- & -- & -- & -- & -- & -- & -- & -- & .20 & .08 \\
\hline & & $04 / 21 / 99$ & -- & .16 & .09 & -- & -- & .21 & .06 & -- & -- & -- & -- & -- & -- & -- & -- & .27 & .12 \\
\hline & & 05/04/99 & -- & .12 & .10 & -- & -- & .06 & $<.05$ & -- & -- & -- & -- & -- & -- & -- & -- & .20 & .10 \\
\hline & & 06/01/99 & -- & $<.05$ & $<.05$ & -- & -- & $<.05$ & $<.05$ & -- & -- & -- & -- & -- & -- & -- & -- & $<.05$ & $<.05$ \\
\hline & & 06/02/99 & -- & $<.05$ & $<.05$ & -- & -- & $<.05$ & $<.05$ & -- & -- & -- & -- & -- & -- & -- & -- & $<.05$ & $<.05$ \\
\hline & & 06/16/99 & -- & .42 & .68 & -- & -- & .05 & .06 & -- & -- & -- & -- & -- & -- & -- & -- & .28 & .18 \\
\hline & & 07/07/99 & -- & .10 & .11 & -- & -- & .07 & $<.05$ & -- & -- & -- & -- & -- & -- & -- & -- & .16 & .07 \\
\hline & & 08/03/99 & -- & .15 & $<.05$ & -- & -- & $<.05$ & $<.05$ & -- & -- & -- & -- & -- & -- & -- & -- & $<.05$ & $<.05$ \\
\hline & & $08 / 31 / 99$ & -- & $<.05$ & .15 & -- & -- & $<.05$ & $<.05$ & -- & -- & -- & -- & -- & -- & -- & -- & $<.05$ & $<.05$ \\
\hline & & $11 / 03 / 99$ & -- & $<.05$ & $<.05$ & -- & -- & $<.05$ & $<.05$ & -- & -- & -- & -- & -- & -- & -- & -- & $<.05$ & $<.05$ \\
\hline & & $12 / 08 / 99$ & -- & $<.05$ & $<.05$ & -- & -- & $<.05$ & $<.05$ & -- & -- & -- & -- & -- & -- & -- & -- & $<.05$ & $<.05$ \\
\hline & & 01/05/00 & -- & $<.05$ & $<.05$ & -- & -- & $<.05$ & $<.05$ & -- & -- & -- & -- & -- & -- & -- & -- & $<.05$ & $<.05$ \\
\hline & & $02 / 02 / 00$ & -- & $<.05$ & $<.05$ & -- & -- & $<.05$ & $<.05$ & -- & -- & -- & -- & -- & -- & -- & -- & $<.05$ & $<.05$ \\
\hline
\end{tabular}


Table 6. Analytical results for selected acetamide herbicides and their degradation products, ethanesulfonic acid, oxanilic acid, and sulfinyl acetic acid, in surface-water samples, 1993-2003.-Continued

[OGRL, U.S. Geological Survey Organic Geochemistry Research Laboratory, Lawrence, Kansas; ESA, ethanesulfonic acid; OXA, oxanilic acid; SAA, sulfinyl acetic acid; --, no data; <, less than]

\begin{tabular}{|c|c|c|c|c|c|c|c|c|c|c|c|c|c|c|c|c|c|c|c|}
\hline \multirow[b]{2}{*}{ Site number } & \multirow[b]{2}{*}{ Site location } & \multirow[b]{2}{*}{$\begin{array}{c}\text { Date of } \\
\text { collection } \\
\text { (month/day/ } \\
\text { year) }\end{array}$} & \multicolumn{17}{|c|}{ Concentrations, in micrograms per liter } \\
\hline & & & $\begin{array}{l}\text { Aceto- } \\
\text { chlor } \\
(\mu \mathrm{g} / \mathrm{L})\end{array}$ & $\begin{array}{l}\text { Aceto- } \\
\text { chlor } \\
\text { ESA } \\
(\mu \mathrm{g} / \mathrm{L})\end{array}$ & $\begin{array}{c}\text { Aceto- } \\
\text { chlor } \\
\text { OXA } \\
(\mu \mathrm{g} / \mathrm{L})\end{array}$ & $\begin{array}{l}\text { Aceto- } \\
\text { chlor } \\
\text { SAA } \\
(\mu \mathrm{g} / \mathrm{L})\end{array}$ & $\begin{array}{l}\text { Ala- } \\
\text { chlor } \\
(\mu \mathrm{g} / \mathrm{L})\end{array}$ & $\begin{array}{l}\text { Ala- } \\
\text { chlor } \\
\text { ESA } \\
(\mu \mathrm{g} / \mathrm{L})\end{array}$ & $\begin{array}{l}\text { Ala- } \\
\text { chlor } \\
\text { OXA } \\
(\mu \mathrm{g} / \mathrm{L})\end{array}$ & $\begin{array}{l}\text { Ala- } \\
\text { chlor } \\
\text { SAA } \\
(\mu \mathrm{g} / \mathrm{L})\end{array}$ & $\begin{array}{c}\text { Di- } \\
\text { meth- } \\
\text { enamid } \\
(\mu \mathrm{g} / \mathrm{L})\end{array}$ & $\begin{array}{c}\text { Dimeth- } \\
\text { enamid } \\
\text { ESA } \\
(\mu \mathrm{g} / \mathrm{L})\end{array}$ & $\begin{array}{c}\text { Dimeth- } \\
\text { enamid } \\
\text { OXA } \\
(\mu \mathrm{g} / \mathrm{L})\end{array}$ & $\begin{array}{c}\text { Flufen- } \\
\text { acet } \\
(\mu \mathrm{g} / \mathrm{L})\end{array}$ & $\begin{array}{c}\text { Flufen- } \\
\text { acet } \\
\text { ESA } \\
(\mu \mathrm{g} / \mathrm{L})\end{array}$ & $\begin{array}{c}\text { Flufen- } \\
\text { acet } \\
\text { OXA } \\
(\mu \mathrm{g} / \mathrm{L})\end{array}$ & $\begin{array}{l}\text { Meto- } \\
\text { lachlor } \\
(\mu \mathrm{g} / \mathrm{L})\end{array}$ & $\begin{array}{l}\text { Meto- } \\
\text { lachlor } \\
\text { ESA } \\
(\mu \mathrm{g} / \mathrm{L})\end{array}$ & $\begin{array}{c}\text { Meto- } \\
\text { lachlor } \\
\text { OXA } \\
(\mu \mathrm{g} / \mathrm{L})\end{array}$ \\
\hline \multicolumn{20}{|c|}{ National Water-Quality Assessment Program (NAWQA) (fig. 3)—Continued } \\
\hline \multicolumn{20}{|c|}{ Upper Illinois River Basin (UIRB/BET)—Continued } \\
\hline \multirow[t]{5}{*}{05517120} & \multirow{5}{*}{$\begin{array}{l}\text { Pitner Ditch at State } \\
\text { Highway } 8, \\
\text { Lacrosse, IN }\end{array}$} & 03/03/99 & -- & $<0.05$ & $<0.05$ & -- & -- & 6.6 & 0.37 & -- & -- & -- & -- & -- & -- & -- & -- & 6.3 & 3.2 \\
\hline & & 04/08/99 & -- & $<.05$ & $<.05$ & -- & -- & 2.4 & .22 & -- & -- & -- & -- & -- & -- & -- & -- & 3.4 & 3.1 \\
\hline & & $04 / 20 / 99$ & -- & $<.05$ & $<.05$ & -- & -- & 2.3 & .21 & -- & -- & -- & -- & -- & -- & -- & -- & 3.3 & 3.0 \\
\hline & & $04 / 23 / 99$ & -- & $<.05$ & $<.05$ & -- & -- & 2.0 & .35 & -- & -- & -- & -- & -- & -- & -- & -- & 2.9 & 2.4 \\
\hline & & 03/08/00 & -- & .12 & .07 & -- & -- & .08 & $<.05$ & -- & -- & -- & -- & -- & -- & -- & -- & .48 & .25 \\
\hline \multirow{19}{*}{05525500} & \multirow{7}{*}{$\begin{array}{l}\text { Sugar Creek at } \\
\text { Milford, IL }\end{array}$} & $04 / 17 / 00$ & -- & $<.05$ & $<.05$ & -- & -- & $<.05$ & $<.05$ & -- & -- & -- & -- & -- & -- & -- & -- & .08 & $<.05$ \\
\hline & & $05 / 03 / 00$ & -- & .27 & .06 & -- & -- & .23 & .06 & -- & -- & -- & -- & -- & -- & -- & -- & .41 & .28 \\
\hline & & 05/04/99 & -- & .55 & .12 & -- & -- & .14 & .07 & -- & -- & -- & -- & -- & -- & -- & -- & 2.0 & .45 \\
\hline & & $05 / 10 / 99$ & -- & 1.3 & .66 & -- & -- & $<.05$ & $<.05$ & -- & -- & -- & -- & -- & -- & -- & -- & 2.1 & .56 \\
\hline & & 05/13/99 & -- & .77 & .17 & -- & -- & .18 & .07 & -- & -- & -- & -- & -- & -- & -- & -- & 2.3 & .39 \\
\hline & & $05 / 17 / 99$ & -- & 2.0 & 2.6 & -- & -- & .46 & .10 & -- & -- & -- & -- & -- & -- & -- & -- & 2.5 & .54 \\
\hline & & $05 / 26 / 99$ & -- & 2.2 & 3.7 & -- & -- & .70 & $<.05$ & -- & -- & -- & -- & -- & -- & -- & -- & 2.6 & .58 \\
\hline & & $06 / 02 / 99$ & -- & 1.4 & 2.2 & -- & -- & .38 & $<.05$ & -- & -- & -- & -- & -- & -- & -- & -- & 2.8 & .68 \\
\hline & & 06/07/99 & -- & 22 & 37 & -- & -- & .58 & .28 & -- & -- & -- & -- & -- & -- & -- & -- & 8.5 & 1.3 \\
\hline & & 06/13/99 & -- & .75 & 1.1 & -- & -- & .35 & .39 & -- & -- & -- & -- & -- & -- & -- & -- & 1.2 & 1.0 \\
\hline & & $06 / 15 / 99$ & -- & 1.6 & 2.8 & -- & -- & .16 & .42 & -- & -- & -- & -- & -- & -- & -- & -- & 1.5 & .63 \\
\hline & & $06 / 30 / 99$ & -- & 2.8 & 1.4 & -- & -- & .43 & .12 & -- & -- & -- & -- & -- & -- & -- & -- & 1.1 & .48 \\
\hline & & 07/07/99 & -- & .83 & .57 & -- & -- & .16 & .05 & -- & -- & -- & -- & -- & -- & -- & -- & 1.5 & .14 \\
\hline & & $07 / 12 / 99$ & -- & .31 & .28 & -- & -- & .55 & .07 & -- & -- & -- & -- & -- & -- & -- & -- & 1.3 & .18 \\
\hline & & 07/19/99 & -- & .19 & .06 & -- & -- & .16 & $<.05$ & -- & -- & -- & -- & -- & -- & -- & -- & .79 & .05 \\
\hline & & 07/28/99 & -- & .11 & .07 & -- & -- & .21 & $<.05$ & -- & -- & -- & -- & -- & -- & -- & -- & 1.1 & .12 \\
\hline & & 08/05/99 & -- & .07 & .05 & -- & -- & .43 & $<.05$ & -- & -- & -- & -- & -- & -- & -- & -- & .75 & .12 \\
\hline & & 08/13/99 & -- & .10 & .07 & -- & -- & .33 & $<.05$ & -- & -- & -- & -- & -- & -- & -- & -- & .57 & .15 \\
\hline & & 08/16/99 & -- & .11 & .06 & -- & -- & .18 & $<.05$ & -- & -- & -- & -- & -- & -- & -- & -- & .51 & .12 \\
\hline
\end{tabular}


Table 6. Analytical results for selected acetamide herbicides and their degradation products, ethanesulfonic acid, oxanilic acid, and sulfinyl acetic acid, in surface-water samples, 1993-2003.-Continued

[OGRL, U.S. Geological Survey Organic Geochemistry Research Laboratory, Lawrence, Kansas; ESA, ethanesulfonic acid; OXA, oxanilic acid; SAA, sulfinyl acetic acid; --, no data; <, less than]

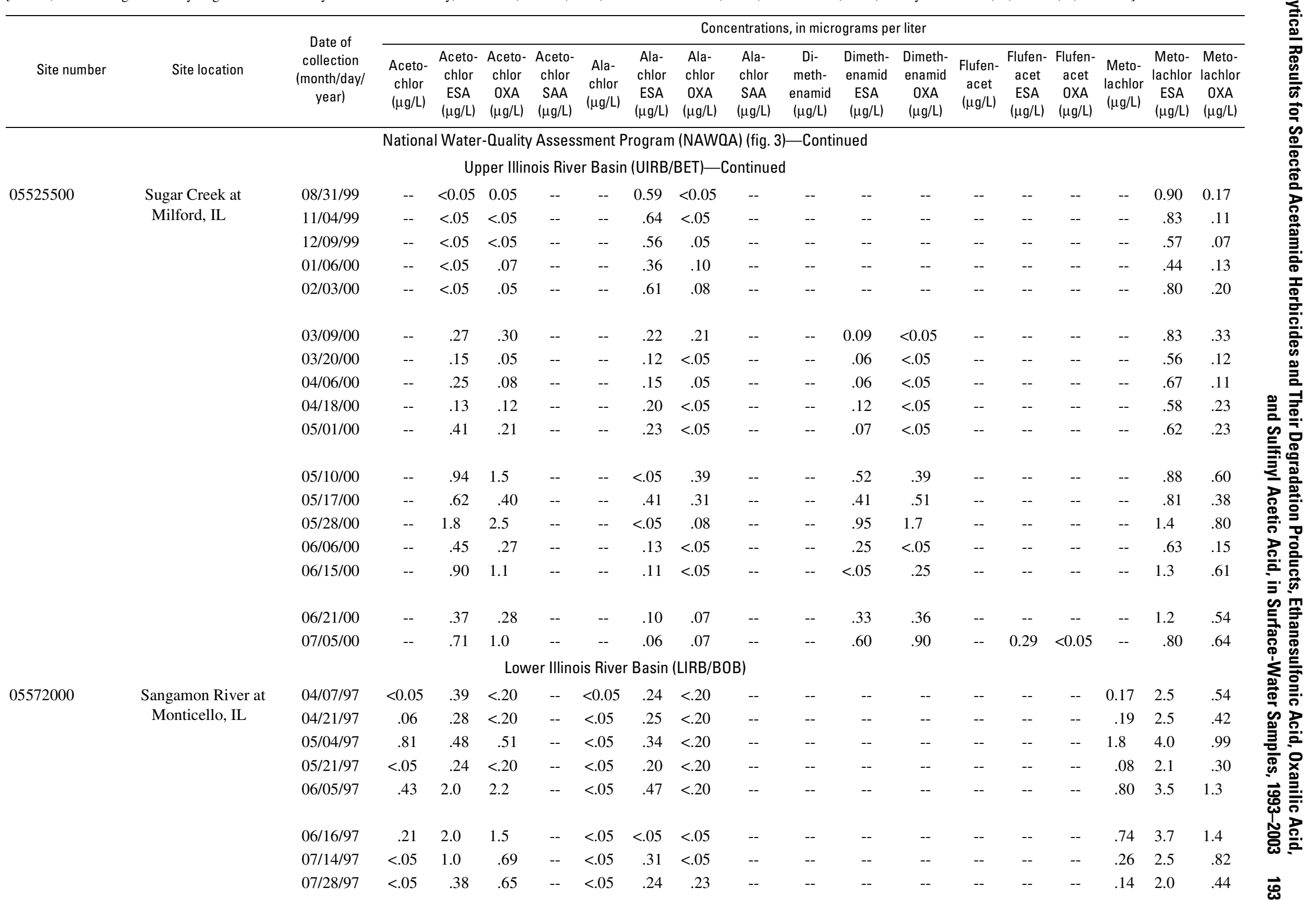


Table 6. Analytical results for selected acetamide herbicides and their degradation products, ethanesulfonic acid, oxanilic acid, and sulfinyl acetic acid, in surface-water samples, 1993-2003.-Continued

[OGRL, U.S. Geological Survey Organic Geochemistry Research Laboratory, Lawrence, Kansas; ESA, ethanesulfonic acid; OXA, oxanilic acid; SAA, sulfinyl acetic acid; --, no data; <, less than]

\begin{tabular}{|c|c|c|c|c|c|c|c|c|c|c|c|c|c|c|c|c|c|c|c|}
\hline \multirow[b]{2}{*}{ Site number } & \multirow[b]{2}{*}{ Site location } & \multirow[b]{2}{*}{$\begin{array}{c}\text { Date of } \\
\text { collection } \\
\text { (month/day/ } \\
\text { year) }\end{array}$} & \multicolumn{17}{|c|}{ Concentrations, in micrograms per liter } \\
\hline & & & $\begin{array}{l}\text { Aceto- } \\
\text { chlor } \\
(\mu \mathrm{g} / \mathrm{L})\end{array}$ & $\begin{array}{l}\text { Aceto- } \\
\text { chlor } \\
\text { ESA } \\
(\mu \mathrm{g} / \mathrm{L})\end{array}$ & $\begin{array}{l}\text { Aceto- } \\
\text { chlor } \\
\text { OXA } \\
(\mu \mathrm{g} / \mathrm{L})\end{array}$ & $\begin{array}{l}\text { Aceto- } \\
\text { chlor } \\
\text { SAA } \\
(\mu \mathrm{g} / \mathrm{L})\end{array}$ & $\begin{array}{l}\text { Ala- } \\
\text { chlor } \\
(\mu \mathrm{g} / \mathrm{L})\end{array}$ & $\begin{array}{l}\text { Ala- } \\
\text { chlor } \\
\text { ESA } \\
(\mu \mathrm{g} / \mathrm{L})\end{array}$ & $\begin{array}{l}\text { Ala- } \\
\text { chlor } \\
\text { OXA } \\
(\mu \mathrm{g} / \mathrm{L})\end{array}$ & $\begin{array}{l}\text { Ala- } \\
\text { chlor } \\
\text { SAA } \\
(\mu \mathrm{g} / \mathrm{L})\end{array}$ & $\begin{array}{l}\text { Di- } \\
\text { meth- } \\
\text { enamid } \\
(\mu \mathrm{g} / \mathrm{L})\end{array}$ & $\begin{array}{c}\text { Dimeth- } \\
\text { enamid } \\
\text { ESA } \\
(\mu \mathrm{g} / \mathrm{L})\end{array}$ & $\begin{array}{c}\text { Dimeth- } \\
\text { enamid } \\
\text { OXA } \\
(\mu \mathrm{g} / \mathrm{L})\end{array}$ & $\begin{array}{l}\text { Flufen- } \\
\text { acet } \\
(\mu \mathrm{g} / \mathrm{L})\end{array}$ & $\begin{array}{c}\text { Flufen- } \\
\text { acet } \\
\text { ESA } \\
(\mu \mathrm{g} / \mathrm{L})\end{array}$ & $\begin{array}{c}\text { Flufen- } \\
\text { acet } \\
\text { OXA } \\
(\mu \mathrm{g} / \mathrm{L})\end{array}$ & $\begin{array}{l}\text { Meto- } \\
\text { lachlor } \\
(\mu \mathrm{g} / \mathrm{L})\end{array}$ & $\begin{array}{l}\text { Meto- } \\
\text { lachlor } \\
\text { ESA } \\
(\mu \mathrm{g} / \mathrm{L})\end{array}$ & $\begin{array}{l}\text { Meto- } \\
\text { lachlor } \\
\text { OXA } \\
(\mu \mathrm{g} / \mathrm{L})\end{array}$ \\
\hline \multicolumn{20}{|c|}{ National Water-Quality Assessment Program (NAWQA) (fig. 3)—Continued } \\
\hline \multirow{5}{*}{05572000} & \multirow{5}{*}{$\begin{array}{l}\text { Sangamon River at } \\
\text { Monticello, IL }\end{array}$} & & & & wer Illin & nois Rive & er Basin & (LIRB/L & BOB)- & ontinue & & & & & & & & & \\
\hline & & $08 / 11 / 97$ & $<0.05$ & 0.27 & 0.20 & -- & $<0.05$ & 0.24 & $<0.05$ & -- & -- & -- & -- & -- & -- & -- & 0.07 & 1.5 & 0.35 \\
\hline & & $08 / 25 / 97$ & $<.05$ & 1.1 & .78 & -- & $<.05$ & .43 & .18 & -- & -- & -- & -- & -- & -- & -- & .24 & 2.9 & 1.1 \\
\hline & & 09/08/97 & $<.05$ & .38 & $<.05$ & -- & $<.05$ & .28 & $<.05$ & -- & -- & -- & -- & -- & -- & -- & .07 & 1.4 & .47 \\
\hline & & $09 / 22 / 97$ & $<.05$ & -- & -- & -- & $<.05$ & -- & -- & -- & -- & -- & -- & -- & -- & -- & .15 & -- & -- \\
\hline \multicolumn{20}{|c|}{ Lower Illinois River Basin (PTI) } \\
\hline 05586598 & $\begin{array}{l}\text { Apple Creek at } 900 \\
\text { E near Haypress, } \\
\text { IL }\end{array}$ & 08/07/97 & $<.05$ & $<.20$ & $<.20$ & -- & $<.05$ & .20 & $<.20$ & -- & -- & -- & -- & -- & -- & -- & $<.05$ & .40 & .25 \\
\hline 05556500 & $\begin{array}{l}\text { Big Bureau Creek at } \\
\text { Princeton, IL }\end{array}$ & 08/11/97 & .06 & .47 & $<.20$ & -- & $<.05$ & .47 & .45 & -- & -- & -- & -- & -- & -- & -- & .16 & 1.2 & .47 \\
\hline 05569860 & $\begin{array}{l}\text { Cedar Creek near } \\
\text { Avon, IL }\end{array}$ & 08/05/97 & $<.05$ & .17 & .35 & -- & $<.05$ & .23 & $<.20$ & -- & -- & -- & -- & -- & -- & -- & .11 & 1.5 & .29 \\
\hline 05559500 & $\begin{array}{l}\text { Crow Creek near } \\
\text { Washburn, IL }\end{array}$ & $08 / 12 / 97$ & $<.05$ & $<.20$ & $<.20$ & -- & $<.05$ & $<.20$ & $<.20$ & -- & -- & -- & -- & -- & -- & -- & $<.05$ & .76 & $<.20$ \\
\hline 05575850 & $\begin{array}{l}\text { Horse Creek at } \\
\text { Springfield, IL }\end{array}$ & 08/08/97 & $<.05$ & .26 & $<.20$ & -- & $<.05$ & .21 & $<.20$ & -- & -- & -- & -- & -- & -- & -- & .08 & 1.7 & .36 \\
\hline \multirow[t]{4}{*}{05553500} & \multirow{4}{*}{$\begin{array}{l}\text { Illinois River at } \\
\text { Ottawa, IL }\end{array}$} & 07/09/98 & .13 & 2.2 & 1.7 & -- & $<.05$ & 1.0 & $<.20$ & -- & -- & -- & -- & -- & -- & -- & .45 & 2.6 & 1.3 \\
\hline & & 08/06/98 & .05 & .84 & .85 & -- & $<.05$ & .95 & $<.20$ & -- & -- & -- & -- & -- & -- & -- & .09 & 1.3 & .48 \\
\hline & & 08/20/98 & $<.05$ & .56 & $<.20$ & -- & $<.05$ & .82 & $<.20$ & -- & -- & -- & -- & -- & -- & -- & $<.05$ & 1.1 & .45 \\
\hline & & 09/03/98 & $<.05$ & .24 & $<.20$ & -- & $<.05$ & .76 & $<.20$ & -- & -- & -- & -- & -- & -- & -- & $<.05$ & .80 & .29 \\
\hline \multirow[t]{7}{*}{05586100} & \multirow{7}{*}{$\begin{array}{l}\text { Illinois River at } \\
\text { Valley City, IL }\end{array}$} & $07 / 07 / 98$ & .13 & 1.3 & 1.1 & -- & $<.05$ & .79 & $<.20$ & -- & -- & -- & -- & -- & -- & -- & .60 & 2.1 & .93 \\
\hline & & $07 / 21 / 98$ & .06 & 1.4 & 1.1 & -- & $<.05$ & .79 & $<.20$ & -- & -- & -- & -- & -- & -- & -- & .36 & 2.5 & 1.1 \\
\hline & & 07/23/98 & $<.05$ & .56 & $<.20$ & -- & $<.05$ & 1.0 & $<.20$ & -- & -- & -- & -- & -- & -- & -- & .10 & 1.8 & $<.20$ \\
\hline & & 08/04/98 & $<.05$ & 1.1 & .60 & -- & $<.05$ & .83 & $<.20$ & -- & -- & -- & -- & -- & -- & -- & .10 & 2.9 & .66 \\
\hline & & 08/18/98 & $<.05$ & .83 & .37 & -- & $<.05$ & .89 & $<.20$ & -- & -- & -- & -- & -- & -- & -- & .07 & 1.8 & .51 \\
\hline & & 09/01/98 & $<.05$ & .56 & .22 & -- & $<.05$ & .76 & $<.20$ & -- & -- & -- & -- & -- & -- & -- & .07 & 1.5 & .55 \\
\hline & & 09/15/98 & $<.05$ & .25 & $<.20$ & -- & $<.05$ & .55 & $<.20$ & -- & -- & -- & -- & -- & -- & -- & $<.05$ & .72 & .20 \\
\hline
\end{tabular}


Table 6. Analytical results for selected acetamide herbicides and their degradation products, ethanesulfonic acid, oxanilic acid, and sulfinyl acetic acid, in surface-water samples, 1993-2003.-Continued

[OGRL, U.S. Geological Survey Organic Geochemistry Research Laboratory, Lawrence, Kansas; ESA, ethanesulfonic acid; OXA, oxanilic acid; SAA, sulfinyl acetic acid; --, no data; <, less than]

\begin{tabular}{|c|c|c|c|c|c|c|c|c|c|c|c|c|c|c|c|c|c|c|c|}
\hline \multirow[b]{2}{*}{ Site number } & \multirow[b]{2}{*}{ Site location } & \multirow[b]{2}{*}{$\begin{array}{c}\text { Date of } \\
\text { collection } \\
\text { (month/day/ } \\
\text { year) }\end{array}$} & \multicolumn{17}{|c|}{ Concentrations, in micrograms per liter } \\
\hline & & & $\begin{array}{l}\text { Aceto- } \\
\text { chlor } \\
(\mu \mathrm{g} / \mathrm{L})\end{array}$ & $\begin{array}{l}\text { Aceto- } \\
\text { chlor } \\
\text { ESA } \\
(\mu \mathrm{g} / \mathrm{L})\end{array}$ & $\begin{array}{l}\text { Aceto- } \\
\text { chlor } \\
\text { OXA } \\
(\mu \mathrm{g} / \mathrm{L})\end{array}$ & $\begin{array}{l}\text { Aceto- } \\
\text { chlor } \\
\text { SAA } \\
(\mu \mathrm{g} / \mathrm{L})\end{array}$ & $\begin{array}{l}\text { Ala- } \\
\text { chlor } \\
(\mu \mathrm{g} / \mathrm{L})\end{array}$ & $\begin{array}{l}\text { Ala- } \\
\text { chlor } \\
\text { ESA } \\
(\mu \mathrm{g} / \mathrm{L})\end{array}$ & $\begin{array}{c}\text { Ala- } \\
\text { chlor } \\
\text { OXA } \\
(\mu \mathrm{g} / \mathrm{L})\end{array}$ & $\begin{array}{l}\text { Ala- } \\
\text { chlor } \\
\text { SAA } \\
(\mu \mathrm{g} / \mathrm{L})\end{array}$ & $\begin{array}{l}\text { Di- } \\
\text { meth- } \\
\text { enamid } \\
(\mu \mathrm{g} / \mathrm{L})\end{array}$ & $\begin{array}{c}\text { Dimeth- } \\
\text { enamid } \\
\text { ESA } \\
(\mu \mathrm{g} / \mathrm{L})\end{array}$ & $\begin{array}{c}\text { Dimeth- } \\
\text { enamid } \\
\text { OXA } \\
(\mu \mathrm{g} / \mathrm{L})\end{array}$ & $\begin{array}{l}\text { Flufen- } \\
\text { acet } \\
(\mu \mathrm{g} / \mathrm{L})\end{array}$ & $\begin{array}{l}\text { Flufen- } \\
\text { acet } \\
\text { ESA } \\
(\mu \mathrm{g} / \mathrm{L})\end{array}$ & $\begin{array}{c}\text { Flufen- } \\
\text { acet } \\
\text { OXA } \\
(\mu \mathrm{g} / \mathrm{L})\end{array}$ & $\begin{array}{l}\text { Meto- } \\
\text { lachlor } \\
(\mu \mathrm{g} / \mathrm{L})\end{array}$ & $\begin{array}{l}\text { Meto- } \\
\text { lachlor } \\
\text { ESA } \\
(\mu \mathrm{g} / \mathrm{L})\end{array}$ & $\begin{array}{l}\text { Meto- } \\
\text { lachlor } \\
\text { OXA } \\
(\mu \mathrm{g} / \mathrm{L})\end{array}$ \\
\hline \multicolumn{20}{|c|}{ National Water-Quality Assessment Program (NAWQA) (fig. 3)—Continued } \\
\hline \multicolumn{20}{|c|}{ Lower Illinois River Basin (PTI)_Continued } \\
\hline 05568800 & $\begin{array}{l}\text { Indian Creek near } \\
\text { Wyoming, IL }\end{array}$ & 08/04/97 & $<0.05$ & $<0.20$ & 0.45 & -- & $<0.05$ & $<0.20$ & $<0.20$ & -- & -- & -- & -- & -- & -- & -- & 0.05 & 2.3 & $<0.20$ \\
\hline 05580000 & $\begin{array}{l}\text { Kickapoo Creek at } \\
\text { Waynesville, IL }\end{array}$ & $08 / 14 / 97$ & $<.05$ & $<.20$ & $<.20$ & -- & $<.05$ & $<.20$ & $<.20$ & -- & -- & -- & -- & -- & -- & -- & .08 & 1.4 & .29 \\
\hline 05563000 & $\begin{array}{l}\text { Kickapoo Creek } \\
\text { near Kickapoo, IL }\end{array}$ & 08/12/97 & $<.05$ & .35 & .72 & -- & $<.05$ & $<.20$ & $<.20$ & -- & -- & -- & -- & -- & -- & -- & .19 & .82 & .35 \\
\hline \multirow[t]{12}{*}{05584500} & \multirow{12}{*}{$\begin{array}{l}\text { Lamoine River at } \\
\text { Colmar, IL }\end{array}$} & 08/05/97 & $<.05$ & .25 & .29 & -- & $<.05$ & $<.20$ & $<.20$ & -- & -- & -- & -- & -- & -- & -- & $<.05$ & .61 & $<.20$ \\
\hline & & 07/07/98 & .12 & 1.2 & .85 & -- & $<.05$ & .35 & $<.20$ & -- & -- & -- & -- & -- & -- & -- & .38 & .92 & .42 \\
\hline & & $07 / 14 / 98$ & $<.05$ & 1.4 & .36 & -- & $<.05$ & .44 & $<.20$ & -- & -- & -- & -- & -- & -- & -- & .05 & 1.6 & .37 \\
\hline & & $07 / 21 / 98$ & $<.05$ & .75 & $<.20$ & -- & $<.05$ & .39 & $<.20$ & -- & -- & -- & -- & -- & -- & -- & $<.05$ & 1.3 & $<.20$ \\
\hline & & $07 / 28 / 98$ & $<.05$ & .63 & .20 & -- & $<.05$ & .35 & $<.20$ & -- & -- & -- & -- & -- & -- & -- & $<.05$ & 1.3 & .33 \\
\hline & & 08/05/98 & $<.05$ & 1.0 & .58 & -- & $<.05$ & $<.20$ & $<.20$ & -- & -- & -- & -- & -- & -- & -- & $<.05$ & 1.0 & $<.20$ \\
\hline & & $08 / 12 / 98$ & $<.05$ & .40 & $<.20$ & -- & $<.05$ & .34 & $<.20$ & -- & -- & -- & -- & -- & -- & -- & $<.05$ & .84 & $<.20$ \\
\hline & & 08/18/98 & $<.05$ & .65 & .38 & -- & $<.05$ & .42 & $<.20$ & -- & -- & -- & -- & -- & -- & -- & $<.05$ & 1.5 & $<.20$ \\
\hline & & $08 / 25 / 98$ & $<.05$ & .35 & $<.20$ & -- & $<.05$ & .33 & $<.20$ & -- & -- & -- & -- & -- & -- & -- & $<.05$ & 1.0 & $<.20$ \\
\hline & & 09/01/98 & $<.05$ & -- & -- & -- & $<.05$ & -- & -- & -- & -- & -- & -- & -- & -- & -- & $<.05$ & -- & -- \\
\hline & & 09/08/98 & $<.05$ & .22 & $<.20$ & -- & $<.05$ & .25 & $<.20$ & -- & -- & -- & -- & -- & -- & -- & $<.05$ & .57 & $<.20$ \\
\hline & & 09/15/98 & $<.05$ & $<.20$ & $<.20$ & -- & $<.05$ & $<.20$ & $<.20$ & -- & -- & -- & -- & -- & -- & -- & $<.05$ & .35 & $<.20$ \\
\hline 05568000 & $\begin{array}{l}\text { Mackinaw River at } \\
\text { Green Valley, IL }\end{array}$ & 08/13/97 & $<.05$ & $<.20$ & $<.20$ & -- & $<.05$ & $<.20$ & $<.20$ & -- & -- & -- & -- & -- & -- & -- & .42 & .37 & $<.20$ \\
\hline 05567500 & $\begin{array}{l}\text { Mackinaw River } \\
\text { near Congerville, } \\
\text { IL }\end{array}$ & 08/13/97 & $<.05$ & $<.20$ & $<.20$ & -- & $<.05$ & $<.20$ & $<.20$ & -- & -- & -- & -- & -- & -- & -- & .06 & .78 & $<.20$ \\
\hline 05564300 & $\begin{array}{c}\text { Mackinaw River } \\
\text { near Kappa, IL }\end{array}$ & 08/14/97 & $<.05$ & $<.20$ & $<.20$ & -- & $<.05$ & .22 & $<.20$ & -- & -- & -- & -- & -- & -- & -- & $<.05$ & 1.0 & .24 \\
\hline 05586645 & $\begin{array}{l}\text { Macoupin Creek } \\
\text { near Carlinville, } \\
\text { IL }\end{array}$ & 08/07/97 & $<.05$ & .30 & $<.20$ & -- & $<.05$ & .44 & $<.20$ & -- & -- & -- & -- & -- & -- & -- & .08 & 2.6 & 69 \\
\hline
\end{tabular}


Table 6. Analytical results for selected acetamide herbicides and their degradation products, ethanesulfonic acid, oxanilic acid, and sulfinyl acetic acid, in surface-water samples, 1993-2003.-Continued

[OGRL, U.S. Geological Survey Organic Geochemistry Research Laboratory, Lawrence, Kansas; ESA, ethanesulfonic acid; OXA, oxanilic acid; SAA, sulfinyl acetic acid; --, no data; <, less than]

\begin{tabular}{|c|c|c|c|c|c|c|c|c|c|c|c|c|c|c|c|c|c|c|c|}
\hline \multirow[b]{2}{*}{ Site number } & \multirow[b]{2}{*}{ Site location } & \multirow[b]{2}{*}{$\begin{array}{c}\text { Date of } \\
\text { collection } \\
\text { (month/day/ } \\
\text { year) }\end{array}$} & \multicolumn{17}{|c|}{ Concentrations, in micrograms per liter } \\
\hline & & & $\begin{array}{l}\text { Aceto- } \\
\text { chlor } \\
(\mu \mathrm{g} / \mathrm{L})\end{array}$ & $\begin{array}{c}\text { Aceto- } \\
\text { chlor } \\
\text { ESA } \\
(\mu \mathrm{g} / \mathrm{L})\end{array}$ & $\begin{array}{l}\text { Aceto- } \\
\text { chlor } \\
\text { OXA } \\
(\mu \mathrm{g} / \mathrm{L})\end{array}$ & $\begin{array}{c}\text { Aceto- } \\
\text { chlor } \\
\text { SAA } \\
(\mu \mathrm{g} / \mathrm{L})\end{array}$ & $\begin{array}{l}\text { Ala- } \\
\text { chlor } \\
(\mu \mathrm{g} / \mathrm{L})\end{array}$ & $\begin{array}{c}\text { Ala- } \\
\text { chlor } \\
\text { ESA } \\
(\mu \mathrm{g} / \mathrm{L})\end{array}$ & $\begin{array}{c}\text { Ala- } \\
\text { chlor } \\
\text { OXA } \\
(\mu \mathrm{g} / \mathrm{L})\end{array}$ & $\begin{array}{l}\text { Ala- } \\
\text { chlor } \\
\text { SAA } \\
(\mu \mathrm{g} / \mathrm{L})\end{array}$ & $\begin{array}{l}\text { Di- } \\
\text { meth- } \\
\text { enamid } \\
(\mu \mathrm{g} / \mathrm{L})\end{array}$ & $\begin{array}{c}\text { Dimeth- } \\
\text { enamid } \\
\text { ESA } \\
(\mu \mathrm{g} / \mathrm{L})\end{array}$ & $\begin{array}{c}\text { Dimeth- } \\
\text { enamid } \\
\text { OXA } \\
(\mu \mathrm{g} / \mathrm{L})\end{array}$ & $\begin{array}{l}\text { Flufen- } \\
\text { acet } \\
(\mu \mathrm{g} / \mathrm{L})\end{array}$ & 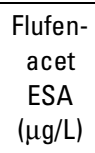 & $\begin{array}{c}\text { Flufen- } \\
\text { acet } \\
\text { OXA } \\
(\mu \mathrm{g} / \mathrm{L})\end{array}$ & $\begin{array}{l}\text { Meto- } \\
\text { lachlor } \\
(\mu \mathrm{g} / \mathrm{L})\end{array}$ & $\begin{array}{l}\text { Meto- } \\
\text { lachlor } \\
\text { ESA } \\
(\mu \mathrm{g} / \mathrm{L})\end{array}$ & $\begin{array}{l}\text { Meto- } \\
\text { lachlor } \\
\text { OXA } \\
(\mu \mathrm{g} / \mathrm{L})\end{array}$ \\
\hline \multicolumn{20}{|c|}{ National Water-Quality Assessment Program (NAWQA) (fig. 3)—Continued } \\
\hline \multicolumn{20}{|c|}{ Lower Illinois River Basin (PTI)_Continued } \\
\hline 05587000 & $\begin{array}{c}\text { Macoupin Creek } \\
\text { near Kane, IL }\end{array}$ & 08/07/97 & $<0.05$ & $<0.20$ & $<0.20$ & -- & $<0.05$ & $<0.20$ & $<0.20$ & -- & -- & -- & -- & -- & -- & -- & $<0.05$ & 0.39 & 0.20 \\
\hline 05585800 & Mckee Creek, IL & 08/06/97 & $<.05$ & .53 & .95 & -- & $<.05$ & .27 & $<.20$ & -- & -- & -- & -- & -- & -- & -- & .16 & .59 & .64 \\
\hline 05554000 & $\begin{array}{l}\text { Vermilion River } \\
\text { near Charlotte, IL }\end{array}$ & $08 / 15 / 97$ & $<.05$ & $<.20$ & $<.20$ & -- & $<.05$ & .31 & .22 & -- & -- & -- & -- & -- & -- & -- & .41 & 1.3 & .53 \\
\hline 05567000 & $\begin{array}{l}\text { Panther Creek near } \\
\text { El Paso, IL }\end{array}$ & 08/14/97 & $<.05$ & $<.20$ & $<.20$ & -- & $<.05$ & .29 & .24 & -- & -- & -- & -- & -- & -- & -- & $<.05$ & .87 & .23 \\
\hline \multirow[t]{12}{*}{05572000} & \multirow{12}{*}{$\begin{array}{l}\text { Sangamon River at } \\
\text { Monticello, IL }\end{array}$} & 06/29/98 & .06 & 2.1 & .98 & -- & $<.05$ & .67 & $<.20$ & -- & -- & -- & -- & -- & -- & -- & .53 & 3.8 & 1.1 \\
\hline & & 07/07/98 & $<.05$ & 1.9 & .73 & -- & $<.05$ & .50 & $<.20$ & -- & -- & -- & -- & -- & -- & -- & .34 & 3.4 & .84 \\
\hline & & 07/14/98 & $<.05$ & 2.6 & .68 & -- & $<.05$ & .58 & $<.20$ & -- & -- & -- & -- & -- & -- & -- & .19 & 4.9 & 1.2 \\
\hline & & 07/20/98 & $<.05$ & 1.6 & .41 & -- & $<.05$ & .44 & $<.20$ & -- & -- & -- & -- & -- & -- & -- & .13 & 4.1 & .92 \\
\hline & & $07 / 27 / 98$ & $<.05$ & 1.7 & .72 & -- & $<.05$ & .56 & .25 & -- & -- & -- & -- & -- & -- & -- & .30 & 4.1 & 1.3 \\
\hline & & 08/04/98 & $<.05$ & 1.9 & .42 & -- & $<.05$ & .57 & $<.20$ & -- & -- & -- & -- & -- & -- & -- & .10 & 5.4 & .88 \\
\hline & & 08/12/98 & $<.05$ & 2.0 & .51 & -- & $<.05$ & .83 & $<.20$ & -- & -- & -- & -- & -- & -- & -- & .13 & 6.1 & 1.5 \\
\hline & & 08/17/98 & $<.05$ & 1.8 & .43 & -- & $<.05$ & .64 & $<.20$ & -- & -- & -- & -- & -- & -- & -- & .09 & 4.7 & .95 \\
\hline & & $08 / 24 / 98$ & $<.05$ & .80 & $<.20$ & -- & $<.05$ & .38 & $<.20$ & -- & -- & -- & -- & -- & -- & -- & $<.05$ & 2.4 & .47 \\
\hline & & $08 / 31 / 98$ & $<.05$ & .43 & $<.20$ & -- & $<.05$ & .33 & $<.20$ & -- & -- & -- & -- & -- & -- & -- & $<.05$ & 2.0 & .31 \\
\hline & & 09/08/98 & $<.05$ & .43 & $<.20$ & -- & $<.05$ & .36 & $<.20$ & -- & -- & -- & -- & -- & -- & -- & $<.05$ & 1.6 & .32 \\
\hline & & 09/14/98 & $<.05$ & .40 & $<.20$ & -- & $<.05$ & .35 & $<.20$ & -- & -- & -- & -- & -- & -- & -- & $<.05$ & 1.5 & .30 \\
\hline 05570910 & $\begin{array}{l}\text { Sangamon River at } \\
\text { Fisher, IL }\end{array}$ & $08 / 15 / 97$ & $<.05$ & .21 & $<.20$ & -- & $<.05$ & .39 & .22 & -- & -- & -- & -- & -- & -- & -- & .06 & 1.1 & .31 \\
\hline 05568830 & $\begin{array}{l}\text { Spoon River near } \\
\text { Elmore, IL }\end{array}$ & 08/04/97 & $<.05$ & .46 & .60 & -- & $<.05$ & .26 & $<.20$ & -- & -- & -- & -- & -- & -- & -- & .14 & 1.9 & .34 \\
\hline 05583900 & $\begin{array}{l}\text { Sugar Creek near } \\
\text { Ray, IL }\end{array}$ & 08/06/97 & $<.05$ & $<.20$ & $<.20$ & -- & $<.05$ & .26 & $<.20$ & -- & -- & -- & -- & -- & -- & -- & .10 & .99 & .31 \\
\hline
\end{tabular}


Table 6. Analytical results for selected acetamide herbicides and their degradation products, ethanesulfonic acid, oxanilic acid, and sulfinyl acetic acid, in surface-water samples, 1993-2003.-Continued

[OGRL, U.S. Geological Survey Organic Geochemistry Research Laboratory, Lawrence, Kansas; ESA, ethanesulfonic acid; OXA, oxanilic acid; SAA, sulfinyl acetic acid; --, no data; <, less than]

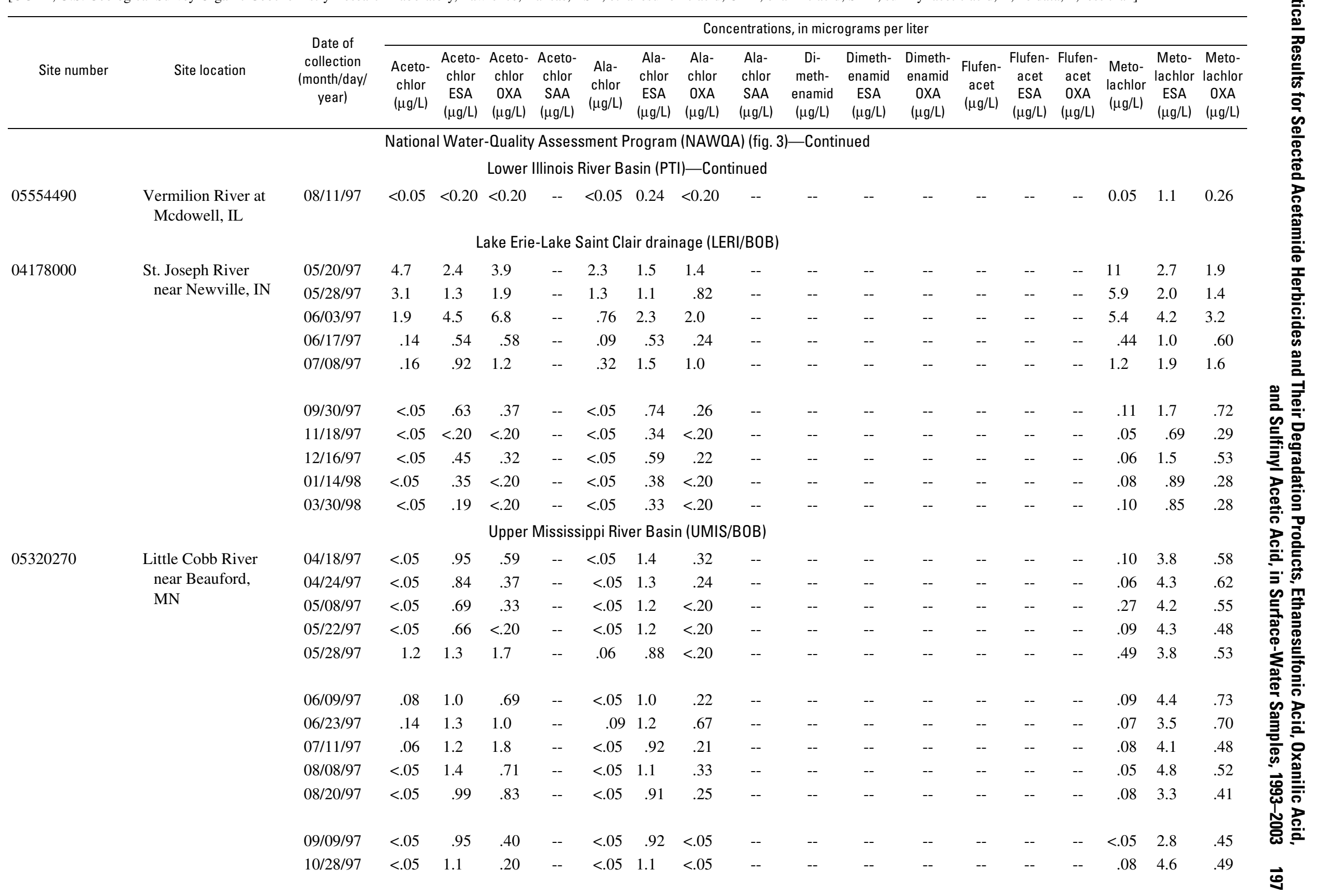


Table 6. Analytical results for selected acetamide herbicides and their degradation products, ethanesulfonic acid, oxanilic acid, and sulfinyl acetic acid, in surface-water samples, 1993-2003.-Continued

[OGRL, U.S. Geological Survey Organic Geochemistry Research Laboratory, Lawrence, Kansas; ESA, ethanesulfonic acid; OXA, oxanilic acid; SAA, sulfinyl acetic acid; --, no data; <, less than]

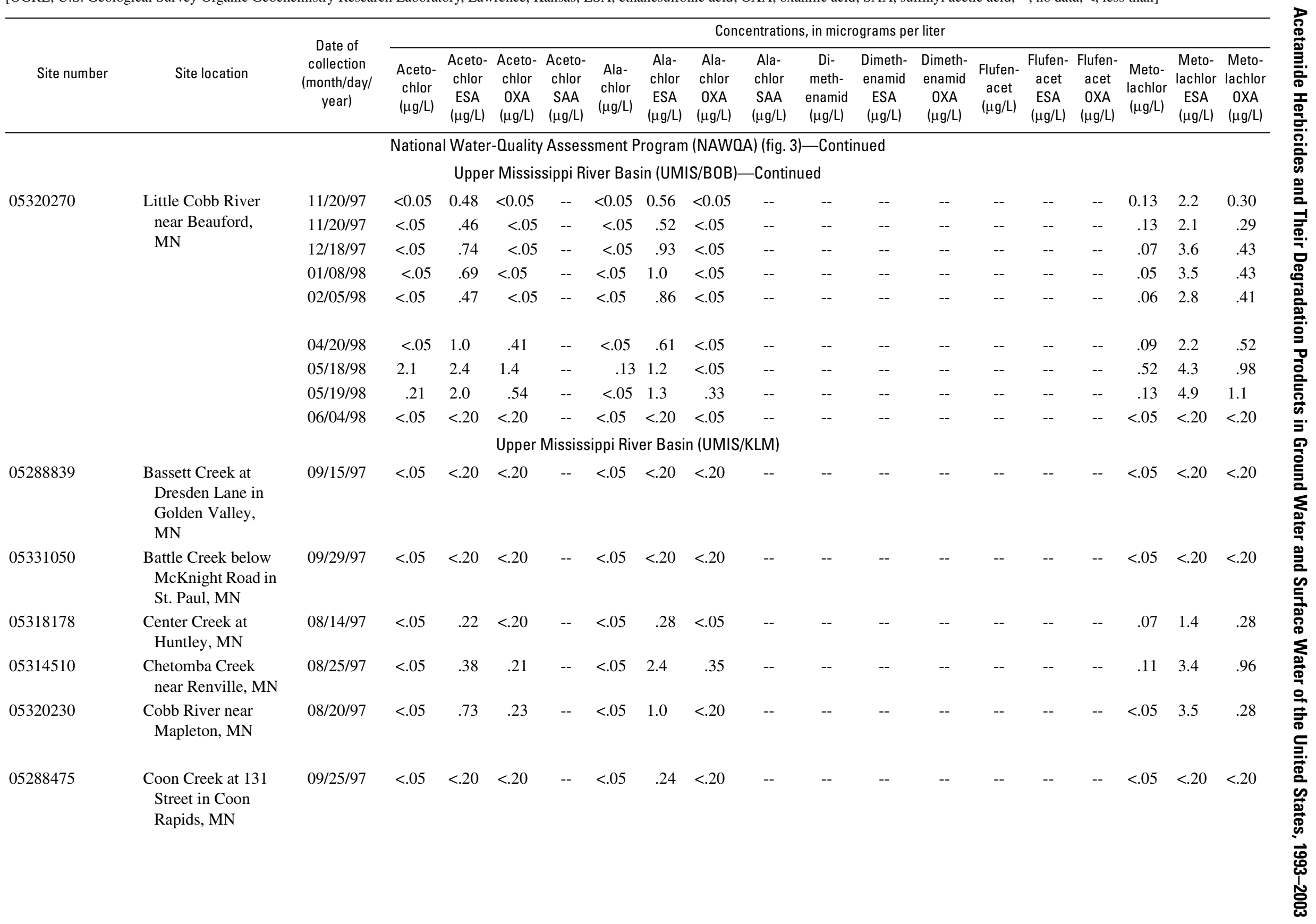


Table 6. Analytical results for selected acetamide herbicides and their degradation products, ethanesulfonic acid, oxanilic acid, and sulfinyl acetic acid, in surface-water samples, 1993-2003.-Continued

[OGRL, U.S. Geological Survey Organic Geochemistry Research Laboratory, Lawrence, Kansas; ESA, ethanesulfonic acid; OXA, oxanilic acid; SAA, sulfinyl acetic acid; --, no data; <, less than]

\begin{tabular}{|c|c|c|c|c|c|c|c|c|c|c|c|c|c|c|c|c|c|c|c|}
\hline \multirow[b]{2}{*}{ Site number } & \multirow[b]{2}{*}{ Site location } & \multirow[b]{2}{*}{$\begin{array}{c}\text { Date of } \\
\text { collection } \\
\text { (month/day/ } \\
\text { year) }\end{array}$} & \multicolumn{17}{|c|}{ Concentrations, in micrograms per liter } \\
\hline & & & $\begin{array}{l}\text { Aceto- } \\
\text { chlor } \\
(\mu \mathrm{g} / \mathrm{L})\end{array}$ & $\begin{array}{l}\text { Aceto- } \\
\text { chlor } \\
\text { ESA } \\
(\mu \mathrm{g} / \mathrm{L})\end{array}$ & $\begin{array}{l}\text { Aceto- } \\
\text { chlor } \\
\text { OXA } \\
(\mu \mathrm{g} / \mathrm{L})\end{array}$ & $\begin{array}{l}\text { Aceto- } \\
\text { chlor } \\
\text { SAA } \\
(\mu \mathrm{g} / \mathrm{L})\end{array}$ & $\begin{array}{l}\text { Ala- } \\
\text { chlor } \\
(\mu \mathrm{g} / \mathrm{L})\end{array}$ & $\begin{array}{l}\text { Ala- } \\
\text { chlor } \\
\text { ESA } \\
(\mu \mathrm{g} / \mathrm{L})\end{array}$ & $\begin{array}{l}\text { Ala- } \\
\text { chlor } \\
\text { OXA } \\
(\mu \mathrm{g} / \mathrm{L})\end{array}$ & $\begin{array}{c}\text { Ala- } \\
\text { chlor } \\
\text { SAA } \\
(\mu \mathrm{g} / \mathrm{L})\end{array}$ & $\begin{array}{l}\text { Di- } \\
\text { meth- } \\
\text { enamid } \\
(\mu \mathrm{g} / \mathrm{L})\end{array}$ & $\begin{array}{l}\text { Dimeth- } \\
\text { enamid } \\
\text { ESA } \\
(\mu \mathrm{g} / \mathrm{L})\end{array}$ & $\begin{array}{l}\text { Dimeth- } \\
\text { enamid } \\
\text { OXA } \\
(\mu \mathrm{g} / \mathrm{L})\end{array}$ & $\begin{array}{c}\text { Flufen- } \\
\text { acet } \\
(\mu \mathrm{g} / \mathrm{L})\end{array}$ & $\begin{array}{l}\text { Flufen- } \\
\text { acet } \\
\text { ESA } \\
(\mu \mathrm{g} / \mathrm{L})\end{array}$ & $\begin{array}{l}\text { Flufen- } \\
\text { acet } \\
\text { OXA } \\
(\mu \mathrm{g} / \mathrm{L})\end{array}$ & $\begin{array}{l}\text { Meto- } \\
\text { lachlor } \\
(\mu \mathrm{g} / \mathrm{L})\end{array}$ & $\begin{array}{l}\text { Meto- } \\
\text { lachlor } \\
\text { ESA } \\
(\mu \mathrm{g} / \mathrm{L})\end{array}$ & $\begin{array}{l}\text { Meto- } \\
\text { lachlor } \\
\text { OXA } \\
(\mu \mathrm{g} / \mathrm{L})\end{array}$ \\
\hline \multicolumn{20}{|c|}{ National Water-Quality Assessment Program (NAWQA) (fig. 3)—Continued } \\
\hline \multicolumn{20}{|c|}{ Upper Mississippi River Basin (UMIS/KLM)—Continued } \\
\hline 05317828 & $\begin{array}{l}\text { Coon Creek at U.S. } \\
\text { Highway } 169 \text { near } \\
\text { Blue Earth, MN }\end{array}$ & 08/20/97 & 0.21 & 0.53 & $<0.20$ & -- & $<0.05$ & 0.81 & $<0.20$ & -- & -- & -- & -- & -- & -- & -- & 0.12 & 4.8 & 0.73 \\
\hline 05304795 & $\begin{array}{c}\text { Dry Weather Creek } \\
\text { near Watson, MN }\end{array}$ & 08/12/97 & $<.05$ & $<.20$ & 1.4 & -- & $<.05$ & .39 & $<.20$ & -- & -- & -- & -- & -- & -- & -- & $<.05$ & .28 & $<.20$ \\
\hline 05318050 & $\begin{array}{l}\text { East Branch Blue } \\
\text { Earth River below } \\
\text { Bricelyn, MN }\end{array}$ & 08/20/97 & $<.05$ & .68 & .26 & -- & $<.05$ & .82 & $<.20$ & -- & -- & -- & -- & -- & -- & -- & $<.05$ & 1.6 & $<.20$ \\
\hline 05287890 & $\begin{array}{l}\text { Elm Creek near } \\
\text { Champlin, MN }\end{array}$ & 09/24/97 & $<.05$ & .30 & .27 & -- & $<.05$ & .52 & $<.20$ & -- & -- & -- & -- & -- & -- & -- & $<.05$ & .21 & $<.20$ \\
\hline 05318240 & $\begin{array}{l}\text { Elm Creek near } \\
\text { Northrop, MN }\end{array}$ & 08/14/97 & $<.05$ & .39 & $<.20$ & -- & $<.05$ & .27 & $<.20$ & -- & -- & -- & -- & -- & -- & -- & $<.05$ & 2.7 & .28 \\
\hline 05286798 & $\begin{array}{l}\text { Ford Brook on } \\
\text { Green Valley } \\
\text { Road in Ramsey, } \\
\text { MN }\end{array}$ & 09/16/97 & $<.05$ & $<.20$ & $<.20$ & -- & $<.05$ & .20 & $<.20$ & -- & -- & -- & -- & -- & -- & -- & $<.05$ & .40 & .23 \\
\hline 05314500 & $\begin{array}{l}\text { Hawk Creek near } \\
\text { Maynard, MN }\end{array}$ & 08/11/97 & $<.05$ & $<.20$ & $<.20$ & -- & $<.05$ & .64 & $<.20$ & -- & -- & -- & -- & -- & -- & -- & $<.05$ & $<.20$ & $<.20$ \\
\hline 05326700 & $\begin{array}{l}\text { High Island Creek } \\
\text { near Arlington, } \\
\text { MN }\end{array}$ & 08/28/97 & $<.05$ & .48 & .36 & -- & $<.05$ & 1.2 & $<.20$ & -- & -- & -- & -- & -- & -- & -- & .09 & 1.7 & .70 \\
\hline 05320080 & $\begin{array}{l}\text { Le Sueur River near } \\
\text { Wilton, MN }\end{array}$ & 08/18/97 & $<.05$ & 1.6 & .55 & -- & $<.05$ & 1.2 & $<.20$ & -- & -- & -- & -- & -- & -- & -- & .10 & 3.8 & .74 \\
\hline 05320270 & $\begin{array}{l}\text { Little Cobb River } \\
\text { near Beauford, } \\
\text { MN }\end{array}$ & 08/18/97 & $<.05$ & 1.3 & .60 & -- & $<.05$ & 1.0 & .21 & -- & -- & -- & -- & -- & -- & -- & .09 & 3.8 & .51 \\
\hline 05317170 & $\begin{array}{l}\text { Little Cottonwood } \\
\text { River near Searles, } \\
\text { MN }\end{array}$ & 08/13/97 & $<.05$ & .33 & $<.20$ & -- & $<.05$ & .75 & $<.20$ & -- & -- & -- & -- & -- & -- & -- & .07 & 1.4 & .27 \\
\hline
\end{tabular}


Table 6. Analytical results for selected acetamide herbicides and their degradation products, ethanesulfonic acid, oxanilic acid, and sulfinyl acetic acid, in surface-water samples, 1993-2003.-Continued

[OGRL, U.S. Geological Survey Organic Geochemistry Research Laboratory, Lawrence, Kansas; ESA, ethanesulfonic acid; OXA, oxanilic acid; SAA, sulfinyl acetic acid; --, no data; <, less than]

\begin{tabular}{|c|c|c|c|c|c|c|c|c|c|c|c|c|c|c|c|c|c|c|c|}
\hline \multirow[b]{2}{*}{ Site number } & \multirow[b]{2}{*}{ Site location } & \multirow[b]{2}{*}{$\begin{array}{c}\text { Date of } \\
\text { collection } \\
\text { (month/day/ } \\
\text { year) }\end{array}$} & \multicolumn{17}{|c|}{ Concentrations, in micrograms per liter } \\
\hline & & & $\begin{array}{l}\text { Aceto- } \\
\text { chlor } \\
(\mu \mathrm{g} / \mathrm{L})\end{array}$ & $\begin{array}{l}\text { Aceto- } \\
\text { chlor } \\
\text { ESA } \\
(\mu \mathrm{g} / \mathrm{L})\end{array}$ & $\begin{array}{c}\text { Aceto- } \\
\text { chlor } \\
\text { OXA } \\
(\mu \mathrm{g} / \mathrm{L})\end{array}$ & $\begin{array}{l}\text { Aceto- } \\
\text { chlor } \\
\text { SAA } \\
(\mu \mathrm{g} / \mathrm{L})\end{array}$ & $\begin{array}{l}\text { Ala- } \\
\text { chlor } \\
(\mu \mathrm{g} / \mathrm{L})\end{array}$ & $\begin{array}{l}\text { Ala- } \\
\text { chlor } \\
\text { ESA } \\
(\mu \mathrm{g} / \mathrm{L})\end{array}$ & $\begin{array}{l}\text { Ala- } \\
\text { chlor } \\
\text { OXA } \\
(\mu \mathrm{g} / \mathrm{L})\end{array}$ & $\begin{array}{l}\text { Ala- } \\
\text { chlor } \\
\text { SAA } \\
(\mu \mathrm{g} / \mathrm{L})\end{array}$ & $\begin{array}{l}\text { Di- } \\
\text { meth- } \\
\text { enamid } \\
(\mu \mathrm{g} / \mathrm{L})\end{array}$ & $\begin{array}{c}\text { Dimeth- } \\
\text { enamid } \\
\text { ESA } \\
(\mu \mathrm{g} / \mathrm{L})\end{array}$ & $\begin{array}{c}\text { Dimeth- } \\
\text { enamid } \\
\text { OXA } \\
(\mu \mathrm{g} / \mathrm{L})\end{array}$ & $\begin{array}{c}\text { Flufen- } \\
\text { acet } \\
(\mu \mathrm{g} / \mathrm{L})\end{array}$ & $\begin{array}{c}\text { Flufen- } \\
\text { acet } \\
\text { ESA } \\
(\mu \mathrm{g} / \mathrm{L})\end{array}$ & $\begin{array}{c}\text { Flufen- } \\
\text { acet } \\
\text { OXA } \\
(\mu \mathrm{g} / \mathrm{L})\end{array}$ & $\begin{array}{l}\text { Meto- } \\
\text { lachlor } \\
(\mu \mathrm{g} / \mathrm{L})\end{array}$ & $\begin{array}{l}\text { Meto- } \\
\text { lachlor } \\
\text { ESA } \\
(\mu \mathrm{g} / \mathrm{L})\end{array}$ & $\begin{array}{c}\text { Meto- } \\
\text { lachlor } \\
\text { OXA } \\
(\mu \mathrm{g} / \mathrm{L})\end{array}$ \\
\hline \multicolumn{20}{|c|}{ National Water-Quality Assessment Program (NAWOA) (fig. 3) -Continued } \\
\hline \multicolumn{20}{|c|}{ Upper Mississippi River Basin (UMIS/KLM)—Continued } \\
\hline 05320450 & $\begin{array}{l}\text { Maple River near } \\
\text { Sterling Center, } \\
\text { MN }\end{array}$ & $08 / 19 / 97$ & 0.07 & 0.65 & 0.31 & -- & $<0.05$ & 0.55 & 0.24 & -- & -- & -- & -- & -- & -- & -- & 0.24 & 2.9 & 0.48 \\
\hline 05326150 & $\begin{array}{l}\text { Middle Branch } \\
\text { Rush River near } \\
\text { New Rome, MN }\end{array}$ & 08/28/97 & $<.05$ & .87 & .38 & -- & $<.05$ & 1.5 & $<.20$ & -- & -- & -- & -- & -- & -- & -- & .12 & 2.8 & 1.1 \\
\hline 05330702 & $\begin{array}{l}\text { Minnesota River } \\
\text { tributary on Beech } \\
\text { Street in Chaska, } \\
\text { MN }\end{array}$ & $09 / 26 / 97$ & $<.05$ & $<.20$ & $<.20$ & -- & $<.05$ & .27 & $<.20$ & -- & -- & -- & -- & -- & -- & -- & $<.05$ & $<.20$ & $<.20$ \\
\hline 05289600 & $\begin{array}{c}\text { Minnehaha Creek at } \\
\text { Nicollet Avenue in } \\
\text { Minneapolis, MN }\end{array}$ & 09/24/97 & $<.05$ & $<.20$ & $<.20$ & -- & $<.05$ & $<.20$ & $<.20$ & -- & -- & -- & -- & -- & -- & -- & $<.05$ & $<.20$ & $<.20$ \\
\hline 05330902 & $\begin{array}{l}\text { Nine Mile Creek } \\
\text { near James Circle } \\
\text { at Bloomington, } \\
\text { MN }\end{array}$ & 09/30/97 & $<.05$ & $<.20$ & $<.20$ & -- & $<.05$ & $<.20$ & $<.20$ & -- & -- & -- & -- & -- & -- & -- & $<.05$ & $<.20$ & $<.20$ \\
\hline 05319360 & $\begin{array}{l}\text { Perch Creek below } \\
\text { Vernon Center, } \\
\text { MN }\end{array}$ & 08/19/97 & $<.05$ & .84 & $<.20$ & -- & $<.05$ & .45 & $<.20$ & -- & -- & -- & -- & -- & -- & -- & $<.05$ & 2.5 & .31 \\
\hline 05330801 & $\begin{array}{l}\text { Purgatory Creek on } \\
\text { Pioneer tributary } \\
\text { in Eden Prairie, } \\
\text { MN }\end{array}$ & $09 / 25 / 97$ & $<.05$ & $<.20$ & $<.20$ & -- & $<.05$ & $<.20$ & $<.20$ & -- & -- & -- & -- & -- & -- & -- & $<.05$ & $<.20$ & $<.20$ \\
\hline 05288598 & $\begin{array}{l}\text { Rice Creek at } \\
\text { Central Avenue, } \\
\text { Fridley, MN }\end{array}$ & $09 / 15 / 97$ & $<.05$ & $<.20$ & $<.20$ & -- & .19 & $<.20$ & $<.20$ & -- & -- & -- & -- & -- & -- & -- & $<.05$ & $<.20$ & $<.20$ \\
\hline 05330750 & $\begin{array}{l}\text { Riley Creek at Eden } \\
\text { Prairie, MN }\end{array}$ & $09 / 25 / 97$ & $<.05$ & $<.05$ & $<.20$ & -- & $<.05$ & $<.20$ & $<.20$ & -- & -- & -- & -- & -- & -- & -- & $<.05$ & $<.20$ & $<.20$ \\
\hline
\end{tabular}


Table 6. Analytical results for selected acetamide herbicides and their degradation products, ethanesulfonic acid, oxanilic acid, and sulfinyl acetic acid, in surface-water samples, 1993-2003.-Continued

[OGRL, U.S. Geological Survey Organic Geochemistry Research Laboratory, Lawrence, Kansas; ESA, ethanesulfonic acid; OXA, oxanilic acid; SAA, sulfinyl acetic acid; --, no data; <, less than]

\begin{tabular}{|c|c|c|c|c|c|c|c|c|c|c|c|c|c|c|c|c|c|c|c|}
\hline \multirow[b]{2}{*}{ Site number } & \multirow[b]{2}{*}{ Site location } & \multirow[b]{2}{*}{$\begin{array}{l}\text { Date of } \\
\text { collection } \\
\text { (month/day/ } \\
\text { year) }\end{array}$} & \multicolumn{17}{|c|}{ Concentrations, in micrograms per liter } \\
\hline & & & $\begin{array}{l}\text { Aceto- } \\
\text { chlor } \\
(\mu \mathrm{g} / \mathrm{L})\end{array}$ & $\begin{array}{l}\text { Aceto- } \\
\text { chlor } \\
\text { ESA } \\
(\mu \mathrm{g} / \mathrm{L})\end{array}$ & $\begin{array}{l}\text { Aceto- } \\
\text { chlor } \\
\text { OXA } \\
(\mu \mathrm{g} / \mathrm{L})\end{array}$ & $\begin{array}{l}\text { Aceto- } \\
\text { chlor } \\
\text { SAA } \\
(\mu \mathrm{g} / \mathrm{L})\end{array}$ & $\begin{array}{l}\text { Ala- } \\
\text { chlor } \\
(\mu \mathrm{g} / \mathrm{L})\end{array}$ & $\begin{array}{l}\text { Ala- } \\
\text { chlor } \\
\text { ESA } \\
(\mu \mathrm{g} / \mathrm{L})\end{array}$ & $\begin{array}{l}\text { Ala- } \\
\text { chlor } \\
\text { OXA } \\
(\mu \mathrm{g} / \mathrm{L})\end{array}$ & $\begin{array}{l}\text { Ala- } \\
\text { chlor } \\
\text { SAA } \\
(\mu \mathrm{g} / \mathrm{L})\end{array}$ & $\begin{array}{l}\text { Di- } \\
\text { meth- } \\
\text { enamid } \\
(\mu \mathrm{g} / \mathrm{L})\end{array}$ & $\begin{array}{c}\text { Dimeth- } \\
\text { enamid } \\
\text { ESA } \\
(\mu \mathrm{g} / \mathrm{L})\end{array}$ & $\begin{array}{c}\text { Dimeth- } \\
\text { enamid } \\
\text { OXA } \\
(\mu \mathrm{g} / \mathrm{L})\end{array}$ & $\begin{array}{c}\text { Flufen- } \\
\text { acet } \\
(\mu \mathrm{g} / \mathrm{L})\end{array}$ & $\begin{array}{c}\text { Flufen- } \\
\text { acet } \\
\text { ESA } \\
(\mu \mathrm{g} / \mathrm{L})\end{array}$ & $\begin{array}{c}\text { Flufen- } \\
\text { acet } \\
\text { OXA } \\
(\mu \mathrm{g} / \mathrm{L})\end{array}$ & $\begin{array}{l}\text { Meto- } \\
\text { lachlor } \\
(\mu \mathrm{g} / \mathrm{L})\end{array}$ & $\begin{array}{l}\text { Meto- } \\
\text { lachlor } \\
\text { ESA } \\
(\mu \mathrm{g} / \mathrm{L})\end{array}$ & $\begin{array}{c}\text { Meto- } \\
\text { lachlor } \\
\text { OXA } \\
(\mu \mathrm{g} / \mathrm{L})\end{array}$ \\
\hline \multicolumn{20}{|c|}{ National Water-Quality Assessment Program (NAWOA) (fig. 3)—Continued } \\
\hline \multicolumn{20}{|c|}{ Upper Mississippi River Basin (UMIS/KLM)—Continued } \\
\hline 05326250 & $\begin{array}{l}\text { South Branch Rush } \\
\text { River near Rush } \\
\text { River, MN }\end{array}$ & $08 / 21 / 97$ & $<0.05$ & 1.2 & 0.42 & -- & $<0.05$ & 2.0 & $<0.20$ & -- & -- & -- & -- & -- & -- & -- & 0.12 & 4.4 & 1.3 \\
\hline 05288487 & $\begin{array}{l}\text { Sand Creek at Xeon } \\
\text { Street in Coon } \\
\text { Rapids, MN }\end{array}$ & $09 / 26 / 97$ & $<.05$ & $<.20$ & $<.20$ & -- & $<.05$ & $<.20$ & $<.20$ & -- & -- & -- & -- & -- & -- & -- & $<.05$ & $<.20$ & $<.20$ \\
\hline 05303900 & $\begin{array}{l}\text { Shakopee Creek } \\
\text { near Louriston, } \\
\text { MN }\end{array}$ & $08 / 11 / 97$ & $<.05$ & $<.20$ & $<.20$ & -- & $<.05$ & $<.20$ & $<.20$ & -- & -- & -- & -- & -- & -- & -- & $<.05$ & $<.20$ & $<.20$ \\
\hline 05288705 & $\begin{array}{l}\text { Shingle Creek at } \\
\text { Queen Avenue in } \\
\text { Minneapolis, MN }\end{array}$ & 09/29/97 & $<.05$ & $<.20$ & $<.20$ & -- & $<.05$ & $<.20$ & $<.20$ & -- & -- & -- & -- & -- & -- & -- & $<.05$ & $<.20$ & $<.20$ \\
\hline 05316985 & $\begin{array}{l}\text { Sleepy Eye Creek } \\
\text { near Springfield, } \\
\text { MN }\end{array}$ & 08/13/97 & $<.05$ & .31 & $<.20$ & -- & $<.05$ & .41 & $<.20$ & -- & -- & -- & -- & -- & -- & -- & $<.05$ & .93 & .22 \\
\hline 05318138 & $\begin{array}{l}\text { South Creek near } \\
\text { Huntley, MN }\end{array}$ & 08/14/97 & $<.05$ & .38 & $<.20$ & -- & $<.05$ & .47 & $<.20$ & -- & -- & -- & -- & -- & -- & -- & $<.05$ & 1.3 & .26 \\
\hline 05319050 & $\begin{array}{l}\text { South Fork } \\
\text { Watonwan River } \\
\text { near St. James, } \\
\text { MN }\end{array}$ & 08/19/97 & $<.05$ & .63 & .33 & -- & $<.05$ & .54 & .21 & -- & -- & -- & -- & -- & -- & -- & $<.05$ & 1.6 & $<.20$ \\
\hline 05312000 & $\begin{array}{l}\text { Spring Creek near } \\
\text { Spring Creek, MN }\end{array}$ & 08/12/97 & $<.05$ & .42 & .59 & -- & $<.05$ & $<.20$ & $<.20$ & -- & -- & -- & -- & -- & -- & -- & $<.05$ & .63 & $<.20$ \\
\hline 05318800 & $\begin{array}{l}\text { St. James Creek } \\
\text { near La Salle, MN }\end{array}$ & $08 / 21 / 97$ & $<.05$ & 1.4 & .32 & -- & $<.05$ & .96 & $<.20$ & -- & -- & -- & -- & -- & -- & -- & .09 & 3.1 & .75 \\
\hline 05318630 & $\begin{array}{l}\text { Watonwan River } \\
\text { near St. James, } \\
\text { MN }\end{array}$ & $08 / 15 / 97$ & $<.05$ & .43 & $<.20$ & -- & $<.05$ & .21 & $<.20$ & -- & -- & -- & -- & -- & -- & -- & $<.05$ & 1.0 & $<.20$ \\
\hline
\end{tabular}


Table 6. Analytical results for selected acetamide herbicides and their degradation products, ethanesulfonic acid, oxanilic acid, and sulfinyl acetic acid, in surface-water samples, 1993-2003.-Continued

[OGRL, U.S. Geological Survey Organic Geochemistry Research Laboratory, Lawrence, Kansas; ESA, ethanesulfonic acid; OXA, oxanilic acid; SAA, sulfinyl acetic acid; --, no data; <, less than]

\begin{tabular}{|c|c|c|c|c|c|c|c|c|c|c|c|c|c|c|c|c|c|c|c|}
\hline \multirow[b]{2}{*}{ Site number } & \multirow[b]{2}{*}{ Site location } & \multirow[b]{2}{*}{$\begin{array}{c}\text { Date of } \\
\text { collection } \\
\text { (month/day/ } \\
\text { year) }\end{array}$} & \multicolumn{17}{|c|}{ Concentrations, in micrograms per liter } \\
\hline & & & $\begin{array}{l}\text { Aceto- } \\
\text { chlor } \\
(\mu \mathrm{g} / \mathrm{L})\end{array}$ & $\begin{array}{l}\text { Aceto- } \\
\text { chlor } \\
\text { ESA } \\
(\mu \mathrm{g} / \mathrm{L})\end{array}$ & $\begin{array}{l}\text { Aceto- } \\
\text { chlor } \\
\text { OXA } \\
(\mu \mathrm{g} / \mathrm{L})\end{array}$ & $\begin{array}{l}\text { Aceto- } \\
\text { chlor } \\
\text { SAA } \\
(\mu \mathrm{g} / \mathrm{L})\end{array}$ & $\begin{array}{l}\text { Ala- } \\
\text { chlor } \\
(\mu \mathrm{g} / \mathrm{L})\end{array}$ & $\begin{array}{l}\text { Ala- } \\
\text { chlor } \\
\text { ESA } \\
(\mu \mathrm{g} / \mathrm{L})\end{array}$ & $\begin{array}{l}\text { Ala- } \\
\text { chlor } \\
\text { OXA } \\
(\mu \mathrm{g} / \mathrm{L})\end{array}$ & $\begin{array}{l}\text { Ala- } \\
\text { chlor } \\
\text { SAA } \\
(\mu \mathrm{g} / \mathrm{L})\end{array}$ & $\begin{array}{l}\text { Di- } \\
\text { meth- } \\
\text { enamid } \\
(\mu \mathrm{g} / \mathrm{L})\end{array}$ & $\begin{array}{c}\text { Dimeth- } \\
\text { enamid } \\
\text { ESA } \\
(\mu \mathrm{g} / \mathrm{L})\end{array}$ & $\begin{array}{c}\text { Dimeth- } \\
\text { enamid } \\
\text { OXA } \\
(\mu \mathrm{g} / \mathrm{L})\end{array}$ & $\begin{array}{c}\text { Flufen- } \\
\text { acet } \\
(\mu \mathrm{g} / \mathrm{L})\end{array}$ & $\begin{array}{l}\text { Flufen- } \\
\text { acet } \\
\text { ESA } \\
(\mu \mathrm{g} / \mathrm{L})\end{array}$ & $\begin{array}{l}\text { Flufen- } \\
\text { acet } \\
\text { OXA } \\
(\mu \mathrm{g} / \mathrm{L})\end{array}$ & $\begin{array}{l}\text { Meto- } \\
\text { lachlor } \\
(\mu \mathrm{g} / \mathrm{L})\end{array}$ & $\begin{array}{l}\text { Meto- } \\
\text { lachlor } \\
\text { ESA } \\
(\mu \mathrm{g} / \mathrm{L})\end{array}$ & $\begin{array}{l}\text { Meto- } \\
\text { lachlor } \\
\text { OXA } \\
(\mu \mathrm{g} / \mathrm{L})\end{array}$ \\
\hline \multicolumn{20}{|c|}{ National Water-Quality Assessment Program (NAWQA) (fig. 3)—Continued } \\
\hline \multicolumn{20}{|c|}{ Upper Mississippi River Basin (UMIS/KLM)—Continued } \\
\hline 05317800 & $\begin{array}{l}\text { West Branch Blue } \\
\text { Earth River above } \\
\text { Elmore, MN }\end{array}$ & $08 / 20 / 97$ & $<0.05$ & 0.27 & $<0.20$ & -- & $<0.05$ & 0.60 & $<0.20$ & -- & -- & -- & -- & -- & -- & -- & 0.10 & 2.6 & 0.48 \\
\hline \multicolumn{20}{|c|}{ Mississippi Embayment study (MISE/BOB) } \\
\hline \multirow[t]{17}{*}{07288650} & \multirow{16}{*}{$\begin{array}{l}\text { Bogue Phalia River } \\
\text { near Leland, MS }\end{array}$} & $03 / 31 / 97$ & $<.05$ & $<.20$ & $<.20$ & -- & $<.05$ & $<.20$ & $<.20$ & -- & -- & -- & -- & -- & -- & -- & $<.05$ & $<.20$ & $<.20$ \\
\hline & & $04 / 15 / 97$ & 2.0 & $<.20$ & .32 & -- & .46 & $<.20$ & $<.20$ & -- & -- & -- & -- & -- & -- & -- & 1.9 & .35 & .40 \\
\hline & & $04 / 28 / 97$ & $<.05$ & $<.20$ & $<.20$ & -- & $<.05$ & $<.20$ & $<.20$ & -- & -- & -- & -- & -- & -- & -- & 5.7 & .84 & .97 \\
\hline & & 05/13/97 & .70 & $<.20$ & $<.20$ & -- & $<.05$ & $<.20$ & $<.20$ & -- & -- & -- & -- & -- & -- & -- & .30 & .30 & .49 \\
\hline & & $05 / 20 / 97$ & $<.05$ & $<.20$ & $<.20$ & -- & $<.05$ & $<.20$ & $<.20$ & -- & -- & -- & -- & -- & -- & -- & .59 & $<.20$ & .20 \\
\hline & & $05 / 28 / 97$ & $<.05$ & $<.05$ & .38 & -- & $<.05$ & $<.05$ & $<.20$ & -- & -- & -- & -- & -- & -- & -- & 2.5 & .23 & $<.05$ \\
\hline & & 06/03/97 & $<.05$ & .21 & $<.20$ & -- & $<.05$ & $<.20$ & $<.20$ & -- & -- & -- & -- & -- & -- & -- & 1.2 & .50 & .44 \\
\hline & & 06/18/97 & $<.05$ & $<.05$ & $<.05$ & -- & $<.05$ & $<.05$ & $<.05$ & -- & -- & -- & -- & -- & -- & -- & .80 & .29 & .65 \\
\hline & & $07 / 14 / 97$ & $<.05$ & $<.05$ & $<.05$ & -- & $<.05$ & $<.05$ & $<.05$ & -- & -- & -- & -- & -- & -- & -- & .09 & $<.05$ & $<.05$ \\
\hline & & 08/11/97 & $<.05$ & $<.05$ & $<.05$ & -- & $<.05$ & $<.05$ & 1.2 & -- & -- & -- & -- & -- & -- & -- & $<.05$ & $<.05$ & $<.05$ \\
\hline & & 08/18/97 & $<.05$ & $<.05$ & $<.05$ & -- & $<.05$ & $<.05$ & 1.1 & -- & -- & -- & -- & -- & -- & -- & $<.05$ & $<.05$ & $<.05$ \\
\hline & & 09/23/97 & $<.05$ & $<.05$ & $<.05$ & -- & $<.05$ & $<.05$ & $<.05$ & -- & -- & -- & -- & -- & -- & -- & $<.05$ & $<.05$ & $<.05$ \\
\hline & & $10 / 07 / 97$ & $<.05$ & $<.05$ & $<.05$ & -- & $<.05$ & $<.05$ & $<.05$ & -- & -- & -- & -- & -- & -- & -- & $<.05$ & $<.05$ & $<.05$ \\
\hline & & $11 / 17 / 97$ & $<.05$ & $<.05$ & $<.05$ & -- & $<.05$ & $<.05$ & $<.05$ & -- & -- & -- & -- & -- & -- & -- & $<.05$ & $<.05$ & .37 \\
\hline & & $12 / 29 / 97$ & $<.05$ & $<.05$ & $<.05$ & -- & $<.05$ & $<.05$ & $<.05$ & -- & -- & -- & -- & -- & -- & -- & $<.05$ & $<.05$ & .20 \\
\hline & & $01 / 28 / 98$ & $<.05$ & $<.05$ & $<.05$ & -- & $<.05$ & $<.05$ & $<.05$ & -- & -- & -- & -- & -- & -- & -- & $<.05$ & $<.05$ & $<.05$ \\
\hline & & & & Sante & River & Basin a & and Coa & stal dra & inages & SANT/B & B) & & & & & & & & \\
\hline \multirow[t]{5}{*}{02174250} & \multirow{5}{*}{$\begin{array}{l}\text { Cow Castle Creek } \\
\text { near Bowman, SC }\end{array}$} & $04 / 16 / 97$ & $<.05$ & $<.20$ & $<.20$ & -- & $<.05$ & .24 & $<.20$ & -- & -- & -- & -- & -- & -- & -- & $<.05$ & $<.20$ & $<.20$ \\
\hline & & 05/06/97 & $<.05$ & $<.20$ & $<.20$ & -- & $<.05$ & $<.20$ & $<.20$ & -- & -- & -- & -- & -- & -- & -- & .93 & .23 & $<.20$ \\
\hline & & 06/10/97 & $<.05$ & $<.20$ & $<.20$ & -- & $<.05$ & $<.20$ & $<.20$ & -- & -- & -- & -- & -- & -- & -- & .26 & $<.20$ & $<.20$ \\
\hline & & 07/10/97 & $<.05$ & $<.20$ & $<.20$ & -- & $<.05$ & .26 & $<.20$ & -- & -- & -- & -- & -- & -- & -- & .05 & .27 & $<.20$ \\
\hline & & 08/04/97 & $<.05$ & $<.20$ & $<.20$ & -- & $<.05$ & $<.20$ & $<.20$ & -- & -- & -- & -- & -- & -- & -- & .05 & $<.20$ & $<.20$ \\
\hline
\end{tabular}


Table 6. Analytical results for selected acetamide herbicides and their degradation products, ethanesulfonic acid, oxanilic acid, and sulfinyl acetic acid, in surface-water samples, 1993-2003.-Continued

[OGRL, U.S. Geological Survey Organic Geochemistry Research Laboratory, Lawrence, Kansas; ESA, ethanesulfonic acid; OXA, oxanilic acid; SAA, sulfinyl acetic acid; --, no data; <, less than]

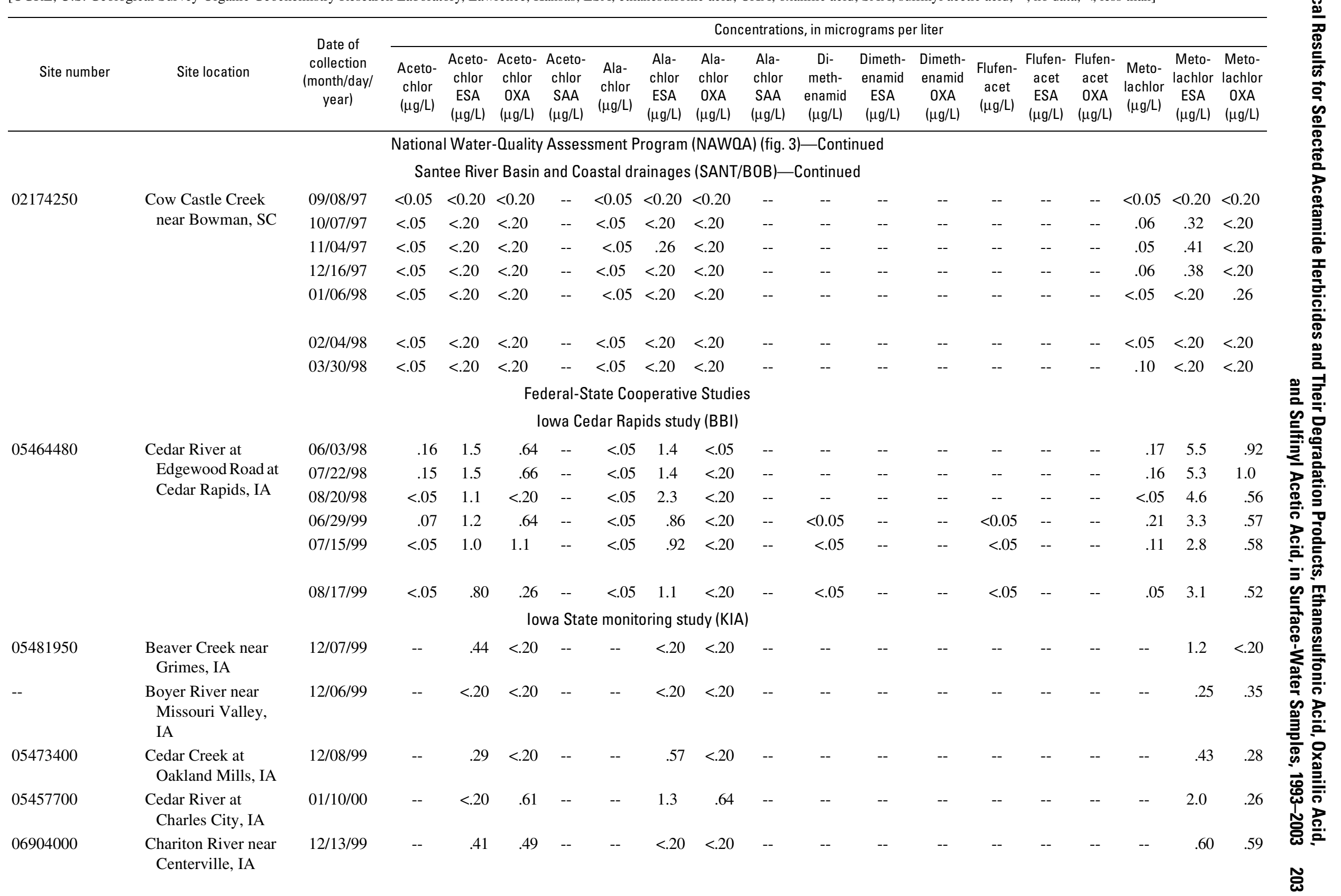


Table 6. Analytical results for selected acetamide herbicides and their degradation products, ethanesulfonic acid, oxanilic acid, and sulfinyl acetic acid, in surface-water samples, 1993-2003.-Continued

[OGRL, U.S. Geological Survey Organic Geochemistry Research Laboratory, Lawrence, Kansas; ESA, ethanesulfonic acid; OXA, oxanilic acid; SAA, sulfinyl acetic acid; --, no data; <, less than]

\begin{tabular}{|c|c|c|c|c|c|c|c|c|c|c|c|c|c|c|c|c|c|c|c|}
\hline \multirow[b]{2}{*}{ Site number } & \multirow[b]{2}{*}{ Site location } & \multirow[b]{2}{*}{$\begin{array}{c}\text { Date of } \\
\text { collection } \\
\text { (month/day/ } \\
\text { year) }\end{array}$} & \multicolumn{17}{|c|}{ Concentrations, in micrograms per liter } \\
\hline & & & $\begin{array}{l}\text { Aceto- } \\
\text { chlor } \\
(\mu \mathrm{g} / \mathrm{L})\end{array}$ & $\begin{array}{l}\text { Aceto- } \\
\text { chlor } \\
\text { ESA } \\
(\mu \mathrm{g} / \mathrm{L})\end{array}$ & $\begin{array}{l}\text { Aceto- } \\
\text { chlor } \\
\text { OXA } \\
(\mu \mathrm{g} / \mathrm{L})\end{array}$ & $\begin{array}{l}\text { Aceto- } \\
\text { chlor } \\
\text { SAA } \\
(\mu \mathrm{g} / \mathrm{L})\end{array}$ & $\begin{array}{l}\text { Ala- } \\
\text { chlor } \\
(\mu \mathrm{g} / \mathrm{L})\end{array}$ & $\begin{array}{l}\text { Ala- } \\
\text { chlor } \\
\text { ESA } \\
(\mu \mathrm{g} / \mathrm{L})\end{array}$ & $\begin{array}{c}\text { Ala- } \\
\text { chlor } \\
\text { OXA } \\
(\mu \mathrm{g} / \mathrm{L})\end{array}$ & $\begin{array}{l}\text { Ala- } \\
\text { chlor } \\
\text { SAA } \\
(\mu \mathrm{g} / \mathrm{L})\end{array}$ & $\begin{array}{c}\text { Di- } \\
\text { meth- } \\
\text { enamid } \\
(\mu \mathrm{g} / \mathrm{L})\end{array}$ & $\begin{array}{c}\text { Di- } \\
\text { meth- } \\
\text { enamid } \\
\text { ESA } \\
(\mu \mathrm{g} / \mathrm{L})\end{array}$ & $\begin{array}{c}\text { Di- } \\
\text { meth- } \\
\text { enamid } \\
0 X A \\
(\mu \mathrm{g} / \mathrm{L})\end{array}$ & $\begin{array}{c}\text { Flufen- } \\
\text { acet } \\
(\mu \mathrm{g} / \mathrm{L})\end{array}$ & $\begin{array}{l}\text { Flufen- } \\
\text { acet } \\
\text { ESA } \\
(\mu \mathrm{g} / \mathrm{L})\end{array}$ & $\begin{array}{l}\text { Flufen- } \\
\text { acet } \\
\text { OXA } \\
(\mu \mathrm{g} / \mathrm{L})\end{array}$ & $\begin{array}{l}\text { Meto- } \\
\text { lachlor } \\
(\mu \mathrm{g} / \mathrm{L})\end{array}$ & $\begin{array}{l}\text { Meto- } \\
\text { lachlor } \\
\text { ESA } \\
(\mu \mathrm{g} / \mathrm{L})\end{array}$ & $\begin{array}{c}\text { Meto- } \\
\text { lachlor } \\
\text { OXA } \\
(\mu \mathrm{g} / \mathrm{L})\end{array}$ \\
\hline \multicolumn{20}{|c|}{ Federal-State Cooperative Studies-Continued } \\
\hline \multicolumn{20}{|c|}{ lowa State monitoring study $(\mathrm{KIA})$ - Continued } \\
\hline-- & $\begin{array}{l}\text { East Fork Des Moines } \\
\text { River near St. } \\
\text { Joseph, IA }\end{array}$ & $12 / 27 / 99$ & -- & 0.47 & 2.0 & -- & -- & 1.1 & 3.3 & -- & -- & -- & -- & -- & -- & -- & -- & 1.2 & 0.38 \\
\hline-- & $\begin{array}{l}\text { East Nishnabotna } \\
\text { River near } \\
\text { Shenandoah, IA }\end{array}$ & $12 / 06 / 99$ & -- & $<.20$ & $<.20$ & -- & -- & $<.20$ & $<.20$ & -- & -- & -- & -- & -- & -- & -- & -- & .65 & .37 \\
\hline-- & $\begin{array}{l}\text { English River at } \\
\text { Riverside, IA }\end{array}$ & $12 / 07 / 99$ & -- & .43 & $<.20$ & -- & -- & .93 & $<.20$ & -- & -- & -- & -- & -- & -- & -- & -- & .92 & 1.0 \\
\hline 06600500 & $\begin{array}{l}\text { Floyd River at James, } \\
\text { IA }\end{array}$ & $12 / 07 / 99$ & -- & $<.20$ & $<.20$ & -- & -- & $<.20$ & $<.20$ & -- & -- & -- & -- & -- & -- & -- & -- & .49 & .26 \\
\hline-- & $\begin{array}{l}\text { Iowa River at } \\
\text { Columbus Junction, } \\
\text { IA }\end{array}$ & $12 / 08 / 99$ & -- & .27 & .30 & -- & -- & .97 & .80 & -- & -- & -- & -- & -- & -- & -- & -- & 1.4 & .43 \\
\hline-- & $\begin{array}{l}\text { Iowa River near } \\
\text { Steamboat Rock, IA }\end{array}$ & $12 / 06 / 99$ & -- & .20 & $<.20$ & -- & -- & 1.3 & $<.20$ & -- & -- & -- & -- & -- & -- & -- & -- & 1.8 & .67 \\
\hline-- & $\begin{array}{l}\text { Little Sioux River near } \\
\text { Smithland, IA }\end{array}$ & $12 / 07 / 99$ & -- & $<.20$ & $<.20$ & -- & -- & .30 & $<.20$ & -- & -- & -- & -- & -- & -- & -- & -- & .48 & .29 \\
\hline 05418500 & $\begin{array}{l}\text { Maquoketa River near } \\
\text { Maquoketa, IA }\end{array}$ & $12 / 06 / 99$ & -- & .22 & $<.20$ & -- & -- & 1.0 & $<.20$ & -- & -- & -- & -- & -- & -- & -- & -- & 1.8 & .52 \\
\hline-- & $\begin{array}{l}\text { North Fork Maquoketa } \\
\text { River at Maquoketa, } \\
\text { IA }\end{array}$ & $12 / 06 / 99$ & -- & .36 & $<.20$ & -- & -- & .96 & $<.20$ & -- & -- & -- & -- & -- & -- & -- & -- & .83 & .58 \\
\hline 05482300 & $\begin{array}{l}\text { North Raccoon River } \\
\text { near Sac City, IA }\end{array}$ & $12 / 08 / 99$ & -- & .27 & $<.20$ & -- & -- & .31 & $<.20$ & -- & -- & -- & -- & -- & -- & -- & -- & .57 & .37 \\
\hline 05486000 & $\begin{array}{l}\text { North River near } \\
\text { Norwalk, IA }\end{array}$ & $12 / 08 / 99$ & -- & $<.20$ & $<.20$ & -- & -- & $<.20$ & $<.20$ & -- & -- & -- & -- & -- & -- & -- & -- & .37 & .22 \\
\hline 05472500 & $\begin{array}{l}\text { North Skunk River } \\
\text { near Sigourney, IA }\end{array}$ & $12 / 07 / 99$ & -- & $<.20$ & $<.20$ & -- & -- & .47 & $<.20$ & -- & -- & -- & -- & -- & -- & -- & -- & .86 & .36 \\
\hline
\end{tabular}


Table 6. Analytical results for selected acetamide herbicides and their degradation products, ethanesulfonic acid, oxanilic acid, and sulfinyl acetic acid, in surface-water samples, 1993-2003.-Continued

[OGRL, U.S. Geological Survey Organic Geochemistry Research Laboratory, Lawrence, Kansas; ESA, ethanesulfonic acid; OXA, oxanilic acid; SAA, sulfinyl acetic acid; --, no data; <, less than]

\begin{tabular}{|c|c|c|c|c|c|c|c|c|c|c|c|c|c|c|c|c|c|c|c|c|}
\hline \multirow[b]{2}{*}{ Site number } & \multirow[b]{2}{*}{ Site location } & \multirow[b]{2}{*}{$\begin{array}{c}\text { Date of } \\
\text { collection } \\
\text { (month/day/ } \\
\text { year) }\end{array}$} & \multicolumn{17}{|c|}{ Concentrations, in micrograms per liter } & \\
\hline & & & $\begin{array}{c}\text { Aceto- } \\
\text { chlor } \\
(\mu \mathrm{g} / \mathrm{L})\end{array}$ & $\begin{array}{l}\text { Aceto- } \\
\text { chlor } \\
\text { ESA } \\
(\mu \mathrm{g} / \mathrm{L})\end{array}$ & $\begin{array}{l}\text { Aceto- } \\
\text { chlor } \\
\text { OXA } \\
(\mu \mathrm{g} / \mathrm{L})\end{array}$ & $\begin{array}{l}\text { Aceto- } \\
\text { chlor } \\
\text { SAA } \\
(\mu \mathrm{g} / \mathrm{L})\end{array}$ & $\begin{array}{l}\text { Ala- } \\
\text { chlor } \\
(\mu \mathrm{g} / \mathrm{L})\end{array}$ & $\begin{array}{l}\text { Ala- } \\
\text { chlor } \\
\text { ESA } \\
(\mu \mathrm{g} / \mathrm{L})\end{array}$ & $\begin{array}{l}\text { Ala- } \\
\text { chlor } \\
\text { OXA } \\
(\mu \mathrm{g} / \mathrm{L})\end{array}$ & $\begin{array}{l}\text { Ala- } \\
\text { chlor } \\
\text { SAA } \\
(\mu \mathrm{g} / \mathrm{L})\end{array}$ & $\begin{array}{c}\text { Di- } \\
\text { meth- } \\
\text { enamid } \\
(\mu \mathrm{g} / \mathrm{L})\end{array}$ & $\begin{array}{c}\text { Di- } \\
\text { meth- } \\
\text { enamid } \\
\text { ESA } \\
(\mu \mathrm{g} / \mathrm{L})\end{array}$ & $\begin{array}{c}\text { Di- } \\
\text { meth- } \\
\text { enamid } \\
0 X A \\
(\mu \mathrm{g} / \mathrm{L})\end{array}$ & $\begin{array}{c}\text { Flufen- } \\
\text { acet } \\
(\mu \mathrm{g} / \mathrm{L})\end{array}$ & $\begin{array}{l}\text { Flufen- } \\
\text { acet } \\
\text { ESA } \\
(\mu \mathrm{g} / \mathrm{L})\end{array}$ & $\begin{array}{c}\text { Flufen- } \\
\text { acet } \\
\text { OXA } \\
(\mu \mathrm{g} / \mathrm{L})\end{array}$ & $\begin{array}{l}\text { Meto- } \\
\text { lachlor } \\
(\mu \mathrm{g} / \mathrm{L})\end{array}$ & $\begin{array}{l}\text { Meto- } \\
\text { lachlor } \\
\text { ESA } \\
(\mu \mathrm{g} / \mathrm{L})\end{array}$ & $\begin{array}{l}\text { Meto- } \\
\text { lachlor } \\
\text { OXA } \\
(\mu \mathrm{g} / \mathrm{L})\end{array}$ & $\begin{array}{l}\bar{n} \\
\frac{1}{2} \\
\frac{c}{0} \\
\frac{D}{D}\end{array}$ \\
\hline \multicolumn{20}{|c|}{ Federal-State Cooperative Studies-Continued } & \\
\hline \multicolumn{20}{|c|}{ lowa State monitoring study $(\mathrm{KIA})$ —Continued } & \\
\hline-- & $\begin{array}{l}\text { Rock River near } \\
\text { Hawarden, IA }\end{array}$ & $12 / 07 / 99$ & -- & $<0.20$ & $<0.20$ & -- & -- & $<0.20$ & $<0.20$ & -- & -- & -- & -- & -- & -- & -- & -- & 0.93 & 0.26 & \\
\hline 06608500 & $\begin{array}{l}\text { Soldier River near } \\
\text { Pisgah, IA }\end{array}$ & $12 / 07 / 99$ & -- & $<.20$ & $<.20$ & -- & -- & $<.20$ & $<.20$ & -- & -- & -- & -- & -- & -- & -- & -- & .22 & $<.20$ & \\
\hline 05484000 & $\begin{array}{c}\text { South Raccoon River } \\
\text { near Redfield, IA }\end{array}$ & $12 / 08 / 99$ & -- & $<.20$ & .26 & -- & -- & $<.20$ & $<.20$ & -- & -- & -- & -- & -- & -- & -- & -- & .54 & $<.20$ & 高 \\
\hline-- & $\begin{array}{l}\text { South Skunk River } \\
\text { near Cambridge, IA }\end{array}$ & $12 / 06 / 99$ & -- & .30 & $<.20$ & -- & -- & .42 & $<.20$ & -- & -- & -- & -- & -- & -- & -- & -- & .88 & .69 & \\
\hline 05412500 & $\begin{array}{l}\text { Turkey River at } \\
\text { Garber, IA }\end{array}$ & $12 / 07 / 99$ & -- & .23 & $<.20$ & -- & -- & .85 & $<.20$ & -- & -- & -- & -- & -- & -- & -- & -- & .79 & .23 & 文 芒 \\
\hline 05388250 & $\begin{array}{l}\text { Upper Iowa River at } \\
\text { Dorchester, IA }\end{array}$ & 12/07/99 & -- & $<.20$ & $<.20$ & -- & -- & .41 & $<.20$ & -- & -- & -- & -- & -- & -- & -- & -- & .73 & $<.20$ & \\
\hline-- & $\begin{array}{l}\text { Volga River at } \\
\text { Elkport, IA }\end{array}$ & $12 / 07 / 99$ & -- & .23 & $<.20$ & -- & -- & .46 & $<.20$ & -- & -- & -- & -- & -- & -- & -- & -- & .97 & .42 & 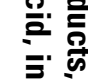 \\
\hline 05422000 & $\begin{array}{l}\text { Wapsipinicon River at } \\
\text { Dewitt, IA }\end{array}$ & $12 / 06 / 99$ & -- & .32 & $<.20$ & -- & -- & 1.4 & $<.20$ & -- & -- & -- & -- & -- & -- & -- & -- & 1.6 & .44 & \\
\hline 05421000 & $\begin{array}{l}\text { Wapsipinicon River at } \\
\text { Independence, IA }\end{array}$ & 01/10/00 & -- & .74 & $<.20$ & -- & -- & 2.3 & .21 & -- & -- & -- & -- & -- & -- & -- & -- & 2.7 & .56 & 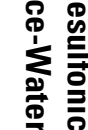 \\
\hline 05458900 & $\begin{array}{l}\text { West Fork Cedar River } \\
\text { near Finchford, IA }\end{array}$ & $12 / 09 / 99$ & -- & .25 & $<.20$ & -- & -- & $<.20$ & $<.20$ & -- & -- & -- & -- & -- & -- & -- & -- & 2.1 & 2.9 & 龸 \\
\hline-- & $\begin{array}{l}\text { West Fork Des Moines } \\
\text { River below } \\
\text { Humboldt, IA }\end{array}$ & $12 / 27 / 99$ & -- & $<.20$ & $<.20$ & -- & -- & .50 & .34 & -- & -- & -- & -- & -- & -- & -- & -- & .83 & $<.20$ & 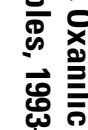 \\
\hline \multicolumn{20}{|c|}{ Minnesota hydrologic study (MLM) } & \\
\hline 06483000 & Rock River at & $03 / 24 / 97$ & $<0.05$ & $<.20$ & $<.20$ & -- & $<0.05$ & $<.20$ & $<.20$ & -- & -- & -- & -- & -- & -- & -- & 0.99 & 1.2 & .35 & \\
\hline & Luverne, MN & $04 / 07 / 97$ & $<.05$ & 1.0 & .67 & -- & $<.05$ & .57 & $<.20$ & -- & -- & -- & -- & -- & -- & -- & .39 & 3.2 & 1.4 & ㅁ \\
\hline
\end{tabular}


Table 6. Analytical results for selected acetamide herbicides and their degradation products, ethanesulfonic acid, oxanilic acid, and sulfinyl acetic acid, in surface-water samples, 1993-2003.-Continued

[OGRL, U.S. Geological Survey Organic Geochemistry Research Laboratory, Lawrence, Kansas; ESA, ethanesulfonic acid; OXA, oxanilic acid; SAA, sulfinyl acetic acid; --, no data; <, less than]

\begin{tabular}{|c|c|c|c|c|c|c|c|c|c|c|c|c|c|c|c|c|c|c|c|}
\hline \multirow[b]{2}{*}{ Site number } & \multirow[b]{2}{*}{ Site location } & \multirow[b]{2}{*}{$\begin{array}{c}\text { Date of } \\
\text { collection } \\
\text { (month/day/ } \\
\text { year) }\end{array}$} & \multicolumn{17}{|c|}{ Concentrations, in micrograms per liter } \\
\hline & & & $\begin{array}{l}\text { Aceto- } \\
\text { chlor } \\
(\mu \mathrm{g} / \mathrm{L})\end{array}$ & $\begin{array}{l}\text { Aceto- } \\
\text { chlor } \\
\text { ESA } \\
(\mu \mathrm{g} / \mathrm{L})\end{array}$ & $\begin{array}{l}\text { Aceto- } \\
\text { chlor } \\
0 X A \\
(\mu \mathrm{g} / \mathrm{L})\end{array}$ & $\begin{array}{l}\text { Aceto- } \\
\text { chlor } \\
\text { SAA } \\
(\mu \mathrm{g} / \mathrm{L})\end{array}$ & $\begin{array}{l}\text { Ala- } \\
\text { chlor } \\
(\mu \mathrm{g} / \mathrm{L})\end{array}$ & $\begin{array}{l}\text { Ala- } \\
\text { chlor } \\
\text { ESA } \\
(\mu \mathrm{g} / \mathrm{L})\end{array}$ & $\begin{array}{c}\text { Ala- } \\
\text { chlor } \\
\text { OXA } \\
(\mu \mathrm{g} / \mathrm{L})\end{array}$ & $\begin{array}{l}\text { Ala- } \\
\text { chlor } \\
\text { SAA } \\
(\mu \mathrm{g} / \mathrm{L})\end{array}$ & $\begin{array}{l}\text { Di- } \\
\text { meth- } \\
\text { enamid } \\
(\mu \mathrm{g} / \mathrm{L})\end{array}$ & $\begin{array}{c}\text { Di- } \\
\text { meth- } \\
\text { enamid } \\
\text { ESA } \\
(\mu \mathrm{g} / \mathrm{L})\end{array}$ & $\begin{array}{c}\text { Di- } \\
\text { meth- } \\
\text { enamid } \\
\text { OXA } \\
(\mu \mathrm{g} / \mathrm{L})\end{array}$ & $\begin{array}{c}\text { Flufen- } \\
\text { acet } \\
(\mu \mathrm{g} / \mathrm{L})\end{array}$ & $\begin{array}{c}\text { Flufen- } \\
\text { acet } \\
\text { ESA } \\
(\mu \mathrm{g} / \mathrm{L})\end{array}$ & $\begin{array}{c}\text { Flufen- } \\
\text { acet } \\
\text { OXA } \\
(\mu \mathrm{g} / \mathrm{L})\end{array}$ & $\begin{array}{l}\text { Meto- } \\
\text { lachlor } \\
(\mu \mathrm{g} / \mathrm{L})\end{array}$ & $\begin{array}{l}\text { Meto- } \\
\text { lachlor } \\
\text { ESA } \\
(\mu \mathrm{g} / \mathrm{L})\end{array}$ & $\begin{array}{l}\text { Meto- } \\
\text { lachlor } \\
\text { OXA } \\
(\mu \mathrm{g} / \mathrm{L})\end{array}$ \\
\hline \multicolumn{20}{|c|}{ Federal-State Cooperative Studies-Continued } \\
\hline \multirow{5}{*}{06483000} & \multirow{5}{*}{$\begin{array}{l}\text { Rock River at } \\
\text { Luverne, MN }\end{array}$} & & & Min & nnesota l & hydrolc & gic stud & dy (MLN & 1)-Con & tinued & & & & & & & & & \\
\hline & & $05 / 20 / 97$ & 0.11 & 0.26 & $<0.20$ & -- & $<0.05$ & 0.32 & $<0.20$ & -- & -- & -- & -- & -- & -- & -- & 0.13 & 3.7 & 0.47 \\
\hline & & 06/03/97 & .09 & $<.20$ & .50 & -- & $<.05$ & .30 & $<.20$ & -- & -- & -- & -- & -- & -- & -- & .09 & 3.7 & .52 \\
\hline & & 06/30/97 & .81 & 4.1 & 6.7 & -- & .10 & 1.5 & 1.3 & -- & -- & -- & -- & -- & -- & -- & 3.4 & 6.3 & 4.5 \\
\hline & & $08 / 28 / 97$ & $<.05$ & .25 & $<.20$ & -- & $<.05$ & .33 & $<.20$ & -- & -- & -- & -- & -- & -- & -- & $<.05$ & 3.0 & .28 \\
\hline \multicolumn{20}{|c|}{ Minnesota hydrologic study (TCM) } \\
\hline \multirow[t]{4}{*}{05475350} & \multirow{4}{*}{$\begin{array}{l}\text { Des Moines River } \\
\text { above Great Bend } \\
\text { near Windom, MN }\end{array}$} & 04/27/99 & $<.05$ & .22 & $<.20$ & -- & $<.05$ & .31 & $<.20$ & -- & $<0.05$ & -- & -- & -- & -- & -- & .06 & 2.1 & .40 \\
\hline & & 09/07/99 & $<.05$ & $<.20$ & $<.20$ & -- & $<.05$ & $<.20$ & 1.2 & -- & $<.05$ & -- & -- & $<0.05$ & -- & -- & $<.05$ & $<.20$ & $<.20$ \\
\hline & & 05/08/00 & $<.05$ & .21 & $<.05$ & -- & $<.05$ & $<.05$ & $<.05$ & -- & $<.05$ & $<0.05$ & $<0.05$ & $<.05$ & -- & -- & $<.05$ & .58 & .23 \\
\hline & & $07 / 25 / 00$ & $<.05$ & .46 & .15 & -- & $<.05$ & .39 & $<.05$ & -- & $<.05$ & $<.05$ & $<.05$ & $<.05$ & $<0.05$ & $<0.05$ & $<.05$ & 1.7 & .31 \\
\hline 05475250 & $\begin{array}{l}\text { Des Moines River } \\
\text { below Great Bend } \\
\text { near Windom, MN }\end{array}$ & 09/08/99 & $<.05$ & $<.20$ & $<.20$ & -- & $<.05$ & .44 & $<.20$ & -- & $<.05$ & -- & -- & $<.05$ & -- & -- & $<.05$ & .57 & $<.20$ \\
\hline \multicolumn{20}{|c|}{ Minnesota hydrologic study (FRG) } \\
\hline 472138092510101 & $\begin{array}{l}\text { HIB site pond near } \\
\text { Hibbing, } \mathrm{MN}\end{array}$ & $09 / 12 / 00$ & $<.05$ & $<.05$ & $<.05$ & -- & $<.05$ & $<.05$ & $<.05$ & -- & $<.05$ & $<.05$ & $<.05$ & $<.05$ & $<.05$ & $<.05$ & $<.05$ & $<.05$ & $<.05$ \\
\hline \multirow[t]{4}{*}{462151093552701} & \multirow{4}{*}{$\begin{array}{l}\text { Horseshoe Lake near } \\
\text { Bay Lake, MN }\end{array}$} & $06 / 11 / 98$ & $<.05$ & $<.20$ & $<.20$ & -- & $<.05$ & $<.20$ & $<.20$ & -- & $<.05$ & -- & -- & $<.05$ & -- & -- & $<.05$ & $<.20$ & $<.20$ \\
\hline & & 06/12/01 & $<.05$ & $<.05$ & $<.05$ & -- & $<.05$ & $<.05$ & $<.05$ & -- & $<.05$ & $<.05$ & $<.05$ & $<.05$ & $<.05$ & $<.05$ & $<.05$ & $<.05$ & $<.05$ \\
\hline & & 08/14/01 & $<.05$ & $<.05$ & $<.05$ & -- & $<.05$ & $<.05$ & $<.05$ & -- & $<.05$ & $<.05$ & $<.05$ & $<.05$ & $<.05$ & $<.05$ & $<.05$ & $<.05$ & $<.05$ \\
\hline & & $10 / 10 / 01$ & $<.05$ & $<.05$ & $<.05$ & -- & $<.05$ & $<.05$ & $<.05$ & -- & $<.05$ & $<.05$ & $<.05$ & $<.05$ & $<.05$ & $<.05$ & $<.05$ & $<.05$ & $<.05$ \\
\hline 461724095480101 & $\begin{array}{l}\text { IWPA site pond near } \\
\text { Underwood, MN }\end{array}$ & $07 / 25 / 00$ & $<.05$ & $<.05$ & $<.05$ & -- & $<.05$ & $<.05$ & $<.05$ & -- & $<.05$ & $<.05$ & $<.05$ & $<.05$ & $<.05$ & $<.05$ & $<.05$ & $<.05$ & $<.05$ \\
\hline \multirow[t]{5}{*}{463712096154001} & \multirow{5}{*}{$\begin{array}{l}\text { JWPA site pond near } \\
\text { Pelican Rapids, MN }\end{array}$} & $07 / 25 / 00$ & $<.05$ & $<.05$ & $<.05$ & -- & $<.05$ & $<.05$ & $<.05$ & -- & $<.05$ & $<.05$ & $<.05$ & $<.05$ & $<.05$ & $<.05$ & $<.05$ & $<.05$ & $<.05$ \\
\hline & & 06/13/01 & $<.05$ & $<.05$ & $<.05$ & -- & $<.05$ & $<.05$ & $<.05$ & -- & $<.05$ & $<.05$ & $<.05$ & $<.05$ & $<.05$ & $<.05$ & $<.05$ & $<.05$ & $<.05$ \\
\hline & & 08/06/01 & $<.05$ & $<.05$ & $<.05$ & -- & $<.05$ & $<.05$ & $<.05$ & -- & $<.05$ & $<.05$ & $<.05$ & $<.05$ & $<.05$ & $<.05$ & $<.05$ & $<.05$ & $<.05$ \\
\hline & & $10 / 01 / 01$ & $<.05$ & $<.05$ & $<.05$ & -- & $<.05$ & $<.05$ & $<.05$ & -- & $<.05$ & $<.05$ & $<.05$ & $<.05$ & $<.05$ & $<.05$ & $<.05$ & $<.05$ & $<.05$ \\
\hline & & $10 / 01 / 01$ & $<.05$ & $<.05$ & $<.05$ & -- & $<.05$ & $<.05$ & $<.05$ & -- & $<.05$ & $<.05$ & $<.05$ & $<.05$ & $<.05$ & $<.05$ & $<.05$ & $<.05$ & $<.05$ \\
\hline \multirow[t]{3}{*}{454753096303301} & \multirow{3}{*}{$\begin{array}{l}\text { TRD Wetland at } \\
\text { Wheaton High } \\
\text { School in Wheaton, } \\
\text { MN }\end{array}$} & $06 / 14 / 01$ & $<.05$ & $<.05$ & $<.05$ & -- & $<.05$ & $<.05$ & $<.05$ & -- & $<.05$ & $<.05$ & $<.05$ & $<.05$ & $<.05$ & $<.05$ & $<.05$ & $<.05$ & $<.05$ \\
\hline & & 08/08/01 & $<.05$ & $<.05$ & $<.05$ & -- & $<.05$ & $<.05$ & $<.05$ & -- & $<.05$ & $<.05$ & $<.05$ & $<.05$ & $<.05$ & $<.05$ & $<.05$ & $<.05$ & $<.05$ \\
\hline & & $10 / 03 / 01$ & $<.05$ & $<.05$ & $<.05$ & -- & $<.05$ & $<.05$ & $<.05$ & -- & $<.05$ & $<.05$ & $<.05$ & $<.05$ & $<.05$ & $<.05$ & $<.05$ & $<.05$ & $<.05$ \\
\hline
\end{tabular}


Table 6. Analytical results for selected acetamide herbicides and their degradation products, ethanesulfonic acid, oxanilic acid, and sulfinyl acetic acid, in surface-water samples, 1993-2003.-Continued

[OGRL, U.S. Geological Survey Organic Geochemistry Research Laboratory, Lawrence, Kansas; ESA, ethanesulfonic acid; OXA, oxanilic acid; SAA, sulfinyl acetic acid; --, no data; <, less than]

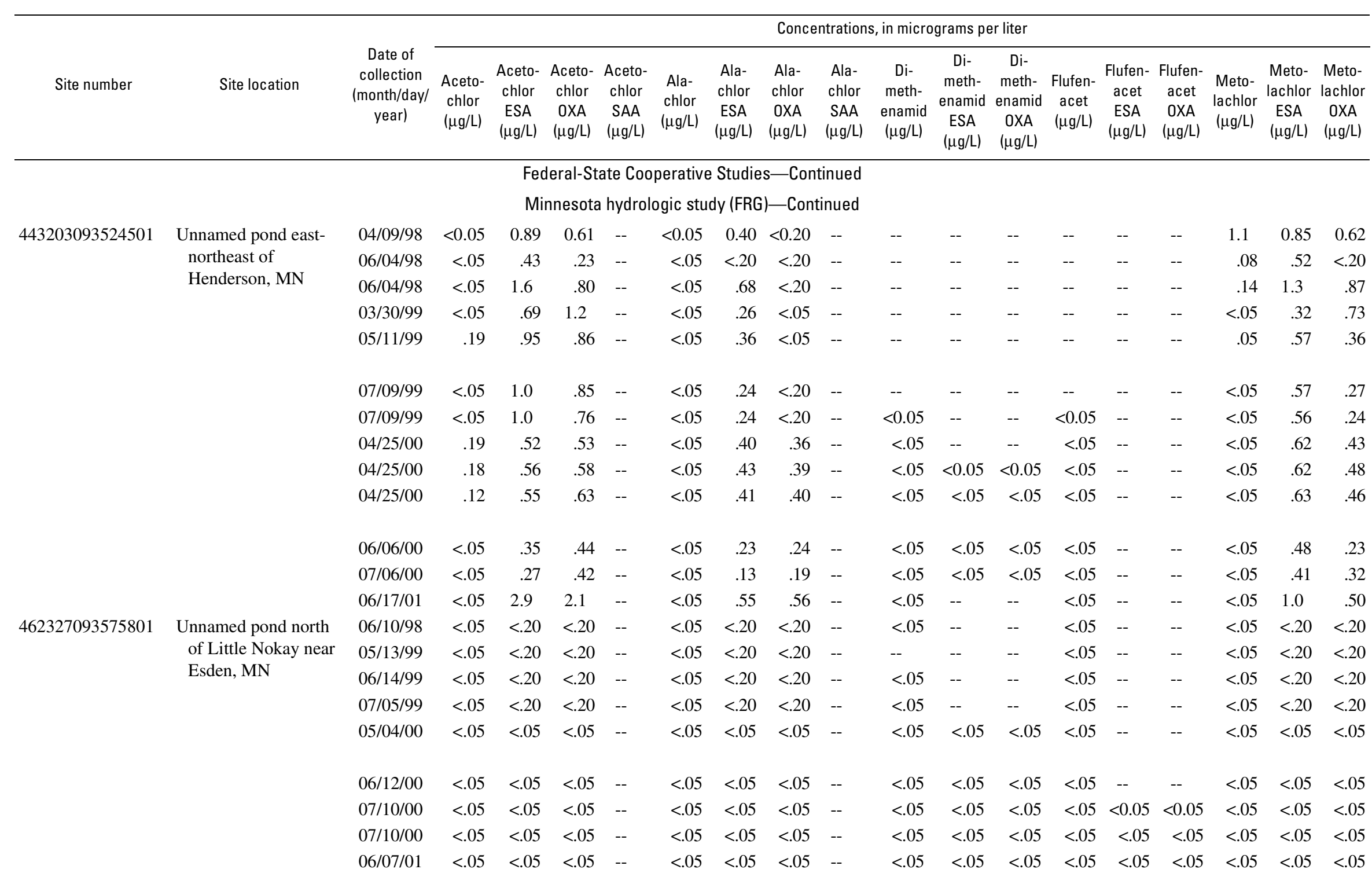


Table 6. Analytical results for selected acetamide herbicides and their degradation products, ethanesulfonic acid, oxanilic acid, and sulfinyl acetic acid, in surface-water samples, 1993-2003.-Continued

[OGRL, U.S. Geological Survey Organic Geochemistry Research Laboratory, Lawrence, Kansas; ESA, ethanesulfonic acid; OXA, oxanilic acid; SAA, sulfinyl acetic acid; --, no data; <, less than]

\begin{tabular}{|c|c|c|c|c|c|c|c|c|c|c|c|c|c|c|c|c|c|c|c|}
\hline \multirow[b]{2}{*}{ Site number } & \multirow[b]{2}{*}{ Site location } & \multirow[b]{2}{*}{$\begin{array}{c}\text { Date of } \\
\text { collection } \\
\text { (month/day/ } \\
\text { year) }\end{array}$} & \multicolumn{17}{|c|}{ Concentrations, in micrograms per liter } \\
\hline & & & $\begin{array}{l}\text { Aceto- } \\
\text { chlor } \\
(\mu \mathrm{g} / \mathrm{L})\end{array}$ & $\begin{array}{l}\text { Aceto- } \\
\text { chlor } \\
\text { ESA } \\
(\mu \mathrm{g} / \mathrm{L})\end{array}$ & $\begin{array}{l}\text { Aceto- } \\
\text { chlor } \\
\text { OXA } \\
(\mu \mathrm{g} / \mathrm{L})\end{array}$ & $\begin{array}{l}\text { Aceto- } \\
\text { chlor } \\
\text { SAA } \\
(\mu \mathrm{g} / \mathrm{L})\end{array}$ & $\begin{array}{l}\text { Ala- } \\
\text { chlor } \\
(\mu \mathrm{g} / \mathrm{L})\end{array}$ & $\begin{array}{l}\text { Ala- } \\
\text { chlor } \\
\text { ESA } \\
(\mu \mathrm{g} / \mathrm{L})\end{array}$ & $\begin{array}{l}\text { Ala- } \\
\text { chlor } \\
\text { OXA } \\
(\mu \mathrm{g} / \mathrm{L})\end{array}$ & $\begin{array}{l}\text { Ala- } \\
\text { chlor } \\
\text { SAA } \\
(\mu \mathrm{g} / \mathrm{L})\end{array}$ & $\begin{array}{l}\text { Di- } \\
\text { meth- } \\
\text { enamid } \\
(\mu \mathrm{g} / \mathrm{L})\end{array}$ & $\begin{array}{l}\text { Di- } \\
\text { meth- } \\
\text { enamid } \\
\text { ESA } \\
(\mu \mathrm{g} / \mathrm{L})\end{array}$ & $\begin{array}{l}\text { Di- } \\
\text { meth- } \\
\text { enamid } \\
\text { OXA } \\
(\mu \mathrm{g} / \mathrm{L})\end{array}$ & $\begin{array}{l}\text { Flufen- } \\
\text { acet } \\
(\mu \mathrm{g} / \mathrm{L})\end{array}$ & $\begin{array}{l}\text { Flufen- } \\
\text { acet } \\
\text { ESA } \\
(\mu \mathrm{g} / \mathrm{L})\end{array}$ & $\begin{array}{l}\text { Flufen- } \\
\text { acet } \\
\text { OXA } \\
(\mu \mathrm{g} / \mathrm{L})\end{array}$ & $\begin{array}{l}\text { Meto- } \\
\text { lachlor } \\
(\mu \mathrm{g} / \mathrm{L})\end{array}$ & $\begin{array}{l}\text { Meto- } \\
\text { lachlor } \\
\text { ESA } \\
(\mu \mathrm{g} / \mathrm{L})\end{array}$ & $\begin{array}{l}\text { Meto- } \\
\text { lachlor } \\
\text { OXA } \\
(\mu \mathrm{g} / \mathrm{L})\end{array}$ \\
\hline \multicolumn{20}{|c|}{ Federal-State Cooperative Studies-Continued } \\
\hline \multicolumn{20}{|c|}{ Minnesota hydrologic study (FRG) —Continued } \\
\hline \multirow[t]{4}{*}{450458094234101} & \multirow{4}{*}{$\begin{array}{l}\text { Unnamed pond } \\
\text { southeast of Little } \\
\text { Darwin near Darwin, } \\
\text { MN (DARP) }\end{array}$} & 05/12/99 & 0.14 & $<0.20$ & $<0.20$ & -- & $<0.05$ & $<0.20$ & $<0.20$ & -- & -- & -- & -- & & -- & -- & $<0.05$ & $<0.20$ & $<0.20$ \\
\hline & & 06/15/99 & $<.05$ & $<.20$ & $<.20$ & -- & $<.05$ & $<.20$ & $<.20$ & -- & $<0.05$ & -- & -- & $<0.05$ & -- & -- & $<.05$ & $<.20$ & $<.20$ \\
\hline & & 07/07/99 & $<.05$ & $<.20$ & $<.20$ & -- & $<.05$ & $<.20$ & $<.20$ & -- & $<.05$ & -- & -- & $<.05$ & -- & -- & $<.05$ & $<.20$ & $<.20$ \\
\hline & & 04/27/00 & $<.05$ & $<.05$ & $<.05$ & -- & $<.05$ & $<.05$ & $<.05$ & -- & $<.05$ & $<0.05$ & $<0.05$ & $<.05$ & $<0.05$ & $<0.05$ & $<.05$ & $<.05$ & $<.05$ \\
\hline \multirow[t]{6}{*}{450458094233501} & \multirow{6}{*}{$\begin{array}{l}\text { Unnamed pond } \\
\text { southeast of Little } \\
\text { Darwin near Darwin, } \\
\text { MN (ROI) }\end{array}$} & 06/05/98 & $<.05$ & $<.20$ & $<.20$ & -- & $<.05$ & $<.20$ & $<.20$ & -- & $<.05$ & -- & -- & $<.05$ & -- & -- & $<.05$ & $<.20$ & $<.20$ \\
\hline & & 05/12/99 & .13 & $<.20$ & $<.20$ & -- & $<.05$ & $<.20$ & $<.20$ & -- & -- & -- & -- & -- & -- & -- & $<.05$ & $<.20$ & $<.20$ \\
\hline & & 06/15/99 & $<.05$ & $<.20$ & $<.20$ & -- & $<.05$ & $<.20$ & $<.20$ & -- & $<.05$ & -- & -- & $<.05$ & -- & -- & $<.05$ & $<.20$ & $<.20$ \\
\hline & & 07/07/99 & $<.05$ & $<.20$ & $<.20$ & -- & $<.05$ & $<.20$ & $<.20$ & -- & $<.05$ & -- & -- & $<.05$ & -- & -- & $<.05$ & $<.20$ & $<.20$ \\
\hline & & 07/07/99 & $<.05$ & $<.20$ & $<.20$ & -- & $<.05$ & $<.20$ & $<.20$ & -- & $<.05$ & -- & -- & $<.05$ & -- & -- & $<.05$ & $<.20$ & $<.20$ \\
\hline & & $04 / 27 / 00$ & $<.05$ & $<.05$ & $<.05$ & -- & $<.05$ & $<.05$ & .14 & -- & $<.05$ & $<.05$ & $<.05$ & $<.05$ & -- & -- & $<.05$ & $<.05$ & $<.05$ \\
\hline \multirow[t]{5}{*}{450500094233401} & \multirow{5}{*}{$\begin{array}{l}\text { Unnamed pond } \\
\text { southeast of Little } \\
\text { Darwin near Darwin, } \\
\text { MN (ROI2) }\end{array}$} & 05/12/99 & .18 & $<.20$ & $<.20$ & -- & $<.05$ & $<.20$ & $<.20$ & -- & -- & -- & -- & -- & -- & -- & .05 & $<.20$ & $<.20$ \\
\hline & & 07/07/99 & $<.05$ & $<.20$ & $<.20$ & -- & $<.05$ & $<.20$ & $<.20$ & -- & $<.05$ & -- & -- & $<.05$ & -- & -- & $<.05$ & $<.20$ & $<.20$ \\
\hline & & 06/15/.99 & $<.05$ & $<.20$ & $<.20$ & -- & $<.05$ & $<.20$ & $<.20$ & -- & $<.05$ & -- & -- & $<.05$ & -- & -- & $<.05$ & $<.20$ & $<.20$ \\
\hline & & 07/07/99 & $<.05$ & $<.20$ & $<.20$ & -- & $<.05$ & $<.20$ & $<.20$ & -- & $<.05$ & -- & -- & $<.05$ & -- & -- & $<.05$ & $<.20$ & $<.20$ \\
\hline & & 07/07/99 & $<.05$ & $<.20$ & $<.20$ & -- & $<.05$ & $<.20$ & $<.20$ & -- & $<.05$ & -- & -- & $<.05$ & -- & -- & $<.05$ & $<.20$ & $<.20$ \\
\hline-- & $\begin{array}{l}\text { Unnamed pond } \\
\text { southeast of Little } \\
\text { Darwin near Darwin, } \\
\text { MN (ROI3) }\end{array}$ & 05/12/99 & .17 & $<.20$ & $<.20$ & -- & $<.05$ & $<.20$ & $<.20$ & -- & -- & -- & -- & -- & -- & -- & .05 & $<.20$ & $<.20$ \\
\hline 450459094232501 & $\begin{array}{l}\text { Unnamed wetland } \\
\text { southeast of Little } \\
\text { Darwin near Darwin, } \\
\text { MN }\end{array}$ & 06/15/99 & $<.05$ & $<.20$ & $<.20$ & -- & $<.05$ & $<.20$ & $<.20$ & -- & $<.05$ & -- & -- & $<.05$ & -- & -- & $<.05$ & $<.20$ & $<.20$ \\
\hline \multicolumn{20}{|c|}{ Nebraska Platte River study (IVL) } \\
\hline \multirow[t]{4}{*}{06801000} & \multirow{4}{*}{$\begin{array}{l}\text { Platte River at } \\
\text { Ashland, NE }\end{array}$} & $06 / 16 / 95$ & $<.05$ & $<.20$ & $<.20$ & -- & .06 & $<.20$ & $<.20$ & -- & -- & -- & -- & -- & -- & -- & .40 & $<.20$ & $<.20$ \\
\hline & & 05/27/96 & 3.2 & $<.20$ & $<.20$ & -- & $<.05$ & $<.20$ & $<.20$ & -- & -- & -- & -- & -- & -- & -- & 4.5 & $<.20$ & $<.20$ \\
\hline & & 04/09/97 & $<.05$ & $<.20$ & $<.20$ & -- & $<.05$ & $<.20$ & $<.20$ & -- & -- & -- & -- & -- & -- & -- & .07 & $<.20$ & $<.20$ \\
\hline & & $05 / 27 / 97$ & .46 & $<.20$ & $<.20$ & -- & $<.05$ & $<.20$ & $<.20$ & -- & -- & -- & -- & -- & -- & -- & .70 & .24 & .21 \\
\hline
\end{tabular}


Table 6. Analytical results for selected acetamide herbicides and their degradation products, ethanesulfonic acid, oxanilic acid, and sulfinyl acetic acid, in surface-water samples, 1993-2003.-Continued

[OGRL, U.S. Geological Survey Organic Geochemistry Research Laboratory, Lawrence, Kansas; ESA, ethanesulfonic acid; OXA, oxanilic acid; SAA, sulfinyl acetic acid; --, no data; <, less than]

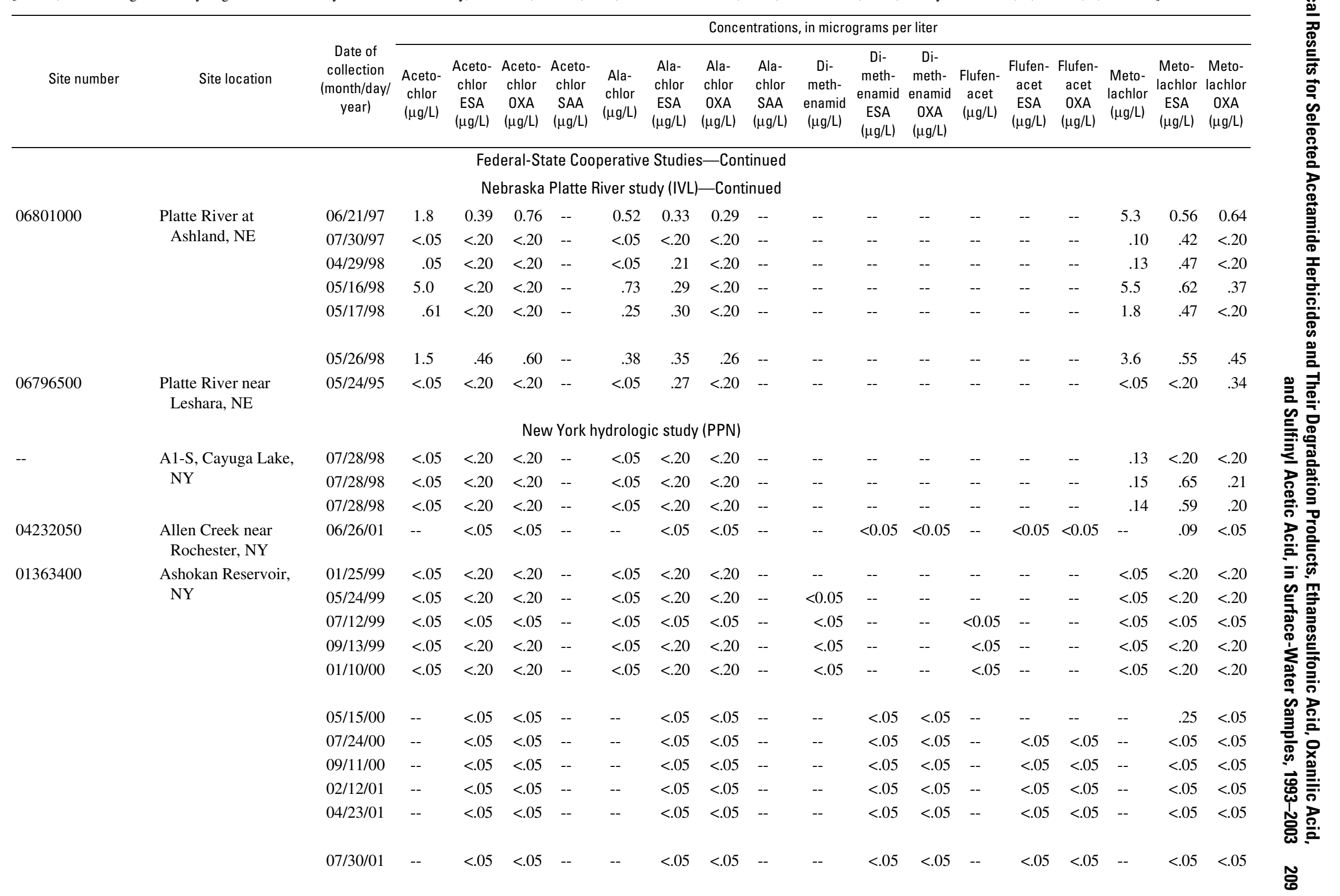


Table 6. Analytical results for selected acetamide herbicides and their degradation products, ethanesulfonic acid, oxanilic acid, and sulfinyl acetic acid, in surface-water samples, 1993-2003.-Continued

[OGRL, U.S. Geological Survey Organic Geochemistry Research Laboratory, Lawrence, Kansas; ESA, ethanesulfonic acid; OXA, oxanilic acid; SAA, sulfinyl acetic acid; --, no data; <, less than]

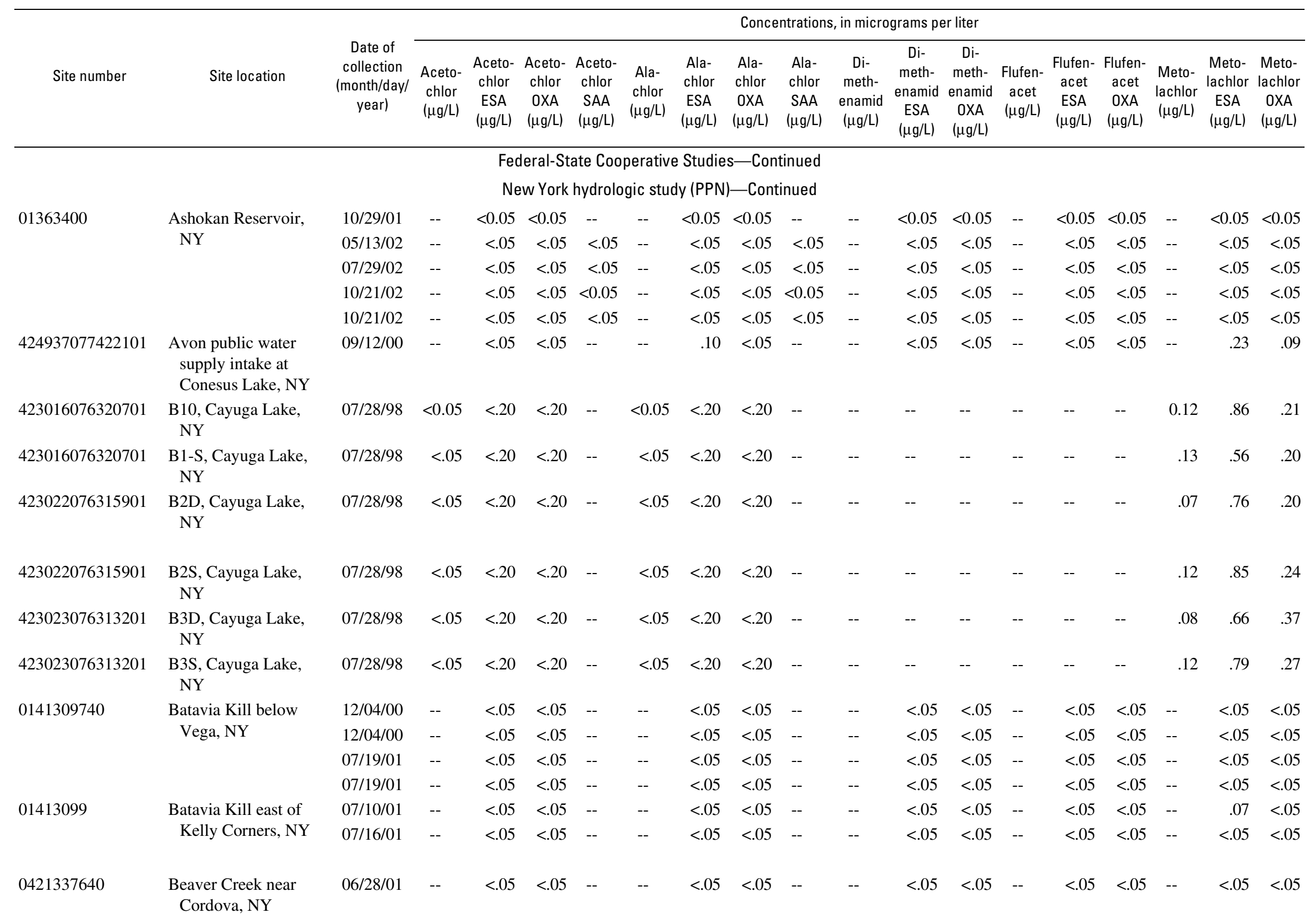


Table 6. Analytical results for selected acetamide herbicides and their degradation products, ethanesulfonic acid, oxanilic acid, and sulfinyl acetic acid, in surface-water samples, 1993-2003.-Continued

[OGRL, U.S. Geological Survey Organic Geochemistry Research Laboratory, Lawrence, Kansas; ESA, ethanesulfonic acid; OXA, oxanilic acid; SAA, sulfinyl acetic acid; --, no data; <, less than]

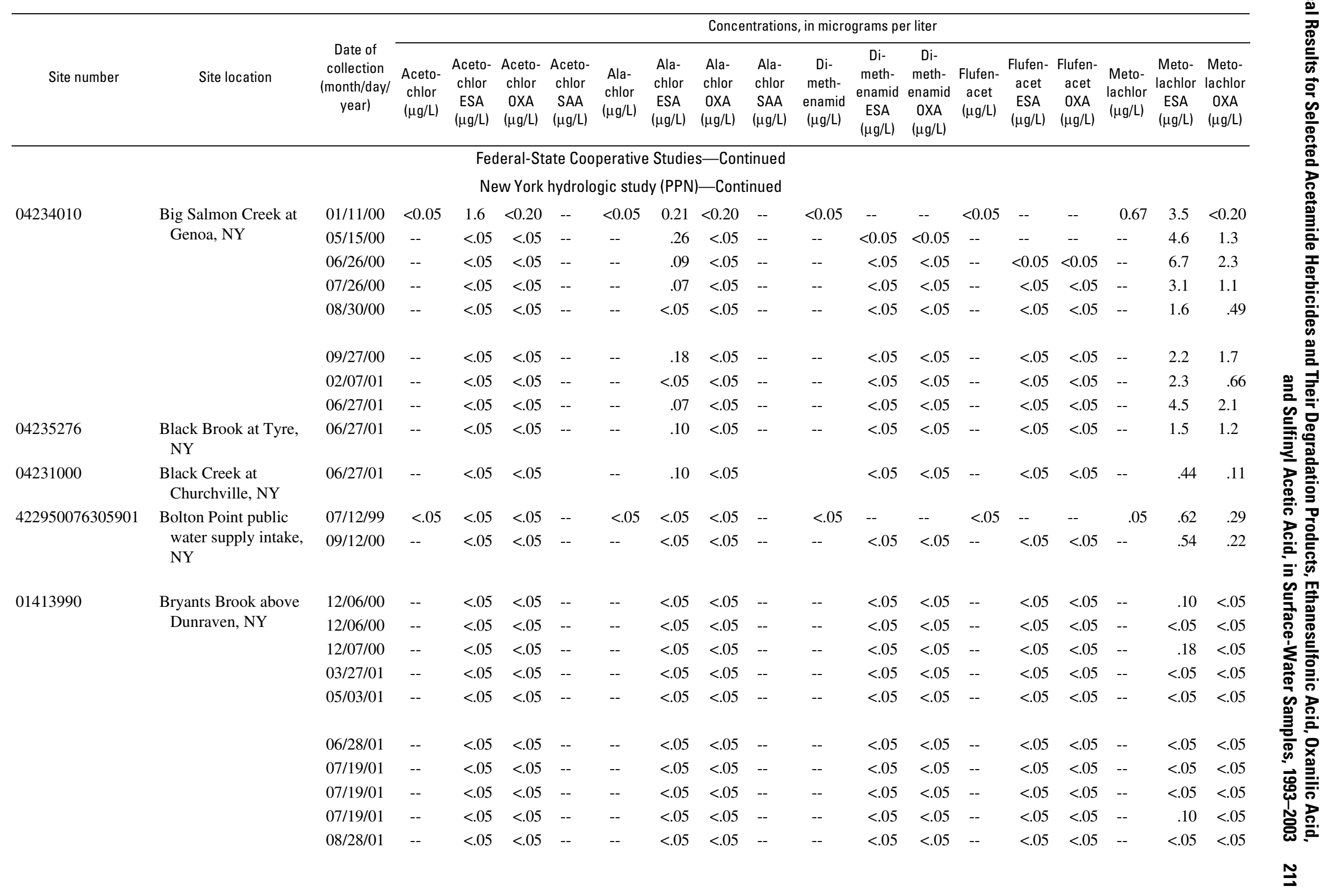


Table 6. Analytical results for selected acetamide herbicides and their degradation products, ethanesulfonic acid, oxanilic acid, and sulfinyl acetic acid, in surface-water samples, 1993-2003.-Continued

[OGRL, U.S. Geological Survey Organic Geochemistry Research Laboratory, Lawrence, Kansas; ESA, ethanesulfonic acid; OXA, oxanilic acid; SAA, sulfinyl acetic acid; --, no data; <, less than]

\begin{tabular}{|c|c|c|c|c|c|c|c|c|c|c|c|c|c|c|c|c|c|c|c|}
\hline \multirow[b]{2}{*}{ Site number } & \multirow[b]{2}{*}{ Site location } & \multirow[b]{2}{*}{$\begin{array}{c}\text { Date of } \\
\text { collection } \\
\text { (month/day/ } \\
\text { year) }\end{array}$} & \multicolumn{17}{|c|}{ Concentrations, in micrograms per liter } \\
\hline & & & $\begin{array}{l}\text { Aceto- } \\
\text { chlor } \\
(\mu \mathrm{g} / \mathrm{L})\end{array}$ & $\begin{array}{l}\text { Aceto- } \\
\text { chlor } \\
\text { ESA } \\
(\mu \mathrm{g} / \mathrm{L})\end{array}$ & $\begin{array}{l}\text { Aceto- } \\
\text { chlor } \\
\text { OXA } \\
(\mu \mathrm{g} / \mathrm{L})\end{array}$ & $\begin{array}{l}\text { Aceto- } \\
\text { chlor } \\
\text { SAA } \\
(\mu \mathrm{g} / \mathrm{L})\end{array}$ & $\begin{array}{l}\text { Ala- } \\
\text { chlor } \\
\text { ( } \mu \mathrm{g} / \mathrm{L})\end{array}$ & $\begin{array}{l}\text { Ala- } \\
\text { chlor } \\
\text { ESA } \\
(\mu \mathrm{g} / \mathrm{L})\end{array}$ & $\begin{array}{l}\text { Ala- } \\
\text { chlor } \\
\text { OXA } \\
(\mu \mathrm{g} / \mathrm{L})\end{array}$ & $\begin{array}{l}\text { Ala- } \\
\text { chlor } \\
\text { SAA } \\
(\mu \mathrm{g} / \mathrm{L})\end{array}$ & $\begin{array}{c}\text { Di- } \\
\text { meth- } \\
\text { enamid } \\
(\mu \mathrm{g} / \mathrm{L})\end{array}$ & $\begin{array}{c}\text { Di- } \\
\text { meth- } \\
\text { enamid } \\
\text { ESA } \\
(\mu \mathrm{g} / \mathrm{L})\end{array}$ & $\begin{array}{c}\text { Di- } \\
\text { meth- } \\
\text { enamid } \\
\text { OXA } \\
(\mu \mathrm{g} / \mathrm{L})\end{array}$ & $\begin{array}{l}\text { Flufen- } \\
\text { acet } \\
(\mu \mathrm{g} / \mathrm{L})\end{array}$ & $\begin{array}{c}\text { Flufen- } \\
\text { acet } \\
\text { ESA } \\
(\mu \mathrm{g} / \mathrm{L})\end{array}$ & $\begin{array}{c}\text { Flufen- } \\
\text { acet } \\
\text { OXA } \\
(\mu \mathrm{g} / \mathrm{L})\end{array}$ & $\begin{array}{l}\text { Meto- } \\
\text { lachlor } \\
(\mu \mathrm{g} / \mathrm{L})\end{array}$ & $\begin{array}{l}\text { Meto- } \\
\text { lachlor } \\
\text { ESA } \\
(\mu \mathrm{g} / \mathrm{L})\end{array}$ & $\begin{array}{l}\text { Meto- } \\
\text { lachlor } \\
\text { OXA } \\
(\mu \mathrm{g} / \mathrm{L})\end{array}$ \\
\hline \multicolumn{20}{|c|}{ Federal-State Cooperative Studies-Continued } \\
\hline \multicolumn{20}{|c|}{ New York hydrologic study (PPN)_Continued } \\
\hline \multirow[t]{3}{*}{01413990} & \multirow{3}{*}{$\begin{array}{l}\text { Bryants Brook above } \\
\text { Dunraven, NY }\end{array}$} & $10 / 03 / 01$ & -- & $<0.05$ & $<0.05$ & -- & -- & $<0.05$ & $<0.05$ & -- & -- & $<0.05$ & $<0.05$ & -- & $<0.05$ & $<0.05$ & -- & $<0.05$ & $<0.05$ \\
\hline & & $10 / 22 / 01$ & -- & $<.05$ & $<.05$ & -- & -- & $<.05$ & $<.05$ & -- & -- & $<.05$ & $<.05$ & -- & $<.05$ & $<.05$ & -- & $<.05$ & $<.05$ \\
\hline & & $11 / 15 / 01$ & -- & $<.05$ & $<.05$ & $<0.05$ & -- & $<.05$ & $<.05$ & $<0.05$ & -- & $<.05$ & $<.05$ & -- & $<.05$ & $<.05$ & -- & $<.05$ & $<.05$ \\
\hline \multirow[t]{15}{*}{425309078542701} & \multirow{15}{*}{$\begin{array}{l}\text { Buffalo public water } \\
\text { supply intake, NY }\end{array}$} & 07/12/99 & $<0.05$ & $<.20$ & $<.20$ & -- & $<0.05$ & $<.20$ & $<.20$ & -- & $<0.05$ & -- & -- & $<0.05$ & -- & -- & $<0.05$ & $<.20$ & $<.20$ \\
\hline & & 09/08/99 & $<.05$ & $<.20$ & $<.20$ & -- & $<.05$ & $<.20$ & $<.20$ & -- & $<.05$ & -- & -- & $<.05$ & -- & -- & $<.05$ & $<.20$ & $<.20$ \\
\hline & & 01/10/00 & $<.05$ & $<.20$ & $<.20$ & -- & $<.05$ & $<.20$ & $<.20$ & -- & $<.05$ & -- & -- & $<.05$ & -- & -- & $<.05$ & $<.20$ & $<.20$ \\
\hline & & $05 / 15 / 00$ & -- & .21 & .06 & -- & -- & .21 & $<.05$ & -- & -- & $<.05$ & $<.05$ & -- & -- & -- & -- & .27 & .08 \\
\hline & & 07/25/00 & -- & .05 & $<.05$ & -- & -- & $<.05$ & $<.05$ & -- & -- & $<.05$ & $<.05$ & -- & $<.05$ & $<.05$ & -- & .11 & .10 \\
\hline & & 09/11/00 & -- & $<.05$ & $<.05$ & -- & -- & $<.05$ & $<.05$ & -- & -- & $<.05$ & $<.05$ & -- & $<.05$ & $<.05$ & -- & .09 & .06 \\
\hline & & 02/05/01 & -- & $<.05$ & $<.05$ & -- & -- & $<.05$ & $<.05$ & -- & -- & $<.05$ & $<.05$ & -- & $<.05$ & $<.05$ & -- & .08 & $<.05$ \\
\hline & & 04/23/01 & -- & $<.05$ & $<.05$ & -- & -- & $<.05$ & $<.05$ & -- & -- & $<.05$ & $<.05$ & -- & $<.05$ & $<.05$ & -- & .09 & .05 \\
\hline & & 05/21/01 & -- & $<.05$ & $<.05$ & -- & -- & $<.05$ & $<.05$ & -- & -- & $<.05$ & $<.05$ & -- & $<.05$ & $<.05$ & -- & .10 & .06 \\
\hline & & 06/18/01 & -- & $<.05$ & $<.05$ & -- & -- & $<.05$ & $<.05$ & -- & -- & $<.05$ & $<.05$ & -- & $<.05$ & $<.05$ & -- & .10 & $<.05$ \\
\hline & & 07/30/01 & -- & $<.05$ & $<.05$ & -- & -- & $<.05$ & $<.05$ & -- & -- & $<.05$ & $<.05$ & -- & $<.05$ & $<.05$ & -- & $<.05$ & $<.05$ \\
\hline & & $10 / 29 / 01$ & -- & $<.05$ & $<.05$ & -- & -- & $<.05$ & $<.05$ & -- & -- & $<.05$ & $<.05$ & -- & $<.05$ & $<.05$ & -- & .08 & $<.05$ \\
\hline & & $01 / 28 / 02$ & -- & $<.05$ & $<.05$ & $<.05$ & -- & $<.05$ & $<.05$ & $<.05$ & -- & $<.05$ & $<.05$ & -- & $<.05$ & $<.05$ & -- & .10 & .05 \\
\hline & & 05/07/02 & -- & $<.05$ & $<.05$ & $<.05$ & -- & .05 & $<.05$ & $<.05$ & -- & $<.05$ & $<.05$ & -- & $<.05$ & $<.05$ & -- & .11 & .07 \\
\hline & & $07 / 22 / 02$ & -- & $<.05$ & $<.05$ & $<.05$ & -- & $<.05$ & $<.05$ & $<.05$ & -- & $<.05$ & $<.05$ & -- & $<.05$ & $<.05$ & -- & .11 & .10 \\
\hline 423241755 & $\begin{array}{l}\text { Bullhorn Creek at } \\
\text { McGrath Point, NY }\end{array}$ & $06 / 25 / 01$ & -- & $<.05$ & $<.05$ & -- & -- & $<.05$ & $<.05$ & -- & -- & $<.05$ & $<.05$ & -- & $<.05$ & $<.05$ & -- & $<.05$ & $<.05$ \\
\hline 422814076304901 & $\begin{array}{l}\text { C1-25, Cayuga Lake, } \\
\text { NY }\end{array}$ & $07 / 21 / 99$ & $<.05$ & $<.20$ & $<.20$ & -- & $<.05$ & $<.20$ & $<.20$ & -- & $<.05$ & -- & -- & $<.05$ & -- & -- & $<.05$ & .74 & .33 \\
\hline 423704076402201 & $\begin{array}{l}\text { C1D, Cayuga Lake, } \\
\text { NY }\end{array}$ & $07 / 28 / 98$ & $<.05$ & $<.20$ & $<.20$ & -- & $<.05$ & .58 & $<.20$ & -- & -- & -- & -- & -- & -- & -- & $<.05$ & .68 & $<.20$ \\
\hline 423704076402701 & $\begin{array}{l}\text { C1S, Cayuga Lake, } \\
\text { NY }\end{array}$ & 07/28/98 & $<.05$ & $<.20$ & $<.20$ & -- & $<.05$ & .31 & $<.20$ & -- & -- & -- & -- & -- & -- & -- & $<.05$ & .26 & $<.20$ \\
\hline
\end{tabular}


Table 6. Analytical results for selected acetamide herbicides and their degradation products, ethanesulfonic acid, oxanilic acid, and sulfinyl acetic acid, in surface-water samples, 1993-2003.-Continued

[OGRL, U.S. Geological Survey Organic Geochemistry Research Laboratory, Lawrence, Kansas; ESA, ethanesulfonic acid; OXA, oxanilic acid; SAA, sulfinyl acetic acid; --, no data; <, less than]

\begin{tabular}{|c|c|c|c|c|c|c|c|c|c|c|c|c|c|c|c|c|c|c|c|c|}
\hline \multirow[b]{2}{*}{ Site number } & \multirow[b]{2}{*}{ Site location } & \multirow[b]{2}{*}{$\begin{array}{c}\text { Date of } \\
\text { collection } \\
\text { (month/day/ } \\
\text { year) }\end{array}$} & \multicolumn{17}{|c|}{ Concentrations, in micrograms per liter } & \\
\hline & & & $\begin{array}{c}\text { Aceto- } \\
\text { chlor } \\
(\mu \mathrm{g} / \mathrm{L})\end{array}$ & $\begin{array}{l}\text { Aceto- } \\
\text { chlor } \\
\text { ESA } \\
(\mu \mathrm{g} / \mathrm{L})\end{array}$ & $\begin{array}{l}\text { Aceto- } \\
\text { chlor } \\
\text { OXA } \\
(\mu \mathrm{g} / \mathrm{L})\end{array}$ & $\begin{array}{l}\text { Aceto- } \\
\text { chlor } \\
\text { SAA } \\
(\mu \mathrm{g} / \mathrm{L})\end{array}$ & $\begin{array}{l}\text { Ala- } \\
\text { chlor } \\
(\mu \mathrm{g} / \mathrm{L})\end{array}$ & $\begin{array}{l}\text { Ala- } \\
\text { chlor } \\
\text { ESA } \\
(\mu \mathrm{g} / \mathrm{L})\end{array}$ & $\begin{array}{l}\text { Ala- } \\
\text { chlor } \\
\text { OXA } \\
(\mu \mathrm{g} / \mathrm{L})\end{array}$ & $\begin{array}{l}\text { Ala- } \\
\text { chlor } \\
\text { SAA } \\
(\mu \mathrm{g} / \mathrm{L})\end{array}$ & $\begin{array}{c}\text { Di- } \\
\text { meth- } \\
\text { enamid } \\
(\mu \mathrm{g} / \mathrm{L})\end{array}$ & $\begin{array}{c}\text { Di- } \\
\text { meth- } \\
\text { enamid } \\
\text { ESA } \\
(\mu \mathrm{g} / \mathrm{L})\end{array}$ & $\begin{array}{c}\text { Di- } \\
\text { meth- } \\
\text { enamid } \\
0 X A \\
(\mu \mathrm{g} / \mathrm{L})\end{array}$ & $\begin{array}{c}\text { Flufen- } \\
\text { acet } \\
(\mu \mathrm{g} / \mathrm{L})\end{array}$ & $\begin{array}{l}\text { Flufen- } \\
\text { acet } \\
\text { ESA } \\
(\mu \mathrm{g} / \mathrm{L})\end{array}$ & $\begin{array}{l}\text { Flufen- } \\
\text { acet } \\
\text { OXA } \\
(\mu \mathrm{g} / \mathrm{L})\end{array}$ & $\begin{array}{l}\text { Meto- } \\
\text { lachlor } \\
(\mu \mathrm{g} / \mathrm{L})\end{array}$ & $\begin{array}{l}\text { Meto- } \\
\text { lachlor } \\
\text { ESA } \\
(\mu \mathrm{g} / \mathrm{L})\end{array}$ & $\begin{array}{l}\text { Meto- } \\
\text { lachlor } \\
\text { OXA } \\
(\mu \mathrm{g} / \mathrm{L})\end{array}$ & $\begin{array}{l}\text { क } \\
\text { 움 } \\
\text { c } \\
\frac{D}{8}\end{array}$ \\
\hline \multicolumn{20}{|c|}{ Federal-State Cooperative Studies-Continued } & \\
\hline \multicolumn{20}{|c|}{ New York hydrologic study (PPN)—Continued } & \\
\hline 423711076395401 & $\begin{array}{l}\text { C2D, Cayuga Lake, } \\
\text { NY }\end{array}$ & $07 / 28 / 98$ & $<0.05$ & $<0.20$ & $<0.20$ & -- & $<0.05$ & 0.41 & $<0.20$ & -- & -- & -- & -- & -- & -- & -- & $<0.05$ & 0.36 & $<0.20$ & \\
\hline 423711076395401 & $\begin{array}{l}\text { C2S, Cayuga Lake, } \\
\text { NY }\end{array}$ & $07 / 28 / 98$ & $<.05$ & $<.20$ & $<.20$ & -- & $<.05$ & .41 & $<.20$ & -- & -- & -- & -- & -- & -- & -- & $<.05$ & .36 & $<.20$ & \\
\hline 423711076395401 & $\begin{array}{l}\text { C3-25, Cayuga Lake, } \\
\text { NY }\end{array}$ & $07 / 21 / 99$ & $<.05$ & $<.20$ & $<.20$ & -- & $<.05$ & $<.20$ & $<.20$ & -- & -- & -- & -- & -- & -- & -- & $<.05$ & .63 & $<.20$ & $\frac{\bar{g}}{\mathrm{~d}}$ \\
\hline 423711076391401 & $\begin{array}{l}\text { C3D, Cayuga Lake, } \\
\text { NY }\end{array}$ & $07 / 28 / 98$ & $<.05$ & $<.20$ & $<.20$ & -- & $<.05$ & .33 & $<.20$ & -- & -- & -- & -- & -- & -- & -- & $<.05$ & .28 & $<.20$ & \\
\hline 424016076411001 & $\begin{array}{l}\text { C4-25, Cayuga Lake, } \\
\text { NY }\end{array}$ & $07 / 21 / 99$ & $<.05$ & $<.20$ & $<.20$ & -- & $<.05$ & $<.20$ & $<.20$ & -- & $<0.05$ & -- & -- & $<0.05$ & -- & -- & $<.05$ & .75 & .32 & $\frac{\Sigma}{D}$ \\
\hline 424547076435601 & $\begin{array}{l}\text { C5-25, Cayuga Lake, } \\
\text { NY }\end{array}$ & $07 / 21 / 99$ & $<.05$ & $<.20$ & $<.20$ & -- & $<.05$ & $<.20$ & $<.20$ & -- & $<.05$ & -- & -- & $<.05$ & -- & -- & $<.05$ & .85 & .33 & \\
\hline 425117076432001 & $\begin{array}{l}\text { C6-25, Cayuga Lake, } \\
\text { NY }\end{array}$ & $07 / 21 / 99$ & $<.05$ & $<.20$ & $<.20$ & -- & $<.05$ & $<.20$ & $<.20$ & -- & $<.05$ & -- & -- & $<.05$ & -- & -- & .05 & .34 & $<.20$ & 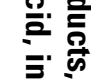 \\
\hline 425602076441401 & $\begin{array}{l}\text { C7-25, Cayuga Lake, } \\
\text { NY }\end{array}$ & $07 / 21 / 99$ & $<.05$ & $<.20$ & $<.20$ & -- & $<.05$ & $<.20$ & $<.20$ & -- & $<.05$ & -- & -- & $<.05$ & -- & -- & $<.05$ & $<.20$ & $<.20$ & \\
\hline 04235000 & $\begin{array}{l}\text { Canadaigua outlet at } \\
\text { Chapin, NY }\end{array}$ & $06 / 27 / 01$ & -- & $<.05$ & $<.05$ & -- & -- & $<.05$ & $<.05$ & -- & -- & $<0.05$ & $<0.05$ & -- & $<0.05$ & $<0.05$ & -- & $<.05$ & $<.05$ & 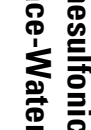 \\
\hline \multirow[t]{5}{*}{01349150} & \multirow{5}{*}{$\begin{array}{l}\text { Canajoharie Creek } \\
\text { near Canajoharie, } \\
\text { NY }\end{array}$} & $04 / 24 / 97$ & $<.05$ & $<.20$ & $<.20$ & -- & $<.05$ & $<.20$ & $<.20$ & -- & -- & -- & -- & -- & -- & -- & $<.05$ & .45 & $<.20$ & is ? \\
\hline & & $05 / 16 / 97$ & $<.05$ & $<.20$ & $<.20$ & -- & $<.05$ & $<.20$ & $<.20$ & -- & -- & -- & -- & -- & -- & -- & $<.05$ & .36 & $<.20$ & 흥 를 \\
\hline & & $05 / 22 / 97$ & $<.05$ & $<.20$ & $<.20$ & -- & $<.05$ & $<.20$ & $<.20$ & -- & -- & -- & -- & -- & -- & -- & $<.05$ & .29 & $<.20$ & \\
\hline & & $06 / 11 / 97$ & $<.05$ & $<.20$ & $<.20$ & -- & $<.05$ & $<.20$ & $<.20$ & -- & -- & -- & -- & -- & -- & -- & $<.05$ & .47 & $<.20$ & \\
\hline & & $06 / 18 / 97$ & $<.05$ & $<.20$ & $<.20$ & -- & $<.05$ & $<.20$ & $<.20$ & -- & -- & -- & -- & -- & -- & -- & $<.05$ & .27 & $<.20$ & 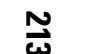 \\
\hline
\end{tabular}


Table 6. Analytical results for selected acetamide herbicides and their degradation products, ethanesulfonic acid, oxanilic acid, and sulfinyl acetic acid, in surface-water samples, 1993-2003.-Continued

[OGRL, U.S. Geological Survey Organic Geochemistry Research Laboratory, Lawrence, Kansas; ESA, ethanesulfonic acid; OXA, oxanilic acid; SAA, sulfinyl acetic acid; --, no data; <, less than]

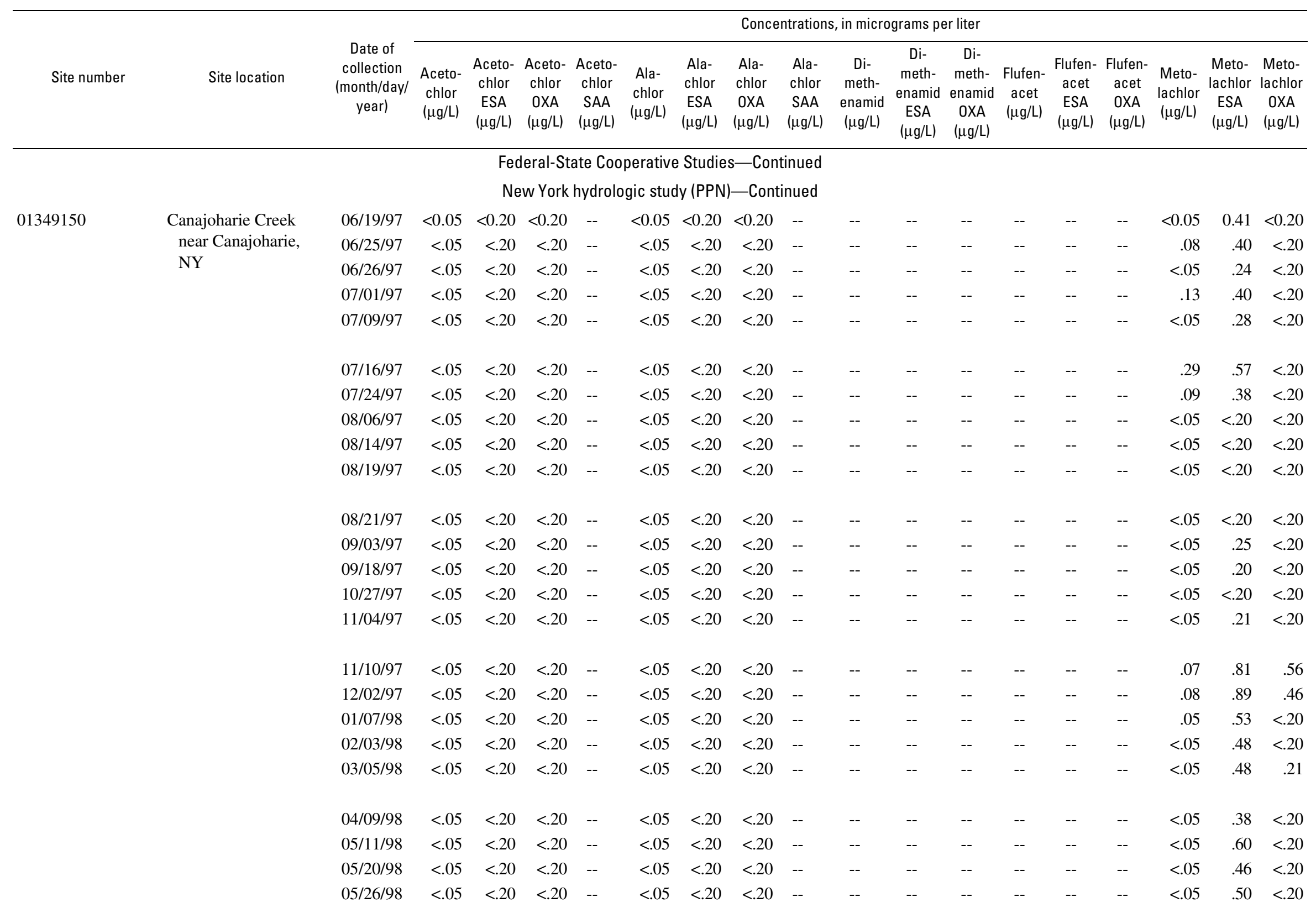


Table 6. Analytical results for selected acetamide herbicides and their degradation products, ethanesulfonic acid, oxanilic acid, and sulfinyl acetic acid, in surface-water samples, 1993-2003.-Continued

[OGRL, U.S. Geological Survey Organic Geochemistry Research Laboratory, Lawrence, Kansas; ESA, ethanesulfonic acid; OXA, oxanilic acid; SAA, sulfinyl acetic acid; --, no data; <, less than]

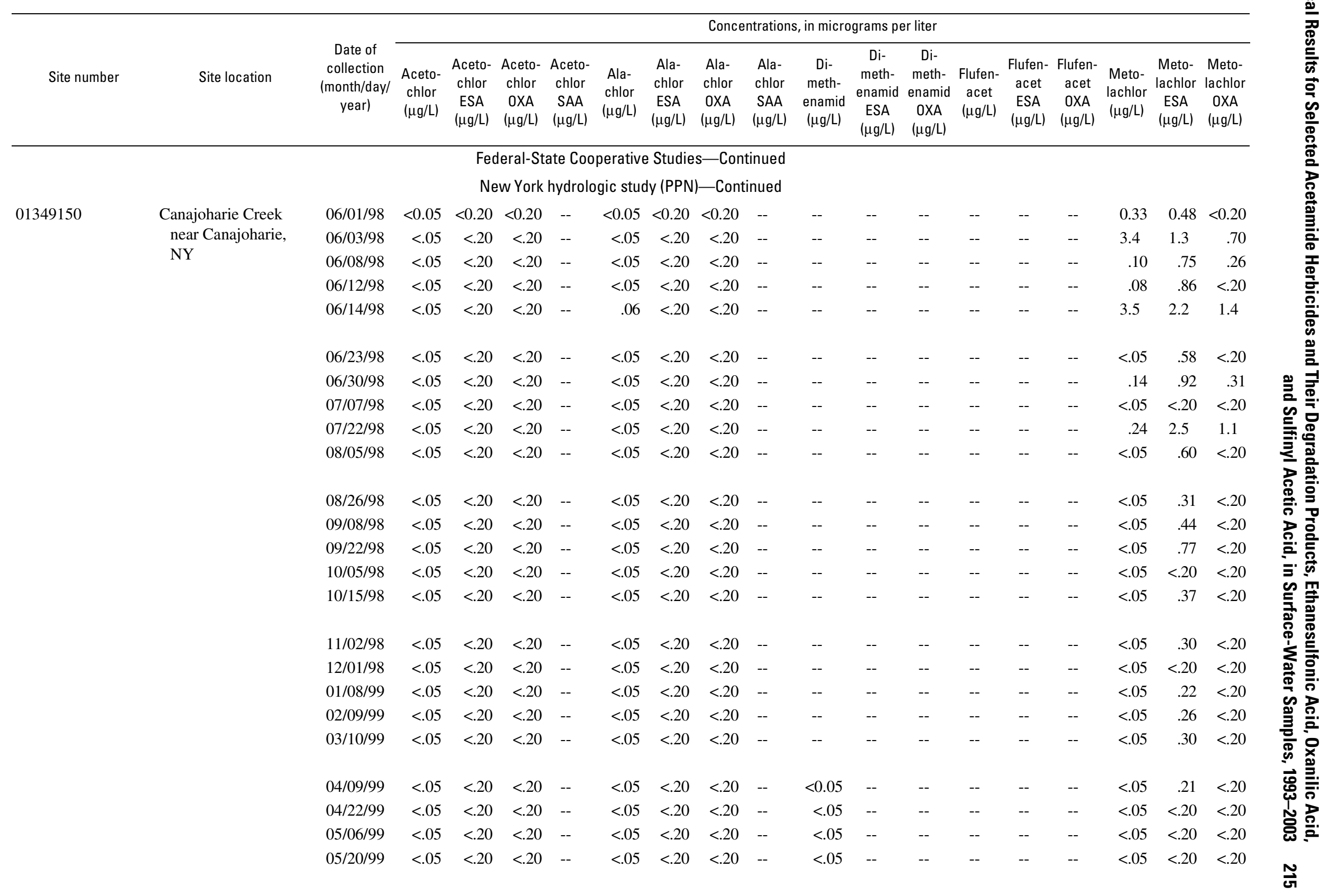


Table 6. Analytical results for selected acetamide herbicides and their degradation products, ethanesulfonic acid, oxanilic acid, and sulfinyl acetic acid, in surface-water samples, 1993-2003.-Continued

[OGRL, U.S. Geological Survey Organic Geochemistry Research Laboratory, Lawrence, Kansas; ESA, ethanesulfonic acid; OXA, oxanilic acid; SAA, sulfinyl acetic acid; --, no data; <, less than]

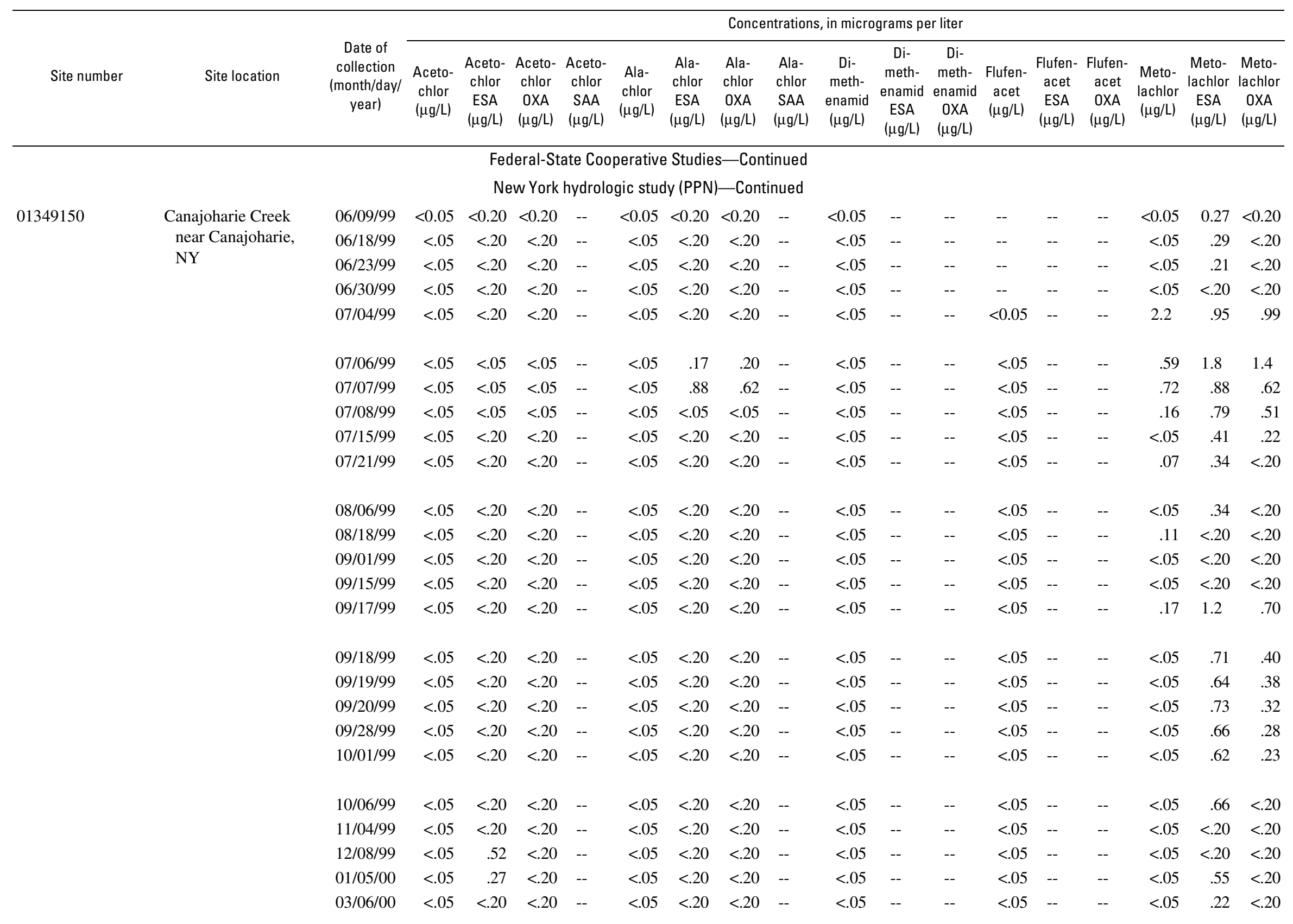


Table 6. Analytical results for selected acetamide herbicides and their degradation products, ethanesulfonic acid, oxanilic acid, and sulfinyl acetic acid, in surface-water samples, 1993-2003.-Continued

[OGRL, U.S. Geological Survey Organic Geochemistry Research Laboratory, Lawrence, Kansas; ESA, ethanesulfonic acid; OXA, oxanilic acid; SAA, sulfinyl acetic acid; --, no data; <, less than]

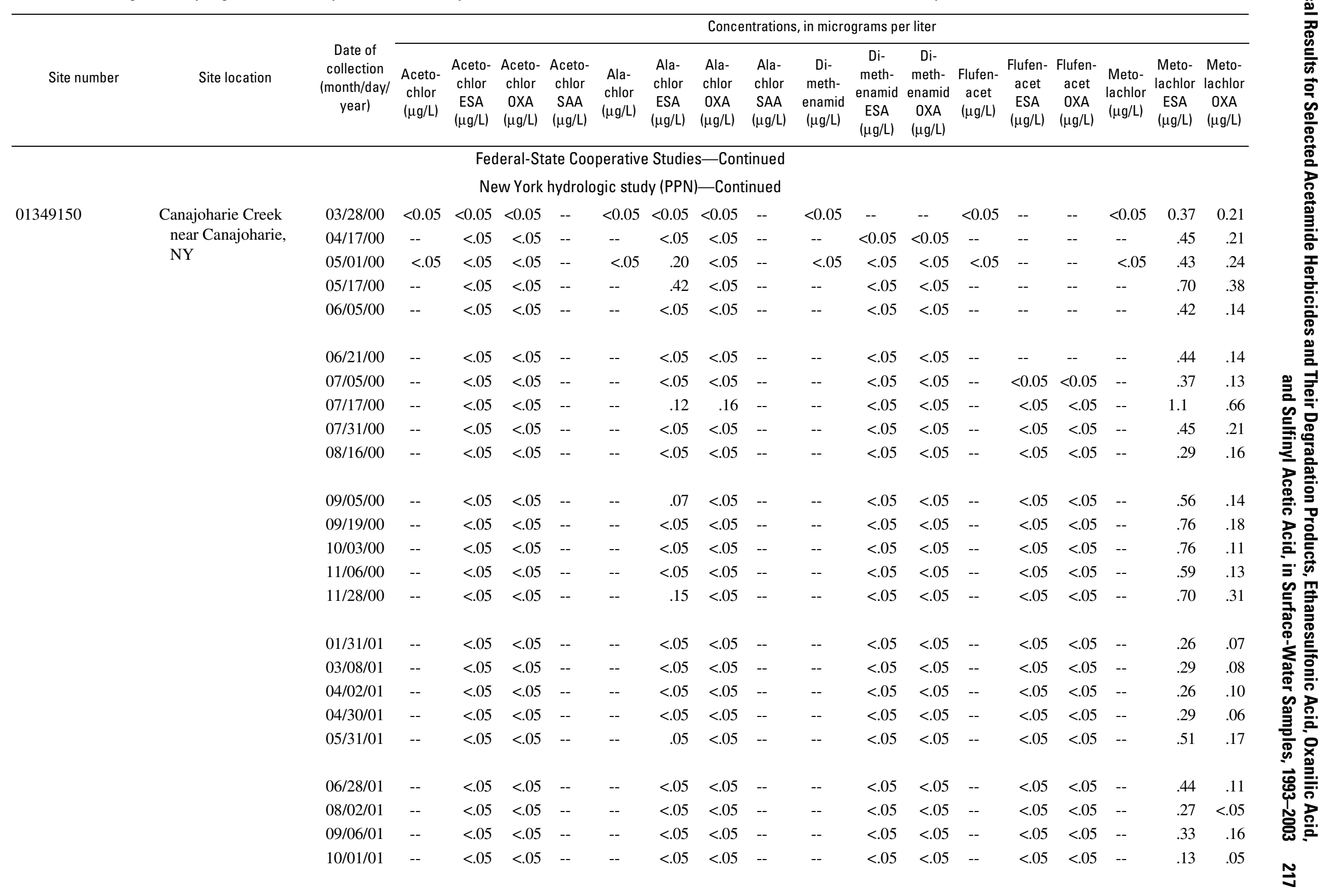


Table 6. Analytical results for selected acetamide herbicides and their degradation products, ethanesulfonic acid, oxanilic acid, and sulfinyl acetic acid, in surface-water samples, 1993-2003.-Continued

[OGRL, U.S. Geological Survey Organic Geochemistry Research Laboratory, Lawrence, Kansas; ESA, ethanesulfonic acid; OXA, oxanilic acid; SAA, sulfinyl acetic acid; --, no data; <, less than]

\begin{tabular}{|c|c|c|c|c|c|c|c|c|c|c|c|c|c|c|c|c|c|c|c|}
\hline \multirow[b]{2}{*}{ Site number } & \multirow[b]{2}{*}{ Site location } & \multirow[b]{2}{*}{$\begin{array}{c}\text { Date of } \\
\text { collection } \\
\text { (month/day/ } \\
\text { year) }\end{array}$} & \multicolumn{17}{|c|}{ Concentrations, in micrograms per liter } \\
\hline & & & $\begin{array}{l}\text { Aceto- } \\
\text { chlor } \\
(\mu \mathrm{g} / \mathrm{L})\end{array}$ & $\begin{array}{l}\text { Aceto- } \\
\text { chlor } \\
\text { ESA } \\
(\mu \mathrm{g} / \mathrm{L})\end{array}$ & $\begin{array}{l}\text { Aceto- } \\
\text { chlor } \\
\text { OXA } \\
(\mu \mathrm{g} / \mathrm{L})\end{array}$ & $\begin{array}{l}\text { Aceto- } \\
\text { chlor } \\
\text { SAA } \\
(\mu \mathrm{g} / \mathrm{L})\end{array}$ & $\begin{array}{l}\text { Ala- } \\
\text { chlor } \\
(\mu \mathrm{g} / \mathrm{L})\end{array}$ & $\begin{array}{l}\text { Ala- } \\
\text { chlor } \\
\text { ESA } \\
(\mu \mathrm{g} / \mathrm{L})\end{array}$ & $\begin{array}{l}\text { Ala- } \\
\text { chlor } \\
\text { OXA } \\
(\mu \mathrm{g} / \mathrm{L})\end{array}$ & $\begin{array}{l}\text { Ala- } \\
\text { chlor } \\
\text { SAA } \\
(\mu \mathrm{g} / \mathrm{L})\end{array}$ & $\begin{array}{l}\text { Di- } \\
\text { meth- } \\
\text { enamid } \\
(\mu \mathrm{g} / \mathrm{L})\end{array}$ & $\begin{array}{c}\text { Di- } \\
\text { meth- } \\
\text { enamid } \\
\text { ESA } \\
(\mu \mathrm{g} / \mathrm{L})\end{array}$ & $\begin{array}{c}\text { Di- } \\
\text { meth- } \\
\text { enamid } \\
\text { OXA } \\
(\mu \mathrm{g} / \mathrm{L})\end{array}$ & $\begin{array}{c}\text { Flufen- } \\
\text { acet } \\
(\mu \mathrm{g} / \mathrm{L})\end{array}$ & $\begin{array}{c}\text { Flufen- } \\
\text { acet } \\
\text { ESA } \\
(\mu \mathrm{g} / \mathrm{L})\end{array}$ & $\begin{array}{c}\text { Flufen- } \\
\text { acet } \\
\text { OXA } \\
(\mu \mathrm{g} / \mathrm{L})\end{array}$ & $\begin{array}{l}\text { Meto- } \\
\text { lachlor } \\
(\mu \mathrm{g} / \mathrm{L})\end{array}$ & $\begin{array}{l}\text { Meto- } \\
\text { lachlor } \\
\text { ESA } \\
(\mu \mathrm{g} / \mathrm{L})\end{array}$ & $\begin{array}{l}\text { Meto- } \\
\text { lachlor } \\
\text { OXA } \\
(\mu \mathrm{g} / \mathrm{L})\end{array}$ \\
\hline \multicolumn{20}{|c|}{ Federal-State Cooperative Studies-Continued } \\
\hline \multicolumn{20}{|c|}{ New York hydrologic study (PPN) —Continued } \\
\hline \multirow[t]{9}{*}{01349150} & \multirow{9}{*}{$\begin{array}{l}\text { Canajoharie Creek } \\
\text { near Canajoharie, } \\
\text { NY }\end{array}$} & $12 / 03 / 01$ & -- & $<0.05$ & $<0.05$ & -- & -- & $<0.05$ & $<0.05$ & -- & -- & $<0.05$ & $<0.05$ & -- & $<0.05$ & $<0.05$ & -- & 0.16 & $<0.05$ \\
\hline & & $02 / 05 / 02$ & -- & $<.05$ & $<.05$ & $<0.05$ & -- & $<.05$ & $<.05$ & $<0.05$ & -- & $<.05$ & $<.05$ & -- & $<.05$ & $<.05$ & -- & .43 & .14 \\
\hline & & $04 / 01 / 02$ & -- & $<.05$ & $<.05$ & $<.05$ & -- & $<.05$ & $<.05$ & $<.05$ & -- & $<.05$ & $<.05$ & -- & $<.05$ & $<.05$ & -- & .49 & .17 \\
\hline & & $05 / 02 / 02$ & -- & $<.05$ & $<.05$ & $<.05$ & -- & $<.05$ & $<.05$ & $<.05$ & -- & $<.05$ & $<.05$ & -- & $<.05$ & $<.05$ & -- & .48 & .14 \\
\hline & & $06 / 12 / 02$ & -- & $<.05$ & $<.05$ & $<.05$ & -- & $<.05$ & $<.05$ & $<.05$ & -- & $<.05$ & $<.05$ & -- & $<.05$ & $<.05$ & -- & .77 & .18 \\
\hline & & 07/01/02 & -- & $<.05$ & $<.05$ & $<.05$ & -- & $<.05$ & $<.05$ & $<.05$ & -- & $<.05$ & $<.05$ & -- & $<.05$ & $<.05$ & -- & .52 & .14 \\
\hline & & 08/06/02 & -- & $<.05$ & $<.05$ & $<.05$ & -- & $<.05$ & $<.05$ & $<.05$ & -- & $<.05$ & $<.05$ & -- & $<.05$ & $<.05$ & -- & .54 & .11 \\
\hline & & $10 / 07 / 02$ & -- & $<.05$ & $<.05$ & $<.05$ & -- & $<.05$ & $<.05$ & $<.05$ & -- & $<.05$ & $<.05$ & -- & $<.05$ & $<.05$ & -- & .69 & .20 \\
\hline & & $12 / 02 / 02$ & -- & $<.05$ & $<.05$ & $<.05$ & -- & $<.05$ & $<.05$ & $<.05$ & -- & $<.05$ & $<.05$ & -- & $<.05$ & $<.05$ & -- & .49 & .10 \\
\hline \multirow[t]{16}{*}{04227000} & \multirow{16}{*}{$\begin{array}{l}\text { Canaseraga Creek near } \\
\text { Shakers Crossing, } \\
\text { NY }\end{array}$} & $05 / 22 / 97$ & $<0.05$ & $<.20$ & $<.20$ & -- & $<0.05$ & $<.20$ & $<.20$ & -- & -- & -- & -- & -- & -- & -- & 0.13 & .29 & $<.20$ \\
\hline & & 06/10/97 & $<.05$ & $<.20$ & $<.20$ & -- & $<.05$ & $<.20$ & $<.20$ & -- & -- & -- & -- & -- & -- & -- & .11 & .37 & $<.20$ \\
\hline & & $06 / 18 / 97$ & $<.05$ & $<.20$ & $<.20$ & -- & $<.05$ & $<.20$ & $<.20$ & -- & -- & -- & -- & -- & -- & -- & .19 & .39 & $<.20$ \\
\hline & & $06 / 24 / 97$ & $<.05$ & $<.20$ & $<.20$ & -- & .06 & $<.20$ & $<.20$ & -- & -- & -- & -- & -- & -- & -- & $<.05$ & .30 & .23 \\
\hline & & $06 / 25 / 97$ & $<.05$ & $<.20$ & $<.20$ & -- & $<.05$ & $<.20$ & $<.20$ & -- & -- & -- & -- & -- & -- & -- & .74 & .55 & .37 \\
\hline & & $07 / 24 / 97$ & $<.05$ & $<.20$ & $<.20$ & -- & $<.05$ & $<.20$ & $<.20$ & -- & -- & -- & -- & -- & -- & -- & .05 & .33 & $<.20$ \\
\hline & & $08 / 21 / 97$ & $<.05$ & $<.20$ & $<.20$ & -- & $<.05$ & $<.20$ & $<.20$ & -- & -- & -- & -- & -- & -- & -- & $<.05$ & $<.20$ & $<.20$ \\
\hline & & $10 / 07 / 97$ & $<.05$ & $<.20$ & $<.20$ & -- & $<.05$ & .26 & $<.20$ & -- & -- & -- & -- & -- & -- & -- & $<.05$ & .81 & .33 \\
\hline & & $12 / 01 / 97$ & $<.05$ & $<.20$ & $<.20$ & -- & $<.05$ & .20 & $<.20$ & -- & -- & -- & -- & -- & -- & -- & $<.05$ & .61 & .23 \\
\hline & & 01/09/98 & $<.05$ & $<.20$ & $<.20$ & -- & $<.05$ & $<.20$ & $<.20$ & -- & -- & -- & -- & -- & -- & -- & .08 & .35 & $<.20$ \\
\hline & & $02 / 02 / 98$ & $<.05$ & $<.20$ & $<.20$ & -- & $<.05$ & $<.20$ & $<.20$ & -- & -- & -- & -- & -- & -- & -- & .09 & .73 & .29 \\
\hline & & $04 / 06 / 98$ & $<.05$ & $<.20$ & $<.20$ & -- & $<.05$ & $<.20$ & $<.20$ & -- & -- & -- & -- & -- & -- & -- & $<.05$ & .55 & .35 \\
\hline & & 05/18/98 & $<.05$ & $<.20$ & $<.20$ & -- & $<.05$ & $<.20$ & $<.20$ & -- & -- & -- & -- & -- & -- & -- & $<.05$ & .57 & $<.20$ \\
\hline & & 06/09/98 & $<.05$ & $<.20$ & $<.20$ & -- & $<.05$ & $<.20$ & $<.20$ & -- & -- & -- & -- & -- & -- & -- & .07 & .50 & .22 \\
\hline & & $06 / 15 / 98$ & $<.05$ & $<.20$ & $<.20$ & -- & $<.05$ & $<.20$ & $<.20$ & -- & -- & -- & -- & -- & -- & -- & $<.05$ & 2.8 & 1.6 \\
\hline & & 06/16/98 & $<.05$ & $<.20$ & $<.20$ & -- & .28 & .25 & $<.20$ & -- & -- & -- & -- & -- & -- & -- & 1.1 & .75 & .36 \\
\hline
\end{tabular}


Table 6. Analytical results for selected acetamide herbicides and their degradation products, ethanesulfonic acid, oxanilic acid, and sulfinyl acetic acid, in surface-water samples, 1993-2003.-Continued

[OGRL, U.S. Geological Survey Organic Geochemistry Research Laboratory, Lawrence, Kansas; ESA, ethanesulfonic acid; OXA, oxanilic acid; SAA, sulfinyl acetic acid; --, no data; <, less than]

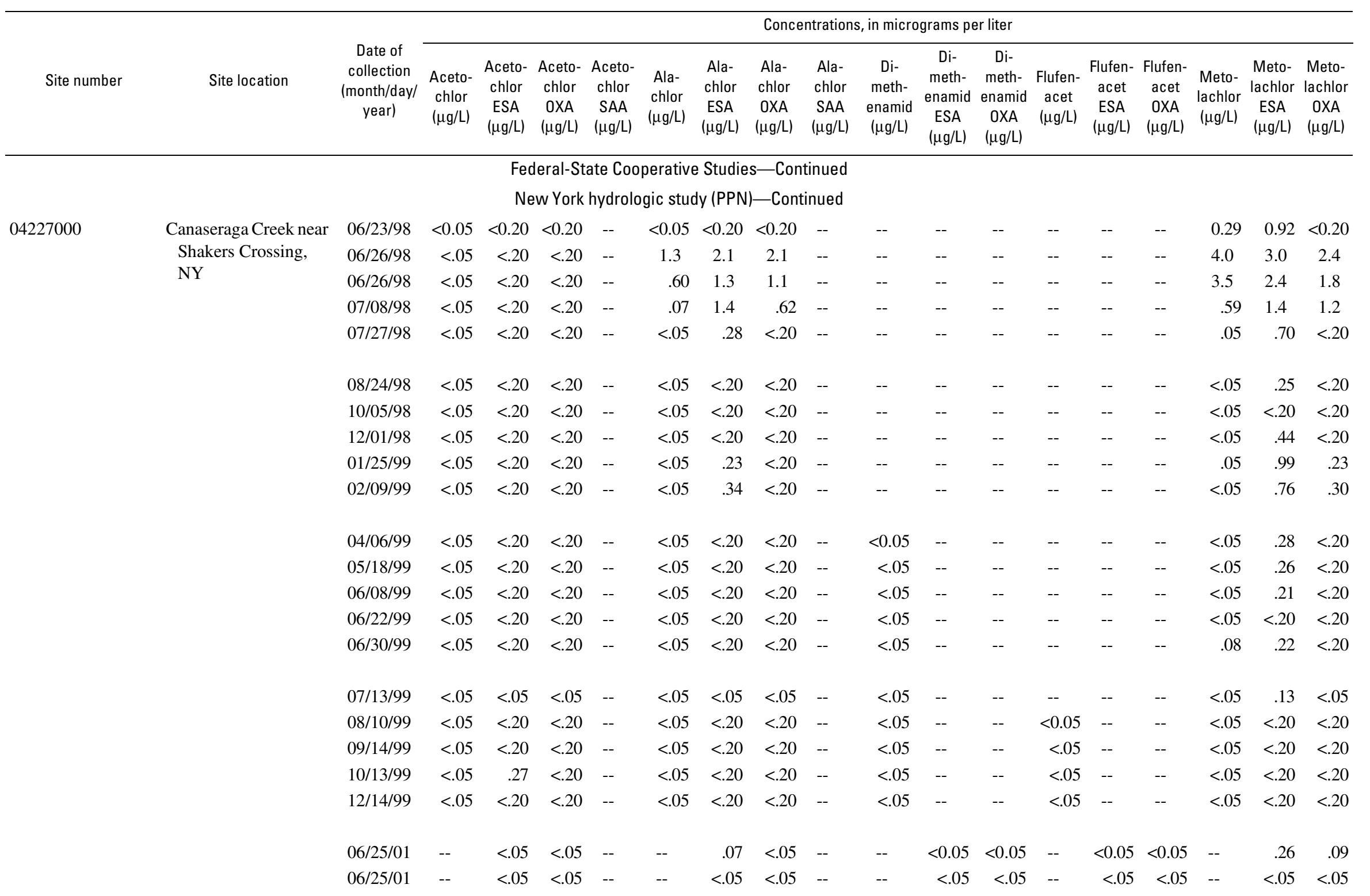


Table 6. Analytical results for selected acetamide herbicides and their degradation products, ethanesulfonic acid, oxanilic acid, and sulfinyl acetic acid, in surface-water samples, 1993-2003.-Continued

[OGRL, U.S. Geological Survey Organic Geochemistry Research Laboratory, Lawrence, Kansas; ESA, ethanesulfonic acid; OXA, oxanilic acid; SAA, sulfinyl acetic acid; --, no data; <, less than]

\begin{tabular}{|c|c|c|c|c|c|c|c|c|c|c|c|c|c|c|c|c|c|c|c|}
\hline \multirow[b]{2}{*}{ Site number } & \multirow[b]{2}{*}{ Site location } & \multirow[b]{2}{*}{$\begin{array}{c}\text { Date of } \\
\text { collection } \\
\text { (month/day/ } \\
\text { year) }\end{array}$} & \multicolumn{17}{|c|}{ Concentrations, in micrograms per liter } \\
\hline & & & $\begin{array}{l}\text { Aceto- } \\
\text { chlor } \\
(\mu \mathrm{g} / \mathrm{L})\end{array}$ & $\begin{array}{l}\text { Aceto- } \\
\text { chlor } \\
\text { ESA } \\
(\mu \mathrm{g} / \mathrm{L})\end{array}$ & $\begin{array}{l}\text { Aceto- } \\
\text { chlor } \\
0 X A \\
(\mu \mathrm{g} / \mathrm{L})\end{array}$ & $\begin{array}{l}\text { Aceto- } \\
\text { chlor } \\
\text { SAA } \\
(\mu \mathrm{g} / \mathrm{L})\end{array}$ & $\begin{array}{l}\text { Ala- } \\
\text { chlor } \\
(\mu \mathrm{g} / \mathrm{L})\end{array}$ & $\begin{array}{l}\text { Ala- } \\
\text { chlor } \\
\text { ESA } \\
(\mu \mathrm{g} / \mathrm{L})\end{array}$ & $\begin{array}{c}\text { Ala- } \\
\text { chlor } \\
\text { OXA } \\
(\mu \mathrm{g} / \mathrm{L})\end{array}$ & $\begin{array}{l}\text { Ala- } \\
\text { chlor } \\
\text { SAA } \\
(\mu \mathrm{g} / \mathrm{L})\end{array}$ & $\begin{array}{l}\text { Di- } \\
\text { meth- } \\
\text { enamid } \\
(\mu \mathrm{g} / \mathrm{L})\end{array}$ & $\begin{array}{l}\text { Di- } \\
\text { meth- } \\
\text { enamid } \\
\text { ESA } \\
(\mu \mathrm{g} / \mathrm{L})\end{array}$ & $\begin{array}{l}\text { Di- } \\
\text { meth- } \\
\text { enamid } \\
\text { OXA } \\
(\mu \mathrm{g} / \mathrm{L})\end{array}$ & $\begin{array}{l}\text { Flufen- } \\
\text { acet } \\
(\mu \mathrm{g} / \mathrm{L})\end{array}$ & $\begin{array}{l}\text { Flufen- } \\
\text { acet } \\
\text { ESA } \\
(\mu \mathrm{g} / \mathrm{L})\end{array}$ & $\begin{array}{l}\text { Flufen- } \\
\text { acet } \\
\text { OXA } \\
(\mu \mathrm{g} / \mathrm{L})\end{array}$ & $\begin{array}{l}\text { Meto- } \\
\text { lachlor } \\
(\mu \mathrm{g} / \mathrm{L})\end{array}$ & $\begin{array}{l}\text { Meto- } \\
\text { lachlor } \\
\text { ESA } \\
(\mu \mathrm{g} / \mathrm{L})\end{array}$ & $\begin{array}{l}\text { Meto- } \\
\text { lachlor } \\
\text { OXA } \\
(\mu \mathrm{g} / \mathrm{L})\end{array}$ \\
\hline \multicolumn{20}{|c|}{ Federal-State Cooperative Studies-Continued } \\
\hline \multicolumn{20}{|c|}{ New York hydrologic study (PPN)—Continued } \\
\hline \multirow[t]{2}{*}{424730077173001} & Canajoharie Lake & 09/04/97 & $<0.05$ & $<0.20$ & $<0.20$ & -- & $<0.05$ & $<0.20$ & $<0.20$ & -- & -- & -- & -- & -- & -- & -- & $<0.05$ & $<0.20$ & $<0.20$ \\
\hline & $\begin{array}{l}\text { Department of } \\
\text { Environment } \\
\text { conservation site, } \\
\text { NY }\end{array}$ & 07/22/98 & $<.05$ & $<.20$ & $<.20$ & -- & $<.05$ & $<.20$ & $<.20$ & -- & -- & -- & -- & -- & -- & -- & $<.05$ & $<.20$ & $<.20$ \\
\hline 424230077340101 & $\begin{array}{l}\text { Candice Lake } \\
\text { Department of } \\
\text { Environment } \\
\text { conservation site, } \\
\text { NY }\end{array}$ & 09/02/97 & $<.05$ & $<.20$ & $<.20$ & -- & $<.05$ & $<.20$ & $<.20$ & -- & -- & -- & -- & -- & -- & -- & $<.05$ & $<.20$ & $<.20$ \\
\hline \multirow[t]{10}{*}{01423900} & Cannonsville & $01 / 25 / 99$ & $<.05$ & $<.20$ & $<.20$ & -- & $<.05$ & $<.20$ & $<.20$ & -- & -- & -- & -- & -- & -- & -- & $<.05$ & $<.20$ & $<.20$ \\
\hline & Reservoir, NY & $05 / 24 / 99$ & $<.05$ & $<.20$ & $<.20$ & -- & $<.05$ & $<.20$ & $<.20$ & -- & $<0.05$ & -- & -- & -- & -- & -- & $<.05$ & $<.20$ & $<.20$ \\
\hline & & 07/12/99 & $<.05$ & $<.05$ & $<.05$ & -- & $<.05$ & $<.05$ & $<.05$ & -- & $<.05$ & -- & -- & $<0.05$ & -- & -- & $<.05$ & .14 & $<.05$ \\
\hline & & 09/13/99 & $<.05$ & $<.20$ & $<.20$ & -- & $<.05$ & $<.20$ & $<.20$ & -- & $<.05$ & -- & -- & $<.05$ & -- & -- & $<.05$ & $<.20$ & $<.20$ \\
\hline & & 01/10/00 & $<.05$ & $<.05$ & $<.05$ & -- & $<.05$ & $<.20$ & $<.20$ & -- & $<.05$ & -- & -- & $<.05$ & -- & -- & $<.05$ & .21 & .22 \\
\hline & & 05/15/00 & -- & $<.05$ & $<.05$ & -- & -- & .22 & $<.05$ & -- & -- & $<0.05$ & $<0.05$ & -- & -- & -- & -- & .39 & .21 \\
\hline & & 07/24/00 & -- & $<.05$ & $<.05$ & -- & -- & $<.05$ & $<.05$ & -- & -- & $<.05$ & $<.05$ & -- & $<0.05$ & $<0.05$ & -- & .10 & $<.05$ \\
\hline & & 09/11/00 & -- & $<.05$ & $<.05$ & -- & -- & $<.05$ & $<.05$ & -- & -- & $<.05$ & $<.05$ & -- & $<.05$ & $<.05$ & -- & .21 & .06 \\
\hline & & $10 / 28 / 02$ & -- & $<.05$ & $<.05$ & $<0.05$ & -- & $<.05$ & $<.05$ & $<0.05$ & -- & $<.05$ & $<.05$ & -- & $<.05$ & $<.05$ & -- & .12 & $<.05$ \\
\hline & & 05/13/02 & -- & $<.05$ & $<.05$ & $<.05$ & -- & $<.05$ & $<.05$ & $<.05$ & -- & $<.05$ & $<.05$ & -- & $<.05$ & $<.05$ & -- & .16 & $<.05$ \\
\hline 01416870 & $\begin{array}{l}\text { Cat Hollow Brook } \\
\text { near Downsville, NY }\end{array}$ & 07/19/01 & -- & $<.05$ & $<.05$ & -- & -- & $<.05$ & $<.05$ & -- & -- & $<.05$ & $<.05$ & -- & $<.05$ & $<.05$ & -- & $<.05$ & $<.20$ \\
\hline \multirow[t]{2}{*}{423331076358501} & Cayuga Lake & 09/11/97 & $<.05$ & $<.20$ & $<.20$ & -- & $<.05$ & $<.20$ & $<.20$ & -- & -- & -- & -- & -- & -- & -- & $<.05$ & .30 & $<.20$ \\
\hline & $\begin{array}{l}\text { Department of } \\
\text { Environment } \\
\text { conservation site, } \\
\text { NY }\end{array}$ & 07/21/99 & $<.05$ & $<.20$ & $<.20$ & -- & $<.05$ & $<.20$ & $<.20$ & -- & $<.05$ & -- & -- & $<.05$ & -- & -- & .05 & .71 & .32 \\
\hline
\end{tabular}


Table 6. Analytical results for selected acetamide herbicides and their degradation products, ethanesulfonic acid, oxanilic acid, and sulfinyl acetic acid, in surface-water samples, 1993-2003.-Continued

[OGRL, U.S. Geological Survey Organic Geochemistry Research Laboratory, Lawrence, Kansas; ESA, ethanesulfonic acid; OXA, oxanilic acid; SAA, sulfinyl acetic acid; --, no data; <, less than]

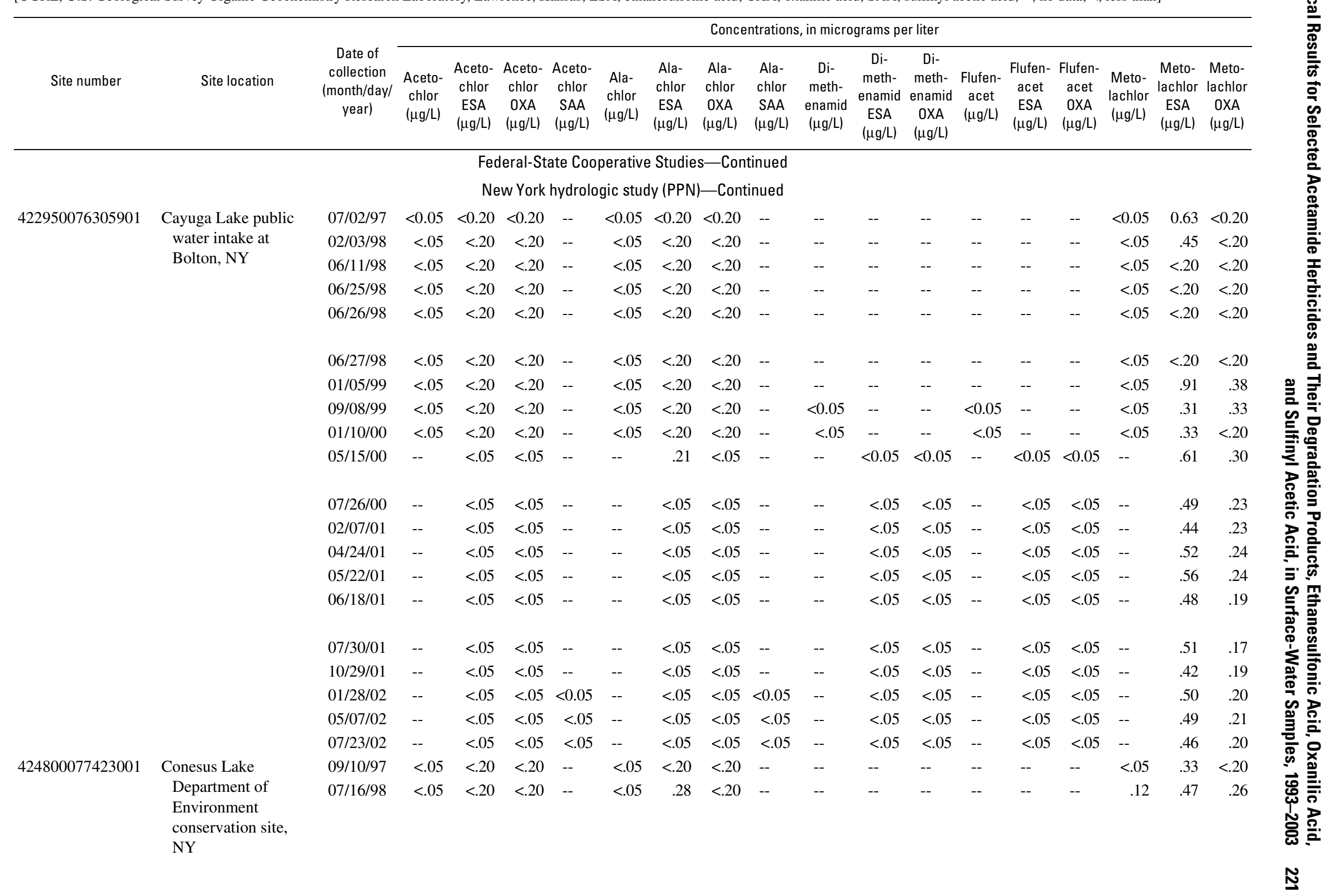


Table 6. Analytical results for selected acetamide herbicides and their degradation products, ethanesulfonic acid, oxanilic acid, and sulfinyl acetic acid, in surface-water samples, 1993-2003.-Continued

[OGRL, U.S. Geological Survey Organic Geochemistry Research Laboratory, Lawrence, Kansas; ESA, ethanesulfonic acid; OXA, oxanilic acid; SAA, sulfinyl acetic acid; --, no data; <, less than]

\begin{tabular}{|c|c|c|c|c|c|c|c|c|c|c|c|c|c|c|c|c|c|c|c|}
\hline \multirow[b]{2}{*}{ Site number } & \multirow[b]{2}{*}{ Site location } & \multirow[b]{2}{*}{$\begin{array}{c}\text { Date of } \\
\text { collection } \\
\text { (month/day/ } \\
\text { year) }\end{array}$} & \multicolumn{17}{|c|}{ Concentrations, in micrograms per liter } \\
\hline & & & $\begin{array}{l}\text { Aceto- } \\
\text { chlor } \\
(\mu \mathrm{g} / \mathrm{L})\end{array}$ & $\begin{array}{l}\text { Aceto- } \\
\text { chlor } \\
\text { ESA } \\
(\mu \mathrm{g} / \mathrm{L})\end{array}$ & $\begin{array}{l}\text { Aceto- } \\
\text { chlor } \\
\text { OXA } \\
(\mu \mathrm{g} / \mathrm{L})\end{array}$ & $\begin{array}{l}\text { Aceto- } \\
\text { chlor } \\
\text { SAA } \\
(\mu \mathrm{g} / \mathrm{L})\end{array}$ & $\begin{array}{l}\text { Ala- } \\
\text { chlor } \\
(\mu \mathrm{g} / \mathrm{L})\end{array}$ & $\begin{array}{l}\text { Ala- } \\
\text { chlor } \\
\text { ESA } \\
(\mu \mathrm{g} / \mathrm{L})\end{array}$ & $\begin{array}{l}\text { Ala- } \\
\text { chlor } \\
\text { OXA } \\
(\mu \mathrm{g} / \mathrm{L})\end{array}$ & $\begin{array}{l}\text { Ala- } \\
\text { chlor } \\
\text { SAA } \\
(\mu \mathrm{g} / \mathrm{L})\end{array}$ & $\begin{array}{l}\text { Di- } \\
\text { meth- } \\
\text { enamid } \\
(\mu \mathrm{g} / \mathrm{L})\end{array}$ & $\begin{array}{c}\text { Di- } \\
\text { meth- } \\
\text { enamid } \\
\text { ESA } \\
(\mu \mathrm{g} / \mathrm{L})\end{array}$ & $\begin{array}{c}\text { Di- } \\
\text { meth- } \\
\text { enamid } \\
\text { OXA } \\
(\mu \mathrm{g} / \mathrm{L})\end{array}$ & $\begin{array}{c}\text { Flufen- } \\
\text { acet } \\
(\mu \mathrm{g} / \mathrm{L})\end{array}$ & $\begin{array}{l}\text { Flufen- } \\
\text { acet } \\
\text { ESA } \\
(\mu \mathrm{g} / \mathrm{L})\end{array}$ & $\begin{array}{c}\text { Flufen- } \\
\text { acet } \\
\text { OXA } \\
(\mu \mathrm{g} / \mathrm{L})\end{array}$ & $\begin{array}{l}\text { Meto- } \\
\text { lachlor } \\
(\mu \mathrm{g} / \mathrm{L})\end{array}$ & $\begin{array}{l}\text { Meto- } \\
\text { lachlor } \\
\text { ESA } \\
(\mu \mathrm{g} / \mathrm{L})\end{array}$ & $\begin{array}{c}\text { Meto- } \\
\text { lachlor } \\
\text { OXA } \\
(\mu \mathrm{g} / \mathrm{L})\end{array}$ \\
\hline \multicolumn{20}{|c|}{ Federal-State Cooperative Studies-Continued } \\
\hline \multicolumn{20}{|c|}{ New York hydrologic study (PPN)—Continued } \\
\hline \multirow[t]{9}{*}{424937077422101} & \multirow{5}{*}{$\begin{array}{l}\text { Conesus Lake public } \\
\text { water supply intake } \\
\text { at Avon, NY }\end{array}$} & $05 / 16 / 00$ & -- & $<0.05$ & $<0.05$ & -- & -- & 0.22 & 0.05 & -- & -- & $<0.05$ & $<0.05$ & -- & -- & -- & -- & 0.34 & 0.21 \\
\hline & & $07 / 25 / 00$ & -- & $<.05$ & $<.05$ & -- & -- & .10 & .06 & -- & -- & $<.05$ & $<.05$ & -- & $<0.05$ & $<0.05$ & -- & .21 & .16 \\
\hline & & $02 / 06 / 01$ & -- & $<.05$ & $<.05$ & -- & -- & .11 & $<.05$ & -- & -- & $<.05$ & $<.05$ & -- & $<.05$ & $<.05$ & -- & .25 & .08 \\
\hline & & $04 / 23 / 01$ & -- & $<.05$ & $<.05$ & -- & -- & .13 & $<.05$ & -- & -- & $<.05$ & $<.05$ & -- & $<.05$ & $<.05$ & -- & .22 & .12 \\
\hline & & $05 / 21 / 01$ & -- & $<.05$ & $<.05$ & -- & -- & .10 & $<.05$ & -- & -- & $<.05$ & $<.05$ & -- & $<.05$ & $<.05$ & -- & .22 & .09 \\
\hline & & $06 / 19 / 01$ & -- & $<.05$ & $<.05$ & -- & -- & .09 & $<.05$ & -- & -- & $<.05$ & $<.05$ & -- & $<.05$ & $<.05$ & -- & .20 & .05 \\
\hline & & $07 / 30 / 01$ & -- & $<.05$ & $<.05$ & -- & -- & $<.05$ & $<.05$ & -- & -- & $<.05$ & $<.05$ & -- & $<.05$ & $<.05$ & -- & .12 & $<.05$ \\
\hline & & $10 / 30 / 01$ & -- & $<.05$ & $<.05$ & -- & -- & .11 & $<.05$ & -- & -- & $<.05$ & $<.05$ & -- & $<.05$ & $<.05$ & -- & .19 & .09 \\
\hline & & $01 / 29 / 02$ & -- & $<.05$ & $<.05$ & $<0.05$ & -- & .09 & $<.05$ & $<0.05$ & -- & $<.05$ & $<.05$ & -- & $<.05$ & $<.05$ & -- & .19 & .06 \\
\hline 424739077430801 & $\begin{array}{l}\text { Conesus Lake, } \\
\text { Geneseo public } \\
\text { water supply intake, } \\
\text { NY }\end{array}$ & $07 / 25 / 00$ & -- & $<.05$ & $<.05$ & -- & -- & .10 & .06 & -- & -- & $<.05$ & $<.05$ & -- & $<.05$ & $<.05$ & -- & .21 & .13 \\
\hline \multirow[t]{13}{*}{01374995} & \multirow[t]{13}{*}{ Croton Reservoir, NY } & $01 / 26 / 99$ & $<0.05$ & $<.20$ & $<.20$ & -- & $<0.05$ & $<.20$ & $<.20$ & -- & -- & -- & -- & -- & -- & -- & $<0.05$ & $<.20$ & $<.20$ \\
\hline & & $05 / 25 / 99$ & $<.05$ & $<.20$ & $<.20$ & -- & $<.05$ & $<.20$ & $<.20$ & -- & $<0.05$ & -- & -- & -- & -- & -- & $<.05$ & $<.20$ & $<.20$ \\
\hline & & 07/13/99 & $<.05$ & $<.20$ & $<.20$ & -- & $<.05$ & $<.20$ & $<.20$ & -- & $<.05$ & -- & -- & $<0.05$ & -- & -- & $<.05$ & $<.20$ & $<.20$ \\
\hline & & $09 / 14 / 99$ & $<.05$ & $<.20$ & $<.20$ & -- & $<.05$ & $<.20$ & $<.20$ & -- & $<.05$ & -- & -- & $<.05$ & -- & -- & $<.05$ & $<.20$ & $<.20$ \\
\hline & & $01 / 11 / 00$ & $<.05$ & $<.20$ & $<.20$ & -- & $<.05$ & $<.20$ & $<.20$ & -- & $<.05$ & -- & -- & $<.05$ & -- & -- & $<.05$ & $<.20$ & $<.20$ \\
\hline & & $05 / 16 / 00$ & -- & $<.05$ & $<.05$ & -- & -- & $<.05$ & $<.05$ & -- & -- & $<.05$ & $<.05$ & -- & -- & -- & -- & $<.05$ & $<.05$ \\
\hline & & $07 / 25 / 00$ & -- & $<.05$ & $<.05$ & -- & -- & $<.05$ & $<.05$ & -- & -- & $<.05$ & $<.05$ & -- & $<.05$ & $<.05$ & -- & $<.05$ & $<.05$ \\
\hline & & $09 / 12 / 00$ & -- & $<.05$ & $<.05$ & -- & -- & $<.05$ & $<.05$ & -- & -- & $<.05$ & $<.05$ & -- & $<.05$ & $<.05$ & -- & $<.05$ & $<.05$ \\
\hline & & $02 / 13 / 01$ & -- & $<.05$ & $<.05$ & -- & -- & $<.05$ & $<.05$ & -- & -- & $<.05$ & $<.05$ & -- & $<.05$ & $<.05$ & -- & $<.05$ & $<.05$ \\
\hline & & $04 / 24 / 01$ & -- & $<.05$ & $<.05$ & -- & -- & $<.05$ & $<.05$ & -- & -- & $<.05$ & $<.05$ & -- & $<.05$ & $<.05$ & -- & $<.05$ & $<.05$ \\
\hline & & 07/31/01 & -- & $<.05$ & $<.05$ & -- & -- & $<.05$ & $<.05$ & -- & -- & $<.05$ & $<.05$ & -- & $<.05$ & $<.05$ & -- & $<.05$ & $<.05$ \\
\hline & & 10/30/01 & -- & $<.05$ & $<.05$ & -- & -- & $<.05$ & $<.05$ & -- & -- & $<.05$ & $<.05$ & -- & $<.05$ & $<.05$ & -- & $<.05$ & $<.05$ \\
\hline & & $05 / 14 / 02$ & -- & $<.05$ & $<.05$ & $<.05$ & -- & $<.05$ & $<.05$ & $<.05$ & -- & $<.05$ & $<.05$ & -- & $<.05$ & $<.05$ & -- & $<.05$ & $<.05$ \\
\hline
\end{tabular}


Table 6. Analytical results for selected acetamide herbicides and their degradation products, ethanesulfonic acid, oxanilic acid, and sulfinyl acetic acid, in surface-water samples, 1993-2003.-Continued

[OGRL, U.S. Geological Survey Organic Geochemistry Research Laboratory, Lawrence, Kansas; ESA, ethanesulfonic acid; OXA, oxanilic acid; SAA, sulfinyl acetic acid; --, no data; <, less than]

\begin{tabular}{|c|c|c|c|c|c|c|c|c|c|c|c|c|c|c|c|c|c|c|c|c|}
\hline \multirow[b]{2}{*}{ Site number } & \multirow[b]{2}{*}{ Site location } & \multirow[b]{2}{*}{$\begin{array}{c}\text { Date of } \\
\text { collection } \\
\text { (month/day/ } \\
\text { year) }\end{array}$} & \multicolumn{17}{|c|}{ Concentrations, in micrograms per liter } & \\
\hline & & & $\begin{array}{l}\text { Aceto- } \\
\text { chlor } \\
(\mu \mathrm{g} / \mathrm{L})\end{array}$ & $\begin{array}{l}\text { Aceto- } \\
\text { chlor } \\
\text { ESA } \\
(\mu \mathrm{g} / \mathrm{L})\end{array}$ & $\begin{array}{l}\text { Aceto- } \\
\text { chlor } \\
\text { OXA } \\
(\mu \mathrm{g} / \mathrm{L})\end{array}$ & $\begin{array}{l}\text { Aceto- } \\
\text { chlor } \\
\text { SAA } \\
(\mu \mathrm{g} / \mathrm{L})\end{array}$ & $\begin{array}{l}\text { Ala- } \\
\text { chlor } \\
(\mu \mathrm{g} / \mathrm{L})\end{array}$ & $\begin{array}{l}\text { Ala- } \\
\text { chlor } \\
\text { ESA } \\
(\mu \mathrm{g} / \mathrm{L})\end{array}$ & $\begin{array}{l}\text { Ala- } \\
\text { chlor } \\
\text { OXA } \\
(\mu \mathrm{g} / \mathrm{L})\end{array}$ & $\begin{array}{l}\text { Ala- } \\
\text { chlor } \\
\text { SAA } \\
(\mu \mathrm{g} / \mathrm{L})\end{array}$ & $\begin{array}{l}\text { Di- } \\
\text { meth- } \\
\text { enamid } \\
(\mu \mathrm{g} / \mathrm{L})\end{array}$ & $\begin{array}{c}\text { Di- } \\
\text { meth- } \\
\text { enamid } \\
\text { ESA } \\
(\mu \mathrm{g} / \mathrm{L})\end{array}$ & $\begin{array}{c}\text { Di- } \\
\text { meth- } \\
\text { enamid } \\
\text { OXA } \\
(\mu \mathrm{g} / \mathrm{L})\end{array}$ & $\begin{array}{l}\text { Flufen- } \\
\text { acet } \\
(\mu \mathrm{g} / \mathrm{L})\end{array}$ & $\begin{array}{l}\text { Flufen- } \\
\text { acet } \\
\text { ESA } \\
(\mu \mathrm{g} / \mathrm{L})\end{array}$ & $\begin{array}{l}\text { Flufen- } \\
\text { acet } \\
\text { OXA } \\
(\mu \mathrm{g} / \mathrm{L})\end{array}$ & $\begin{array}{l}\text { Meto- } \\
\text { lachlor } \\
(\mu \mathrm{g} / \mathrm{L})\end{array}$ & $\begin{array}{l}\text { Meto- } \\
\text { lachlor } \\
\text { ESA } \\
(\mu \mathrm{g} / \mathrm{L})\end{array}$ & $\begin{array}{l}\text { Meto- } \\
\text { lachlor } \\
\text { OXA } \\
(\mu \mathrm{g} / \mathrm{L})\end{array}$ & \\
\hline \multicolumn{20}{|c|}{ Federal-State Cooperative Studies-Continued } & \\
\hline \multicolumn{20}{|c|}{ New York hydrologic study (PPN)—Continued } & \\
\hline 424546076445901 & $\begin{array}{l}\text { D1D, Cayuga Lake, } \\
\text { NY }\end{array}$ & 07/28/98 & $<0.05$ & $<0.20$ & $<0.20$ & -- & $<0.05$ & 0.61 & $<0.20$ & -- & -- & -- & -- & -- & -- & -- & $<0.05$ & 0.57 & 0.20 & \\
\hline 424546076445901 & $\begin{array}{l}\text { D1S, Cayuga Lake, } \\
\text { NY }\end{array}$ & 07/28/98 & $<.05$ & $<.20$ & $<.20$ & -- & $<.05$ & .70 & $<.20$ & -- & -- & -- & -- & -- & -- & -- & $<.05$ & .65 & .32 & \\
\hline 424547076435601 & $\begin{array}{l}\text { D2D, Cayuga Lake, } \\
\text { NY }\end{array}$ & $07 / 28 / 98$ & $<.05$ & $<.20$ & $<.20$ & -- & $<.05$ & .83 & $<.20$ & -- & -- & -- & -- & -- & -- & -- & .17 & .70 & .45 & \\
\hline 424547076435601 & $\begin{array}{l}\text { D2S, Cayuga Lake, } \\
\text { NY }\end{array}$ & 07/28/98 & $<.05$ & $<.20$ & $<.20$ & -- & $<.05$ & 1.0 & $<.20$ & -- & -- & -- & -- & -- & -- & -- & .23 & .70 & .34 & \\
\hline 424546076430601 & $\begin{array}{l}\text { D3D, Cayuga Lake, } \\
\text { NY }\end{array}$ & 07/28/98 & $<.05$ & $<.20$ & $<.20$ & -- & $<.05$ & .58 & $<.20$ & -- & -- & -- & -- & -- & -- & -- & $<.05$ & .38 & $<.20$ & 光 \\
\hline 424546076430601 & $\begin{array}{l}\text { D3S, Cayuga Lake, } \\
\text { NY }\end{array}$ & 07/28/98 & $<.05$ & $<.20$ & $<.20$ & -- & $<.05$ & .76 & $<.20$ & -- & -- & -- & -- & -- & -- & -- & $<.05$ & .54 & .21 & 文 \\
\hline 425425076443901 & $\begin{array}{l}\text { E1S, Cayuga Lake, } \\
\text { NY }\end{array}$ & 07/28/98 & $<.05$ & $<.20$ & $<.20$ & -- & $<.05$ & .65 & $<.05$ & -- & -- & -- & -- & -- & -- & -- & $<.05$ & .34 & .20 & \\
\hline 425426076441801 & $\begin{array}{l}\text { E2S, Cayuga Lake, } \\
\text { NY }\end{array}$ & $07 / 28 / 98$ & $<.05$ & $<.20$ & $<.20$ & -- & $<.05$ & .76 & $<.05$ & -- & -- & -- & -- & -- & -- & -- & $<.05$ & .37 & .32 & \\
\hline 425428076440001 & $\begin{array}{l}\text { E3S, Cayuga Lake, } \\
\text { NY }\end{array}$ & 07/28/98 & $<.05$ & $<.20$ & $<.20$ & -- & $<.05$ & .61 & $<.05$ & -- & -- & -- & -- & -- & -- & -- & $<.05$ & .57 & .41 & 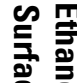 \\
\hline 425032074393901 & $\begin{array}{l}\text { ED3, drainage ditch, } \\
\text { Montgomery Co., } \\
\text { NY }\end{array}$ & 06/01/98 & $<.05$ & $<.20$ & $<.20$ & -- & $<.05$ & $<.20$ & $<.20$ & -- & -- & -- & -- & -- & -- & -- & .14 & 4.1 & .99 & 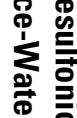 \\
\hline 425030074393902 & $\begin{array}{l}\text { ED3A, drainage ditch, } \\
\text { Montgomery Co., } \\
\text { NY }\end{array}$ & 06/30/98 & $<.05$ & $<.20$ & $<.20$ & -- & $<.05$ & $<.20$ & $<.20$ & -- & -- & -- & -- & -- & -- & -- & $<.05$ & $<.05$ & $<.20$ & 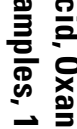 \\
\hline \multirow[t]{3}{*}{04234000} & \multirow{3}{*}{$\begin{array}{l}\text { Fall Creek near Ithaca, } \\
\text { NY }\end{array}$} & 05/22/97 & $<.05$ & $<.20$ & $<.20$ & -- & $<.05$ & $<.20$ & $<.20$ & -- & -- & -- & -- & -- & -- & -- & $<.05$ & .40 & $<.20$ & \\
\hline & & 06/11/97 & $<.05$ & $<.20$ & $<.20$ & -- & $<.05$ & $<.20$ & $<.20$ & -- & -- & -- & -- & -- & -- & -- & $<.05$ & .37 & $<.20$ & \\
\hline & & 06/18/97 & $<.05$ & $<.20$ & $<.20$ & -- & $<.05$ & $<.20$ & $<.20$ & -- & -- & -- & -- & -- & -- & -- & $<.05$ & .32 & $<.20$ & జ \\
\hline
\end{tabular}


Table 6. Analytical results for selected acetamide herbicides and their degradation products, ethanesulfonic acid, oxanilic acid, and sulfinyl acetic acid, in surface-water samples, 1993-2003.-Continued

[OGRL, U.S. Geological Survey Organic Geochemistry Research Laboratory, Lawrence, Kansas; ESA, ethanesulfonic acid; OXA, oxanilic acid; SAA, sulfinyl acetic acid; --, no data; <, less than]

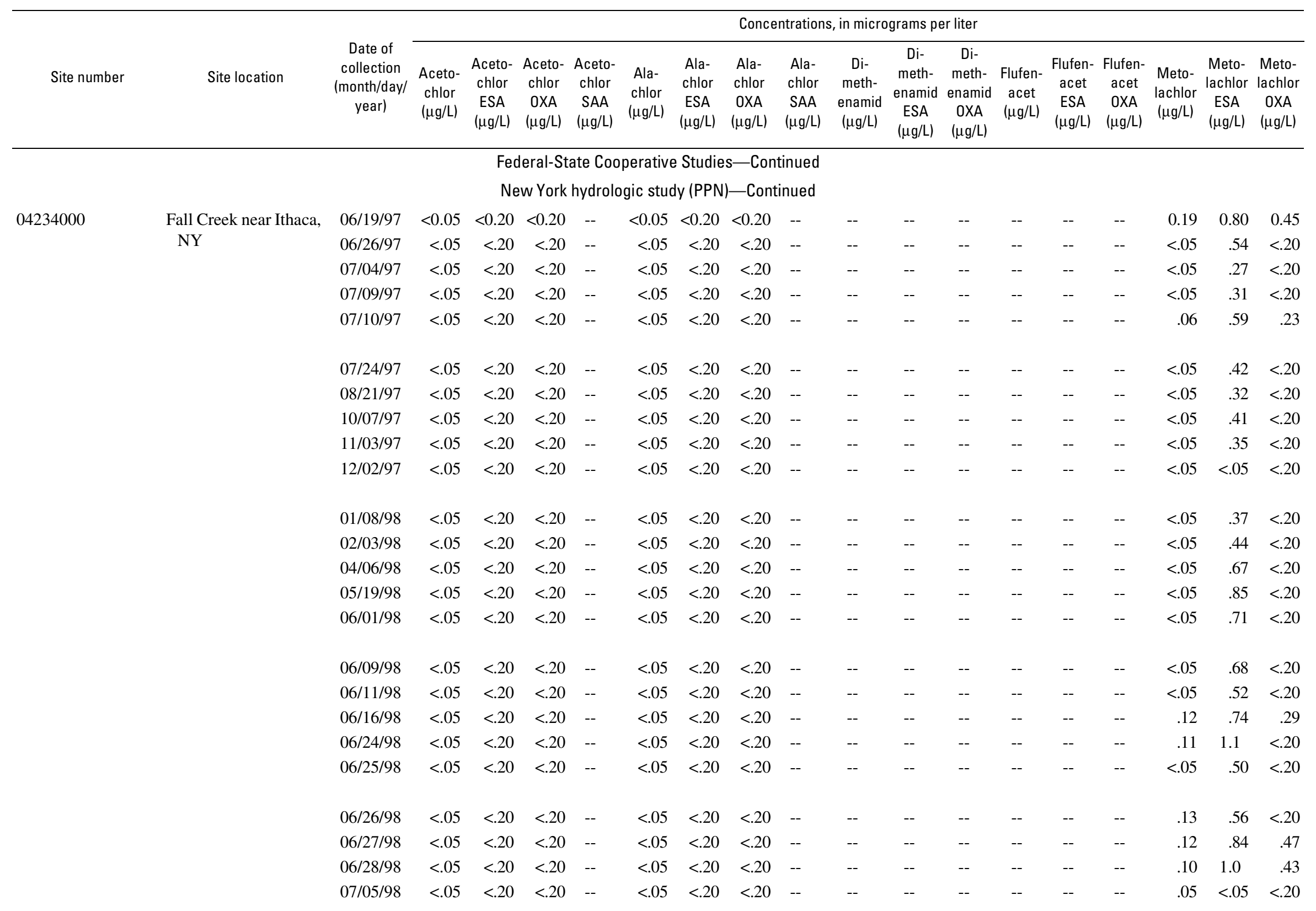


Table 6. Analytical results for selected acetamide herbicides and their degradation products, ethanesulfonic acid, oxanilic acid, and sulfinyl acetic acid, in surface-water samples, 1993-2003.-Continued

[OGRL, U.S. Geological Survey Organic Geochemistry Research Laboratory, Lawrence, Kansas; ESA, ethanesulfonic acid; OXA, oxanilic acid; SAA, sulfinyl acetic acid; --, no data; <, less than]

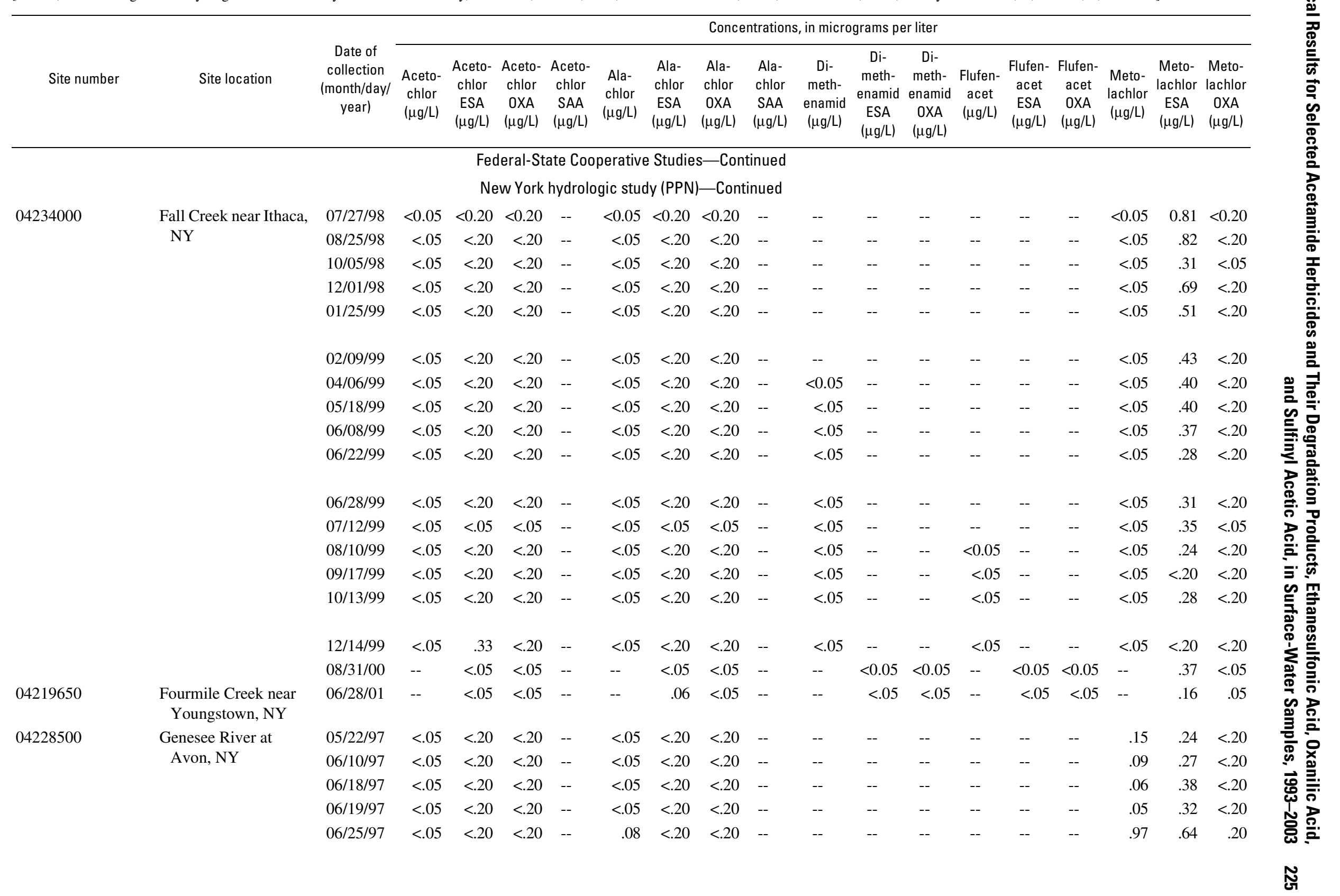


Table 6. Analytical results for selected acetamide herbicides and their degradation products, ethanesulfonic acid, oxanilic acid, and sulfinyl acetic acid, in surface-water samples, 1993-2003.-Continued

[OGRL, U.S. Geological Survey Organic Geochemistry Research Laboratory, Lawrence, Kansas; ESA, ethanesulfonic acid; OXA, oxanilic acid; SAA, sulfinyl acetic acid; --, no data; <, less than]

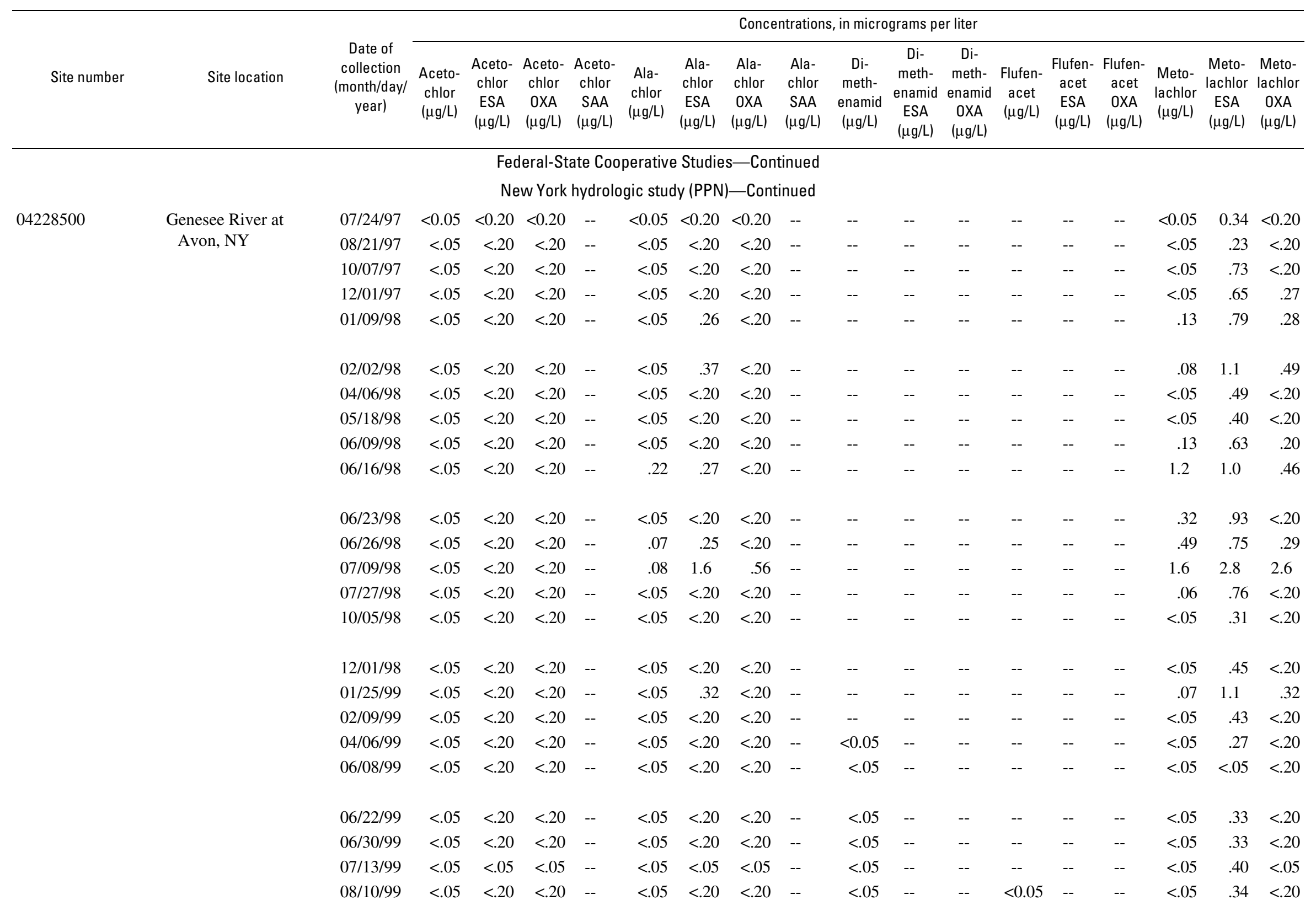


Table 6. Analytical results for selected acetamide herbicides and their degradation products, ethanesulfonic acid, oxanilic acid, and sulfinyl acetic acid, in surface-water samples, 1993-2003.-Continued

[OGRL, U.S. Geological Survey Organic Geochemistry Research Laboratory, Lawrence, Kansas; ESA, ethanesulfonic acid; OXA, oxanilic acid; SAA, sulfinyl acetic acid; --, no data; <, less than]

\begin{tabular}{|c|c|c|c|c|c|c|c|c|c|c|c|c|c|c|c|c|c|c|c|}
\hline \multirow[b]{2}{*}{ Site number } & \multirow[b]{2}{*}{ Site location } & \multirow[b]{2}{*}{$\begin{array}{c}\text { Date of } \\
\text { collection } \\
\text { (month/day/ } \\
\text { year) }\end{array}$} & \multicolumn{17}{|c|}{ Concentrations, in micrograms per liter } \\
\hline & & & $\begin{array}{c}\text { Aceto- } \\
\text { chlor } \\
(\mu \mathrm{g} / \mathrm{L})\end{array}$ & $\begin{array}{l}\text { Aceto- } \\
\text { chlor } \\
\text { ESA } \\
(\mu \mathrm{g} / \mathrm{L})\end{array}$ & $\begin{array}{l}\text { Aceto- } \\
\text { chlor } \\
\text { OXA } \\
(\mu \mathrm{g} / \mathrm{L})\end{array}$ & $\begin{array}{l}\text { Aceto- } \\
\text { chlor } \\
\text { SAA } \\
(\mu \mathrm{g} / \mathrm{L})\end{array}$ & $\begin{array}{l}\text { Ala- } \\
\text { chlor } \\
(\mu \mathrm{g} / \mathrm{L})\end{array}$ & $\begin{array}{l}\text { Ala- } \\
\text { chlor } \\
\text { ESA } \\
(\mu \mathrm{g} / \mathrm{L})\end{array}$ & $\begin{array}{l}\text { Ala- } \\
\text { chlor } \\
\text { OXA } \\
(\mu \mathrm{g} / \mathrm{L})\end{array}$ & $\begin{array}{l}\text { Ala- } \\
\text { chlor } \\
\text { SAA } \\
(\mu \mathrm{g} / \mathrm{L})\end{array}$ & $\begin{array}{l}\text { Di- } \\
\text { meth- } \\
\text { enamid } \\
(\mu \mathrm{g} / \mathrm{L})\end{array}$ & $\begin{array}{c}\text { Di- } \\
\text { meth- } \\
\text { enamid } \\
\text { ESA } \\
(\mu \mathrm{g} / \mathrm{L})\end{array}$ & $\begin{array}{c}\text { Di- } \\
\text { meth- } \\
\text { enamid } \\
\text { OXA } \\
(\mu \mathrm{g} / \mathrm{L})\end{array}$ & $\begin{array}{l}\text { Flufen- } \\
\text { acet } \\
(\mu \mathrm{g} / \mathrm{L})\end{array}$ & $\begin{array}{l}\text { Flufen- } \\
\text { acet } \\
\text { ESA } \\
(\mu \mathrm{g} / \mathrm{L})\end{array}$ & $\begin{array}{l}\text { Flufen- } \\
\text { acet } \\
\text { OXA } \\
(\mu \mathrm{g} / \mathrm{L})\end{array}$ & $\begin{array}{l}\text { Meto- } \\
\text { lachlor } \\
(\mu \mathrm{g} / \mathrm{L})\end{array}$ & $\begin{array}{l}\text { Meto- } \\
\text { lachlor } \\
\text { ESA } \\
(\mu \mathrm{g} / \mathrm{L})\end{array}$ & $\begin{array}{c}\text { Meto- } \\
\text { lachlor } \\
\text { OXA } \\
(\mu \mathrm{g} / \mathrm{L})\end{array}$ \\
\hline \multicolumn{20}{|c|}{ Federal-State Cooperative Studies-Continued } \\
\hline \multicolumn{20}{|c|}{ New York hydrologic study (PPN)—Continued } \\
\hline \multirow[t]{3}{*}{04228500} & \multirow{3}{*}{$\begin{array}{l}\text { Genesee River at } \\
\text { Avon, NY }\end{array}$} & $09 / 14 / 99$ & $<0.05$ & $<0.20$ & $0<.20$ & -- & $<0.05$ & $<0.20$ & $<0.20$ & -- & $<0.05$ & -- & -- & $<0.05$ & -- & -- & $<0.05$ & 0.33 & $<0.20$ \\
\hline & & $10 / 13 / 99$ & $<.05$ & $<.20$ & $<.20$ & -- & $<.05$ & $<.20$ & $<.20$ & -- & $<.05$ & -- & -- & $<.05$ & -- & -- & $<.05$ & .22 & $<.20$ \\
\hline & & $12 / 14 / 99$ & $<.05$ & $<.20$ & $<.20$ & -- & $<.05$ & $<.20$ & $<.20$ & -- & $<.05$ & -- & -- & $<.05$ & -- & -- & $<.05$ & $<.20$ & $<.20$ \\
\hline \multirow[t]{4}{*}{424025076321001} & \multirow{4}{*}{$\begin{array}{l}\text { Genoa-King Ferry } \\
\text { Water District, NY }\end{array}$} & 08/03/99 & $<.05$ & $<.20$ & $<.20$ & -- & $<.05$ & $<.20$ & $<.20$ & -- & $<.05$ & -- & -- & $<.05$ & -- & -- & .25 & 1.0 & $<.20$ \\
\hline & & 07/31/01 & -- & $<.05$ & $<.05$ & -- & -- & $<.05$ & $<.05$ & -- & -- & $<0.05$ & $<0.05$ & -- & $<0.05$ & $<0.05$ & -- & 1.8 & .35 \\
\hline & & $10 / 31 / 01$ & -- & $<.05$ & $<.05$ & -- & -- & $<.05$ & $<.05$ & -- & -- & $<.05$ & $<.05$ & -- & $<.05$ & $<.05$ & -- & 1.6 & .30 \\
\hline & & $01 / 28 / 02$ & -- & $<.05$ & $<.05$ & $<0.05$ & -- & $<.05$ & $<.05$ & $<0.05$ & -- & $<.05$ & $<.05$ & -- & $<.05$ & $<.05$ & -- & .69 & $<.05$ \\
\hline \multirow[t]{2}{*}{01414840} & \multirow{2}{*}{$\begin{array}{l}\text { Gladstone Hollow } \\
\text { Brook near Andes, } \\
\text { NY }\end{array}$} & $12 / 06 / 00$ & -- & $<.05$ & $<.05$ & -- & -- & .07 & $<.05$ & -- & -- & $<.05$ & $<.05$ & -- & $<.05$ & $<.05$ & -- & .16 & $<.05$ \\
\hline & & $07 / 19 / 01$ & -- & $<.05$ & $<.05$ & -- & -- & .06 & $<.05$ & -- & -- & $<.05$ & $<.05$ & -- & $<.05$ & $<.05$ & -- & .11 & $<.05$ \\
\hline \multirow[t]{10}{*}{01414835} & \multirow{10}{*}{$\begin{array}{l}\text { Gladstone Hollow } \\
\text { Brook north of } \\
\text { Andes, NY }\end{array}$} & $12 / 06 / 00$ & -- & $<.05$ & $<.05$ & -- & -- & $<.05$ & $<.05$ & -- & -- & $<.05$ & $<.05$ & -- & $<.05$ & $<.05$ & -- & .06 & $<.05$ \\
\hline & & 05/03/01 & -- & $<.05$ & $<.05$ & -- & -- & $<.05$ & $<.05$ & -- & -- & $<.05$ & $<.05$ & -- & $<.05$ & $<.05$ & -- & $<.05$ & $<.05$ \\
\hline & & $06 / 28 / 01$ & -- & $<.05$ & $<.05$ & -- & -- & .09 & $<.05$ & -- & -- & $<.05$ & $<.05$ & -- & $<.05$ & $<.05$ & -- & .07 & $<.05$ \\
\hline & & 07/19/01 & -- & $<.05$ & $<.05$ & -- & -- & $<.05$ & $<.05$ & -- & -- & $<.05$ & $<.05$ & -- & $<.05$ & $<.05$ & -- & .06 & $<.05$ \\
\hline & & 08/29/01 & -- & $<.05$ & $<.05$ & -- & -- & .08 & $<.05$ & -- & -- & $<.05$ & $<.05$ & -- & $<.05$ & $<.05$ & -- & .15 & $<.05$ \\
\hline & & $10 / 03 / 01$ & -- & $<.05$ & $<.05$ & -- & -- & .05 & $<.05$ & -- & -- & $<.05$ & $<.05$ & -- & $<.05$ & $<.05$ & -- & .08 & $<.05$ \\
\hline & & $10 / 03 / 01$ & -- & $<.05$ & $<.05$ & -- & -- & .05 & $<.05$ & -- & -- & $<.05$ & $<.05$ & -- & $<.05$ & $<.05$ & -- & .08 & $<.05$ \\
\hline & & $10 / 23 / 01$ & -- & $<.05$ & $<.05$ & -- & -- & $<.05$ & $<.05$ & -- & -- & $<.05$ & $<.05$ & -- & $<.05$ & $<.05$ & -- & .09 & $<.05$ \\
\hline & & $11 / 15 / 01$ & -- & $<.05$ & $<.05$ & -- & -- & $<.05$ & $<.05$ & -- & -- & $<.05$ & $<.05$ & -- & $<.05$ & $<.05$ & -- & .07 & $<.05$ \\
\hline & & $11 / 15 / 01$ & -- & $<.05$ & $<.05$ & -- & -- & $<.05$ & $<.05$ & -- & -- & $<.05$ & $<.05$ & -- & $<.05$ & $<.05$ & -- & .08 & $<.05$ \\
\hline 04235820 & $\begin{array}{l}\text { Grout Brook near } \\
\text { Fairhaven, NY }\end{array}$ & $05 / 22 / 97$ & $<.05$ & $<.20$ & $<.20$ & -- & $<.05$ & $<.20$ & $<.20$ & -- & -- & -- & -- & -- & -- & -- & $<.05$ & .32 & $<.20$ \\
\hline 01374494 & $\begin{array}{l}\text { Haviland Hollow } \\
\text { Brook near Putnam } \\
\text { Lake, NY }\end{array}$ & 07/20/99 & $<.05$ & $<.20$ & $<.20$ & -- & $<.05$ & $<.20$ & $<.20$ & -- & -- & -- & -- & -- & -- & -- & $<.05$ & $<.20$ & $<.20$ \\
\hline
\end{tabular}


Table 6. Analytical results for selected acetamide herbicides and their degradation products, ethanesulfonic acid, oxanilic acid, and sulfinyl acetic acid, in surface-water samples, 1993-2003.-Continued

[OGRL, U.S. Geological Survey Organic Geochemistry Research Laboratory, Lawrence, Kansas; ESA, ethanesulfonic acid; OXA, oxanilic acid; SAA, sulfinyl acetic acid; --, no data; <, less than]

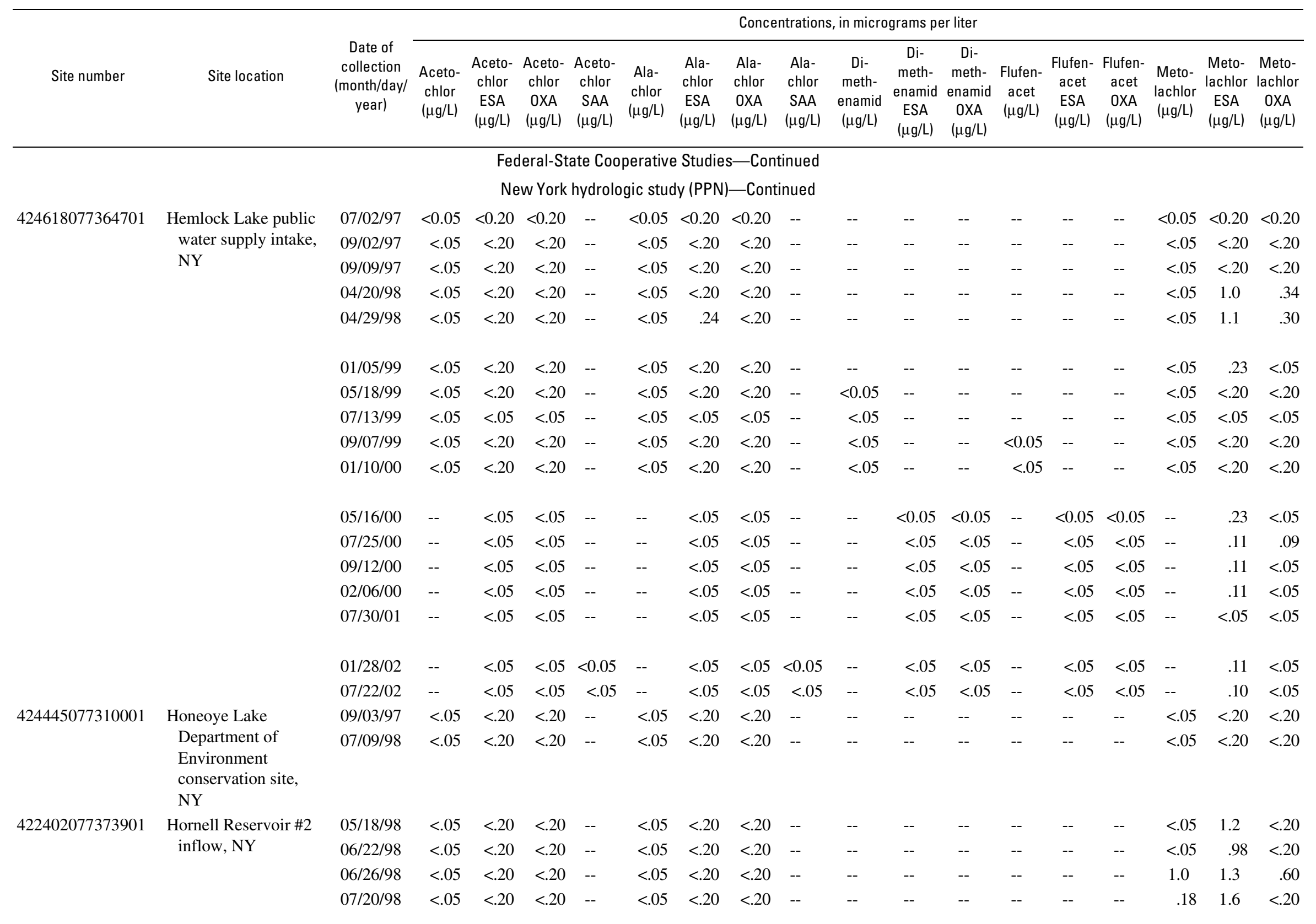


Table 6. Analytical results for selected acetamide herbicides and their degradation products, ethanesulfonic acid, oxanilic acid, and sulfinyl acetic acid, in surface-water samples, 1993-2003._Continued

[OGRL, U.S. Geological Survey Organic Geochemistry Research Laboratory, Lawrence, Kansas; ESA, ethanesulfonic acid; OXA, oxanilic acid; SAA, sulfinyl acetic acid; --, no data; <, less than]

\begin{tabular}{|c|c|c|c|c|c|c|c|c|c|c|c|c|c|c|c|c|c|c|c|}
\hline \multirow[b]{2}{*}{ Site number } & \multirow[b]{2}{*}{ Site location } & \multirow[b]{2}{*}{$\begin{array}{c}\text { Date of } \\
\text { collection } \\
\text { (month/day/ } \\
\text { year) }\end{array}$} & \multicolumn{17}{|c|}{ Concentrations, in micrograms per liter } \\
\hline & & & $\begin{array}{l}\text { Aceto- } \\
\text { chlor } \\
(\mu \mathrm{g} / \mathrm{L})\end{array}$ & $\begin{array}{l}\text { Aceto- } \\
\text { chlor } \\
\text { ESA } \\
(\mu \mathrm{g} / \mathrm{L})\end{array}$ & $\begin{array}{l}\text { Aceto- } \\
\text { chlor } \\
\text { OXA } \\
(\mu \mathrm{g} / \mathrm{L})\end{array}$ & $\begin{array}{c}\text { Aceto- } \\
\text { chlor } \\
\text { SAA } \\
(\mu \mathrm{g} / \mathrm{L})\end{array}$ & $\begin{array}{l}\text { Ala- } \\
\text { chlor } \\
(\mu \mathrm{g} / \mathrm{L})\end{array}$ & $\begin{array}{l}\text { Ala- } \\
\text { chlor } \\
\text { ESA } \\
(\mu \mathrm{g} / \mathrm{L})\end{array}$ & $\begin{array}{l}\text { Ala- } \\
\text { chlor } \\
\text { OXA } \\
(\mu \mathrm{g} / \mathrm{L})\end{array}$ & $\begin{array}{l}\text { Ala- } \\
\text { chlor } \\
\text { SAA } \\
(\mu \mathrm{g} / \mathrm{L})\end{array}$ & $\begin{array}{c}\text { Di- } \\
\text { meth- } \\
\text { enamid } \\
(\mu \mathrm{g} / \mathrm{L})\end{array}$ & $\begin{array}{c}\text { Di- } \\
\text { meth- } \\
\text { enamid } \\
\text { ESA } \\
(\mu \mathrm{g} / \mathrm{L})\end{array}$ & $\begin{array}{c}\text { Di- } \\
\text { meth- } \\
\text { enamid } \\
O X A \\
(\mu \mathrm{g} / \mathrm{L})\end{array}$ & $\begin{array}{c}\text { Flufen- } \\
\text { acet } \\
(\mu \mathrm{g} / \mathrm{L})\end{array}$ & $\begin{array}{l}\text { Flufen- } \\
\text { acet } \\
\text { ESA } \\
(\mu \mathrm{g} / \mathrm{L})\end{array}$ & $\begin{array}{c}\text { Flufen- } \\
\text { acet } \\
\text { OXA } \\
(\mu \mathrm{g} / \mathrm{L})\end{array}$ & $\begin{array}{l}\text { Meto- } \\
\text { lachlor } \\
(\mu \mathrm{g} / \mathrm{L})\end{array}$ & $\begin{array}{c}\text { Meto- } \\
\text { lachlor } \\
\text { ESA } \\
(\mu \mathrm{g} / \mathrm{L})\end{array}$ & $\begin{array}{c}\text { Meto- } \\
\text { lachlor } \\
\text { OXA } \\
(\mu \mathrm{g} / \mathrm{L})\end{array}$ \\
\hline \multicolumn{20}{|c|}{ Federal-State Cooperative Studies-Continued } \\
\hline \multicolumn{20}{|c|}{ New York hydrologic study (PPN)_Continued } \\
\hline \multirow[t]{2}{*}{422402077373901} & \multirow{2}{*}{$\begin{array}{l}\text { Hornell Reservoir \#2 } \\
\text { inflow, NY }\end{array}$} & $09 / 28 / 98$ & $<0.05$ & $<0.20$ & $<0.20$ & -- & $<0.05$ & $<0.20$ & $<0.20$ & -- & -- & -- & -- & -- & -- & -- & $<0.05$ & 0.35 & $<0.20$ \\
\hline & & $01 / 05 / 99$ & $<.05$ & $<.20$ & $<.20$ & -- & $<.05$ & $<.20$ & $<.20$ & -- & -- & -- & -- & -- & -- & -- & $<.05$ & .70 & $<.20$ \\
\hline \multirow[t]{5}{*}{422403077370901} & \multirow{5}{*}{$\begin{array}{l}\text { Hornell Reservoir \#3 } \\
\text { inflow, NY }\end{array}$} & $05 / 18 / 98$ & $<.05$ & $<.20$ & $<.20$ & -- & $<.05$ & $<.20$ & $<.20$ & -- & -- & -- & -- & -- & -- & -- & $<.05$ & 1.2 & $<.20$ \\
\hline & & $06 / 22 / 98$ & $<.05$ & $<.20$ & $<.20$ & -- & $<.05$ & $<.20$ & $<.20$ & -- & -- & -- & -- & -- & -- & -- & $<.05$ & .88 & $<.20$ \\
\hline & & $06 / 26 / 98$ & $<.05$ & $<.20$ & $<.20$ & -- & $<.05$ & $<.20$ & $<.20$ & -- & -- & -- & -- & -- & -- & -- & .82 & 1.4 & .72 \\
\hline & & $07 / 20 / 98$ & $<.05$ & $<.20$ & $<.20$ & -- & $<.05$ & $<.20$ & $<.20$ & -- & -- & -- & -- & -- & -- & -- & $<.05$ & .97 & $<.20$ \\
\hline & & $09 / 28 / 98$ & $<.05$ & $<.20$ & $<.20$ & -- & $<.05$ & $<.20$ & $<.20$ & -- & -- & -- & -- & -- & -- & -- & $<.05$ & $<.20$ & $<.20$ \\
\hline \multirow[t]{3}{*}{422328077374001} & \multirow{3}{*}{$\begin{array}{l}\text { Hornell Reservoir \#3 } \\
\text { outflow, NY }\end{array}$} & $07 / 20 / 98$ & $<.05$ & $<.20$ & $<.20$ & -- & $<.05$ & .20 & $<.20$ & -- & -- & -- & -- & -- & -- & -- & .11 & 1.0 & .47 \\
\hline & & $09 / 28 / 98$ & $<.05$ & $<.20$ & $<.20$ & -- & $<.05$ & $<.20$ & $<.20$ & -- & -- & -- & -- & -- & -- & -- & $<.05$ & .35 & $<.20$ \\
\hline & & $01 / 05 / 99$ & $<.05$ & $<.20$ & $<.20$ & -- & $<.05$ & $<.20$ & $<.20$ & -- & -- & -- & -- & -- & -- & -- & $<.05$ & .63 & $<.20$ \\
\hline \multirow[t]{17}{*}{422306077380401} & \multirow{17}{*}{$\begin{array}{l}\text { Hornell Reservoir } \\
\text { intake, NY }\end{array}$} & $05 / 18 / 98$ & $<.05$ & $<.20$ & $<.20$ & -- & $<.05$ & $<.20$ & $<.20$ & -- & -- & -- & -- & -- & -- & -- & $<.05$ & 1.3 & .39 \\
\hline & & $06 / 22 / 98$ & $<.05$ & $<.20$ & $<.20$ & -- & $<.05$ & 1.4 & $<.20$ & -- & -- & -- & -- & -- & -- & -- & $<.05$ & .73 & .34 \\
\hline & & $07 / 20 / 98$ & $<.05$ & $<.20$ & $<.20$ & -- & $<.05$ & .22 & $<.20$ & -- & -- & -- & -- & -- & -- & -- & .21 & .96 & .51 \\
\hline & & $09 / 28 / 98$ & $<.05$ & $<.20$ & $<.20$ & -- & $<.05$ & $<.20$ & $<.20$ & -- & -- & -- & -- & -- & -- & -- & $<.05$ & $<.20$ & $<.20$ \\
\hline & & 01/05/98 & $<.05$ & $<.20$ & $<.20$ & -- & $<.05$ & $<.20$ & $<.20$ & -- & -- & -- & -- & -- & -- & -- & $<.05$ & .62 & $<.20$ \\
\hline & & 05/18/99 & $<.05$ & $<.20$ & $<.20$ & -- & $<.05$ & $<.20$ & $<.20$ & -- & $<0.05$ & -- & -- & -- & -- & -- & $<.05$ & .56 & $<.20$ \\
\hline & & 07/13/99 & $<.05$ & $<.05$ & $<.05$ & -- & $<.05$ & $<.05$ & $<.05$ & -- & $<.05$ & -- & -- & $<0.05$ & -- & -- & $<.05$ & .55 & $<.05$ \\
\hline & & 09/07/99 & $<.05$ & $<.20$ & $<.20$ & -- & $<.05$ & $<.20$ & $<.20$ & -- & $<.05$ & -- & -- & $<.05$ & -- & -- & $<.05$ & .49 & .21 \\
\hline & & $01 / 11 / 00$ & $<.05$ & $<.20$ & $<.20$ & -- & $<.05$ & $<.20$ & $<.20$ & -- & $<.05$ & -- & -- & $<.05$ & -- & -- & $<.05$ & .54 & $<.20$ \\
\hline & & 05/16/00 & -- & $<.05$ & $<.05$ & -- & -- & .20 & $<.05$ & -- & -- & $<0.05$ & $<0.05$ & -- & $<0.05$ & $<0.05$ & -- & .88 & .35 \\
\hline & & $07 / 25 / 00$ & -- & .10 & $<.05$ & -- & -- & $<.05$ & .06 & -- & -- & $<.05$ & $<.05$ & -- & $<.05$ & $<.05$ & -- & .56 & .33 \\
\hline & & $09 / 11 / 00$ & -- & $<.05$ & $<.05$ & -- & -- & .11 & .08 & -- & -- & $<.05$ & $<.05$ & -- & $<.05$ & $<.05$ & -- & .63 & .12 \\
\hline & & 02/06/01 & -- & $<.05$ & $<.05$ & -- & -- & .10 & $<.05$ & -- & -- & $<.05$ & $<.05$ & -- & $<.05$ & $<.05$ & -- & .65 & .11 \\
\hline & & $04 / 24 / 01$ & -- & $<.05$ & $<.05$ & -- & -- & .10 & $<.05$ & -- & -- & $<.05$ & $<.05$ & -- & $<.05$ & $<.05$ & -- & .56 & .21 \\
\hline & & $05 / 21 / 01$ & -- & $<.05$ & $<.05$ & -- & -- & .09 & $<.05$ & -- & -- & $<.05$ & $<.05$ & -- & $<.05$ & $<.05$ & -- & .58 & .14 \\
\hline & & 06/19/01 & -- & $<.05$ & $<.05$ & -- & -- & $<.05$ & $<.05$ & -- & -- & $<.05$ & $<.05$ & -- & $<.05$ & $<.05$ & -- & .45 & .06 \\
\hline & & 07/30/01 & -- & $<.05$ & $<.05$ & -- & -- & $<.05$ & $<.05$ & -- & -- & $<.05$ & $<.05$ & -- & $<.05$ & $<.05$ & -- & .29 & $<.05$ \\
\hline
\end{tabular}


Table 6. Analytical results for selected acetamide herbicides and their degradation products, ethanesulfonic acid, oxanilic acid, and sulfinyl acetic acid, in surface-water samples, 1993-2003.-Continued

[OGRL, U.S. Geological Survey Organic Geochemistry Research Laboratory, Lawrence, Kansas; ESA, ethanesulfonic acid; OXA, oxanilic acid; SAA, sulfinyl acetic acid; --, no data; <, less than]

\begin{tabular}{|c|c|c|c|c|c|c|c|c|c|c|c|c|c|c|c|c|c|c|c|}
\hline \multirow[b]{2}{*}{ Site number } & \multirow[b]{2}{*}{ Site location } & \multirow[b]{2}{*}{$\begin{array}{c}\text { Date of } \\
\text { collection } \\
\text { (month/day/ } \\
\text { year) }\end{array}$} & \multicolumn{17}{|c|}{ Concentrations, in micrograms per liter } \\
\hline & & & $\begin{array}{l}\text { Aceto- } \\
\text { chlor } \\
(\mu \mathrm{g} / \mathrm{L})\end{array}$ & $\begin{array}{l}\text { Aceto- } \\
\text { chlor } \\
\text { ESA } \\
(\mu \mathrm{g} / \mathrm{L})\end{array}$ & $\begin{array}{l}\text { Aceto- } \\
\text { chlor } \\
\text { OXA } \\
(\mu \mathrm{g} / \mathrm{L})\end{array}$ & $\begin{array}{l}\text { Aceto- } \\
\text { chlor } \\
\text { SAA } \\
(\mu \mathrm{g} / \mathrm{L})\end{array}$ & $\begin{array}{l}\text { Ala- } \\
\text { chlor } \\
(\mu \mathrm{g} / \mathrm{L})\end{array}$ & $\begin{array}{l}\text { Ala- } \\
\text { chlor } \\
\text { ESA } \\
(\mu \mathrm{g} / \mathrm{L})\end{array}$ & $\begin{array}{c}\text { Ala- } \\
\text { chlor } \\
\text { OXA } \\
(\mu \mathrm{g} / \mathrm{L})\end{array}$ & $\begin{array}{l}\text { Ala- } \\
\text { chlor } \\
\text { SAA } \\
(\mu \mathrm{g} / \mathrm{L})\end{array}$ & $\begin{array}{l}\text { Di- } \\
\text { meth- } \\
\text { enamid } \\
(\mu \mathrm{g} / \mathrm{L})\end{array}$ & $\begin{array}{c}\text { Di- } \\
\text { meth- } \\
\text { enamid } \\
\text { ESA } \\
(\mu \mathrm{g} / \mathrm{L})\end{array}$ & $\begin{array}{c}\text { Di- } \\
\text { meth- } \\
\text { enamid } \\
\text { OXA } \\
(\mu \mathrm{g} / \mathrm{L})\end{array}$ & $\begin{array}{l}\text { Flufen- } \\
\text { acet } \\
(\mu \mathrm{g} / \mathrm{L})\end{array}$ & $\begin{array}{l}\text { Flufen- } \\
\text { acet } \\
\text { ESA } \\
(\mu \mathrm{g} / \mathrm{L})\end{array}$ & $\begin{array}{l}\text { Flufen- } \\
\text { acet } \\
\text { OXA } \\
(\mu \mathrm{g} / \mathrm{L})\end{array}$ & $\begin{array}{l}\text { Meto- } \\
\text { lachlor } \\
(\mu \mathrm{g} / \mathrm{L})\end{array}$ & $\begin{array}{l}\text { Meto- } \\
\text { lachlor } \\
\text { ESA } \\
(\mu \mathrm{g} / \mathrm{L})\end{array}$ & $\begin{array}{l}\text { Meto- } \\
\text { lachlor } \\
\text { OXA } \\
(\mu \mathrm{g} / \mathrm{L})\end{array}$ \\
\hline \multicolumn{20}{|c|}{ Federal-State Cooperative Studies-Continued } \\
\hline \multicolumn{20}{|c|}{ New York hydrologic study (PPN)—Continued } \\
\hline \multirow[t]{3}{*}{422306077380401} & \multirow{3}{*}{$\begin{array}{l}\text { Hornell Reservoir } \\
\text { intake, NY }\end{array}$} & $10 / 30 / 01$ & -- & $<0.05$ & $<0.05$ & -- & -- & $<0.05$ & $<0.05$ & -- & -- & $<0.05$ & $<0.05$ & -- & $<0.05$ & $<0.05$ & -- & 0.27 & 0.05 \\
\hline & & $01 / 29 / 02$ & -- & $<.05$ & $<.05$ & $<0.05$ & -- & $<.05$ & $<.05$ & $<0.05$ & -- & $<.05$ & $<.05$ & -- & $<.05$ & $<.05$ & -- & .39 & .14 \\
\hline & & 05/07/02 & -- & $<.05$ & $<.05$ & $<.05$ & -- & .14 & $<.05$ & $<.05$ & -- & $<.05$ & $<.05$ & -- & $<.05$ & $<.05$ & -- & .67 & .18 \\
\hline 0150273301 & $\begin{array}{l}\text { Hotchkiss Creek near } \\
\text { Windsor, NY }\end{array}$ & $11 / 26 / 01$ & -- & $<.05$ & $<.05$ & $<.05$ & -- & $<.05$ & $<.05$ & $<.05$ & -- & $<.05$ & $<.05$ & -- & $<.05$ & $<.05$ & -- & $<.05$ & $<.05$ \\
\hline 414318073562501 & $\begin{array}{l}\text { Hudson River at } \\
\text { Poughkeepsie (raw), } \\
\text { NY }\end{array}$ & 09/10/01 & -- & $<.05$ & $<.05$ & -- & -- & .06 & $<.05$ & -- & -- & $<.05$ & $<.05$ & -- & $<.05$ & $<.05$ & -- & .07 & .06 \\
\hline 414318073562502 & $\begin{array}{l}\text { Hudson River at } \\
\text { Poughkeepsie } \\
\text { (finished), NY }\end{array}$ & 09/10/01 & -- & $<.05$ & $<.05$ & -- & -- & .06 & $<.05$ & -- & -- & $<.05$ & $<.05$ & -- & $<.05$ & $<.05$ & -- & .07 & .05 \\
\hline \multirow[t]{15}{*}{01301900} & \multirow{15}{*}{$\begin{array}{l}\text { Kensico Reservoir } \\
\text { along Delaware } \\
\text { Aqueduct 18, NY }\end{array}$} & 01/26/99 & $<0.05$ & $<.20$ & $<.20$ & -- & $<0.05$ & $<.20$ & $<.20$ & -- & -- & -- & -- & -- & -- & -- & $<0.05$ & $<.20$ & $<.20$ \\
\hline & & 05/25/99 & $<.05$ & $<.20$ & $<.20$ & -- & $<.05$ & $<.20$ & $<.20$ & -- & $<0.05$ & -- & -- & -- & -- & -- & $<.05$ & $<.20$ & $<.20$ \\
\hline & & 07/13/99 & $<.05$ & $<.05$ & $<.05$ & -- & $<.05$ & $<.05$ & $<.05$ & -- & $<.05$ & -- & -- & $<0.05$ & -- & -- & $<.05$ & .05 & $<.05$ \\
\hline & & 09/14/99 & $<.05$ & $<.20$ & $<.20$ & -- & $<.05$ & $<.20$ & $<.20$ & -- & $<.05$ & -- & -- & $<.05$ & -- & -- & $<.05$ & $<.20$ & $<.20$ \\
\hline & & 01/11/00 & $<.05$ & $<.20$ & $<.20$ & -- & $<.05$ & $<.20$ & $<.20$ & -- & $<.05$ & -- & -- & $<.05$ & -- & -- & $<.05$ & $<.20$ & $<.20$ \\
\hline & & $05 / 16 / 00$ & -- & $<.05$ & $<.05$ & -- & -- & $<.05$ & $<.05$ & -- & -- & $<.05$ & $<.05$ & -- & -- & -- & -- & .27 & $<.05$ \\
\hline & & $07 / 25 / 00$ & -- & $<.05$ & $<.05$ & -- & -- & $<.05$ & $<.05$ & -- & -- & $<.05$ & $<.05$ & -- & $<.05$ & $<.05$ & -- & $<.05$ & $<.05$ \\
\hline & & 09/12/00 & -- & $<.05$ & $<.05$ & -- & -- & $<.05$ & $<.05$ & -- & -- & $<.05$ & $<.05$ & -- & $<.05$ & $<.05$ & -- & $<.05$ & $<.05$ \\
\hline & & $02 / 13 / 01$ & -- & $<.05$ & $<.05$ & -- & -- & $<.05$ & $<.05$ & -- & -- & $<.05$ & $<.05$ & -- & $<.05$ & $<.05$ & -- & $<.05$ & $<.05$ \\
\hline & & 04/24/01 & -- & $<.05$ & $<.05$ & -- & -- & $<.05$ & $<.05$ & -- & -- & $<.05$ & $<.05$ & -- & $<.05$ & $<.05$ & -- & .08 & $<.05$ \\
\hline & & 07/31/01 & -- & $<.05$ & $<.05$ & -- & -- & $<.05$ & $<.05$ & -- & -- & $<.05$ & $<.05$ & -- & $<.05$ & $<.05$ & -- & $<.05$ & $<.05$ \\
\hline & & $10 / 30 / 01$ & -- & $<.05$ & $<.05$ & -- & -- & $<.05$ & $<.05$ & -- & -- & $<.05$ & $<.05$ & -- & $<.05$ & $<.05$ & -- & $<.05$ & $<.05$ \\
\hline & & $05 / 14 / 02$ & -- & $<.05$ & $<.05$ & $<.05$ & -- & $<.05$ & $<.05$ & $<.05$ & -- & $<.05$ & $<.05$ & -- & $<.05$ & $<.05$ & -- & .06 & $<.05$ \\
\hline & & $07 / 16 / 02$ & -- & $<.05$ & $<.05$ & $<.05$ & -- & $<.05$ & $<.05$ & $<.05$ & -- & $<.05$ & $<.05$ & -- & $<.05$ & $<.05$ & -- & .06 & $<.05$ \\
\hline & & $10 / 22 / 02$ & -- & $<.05$ & $<.05$ & $<.05$ & -- & $<.05$ & $<.05$ & $<.05$ & -- & $<.05$ & $<.05$ & -- & $<.05$ & $<.05$ & -- & .05 & $<.05$ \\
\hline
\end{tabular}


Table 6. Analytical results for selected acetamide herbicides and their degradation products, ethanesulfonic acid, oxanilic acid, and sulfinyl acetic acid, in surface-water samples, 1993-2003.-Continued

[OGRL, U.S. Geological Survey Organic Geochemistry Research Laboratory, Lawrence, Kansas; ESA, ethanesulfonic acid; OXA, oxanilic acid; SAA, sulfinyl acetic acid; --, no data; <, less than]

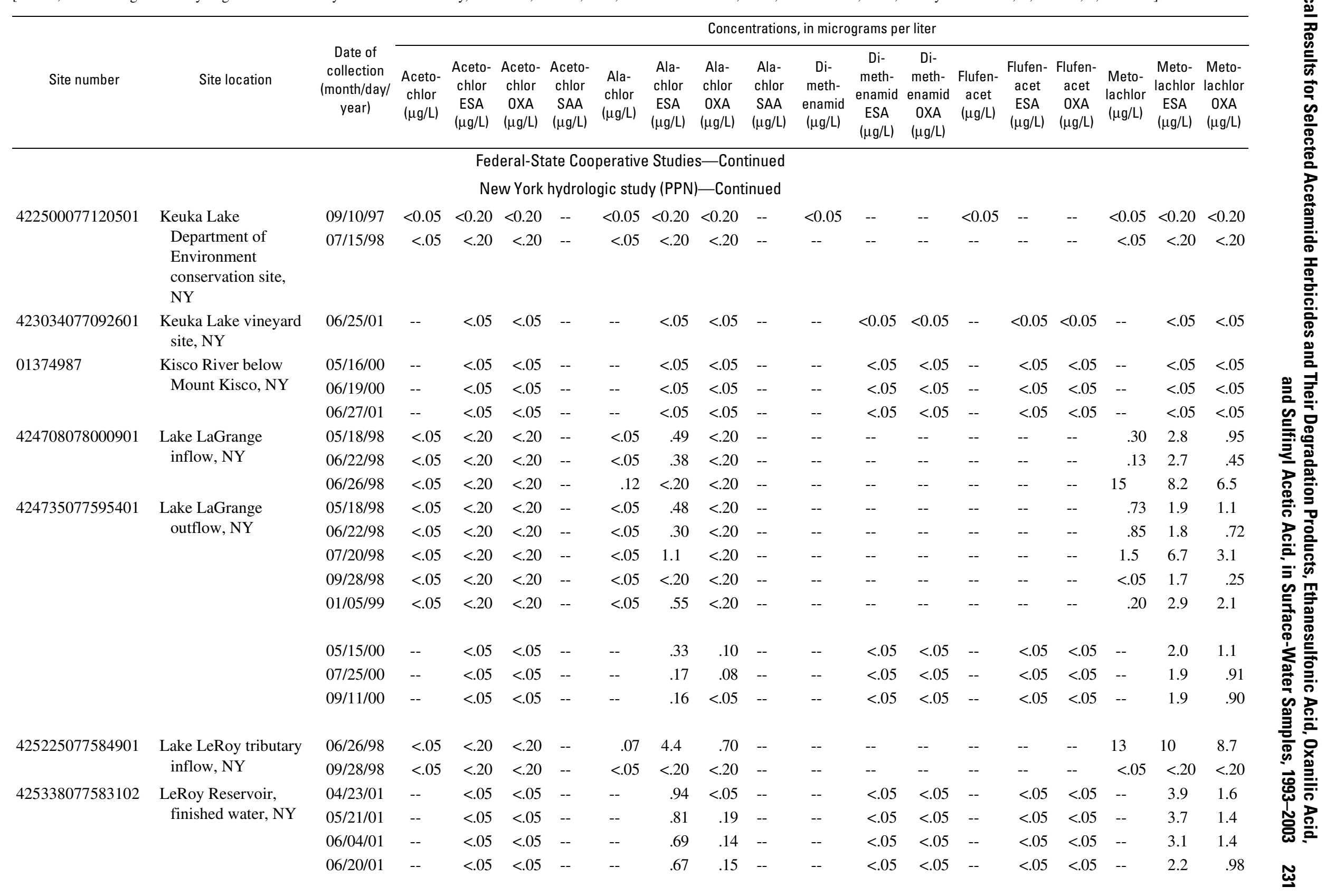


Table 6. Analytical results for selected acetamide herbicides and their degradation products, ethanesulfonic acid, oxanilic acid, and sulfinyl acetic acid, in surface-water samples, 1993-2003.-Continued

[OGRL, U.S. Geological Survey Organic Geochemistry Research Laboratory, Lawrence, Kansas; ESA, ethanesulfonic acid; OXA, oxanilic acid; SAA, sulfinyl acetic acid; --, no data; <, less than]

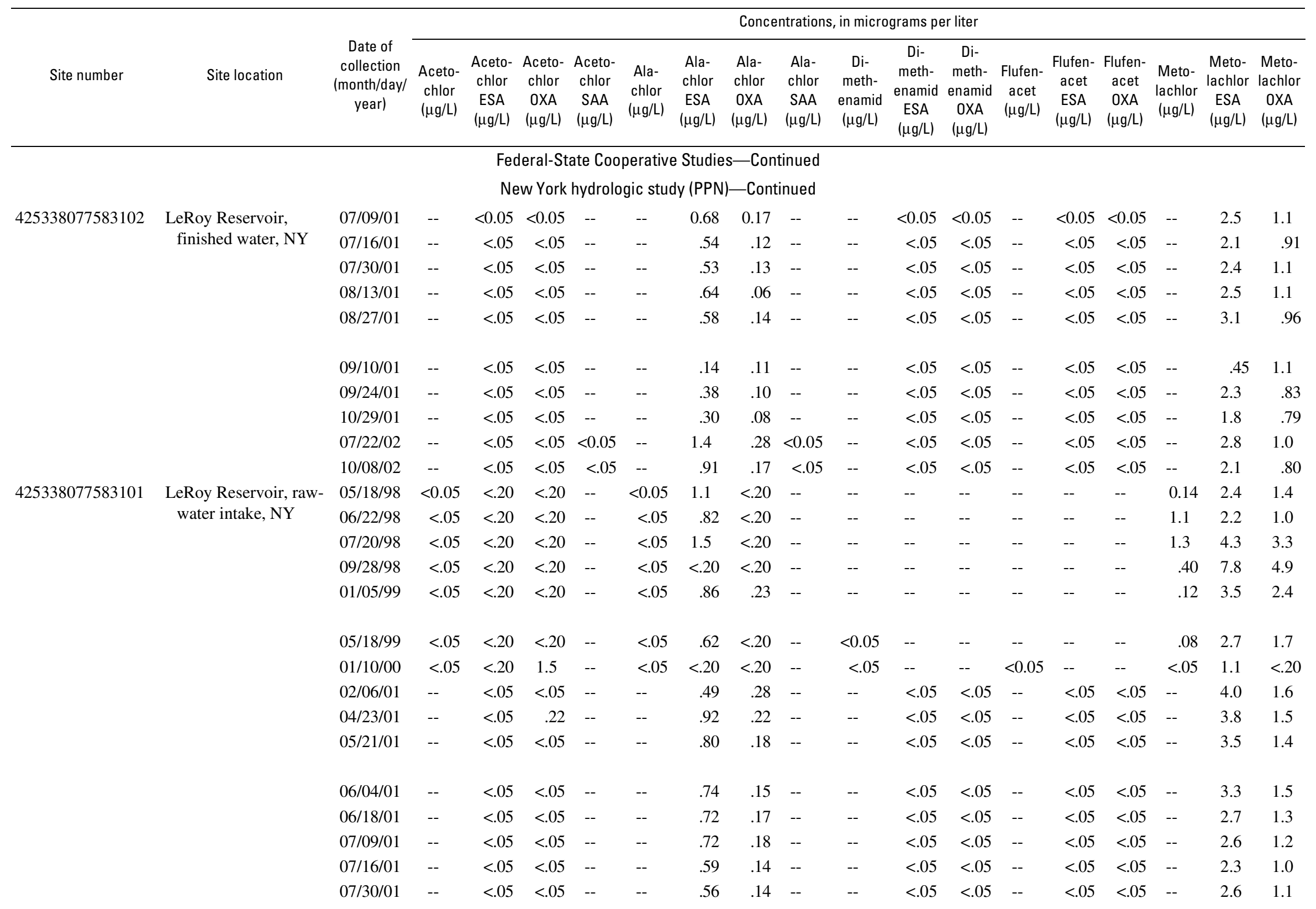


Table 6. Analytical results for selected acetamide herbicides and their degradation products, ethanesulfonic acid, oxanilic acid, and sulfinyl acetic acid, in surface-water samples, 1993-2003.-Continued

[OGRL, U.S. Geological Survey Organic Geochemistry Research Laboratory, Lawrence, Kansas; ESA, ethanesulfonic acid; OXA, oxanilic acid; SAA, sulfinyl acetic acid; --, no data; <, less than]

\begin{tabular}{|c|c|c|c|c|c|c|c|c|c|c|c|c|c|c|c|c|c|c|c|c|}
\hline \multirow[b]{2}{*}{ Site number } & \multirow[b]{2}{*}{ Site location } & \multirow[b]{2}{*}{$\begin{array}{c}\text { Date of } \\
\text { collection } \\
\text { (month/day/ } \\
\text { year) }\end{array}$} & \multicolumn{17}{|c|}{ Concentrations, in micrograms per liter } & \\
\hline & & & $\begin{array}{l}\text { Aceto- } \\
\text { chlor } \\
(\mu \mathrm{g} / \mathrm{L})\end{array}$ & $\begin{array}{l}\text { Aceto- } \\
\text { chlor } \\
\text { ESA } \\
(\mu \mathrm{g} / \mathrm{L})\end{array}$ & $\begin{array}{l}\text { Aceto- } \\
\text { chlor } \\
\text { OXA } \\
(\mu \mathrm{g} / \mathrm{L})\end{array}$ & $\begin{array}{l}\text { Aceto- } \\
\text { chlor } \\
\text { SAA } \\
(\mu \mathrm{g} / \mathrm{L})\end{array}$ & $\begin{array}{l}\text { Ala- } \\
\text { chlor } \\
(\mu \mathrm{g} / \mathrm{L})\end{array}$ & $\begin{array}{l}\text { Ala- } \\
\text { chlor } \\
\text { ESA } \\
(\mu \mathrm{g} / \mathrm{L})\end{array}$ & $\begin{array}{l}\text { Ala- } \\
\text { chlor } \\
\text { OXA } \\
(\mu \mathrm{g} / \mathrm{L})\end{array}$ & $\begin{array}{l}\text { Ala- } \\
\text { chlor } \\
\text { SAA } \\
(\mu \mathrm{g} / \mathrm{L})\end{array}$ & $\begin{array}{c}\text { Di- } \\
\text { meth- } \\
\text { enamid } \\
(\mu \mathrm{g} / \mathrm{L})\end{array}$ & $\begin{array}{c}\text { Di- } \\
\text { meth- } \\
\text { enamid } \\
\text { ESA } \\
(\mu \mathrm{g} / \mathrm{L})\end{array}$ & $\begin{array}{c}\text { Di- } \\
\text { meth- } \\
\text { enamid } \\
0 X A \\
(\mu \mathrm{g} / \mathrm{L})\end{array}$ & $\begin{array}{c}\text { Flufen- } \\
\text { acet } \\
(\mu \mathrm{g} / \mathrm{L})\end{array}$ & $\begin{array}{l}\text { Flufen- } \\
\text { acet } \\
\text { ESA } \\
(\mu \mathrm{g} / \mathrm{L})\end{array}$ & $\begin{array}{l}\text { Flufen- } \\
\text { acet } \\
\text { OXA } \\
(\mu \mathrm{g} / \mathrm{L})\end{array}$ & $\begin{array}{l}\text { Meto- } \\
\text { lachlor } \\
(\mu \mathrm{g} / \mathrm{L})\end{array}$ & $\begin{array}{l}\text { Meto- } \\
\text { lachlor } \\
\text { ESA } \\
(\mu \mathrm{g} / \mathrm{L})\end{array}$ & $\begin{array}{l}\text { Meto- } \\
\text { lachlor } \\
\text { OXA } \\
(\mu \mathrm{g} / \mathrm{L})\end{array}$ & $\begin{array}{l}\overline{\bar{m}} \\
\overline{0} \\
\frac{0}{c} \\
\frac{0}{D} \\
\frac{D}{10}\end{array}$ \\
\hline \multicolumn{20}{|c|}{ Federal-State Cooperative Studies-Continued } & \\
\hline \multicolumn{20}{|c|}{ New York hydrologic study (PPN)_Continued } & \\
\hline \multirow[t]{9}{*}{425338077583101} & \multirow{9}{*}{$\begin{array}{l}\text { LeRoy Reservoir, raw- } \\
\text { water intake, NY }\end{array}$} & 08/13/01 & -- & $<0.05$ & $<0.05$ & -- & -- & 0.68 & 0.07 & -- & -- & $<0.05$ & $<0.05$ & -- & $<0.05$ & $<0.05$ & -- & 2.6 & 1.1 & \\
\hline & & $08 / 27 / 01$ & -- & $<.05$ & $<.05$ & -- & -- & .35 & .08 & -- & -- & $<.05$ & $<.05$ & -- & $<.05$ & $<.05$ & -- & 1.8 & 65 & \\
\hline & & 09/10/01 & -- & $<.05$ & $<.05$ & -- & -- & .14 & .10 & -- & -- & $<.05$ & $<.05$ & -- & $<.05$ & $<.05$ & -- & .50 & 1.2 & \\
\hline & & $09 / 24 / 01$ & -- & $<.05$ & $<.05$ & -- & -- & .37 & .10 & -- & -- & $<.05$ & $<.05$ & -- & $<.05$ & $<.05$ & -- & 2.2 & .83 & \\
\hline & & $10 / 29 / 01$ & -- & $<.05$ & $<.05$ & -- & -- & .35 & .09 & -- & -- & $<.05$ & $<.05$ & -- & $<.05$ & $<.05$ & -- & 2.1 & .92 & \\
\hline & & $01 / 28 / 02$ & -- & $<.05$ & $<.05$ & $<0.05$ & -- & .55 & .21 & $<0.05$ & -- & $<.05$ & $<.05$ & -- & $<.05$ & $<.05$ & -- & 1.8 & .81 & \\
\hline & & $06 / 11 / 02$ & -- & $<.05$ & $<.05$ & $<.05$ & -- & $<.05$ & $<.05$ & $<.05$ & -- & $<.05$ & $<.05$ & -- & $<.05$ & $<.05$ & -- & $<.05$ & $<.05$ & \\
\hline & & $07 / 22 / 02$ & -- & $<.05$ & $<.05$ & $<.05$ & -- & 1.5 & .29 & $<.05$ & -- & $<.05$ & $<.05$ & -- & $<.05$ & $<.05$ & -- & 2.9 & 1.0 & \\
\hline & & $10 / 08 / 02$ & -- & $<.05$ & $<.05$ & $<.05$ & -- & 1.0 & .19 & $<.05$ & -- & $<.05$ & $<.05$ & -- & $<.05$ & $<.05$ & -- & 2.4 & .92 & \\
\hline \multirow[t]{2}{*}{04228908} & \multirow{2}{*}{$\begin{array}{l}\text { Limekiln Creek north } \\
\text { of Springwater, NY }\end{array}$} & 07/09/97 & $<0.05$ & $<.20$ & $<.20$ & -- & $<0.05$ & $<.20$ & $<.20$ & -- & -- & -- & -- & -- & -- & -- & $<0.05$ & $<.20$ & $<.20$ & \\
\hline & & $07 / 23 / 97$ & $<.05$ & $<.20$ & $<.20$ & -- & $<.05$ & $<.20$ & $<.20$ & -- & -- & -- & -- & -- & -- & -- & $<.05$ & $<.20$ & $<.20$ & \\
\hline \multirow[t]{3}{*}{01356190} & \multirow{3}{*}{$\begin{array}{c}\text { Lisha Kill northwest } \\
\text { of Niskayuna, NY }\end{array}$} & $06 / 26 / 01$ & -- & $<.05$ & $<.05$ & -- & -- & $<.05$ & $<.05$ & -- & -- & $<.05$ & $<.05$ & -- & $<.05$ & $<.05$ & -- & $<.05$ & $<.05$ & \\
\hline & & $12 / 05 / 01$ & -- & $<.05$ & $<.05$ & $<.05$ & -- & $<.05$ & $<.05$ & $<.05$ & -- & $<.05$ & $<.05$ & -- & $<.05$ & $<.05$ & -- & $<.05$ & $<.05$ & \\
\hline & & $02 / 06 / 02$ & -- & $<.05$ & $<.05$ & $<.05$ & -- & $<.05$ & $<.05$ & $<.05$ & -- & $<.05$ & $<.05$ & -- & $<.05$ & $<.05$ & -- & $<.05$ & $<.05$ & \\
\hline 01309500 & $\begin{array}{l}\text { Massapequa Creek at } \\
\text { Massapequa, NY }\end{array}$ & $06 / 26 / 01$ & -- & $<.05$ & $<.05$ & -- & -- & $<.05$ & $<.05$ & -- & -- & $<.05$ & $<.05$ & -- & $<.05$ & $<.05$ & -- & $<.05$ & $<.05$ & \\
\hline \multirow[t]{2}{*}{01374654} & \multirow{2}{*}{$\begin{array}{l}\text { Middle Branch Croton } \\
\text { River near Carmel, } \\
\text { NY }\end{array}$} & $05 / 16 / 00$ & -- & $<.05$ & $<.05$ & -- & -- & $<.05$ & $<.05$ & -- & -- & $<.05$ & $<.05$ & -- & $<.05$ & $<.05$ & -- & $<.05$ & $<.05$ & \\
\hline & & $06 / 19 / 00$ & -- & $<.05$ & $<.05$ & -- & -- & $<.05$ & $<.05$ & -- & -- & $<.05$ & $<.05$ & -- & $<.05$ & $<.05$ & -- & $<.05$ & $<.05$ & \\
\hline \multirow[t]{4}{*}{01414280} & \multirow{4}{*}{$\begin{array}{l}\text { Mill Brook east of } \\
\text { Belle Ayr, NY }\end{array}$} & $12 / 07 / 00$ & -- & $<.05$ & $<.05$ & -- & -- & $<.05$ & $<.05$ & -- & -- & $<.05$ & $<.05$ & -- & $<.05$ & $<.05$ & -- & $<.05$ & $<.05$ & \\
\hline & & $07 / 20 / 01$ & -- & $<.05$ & $<.05$ & -- & -- & $<.05$ & $<.05$ & -- & -- & $<.05$ & $<.05$ & -- & $<.05$ & $<.05$ & -- & $<.05$ & $<.05$ & \\
\hline & & $07 / 20 / 01$ & -- & $<.05$ & $<.05$ & -- & -- & $<.05$ & $<.05$ & -- & -- & $<.05$ & $<.05$ & -- & $<.05$ & $<.05$ & -- & $<.05$ & $<.05$ & \\
\hline & & $08 / 29 / 01$ & -- & $<.05$ & $<.05$ & -- & -- & $<.05$ & $<.05$ & -- & -- & $<.05$ & $<.05$ & -- & $<.05$ & $<.05$ & -- & $<.05$ & $<.05$ & జ్ల్ల \\
\hline
\end{tabular}


Table 6. Analytical results for selected acetamide herbicides and their degradation products, ethanesulfonic acid, oxanilic acid, and sulfinyl acetic acid, in surface-water samples, 1993-2003.-Continued

[OGRL, U.S. Geological Survey Organic Geochemistry Research Laboratory, Lawrence, Kansas; ESA, ethanesulfonic acid; OXA, oxanilic acid; SAA, sulfinyl acetic acid; --, no data; <, less than]

\begin{tabular}{|c|c|c|c|c|c|c|c|c|c|c|c|c|c|c|c|c|c|c|c|}
\hline \multirow[b]{2}{*}{ Site number } & \multirow[b]{2}{*}{ Site location } & \multirow[b]{2}{*}{$\begin{array}{c}\text { Date of } \\
\text { collection } \\
\text { (month/day/ } \\
\text { year) }\end{array}$} & \multicolumn{17}{|c|}{ Concentrations, in micrograms per liter } \\
\hline & & & $\begin{array}{l}\text { Aceto- } \\
\text { chlor } \\
(\mu \mathrm{g} / \mathrm{L})\end{array}$ & $\begin{array}{l}\text { Aceto- } \\
\text { chlor } \\
\text { ESA } \\
(\mu \mathrm{g} / \mathrm{L})\end{array}$ & $\begin{array}{l}\text { Aceto- } \\
\text { chlor } \\
\text { OXA } \\
(\mu \mathrm{g} / \mathrm{L})\end{array}$ & $\begin{array}{l}\text { Aceto- } \\
\text { chlor } \\
\text { SAA } \\
(\mu \mathrm{g} / \mathrm{L})\end{array}$ & $\begin{array}{l}\text { Ala- } \\
\text { chlor } \\
(\mu \mathrm{g} / \mathrm{L})\end{array}$ & $\begin{array}{l}\text { Ala- } \\
\text { chlor } \\
\text { ESA } \\
(\mu \mathrm{g} / \mathrm{L})\end{array}$ & $\begin{array}{l}\text { Ala- } \\
\text { chlor } \\
\text { OXA } \\
(\mu \mathrm{g} / \mathrm{L})\end{array}$ & $\begin{array}{l}\text { Ala- } \\
\text { chlor } \\
\text { SAA } \\
(\mu \mathrm{g} / \mathrm{L})\end{array}$ & $\begin{array}{c}\text { Di- } \\
\text { meth- } \\
\text { enamid } \\
(\mu \mathrm{g} / \mathrm{L})\end{array}$ & $\begin{array}{c}\text { Di- } \\
\text { meth- } \\
\text { enamid } \\
\text { ESA } \\
(\mu \mathrm{g} / \mathrm{L})\end{array}$ & $\begin{array}{c}\text { Di- } \\
\text { meth- } \\
\text { enamid } \\
0 X A \\
(\mu \mathrm{g} / \mathrm{L})\end{array}$ & $\begin{array}{l}\text { Flufen- } \\
\text { acet } \\
(\mu \mathrm{g} / \mathrm{L})\end{array}$ & $\begin{array}{l}\text { Flufen- } \\
\text { acet } \\
\text { ESA } \\
(\mu \mathrm{g} / \mathrm{L})\end{array}$ & $\begin{array}{l}\text { Flufen- } \\
\text { acet } \\
\text { OXA } \\
(\mu \mathrm{g} / \mathrm{L})\end{array}$ & $\begin{array}{l}\text { Meto- } \\
\text { lachlor } \\
(\mu \mathrm{g} / \mathrm{L})\end{array}$ & $\begin{array}{l}\text { Meto- } \\
\text { lachlor } \\
\text { ESA } \\
(\mu \mathrm{g} / \mathrm{L})\end{array}$ & $\begin{array}{c}\text { Meto- } \\
\text { lachlor } \\
\text { OXA } \\
(\mu \mathrm{g} / \mathrm{L})\end{array}$ \\
\hline \multicolumn{20}{|c|}{ Federal-State Cooperative Studies-Continued } \\
\hline \multicolumn{20}{|c|}{ New York hydrologic study (PPN)—Continued } \\
\hline \multirow[t]{3}{*}{01414280} & \multirow{3}{*}{$\begin{array}{l}\text { Mill Brook east of } \\
\text { Belle Ayr, NY }\end{array}$} & $10 / 03 / 01$ & -- & $<0.05$ & $<0.05$ & -- & -- & $<0.05$ & $<0.05$ & -- & -- & $<0.05$ & $<0.05$ & -- & $<0.05$ & $<0.05$ & -- & $<0.05$ & $<0.05$ \\
\hline & & $10 / 22 / 01$ & -- & $<.05$ & $<.05$ & -- & -- & $<.05$ & $<.05$ & -- & -- & $<.05$ & $<.05$ & -- & $<.05$ & $<.05$ & -- & $<.05$ & $<.05$ \\
\hline & & $11 / 15 / 01$ & -- & $<.05$ & $<.05$ & $<0.05$ & -- & $<.05$ & $<.05$ & $<0.05$ & -- & $<.05$ & $<.05$ & -- & $<.05$ & $<.05$ & -- & $<.05$ & $<.05$ \\
\hline \multirow[t]{4}{*}{01414500} & \multirow{4}{*}{$\begin{array}{r}\text { Mill Brook near } \\
\text { Dunraven, NY }\end{array}$} & 07/10/01 & -- & $<.05$ & $<.05$ & -- & -- & $<.05$ & $<.05$ & -- & -- & $<.05$ & $<.05$ & -- & $<.05$ & $<.05$ & -- & .08 & $<.05$ \\
\hline & & $07 / 13 / 01$ & -- & $<.05$ & $<.05$ & -- & -- & $<.05$ & $<.05$ & -- & -- & $<.05$ & $<.05$ & -- & $<.05$ & $<.05$ & -- & .31 & $<.05$ \\
\hline & & $07 / 16 / 01$ & -- & $<.05$ & $<.05$ & -- & -- & $<.05$ & $<.05$ & -- & -- & $<.05$ & $<.05$ & -- & $<.05$ & $<.05$ & -- & .08 & $<.05$ \\
\hline & & $07 / 17 / 01$ & -- & $<.05$ & $<.05$ & -- & -- & $<.05$ & $<.05$ & -- & -- & $<.05$ & $<.05$ & -- & $<.05$ & $<.05$ & -- & $<.05$ & $<.05$ \\
\hline \multirow[t]{2}{*}{01414290} & \multirow{2}{*}{$\begin{array}{l}\text { Mill Brook tributary } \\
\text { west of Belle Ayr, } \\
\text { NY }\end{array}$} & $12 / 07 / 00$ & -- & $<.05$ & $<.05$ & -- & -- & $<.05$ & $<.05$ & -- & -- & $<.05$ & $<.05$ & -- & $<.05$ & $<.05$ & -- & $<.05$ & $<.05$ \\
\hline & & $07 / 20 / 01$ & -- & $<.05$ & $<.05$ & -- & -- & $<.05$ & $<.05$ & -- & -- & $<.05$ & $<.05$ & -- & $<.05$ & $<.05$ & -- & $<.05$ & $<.05$ \\
\hline \multirow[t]{15}{*}{01357500} & \multirow{15}{*}{$\begin{array}{l}\text { Mohawk River at } \\
\text { Cohoes, NY }\end{array}$} & $04 / 24 / 97$ & $<0.05$ & $<.20$ & $<.20$ & -- & $<0.05$ & $<.20$ & $<.20$ & -- & -- & -- & -- & -- & -- & -- & $<0.05$ & $<.20$ & $<.20$ \\
\hline & & $05 / 22 / 97$ & $<.05$ & $<.20$ & $<.20$ & -- & $<.05$ & $<.20$ & $<.20$ & -- & -- & -- & -- & -- & -- & -- & $<.05$ & $<.20$ & $<.20$ \\
\hline & & $05 / 24 / 97$ & $<.05$ & $<.20$ & $<.20$ & -- & $<.05$ & $<.20$ & $<.20$ & -- & -- & -- & -- & -- & -- & -- & $<.05$ & $<.20$ & $<.20$ \\
\hline & & $05 / 28 / 97$ & $<.05$ & $<.20$ & $<.20$ & -- & $<.05$ & $<.20$ & $<.20$ & -- & -- & -- & -- & -- & -- & -- & $<.05$ & $<.20$ & $<.20$ \\
\hline & & $06 / 04 / 97$ & $<.05$ & $<.20$ & $<.20$ & -- & $<.05$ & $<.20$ & $<.20$ & -- & -- & -- & -- & -- & -- & -- & $<.05$ & $<.20$ & $<.20$ \\
\hline & & $06 / 11 / 97$ & $<.05$ & $<.20$ & $<.20$ & -- & $<.05$ & $<.20$ & $<.20$ & -- & -- & -- & -- & -- & -- & -- & $<.05$ & $<.20$ & $<.20$ \\
\hline & & $06 / 16 / 97$ & $<.05$ & $<.20$ & $<.20$ & -- & $<.05$ & $<.20$ & $<.20$ & -- & -- & -- & -- & -- & -- & -- & $<.05$ & $<.20$ & $<.20$ \\
\hline & & $06 / 18 / 97$ & $<.05$ & $<.20$ & $<.20$ & -- & $<.05$ & $<.20$ & $<.20$ & -- & -- & -- & -- & -- & -- & -- & $<.05$ & $<.20$ & $<.20$ \\
\hline & & $06 / 25 / 97$ & $<.05$ & $<.20$ & $<.20$ & -- & $<.05$ & $<.20$ & $<.20$ & -- & -- & -- & -- & -- & -- & -- & $<.05$ & $<.20$ & $<.20$ \\
\hline & & $07 / 02 / 97$ & $<.05$ & $<.20$ & $<.20$ & -- & $<.05$ & $<.20$ & $<.20$ & -- & -- & -- & -- & -- & -- & -- & $<.05$ & $<.20$ & $<.20$ \\
\hline & & $07 / 10 / 97$ & $<.05$ & $<.20$ & $<.20$ & -- & $<.05$ & $<.20$ & $<.20$ & -- & -- & -- & -- & -- & -- & -- & $<.05$ & $<.20$ & $<.20$ \\
\hline & & $07 / 17 / 97$ & $<.05$ & $<.20$ & $<.20$ & -- & $<.05$ & $<.20$ & $<.20$ & -- & -- & -- & -- & -- & -- & -- & $<.05$ & $<.20$ & $<.20$ \\
\hline & & $07 / 24 / 97$ & $<.05$ & $<.20$ & $<.20$ & -- & $<.05$ & $<.20$ & $<.20$ & -- & -- & -- & -- & -- & -- & -- & $<.05$ & $<.20$ & $<.20$ \\
\hline & & $08 / 20 / 97$ & $<.05$ & $<.20$ & $<.20$ & -- & $<.05$ & $<.20$ & $<.20$ & -- & -- & -- & -- & -- & -- & -- & $<.05$ & $<.20$ & $<.20$ \\
\hline & & 09/03/97 & $<.05$ & $<.20$ & $<.20$ & -- & $<.05$ & $<.20$ & $<.20$ & -- & -- & -- & -- & -- & -- & -- & $<.05$ & $<.20$ & $<.20$ \\
\hline
\end{tabular}


Table 6. Analytical results for selected acetamide herbicides and their degradation products, ethanesulfonic acid, oxanilic acid, and sulfinyl acetic acid, in surface-water samples, 1993-2003.-Continued

[OGRL, U.S. Geological Survey Organic Geochemistry Research Laboratory, Lawrence, Kansas; ESA, ethanesulfonic acid; OXA, oxanilic acid; SAA, sulfinyl acetic acid; --, no data; <, less than]

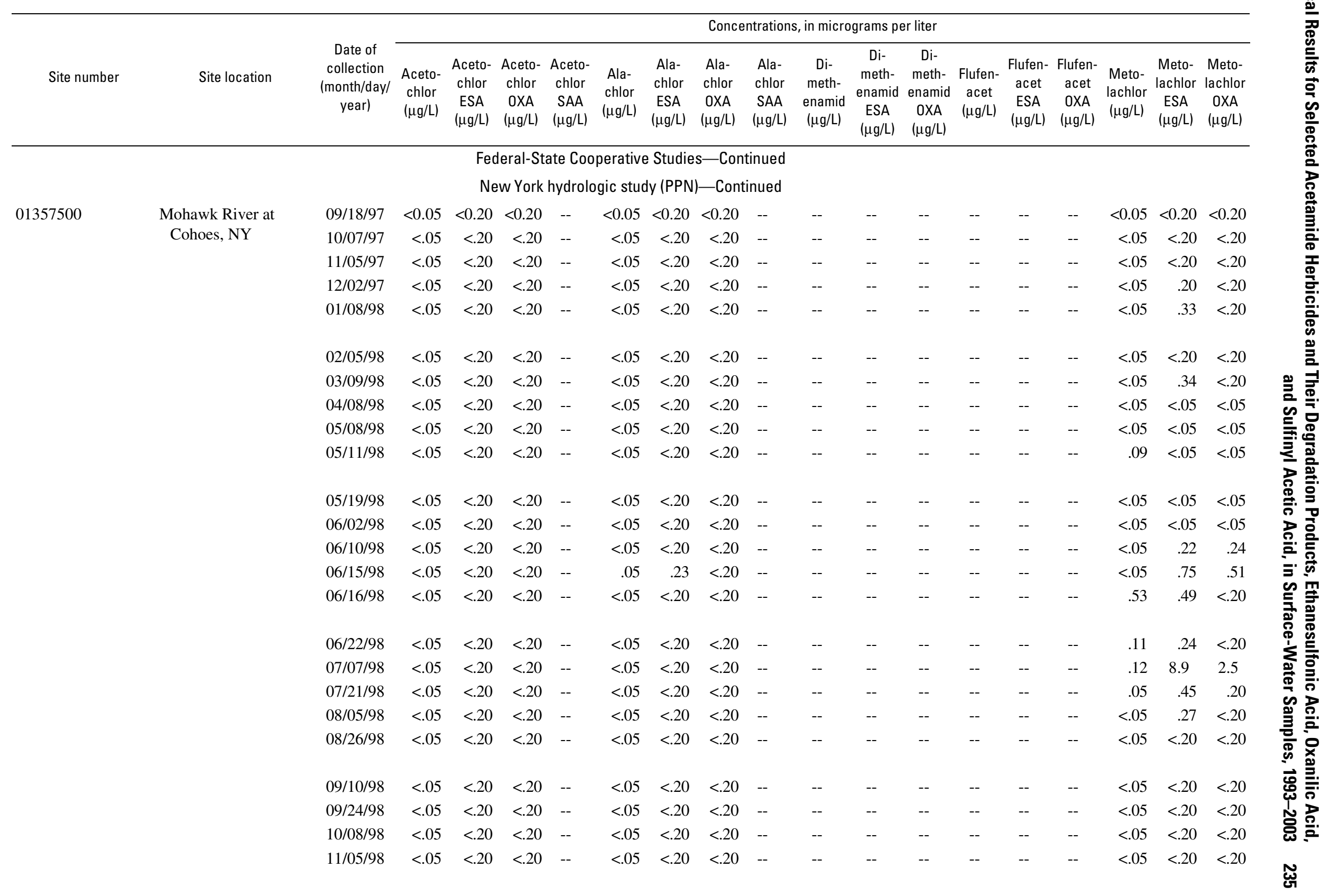


Table 6. Analytical results for selected acetamide herbicides and their degradation products, ethanesulfonic acid, oxanilic acid, and sulfinyl acetic acid, in surface-water samples, 1993-2003.-Continued

[OGRL, U.S. Geological Survey Organic Geochemistry Research Laboratory, Lawrence, Kansas; ESA, ethanesulfonic acid; OXA, oxanilic acid; SAA, sulfinyl acetic acid; --, no data; <, less than]

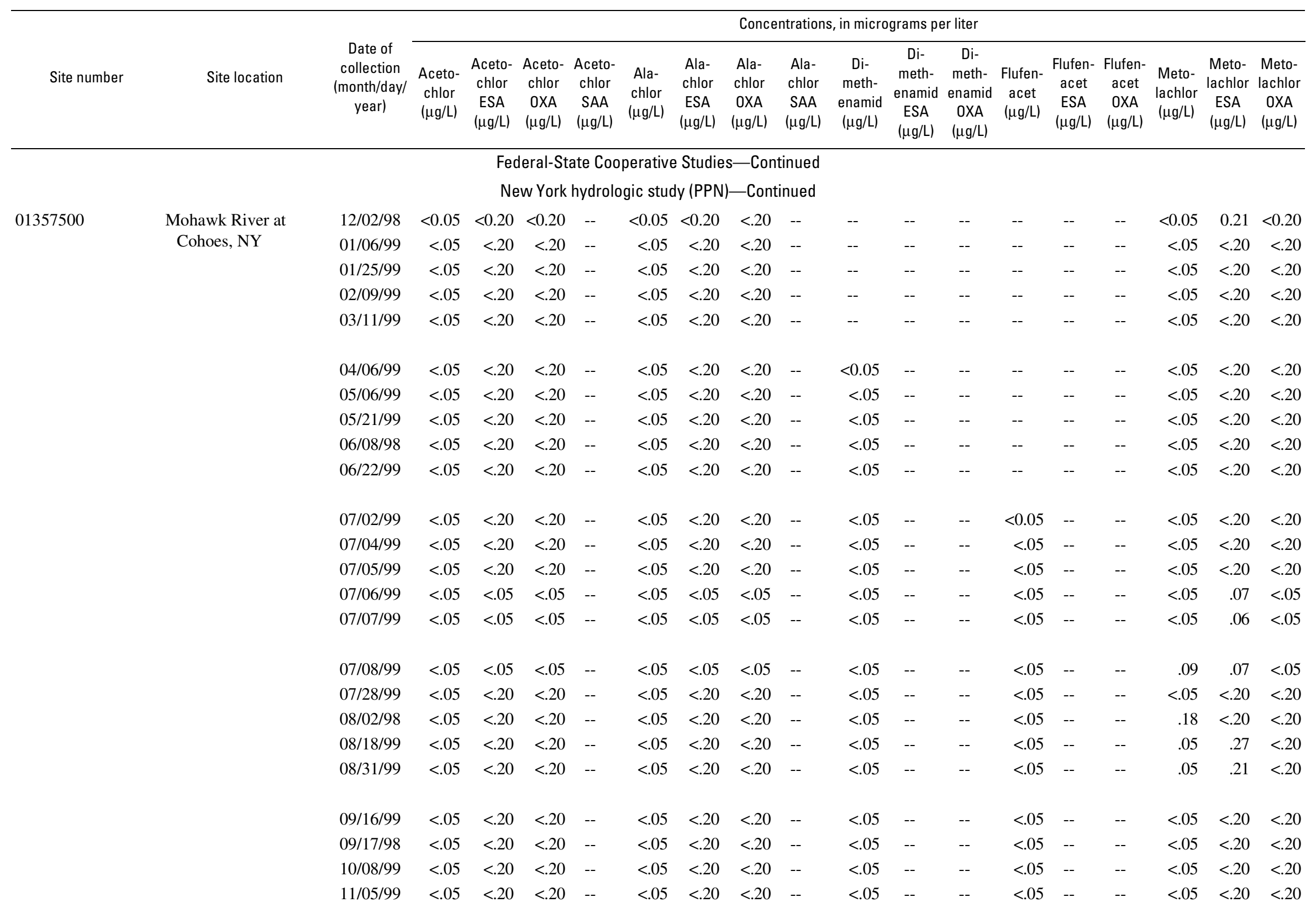


Table 6. Analytical results for selected acetamide herbicides and their degradation products, ethanesulfonic acid, oxanilic acid, and sulfinyl acetic acid, in surface-water samples, 1993-2003.-Continued

[OGRL, U.S. Geological Survey Organic Geochemistry Research Laboratory, Lawrence, Kansas; ESA, ethanesulfonic acid; OXA, oxanilic acid; SAA, sulfinyl acetic acid; --, no data; <, less than]

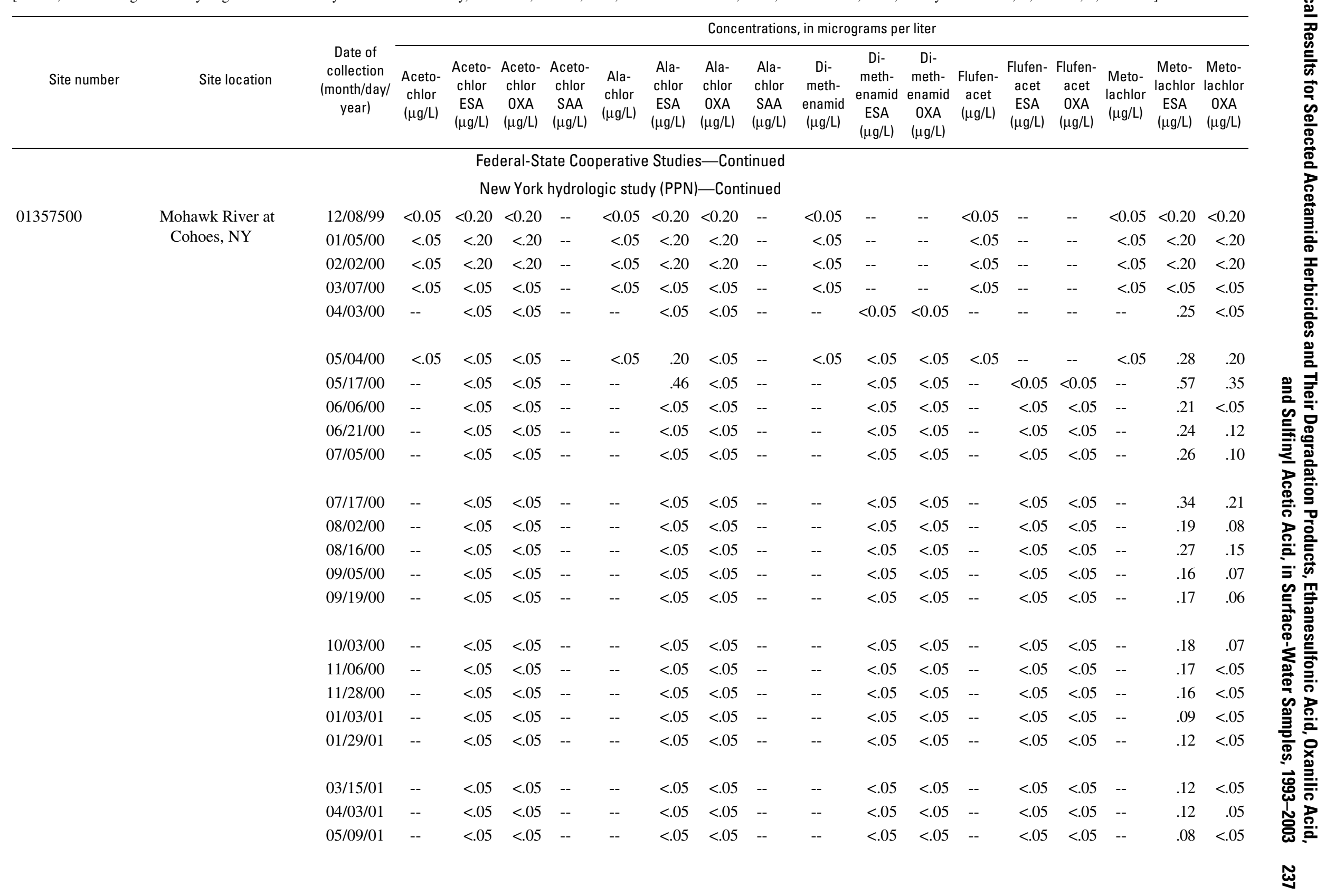


Table 6. Analytical results for selected acetamide herbicides and their degradation products, ethanesulfonic acid, oxanilic acid, and sulfinyl acetic acid, in surface-water samples, 1993-2003.-Continued

[OGRL, U.S. Geological Survey Organic Geochemistry Research Laboratory, Lawrence, Kansas; ESA, ethanesulfonic acid; OXA, oxanilic acid; SAA, sulfinyl acetic acid; --, no data; <, less than]

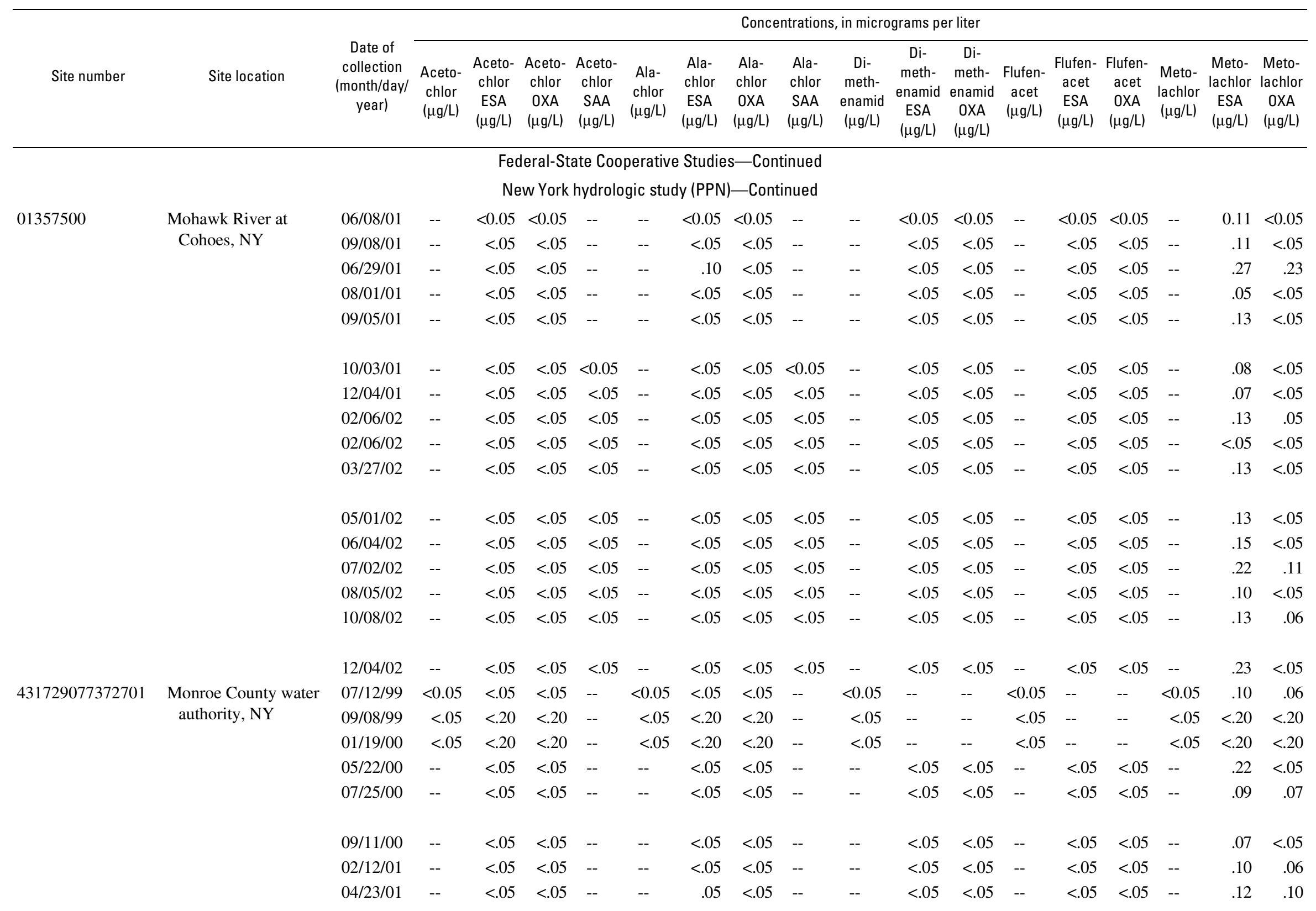


Table 6. Analytical results for selected acetamide herbicides and their degradation products, ethanesulfonic acid, oxanilic acid, and sulfinyl acetic acid, in surface-water samples, 1993-2003.-Continued

[OGRL, U.S. Geological Survey Organic Geochemistry Research Laboratory, Lawrence, Kansas; ESA, ethanesulfonic acid; OXA, oxanilic acid; SAA, sulfinyl acetic acid; --, no data; <, less than]

\begin{tabular}{|c|c|c|c|c|c|c|c|c|c|c|c|c|c|c|c|c|c|c|c|c|}
\hline \multirow[b]{2}{*}{ Site number } & \multirow[b]{2}{*}{ Site location } & \multirow[b]{2}{*}{$\begin{array}{c}\text { Date of } \\
\text { collection } \\
\text { (month/day/ } \\
\text { year) }\end{array}$} & \multicolumn{17}{|c|}{ Concentrations, in micrograms per liter } & \\
\hline & & & $\begin{array}{c} \\
\text { Aceto- } \\
\text { chlor } \\
(\mu \mathrm{g} / \mathrm{L})\end{array}$ & $\begin{array}{l}\text { Aceto- } \\
\text { chlor } \\
\text { ESA } \\
(\mu \mathrm{g} / \mathrm{L})\end{array}$ & $\begin{array}{l}\text { Aceto- } \\
\text { chlor } \\
\text { OXA } \\
(\mu \mathrm{g} / \mathrm{L})\end{array}$ & $\begin{array}{c}\text { Aceto- } \\
\text { chlor } \\
\text { SAA } \\
(\mu \mathrm{g} / \mathrm{L})\end{array}$ & $\begin{array}{l}\text { Ala- } \\
\text { chlor } \\
(\mu \mathrm{g} / \mathrm{L})\end{array}$ & $\begin{array}{l}\text { Ala- } \\
\text { chlor } \\
\text { ESA } \\
(\mu \mathrm{g} / \mathrm{L})\end{array}$ & $\begin{array}{c}\text { Ala- } \\
\text { chlor } \\
\text { OXA } \\
(\mu \mathrm{g} / \mathrm{L})\end{array}$ & $\begin{array}{l}\text { Ala- } \\
\text { chlor } \\
\text { SAA } \\
(\mu \mathrm{g} / \mathrm{L})\end{array}$ & $\begin{array}{c}\text { Di- } \\
\text { meth- } \\
\text { enamid } \\
(\mu \mathrm{g} / \mathrm{L})\end{array}$ & $\begin{array}{c}\text { Di- } \\
\text { meth- } \\
\text { enamid } \\
\text { ESA } \\
(\mu \mathrm{g} / \mathrm{L})\end{array}$ & $\begin{array}{c}\text { Di- } \\
\text { meth- } \\
\text { enamid } \\
\text { OXA } \\
(\mu \mathrm{g} / \mathrm{L})\end{array}$ & $\begin{array}{c}\text { Flufen- } \\
\text { acet } \\
(\mu \mathrm{g} / \mathrm{L})\end{array}$ & $\begin{array}{c}\text { Flufen- } \\
\text { acet } \\
\text { ESA } \\
(\mu \mathrm{g} / \mathrm{L})\end{array}$ & $\begin{array}{c}\text { Flufen- } \\
\text { acet } \\
\text { OXA } \\
(\mu \mathrm{g} / \mathrm{L})\end{array}$ & $\begin{array}{l}\text { Meto- } \\
\text { lachlor } \\
(\mu \mathrm{g} / \mathrm{L})\end{array}$ & $\begin{array}{c}\text { Meto- } \\
\text { lachlor } \\
\text { ESA } \\
(\mu \mathrm{g} / \mathrm{L})\end{array}$ & $\begin{array}{c}\text { Meto- } \\
\text { lachlor } \\
\text { OXA } \\
(\mu \mathrm{g} / \mathrm{L})\end{array}$ & 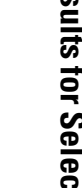 \\
\hline \multicolumn{20}{|c|}{ Federal-State Cooperative Studies-Continued } & \\
\hline \multicolumn{20}{|c|}{ New York hydrologic study (PPN)_Continued } & \\
\hline \multirow[t]{6}{*}{431729077372701} & \multirow{6}{*}{$\begin{array}{l}\text { Monroe County water } \\
\text { authority, NY }\end{array}$} & $05 / 21 / 01$ & -- & $<0.05$ & $<0.05$ & -- & -- & $<0.05$ & $<0.05$ & -- & -- & $<0.05$ & $<0.05$ & -- & $<0.05$ & $<0.05$ & -- & 0.10 & 0.07 & \\
\hline & & 06/18/01 & -- & $<.05$ & $<.05$ & -- & -- & $<.05$ & $<.05$ & -- & -- & $<.05$ & $<.05$ & -- & $<.05$ & $<.05$ & -- & .08 & $<.05$ & \\
\hline & & $07 / 30 / 01$ & -- & $<.05$ & $<.05$ & -- & -- & $<.05$ & $<.05$ & -- & -- & $<.05$ & $<.05$ & -- & $<.05$ & $<.05$ & -- & $<.05$ & $<.05$ & \\
\hline & & $10 / 29 / 01$ & -- & $<.05$ & $<.05$ & -- & -- & $<.05$ & $<.05$ & -- & -- & $<.05$ & $<.05$ & -- & $<.05$ & $<.05$ & -- & .09 & .06 & \\
\hline & & $01 / 28 / 02$ & -- & $<.05$ & $<.05$ & $<0.05$ & -- & $<.05$ & $<.05$ & $<0.05$ & -- & $<.05$ & $<.05$ & -- & $<.05$ & $<.05$ & -- & .12 & .06 & $\frac{1}{8}$ \\
\hline & & 05/07/02 & -- & $<.05$ & $<.05$ & $<.05$ & -- & $<.05$ & $<.05$ & $<.05$ & -- & $<.05$ & $<.05$ & -- & $<.05$ & $<.05$ & -- & .14 & .09 & $\bar{z}$ \\
\hline \multirow[t]{12}{*}{01435800} & \multirow{12}{*}{$\begin{array}{l}\text { Neversink Reservoir, } \\
\text { NY }\end{array}$} & $01 / 25 / 99$ & $<0.05$ & $<.20$ & $<.20$ & -- & $<0.05$ & $<.20$ & $<.20$ & -- & -- & -- & -- & -- & -- & -- & $<0.05$ & $<.20$ & $<.20$ & \multirow{12}{*}{ 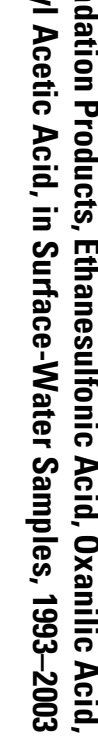 } \\
\hline & & $05 / 24 / 99$ & $<.05$ & $<.20$ & $<.20$ & -- & $<.05$ & $<.20$ & $<.20$ & -- & $<0.05$ & -- & -- & -- & -- & -- & $<.05$ & $<.20$ & $<.20$ & \\
\hline & & 07/12/99 & $<.05$ & $<.05$ & $<.05$ & -- & $<.05$ & $<.05$ & $<.05$ & -- & $<.05$ & -- & -- & $<0.05$ & -- & -- & $<.05$ & .18 & $<.05$ & \\
\hline & & 09/13/99 & $<.05$ & $<.20$ & $<.20$ & -- & $<.05$ & $<.20$ & $<.20$ & -- & $<.05$ & -- & -- & $<.05$ & -- & -- & $<.05$ & $<.20$ & $<.20$ & \\
\hline & & 01/10/00 & $<.05$ & $<.20$ & $<.20$ & -- & $<.05$ & $<.20$ & $<.20$ & -- & $<.05$ & -- & -- & $<.05$ & -- & -- & $<.05$ & $<.20$ & $<.20$ & \\
\hline & & $01 / 15 / 00$ & -- & $<.05$ & $<.05$ & -- & -- & $<.05$ & $<.05$ & -- & -- & $<.05$ & $<.05$ & -- & -- & -- & -- & $<.05$ & $<.05$ & \\
\hline & & $07 / 24 / 00$ & -- & $<.05$ & $<.05$ & -- & -- & $<.05$ & $<.05$ & -- & -- & $<.05$ & $<.05$ & -- & $<.05$ & $<.05$ & -- & $<.05$ & $<.05$ & \\
\hline & & 09/11/00 & -- & $<.05$ & $<.05$ & -- & -- & $<.05$ & $<.05$ & -- & -- & $<.05$ & $<.05$ & -- & $<.05$ & $<.05$ & -- & $<.05$ & $<.05$ & \\
\hline & & $04 / 23 / 01$ & -- & $<.05$ & $<.05$ & -- & -- & $<.05$ & $<.05$ & -- & -- & $<.05$ & $<.05$ & -- & $<.05$ & $<.05$ & -- & $<.05$ & $<.05$ & \\
\hline & & $07 / 30 / 01$ & -- & $<.05$ & $<.05$ & -- & -- & $<.05$ & $<.05$ & -- & -- & $<.05$ & $<.05$ & -- & $<.05$ & $<.05$ & -- & $<.05$ & $<.05$ & \\
\hline & & $07 / 22 / 02$ & -- & $<.05$ & $<.05$ & $<.05$ & -- & $<.05$ & $<.05$ & $<.05$ & -- & $<.05$ & $<.05$ & -- & $<.05$ & $<.05$ & -- & $<.05$ & $<.05$ & \\
\hline & & $10 / 28 / 02$ & -- & $<.05$ & $<.05$ & $<.05$ & -- & $<.05$ & $<.05$ & $<.05$ & -- & $<.05$ & $<.05$ & -- & $<.05$ & $<.05$ & -- & $<.05$ & $<.05$ & \\
\hline
\end{tabular}


Table 6. Analytical results for selected acetamide herbicides and their degradation products, ethanesulfonic acid, oxanilic acid, and sulfinyl acetic acid, in surface-water samples, 1993-2003.-Continued

[OGRL, U.S. Geological Survey Organic Geochemistry Research Laboratory, Lawrence, Kansas; ESA, ethanesulfonic acid; OXA, oxanilic acid; SAA, sulfinyl acetic acid; --, no data; <, less than]

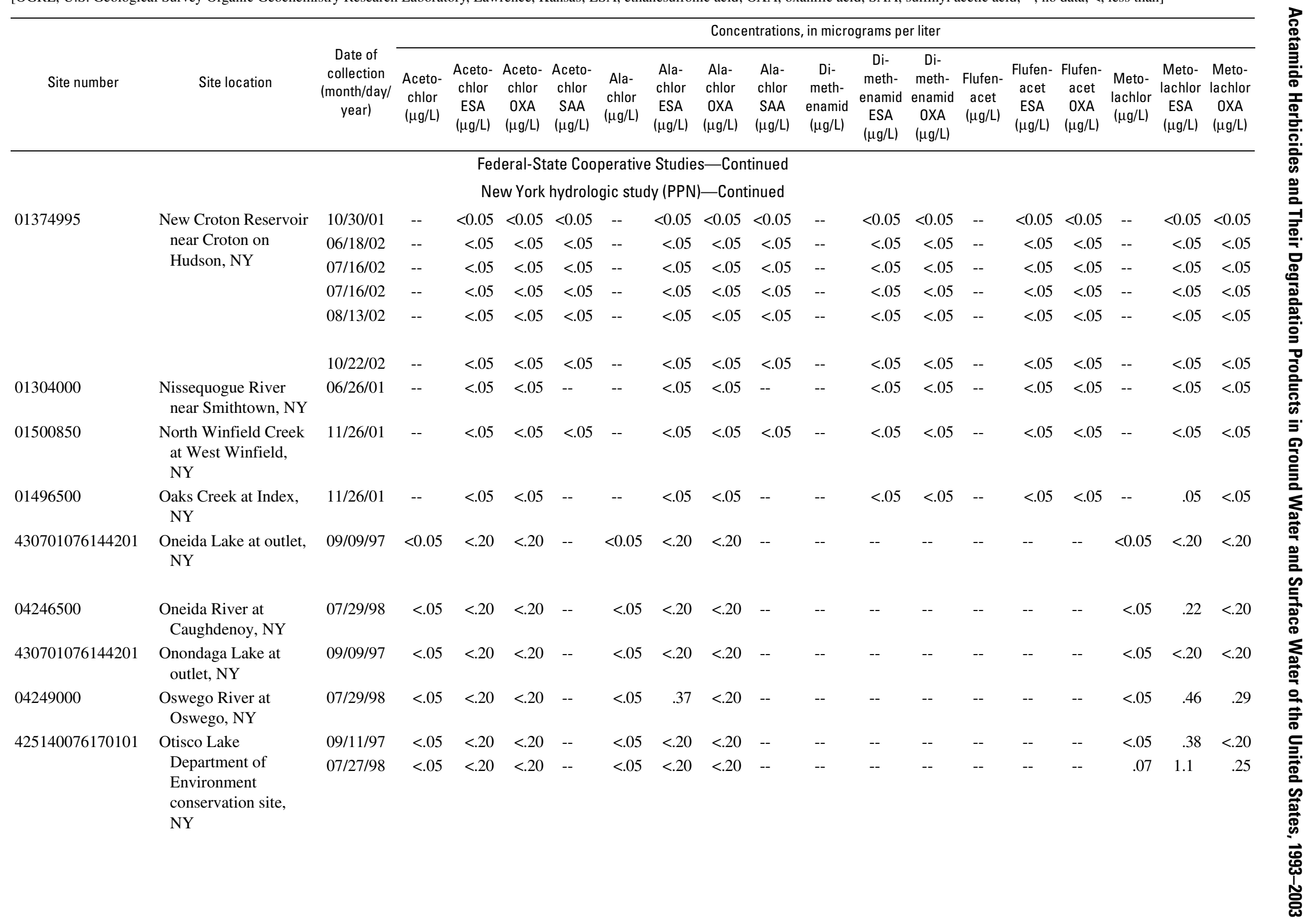


Table 6. Analytical results for selected acetamide herbicides and their degradation products, ethanesulfonic acid, oxanilic acid, and sulfinyl acetic acid, in surface-water samples, 1993-2003.-Continued

[OGRL, U.S. Geological Survey Organic Geochemistry Research Laboratory, Lawrence, Kansas; ESA, ethanesulfonic acid; OXA, oxanilic acid; SAA, sulfinyl acetic acid; --, no data; <, less than]

\begin{tabular}{|c|c|c|c|c|c|c|c|c|c|c|c|c|c|c|c|c|c|c|c|}
\hline \multirow[b]{2}{*}{ Site number } & \multirow[b]{2}{*}{ Site location } & \multirow[b]{2}{*}{$\begin{array}{c}\text { Date of } \\
\text { collection } \\
\text { (month/day/ } \\
\text { year) }\end{array}$} & \multicolumn{17}{|c|}{ Concentrations, in micrograms per liter } \\
\hline & & & $\begin{array}{l}\text { Aceto- } \\
\text { chlor } \\
(\mu \mathrm{g} / \mathrm{L})\end{array}$ & $\begin{array}{l}\text { Aceto- } \\
\text { chlor } \\
\text { ESA } \\
(\mu \mathrm{g} / \mathrm{L})\end{array}$ & $\begin{array}{l}\text { Aceto- } \\
\text { chlor } \\
\text { OXA } \\
(\mu \mathrm{g} / \mathrm{L})\end{array}$ & $\begin{array}{l}\text { Aceto- } \\
\text { chlor } \\
\text { SAA } \\
(\mu \mathrm{g} / \mathrm{L})\end{array}$ & $\begin{array}{l}\text { Ala- } \\
\text { chlor } \\
(\mu \mathrm{g} / \mathrm{L})\end{array}$ & $\begin{array}{l}\text { Ala- } \\
\text { chlor } \\
\text { ESA } \\
(\mu \mathrm{g} / \mathrm{L})\end{array}$ & $\begin{array}{l}\text { Ala- } \\
\text { chlor } \\
\text { OXA } \\
(\mu \mathrm{g} / \mathrm{L})\end{array}$ & $\begin{array}{l}\text { Ala- } \\
\text { chlor } \\
\text { SAA } \\
(\mu \mathrm{g} / \mathrm{L})\end{array}$ & $\begin{array}{l}\text { Di- } \\
\text { meth- } \\
\text { enamid } \\
(\mu \mathrm{g} / \mathrm{L})\end{array}$ & $\begin{array}{c}\text { Di- } \\
\text { meth- } \\
\text { enamid } \\
\text { ESA } \\
(\mu \mathrm{g} / \mathrm{L})\end{array}$ & $\begin{array}{c}\text { Di- } \\
\text { meth- } \\
\text { enamid } \\
\text { OXA } \\
(\mu \mathrm{g} / \mathrm{L})\end{array}$ & $\begin{array}{c}\text { Flufen- } \\
\text { acet } \\
(\mu \mathrm{g} / \mathrm{L})\end{array}$ & $\begin{array}{l}\text { Flufen- } \\
\text { acet } \\
\text { ESA } \\
(\mu \mathrm{g} / \mathrm{L})\end{array}$ & $\begin{array}{l}\text { Flufen- } \\
\text { acet } \\
\text { OXA } \\
(\mu \mathrm{g} / \mathrm{L})\end{array}$ & $\begin{array}{l}\text { Meto- } \\
\text { lachlor } \\
(\mu \mathrm{g} / \mathrm{L})\end{array}$ & $\begin{array}{l}\text { Meto- } \\
\text { lachlor } \\
\text { ESA } \\
(\mu \mathrm{g} / \mathrm{L})\end{array}$ & $\begin{array}{l}\text { Meto- } \\
\text { lachlor } \\
\text { OXA } \\
(\mu \mathrm{g} / \mathrm{L})\end{array}$ \\
\hline \multicolumn{20}{|c|}{ Federal-State Cooperative Studies-Continued } \\
\hline \multicolumn{20}{|c|}{ New York hydrologic study (PPN)_Continued } \\
\hline \multirow[t]{2}{*}{425232076314001} & Owasco Lake & 09/11/97 & $<0.05$ & $<0.20$ & $<0.20$ & -- & $<0.05$ & 0.21 & $<0.20$ & -- & -- & -- & -- & -- & -- & -- & $<0.05$ & 0.31 & $<0.20$ \\
\hline & $\begin{array}{l}\text { Department of } \\
\text { Environment } \\
\text { conservation site, } \\
\text { NY }\end{array}$ & 07/27/98 & $<.05$ & $<.20$ & $<.20$ & -- & $<.05$ & $<.20$ & $<.20$ & -- & -- & -- & -- & -- & -- & -- & .06 & .77 & .23 \\
\hline \multirow[t]{3}{*}{04234044} & Paines Creek near & 06/11/98 & $<.05$ & $<.20$ & $<.20$ & -- & $<.05$ & .20 & $<.20$ & -- & -- & -- & -- & -- & -- & -- & 1.3 & 5.3 & .70 \\
\hline & Aurora, NY & 06/17/98 & $<.05$ & $<.20$ & $<.20$ & -- & $<.05$ & .38 & $<.20$ & -- & -- & -- & -- & -- & -- & -- & .93 & 5.1 & 1.5 \\
\hline & & 06/18/98 & $<.05$ & $<.20$ & $<.20$ & -- & .05 & $<.05$ & $<.05$ & -- & -- & -- & -- & -- & -- & -- & 5.4 & 16 & 9.5 \\
\hline \multirow[t]{15}{*}{01415200} & Pepacton Reservoir, & $01 / 25 / 99$ & $<.05$ & $<.20$ & $<.20$ & -- & $<.05$ & $<.20$ & $<.20$ & -- & -- & -- & -- & -- & -- & -- & $<.05$ & $<.20$ & $<.20$ \\
\hline & NY & $05 / 24 / 99$ & $<.05$ & $<.20$ & $<.20$ & -- & $<.05$ & $<.20$ & $<.20$ & -- & $<0.05$ & -- & -- & -- & -- & -- & $<.05$ & $<.20$ & $<.20$ \\
\hline & & 07/12/99 & $<.05$ & $<.05$ & $<.05$ & -- & $<.05$ & $<.05$ & $<.05$ & -- & $<.05$ & -- & -- & $<0.05$ & -- & -- & $<.05$ & $<.05$ & $<.05$ \\
\hline & & 09/13/99 & $<.05$ & $<.20$ & $<.20$ & -- & $<.05$ & $<.20$ & $<.20$ & -- & $<.05$ & -- & -- & $<.05$ & -- & -- & $<.05$ & $<.20$ & $<.20$ \\
\hline & & 01/10/00 & $<.05$ & $<.20$ & $<.20$ & -- & $<.05$ & $<.20$ & $<.20$ & -- & $<.05$ & -- & -- & $<.05$ & -- & -- & $<.05$ & $<.20$ & $<.20$ \\
\hline & & $05 / 15 / 00$ & -- & $<.05$ & $<.05$ & -- & -- & $<.05$ & $<.05$ & -- & -- & $<0.05$ & $<0.05$ & -- & -- & -- & -- & .24 & $<.05$ \\
\hline & & $07 / 24 / 00$ & -- & $<.05$ & $<.05$ & -- & -- & $<.05$ & $<.05$ & -- & -- & $<.05$ & $<.05$ & -- & $<0.05$ & $<0.05$ & -- & $<.05$ & $<.05$ \\
\hline & & $09 / 11 / 00$ & -- & $<.05$ & $<.05$ & -- & -- & $<.05$ & $<.05$ & -- & -- & $<.05$ & $<.05$ & -- & $<.05$ & $<.05$ & -- & $<.05$ & $<.05$ \\
\hline & & 02/12/01 & -- & $<.05$ & $<.05$ & -- & -- & $<.05$ & $<.05$ & -- & -- & $<.05$ & $<.05$ & -- & $<.05$ & $<.05$ & -- & $<.05$ & $<.05$ \\
\hline & & $04 / 23 / 01$ & -- & $<.05$ & $<.05$ & -- & -- & $<.05$ & $<.05$ & -- & -- & $<.05$ & $<.05$ & -- & $<.05$ & $<.05$ & -- & $<.05$ & $<.05$ \\
\hline & & 07/30/01 & -- & $<.05$ & $<.05$ & -- & -- & $<.05$ & $<.05$ & -- & -- & $<.05$ & $<.05$ & -- & $<.05$ & $<.05$ & -- & $<.05$ & $<.05$ \\
\hline & & $10 / 29 / 01$ & -- & $<.05$ & $<.05$ & -- & -- & $<.05$ & $<.05$ & -- & -- & $<.05$ & $<.05$ & -- & $<.05$ & $<.05$ & -- & $<.05$ & $<.05$ \\
\hline & & 05/13/02 & -- & $<.05$ & $<.05$ & $<0.05$ & -- & $<.05$ & $<.05$ & $<0.05$ & -- & $<.05$ & $<.05$ & -- & $<.05$ & $<.05$ & -- & $<.05$ & $<.05$ \\
\hline & & $07 / 22 / 02$ & -- & $<.05$ & $<.05$ & $<.05$ & -- & $<.05$ & $<.05$ & $<.05$ & -- & $<.05$ & $<.05$ & -- & $<.05$ & $<.05$ & -- & $<.05$ & $<.05$ \\
\hline & & $10 / 28 / 02$ & -- & $<.05$ & $<.05$ & $<.05$ & -- & $<.05$ & $<.05$ & $<.05$ & -- & $<.05$ & $<.05$ & -- & $<.05$ & $<.05$ & -- & .05 & $<.05$ \\
\hline 01416895 & $\begin{array}{l}\text { Pepacton Reservoir } \\
\text { west end tributary } \\
\text { near Downsville, NY }\end{array}$ & 07/19/01 & -- & $<.05$ & $<.05$ & -- & -- & $<.05$ & $<.05$ & -- & -- & $<.05$ & $<.05$ & -- & $<.05$ & $<.05$ & -- & $<.05$ & $<.20$ \\
\hline
\end{tabular}


Table 6. Analytical results for selected acetamide herbicides and their degradation products, ethanesulfonic acid, oxanilic acid, and sulfinyl acetic acid, in surface-water samples, 1993-2003.-Continued

[OGRL, U.S. Geological Survey Organic Geochemistry Research Laboratory, Lawrence, Kansas; ESA, ethanesulfonic acid; OXA, oxanilic acid; SAA, sulfinyl acetic acid; --, no data; <, less than]

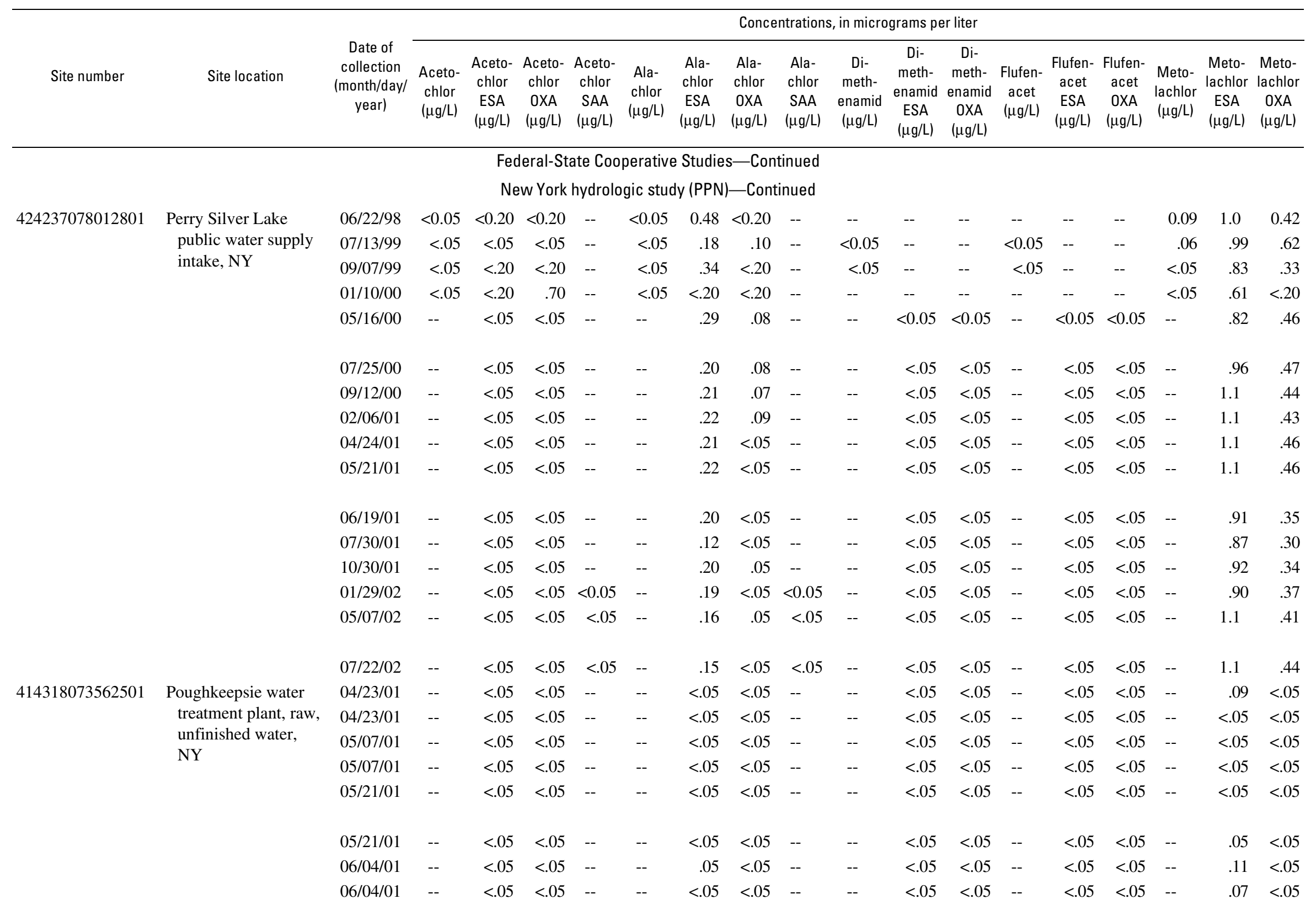


Table 6. Analytical results for selected acetamide herbicides and their degradation products, ethanesulfonic acid, oxanilic acid, and sulfinyl acetic acid, in surface-water samples, 1993-2003.-Continued

[OGRL, U.S. Geological Survey Organic Geochemistry Research Laboratory, Lawrence, Kansas; ESA, ethanesulfonic acid; OXA, oxanilic acid; SAA, sulfinyl acetic acid; --, no data; <, less than]

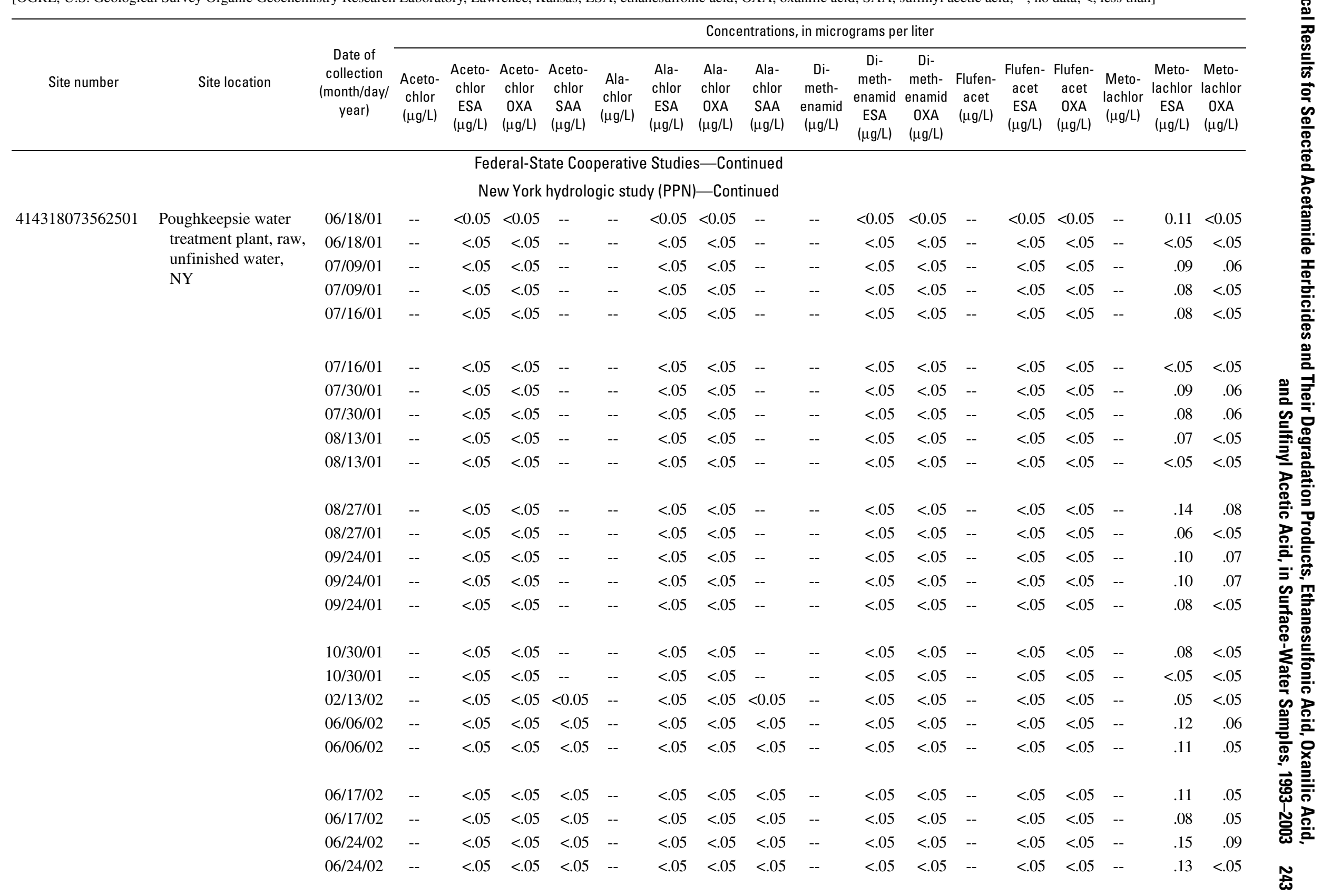


Table 6. Analytical results for selected acetamide herbicides and their degradation products, ethanesulfonic acid, oxanilic acid, and sulfinyl acetic acid, in surface-water samples, 1993-2003.-Continued

[OGRL, U.S. Geological Survey Organic Geochemistry Research Laboratory, Lawrence, Kansas; ESA, ethanesulfonic acid; OXA, oxanilic acid; SAA, sulfinyl acetic acid; --, no data; <, less than]

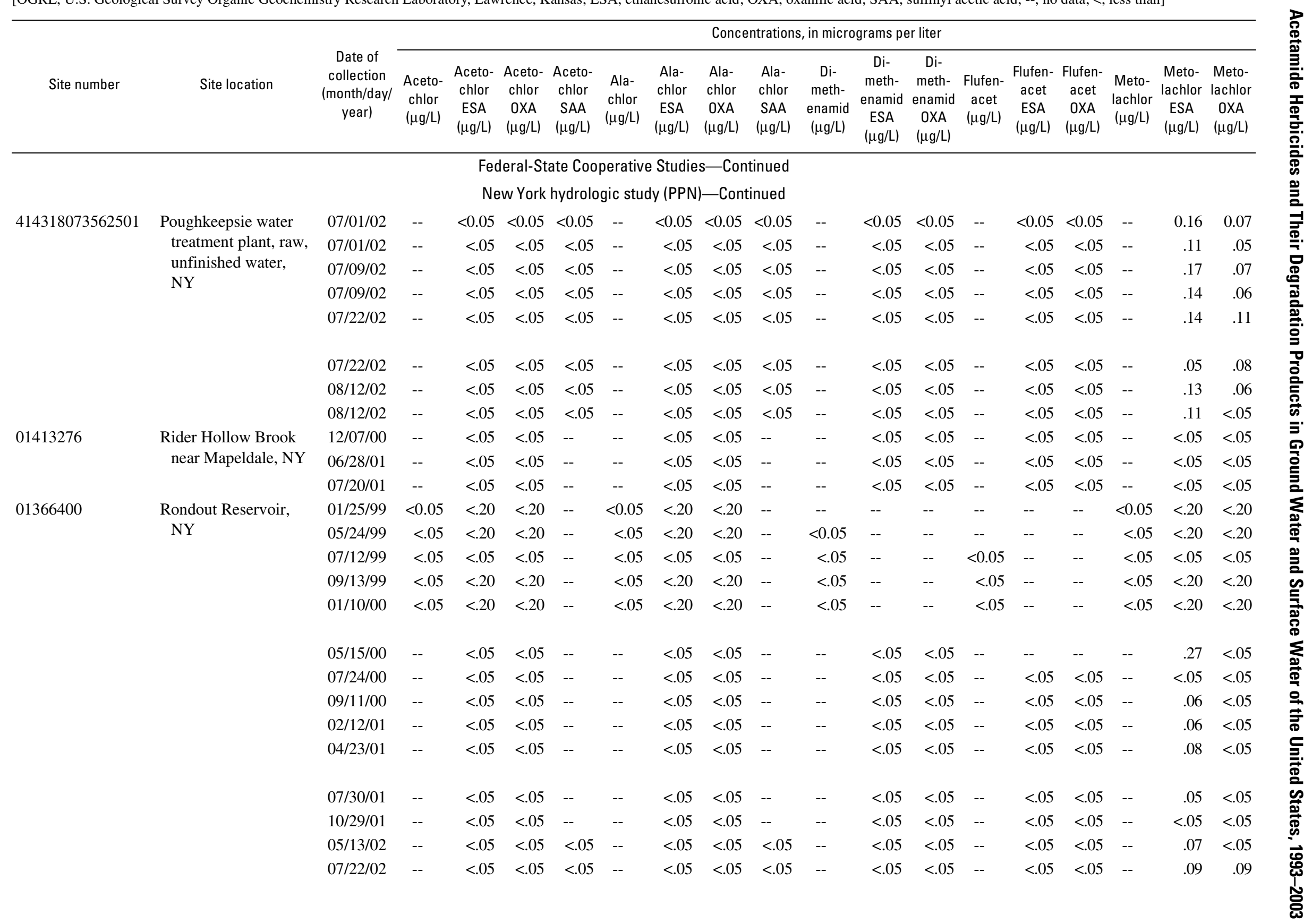


Table 6. Analytical results for selected acetamide herbicides and their degradation products, ethanesulfonic acid, oxanilic acid, and sulfinyl acetic acid, in surface-water samples, 1993-2003.-Continued

[OGRL, U.S. Geological Survey Organic Geochemistry Research Laboratory, Lawrence, Kansas; ESA, ethanesulfonic acid; OXA, oxanilic acid; SAA, sulfinyl acetic acid; --, no data; <, less than]

\begin{tabular}{|c|c|c|c|c|c|c|c|c|c|c|c|c|c|c|c|c|c|c|c|c|}
\hline \multirow[b]{2}{*}{ Site number } & \multirow[b]{2}{*}{ Site location } & \multirow[b]{2}{*}{$\begin{array}{c}\text { Date of } \\
\text { collection } \\
\text { (month/day/ } \\
\text { year) }\end{array}$} & \multicolumn{17}{|c|}{ Concentrations, in micrograms per liter } & \\
\hline & & & $\begin{array}{c}\text { Aceto- } \\
\text { chlor } \\
(\mu \mathrm{g} / \mathrm{L})\end{array}$ & $\begin{array}{l}\text { Aceto- } \\
\text { chlor } \\
\text { ESA } \\
(\mu \mathrm{g} / \mathrm{L})\end{array}$ & $\begin{array}{l}\text { Aceto- } \\
\text { chlor } \\
\text { OXA } \\
(\mu \mathrm{g} / \mathrm{L})\end{array}$ & $\begin{array}{l}\text { Aceto- } \\
\text { chlor } \\
\text { SAA } \\
(\mu \mathrm{g} / \mathrm{L})\end{array}$ & $\begin{array}{l}\text { Ala- } \\
\text { chlor } \\
(\mu \mathrm{g} / \mathrm{L})\end{array}$ & $\begin{array}{l}\text { Ala- } \\
\text { chlor } \\
\text { ESA } \\
(\mu \mathrm{g} / \mathrm{L})\end{array}$ & $\begin{array}{c}\text { Ala- } \\
\text { chlor } \\
\text { OXA } \\
(\mu \mathrm{g} / \mathrm{L})\end{array}$ & $\begin{array}{l}\text { Ala- } \\
\text { chlor } \\
\text { SAA } \\
(\mu \mathrm{g} / \mathrm{L})\end{array}$ & $\begin{array}{c}\text { Di- } \\
\text { meth- } \\
\text { enamid } \\
(\mu \mathrm{g} / \mathrm{L})\end{array}$ & $\begin{array}{c}\text { Di- } \\
\text { meth- } \\
\text { enamid } \\
\text { ESA } \\
(\mu \mathrm{g} / \mathrm{L})\end{array}$ & $\begin{array}{c}\text { Di- } \\
\text { meth- } \\
\text { enamid } \\
0 X A \\
(\mu \mathrm{g} / \mathrm{L})\end{array}$ & $\begin{array}{c}\text { Flufen- } \\
\text { acet } \\
(\mu \mathrm{g} / \mathrm{L})\end{array}$ & $\begin{array}{c}\text { Flufen- } \\
\text { acet } \\
\text { ESA } \\
(\mu \mathrm{g} / \mathrm{L})\end{array}$ & $\begin{array}{c}\text { Flufen- } \\
\text { acet } \\
\text { OXA } \\
(\mu \mathrm{g} / \mathrm{L})\end{array}$ & $\begin{array}{c}\text { Meto- } \\
\text { lachlor } \\
(\mu \mathrm{g} / \mathrm{L})\end{array}$ & $\begin{array}{c}\text { Meto- } \\
\text { lachlor } \\
\text { ESA } \\
(\mu \mathrm{g} / \mathrm{L})\end{array}$ & $\begin{array}{c}\text { Meto- } \\
\text { lachlor } \\
\text { OXA } \\
(\mu \mathrm{g} / \mathrm{L})\end{array}$ & $\begin{array}{l}\overline{\text { क }} \\
\overline{0} \\
\frac{1}{c} \\
\frac{\Phi}{0}\end{array}$ \\
\hline \multicolumn{20}{|c|}{ Federal-State Cooperative Studies-Continued } & \\
\hline \multicolumn{20}{|c|}{ New York hydrologic study (PPN) — Continued } & \\
\hline 01366400 & $\begin{array}{l}\text { Rondout Reservoir, } \\
\text { NY }\end{array}$ & $10 / 28 / 02$ & -- & $<0.05$ & $<0.05$ & $<0.05$ & -- & $<0.05$ & $<0.05$ & $<0.05$ & -- & $<0.05$ & $<0.05$ & -- & $<0.05$ & $<0.05$ & -- & 0.06 & $<0.05$ & E \\
\hline 01502702 & $\begin{array}{l}\text { Route } 41 \text { Street south } \\
\text { of Afton, NY }\end{array}$ & $11 / 26 / 01$ & -- & $<.05$ & $<.05$ & $<.05$ & -- & $<.05$ & $<.05$ & $<.05$ & -- & $<.05$ & $<.05$ & -- & $<.05$ & $<.05$ & -- & 1.3 & 1.2 & $\square$ \\
\hline \multirow[t]{4}{*}{04234018} & \multirow{4}{*}{$\begin{array}{l}\text { Salmon Creek at } \\
\text { Ludlauville, NY }\end{array}$} & $06 / 11 / 98$ & $<0.05$ & $<.20$ & $<.20$ & -- & $<0.05$ & .32 & $<.05$ & -- & -- & -- & -- & -- & -- & -- & 0.77 & 4.3 & .46 & $\bar{a}$ \\
\hline & & $06 / 17 / 98$ & $<.05$ & $<.20$ & $<.20$ & -- & $<.05$ & .63 & $<.05$ & -- & -- & -- & -- & -- & -- & -- & 1.5 & 5.3 & 1.5 & 7 \\
\hline & & 06/18/98 & $<.05$ & $<.20$ & $<.20$ & -- & .23 & 1.9 & .68 & -- & -- & -- & -- & -- & -- & -- & 4.8 & 8.3 & 4.4 & \\
\hline & & 06/19/98 & $<.05$ & $<.05$ & $<.05$ & -- & .10 & 1.0 & .50 & -- & -- & -- & -- & -- & -- & -- & 2.0 & 5.2 & 2.7 & $\cong$ \\
\hline 01376500 & $\begin{array}{l}\text { Saw Mill River at } \\
\text { Yonkers, NY }\end{array}$ & $06 / 09 / 97$ & $<.05$ & $<.20$ & $<.20$ & -- & $<.05$ & $<.20$ & $<.20$ & -- & -- & -- & -- & -- & -- & -- & $<.05$ & $<.20$ & $<.20$ & \\
\hline 04216214 & $\begin{array}{l}\text { Scajaquada Creek at } \\
\text { Buffalo, NY }\end{array}$ & $06 / 28 / 01$ & -- & $<.05$ & $<.05$ & -- & -- & $<.05$ & $<.05$ & -- & -- & $<.05$ & $<.05$ & -- & $<.05$ & $<.05$ & -- & .05 & $<.05$ & \\
\hline \multirow[t]{10}{*}{01362230} & \multirow{10}{*}{$\begin{array}{l}\text { Schoharie Reservoir, } \\
\text { NY }\end{array}$} & $05 / 24 / 99$ & $<.05$ & $<.20$ & $<.20$ & -- & $<.05$ & $<.20$ & $<.20$ & -- & $<0.05$ & -- & -- & -- & -- & -- & $<.05$ & $<.20$ & $<.20$ & \\
\hline & & $07 / 12 / 99$ & $<.05$ & $<.05$ & $<.05$ & -- & $<.05$ & $<.05$ & $<.05$ & -- & $<.05$ & -- & -- & $<0.05$ & -- & -- & $<.05$ & $<.05$ & $<.05$ & \\
\hline & & 09/13/99 & $<.05$ & $<.20$ & $<.20$ & -- & $<.05$ & $<.20$ & $<.20$ & -- & $<.05$ & -- & -- & $<.05$ & -- & -- & $<.05$ & $<.20$ & $<.20$ & \\
\hline & & $01 / 10 / 00$ & $<.05$ & $<.20$ & $<.20$ & -- & $<.05$ & $<.20$ & $<.20$ & -- & -- & -- & -- & -- & -- & -- & $<.05$ & $<.20$ & $<.20$ & \\
\hline & & $05 / 15 / 00$ & -- & $<.05$ & $<.05$ & -- & -- & $<.05$ & $<.05$ & -- & -- & $<.05$ & $<.05$ & -- & -- & -- & -- & .27 & $<.05$ & \\
\hline & & $07 / 24 / 00$ & -- & $<.05$ & $<.05$ & -- & -- & $<.05$ & $<.05$ & -- & -- & $<.05$ & $<.05$ & -- & $<.05$ & $<.05$ & -- & $<.05$ & $<.05$ & \\
\hline & & 09/11/00 & -- & $<.05$ & $<.05$ & -- & -- & $<.05$ & $<.05$ & -- & -- & $<.05$ & $<.05$ & -- & $<.05$ & $<.05$ & -- & $<.05$ & $<.05$ & 를 을 \\
\hline & & $02 / 12 / 01$ & -- & $<.05$ & $<.05$ & -- & -- & $<.05$ & $<.05$ & -- & -- & $<.05$ & $<.05$ & -- & $<.05$ & $<.05$ & -- & .05 & $<.05$ & \\
\hline & & $05 / 13 / 02$ & -- & $<.05$ & $<.05$ & $<.05$ & -- & $<.05$ & $<.05$ & $<.05$ & -- & $<.05$ & $<.05$ & -- & $<.05$ & $<.05$ & -- & $<.05$ & $<.05$ & \\
\hline & & 07/29/02 & -- & $<.05$ & $<.05$ & $<.05$ & -- & $<.05$ & $<.05$ & $<.05$ & -- & $<.05$ & $<.05$ & -- & $<.05$ & $<.05$ & -- & $<.05$ & $<.05$ & 害 \\
\hline
\end{tabular}


Table 6. Analytical results for selected acetamide herbicides and their degradation products, ethanesulfonic acid, oxanilic acid, and sulfinyl acetic acid, in surface-water samples, 1993-2003.-Continued

[OGRL, U.S. Geological Survey Organic Geochemistry Research Laboratory, Lawrence, Kansas; ESA, ethanesulfonic acid; OXA, oxanilic acid; SAA, sulfinyl acetic acid; --, no data; <, less than]

\begin{tabular}{|c|c|c|c|c|c|c|c|c|c|c|c|c|c|c|c|c|c|c|c|}
\hline \multirow[b]{2}{*}{ Site number } & \multirow[b]{2}{*}{ Site location } & \multirow[b]{2}{*}{$\begin{array}{c}\text { Date of } \\
\text { collection } \\
\text { (month/day/ } \\
\text { year) }\end{array}$} & \multicolumn{17}{|c|}{ Concentrations, in micrograms per liter } \\
\hline & & & $\begin{array}{l}\text { Aceto- } \\
\text { chlor } \\
(\mu \mathrm{g} / \mathrm{L})\end{array}$ & $\begin{array}{l}\text { Aceto- } \\
\text { chlor } \\
\text { ESA } \\
(\mu \mathrm{g} / \mathrm{L})\end{array}$ & $\begin{array}{c}\text { Aceto- } \\
\text { chlor } \\
0 X A \\
(\mu \mathrm{g} / \mathrm{L})\end{array}$ & $\begin{array}{c}\text { Aceto- } \\
\text { chlor } \\
\text { SAA } \\
(\mu \mathrm{g} / \mathrm{L})\end{array}$ & $\begin{array}{l}\text { Ala- } \\
\text { chlor } \\
(\mu \mathrm{g} / \mathrm{L})\end{array}$ & $\begin{array}{l}\text { Ala- } \\
\text { chlor } \\
\text { ESA } \\
(\mu \mathrm{g} / \mathrm{L})\end{array}$ & $\begin{array}{c}\text { Ala- } \\
\text { chlor } \\
\text { OXA } \\
(\mu \mathrm{g} / \mathrm{L})\end{array}$ & $\begin{array}{l}\text { Ala- } \\
\text { chlor } \\
\text { SAA } \\
(\mu \mathrm{g} / \mathrm{L})\end{array}$ & $\begin{array}{c}\text { Di- } \\
\text { meth- } \\
\text { enamid } \\
(\mu \mathrm{g} / \mathrm{L})\end{array}$ & $\begin{array}{c}\text { Di- } \\
\text { meth- } \\
\text { enamid } \\
\text { ESA } \\
(\mu \mathrm{g} / \mathrm{L})\end{array}$ & $\begin{array}{c}\text { Di- } \\
\text { meth- } \\
\text { enamid } \\
\text { OXA } \\
(\mu \mathrm{g} / \mathrm{L})\end{array}$ & $\begin{array}{c}\text { Flufen- } \\
\text { acet } \\
(\mu \mathrm{g} / \mathrm{L})\end{array}$ & $\begin{array}{c}\text { Flufen- } \\
\text { acet } \\
\text { ESA } \\
(\mu \mathrm{g} / \mathrm{L})\end{array}$ & $\begin{array}{c}\text { Flufen- } \\
\text { acet } \\
\text { OXA } \\
(\mu \mathrm{g} / \mathrm{L})\end{array}$ & $\begin{array}{l}\text { Meto- } \\
\text { lachlor } \\
(\mu \mathrm{g} / \mathrm{L})\end{array}$ & $\begin{array}{l}\text { Meto- } \\
\text { lachlor } \\
\text { ESA } \\
(\mu \mathrm{g} / \mathrm{L})\end{array}$ & $\begin{array}{c}\text { Meto- } \\
\text { lachlor } \\
\text { OXA } \\
(\mu \mathrm{g} / \mathrm{L})\end{array}$ \\
\hline \multicolumn{20}{|c|}{ Federal-State Cooperative Studies-Continued } \\
\hline \multicolumn{20}{|c|}{ New York hydrologic study (PPN)—Continued } \\
\hline 01362230 & $\begin{array}{l}\text { Schoharie Reservoir, } \\
\text { NY }\end{array}$ & $10 / 21 / 02$ & -- & $<0.05$ & $<0.05$ & $<0.05$ & -- & $<0.05$ & $<0.05$ & $<0.05$ & -- & $<0.05$ & $<0.05$ & -- & $<0.05$ & $<0.05$ & -- & 0.07 & $<0.05$ \\
\hline 422428076523101 & $\begin{array}{l}\text { Seneca Lake S1-2D, } \\
\text { NY }\end{array}$ & $07 / 22 / 99$ & $<0.05$ & $<.20$ & $<.20$ & -- & $<0.05$ & $<.20$ & $<.20$ & -- & $<0.05$ & -- & -- & $<0.05$ & -- & -- & $<0.05$ & $<.20$ & $<.20$ \\
\hline 422428076523101 & $\begin{array}{l}\text { Seneca Lake S1-2S, } \\
\text { NY }\end{array}$ & $07 / 22 / 99$ & $<.05$ & $<.20$ & $<.20$ & -- & $<.05$ & $<.20$ & $<.20$ & -- & $<.05$ & -- & -- & $<.05$ & -- & -- & $<.05$ & $<.20$ & $<.20$ \\
\hline 422954076540401 & $\begin{array}{l}\text { Seneca Lake S2-2D, } \\
\text { NY }\end{array}$ & $07 / 22 / 99$ & $<.05$ & $<.20$ & $<.20$ & -- & $<.05$ & $<.20$ & $<.20$ & -- & $<.05$ & -- & -- & $<.05$ & -- & -- & $<.05$ & $<.20$ & $<.20$ \\
\hline 422954076540401 & $\begin{array}{l}\text { Seneca Lake S2-2S, } \\
\text { NY }\end{array}$ & $07 / 22 / 99$ & $<.05$ & $<.20$ & $<.20$ & -- & $<.05$ & $<.20$ & $<.20$ & -- & $<.05$ & -- & -- & $<.05$ & -- & -- & $<.05$ & $<.20$ & $<.20$ \\
\hline 423619076534901 & $\begin{array}{l}\text { Seneca Lake S3-2D, } \\
\text { NY }\end{array}$ & $07 / 22 / 99$ & $<.05$ & $<.20$ & $<.20$ & -- & $<.05$ & $<.20$ & $<.20$ & -- & $<.05$ & -- & -- & $<.05$ & -- & -- & $<.05$ & $<.20$ & $<.20$ \\
\hline 423190765349011 & $\begin{array}{l}\text { Seneca Lake S3-2S, } \\
\text { NY }\end{array}$ & $07 / 22 / 99$ & $<.05$ & $<.20$ & $<.20$ & -- & $<.05$ & $<.20$ & $<.20$ & -- & $<.05$ & -- & -- & $<.05$ & -- & -- & $<.05$ & $<.20$ & $<.20$ \\
\hline 423937076531801 & $\begin{array}{l}\text { Seneca Lake S4-2D, } \\
\text { NY }\end{array}$ & $07 / 22 / 99$ & $<.05$ & $<.20$ & $<.20$ & -- & $<.05$ & $<.20$ & $<.20$ & -- & $<.05$ & -- & -- & $<.05$ & -- & -- & $<.05$ & $<.20$ & $<.20$ \\
\hline 423927076531801 & $\begin{array}{l}\text { Seneca Lake S4-2S, } \\
\text { NY }\end{array}$ & $07 / 22 / 99$ & $<.05$ & $<.20$ & $<.20$ & -- & $<.05$ & $<.20$ & $<.20$ & -- & $<.05$ & -- & -- & $<.05$ & -- & -- & $<.05$ & $<.20$ & $<.20$ \\
\hline 424603076565701 & $\begin{array}{l}\text { Seneca Lake S5-2D, } \\
\text { NY }\end{array}$ & $07 / 22 / 99$ & $<.05$ & $<.20$ & $<.20$ & -- & $<.05$ & $<.20$ & $<.20$ & -- & $<.05$ & -- & -- & $<.05$ & -- & -- & $<.05$ & $<.20$ & $<.20$ \\
\hline 424603076565701 & $\begin{array}{l}\text { Seneca Lake S5-2S, } \\
\text { NY }\end{array}$ & $07 / 22 / 99$ & $<.05$ & $<.20$ & $<.20$ & -- & $<.05$ & $<.20$ & $<.20$ & -- & $<.05$ & -- & -- & $<.05$ & -- & -- & $<.05$ & $<.20$ & $<.20$ \\
\hline 458041076573601 & $\begin{array}{l}\text { Seneca Lake S6-2D, } \\
\text { NY }\end{array}$ & $07 / 22 / 99$ & $<.05$ & $<.20$ & $<.20$ & -- & $<.05$ & $<.20$ & $<.20$ & -- & $<.05$ & -- & -- & $<.05$ & -- & -- & $<.05$ & $<.20$ & $<.20$ \\
\hline 425041076573601 & $\begin{array}{l}\text { Seneca Lake S6-2S, } \\
\text { NY }\end{array}$ & $07 / 22 / 99$ & $<.05$ & $<.20$ & $<.20$ & -- & $<.05$ & $<.20$ & $<.20$ & -- & $<.05$ & -- & -- & $<.05$ & -- & -- & $<.05$ & $<.20$ & $<.20$ \\
\hline \multirow{2}{*}{04237500} & \multirow{2}{*}{$\begin{array}{l}\text { Seneca River at } \\
\text { Baldwinsville, NY }\end{array}$} & $05 / 22 / 97$ & $<.05$ & $<.20$ & $<.20$ & -- & $<.05$ & $<.20$ & $<.20$ & -- & -- & -- & -- & -- & -- & -- & .08 & .47 & $<.20$ \\
\hline & & $06 / 11 / 97$ & $<.05$ & $<.20$ & $<.20$ & -- & .12 & $<.20$ & $<.20$ & -- & -- & -- & -- & -- & -- & -- & .14 & .35 & $<.20$ \\
\hline
\end{tabular}


Table 6. Analytical results for selected acetamide herbicides and their degradation products, ethanesulfonic acid, oxanilic acid, and sulfinyl acetic acid, in surface-water samples, 1993-2003.-Continued

[OGRL, U.S. Geological Survey Organic Geochemistry Research Laboratory, Lawrence, Kansas; ESA, ethanesulfonic acid; OXA, oxanilic acid; SAA, sulfinyl acetic acid; --, no data; <, less than]

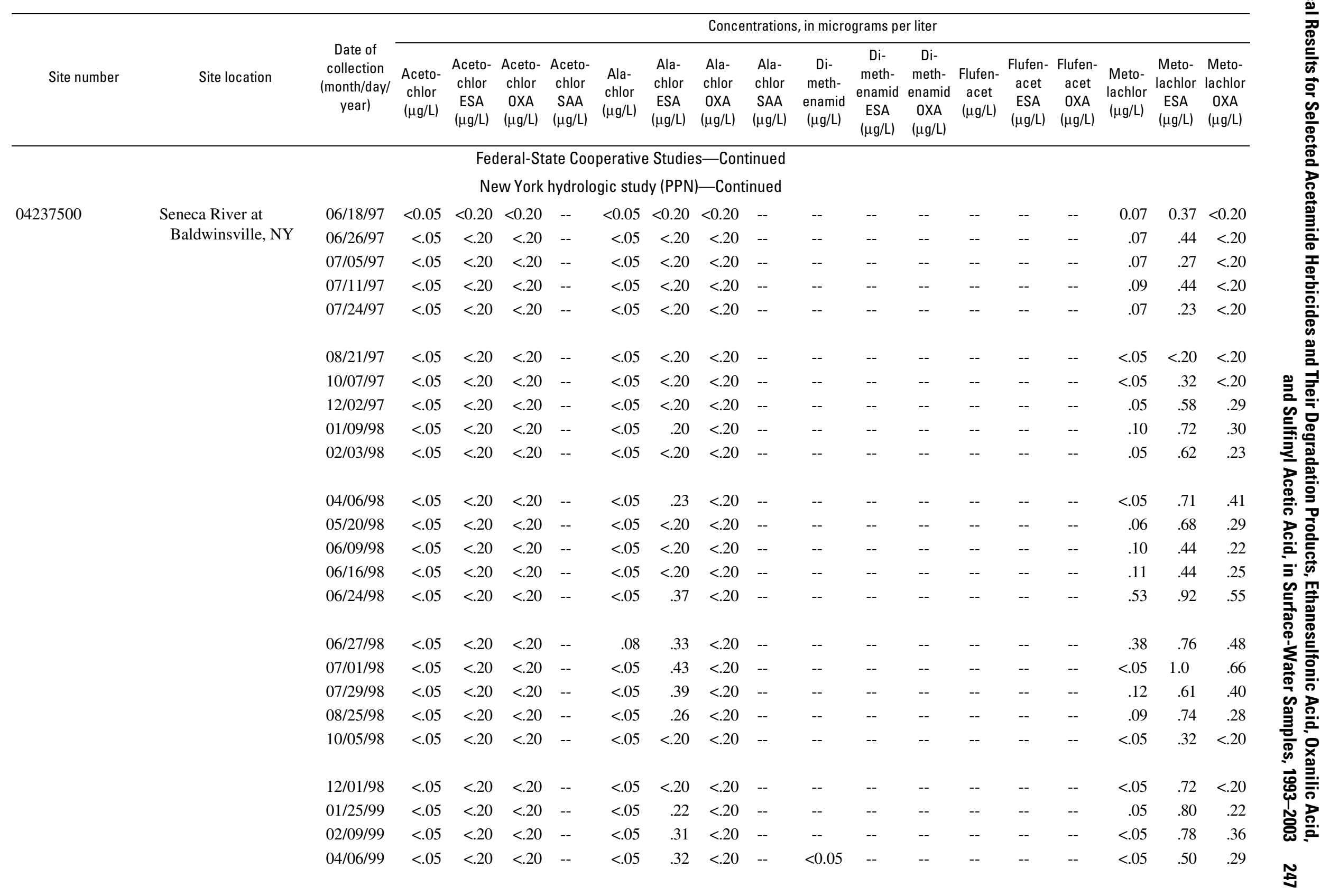


Table 6. Analytical results for selected acetamide herbicides and their degradation products, ethanesulfonic acid, oxanilic acid, and sulfinyl acetic acid, in surface-water samples, 1993-2003.-Continued

[OGRL, U.S. Geological Survey Organic Geochemistry Research Laboratory, Lawrence, Kansas; ESA, ethanesulfonic acid; OXA, oxanilic acid; SAA, sulfinyl acetic acid; --, no data; <, less than]

\begin{tabular}{|c|c|c|c|c|c|c|c|c|c|c|c|c|c|c|c|c|c|c|c|}
\hline \multirow[b]{2}{*}{ Site number } & \multirow[b]{2}{*}{ Site location } & \multirow[b]{2}{*}{$\begin{array}{c}\text { Date of } \\
\text { collection } \\
\text { (month/day/ } \\
\text { year) }\end{array}$} & \multicolumn{17}{|c|}{ Concentrations, in micrograms per liter } \\
\hline & & & $\begin{array}{l}\text { Aceto- } \\
\text { chlor } \\
(\mu \mathrm{g} / \mathrm{L})\end{array}$ & $\begin{array}{l}\text { Aceto- } \\
\text { chlor } \\
\text { ESA } \\
(\mu \mathrm{g} / \mathrm{L})\end{array}$ & $\begin{array}{c}\text { Aceto- } \\
\text { chlor } \\
\text { OXA } \\
(\mu \mathrm{g} / \mathrm{L})\end{array}$ & $\begin{array}{c}\text { Aceto- } \\
\text { chlor } \\
\text { SAA } \\
(\mu \mathrm{g} / \mathrm{L})\end{array}$ & $\begin{array}{l}\text { Ala- } \\
\text { chlor } \\
(\mu \mathrm{g} / \mathrm{L})\end{array}$ & $\begin{array}{l}\text { Ala- } \\
\text { chlor } \\
\text { ESA } \\
(\mu \mathrm{g} / \mathrm{L})\end{array}$ & $\begin{array}{l}\text { Ala- } \\
\text { chlor } \\
\text { OXA } \\
(\mu \mathrm{g} / \mathrm{L})\end{array}$ & $\begin{array}{l}\text { Ala- } \\
\text { chlor } \\
\text { SAA } \\
(\mu \mathrm{g} / \mathrm{L})\end{array}$ & $\begin{array}{c}\text { Di- } \\
\text { meth- } \\
\text { enamid } \\
(\mu \mathrm{g} / \mathrm{L})\end{array}$ & $\begin{array}{c}\text { Di- } \\
\text { meth- } \\
\text { enamid } \\
\text { ESA } \\
(\mu \mathrm{g} / \mathrm{L})\end{array}$ & $\begin{array}{c}\text { Di- } \\
\text { meth- } \\
\text { enamid } \\
\text { OXA } \\
(\mu \mathrm{g} / \mathrm{L})\end{array}$ & $\begin{array}{c}\text { Flufen- } \\
\text { acet } \\
(\mu \mathrm{g} / \mathrm{L})\end{array}$ & $\begin{array}{c}\text { Flufen- } \\
\text { acet } \\
\text { ESA } \\
(\mu \mathrm{g} / \mathrm{L})\end{array}$ & $\begin{array}{c}\text { Flufen- } \\
\text { acet } \\
\text { OXA } \\
(\mu \mathrm{g} / \mathrm{L})\end{array}$ & $\begin{array}{c}\text { Meto- } \\
\text { lachlor } \\
(\mu \mathrm{g} / \mathrm{L})\end{array}$ & $\begin{array}{l}\text { Meto- } \\
\text { lachlor } \\
\text { ESA } \\
(\mu \mathrm{g} / \mathrm{L})\end{array}$ & $\begin{array}{c}\text { Meto- } \\
\text { lachlor } \\
\text { OXA } \\
(\mu \mathrm{g} / \mathrm{L})\end{array}$ \\
\hline \multicolumn{20}{|c|}{ Federal-State Cooperative Studies-Continued } \\
\hline \multirow{12}{*}{04237500} & \multirow{12}{*}{$\begin{array}{l}\text { Seneca River at } \\
\text { Baldwinsville, NY }\end{array}$} & & & & ew York & kydrolo & ogic stuc & dy (PPN) & 1)-Con & inued & & & & & & & & & \\
\hline & & 05/18/98 & $<0.05$ & $<0.20$ & $<0.20$ & -- & $<0.05$ & $<0.20$ & $<0.20$ & -- & $<0.05$ & -- & -- & -- & -- & -- & $<0.05$ & 0.35 & 0.20 \\
\hline & & 06/08/99 & $<.05$ & $<.20$ & $<.20$ & -- & $<.05$ & $<.20$ & $<.20$ & -- & $<.05$ & -- & -- & -- & -- & -- & $<.05$ & .35 & $<.20$ \\
\hline & & $06 / 22 / 99$ & $<.05$ & $<.20$ & $<.20$ & -- & $<.05$ & $<.20$ & $<.20$ & -- & $<.05$ & -- & -- & -- & -- & -- & .06 & .24 & $<.20$ \\
\hline & & 07/01/99 & $<.05$ & $<.20$ & $<.20$ & -- & $<.05$ & $<.20$ & $<.20$ & -- & $<.05$ & -- & -- & $<0.05$ & -- & -- & $<.05$ & .33 & $<.20$ \\
\hline & & $07 / 12 / 99$ & $<.05$ & $<.05$ & $<.05$ & -- & $<.05$ & .06 & $<.05$ & -- & $<.05$ & -- & -- & $<.05$ & -- & -- & .05 & .36 & .13 \\
\hline & & 08/06/99 & $<.05$ & $<.20$ & $<.20$ & -- & $<.05$ & $<.20$ & $<.20$ & -- & $<.05$ & -- & -- & $<.05$ & -- & -- & .05 & .22 & $<.20$ \\
\hline & & 09/14/99 & $<.05$ & $<.20$ & $<.20$ & -- & $<.05$ & $<.20$ & $<.20$ & -- & $<.05$ & -- & -- & $<.05$ & -- & -- & $<.05$ & $<.20$ & $<.20$ \\
\hline & & $10 / 13 / 99$ & $<.05$ & $<.20$ & $<.20$ & -- & $<.05$ & $<.20$ & $<.20$ & -- & $<.05$ & -- & -- & $<.05$ & -- & -- & $<.05$ & $<.20$ & $<.20$ \\
\hline & & $12 / 14 / 99$ & $<.05$ & .61 & $<.20$ & -- & $<.05$ & $<.20$ & $<.20$ & -- & $<.05$ & -- & -- & $<.05$ & -- & -- & $<.05$ & $<.20$ & .33 \\
\hline & & $01 / 09 / 00$ & $<.05$ & $<.20$ & $<.20$ & -- & $<.05$ & $<.20$ & $<.20$ & -- & $<.05$ & -- & -- & $<.05$ & -- & -- & $<.05$ & $<.20$ & $<.20$ \\
\hline & & $01 / 11 / 00$ & $<.05$ & $<.20$ & $<.20$ & -- & $<.05$ & $<.20$ & $<.20$ & -- & $<.05$ & -- & -- & $<.05$ & -- & -- & $<.05$ & $<.20$ & .26 \\
\hline 0423406130 & $\begin{array}{l}\text { Seneca River at Free } \\
\text { Bridge Corners, NY }\end{array}$ & $07 / 29 / 98$ & $<.05$ & $<.20$ & $<.20$ & -- & $<.05$ & $<.20$ & $<.20$ & -- & -- & -- & -- & -- & -- & -- & $<.05$ & $<.20$ & $<.20$ \\
\hline 04235600 & $\begin{array}{l}\text { Seneca River near Port } \\
\text { Byron, NY }\end{array}$ & 07/29/98 & $<.05$ & $<.20$ & $<.20$ & -- & $<.05$ & .27 & $<.05$ & -- & -- & -- & -- & -- & -- & -- & .06 & .58 & .34 \\
\hline 04232730 & $\begin{array}{l}\text { Seneca River near } \\
\text { Seneca Falls, NY }\end{array}$ & 07/29/98 & $<.05$ & $<.20$ & $<.20$ & -- & $<.05$ & $<.20$ & $<.20$ & -- & -- & -- & -- & -- & -- & -- & $<.05$ & $<.20$ & $<.20$ \\
\hline 01496330 & $\begin{array}{l}\text { Shadow Brook at East } \\
\text { Springfield, NY }\end{array}$ & $11 / 26 / 01$ & -- & $<.05$ & $<.05$ & -- & -- & $<.05$ & $<.05$ & -- & -- & $<0.05$ & $<0.05$ & -- & $<0.05$ & $<0.05$ & -- & .33 & $<.05$ \\
\hline \multirow[t]{3}{*}{04234038} & \multirow{3}{*}{$\begin{array}{l}\text { Sheldrake Creek at } \\
\text { Sheldrake, NY }\end{array}$} & $06 / 11 / 98$ & $<.05$ & $<.20$ & $<.20$ & -- & $<.05$ & $<.20$ & $<.20$ & -- & -- & -- & -- & -- & -- & -- & $<.05$ & .56 & $<.20$ \\
\hline & & 07/05/98 & $<.05$ & $<.20$ & $<.20$ & -- & $<.05$ & $<.20$ & $<.20$ & -- & -- & -- & -- & -- & -- & -- & $<.05$ & 13 & 4.0 \\
\hline & & 07/08/98 & $<.05$ & $<.20$ & $<.20$ & -- & $<.05$ & .36 & $<.20$ & -- & -- & -- & -- & -- & -- & -- & $<.05$ & 1.1 & .31 \\
\hline \multirow[t]{5}{*}{424318078014601} & \multirow{5}{*}{$\begin{array}{l}\text { Silver Lake inlet near } \\
\text { Perry, NY }\end{array}$} & 05/18/98 & $<.05$ & $<.20$ & $<.20$ & -- & $<.05$ & 1.2 & $<.20$ & -- & -- & -- & -- & -- & -- & -- & .37 & 3.8 & .94 \\
\hline & & $06 / 22 / 98$ & $<.05$ & $<.20$ & $<.20$ & -- & $<.05$ & 1.2 & $<.20$ & -- & -- & -- & -- & -- & -- & -- & .07 & 2.5 & .62 \\
\hline & & $06 / 26 / 98$ & $<.05$ & $<.20$ & $<.20$ & -- & .45 & 2.0 & .72 & -- & -- & -- & -- & -- & -- & -- & 8.0 & 3.4 & 2.4 \\
\hline & & 07/20/98 & $<.05$ & $<.20$ & $<.20$ & -- & $<.05$ & 1.9 & .48 & -- & -- & -- & -- & -- & -- & -- & .22 & 3.6 & 2.0 \\
\hline & & $09 / 28 / 98$ & $<.05$ & $<.20$ & $<.20$ & -- & $<.05$ & .61 & .34 & -- & -- & -- & -- & -- & -- & -- & .08 & 1.8 & .45 \\
\hline
\end{tabular}


Table 6. Analytical results for selected acetamide herbicides and their degradation products, ethanesulfonic acid, oxanilic acid, and sulfinyl acetic acid, in surface-water samples, 1993-2003.-Continued

[OGRL, U.S. Geological Survey Organic Geochemistry Research Laboratory, Lawrence, Kansas; ESA, ethanesulfonic acid; OXA, oxanilic acid; SAA, sulfinyl acetic acid; --, no data; <, less than]

\begin{tabular}{|c|c|c|c|c|c|c|c|c|c|c|c|c|c|c|c|c|c|c|c|c|}
\hline \multirow[b]{2}{*}{ Site number } & \multirow[b]{2}{*}{ Site location } & \multirow[b]{2}{*}{$\begin{array}{c}\text { Date of } \\
\text { collection } \\
\text { (month/day/ } \\
\text { year) }\end{array}$} & \multicolumn{17}{|c|}{ Concentrations, in micrograms per liter } & \\
\hline & & & $\begin{array}{c}\text { Aceto- } \\
\text { chlor } \\
(\mu \mathrm{g} / \mathrm{L})\end{array}$ & $\begin{array}{l}\text { Aceto- } \\
\text { chlor } \\
\text { ESA } \\
(\mu \mathrm{g} / \mathrm{L})\end{array}$ & $\begin{array}{l}\text { Aceto- } \\
\text { chlor } \\
\text { OXA } \\
(\mu \mathrm{g} / \mathrm{L})\end{array}$ & $\begin{array}{l}\text { Aceto- } \\
\text { chlor } \\
\text { SAA } \\
(\mu \mathrm{g} / \mathrm{L})\end{array}$ & $\begin{array}{l}\text { Ala- } \\
\text { chlor } \\
(\mu \mathrm{g} / \mathrm{L})\end{array}$ & $\begin{array}{l}\text { Ala- } \\
\text { chlor } \\
\text { ESA } \\
(\mu \mathrm{g} / \mathrm{L})\end{array}$ & $\begin{array}{l}\text { Ala- } \\
\text { chlor } \\
\text { OXA } \\
(\mu \mathrm{g} / \mathrm{L})\end{array}$ & $\begin{array}{l}\text { Ala- } \\
\text { chlor } \\
\text { SAA } \\
(\mu \mathrm{g} / \mathrm{L})\end{array}$ & $\begin{array}{c}\text { Di- } \\
\text { meth- } \\
\text { enamid } \\
(\mu \mathrm{g} / \mathrm{L})\end{array}$ & $\begin{array}{c}\text { Di- } \\
\text { meth- } \\
\text { enamid } \\
\text { ESA } \\
(\mu \mathrm{g} / \mathrm{L})\end{array}$ & $\begin{array}{c}\text { Di- } \\
\text { meth- } \\
\text { enamid } \\
0 X A \\
(\mu \mathrm{g} / \mathrm{L})\end{array}$ & $\begin{array}{l}\text { Flufen- } \\
\text { acet } \\
(\mu \mathrm{g} / \mathrm{L})\end{array}$ & $\begin{array}{l}\text { Flufen- } \\
\text { acet } \\
\text { ESA } \\
(\mu \mathrm{g} / \mathrm{L})\end{array}$ & $\begin{array}{l}\text { Flufen- } \\
\text { acet } \\
\text { OXA } \\
(\mu \mathrm{g} / \mathrm{L})\end{array}$ & $\begin{array}{l}\text { Meto- } \\
\text { lachlor } \\
(\mu \mathrm{g} / \mathrm{L})\end{array}$ & $\begin{array}{l}\text { Meto- } \\
\text { lachlor } \\
\text { ESA } \\
(\mu \mathrm{g} / \mathrm{L})\end{array}$ & $\begin{array}{c}\text { Meto- } \\
\text { lachlor } \\
\text { OXA } \\
(\mu \mathrm{g} / \mathrm{L})\end{array}$ & $\begin{array}{l}\overline{5} \\
\overline{0} \\
\frac{0}{2} \\
\frac{1}{D} \\
\frac{D}{D}\end{array}$ \\
\hline \multicolumn{20}{|c|}{ Federal-State Cooperative Studies-Continued } & \\
\hline \multicolumn{20}{|c|}{ New York hydrologic study (PPN)—Continued } & \\
\hline \multirow[t]{2}{*}{424318078014601} & \multirow{2}{*}{$\begin{array}{l}\text { Silver Lake inlet near } \\
\text { Perry, NY }\end{array}$} & $01 / 05 / 99$ & $<0.05$ & $<0.20$ & $<0.20$ & -- & $<0.05$ & 0.58 & 0.20 & -- & -- & -- & -- & -- & -- & -- & 0.06 & 1.4 & 0.54 & \\
\hline & & 05/18/99 & $<.05$ & $<.20$ & $<.20$ & -- & $<.05$ & .45 & $<.20$ & -- & $<0.05$ & -- & -- & -- & -- & -- & .06 & .91 & .46 & \\
\hline \multirow[t]{13}{*}{425549076250201} & \multirow{13}{*}{$\begin{array}{l}\text { Skaneateles Lake } \\
\text { intake \#1, NY }\end{array}$} & $07 / 02 / 97$ & $<.05$ & $<.20$ & $<.20$ & -- & $<.05$ & $<.20$ & $<.20$ & -- & -- & -- & -- & -- & -- & -- & $<.05$ & $<.20$ & $<.20$ & \\
\hline & & 09/11/97 & $<.05$ & $<.20$ & $<.20$ & -- & $<.05$ & $<.20$ & $<.20$ & -- & -- & -- & -- & -- & -- & -- & $<.05$ & $<.20$ & $<.20$ & \\
\hline & & $07 / 27 / 98$ & $<.05$ & $<.20$ & $<.20$ & -- & $<.05$ & $<.20$ & $<.20$ & -- & -- & -- & -- & -- & -- & -- & $<.05$ & $<.20$ & $<.20$ & \\
\hline & & $01 / 05 / 99$ & $<.05$ & $<.20$ & $<.20$ & -- & $<.05$ & $<.20$ & $<.20$ & -- & -- & -- & -- & -- & -- & -- & $<.05$ & $<.20$ & $<.20$ & \\
\hline & & 05/18/99 & $<.05$ & $<.20$ & $<.20$ & -- & $<.05$ & $<.20$ & $<.20$ & -- & $<.05$ & -- & -- & -- & -- & -- & $<.05$ & $<.20$ & $<.20$ & \\
\hline & & 09/07/99 & $<.05$ & $<.20$ & $<.20$ & -- & $<.05$ & $<.20$ & $<.20$ & -- & $<.05$ & -- & -- & $<.05$ & -- & -- & $<.05$ & $<.20$ & $<.20$ & \\
\hline & & $01 / 19 / 00$ & $<.05$ & $<.20$ & $<.20$ & -- & $<.05$ & $<.20$ & $<.20$ & -- & $<.05$ & -- & -- & $<.05$ & -- & -- & $<.05$ & $<.20$ & $<.20$ & \\
\hline & & $05 / 15 / 00$ & -- & $<.05$ & $<.05$ & -- & -- & .20 & $<.05$ & -- & -- & $<0.05$ & $<0.05$ & -- & -- & -- & -- & .26 & .05 & \\
\hline & & $07 / 25 / 00$ & -- & $<.05$ & $<.05$ & -- & -- & $<.05$ & $<.05$ & -- & -- & $<.05$ & $<.05$ & -- & $<0.05$ & $<0.05$ & -- & .07 & .06 & \\
\hline & & 09/11/00 & -- & $<.05$ & $<.05$ & -- & -- & $<.05$ & $<.05$ & -- & -- & $<.05$ & $<.05$ & -- & $<.05$ & $<.05$ & -- & $<.05$ & $<.05$ & \\
\hline & & $02 / 05 / 01$ & -- & $<.05$ & $<.05$ & -- & -- & $<.05$ & $<.05$ & -- & -- & $<.05$ & $<.05$ & -- & $<.05$ & $<.05$ & -- & .05 & $<.05$ & \\
\hline & & $07 / 30 / 01$ & -- & $<.05$ & $<.05$ & -- & -- & $<.05$ & $<.05$ & -- & -- & $<.05$ & $<.05$ & -- & $<.05$ & $<.05$ & -- & $<.05$ & $<.05$ & \\
\hline & & $01 / 28 / 02$ & -- & $<.05$ & $<.05$ & $<0.05$ & -- & $<.05$ & $<.05$ & $<0.05$ & -- & $<.05$ & $<.05$ & -- & $<.05$ & $<.05$ & -- & .05 & $<.05$ & \\
\hline \multirow[t]{4}{*}{04228900} & \multirow{4}{*}{$\begin{array}{l}\text { Springwater Creek } \\
\text { near Springwater, } \\
\text { NY }\end{array}$} & $07 / 09 / 97$ & $<.05$ & $<.20$ & $<.20$ & -- & $<.05$ & $<.20$ & $<.20$ & -- & -- & -- & -- & -- & -- & -- & .15 & .55 & .22 & \\
\hline & & $07 / 23 / 97$ & $<.05$ & $<.20$ & $<.20$ & -- & $<.05$ & $<.20$ & $<.20$ & -- & -- & -- & -- & -- & -- & -- & .05 & .40 & $<.20$ & \\
\hline & & $06 / 26 / 98$ & $<.05$ & $<.20$ & $<.20$ & -- & $<.05$ & $<.20$ & $<.20$ & -- & -- & -- & -- & -- & -- & -- & .95 & 6.0 & 4.3 & \\
\hline & & $07 / 16 / 98$ & $<.05$ & $<.20$ & $<.20$ & -- & $<.05$ & .32 & $<.20$ & -- & -- & -- & -- & -- & -- & -- & .18 & 1.4 & .59 & \\
\hline 01500628 & $\begin{array}{l}\text { Smokey Kill west of } \\
\text { Sidney Center, NY }\end{array}$ & $11 / 26 / 01$ & -- & $<.05$ & $<.05$ & $<.05$ & -- & $<.05$ & $<.05$ & $<.05$ & -- & $<.05$ & $<.05$ & -- & $<.05$ & $<.05$ & -- & $<.05$ & $<.05$ & \\
\hline 01374921 & $\begin{array}{c}\text { Stone Hill tributary at } \\
\text { Bedford Hills, NY }\end{array}$ & $06 / 27 / 01$ & -- & $<.05$ & $<.05$ & -- & -- & $<.05$ & $<.05$ & -- & -- & $<.05$ & $<.05$ & -- & $<.05$ & $<.05$ & -- & $<.05$ & $<.05$ & N \\
\hline
\end{tabular}


Table 6. Analytical results for selected acetamide herbicides and their degradation products, ethanesulfonic acid, oxanilic acid, and sulfinyl acetic acid, in surface-water samples, 1993-2003.-Continued

[OGRL, U.S. Geological Survey Organic Geochemistry Research Laboratory, Lawrence, Kansas; ESA, ethanesulfonic acid; OXA, oxanilic acid; SAA, sulfinyl acetic acid; --, no data; <, less than]

\begin{tabular}{|c|c|c|c|c|c|c|c|c|c|c|c|c|c|c|c|c|c|c|c|}
\hline \multirow[b]{2}{*}{ Site number } & \multirow[b]{2}{*}{ Site location } & \multirow[b]{2}{*}{$\begin{array}{c}\text { Date of } \\
\text { collection } \\
\text { (month/day/ } \\
\text { year) }\end{array}$} & \multicolumn{17}{|c|}{ Concentrations, in micrograms per liter } \\
\hline & & & $\begin{array}{l}\text { Aceto- } \\
\text { chlor } \\
(\mu \mathrm{g} / \mathrm{L})\end{array}$ & $\begin{array}{l}\text { Aceto- } \\
\text { chlor } \\
\text { ESA } \\
(\mu \mathrm{g} / \mathrm{L})\end{array}$ & $\begin{array}{l}\text { Aceto- } \\
\text { chlor } \\
\text { OXA } \\
(\mu \mathrm{g} / \mathrm{L})\end{array}$ & $\begin{array}{l}\text { Aceto- } \\
\text { chlor } \\
\text { SAA } \\
(\mu \mathrm{g} / \mathrm{L})\end{array}$ & $\begin{array}{l}\text { Ala- } \\
\text { chlor } \\
(\mu \mathrm{g} / \mathrm{L})\end{array}$ & $\begin{array}{l}\text { Ala- } \\
\text { chlor } \\
\text { ESA } \\
(\mu \mathrm{g} / \mathrm{L})\end{array}$ & $\begin{array}{l}\text { Ala- } \\
\text { chlor } \\
\text { OXA } \\
(\mu \mathrm{g} / \mathrm{L})\end{array}$ & $\begin{array}{l}\text { Ala- } \\
\text { chlor } \\
\text { SAA } \\
(\mu \mathrm{g} / \mathrm{L})\end{array}$ & $\begin{array}{c}\text { Di- } \\
\text { meth- } \\
\text { enamid } \\
(\mu \mathrm{g} / \mathrm{L})\end{array}$ & $\begin{array}{c}\text { Di- } \\
\text { meth- } \\
\text { enamid } \\
\text { ESA } \\
(\mu \mathrm{g} / \mathrm{L})\end{array}$ & $\begin{array}{c}\text { Di- } \\
\text { meth- } \\
\text { enamid } \\
0 X A \\
(\mu \mathrm{g} / \mathrm{L})\end{array}$ & $\begin{array}{c}\text { Flufen- } \\
\text { acet } \\
(\mu \mathrm{g} / \mathrm{L})\end{array}$ & $\begin{array}{l}\text { Flufen- } \\
\text { acet } \\
\text { ESA } \\
(\mu \mathrm{g} / \mathrm{L})\end{array}$ & $\begin{array}{l}\text { Flufen- } \\
\text { acet } \\
\text { OXA } \\
(\mu \mathrm{g} / \mathrm{L})\end{array}$ & $\begin{array}{l}\text { Meto- } \\
\text { lachlor } \\
(\mu \mathrm{g} / \mathrm{L})\end{array}$ & $\begin{array}{l}\text { Meto- } \\
\text { lachlor } \\
\text { ESA } \\
(\mu \mathrm{g} / \mathrm{L})\end{array}$ & $\begin{array}{c}\text { Meto- } \\
\text { lachlor } \\
\text { OXA } \\
(\mu \mathrm{g} / \mathrm{L})\end{array}$ \\
\hline \multicolumn{20}{|c|}{ Federal-State Cooperative Studies-Continued } \\
\hline \multicolumn{20}{|c|}{ New York hydrologic study (PPN)—Continued } \\
\hline 01503000 & $\begin{array}{l}\text { Susquehanna River at } \\
\text { Conklin, NY }\end{array}$ & $11 / 26 / 01$ & -- & $<0.05$ & $<0.05$ & $<0.05$ & -- & $<0.05$ & $<0.05$ & $<0.05$ & -- & $<0.05$ & $<0.05$ & -- & $<0.05$ & $<0.05$ & -- & 0.30 & $<0.05$ \\
\hline 01500500 & $\begin{array}{l}\text { Susquehanna River at } \\
\text { Unadilla, NY }\end{array}$ & $11 / 26 / 01$ & -- & $<.05$ & $<.05$ & $<.05$ & -- & .06 & $<.05$ & $<.05$ & -- & $<.05$ & $<.05$ & -- & $<.05$ & $<.05$ & -- & .16 & $<.05$ \\
\hline \multirow[t]{4}{*}{0423403010} & \multirow{4}{*}{$\begin{array}{l}\text { Taughannock Creek } \\
\text { near Taughannack } \\
\text { Falls, NY }\end{array}$} & $06 / 11 / 98$ & $<0.05$ & $<.20$ & $<.20$ & -- & $<0.05$ & $<.20$ & $<.20$ & -- & -- & -- & -- & -- & -- & -- & 0.05 & 1.7 & .28 \\
\hline & & 07/05/98 & $<.05$ & $<.20$ & $<.20$ & -- & $<.05$ & $<.20$ & $<.20$ & -- & -- & -- & -- & -- & -- & -- & .12 & $<.20$ & $<.20$ \\
\hline & & $07 / 08 / 98$ & $<.05$ & $<.20$ & $<.20$ & -- & $<.05$ & .62 & $<.20$ & -- & -- & -- & -- & -- & -- & -- & $<.05$ & 2.0 & .49 \\
\hline & & 07/09/98 & $<.05$ & $<.20$ & $<.20$ & -- & $<.05$ & 1.3 & $<.20$ & -- & -- & -- & -- & -- & -- & -- & .13 & 1.9 & .66 \\
\hline 04218000 & $\begin{array}{l}\text { Tonawanda Creek at } \\
\text { Rapids, NY }\end{array}$ & $06 / 27 / 01$ & -- & $<.05$ & $<.05$ & -- & -- & .08 & $<.05$ & -- & -- & $<.05$ & $<.05$ & -- & $<.05$ & $<.05$ & -- & .31 & .10 \\
\hline \multirow[t]{7}{*}{01421618} & \multirow{7}{*}{$\begin{array}{l}\text { Town Brook southeast } \\
\text { of Hobart, NY }\end{array}$} & $01 / 24 / 99$ & $<.05$ & $<.20$ & $<.20$ & -- & $<.05$ & $<.20$ & $<.20$ & -- & -- & -- & -- & -- & -- & -- & $<.05$ & .36 & $<.20$ \\
\hline & & $05 / 25 / 99$ & $<.05$ & $<.20$ & $<.20$ & -- & $<.05$ & $<.20$ & $<.20$ & -- & $<0.05$ & -- & -- & -- & -- & -- & .08 & .25 & $<.20$ \\
\hline & & 06/09/99 & $<.05$ & $<.20$ & $<.20$ & -- & $<.05$ & $<.20$ & $<.20$ & -- & $<.05$ & -- & -- & -- & -- & -- & $<.05$ & .49 & $<.20$ \\
\hline & & $06 / 15 / 99$ & $<.05$ & $<.20$ & $<.20$ & -- & $<.05$ & $<.20$ & $<.20$ & -- & $<.05$ & -- & -- & -- & -- & -- & $<.05$ & .54 & $<.20$ \\
\hline & & $06 / 28 / 99$ & $<.05$ & $<.20$ & $<.20$ & -- & $<.05$ & $<.20$ & $<.20$ & -- & $<.05$ & -- & -- & -- & -- & -- & $<.05$ & .34 & $<.20$ \\
\hline & & 06/29/99 & $<.05$ & $<.20$ & $<.20$ & -- & $<.05$ & $<.20$ & $<.20$ & -- & $<.05$ & -- & -- & -- & -- & -- & $<.05$ & .44 & .22 \\
\hline & & 06/30/99 & $<.05$ & $<.20$ & $<.20$ & -- & $<.05$ & $<.20$ & $<.20$ & -- & $<.05$ & -- & -- & -- & -- & -- & $<.05$ & .54 & $<.20$ \\
\hline \multirow[t]{5}{*}{01421618} & \multirow{5}{*}{$\begin{array}{l}\text { Town Brook southeast } \\
\text { of Hobart, NY }\end{array}$} & 07/02/99 & $<.05$ & $<.20$ & $<.20$ & -- & $<.05$ & $<.20$ & $<.20$ & -- & $<.05$ & -- & -- & $<0.05$ & -- & -- & $<.05$ & .56 & $<.20$ \\
\hline & & 07/03/99 & $<.05$ & $<.05$ & $<.05$ & -- & $<.05$ & $<.05$ & $<.05$ & -- & $<.05$ & -- & -- & $<.05$ & -- & -- & .09 & .48 & .28 \\
\hline & & 07/04/99 & $<.05$ & $<.20$ & $<.20$ & -- & $<.05$ & $<.20$ & $<.20$ & -- & $<.05$ & -- & -- & $<.05$ & -- & -- & .10 & .34 & .20 \\
\hline & & 07/06/99 & $<.05$ & .25 & $<.20$ & -- & $<.05$ & $<.20$ & $<.20$ & -- & $<.05$ & -- & -- & $<.05$ & -- & -- & .11 & .46 & $<.20$ \\
\hline & & 07/07/99 & $<.05$ & $<.05$ & $<.05$ & -- & $<.05$ & $<.05$ & $<.05$ & -- & $<.05$ & -- & -- & $<.05$ & -- & -- & .09 & .93 & .52 \\
\hline 01500832 & $\begin{array}{l}\text { Unadilla River at } \\
\text { Miller's Mill, NY }\end{array}$ & $11 / 26 / 01$ & -- & $<.05$ & $<.05$ & $<.05$ & -- & $<.05$ & $<.05$ & $<.05$ & -- & $<.05$ & $<.05$ & -- & $<.05$ & $<.05$ & -- & .44 & $<.05$ \\
\hline 01502500 & $\begin{array}{c}\text { Unadilla River at } \\
\text { Rockdale, NY }\end{array}$ & $11 / 26 / 01$ & -- & $<.05$ & $<.05$ & $<.05$ & -- & $<.05$ & $<.05$ & $<.05$ & -- & $<.05$ & $<.05$ & -- & $<.05$ & $<.05$ & -- & .47 & .11 \\
\hline
\end{tabular}


Table 6. Analytical results for selected acetamide herbicides and their degradation products, ethanesulfonic acid, oxanilic acid, and sulfinyl acetic acid, in surface-water samples, 1993-2003.-Continued

[OGRL, U.S. Geological Survey Organic Geochemistry Research Laboratory, Lawrence, Kansas; ESA, ethanesulfonic acid; OXA, oxanilic acid; SAA, sulfinyl acetic acid; --, no data; <, less than]

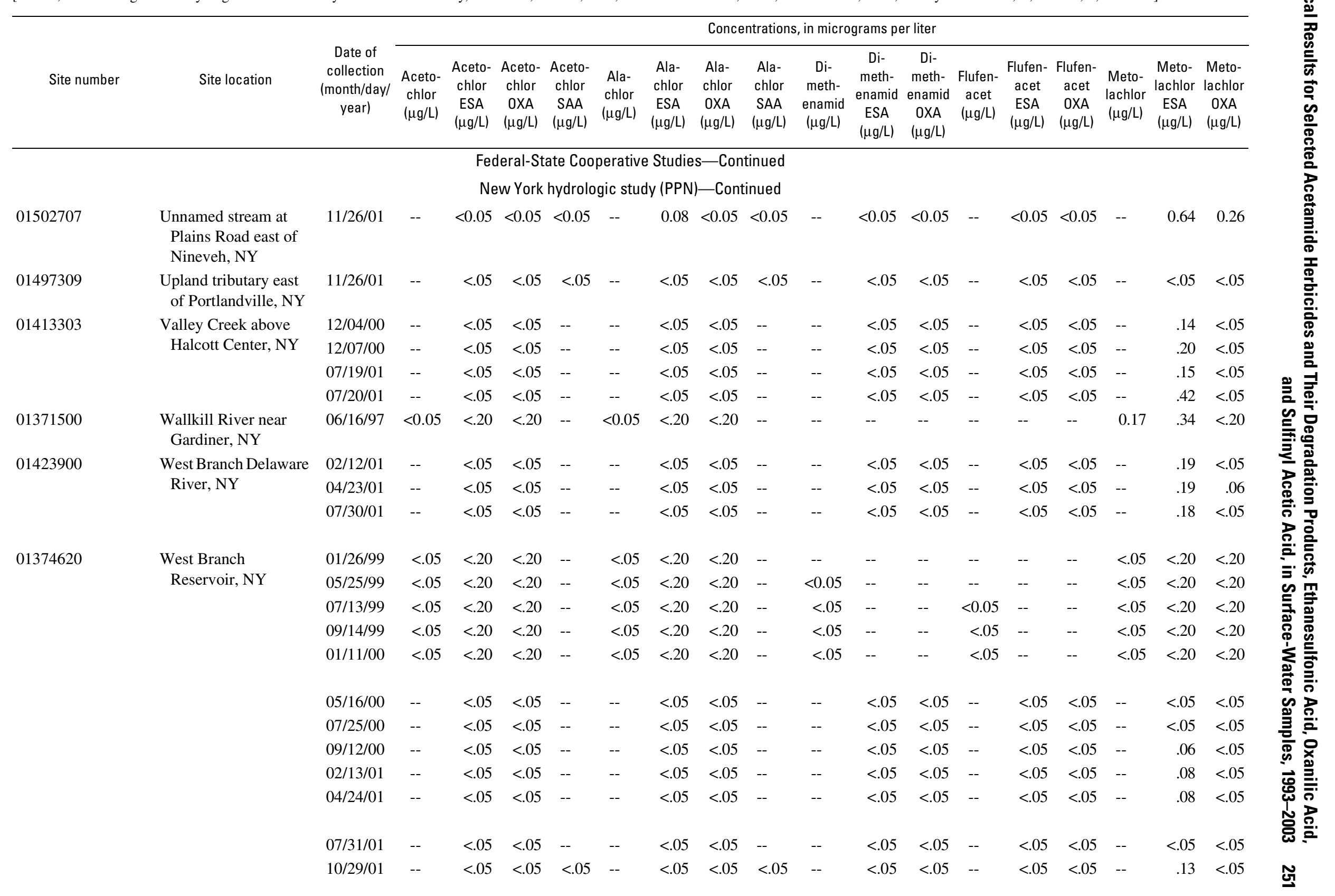


Table 6. Analytical results for selected acetamide herbicides and their degradation products, ethanesulfonic acid, oxanilic acid, and sulfinyl acetic acid, in surface-water samples, 1993-2003.-Continued

[OGRL, U.S. Geological Survey Organic Geochemistry Research Laboratory, Lawrence, Kansas; ESA, ethanesulfonic acid; OXA, oxanilic acid; SAA, sulfinyl acetic acid; --, no data; <, less than]

\begin{tabular}{|c|c|c|c|c|c|c|c|c|c|c|c|c|c|c|c|c|c|c|c|}
\hline \multirow[b]{2}{*}{ Site number } & \multirow[b]{2}{*}{ Site location } & \multirow[b]{2}{*}{$\begin{array}{c}\text { Date of } \\
\text { collection } \\
\text { (month/day/ } \\
\text { year) }\end{array}$} & \multicolumn{17}{|c|}{ Concentrations, in micrograms per liter } \\
\hline & & & $\begin{array}{c}\text { Aceto- } \\
\text { chlor } \\
(\mu \mathrm{g} / \mathrm{L})\end{array}$ & $\begin{array}{l}\text { Aceto- } \\
\text { chlor } \\
\text { ESA } \\
(\mu \mathrm{g} / \mathrm{L})\end{array}$ & $\begin{array}{l}\text { Aceto- } \\
\text { chlor } \\
\text { OXA } \\
(\mu \mathrm{g} / \mathrm{L})\end{array}$ & $\begin{array}{l}\text { Aceto- } \\
\text { chlor } \\
\text { SAA } \\
(\mu \mathrm{g} / \mathrm{L})\end{array}$ & $\begin{array}{l}\text { Ala- } \\
\text { chlor } \\
(\mu \mathrm{g} / \mathrm{L})\end{array}$ & $\begin{array}{l}\text { Ala- } \\
\text { chlor } \\
\text { ESA } \\
(\mu \mathrm{g} / \mathrm{L})\end{array}$ & $\begin{array}{c}\text { Ala- } \\
\text { chlor } \\
\text { OXA } \\
(\mu \mathrm{g} / \mathrm{L})\end{array}$ & $\begin{array}{l}\text { Ala- } \\
\text { chlor } \\
\text { SAA } \\
(\mu \mathrm{g} / \mathrm{L})\end{array}$ & $\begin{array}{l}\text { Di- } \\
\text { meth- } \\
\text { enamid } \\
(\mu \mathrm{g} / \mathrm{L})\end{array}$ & $\begin{array}{c}\text { Di- } \\
\text { meth- } \\
\text { enamid } \\
\text { ESA } \\
(\mu \mathrm{g} / \mathrm{L})\end{array}$ & $\begin{array}{c}\text { Di- } \\
\text { meth- } \\
\text { enamid } \\
\text { OXA } \\
(\mu \mathrm{g} / \mathrm{L})\end{array}$ & $\begin{array}{c}\text { Flufen- } \\
\text { acet } \\
(\mu \mathrm{g} / \mathrm{L})\end{array}$ & $\begin{array}{c}\text { Flufen- } \\
\text { acet } \\
\text { ESA } \\
(\mu \mathrm{g} / \mathrm{L})\end{array}$ & $\begin{array}{c}\text { Flufen- } \\
\text { acet } \\
\text { OXA } \\
(\mu \mathrm{g} / \mathrm{L})\end{array}$ & $\begin{array}{l}\text { Meto- } \\
\text { lachlor } \\
(\mu \mathrm{g} / \mathrm{L})\end{array}$ & $\begin{array}{l}\text { Meto- } \\
\text { lachlor } \\
\text { ESA } \\
(\mu \mathrm{g} / \mathrm{L})\end{array}$ & $\begin{array}{c}\text { Meto- } \\
\text { lachlor } \\
\text { OXA } \\
(\mu \mathrm{g} / \mathrm{L})\end{array}$ \\
\hline \multicolumn{20}{|c|}{ Federal-State Cooperative Studies-Continued } \\
\hline \multirow{6}{*}{01374620} & \multirow{5}{*}{$\begin{array}{l}\text { West Branch } \\
\text { Reservoir, NY }\end{array}$} & & & & w York & hydrolo & gic stud & y (PPN & 1-Cont & inued & & & & & & & & & \\
\hline & & $10 / 30 / 01$ & -- & $<0.05$ & $<0.05$ & $<0.05$ & -- & $<0.05$ & $<0.05$ & $<0.05$ & -- & $<0.05$ & $<0.05$ & -- & $<0.05$ & $<0.05$ & -- & $<0.05$ & $<0.05$ \\
\hline & & $05 / 14 / 02$ & -- & $<.05$ & $<.05$ & $<.05$ & -- & $<.05$ & $<.05$ & $<.05$ & -- & $<.05$ & $<.05$ & -- & $<.05$ & $<.05$ & -- & .06 & $<.05$ \\
\hline & & 07/16/02 & -- & $<.05$ & $<.05$ & $<.05$ & -- & $<.05$ & $<.05$ & $<.05$ & -- & $<.05$ & $<.05$ & -- & $<.05$ & $<.05$ & -- & .09 & $<.05$ \\
\hline & & $07 / 22 / 02$ & -- & $<.05$ & $<.05$ & $<.05$ & -- & $<.05$ & $<.05$ & $<.05$ & -- & $<.05$ & $<.05$ & -- & $<.05$ & $<.05$ & -- & .17 & .10 \\
\hline & \multirow{4}{*}{$\begin{array}{r}\text { Yawger Creek near } \\
\text { Cayuga Lake, NY }\end{array}$} & $10 / 22 / 02$ & -- & $<.05$ & $<.05$ & $<.05$ & -- & $<.05$ & $<.05$ & $<.05$ & -- & $<.05$ & $<.05$ & -- & $<.05$ & $<.05$ & -- & .05 & $<.05$ \\
\hline \multirow[t]{3}{*}{04234060} & & 06/11/98 & $<0.05$ & $<.20$ & $<.20$ & -- & $<0.05$ & $<.20$ & $<.20$ & -- & -- & -- & -- & -- & -- & -- & 0.08 & 1.7 & $<.05$ \\
\hline & & 06/17/98 & $<.05$ & $<.20$ & $<.20$ & -- & .28 & $<.20$ & $<.20$ & -- & -- & -- & -- & -- & -- & -- & 13 & 7.5 & 6.1 \\
\hline & & 06/18/98 & $<.05$ & $<.20$ & $<.20$ & -- & $<.05$ & $<.20$ & $<.20$ & -- & -- & -- & -- & -- & -- & -- & 10 & 20 & 17 \\
\hline
\end{tabular}

